
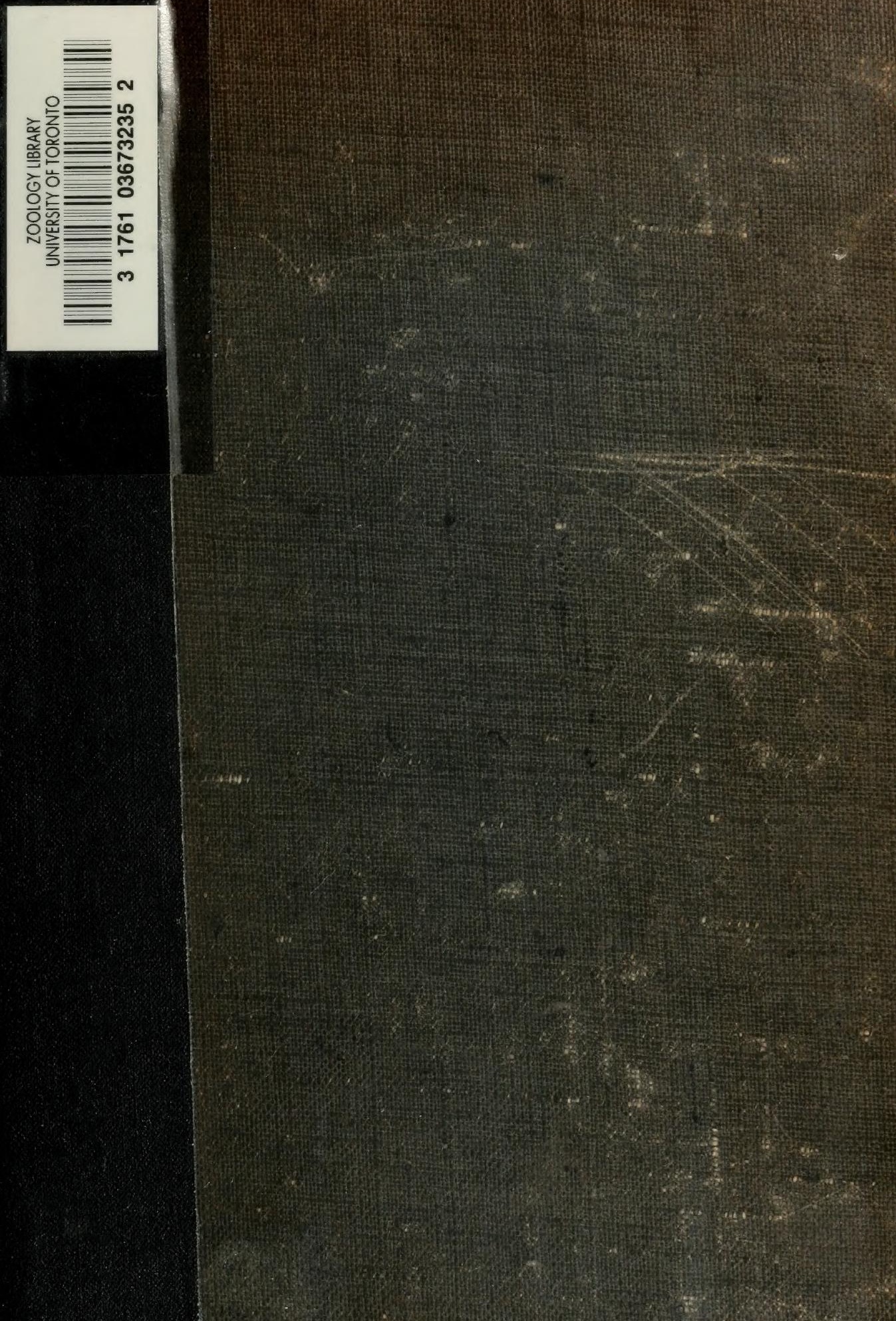

DEPGRTAENT OF ZOOLOCY

LBRARY

$$
\begin{aligned}
& 9 L \\
& 627 \\
& y \\
& 2001 \\
& \text { bol. } 4
\end{aligned}
$$


Digitized by the Internet Archive in 2010 with funding from University of Toronto 


\section{SMTTHSONIAN INSTITUTION.}

UNITED STATES NATIONAL MUSEUM.

\section{BULLETIN}

OF THE

\section{UNITED STATES NATIONAL MUSEUM.}

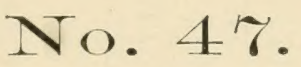

\section{THE FISHES}

OF

\section{NORTH AND MIDDLE AMERICA:}

A DESCRIPTIVE CATALOGUE OF THE SPFCIES OF FISH-LIKE VERTEBRATES FOUND IN THE WATERS OF NORTH AMERICA, NORTH OF THE ISTHMUS OF PANAMA.

PY

DAVID STARR JORDAN, Ph. D.,

PRESIDENT OF THE LELAND STANFORD JUNIOR UNIVERSITY AND OF THE

CATIFORNIA ACADEMY OF SCIENCES,

AND

BARTON WARREN EVERMANN, Ph. D, ICHTHYOLOGIST OF THE UNITED STATES FISH COMMISION.

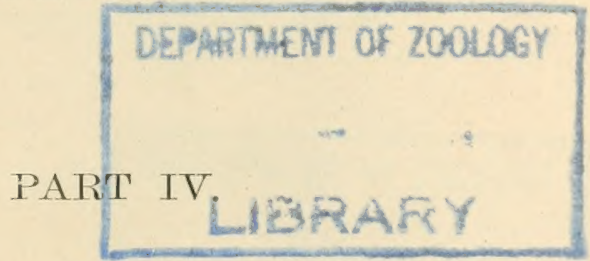

WASHINGTON :

GOVIRNMENT PRINTING GFICE.

1900 . 



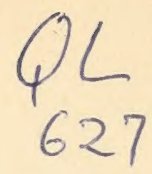

SMITHSONIAN INSTITUTION. UNITED STATES NATIONAL MUSEUM.

\section{THE FISHES}

$\mathrm{OF}$

\section{NORTH AND MIDDLE AMERICA:}

\section{A DESCRIPTIVE CATALOGUE}

OF THE

SPECIES OF FISH-LIKE VERTEBRATES FOUND IN THE WATERS OF NORTH AMERICA, NORTH OF THE ISTHMUS OF PANAMA.

$\mathrm{BY}$

DAVID STARR JORDAN, PH. D.,

PRESIDENT OF THE LELAND STANFORD JUNIOR UNIVERSITY AND OF THE CALIFORNIA ACADEMY OG SCIENCES,

AND

BARTON WARREN EVERMANN, PH. D., ICHTHYOLOGIST OF THE UNITED STATFS FISH COMMISSION.

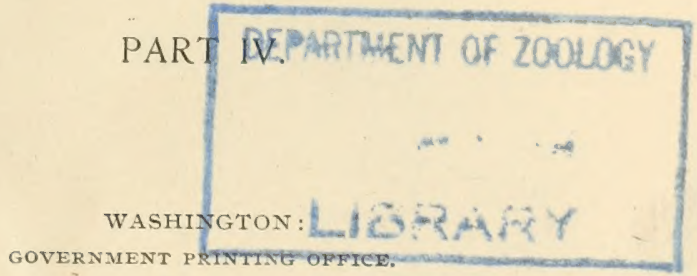

I 900 . 
LIBRARY

OCT 191993

UViversity of iukUN" 


\section{PREFACE.}

This volume is fourth and last of a descriptive catalogue of the fishes and fish-like vertebrates of North and Middle America. Part I, Branchiostomatidx to Priacanthidix inclusive (pp. 1 to 1240), was published October 3, 1896; Part II, Lutianidx to Cephalicanthidx inclusive (pp. 1241 to 2183), was published October 3, 1898; Part III, Callionymidx to Ogcocephalidx inclusive, including also "Addenda," an "Artificial Key to the Families of the True Fishes," a "Glossary of Technical Terms," and an "Index" complete for Parts I, II, and III (pp. 2183a to 3136), was published November 28, 1898; and Part IV, the present volume, appears on June 26, 1900.

Since the publication of Part III, investigations by Dr. Jordan, in Mexico, and by Dr. Fvermann, in Puerto Rico, have added greatly to our knowledge of the fishes of those regions, and have made it desirable to incorporate in the present volume still further addenda.

Recent studies by Dr. Günther, of certain Linnæan types, show that some changes in nomenclature are necessary, which are also included.

David Starr Jordan.

Barton Warren Evermans.

LELAND STANFord JUNTOR UNIVERSTT, Palo alto, santa Clara County, Cahforya.

Wareh 15, 1900. 



\section{A STSEMATIC ARRATGEMENT OF THE FISHES OF IORTH ANI IIIDDLE ANERICA.}

In these pages the authors exhibit. as clearly as the method will permit, their present views as to the genetic relations of the fishes and fish-like rertebrates of North and Middle America. This list is, in fact, a 'Table of Contents complete for the four volumes and corrected to include the Addenda (pp. 2745-2873 of Part III and pp. 3137-3197 of PartI Y). From this Systematic Arrangement it is seen that the fish fauna of North and Middle America, as now understood by the present authors, embraces 3 classes, 30 orders, 225 families, 1113 genera, 325 subgenera, 3263 species, and 133 subspecies.

Fumily Birenchirstumide.

Genus Branchiostoma, Costa................................... 3

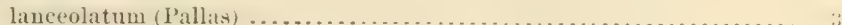

caribrum, Sundevall.......................................

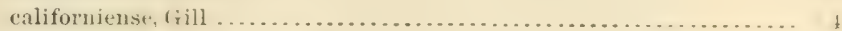

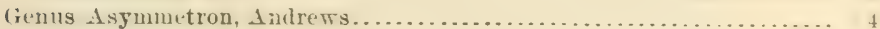

lucayanum. Antrews ..................................... 4

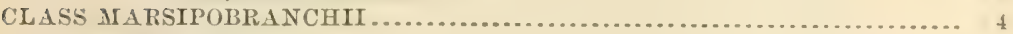

ORder HYPERTTIETI . . . . . . . . . . . . .

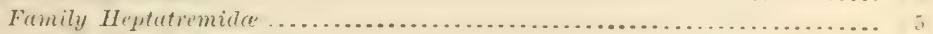

Grenus I'olistotrema, Gill....................................... it

stonti (Lockington) ........................................... i

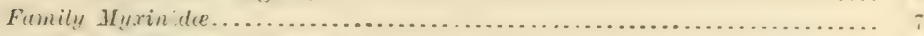

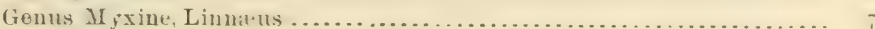

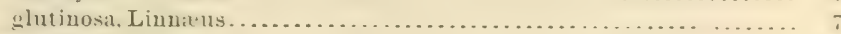

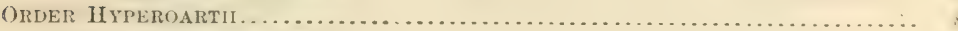

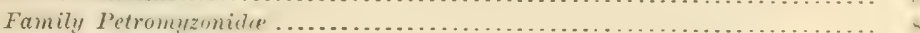

Genus Bathymyzon, Gill..................................... y

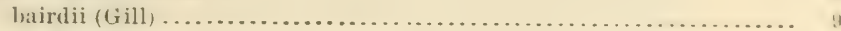

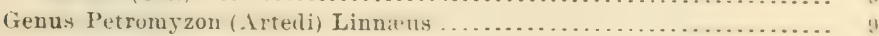

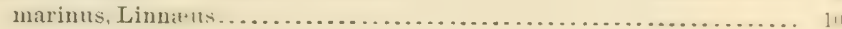

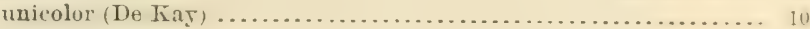

Genus Ichthyomyzon, Girard.................................... 10

coneolor (Kirtlanh) ..................................... 11

castaneus, Girard .......................................... 11

Genus Entosphenus, Gill ...................................... 11

tridentatus (Gatidner) ..................................

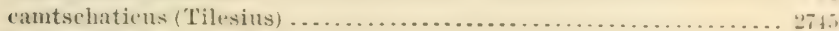

Genus Lampetra, Gray ....................................... 12

aurea (Bean) ................................................ 13

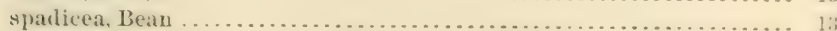

cibaria (Girard) ............................................. 13

wilderi, Gage ..........................................

CLASS PISCES .......................................................

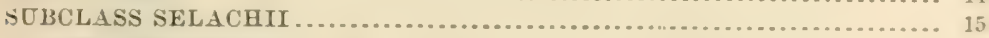

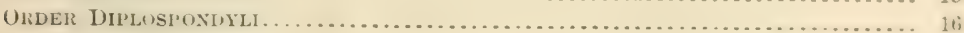

Family Chlamydoselachide ........................................ 16

Genus Chlamydoselachus, Garman ............................ 16

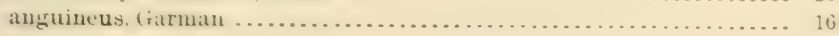


CLASS PISCES-Contimeil.

SIIJBCLA.SS SELACHII-Continned.

O)

Family Hexunchide ......................................... 17

Genus Notorlynchus, lyreg.................................. in

пан

fiemu Hexanehus, latinesque............................. In

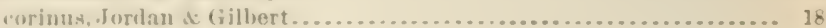

"risens (Gmelin)............................................ 19

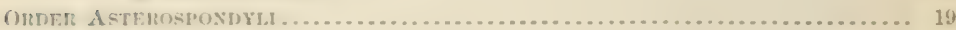

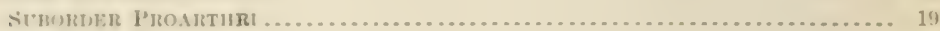

Fumily Heterodontide.......................................... 19

Genus Gyropleurolus, Gill .................................... 20

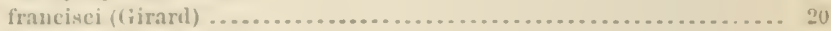

quovi (Frúminville)........................................ 21

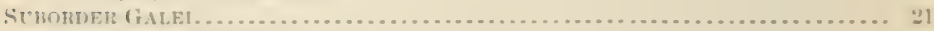

Family, senlliorninile ........................................

Genus seytliorhinus, Blainville ............................. 22 profumdorum, Gomle of Bean ............................... 22

Genus Catulus, Smith ......................................... 23

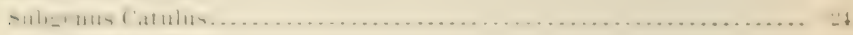

samiurus, (rilhert ........................................ 24

brunens, Gilbert ......................................... 24

cephalus, (rilbert........................................... 24

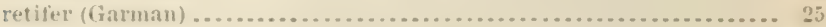

sulgemus Cephalosegllium, Gill................................ 25

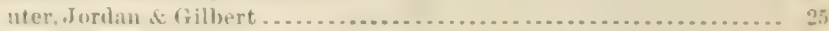

Family Ginglymogtomide ..................................... 25

Genus Ginglymostuma, Miiller of Henle ......................... 26

rirratum (Gmeliu) ..................................... 20

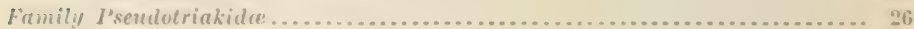

Genus l'seudotriakis, Capello ..................................

microllon, Capello .........................................

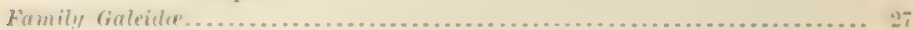

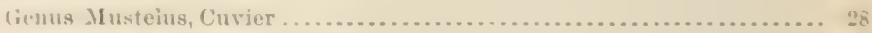

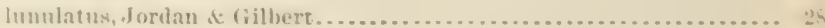

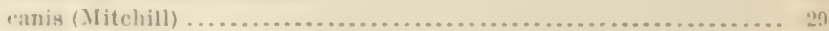

Genus Galent, liatimesque ....................................

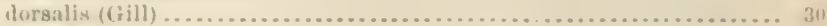

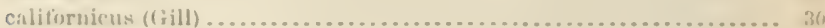

(ienus lihinotriacis, (iill...................................... 30

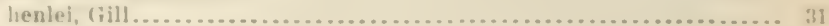

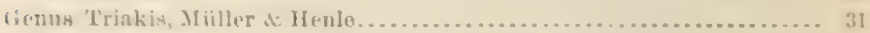

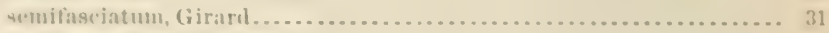

fienus (inleorhinus, lilainvillo................................ 31

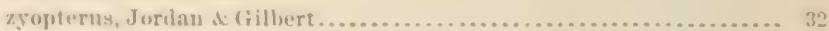

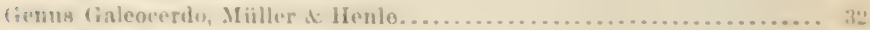
tigrinu, Miiller of Hınlo ................................. 32

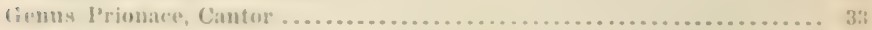

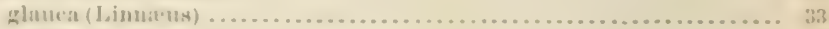

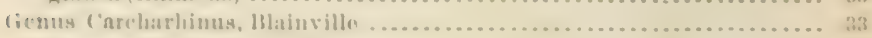

Subgenus Platy jumlon, (iill.................................. 35

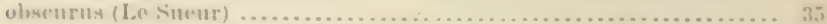

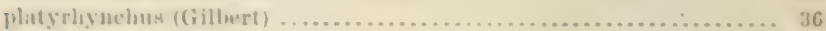

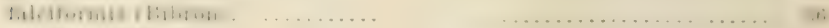

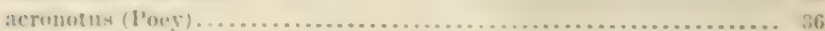

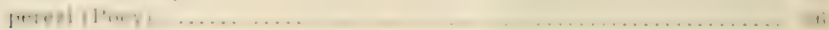


CLASS PISCES-Contiuued.

SU BCLASS SELACHII-Continued.

Order Asterospondyli-Continued.

SUBOKDER GAIEI-Continued.

Family Galeida-Continued.

Genus Carcharhinus, Blainville-Continued.

Subgenus Platypodon, Gill-Continued.

remotus (Valenciennes) . .................................... 37

henlei (Valeuciennes) ........................................ 37

cerdale, Gilbert ........................................... 2746

Subgenus Carcharhinus ....................................... $3 \pi$

milberti (Miiller \& Henle) ..................................... 37

lamiella (Jordan \& Gilbert) .................................. 37

lamia (Rafinesque) ............................................ 38

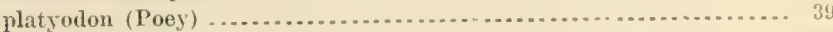

fronto (Jordan \& Gilbert) ................................ 39

nicaraguensis (Gill \& Bransford) ............................

Subgenus Isogompholon, Gill . ............................... 40

rethalorus (Jordan \& Gilbert) .................................. 40

limbatus (Mïller \& Henle) .................................... 40

oxyrhynchus (Müller \& Henle) ............................... 40

velox, Gilbert.......................................... 2747

Genus Hypoprion, Miiller \& Henle.............................. 41

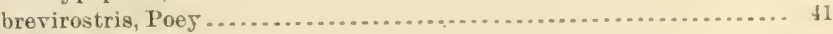

signatus, Poey ...........................................

Genus Aprionodon, Gill .........................................

isodon (Miiller \& Henle) ................................... 4 ?

Genus Scoliodon, Müller \& Henle............................... 42

longurio (Jordan \& Filbert) ................................ 42

terræ-novi (Richardson) ................................. 43

Family sphyrnide................................................

Genus Sphyrna, Rafinesque ....................................

Subgenus Reniceps, Gill ....................................... 4

tiburo (Linnaus) .............................................. 44

Subgenus Platysqualus, Swainson.............................. tudes (Cuvier) ..........................................

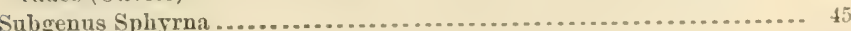

zygæna (Linnreus) ......................................... 45

Family Alopiide ............................................... 45

Genus Alopias, Rafinesque.................................. 45

vulpes (Gmelin) ............................................. 45

Family Carchariide ................................................. 40

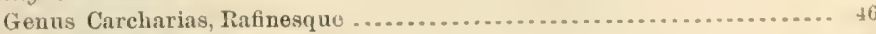

Subgenus Eugomphodus, Gill .................................... 46

littoralis (Mitchill) ...................................

Family Lamnide................................................. th

Genus Isurus, Rafinesque...................................... t7

Subgenus Isuropsis, Gill .................................... to to

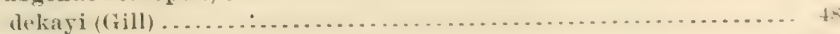

Subgenus Isurus ............................................... 48

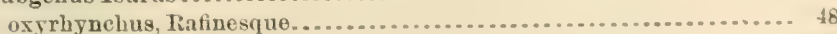

Genus Lamna, Curier...........................................

cornubica (Gmelin)..........................................

Genus Carcharodon, Smith........................................ 50

carcharias (Linnatus) ........................................ 50

Family Cetorhinide ............................................ 50

Genus Cetorhiuus, Blainville ................................. 51

maximus (Gunner) 
CIASS JISCES-Continned. SITBCLASS SELACIII-Continmed.

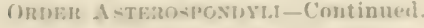

Sirsunturar (iALEI-C'ontinurel.

Family Rhinotontide........................................... 5 ?

firnus Micristodus fill ...................................... 52

prunctatus, fill ............................................

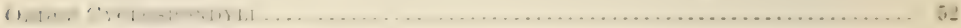

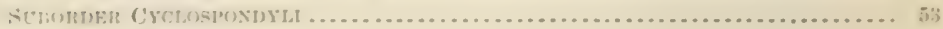

Family siqualide ................................................ 58

1ienus siqualus (Artedi) Linuatus................................. 53

acanthias, Linneus ........................................ 54

sucklii (Girarll) ......................................... 54

fierus C'entroscymuns. Bocage s. Capello.......................... 5

crilolepis, Bocage of Capello ................................ 55

Ginus Etmopterus, liatinesıuo ............................... 55 musillus (Luwe) ........................................... is

Genus Centroserllium, Müller \& Henle.............................. 56

falbricii (leinharlt) .................................... 56

Family Dalatide ................................................... 56

Genus Somniosus, Le Sueur .................................. 56

mierocephalus (Bloch) ...................................... 57

Family Echinurhinidle ............................................... it

Genus Echinorhinus, Blainville ............................... 5i

spinosus (Gmelin)...................................... 58

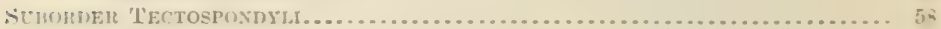

Family siruatinider............................................ 58

Genus sंquatıa, Duméril......................................... ss

squatina (Linnteus) ............................................ 5 t

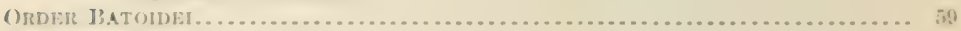

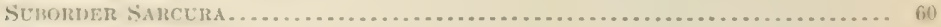

Family l'ristida ................................................... Gu

Genns l'ristis, Latham.................................... 60 zephyreus, Jordan \& Starks .............................60; 2749 pectinatus, Latham ....................................... 60

Family lihinebatide ........................................... 61

Genus Jhinobatus, Bloch $s$ Schweiler........................... 61

lentiginosus, Garman ...................................... 6"

stellio, Jorklan \& Rutter................................... 2750

flaucostigma, Jorlan of Gilbert.............................. 62

Jencorhynchus, Gïnther .................................... 62

productus, $A$ yres . . ...................................... 63

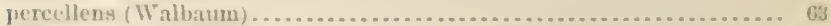

spiusus, (iinther..........................................

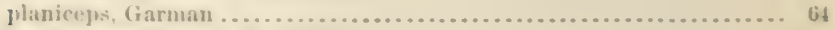

Genns Zapteryx, Jordan \& Gilbert............................... Gt

exasperatus (Jordan \& Gilbert) ............................ ob

xyster, Jorlan d Evermann ............................. 65

ficuns I'latyrhinwilis, Garman..................................

trimeriatus (Jorkan of (iblhert) ............................. 65

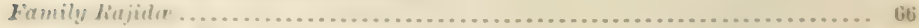

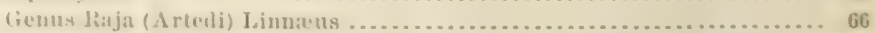

rrin:4en, Mtehill ........................................ 68

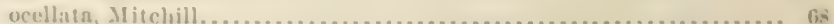

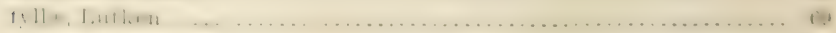

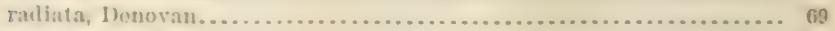

pluturia, Garman........................................ 60

ackleyi, Garman ........................................... in

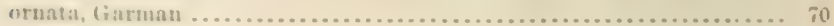


CLASS PISCES-Continued.

SUBCLASS SELACHII-Continued.

Page.

Order BatoIDEI-Continued.

SUBORDER SARCURA-Continued.

Family Rajida-Continued.

Genus Raja (Artedi) Linnzeus-Continued.

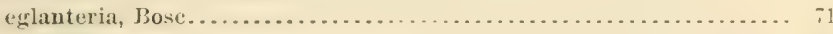

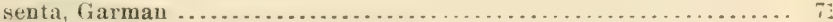

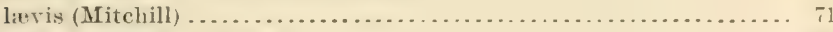

rlina, Jordan of filluert . . . . . . . . . . . . . . . . . .

binoculata, Girart ................................

inornata, Jordan \& Gilbert............................ 73

equatorialis, Jordan of Jollman ....................... it

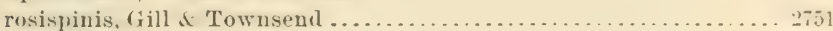

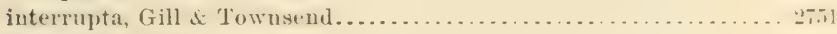

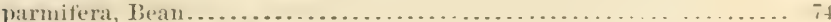

stellulata, Jorlau of (iilbert.............................

alentica, (illbert . . . . . . . . . . . . . . . . . . . . . . . . .

trachiıra. Gilbert........................................ 75

abyssicola, Gilhert ...........................

Family Varcobatide . . . . . . . . . . . . . . . . . . . . . . . . . . . . . . .

(itenus Tetronarce, Gill . . . . . . . . . . . . . . . . . . . . . .

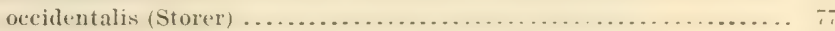

californica $($ I yres) . . . . . . . . . . . . . . . . . . . . . . . . . .

Genus Narcine, Henle. . . . . . . . . . . . . . . . . . . . . . . .

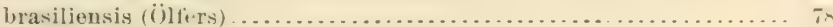

eutemedor, Jordan \& Starks ............................... 2751

Genus Discopyge, Tschudi.................................... to

ommata, Jordan $\&$ Gilbert ..................................

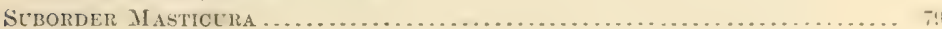

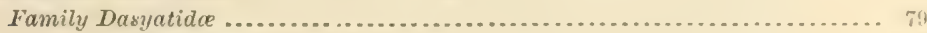

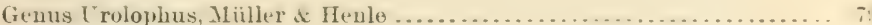

halleri, Cooper ............................................. 80

nebulusus, Garman. . . . . . . . . . . . . . . . . . . . . . . . . . .

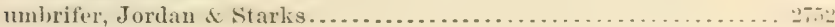

jamaicensis (Cuvier) ...................................... 81

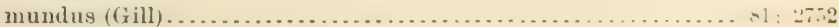

goolei. Jurtan of Bollman ................................

aspidurus, Jordan \& Gilbert ............ . . . . . . . . . . . . . . . . . . 81

rogersi, Jordai \& Starks . . . . . . . . . . . . . . . . . . . . . . . . . . . . 275.

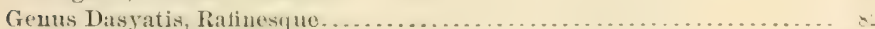

Subgenus Hemitrygon, Müller \& Hevle........................ 83

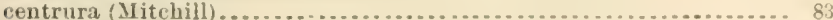

Subgenus Dasyatis..............................

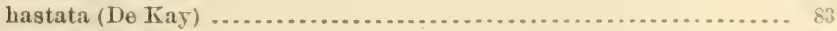

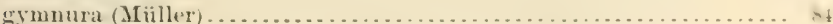

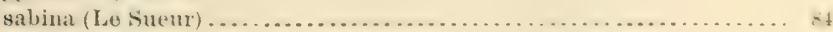

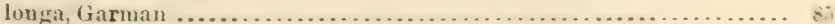

dinterura, Jordan \& Gillert. ...........................

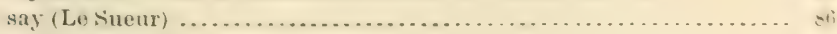

Genus I'toroplatea, Miiller st Menle ............................... 80

maclura (Le Suetr) ........................................ 80

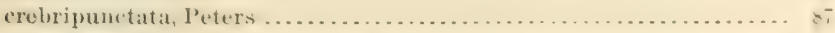

marmorata, Couper. . . . . . . . . . . . . . . . . . . .

ravil, Jordan \& Starkis . . . . . . . . . . . . . . . . . . . . . . .

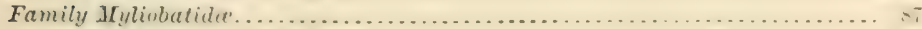

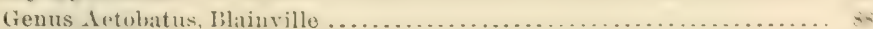

narinari (Euphrisen) ......................... 
CLASS PISCHE-Cimtinneri.

SLIBCLASS SELACHLL-Contimed.

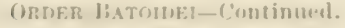

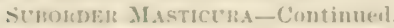

Family Myliwbatile-Continued.

(ienus Myliohatis. 1) méril .................................... 89

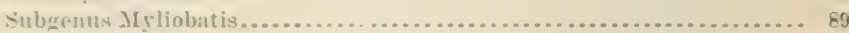

ireminvillei, I.0. Nuenr................................. 89

sulgenus Holorhinus, Gill ................................. 89

ealifurnisus, (iill ......................................... 89

asperrimms, (jilbert .......................................... 2754

quollei, Garman ........................................... 2755

Genus Lihinoptera, Kúnl..................................... 90

subgenus lihinoptera ........................................ 9. 90

honasus (Mitchill) ..................................... 90

steindachneri, Evermann \& Jenkins ......................... 91

Subgenus Jicromesus, Gill .................................. 91

ellsenadie, Losa Smith ..................................... 91

Fumily dodontidw. ..................................... $91 ; 2750$

(ienus Aodon, Lacépède ........................................ 91

hypostomus (Bancroft) ...................................... 92

Genus Ceratobatis, Boulenger................................. 2756 rolertsii, Boulenger..................................... 2750

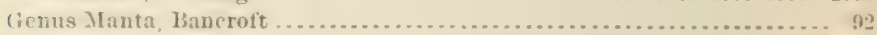

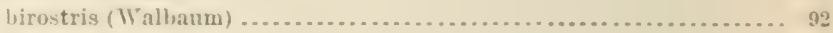

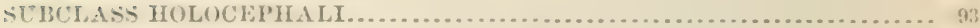

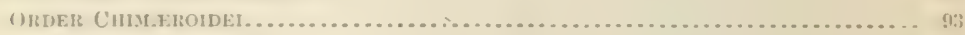

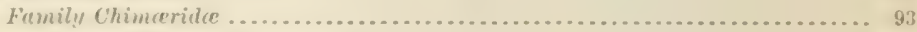

(ienus C'himiera. Linneus ...................................... 94

munstrosa, Linnats ...................................... 98

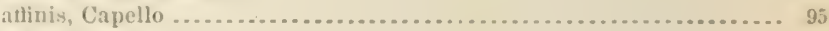

Genus Hydrolagus, Gill.......................................... 95

colliei (Lay it I lenvett) ...................................... 95

(ienus Harriotta, Goode \& Bean................................. 96

raleighana, Goole of Bean .................................... 96

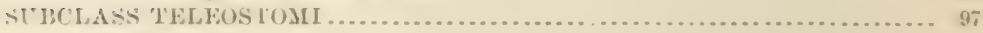

series dianoilei ..................................................... 101

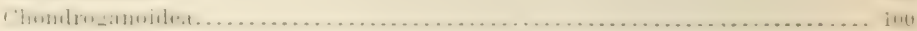

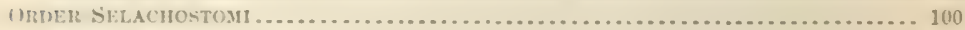

Frenily Polyodontide ............................................... 101

Genus P'olyodon, Lacépède....................................... 101

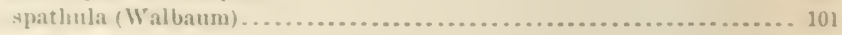

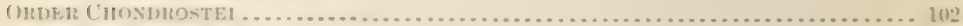

Fumily . teipenseride ............................................ 102

Genus Acipenser, Linneus...................................... 103

transmontanus, lichardson ............................... 104

medirustris, A yres ......................................... 10 s

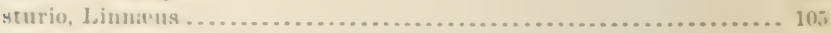

rubieunlus, Le Suenr .................................... 106

hrevirostrum, Lu Sueur.................................... 106

(ienus Seaphirhynehus, Ireclied.................................. 107

flatorynchus (liatinesųut) ................................. 10

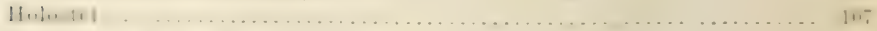

()

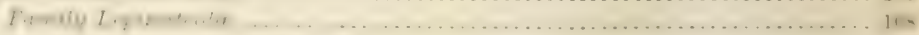

(ienns Lepianstus, Laccipicio.................................. 109

suligenus Leppisesteus....................................... 10

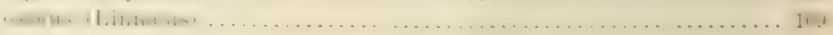


CLASS PISCES-Continued.

SUBCLASS TELEOSTOMI_Continued.

ORDER RHOMBOGaxoIDEA-Continued.

Family Lepisosteide-Continued.

Genus Lepisosteus, Lacépè̀le-Continued.

Subgenus Cylindrosteus, Rafinesfiue........................... 110

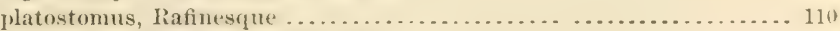

Subgenus Atractosteus, Rafinesque............................ 111

tristrechus (Bloch it sichneider) ............................ 111

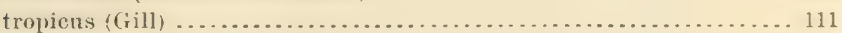

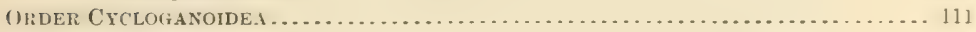

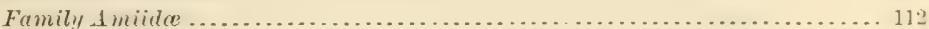

Genus Amia, Linnaus ..................................... 112

calva, Linnitus......................................... 113

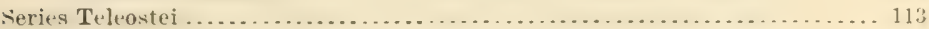

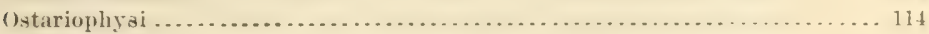

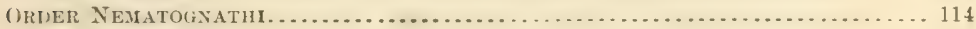

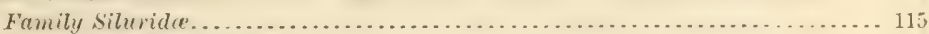

Genus Felichthęs, swainsun ................................. 116

panamensis (Gill) ............................................. 117

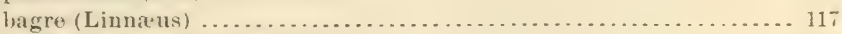

pinnimaculatus (steindaclıner) ............................. 117

exdouxii (Cuvier \& Valenciennes) ........................... 11 .

filamentosus, swainson ................................. 118

felis (Linnæus) .......................................... 118; 3197

bahiensis (Castelnan) ...................................... 11s

Genus Galeichthys, Cuvier \& Valenciennes..................... 122; 2770

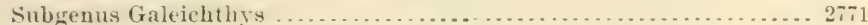

lentiginosus (Figenmann \& Eigenmann) ................... 122; 2771

peruanus, Lütken .................................. 122; 271

Subgeuus Hexanematichthys, Bleeker .................... 128; 2772

milberti (Cuvier \& Valenciennes) ......................... 128; 3197

seemanni (Günther) ..................................... 128; 2772

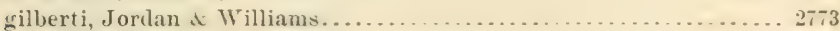

jordani (Eigenmanu \& Eigenmann)...................... 128; 2774

azureus, Jordan \& Williams ................................ 2775

('ierulescens (Gïnther) ............................... 129:

xenauchen, Gilbert........................................ 2777

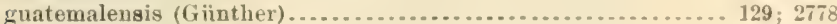

surinamensis (Bleeker) .................................. 129; 2780

dasyeephalus (Giinther) ............................. 130; $27=0$

longiceplualus (Eigenmann \& Eigeumann) ................. 130; 2781

rugispinis (Curier \& Valencienues) ......................... 130; 2781

labiatus (Boulenger) . . . . . . . . . . . . . . . . . . . . . . . . . . 31:it

phrvgiatus (Cuvier \& Valenciennes) ..................... 130; 2782

dienus Seiadeichtlys, bleektr.............................. 122 troscheli (Gill) .......................................... 122

emphrsetus (Miiller \& Troschel) .............................. 122

temminckianus (Cuvier $\&$ Valenciennes) . ....................... 122

flavescens (Cuvier \& Talenciennes) ............................... 123

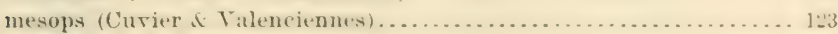

proops (Cuvier \& Valenciennes) ........................... 12:?

passany (Cuvier ot Valentiennes) . . . . . . . . . . . . . . . . . 12t

albieans (f'uvier \& Valeneiennes) . . . . . . . . . . . . . . . . . . .

Genus Aspistor, Jordan \& Evermann............................... 2763

luniscutis (Cuvier \& Valenciennes)....................... 125; 2763

Genus Selenaspis, Bleeker ..................................... 124

herzbergii (Bloch) ............................................ 124 
CLASS JPSCF-COntinn: SUBCLASS TELFOSTOMI--Continuen?

OnLE: Nimatorisatu-Contimenel.

Family silurilu-Continuel.

Genus Selenaspis, Blecker-Continuerl. dowi (fijll)

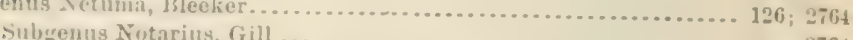

subgenus Notarius, Gill .................................... 2764

\&randicassis (Cuvier \& Valenciennes) . .................... 126; 2701

stricticassis (Cuvier \& Valenciennes) . . . . . . . . . . . . . . . 126; 2765

Subenus Netuma ....................................... 2765

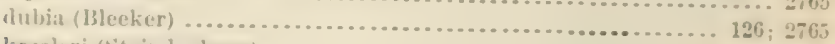

kessleri (Steindacluer) ................................ 127; 2705

inseulpta (Jordan \& Gilbert) ............................ 127; 2765

Hlanicens (Stuindachner)............................. 127; 2760

platypogon (Giinther) ................................. 127; 2760

nscula (Jortan \& Gilbert)............................... 127;

elattura (Jorlan of Gilbert) . ............................ 128; 2769

insularum, Flora Hartley Greene........................... 2770

(ienuts 'Tanhysurus, Lacépede.............................. 131; 278?

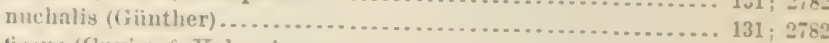

fisuns (Cuvicr \& Talenciennes) ........................ 131; 278

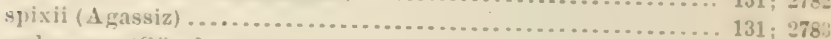

melanopus (Giinther) ................................... 132; 2784

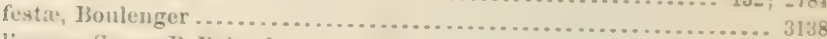

liropus, Susan B. Bristol ....................................... 278

('mumelawe, Gilbert ............................................

furthii (Steindachuer) . ................................ 132; 2788

variolosus (Cuvier \& Valenciennes) .......................... 132; 278s

multiridiatus (Giinther) .............................. 132; 2788

Genus Cathorops, Jordan \& Gilbert ........................... 133; 2788

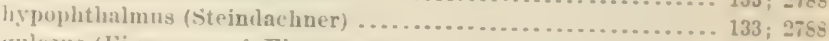

„ulosus (Eigenmann \&. Eigenmann) ........................... 133

Genus Ictalurus, IRatinesque............................. 133; 2788

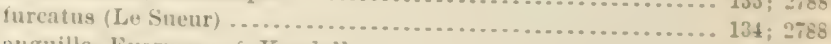

anguilla, Evermamn of Kentall ............................... 2788

punctatus (liatinesque) …........................... 134; 9780

meridlionalis (Gïnther) ......................................

fimnt Istarius, Jordan of Snyder................................. 3138

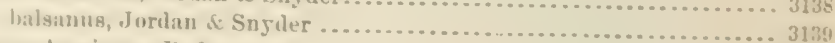

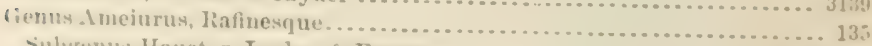

¿ulugenus Haustor, Jordan \& Erermanu ..................... 13i; 3197

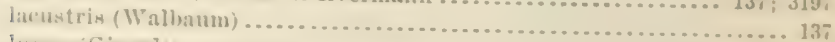

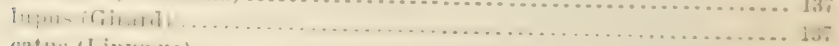

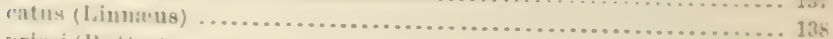

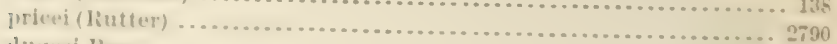

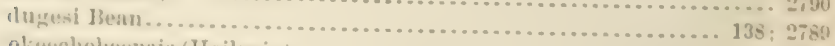

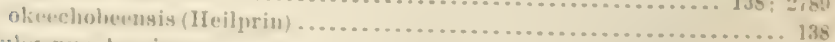

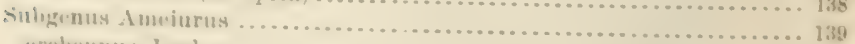

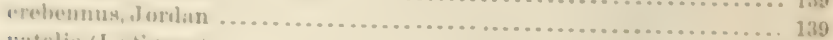

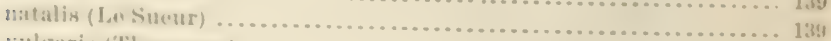

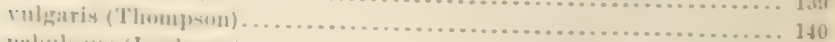

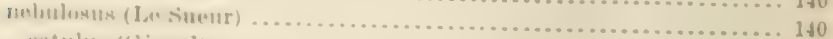

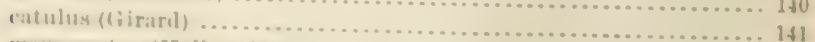

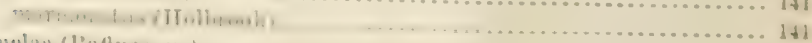

melaq (lintlnem

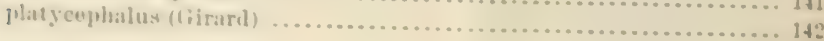


SUBCLASS TELEOSTOMI-COntinued.

Order Neyatognathi-Continued.

Family Silurida-Continued.

Genus Ameiurus, Rafinesque-Continued.

Subgenus Gronias, Cope ..................................... 142

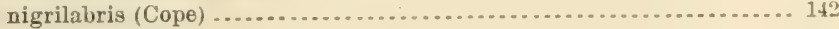

Genus Leptops, Rafinesque ....................................... 14

olivaris (Rafinesque) . . ........................................ 143

Genus Noturus, Rafinesque...................................... 143

flavus, Rafinesque............................................ 144

Genus Schilbeodes, Bleeker...................................... It

Subgenus Schilbeodes ....................................... 140

घyrinus (Mitchill) . . . . . . . . . . . . . . . . . . . . . . . . . . 110

leptacanthus (Jordan) ..................................... 140

Subgenus Rabida, Jordan \& Erernann :......................... 146

nocturnus (Jordan \& Gilbert) ................................... 146

funebris (Gilbert \& Swain) ................................ 147

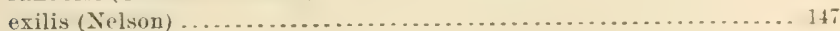

insignis (Richardson) ....................................... 147

gilberti (Jordan \& Erermann) .................................. 148

eleutherus (Jordan\} ...................................... 143

miurus (Jorlan) ...........................................

furiosus (Jordan \& MTeek) ..................................... 149

Genus Rhamelia, Bleeker....................................... 149

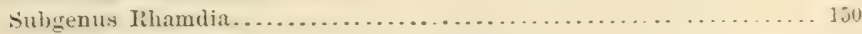

wagneri (Günther) .............................................. 150

Subgenus Rhamitella, Eigenmann \& Eigenmann ................... 151

baronis-mulleri (Troschel) .................................... 151

motaguensis (Günther) ...................................... 151

brachyptera (Cope) ............................................ 151

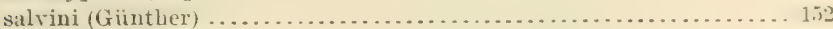

hrpselurus (Günther).................................... 15"

laticauda (Heckel) . . . . . . . . . . . . . . . . . . . . . . . . . . .

godmani (Güntler) . ........................................... 152

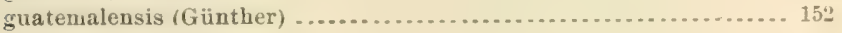

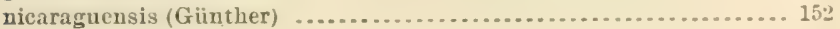

microptera (Giinther) ........................................ 153

managuensis (Günther) ..................................... 153

polycaulus (Günther) ........................................... 153

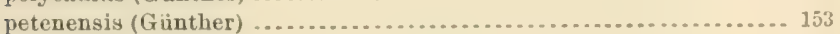

parryi (Eigenmann) ....................................... 153

Genus Pimelodella, Eigenmann \& Eigenmann ...................... 153

chagresi (Steindithner) ................................... 154

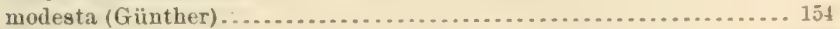

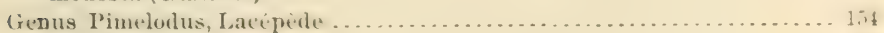

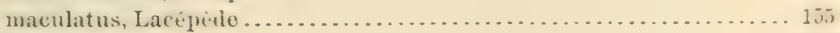

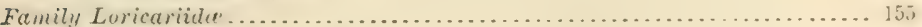

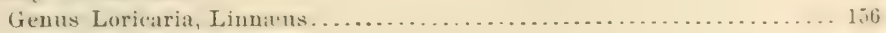

Subgenus Hemiodon, Kner.................................

panamensis, Eigenmann \& Eigenmann ...................... 15i

Subgenus Sturisoma, Swainson ............................. 157

rostrata, spix .........................................

Subgenus Riueloricaria, Bleeker.............................. 158

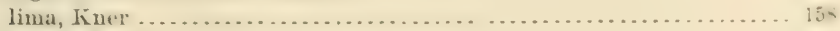

bransfordi. (iill .........................................

Subgenus Parahemiodon, Bleeker............................. 15s

uracantha, Kiner \& Steindacluner............................. 158 
('MASS I'ISCES-Contimme日. SLIBCY.ASS TELAOST()MI-Continmenl.

(H:LER Ni:vatugNatu-Contimuerl.

Family Loricariele-Continued.

(iеnus Loriearia, Linnams-Continuen.

Sub.

varienata, Steimlachner .................................. 159

fienus II -miancistru, Blevker ............................... 159

unacharote (Cuvier d Valenereunes) ......................... 159

aspldolepis (Günther) ...................................... 159

(iмnus Chartostomus, Kner ................................... 160

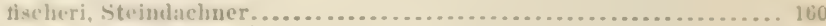

(ivnus Ancistrus, Kner .................................... 16u

chagresi, Eigenmann of Eigemuann........................ 160

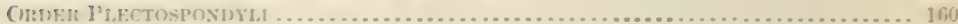

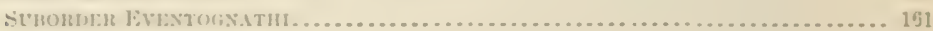

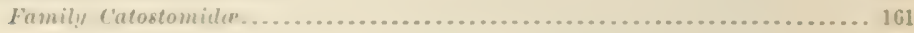

(ienus Ictiolı, Jatineąุuอ..................................... 168

Subgenus Sclerognathus, Cuvier $\&$ Valeneienues................... 163

cyprinella (Cuvier \& Valenciennes) ............................ 163

Sulgenus Ietiubus ....................................... 16t

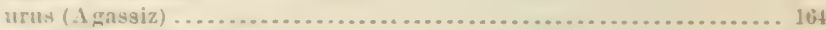

meridlonalis (Gïnther) .................................... 164

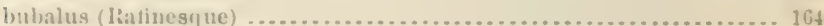

Genus Carpiodes, Kalinesgue .................................... 105

carpio (latinesifue) ......................................... 160

thtlormis, Copte............................................ 166

thom jesoni, .1 massiz ............................................ 10

velifer (latlizesque) ........................................ 10

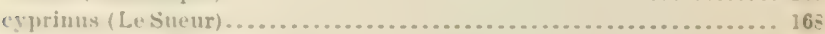

Genus Cyeleptus, IRatinesque................................. 168

(Hongatus (L.e Sueur) ....................................... $100^{\circ}$

Gents I'untustens, Cope ....................................... 109

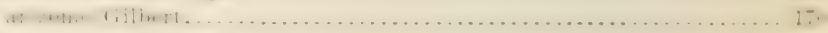

generosus (lirard) ...................................... 170

plebeins (Bairol of Girard) ................................... 170

delphinus (Cope) . ............................................... 171

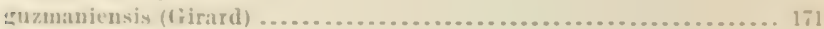

jordani, Evermanu........................................... 171

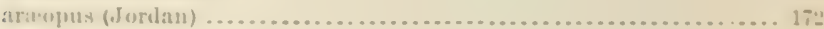

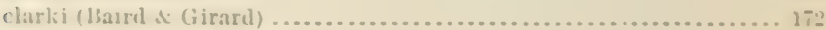

lienus Cintostomus, Lo Sueur .................................. 173

Sulogrnus Catostomus ......................................... 174

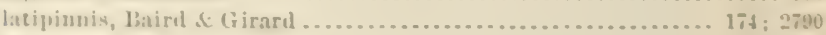

лiмcobulus, Cope .................................... 175;

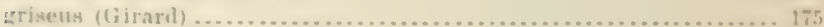

retropinnis, Jurdin ........................................

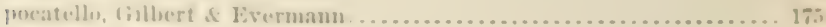

catestemus (Furater) a.

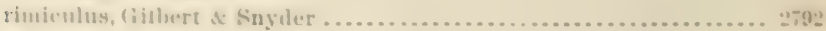

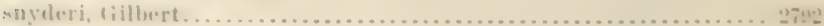

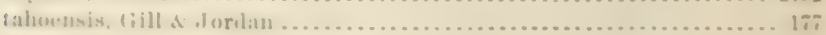

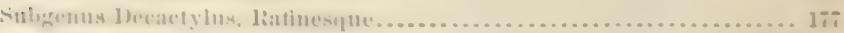

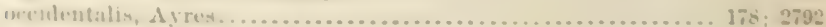

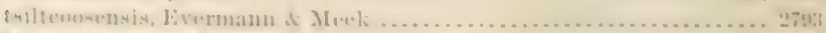
tел-

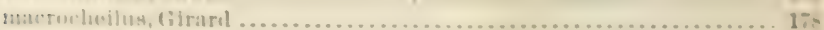

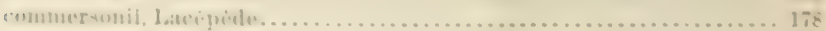

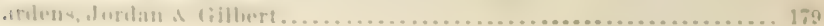


CLASS PISCES-Continued.

SUBCLASS TELEOSTOMI-Continuer.

Order Plectospondyli-Continued.

Suborder Eventognathi-Continued.

Fanily Catostomido-Continued.

Genus Catostomus, Le Sueur-Continued.

Subgenus Decactrylus, Rafinesque-Continued.

gila, Kirsch ......................................... 18

insignis, Baird \& Girard . . . . ................................. 180

Subgenus Hypentelium, Rafinesque . . . . . . . . . . . . . . . . . . . . . . . 181

nigricans, Le Sueur ............................................ 181

rhothocus, Thoburn ........................................ 181

Genus Deltistes, Seale......................................... 2794

luxatus (Cope) ......................................... 183; 2794

Genus Chasmistes ............................................... 182

fecundus (Cope of Yarrow) .............................. 180; 2794

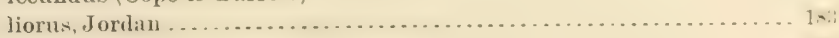

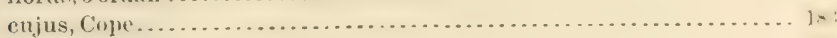

brevirostris, Cope ......................................... 183

stomias, Gilbert .......................................... $27 q_{4}$

copei, Evermann \& Meek ................................... 2795

Genus Xyrauchen, Eigenmann \& Kirsch........... . . . . . . . . . . . . . . . 18

cypho (Lockington) . . . . . . . . . . . . . . . ........................ 181

uncompahgre, Jordan \& Evermann ............................ 184

Genus Erimyzon, Jorian........................................... 185

sucetta (Lacépède) ........................................... 185

oblongus (Mitehill) ....................................... 186

Genus Minytrema, Jordan ............................................... 180

melanops (Ratinesquc) ....................................... 187

Genus Moxostoma, Rafinesque ................................... 187

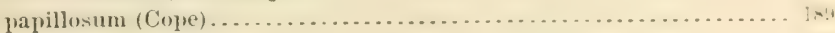

anisurum (Rafinesque) ...................................... 190

collapsum (Cope) ............................................... 190

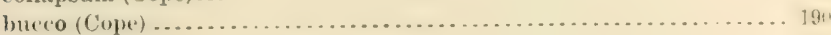

pidiense (Cope) ............................................. 191

coregonus (Cope) ............................................. 191

album (Cope) ................................................. 191

thalassinum $(\mathrm{Cope})$. . . . . . . . . . . .............................. 191

congestum (Baird \& Girard) ..................................... 19?

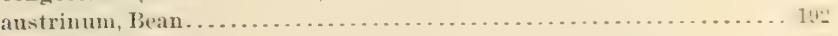

aureolum (Le Sueur) ........................................ 19:

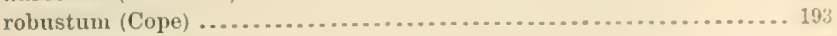

macrolepidotum (Le Sueur) ................................ 193

crassilabre (Cope) ................................................ 194

lesneuri (Richarlson) ................................... 144

breviceps (Cope) .............................................. 196

conus $($ Cope $)$................................................... 196

pœcilurum (Jordan) ........................................... 196

rupiscartes, Jorklan \& Jenkins . . . . . . . . . . . . . . . . . . . . . . . . . . . 196

cervinum (Cope) .......................................... 197

Genus Placopharynx, Cope.................................... 197 duquesnii (Le Sueur) .................................... 198

Genus Lagochila, Jordan of Brajton .............................. 19s

lacera, Jordan \& Brayton . . . . . . . . . . . . . . . . . . . . . . . . . . . . . . . 199

Family Cyprinide ................................................ 199

Gonus Campostoma, A gussiz................................... 204

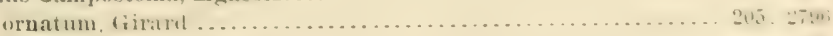

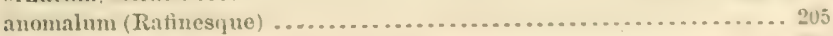

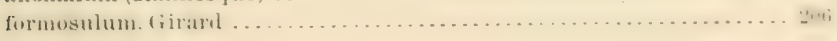




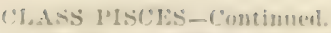

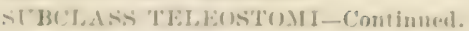

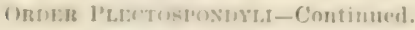

stmomat Fivistorisatu-Continuel.

Family C'yprinide-Continuell.

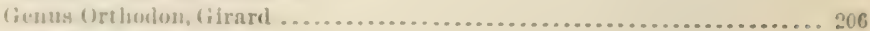

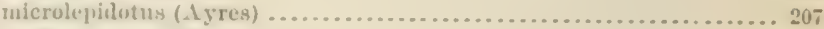

(ienus (1xygeneum, Forbes...................................... 207

pulverulentum, Forbeg .................................. 207

(iemus Actocheilus, $A$ gassiz .................................. 20 .

alutuerus, $A$ gagsiz \& Pickering................................ 208

Genus Lavinia, Girard ....................................... 208

exilicaula, Baird \& Girard .................................... 209

(ientu Clurosomus, laatinesque ................................... 209

1.rythrogaster, liatinesque................................. 209

eos (Cone) ................................................... 210

llakotersis, Evermann of Cox ................................ 210

oreas, Cine............................................... 211

Gums Algansen, Girard ...................................... 211

tincella (Cuvier .t. Valenciennes) .............................. 211

lacustris, steindachner................................... 3140

farasc orum, Steindachner . ................................. 270.

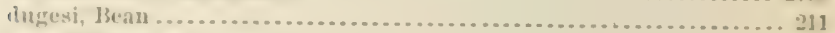

sallari (Gïnther) ............................................ 212

fienus Hybognathus, Agassiz................................... 212

sulggenus Hybognathus ........................................ 213

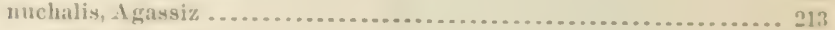

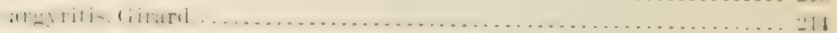

hay i, Jorklan ................................................ 214

subgenus 1)ionla, Girard ..................................... 214

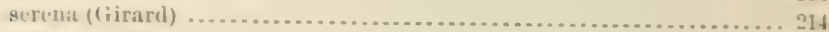

cpiscolna (Giraril) . .......................................... 214

nubila (Forlues) .............................................. 215

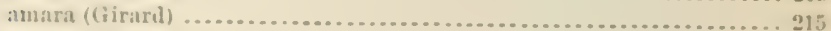

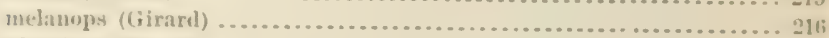

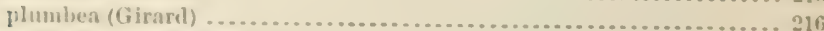

Genus P'ineplates, Ratinesque ................................... 216

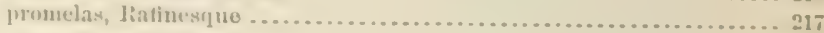

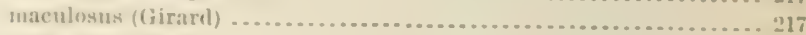

vonfertus (Giraril) .......................................... 217

notatus (liatinesque) ....................................... 218

(ienus Mylerpharodon, 1 yres ................................... 218

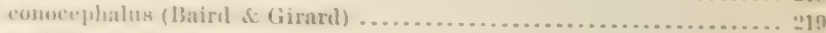

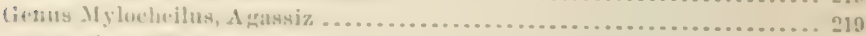

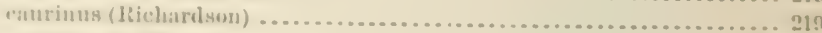

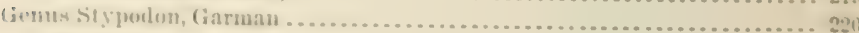

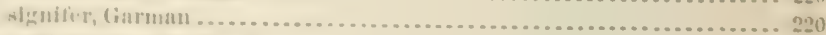

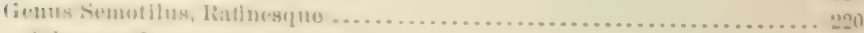

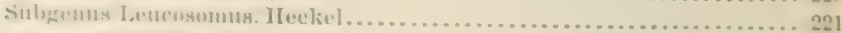

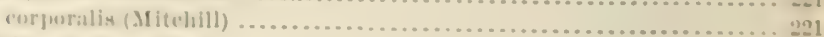

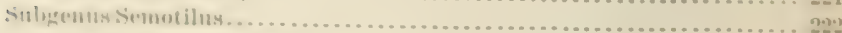

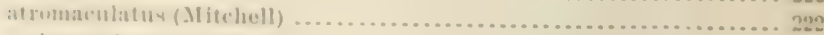

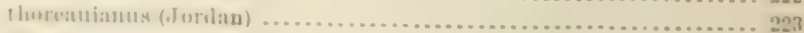

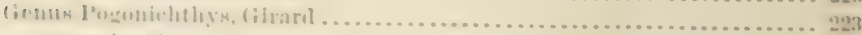

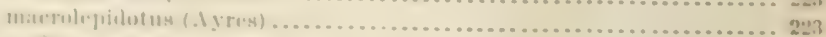

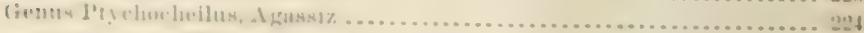

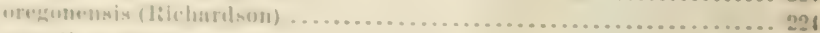

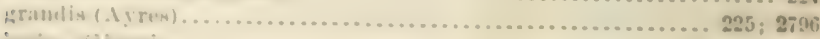

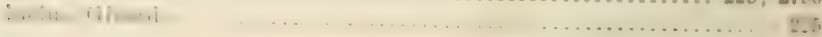


CLASS PISCES-Continued.

SUBCLASS TELEOSTOMI-Continued.

Order Plectospondyli-Continuer.

SUborder EveNTOGNathi-Continued.

Family Cyprinida-Continuel.

Genus Gila, Baird \& Girard

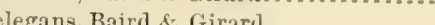

.

seminuda, Cope \& Yarrow . ..................................... 228

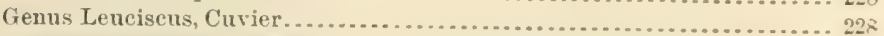

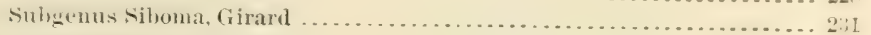

crassicanda (Baird \& Girard) . . . . . . . . . . . . . . . . . . . . . . . . 231

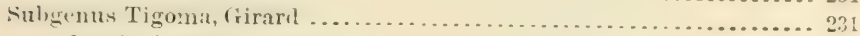

couformis (Baird \& Girard) . ................................ 231

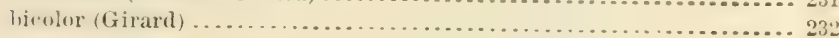

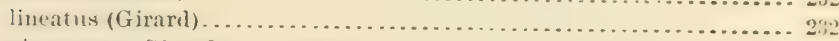

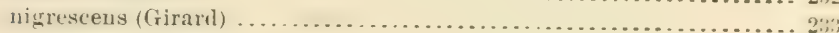

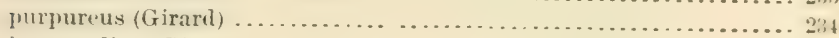

intermedius (Girard) ......................................... 235

niger (Cope) ................................................ 235

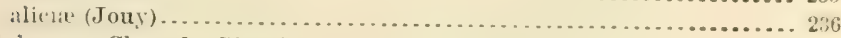

Subgenus Cheonda, Girari..................................... 236

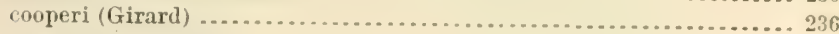

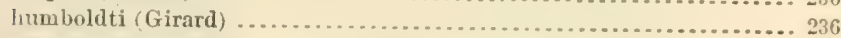

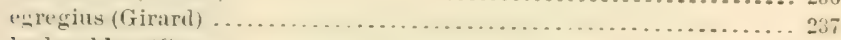

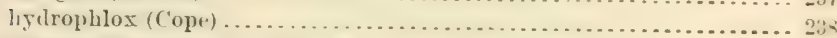

Sulgemus Richardsmius, firard . . . . . . . . . . . . . . . . . . . .

balteatus (Richardson) ....................................... 238

sinslawi, Evermann d Meek .............................. 2797

Subgenus Clinostomus, Girard ................................... 239

vandoisulus, Cuvier $\&$ Valenciennes . . . . . . . . . . . . . . . . . . . . . 239

elongatus (Kirtland) ........................................ 240

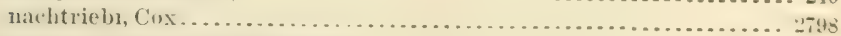

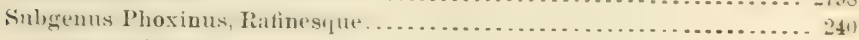

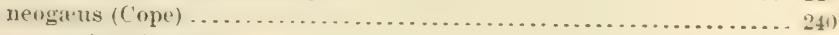

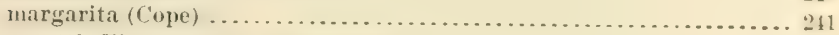

orcutti (Eigenmann \& Eigenmann) .......................... 241

Subgenus Hemitrenia, C'ope .............................. 212

milnerianus (Cope) .......................................... 242

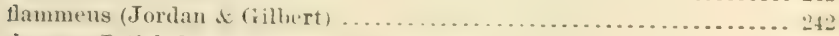

Subgenus Iotichthys, Jorkan \& Evermann .......................... 243

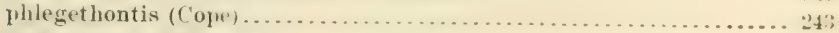

Genus liutilus, Ia tinesune..................................... 213

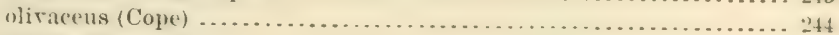

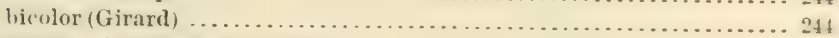

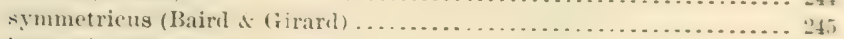

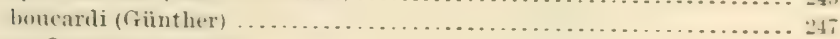

Genus Opsopœorlus, Hay ......................................... 247

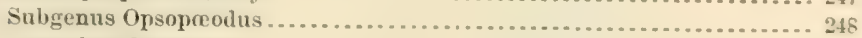

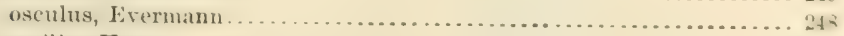

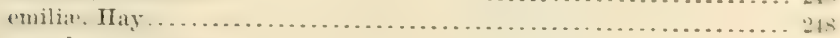

megalops (Forbes) ....................................... 248

Subgenus Opsopœa, Jorkan of Evermann . . . . . . . . . . . . . . . . . . 249

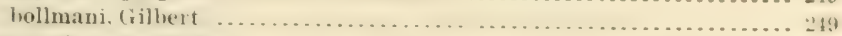

Gemus Abramis, Guvier..................................

Subgenus Jotemigonus, Rafinesque............................. $25_{0}$

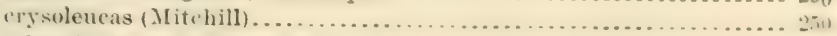

bosci (Curier $\&$ Valenciennes) ................................... 251

gardoneus (Curier \& Valenciennes) ............................ 251

Bull. No. 47, pt. 4-II 


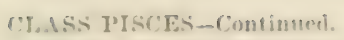

S1'BST.ASS TEIABOSTOALI-Continued,

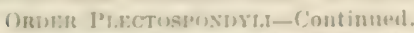

․ 1-

Famil!" C'yprinida-Contimuel.

Gemus C'ochlognathus, Bairel of Girarel . .......................... 251

urnata, Bairul \&: Giraril..................................... 25:

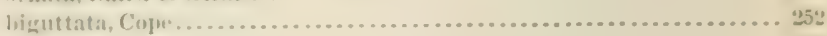

fienur Cliola, Girarıl . ....................................... 252

vigilax (Baird \& Giraral) ................................... 25

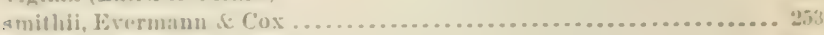

Gemus Votropis, Rafiuesque................................. 25t

sulugesus of ztecula, Jordan of Lvermann.................... 258; 2799

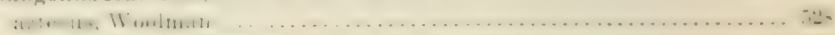

Sulıenus Chriope, Jortan .................................. 258

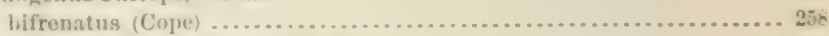

fordani, Jigenuann of Eigenmanu ............................. 259

maculatus (Hay) ............................................. 259

nnogenus, Forlies ........................................ 259

cayuera, . Mcek .............................................. 260

atrocaudalis, Fivermann ...................................260

luteroton (Cope) ............................................ 261

ralientis, Jorlan s. Suyder .................................... 3197

muskoka, Meek ........................................... 3141

wolaka, Evermann of Kiemlall.................................. 2799

Subgenus Alburnops, Girari.................................... 261

fretensıs (Cope) ............................................ 261

blemnius (firard) .......................................... 261

buchanani, Mesk........................................ 2800

sabinat, forilan \& Gilluert ................................... \$6?

volucellus $($ (iope $)$............................................ 263

s! 1 lia "

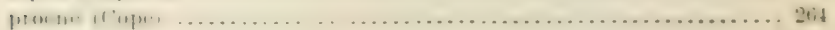

rasconis, Jumlan \& Snyder ................................. 3141

nigrotinatus (Gïnther) . ............................... 264

kanaw ha, Jorlan of Jenkiws..................................... 26t

braytuni, Jurdan of Lvermann ................................ net

spectrunculns (Cope) ....................................... 2ti5

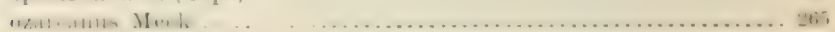

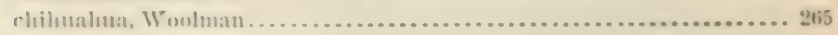

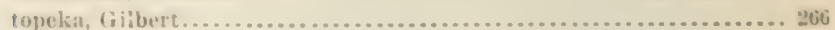

Subgenus II ulswnils, dirart................................. 266

gilberti, Jordan o dhok .................................... 266

quiptol.pis (Cojw) ............................................ 266

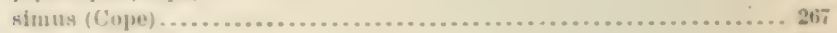

longirustris (1lay) ............................................. 200

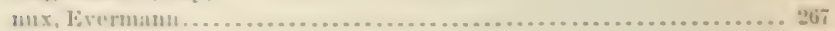

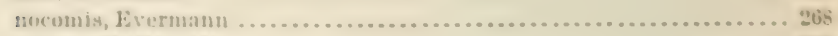

.

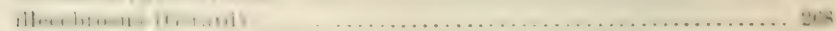

hu\}

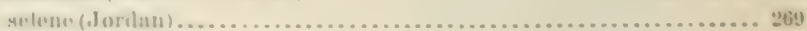

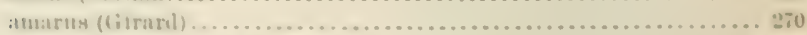

snludamus (Jord:an \& Ibrayton) ............................ 28.

Sin

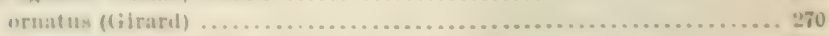

Suhgenus Mloniana, (iirard ............................... 271

:

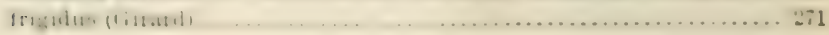


CLASS PISCES-Continuer.

SUBCLASS TELEOSTOMI-Continued.

Order Plectospondyli-Continued.

Suborder EventogNathi-Continuerl.

Family Cyprinida-Continued.

Genus Notropis, Rafinesque-Continued.

Subgenus Moniana, Girard-Continued.

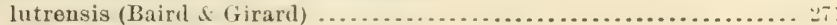

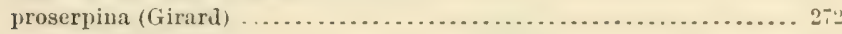

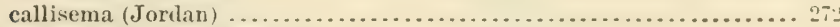

Subreuug Crprinella, Girari ................................

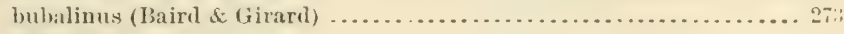

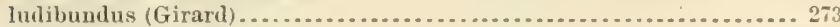

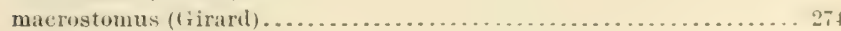

texanus (Girard) ........................................ 27t

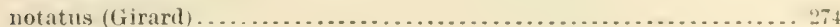

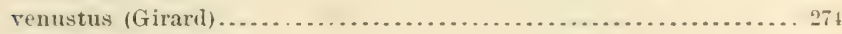

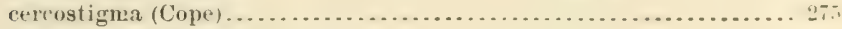

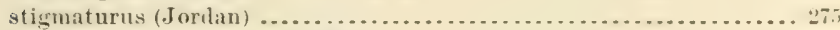

trichroistius (Jordan \& Gilbert) . . . . . . . . . . . . . . . . . 275

callistius (Jordan) .......................................... 270

eurystomus (Jordan) ..........................................

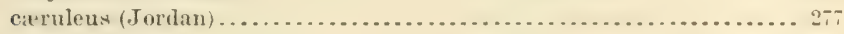

nivets (Cope) ...............................................

chloristius (Jordan \& Brayton) ........................... 27

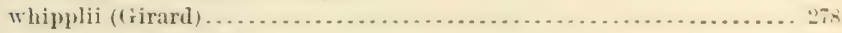

analostimus (Girard) . . ....................................... 27

galacturus (Cope) . ....................................... 279

camurus (Jordan \& Meek) .................................... 279

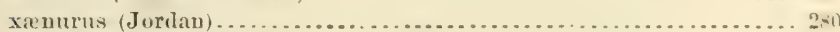

hypselopterus (Giinther) ................................... 280

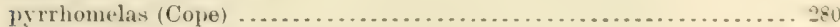

garmani, Jorklan . . . . . . . . . . . . . . . . . . . . . . . . . . . . . . . 281

Sulgenus Luxilus, Rafinesque ............................... 2si

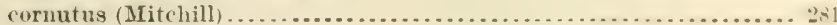

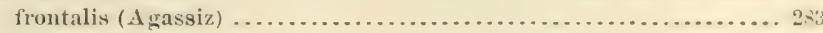

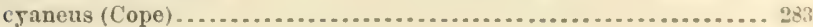

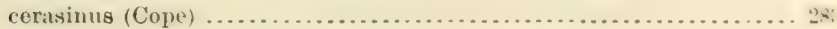

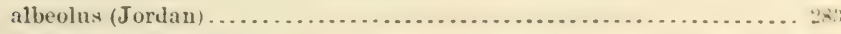

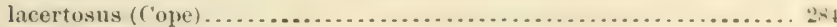

Subgeuus Hydrophlox, Jordan................................. 2nt

mactonaldi, Jordan denkins .............................. 2sit

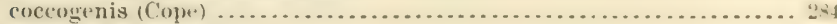

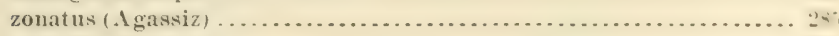

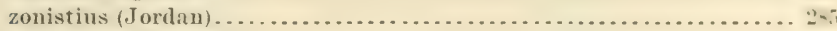

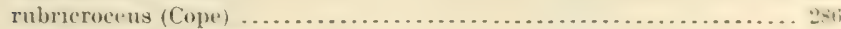

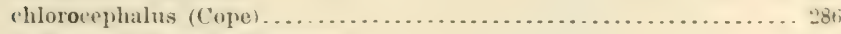

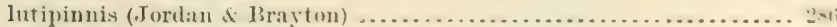

chamberlaini, Evermann ...................................... 2800

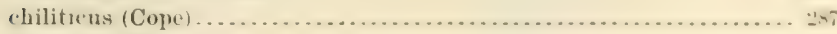

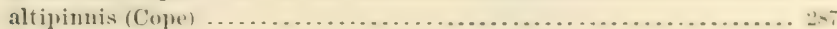

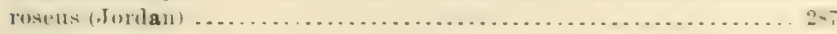

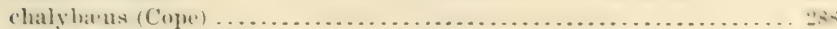

chrosomus (Jorilan) . . . . . . . . . . . . . . . . . . . . . . . . . . . .

xanoceplalus (Jordan) .................................. 28?

Subgenus Oreula, Jordan \& Evermann....................... 289; 3140

oren, Woolman................................................ 889

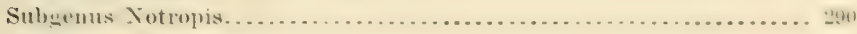

ariommus (Cope) ................................................. 290 
('L.ASS PISCES-Comtinuml.

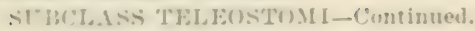

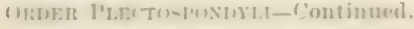

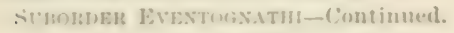

Famil! Conjrinide-Continuel.

(ietuns Dorropis, IRatines!ne-Continuet.

sulı-nus Vivtropis-Continned.

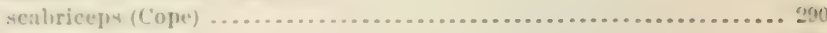

jejunus (Forlues) ........................................ 890

мени

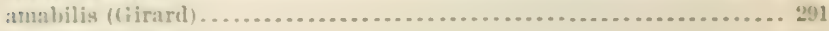

Ienciodus (Cope) ......................................... 291

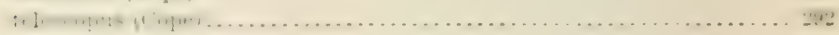

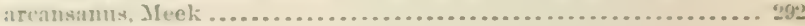

socins (lirarl) ........................................... 292

notemigonoiles, Evermann................................

stilbius, Jordan ..............................................

atlıerinoldes, Iatinesqque ..................................... 293

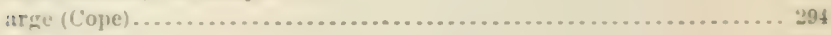

dilectus (Giraril) ........................................... 294

lıuisiant, Everminn ....................................... 2£01

fumens, Evermann.................................... 904

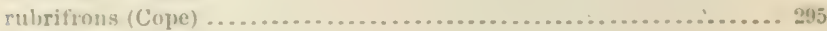

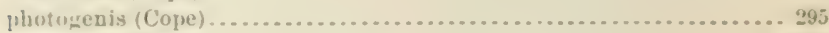

.

seepticus (.Jordan of Gilhert) ............................... 290

mieropteryx (Cope) .......................................... 2406

metallieus, Jorlan d. Meek ....................................... ${ }^{299}$

Sul,genus Lythrurus, Jordan. . . . . . . . . . . . . . . . . . . . . . . . . . . . . . 297

bellus (Hay) ........................................... 297

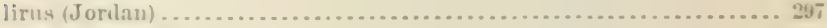

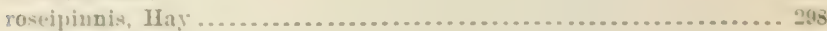

umliratilis ((i) irard) ............................................

umbratilis (Giraril) .......................................... 299

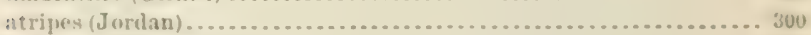

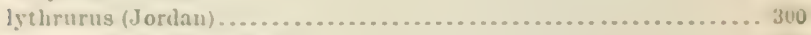

cyanoecphalus (Copelant) .................................... \$ \$0

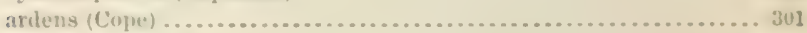

fusciolaris, (illbert ........................................ 301

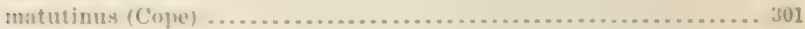

gunetulatus (11ay) ............................................. 301

(ienus Xyмtrusts, Jorelan o. Snyter ............................ 3142

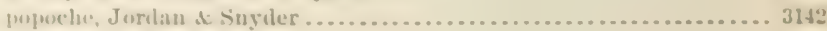

1,emus Ericymba, Cope ...................................... 302

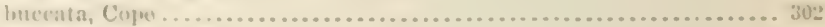

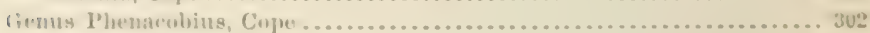

teretulus, ('opee............................................ s03

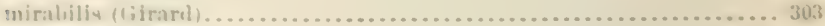

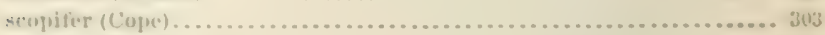

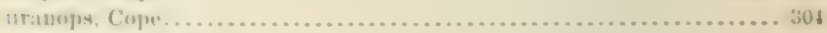

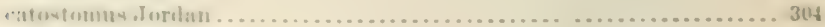

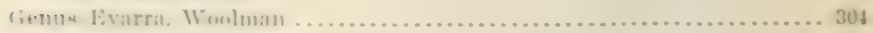

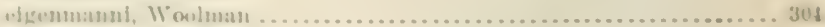

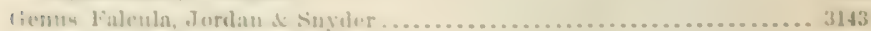

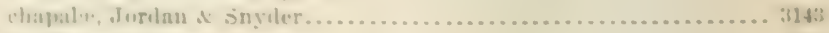

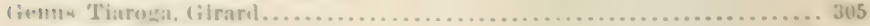

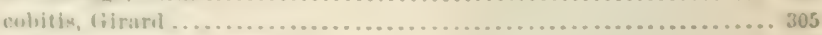

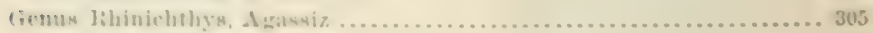

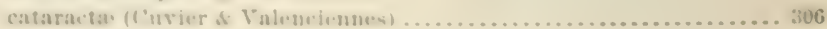

Iulcin (liiraril)....................................... 300 
CLASS PISCES-Continued.

SUBCLASS TELEOSTOMI-Continned.

Order Plectospondyli-Continued.

Suborner Evextogiathi-Continued.

Family Cyprinidee-Continued.

Genus Rhinichthys, Agassiz-Continued.

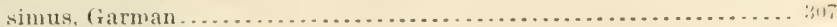

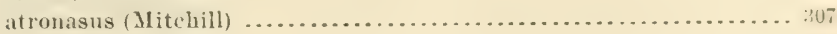

croceus (Storer) ................................................ 308

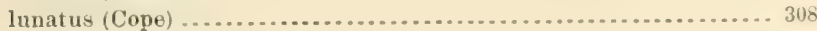

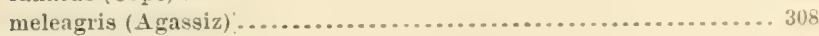

Genus Agosia, Girard ............................................ 308

Sulgenus A pocope, Cope.................................... 309

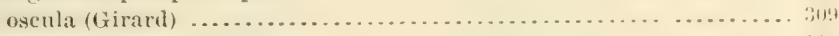

Jarrowi, Jordan \& Evermann............................... 309

conesii (Tarrow) ............................................

adobe. Jordan \& Evermann .................................. 310

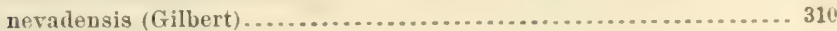

nubila (Girard) . ........................................... 311

carringtonii (Cope) ......................................... 311

klamathensis, Evermann \& Meek ............................. 3144

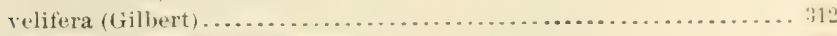

nmatilla. Gilhert \& Evermann ...............................

falcata, Eigermann \& Eigenmann .......................... 313

Subgenus Agosia ................................................. 313

chrysogaster. Girard ........................................... 313

Genus Hybopsis, A gassiz .................................... 314

Subgenus Erimystax, Jordan ...................................... 315

tetranemus, (iilbert.........................................

irstivalis (Girarl) ....................................... $3 ! 6$

marconis, Jordan \& Gilbert.................................... 316

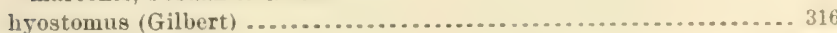

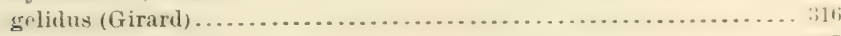

meeki, Jordan \& Evermann .................................... 317

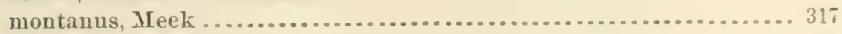

cumingii (Günther) ........................................... 318

monacus $(\mathrm{Cope})$................................................. 318

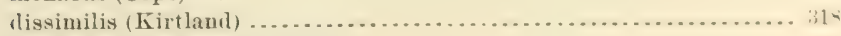

watauga, Jordan \& Evermann .................................. 319

Subgenus Hybopsis. . . . . . . . . . . . . . . . . . . . . . . . . . . . . . . . 319

labrosus (Coper ............................................

hypsinotus (Cope) ..............................................

rubrifrous (Jordiun) . . .......................................

amblops (Rafinesque) .......................................

storerianus (Kirtland) ................................................ 321

Subgenus Iuriria, Jordan of Erermanu . . . . . . . . . . . . . . . . . . . . . . . 321

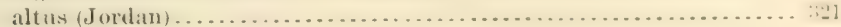

Subgenus Nocowis, Girard .......................................

kentuckiensis (Ratinesque) ................................ 32..

Genus Couesius, Jordan ............................................. 32

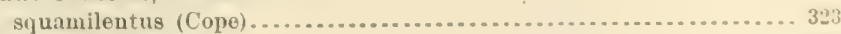

plumbeus (A gassiz) ........................................ 323

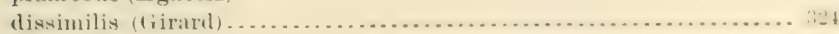

kreeni, Jordin ..........................................

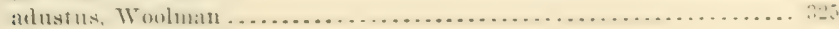

Genus Platygobio, Gill .......................................... 325

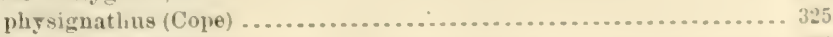

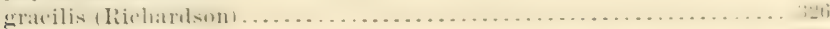

prallidus, Forbes...................................... 


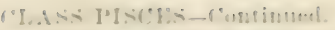

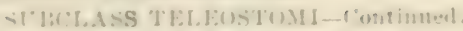

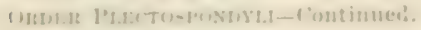

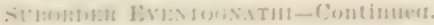

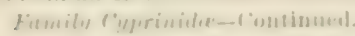

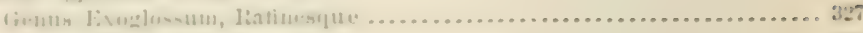

maxillingma (Le suenr) ................................. 327

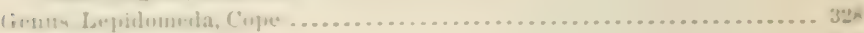

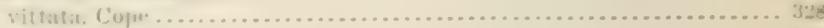

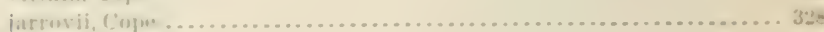

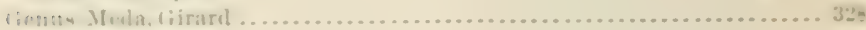

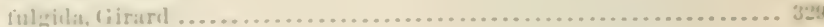

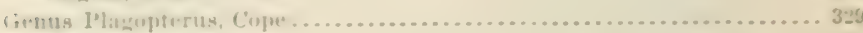

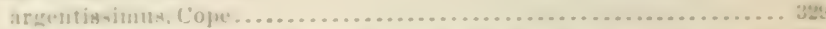

$\rightarrow$ с

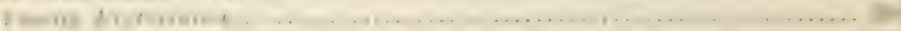

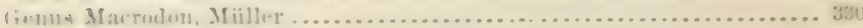

mierulevin, (iiinther........................................... ssu

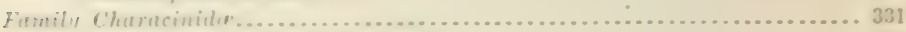

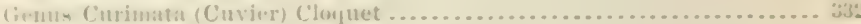

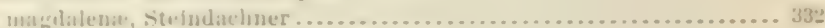

livmu l'iabueina, ('uvier of Valencienne.s ........................

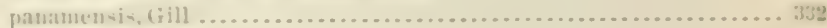

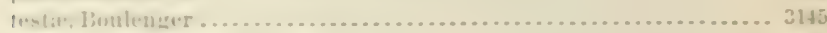

(innus Titranmupterus, Curier ................................. 335

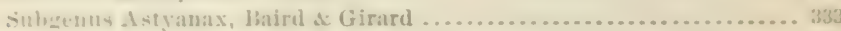

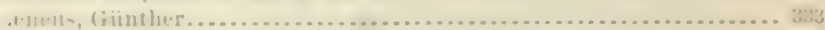

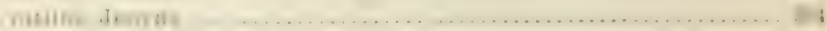

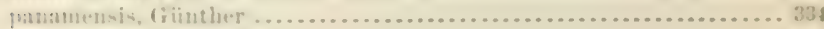

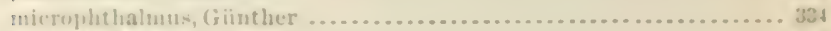

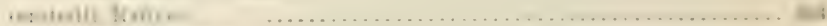

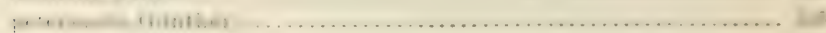

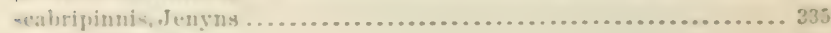

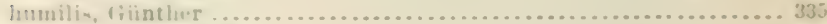

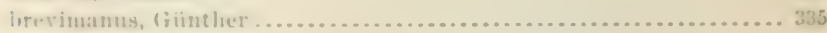

neexicanus, Filippi ...........................................

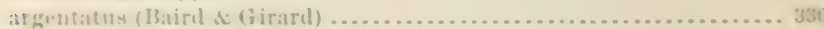

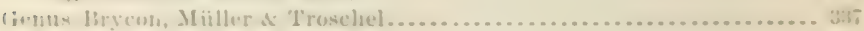

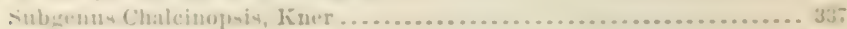

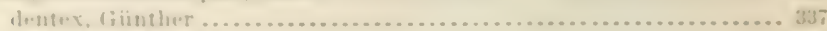

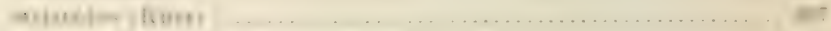

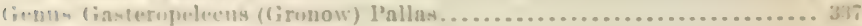
- -

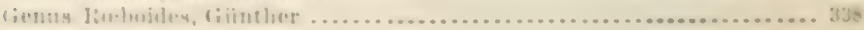

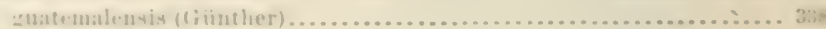

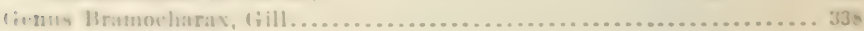

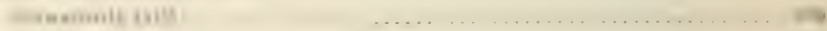

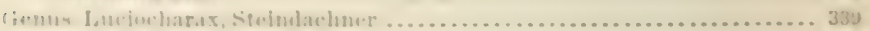

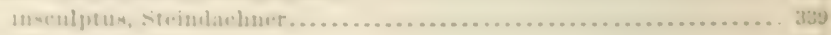

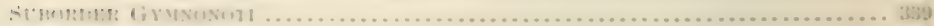

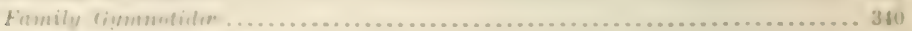

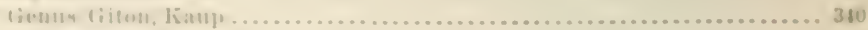

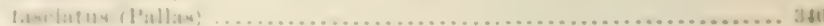

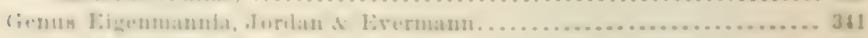

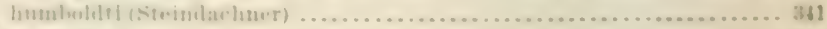

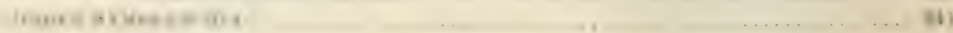

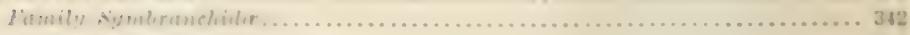

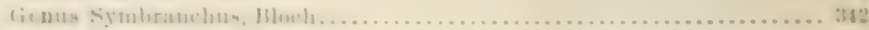

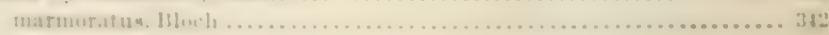


CLASS PISCES-Continmed

SUBCLASS TELEOSTOMI-Continmed.

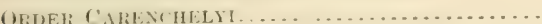

Family Derichthyidce ............................................. $3 t^{3}$

Genus Derichthys, Gill ......................................... 343

serpentinus, Gill ........................................... $34{ }^{3}$

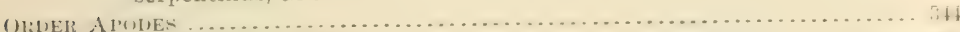

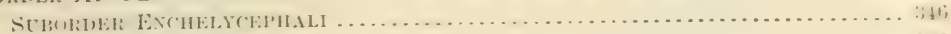

Family drguillide .............................................

Genus $\Delta$ uguilla, Shaw ......................................... 347

chrysya, Rafinesque ....................................... 318

Fumily simcuchelyide......................................

Genus Simenchelys, Gill ..................................... 349

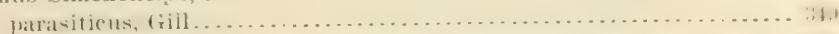

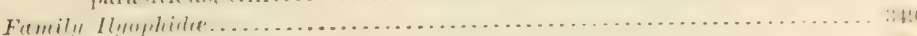

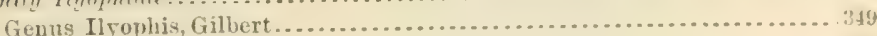

brunneus, Gilbert .......................................... 350

Family Synaphobranchida ........................................ 350

Gienus Synaphobranchus, Johnson............................... 35

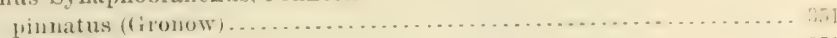

Genus Histiobranchus, Gill.................................... 351

bathrbius, Gïnther ..................................... 35

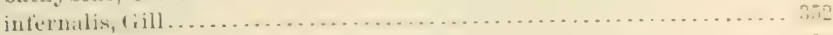

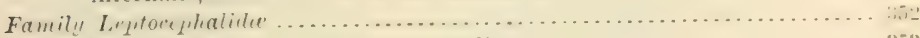

Gepus Leptocephalus (Gronow) Scopoli............................ 353

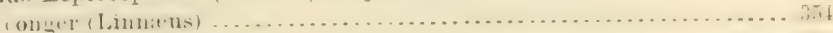
caudilimbatus (Poey) ...................................... 355

Genus Congrellus, Ogilby.................................. 355; 2801 balearjeus (De la lioche) ..................................... 350 macrurus (Gilbert) ........................................ 356

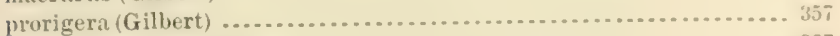

niteus (Jordan \& Bollman) ..................................... 35

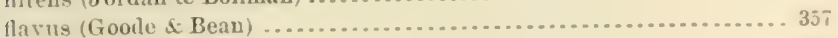

Genus Uroconger, Kaup ....................................... 358 viciuns, Vaillant.......................................... 358

Family Murenesocide ............................................ 358

Geuns Muratnesox, McClelland .................................. 359

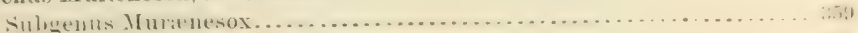

coniceps, Jorlan \& Gilbert ................................. 350

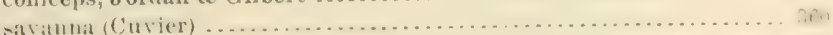

Gemus Xenomystax, Gilbert .................................... : atrarins, Gilbert .............................................

Genus Hoplunis, Kaup ...................................... 361

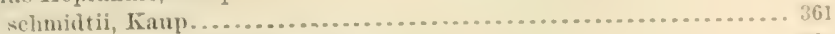

diomedianus, Goode \& Bean................................. s61

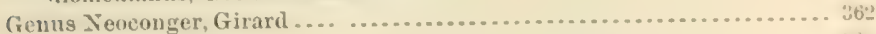
mucronatus, Girard .......................................... $36^{\circ}$ vermiformis, Gilbert....................................... $3 \theta^{4}$

Gienus Leptoconger, Poes .......................................... 30.

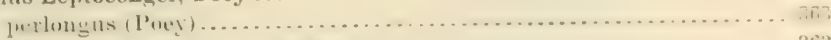

Genus Stilbiscus, Jortan \& Bollman ............................ 303

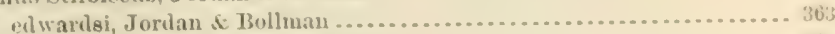

Gemus Gorlichthrs. Jordan of Davis ............................. 30.3 irretitus, Jordan \& Daris.................................... 30.

Family Nettastomille ............................................. 30.4

Genus Chlopsis, Matiuesquo .................................... 364 equatorialis, filbert ...................................... 36

Genus Tenefica. Jortan \&. Daris ............................. 36. procera, Goote s. Bean ................................... $300^{\circ}$ 


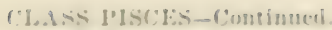

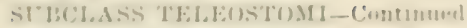

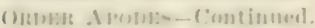

-

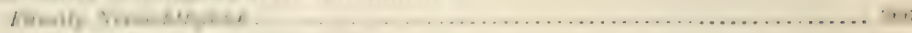

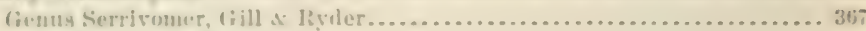

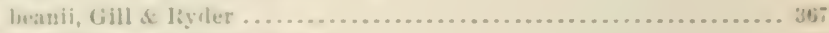

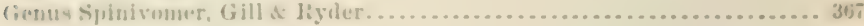

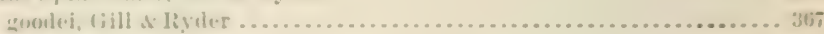

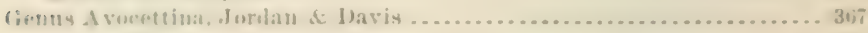

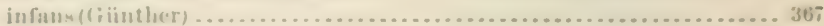

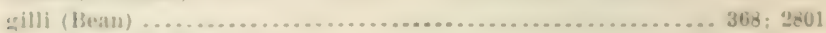

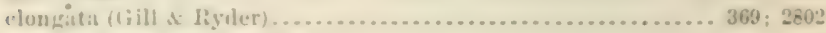

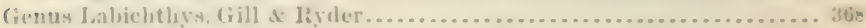

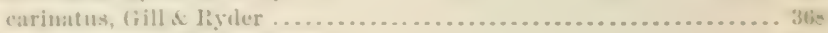

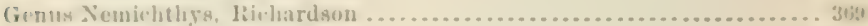

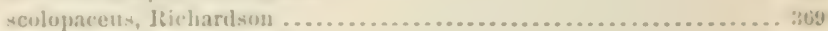

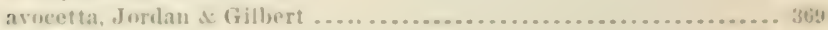

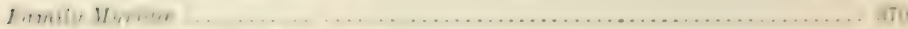

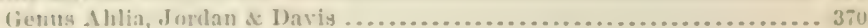

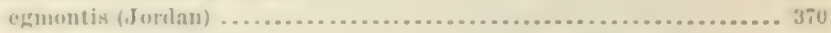

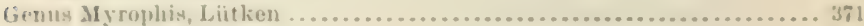
punctatıs, Liitken ................................. 271

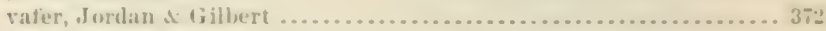

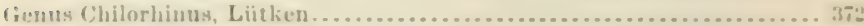

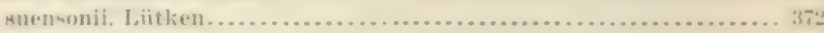

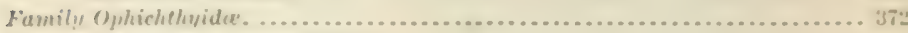

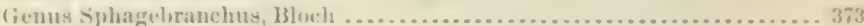

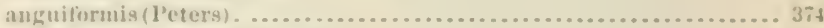
selachopis (Jorklan \& Gilbert) ................................. Jit

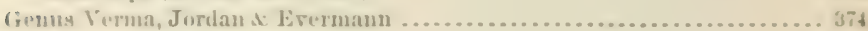

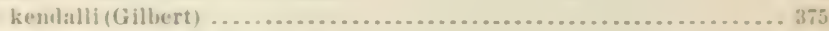

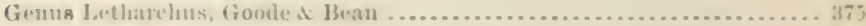

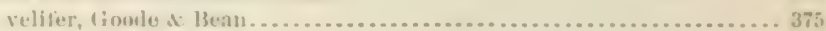

Genus Myrielıtlyg, firaml ................................ 375 tiдriun, li

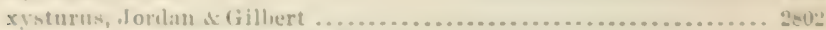

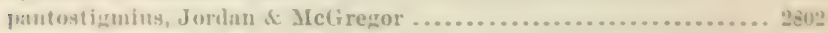

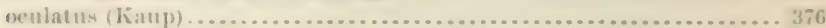

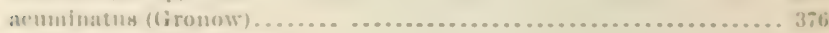

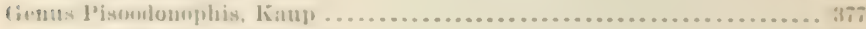

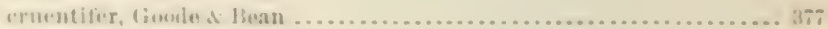

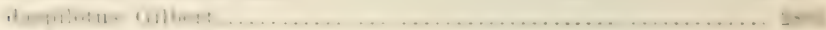

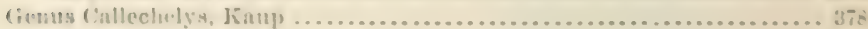

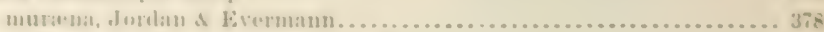

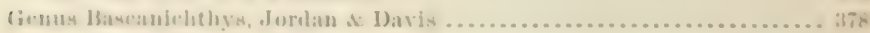

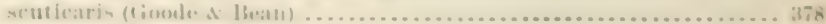

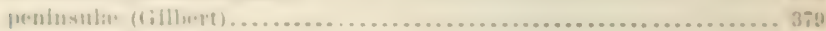

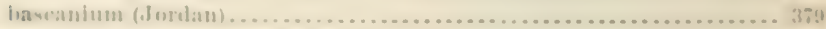

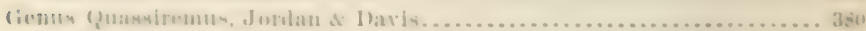

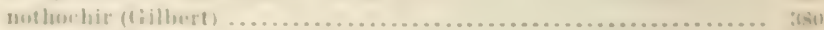

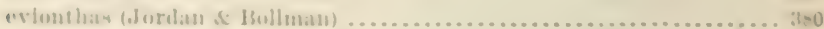

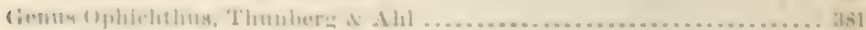

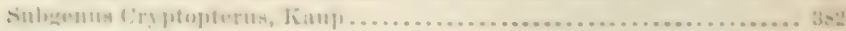

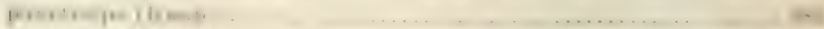

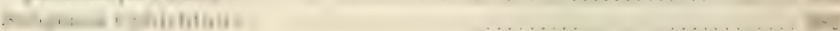

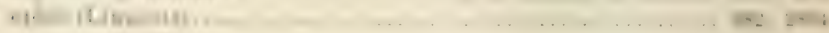

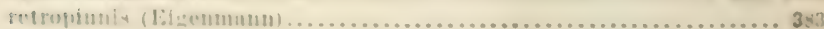


CLASS PISCES-Continued.

SUBCLASS TELEOSTOMI - Continuerl.

ORDER APODES-Continued.

SUBORDER ENCHELXCEPHALI-Contimued.

Family Ophichthyida-Continued.

Genus Ophichthus, Thunberg \& Alul-Continued.

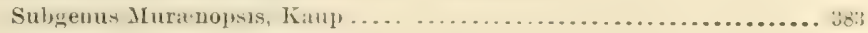

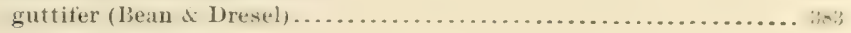

ocellatus (Le Sueur) ........................................ 383

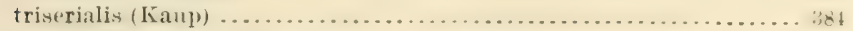

Suhgenus seytalophis, Kanp...............................

gomesii (Castelnau) ................................................. 384

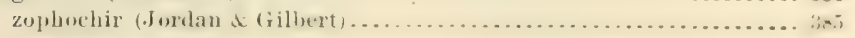

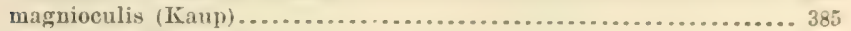

parilis (Richardson) . . . . . . . . . . . . . . . . . . . . . . . . . . . . .

Genus Mystriophis, Kaup . . ................................... 386

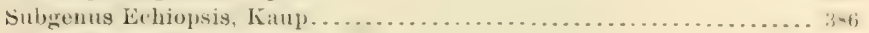

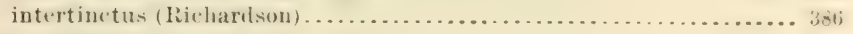

Genus Scytalichthys, Jordan \& Davis ............................ 387 miurus (Jordan \& Gilbert) .................................... 387

Genus Brachysomophis, kiau ............................... 3-7

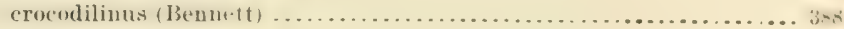

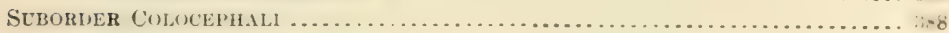

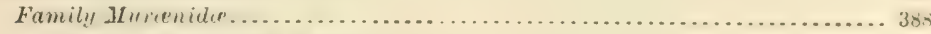

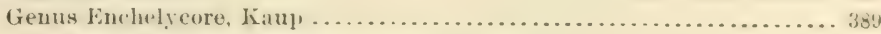

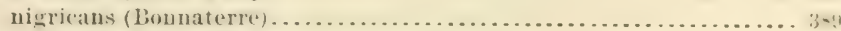

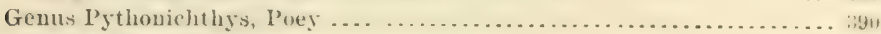
satngnine

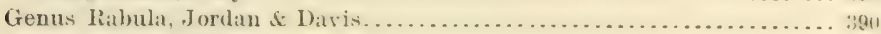
aquad dulcis (Cope) ............................................

marmorea (Valenciennes) ..........................................

panamensis (Steindachuer) .................................... 391

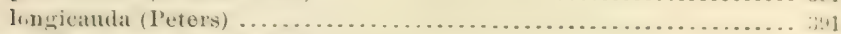

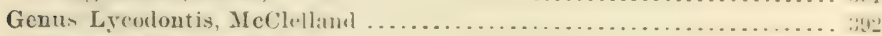

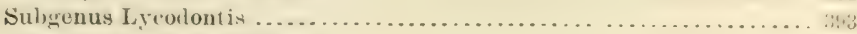

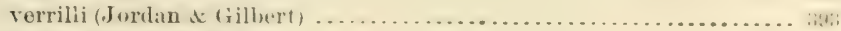

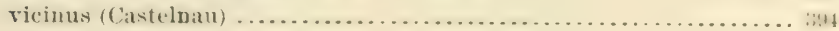

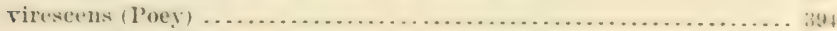

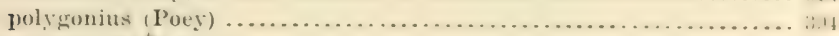

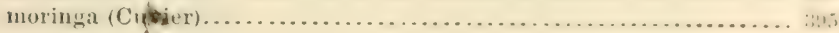

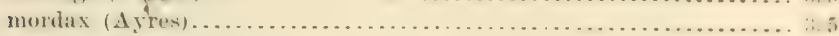

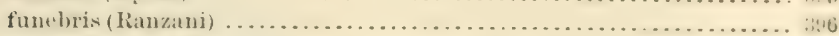

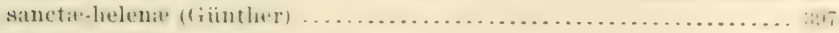

castaneus (Jordan \& Gilbert) .......................... 396; 2804

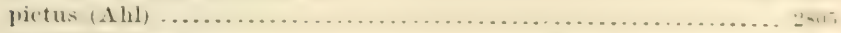

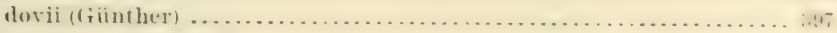

(m)

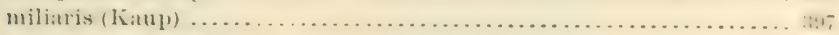

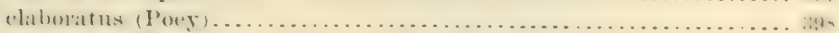

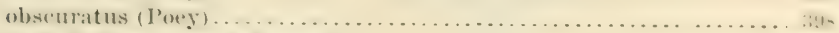

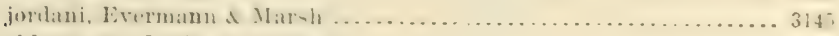

chlevastes (Jordan \& Gilbert)................................ 398

Subgenus P'riodonophis, Kaup . . . . . . . . . . . . . . . . . . . . . . . . 399

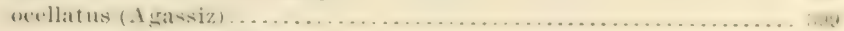

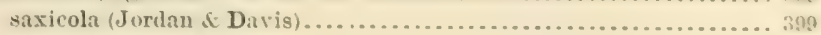

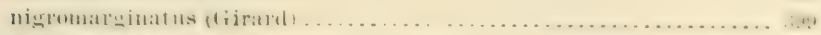

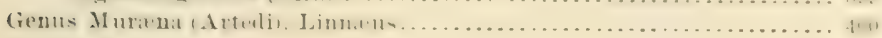

insularum, Jordan it Davis ................................ 400 
QT.AST ISICEL-Continuet.

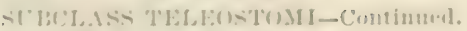

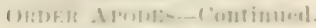

s(r)

fimily Muraniler-Continued.

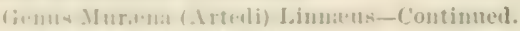

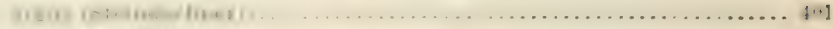

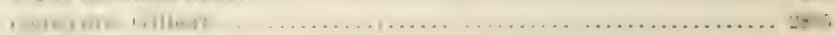

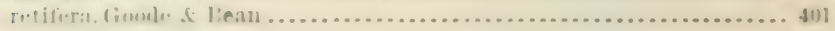

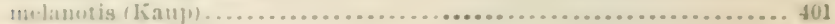

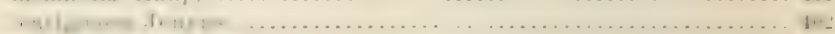

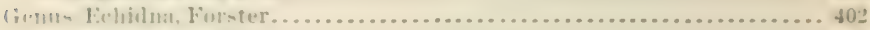

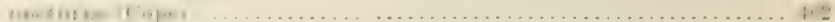

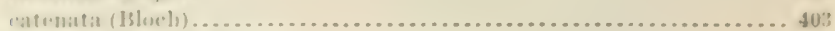

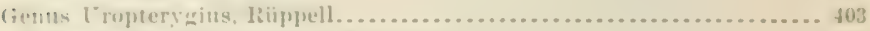

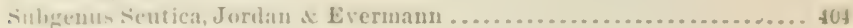

neeturus (forelan of (iillert) ............................... t14

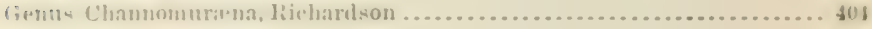

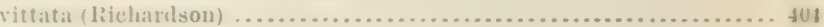

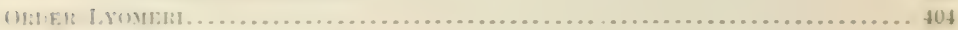

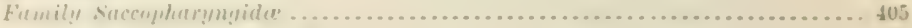

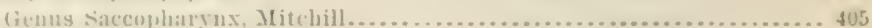

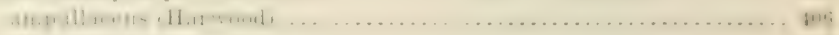

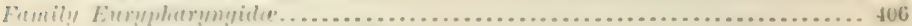

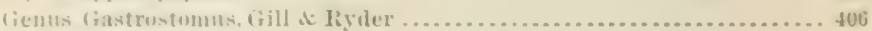

hairalii, (iill st liveler ....................................... 406

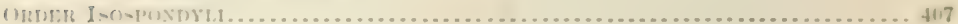

Family Lilopider .................................................. 408

Vienus Tarpon, Jurtan of Evermann .............................. tU9

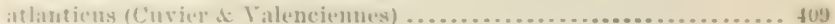

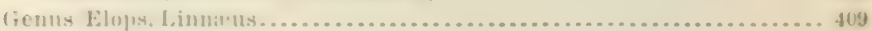

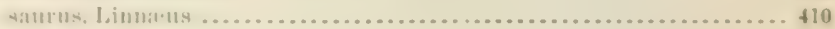

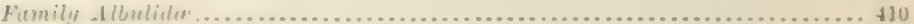

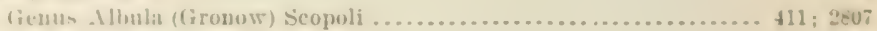

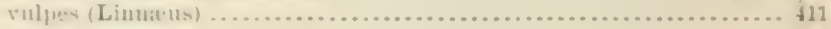

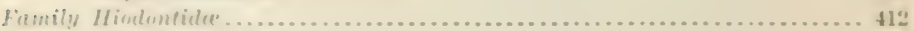

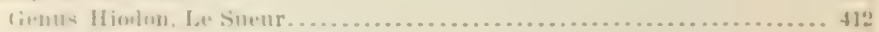

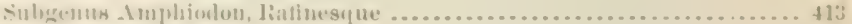

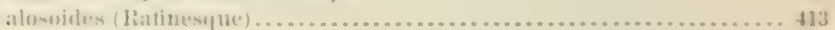

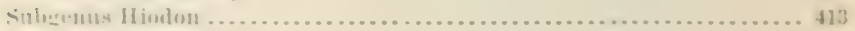

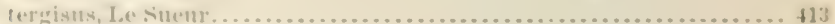

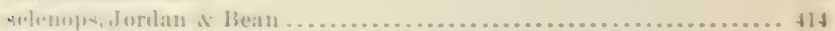

Family thunider................................................

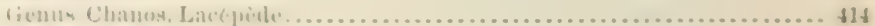

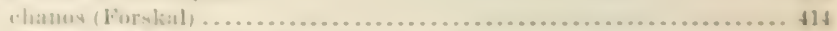

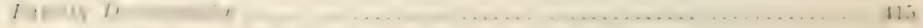

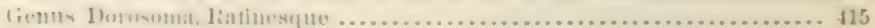

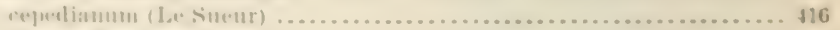

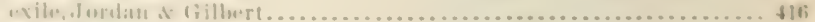

m.

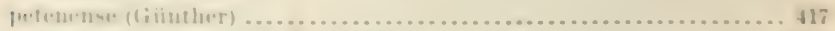

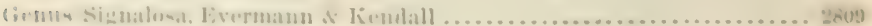

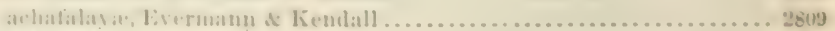

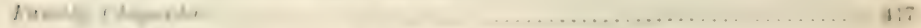

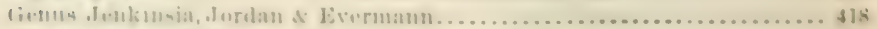

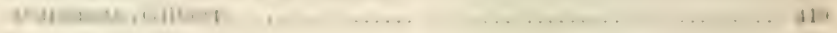

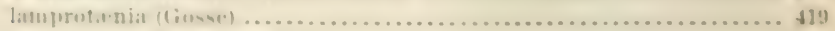

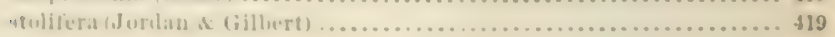


CLASS PISCES-Continued.

SUBCLASS TELEOSTOMI-Continued.

Order IsospondyL-Continued.

Family Clupeida-Continued.

Genus Etrumeus, Bleeker..................................... $\$ 19$

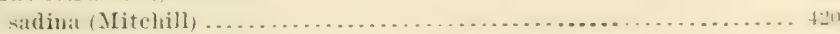

Genus Perkinsia, Rosa Suith Eigenmann .......................... 420

othonops, R. S. Eigenmann................................. 420

Genus Clupea (Artedi) Linnaens . . . . . . . . . . . . . . . . . . . . . . . . . . . . . . . 421

Larengus, Linnreus ......................................... 421

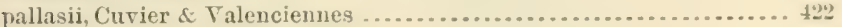

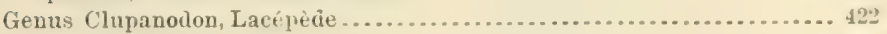

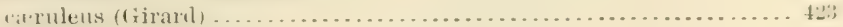

pseudohispanicus (P'oey) .................................. 423

Genus Pomolobus, Rafinesque .................................. 424

chrysochloris, Rafinesque .................................. 425

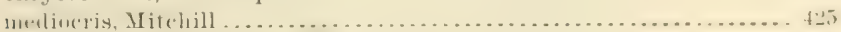

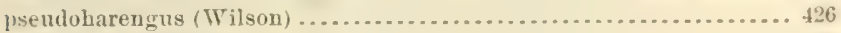

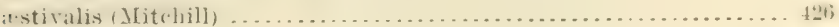

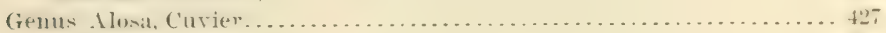

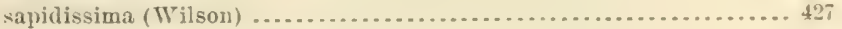

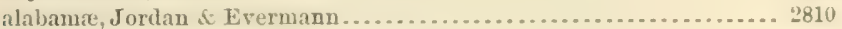

Genus Sardinella, Cuvier \& Valencieunes........................ 428

Subrenus Sardinella ........................................ 429

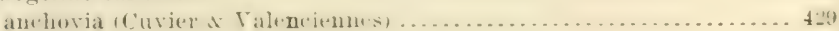

clupeola (Cuvier $\&$ Valenciennes) ............................ 429

apicalis (Mïller \& Troschel) . . . . . . . . . . . . . . . . . . . . . . . . . . . . 429

bishopi (Müller \& Troschel) ................................. . 430

Subgenus Harengula, Cuvier \& Valenciennes.... . . . . . . . . . . . . . . $\$ 30$

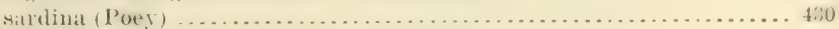

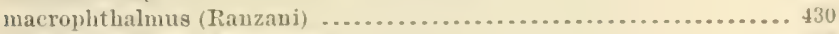

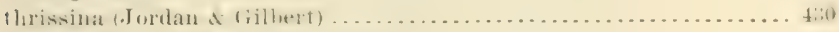

lumeralis (Cuvier \& Valenciennes) .......................... 431

Subgenus Tile, Jorlan \& Evermann . . . . . . . . . . . . . . . . . . . . . . . . 431

stolitera (Jortan of Gilbert) ................................ 431

Genns Opisthonema, Gill........................................ 432

oglinum $($ Le Sueur) ...................................... 432

lihertate (tiinther) ...................................

Gemus lirerentia, (rill. . . . . . . . . . . . . . . . . . . . . . .

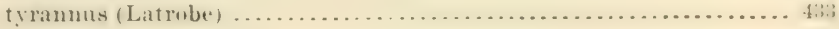

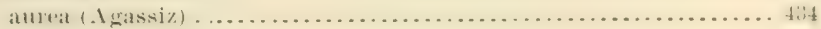

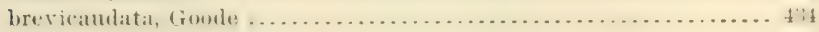

patronus, Goole............................................. 434

Genus Chirocentrodon, Giinther ............................... 435

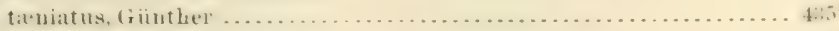

(iemus Ilisha, diray. . . . . . . . . . . . . . . . . . . . . . . . . .

flavipinnis (Valenciennes) ................................. 435

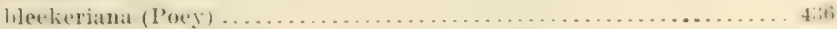

firthi (Steindachner) ................................. 430

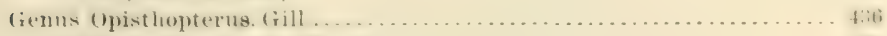

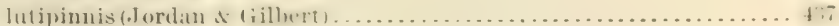

dovii (Gïnther) ......................................... 437

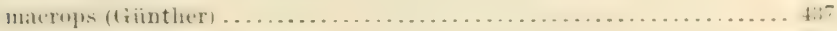

Genus Odontognathus, Lacépìde.............................. 437

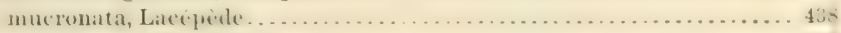

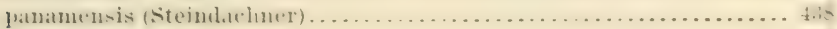

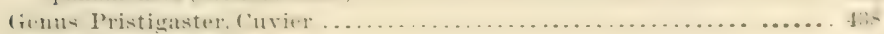

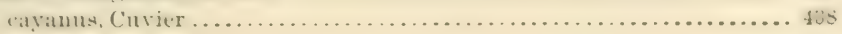




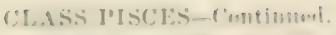

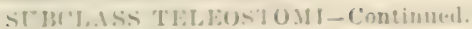

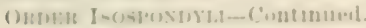

Fimily Lnurumlidilw.......................................... 439

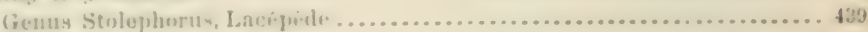

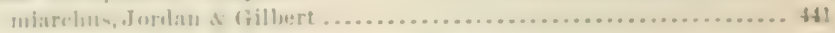

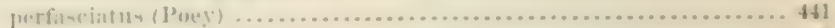

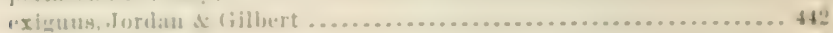

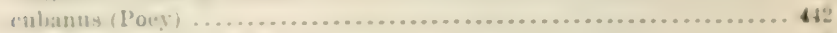

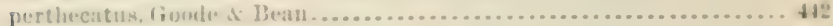

i=chanm, Jordan . (iilhert..............................

browsii (1) (inelin) ......................................... 43

suleratuq, Gillawt ..................................... $4 \$$

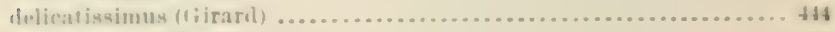

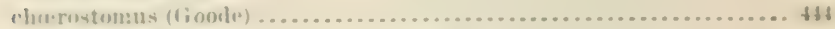

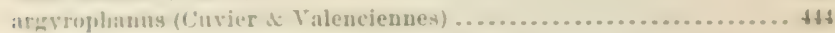

('urtns, Jurtan di dillert ................................ $\$ 45$

operenlaris, Jurblan \& Gilhert ............................ 4 \$5

mituhilli (Cuvior \& Vislencionnes) ........................... tsc

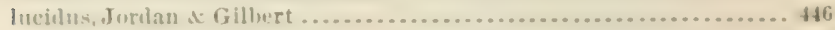

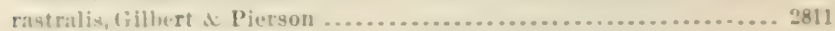

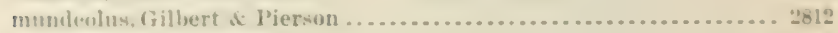

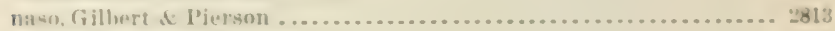

starkili. (ijlhert \& l'jerson ................................... 2518

cluprobiles (swrinson) ................................... 4 .

prouluctus (l'ney) ........................................ 48.

qillerti, Evermann of Marsh ................................. $31 \$ 0$

Earmani, Everman o Marsh ................................. 31 \$1

conupressus (Girarhl) ......................................

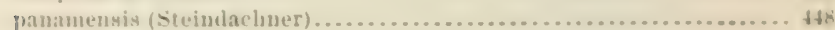

spinifer (Cuvier o Valencienues) ........................... t4s

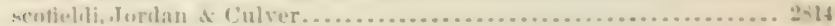

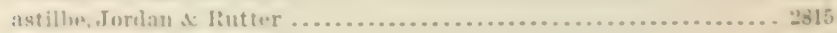

rulwrtwi, Jurlan of liutter .................................. 2515

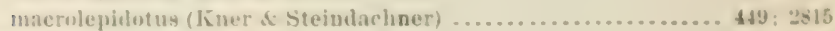

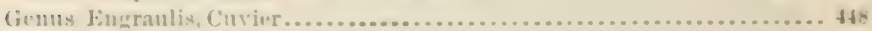

mordax, didrard......................................... 48 .

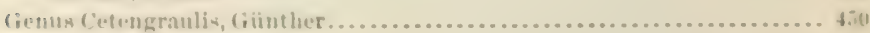

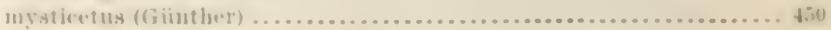

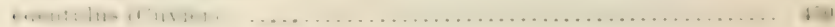

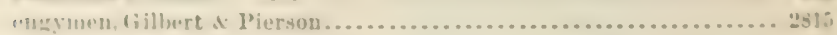

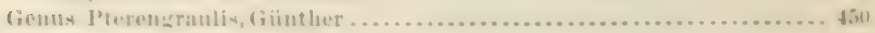

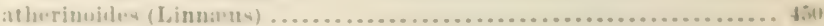

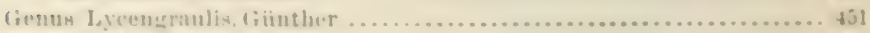

guse-

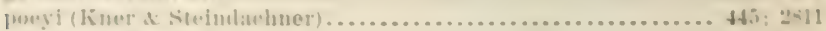

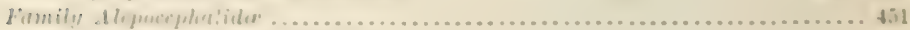

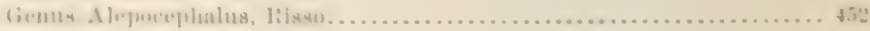

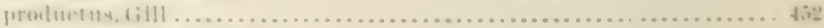

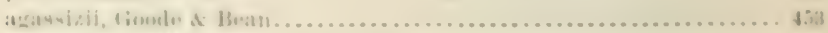
. .

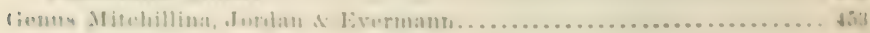

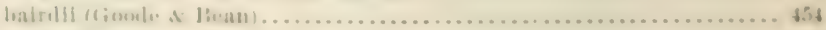

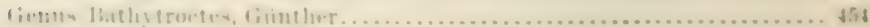

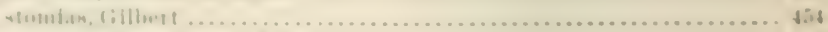

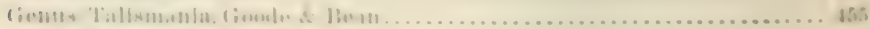

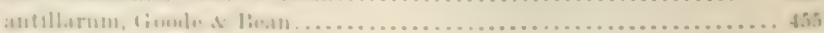

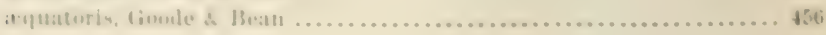


CLASS PISCES-Continued.

SUBCLASS TELEOSTOAII-Continued.

Page.

Order Isospondy -Continued.

Family Alepocephatida-Continued.

Genus Conocara, Goote \& Bean.................................... 456

mactonathli, Gonde of Betin ................................ 457

macroptera (Vaillant) ..................................... 45

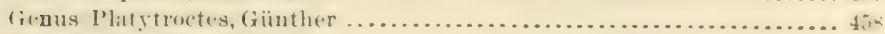

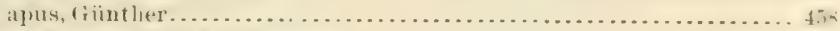

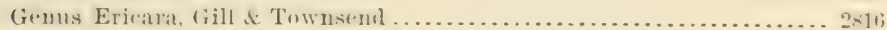

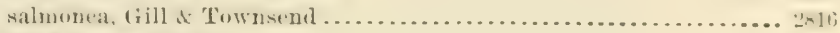

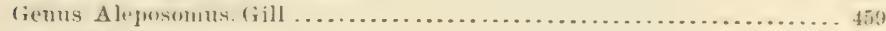
ropei, (rill ................................................ 459

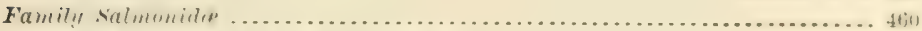

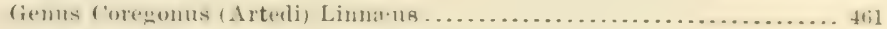

sul,

coulterii, Eigenmann $\&$ Eigenmann ........................... 462 williamsoni, (airard ................................... 4 .

rismontanus, Jordan . . . . . . . . . . . . . . . . . . . . . . . . . . . 4 ti:;

kennientti, Milner........................................... thit

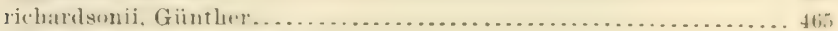

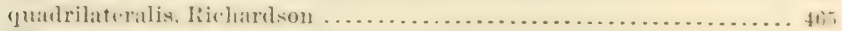

Subgenus Coregonus.....................................4 465

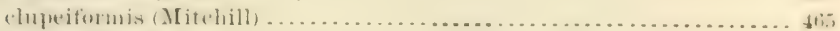

nelsonii,

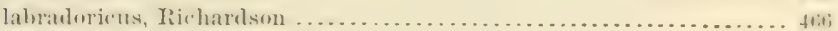

(ienus Aly

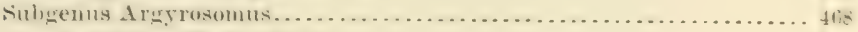

osmeritormis (II. M. Smith) ................................. the

artedi (Le sneur) ................................................ the

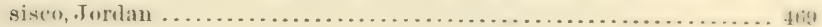

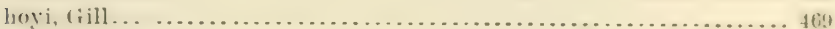

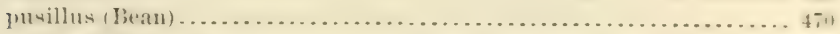

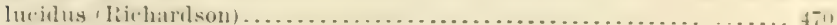

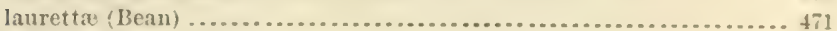

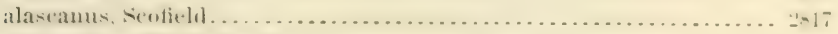

prognathus (H. M. Smith) ............................... $4 i 1$

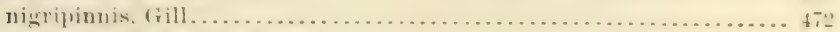

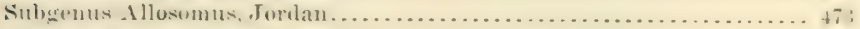

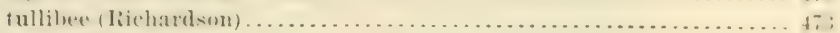

hisselli, lollunan ...................................

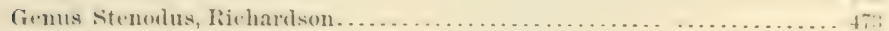

mackenzii (Richardson) .................................. 4 it

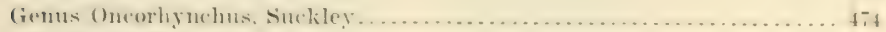

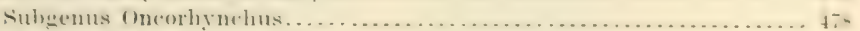

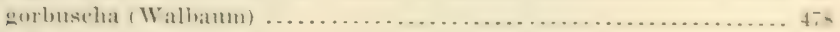

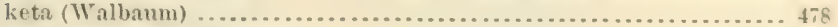

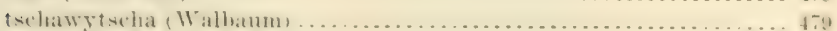

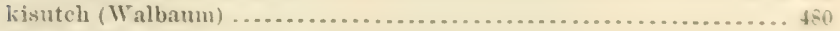

Subgenus Hypsifario, Gill .................................... 4 \&1

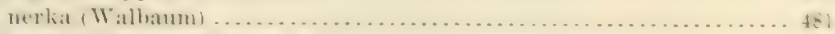

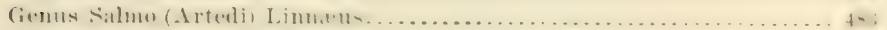

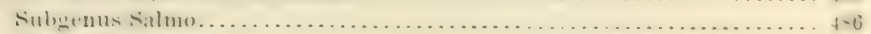

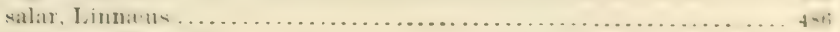

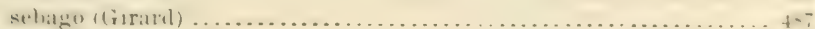

omanauiche, IIcCarthy .................................... 4s

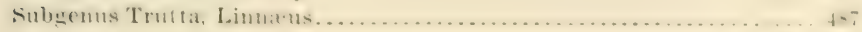
mykiss. Walbaum.............................. $457 ; 492 ; 2318$ 


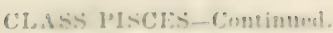

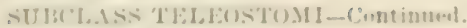

(O)

Family Sulmoniler-rontinutest.

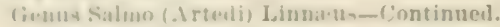

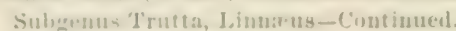

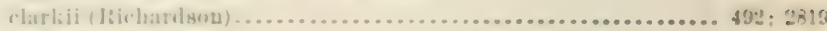

lewiqi (1iirarl) ........................................ 493:

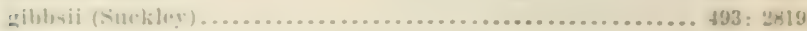

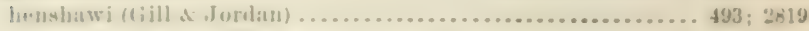

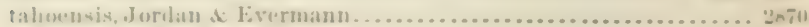

virginalis (Giraral) ................................ $495 ; 2819$

spilurus (Cupe) ........................................ 495: 2819

Heuriticu (Cope) .................................... 496 ; 2819

1... 1.

stumias (C) (upe) .................................... 497; 2819

mactunaldi, Jomian d Evermann ....................... 497; 2819

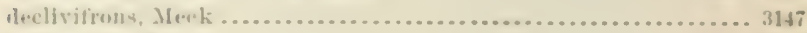

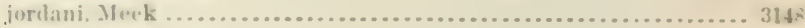

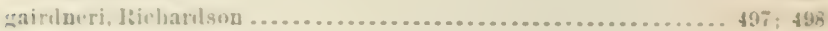

kamloeps (Jorlau) .................................... 499

bearilaleei, Jortan of Seale................................ 2819

crescentis, Jordan of Meardslee............................. 2221

hatheretor, Meek ....................................... 3149

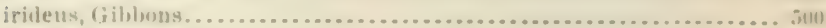

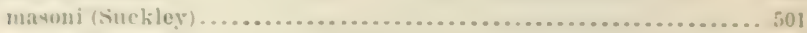

shasta (Jerilan) ......................................... 50.

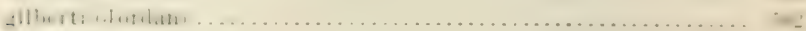

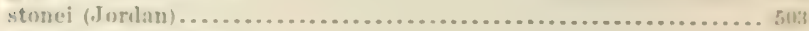

arna-bunita (Jurilan) ..................................... 5013

Genus Cristivemer, Gill \& Inrdan .............................. sos

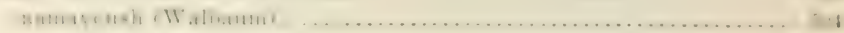

aiscowet (Agassiz) ...................................... 50 ;

Genus Salvelinus (Xilsson) Kicharelson........................ 5ne

funtiualis (Mitehill) ..................................... sof,

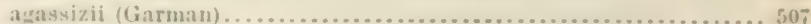

malma (Wallıam) ................................. 507; 2903

parkei (Surliley) .................................. 507; 2323

kumlacla $\left(1^{2}\right.$ allas).......................................

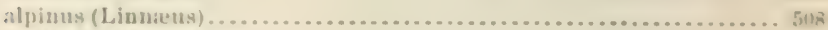

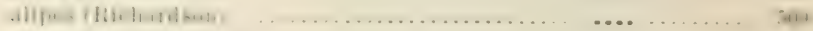

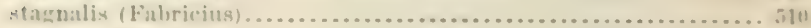

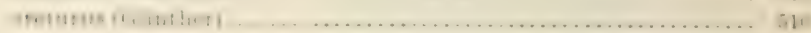

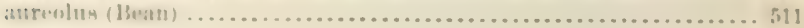

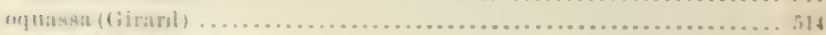

:anoll1.

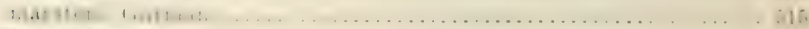

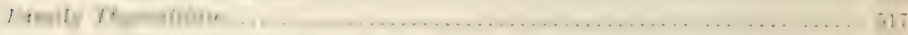

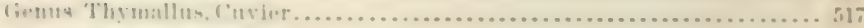

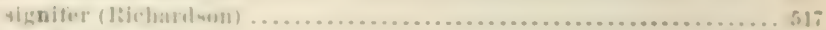

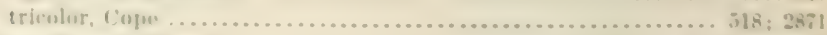

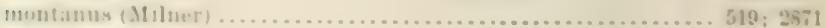

$l$ mov inure,

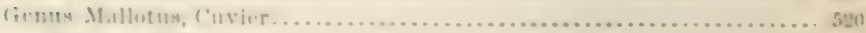

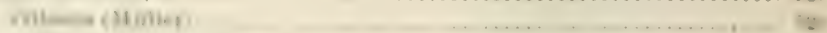

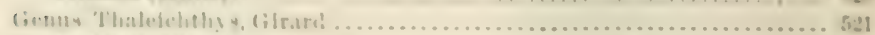

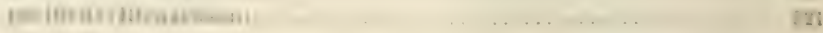


CLASS PISCES-Continued.

SUBCLASS TELEOSTOMI-Continued.

ORDER ISOSPONDYLI-Continued.

Family Argentinida-Continued.

Genus Osmerus (Artedi) Linureus ................................ 5 522

Subgenus Spirinchus, Jorilan \& Erermann ........................ 52:

thaleichthys, $\Delta$ yres ...................................... 52?

attenuatus, Lockington ............................................. 523

Subgenus Osmerus......................................... 523

$\operatorname{mordax}($ Mitehill)......................................... 523

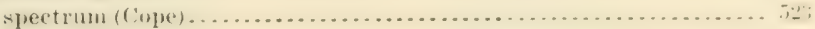

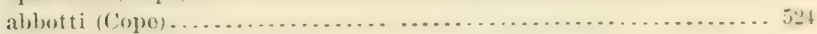

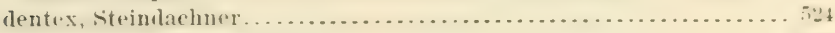

albatross1s, Jordan \& Gilbert................................ 28.63

Genus Mesopus, Gill.......................................... 521

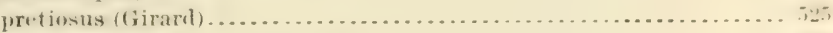

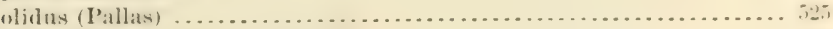

Genus Argentina (Artedi) Linnreas ................................. 525

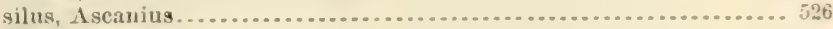

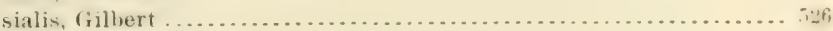

striata, Goode \& Bean....................................... 526

Genus Leuroglossus, Gilbert ..................................... 527

stilbins, Gilbert ............................................... 527

Family Jicrostomide............................................... 527

Genus Nansenia, Jordan \& Erermann ................................ 528

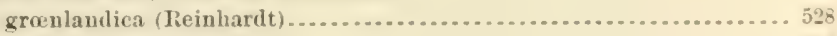

Genus Bathylagus, Günther ........................................ 598

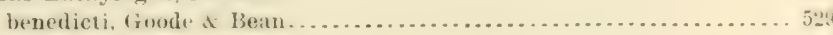

euryops, Goole \& Bean ...................................... 529

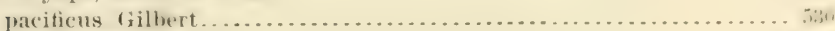

borealis, (illbert .......................................... 821

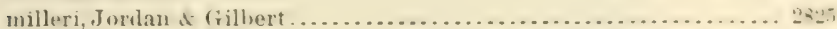

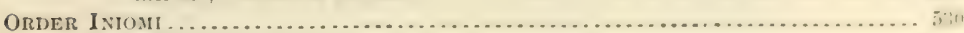

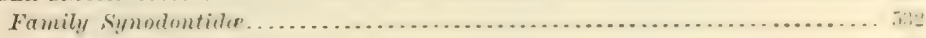

Genus Trachinocephalus, Gill .................................... 533

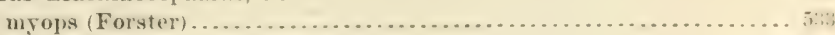

Genus Synodus (Gronow) Scopoli .......................... 533; 2807

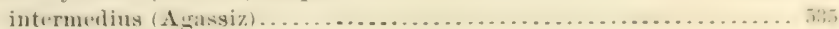
evermanni, Jordan of Ibollman ............................

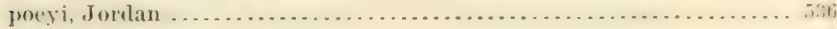

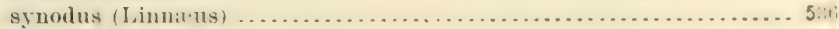

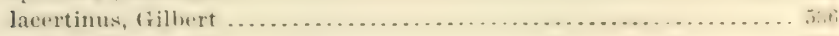

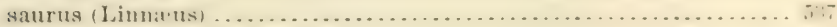

scituliceps, Jordan \& Gilbert ................................. 537

jenkinsi, Jorktan a Jollman .............................. is :

firtens (Linntus) . . . . . . . . . . . . . . . . . . . . . . . . . . .

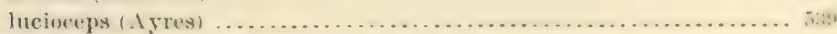

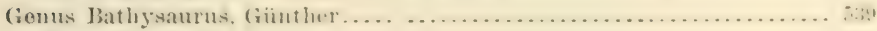
ferox, Günther .......................................... 539

Genus Bathylaco, Goode of Bean................................. 540

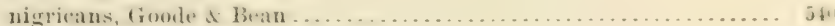

Family Aulopida ............................................ 541

Genus Chlorophthalmus, Bonaparte ............................. 541

agassizii, Bonawarte.......................................... 541

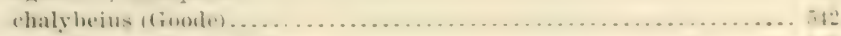

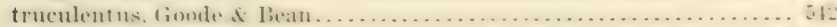

Family Benthosauridex............................................. 543

Gonus Benthosaurus, froote id Bean ............................ 543 grallator, Gookle of Boan...................................... 543 


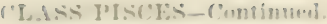

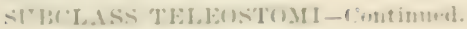

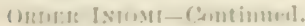

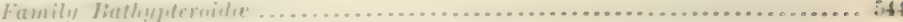

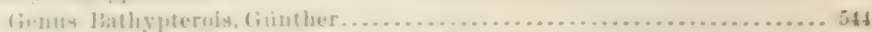

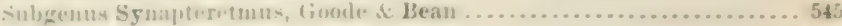

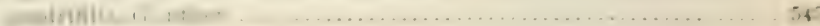

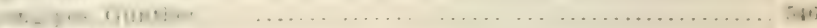

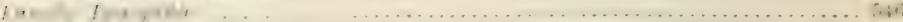

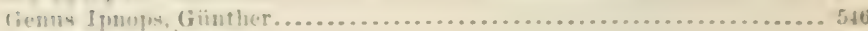

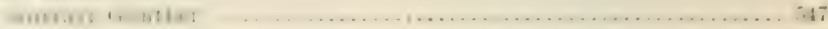

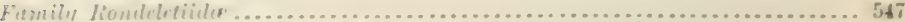

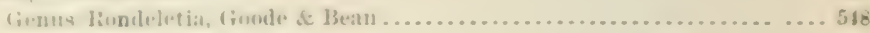

hisolur, limole of Ibean ................................. $5 \$ 8$

Fimili felomimidle ........................................ 548

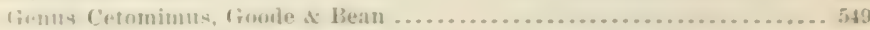

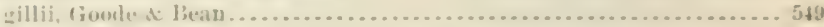

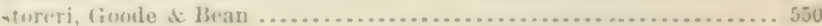

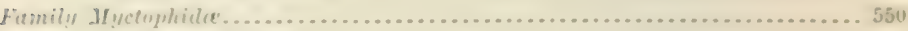

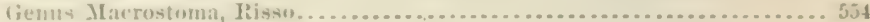

1)

matgaritifermu (foole $\mathrm{A}$ Bean) $\ldots \ldots \ldots \ldots \ldots \ldots \ldots \ldots \ldots \ldots \ldots \ldots \ldots . \ldots \ldots 5$

m

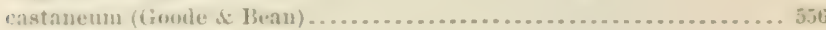

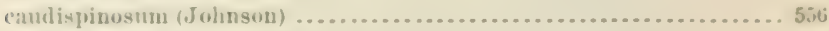

Genus Ceratoscopelus, Giimther..................................... 55 .

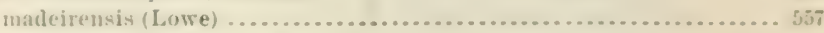

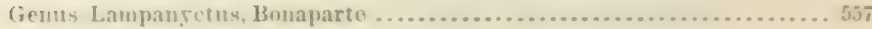

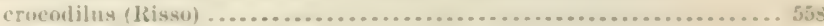

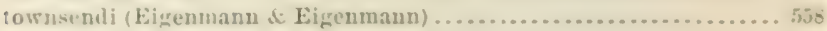

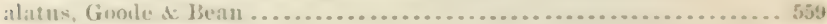

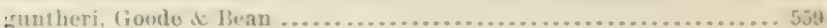

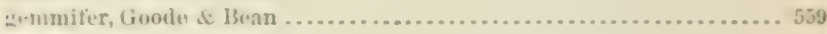

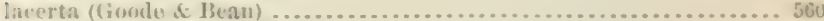

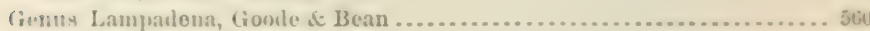

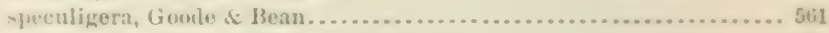

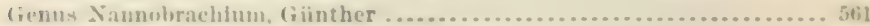

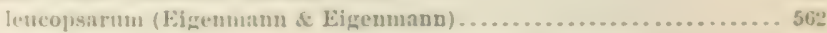

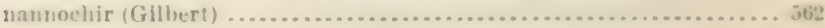

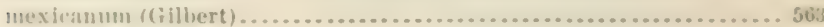

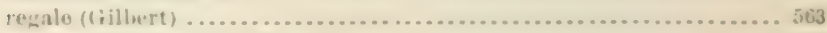

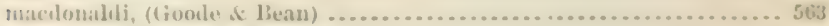

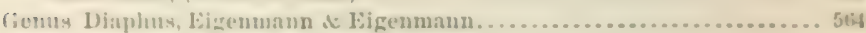

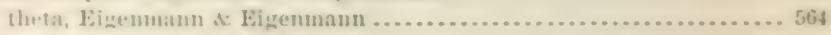

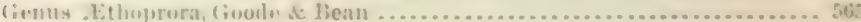

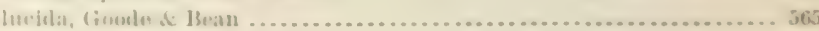

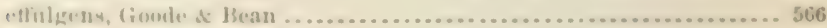

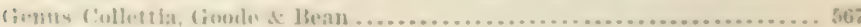

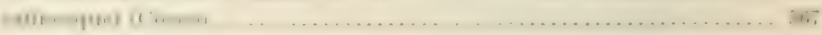

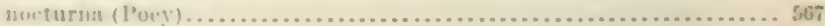

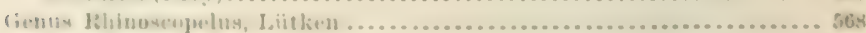

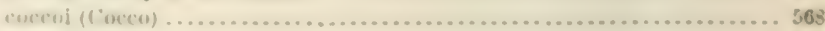

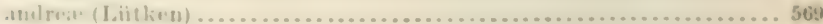

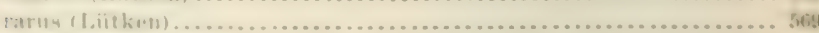

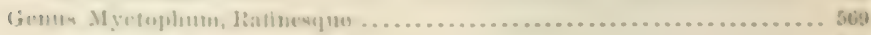

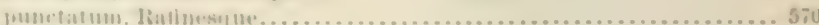

:

up

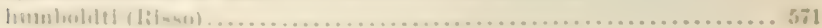


CLASS PISCES-Continued.

SUBCLASS TELEOSTOMI-Continuer.

ORDER INIOMI-Continued.

Family IIyctophide-Continued.

Genus Myetophum, Rafinesquue-Continued.

californiense, Eigenmann \& Eigenmanמ......................... 572

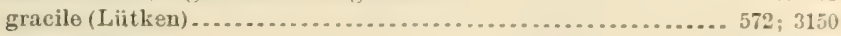

benoiti (Cocco) .................................................. 573

hygomii (Lütken) . ......................................... 573

Genus Benthusema, Goude \& Be:m . . . . . . . . . . . . . . . . . . 5i:

mulleri (Gmelin) ....................................... $5{ }^{4}$

areticum (Liitken) ........................................... $5 i 4$

Genus Dasyseopelus, Gïnther ............................. 5 Tt $^{4}$

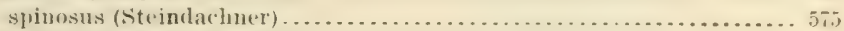

Geuns Tarletonbeania, Eigenmann $\&$ Eigenmann.................... 575

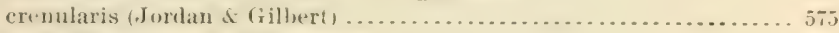
tenuil, Eigrenmann \& Eigenmann . . . . . . . . . . . . . . . . . . . .

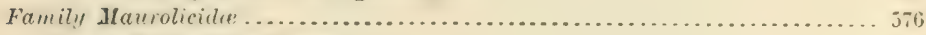

Genus Manrolicus, Coceo ....................................... pennanti (Walbaum) ...................................... 577

Genus Vinciguerria, Jordan \& Erermann ......................... 577 attenuata $($ Cocco) .............................................. 577

Genus Yalenciennellus, Jordan \& Evermann ...................... 577 tripunctulatus (Esmark) ................................

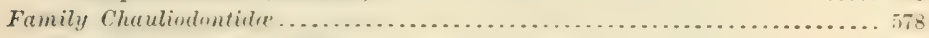

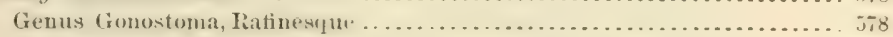

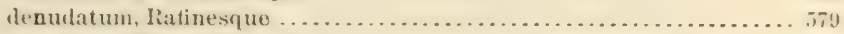
brevidens, Fner of Steimlachner . . . . . . . . . . . . . . . . . . 579

Geuus Zaphotias, Goode \& IBean ........................... 580; 2826 perlaliotus ((ioode $\mathbb{A}$ liean) ......................... 5\&0; $2 \times 26$

Genus Cyclothone, Goode \& Bean ................................ 581

Subgenus Cyelethone ................................. 58:

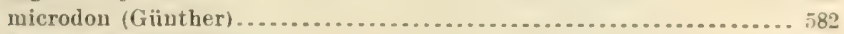
bathyphila (Vaillant) ...................................... $58: 2$

Subgenus sigmops, Gill. .............................. 583

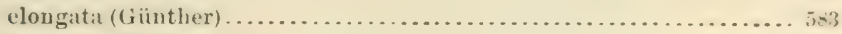
megalops Liitken ...................................... 3150

Genus Yarrella, Goode \& Bean................................... 583 blackforeli, Goode of Bean ... . . . . . . . . . . . . . . . . . . . . . . . . . . . . 584

Genus Clauliolus, Bloch a schueider ...................... ist sloanei, Bloch of sehneider ............................... in 5 macouni, Bean............................................ 585

Family Astronesthida........................................... 586

Genus $A$ stronesthes, Richaritson ......................... $5=6$

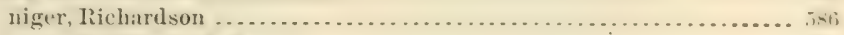

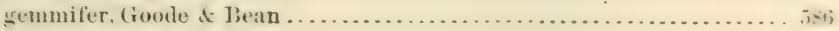

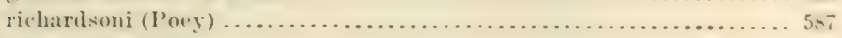

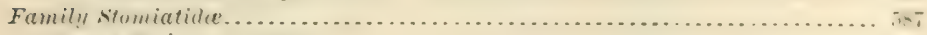

Genus Stoinfas, ('uvier...................................... 5-s

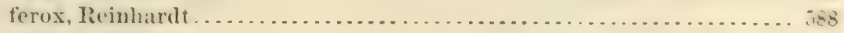

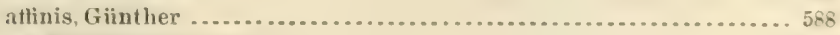

(ienus Echiostoma, Lowe ..................................... is! barbatum, Lowe............................................. 589 margarita, Goode $\&$ Bean.................................... 589

Genus Grammatostomias, Goolo if Bean . . . . . . . . . . . . . . . . . . . . . 590 dentatus, Goole of Bean .................................... 590

Genus. Photonectes, Giinther........................................ 591 gracilis, Goode of Bean ........................................ 591

Bull. No. 47 , pt. $4-\mathbf{I I}$ 


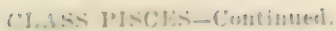

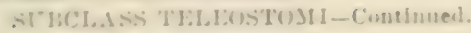

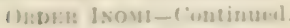

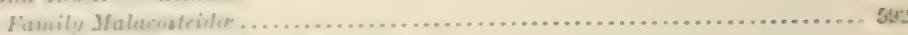

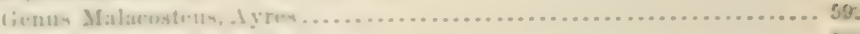

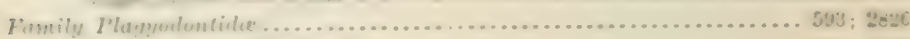

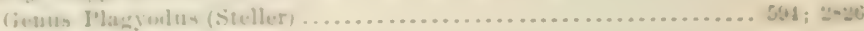

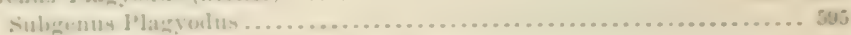

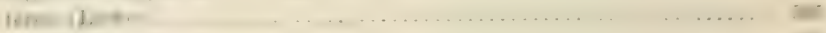

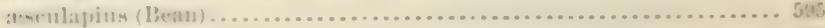

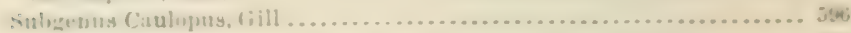

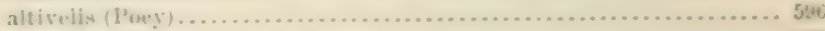

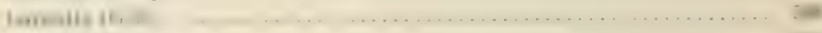

are

fiamily ditontostomiler........................................

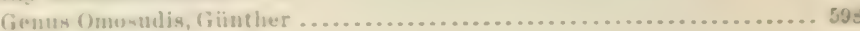

lowii, (Gïnth.r......................................... sose

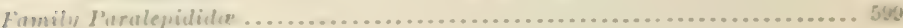

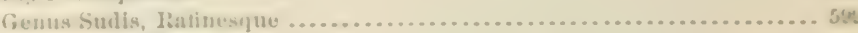

intermediun (l'oey) ........................................... 600

ringen*, Jorklan oc Gilbert.................................... 60u

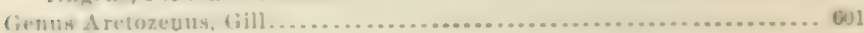

horealis (lieinhardt) ....................................... Gul

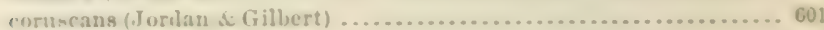

Cemute I'aralepis, IRisso .................................... 602

cormponoides, lisso ........................................ c02

Fanily sternoplyehilie ............................................ 603

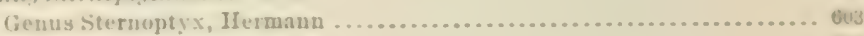

diaphana, Hermann....................................... E

(ienus .Irryrupulecus, Cocco ................................... Gus

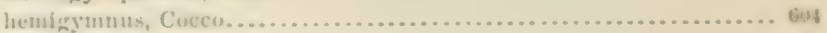

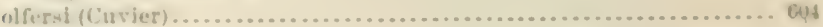

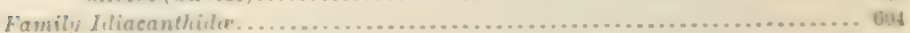

(ivnus Inliaennthus, Feters.................................... coj

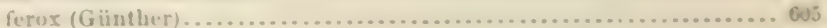

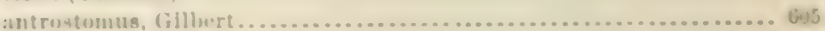

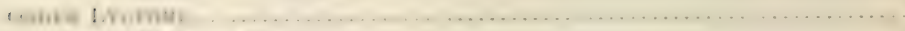

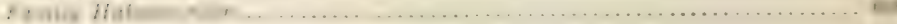

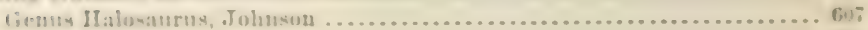

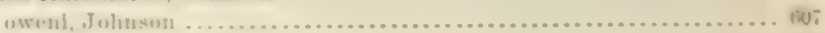

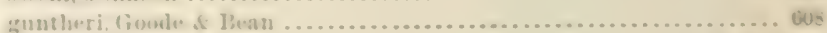

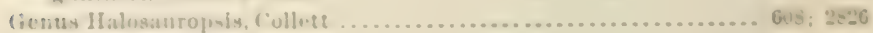

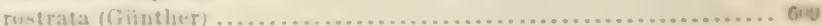

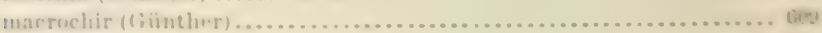

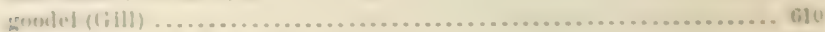

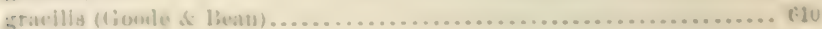

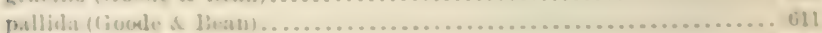

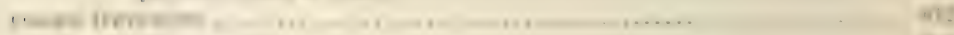

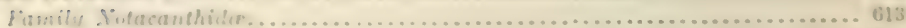

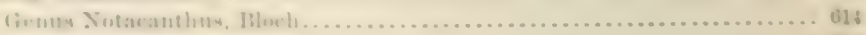

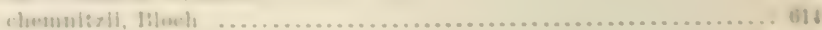

allitili

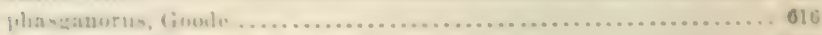

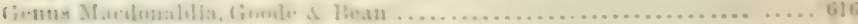

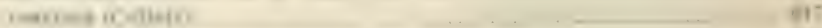

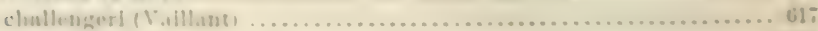

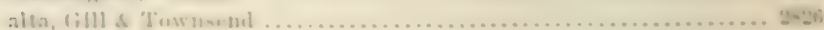

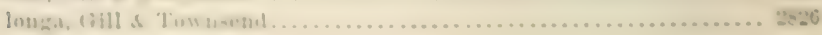


CLASS PISCES-Continued.

SUBCLASS IELEOSTOMI-Continued.

ORDER Heteromi-Continued.

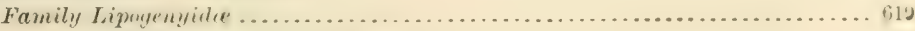

Genus Lipogenys, Goode \& Bean ................................... 619 gillii, Goodo \& Bean .................................... 619

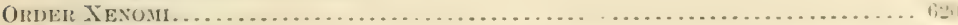

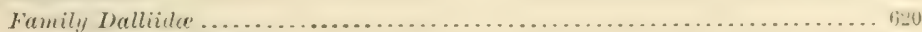

Genus Dallia. Itean ..................................... 621 pectoralis, liean....................................

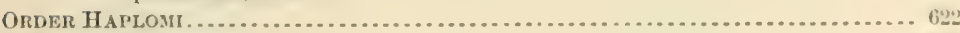

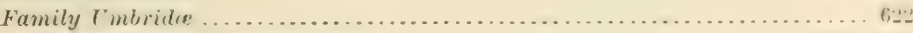

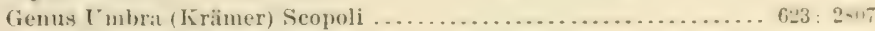

Subgenus Melanura, Agassiz............................... 625

limi $($ Kirtlanil).............................................. 623

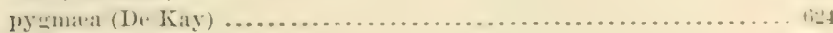

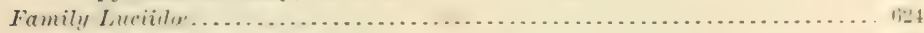

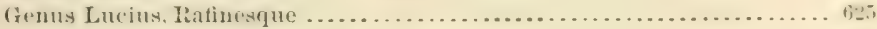

Subgenus Kenoza, Jorủan \& Evermann .......................... (206

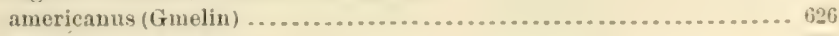

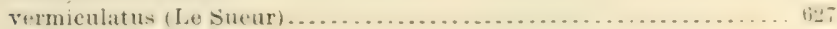

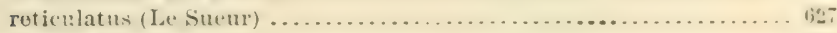

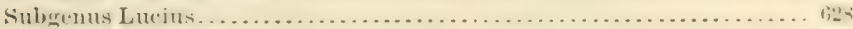

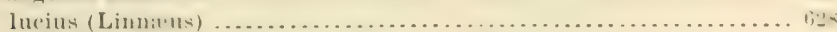

Subgenus Mascalongus, Jordan ............................... 629

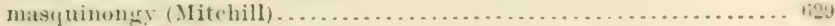

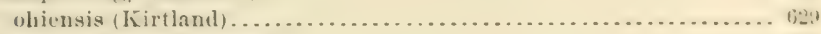

immaculatus (Garrard) ...............................

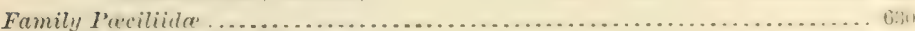

Genus Funduluv, Lace pedo................................

Subgenus Fumlulus ..................................

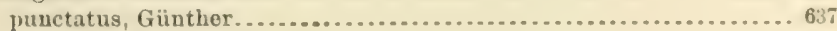

vinctus, Jordan \& Gilbert ................................... $63 \pi$

palliclus, Evermann.......................................... 638

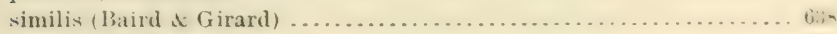

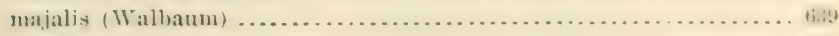

parvipinnis, Girard .........................................610

heteroclitus (Linnous) ....................................6tu

macrolepirlotus (Walbauns) ................................. 64

badius, Garman ......................................... 28.7

grandis (Baird \& Girard) ..............................611; 2827

fonticola, Curier \& Valenciennes ............................. 643

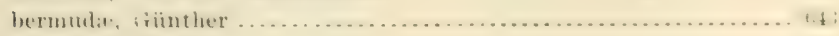

robustus, Bean ............................................ 644

labialis, Giinther....................................................

Subgenus Fontinus, Jordan \& Erermann ......................... 645

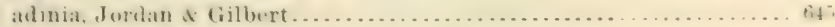

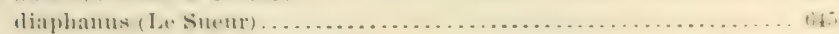

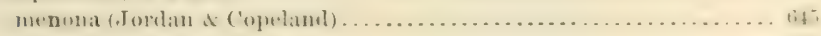

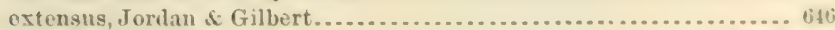

seminolis, Girarl.....................................6 647; 2828

Subgenus 1'lancterus, Garmau................................ 25... zebrinus, Jorkan \& Gilbert............................. 616; 2s.2.

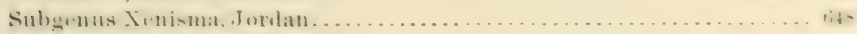

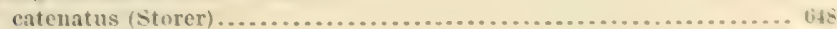

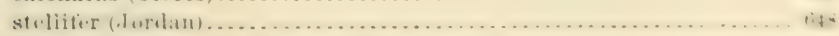

Subgenus Gambusinus, Jordan \& Erermann.................... 6 6t!

rathbuni, Jorilan of Meek ...................................... big 


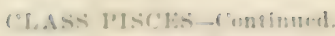

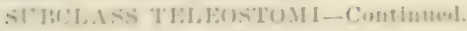

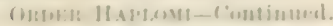

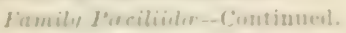

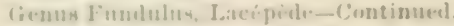

Sulugum Gaulunimu, Jurdan o. Evernann-Continued.

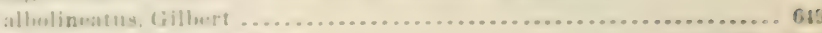

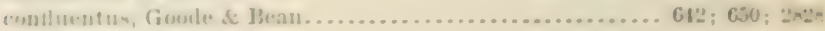

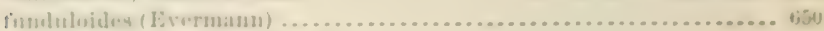

Sulgentus $\%$ ygonet

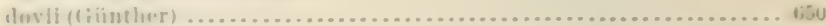

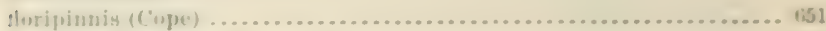

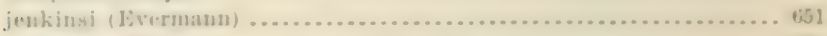

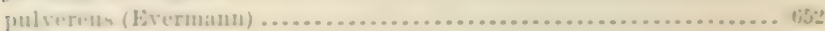

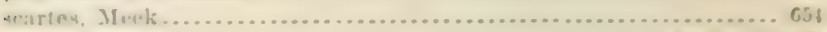

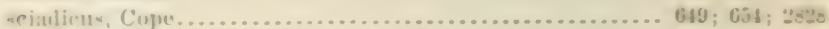

lowim nuнан

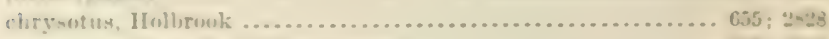

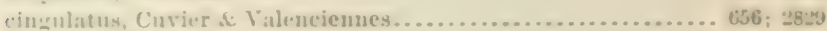

:

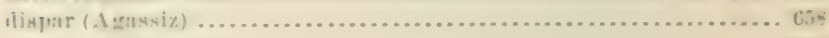

notatus (liatinesque) ........................................ voy

qunculei (Jurilan) .................................... 601; 2331

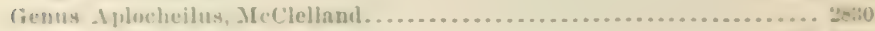

devii (Gibutler) ....................................6. 650; $25 \$ 4$

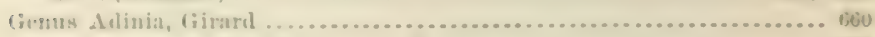

Enatenalensiv (Giinther) ................................ ven

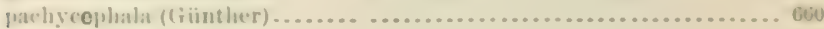

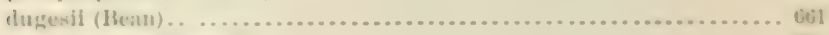

multifanciata, (ifrarif...................................... Gi]

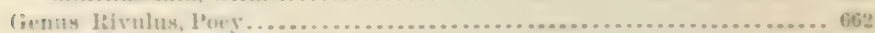

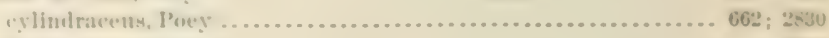

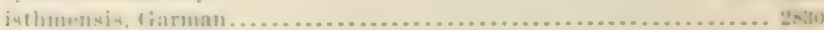

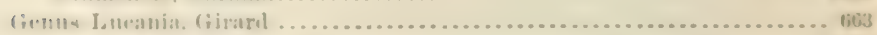

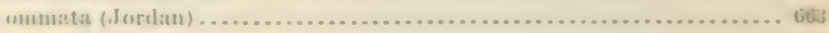

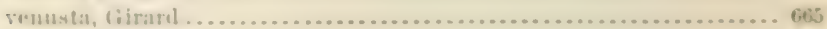

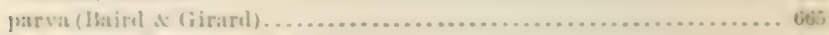

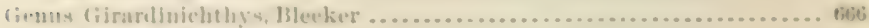

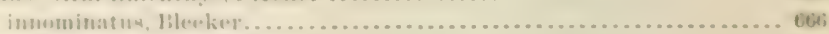

timus Finputrichthys, fillhert............................... Gite

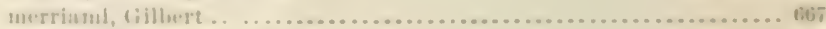

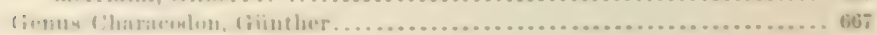

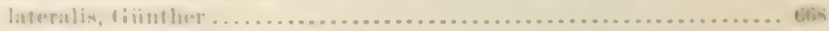

hilinusu

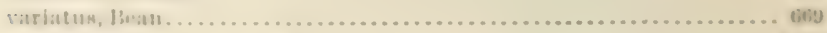

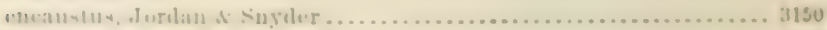

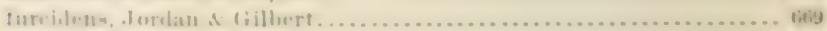

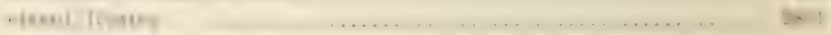

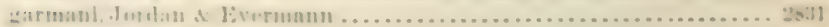

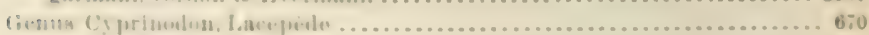

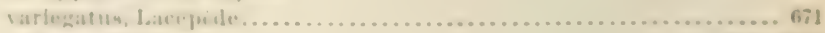

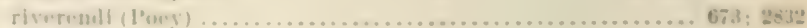

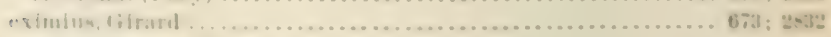

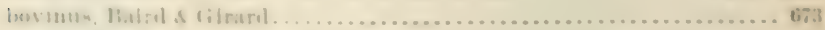

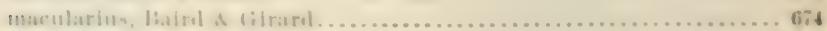

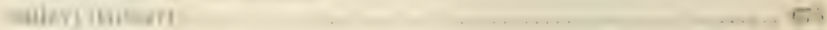

ane qu=11

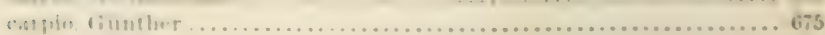


CLASS PISCES-Continued.

SUBCLASS 'TELEOSTOMI-Continned.

ORDER HAPLOMI-Continued.

Family Pociliidoe-Continued.

Genus Cyprinodon, Lacépede-Continued.

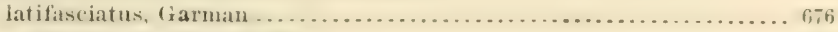

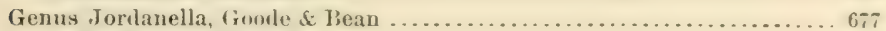
floridx, Goorle \& Bean...................................... 677

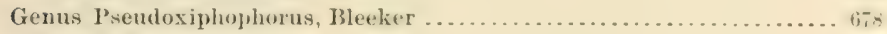
bimarulatus (Heckel) ..................................... ti:

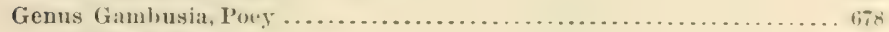
punctata, Poey.......................................... 679

puneticulata. Poes ..................................... tian

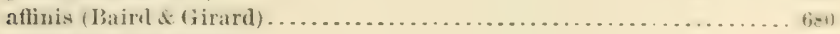

tridentiger, Garman.......................................... 2833

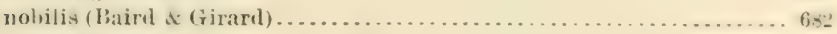

niearaguensis, (iunther ................................. tis?

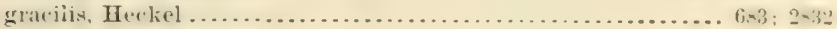

episcopi, Steindachuer..................................6. 683

melapleura (Gosse) .................................... 659; 2830

Genus Belonesox, Kéner ....................................... 684

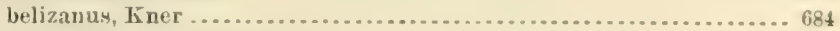

Genus Anableps (Artedi) Scopoli .......................... 68t; 2807 dovii, Gill ................................................. 685

Genus Goodea, Jordan .......................................... 685 atripinnis, Jordan .......................................... 655

Genus Xenendum, Jordan \& Snyder............................. 3151 calierte, Joralan a snyder.............................. xaliscone, Jordan \& Snyder ................................... 3153 Initpoldii, Steindachner ............................ 2832; 3152

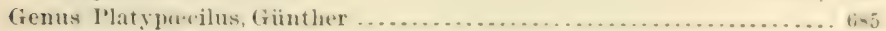

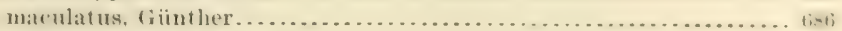
quit zenensis, 13. A. Bean ................................ $2-73$ mentalis, (rill.............................................. tiat

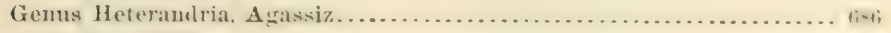

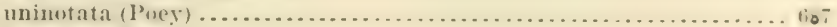

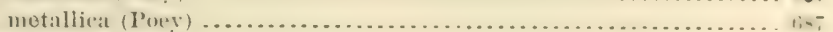

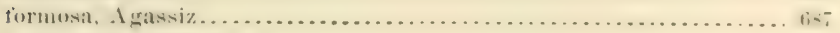

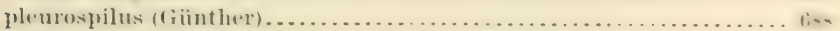

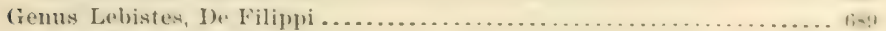

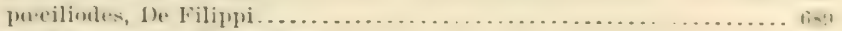

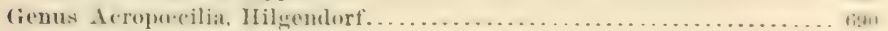

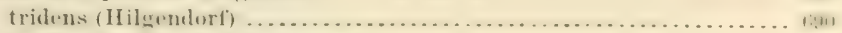

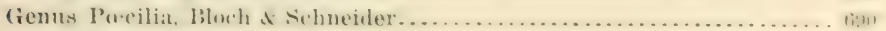
viripara, Bloch of Schneiter................................. 691 butleri, Jortan ........................................... 691

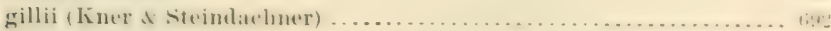
vittata, Guichenot................................... 692: 2333 mexic:ana, sleimlat huer . . . . . . . .

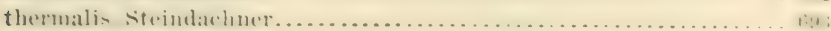
chisoyensis, Giinther.................................... 69. petenensis, Gïnther........................................... 69 .

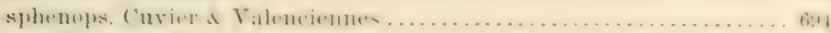

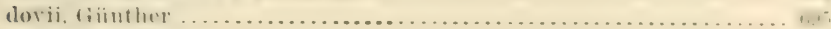

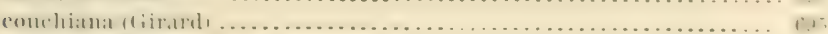

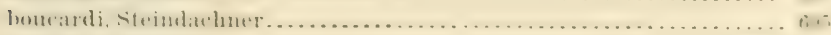

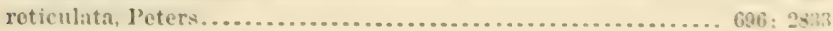
arubensis, Vim Liath de Jeule ............................. 
("I.ASS riscl:S-Cominned.

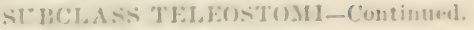

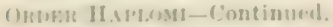

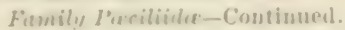

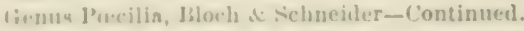

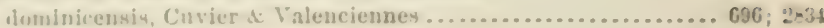

-pilurus, (iiintler ................................... 69 .

- longata, biinther...........................................

frremilionis, Jordan \& Culver............................ 697

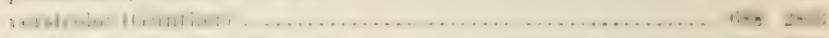

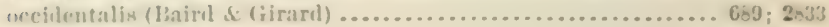

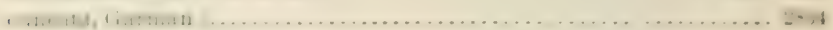

limantonri, Jertan o snyder ............................ 315

fingus Mollienisia. Lo Sueur ............................... 69.5

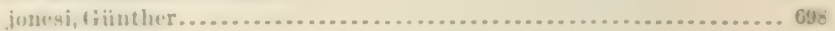

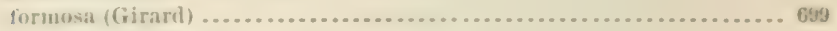

latipinua, Le sueur ........................................ 699

petenensis, Giinther.......................................... 700

(ienus Xiphnphorus, Heckel.................................... 701

brlleri, Heekel .......................................... 701

montezuma, Jordan \& Snyter .............................. 3154

¿nutheri, Jorilan s. Evermann ................................. 70

Fimily 1 mulyoprille ........................................... ive

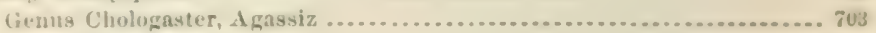

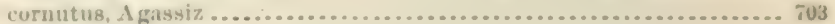

agassizii, P'utnam ........................................... 70

papilliferus, Forbes ...................................... 704

Genns Typhlichthys, Girard....................................... 704

subterranent, (iirard...................................... ius

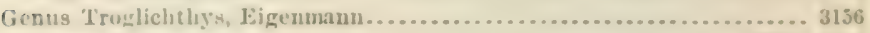
rosat: Eigenmann........................................ 2835; 3150

(jenns Amblyopis, De Kay................................... 706

speliens, De Kay ....................................... 706

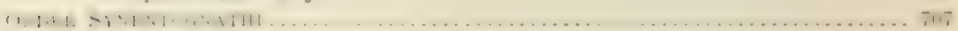

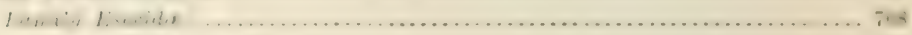

lienus 'Tylosurus, Cocco........................................ T0s

notatus (1'oey) ..............................................

*eapularin, Jordan d Gilbert .............................. 611

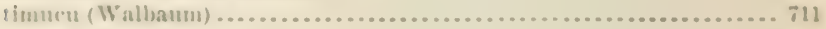

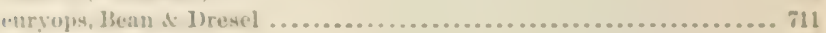

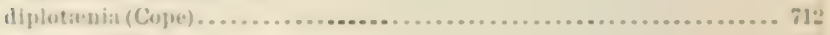

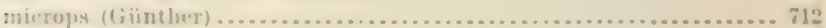

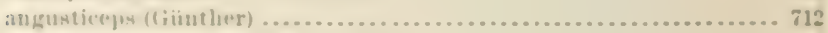

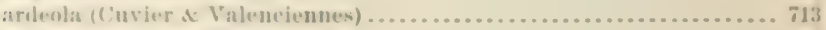

stelzmand (steimlachner) .................................. 813

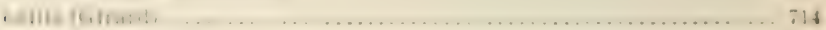

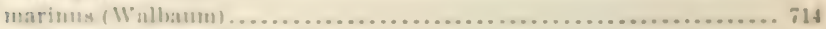

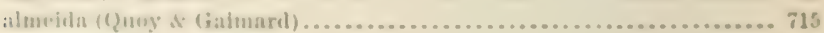

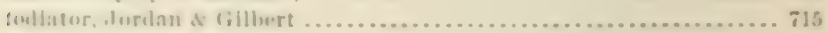

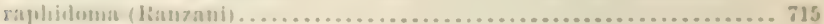

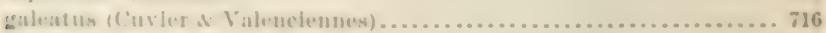

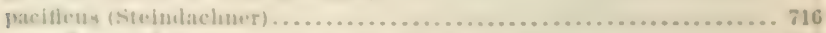



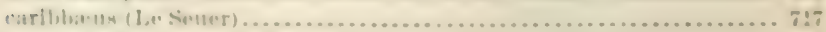

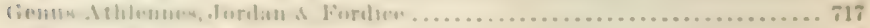

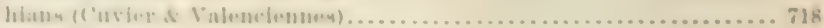

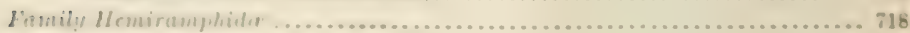

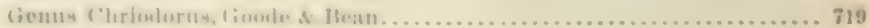

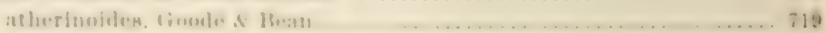




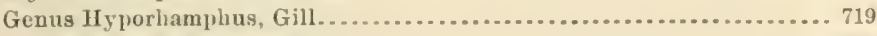

unifasciatus (Ranzani)................................

roberti (Curier \& Valenciennes) ............................. 721

rosi. (Jordan di Gilhert) ..................................... 721

Genus Heniramphus, Cuvier.............................. 722

brasiliensis (Linna'us) ............................... 722

balao, Le Sueur............................................ 723

Geuus Euleptorhamphus, Gill.................................. 723

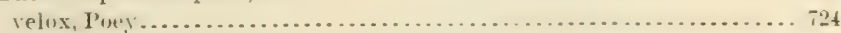

Family Scombresncide ........................................... 72.

Genus scombresox, Lacipide ................................ 725

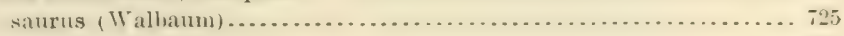

(ienus Cololabis, Gill ......................................

brevirostris (Peters) ......................................

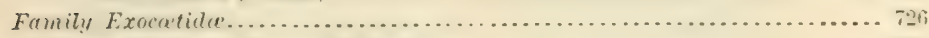

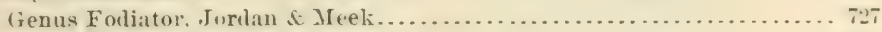
acutus (Cuvier \& Valenciennes) ............................ 728

Genus Paroxocctus, Blecker................................... 728 mesogaster $($ Bloch) ........................................... 728

Genus Exocœtus, Linnaens............................... 730; 2835 rolitans, Linnæus............................... $729 ; 734 ; 2835$

Genus Cypsilurus, Strainson ..................................... 2835

heterurus (Rafinesque) ............................... 735; 2836

lutkeni (Jordan \& Evermann) . . . . . . . . . .................. 736; 2836

fureatus (Mitrhill) .............................. 737 ; 28.31 ;

nigricans (Bennett) ............................. $737 ; 2836$

xenopterus (Gilbert) .................................. 738; 2836

lineatus (Cuvier \& Valenciennes) ........................ 739; 2836

cyanopterus (Cuvier \& Valenciennes) .................... 739; 2836

bahiensis (Ranzani) ..................................... 739; 2830

califoruicus (Coper) ............................. $7+11: 28,66$

calliopterus (Güinther)............................. 740; 2836

gibbifrons (Cuvier \& Valenciennes)..................... 741; 2836

Gemus Exonantes, Jordan \& Evermann ............................. 2835

exsiliens (P. L.S. Mitiller)............................... 732; 2830

rondeletii (Cuvier \& Valenciennes) ....................... 733; 2836

vinciguerro (Jorlan $\&$ Meek) .......................... 734; 28.36

rufipinnis (Curier \& Valenciennes) ...................... 735; 2836

aftinis (Giintber) .......................................

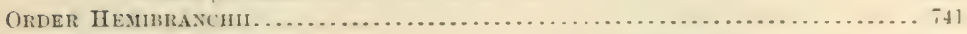

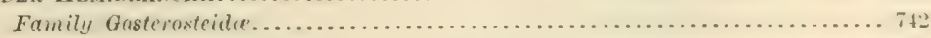

Genus Eucalia, Jordan...................................... 743

inconstans (Kirtland) .............................. $74 ; 3157$

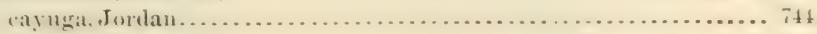

pygmica (Agassiz) ....................................... 74

Genus Prgosteus, Brevoort ...................................... 745

pungitius (Linneus) ...................................... 745

brachrpoda (Bean) ....................................... $t 40$

Genus Gasterosteus (Artedi) Linnæus ............................. t .

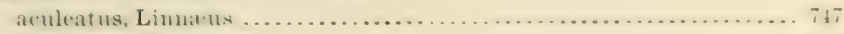

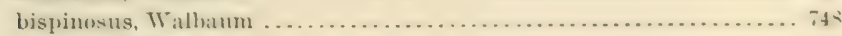

atkinsii (Bean) ........................................... 74

curieri (Giram) ......................................... it?

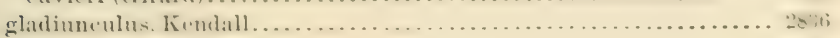

cataphractus (Pallas) .................................. 749 


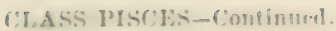

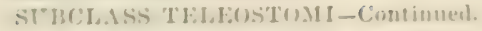

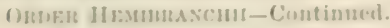

Fanily Gasteruste ider-Continumi.

firnus Gasterostrus (Artedi) L.innarns-Continued.

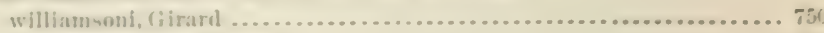

mirroeephalus ((iirarl) ................................. 751

(ienus A preltes. 1)e Kay ......................................... 75:

quatracus (Jitchill) ..................................... 752

Fismily .1 ulorhynchide ........................................... 752

Gients Anlwrlynuhus, Gill ....................................... 753

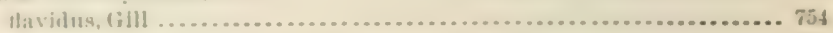

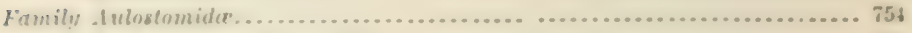

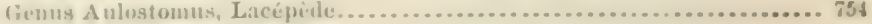

marulatus, Valenciennes...................................... 154

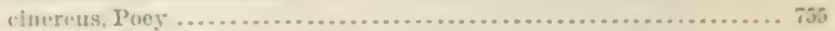

Family Fidulariule.............................................. 75

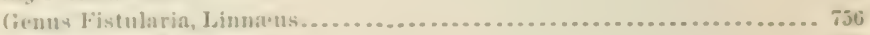

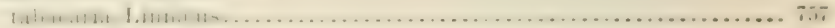

depressa, (iintler.......................................... 757

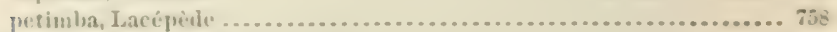

Family Macrorhamphosidw .......................................... 75

Geuns Macrorhamphosus, Lacé pèlo .............................. 750

Acolopax (Linnæus) .................................... 75

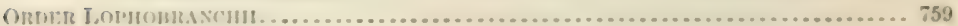

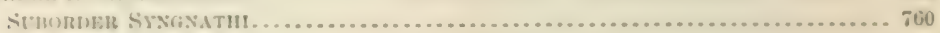

Family simmathide ........................................... $7 \sigma_{0}$

Cienus Siplustoma, Rafinegque ................................ T61

Sulyenus Dermatostethus, Gill .............................. 763

punetipinne (Gill) ......................................... 76 .

Suluesus Siphostoua..................................... ti

carinatum, (jillert ....................................... 7ن

(nalibrniense (storer) ....................................... 76t

griseolineatum (Ayreg) ................................... 664

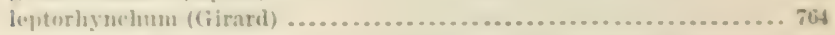

fiмtulatum (l'eters) ...................................... i

burbara", swain ............................................ tus

mackuy Swain o Meek.................................. 766

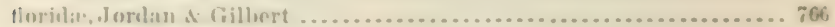

poexi, Jordan \& Evermunn.................................... 766

anliмсия, Swaiı ..............................................

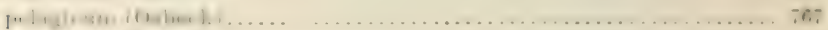

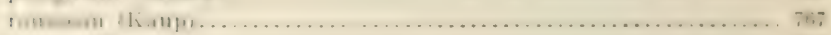

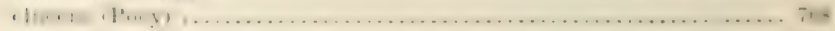

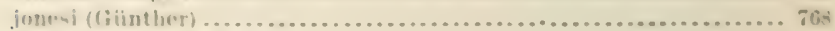

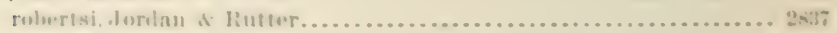

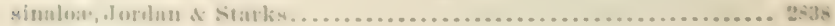

|rath

all . I I

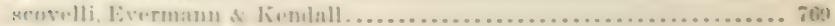

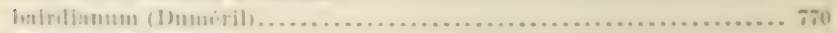

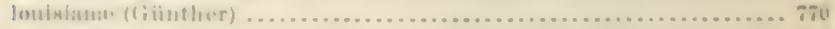

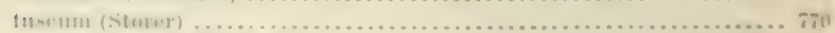

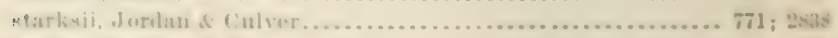

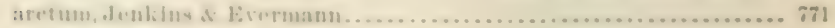

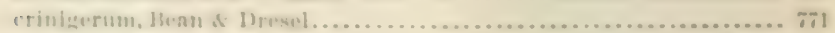

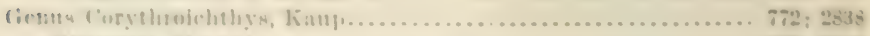

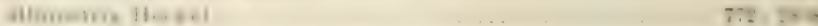

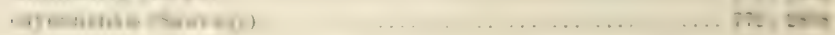


CLASS PISCES-Continued.

SUSCLASS TELEOSTOMI-Continued.

ORDER-LOPHOBRanCHI-Continued.

Suborder Sragnathi-Continued.

Family Syngnathide-Continued.

Genus Corythroichthys, Kaup-Continued.

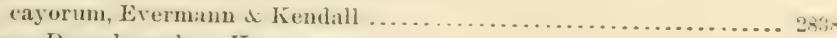

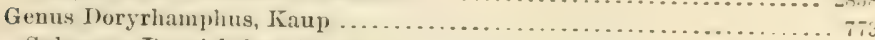

Subgenus Doryichthys, Kaup.................................

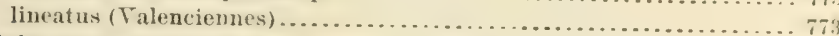

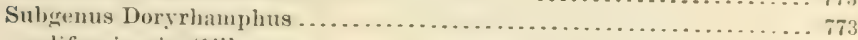

ealiforniensis, Gill .........................................

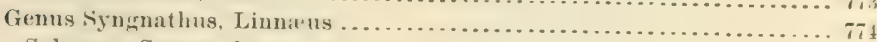

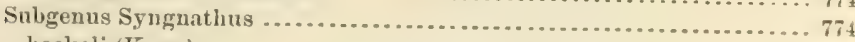

heckeli (Kaup) .........................................

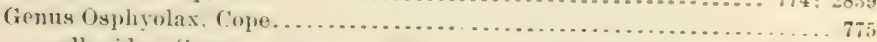

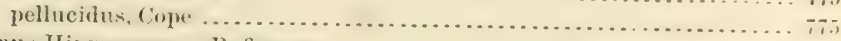

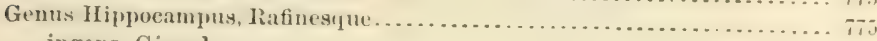

ingens, Girard ............................................ 776

hudsonius, In Kay . . . . . . . . . . . . .

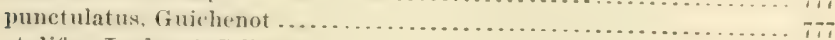

stylifer, Jordan \& Gilbert....................................... . 778

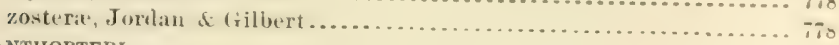

ORDER ACANTHOPTERT . . . . . . . . . . . . . .

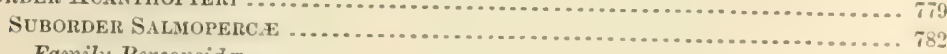

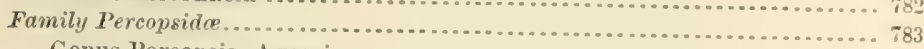

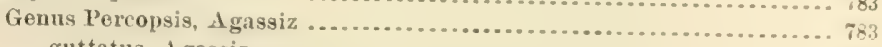

guttatus, 1 gassiz ........................................... $78 t$

Genus Columbia, Eigenmann $\&$ Eigenmamn ............................ 784

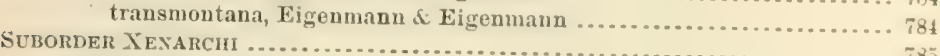

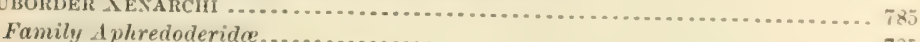

Genus A phredoderus, Lo Sneur

sayanus (Gilliams).

Suborder Percesoces.

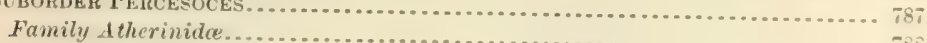

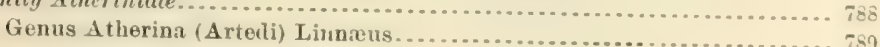

stipes, Miiller \& Troschel

............. 790

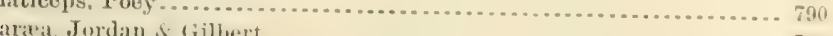

harringtonensis, Goode ......................................

carolina, Curier \& Valenciennes................................... 791

microps, Poey ............................................... 791

Genus Chirostoma, Swairson ............................ $792 ;-839$

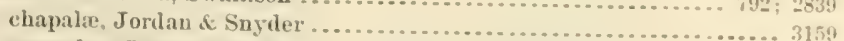

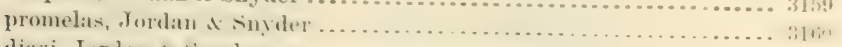

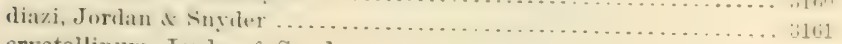

crystallinum, Jordan $\&$ Snyder ................................ 3160

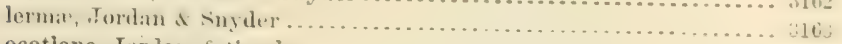

ocotiane, Jorlan of suyder...................................... 3163

estor, Jordan........................................ 792; $2839 ; 316$.

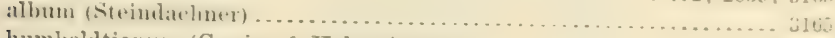

humboldtianm (Cuvier \& Talenciennes) .................. 793 ; 2839 grandocule (Steindachner) . . . . . . . . . . . . . . . . . . . . . . . . . . 2839

Genus Eslopsarmm, Jordan ․ Evermann . . . . . . . . . . . . . . . . . . . . . 2840

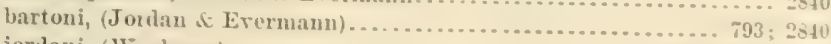
jorlani, (Woolman) ............................... $793 ; 2840 ; 3159$ arge, Jordan \& Snyiler................................... 3158 


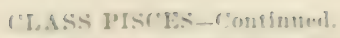

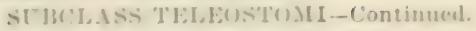
(moms Acaxthoptust-Continued.

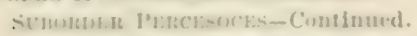

Fitmilu Atherinile-Consinuet.

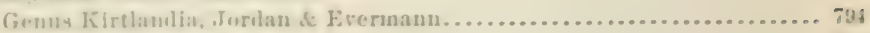

vagraus (finde \& Bean) ................................ 794

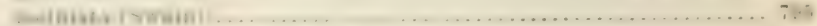

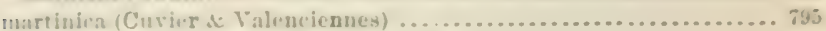

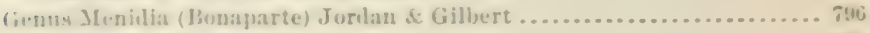

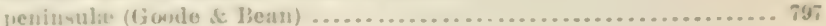

"racilis (liïnther) .................................... 797

lneryllina (cope) ..................................... 7q

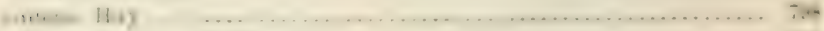

Eflle.rt, Jurdan of Bollman ............................. 790

sardina (Jenkins of Evernann) . ......................... 799

menielia (I.innatus) ...................................... 800

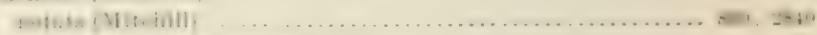

clara, Evrrnann di Jenkins............................... 8us

Ginns I.

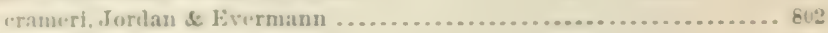

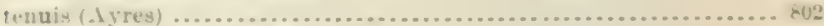

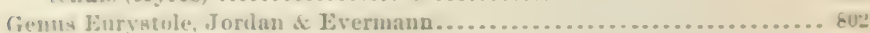

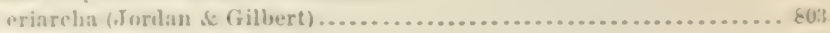

Gicun Thvrina, Jorilan \& C'ulver................................... dus

everuanu, Jurdan of Culver............................... 8us

crvatallina, Jorilan of Culver............................... cut

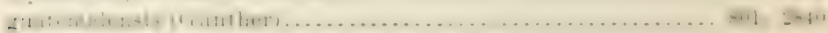
pachylepis (tiinther) .............................. 801; 2840

fienny Atherinella, Steindachner................................ d do

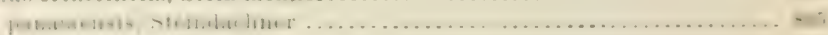

fienus I.alidesthem, Cope ..................................... eus

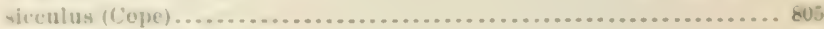

(intur Atherinopsis, Girard ..................................... sot californiensis, Girark .................................... 806

firmus Atherinops, steindachner ............................... 80 i

виния

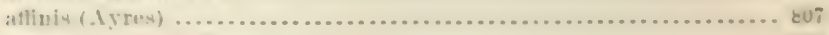

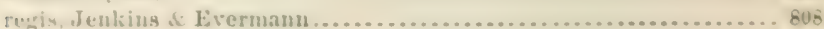

fintily, Jfurilith ........................................... vus

(iemu Mlugil (Arteli) Linnatus.............................. 809 lrambllensis. $A$ gassiz........................................ 810

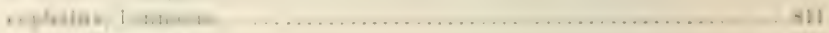

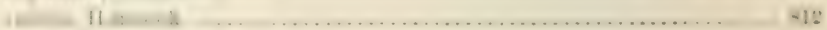

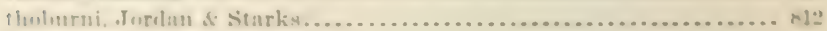

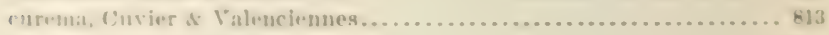
bumpes, Jurdan of Ginlver..................................... sis

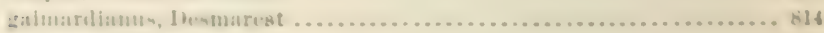

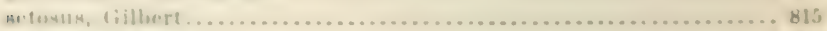

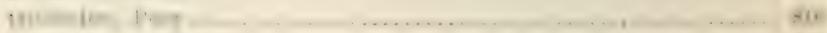

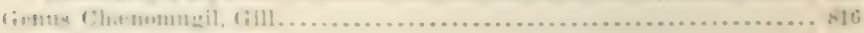

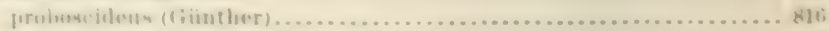

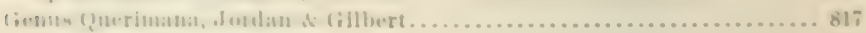

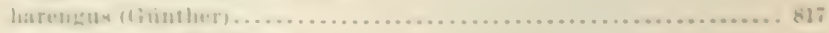

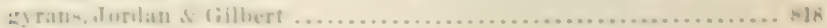

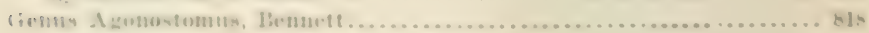

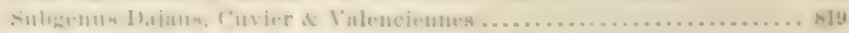

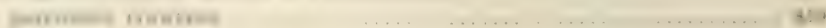


CLASS PISCES-Continued.

SUBCLASS TELEOSTOMI-Continuel.

Page.

Order acaNthopter?-Continued.

SUBORDER P'ERCESOCES-Continued.

Family II gilide-Continued.

Genus Agonostomus, Bennett-Continued.

Subgenus Dajaus, Cuvier \& Valeneiennes-Continued.

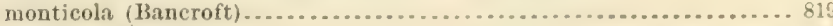

nisutus, (iinther . . . . . . . . . . . . . . . . . . . . . . . . . . . 819

microps, Günther ............................................. 820

Genns Joturus, Poey........................................... 820

pichardi, Poey ......................................... 21

Genus Neomugil, Vaillant ..................................... 3165

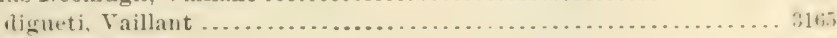

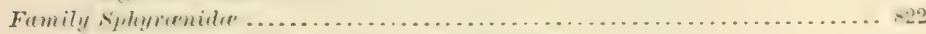

Genus Sphyriena (Artedi) Bloch \& Schneider....................... 823 barracuda (Walbaum) ................................ 823; 2841

eusis, Jorilan \& (ilbert ...................................

guachancho, Curier \& Valenciennes......................... 824

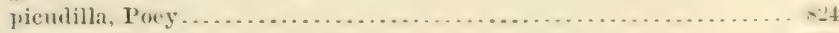

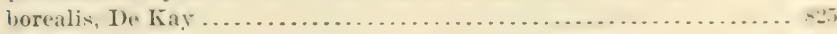

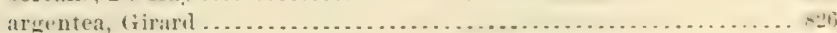

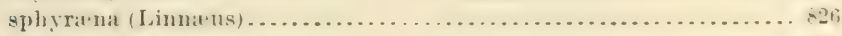

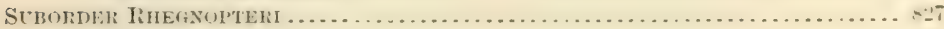

Family Polynemide ................................................ 827

Genus Polynemus (Gronow) Linnæus.............................. 828

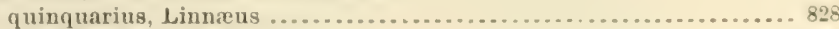

(ienus Polydatylus, Lacepede............................. 8 : approximans (Lay of Bemett) ............................

virginicus (Linneus) ................................

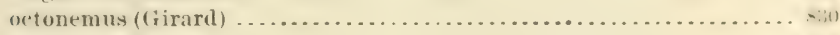

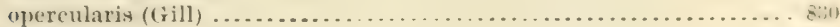

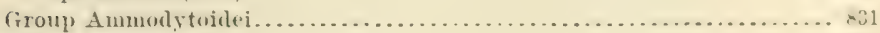

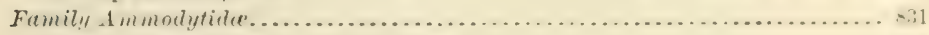

Genus Ammodytes (Artedi) Linnaus............................... 832

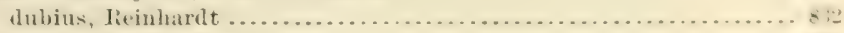
amerieanus, De Kay .....................................

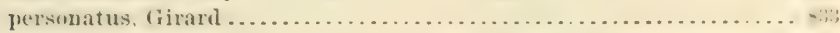

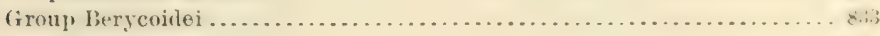

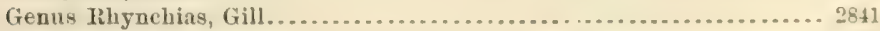

setipinnis (Pallas) .......................................

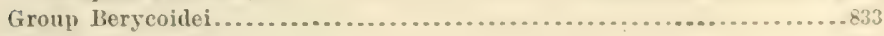

Family Bathyclupeide ............................................. 83

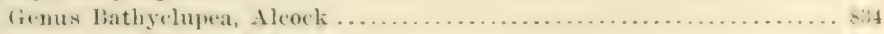

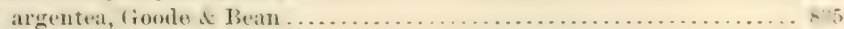

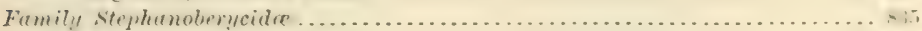

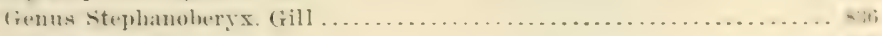

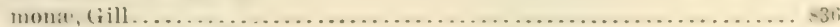

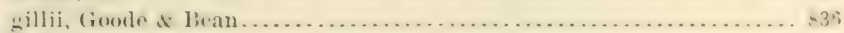

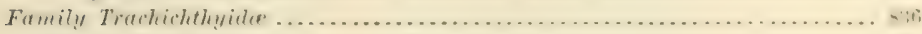

Genus Hoplostethns, Cuvier of Talenciennes ........................ 837 meliterraneus, Curjer $\&$ Valenciennes...................... 837

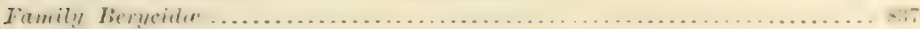

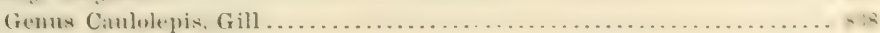

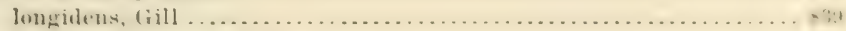

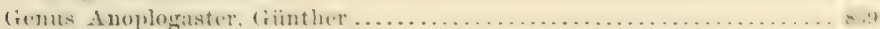

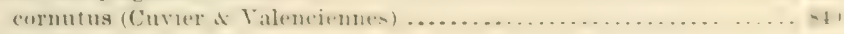

Genus Poromitra, Goode of Benn................................ 840

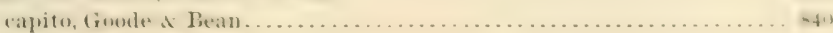




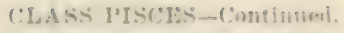

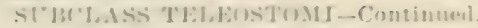

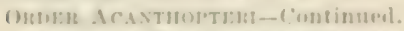

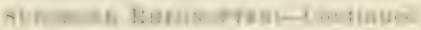

Family Jierncider-Cominuml.

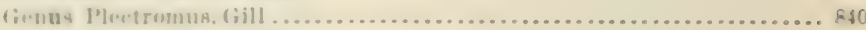

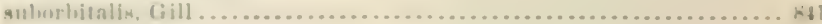

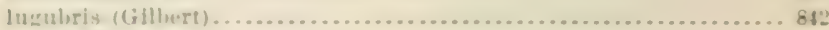

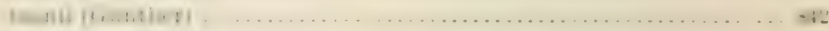

crasslerpa, diinther ...................................... 813

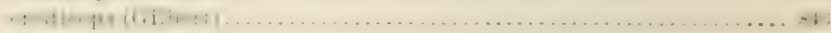

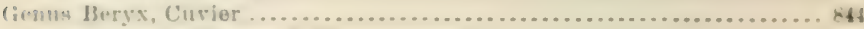

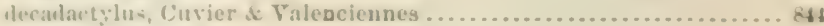

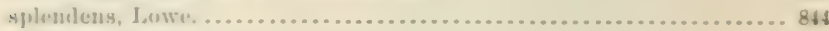

Family Jlulocentrider ............................................. $8 \$ 5$

(ienus Ilvripristis, Cuvier.................................... $88_{8}$

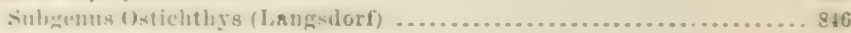

irachypoma, (iintler ........................................... \&

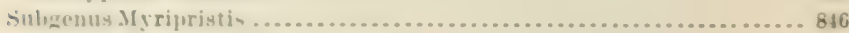

.jacolus, Cuvier s Valencienues............................ 8 .

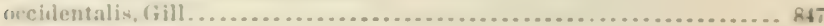

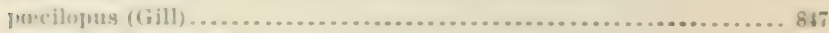

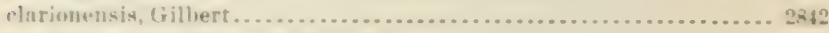

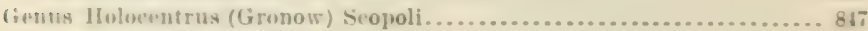

ascennionis (1)sbeck) ........................................ 818

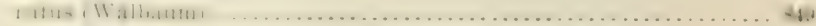

sleciter, Copre............................................. 848

sulorbitalis, Gill ........................................ $8=0$

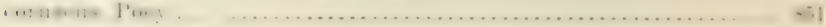

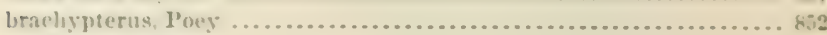

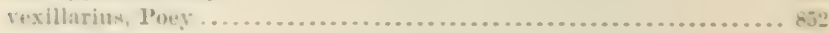

osenlus, l'oev . ............................................. 853

sancti.pauli, (riinther ..................................... 853

(iemus Flammen, Jorilan \& Fvermann .................... 852; 2571 marianus (Cuvier of Yoleneipnneg) ........................ 852: 2571

fienus Plectrypeps, (iill .................................... 853

retronpinis (liniehenut) ...................................... $85:$

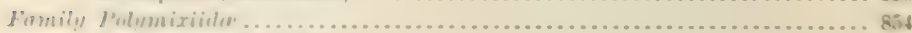

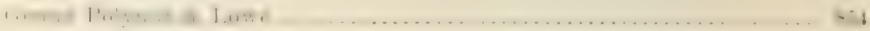

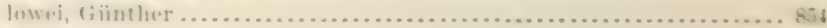

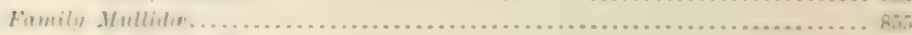

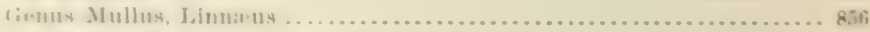

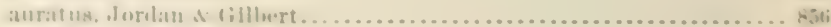

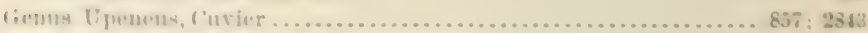

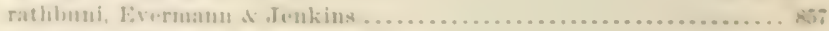

mank

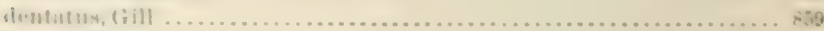

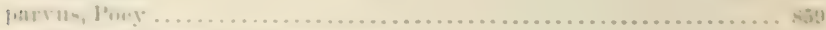

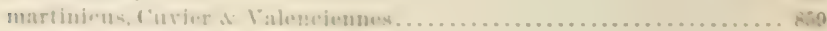

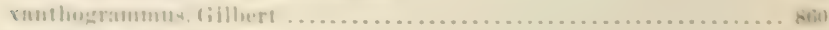

:

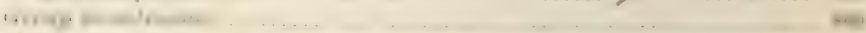

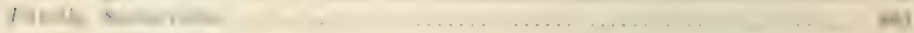

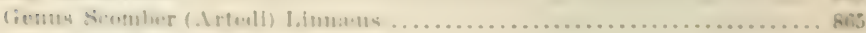

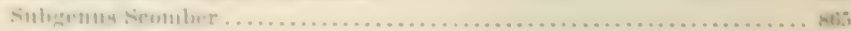

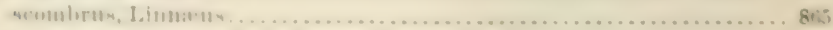

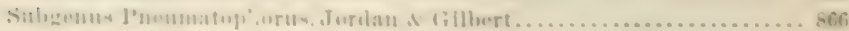

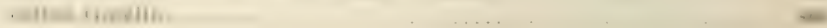


CLASS PISCES-Continued.

SUBCLASS TELEOSTOMI-Continuerl.

Order ACANTHOpteri-Continued.

Suborder RHegnopteri-Continued.

Family Scombrida-Continued.

Genus duxis, Cuvier.

thazard (Lacépède)

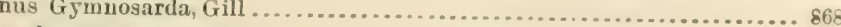

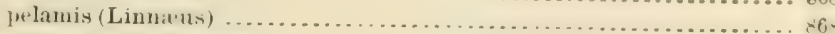

alleterata (IRatine-sque) . . . . . . . . . . . . . . . . . . . . . . . 869

Genus Thunnus, South........................................ 869

thynmus (Linnilus) . . . . . . . . . . . . . . . . . . . . . . . . . . . biu

Genus Germo, Jorlan. .............................................. 870

alalunga (Gmelin) ......................................... 871

Genus Sarda, Cuvier.............................................. 871

sarda (Bloch) ............................................. 872

chilensis, Cuvier \& Valenciennes............................... 872

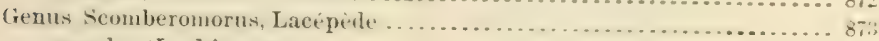
concolor (Lockington) . . ................................... 873

maculatus (Mitchili) ......................................

sierra, Jorlan d Starks................................ 8 it

regalis (Bloch) ........................................... 875

cavalla (Cuvier \& Valeuciennes) .................................. 875

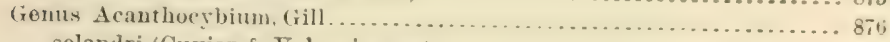

solandri (Cuvier \& Valenciennes) ................................ 876

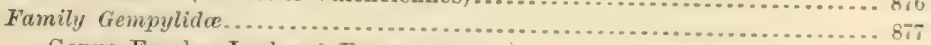

Genus Escolar, Jordan \& Eremann .......................... $878 ; 2843$ violaceus (Bean) ...................................... $878 ; 2843$

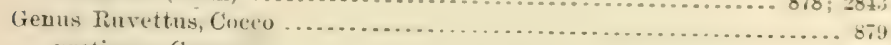

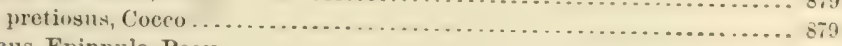

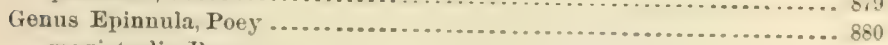
magistralis, Poey ............................................. 880

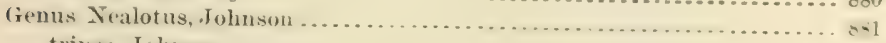

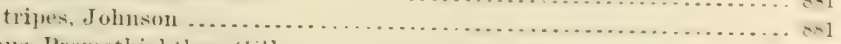

Genus Promethichthy, (iill . . . . . . . . . . . . . . . . . . . . . . prometheus, Cuvier $\&$ Valencienues.............................. 882 parvipinnis (Goode \& Bean) . . .................................... 883

Genus Gempslus, Cuvier \& Valenciennes. . . . . . . . . . . . . . . . . . . . . . 883 serpens, Cuvier \& Valenciennes .................................. 8 s $_{4}$

Family Lepidopide ................................................... 884

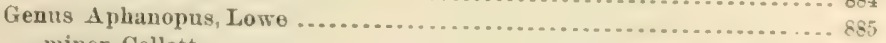
minor, Collett...................................................... 885

Gemus Evoxymetopon, Poey . . . . . . . . . . . . . . . . . . . . .

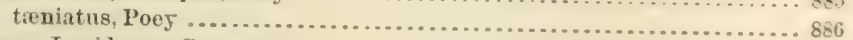

Genus Lepidopus, Gouan ........................................... 886

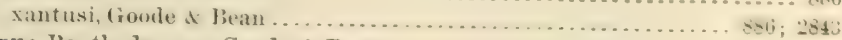

Genus Benthodesmus, Goode \& Bean ................................ 88 . atlanticus, Goode if Bean ............................................ 88

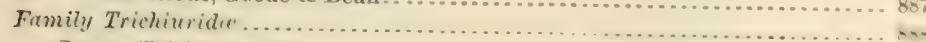

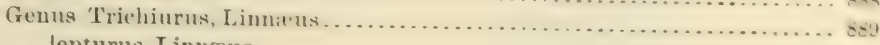
lepturus, Linnreus............................................. 889

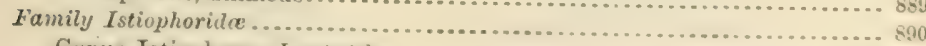

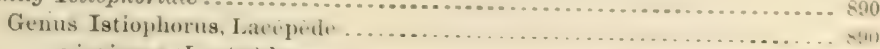

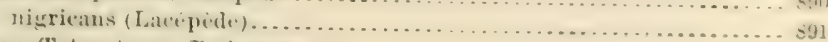

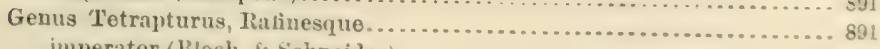
imperitor (Bloch of Schneider) ............................... 890

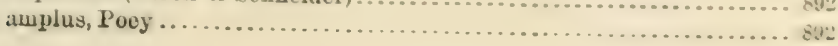


r:P.ASS J'ISCRS-COntinued

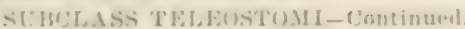

(omen . JeAsthol't:M-C'antisme-

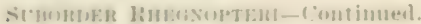

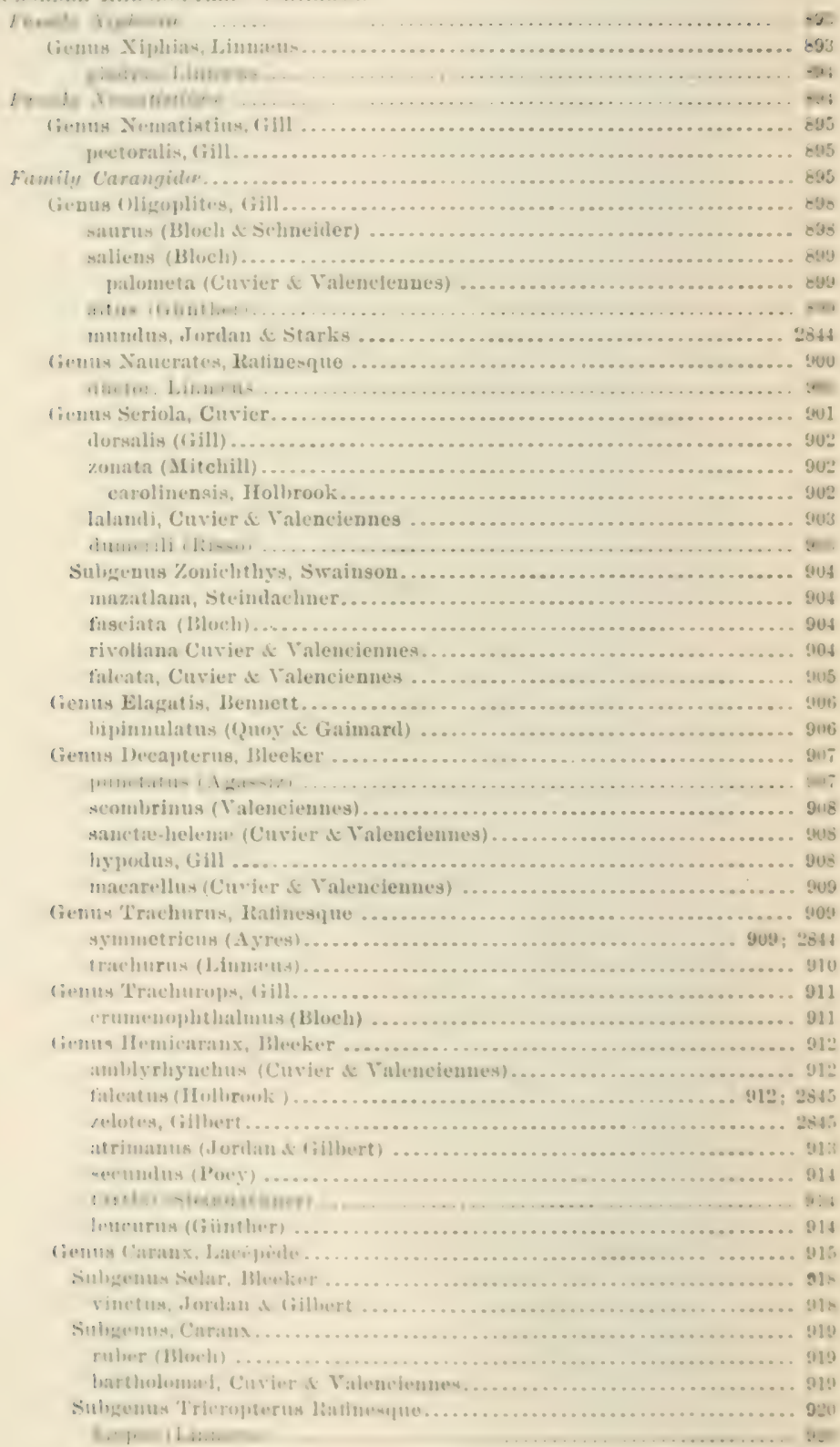


Order lCanthopteri-Continued.

SUBORDER RHEGNOPTERI-Continued.

Family Carangida-Continued.

Genus Caranx, Lacépède-Continued.

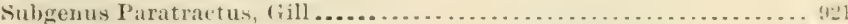

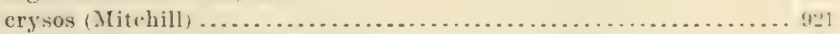

pisquetus, Cuvier \& Valenciennes....................... 921; 2816

caballus (tiinther) . .................................... $y_{21}$

Subgenus Carangichthys, Bleeker............................... 922

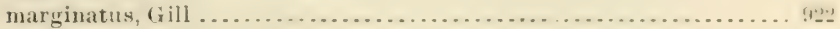

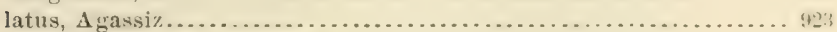

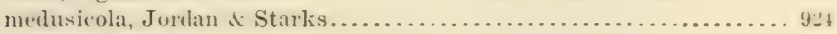

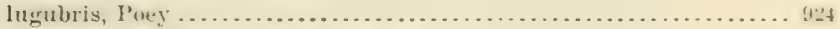

melampynus, Cuvier \& Valencjemes. .......................

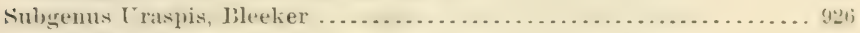

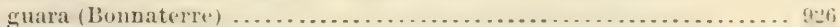

Genus Ginathanodon, Blecker.............................. 927

speciosus (Forskál) ............................................ 928

Genus Carangoilles. Bleeker ................................ gyzh

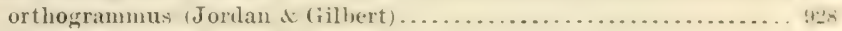

Genus Citula, Cuvier .......................................

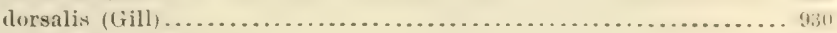

Genus Alectis, Ratinesque.................................... 931 ciliaris $($ Bloch) ................................................. 931

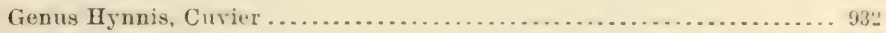

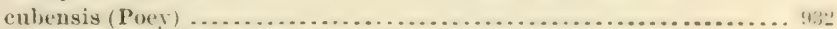

hopkinsi, Jorlan \& Starks................................... 93:

Genus Vomer, Cuvier \& Valenciennes ........................ dorsalis, Gill ..............................................

setipimis (Mitcinill) ...................................... 134

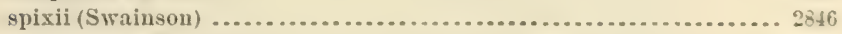

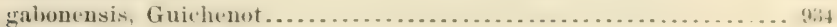

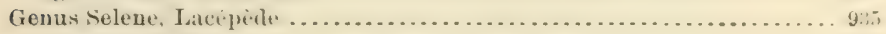

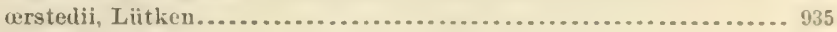

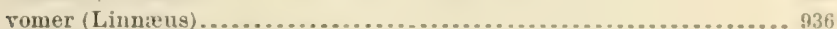

Genns Chloroscombrus, firard . ................................

orqueta, Jordan \& Gilbert .................................... 937

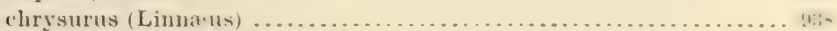

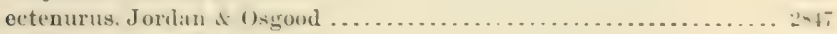

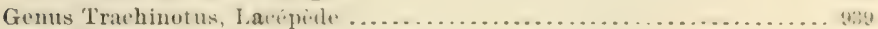

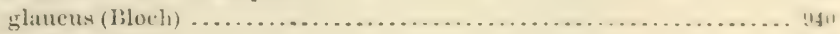

rhodopus, (iill .........................................

falcatus (Linnatus) ....................................... 911

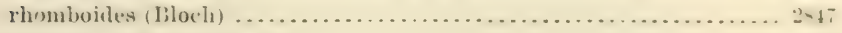

culreri, Jordan \& Starks..................................... 94:

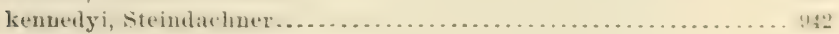

goodei, Jorilan \& Evermann................................. 943

argenteus, Cuvier $\&$ Yalenciennes ............................ 94t

earolinus (Linnitus) .....................................

paloma, Jurdan it Starlis................................

cayemensis, Curier \& Valenciennes ............................. 945

Genus Zalocys, Jordan \& J JeGregor .............................. $2 s \pm 8$

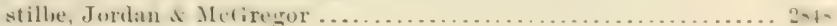

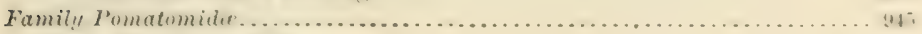

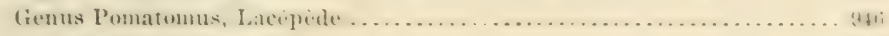

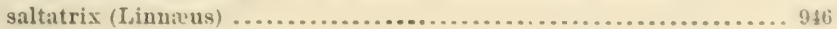




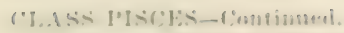

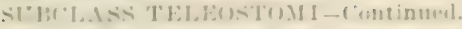

()

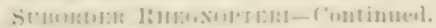

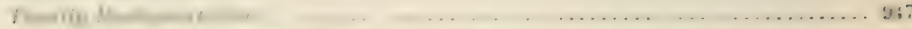

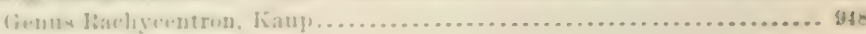

.

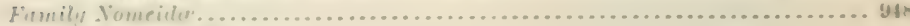

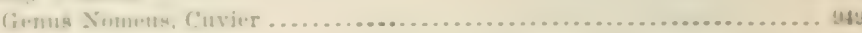

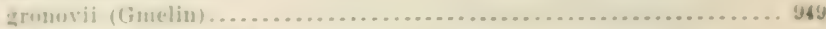

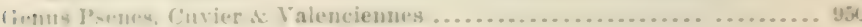

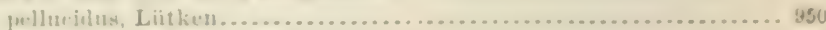

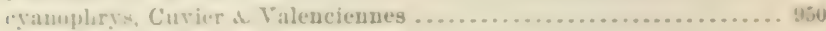

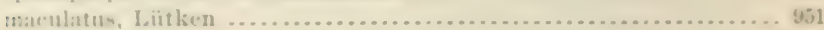

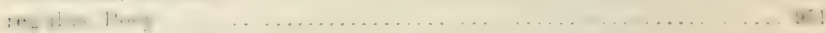

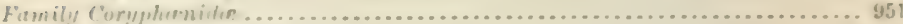

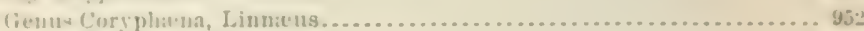

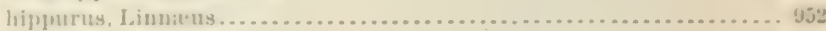

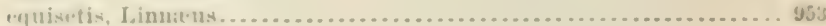

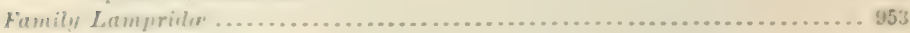

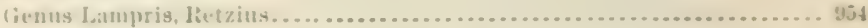

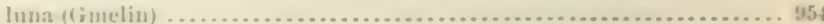

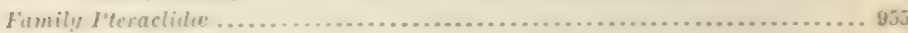

fienus l'feraclis, fironuw ...................................... 955

(arolisus, Cuvi.r \& Valenciennes.......................... 456

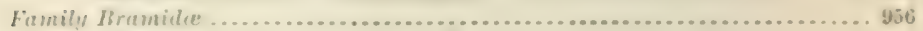

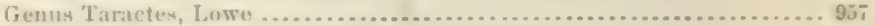

saussurii (Iaunel) ......................................

(ienus Jirama, Blueh \& Schneiler................................ 958

agasvizii, I'oty ....

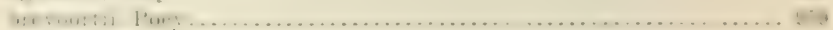

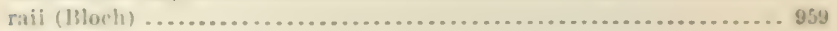

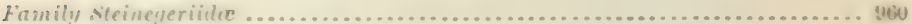

lienus Steinegeria, Jorlan \&. Evermann ......................... 860

ruluscens, Jurdan \& Evermann............................. 901

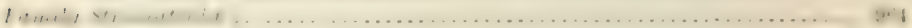

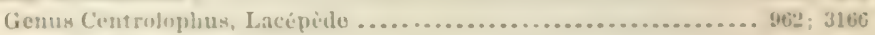

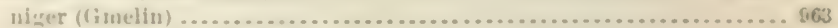

(ienus l'alimurichthys, blecker ............................... vo

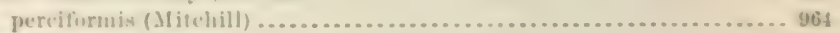

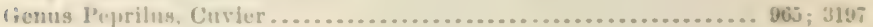

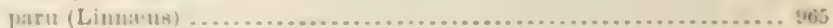

xintlurum (1)

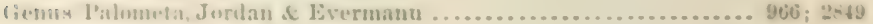

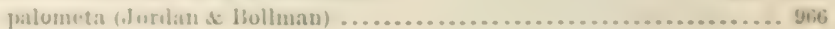

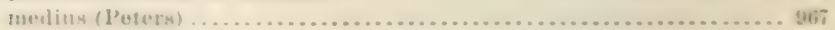

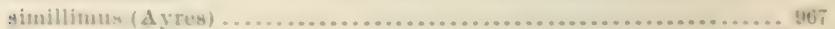

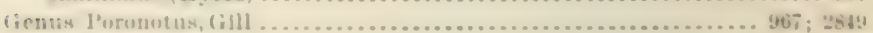

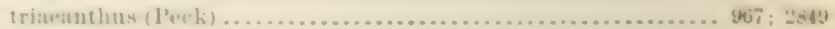

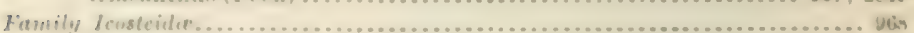

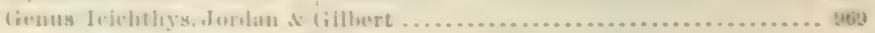

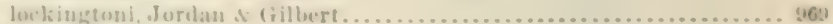

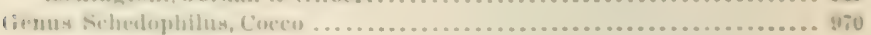

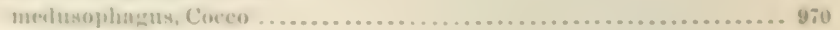

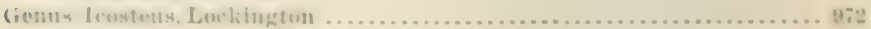

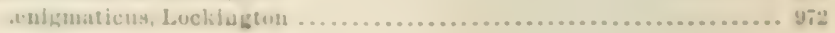


ORDER ACANTHOPTERT-Continued.

SURoRder RhegNopteri-Continued.

Fumily derutille..................................... 973; 2814

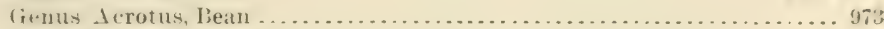

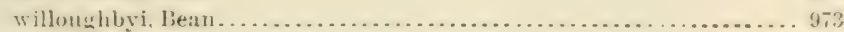

Family Zuprorile ..........................................

lirnus Zaprorat. Jordan ....................................

silenus, Jordan . .......................................... . . 2850

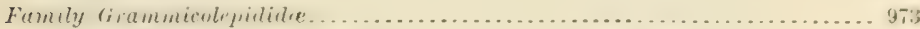

(iknus lirammicol pis. Poey................................. 974

brachiusedulus, Poey................................. 974

Fumil" Tetroutonuride ............................................ 975

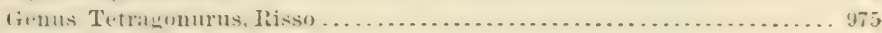

"urieri, liisso. ............................................ 976

Family Pempherida ................................................. 977

Geuns Pempheris, Cuvier \& Valenciennes ........................... 977

mexicanus, Curier \& Valenciennes............................... 978

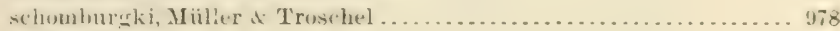

mulleri, Ioey .............................................

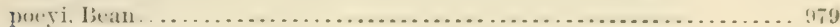

Group I'ercoidea ................................................. 979

Family Elqswmides .........................................

Genus Elassuma, Jordan. . . . . . . . . . . . . . . . . . . . . . .

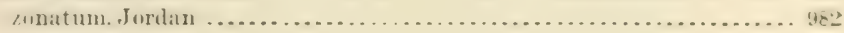

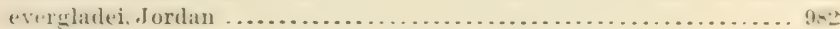

Family rentrarchide .............................................

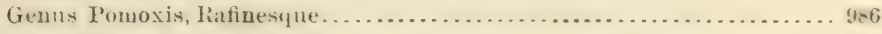

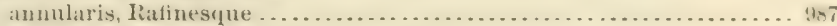
sparoilles (Lacépuede) .................................... gs

Genus Centrarchus, Cuvier \& Valenciennes ....................... 988

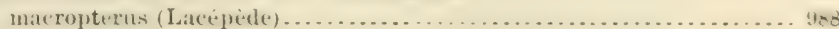

Gienus Acautharchus, Gill ........................................ $y=9$

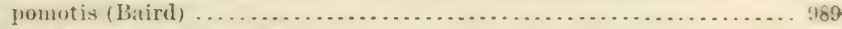

(ienus drubluplites, Ratinesyue ............................. 95? rupestris (Rafinesque) .................................... "atrifons, Cope .......................................

Genus Archoplites, Gill ......................................... 990

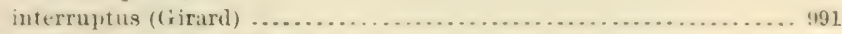

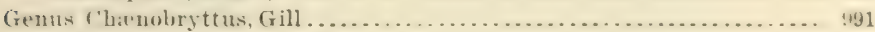
gulosus (Cuvier \& Valenciennes) ............................. 992

Genus Enneacanthus, Gill ...................................... 992

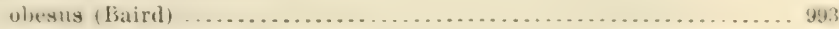

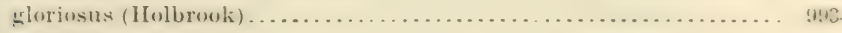

Geuns Mesogonistius, Gill ................................... 994

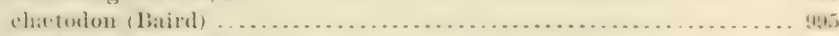

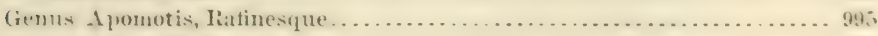
cyauelluy (Ratinesque) .................................... 996 ischyrus (Jordan of Yelson) ................................

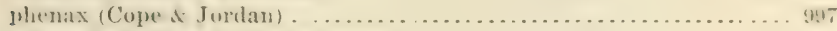
punctatus (Curier \& Valenciennes) ............................ 997 symmetricus (Forbes) ...................................... 998

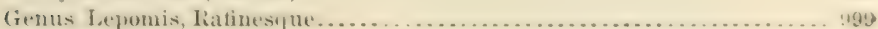
Subgenus Lepomis.............................................. 1001

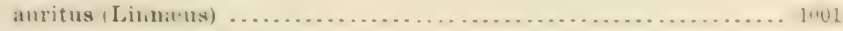
solis (Cuvier \& Valenciennes) ............................. 100 miniatus, . Turdan ................................... In

Bull. No. 47 , pt. $4-$ IV 
CIAST IDLCE-Comtinued.

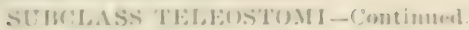

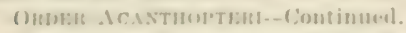

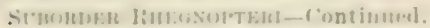

Familu fintrarchiler-Contimment.

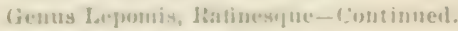

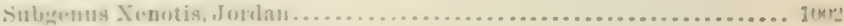

yarmatui, lorlues .......................................... 100

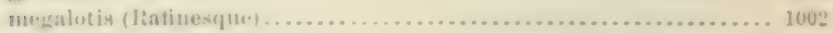

Sulizegnu Ileliquerca, Jurdan................................ 1004

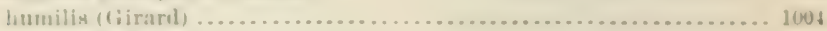

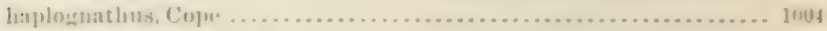

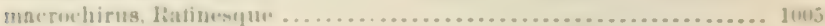

pallialus (Mitchill) .......................................... 100:

(iemus Eupemotis, fill ak Jordan ................................... 1006

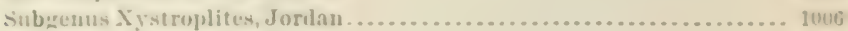

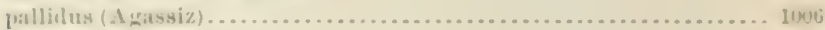

Subgenus Enpomotis ......................................... 100

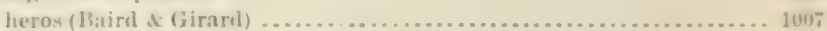

Jubluruki (Cuvier \& Valenciennes) .......................... 1008

euryurus (J ckay) . ........................................ Jong

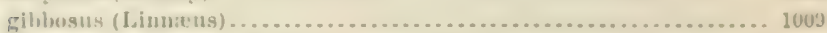

(ieun. Micropterus, Lacé piele ................................ 1010

dolonitur, Lacipede ....................................... 1011

salmointes (Lacépiede) ........................................ 1012

Fanily fuhlide ........................................... 1013

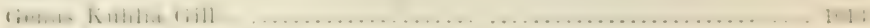

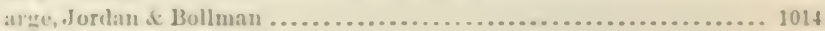

xesura (Jorklan \& Gilbert) ............................... 1015

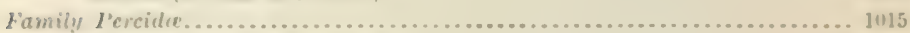

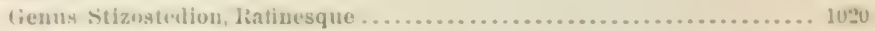

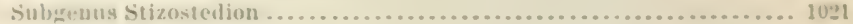

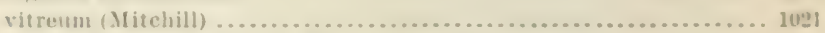

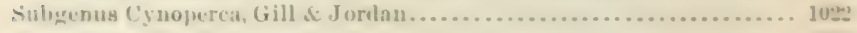

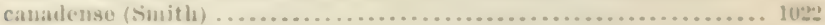

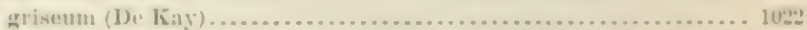

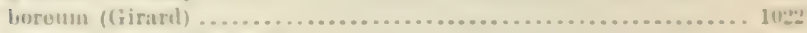

(iemus Porea (Artedi) I.innatis ............................... 1023

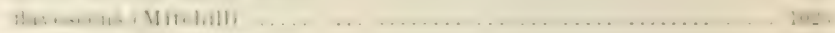

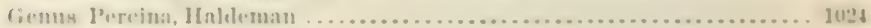

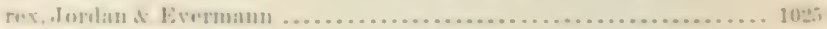

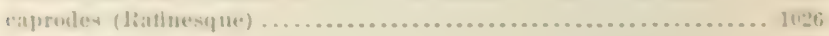

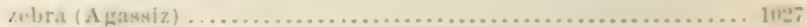

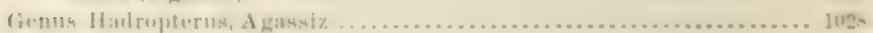

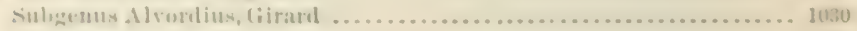

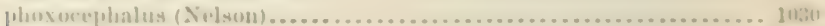

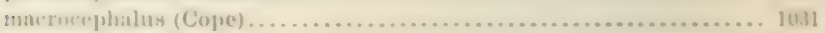

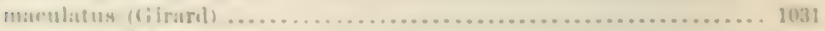

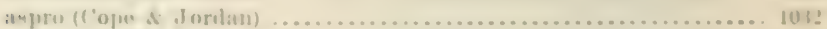

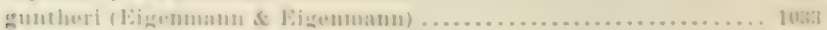

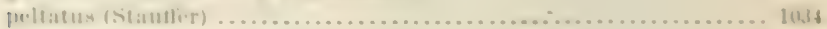

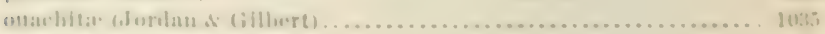

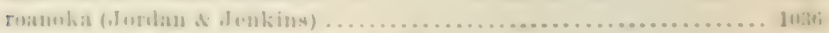

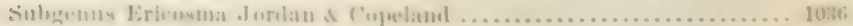

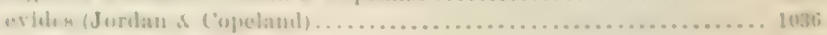

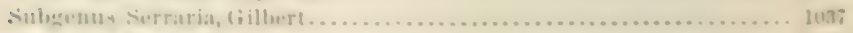

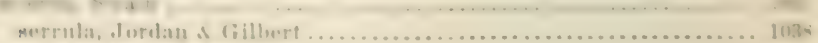

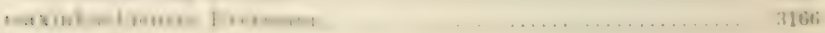


CLASS PISCES-Continued.

SUBCLASS TELEOSTOMI_Continuer.

ORDER ACANTHOPTERI-Continued.

Suborder Rhegnopteri--Continuer.

Family P'ercidoe-Continued.

Genus Hadropterus, $\Lambda$ gassiz-Continued.

Subgenus Iladropterus.................................. 10.,

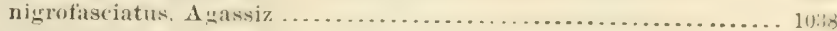

Genus Hypohomms, Cope ........................................ 1039

Subgenus Swaiuia, Jordan \& Erermann......................... 1040

squamatus (Gilbert d Swain) . . ............................... 10 . . . . . 1040

Sulgenus Hypohomus....................................... 1040

aurantiacus (Cope) ........................................ 1010

cymatotrenia (Gilbert \& Meek) .............................. Intl

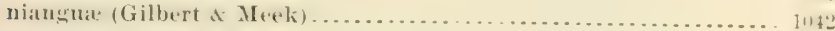

spilotus (Gilbert) ........................................ 1043

Gezus Cottogaster, Putnam . . . . . . . . . . . . . . . . . . . . . . .

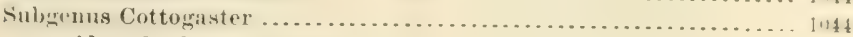

uranidea (Jordan \& Gilbert) ..................................... 104t

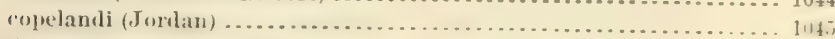

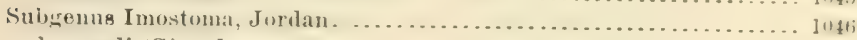

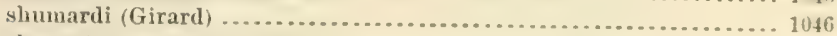

cheneyi, Evermann a Kiemlall ..............................

Gonus Ulocentra, Jordan .................................. 1114

stigmax (Jordan) ........................................... 10 17

gillert, Frerman of Thohurn ......................... 1014

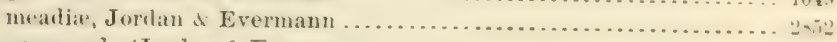

verecunda (Jorlan \& Evermann) ............................ 104?

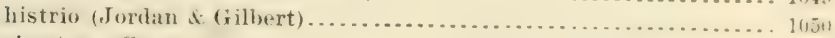

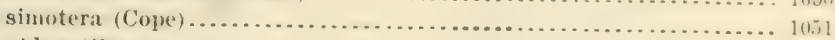

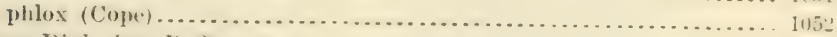

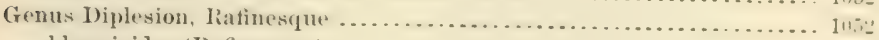

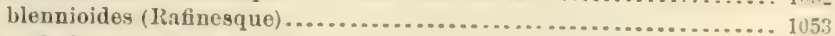

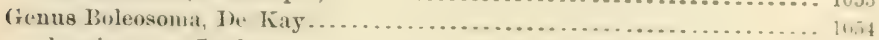

longimanus (Jorlan) ....................................... 1054

podostemone (Jordan $\mathbb{f}$ Jenkins) .............................. 1055

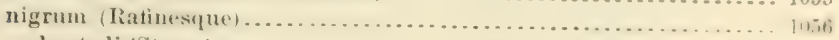

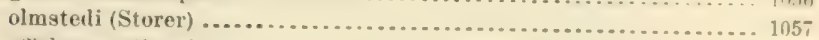

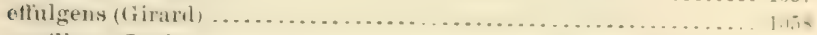

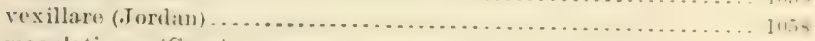

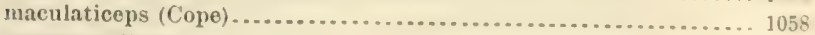

mes:

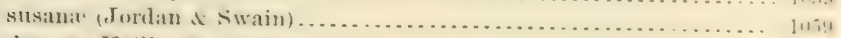

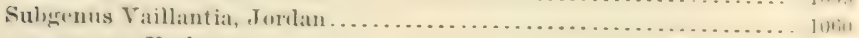

camurum, Forbes ............................................ 10 100

Genus Crystallaria, Jorlan \& Gilbert................................ 1060

asprella (Jordan) ............................................. 1061

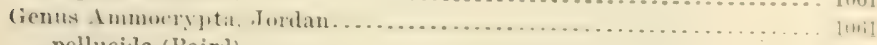

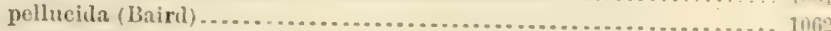

clara (Jordan \& Mreek) .................................... 106

vivax $($ Hag) . ............................................. 1063

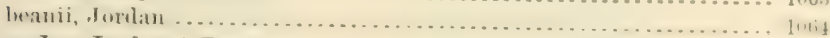

Genus Ioa, Jorklan of Brayton..................................... 10ht

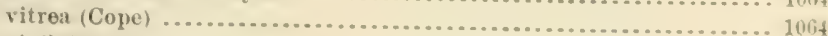

vigil, Hay .................................................. 100,

Genus litheostonta, Ratinesque................................... lotiti

Subgenus P'ocilichthys, Agassiz ............................... 106?

variatum, Firtland ..................................... 1069 


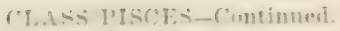

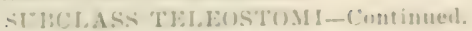

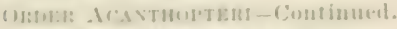

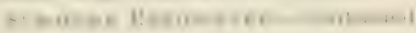

Fitmily Percide-Consimusl.

(ivenus Etlueentesuat. Liatinespue-Continued.

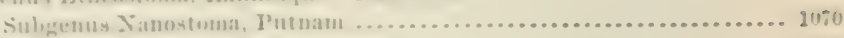

чw:

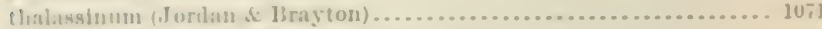

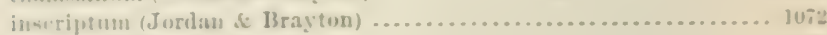

hemulus, filleert s. simain .............................. 10

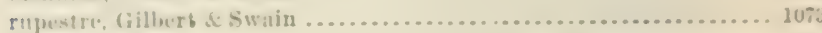

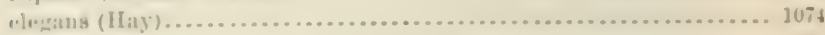

zomale (Cope) .............................................. 10

areansan

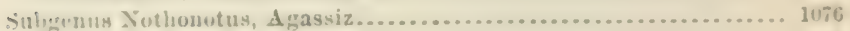

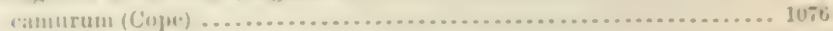

...

maculatum, Kirsland .................................... 107

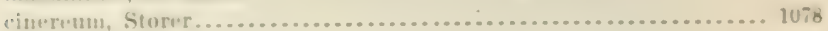

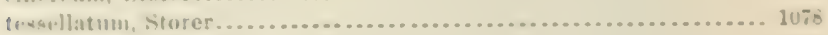

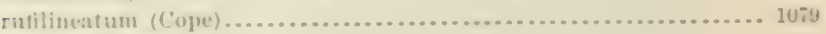

jurilani, (illhert ......................................... 1079

Sub erens Torrentaria, Jorian of Erermann .................... 1080

wilgitta, Jortan of Swain.................................... 1usu

altwtrale, Jorilau ............................................ 1031

Sutigenus Xivicoln, Jordan \& Evermann......................... 10s?

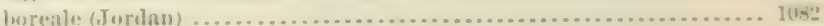

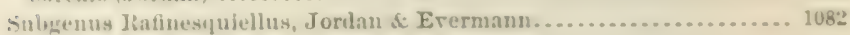

juttsii ((iirart) .......................................... 105"

Suigenus Oliencephalus, Girard ............................. 1083

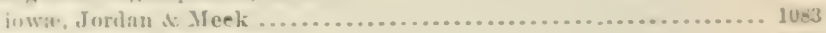

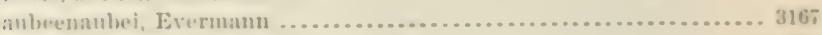

Jessia (Jordan of Brnytou) ................................ 10s

luteovinctum, dilloert $\&$ Swain............................... 1use

lepilogeny, Evermann of Kemlall ......................... 1usi

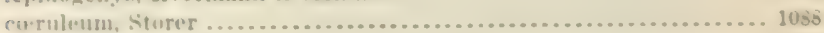

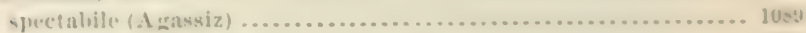

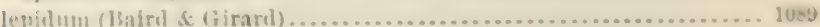

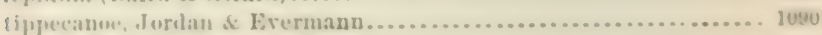

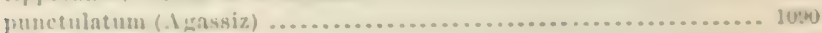

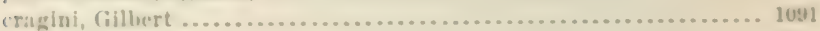

uhe

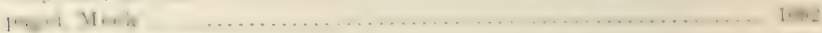

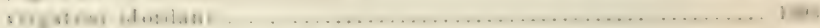

Sulumu Claricula, Jorlan \& Evrmanu ...................... 1093

julial. Merk.............................................. 1003

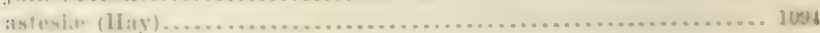

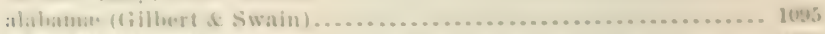

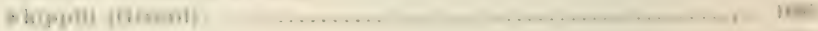

qup

- -

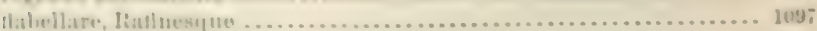

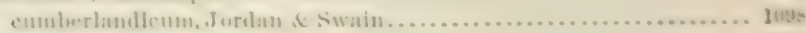

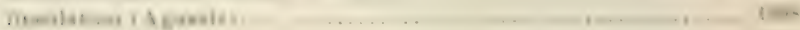

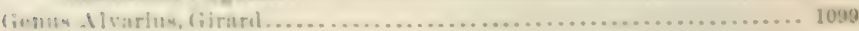

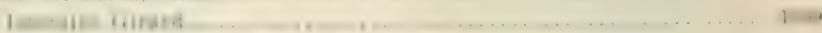

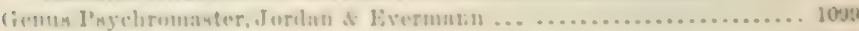

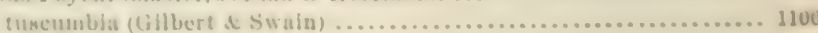


CLASS PISCES-Continued.

SUBCLASS TELEOSTOMI-Continuer?

ORDER ACANTHOPTERI-Continued.

SOBORDER liHegNopteri-Continued.

Family Percide-Continued.

Genus Copelandellus, Jordan \& Evermann.......................... 1100

qдіенсен (Jorlan...........................................

Genus Boleichthys, firart............................... 1111

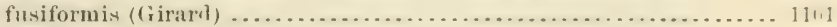

exilis, Girard............................................. 114;

Genus Microperca, Putnam ................................... 1103

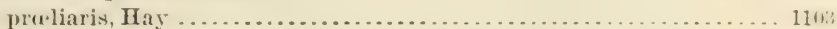

punctulata, Putnam......................................

fontieolat (Jordan of Gilleert) ........................... 1111t

Family Cheilndipteride .. ........................................ 110.;

Genus Apogon Lacépèrle.................................... 1106

imberbis (Linnitus) ..................................... 1111 ;

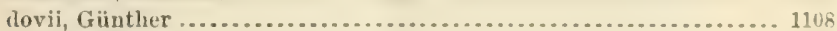

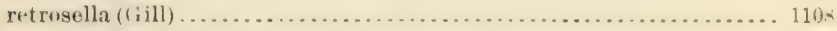

maculatus (Poey) ........................................ 110

binotatus (Poey) ........................................ 11010

pigmentarius (Poey) ....................................... 1109

atricaudus, Jordan of MeGregor. . . . . . . . . . . . . . . . . . . . . . . . . 2853

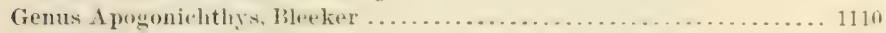

alutus (Jordan \& Gilbert) ................................ 1110

stellatus, Cope ........................................... 1110

puncticulatus, P'oty.................................... 1111

Gemus Glossamia, (rill ................................... 1111

pantionis (Goode \& Bean) ...................................... 1111

Genus Eligonus, Ratinest ue .............................. 1111

occidentalis, fioode of Bean .............................. 1112

Genus Cheilodipterus, Lacépèdo ................................ 1112

atlinis, Poey .......................................... 111 .

Genus Amiichtlys, Poey.................................... 1113

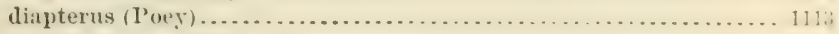

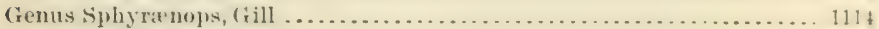

batrdianus, Poey .......................................... 1114

Genus Scombrops, Temminck \& Schlegel .......................... 1114

Subgemus Latebrus, Poey ................................. 111 .

oculatus (Poes)................................................ 1114

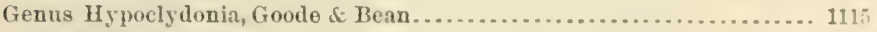

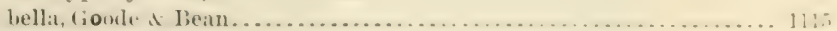

Family Centromitre...................................... 1110

Genus Centropomus, Lacépèdo.................................... 1117

viridis, Lockington ........................................

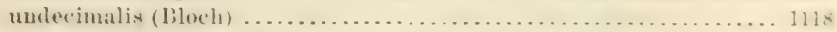

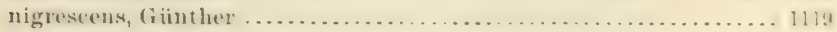

pedimacula. Pory..................................... 1119

gramdoculatus, Jeukins of Evermann........................ 1120

eurjeri, Bocourt ............................................. 1121

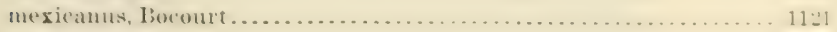

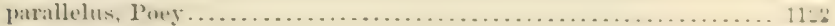

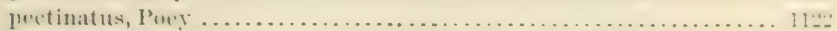

unionensis, bocourt .........................................

armatus, Gill....................................................

robalitu, Jorilan of Gillert . . . . . . . . . .

ensiforus, Poeg....................................... 1125; $2 \$ 53$ 


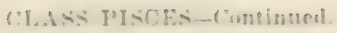

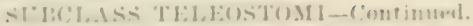

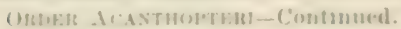

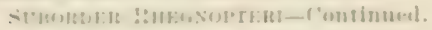

Fiunily serranider.............................................. 1120

Virnus Roerns, Mitchill . .......................................... 11'1

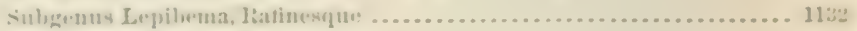

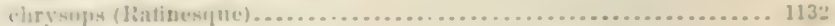

sub;

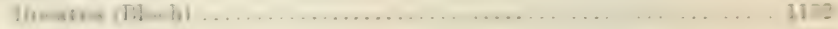

(ienus Morme, Mitchill. ..................................... 1133

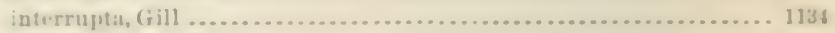

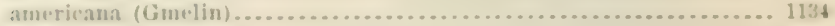

Coetutt I,iopropoma, Gill ....................................... 1135

alierman (Puey) ......................................... 1136

Civmus f'horistiatium, Gill..................................... 1136

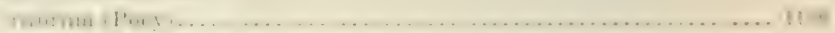

Gemus Stereuleyis, Lytes .................................. 1127

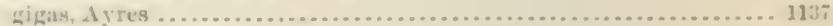

(icmus Polyprion, Cavier..................................... 1139

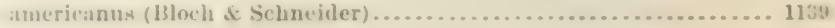

fienus (ionioplectrus, (iill................................... 1139

hispanus (Cuvier of Valenciennes)........................... 1140

fienus l'etrometopon, Gill....................................... 1140

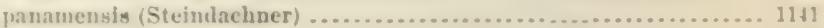

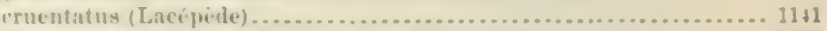

coronatus (Curier of Valeuciennes) .......................... Iit:

Geuns Ihulianus, Bloch ......................................... $11 \downarrow 3$

tuniops (Cuvier \& Valencieunes) ........................... 114

julvus (Linnitus) .......................................... 11th

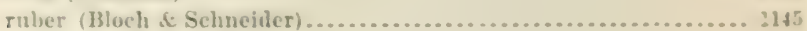

punctatus (Linn:tus) .................................... $11 \$ 6$

Sulgenus XI nuphorus, Poey............................... 1146

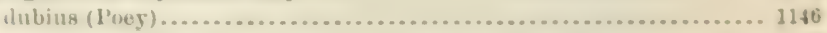

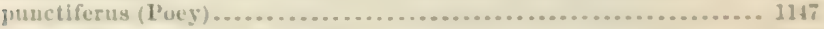

Subgenus İnneistus, Jordan of Evermann ....................... 1147

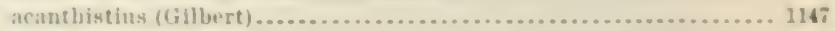

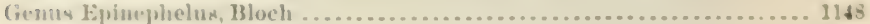

Sub renum Schistorus, Gill................................... 1151

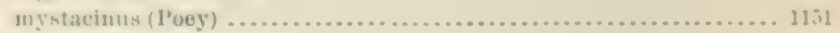

Sulnemus Epinephelus....................................... 185"

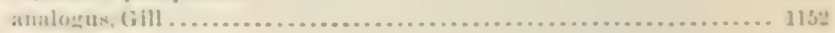

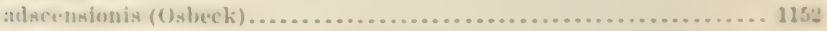

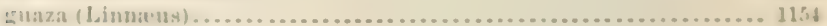

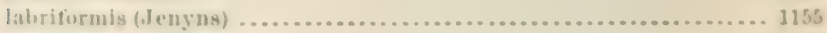

thavulimbatus, l'oey .......................................... 1155

nlphohler, Gilliert \& starks................................ $2=53$

niventur (Cuvie: \& Valenclennes) ........................... 11:0

Atrintus $($ H)

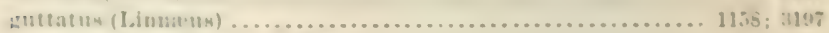

drum

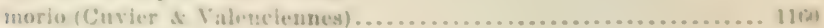

(iemu (iarruph, Jurilun ....................................... 1101

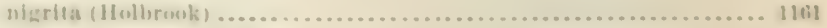

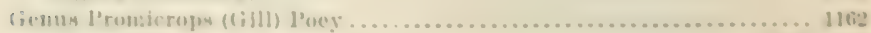

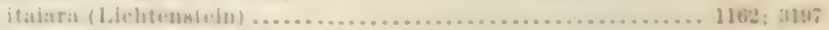

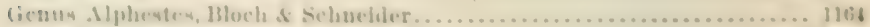

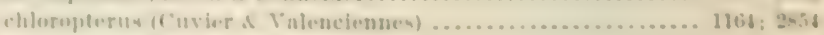
mublgut 
CLASS PISCES-Continued.

SUBCLASS 'IELEOSTOMI-Continuer.

ORDER ACANTHOPTERI-Continued.

SUbORDER RHEgNopteri-Continued.

Family Serranida-Continued.

Gemus Dermatolepis, (Gill .................................... 11 the

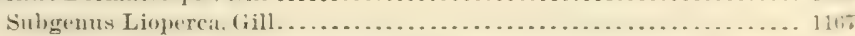

inermis (Cuvier \& Valeneiennes) .......................... 116 \%

zanclus, Evermann \& Kendall ................................ . 2854

Sulgenus Dermatolepis .................................. 116

punctatus, (iill .......................................... 11tie

Genus II yeteroperea, (sill .................................. 116 ?

Subgenus Archoperca. Jordan of Erermann..................... 1171

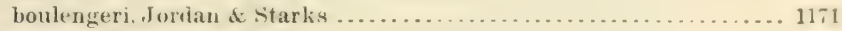

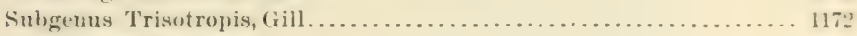

veuenosa (Linna

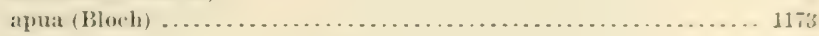

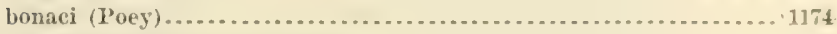

xanthosticta, Jordan \& Swain........................... 1176

jorlani (Jonkins of Evermann) ........................... 1176

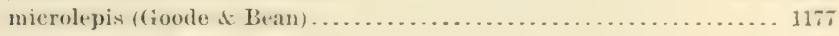

interstitialis (Poey) ......................................... 11 .

dimidiata (Poey)................................................. 1179

yenarcha, Jordan . . . . . . . . . . . . . . . . . . . . . . . . . 1184!

Subgenus Parepinephelus, Bleeker... ........................... 1180

rubra $($ Bloch) ........................................... 1180

Subgenus Xystroperca, Jordan \& Erermann........................ 118T.

pardalis, (Gilbert ..................................... 11 .

Subgenus Mycteroperea ................................... 1183.

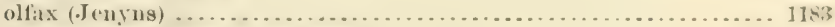

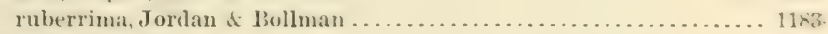

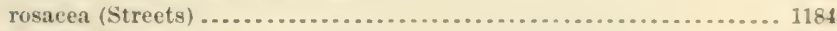

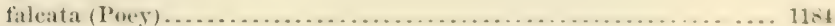

phenax, Jordan \& swain .............................. 118.

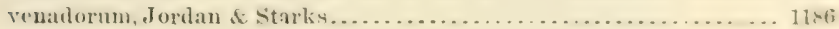

calliura, Poey ............................................. 1186

hopkinsi, Jordan di IRter. . . . . . . . . . . . . . . . . . .

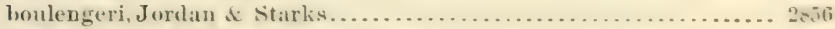

tigris (Cuvier \& Valenciennes) ............................

camelopardalis (Poey) .................................. 11si

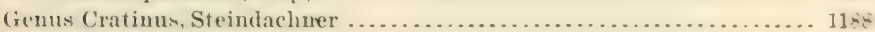

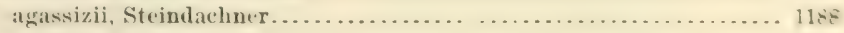

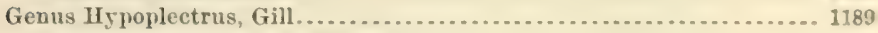

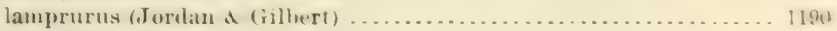

unicolor (Walbaum) ....................................... 1190

puella (Cuvier of Valeneiennes) . . . . . . . . . . . . . . . . . . . 119z

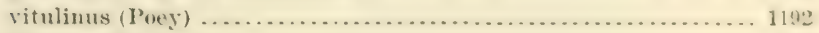

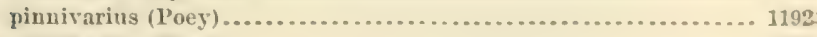

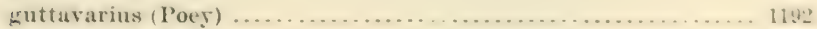

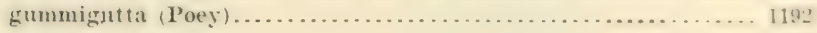

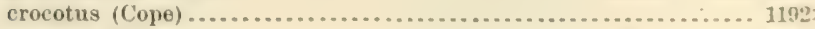

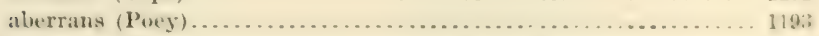

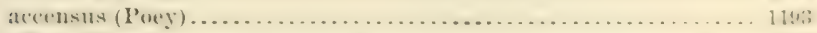

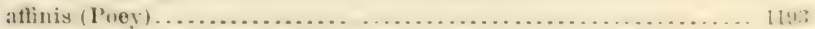

chlorurus (Curier \& Valenciennes) ........................ 1193

nigricans (Poer) . . . . . . . . . . . . . . . . . . . . . . . . . . 119 ;

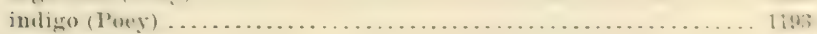

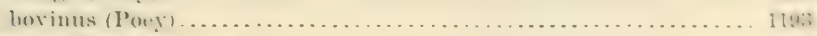

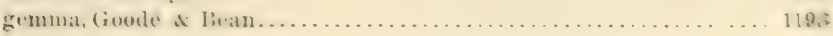




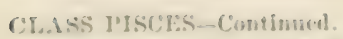

SUBCLASS TELEASTOM - Contintues.

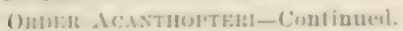

Strusuen IRnEcinotran-Continuen.

Family Serronida-Continued.

Genuss l'aralabrax, (irarl. .................................... 119

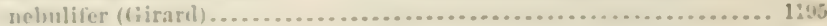

maculatofasciatus (Steinulachner) ........................... 1196

lumm ralis (Cuvier $\&$ Valenciennes) ........................... 1196

rlathratus (Giraril) ........................................ 1197

Gienns Centropristes, Cuvier ................................... 1198

Subzenus Centropristeg....................................... 1199

rufus, ('uvier \& Valenciennes.................................... 1190

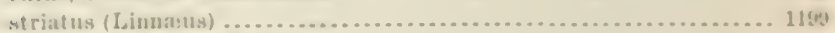

wevurus (Jortan o Evermann) .............................. 12un

Sulıenus Triloburus, Gill................................ 1201

philalelphicus (Linnieus) ............................... 18nd

Genus I)iplectrum, Holbrook ..................................... 120

Sulyenus Inaliperea, Gill ................................. 1sut

sciurus, Gilbert.............................................. 120

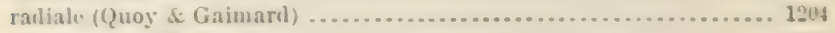

macropuma (Gïnther) .................................... 1:05

euryplectrum, Jordau \& Bollman ............................ 1266

Sulıgenus Diplectrum .................................... 120.

formosum (Linnatus) ............................................. 120

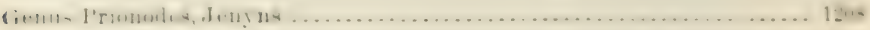

Suhrenus Prionoles....................................... 1210

aiquidens (Gilbert) . ........................................... 1210

tusculus (1'oey) ......................................... 1211

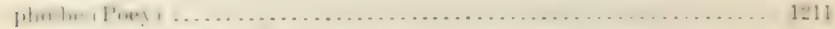

balılwini, Evermann \& Marsh................................... 310

fascintus, Jenyns ........................................... 1212

bulleri (loulenger).......................................... 121:

Sulugenus dentiperca, Gill ................................. 1214

tigrinus (Bloch) ........................................... 121t

Iuburius (Cuvier \& Valeneiennes) ......................... 1:15

thaveens (Cuvier of Valenciennes) ............................. 1215

luciopercums (1'oey) ....................................... 1:16

ntillowstigma, Jordan of IBollman ........................... 1216

Genus 1)ules, Cuvier.......................................... 121

subligarins (Cope) ........................................ 121s

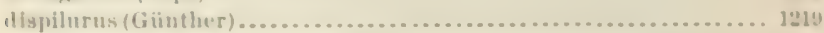

auriga, Cuvier ot Valeneiennes............................... 1:200

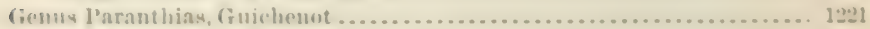

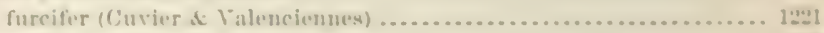

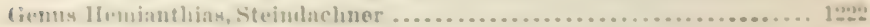

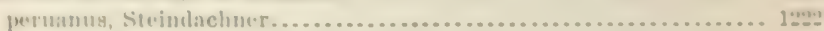

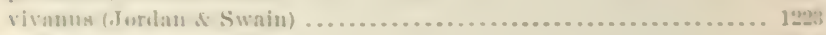

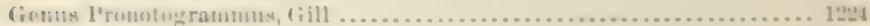

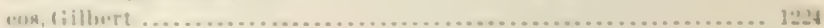

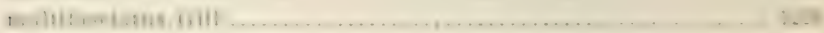

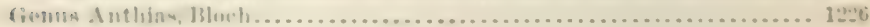

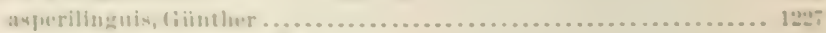

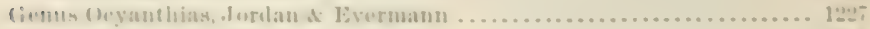
martinimensis (linichenut) ................................ 12us

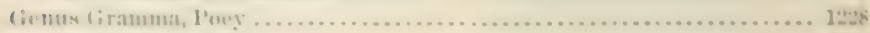

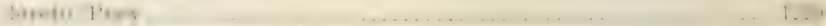

(i) thuแ 
CLASS PISCES-Continued.

SUBCLASS TELEOSTOMI-Continner.

ORDER ACANTHOPTERI-Continued.

SUBORder RHEgNopteri-Continued.

Family Serranida-Continued.

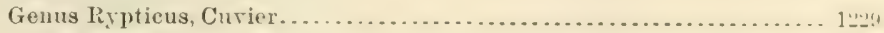

xanti, (rill..........................................

bicolor (Valenciennes) ....................................

saponaceus (Bloch \& Silmeicler) .......................... 1

arenatus, Curier \& Valencienues ........................ 12:2

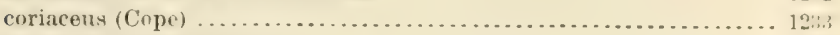

Subgenus Promicropterus, Gill............................... 1233

bistrispinus (Mitchill) ....................................... 123i

nigripinnis, Gill ......................................... 1234

Family Lobotidae ................................................. 1235

(renus Lobotes, Curirr....................................... 1:55

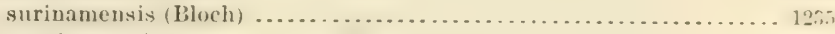

pacificus, Gilbert........................................... 28.5

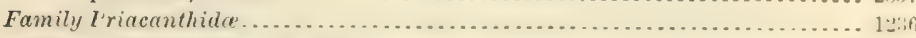

Geuus Priacanthus, Curier ................................. 12:;

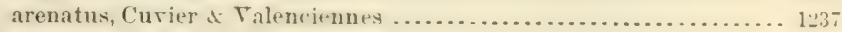

cruentatus (Lacépède) ..................................... 1238

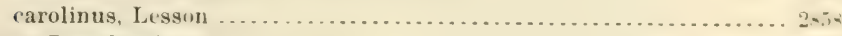

Genus Pseudopriacanthus, Bleeker ............................... 1239

serrula, Gilbert . . . . . . . . . . . . . . . . . . . . . . . . . . . . . . . . . . . 1239

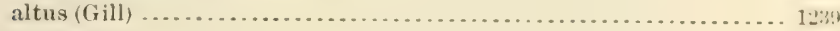

Family Lutianida . ............................................... 124]

Genus Hoplopagrus, Gill ................................... 124t

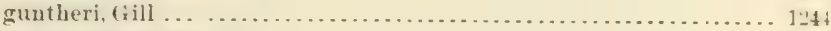

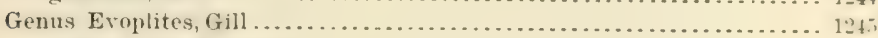

viridis (Talenciennes) .......................................

Genus Neomanis, Girard ....................................... 12t7

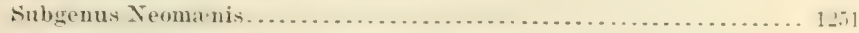

,jorlani, Gilhert ..................................... . .

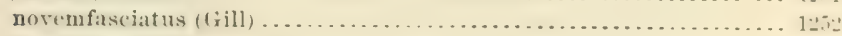

cyanopterus (Cnvier \& Valenciennes) ......................... 1254

griseus (Linnerts) . .......................................... 1255

jocu (Bloch \& Schueider) .................................... 125

apodus (Walbaum) ...................................... 1258

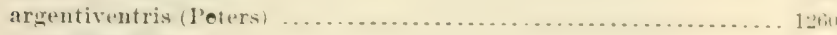

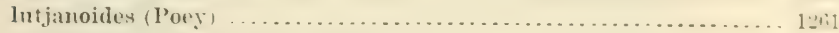

buccanella (Cuvier \& Talenciennes) ......................... 1261

riranus (Curier \& Valenciennes) ............................. 1262

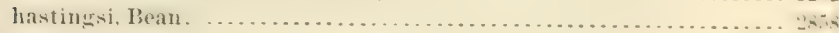

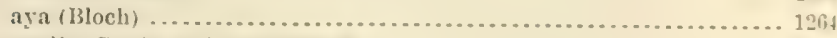

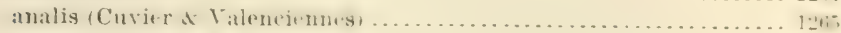

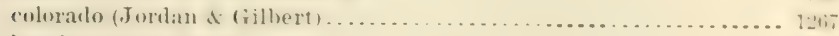

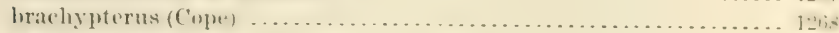

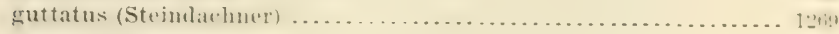

synagris (Linnæus) ....................................... 12711

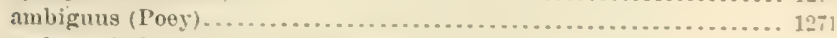

mahogoni (Curier \& Valenciennes) . . . . . . . . . . . . . . . . . . . 12

Subgenus Raizero, Jordan \& Fesler............................. 1273

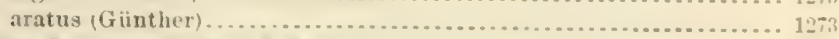

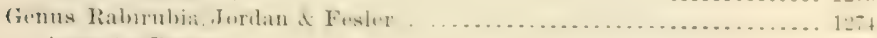

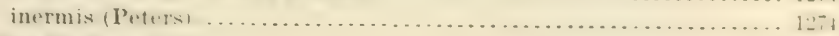

(ienus ()

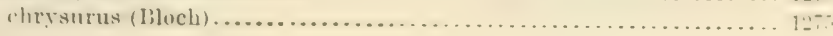




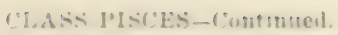

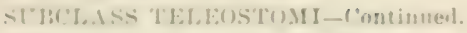

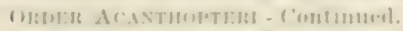

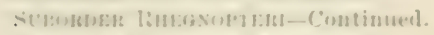

Family Sutianidu-Continued.

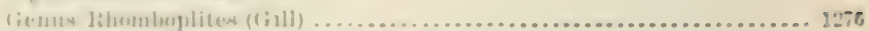

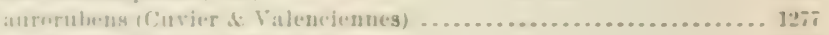

licuus Aquilus, Cuvier se Valenviennes ......................... 1288

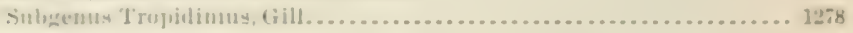

dentasur, diniclenot ........................................ 1278

(ienus Aprien, Cuviur \& Valenciennes........................... 1279

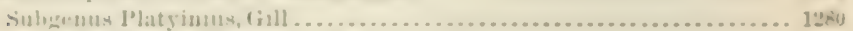

mucrephlithalmus (Miiller of Troschel) ....................... 1200

Genus Elelin, Cuvier A Valenciennes.............................. 1"81

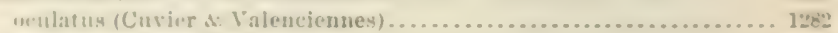

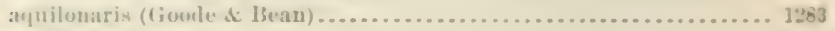

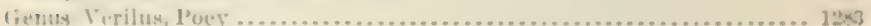

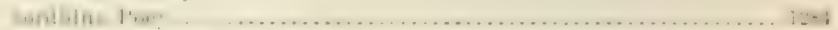

(ienus Xinoey's, Jorkan . E Bollman............................. 1285

jessial, forlan di Bollman .................................... 12e5

(ienus Xinistius, Jordan \& Gilbert............................. 1286

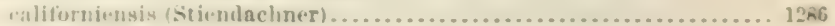

(ienus Xenichthys, Gill................................. 128

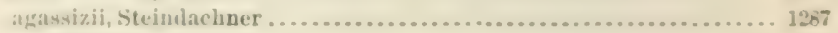

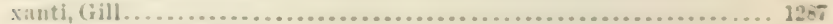

Cienus Xemipterus, Swainson .................................. 138

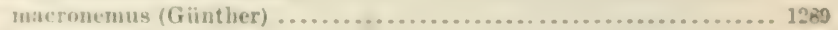

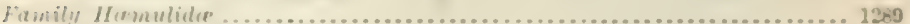

Cienu. Ilamulon, Cuvier...................................... 1291

46.xlasciatum, Gill......................................... 1234

allum, Cuvier of Valenciennes . . . . . . . . . . . . . . . . . . . . . 1205

macrost

bonariense, Cuvier of Valenciennes ......................... 129

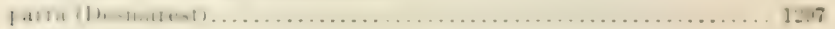

senthleri, Gill......................................... 1290

fielenit, Boulenger ....................................... 31 .

enrhomarim, Poey ......................................... 1300

steinelachmeri (Jordan \& Gilhert) ............................ 1301

melanurum (Linua'us) .................................. 1312

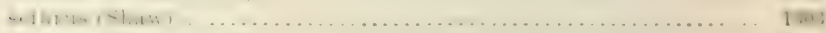

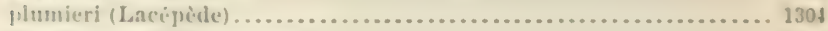

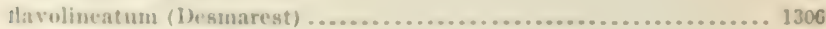

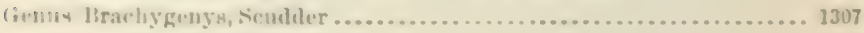

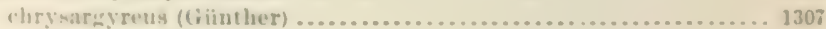

lienus lhathymtoma, Setulder................................ 130

risuttur (Jurdan o. Swnin) ................................ 1308

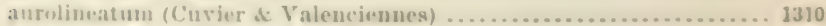

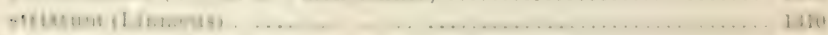

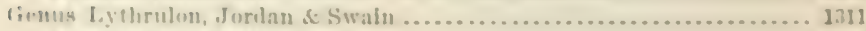

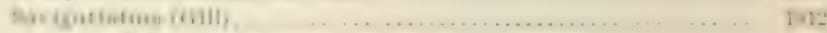

upalescens, durilun \& Starks............................... 1312

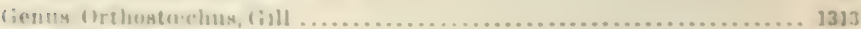

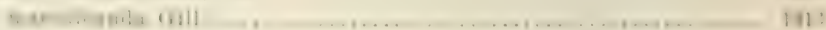

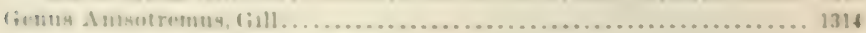

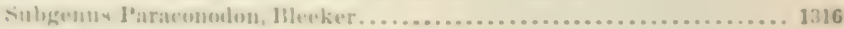

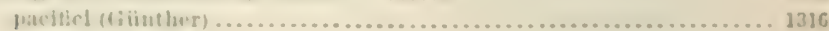

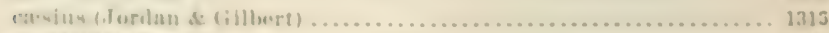

.n 
CLASS PISCES-Continuel.

SUBCLASS TELEOSTOMI-Continued.

Order acanthopteri-Continuer.

SUBOHDER RHEGNOPTERI-Continued.

Family Hamulide-Continued.

Genus Anisotremus, Gill-Continued.

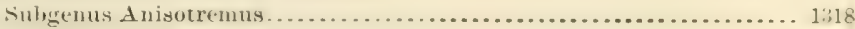

surinamensis (IBloch) . . . . . . . . . . . . . . . . . . . . . . . . 131s

interruptus (Gill) ............................................. 1319

hicolor (Castelnat1) . . . . . . . . . . . . . . . . . . . . . . . . . 1:19

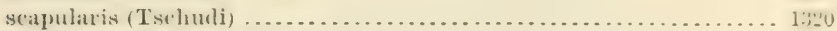

daridsonii (Steimlachner) ............................... 1

spleniatus (Poey) ........................................... 1;

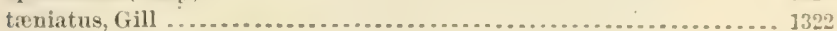

virginicus (Linneus) .................................... 1322

serrulat (Cuvier of Talenciennes) . . . . . . . . . . . . . . . . . . . 13:3

Genus Conodon, Cuvier \& Valenciennes .......................... 1324

mobilis (Lindetus) ...................................... 1324

serrifer, Jordan \& Gilbert.... . . . . . . . . . . . . . . . . . . . . . . . . . . 1924

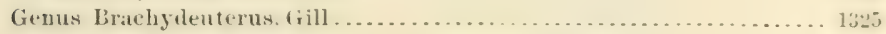

nitilus (Steinulathner) ................................ 1:20;

corvineformis (Steindachner) ................................ 1326

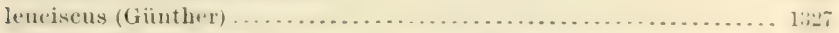

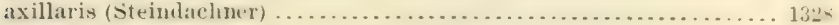

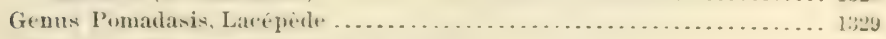

Subgenus lihencus, Jordan \& Evermann . . . . . . . . . . . . . . . . . . 1331

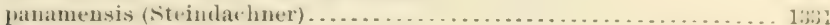

Subgenus Pristipoma, Cuvitr ........................... $13: 31$

bayanus, Jordan d. Evermann . . . . . . . . . . . . . . . . . . . 13381

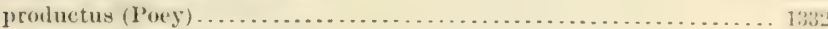

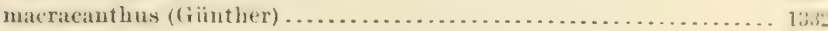

andrei (Sauvage) ..........................................

Subgenus Rhonciscus, Jorlan \& Evermann ....................... 1333

crocro (Cuvier \& Valenciennes) ................................. 1333

hranicki (Steindacher) ................................. 10.9

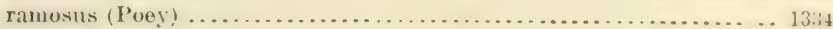

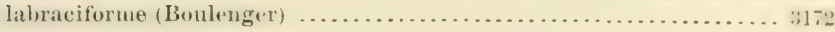

Genus Orthopristis, Girard ....................................... 133

Sulggenus Orthopristis . ....................................

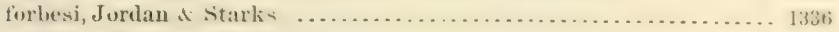

reddingi, Jordan $\mathbb{S}$ Jicharilsom............................... 1336

chalceus (Günther) ....................................... 1337

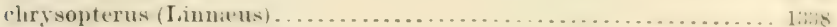

poeyi, Scudder .............................................

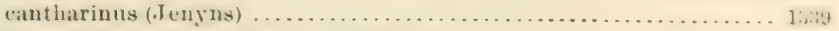

Subgenus Evapristis, Jordan \& Evermann . . . . . . . . . . . . . . . . . . . 13 130

lethopristis, Jord:m of Feslex . . . . . . . . . . . . . . . . . .

(ienus Isariela. Jortan s Fesler . . . . . . . . . . . . . . . . . . divu

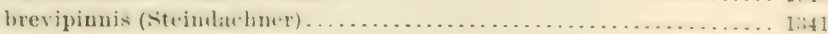

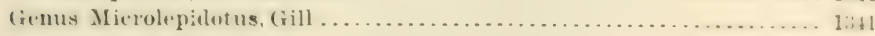
inornatus, (iill . . . . . . . . . . . . . . . . . . . . . . . . . . . . . .

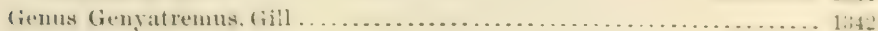

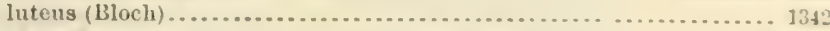

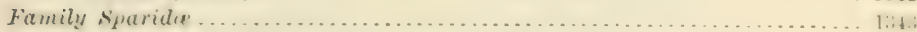

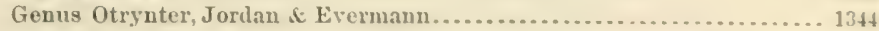

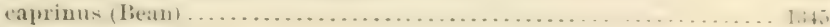

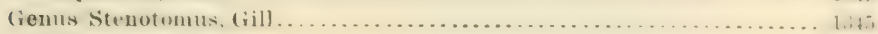

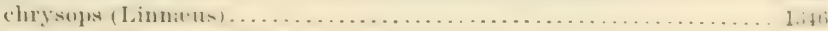
aculeatus (Cuvier \& Valencienues) .......................... 1340 


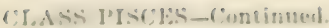

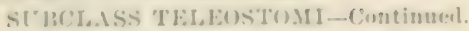

ommar dravimesman-Continued.

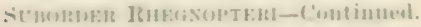

Fumiles syerider-Contimul.

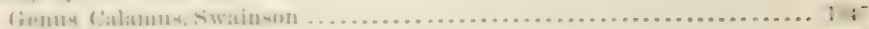

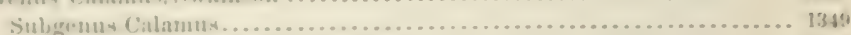

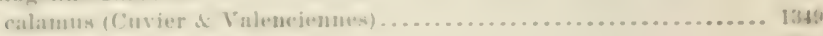

proricleny Jordan o (iilhert ............................... 1350

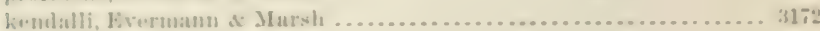

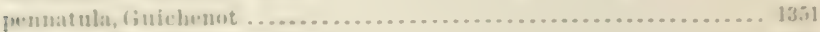

bajonale (Bloch \& Schneder) ............................... 135:

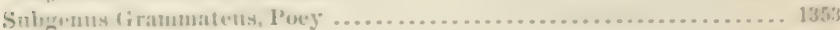

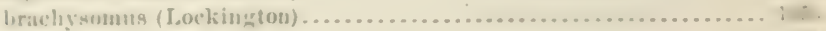

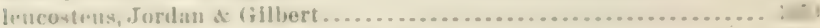

:

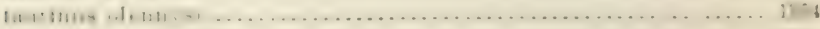

penna (Cuvier \& Valenciennes) ............................ 1

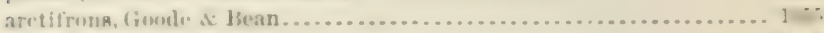

מй

(iеnus l'aurum, Cuvier .....................................

pagrus (I.inuatus) ...................................... it ${ }^{*}$

fiemus I.agudun, Holbrook ..................................... n

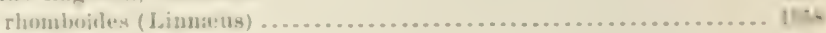

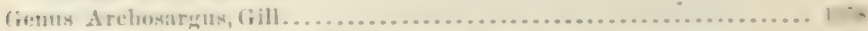

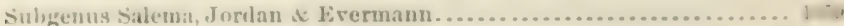

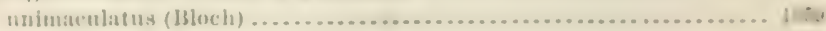

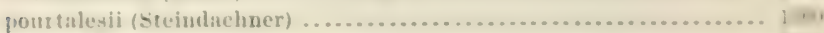

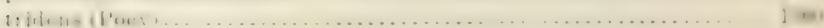

Subgenus Archosargus..................................... int

proliatecephatus (Walbanm) ............................... 166t

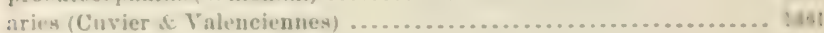

Ciersus Diplodus, liatinestjue................................. 1.2

hollorogkii (Bean) .......................... i

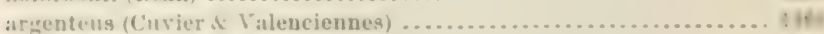

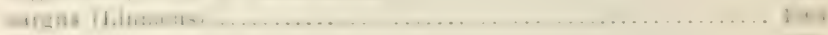

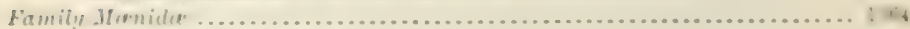

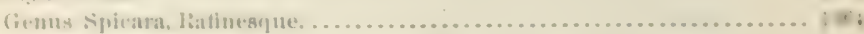

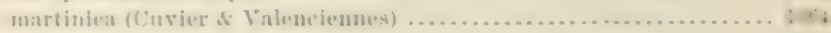

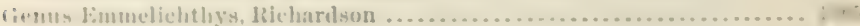

Sul,

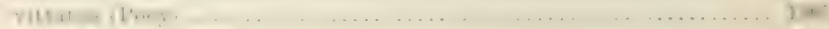

I, a

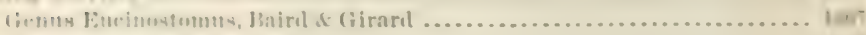

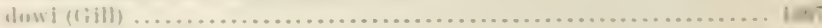

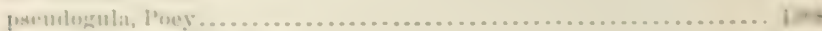

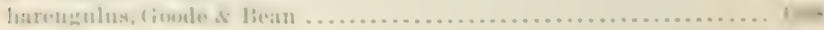

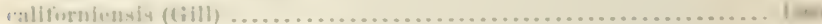

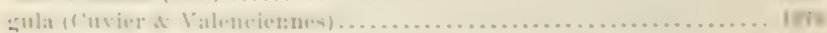

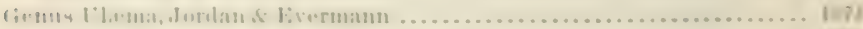

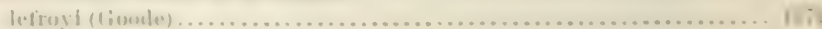

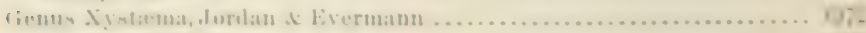

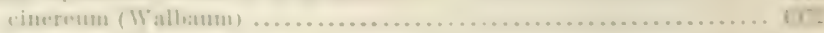

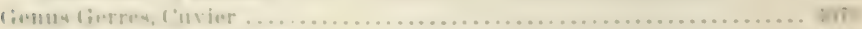

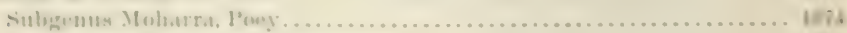

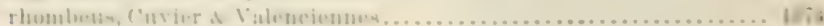

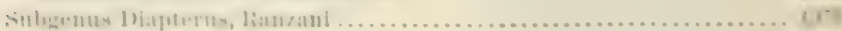

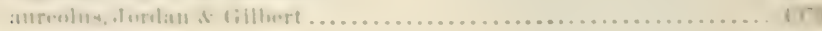


CLASS PISCES-Continued.

SUBCLASS TELEOSTOMI-Continued.

Order ACANTHOpteri-Continuerl.

SUbORDER liHegNopterI-Continuetl.

Family Gerrida-Continued.

Genus Gerres, Cuvier-Continued.

Subgenus Diapterus, Ranzani-Continued.

peruvianus, Curier \& Talenciennes ........................... 1376

olisthostomus, Goode \& Bean ................................. 1376

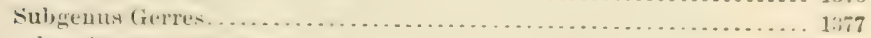

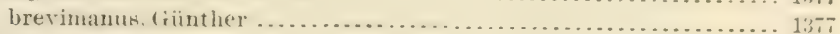

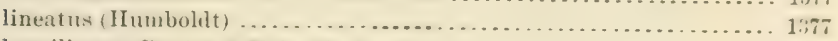

brasilianus, Cuvier \& Valeneiennes .......................... 137

embryx, Jordan di St:arlis . . . . . . . . . . . . . . . . . . . . . . . . $1 ; 79$

plumieri, Cuvier di Valeneienues . . . . . . . . . . . . . . . . . . .

mexicanus, Steindachner..................................... 1380

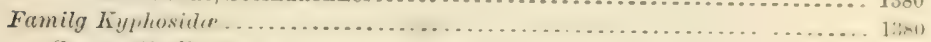

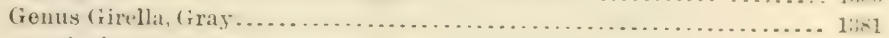

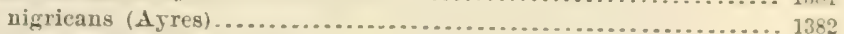

Genus Doydixodon, Talenciennes . . . . . . . . . . . . . . . . . . . . . . . . . . . 1382

freminvillej, Talenciennes................................ 13F.

Genus Hermosilla, Jenkins \& Evermann.... . . . . . . . . . . . . . . . . . . 1383

azurea, Jenkins \& Evermaun . . . . . . . . . . . . . . . . . . . . . . . . . . . 1383

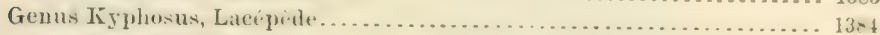

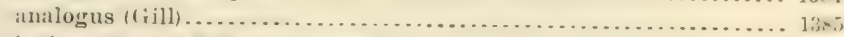

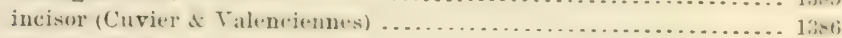

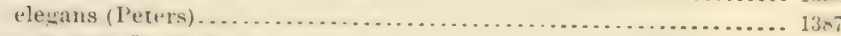

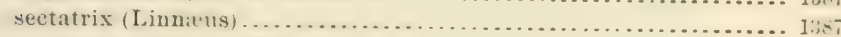

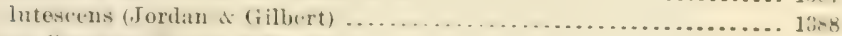

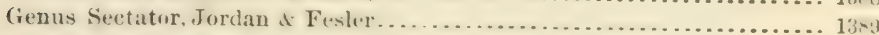

ocyurus (Jordan \& Gilbert) ................................ 1389

Genus Medialuna, Jordan \& Fesler . . . . . . . . . . . . . . . . . . . . . . . 1390

californiensis (Steindachner) ................................... 13:41

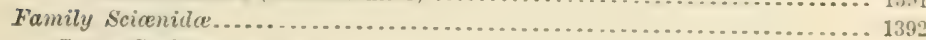

Genus Seriphus, dyres ..................................... 1397 politus, $\Delta y$ res................................................ 1397

Genus Isopisthus, Gill . . . . . . . . . . . . . . . . . . . . . . . . . . . . . . . . 1398 remifer, Jordan \& Gilbert...................................... 1398 parvipinnis (Cuvier \& Valenciennes) .......................... 1399

Genus Buccone, Jordan \& Evermann ............................ 1100

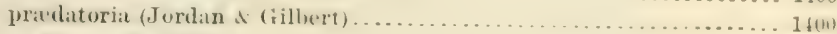

Genus Cynoseion, Gill . . . . . . . . . . . . . . . . . . . . . .

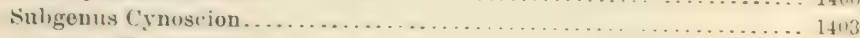
acoupa (Lacépède) ......................................... 1403

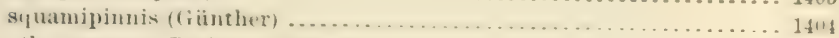

othonopterus, Jordan \& Gilbert................................. 1404

obliquatus (Valenciennes) ................................... 1405

jamaicensis (Vaillant S. Bocourt) ........................... 1406

nothus (Holbrook) ....................................... 1406

regalis (Bloch $x$ Schneider) ..................................... 1407

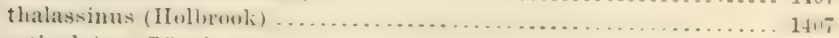

reticulatus (Giinther) . ........................................... 1408

nebulosus (Cuvier \& Valenciennes) .............................. 1409

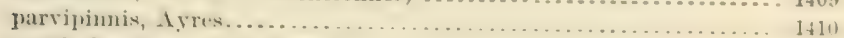

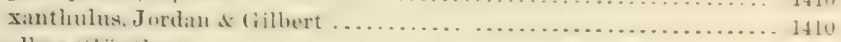

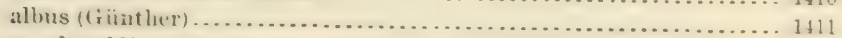

macdonaldi, Gilbert........................................... 1411

stolzmauni (Steiudachnerı................................ $1+12$ 


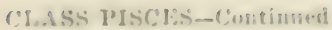

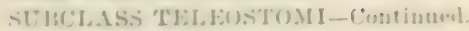

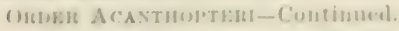

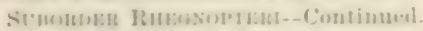

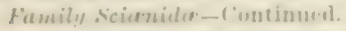

Genus Conuseion, dill-Continnesl.

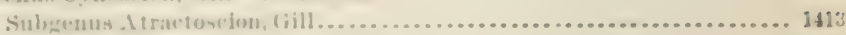

nubilis $($ Ayres) ............................................ 1413

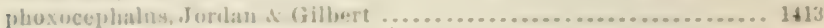

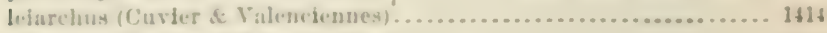

vireacens (Cuvier \& Valenciennes) ........................... 1115

ulerulepiflotus (Cuvier \& Valencienues) ...................... 1415

(ienum Sagenichthyн, Berg................................... 1+16

ancylulun (IBloch s seluneider) ................................. 1416

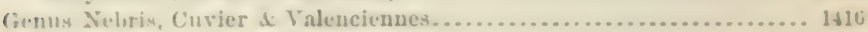

microps, Cuvier \& Valencienues............................. 1417

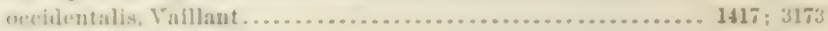

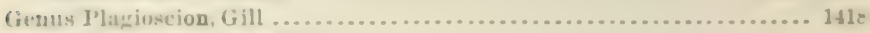

squanosissimus (Heckel) ................................ 1\$18

heturulepls (Bleeker) ....................................... 1419

surinanensis (Bleekir) ................................... 1\$18

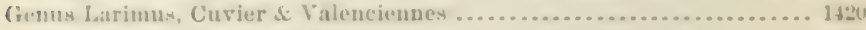

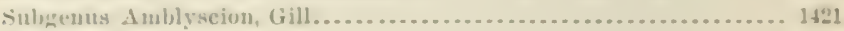

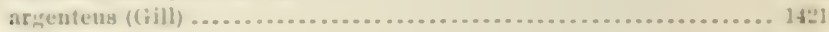

Suhgenus Loarinus............................................. 14:1

eflul „rens, (iilbert ....................................... 1\$21

aceliviн, Jorilan \& Bristol ................................ 142:

breviceps, Cuvier of Valonciennes ............................ 14:3

pacitleus, Jordiu of Bollman ................................... 1

finciatus, Holbrouk ..................................... 1t2d

(ienus (hlontustien, diill ...................................... 1425

lentex (Cuvier of Valenciennew) ............................ 1425

xanthops, (iillnert ........................................ 14:0

Gemus Corvula, Jorulan of Eigeumanu .............................. 14:7

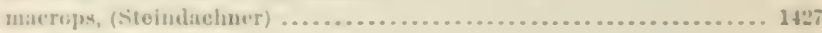

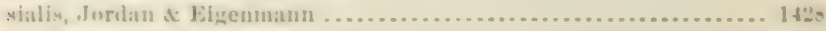

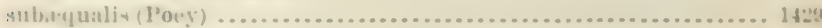

м:anesu. Ine in, fordan ...................................... $14: 9$

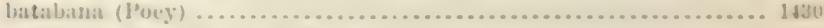

(ienus Elattarchus, Jordan \& Evermann ........................ $\$ \$ 31$

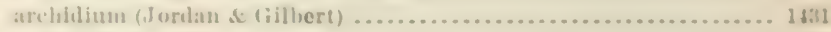

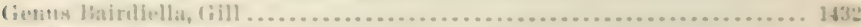

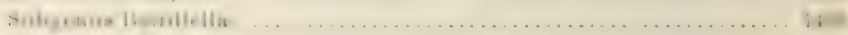

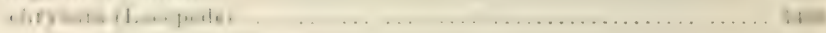

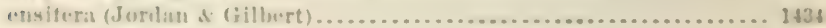

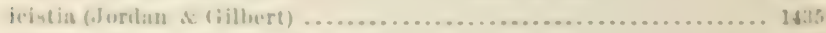

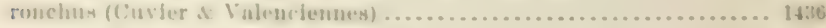

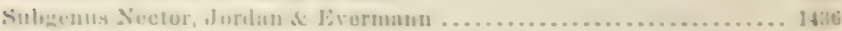

amoshs will

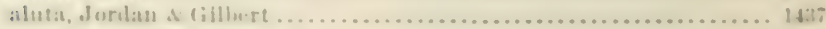

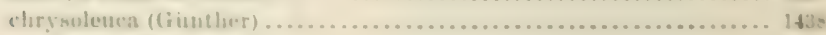

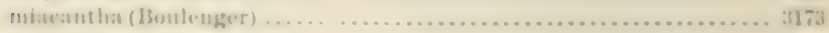

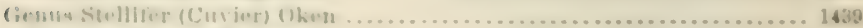

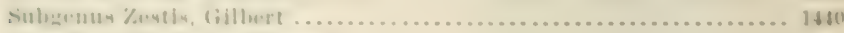

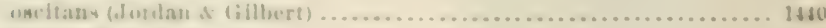

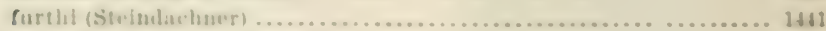

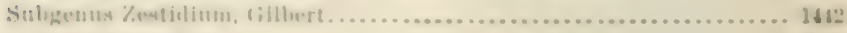

illecelormsus, Gillert ..................................... 14t: 
ULASS PISCES-Continued.

SUBCLASS T'ELEOSTOMI-Continued.

ORder ACANTHOPteri-Continued.

SUboRder RHEUNopterl-Contimer?.

Family Scianida-Continued.

Genus Stellifer (Cuvier) Oken-Continued.

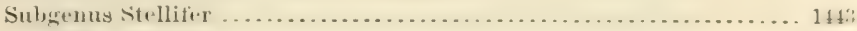

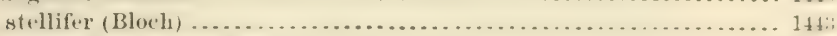

linceolatus (Ifolbrook) ................................... 14t:

erieymba (Jordan of (iilhert) ............................... 1444

microns (Steintachner) ....................................... 144

Subgenus Stelliearens, filbert...........................

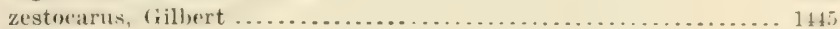

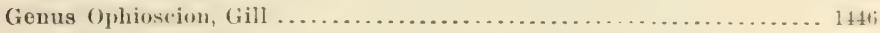

Sulgenus Ophioscion ........................................ 1447

adustus (Agassiz) ...................................................

typicus, Gill ............................................... 1418

trabo, (iilbert ...............................................

simulus, Gilbert ............................................. 1449

imiceps (Jordan \& Gilbert) ................................... 1451

scierus (Jordan \& Gilbert).................................... 1452

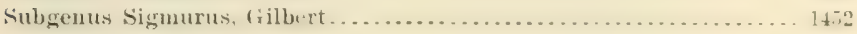

vormicularis (Günther) ................................... 1452

Genus Scirnoprs, Gill ......................................... 1433

ocollatus (Limitus) ......................................

Genus Scirna (Artedi) Linnæus . .................................. 1454

Sulgenus Callaus, Jordan .................................. 14.

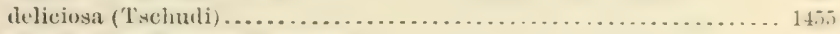

Sul,genus Cheilotrma, 'Tschuti .............................. 115

saturna (Girard) ............................................... 1456

Genus Roncador, Jordan \& Gilbert .............................. 1457

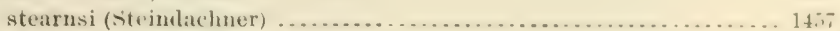

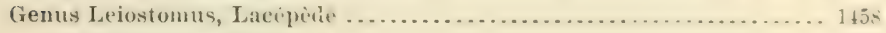

xanthurus, Latcipiele.....................................

(ienus Pachypup, (rill...................................... 14.

furcratus (Lace pèlu) . ................................... 1459

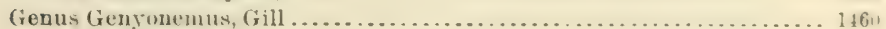

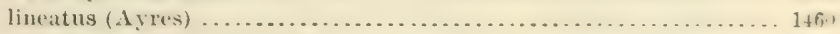

Genus Micropogon, Cuvier \& Valenciennes ........................ 1461

undulatus (Linnteus) ..............................

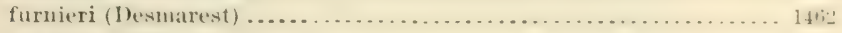

megalops, (iillert.....................................

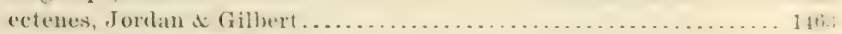

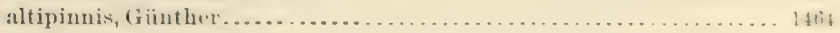

Geuus Umbrina, Cuvier.......................................... . . 1465

broussonetii, Cuvier of Valenciennes........................ 146ti

coroides, Cuvier \& Valenciennes............................... 1466

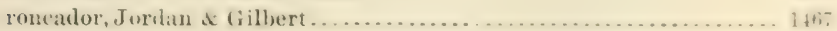

xanti, Gill...................................................

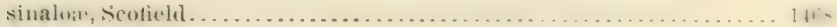

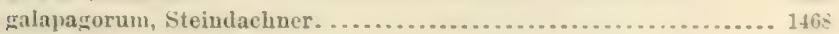

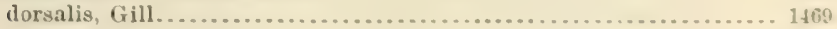

Genus Menticirrhus, Gill ...................................... 1469

simus, Jortau of Eigenmann . . . ..................................

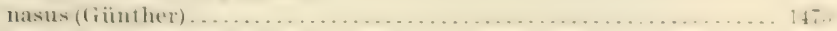

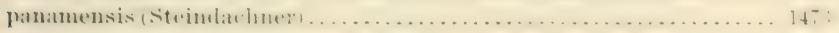

martinicensis (Cuvier ot Valenciennes) ....................... 1473

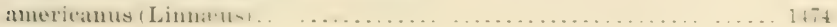


CLASS I'ISCES- Entinumi.

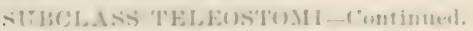

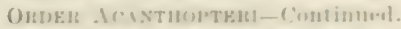

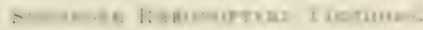

Fitmily scirmider-Continuent.

dienus Menticirrhum. (iill-Cont inued.

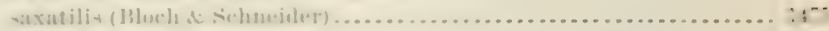

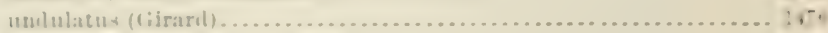

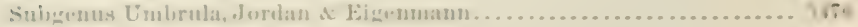

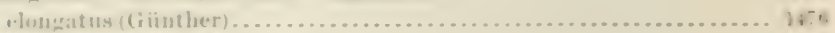

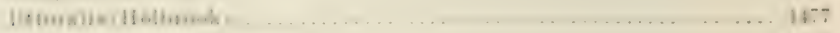

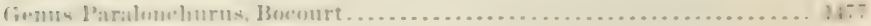

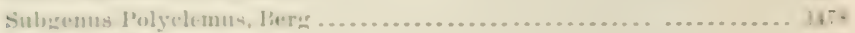

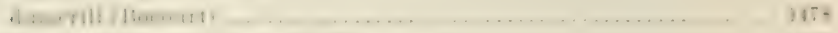

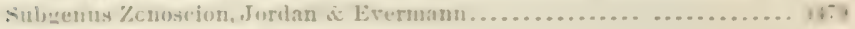

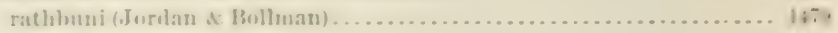

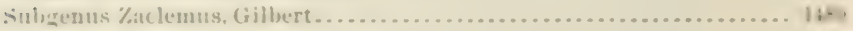

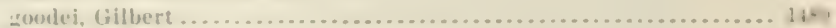

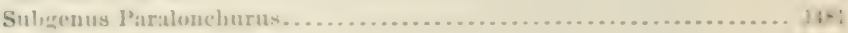

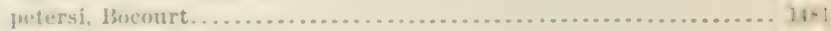

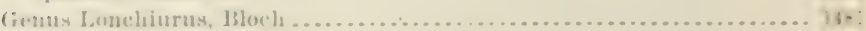

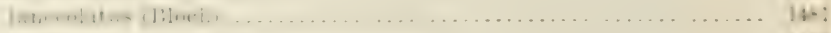

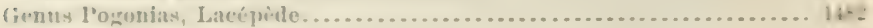

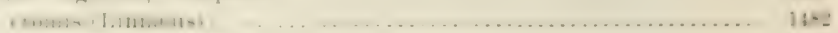

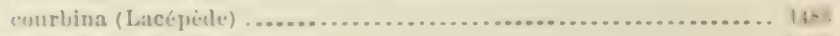

Ferus Aplodinotus, liatinesque............................. \& \&

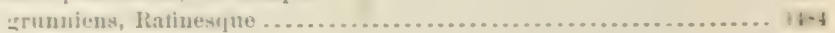

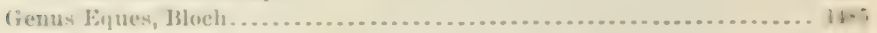

Subuenus I'areques, Gill ................................... I4...

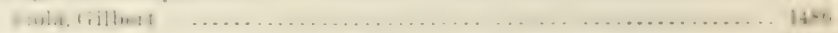

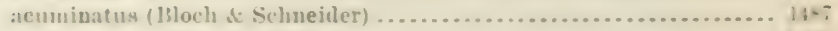

umbrusus, Jordan di Eigenmann............................ 14-:

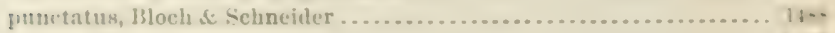

pulcher, steindacliner..................................... $14-$.

sulgenus dipues ..............................................

lancemblatus (Linnatus) ................................... 1:

Gruny Cirrhituidei ......................................... Ger

Family Cirrhitile .............................................

(ienus Cirrhites, daté pèle.................................... \& rivulatus, Valenciennes.................................... 8 .

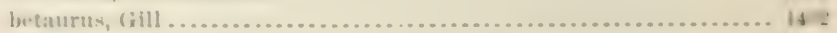

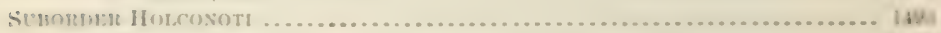

family Embiotocider ...........................................

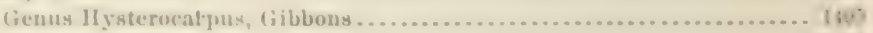

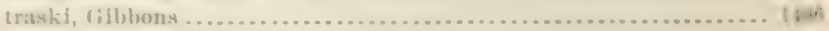

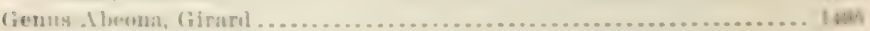

minimn (bibbens) ........................................ 147

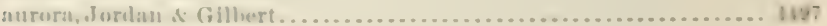

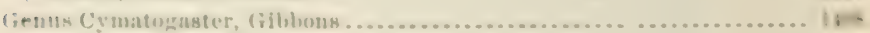

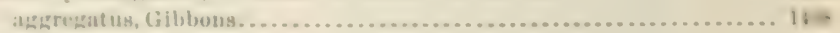

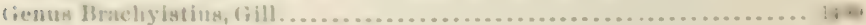

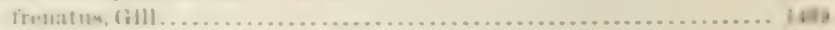

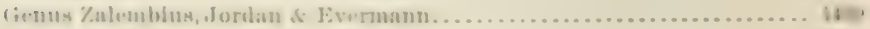

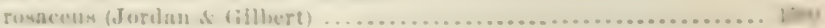

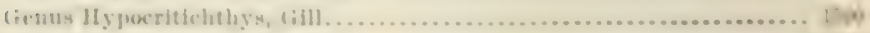

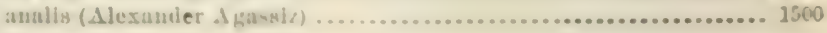


Order ACanthopteri-Continued.

SUbormer Holcoxoti-Continner.

Family Embiotocidce-Continued.

Genus Hyperprosopon, Gibluns......................... 1:m

argentens, Gilbons. ...................................... 1501

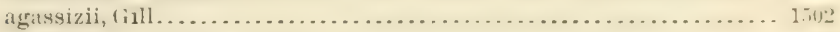

Genus Holconotus, Agassiz .................................... 1502

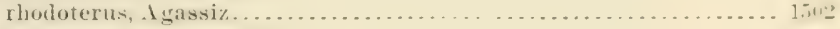

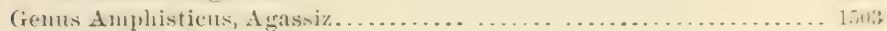

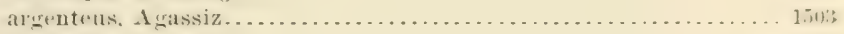

Genus Embiotoca, Agassiz .................................... 1504

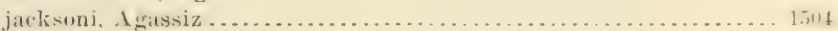

Genus T'rniotoca, Alexander $\Delta$ gassiz............................ $15(15$

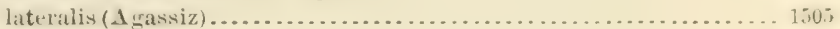

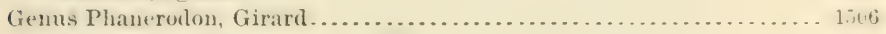
furcatus, Girart .............................................. 1:06

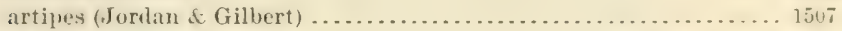

(ienus lihacochilus, Agassiz .................................. toxotes, $\Delta$ gassiz......................................... 1507

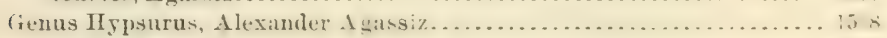

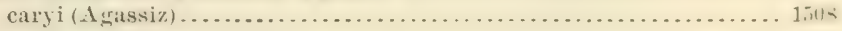

Genus Damalichthys, Girard.................................... 1509

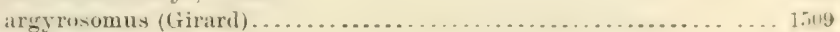

Subonder CHRonides................................................. 1511

Fumily richlide ............................................ 1512

Genus I'tenia, Giinther.................................. 1513 splendiclia. Gïnther........................................ 1.i1:;

Genus Equidens, Eigenmann \& Bray.............................. 1513 coeruleopunctatus (Kner \& Steindachner) ....................... . 1514

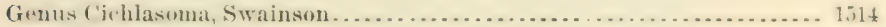

Sulgenus Cichlasoma ...................................... 1515

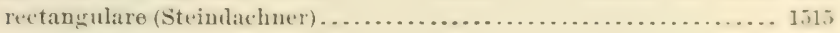

bartoni (Bean) ................................................ 1515

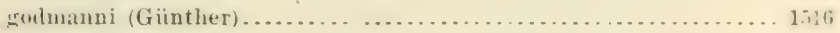

sieboldii (Kner \& Steindachner) . . . . . . . . . . . . . . . . . . . . . 1516

intermelium (Gïnther).................................. 1517

anguliferum (Giinther) . . . . . . . . . . . . . . . . . . . . . . . . . .

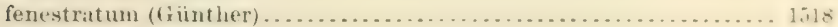

montezuma (Heckel) ......................................... 1518

macracanthum (Giinther) ................................. 1518

parma $($ xiintherı......................................... 1519

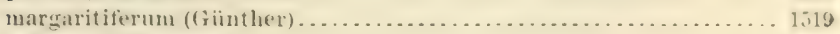

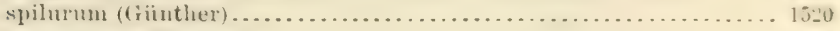

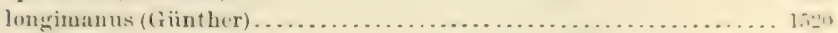

bifasciatum (Steindachner) . . . . . . . . . . . . . . . . . . . . . . . . 1521

helleri (Steindachner) . . ..................................... 1521

balteatum (Gill \& Bransford) . . . . . . . . . . . . . . . . . . . . . . . . . 1521

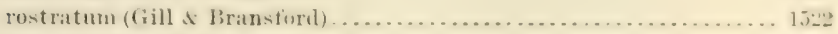

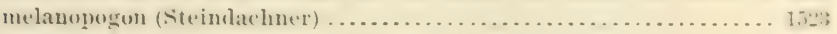

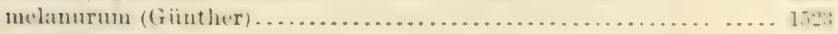

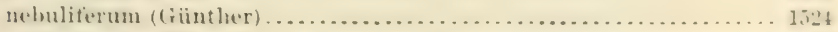

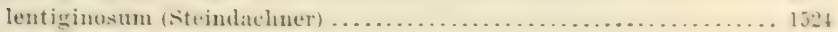

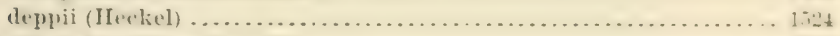

Subgemus Irehoeentrus. (rill.............................

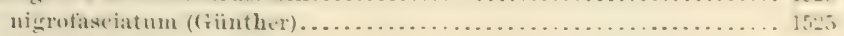

Bull.. No. 47 , pt. $4-\mathrm{r}$ 
CI.As Histes-Continueal.

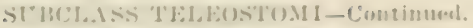

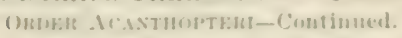

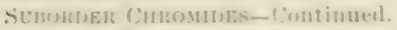

Family Cichlider-Continued.

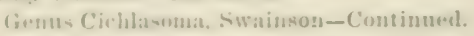

Sulugerus Arehocenstus, Gill-C'outinued.

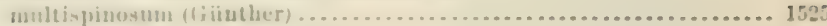

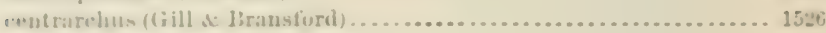

qq.indaclaneri, Jorlan of snveler.............................. 317

Genux Il ros, Heckel .......................................... 1586

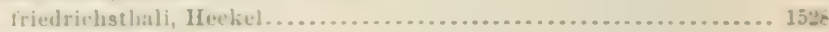

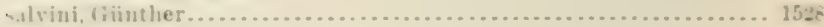

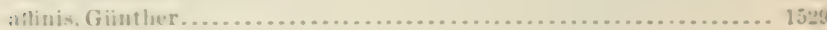

mueulijuinnis, Steindachuer.............................. $15: 9$

irinaculatus, (iiinther...................................... 1598.

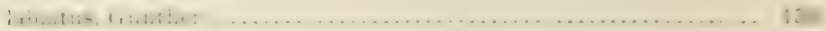

lobochilus, Giinther...................................... 1530

rrythratus, (jiinther..................................... 1531

basilaris, (jill s. Bransfort................................ 153"

nientragu nsis, Giinther ......................................... 153:

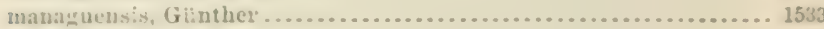

aurens, linuther .......................................... 1533

citrimellus, Gïnther......................................... 1534

motaguensis, Gïnther..................................... 1534

oblongus, liinther........................................... 1535

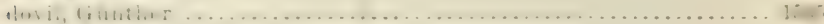

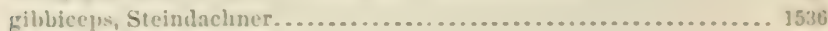

microphthalmus, Gïnther. .............................. 15:36

urophthadusus, Giinther.................................... 1537

troscheli, steiudachner................................... 1537

cyanoguttatus (Baird \& Girard) ........................... 153i

ma ronaceus, (iarman........................................ 153

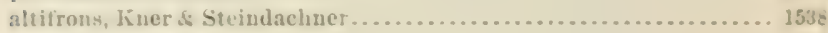

beani, fordan ........................................... 1538

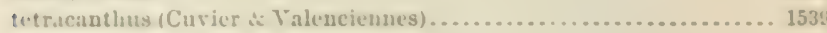

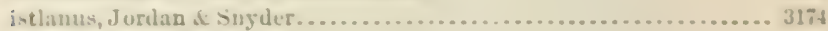

(icuns Theraps, Ginther........................................ 15s0

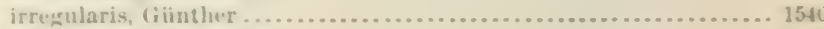

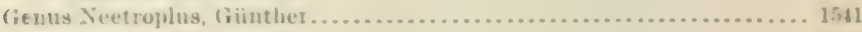

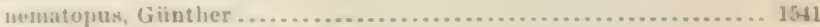

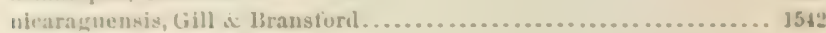

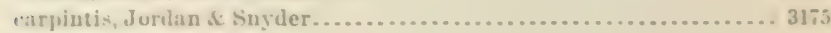

lienus Satanoperea, (jinther................................... 15»2

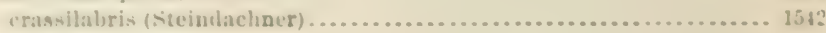

F'smily l'macenerider .......................................... 1513

(ienus Azurina, Jorilan \& Mctiregor............................ 15st

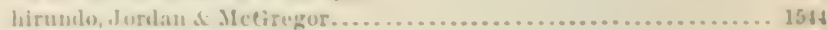

Gemus Cloromis. Cuvier......................................... 1545

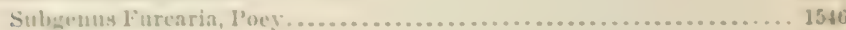

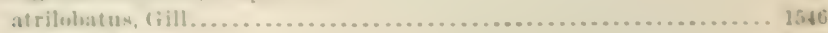

4, ale

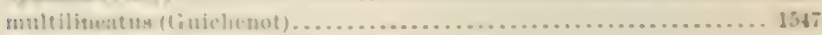

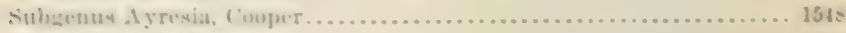

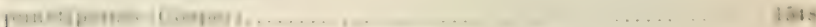

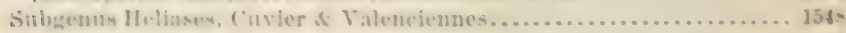

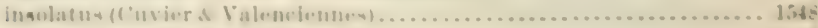

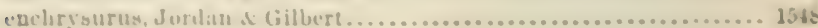


CLASS PISCES-Continued.

SUBCLASS TELEOSTOMI-Continuet.

Order Acanthopteri-Continuer.

Suborder Chromides-Continuel.

Family Pomacentrida-Contimel.

Genus Eupomacentrus, Ijletier.............................. 15t!

Subgenus Eupomacentrus........................................ 1551

leucorus (Gilbert) . . . . . . . . . . . . . . . . . . . . . . . . . . . . . . . . . . . 1551

adustus (Troschel) . . . . . . . . . . . . . . . . . . . . . . . . . . 1551

fuscus (Cuvier \& Talencionnes) ................................ 1552

diencexs, Jorian \& Rutter . . . . . . . . . . . . . . . . . . .

rectifrenum $($ Gill $)$.................................. 1553; 3176

analis (Poey) ................................................. 1554

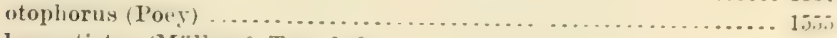

leucostictus (Müller \& Troschel) ............................. 1555

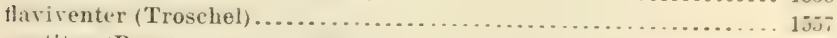

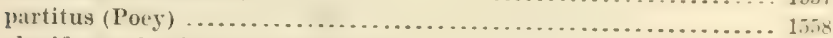

planifrons (Cuvier \& Valenciennes) ........................... 1559

Genus Nexilarius, Gilbert ....................................

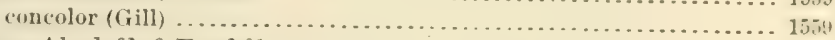

Genus Abudefuf, Forskål ........................................... 1560

Subgenus Glyphisodon, Lacépede................................ 1501

saxatilis (Linnæus) ........................................ 1561

Subgenus Euschistedus, Gill .............................. 15t:

declivifrons (Gill) ....................................... 1562

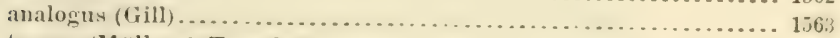

taurus (11 iller \& Trosehel) . . . . . . . . . . . . . . . . . . . . . . 1.tis

rudis $($ Poes) . . . . . . . . . . . . . . . . . . . . . . . . . . . . . . .

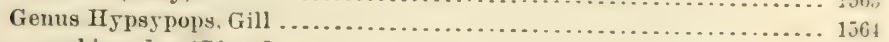
rubicundus (Girard) . ....................................... 1504

Genus Microspathodon, Günther . . . . . . . . . . . . . . . . . . . . . . . . 1565

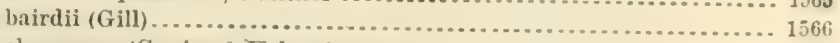

chrysurus (Curier \& Taleneiennes) ........................... 1567

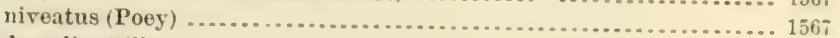

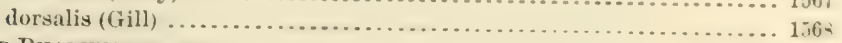

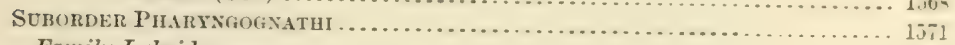

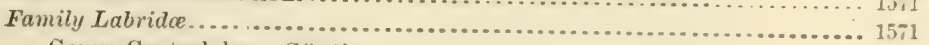

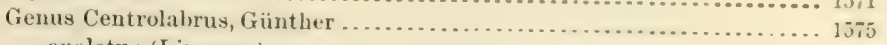

exoletus (Linnwus) ................................... 15919

Genus Tautogolabrus, Günther ................................... 1576

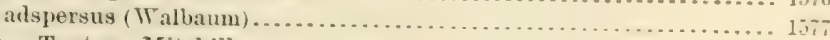

Genus Tautoga, Mitchill . . . . . . . . . . . . . . . . . . . . . . . . . . . . . . . 157

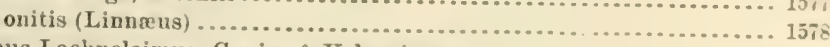

Genus Lachnolaimus, Cuvier \& Valenciennes . . . . . . . . . . . . . . . . . . . 1579 maximus (Walbaum) ........................................ 1579

Genus Harpe, Lacipiele..................................... $15=1$ diplotænia, Gill .......................................... 158.

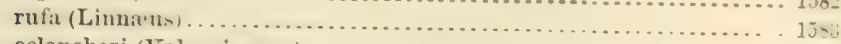

elancheri (Valenciennes) ................................... 1583

pulchella (Poey) .................................................. 1584

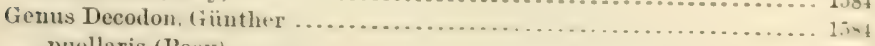

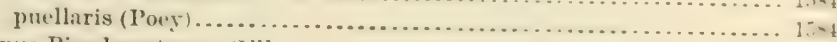

Genus Pimelometopon, Gill . . . . . . . . . . . . . . . . . . . . . . . .

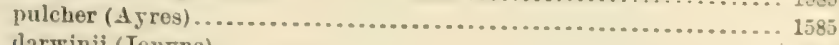

darwinii (Jenyns) .............................................. 1586

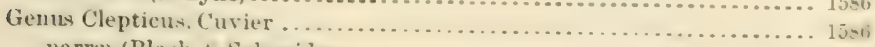
parrit (Bloch \& Sehneiler) ............................. 1isti 


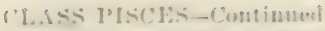

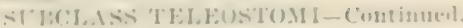

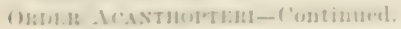

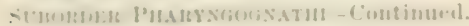

Family Labride-Consinutul.

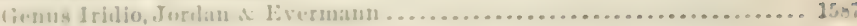

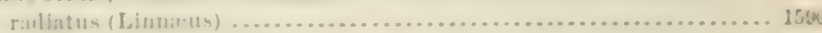

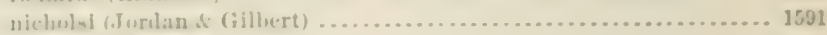

melliter (liilhert) ....................................... 1592

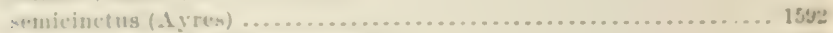

marnoti (Cuvier de balencienues) ........................... 159:

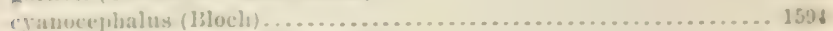

maculipinna (Jfïlet \& Trosclıcl) ......................... 159

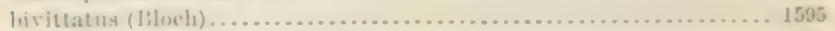

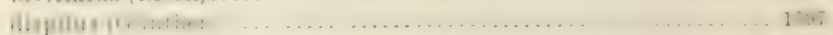

kirsehif, Jortan s Evermann ............................ 159s

nueyi (strindachaer) . .................................. 1599

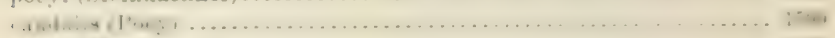

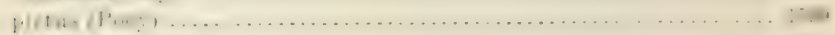

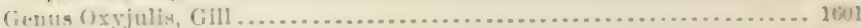

(ailifurnicus (bïnther) ..................................... 1e01

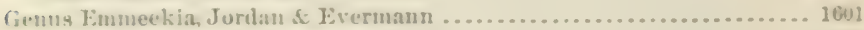

venusta (Jenking . E. Evermanu) ............................ 1602

Gums Julidlio, Jurdan of Evermann ............................ 160

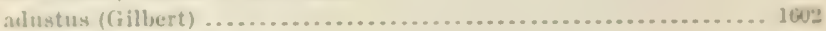

notospilus (tiinther) ...................................... J60

Genus I'sendojulis, lileeker .................................... 160

isornatus, (illbert....................................... 10.

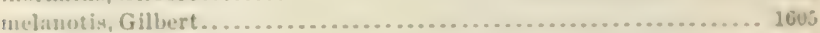

Genथs Thalassoma, Swainsou ......................... 1645; ¿e5!

lucasaum ((jill) ................. 1607; 2859

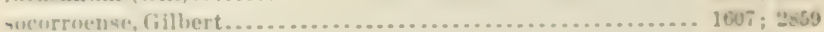

sititlum (Giinther) ................................ 16us; :s5s

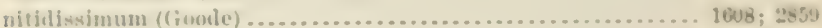

steindachneri (Jurdan) ................................. 16erg; 2859

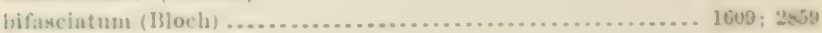

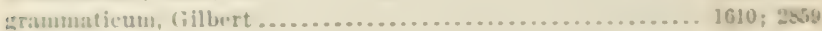

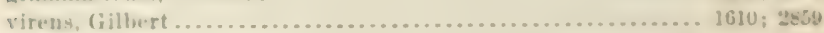

Giems ] doratunutus, fiinther ................................. 1011

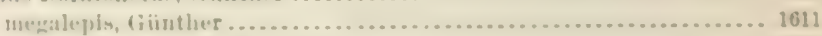

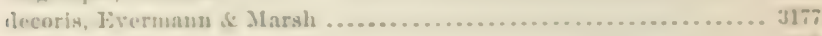

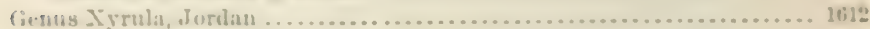

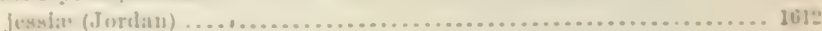

(ienus Xovacullehthys, Ibleeker ............................. 1613

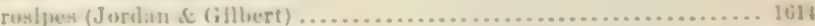

veutralis (Bean) ............................................ 1615

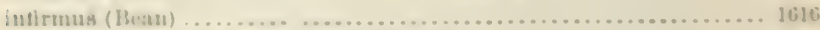

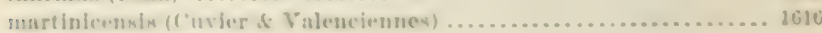

Gernus Ryricheliyn, Cuvier.................................... 1617

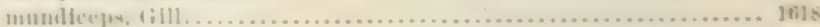

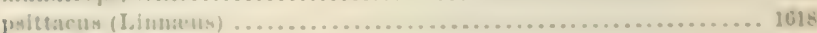

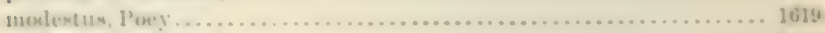

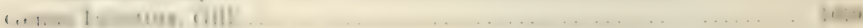

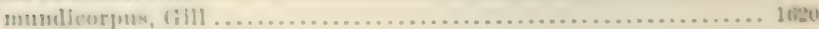

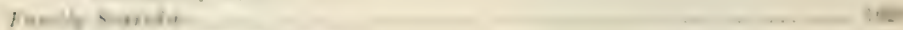

(ienus Cryptututnus, Copre ................................... 16:1

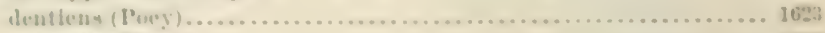

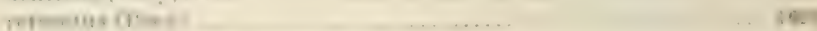

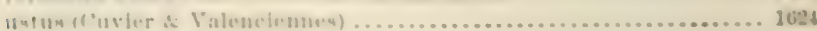


CLASS PISCES-Continued. SUBCLASS TELEOSTOMI-Continued.

Order acanthopteri-Contimued.

Suborder Pharygognathi-Contimued.

Family Scarido-Continued.

Genus Cryptotomus, Cope-Continued.

auropunctatus (Cuvier \& Valeneiennes) ........................ 1624

beryllinu, Jorkan it Swain . . . . . . . . . . . . . . . . . . . 16,

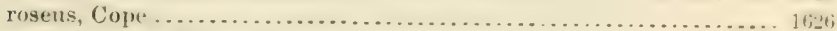

Genus Calotomus, (rilbert ................................. 1ficti

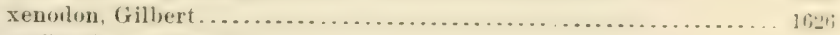

Genus Sparisoma, Swainson ................................ 16:7

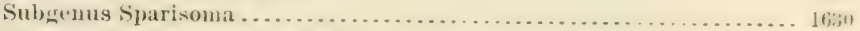

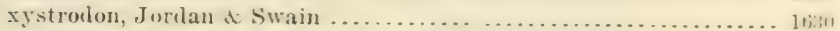

atomarium (Poey) ............................................ 1631

radians (Cuvier \& Valencienues) ............................. 1631

hoplomystax $(\mathrm{Cope})$....................................... 163?

niplıobles, Jordan \& Bollman ........................... 16.;.;

aurofrenatum (Cuvier \& Valenciennes) ....................... 1634

oxybrachium (Рову) ........................................ 16. . . . . . . .

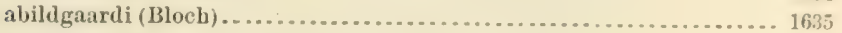

distinctum (Poey) ............................................ 163j

chrysopterum (Bloch \& Schneider)............................ 16.3f

lorito, Jordan of Swain ..................................

virile (Bmnaterre) ..................................... 14,

Subgenus Euscarus, Jordan \& Evermann........................ 1639

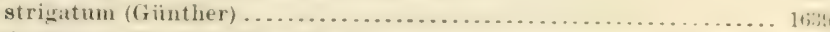

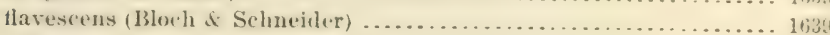

rubripinne (Cuvier \& Valenciennes) .......................... 1040

brachiale (l'oey) ...................................... 10.1

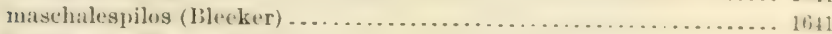

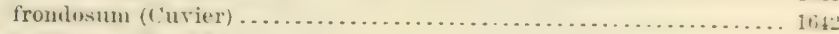

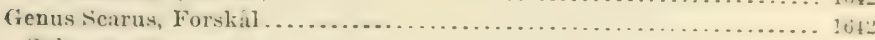

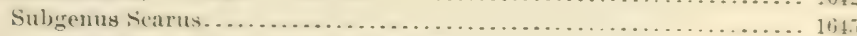

punctulatus (Cuvier \& Valenciennes) ........................ 16t5

hollmani, Jordan \& Evermana.......................... 14it

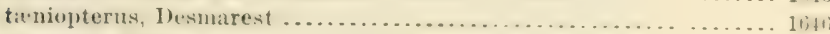

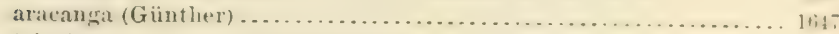

trispinosus, Cuvier \& Valenciennes ........................... 1048

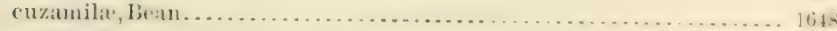

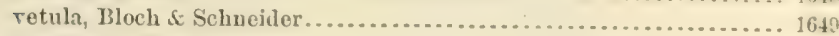

gnathodus, Poey .......................................... 1650

Subgenus Calliodon (Gronow) Sehneider........................ 1650

croicensis (Lloch) ........................................... 1651)

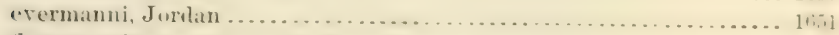

flavomarginatus, Cuvier of Valenciennes ....................... 1652

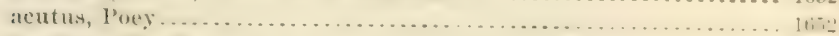

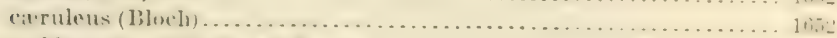

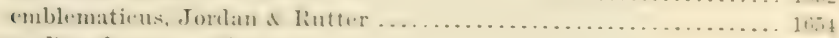

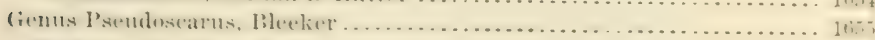

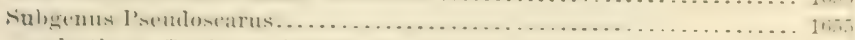

eolestinus (Cuvier \& V"alencionnes) ........................... 16, . . . . .

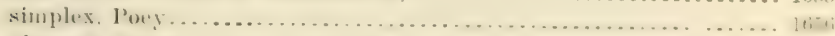

J)

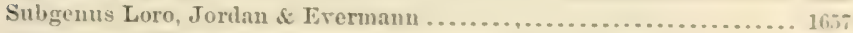

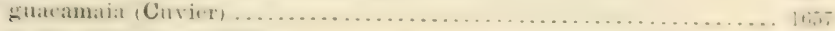

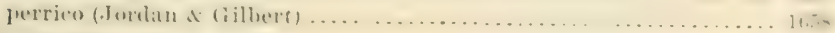

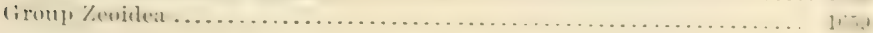


1.t.

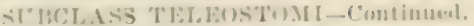

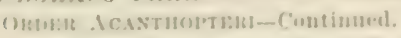

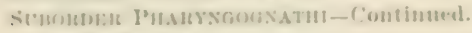

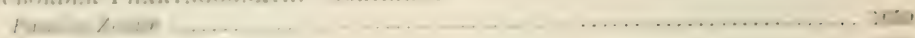

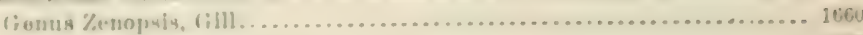

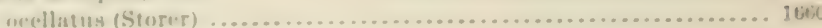

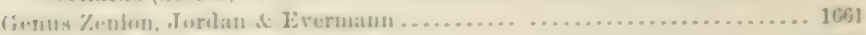

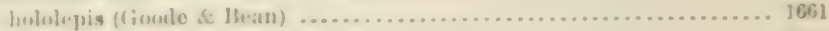

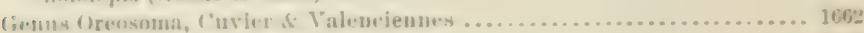

atlanticum, Cuvier \& Vialenciennes........................ 106:

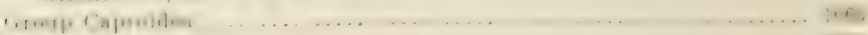

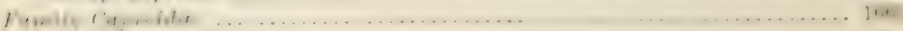

(ivnns Antigunla, Lowe.................................... lout

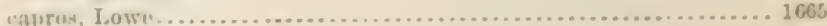

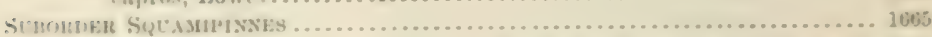

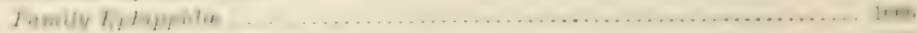

fornus Chretolipterus, Laté pede............................. 1667

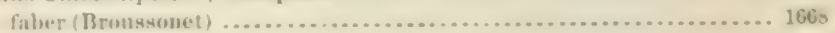

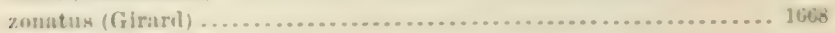

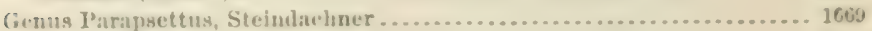

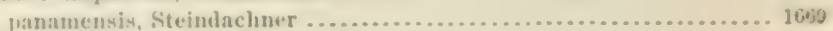

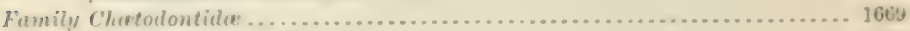

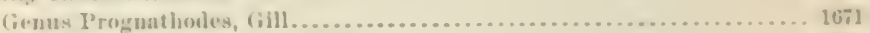

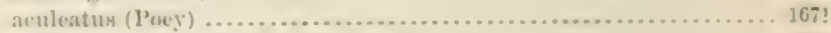

Genus Forcipiger, Jordan \& McGregor............................ 1671 Havissimus, Jordan .t. MeGregor ........................... 1671

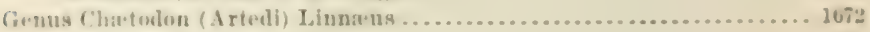

subgenus Chetolontups, Bleeker.......................... 1673

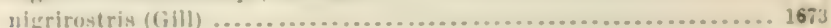

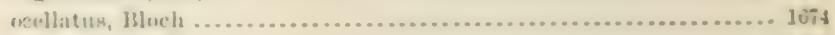

humeralls, (iiinther ....................................... 16it

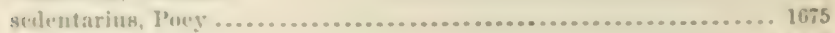

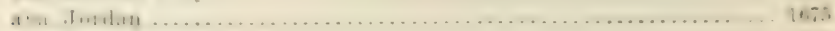

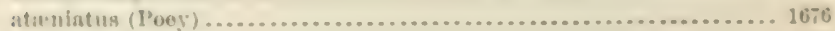

af riatus, I. innipus ....................................... 16 .

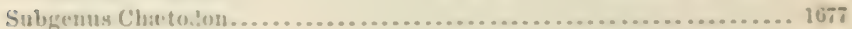

apintratus, Linnatus......................................... 167

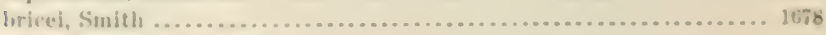

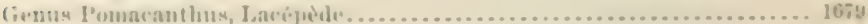

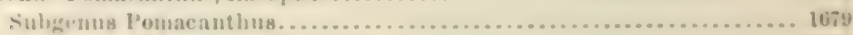

аними

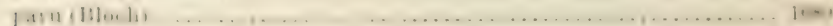

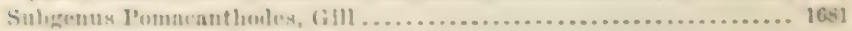

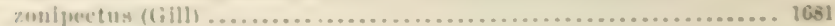

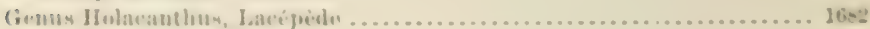

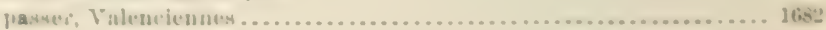

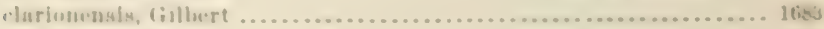

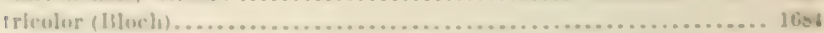

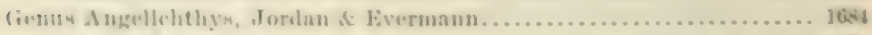

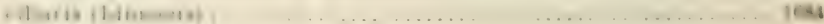

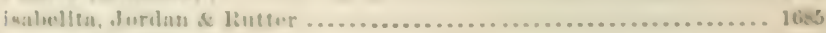

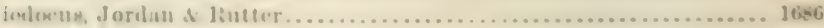

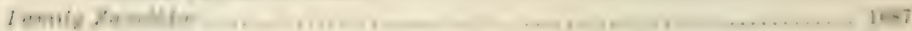

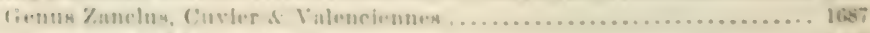

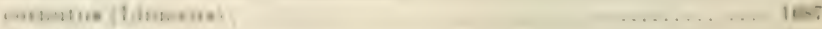

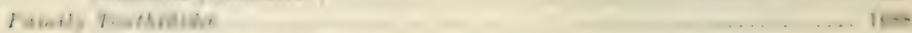

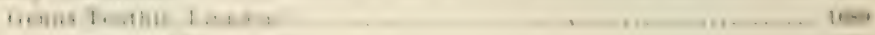

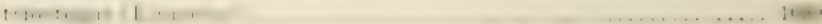


CLASS PISCES-Continued.

SUBCLASS TELEOS'TOMI-Continued.

Order ACANTHOpteri-Contimuel.

SUborder SQQuAMPINNES-Contiuned.

Family Teuthidide-Continued.

Genus Teuthis, Linneus-Continued.

(atruleus (Bloch of Schneider) ........................... 1691

hepatus, Linna'us .................................. 691

erestonis, Jordan . Starks............................ 1692

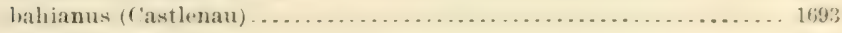

aliala (Lesson) . . . . . . . . . . . . . . . . . . . . . . . . . . . . . 169:

Genus Xesurus, Jordan \& Evermann ............................ 1694

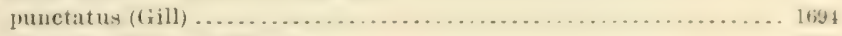

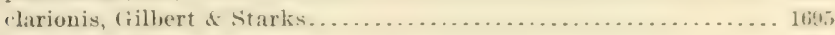

laticlavius (Valenciennes) . . . . . . . . . . . . . . . . . . . . 16!

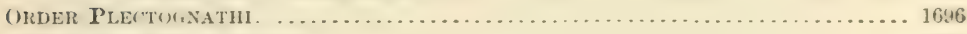

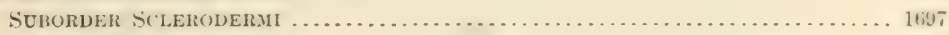

Family Triatenthide ....................................... 169i

Genus IIollardia, Poey ..................................... 169

hellardi, Poey ........................................ 169k

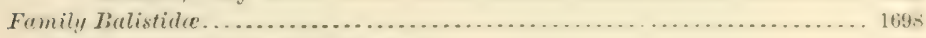

Genus Balistes (Artedi) Linna us. . . . . . . . . . . . . . . . . . . . . . . . . . . 1699

Subgenus Capriscus, Ratimesque .......................... 1700

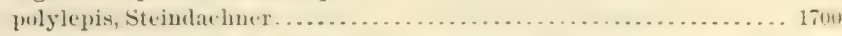

nautragium, Jordan it Starks .......................... 17um

citrolinensis, (imelin................................ 17

forcipatus, (imelin .................................... 1742

Subgenus Balistes........................................ 1703

vetula, Linnieus........................................ 17tw;

Genus Pachynathus, Swainson .............................. 1in: capistratus (Shaw) .......................................... 1704

Genus Canthidermis, Swainson............................... 1705

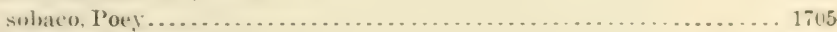

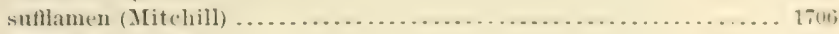

maculatus (Bloch) ..................................... 1706

willughbeii (Lay \& Bennett) ................................ 1707

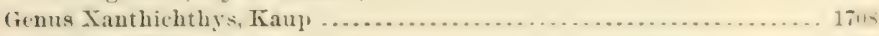
ringens (Linn:eus) . . . . . . . . . . . . . . . . . . . . . . . . . . .

mento (Jordan \& (Billert) ................................ . . 17111

fiemus Mfelichthys, swainson............................... 171 piceus (Pooy)...................................................... 1711

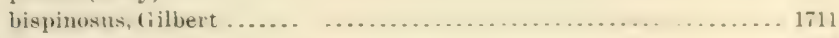

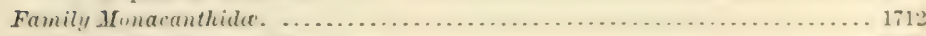

(ienus Cantherimes, Swainson ............................ 1:1:

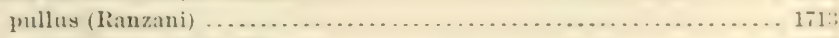

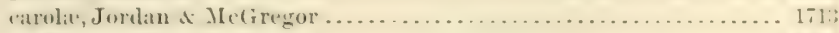

Genus Xonateanthus, Curier.................................. 1714

ciliatus (Mitchill) .......................................... 171t

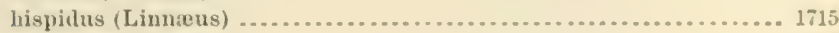

spilonotus, Cope...................................... 1716

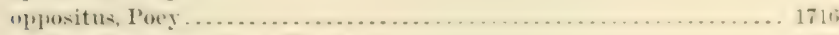

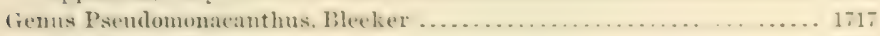

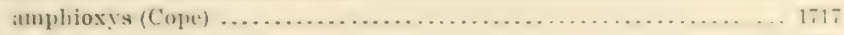

Genus Ceratacanthus, Gill.............................. 1718; 2860

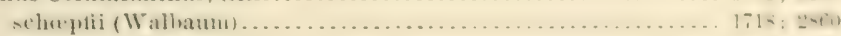

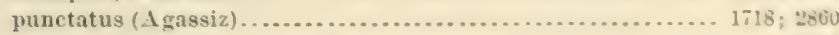

Subgenus Osbeckia. Jordan \& Evermann ....................... 1719

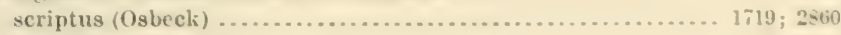

Genus Alutera, Cuvier ..................................... 1717; 2800 monoceros (Oslreck) ........................... 1720: $2660 ; 31$ is 


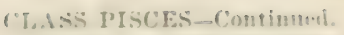

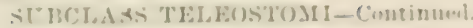

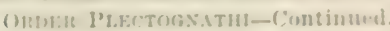

sтum

Fomily ()straciuder ............................................. 1721

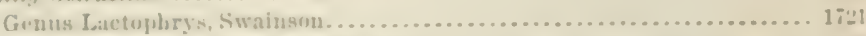

Sul,genus Rhinesumus, Swainson .......................... 17te.

1:.,

Subgenn Chapinn, Jordan \& Evermann ..................... 17:2!

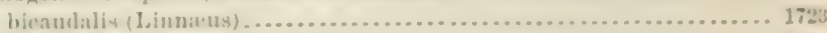

Suhgesus Lactuphrys................................... 17:

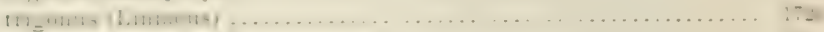

Sub renuy Acunthost racion, Jleeker.......................... 172 trjornis (Limuans) ...................................... 18.

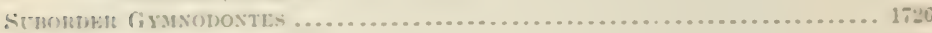

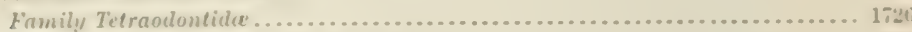

Genus Laggocephalus, Swainson ............................. 1727

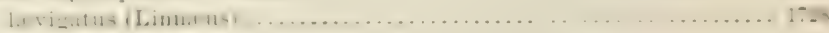

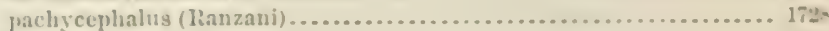

Gonus Spheroilles, Lacepielo .................................. 17:

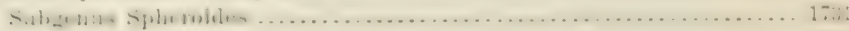

angusticr.ps (Jenrns) .................................. 1731

lobatus (Steindachner) .................................. 1731

spengleri (Bloch) ...................................... 1739

maculatus (Bloclt s. Selıneider) ............................ 1733

nephelus (Goole \& Beau) ............................... 1733; 317 ह

marmoratus (lanzani) ..................................... 1733

Sulgenus Cheilielathys, Müller ............................. 1734

testudineus (Linneus) .................................... 1734

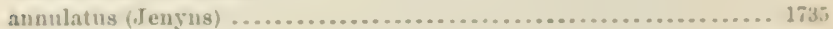

politus (Giraril) . . ......................................... 1736

formosus (fiinther) ...................................... 1736

furthi (Steintachner) ..................................... 173i

trichocephalus (Cope) .................................. 170.

prachygaster (Miiller of 'Troschel) .......................... 173s

(ienns () videles, Laciphele ..................................... 173s

rerethizon (Jorilan of Gilbert) ............................ 1739

setosus (Iosa Smith) .................................... 17isg

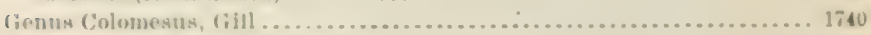

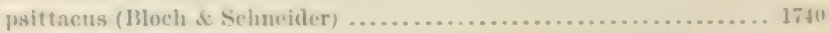

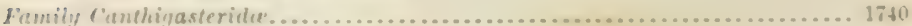

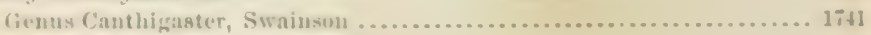

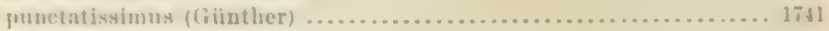

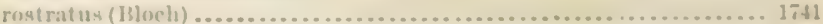

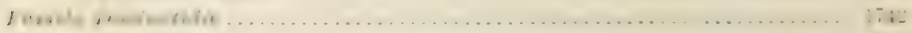

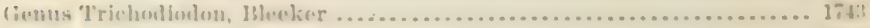

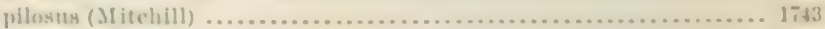

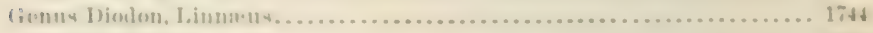

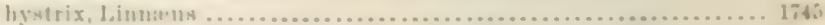

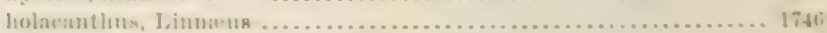

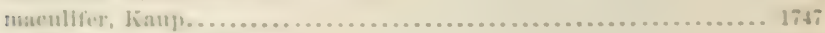

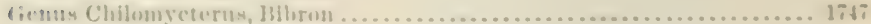

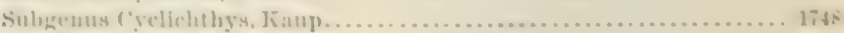

scho.

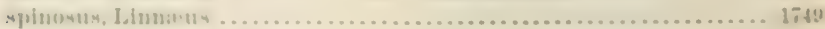

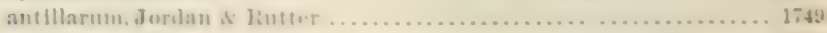

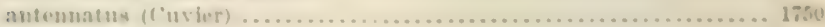

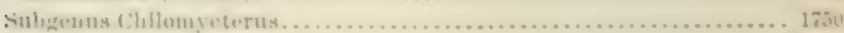

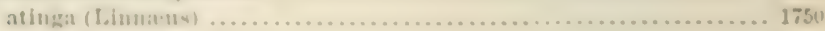

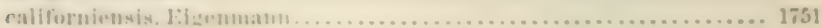


CLASS PISCES-Continued.

SUBCLASS TELEOS'OMI-Continuer.

ORDER PleCTOGNATHI--Continued.

SUBORDER GYMNODONTES-Continued.

Farnily Diodontida-Continued.

Genus Ljosphaera, Evermanu \& Tendall. ......................... 1751

globosa, Evermann \& Kendall. . . . . . . . . . . . . . . . . . . . . . . . . . . 1751

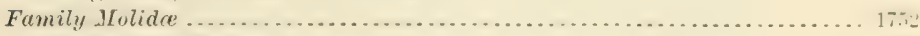

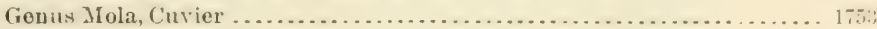

mola (Linna us) ........................................... 1753

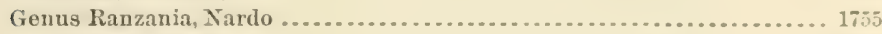

truncatil (Retzius) . ......................................

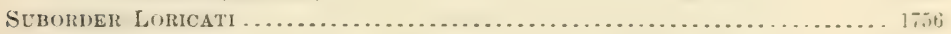

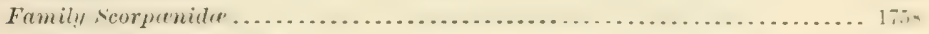

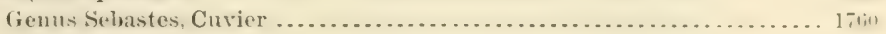

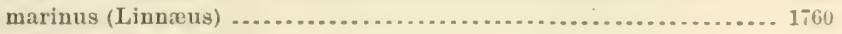

Genus Sébastulohus, Gill . . . . . . . . . . . . . . . . . . . . . . . . 1; til

. alascanus, Bean ........................................... 1761

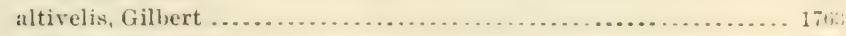

Genus Sebastodes, Gill... . . . . . . . . . . . . . . . . . . . . 1,

Subgenus Emmelas, Jordan de Evermann ... . . . . . . . . . . . . . . . . 177i

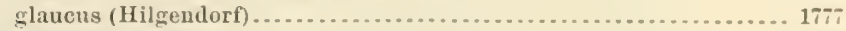

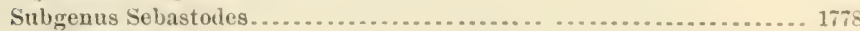

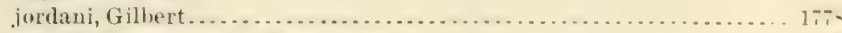

goolei, Eigemmann \& Eigenmann ........................

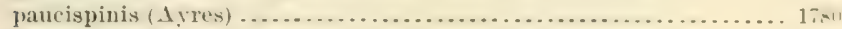

Silbenus Selnastosonus, Gill ... . . . . . . . . . . . . . . . . . . . . 17-1

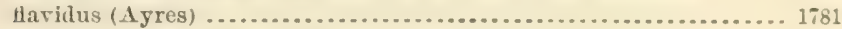

serranoides, Eigenmann \& Eigenmann ........................ 1782

melanops (Girard) ........................................... 1782

Subgenus I'rimospina, Eigenmann \& Beeson ..................... 1783

ciliatus (Tilesin.s) . . . . . . . . . . . . . . . . . . . . . . . . . . .

mystinus, Jorkan \& Gillert . . . . . . . . . . . . . . . . . . . $17-1$

Subgenus Acutomentum, Eigenmann of Beeson .................. 1785

entomelas (Jorlan \& Gilbert) ... . . . . . . . . . . . . . . . . . . . . 1785

rufus, Eigemmann A Eigenmann . . . . . . . . . . . . . . . . .

macionaldi (Eigenmann \& Beeson) ........................... 1786

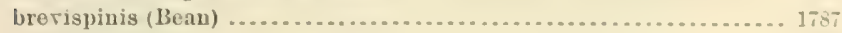

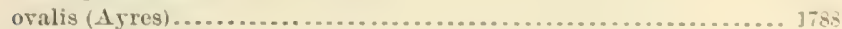

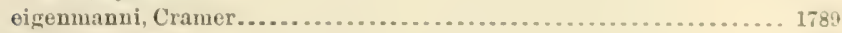

hopkinsi, Cramer. . . . . . . . . . . . . . . . . . . . . . . . . . . . . . . . . 1789

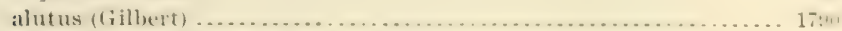

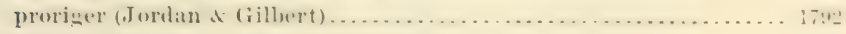

Subgenus liosicola, Jordan . E Erermamn . . . . . . . . . . . . . . . . . . . . . 1793

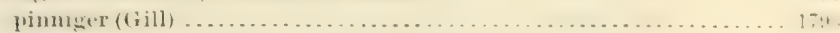

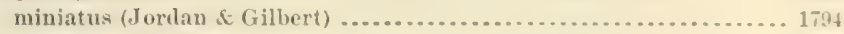

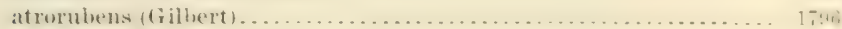

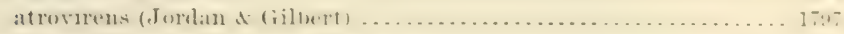

Subgenus Zalopyr, Jordan \& Ivermann . . . . . . . . . . . . . . . 1795; 2860 alentianus, Jordan \& Evermam ......................... 1795; 28nแ

Subgenus Eosehastes, Jorlan of Evermanu . . . . . . . . . . . . . . . . . . . 1798

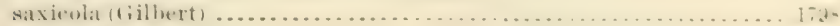

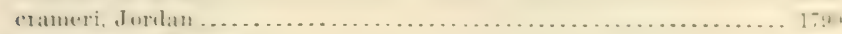

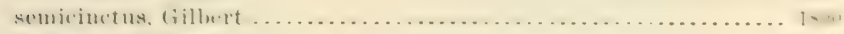

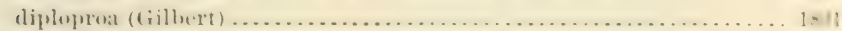

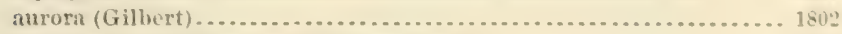

molanostomus, Eigenmann \& Eigenmann ...................... 1803

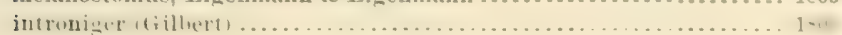


r.I.ds.' PISCLS-Continued.

S! IBCLASS TELEOSTOMI-Continued.

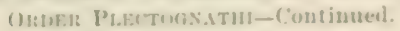

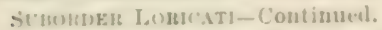

Fumily Sconpeniele-Continucal.

dienns Selnastudes, fill-Continued.

Sulggenus Seloastomus, Gill ............................... 180

ruberrimus, Cramer....................................... 18us

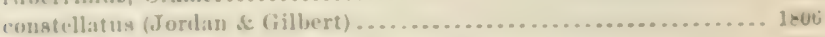

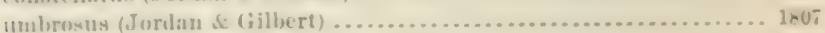

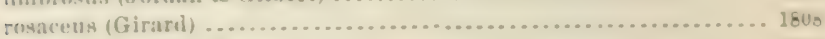

ayrenii, Gilhert \& Cramer .................................... leve

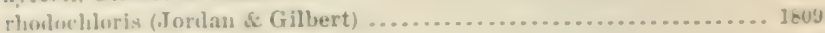

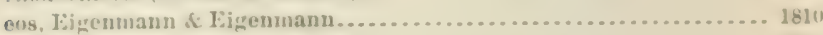

gilli, Eigenuann \& Eigenmann ........................... 1811

Ghlorostictus (Jurdan \&. Gilbert) ............................... 1811

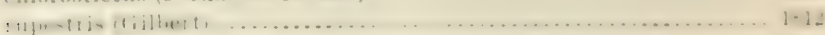

Subgenus Hispaniscus, C'ramer .............................. 1813

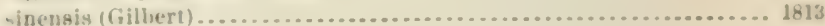

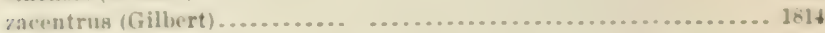

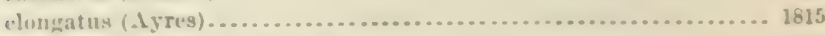

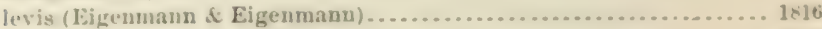

ruhrivinetus (Jordan \& Gilbert) ............................ 1k17

Subgums A uetospina, Eigenmanu \& Beeson ...................... 1817

anriculatus $(\mathrm{Girard})$........................................ 1817

dallii (Eigenmann o Beeson) ............................. 181n

Sulgenus Pteropolug, Eigenmann \& Beeson...................... 1819

rastrelliger (Jordan \& Gilbert) ........................... 1619

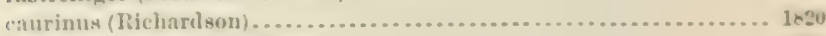

vexillaris (Jorulan of Gillert) .............................. 1821

maliger (Jortan . G Gilber1) ................................. 18:2

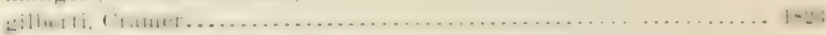

varnatus (Jurilan o. Eilhert) ............................. 1824

clirysumelas (Jorelan \& Gilbert) ............................ 18ะ5

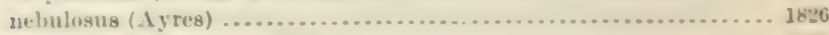

Subgenus Sebastichthys, Gill.............................. 1k:

serriceps (Jordan \& Gilbert) .............................. 16:7

nigrveinetus (Ayres) ................................... 18:

Subzenus Sebastosomus, Gill ......................... 1889; 2864 tacranowskii (Steindachner) ............................ 18:31; 2sco

Gemus Sichastopsis, Gill..................................... 183i

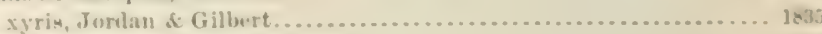

Genn Helicolenus, Givolle of Bean ............................. 1836

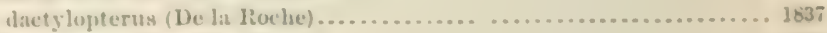

malerensim, fivole of lkean ................................. 1837

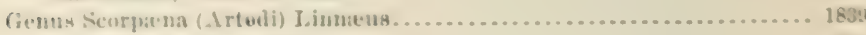

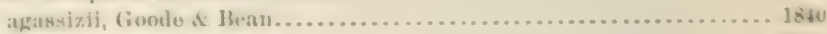

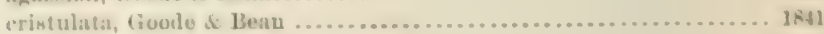

lirasiliensin, Cuvier of Vilencienues........................... 18\$?

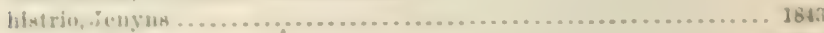

panuosi, Crinner........................................... 184t

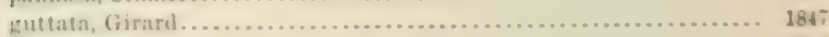

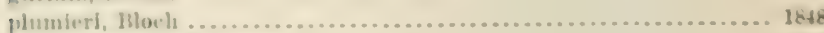

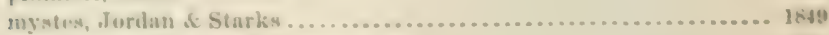

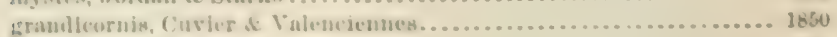

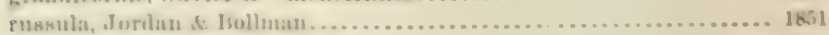

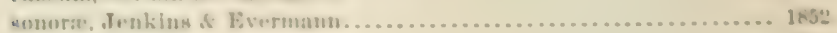

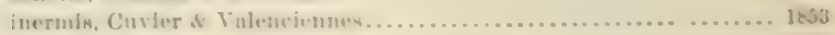

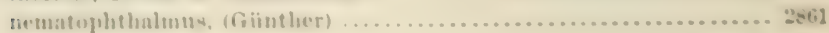


CLASS PISCES-Continued.

SUBCLASS TELEOSTOMI-Continued.

Order Plectognatui-Continued.

SUBORder Loricati-Continued.

Family Scorpcenida-Continued.

Genus Pontinus, Puer .................................... 1 184

macrolepis, froole \& Bean.................................. 185.

castor, Poer................................................. 1856

pollux, Poey ................................................ 1857

rathbuni, Goode \& Bean . . . . . . . . . . . . . . . . . . . . . . 1

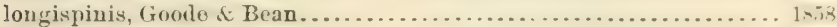

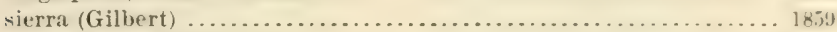

Genus Setarches, Johnson ................................... 18kio

parmatus, Goode ....................................... 18 tit

Family Anoplopomatide . ................................... 1etil

Genus Anoplopona, Ayres................................ 1 $₫ 61$

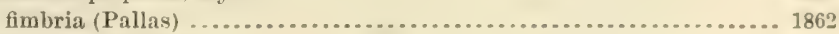

Genus Erilepis, (iill.................................... 186?

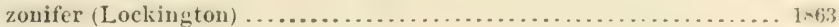

Family Hexagrammide ..................................... 1,6?

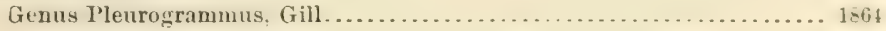
monoptersgins (Pallas) ................................... 1864

Genus Hexagrammos (Steller) Tilesius ........................ 1866 doeagrammus (Pallas) . .................................... $1 \times f_{i}$ octogrammus (I'allas) . . . . . . . . . . . . . . . . . . . . . . 1800 stelleri, Tilesius.......................................

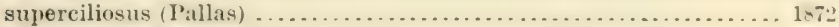
lagocephalus (Pallas) ..................................... 1873

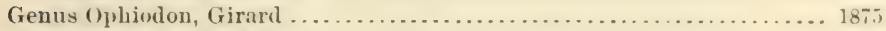
elongatus, Girard .................................................

Genus Zaniolepis, Girard ................................ 1876

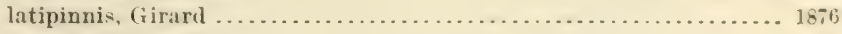
frenatus, Eigenmann................................... 1877

Genus (Oxylebius, Gill .................................. 18,8 pictus, Gill...........................................

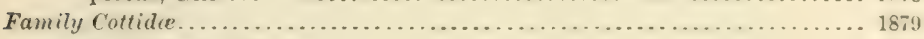

Genus Jordania, Starks .................................. 1n

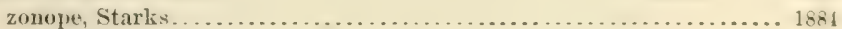

Genus Paricelinus, Eigenmann of Eigenmann .................... 1885 hopliticus, Eigenmann \& Eigeumann ......................... 1886

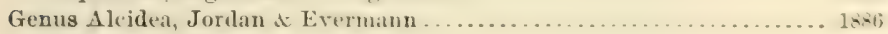

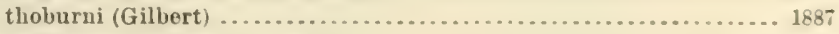

Genus Scorpanichthys, Girard ........................... 1899 marmoratus (Avres) . . . . . . . . . . . . . . . . . . . . . . . 1889

Genus Chitonotus, Lockington........................... lns!

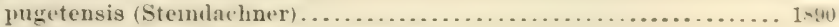

Genus Tarandichthys, Jordan ․ Evermann....................... 1891

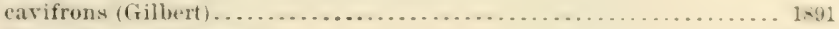
filamentosus (trilbert) . . . . . . . . . . . . . . . . . . . . . 1892

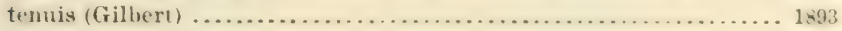

Genus Icelinus, Jordan....................................... 189 fimbriatus, Gilbert ....................................... 1894

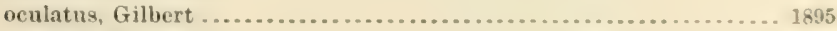

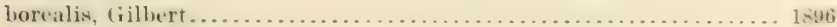

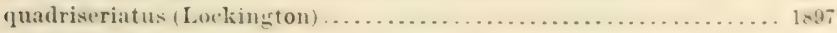

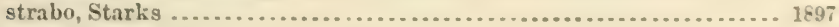

Genus Astrolytes, Jorlan of Starks...... . . . . . . . . . . . . . . . . . 1898

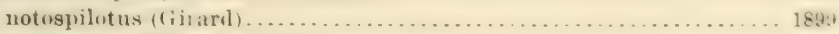
fenestralis (Jordan s: Gilbert)................................. 1899 


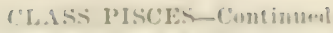

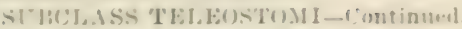

()

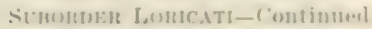

Family Cotfider-Continuet.

dienus Arehises, Turelan of Gillu.rt ........................... 1900

plumarius, Jurdau of (iilhert.............................. 1900

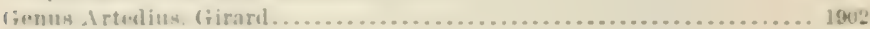

lateralis (lirart) ....................................... 19402

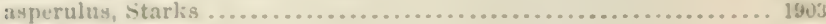

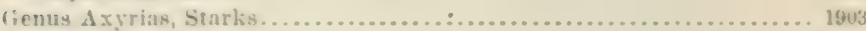

harringtoni, Starks..................................... 1tw

firnus Irtediellus, Jurulan ................................... 1505

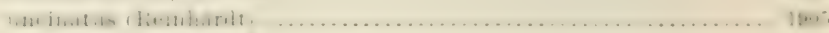

atlanticus, Jordan \& Evermann .......................... J900

naciticus, Gillyert. . ....................................... 1900

Genus liusearius, Jordan of Starks ............................ 1908

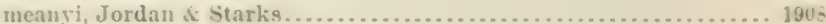

Genus lastrinus, Jorlan of Evermann............................. 1909

scutiger (Benn) . . . ............................................. 1909

Ginus Jeelus, lírörer ............................................ 1911

bicornis (lieinhardt) ....................................... 1911

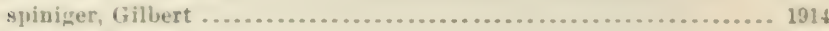

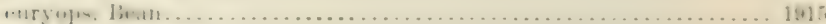

vicinalis, Gilbert......................................... 1910

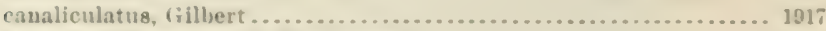

australis, Eiqenmann of Eigenmann......................... 1910

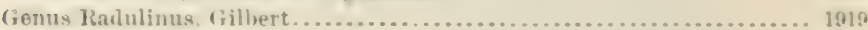

beleoillen, Gilbert ......................................... 1919

asprellus, Gilhert ......................................... 1020

Genus Stelgistrum, Jordan of Gilbert............................. 1921

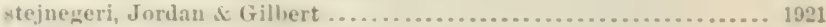

(ienus 'Triglops, lieinhardt..................................... 1923

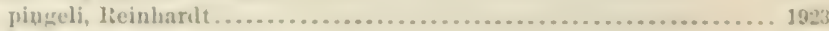

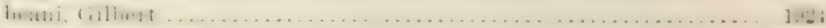

-

(ienus Sternias, Jorlan of Evermann . . . . . . . . . . . . . . . . . . . . . 1026 xemestethus (Gillert) . . . . . . . . . . . . . . . . . . . . . . . . . . 1027

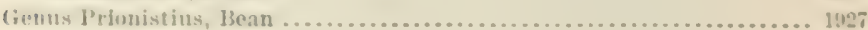

macr.llus, likan........................................ 192a

Gicnns Elanura, (illiert ...................................... 1930 furlicati, (iilbert .......................................... 19830

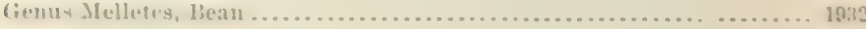

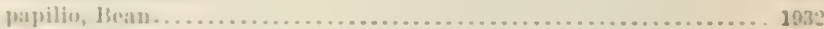

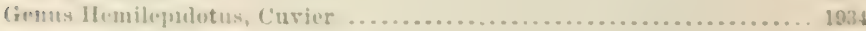
jurilani, Dean ...........................................

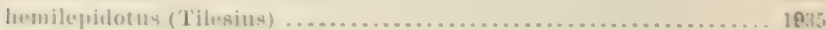

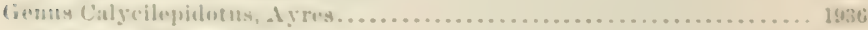

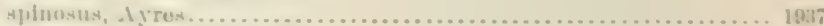

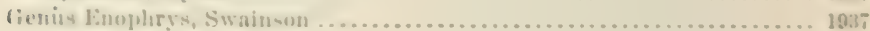

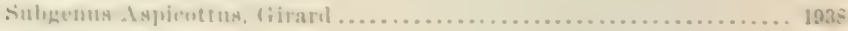

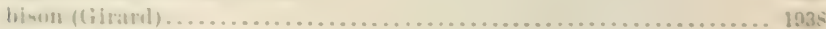

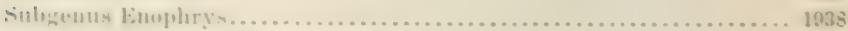

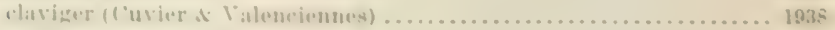

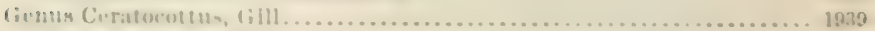

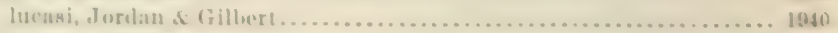

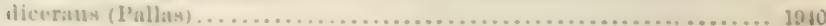


Fomily Cottidce-Continued.

Genus Cottus (Artedi) Linnaus ................................. 1941

Subgenus Pegedictis, Rafinesque .............................. 1944

asper, Richardson. ......................................... 1944

gulusns (Girarul) ......................................... 1:4t

evermanni, Gilbert.......................................... 1945

rhotheus, Rosa smith ................................. 1946

shasta, Jordan \& Starks .................................... 1947

punctulatus (Gill) ....................................... 1948

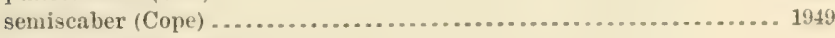

ictalops (Rafinesque) ....................................... 1950

Subgenus Tauridea, Jordan \& Rice.............................. 1952

ricei, Nelson......................................... 1952

Sulgeuus Cottus. . . . . . . . . . . . . . . . . . . . . . . . . . .

onychus, Eigenmann \& Eigenmann ......................... 1953

pollicaris (Jordan \& Gilbert) .................................... 1953

cognatus, Richardson .................................... 195 . . . . .

perplexus, Gilbert \& Erermann.............................. 1955

klamathensis, Gilbert....................................... 195

aleuticus, Gilbert ...................................... 195

minutus, Pallas ......................................... 1958

veldingii, Eigenmann \& Eigenmann ....................... 1958

philonips, Eigenmann \& Eigenmann .......................... 1959

amnir, Jorklan \& starks.................................. litei

spilotus (Copw) ..................................................

leiopomus, Gilbert \& Evermann .......................... 1962

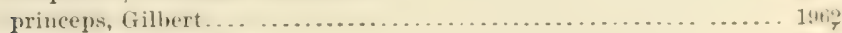

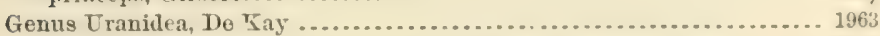

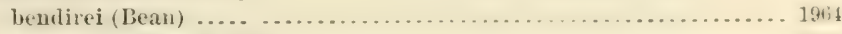

greenei, Gilbert \& Culver ................................ 196.

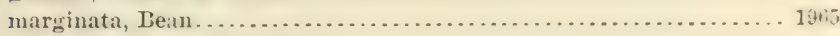

tenuis, Evermann \& Meek .................................. 1969

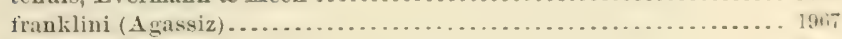

kumlienii, Hoy.................................... $14 n_{i i}$

gracilis (Heckel) .............................................

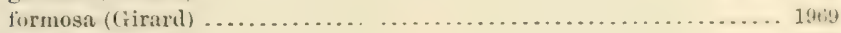

loyi, I'ntnam ..................................... $1: 4,9$

Genus II roxocephalus (Steller) Tilesins ........................ 1970

Subgenus Acanthocottus, Girarl . . . . . . . . . . . . . . . . . . . . . . . . . 1971

hubalis (Euphrasen) . . . . . . . . . . . . . . . . . . . . . . 19:1

a neus (Xlitchill) . . . . . . . . . . . .

scorpioides (Fabricins) .................................. 1973

scorpius (Linneus) ......................................... i 97 ts

groulandicus (Cuvier \& Valenciennes) ......................... 19it

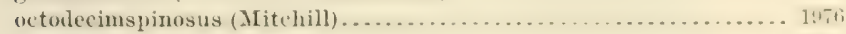

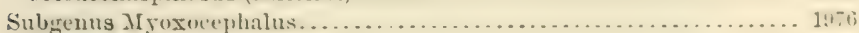

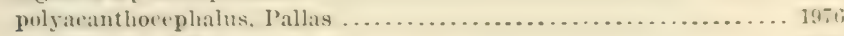

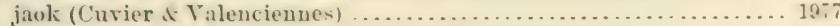

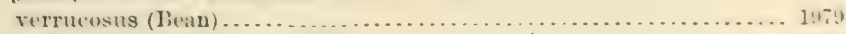

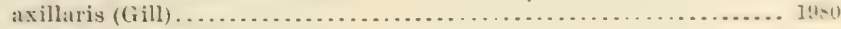

stelleri, Tilesius................................................... 1981

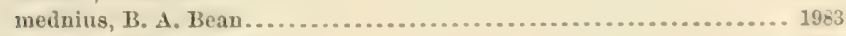

nivosus (Herzenstein) ................................. 19-4

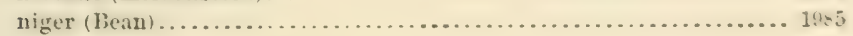




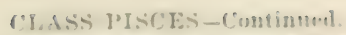

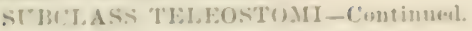

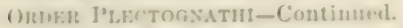

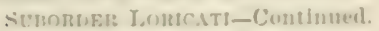

Fonmily Collider-Continued.

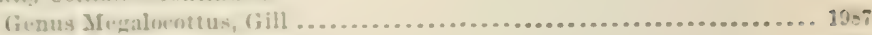

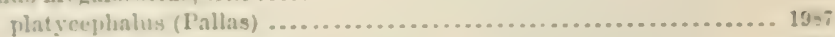

latiengs (Gilluet) ...................................... Ithe

Cinnus Zesticelns, Jurdan \& Evernann .............................. 1990

profundorum (Gille.rt) .................................. 1990

Genus Dasycottus, Bean ...................................... 1901

s.tiger, Beran .............................................. 19:11

Gerns Cottuneulus, Collett ................................. 19:42 microps, Collett ........................................... 1950.

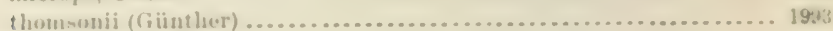

(ienns . Ialacocuttus, Jhean ................................... 19:4t

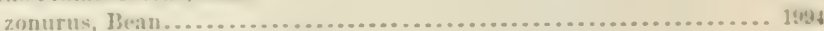

Genuts Areyrocottus, Iferzenstein ............................. 1995

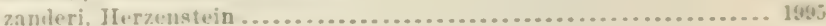

Gienua Puroenttus, Gill ....................................... 1480

s.llaris (Gilbert) ........................................ 1990

quadratus, B. 1. Bean .................................... 199.

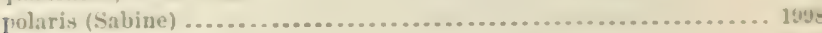

yuadrifilis, Gill ............................................ 1999

tentaculatus (Kner).........................................

braulfordi, Inutter ...........................................

G*nug Oncocottus, Gill ...................................... 2000

rualricornis (Linnerus) ....................................... 2001

heraconuis (Richardson) .................................... vou:

Gems Triglopsis, Girard ..................................... $2000^{5}$

thompsoni, Girard ....................................... 2015

Genus Gymuocanthus, Swainson .............................. 2000

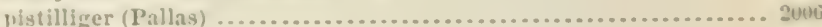

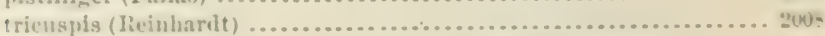

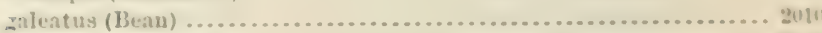

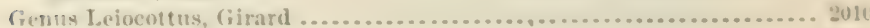

hirumlo, Girard............................................ : o11

firnus L.rptecottus, Girart..................................... 2011

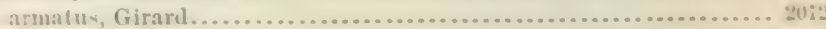

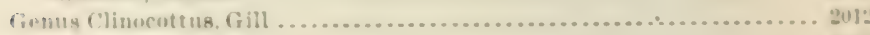

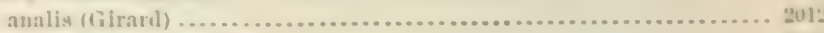

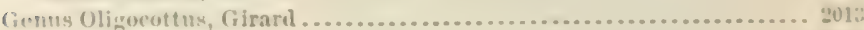

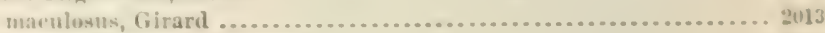

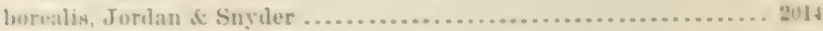

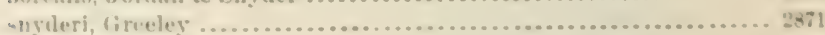

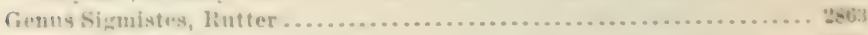

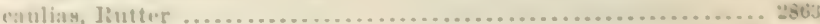

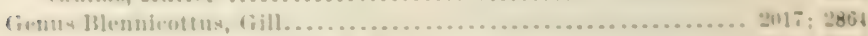

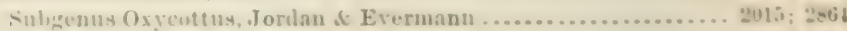

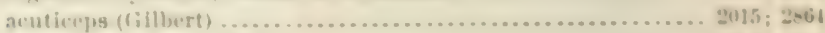

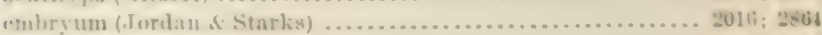

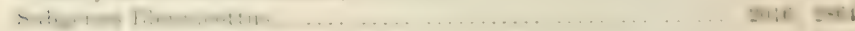

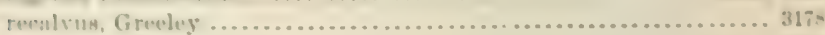

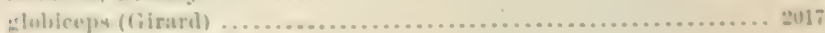

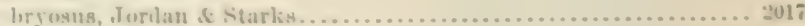

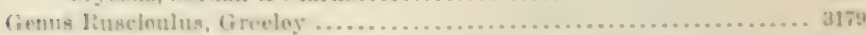

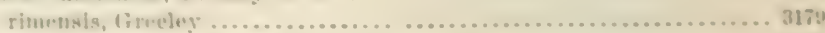

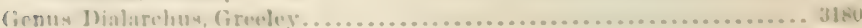

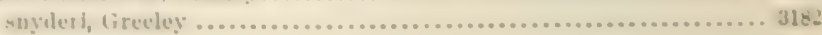


CLASS I'ISCES-Continued. SUBCLASS TELEOSTOMI-Continued.

ORder Plectognathi-Continued.

St'Border Loricati-Continued.

Family Coltidoe-Continued.

Genus Eximia, Greeley ........................................ 318

rubelliv, Greeley ..........................................

Genus Histiocottus, Gill .................................... 2018 bilobus (Cutier of Talencicines) .............................

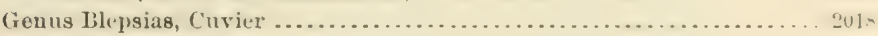

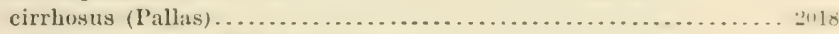

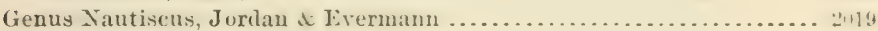

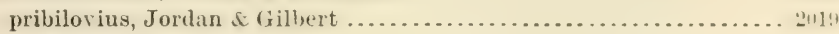

Genus Nautichthys, Girard ...................................... 2020 oculofasciatus (Girard) ...................................

Genus L'lea, Jordan of Evermanu . . . . . . . . . . . . . . . . . . . . . 2u:1

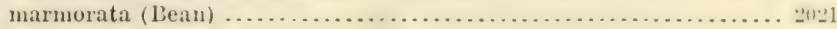

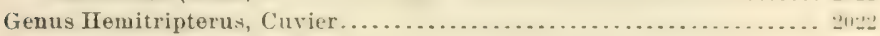
americanus (Gmelin) . . . . . . . . . . . . . . . . . . . . . . . . . .

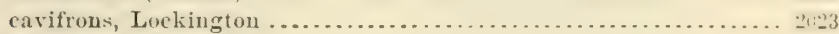

Genus Synchirus, Beau . . . . . . . . . . . . . . . . . . . . . . .

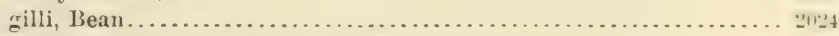

Genus Ascelichthys, Jordan \& Gilbert ............................ 202t rhodorus, Jordan \& Gilbert ................................ 2025

Genus Psychrolntes, Günther ................................... paradoxus, Günther .......................................

Genus Gilbertidia, Berg ............................... 2027; 3183

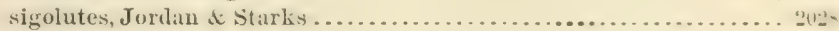

Family Ramphocottidce ............................................. 2029

Genus Ramphocottus, Günther ................................. 2030 richardsoni, Güuther ........................................ 2030

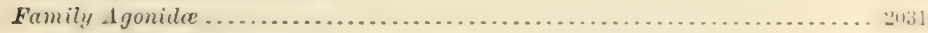

Genus Percis, Scopoli............................................. 2033 japonicus (Pallas) ...........................................

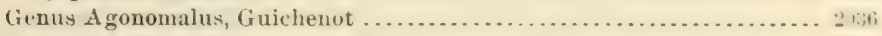
proboseidalis (Valencieune:s) .............................

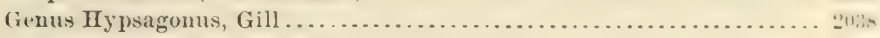

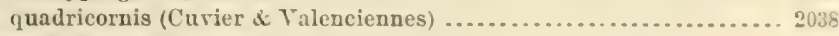

Genus Stellerina, Cramer .... ............................. 20 xyosterna (Jorlan d (iilhert) ................................ 201:

Genus Occa, שordan \& Evermann ............................... 2013 verrucosa (Lockington) ....................................... 2043 dodecaedron (Tilesius) ....................................

Genus Brachyopsis. (iill ...................................

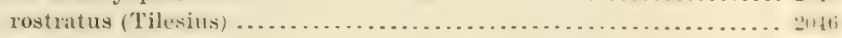

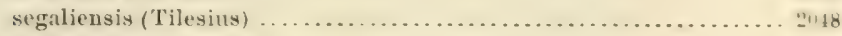

Genus Pallasina, Cramer ................................... barbata (Steiulachner) ................................... 244 aix, Starks.................................................... 2050

(ienus Leptagonus, Gill..................................... decagonus (Bloch \& Schneider) ............................ 2052

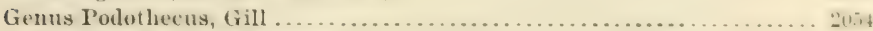

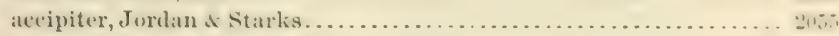

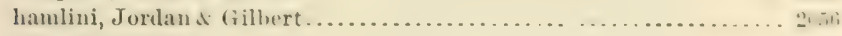
gilberti (Collett) ............................................... 2058

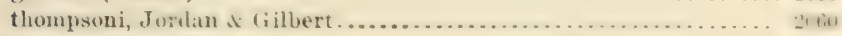

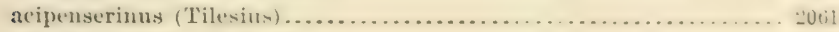

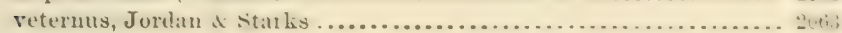


(Y.A.S IPISCLE-Cinneinued.

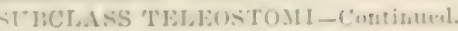

Gurma P’lat Turisath- 'ontinued.

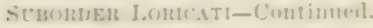

Fomily denter-Continuted.

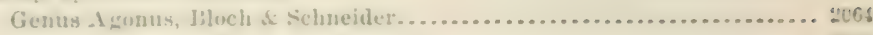
cataphractus, Limuatus.................................. 206

(ienus Stelyis, Cranuer ........................................ 206:

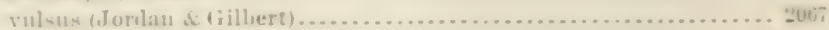

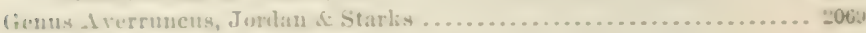

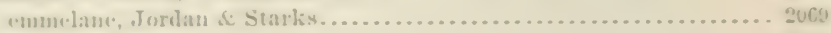

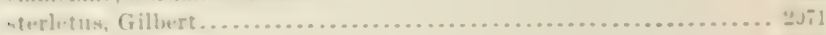

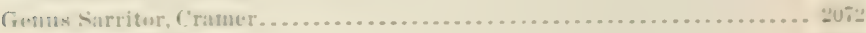

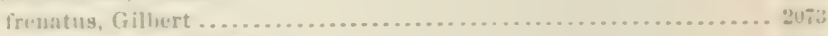
leptorhynchus (Gilhert) .................................... 2075

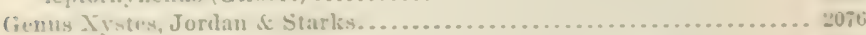
axinoplirys, Jurdan se starks .............................. 20ic

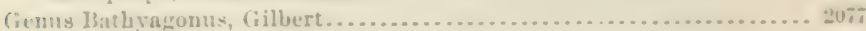
nigripinnis, Gilbert ......................................

(ienus Kienochirus, Gilbert ...................................

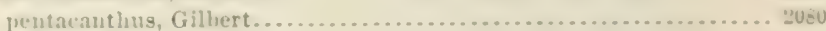
alase: latifruns, Gilbert........................................ 2us: triacautlus, Gilbert....................................... 2084

Genus Oluntolyxis, Lockington.................................. 2us triphinosus, I.vekington................................. 2uEs

Gerns Butlıragonus, Gill.................................. 2u 206 swanii (Steindachner) . .........................................

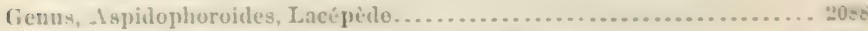

Sul,zenus Cleina, Cramer................................. $20=0$

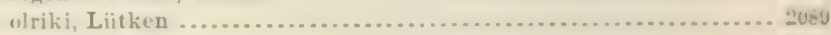

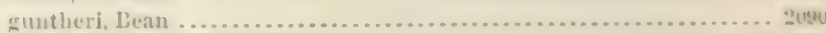

nonopterygius (Blocli) ....................................... 2091

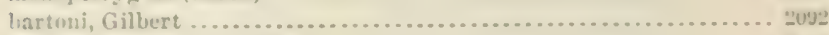

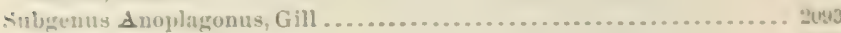

inermis, Ginntler............................................. 20.63

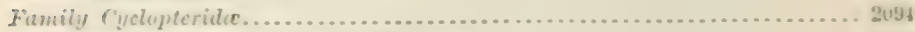

Genut C'yclopterus (Artedi) Linn:eus............................ lumpun, Linuietus ........................................

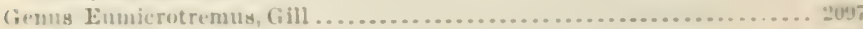
ipinusus (Miiller) .......................................... s080 urbis (Gïnther) . ............................................

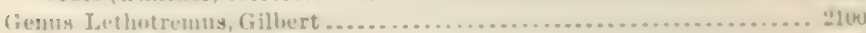

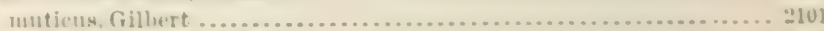

vinulentus, Jordan of Starks ................................ v101

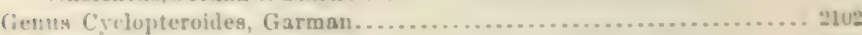

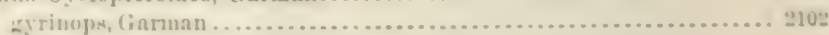

fienum ("yclopterichthys, Steindachmer ......................... 2103

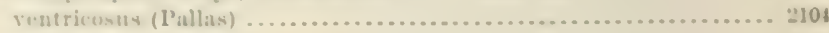

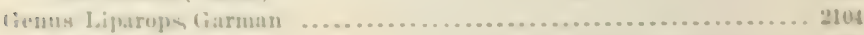

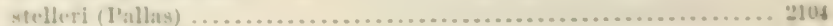

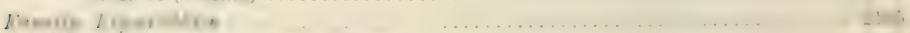

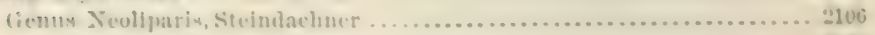

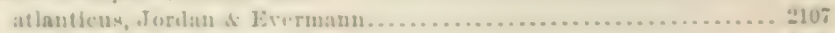
rulteri, Gillert is suster ................................. $210 \mathrm{~s}$

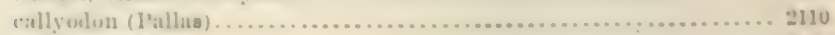

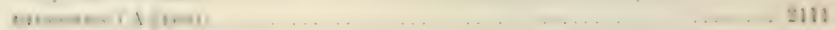

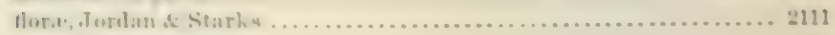

Lreedi, Jurilun of Starkis .................................. 
CLASS PISCES-Continued.

SUBCLASS TELEOSTOMI-Continued.

Order Plectognathi-Continued.

Suborder Loricati-Continued.

Family Liparidide-Continnerl.

Genus Neoliparis, Steindachner-Continued.

tissuratus, Starks

Genus Liparis (Arterli) Scopolit

Sub

liparis $\left(\mathrm{L}_{\mathrm{i}}\right.$

cyclopus, Giixtluer

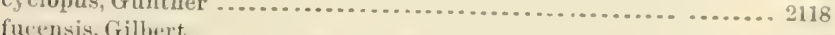

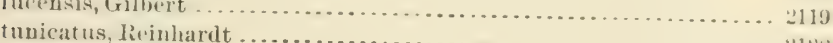

asassizii, ${ }^{\prime}$

herschelinus, Scofeld

dennyi, Jordan \& Starks ...................................... 2123

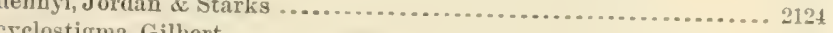

Subgenus L Loliparis, Jor........................................ 2125

pulchellus, $A$ yris, Jordan \& Evermann......................... 2126

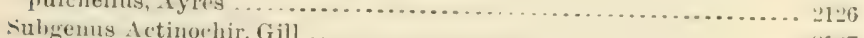

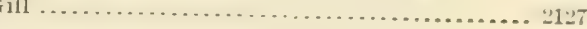

Genus Crystallichthys Jorinn

.

mirabilis, Jordan \& Gilbert ...................................... . . 2865

Genus Bathyphasma, Gilbert ................................... 2128

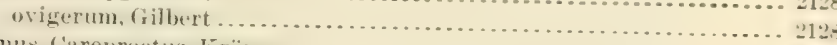

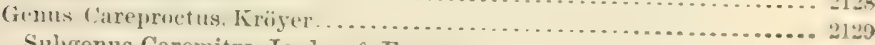

Subgenus Caremitra, Jordan \& Evernann............................ 2131

simus, Gilbert.................................................. 2131

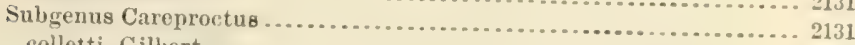

colletti, Gilbert ........................................... 2131

phasma, Gilbert .................................................. 2132

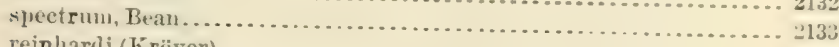

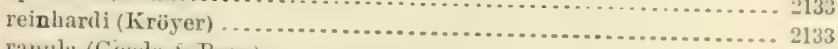

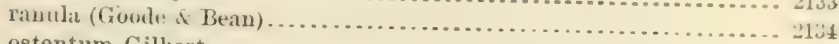

ostentum, Gilbert .......................................... 213t

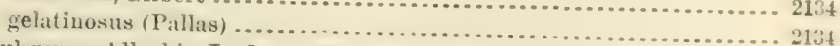

Sulggenus Allochir, Jordan \& Evermann ............................. 2135

melanurus, fillbert ....................................... 2135

Subgenus Allinectes, Jorlan \& Evermann . . . . . . . . . . . . . . . . 2136; 2866

ectener, Gilbert ........................................ $21060^{\circ}$

Genus Prognurus, Jordan \& Evermann ................................ . 28660

cypselurus, Jordan \& Gilbert ...................................... 2866

Genus Gyrinichthys, Gilbert.................................. 2137

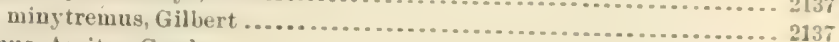

Genus Amitra, Goole......................................... 2138

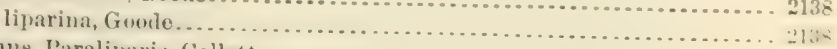

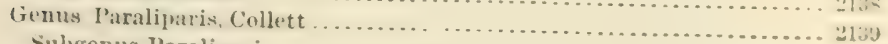

Subgenus Paraliparis ......................................... 2140

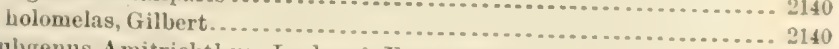

Subgenus Amitrichthys, Jordan \& Evermann........................ 2141

cephahus, Gilbert........................................... 211

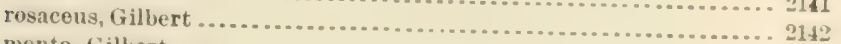

mento, Gilbert ............................................... 2142

copej, Goodo \& IBean ........................................... 2143

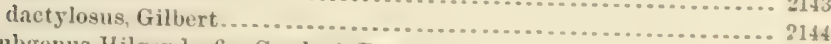

Subgeuus Hilgendorfia, Goode \& Bean.............................. 214t

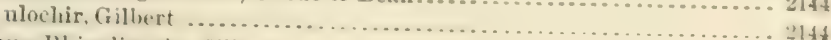

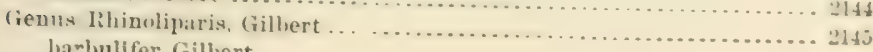

barbullfer, Gilbert ...................................... 2145

Bull. \o. 47, pt. 4 - vi 


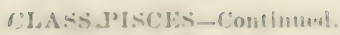

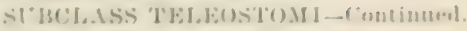

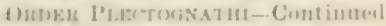

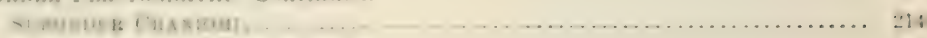

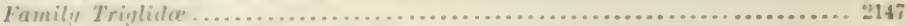

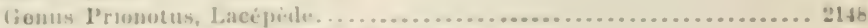

subgenus fiurnarilum, Jorilan a Evermann ...................... 2152

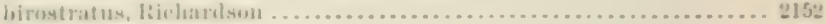

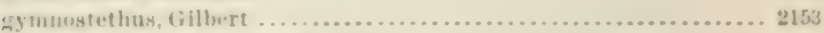

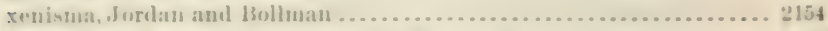

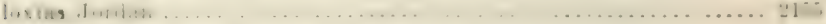

Subenus Sterulinus, Joriau \& Evermanu ................... 215t

c.

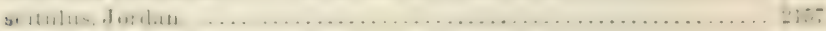

Fuseu, Jorklan A Evermanu............................... 215e

alatus, firsete \& Bean .................................... 2159

Subgenus l'rionotus ......................................... vibu

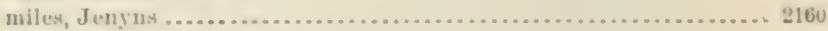

Atephanophers, bockingtou ................................. 2161

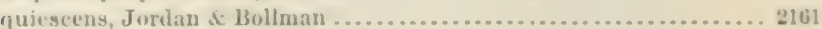

albirestris, Jordan \& Jollman............................. 2lé3

ruluio, dordan ................................................ 210

ophryats, Jorlan of Swait .................................... 2104

stearngi, Jurilan \& Swain ................................. 2106

strigatus (Cuvier of Valencleunes) ........................... 216i

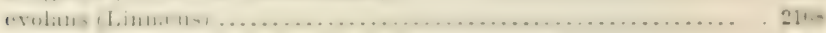

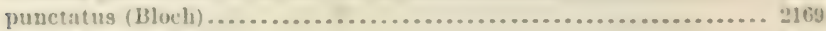

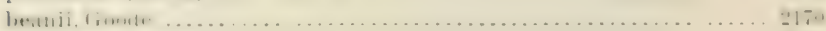

tribulus (Cuvier) ........................................ 2171

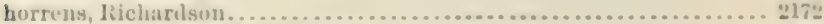

Genus Bellator, Jorilan it Evermann ............................. 2173

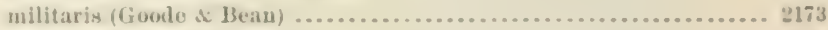

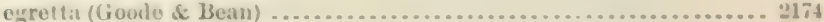

Genus Trigla (Artedi) Linnates .................................. 2176

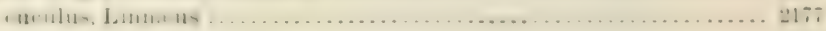

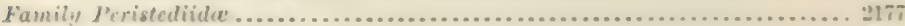

liemus P'eristedion, Lacépiele ................................. 2lie

miniatum, (iovele.......................................... 21 is

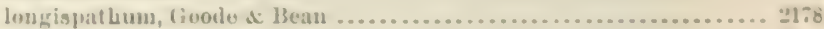

graciln, (iooto of Bean ......................................... 2179

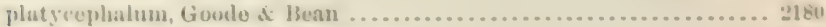

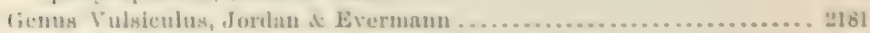

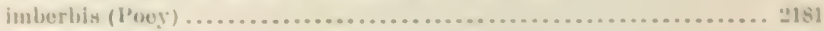

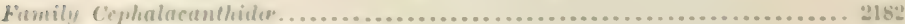

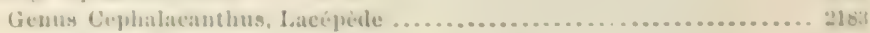

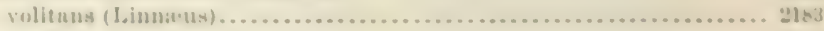

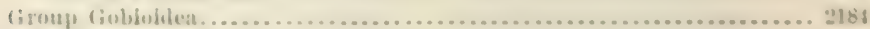

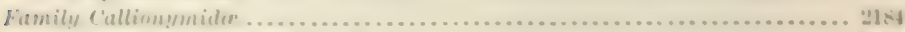

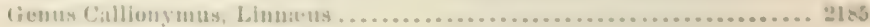

Inirdi, Jurdan. ......................................... 2185

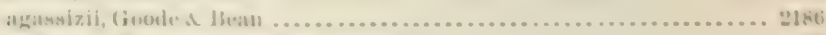

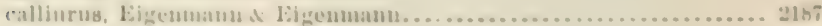

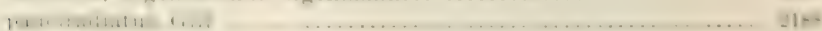

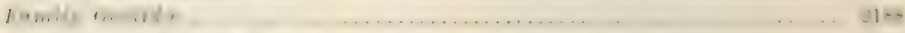

(iеним I0

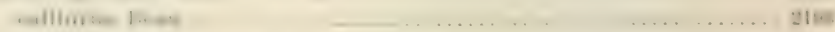

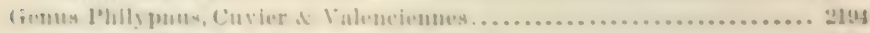

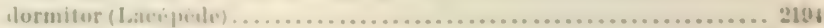

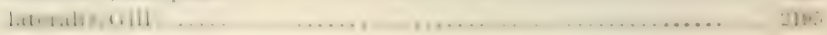


CLASS PISCES-Continuer.

SUBCLASS TELEOSTOMI_-Continued.

ORdER I'LECTOGNATHI-Continued.

Suborder Craniom-Continuerl.

Family Goliida-Continued.

Genus Dormitator, (\$ill........................................ . 110

maculatus (Bloch) .........................................

Genus Guavina, Bleeker........................................ 219

guavina (Cuvier \& Valencienues) . ........................... 219.

Genus Eleotris (Gronow) Bloch \& Schneider....................... 2199

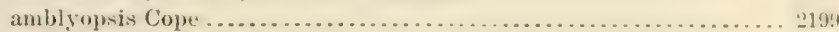

abacturus, Jordan \& Gilbert.................................... 2200

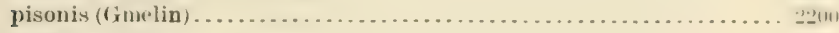

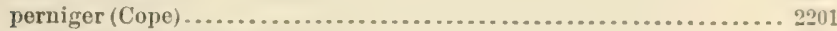

pictus (Kner \& Steinduchner) .............................. 2201

Genus Alexurus, Jordan . . . . . . . . . . . . . . . . . . . . . . . . . .

armiger, Jordan \& Richardson................................ 2203

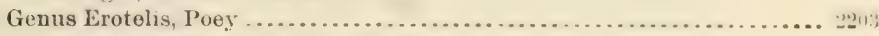
smaragdus (Cuvier \& Valenciennes) ............................. 2204

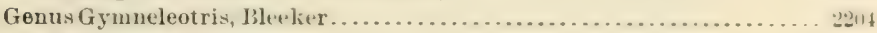

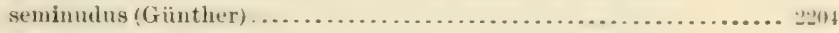

Genus Chriolepis, Gilbert........................................ 2205

minutillus, Gilbert .........................................

Genus Sicydium, Cuvier \& Valenciennes.......................... 220

plumieri (Bloch) .........................................

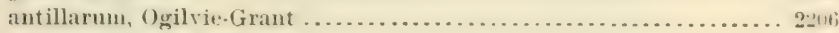

vincente, Jorlan \& Evermann.................................. 2207

punctatum, Perugia ..........................................

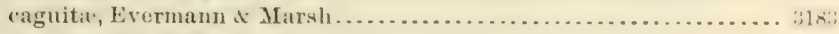

Genus Cotylopus, Guichenot ................................... 2207

Subgenus Sicyosus, Jordan \& Evermann................... 2207; 2867

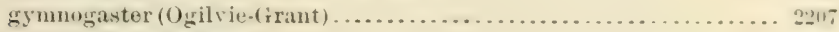
salvini (0)ilvie-Grant) .........................................

Genus Evorthodus, Gill.............................................

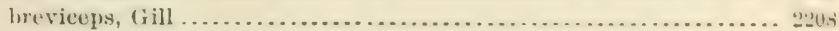

Genus Lophogobius, Gill........................................ 2209

cyprinoides (Pallas) ........................................ 2209

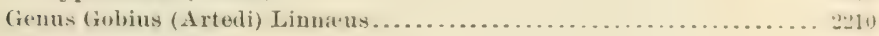

Subgenus Gobius ......................................... "2216

soporator, Curier \& Valenciennes............................. 2216

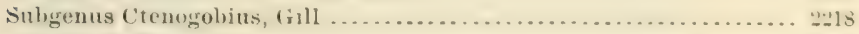

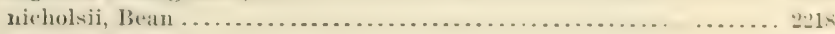

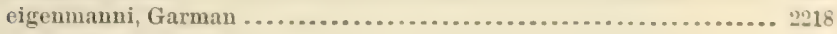

glaucofrænum (Gill) ........................................... 2219

manglicola, Jordan \& Starks............................. 22:20

stigmaturus, Gookte \& Bean . . . . . . . . . . . . . . . . . . . . . .

qualriporus, Cuvier st Valenciennes.......................... 2021

shufeldti, Jordan \& Eigenmann ............................... .

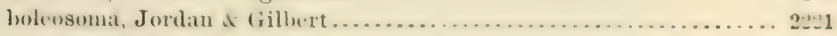

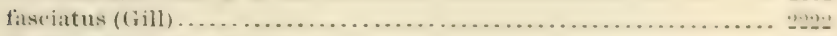

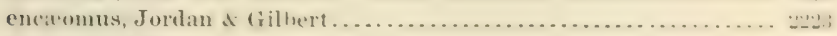

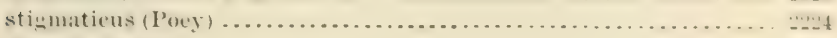

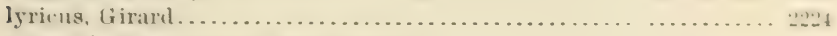

garmani, Eigemmanu \& Eigenmann ........................... 2225

zebra, Gilbert ...................................... 2220. 2867

Subgenus Euctenogobins, Gill ................................ 2...226

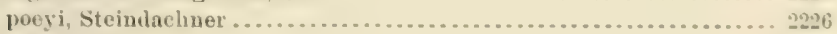

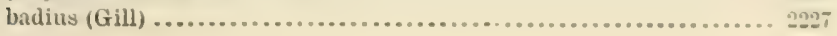


CI.ASS IJIACLS-CominnemL.

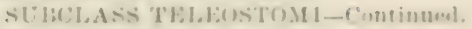

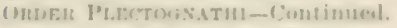

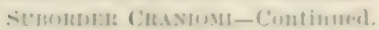

family disbiallé-Continuesl.

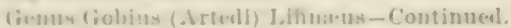

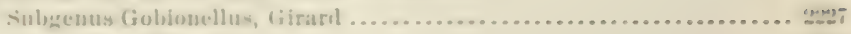

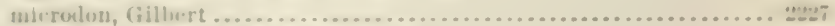

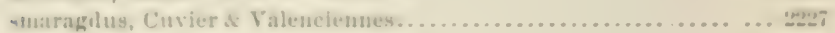

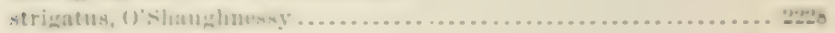

sagittula $($ (iïutlurs) ......................................

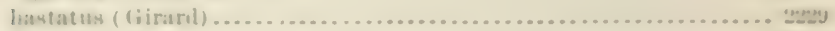

mesanicus, Pallas.............................................. "

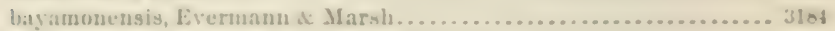

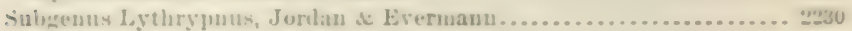

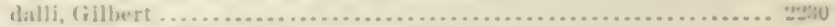

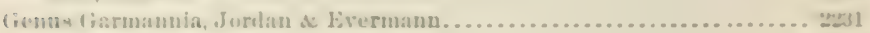

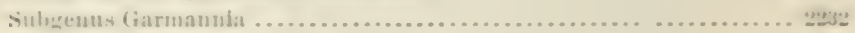

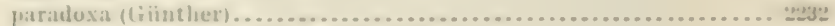

he-นiдy

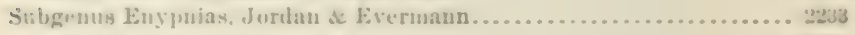

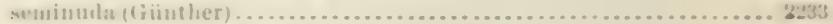

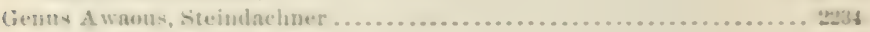

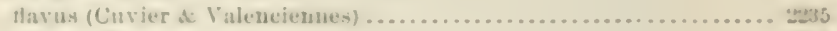

nelsoni, Everman ..........................................

taiasica (Lichtenstein) . . .......................................

mexieanus (liinther) ...................................

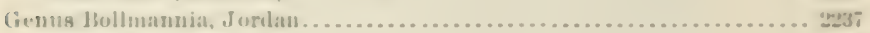

ocellata, tilliert ...........................................

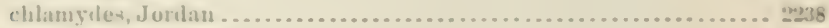

macroprusu, (iilbert ....................................

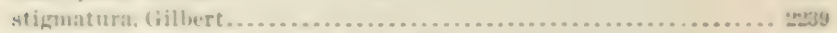

lиquerouedsis, Everman \& Marsh........................... \$18

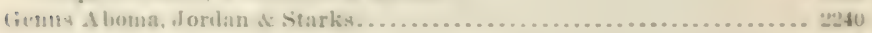

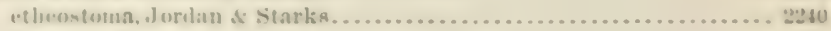

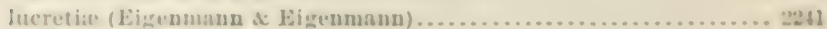

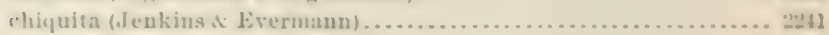

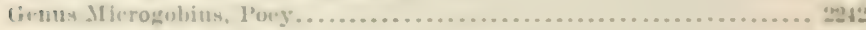

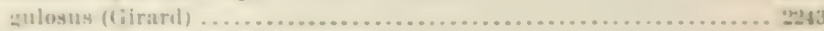

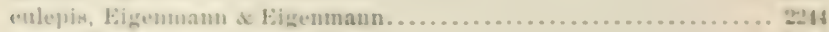

meki, bvermann o darsh ..............................

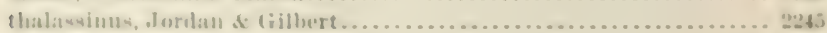

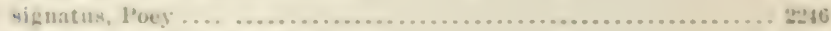

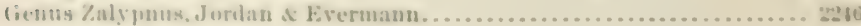

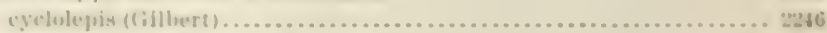

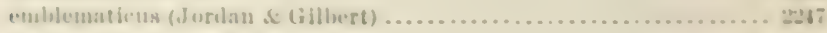

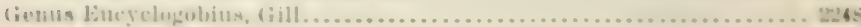

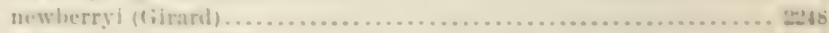

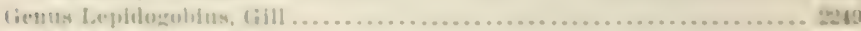

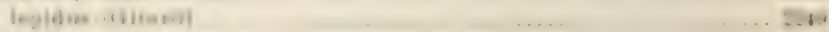

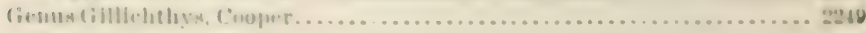

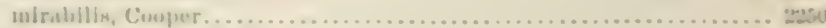

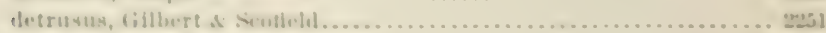

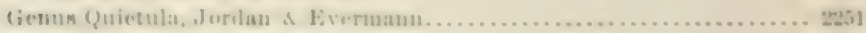

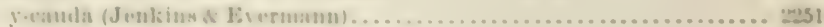

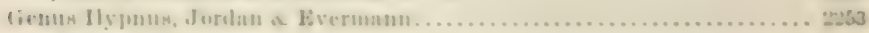

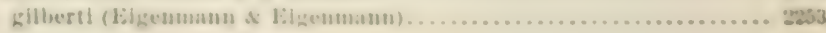


CLASS PISCES-Continued.

SUBCLASS TELEOSTOMII-Continuer.

Order Plectognathi-Continued.

Suborder Craniomi-Continued.

Family Gobiide-Continued.

Genus Clevelandia, Eigeumann \& Eigenmann

ios (Jordan \& Gibert).

rose, Jordau \& Everuman

Genus Evermannia, Jordam.

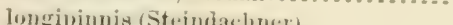

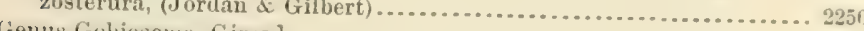

histrio, Jordan

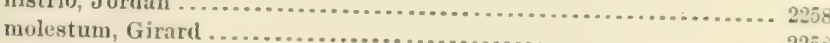

bosci (Lacépìde) .............................................. 225

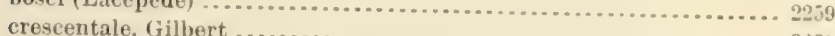

multifasciatum, Steindachner

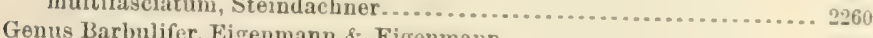

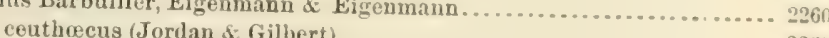

couthoecus (Jordan \& Gilbert) ......................... 2260

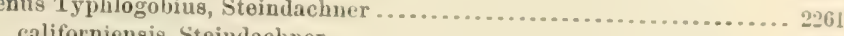

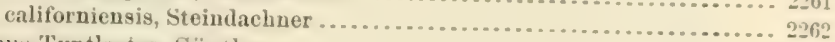

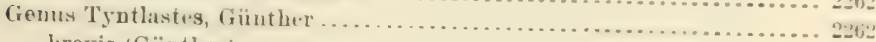

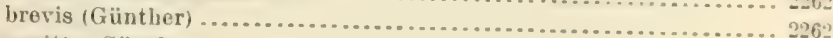

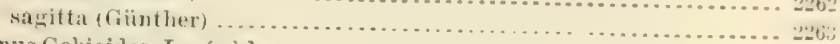

Genus Gobioides, Lacépède.................................. 2263; 2868

broussonnetii, Lacépède......................................... 2263

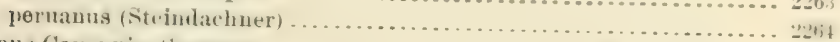

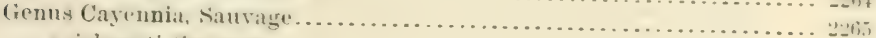

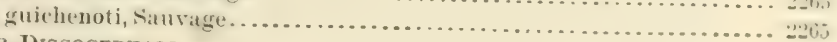

SUBORDER DISCOCEMHALI .......................................... 2265

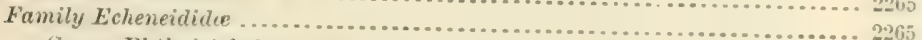

Genus Phtheirichthys, Gill . ....................................... 220

lineatus (Menzies) .......................................... 2206

Genus Echeneis (Artedi) Linureus..................................... . . . 2268

naucrates, Linnaeus ............................................

naucrateoides, Zuiew. .......................................

Genus Remilegia, Gill..........................................

australis (Benuett) ..........................................

Genus Remora, Gill ..........................................

Subgenus Remora..................................................

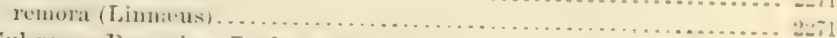

Subgenus liemorina, Jordan \& Evermamn ......................... 2...

albescens (Temminck \& Schlegel) ............................. 227

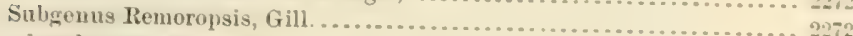

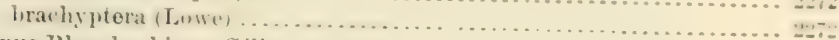

Genus Rhombochirns, Gill.........................................

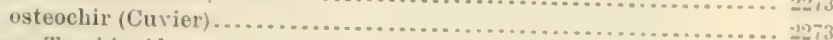

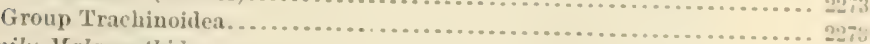

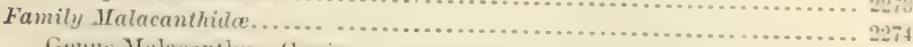

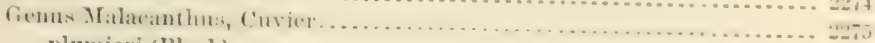

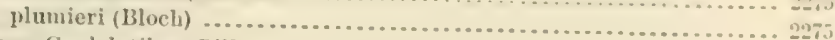

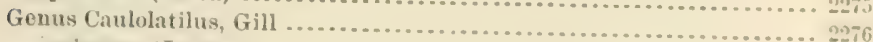

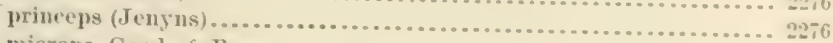

microps, Goodlo s Bean .........................................

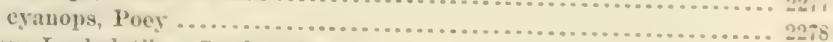

Genus Lopholatilus, Goole of Bean . . . . . . . . . . . . . . . . . . . . . . . . . . 2278

chamaleonticeps, Goode of Bean ................................... 


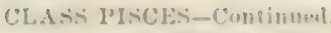

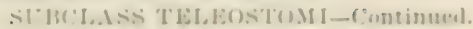

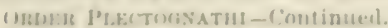

4Pe -

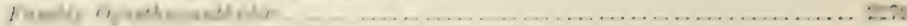

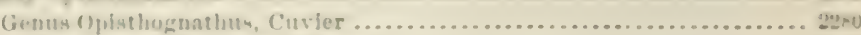

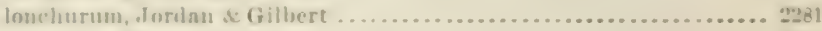

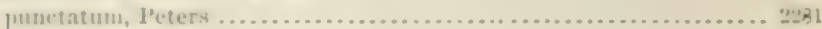

macrugnathum, l'oeg......................................

oumatum, Junkins \& Evermann .......................... 228

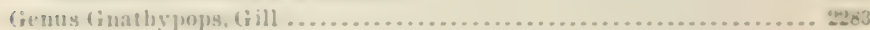

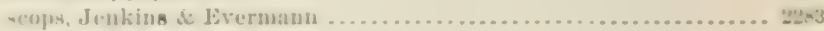

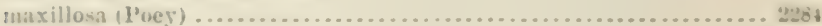

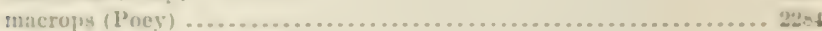

rhmualea (Jurilan \& (iilhert) ............................ 2285

snyderi, Jordan d Everuann ............................... 29s

nus

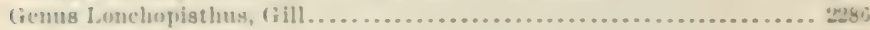

micrognathus (1)

Family liathymasteridic............................................

Cienus bathymaster, Cope ........................................ 2.2es

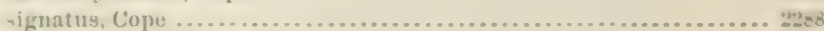

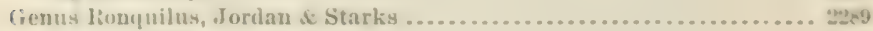

jorlani (liblbert) ............................................

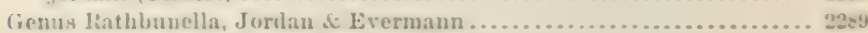

hyjoplecta (Gilbert) ....................................20

Family Chiaxmotontide ...........................

(ienus Chiasmodon, Johnson................................... 2201

nigrer, Johnson ..........................................

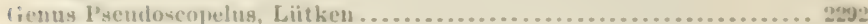

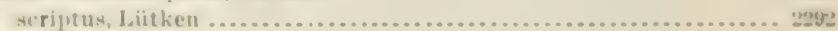

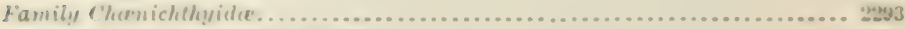

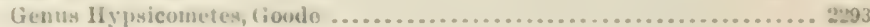
-

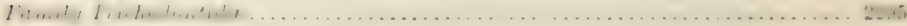

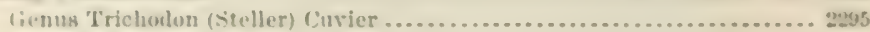

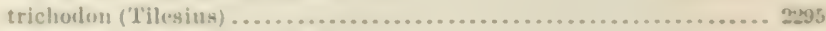

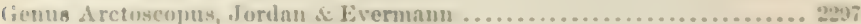

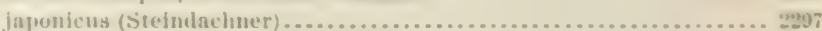

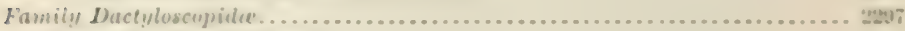

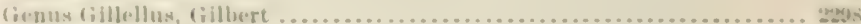

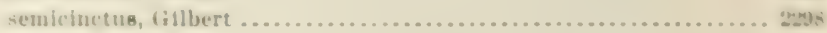

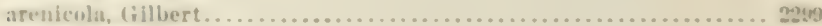

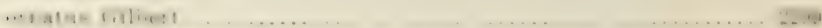

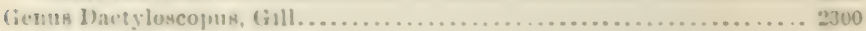

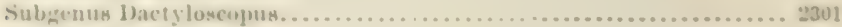
pectoralis, Gill .......................................

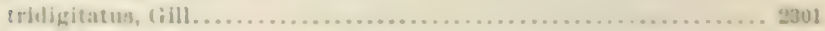

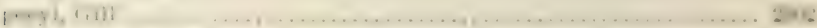

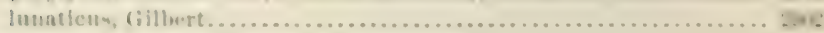

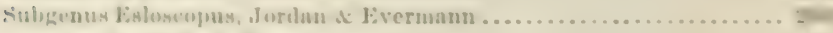

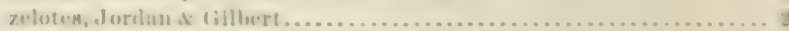

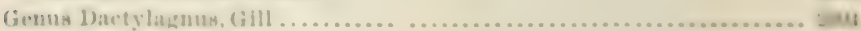

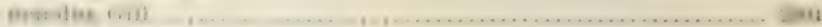

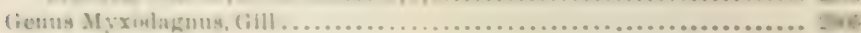
masidere 
CLASS PISCES-Continued.

SUBCLASS 'TELEOSTOMI-Continned.

Order Plectognathi-Continuerl.

SUBORDER DiscocepHALI-Continted.

Family ronoscopirle. . . . . . . . . . .

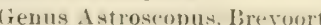

y-grecum (Cuvier \& Valenciennes) ........................... 2307

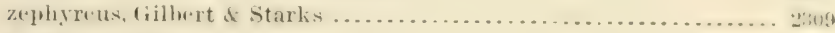

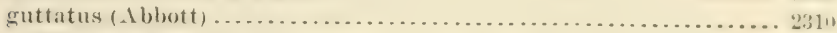

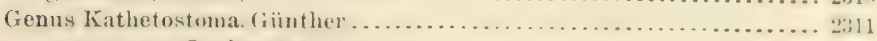

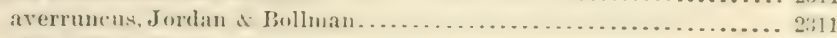

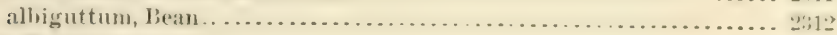

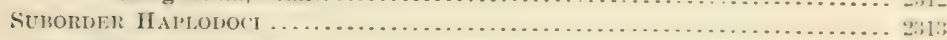

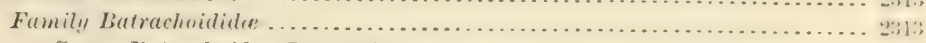

Genus Batrachoides, Lacépède ............................. 231£; 2868

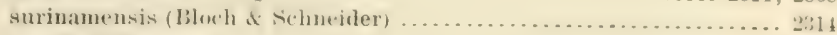

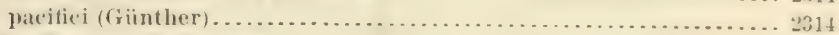

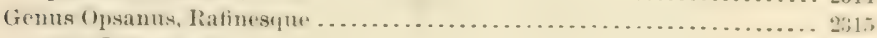

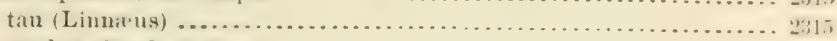

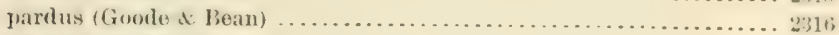

Genus Porichthys, firarl. . . . . . . . . . . . . . . . . . . . . . . 2:17

porusissimus (Cuvier \& Valenciennes) ....................... 2319

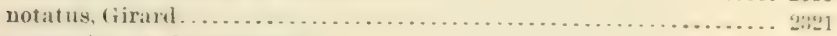

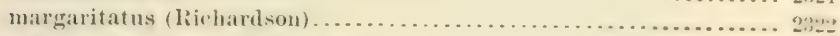

Genus Thalassophryne, Giinther. . . . . . . . . . . . . . . . . . .

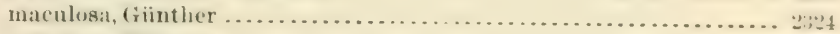

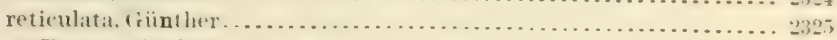

Genus Diector, Jordan \& Evermann . . . . . . . . . . . . . . . . . . . . . . . . 2325

dowi (Jordin A (iillert) . . . . . . . . . . . . . . . . . . . . . . . .

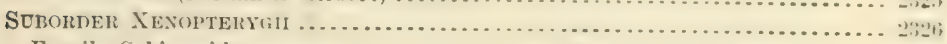

Family Gobiesocide .............................................. 2326

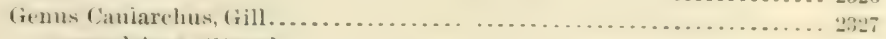

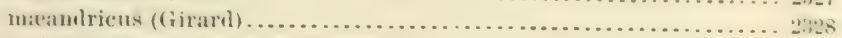

Genus IBryssetæeres, Jorlan de Evermann... ..... . . . . . . . . . . . . . . . 2328 pinniger (Gilbert) . . . . . . . . . . . . . . . . . . . . . . . . . . . . . . . . . 2328

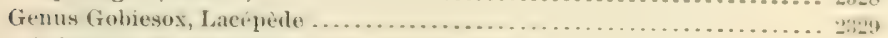

Subgenus Bryssophilus, Jordan \& Evermann ...................... 2330

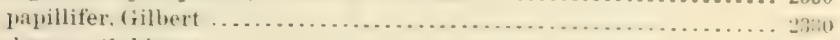

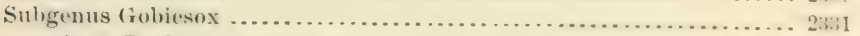

gyrinus, Jorlan \& Evermann . . . . . . . . . . . . . . . . . . . .

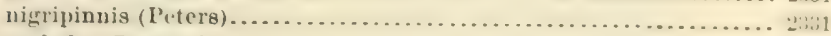

cephalus, Lacépede.......................................... 2332

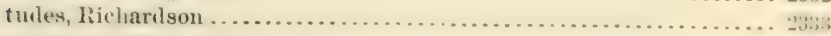

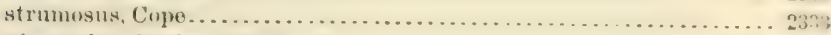

virgatulus, Jorkan \& Gilbert................................. 2333

adıstus, Jorlan \& Gilbert . . . . . . . . . . . . . . . . . . . . . .

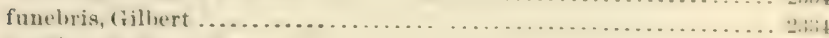

pueilophthalmus, Jenyns ......................

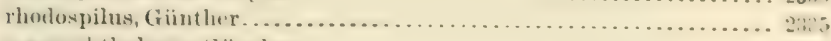

macrophthalmus, fiinther . . . . . . . . . . . . . . . . . . . . .

cerasinus, Cope............................................ 233t;

Subgenus Sicyases, Miiller o Troschel . . . . . . . . . . . . . . . . . . . . . . 2336

erytlirops, Jordan \& Gilhert .............................

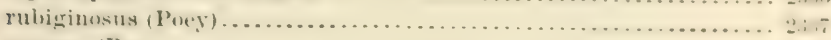

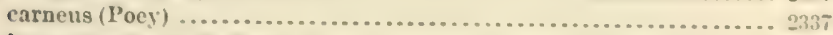

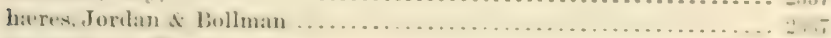

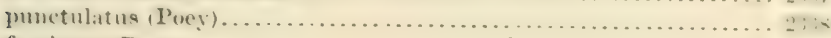

fasciatus (Peters) . ...................................... 2338 
CLISS I'ISCFS-Continued.

SI HCLASS TELAOSTOSH - Contínuesl.

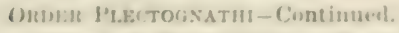

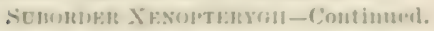

Family limbiesocider-Cons innesl.

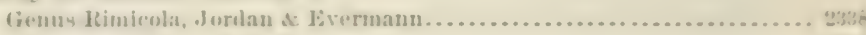

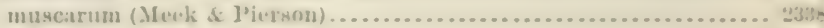

с.іgenuanui (Gilluert) ................................. 2309

Genus Artaciona, Jordan of Fivermann......................... 2940

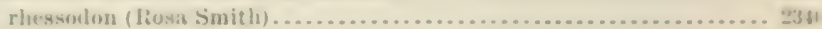

huneralin (bilbert) .....................................

muestris (Poey) .............................................

qebra (Jorilan d Gilluert) ................................... 2341

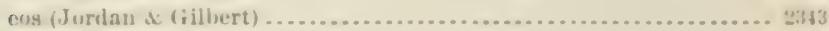

Group) Blenuitoidea .............................................. 2143

Family lblenniider.................................................

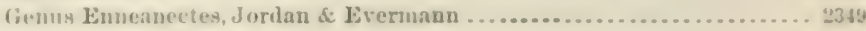
(arminalis (Jordlan d (illbert)............................... v35t

(ienup (illias, Evermann \& Marsh................................ 31 jordani, Evermanu of Jarsh ............................... 3100

(ienus Dialonums, (iilhert...................................... getip

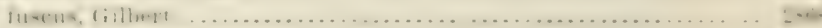

Gemus Heterostiehus, (iirart ............................... 233) rostratus, liirard............................................. 2351

Genus Gilhbongia, Cooper ......................................

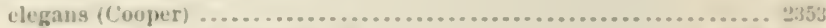

evides (bordan of (iilbert).............................. 2352; $2=69$

Frrmus Nenclinus, Girark ................................... 2354

Subgenus Jievclinus ...........................................

blanchardi, Giraril......................................... 29358

Subgenus l'terounat hus, Girard.............................. satiricus, (ijrart ........................................... 2555

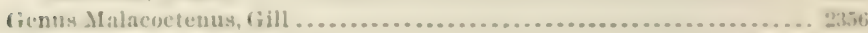
ocellatus (Steindachner) ........................... 2356; 2860

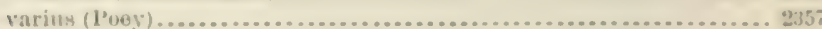
macropus (1'oeg) ..............................................

lugubris (1'oey) .............................................. 9359

culebrat, Evermann o Marsh .............................. 318

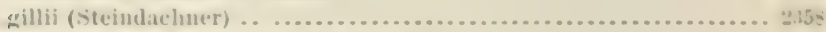
juertoricensis, Еvermann \& Marsh.......................... 315! himaculatus (Steimlaclaner) ................................

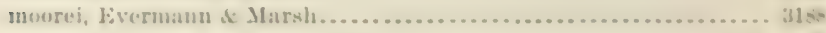
thelalandi (Cuvier o Valenciennes) ............................ 2yss versicolor (Poey) ............................................

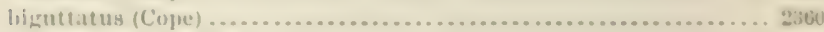

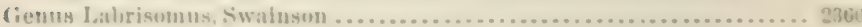
Jurminier (1.n Suenr) ......................................... 2:161

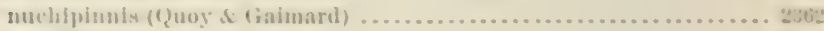

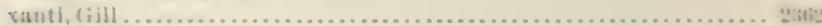
lucciterus, l'nesy...........................................

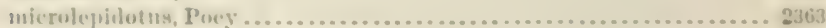

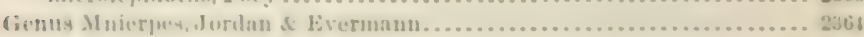

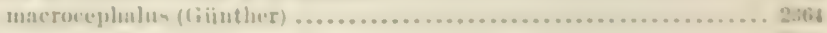

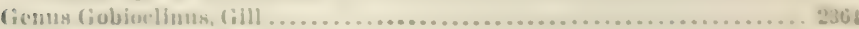

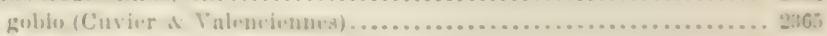

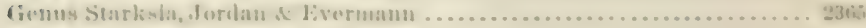

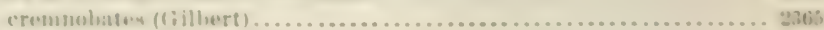

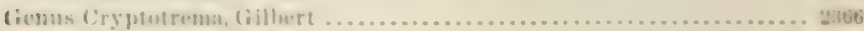
corallium, (illlert....................................... 
CLASS PISCES-Continued.

SUBCLASS TELEOSTOMI-Continuer.

Order Plectognatui-Continued.

SUBORDER XeNOPTERYGII-Continued.

Family Blenniida-Continued.

Genus Exerpes, Jordan \& Evermaun ........................... 2367 asper (Jenkins of Evermaun) . ...................................

Genus Auchenistius, Evermam \& Marsh . . . . . . . . . . . . . . . . . . . . 3190 stahli, Evermann \& Marsh .............................

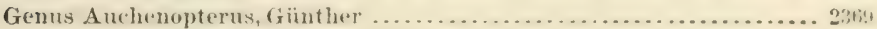

Subgenus Corallicola, Jordan \& Evermann ........................ 2369

nigripinnis (Steindachner) .................................. 2369

altivelis (Lockington) .......................................... 2370

marmoratus (Steindachner) ..................................

Sulgenus Auchenopterus......................................

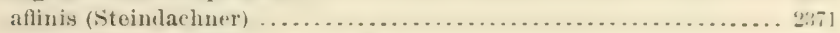

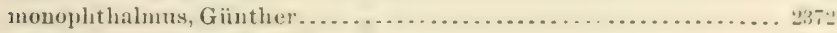

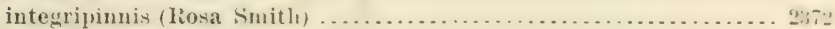

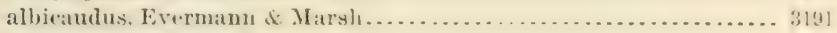

rubescens, Evermanu \& Marsh ........................... 31!1]

cingulatus, Evermann \& Marsh..........................

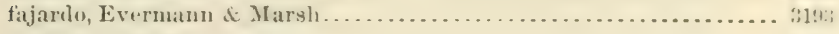

fasciatus (Stiendachner) .................................. 2373

nox (Jordan \& Gilbert) ...................................... 2373

Genus Paraclinus, Mrequard.................................. chaperi, Moequard ............................................ 237t

Genus Emmnion, Jordan . ..................................... 2375 bristole, Jordan ............................................. 2375

Gemus Atoporlinus, Taillant ....................................

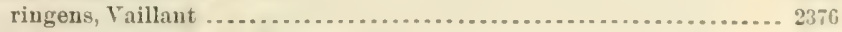

Gemus Rumula, Jurdan of lollman . . . . . . . . . . . . . . . . . . . . azalea, Jurilan \& Bollman . . . . . . . . . . . . . . . . . . . . . . . .

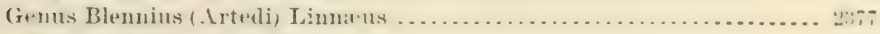

Subgenus Lipophrys, Gill............................. 2:iz

earolinus (Cuvier \& Valencirnnes) .............................

fucorum, Cuvier \& Valenciennes .............................. 2370

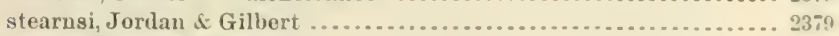

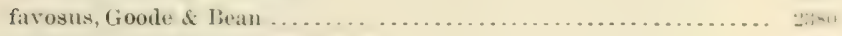

pilicornis, Cuvier \& Valenciennes ............................. 2380

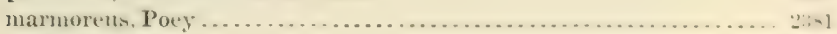

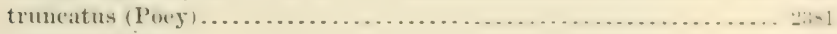

vinctus, Pooy ................................................. 2382

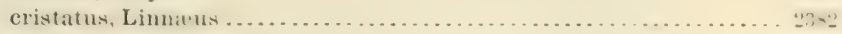

Genus Scartella, Jorlan ......................................... 2384

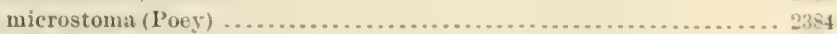

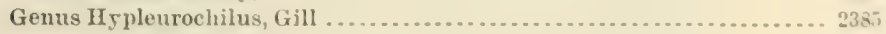

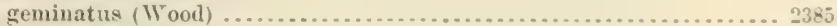

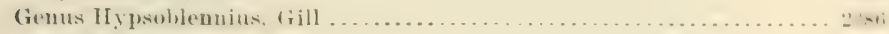

Subgenus Hypsoblennius................................... 2386

gillberti (Jordan) .............................................. 2386

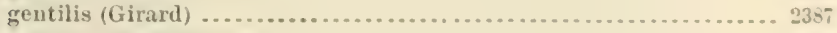

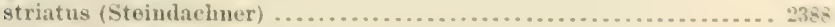

ionthas (Jordan \& Gilbert) . . . . . . . . . . . . . . . . . . . . . . . . . 238.

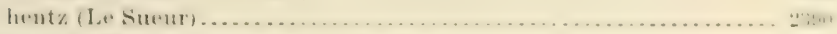

Subgenus Blemiolıs, Jordan it Evermann........................ 2390

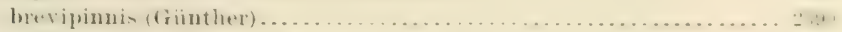

Genus Chasmotes, Curier of Valenciennes ......................... 2391

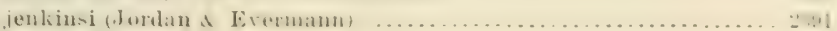

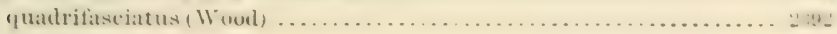




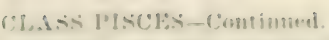

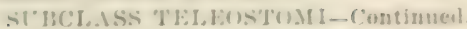

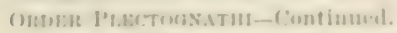

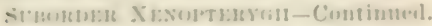

Famiby dilenniale-C'ontinued.

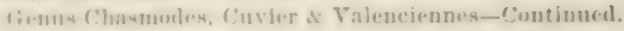

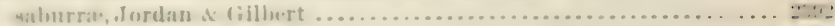

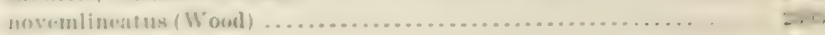

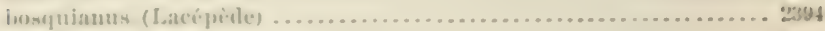

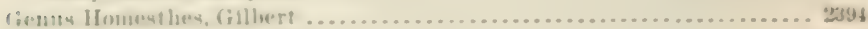

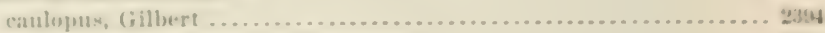

dicsun Seartichthys, Jordan of Evermann ........................... 2395

rubrupunctatus (C'nvier of Valenciennes) ....................... 2390

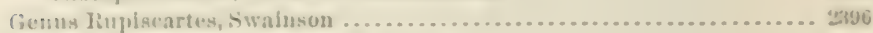

uthanticus (Cuvior of Valenciennem) ............................ 2397

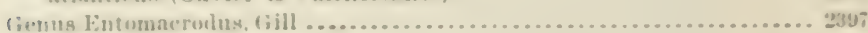

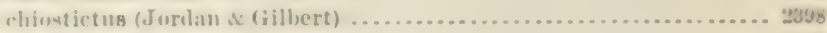

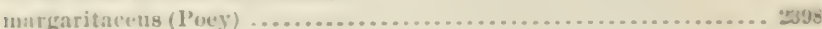

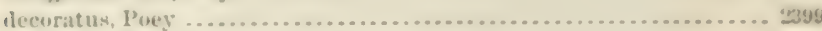

п1 - ...

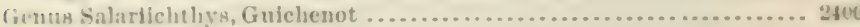

textilis $($ Quey of (inimard) ................................ 2400

Cienus Coralliozetus, Evermanu .t Marsh........................... 3194

cardemat, Evernann of Marsh ............................... 3194

(ienuix Ophioblennius, Gill ................................... 2400

webbil (Valenejenues) ................................... 2s

uteimlaclumeri. Jordan \& Evermann........................... *tol

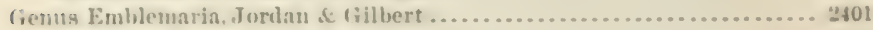

atlantica, Jordan of Evermann ............................. stu2

nivipes, Jordan \& Gilbert .................................. 2suz

mulocirris. Jortan ....................................... 2403

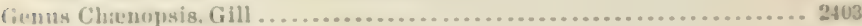

medlatus, loey ........................................... 2403

(ienus Lucioblennius, Gilbert...................................... 280

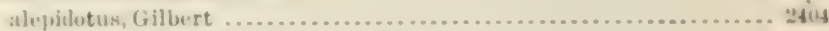

Iienus Pholitlichthys, Bleeker ............................... "40 angulliformis, Luckington ...................................

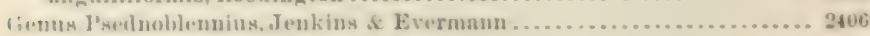

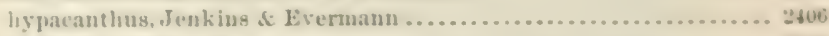

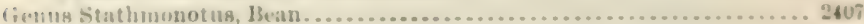

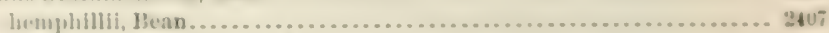

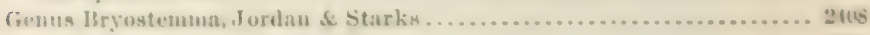

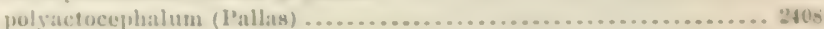

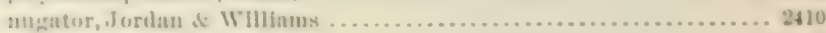

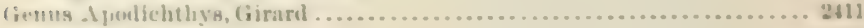
Havidlus, lilrarel ........................................... 2\$11

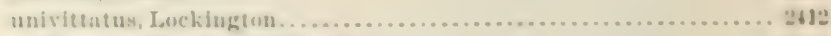

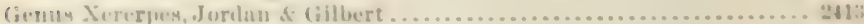

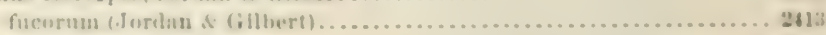

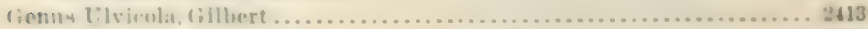

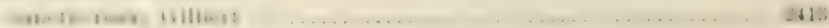

lienus flublis (lironuw) scopoli ............................. 2016

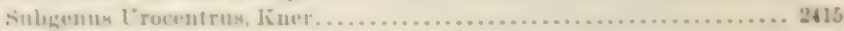
1.

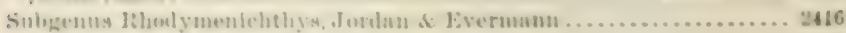

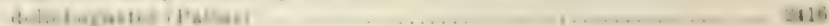

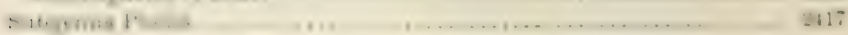

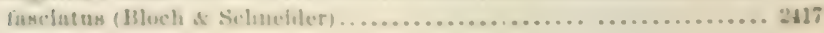


CLASS PISCES-Continuer.

SUBCLASS TELEOSTOMI-Continued.

order Plectogsathi-Continued.

Suborder Tenopterygli-Continued.

Family Blenniudce-Continued.

Genus Pholis (Gronow) Scopoli-Continued.

Subgenus Pholis-Continued.

gunnellus (Linnæus)....................................... 2419

ornatus (firart) ....................................... 2419

Genus Gunnellops, Bleeker........................................ 2420

roseus (Pallas) .............................................. 2420

Genus Asternopteryx, Rïppell.................................. 24:0 gunnelliformis, Riippell ....................................... 2424

Genus Anoplarchus, Gill ...................................... 2421 atropurpureus (Kittlitz) ............................ 2422; 2869

Genus Alectrias, Jordan \& Evermann . . . . . . . . . . . . . . . . . . . . . . . . . . 2869 alectrolophus (Pallas) .............................. 2421; 2869

Genus Xiphistes, Jordan \& Starks ............................... 2423

ulve, Jordan \& Starks ...................................... 2423

chirus (Jortan \& Gilbert) . . . ................................ 2424

Genus Xiphidion, Girard ....................................... 2424

mucosmm, Girard . . . . . . . . . . . . . . . . . . . . . . . . .

rupestre (Jordan \& Gilbert) . . . . . . . . . . . . . . . . . . . . . . . 2426

Genus Cebedichthys, Arres .................................. 2426

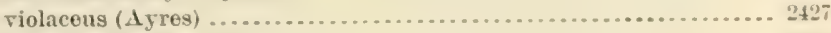

Genus Plagiogrammus, Bean ................................. 2427 hopkinsi, Bean............................................

Genus Opisthocentrus, Kner .................................. 2428

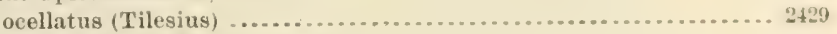

Genus Pholidapus, Bean \& Bean . . . . . . . . . . . . . . . . . . . . . . . . 2430 dybowskii (Steindachner) ..................................2 430

Genus Plectobranchus, Gilbert ................................ 2431 evides, Gilbert.......................................... 2432

Genus Leptoclinus, Gill ........................................ 2432 maculatus (Fries) .......................................... 2433

Genus Poroclinus, Bean ....................................... 2433

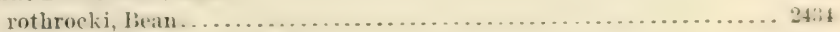

Genus Lumpenus, Reinharit .................................. 2435

Subgenus Anisarchus, Gill. .................................. 2435 medius (Reinbarelt) ......................................... 2435

Subgenus Lumpenus.......................................... . . 436 anguillaris (Pallas) ......................................... 2436

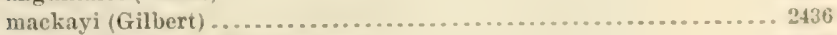

fabricii (Cuvier of Talenciennes) .............................. 2437 lampetrieformis (Walbaum) ............................... 2438

Genus Sticheus, Reinhardt.................................. 2439

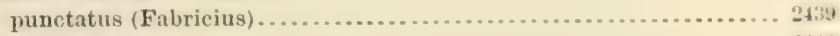

Genus Ulvaria, Jordan $\&$ Evermann .............................. $\$ 40$

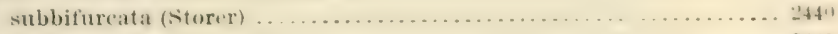

Genus Eumesogrammus, Gill ................................ 24t1

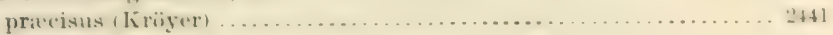

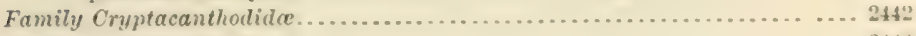

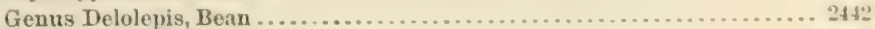

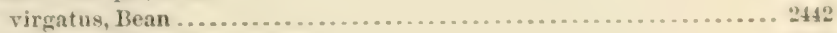

Genus Cryptacantholes, Storer ................................. 2443

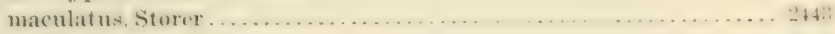

Genus Lyconectes, Gilbert ................................. 2444 aleutensiм tiblhert ................................ 


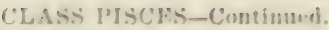

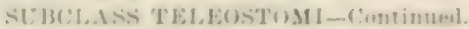

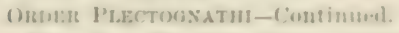

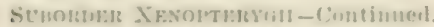

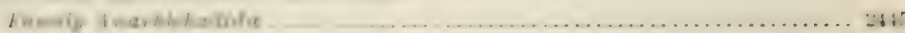

(ienus Anurhichas (Artenli) Linn:1+us .......................... 2\$65

latitrons. Steenstrup of Hallerimsson ........................ 2t\$t;

minor. ()latisen........................................ 2446

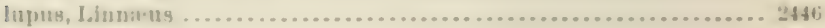

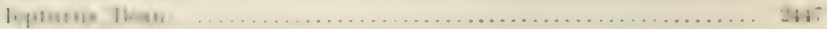

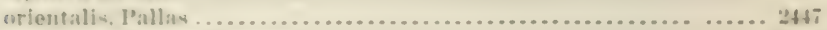

fiemus Anurrlichaliys, dvres ..................................... 24ti

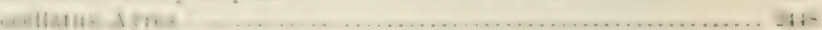

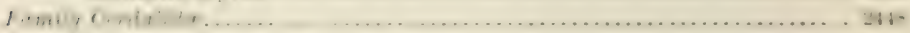

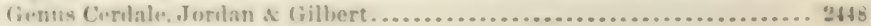

ionthas, Jurdan of dillbert . ................................ 24t9

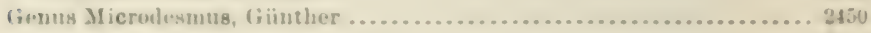

dipus, (iinther ............................................

retropinnis, Jorklan \& Gilhert ................................ 2450

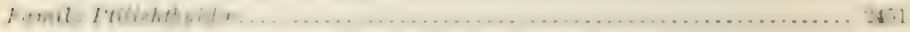

(ienus l'tilichthys, Jean ..................................... $445 \%$

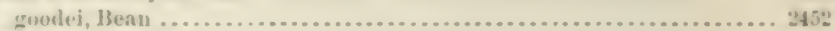

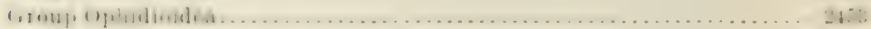

Family scylalinide .............................................. 2453

Gents Sertalima, Jorılan d Gilbert ............................ 245t crerlale, Jordan of (iillert ................................ 2\$5

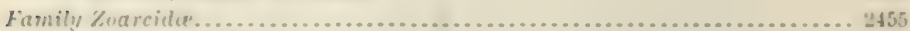

(ienus \%oarces, Gill ............................................ 245t6

iubgeuns di acruzuarces, (ill ............................... 245i

anguillaris (lect) ........................................ 245i

(ie'nus Emloryx, Jorlan \& Evermaun ............................ 2458

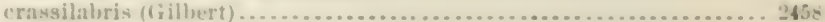

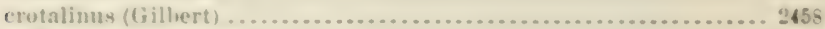

Ciemus Lycotoprsis, Collett .................................... 460 paciticus (Collett) ....................................... 2s

(it.nus $A$ prolon, tillhert ....................................... $8 \$ 60$

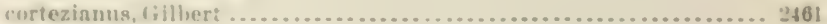

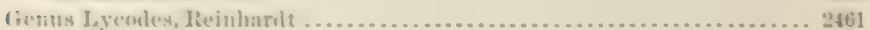

Subermus Lycoles .......................................... 2463

emarkii, ('ollett .......................................... 2463

vahlii, lievinhardt........................................ $24 n 3$

concolor, (iill \& 'lownsent ................................ 2to

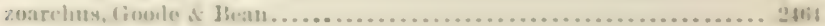

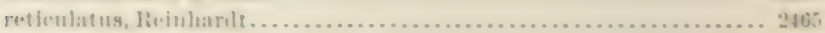

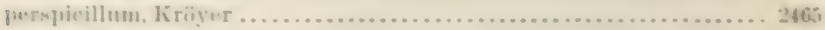

iriegilus, (collet .........................................

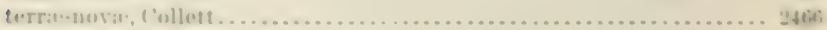

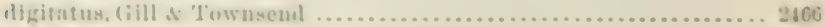

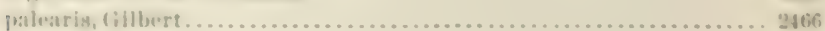

brevјрея, J

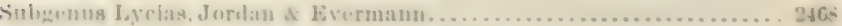

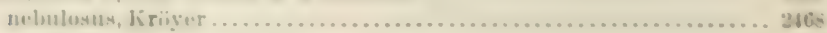

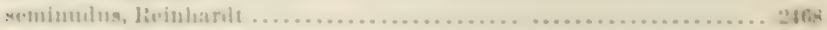

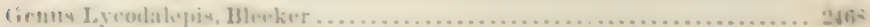

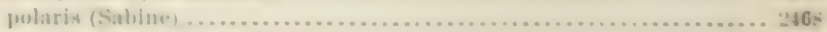

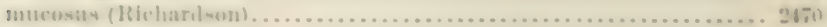

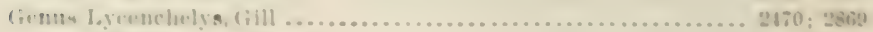
verrillit (lioule \& liwant .............................. 
CLASS PISCES-Continned.

SUBCLASS TELEOSTOAI-Continued.

Order Plectognathi-Continued.

Suborder Xenopteryail-Continued.

Family Zoarcida-Contimed.

Genus Lycenchelys, Gill-_Continued.

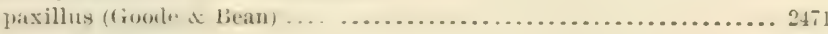

murxma (Collett) ........................................ 3195

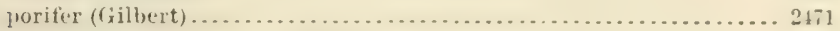

Genus Furcimanus, Jordan \& Evermann. . . . . . . . . . . . . . . . . . . 2472

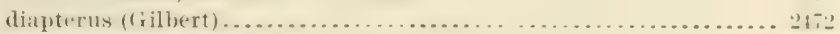

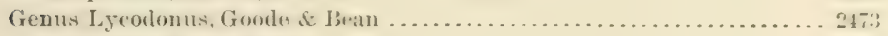

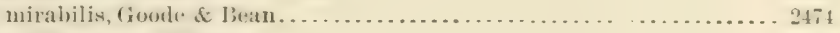

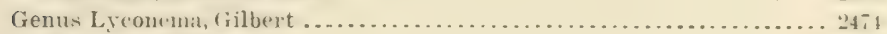

harbatum, (iilhert .....................................

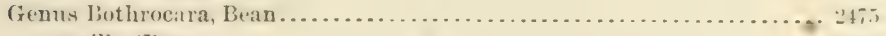

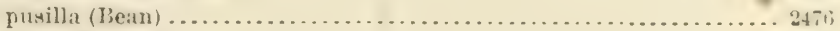

mollis, bean .................................................

(ienus Gymmelis, litinhardt . . . . . . . . . . . . . . . . . . . . . .

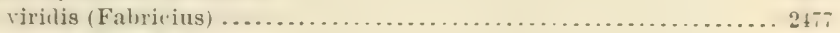

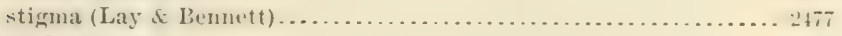

(ienus Lycocara, fill .........................................

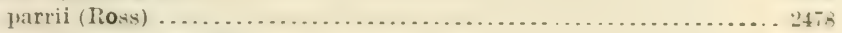

fienus Melanostigma, Gïnther.................................

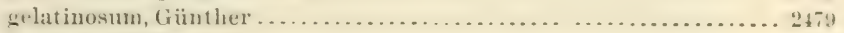

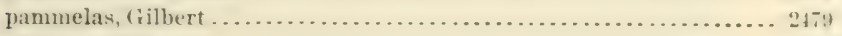

Family Derepodichthyides...................................... 2480

(icnus I) repoulichthys, Gilbert ............................ $2 t-1$ alepridotus, Gilhert ......................................

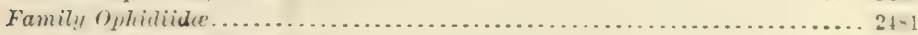

Gemes Lepophitium, Gill............................. $2 t_{n-2}$

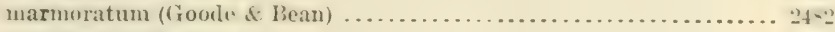

emmelas (Gilbert) ....................................... 248.

stigmatistium (Gilbert) .................................. 2183

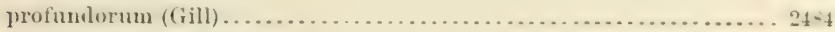

cervinum (frooled d Bean) ............................ 2t-t

prorates (Jordan of Bolluan) ............................ 24-3

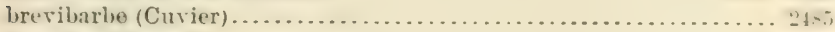

parilale (Gilbert) ...................................

microlepis (Gilbert) .......................................... 2480

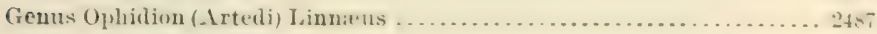

beani. Jurdin o Gilbert. ............................... 24-7

holbrooki(Putnam) ..................................... 248i

griellsi, P'o y.............................................

Genus Chilarn, Jordan \& Evermamn ............................ 2488

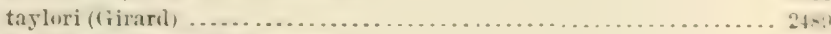

Genus Rissola, Jordan $\&$ Evermann ............................. 248

marginata (1) kay) ................................. $24 \cdot 4$

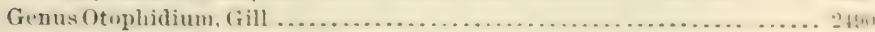

omostignum (Jordan of Gilbert) ............................ 2490

indefatigabile, Jordau \& Bollman ............................ 2490

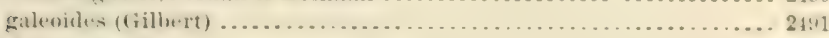

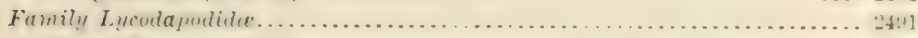

Gemus Lyculajus, Gilbert . . . . . . . . . . . . . . . . . . . . . 24:?

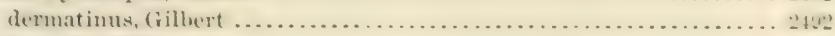

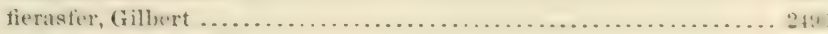

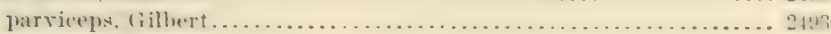

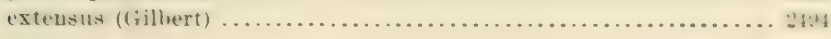


C1.ASs IPISCRS-Continum?.

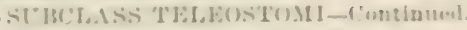

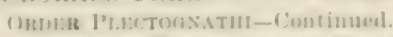

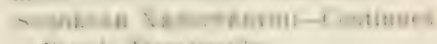

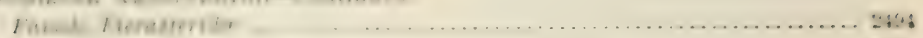

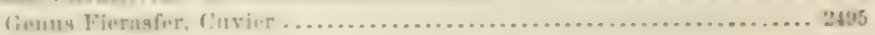

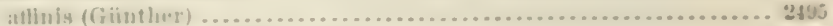

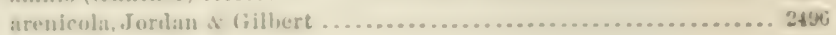

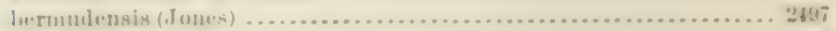

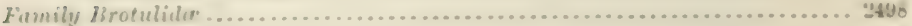

Cirmus Brotula, Cuvier ..................................... 2500

harhata (Bloch of Sehneiler) ................................. 2500

(ienur Sivgleola, Gill ...................................... 2000

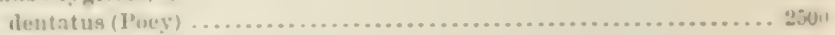

(iеnus Lucifuga, Jory ......................................

subterranens, loey ...................................... 2501

- Gimus Hrosmoplyycis, Gill ................................... 250?

marninatus (Avres) .........................................

(ivmun f)gilbia, Jurdian of Evermant ............................. 250?

ventralis (Gill) .......................................... 2503

cavorum, Evermann of líentall .............................

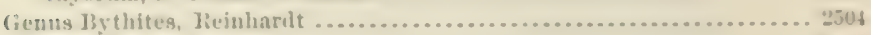

fuscus, Iivinhardt ........................................

Germs Cattut

ruluirustris, Gilluert ...................................... 1505

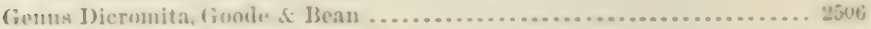

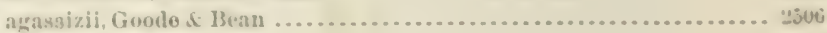

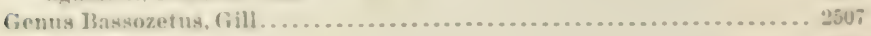

murmalis, Gill ........................................... "954

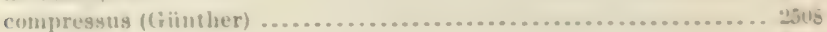

catena, (iomle of Bean ......................................... 2502

turnia (fiinther) ...............................................

Gums Muhia, Gomlu of Iheas .................................. 2510

promelas (Gillert) ....................................... 2511

Genus Neobrthites, froote o. Benn .............................. 5512

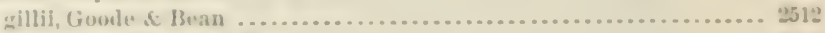

masir..inatus, Goorio s. Bean .................................. 2510

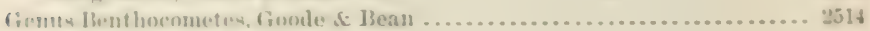

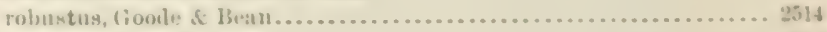

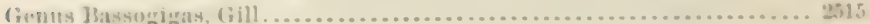

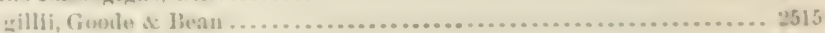

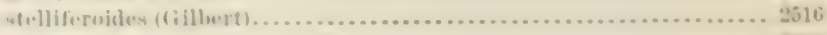

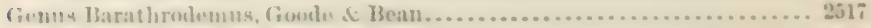

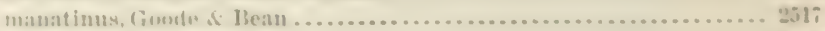

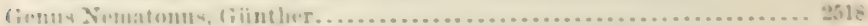

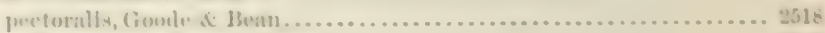

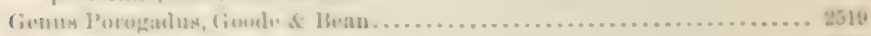

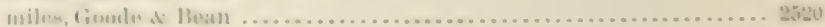

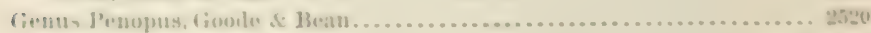

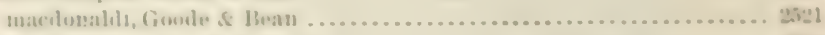

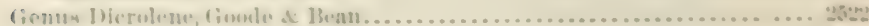

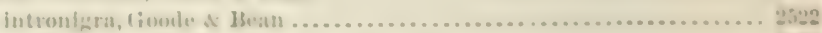

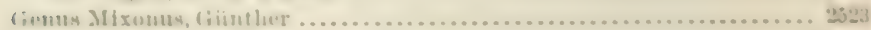

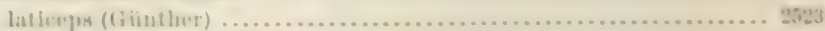

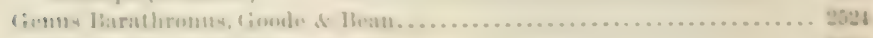

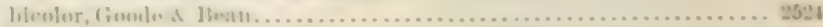

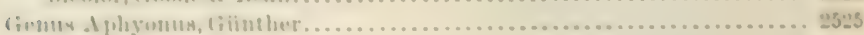

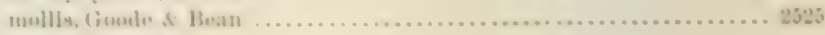


CLASS PISCES-Continued.

SUBCLASS TELEOSTOMI-Continuer.

Order Plectognathi-Continuerl.

Suborder Tenopterygil-Contimuerl.

Family Bregmacerotide

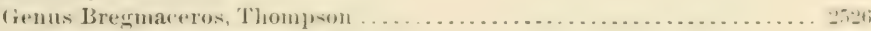

marclellanrlii, Thompon . . . . . . . . . . . . . . . . . . . . . .

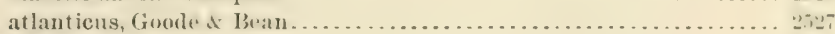

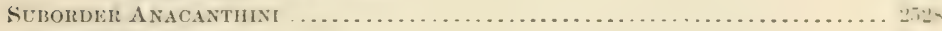

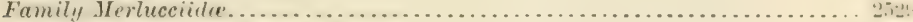

Genus Merlucrius, Ratinesque . . . . . . . . . . . . . . . . . . . . .

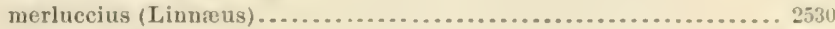

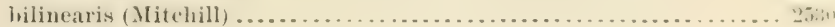

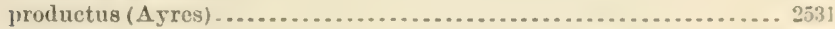

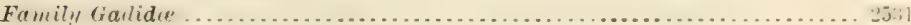

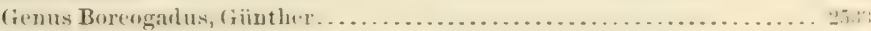

saida (Lepechin) ......................................... . 2533

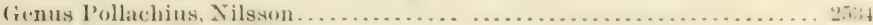

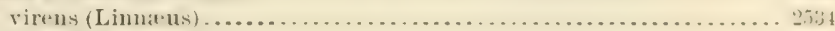

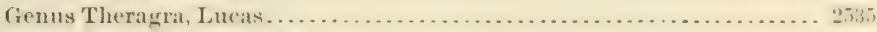

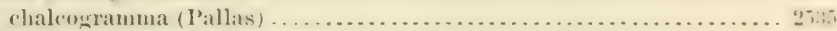

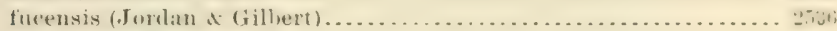

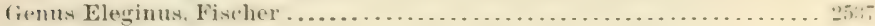

navaga (Kolrenter) . . . . . . . . . . . . . . . . . . . . . . . .

Genus Microgadus, Gill. ... ................................ 2538

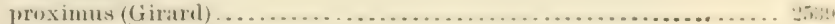

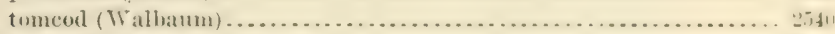

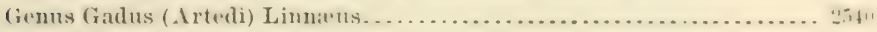

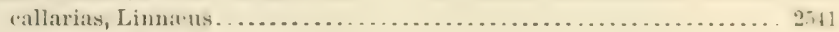

macrocephalus, Tilesıus................................... 2541

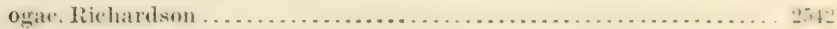

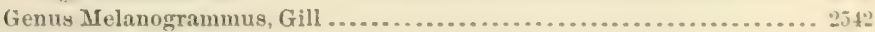

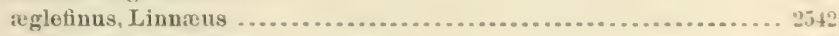

Genus Lepedion, Swainson . . . . . . . . . . . . . . . . . . . . . .

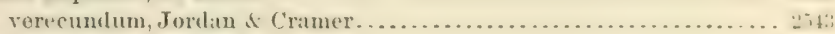

Genus Antimom. Giinther. . . . . . . . . . . . . . . . . . . . . . . .

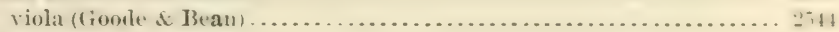

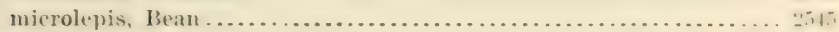

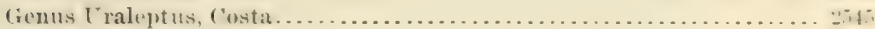

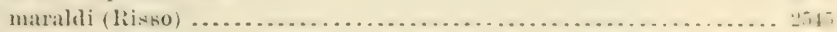

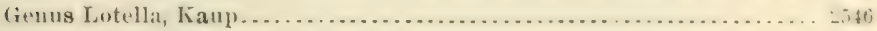

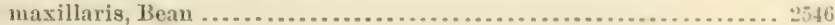

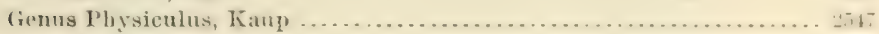

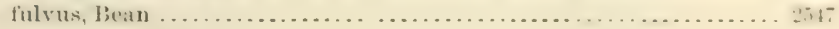

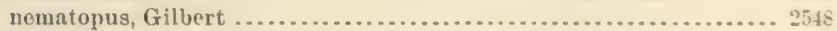

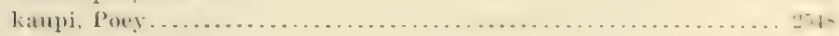

rastrelliger, Gilbert..................................... 2540

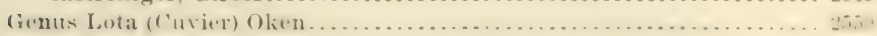

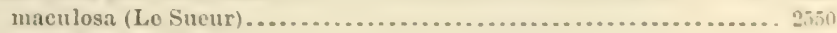

Genus Molva, Fleming. . . . . . . . . . . . . . . . . . . . . . . . . . . . . . . 2. 255] molva $($ Linmaнин) . . . . . . . . . . . . . . . . . . . . . . . . . . . . .

Genus Urophyeis, Gill. . . . . . . . . . . . . . . . . . . . . . . . . . . . . . 255?

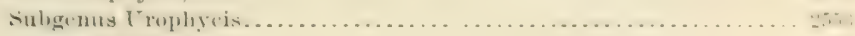

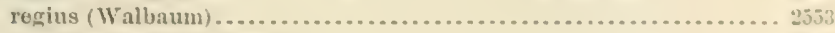

cirratus (Goole s 130an) .................................... 2553

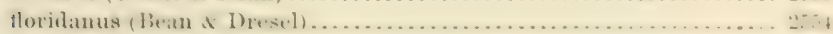

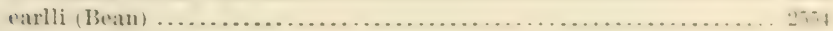

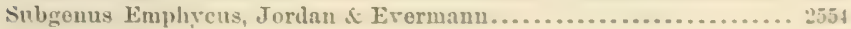

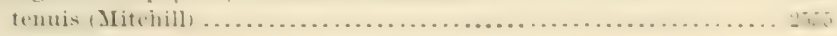




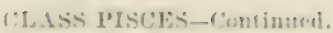

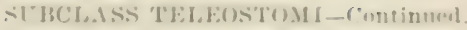

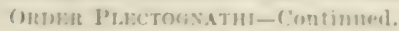

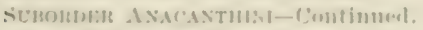

framily diadiele-Contisued.

(ienus trophycis, fill-Continued.

Sulgemus Empliycus, Jurdan A Evermann-Comtinued.

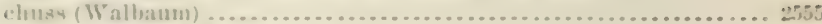

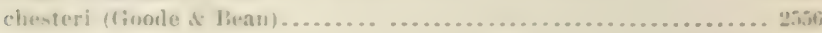

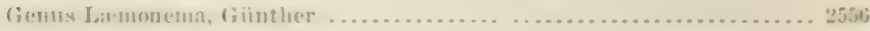

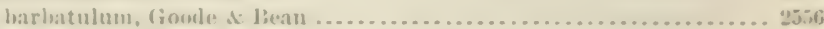

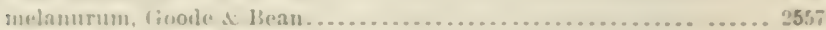

(jenus Gatilropararı, lanthesque ............................... 2557

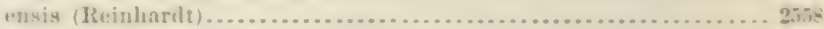

arementatus (lieinharit) .................................. 2559

septentrionalis (Collett) ..................................

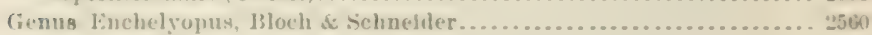

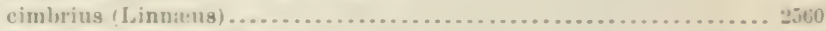

(ienus Iirosme (Cuvier) Oken..................................... "556!

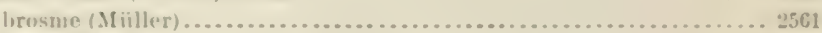

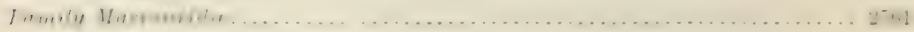

Genus lBathygadus, Giinther................................

arenatus, livede \& 13ean .....................................

fiavosus, froole \& Bean ...................................... 2505

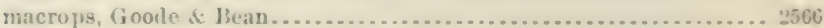

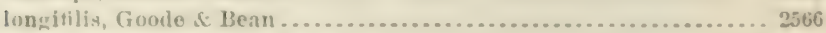

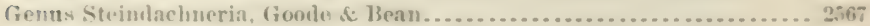
ar.enten, Gnode of Jean....................................... 2568

Genus 'Trachyriucus, Giorna................................ 9568 helolepis, (illbert ......................................... 2569

Giemu Malacocephalıs, Giinther.............................. 9569 occiclentalis, (iomle of Bean .............................. 2570

Guns Museleya, Goorle of Bean................................. 2550

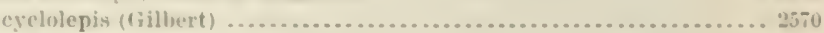

(ienus Xiematonurus, Giinther....................................

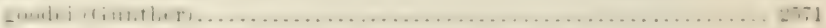

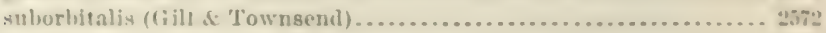

fienus Alhatrossia, Jorllan s. Evermann .......................... 2573

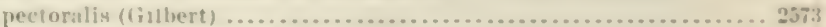

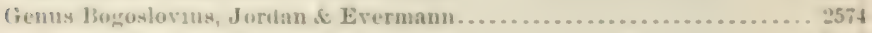
elarki, Jorilan of Gillert..................................... 2575

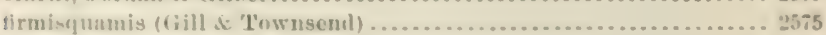

Genus Chalinura, dionde of Benn .............................. 2576

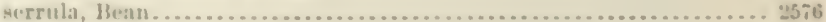

fllitera, (iillert ..........................................

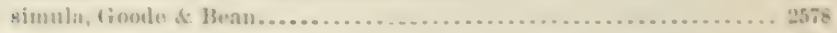

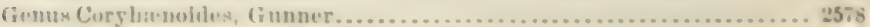

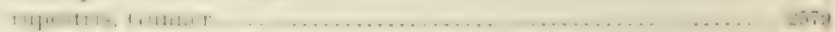

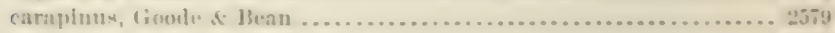

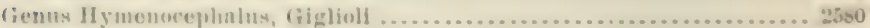

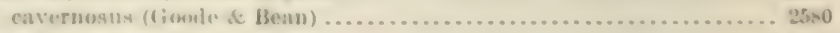

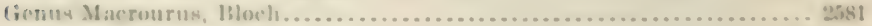

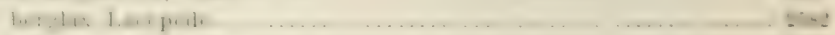

holnt rathyn, fiinther ................................... 2582

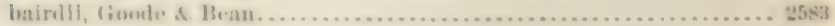

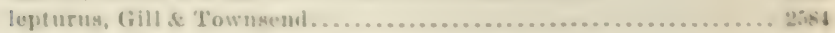

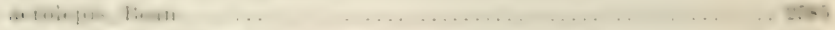

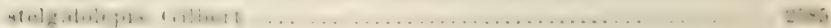


CLASS PISCES-Contmined. SUBCLASS TELEOSTOAII-Continued.

Order Plectognathi-Continued.

SUBORder ANaCANTHINi-Continterl.

Family IIacrouride-Continued.

Genus Macronrus, Bloch-Continued.

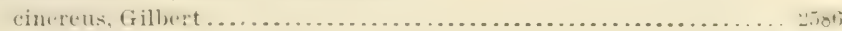

in_olli, Liitlitn . . . . . . . . . . . .

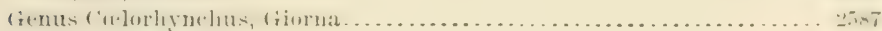

occa (Goorie of Beau) ......................................... 2588

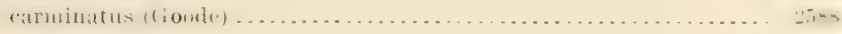

caribbens (Goorle \& Bean) . . . . . . . . . . . . . . . . . . . . . . . . . . . . . . 2 2589

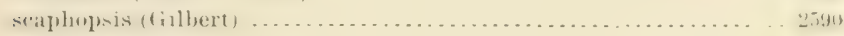

Genus 'Trachonurus, Giinther. . . . . . . . . . . . . . . ................ 2591

sulcatus (Goote of Bean) ........................................ . 2591

Genus Lionurus, Gïuther .................................... 2592

filicauda (Güntler) ...................................... 2592

liulepis, (xillurt . . . . . . . . . . . . . . . . . . . . . . . . . . .

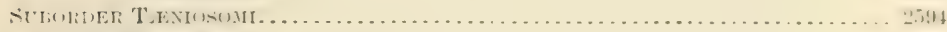

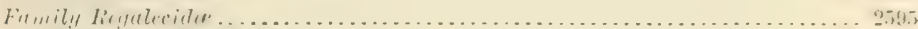

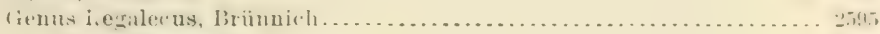

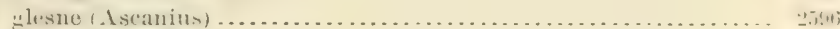

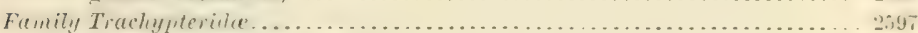

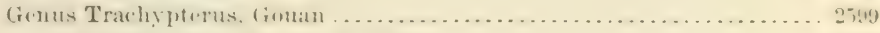

rex-salmonorum, Jorkan \& Gilbert. . . . . . . . . . . . . . . . . . . . . 2599

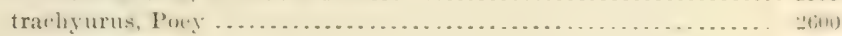

Famity stylephoride .............................................. 2601

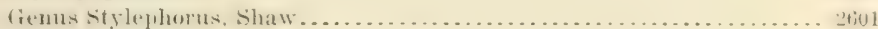

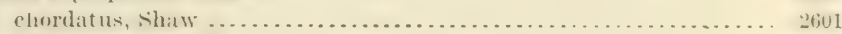

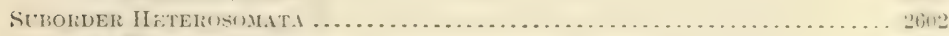

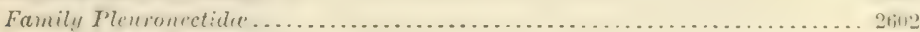

Genus Atheresthes, Jordan \& Gilbert. ............................. 2609

stomias (Jorkan \& Gilbert) .................................... 2609

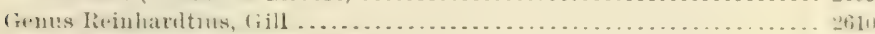

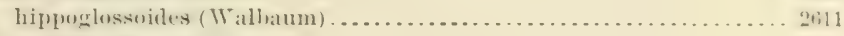

Genus Hippenlossus, Cuvier . . . . . . . . . . . . . . . . . . . . . . . . . 2611]

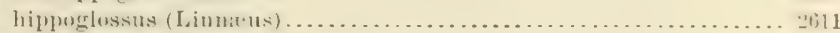

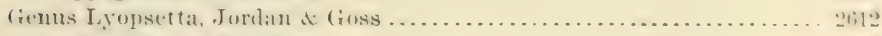

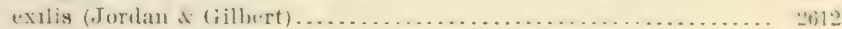

Genus Eopsetta, Jordan \& Goss................................... 2013

jordani (Lockington) . ............................................ 2613

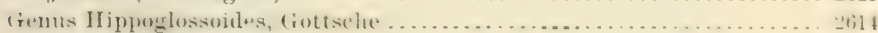

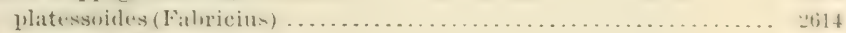

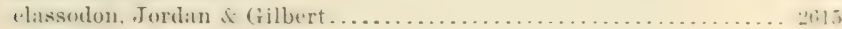

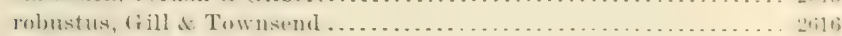

hamiltoni, Jordan \& Gilbert ............................. 2616

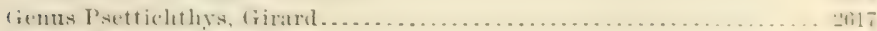

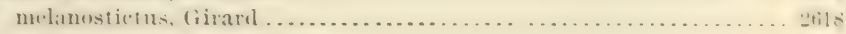

Genus Verasper, Jorlan is Gilbert................................ 2018

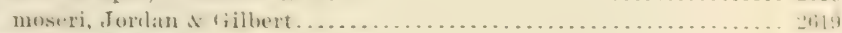

Gonus Ifippoglossina, Steintachner............................ 2620

stomata, Eigenmaun \& Eigenmam ............................. 2020

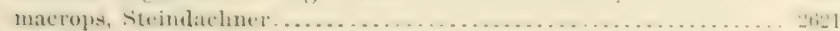

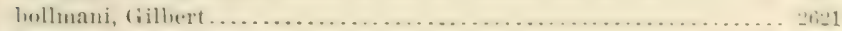

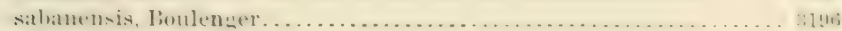

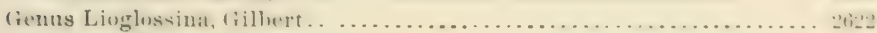

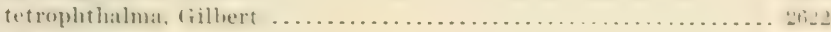

Bull. No. 47 , pt. $t$ - vil 


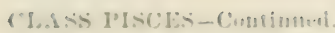

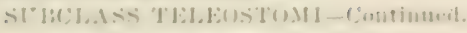

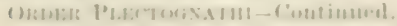

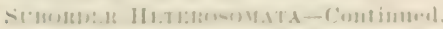

Familı I'temsnetide-Coutumed.

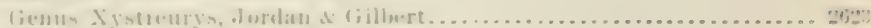

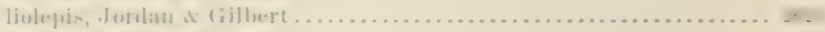

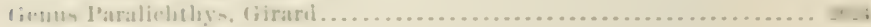

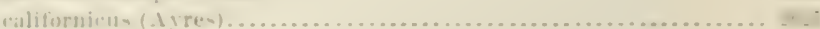

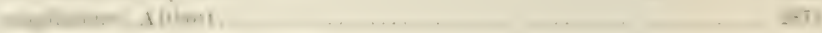

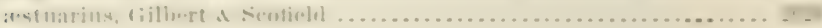

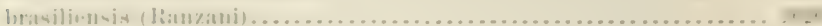

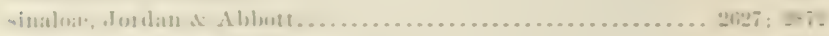

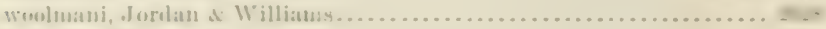

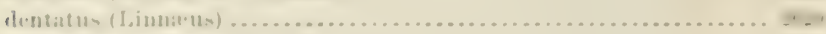

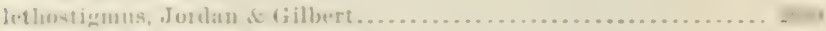

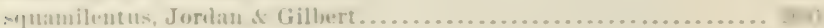

alliguttus, Jurilan of Gilbert................................

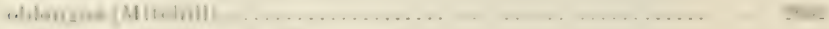

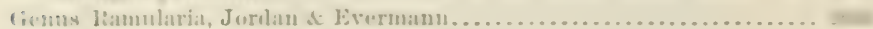
ilemulritica $(1$ illhert) ....................................

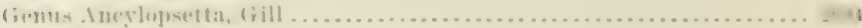
quitrucellat:t, Gill .........................................

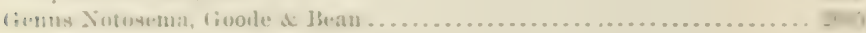

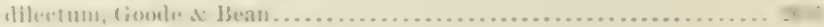

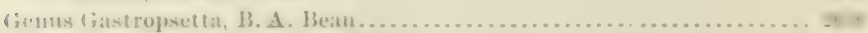

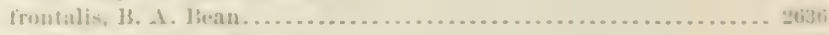

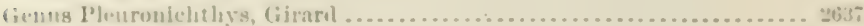

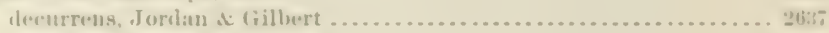

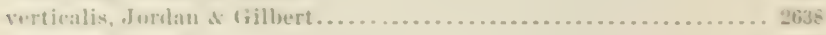

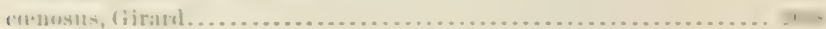

Gientus Hy

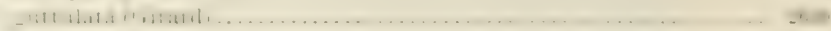

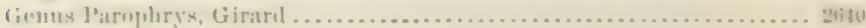

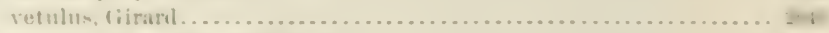

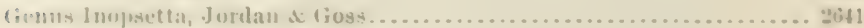

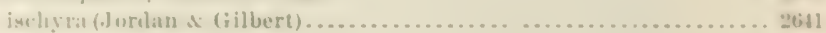

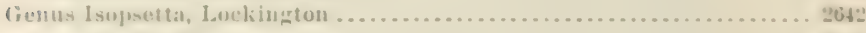

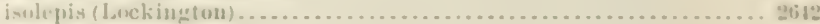

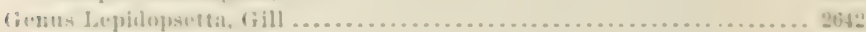

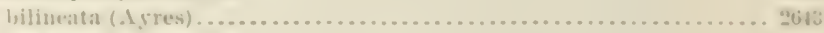

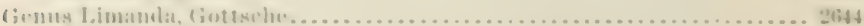
hrmus... - -

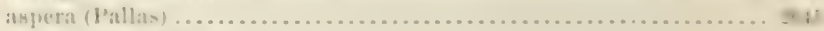

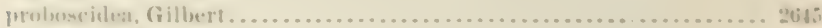
І.

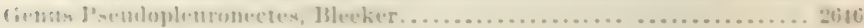

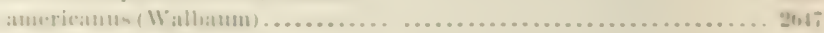

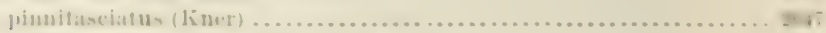

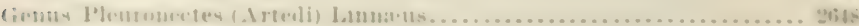

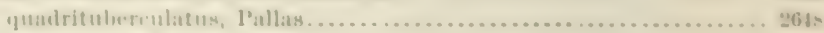

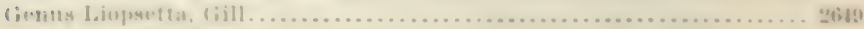

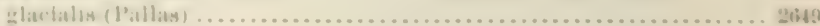

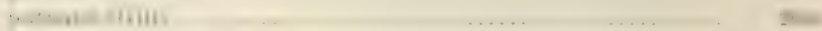

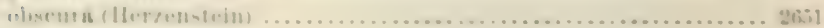

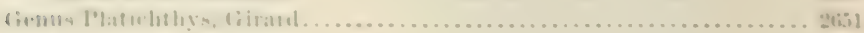

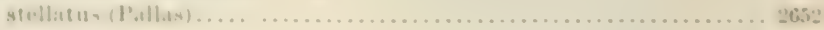


CLASS PISCES-Continuel.

SUBCLASS TELEOSTOMI-Continued.

Order Plectognathi-Continued.

SLBORder Heterosoyata-Continued

Family I'leuronectide-Continued.

Genus Microstomus, Gottsche .................................. 265:

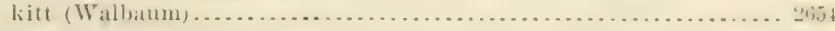

pacificus (Lockington) ........................................ 2055

Genus Enbassichthys, Jordan \& Evermann.......................... 2055

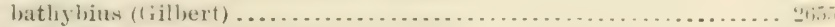

Genus Glyptocephalus, Gottsche .............................. 2050

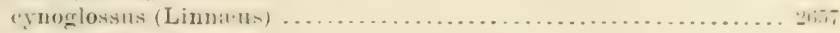

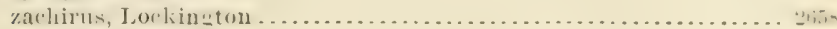

Genus Lophopsetta, Gill ...................................... 2659

maculata (Jitchill) ........................................... 2660

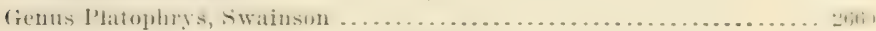

spinosus (l'oey) ......................................... 266?

constellatus, Jordan ...................... . . . . . . . . . . . . . . . . . . 2603

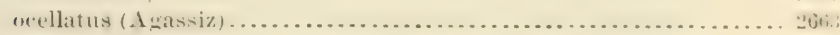

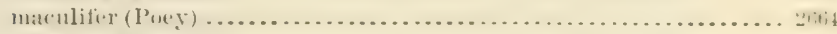

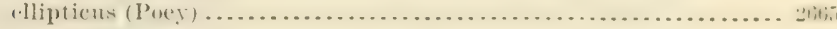

lunatus (Linnitus) ........................................

leopardinus (Günther) ................................... 2660

Genus Perissias, Jordan \& Evermann ............................. 266

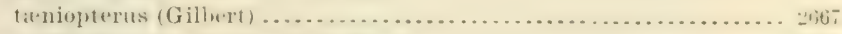

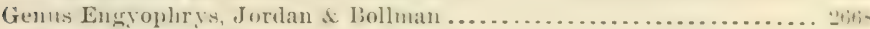

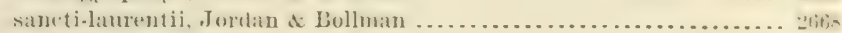

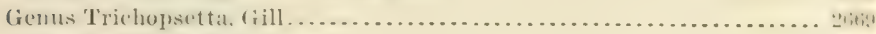

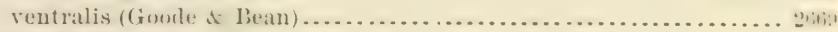

Genus Syacium, Irnzani ....................................... 2670

papillosum (Linneus) ........................................ 2071

micrurum, Ranzani ..................................

latifrons (Jordan \& Gilbert) ............................. 2 673

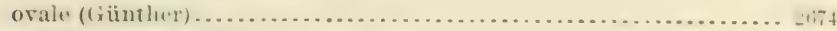

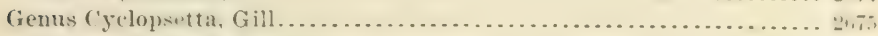

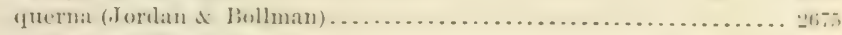

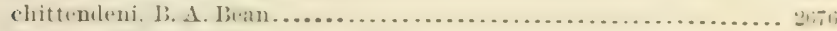

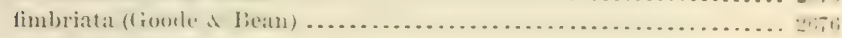

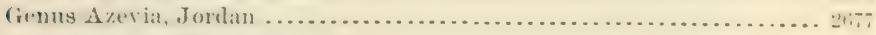
panamensis (Steindachner) ...................................

(ienus ('ithariehthys, blevelier...............................

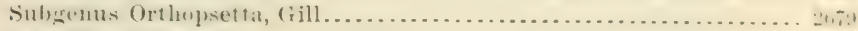

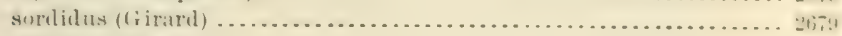

fragilis, Gilbert ...........................................

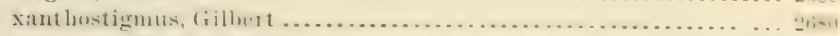

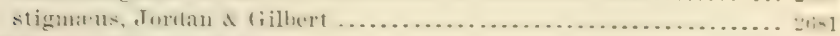

Subgenus Citharichthys .................................... 2682

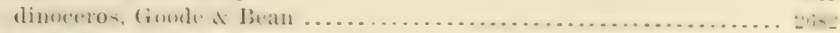

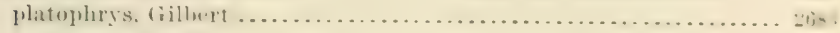

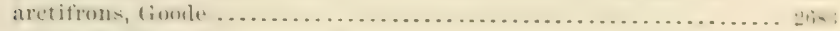

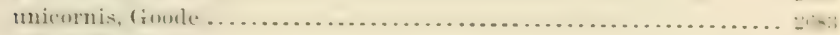

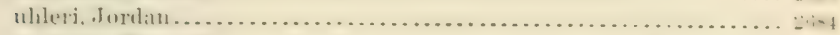

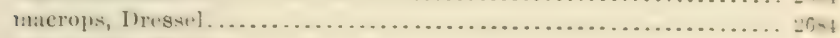

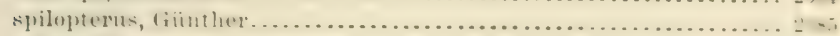

gilberti, Jenkins \& Erermann ................................. 2686

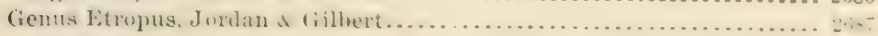

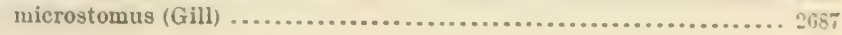




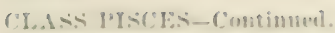

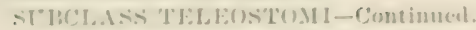

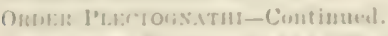

- rove 11 ..

Fitmily J'leuronetide"-Continued.

Genus Letrupur, Jordin se filbert-Continued.

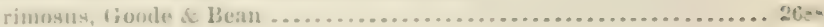

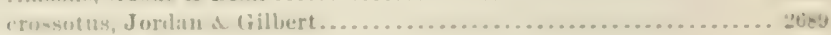

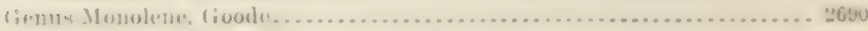

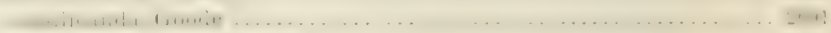

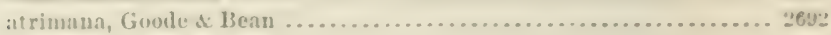

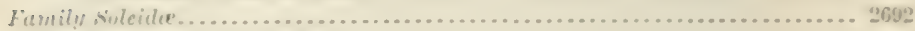

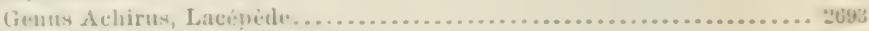

Sulyenus Baiostuma, Bean.................................. 2065

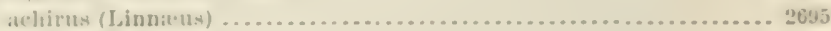

inseriptus, (jus.te........................................

lilunzingeri (steindacbuer) .............................

Jineatus (Linneus) . ....................................... 20.6

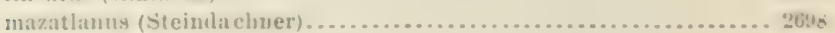

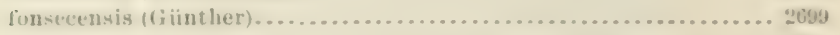

fischeri (titeindachner) . ................................. 2009

- итин

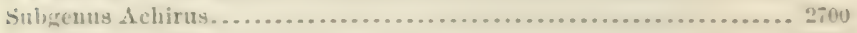

limbriatus (Gïnther) ....................................... 2700

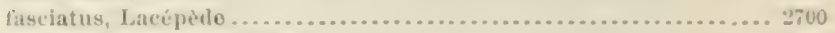

pantmeusis (Steiudacher) ................................. 270:

fienus $\Delta$ pionichthys, Kaup ................................ gั.

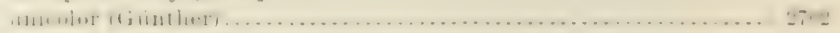

Gonн Ggmnachirns, Kaup ................................. 2:03 fascintus, Giinther ....................................... "us

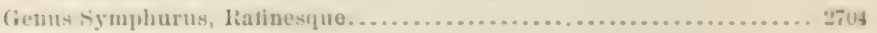

subgenus symphurus....................................... zing

piger (Goole d Bean) .................................... 2ien

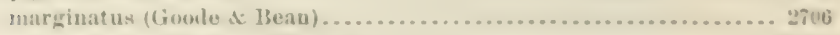

atramesntatus, Jortan \& Bollman............................ g7vo

fusciolaris, Gilbert .......................................

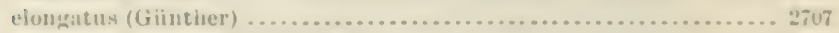

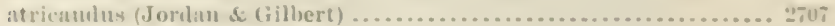

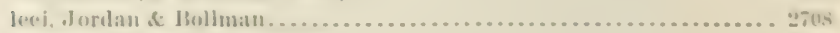

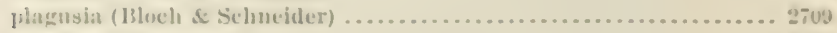

platrinsa (Linntus) ....................................... 2710

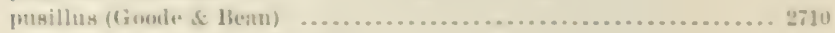

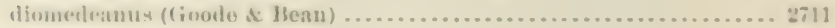

willinuni, Jortan at Culver ................................ 2711

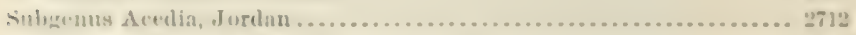

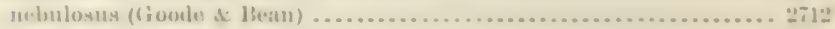

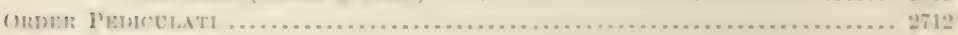

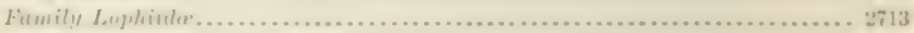

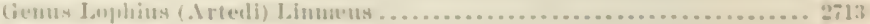

pincaterius, limunuн..................................... 2713

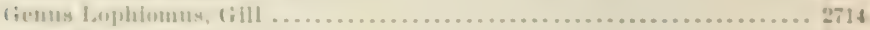

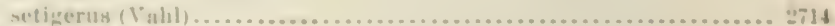

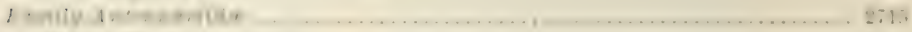

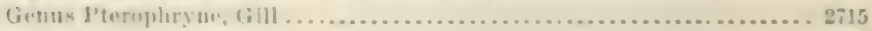

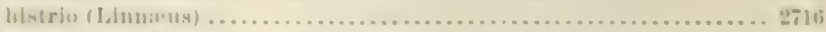

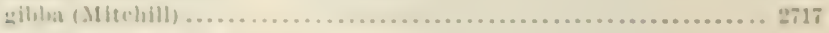

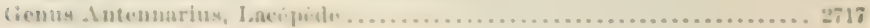

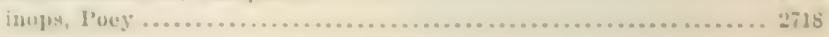


CLASS PISCES-Coutinued.

SUBCLASS TELEOSTOMI-Continned.

Order P'Ediculati-Continued.

Family Antennariidoe-Continued.

Genus Antennarius-Continuerl.

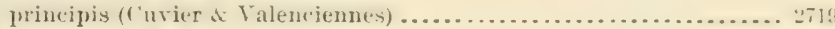

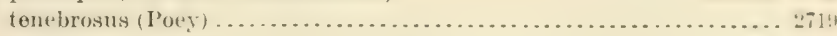

reticularis, fillbert . . . . . . . . . . . . . . . . . . . . . . . . . . . .

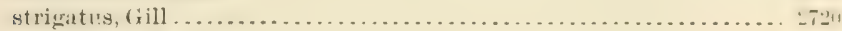

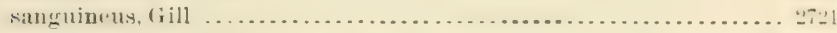

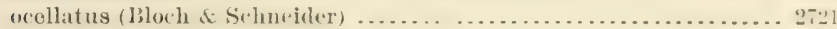

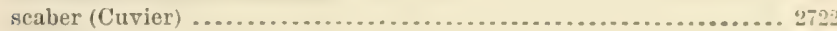

tigris, Poey . . . . . . . . . . . . . . . . . . . . . . . . . . . . . . . . . .

nuttingii, Garman . . . . . . . . . . . . . . . . . . . . . . . . . .

multiocellatus (Cuviér \& Valenciennes) ........................ 272 .

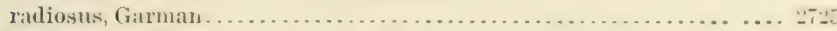

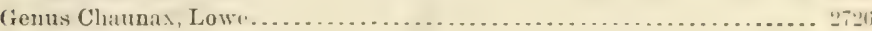

jictus, Lowe . . . . . . . . . . . . . . . . . . . . . . . . . . . .

nuttingii, Garman....... . . . . . . . . . . . . . . . . . . . . . . . . . . 2727

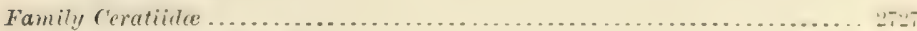

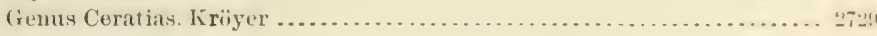

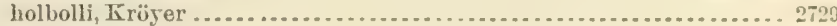

Genus Mancalius, Gill . . . . . . . . . . . . . . . . . . . . . . . . . . . . . . 2729

uramoscopus (Murray) . . . . . . . . . . . . . . . . . . . . . . . . . . . .2!

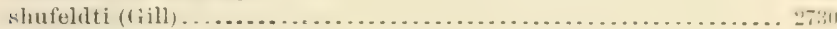

(irnus Cryptopsaras, (iill ....................................

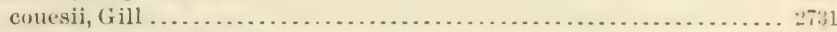

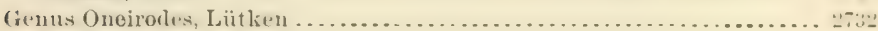
eschrichtii, Liitken ......................................... .

Genus Himantolophus, Rivinharelt . . . . . . . . . . . . . . . . . . .7iz

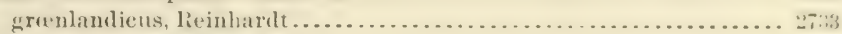

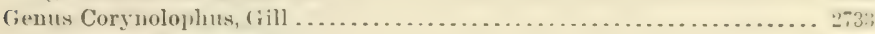

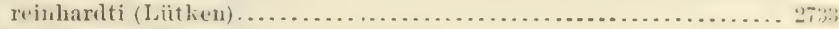

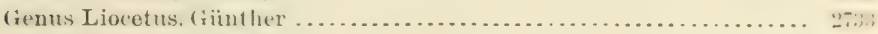
murrayi (Günther) . . . . . . . . . . . . . . . . . . . . . . . . . . . . . 2733

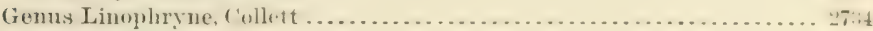
Ineifer, Collett ......................................

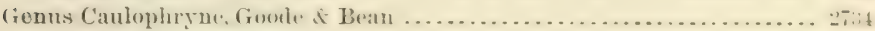
jordani, Goode \& I3ean .................................... .

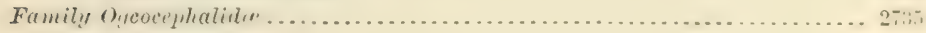

Genus Ogcocephalus, Fischer ................................ 2736

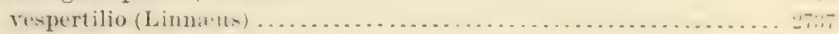

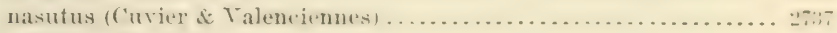

radiatus (Mitchill) ........................................ 2738

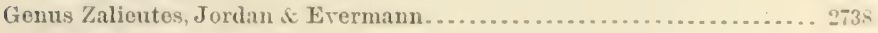

elater (Jordan \& Gilbert) . . . . . . . . . . . . . . . . . . . . . . . . . . .

Genus Halieutichthys, Poey .................................. . $273 n$

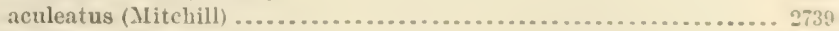

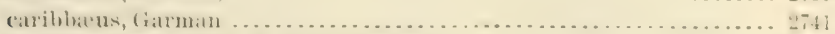

Geurs Halientaen, Curier of Taloncieunes......................... 2741

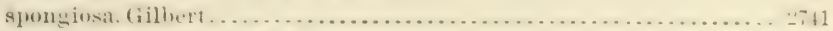

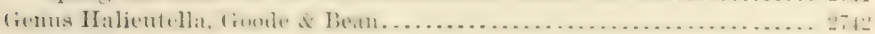

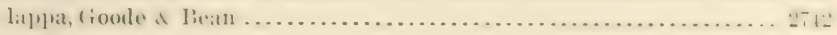

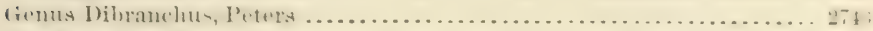

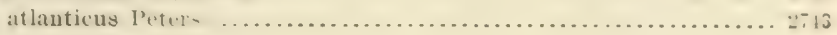





\section{ADDITIONAL ADDENDA.}

Since the publication of Part III of this work a number of new genera and species of fishes have been described from localities within ou limits, and onr kmoledge regarling the relations and distribution of previously known species has been enlarged. These additions and corrertions ars given in the folìowing pages:

Page 130. After Hexamenatichthys rugispinus (Cuvier of Valenciennes), insert the following:

\section{5 (a). (HIALCHTIY's LABITU's (Boulenger).}

Head 31; wilth of head $1^{\frac{5}{5}}$ in its length; depth 5 ; eyo 9 in heal. D. I, $7 ; A .17$. Band of premaxillary teeth 4 times louger than wide, the width half the eso; palatine tooth conical, in 2 small obliquo groups, much smaller than eye, and separated by a space equal to $\frac{x}{4}$ the length of premaxillary band; eye situated abore lerel of mouth, in anterior half of head, 4 in interocular space; oceiput and oceipital prolongation granular, this last a little longer than witle, with an obtuse spine; fontanelle narrow, not distinct, followed ly a groove which does not reach the occipital prolongation; lips thick, the upper overhanging the lower, as in G. rugispinus, to which this species is elosely related; barbels tlattened; maxillary $1 \frac{2}{3}$ in lread, not reaching elgo of operele; exterual mandible $1 \frac{1}{2}$ times as long as internal, 2 in head; gill membrane with a free, unbroken edge; dorsal spine with indistinct serrations, covered with skin, its length a little less than $\frac{1}{2}$ head; adlipose dorsal as long at base as rayed dorsal, twice as far from it as from the caulal; humeral prolongation smooth; pectoral spine $2 \frac{1}{2}$ in head, enveloped in skin like tho dorsal spine, much shorter than the soft rays; ventral shorter, reaching origin of aual; candal deeply forked, with pointed lobes; candal pedunclo $2 \frac{1}{3}$ times longer than deep; no maxillary pore. Blackish gray above, silvery below; all the fins blackish. Length $470 \mathrm{~mm}$. Known only from a singlo specimen from Rio Peripa, Eeuador. (Bonlenger.) (labiatus, having large lips.)

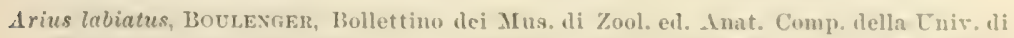
Torino, Vol. xm, ㅅo. 329, 6, Dec. 2, 1898, Rio Peripa, Ecuador. (Coll. I)r. Enricu Festa.) 
Page 132. Aiter Tuchysurus melenopus (fiintles), innert the following:

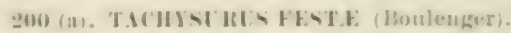

Head 4: depth 1 : ere 63, above level of month, in anterior part-of hond. 10.17: 1. 23. J'remaxillary bamel of seeth 4 times lomger than broal, i a wilth 28 in eye. of eyual to wiolth of gromps of vomer aud palatine

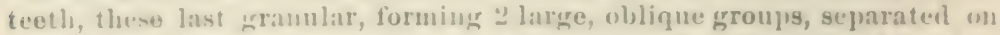
the median line by a space cumal to t diameter of eye; width of luead $1 \frac{1}{8}$ in its length; occiput aud occipital prolongation granular, the last as long as wisle, and with blunt spine; fontanelle narrow, commeneing in a gronve between the anterior nostrild and reaching oceiput, not roaching wecipital prolumgation; larbels flattened; maxillary as iong as head, reach. ing luevoml hase of pectoral spine; extermal maudible a little shorter, as longr as the internal; gill-memlunane with a freo logler broken at the isthmus; anal spine striated, $1 \frac{1}{b}$ in heal, its anterior edge feeln! y touthed except at extremity, which has, like the posterior elge, stronger and move retrorse teeth; alipose dorsal twice as far from rayed dursal as from canulal, its hase $1 \frac{1}{2}$ in base of rayed dorsal; pectoral spine $1 \frac{1}{2}$ in lueacl, its external erlge furnisherl with a series of soft, suall tuhercles on its basal half, anterior engu armed with feeblo retrorse teoth; ventual shorter, reaching origin of anal; caudal deeply forked; caudal peduncle twice as loner as reep; axillary poro present, much smaller tlan the nostril. Color steel hiue above, silvery below. 'This species closely resembles Tachysurus liropux, Bristol. Length 210 mm. One specimen knowu, from Narrangal, Evuador. (Boulenger.) (Xamed fur the collector of the type, 1)r. Enrico Festia.)

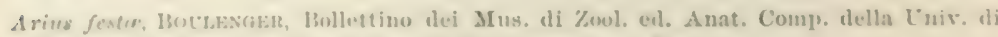

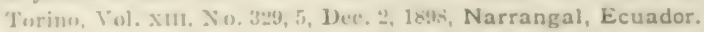

Page 135. Iftur Ictalurns meridionalix (Giintluer), insert:

76 (a). ISTLARIUS, Jorlan a Snyder.

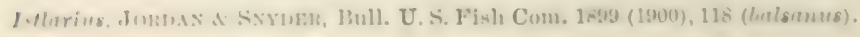

Body ratles derep and compressed; head not widesued, mor great!s depresseul: "yo latge; lower jaw included; teeth in villiform baucls on promaxillaries and alentavies; the promaxillary hamel convex anteriorly. with a short angular posterior extension on each side, no division of

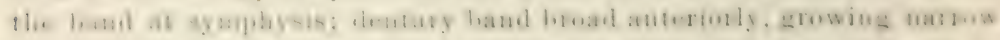
ambl pointed posteriorly, with a listinet median elivision; no teeth on voumer or palatiues; villiform tenth on myper and lower plasrugeals;

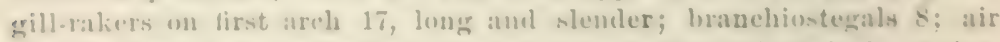
bladeler very larege, extemding alumst to pusterior end of holy raviry, dividerl ly a transverse eonstriefion into two parts of nearly enual

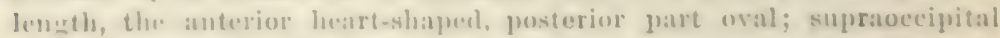
hone: wielely separaterl from interspinal; humeral prosess short, almont hidelen by the sliu; lateral lime extendiug from bolow insertion of

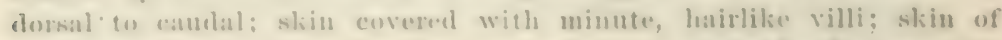

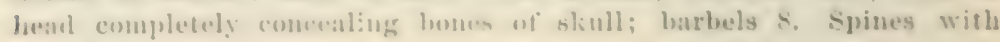


distal parts soft, not brauched, continuous with the hard parts; lasal part of pectoral spine grooved posteriorly, weakly serrate above the groove.

Istlarius has some of the characters of the gen:s Leptops, notably the dentition of the upper jaw and the weakness of the fin-spines; but it more closely resembles Ictalurus, and its relationship is probably with that genus.

(Named for the Rio Ixtla, the type locality of the type species.)

„OS (a). ISTLARIUS BALSAXLs, Jordan se Suyter.

Ilead 4 ; depth $4 \frac{2}{3}$; depth of eaudal peduncle $2 \frac{1}{2}$ in head; eye $5 \frac{1}{2}$; snout 23 ; distance between eyes $2 \frac{1}{2}$; height of dorsal $1 \frac{3}{5}$; length of hase of dorsu? 3 ; height of anal $1 \frac{2}{8}$; length of base of aual 1 ; length of pectoral $1 \frac{1}{2}$; ventrals $1 \frac{2}{3}$; caudal 1 ; D. I, 6 ; A. 24. Body deep and somewhat compressed, deepest above ventrals, widest between pectorals; head narrow, not greatly depressed; eye large, nearer tip of snont than to posterior elge of opercle a distance equal to diameter of eye; iuterorbital space convex; width of mouth $2 \frac{1}{2}$ in liead, lower jaw included; upper jaw projecting a distance equal to diameter of pupil; barbels 8 ; of the inferior ones the median pair shorter; distance between their bases equal to the diameter of pupil; the outer pair when extended directly back ward reach the edges of gill covers; maxillary barbels longest, reachin: upper angle of gill opening; nostril barbels reaching middle of pupil. Tecth in broad villiform bands on premaxillaries and dentaries, the band on npper jaw convex anteriorly, with a short, angular posterior extension on each side, no apparent division of the band at symphysis; band on lower jaw broad anteriorly, narrow and pointed posteriorly, a distinct median division: no teeth on romer or palatines; upper pharyngeal teeth in oval villiform bands; lower pharyngeal teeth in 2 narrow ollong bands; gill-rakers on first arch 17, long and slencler; branchiostegals 8; anterior nostril tubular, the posterior with a raised rim estending on each side from the barbel to the posterior elge of the opening, where it is divided; skin of head completely concealing the hones of skull; supraoccipital hone not in contact with interspinal; humeral process ahout as long as vertical diameter of ejo, almost hidden by the thick skin; lateral line extending from a perpendicular throngh insertion of dorsal to candal; slin covered with minute, laair-like villi; dorsal spine with its distal third soft, preceded by a small, angular, immature spine; first branched ray longest, the others gradually shorter; adipose fin large, ahove middle of anal; length of the posterior free eilge 3 times diameter of pupil; third or fourth anal ray longest, edge of fin rounded posteriorly; caudal deeply forked, the lower lobo rounded, the upper rather prointed; pectoral rays 1, 11, distal two-thirds of spine soft, not brancher, continuons with the hard part: basal part groovel posteriorly, weakly serrate above the groove: ventrals reaching origin of anal. Color bluish slate above. light silvery helow; a few small dark spots on tho head and body; fins with dusky coloriug: inferior barbels light; maxillary barbels with mpper half dark; nasial barbules with light edges. Some specimens lave many well-eletined color 
spots, while others have few or mone. The youmg have no spors. One sperimens virg in lengrh from 10 to to centimetors.

Ixllarius bulanus has a larges and rather complex air bladder. It lies (c)en to the spinal colum and extends almost to the posterior encl of the boly cavity. It is divided by a deep transverse constriction into two halves. The anterior part is heart-shaped, constricted dorso-ventrally. It is separated by it T-shajuel partition into 3 chambers. Of these the anterior, transvers: chamber is partly diviled on the median line ly a fold of the dorsal wall of the blatiler over the vertebral column. The transicerse chamler is connected on each siclo with the 2 posterior lateral "lamulers hy large openings in the ends of the transverse wall. The posterior hall of the hladeler is withont partitions. It is connected with the left lateral (hamler only by a small opening. (Jordan d. Snyler.)

knuwn only from lio Ixtlat at l'uente de Ixtla, Morelos, Mexico. ('Yype,

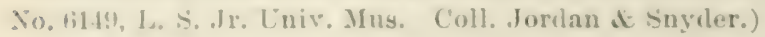

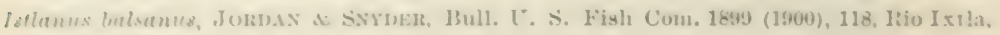
Mnteren. Mexion.

\section{Page 211. Atter Algansen tincella (C'uvier d Villenciennes), insert:}

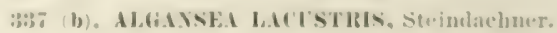

Hend 3.67: deptlo 5 ; eye 4.75; snout 3.6; interorlital 3. I). III, 7: . .

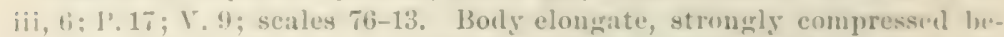
hind: catulal perhunclo long and siender, its length 5 in hear, its depth 10); hear printed, rounled above, greatly increasing in width behind the eyes where the width is hati tho heal's length; mouth slightly oblique, jaws equal; tip of chin slightly thickened; maxillary nearly reaching eye; mapn \& in heal: preorhital boat-shaped, concavo above, convex below, equal to eye, w' 1.6 longer than wide; teeth $4-1$, hooked, with compresied "rowns. Origin of clorsal midway between anterior borler of eye aud hats. of candial, al little in front of ventrals, whose origin is equally distant between center of ere and hase of camblal height of dorsal 1.75 in head, its hase half its leeight; height of anal 2.14 in heal, or twice its

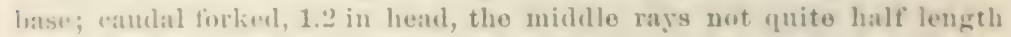
of longest; ventrals short, 2.17 in heasl, their tips not reaching vent ; peetoral 1.6 in heal. Seales firm, half-oval in slanpe, larger posteriorly: latoral lin. on mildles of side, deseending somewhat anteriorly. Color dark lorownish gray above, a darker band along middle of side; color ledow bateral line lecoming aloruptly lighter gray, merming into silvery un unler piats. One example $20 \mathrm{~cm}$, long from Lake Patzenaro, Mexieo. (Stovintiacluner.)

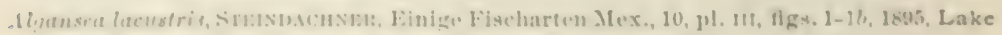
Pátzcuaro, Mcxico. (Coll. I'rinceses Thetesa ven Bayern.)

Page 254. Mreellu, propused by us for a sulugems in lotropis, is pre-

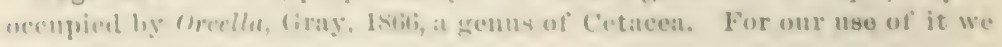
nilsititute circulu.

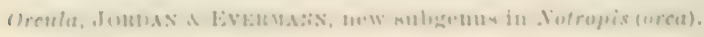


Page 260. After Lotropis anogenus, Forbes, insert:

f0t (a). Notropis MLshoki, Meek.

Hear 4 ; depth 5.5 ; eye 3.25 ; snont $3.75 ; 1.8$; scales 36,10 in series from dorsal to ventral fin; longest dorsal ray 1.34 in head; pectoral 1.5 ; ventral 2, reaching anal. Teeth 4-1, slightly hooked, grinding surface narrow.

Borly rather terete and slender; snout bluntish; mouth small and slightly oblique; lower jaw the shorter, slightly included; posterior end of maxillary scarcely reaching vertical from the front of orbit. Eighteen scales between nape and dorsal fin, the seales in this region being smaller and more crowded than on sides and poskerior dorsal region, much resembling I'imephales notatus in this respect; lateral line incomplete, not extending beyond the fourth scale on each side. Origin of the first ray of dorsal fin midway between the base of the candal fin au! the tip of the snout; the longest (anterior) dorsal rays nearly three times the length of the posterior ones, the tips of the former extending considerably beyond the tips of the latter when the fin is deflexed. Color olivaceons, darker above, very pale below; a dark band abont $\frac{2}{3}$ diameter of eje around snout and on sicle to base of eandal fin; on the snont this loand is confined to the upper jaा; between this band and the darker color on the dorsal region is a lighter band of about the same width; a dark vertebral line present, also a similar one from base of anal to caudal fin. In some specimens the Iateral line is absent on the firstscales and appears at intervals on abont 12 scales, but it is occasionally absent altogether. It is less developecl in this'species than in auy other of the genus.

Close to $\lambda$. caynga, from which it differs in the reduced size of the scales before the dorsal, the more sleuder body, the sharper snont, the slightly larger and more oblique month, and the more incomplete lateral line. It is also a larger tish than $\mathrm{X}$. caynga.

linown only from Gull Lake, near Muskoka Lake, Ontario, whero 24 specimens were obtained by Dr. Meek in September, 1899. They vary in leugth from 1.31 to 2.91 inches. (Named for Minskoka Lake.)

Sotropis muskoka. MEEk. Totes on a Collection of Fishes and Amphibians from Muskolia and Gull Lakes: P'ublication 41, Fielt Columbian IInemm, Zoological Series, Yol. I, No. 17, 308, Norember, 1899, Gull Lake, Ontario. (Type, No. 2964, Field Columbian Museum. Coll. Dr. Mrek.)

Page 264. After Yotropis procne (Cope), insert:

11: (a). Notropis Riscoxis, Jordan if Snyler.

Head 4; depth $3 \frac{1}{2}$; eyo $2 \frac{2}{3}$ in heal; snout $3 \frac{1}{3}$; interorbital spaco 3 ; depth of caudal peduncle $2 \frac{1}{3}$; height of dorsal 4 t in length; anal 5 ; leugth of pectoral $5 \frac{1}{3}$; ventral $5 \frac{7}{4}$; cantal $3 \frac{1}{2}$; D. I, 8; A. I, 8; P. 12 ; scales 33,15 between dorsal and oceiput, 10 between dorsal and rentral fins. Dorsal oitline of body evenly ronniled from snout to insertion of dlorsal fin. slightly concare from latter point to base of caudal; rentralontline evenly rounded to end of anal base; deepest part of body just anterior to insertion of dorsal; willth of body $\frac{1}{7}$ of its length. Snout pointed: mouth 
oblique, lower jaw inclubled; maxillary not duite reaching vertical from anterior maryin of orluit. Teeth $4-4$, frail and easily detached from the arch, slightly hoolsew, no evident grinding surface; grill-raliers short atul blunt, reduced to slight elevations on lower part of first arch. First ray of dorsal tin longest. last ray murle shorter; when the fin is elevated, the posterior marin is straight, when clepressed, it is somowhat falcate; anal fin similar in shape; pectural pointed; tips of ventrals reaching anal; caulal doeply forked, the lohes pointed. Color silvery, a dark lateral band, the willth of a seale, extending from tip of suont through eye to hase of caulal, where it encls in an elongate, black spot; body above lateral batnd stippled with black, the dots grouped closely together on top of heat, and in a narrow dorsal hand extending from oceiput to insertion of clorsal, in at sharply defined lolack line along base of dorsal fin, aud also on the edres of the dorsal scales; hody below dark baud immaculate, exeept a dusky line along loase of anal fin; all the fin rays dusky, especially the partly developed camblal rays, which are uoticeably darker than the otliers.

Nolropis rasconis is closely related to. $\mathrm{I}$. nigrotentutus, from which it difters in having a more compressed houly, a longer snout, larger eye, longer and more pointed fins, and in having the color-band narrower on the body and more marked on the snont. (Jordan \& Snycler.)

Known only from the live Verele near Rascon, San Luis Potosi, Jexico, where the type (Xo. 6153, L. S. Jr. Lniv. Mus.) was collected by J. O. sinviler.

(Xamed for liascon, near which place the type was obtained.)

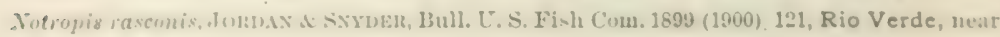
Rascon, Mexico.

Page 302. Jefore Liricymba, Cope, insert:

123 (a). XYSTROSUS, Jordan \& Sny̧iler.

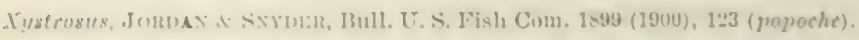

Buly long, compressed; interorbital space low and flat; mouth terminal. oblinue; jaws subeepual; yremaxillaries protractile; no barbel; no prentubranchiar; will-ritiers $6+13$, long, slender, crowled on arch; teeth $4-4$. hooked, grinling surfice oblipno, grooved; alinentary canal about twice as longas ludy ; peritoneum dusky; lateral line complete, decurved alove pectoral; seales dil, evenly distributed over boly; tins falcate; dorsal inserted directly above insertion of ventral; andal deeply forked; anal batsis short. Allied to . Votropix; diflering in the long grll-raliers, the suall

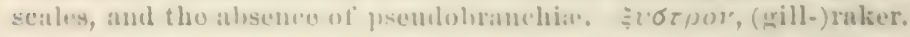

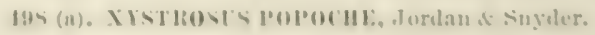

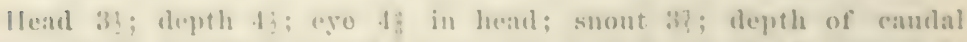

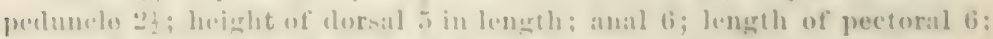

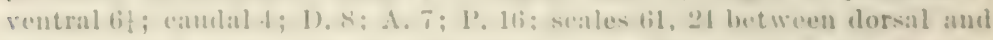
oer:iput, li leetween dorsal and lateral line. lbody long and slender:

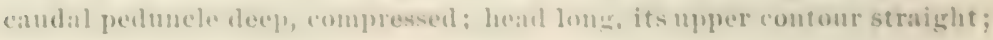


interorbital space broal and flat; eye large; its longitudinal diameter contained 2 times in interorbital space; snout sharp, slightly turnerl up, at end; month large, oblique; lower jaw included; lips thin; maxillary not quite extending to orbit; gill-rakers $6+6$, on first arch, close together, sleuder, the longest $\frac{1}{2}$ diameter of orbit. 'Teeth $1-1$, strong, hooked, grinding surface oblique, narrow, grooved, a notch just below the hook; alimentary canal almost $t$ wice the length of boly; peritoneum dusky. Scales not crowded anteriorly, evenly distributed over body; lateral line shaped like the contour of body, except above pectoral fin, where it is sharp]y decurved; dorsal inserted directly above ventral, first ray lighest, nearly 3 times height of last; when depressed, the fin is falcate; when elerated, its edge is concare; anal similar in shape; candal deeply forked, the tips sharp; ventrals pointed, not reaching vent; pectoral slightly rounded. Color silvery; darker above, especially on median dorsal area, where a more or less dusky band extends the length of the body; rays of dorsal fin and tips of caudal dusky; lower fins white.

Exact measurements of the only specimen obtained are here given: Length of body in millimeters 92 ; depth of body, expressed in hundreaths of length, 23; depth of caudal peduncle $11 \frac{1}{2}$; length of head $28 \frac{1}{2}$; width of interorbital space $11 \frac{1}{2}$; length of snout 8 ; diameter of orbit 6 ; distance from snout to dorsal fin 52; height of longest dorsal rays 20; distance from snout to anal fin 73 ; height of longest anal rays 17 ; distance from anal to caudal fin 21; length of caudal fin 25; length of pectoral fin 18; distance from snout to ventral fin 53 ; length of ventral fin 16. (Jordan \& Snjder.)

Only the type known (No.6151, L. S. Jr. Unir. Mus.) collected by J. o. Snyder in Laguna de Chapala, near Ocotlan, Jalisco, Mexico.

Tystrosus popoche, Jordax \& Sxider, Bull. U. S. Fisl Com. $1899(1900), 123$, Laguna de Chapala, Mexico.

I23 (b). FALCULA, Jordan \& Snyder.

Falcula, Jordax \& STrDer, Bull. U. S. Fish Com. 1899 (1900), 12 4 (chapalce).

Body long, compressed; caudal slender; mouth large, lips thin, promaxillaries protractile; no barbel; teeth in 1 row, 1-1, hooked, grinding surface grooved; gill-rakers fow, short, far apart; alimentary caual short; peritoneum silvery; lateral line complete; scales rather small, about 50 in lateral series. Fins ligh; falcate; dorsal inserted directly over ventrals; anal basis short; first simple rass of dorsal and anal rudimentary and closely adnate to fixst brauched ray; caudal long, deeply notehed.

The genus Falcula is related to Notropis, from which it difiers in its small scales and in its vory high falcate fins. (falx, scythe.)

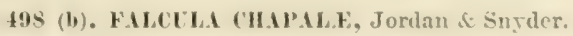

Head 4; depth $4 \frac{1}{2}$; eye $3 \frac{2}{5}$ in head; suout $3 \frac{8}{5}$; interorbital space $3 \frac{5}{5}$; depth of caudal peduncle $9 \frac{1}{2}$; height of dorsal 1 in length; anal 5 ; length of pectoral $4 \frac{4}{5}$; ventral $5 \frac{9}{4} ;$ caulal $3 \frac{1}{4} ;$ D. $8 ; A .8 ;$ P. $17 ;$ scales 50 , 19 between dorsal and occiput, 16 between dorsal anil veutral tius. 
Bonly long, flenulur, compuresent; suont jointed, its length ergual to diamoter of orloit or to interurhifal space; month almost horizontal, its cleft extending to vertical from anterior edgo of orbit; lips thin ; tcoth 1-1, strong, siliglitly lowoked, the loosk barely evident on lower tooth; threo 1 plper teoth with a growvel grinding surface; gill-rakers $3+\bar{\imath}$, short, pymulal, and far apart; alimentary cunal short; peritonenm silvery: lateral line complete, decurved to a point in a vortieal midway between pectoral and ventral fins, from which it extends in at straight line a litlle. bulow middle of lody to the eaudal; fins all very ligh and pointed: flursal inserterl directly over wrigin of ventmals, falcate when depressed, its lirst ray longrest; anal nimilar in shape, inserted at a point of the distance frou tip of snout to hase of candal; ventrals 'stending to vent, tigl of pectoral reaching ventrals; caudial deeply torked. Color silvery; a narrow, dark median bamol extending down from creciput to base of candal; dorsal srales with lino dots which givo their edges a dusky color.

Ono specinun linown, careful measurements of which aro given: Length of body in millimeters 7.1 ; apth of hody, expressed in lumirenths of leneth, 25 ; depth of caulal peduncle 11 ; length of head 25 ; width of interorlutal space 7 ; length of suont 7 ; diameter of orbit 7 ; distance from snout to dorsal fin 47 ; lieight of longest dorsal rays 26 ; distance from snunt tu inul lin 67 ; height of longest anal rays 21; distance from an:al to caudal tin 27; length of caudal fin $3 \frac{1}{2}$; length of pectoral tin 22 ; distance from snout to ventral lin 62; length of ventral tin 21. (Jordan d: Suyder.)

Only the type known (Yo, 6152, I. S. Jr. Vnir. Mus.) collected lyg J. U. snyider in Layuna de Chanala, near Ocothan, Jalisen, Mexico.

(Xamerl for the tripe loeslity.)

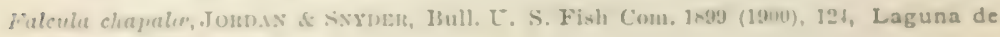
Chapala, Mexico

Page 311. Beforo flymin unbila (firaril), insert the following:

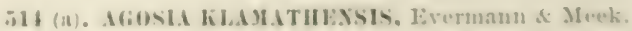

Hent 1 ; deptli 4 : eye $1 \frac{1}{2}$ in heal; suout 31 ; 1). I, \&; A. I, 7; acales abmat $11-7,-10$ (average, 73 in 49 -pecimens).

Body rohust, subeylinclrieal, back somewhat elevated ; suout rather long, month inferior, littlo obliyun, thu lower jaw included; maxillary not reaching front of orbit; upper liz̧ without frenum; barbel present, luu small. Lateral line incomplete, interrupted in many places, about 30 pores developurl. Origin of alorsal tin midway lotween front of pupil and hase of eaudil tin; jectoral rather short, reaching alont three-fourths the distance to biave of ventrals; ventrals reaching vent; anal large, its longest ray 1 t in head; candal willely forlecel.

Color in alcohol: Hlivaceons, mottles and blotehed witls darker on back and side; under parts quile; an olsouru palo streak from eye to base of

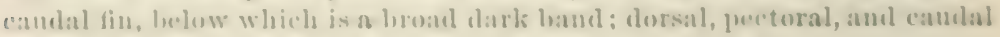

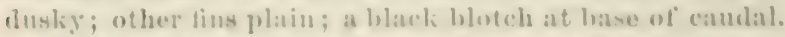

An examination of fs speimens shows all the inportant characters to 
be fairly constant. The barbel is in a fer cases obscure or possibly absent. The number of scales in a transverse line raries from 68 to 78 , the average being 73 . The lateral line is in all cases incomplete, though in different degrees. Sometimes it is continuous for only 6 or 8 scales, after which there are several interruptious and only 8 to 10 more pores. In others there are 20 to 30 pores in a continuous series.

Close to A. nubila.

Upper Klamath Lake, Oregon.

Agosia klamathensis, EverMaxx \& MEEk, Bull. U. S. Fish Com. 1897 (Jan. 6, 1898 is fig. 5, Pelican Bay, Upper Klamath Lake, Oregon. (Trpe, No. 48225, U.S. X. II Coll. Meek \& Alexander.)

Page 333. After Piabucina panamensis, Gill, insert the following:

5ั๊ (a). PIHC

Head $4 \frac{1}{3}$; depth 4 ; eye $4 \frac{1}{2}$; suont $4 \frac{1}{2}$; interorbital width $9 ;$ D. $9 ; 1.11$; seales 28-8; lower jaw longer than snout; teeth 26 above, 32 in external mandibular series; maxillary reaching eye; origin of dorsal equally distant between end of snout and caudal sinus, a little behind hase of ventrals; adipose dorsal very small; anal with 8 brauched rajs; pectoral $1 \frac{1}{3}$ in head, louger than ventrals; caudal strongly emarginate, scaled on basal half. Very deep olive brown above, whitish below; fins uniformiy gray. Length $160 \mathrm{~mm}$. (Bonlenger.) From tributary of Lake Pita. Darien; only the type known. (Named for the collector, Dr. Enrico Festa.

Piabucina festa, BoulexaEr, Bollettino dei MIus. di Zool. ed Anat. Comp. della Lnir. de

Torino, Vol. xIV, No. 346, April 29, 1899, Rio Sabina, Darien. (Coll. Dr. Enrico Festa.)

Page 367. Serriromer beanii has recently been taken by the Ingolf at Stations 12 and 20 , lat. $64^{\circ} 38^{\prime} \mathrm{N}$., long. $32^{\circ} 37^{\prime} \mathrm{W}$., and lat. $58^{\circ} 20^{\prime} \mathrm{N}$. long. $40^{\circ} 48^{\prime} \mathrm{W}$., in Denmark Strait and SSE. of Cape Farewell, in 1,040 and 1,695 fathoms, respectively.

Page 398. After Lycodontis obscuratus (Poey), insert:

656 (a). HYcomoxts Jok1DINI, Evermanu \& Marsh.

Head 7 in total length; depth about 14 ; eye 8 in head; snout 5 ; gape 2.2 ; interorbital a littlo less than snout. Teoth miserial, strong, sharp. not close set, all entire and without basal lobes; tail considerably longer than rest of body; gill-opening smaller than eye; snout rather pointed. lower jaw the shorter, the month capable of being completely closed. Dorsal fin high, much higher than anal; nasal tube long, about 3 in ere.

Color: Tawny-ochraceous, paler below; upper jaw gray; iris blue: longitudinal brown stripes on side of head in front of gill-opening; heal and body covered with mumerons small, round, white spots, those on head smallest; a series of larger ones along upper part of side, and 1 or ? irregular series of large ones on side of belly; botween these on the middle of side the spots are smaller; dorsal with an irregular series of small white spots aloug the base, and another series of about 16 much larger, more quadrate spots of same color along elge of fin, some of the spots cutting the border, which is black; anal similarly spotted and with black 


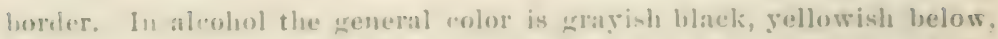
the tawny-uchraceons or sellow lonoming darlier, almost black, and the white sputs on hooly hecoming yellowish.

'Jhis species seems to lor related to L. olsscuratus (l'oey), but differs from it markivly in colox. I'nerto lieo; only the type known, at specinen 15

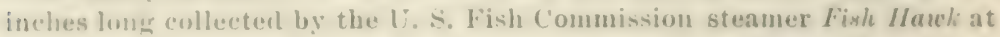
Mayagie\%, January 20), 18!99. (Vamed for Davil Starr Jorian.)

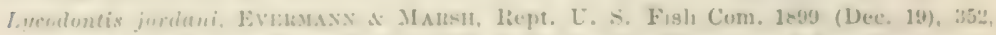
Mayagücz, P. R. (Type, Lil. 4935s, L. S. X. I. Coll. Evernann \& Marsh.)

Page 447. Iftur Ntolephorus productus (P'oes), iasert:

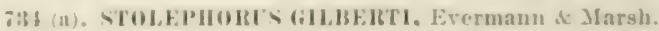

Head 2.25 ; deplth 3.4 ; eye 4 : snont 6 ; maxillary 1.7 ; mandible 1.7 ; inter-

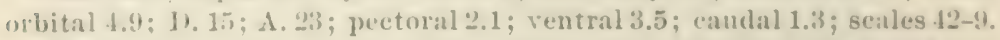

Bonly comparatively (leep) and strungly compressed, the belly trenchant, withont serrations; snout thick, much projecting; maxillars reaching nearly to root of mandible, scarcely serrate; ese moderate; tip of lower jaw realuhug vertical from front of eye; distance from lower posterior amgle of check to vertical from posterior margin of opercle much less than from same point to oye; dorsal inserted far in advance of anal, just behiud insertion of ventrals, midway between anterior edge of oyo and inase of raurlal.

f'olor in spirits: Back light olivaceous with dark punctulations; rest of body below a line from shoulder to upper base of caulal silvery; faint traces of golien behind eve; no lateral band.

This species is very elose to stolephorns garmani, Evermann of Marsh, diftering chicfly in the much smaller eje, tho more miform color of the back, the somewhat moro sharply compressed helly, and tho moro nearly entire maxillary.

Pnerto Rica; only the type, a specimen 4.j inches long, known. This was collected hy the Li. S. Fish Commission expedition to buerto lieu, at Palo Soco, near san Juan, January 13, 1s99, associated with \&. produclus. (Xamed for Dr. Charles Henry (ilbert.)

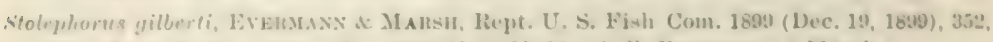
Palo Seco, P. R. ('TYque, Xo). 49359, C. S. X. MI. Cull. Everuanu A Mnrsh.)

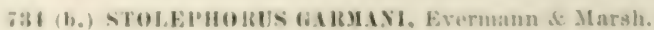

Heat 3.2; llepth 3.3; eyo 3.5; suont is. ; maxillary 1.7 ; mandible 1.7 ; interorbital 5 ; 11.11 ; 1.23 ; pectoral 2 ; ventral 3.5 ; caudal 1.3 ; scales $12-4$.

borly compuratively deop and strongly compressed; the bolly not strongly tronchant, withunt serrulations: snout thiek, mueh projecting; maxillary reaching uearly to root of mandiblo, very tinely and wealy sermate; cyo labge; tip of lower jaw reaching vertical from front of eye; distane from lower posterior angle ot rheek to vertical from posterior mirgin of uperele much lesis than from same point to cye; dorsal inserted fitr in alvance of anal, just buhind insertion of ventrals, midway lotween auterior ealge of pupil and base of camelal. 
Color in spirits: Back dark near the median line, below this somewhat reddish; rest of body below a line from shoulder to upper base of caudal silvery; some golden on snont and behind ese; no lateral band.

This species has a general resemblance to Stolephorus produchs, but is unquestionably distinct from it; the anal is much shorter and inserted farther back, the body is deeper, the eye larger, and the snont longer. It is very close to Stolephorus gilberti, Evermann \& Marsh, difiering eliefly in the larger eve, in the color of the back, and the somewhat less sharply compressed belly.

Puerto Rico; only the type, a specimen 4.5 inches long, known. This was collected by the U. S. Fish Commission expedition to P'uerto Rico at Puerto Real, Jamuary 27, 1899. (Named for Prof: Samuel Garman, of the Museum of Comparative \%oology。)

Stolephoms garmani, EverMaNi \& MARSH, liept. U. S. Fish Com. 1899 (Iec. 19, 1899), 352, Puerto Real, P. R. (Type, No.49360, U. S. N. M. Coll. Evermann \& Marsl.)

Page 497. Before Salmo gairdneri, Richarison, insert the following:

7S0 (k). SALMO CLARKII IECLIVIFIOXS, Jeek.

(SALMON 'IROUT OF LAKE SOUTHERLAND.)

Head 3.8 ; depth 4.6 ; eje 5.14 ; snout 4.5 ; maxillary 1.6 ; scales 148 ; D. 10 ; A. 11 ; branchiostegals 10 ; gill-rakers $7+10$. Body elongate, back elevated, anterior profile much decurved, especially so from napo forward; tip of snout below axis of body; margin of upper lip on a level with lower margin of orbit; gape nearly horizontal, more so than in other trout; maxillary broad, its greatest width 5 in its length, its posterior end reaching beyond eye; dentition strong; posterior margin of dorsal fin straight; tip of second ray roaching mildle of last ray in the depressed fin, tho last ray $2 \frac{1}{3}$ in longest ray; peetoral 1.67 in hearl ventral 2.25 . Color dark blue above and on side to lateral line anteriorly, and to a short distance above lateral line posteriorly, then becoming abruptly silvery; belly nearly white; no spots on head or body, nono on any of the fins, except a fow on candal tin; upper margin of lower jaw black, a dark blue patch on eheek, extending obliquely upward and backward to near top of opercle; pectoral, ventrals, and anal jellowish.

'The general color of this species much resembles that of the Blue IBack of Lake Crescent. It is some darker, has no spots, except on candal fin, and the upper anterior protile is much more curverl.

P'rofessor Elliot gives the following note on this trout:

"This trout is oecasionally taken in Lake Sontherland, and is callec. the 'Salmon 'Trout.' It is easily recoguizal,le, not only by the sharply curved upper outline of the fore part of the body, but also by its quito different style of coloration, resembling, as stated above, somerr hat the style of the Blne Back of Lake Creseent. Is there is no water connection between these lakes, and Lake Sontherland is 75 feet lower than Crescent, and, moreover, the fish of that lake having no communication with the sea, on account of a very high precipitons fall a short distance from its ontlet, it can not be supposed that these two forms are in any way identical. Out Bull. No. t7, pt. 1 - VIII 


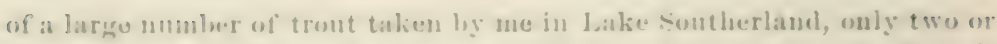
the specimens of this form were poucherl, and they were all of small

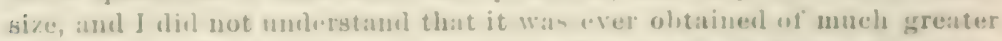
dimensions. 'This could not he the famle of the latke, which is exceedingly

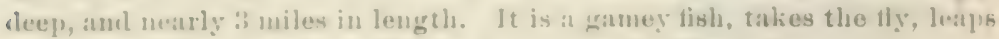
ont of water, and is a good fighter for it sire." (Meek.)

Lengerth alount 10 inclaes.

Known wnly trom Sontherlaul lake, Washington, where 2 or 3 speetmens, only 1 of whiele was preserverl) were taken hy l'rofessor Elliot in 1895. (declirns, steep); frons, forehe:ald.

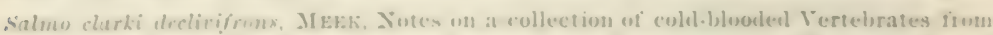

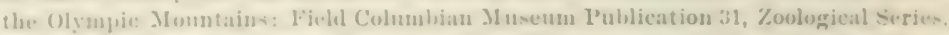

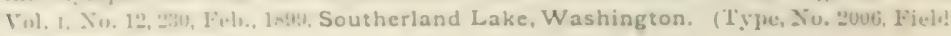
Columbian Muะเum.

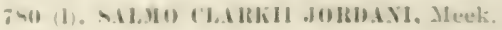

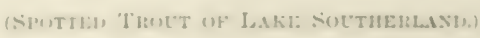

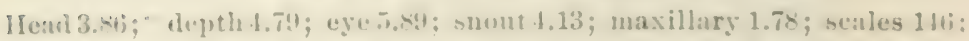
1). 10; 1. 11; lrameliostrinals 10 or 11. Body clongate, not much compressen; heal short, maxillary rather hroad, not extending fur hehind orbit-its greatest wilth $1 \frac{1}{2}$ in its lenesth, in some specimens ahout 5 slinht curvo dnwawarl nuler the oge; dentition not sustrong as in the Spreckled lrout of Ialke C'roseent; gill-rakers ratler loug, longer than in

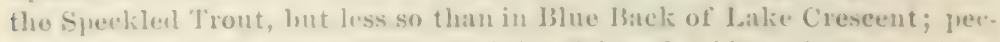
tomal in heal, 1.8x: ventral 2.21; origrin of clorsal miclway luetween tip m suont and hase of caulal, or slightly nearel tip of suont; origin of ventrals under first to third clorsal rays: unargin of dorsal tin convex, it. base 1.32 in longest ray ; snont bluntish. Color in alcohol, dark steel-blue abuve, pater helow, beroming nearly white on the luelly; back, sirles, amb Inat profusely spotted with hlack; some specimems with black sputs on the lielly and on all tius; pecetorals amel ventrals usually without spots: super hall of lower jaw blatek, reel nueler dentary buses. The life rulor: are eriven by l'rofesurer Ellient in the following mote:

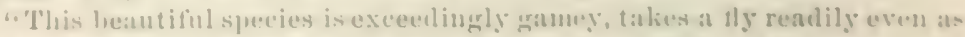

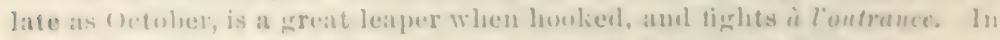

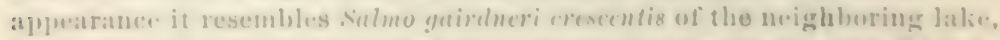

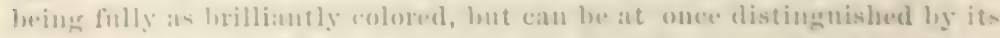

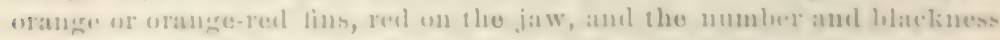

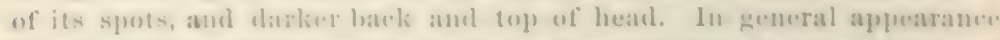
there is 30 the slightest similatity hetweren this speries and the spere

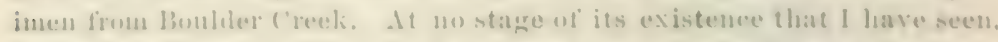

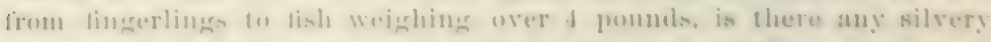

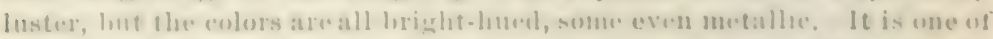

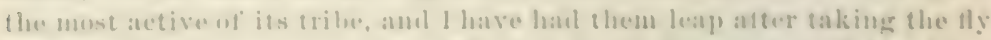

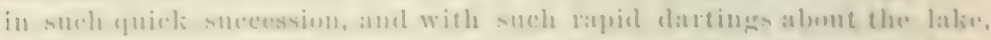

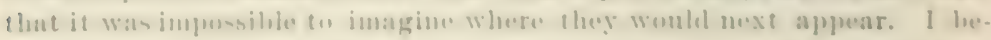

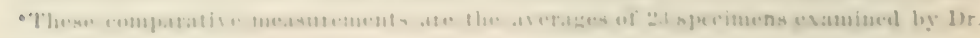
M...h. 
lieve it spawns in the spring, as in the middle of October, when I left Lake Southerland, the eggs of the females we caught were not enlarged, and no indication of the approach of the spawning season." (Meek.)

Length 1 to 2 feet.

Known only from Sontherland Lake, Washington, where 23 specimens were collected in 1898 by Professor Lilliot.

("Named for Dr. D. S. Jordan, president of Stanford Luirersity, who, more than anyone else, has studied our Western tront.") (Meek.)

Salmo clarkii jordani, MEEK, Notes on a collection of cold-blooted Vertebrates from the Olympic Mountains: Field Columbian Mfuseum I'ublication 31, Zoological Series. Vol. I; No. 12, 229, February, 1899, Southerland Lake, Washington. ('IY'e, Nio. 201:. Field Columbian Museum.)

Page 500. Before Salmo irideus, Giblsons, insert the following:

180 (a). SALMO BATHECETOR, Meek.

(LONG-Headed 'Trout of Crescext I.AKE.)

Head 3.5 to 3.8 ; depth 5.10 to 5.75 ; esce 6.75 to 7.60 ; smout 3.33 ; maxillary 1.66 ; scales 150 to 152 ; gill-rakers 7 or $8+11$ to 13 ; liranchiostegals 9 to $11 ; \mathrm{D} .10 ;$ A. 11 . Body elongate, slender; head much pointerl; anterior margin of upper jaw slightly above axis of the body; maxillary very long and very slender, reaching considerably beyond posterior part of the orbit, its greatest wilth 7 in its length; about 24 teeth on maxillary; teeth on jaws, vomer, and palatines large, the dentition much stronger than in specimens of Salmo gairdneri crescentis of same size; mandible very strong; opercle very l,road and contains the eye $1 \frac{1}{2}$; preoperculum narrow, less than diameter of the eye; gill-rakers very short and thick: last ray of dorsal less than half longest rays, third ray louger than hase of fin; when depressed, second and third ray tips reach beyond middle of last dorsal ray; pectoral $1 \frac{8}{4}$ in head; ventrals $2 \frac{1}{2}$. Color much as in $S$. gairdnericrescentis, except lighter. Head, body, and tail profusely spotted with black spots; ventrals and peetorals dark. No. "2036 (Fich Museun) las fewer spots, none on anal and pectoral; anterior part of upper ja wery dark, darker than in the larger specimen. This species dillers from $s$. gairdneri crescentis in being much moro slender, its back much less elovated. head more slender and pointed, gill-rakers shorter, maxillary straighter, narrower, and longer. The general color pattern is the same, except that this species is less spotted and lighter. No red on the under jaw: the dentition is much stronger in this species than in S. gairdnericrescentis. (Meek.)

Prof. D. G. Elliot gires the following interesting note ou this tront:

"This is a deep-water fish, keeping always near the hottom. Lalie" Crescent is of great depth, in some places over 700 feot, and doultless much more in others not yet aseertained. The present species. mnlike other tront, dloes not come to the surface, as I was informed, at any season of the rear, and will not of course take a ily, or iucleed a spoon, or any kind of lure. The onls way it ean be captured is by set-lines sum within a foot of the bottom, and it seems that there are only a few places in the lake where it can be canght even by this means. The specimens 


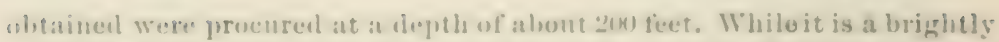

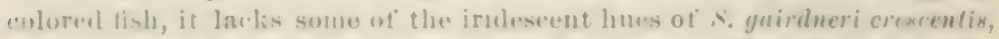
and rouserfuenty is less attrartive in appeadance. It is known as the loner-nuser, or lomp-loendeal erout." (Meek.)

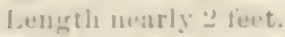

known anly from Crestent laties, Washingron. where two specimens

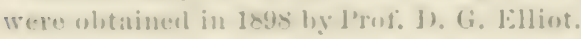

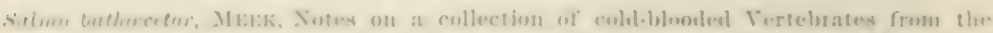

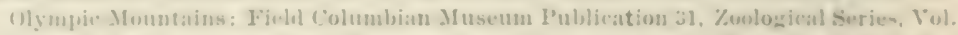

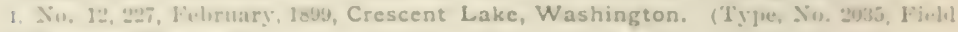
('ulumbiau М Мse.um.)

Page 572. Myclophum gracilis (Liitien) is reported hy I.fitken firmu Ihemmark strat, west ut Icelamol.

Page 583. Betore Lurrellu, (ioode d Bean, insert the following:

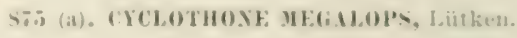

Thesether with a great number of Cyclothome microdun calptuled at station

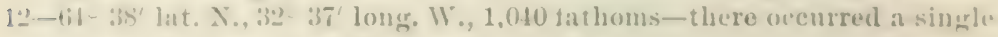
specimen of a length of $70 \mathrm{~mm}$. habitually lobking much liko the said species, but difloriner by tho eyes not heing farticularly small, and by totally wanting tho light glands or "pliotospheres." It can, therofore. apparently. lambly he referred to the same genus. The clorsal and anal

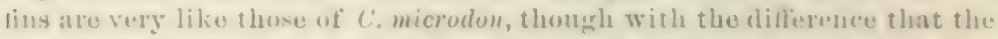
torsal tin begins somewhat hefore the anal fin, while this, on the other hand, emels somewhat farther back than the dorsal tin. (quite linek. A

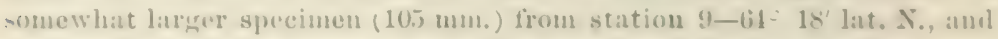

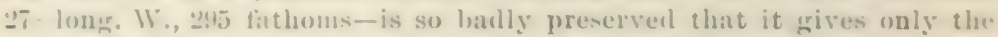
intormation that tho eyes are not small and that both jaws are armed with small teeth directed oblinuely backward, with a few longer unes in the foremest part of the lower jaw and the foremost part of the palate or the intermaxillary. The neater clotermination of this sperimen must le reserven for a finture discovery.

It serems revilent that these specimens helong (n species olse mninown, lut as the material is so scanty I slatll limit myselt to the short prelimimary untes males alsover. (1,iitien。)

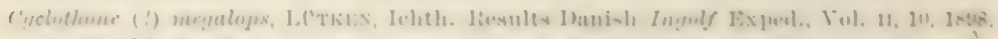
west of Iceland.

Page 617. Mordonuldia rostrulu was taken in 18!5 ly the Ingulf expedition west of lonlanel.

Page 669. Aftre I 'unterulun ruriatus, Bean, insert:

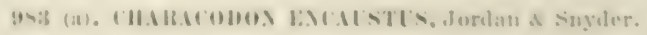

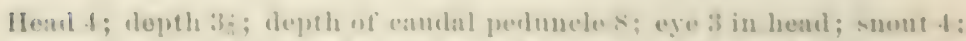

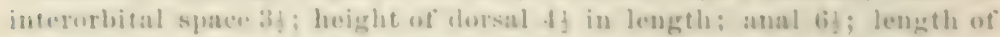

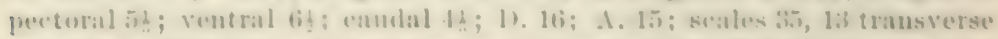

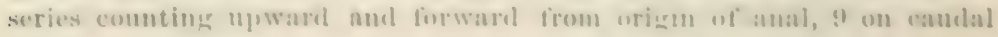


peduncle. Body deep, compressed, dorsal outline almost straight from snout to origin of dorsal, concave from the latter point to base of caudal; ventral outline evenly curved from suout to posterior part of base of anal; eve very large, nearer suout than to posterior edge of opercle by a distance orpal to longitudinal diameter of pupil; month small, its width equal to 2 diameter of pupil; maxillary protractile; lower jaw projecting; teeth in 2 series, the onter series small, bicuspid, in a single rom, rather firmly attacherl; inner series minute, iu small patches; gill-opening restricted, not extending above base of pectoral fin; gill-rakers slender, equal in length to $\frac{1}{2}$ diameter of pupil. Alimentary canal short; air-bladder large, extending posteriorly to a point above origin of anal; dorsal fin inserted halfway between tip of snout and base of caudal, length of base a little less than height of fin; aual inserted below middle of dorsal, its edge rounded; pectoral extending to base of ventrals; ventrals extending to vent. Scales on body large; upper posterior part of head and a narrow space below and posterior to ere with scales; other parts of head naked; small scales on basal part of caudal fin; a row of large pores above eje and at lower edge of suborbital patch of scales; no lateral line. Color in alcohol light, yellowish olive; 9 short and narrow vertical bands on median part of body, the first above base of pectoral, the ninth at hase of caudal; scales on dorsal region of body edgen with black dots; upper part of head dark; npper half of orbit black; opercles silvery; clorsal fin with a little dusky; other fins withont dark color.

This species somewhat resembles $C$. eiseni in appearance. It diflers in having more rays in the dorsal and anal fins, smaller scales, a more compressed body, and less dark color on the body. (Jordan \& Surder.)

Known only from Lagmua do Chapala, near Ocotlan, Jalisco, Mexico. where J. O. Snyder collected the type, a female, No, 6163, L. S. Jr. Unir.

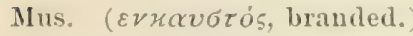

Characodon encaustus, JoRDAx \& Sxyder, 13ull. U. S. Fisl Com, 1899 (1900), 126, Laguna de Chapala, Mexico.

Page 685. After Gooden atripinnis, Jordau, insert:

313 (a). XENENDUM, Jordan \& Snyter.

Tenendum, Jomdax \& Sxyder, Bull. L. S. Fish Com. 1899 (1900), 127 (caliente).

Body deep, not much compressed; males and females of about tho same size. Eye normal; month vertical, lower jaw projectiug; teeth loosely attached, in 2 series, the first series nat, bicuspin, in 2013 rows on each jaw; the second series minute, in villiform bumis, sometimes absent; gill opening not restricted, extending above tho pectoral fin a distanco equal to the diameter of pupil. Alimentary canal loug, with many convolutions; air-bladeler present, large. Seales large; no lateral line. Dorsal and anal inserted posteriorly, the one directly over the other, their bases short; anal very slightly modilied in male, first rașs shorter, edere doubleconvex-the noteh being between smaller and larger groups of rars: ventral fins prosent; candal rounded, aud not molified in male.

Venendum ditiers notably from Characodon in having tho bieuspid teeth loosely attached and in more than one sories. Characolon has the bieuspid 
freth liraly atfached and in a single sures. Its actual aflinites are with

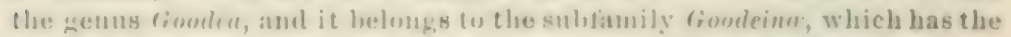
uneneral characters of the l'u cilien, lut with hifureate or trifurcate teath,

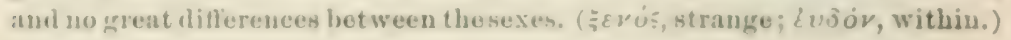

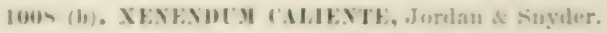

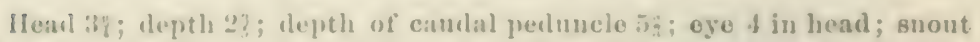
:3 ; interorlital space 23 height of dorsal 5i in length; anal 6f; length of petoral is; ventral 7 ; caudal 5 ; 1). 13; 1.11 ; scales 36 , in transverse eries connting "pward and forward from origin of anal, 14; on candal

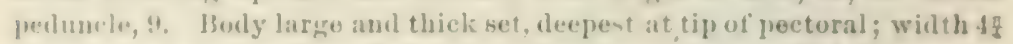
times in lesgtls; learl pointed; interorbital space broal, slightly convex; lesgth of snout about equal to diameter of orbit; mouth vertical, its wirlt enual to length of smont; maxillary verg protractile; teeth looss ly attacherl, in : series, those of the lirst series larger, flat, and noteherl, in "2 rows on 11 plec jaw, 3 rows on lower, the indiviclual teeth of each row alternating in position with those of tho next, those of the second series very minuto, in a villiform hamd; gill-openimg not restricted, extending above the pectoral a ristanco enual to dianeter of pupil ; gill-raliers long, slender, and close togethor, 40 on first arch. Alimentary canal long (in one specimm $1 \frac{1}{2}$ times tlum length of body), coiled many times; perifonem lilack; grnital opening close to base of anal, covered by a thick, notched mal. Dorsal din inserted posteriorly, roumled, the base sloort, its lenglh less than height of tin; anal iuserted under clorsal, dirst 5 rays crowed ingrether and shortened; edge of fin dinble-comvex, the notch beimg between tho shorter and longer sets of rays; pectorals and rentrals with romuled aldes; candal evenly roumled; bouly and head overywhore, rxerpt jaws aml preurlital area, with seales; no lateral line. Colur light. wlive, errowing darlier alove; medium dorsal area blackish, each seale. with a dark, angular hame, thuso of the sixth series helow the dorsal darkel, making an indistinct, narrow lateral bant; all the lins, except. verulrals, dusky.

The temales difler but slightly from the males. The bouly is more thick set, the caublal perlunele a little less deep, the tins a little lower, and the :nal evenly roumbled. The young aro somewhat mottled in color.

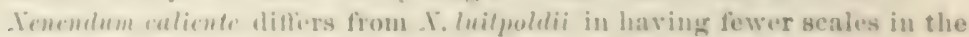
latrral and transverse series and on the caulal pechnele, nud in a similar way from - I. raliscone, besioles having villiform toeth, which aro ulsent in 1. raliseone. (Jurdan ol snyuler.)

kinwn only from kio Vurele, near Agmas Calientes, Mexico. (Type, a

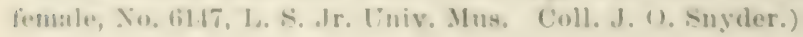

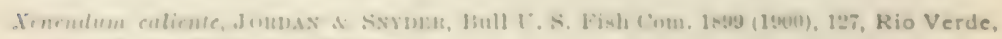
near Aguas Calientes, Mexico.

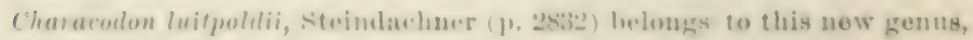
and mlowslil stand nu

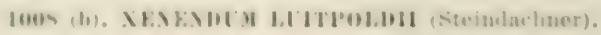


1008 (c). XEXEXDUM XALACONE, Jorian \& Snyter.

Head $4 \frac{2}{3}$; depth $3 \frac{1}{3}$; depth of caudal peduncle $6 \frac{1}{2}$; ege $3 \frac{4}{3}$ in head; snout 3 ; interorbital space 1 ; ; height of dorsal $6 \frac{1}{3}$ in length; anal 9; leugth of pectoral $5 \frac{1}{4}$; vontral $7 \frac{1}{2}$; catudal 5 ; D. 13 ; A. 14; scales 42 , transverse series, counting npwarl and forward from origin of anal, 17; on caudal peclumcle 12. Body thick set, deepest at origin of ventrals, widest at bases of pectorals; caudal peduncle deep and long; head large and pointed; interorbital space broad, slightly convex; mouth vertical, its width equal to length of suout; maxillary very protractile; teeth loosely attachen, broader at distal ends than at bases, bieuspid, in two rows on each jaw ; no villiform teeth present; gill openings extending above base of pectorals a distanco about erfual to diameter of pupil; gill-rakers long, flat, very close together, 56 on first arch. Alimentary canal long, in many folds; peritoneum black. Dorsal fin inserted posteriorly, first ras simple, closely attached to second; edgo of fin rounded; anal inserted on a vertical passing through the base of fourth dorsal ray, similar to dorsal in shape; pectoral and ventral fins rounded; edge of caudal a little convex; basal fourth with scales. Scales large, ererywhere on body and heal, except lower jaw and preorbital area; no lateral line. Color plain, dark alove, light below, the dark color leaving oft rather abruptly on the head aloug a line passing through lower elge of eye; on cho body, along a line passing from lower edge of base of pectoral to candal, loaving lower one-fifth of caudal peduncle light; faint traces of a dark spot at base of each seale on dorsal region of boly; all the fius, except ventrals, dusky. Laguna de Chapala, Mexico.

Ono malo individual was taken. It resembles tho femalo in generil appearance. Tho anal fin is not advanced nor modified into an intromit- tent organ. Nlthough it is injured, it shows that the first 5 or 6 rays wero close torrether aud shortened.

Venendum xaliscone differs from $\mathrm{X}$. caliente in not having villiform teeth, in having morescales in the lateral and transverse sevies, and on the caudal peduncle. It tiffers from $\mathbf{X}$. luitpoldii (Steindachner), which is the third known species of the genus, in having a much longer snout, a moro pointed lead, and in not having villiform teeth. (Jordan di Snjder.)

Knowu only from Laguna de Clapala, neax Ocotlan, Jalisco, Mexico. (Type, a female, No.6148, L. S. Jr. Univ. Mus. Coll.J. O. Suyder.)

(Name from Jalisco, the typo locality.)

Tenendum xaliseme, Jordas \& Sxyoen, Bull. L. S. Fish Com. 1899 (1900), 129, Laguna de Chapala, Mexico.

Page 698. Beforo Mollienisia, Le Suenr, insert:

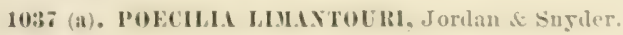

Iread $3 \frac{1}{2}$ in length; depth 3 ; depth of caudal peduncle 43 ; eye $3 \frac{3}{2}$ in head; snout. 3 ; interorbital spaco 2); height of dorsal 5 in length; anal

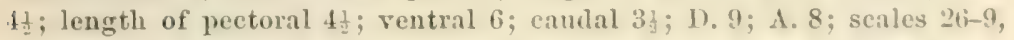
8 on caudal peduncle. Boly rather deep and compressed; dorsal outline angular, its highest point at insertion of dorsal; lowest point of 
ventral ontline at hases of ventrals. Ilead pointed, interurlital spatee will. and flat; ey. large, nearır tip of smout than to posterior enge of operclo l,y a distance epual to dianeter of jujpl; mouth verg obligue: its wilth equal to 2 times diameter of pupil; premaxilaries protractil.; distal end of maxillary visible; lower jaw junjectimg; teeth in two scries on earh jaw, thu outur series in at sinerlo row, small, pointen, locsely attalled; second serius searcely discernible, in bauds; gill-openings extembing aluove base of peetoral a distance equal to diametur of orlst; yill-raliers on first arch 20, suall and sleuder; alisucntars canal very lon: and slemler. Iboly and entire heal, except jreorbital area, lijus, and lower jaw, covered with Jargo scales; 3 rows of sealeon baso of candal; small scales extending on inter-radial membranes of candal, a distance heyoud the basal scales about equal to diameter of the eye. Horsal fin inserted halfway hetween hase of caudal and anterior edge of pupil, its base contaimed $6_{3}$ times in length of head aud bouly, its heinht $5:$, the last rays a little higher than the tirst; anal aulvaced close to haso of ventrals, the first and secoud rays short, closels attached to the next, thirl ray ureatly enlarged and lengthened, a loosely at taclued ovate, Hesly pad uear its tip; fourth and fifth rags blender, as long as third; tijus of thiml aud fifth rays bent toward that of the fourth; sixth to eirhth rays about $₫$ as long as third; caulal rounded, its length contained 3t times in loeal and body; pectoral rounded, tho length contained $1 \frac{1}{\frac{1}{4}}$ times in hual; ventrild pointed, extending to millle of longest anal ray. Color in alcolıol, light yellowish olive, much lighter on breast and ventral part of heal; pusterior exleres of scales dark; lower jaw, jreorlital area, "puper part of heal, and a marrow, median dorsal stripe, dark: basal of af dorsal fin black, distal patt of fiu white, tho hommelars betwern the whito and black more definite on tho anterior than on posforior part of tiu; hasal of of caudal dusliy, distal part without color.

(1)ther male examples have only a fow small dark sputs on dorsal and

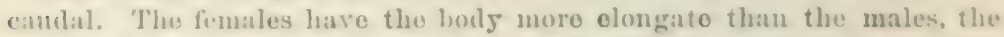
depth of the camblal pedunclo $5 \%$ in length. 'The dorsal tin is inserted in alvance of the anal, its origin above anal opening, the first rays highest. The ventrals extend to the posterior edge of the vent, but do not reach the anal. The dorsal and ramelal have a litelo dusky coloring.

Lase of material for comparison provents our commentiner on the prols.

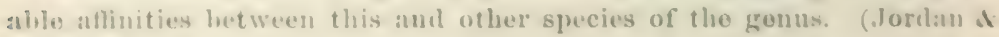
Sinvilor.)

Knmwn mnly tron dio Tamesen, near Tampico, 'Tamanlipas, Mexico,

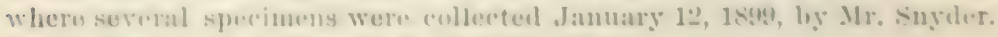

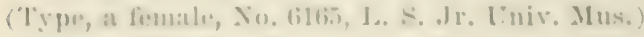

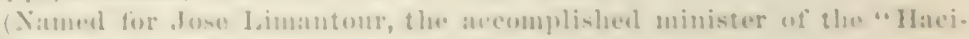

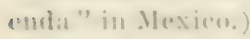

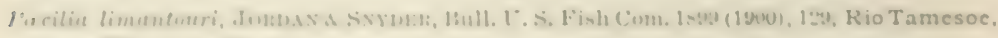
near Tampico, Mexico.

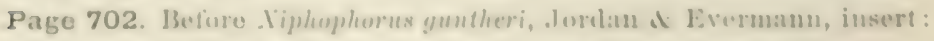

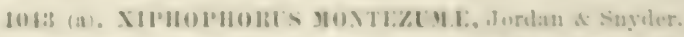

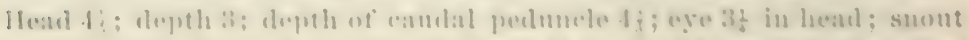

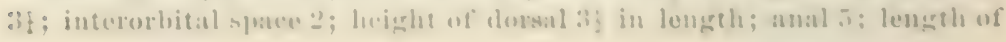


lectoral 4 ; ventral $4 \frac{1}{2}$; upper lajs of candal $3 \frac{1}{3}$, lower rays $1_{11} ; 10.13$; A. 7 ; scales 29-9, 7 on caudal pelnucle. Body deep, compressed, dorsil contour arched, its highest point at insertion of dorsal; caudal peduncle narrow and very deep; head small, pointerl; interorbital space wide, slightly convex; eye large, a little nearer tip of snont than to posterion. edge of opercle; mouth vertical; teeth in two series, tho first in a single row, minute, flat, and pointed, the second in a villiform band, minch smaller and narrower than the first, brownish-colored, strougly curved backward; gill openiug extendiug above base of pectoral a ristance equil to diameter of pupil; gill-rakers on first arch 19, slender, the length of longest equal to half diameter of eje; intestinal tract slender and long; peritoneum black; seales on head and boly large, 1 large, round scale ou interorbital space, followed by 2, a row of 11 from the latter to first dorsal ray; 3 rows ou base of caudal fin; base of dorsal fin short, 4 in bods, first riag shortest, the others graduated to the eighth, which is longest; ninth, tenth, and eleventh shorter; twelfth and thirteenth longer; the abrupt shortening of the ninth, tenth, and elerenth rays makes a notch in the outline of fin; anal alvancer, its origin under thirel ray of dorsal; first ray greatly cnlarged and lengthened; second and third equally lengthened, but more slender, these three with their connecting membranes forming a luif tube with a pointed end; other rays lialf the length of first; upper lobe of caudal rounded, 5 lower rays forming a very loug, blunt appendage; veutrals pointed, extending almost to tip of anal; pectoral sluaply rounded; co'or gellowish olive, marked with black; during life there were 4 narow, longitudinal orange bands, each extending along a row of scales on bodt; top of hearl, and a median dorsal baud extending to candal, dusks; a marrow, dusliy band on edge of lower jaw; 2 short, vertical bands ou snont; 6 upper rows of scales edged with black or dusky; a fow black spots irregularly arranged ou body above ventrals; a large black spot at base of eaudal, its color extending along upper edge of prolongation; a dark line extending along lower edge of caudal pectunele to end of lomer caudal rass, the cauclal extension with a light central portion bolelered with black, the lower border wider; underpart of head and belly without dark color; dorsal fin with black dots and lines; pectorals, ventrals, and anal plain.

Considerable variation in shape of fins and in color is shown amoun other male examples (cotypes, males aul fomales, No. 6i146, L. S. Jr. Univ. Mus.). In some the fius are low and short, the caubla oruament represented ouly bF a slight lengtheniug of tho lower rays. Among incliviluals, appatently fully grown, there is every graluation from the undereloper to the very long candin extension. In every easo the scales aro conspicuously dark edgenl. In sumo examples black spots, crowiled together, form a moro or less dark line from eyo to caulal, whilo belom this lino aro large, irregular black blotehes. Othors have no black spots. aud tho dark caudal patch has almost disappeared.

Tho females havo tho fins low or short, and without special modifica tions, tho posterior edgo of camdal 2-shaped, tho upper part romuled: the lower pointed, tho scales dark edged; a narrow, indefinite, diali colur band usualy present along median line of sieles; tho darli eaudal patel 
raroly absent. Fully grown males ate srarce, a larece cateh cousisting mustly ul femules and young.

Diphuphorus montezmme is distingnisherl from the other known species of tho gemus ly having 7 anal rays, tho seales with conspicuons dark ulges, a large brown caudal spot, aud tho caudal appendago not swordshapeel, but with its emel enlarged ambl blunt. (Jurdan of Snycler.)

Kuown only from lio Verle, near Rascon, San Luis Potusi, Mexico. (Tyle, a male, Jo.61.5, L. S. Jr. Lnir. Mus. Coll. J. O. Snyrler.)

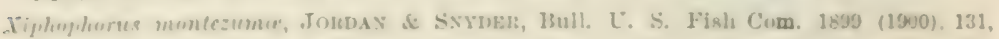
Rio Verde, near Rascon, Mexico.

Page 702. The recent stulies of the eyes of American blind tislses by Dr. C. II. Eigenman have shown that tho species occurring in the caves of soutluwestern Missouri which las usually heen illentifiod as Typhlichthys sulfertumex, (iirarl, but which l)r. Eigronuan described as a new species muler tho namo T!llhlichthy. rosu: (p. 28:35), has had an ancestry quite distinct from that of Ty/lhlichthys. It thereforo is generically distinct from Typhlichthys, and has heen made the type of the new genus Troylichlhys by ligigenmann.

\section{2 (a). TROGLICHTHYS, Figemmann.}

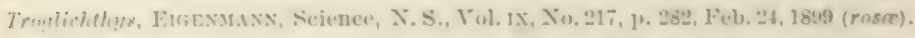

scleral cartilages present, pigment in the pirment epichelium; ritreal atvity obliterated, no lyyaloid membrane; pupil closed, somo of tho eyo muscles revelojed; no onter reticular layer; outer and inner nuclear layrs mererel into one: evo in alult not conuected with the lirain; pigment epithelium developer on the distal faco of the eye, rarely over the siles a:ul back; 110 cones; nuclear layers mero restiges; ganglionic layer restricted to the anterior face of the eyo just within the pigmented

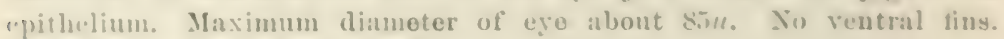

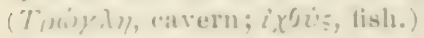

The erenera an l species of Amblyopsicla may be determined by the following ley (from Eigenmann), basul largely on the character of tho eyes:

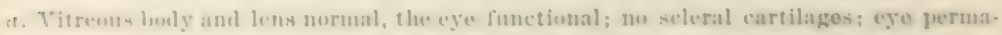

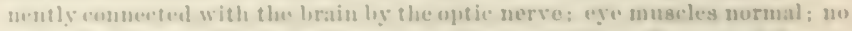

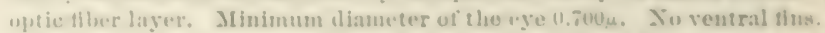

CHot.r.ist:n, 3:1.

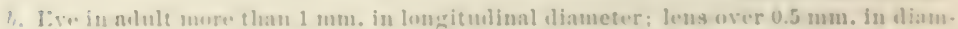
"eter; retina very simple, its maximum thiclisess $83.5 \mu$ in the old; the onter

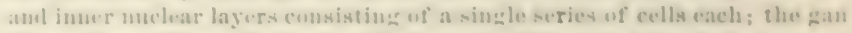

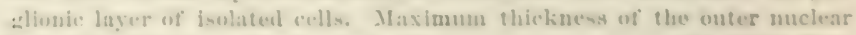

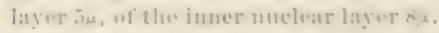

conist'ts, Juts.

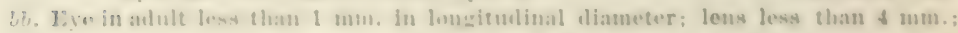

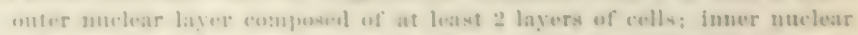

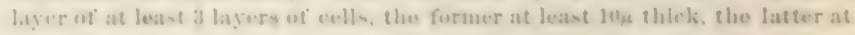
leas losa.

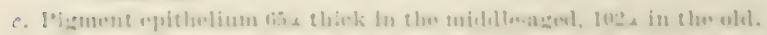

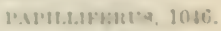

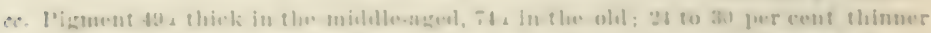

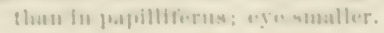
Mis-sizi1, 1045. 
$a a$. The eye a vestige, not functional; vitreous boly and lens mere restiges, the eye collapsed, the inner faces of the retina in contact. Maximum diameter of eye about $200 \mu$.

d. No scleral cartilages, no pigment in the pigment epithelium; a minute vitreal cavity; hyaloid membraue with blood ressels ; pupil not closed; outer nuclear, outer reticular, inner nuclear, inner reticular, ganglionic, aud jigment epithelial layers differentiated; cones probably none; no ese muscles. Jaximum dianeter of eye $180 \mu$ Eye probably connected with brain thronghout life.

'T'TPH.ICHTHY', 32:

dd. Scleral cartilages present; pigment in the pigment epithelium; vitreal cavity obliterated, no hyaloid membrane; pupil closed; some of the eye muscles developed; no outer reticular layer; outer und inuer muelear layers merged into one. Lye in adult not connected with the urain.

e. Pigment epithelium well dereloped; cones well developed; ganglionic cells forming a funnel-shaped mass through the center of the eve; pigmented epithelimm over the front of the eye witheut pigment. Maximum diameter of eve about $y 00 \mu$. Ventral fins present.

AMBLYorsis, 323

ee. Pigment epithelium dereloped over the distal fice of the eye, rarely over the sides and back; no coues; nuclear layers mere restiges; the ganglionic Jayer restricted to the anterior face of the ere, just within tho pigmented epithelium. Maximum diameter of the eve abont $85 \mu$. No ventral fins.

Troglichtixs, 322 (a).

Page 744. To the synonymy of Eucalia inconstans (Kirtland), add the following:

Gasterosteus gymnetes, Dawsox, Canadian Naturalist, Vol. 1V, No. 5, Octoher, 1859, 321. 324, tigs. 1-3, Montreal, Canada. (Coll. I'rof.J. Wr. Dawson.)

Page 789. Recent investigations made by Dr. Jorlan in certain freshwater streams and lakes of Mexico resulted in the discovery of several new species of Atherinida and new facts regarding other species:

\section{5\%. ESLOPSARUM JORIIII (Woolman).}

Locality, Rio Verde, Aguas Calieutes, Mexico.

Two specimens of Eslopsarum jordani were collected, together witl numerous individuals of E. arge. Chirostoma breve, Steindacher, is probably identical with $E$. jordani, as alrealy supposed by us.

An examination of a number of species of Eslopsarum and of Chirostoma shows that the number of vertebre, in addition to the charaster of the scales, furuishes a distinguishing generic feature.

\begin{tabular}{|c|c|}
\hline Species. & Tertebrie. \\
\hline 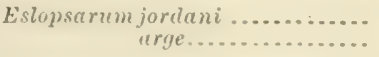 & $\begin{array}{l}38 \\
38\end{array}$ \\
\hline 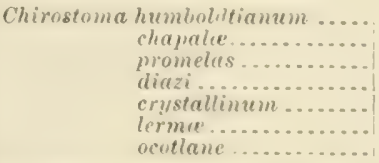 & $\begin{array}{l}44 \\
45 \\
45 \\
14 \\
44 \\
14 \\
44\end{array}$ \\
\hline
\end{tabular}

Chirostoma contains two very marked types of species. The one represented by the typical species, Chirostoma humboldtianum, lats the flesh firm 
and opanue in lite. "The other (Lethostele', typutiell by thirontoma extor, has the thesh this, transluent, and very pale. There are eorrelated differences in the limmess of the bunes and scales, hut thus far wo have foumb un tangihle character on which to separate Lethostole as a genus from Chirostoma. The kuown specius of lechostole are estor, alhum, chapale, granducule, promelas, diazi, crystallinum, lerme, and ocollane.

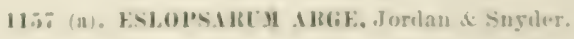

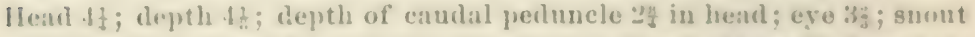

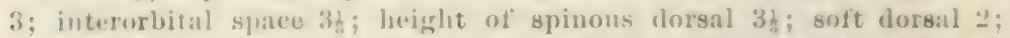
anal 2; longth of pretoral $1 \frac{1}{2}$; ventrals $2 \frac{1}{2}$; caudal $1 \frac{1}{3} ; 1$. $11-8 ; 1.15$; 1. 13: senles f0-11, 5 between the dorsals. Body rather thiekset, its leepest fiart just anterior to base of rentrals; width of body equal to distance from posterion elge of orhit to tip of snont; eye nearer to tip of snont than to posterior odge of operele hy a distance eymal to dianeter of pupil ; interorbital space convex; width of preorlital area equal to diamoter of pupil; tip of lower jaw jrojecting beyond that of upper; month Iarge, oblique; lips not much thickened posteriorly, the luwer not distim.1 fo folded over the mpuer at their angle; maxillary extending posteriorly to a perpendienlar passing through anterior eolge of orbit, its olistal oul below the level of eye; teeth large, sharp, projecting backwards, in 2ulufinite rows on each jaw, none on vomer or palatiues; gill-rakers on. list arch 11, long and slenker; air-bladder extendiug posteriorly to a puint a little past insertion of anal; jeritomenm black; vertebra 37. Lateral line represented on the fifth row of scales below the dorsal by a meries of partly developed pores; scales larere, entire, covering head and bunly except snout, lower jaw, preorbital area, amd a small space around base of pectoral; small seales extending for a short distance on inter. ralial membrames of candal; first 3 dorsal spines of ahout the same lu.jelit, the fourth shurter; first dorsal ray lomgest, the others gramally shorfer; edgo of lin straight; anal inserted on a perpendicular, passing

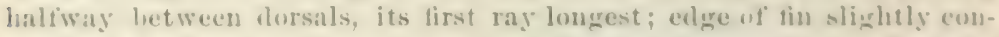

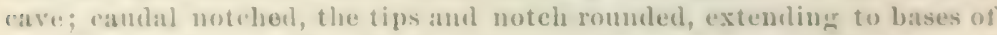
ventrals; ventrals falliug shurt of vent a distance exmal to diameter of

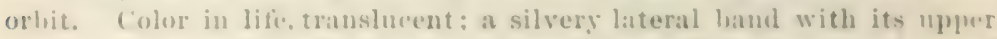
culge dark, extending from upper part of base of pectoral to luase of eamulal, the hamb less distinet in the region of the pretoral fin; scales we lack edged with line, dark specks; suout, lower jaw, top of hear, and upper part of eye dusliy: dorsal and caublal tius with a little dusliy (r)lorings.

Fiperimens of $\mathrm{E}$, arge were anght in the same seine-hanl with $\mathrm{E}$. jorlani. The former species difless from the latter in having a thicker boly, a longer snomt, a larerer and less ublipues momsh, a larerer eyo, and a wider (relor liand.

In the drawing aceompanying the original oleseription of Eslogsarmm jordani the montle in wrongly represented. (ne the sperimens oxamined.

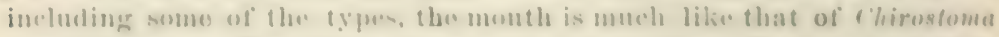

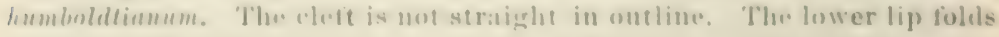

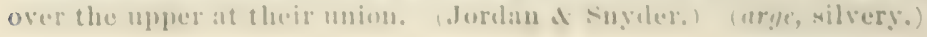


Known only from the Rio Verde, near Aguas Calientes, Mexico. (Type, No. 6154, L. S. Jr. Univ. Mus. Coll.J. O. Suyder.)

Eslopsarum arge, Jordar s. Sxyder, Bull. U. S. Fish Com. 1899 (1900), 133, Rio Verde, near Aguas Calientes, Mexico.

\section{7 (b). CHIROSTOMA CH.AP.L. Jortan st Snyler.}

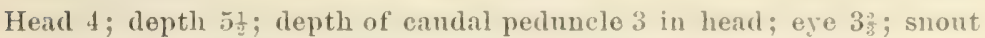
$3 \frac{1}{;}$; interorbital space 4 ; height of spinous dorsal 3; soft dorsal 13; anal $1 \frac{3}{5}$; leugth of pectoral $1 \frac{1}{4}$; ventrals $2 \frac{1}{5}$; cautal $?^{y} ;$ D. IV-10; A. 20; pectoral 14 ; seales $47-13,6$ between dorsals. Body slender, compresserl, its deepest part below first dorsal; ere large, nearer tip of snont than to posterior edge of opercle by a distanco equal to $1 \frac{1}{2}$ times the diameter of pupil; interorbital space convex, its width about equal to diameter of pupil or to preorlital area; symphysis of lower jaw projecting a little beyond tip of upper, the lips meeting; month oblinue, lips thickened posteriorly, the lower folding over tho upper at their union; angle of month on a level with center of pupil; maxillary nearly vertical, its distal end in adrance of a vertical from anterior edge of orbit, it distance equal to $\frac{2}{3}$ the diameter of pupil. 'Teeth minute, in bauds, not arranged in definite rows; no teeth on vomer or palatines; gill-rakers on first arch 30 , very slender, the length of longest equal to diameter of orbit; pexitoneum black; air-bladkler very large, extending posteriorly to a point above middle of anal fin; vertehra 45. A well-defined lateral line extendiug aloug the body on the eighth row of scales below the first lorsal. Scales large, crenate, not notahly reduced in sizo nor closely crowled together on any part of the borly; those on post-oceipital region and auterior to pectorals smali; scales extending on hasal of inter-radial membranes of candal; lower jaw, snout, and preorbital space naked: first 2 spines of tlorsal higbest, the following 2 a little shorter; first rily of second ilorsal highest, others gradually shorter; anal inserterl a little anterior to a perpendicular passing halfway between the origins of dorsals, first ray longest, others snccessively shorter; when the fin is elevated, its edge is concave; eaulal deeply forlied, the tips pointed; pectoral notably pointed, extending past base of ventral a distanco equal to diameter of orbit; ventrals oxtending to vent. Color in life, translncont; a silvery lateral band 1 seale wide, bright, and distinct posteriorly, becoming indistinct anteriorly; upper edge of lateral band with dusli: scales on dorsal part of body edged with dark dots; upper and lower jaws with dark dots; uplee part of eye black, the dark, pigmenter arachnoid showing through the thin skull. (Jorkan d Snycler.)

C. chapale is closely related to $C$. grandocule. Steindachner. It difters in haviug a smalier eye ambl larger scales. The former has 44 to 51 scales in tho lateral series, and 12 to 14 in a trausverge series, while $C$ grandocule has 60 to 62 scales in tho lateral series and 15 or 16 in a transverse series.

Ǩnown only from Laguma do Chapala, near Ucotlan, Jalisco, Mexico. (Type, No.6165, L.S. Jr. Unir. Mus. Coll.J. O. Suýder.)

Chirostoma chapalae, JoRdAx A Sxrder, Bull. U. S. Fish (Com. 1899 (1900), 135, Laguna de Chapala, Mexico. 


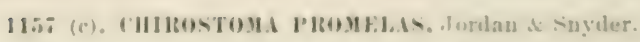

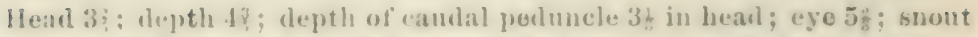
21 ; interorbital space $4 \frac{1}{3}$; loreight of spiums dorsal 33 ; solt dorsal 23 ; aual 2 ; lengtlo of pectoral 1 ? ; ventral 21 ; cauclal $1 \frac{1}{2} ; 1$. IV -11 ; A. 19; pectural 15: scales 5ij-16.9 hetween dorsals. Heal slender, triangular; eyo small, mearer tip of suont than to enge of opercle ly a distance equal to hali diamoter of pupil: width of jreorbital area somewhat greater than diameter of pupil; interorbital space slighty convex: suont pointerl: npler jitw projecting a little besond the lower; cleft of month almost horizontal; li js enlarged posteriorly, tho lower lip folding over the upper at their junction; angle of month on a level with lower part of pupil; maxillary almost vertionl in position, its distal end not extenuling laukwarl as far as auterior enlge of orbit; teeth large, curvel inward, not arrangel in detinite rows; nome on vomer or palatimes; vertebra 45 . A tol'rab]y well-definorl lateral line extemeling along the hody about 8 scales below the first iorsal, the pores alssent on some of the seales; scales cremate, largest alongr lateral culor baud; a post-occipital pateh of uiunte, clusely-rowled scales extending backward nearly to a line connecting hases of pectorals; a marrow baul of similar scales just posterior to gillopenings, and on base of caudal, the latter extending on inter-radial membranes half their length; scales letweeu dorsals not abruptly sualler than thoso near hy, nor croweled closely together; head with scales except on snout, preorbital region, and on lower jaw; first 3 spines of dorsal nearly equal in length, the fouxth a little shorter; first dorsal ray lougest, tho others gralually shortex: anal inserted on a perpeudicular passiug milway butween dorsals, first ray longest, others successively shorter: edge of tin slightly concave; pectoral pointed; extending beyoud base of ventrals a digtance equal to diameter of pupil ; ventrals not quito reaching rent. Color in alcohol, yellowish olive; a distinct silvery lateral bamb. the light color of which is mulerlad with dark pigment, extending from npper pate of hase of jectoral to cauclal, wider and hrighter in color between dorsil and anal, growiug narrower on camblal pedumele, widlening at its end: seales uf upper part of bokly with alusky coloring on their edere; clorsals, peetoral, and candal with dark color: eye dusky abure. a dark band on interorbital space; smout and jaws black.

rhirostuma momelus is distinguishable from other known speries of the genus hy the projecting uppos jat and the black snout.

One specimen other than the type was olstaned. In it the projection of the upper faw is mure promouncel than in the type. The gill-raliers amd ablominal visera hat heen removed from huth. (Jordan of suyler.)

Kuown only from Cinalalajara, Jaliseo, Mexico, where 2n specinens were

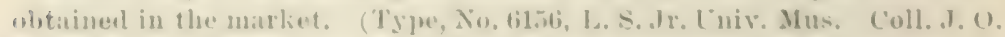

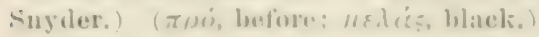

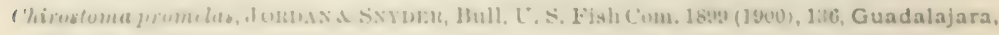
Mexico. 
1157 (d). CHIRostoma DIAZI, Jordan \& Suyler.

Head $3 \frac{1}{3}$; depth 5 ; depth of caudal peduncle $3 \frac{1}{1}$ in hear ; eye $5 \frac{1}{3}$; snout $2 \frac{1}{2}$; interorbital space $1 \frac{1}{2}$; height of spinous dorsal $\frac{12}{3}$; soft dorsal $2 \frac{1}{2}$; anal $2 \frac{2}{3}$; length of pectoral $1 \frac{3}{5}$; ventral 3 ; caudal $1 \frac{2}{3} ;$ D. V-11;A. 20; P. 15; scales 69-20, 22 between dorsals. Body long, deeper and more compressed than that of $C$. humboldtianum; head large, its dorsal contour straight from tip of snout to occiput; viewed from above, the head is much compressed, the upper jaw is sharply pointed and included by the lower; interorbital space slightly convex; eye nearer tip of snout than to edge of operele by a distance equal to diameter of pupil; preorbital area a little wider thau diameter of orbit; month oblique, its cleft extending to a horizontal from lower edge of orbit; lips thickened posteriorls, the lower forming a fold across the upper at angle of mouth; lower jaw projecting, the teeth just passing the elge of the upper; maxillary extendiny to a vertical from anterior elgo of orbit, its distal end augular; teeth large anteriorly, growing gradually smaller posteriorly, canine-like, sharp, projecting backward, not arranged in definite rows, mone on vomer or palatines; vertebre 44. Scales crenate, larger in region of lateral color band, growing smaller dorsally and ventrally, abruptly smaller aud closely crowded together in a region anterior to the pectoral fin, extendiug from the isthmus to the occiput, also between the dorsal fins and aloug bases of dorsals, anal and caudal; inter-radial membranes of the latter with scales extenting $\frac{2}{3}$ its length; head with scales except on jaws, upper part of snout, and on preorbital area; two anterior spines of lorsal longest, others a little shorter; first dorsal ras lougest, others gradually shorter; auil similar to soft dorsal in shape, except that its base is much longer; insertion of anal on a perpendicular passing halfwaly between dorsals; caudal deeply forked, the lobes pointed; pectoral sharp, extending past base of ventrals a distance equal to diameter of orbit; edge of pectoral, when oxtended, straight. Bocly, duxing life, trauslucent; in alcohol, greonish olive; a silvery lateral band, in which the silvor is not underlaid with black pigment, extending from axil to base of caudal; dorsal seales narrowly edged with dusky; upper part of eyo dark. Jaliseo, Mexico.

Our specimens are all from the market at Guadalajara. The gill-arehes and viscora had been romoved. The flesh is somewhit shrunken, which probably causes the teeth to appear more prominent than in life.

Chirostoma dia $i$ may be easily distinguished from the other known species of the gemus by the small seales crowled closely tomether between the dorsal fins. (Jordan of Snyder.)

Known only from the markot at Guadalajara, Jalisco, Mexico. (Trpe, No. 6157, L. S. Jr. Univ. Mus. . Coll. J. O. Snyder.)

(Named for Porfirio Diaz, the honored I'resident of the Republic of Mexico, in recognition of his interest in the progress of seience.)

Chirostoma cliazi, Jondas s Sxyder, Bull. U. S. Fisl Com. 1899 (1900), 137, Guadalajara, Mexico. 


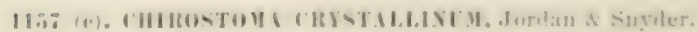

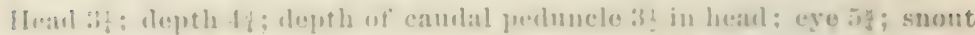

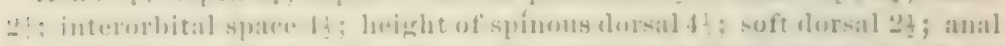

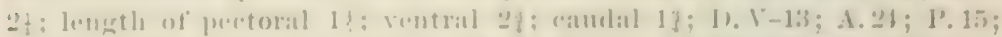

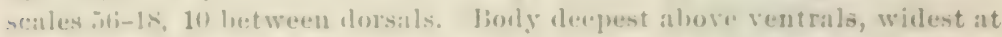
insertion of peretorals; leaul long and pointwel, its upper contour straight; mont, viowell from above, sharply pointed, the lower jaw projecting lacenul upper at distance enual to not more than half dianeter of orbit; urre nearer tip of snout than to justerior "dige of opercle a distance refual to dianeter of pupil; month oblique, the clest extending downwarl to al level with lower erlge of pupil; lips thickened posteriorly, the lower folding over the upper at angle of month: teeth minte, in wide fatthes, not arranged in definite rows, on mpper and lower jaws; no teeed un voner or palatines: grill-ratiers on lirst arch 27 , slender, the length of longest equal to diameter of pupil: vertebra H. An indefinite lateral line extending along tho hody abont ! scales below tirst clorsal, the pores absent on many of the seales; seales crenate, largest along color-band, urowing smaller ahove and below, murh smaller and closely crowderl on the holy anterior to the pectoral tin, from the isthmus to the oceiput; a fow small scales at mpler emil of opercle, along hases of fins, and on the inter-radial memhranes of callal for half its lugth; seales between dorsals latre and not crowided togrether; lower jaw and npper part of inont nalkel; first 3 spines of dorsal abont the sume length, raching. when depresse:l, to th their leneth of insertion of suft dorsal ; first dersal ray longest, others gratually shorter; anal inserted on al perpendicular passingr ilurough a point half way between origrins of dorsals, base much longer than that of dursal; first dorsal ray longest, of hers gradnally shorter to midhle of tin, all remaining rays of ahont the same height; eaudal deeply forked, the tipi rounded; pectoral pointed, extending a littlo past hase of ventrals; tipes of wentrals extending to vent. Borly, during life, translueent, wifh a slightly bluish tinge: in alcohol, yellowish; upper sontes with dusky edges; top of head dusky; upper part of eve dark: tins, exent ventrals and anal, with a little dusky color; a silvery eolor hand exteuding from upper part of pectoral base to cambal, narrower on caudal, wideniug at bates of caublal.

C. erystallinum closely remsembles C.omllane in general appearance. The much shorter lower fitw and the smaller eyo of $C^{\prime}$. cryshollinum are at once distinguishing characters, (inadalajara aud Laguna do Chapala, Mexico. (. doralan of sinyeler.

Two speetimens from the (inalahạara market are shrunken so that the orbit is larger, and the teeth more evident than in those taken at ceotlan.

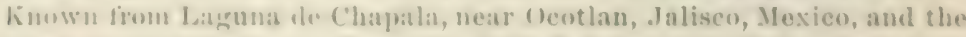

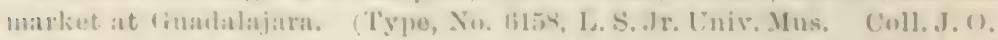
Sinyiler.)

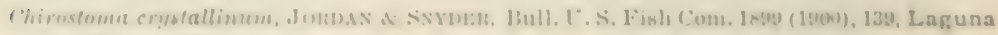
de Chapala, Mexico. 
1157 (f). ('IIROSTOHA IERH.E, Jordan \& sinyler.

Head 3 ; ; depth $5 \frac{1}{3}$; depth of caudal pertunclo $3 \frac{3}{3}$ in head; eyo $5 \frac{\pi}{2}$; snont $2 \%$ interorbital space $5 \frac{1}{2}$; height of spinous dorsal $4 \frac{1}{2}$; soft dorsal $2 \frac{1}{2}$; anal $2 \frac{1}{1}$; length of pectoral 1 ; ventrals 3 ; candal $13 ; 1.1 \mathrm{~V}-11 ; 1.20$; scales $58-20,11$ between dorsals. Body slender, deepest part in region of ventrals; caudal peduncle narrow; snout long and pointed; lower jaw slightly projecting, but not enough to include the upper; eye largo, nearer tip of snout than to posterior orgo of operclé, a distance equal to diameter of orbit, or to width of preorbital space; cleft of mouth extending to a liorizontal throngh lower erlgo of orbit; lower lip folded over the upper at their union; maxillary extending posteriorly almost to a perpendicular from anterior edge of orbit, its distal end angular; teeth large and strong, curved backward and inward, arranged in 2 detinite rows, those of the inner row of the upper jaw and of the outer row of the lower jaw larger, none on vomer or palatines; vertebra 44. An indefinite lateral line extending along bolly abont 10 scales belor first dorsal, the pores alsent ou many of the scales; scales crenate, largest along the lateral color-band, much smaller between occiput and first dorsal, those immediately posterior to oceiput, minute and very closely crowded; a narrow edging of similar seales along tho gill-openings, extending ventrally to the isthmus; scales between dorsals not much rednced in sizo or crowded together; basal half of inter-radial membranes of caudal with scales; first 2 spines of clorsal longest, third shorter, fourth about $\frac{\circ}{3}$ as long as tho first; first dorsal ray longest, others gradually shorter, colge of fin straight; insertion of anal on a perpeudicular passing through a point half way between origins of clorsals, first ray longest, others successively shorter, last ray one-third the leugth of first; caudal deeply forked, its tips rather pointed; pectoral pointed, extending beyond origin of ventrals a distance erpual to diameter of pupil; ventrals reaching vont. Color in aleohol, light olise, a silvery lateral band equal to a scale in width extendiug from upper part of base of pectoral to base of caudal; edges of upper scales dusliy; a little dark color on dorsals, candal, and pectoral; npper and lower jaws and top of head with minute dark dots; upper part of eye dark.

$C$. lerma closely resembles $C$. crystallinum. It difters markerily in having large teeth, which aro arrangod in two rows, a shorter lower jaw and a larger eye. Jalisco, Mexico. (Jordan of Snycler.)

Our specimens of $C$. lerme are all from the market of Gualalajara. The gill-arehes and viscera had been removed and the bodies were somewhat shrunlien. The specimens are sail to hare como from Laguma de Chapala, Jaliseo, Mexico. ('Type, No.6159, L.S. Jr. Univ. Mus. Coll. J. O. Snyder.)

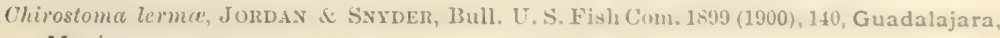
Mexico.

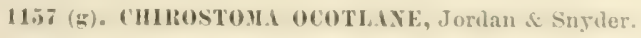

Hear $3 \frac{1}{3}$; depth $4 \frac{1}{2}$; depth of caudal pectunclo $3 \frac{1}{3}$ in heal ; oye $1 \frac{1}{2}$ : snont 23; interorbital space 5 ; height of spinous dorsal $3 \frac{1}{2}$; soft dorsal $2 \frac{1}{1}$; aual 2 ; length of pectoral $1 \frac{3}{5}$; ventral 21; caudal 11; 1). V-12; A. 20; scales $51-19,11$ between dorsals. Body long, rather slender, deepest above Bull. No. 47, pt. $1-1 x$ 
ventrals; Luad long, puinted, its dorsal contour straight from tip of

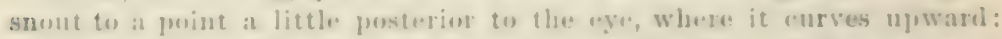

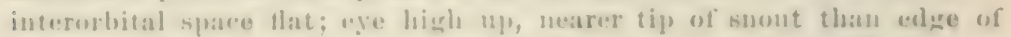

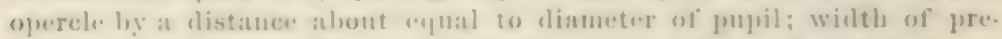

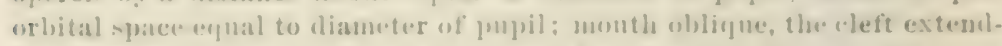
ing lownward to a point opposise lowererlge of pupil; lips growing more

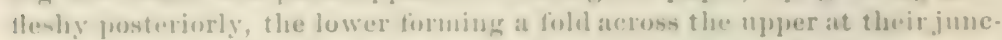
tion: distal cul of maxillary aurnlar, extendiug almost tu a vertical from anterior enlye of orlit; lower jaw very loug, frojectims luegond upper a distance repual to diambler of jupil; viewed from alove, both jaws are a little more pointed than are those of $E$ estor or of $r$. humboldtianum. Teeth an jaws in bands, minnte, projecting backward: no teeth on voner or julatines; gill-raliers sleneler, close together, length of longest enual to diameter of jupil: vertobra 11. I rather indefinite lateral line extemuling along boily, 10 sales helow ilu first dorsal, the pores alosent on many of theseales: seales crenate, growing sualler clorsally, larger ventrally, thuse on posterios part of oreiput, on sape, on region anterior to peceforals-alove and helow, and on hase of caudal, very small aud croweled together, those in the region of dorsals not reduced in size or crowderi exwet at insertion of fins, where there are 1 very small ones; cliceli with rows: lower jaw and แpper part of suont naked; lasal half of intelratial menloranes of caudal. with minute seales; tirst 3 spines of dursal highest, extmuling when depressed within halt their length of origin of soft dorsal; first dorsal ray preceded ly a slorter, simple, closely attached one; other rays sucessively shorter than tirst; edge of fin slightly coneavo; anal similar to soft dorsal in shape, its basis 1 times as long as that of suft dorsal; atfachuent of first ray umler a point half way between

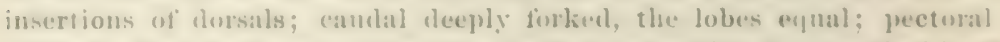
pointesl when depresisel, mplur rays longest, extendiug to a vertical half way lostwen insertious of lirst dorsal and ventrals. Body, during life. aluost transluent, with a hluish timge of color: in alcolonl, the eolor is a light wlive yollow: a silvery lateral bamb extending from axil to loase uf randal, the luand wider and brighter in color letween dorsal and anal,

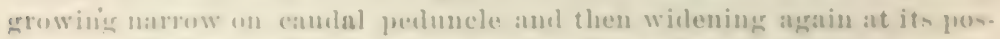

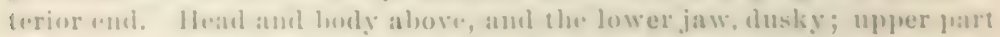
of eye elarli: enlere uf seales alove lateral hamel with suall black dofs: raudal somewlat duskyon its hasal thire: other lins with littles or mo dark rolor.

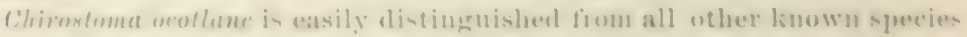

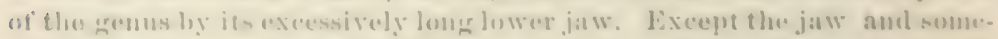

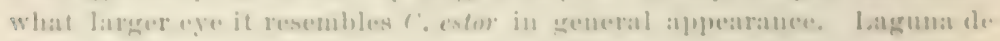

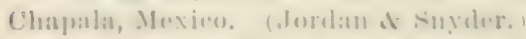

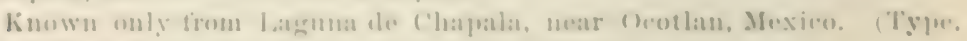

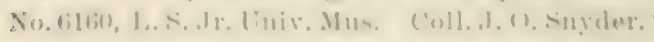

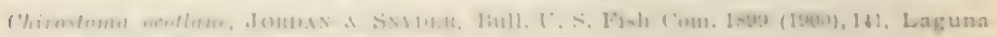
de Chugnala, near Ocollan, Mexico. 
1154. CHIROSTOMA ESTOIR, Jordan.

We have examined the type specimen of Chirostoma cstor. The scales are small and closely crowded on the region anterior to the pectoral from the occiput to the isthmus; they are not much reduced in size nor closely crowded together between the dorsals. The teeth on the jaws are large and numerous, not arranged in definite rows; there are 3 vomerine teeth almost as large as those on the jaws.

Some exact measurements of the typo, expressed in hundredths of the length of the body, are here given:

Length of body in millimeters 212 ; depth of candal peduncle 7 ; lengtl of head $31 \frac{1}{2}$; distance from snout to oceiput 23 ; tip of lower jaw to oceiput 24 ; width of interorbital space 7 ; length of snout 12 ; diameter of orbit 5 ; distance from snont to spinous dorsal $56 \frac{1}{2}$; insertion of spinous to soft

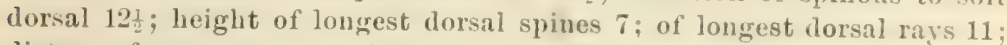
distance from suont to anal $62 \frac{1}{2}$; height of longest anal rays 12; distance from anal to candal 22 ; length of eaudal fin $18 \frac{1}{2}$; of pectoral fin 17 ; distance from snout to ventral $45 \frac{2}{2}$; length of ventral fin $10 \frac{1}{2} ; \mathrm{D}, \mathrm{V}-12: \mathrm{A} .18$; P. 14; scales 72-19, 9 between dorsals.

\section{5t (a). CHmostomi AIBUM (Steintachner).}

The type of Chirostoma estor agrees externally almost perfectly with Steindachner's account of Chirostoma album from Lake Pátzcuaro, a species which he later places in the synonyuy of $C$. estor. But the type localities are widely separated, and Steindachner found no trace of the large vomerine teeth so conspicuous in $C$. estor. We therefore regaril $C$. album as probably a valid species, not identical with $C$. esior.

Page 821. After Joturus pichardi, Poey, add:

$$
368 \text { (a). NEOMUGIL, Vaillant. }
$$

Neomugil, Varluaxt, IBull. Soc. Philon., Iv, 1893-1894 (June, 1894), 72 (dimueti).

General aspect of Mugil. Month witely cleft, maxillary extending beyoud anterior edge of orbit. Fine teeth in many series in each jatw, those of outer series on intermaxillary most developed, all conical, simple, small, but distinct; villiform teeth on vomer and palatines. Stomach siphonal, walls membranons, very thin in pyloric region, hence not gizzarellike; a large pyloric cuca and a second smaller oue; psendobranchia distinet.

This genus resembles Joturus, which has the teoth tlattenerl and serrated, with only 1 series on the intermaxillary. The maxillary in foturns extends not so fir back. (Vaillant.) (v'os, new; Mugil.)

\section{5 (a), XEOHUGI, MIfIETI. Vaillant.}

Head 3 ; depth 4; eye 6; interorhital 2.75. 13.6; I). IV-I, s; A. I, 9; V. I, 5; seales $3-13-11$; candal tin 4. Maxillary reaching pupil; mouth deeply eleft; npler jaw the longer; no aclipose eyelicl. Origin of first dorsal at middle of body, the first spine"2 in dopth of body ; origin of anal 
a little in front of secomil dorsal. Whole heal, excejt preocular region. sealed lilie the houly, the sealles ctenoinl.

known from 20 ) examples from 99 to $19 !$ mm. long, from Lower California, south of' Lit l'ak.

(Xanded tor the collectur of the igle, M. Lion Diguet.)

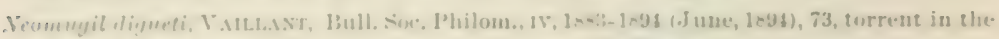
Sierra de las Cacachilas de Santa Cruz, Lower California.

Page 903. In the description of . Seriolu lulandi for “. A. I, 27" real "1. 1, 21."

Page 962. Centrolophidu can not be maintained as a distince family. It belongs with the Stromateidu.

Page 1038. Afur Iludroplerus scierus servulu, insert:

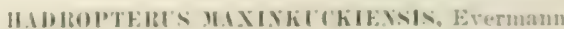

Hearl 3.75: depth 6; eye 1 ; snout 4.2; maxillary 3.25; mandible 2.75: inferorlutal fi; pectoral 1.25; ventral 1.3 ; I. XIV, 13; A. II, 8: scales 7-til- 111 ,

Body ratler long, slemler, and subterete; candal pealuncle somewhint compressud, its least wirlth one-half its least depth: head rather long, suout pointenl; mouth molerately large, oblique, maxillary reaching past anterior part of eye, lower jaw included; eyo ratler large, slightly above axis of holy; interorbital moderately wide, nearly tlat; gill-membranes free from each other and from the isthmus; operelo with a rather long flap and stunt spines; jremaxillaries not protractile; fius rather large: clistance from orimin of spinous clorsal to tip of snout slightly greater than hase of spinuts dorsal or nearly twice base of soft dorsal; longest alorsal spine 2.25 in head; soft dorsal higher than spinous portion, 22 in head, the freve velge gently arved; arigin of anal under that of soft dorsal, its base 2.1 in liead: eamdal rounded or slightly emarginate.

Sules firm and strongly ctenoid, lateral line complete and straight, luemimnime oreg operenlar spine; top of heal and an olılong area on nape nalivel; space in front of spiumus dorsal with small emberlded scales; operolo with alunt 7 rowg of seales; eheek with a few small embedrled seales.

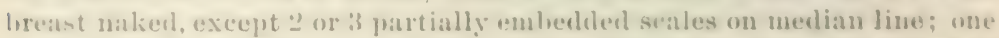
latren seales leetween ventrals: belly nalied antoriosly, hut with alout 10 enlareret, stellate seales posteriorly; space leetwen ventrals hroal, equal to widele of lrise of ventral: preoperele smouth.

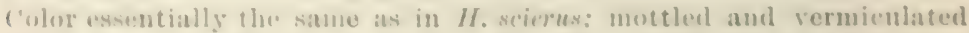
with light amb rlark hrown or blackish, the midelle line of lack with alume ! lature, roumblish, dark, emolluent areas, each surrounded by a wasy, whitish lime; misble of sisle with about 7 larere, conduent dark sputs, the anteriur 2 lareest and longess, the thirel small, the fonrth large. and the rematuing 3 progressively sualler: mmler parts yellowish white:

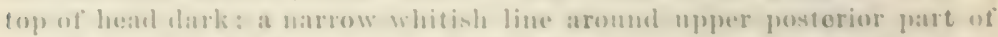

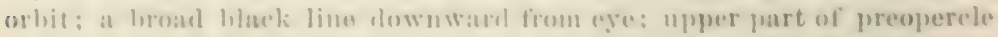

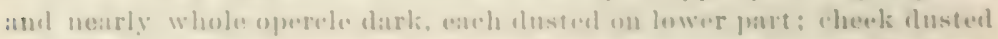

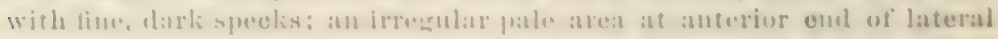


line; spinous dorsal ashy, the first 3 spines black on the middle portion, the other spines dark, but not so distiuctly so; tips of last fer spines dark; soft dorsal light browuish or grayish, crossed near the base series of dark spots and above by 2 series of whitish spots; candal spotted with white and brown; anal white, dusted with brownish; rentrals whitish, with fine, dark dustings; pectoral whitish, yellowish at base, followed by alternating series of dusky and whitish spots.

This species is related to $H_{0}$ scierus, from which it difiers chiefly in haring the dorsal fins united, in having the maxillary reaching beyonel front of orbit, in the larger seales, the free gill-membranes, and in the smooth preoperele.

Only the type known, an example, 3.5 inches long, taken in Aubeenanbee Creek, the eastern inlet of Lake Maxinkuckee, Indiana, abont $\frac{1}{2}$ mile from the lake, August 4, 1899. Type No. 49378, U. S. N. I. Coll. Evermann \& Scovell.

Hadroptenus maxinkuchiensis, Everanans, Rept. T. S. Fish Com. 1899 (1900), 366, with \}late, Aubeenaubee Creek, Lake Maxinkuckee, Indiana.

Page 1084. Before Etheostoma jessict, insert:

ETHEostouA AUBEEIUBE, Eremann.

Ilear 3.6 ; depth 5.5 ; eye 4.5 ; snout 4.5 ; maxillary 3 ; interorbital 5 ; 1 . $\mathrm{X}-10 ; \Lambda . \mathrm{I}, 7$; scales $4-55$ to $58-7,8$ to 24 pores.

Body rather elongate, not much compressed except.posteriorly; head rather short; snout short, somewhat decurved; month moderate, slightly oblique, lower jaw included, maxillary reaching front of eye; eye small, above axis of body; premaxillaries not protractile; gill-membranes free from the isthmus and each other. Fins not large, the dorsals usnally distinctly, but narrowly separated, sometimes scarcely separate; origin of spinous dorsal one-third distance from tip of snout to base of caudal; ontline of spinous dorsal gently rouncled, the longest spine about 3 in head; longest dorsal ray about 2; first anal spine longer, and slightly stronger than second, 3 to 3.5 in hearl; longest anal rac about 2; pectoral short, about 1.3 in head; rentrals close together, about 2 in head; caulal rounted, 1.5 in head. Scales rather small, rongh-ctenoid; lateral lino incomplete, usually developed on only 8 to 24 scales at anterior end; cheek usually naked or with a few small, more or less embedrled scales; opercle usually abont half scaled, sometimes with but few scales; hreast always naked; belly with ordinary scales; nape usually densely and regularly scaled, some scales sometimes embedded; preopercle entire; opercular llap moderate, broad; opercular spine rather small; no humeral spot or process.

Color in alcohol, greenish brown above; side with about 12 or 13 rerti. cal, dark blotches, separated by pale orauge red areas of similar size; another series of similar but smaller orango blotehes along lower part of side anterior to anal fin; under parts whitish; candal peduncle grarish; head dark above; opercle and cheek dark, with greenish shate; a dark line downward from eve; snont grayish; spinous dorsal with a narrow dark border, below which is a broad orange baud, then a broad but irregu- 
lar dark band along hase of lin: soft dorsal and caulal barred with white and grayish, the latter in spots on the rays; anal and ventrals without markings: pectural souewhat dusky.

This sprecies is close to $\mathrm{L}$. innew, from which it seems to difler in the almost naked cheok, the less complete sealing of the opereles, the somewhat longer maxillary, more ohlique month, closer approximation of the forsal lins, and the coloration. Lalke Maxinkuckee, Indiana.

Many examples, each ahout '2 inches long, taken in Anbeenaubee Creek, the east inlet of Lake Maxinkuckee, August 4, and on other diys in Angust ancl september, 1899\%. E. ioner is a common species in the lake, lout lows not orenr in tho inlet, nor were we able to find any specimens of E. anheenable anywhere except in this small creek. Type Xo. 49379, I.C.X.M. Coll. Evermann os Scovell.

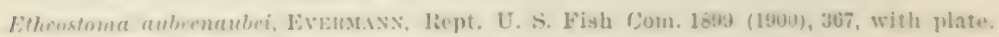
Aubeenaubee Creek, Lake Maxinkuckee, Indiana.

Page 1212. Hetore I'rionodes fasciatus, Jonyns, insert:

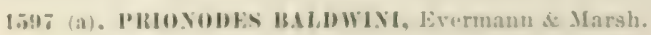

IIrat 2..5: depth 3.2; aye 4 ; snont 4.6 ; uaxillary 2.4; mandilule 2; interorhital 7 ; D. $x, 12$; $A .11$, 7 ; pectoral 1.4 ; ventral 1.3 ; cauclal 1.7 ; scalles 4-1:2-12.

Body elongate, moderately compresserl, not elevated, covered with ctenoid seales; dorsal aml ventral ontlines alike , head moderato, pointed, naked ahove and below : ere large, greater than length of snont, high in frosition; month terminal, slightly olslique, the maxillary reaching midelle of eye or somewhat heromi; gill-rakers short, 6 developerl on lower limb; treth small, conical, and sharp, on vomer and palatines and in several sories in each jaw, with weak caniues in front and a fow eanine-like teeth an milldle of sille of lower jaw; cheek with abont 7 rows of scales; preoperele finely serrate; "npercle ending in 3 sharp. 1lat spines, the midelle one largest, a membanons pointed llap projecting begond; fins all naked, the dorsal continuous, with a slight emargination, the spines slenter and pmagent, tho first $f$ or is grachated, the rest subequal, 3.2 in head, lower than the soft rays which are contained alout 2.5 in head: anal tin short, the second spine longest and strongest, 3 in head, tho soft part high, the fifth or sixth ray longrest, reaching almost to tront of anal, 2 in head; ventral with second ray prouluced, reaching vent; candal truncate, or with uidlile rays very slightly shorter, making the margin slightly comcave.

folor in lifis: Dotsal half of luead and trunk and all of eablal peduncle. searlet, veintral portion pale hlue, almost white: a yellow longitudinal band nearly as wible as pupil from preoperenlar margin straghte acroms operele and along lowly to lateral line under last dorsal rays; I quadrate. or oblong black blotehes just under this bamb, the first about under midelle of spinous dorsal, secoml umler lant spines, third under first rays, fourth moder last mys: from eath uf the lirst thee of these blotches a square,

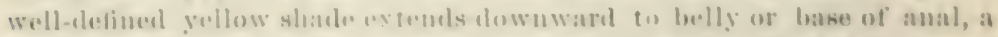

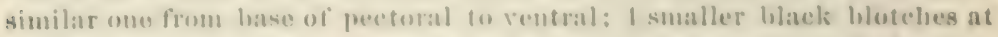

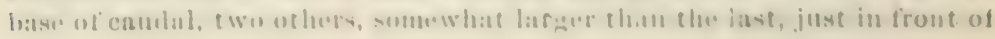


them on caudal peduncle; a row of 9 round black spots on each side at base of dorsal fin, the first one smallest, opposite membrane of lirst spine, the other 8 separated somewhat obsenrely into pairs, the first pair under middle spines, second under last spines, third under first rass, fourth under last rays; 2 or 3 very small black dots on upper edge of caudal peduncle; 2 or 3 more in front of dorsal on median line, each accompanied lyy a similar one on either side; in some specimens a few seattering ones on top of head behind eyes, sometimes regularly arranged; a few darkbrown apots behind eye; various dark markings on side of head, without very definite pattern, but usually 2 obliqne stripes on eheek, a heavy black blotch on interopercle and 2 on the ramus of the mandible, which, with their fellows of the other side, make distinct crossbars on lower side of head usually extending across maxillars; chin and lower part of opercle with dark spots; lateral line white, with a few broken spots, comparatively faint, just below it; iris red, with an inner ring of white surrounding the pupil; spinons dorsal pale, the edge of the membrane black, this color bordered below with faint yellow; soft dorsal pale, spotted throughout with light orange, with a marginal band of the same, outside of which is a very narrow pale-bhe edge; ventral very pale blue, the producerl ray somewhat yellow; anal pale blue with some light orange on last rays; pectoral and candal uniform pale reddish, unmarked.

In spirits all the red and yellow markings disappear, the dark persists, aud additional markings are brought out as follows: Aloug the anterior and upper part of trunk and erossing the lateral line are dark-brown vertical bars, diffuse and running together, or separated aud broken into ronnd or quadrate blotches; in the middle part of the conrse of the yellow longituclinal band appears a row of very small black points; spots on soft dorsal dusky; dark mottlings on caudal; upper and lower baso of pectoral, aud sometimes axil, dusky.

Puerto Rico. A beautiful and strongly marked species; 2 specimens dredged and 33 others, ranging in size from 0.55 to 2 inches, caught in the taugle, oft Culebra and Vieques islands, from coral bottom, in depths of 15 and 16 fathoms; the type, 2 inches long, taken in the tangle at Fish Hawk Station 6093, off Culebra Island, 5.25 miles sonthwest of Culebritas light-honse, February 8, 1899, in 15 fathoms, by the U. S. Fish Commission expedition to Puerto Rice. ("Named for Mr. Allertus II. Baldwin, the artist of the U.S. Fish Commission expeclition to Pnerto Rico, in recognition of his excollent drawings and paintings of American fishes.")

Prionodes baldwini, Everas. A MARSH, Iieport U. S. Fish Com. 1899 (Decemher 19, 1899), 353, oft Culebra Island, P. R. ('Type, No. 19361, U.S.N.M. Coll. Erermann d Marsh.)

Page 1229. After Gramma loreto, Poey, insert:

\section{8 (a). RHEGMA, Gilbert, new genus.}

Rhegma, Giubert, new genus of Serranidio (thaumasium).

Allied, but not closels, to Rypticus, Grummistes and Gramma, having the large, ctenoid scales and interrupted lateral line of Gremmu and the peenliar physiognomy, attachment of gill-mombranes, and fin structure of the liyplicine? 
Seales of molerate size, thin, not embededed, minutely ciliated ; Jatural lines 2, the apper near hase of dorsal, ceasing under orignin of posterior fifth of soft dorsal, the lower line bugiming alightly in front of the end of the upper line, ruming along midhle of caudal peduncle; tho tubes very short, borme on much sualler intercalited seales, and not forming a continuons line; hoal largely sealeul, the suont and jaws nalied; gill-memhranes united anteriorly, forming a narrow free fold across the isthmus; branchinstegals 7 ; peudolmandhia well developed; a wibe slit hehind last grill-arch; gill-raliers shore, broally triangular, strongly toothed. Lpper materin of operele, alove its angle, wholly attached loy menbrauce to the shoulder girlle, as in the liyplicina. Nouth large, protraetile, the lower jaw protruding, the maxillary broadly exposed, with a narrow supplemental lone along its npper edge. Teeth all villiform, in broad bands on jaws, vommen and palatines, the inner teeth on jaws slightly longer than the ot hers and ilepressible. Tongne smooth. Large mueous pores on under sille of mandible, and slit-like pores present on edge of preorbital and aromind front of eye. Ant rior nustril near edge of preorbital, provided with is short tube: posterior nostril without tule or raised rim, immediafely in front of eye; a slourt free triangular llap ou upper edge of each orbit. lpper portion of preopercle with a single strong plectroid spine, directed backward and downwad; hones of tho head otherwise muarmed, the preorhital amd prenperelo with entire edges, the operelo withont spines or riblges. Ventrals suall and anterior in position, as in the liypticine, "onsistiug each of one strong spine, and five branched rays, their base being in front of base of pectorals; no enlarged seale behind base of ven1mals; vertical fins low, with rounded lobes, their basal portions well sraled; dorsal with $T$ low strong spines and 2: profusely hanched rays: anal wilh:3 spines and 18 rays. One speeies, apparently the type of a distinct subfiunily, lihegmalinat.

(iii)/uct, fracture; referring to (be: interrupted lateral line.)

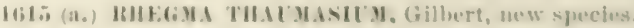

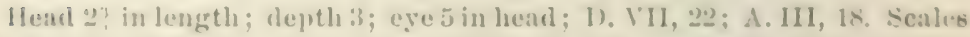
Ai in a longitudinal series along middlo of siclo. Buly elongate, moderately compressed, with very short, deep, candibl pedunelo; anterior profile strongly arched, slightly depressed ahowe orbits; interorbital space very narrow, convex, its width twe-thirels diameter of orbit. Month large, slighty obligne; lower jaw the longer, its tip entering the protile. Dorsal spines low, strong, increasing lackwarl, the last spine a tritle lomser than diameter of orhit; anal spines short and strong, the midlle spine longest. Lateral line surved strongly npward from its origin tu below third dorsal spine, thene rouning parallel with the hack to below midldle of second dorsal, from which point it gradually approaches the lasen of the dersal, where it terminates moder the fifth ray fron the last: along its anterion conros it is separated from hase of clossal by from of to fi

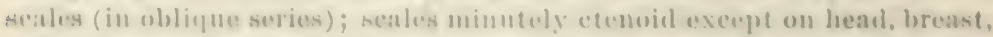
and leelly, on nape under anterior dorsal spines, and on hase of peetoral:

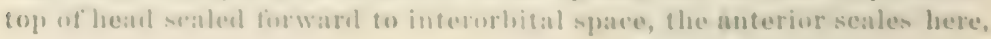


as well as those on cheek, much reduced in size, embedded, so that their outlines can not be distinguished.

The following measurements of the type specimen are given:

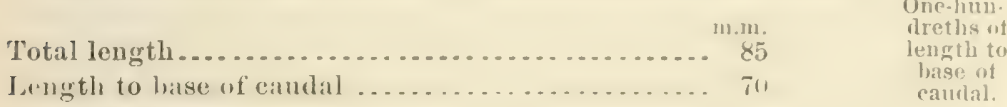

(ireatest depth .....................................

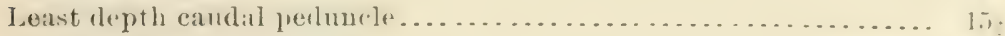

Lenerth of caudial pedunule............................

Distance from tip of upper jaw to ond of operenlar 11 ap . . . . . . . .

Length of snont . . . . . . . . . . . . . . . . . . . . . . . . .

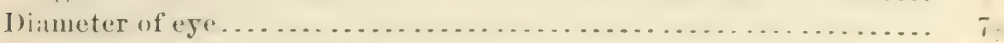

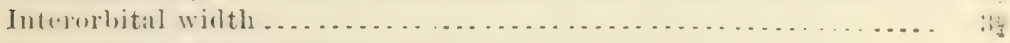

Tip of snout to end of maxillary . . . . . . . . . . . . . . . . . . . $1 !$

Length of pectorill............................ . . . .

Tip of snout to base of upper pectoral ray .................. . . . .

'T'ip of snout to base of ventrals ........................

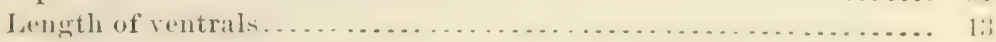

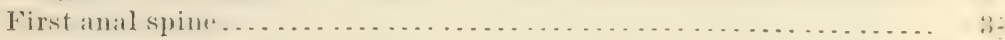

Ferond anal spine. . . . . . . . . . . . . . . . . . . .

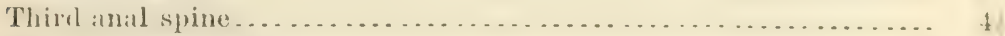

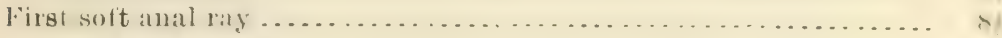

Lungest soft atual may . . . . . . . . . . . . . . . . . . . . . . .

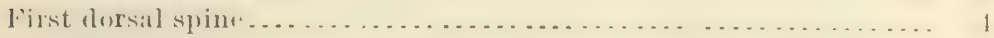

Last dorsal spine . . . . . . . . . . . . . . . . .

Longest dorsal rity . . . . . . . . . . . . . . . . . . . . . . . . .

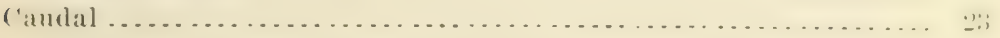

Color, nearly uniform warm brown on head, bods, and fins; a duslis opereular blotch; soft dorsal, anal and caudal only narrowly margined with white.

One specimen known, from Pauama.

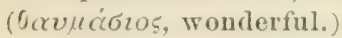

Khegma thaumasium, GiLl3ËT MS., 1'anama (Coll. C. H. Gilbert. Typu, No, 5978, L. S.Jr. Univ. Mtus.)

Page 1300. After Hamulon scudderi, Gill, insert the followiug:

1667 (a). HEMLIOX IIELEX.., IBoulenger.

Heal $3 \pm$; depth 24 ; ore $3 \pm$ in liead; interorbital width $3 \frac{1}{2}$ I). XIII. 16: A. III, 12; scales $11-87-20,67$ pores.

Proble very much arched from snont to origrin of dorsal; muzzle not reaching beyond lower jaw, little shorter than oye; month little ohligne; maxillary reaching anterior $\frac{1}{d}$ of eye; teoth very small ; - very small pores in chin; preopercle very feobly serrate; heal sealoul, except snout and chin: grill raliers very short, 15 on lower arm of areh; dorsal spines fereble, increasing to the fourth, which almost eymals \pm length of hean, then ilecreasing to the last, which is contained times in hearl; soft dorsal low. covored with seales; pectoral falciform, longer thau head, 13 times ventral: 
anal spines small, increasing to the third, which is 5 in heal; soft anal inw and sealy; andal almost completely scaled, deeply emarginate, the median rays less than half the exterual ones; cambl peduncle th times longer than deep; seales alowe lateral lino in very obligute series. Grayish above, with olshigue brown lines, sonewlat undulating; silvers below. Length 22 em. One specimen from Bay of Sintal Llena, Ecualor. (Bonlinger.) (Named for the type locality.)

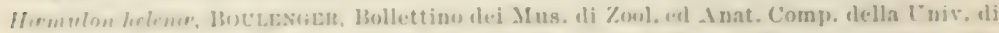

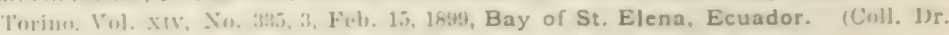
Enricu lientat.)

Page 1334. Niter I'omadasis ramosus (l'oty), insert the following:

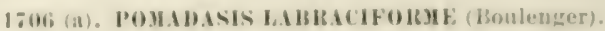

Ifend 3; depth 3t; eve 4; snout slightly longer than mandible, by $\frac{1}{4}$ diameter of eye, which is little more than interorbital widtl, maxillary rearhing anterior $t$ of eye; 1 . XIII, $12 ;$ A. III, 7 ; scales 8 or $4-68-2$; teetl very suall; 2 pores in chin; preopercle strongly serrate, strongest at angle: head sealerl, except snout and lips; gill-rakers 13 on low.r arm. of tirst arch, the longest equaling $\frac{1}{3}$ length of gill-filaments. Dorsal spine strong, the fifth longest, 2 in luad, from which they decrense to next to last, which is $1 \pm$ in head, and a little shorter than last; soft dorsal scaleul at base, its longest rays a little shorter than longest spine; pectoral 1 in leat, senreely shorter than ventrals; secomb anal spine very strong, 3 in fread, third 2 in head; longest anal rays shorter than anal spine; candal peduncle longer than deep; seales arranged in series searcely oblique ahove the lateral line, which has in pores. Color uniformsilvory. Length $17 \mathrm{~cm}$. (He: specumen known, from the Bay of St. Elena, Ecuador. (Boulenger.) (Lalures: formu, form.)

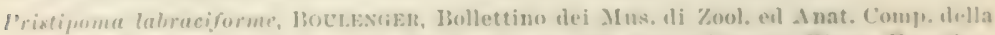
Fuiv, di 'Torine, Vol, XIV. X̌o. 335, 3, Fob. 15, 1899, Bay of Santa Elena, Ecuador. (cull. I) Enrieo listat.)

Page 1351. After falumus proridens, Jorlan d (iilhert, insert:

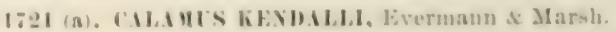

(I'T.uMA.)

Heal 3.1 ; depth 2.1 ; eye 3.5 ; snout 1.5 ; maxillary 2.4; interorbital 3.5 ; preorbital 2.1; 1). XII, 12; A. III, 10; pectoral 1; ventral 1.8; caulal 1.3: 4eales $7-i, i 3-11 i$.

Bubly deep, back strongly olovated, more so than in C. bajonado, but less

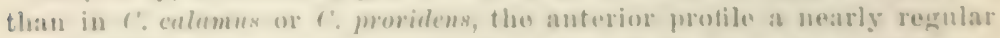
enrve, lacking the abmupt unchat elevation of these species; eye large. larger than in C providens; 7 or \& rows of senles on cheek: teeth about an iss C. propidens: molars in - or more rows on sides, flose of inner row much the largest, those in front bremuing more numerons not merging into cardiform teeth, the most anterior of which, in oach jaw, aro somewhat enlarged; in front of upper jaw are 2 much colarged antrorse canises, onrvesl slighly upward: highest dorsal sp̧ine 2.7 in head, secomd aual spille f.ti. 
Color in spirits: Silvery, side with bluish longitudinal lines following the rows of seales, plainest above; a pale-blue line bordering the orbit below; some blue lines on preorbital, not evidently reticulated and not as numerons as in C.proridens; iris yellow; otherwise as in C.proridens, to which this species is very close.

Puerto Rico; known from the type, a specimen 10.5 inches long, from Mayagiez, Jannary 20, 1899, and 2 cotypes, each 8.5 inches long, one from Mayagiez, the other from Arroyo, all collected by the U.S. Fish Commission expedition to Puerto Rico. (Named for Dr. William Converse Ken(lall, assistant, U. S. Fish Commission.)

Calamus kendalli, Evernann of Marsh, liept. U. S. Fish Com. 1899 (Dec. 19, 1899), 35t, Mayagiez, Puerto Rico, (Type, No 19362, U.S.N.M. Coll. Evermann d Marsh.)

Page 1417. For Nebris zestus, Jordin of Starks, sulustitute Nébris occidentalis, Vaillant.

The synonymy of this species is as follows:

Nebris occidentalis, VAlLLANT, Notes from the Leyden Aluseum, XX, 20, Oetober, 1897, Panama; after deseription by Jorlan \& Eigenmann.

Nebris zestus, Jondan \& Stanks, in Jordan \& Evermamn, Fisheg North \& Middle America, Fart II, 1417, October 3, 1898, Panama. (Trpe, No.433, L. S. Jr. Univ. Mus. Coll. Dr. C. H. Gilbert.)

Page 1439. After Bairdiella chrysolenca (Ginther), insert the following:

1520 (a). BARDIELA MACANTA (Bonlenger).

Head $3 \frac{1}{3}$ to $3 \frac{1}{2}$; depth $3 \frac{1}{3}$ to $3 \frac{1}{2}$; eye $4 \frac{1}{3}$ to $4 \frac{1}{2}, 1$ to $1 \frac{1}{5}$ in interorbital wisth. D. XI, 21 or 22 ; A. II, 7 or 8 ; scales 5 or $6-45$ to $43-11$ or 12,50 to 55 pores. Snout round, scarcely longer than eye; mouth in ferior, compressed, scarcely oblique, with simple subequal teeth, the gape reaching anterior $\frac{1}{4}$ or $\frac{1}{3}$ of eyo; 2 very strong spines at angle of preoperele, the lower directed dowuward; gill-rakers very short, 10 or 11 on lower arm of first arch; third dorsal spine longest, $\frac{3}{3}$ to $\frac{2}{3}$ length of hearl; pectoral $1 \frac{1}{4}$ in head; first anal spine very small, the second small, $2 \frac{1}{2}$ to 3 in head, or $1 \frac{1}{2}$ in longest soft ray; caudal acuminate; caudal peduncle a little longer than deep. Grayish above, with faint brown stripes following series of seales; silvery below. Close to $B$, chrysolenca, Giinther, from which it is suficiently distinguished by the less development of the second anal spine, the smaller month, and the feebler dentition. Length $21 \mathrm{~cm}$. Three specimens known from the port of Guayaruil. (Boulenger.)

Cortina miacanthus, Bovievger, Bollettino dei Mus. di Zool. ell Anat. Comp. della Univ. di Torino, Vol. XIv, No. 335, 5, Feb. 15, 1899, poit of Guayaqui'. (Coll. Dr. Enrico Festa.)

Page 1526. After Cichlasoma centrarchus (Gill d Bransford), insert:

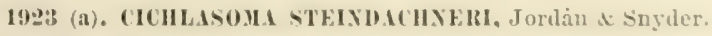

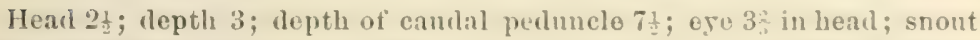
2f; interorbital space 5; longest dorsal spine 4, ray 22; longest anal spino $2 \frac{2}{3}$, ray 2 ; length of pectorals $1 \frac{1}{2}$; ventrals 1 ; ; caudal $1 \frac{1}{2} ; \mathrm{D} . \mathrm{XVI}, 10 ; \mathrm{A} . \mathrm{V}$,

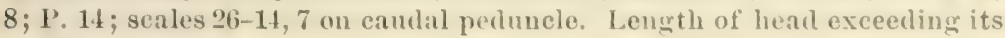
depth a distance equal to diameter of orbit; bolls elongate, deepestaboro 
vontrals; "urve of dorsal ontline interrupted ly a slight elevation abore ego and a rather rapiel descent at luses of suft dorsal; ventral ontline less curvel than lorsal: inferorbital spare convex; orlsit somewhat chongate laterally, locateul slightly mearer tip of suont than to pusterior edge of operele, its lower edge a liftle: above a lorizontal fom amoth to milalle

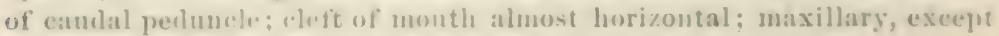
distal and. concealed ly preurbital; lips thiele, the lower with a narrow fremm; jaws efpal, the upper moblerately protractile; teeth iu 2 series. on each jaw, anter series in a singlu row, largo, canine-like, fiar apart: inuer sories minute, in bauds; tips of teeth brown-colored; no teeth on vomer or yalatines. Cill-membrames forming a fold across the isthmus: grill-raliers on lirst areh 10, short aud hlumt. Hody covered with large weakly-ctunuid sirales: head with eycloid scales; upper part of heal anterior to miblle of orbit, smout, preorbital area, and ventral part of lueacl, nalied; a single row of suall seales along hases of florsal and aual fins: small salus un basal part of inter-radial meubranes of caublal; lateral line intermpted at the fourteeuth seale, herginning again 3 seales lower and "xtending to baso of eaudal; first olorsal spine very short, others gradually longer to the sixtl or seventh, atter which tho spunes are abont the same lenerth; lifth and sixtl dorsal rays longest, abont 1 times the length of longest spine, depressed tin extruling to posterior edge of darli raulal spot; lirst anal spine slurtest, oue-tifth as long as fifth spine, thirol, funrth, amb fifth rays longest: depressed fin extemdiug to a terior edge of

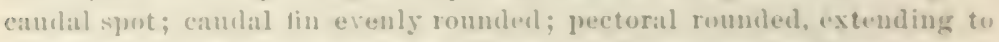
a vertical from vent; untre rays of puetoral longest, extendiug to vent. Color in aleohol, liglit olive, darker above than below : an indistinet, dark lateral band exfending from snout to cambl: sor 9 scarcely elistineruisleable dark vertical bands on side of borly; irregular dark spots at inter. nection of lateral aud vertical luands; a suall, dark spot at base of caudal; small, distinct dark dots on anterior dorsal region of heal.

The tutal length of the type is til mus. Younger examples measuring about di3 mu. have the body a little leeper and the hearl shorter. The vertieal colur hausls on posterior half wh body are more slistinct on the

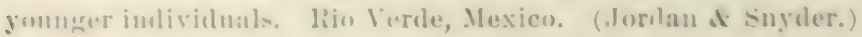

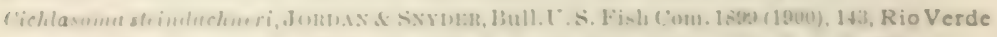

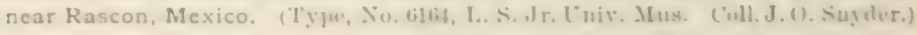

Page 1540. Before Theretp, (iintler, insert the following:

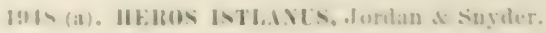

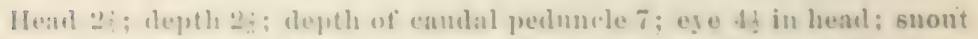

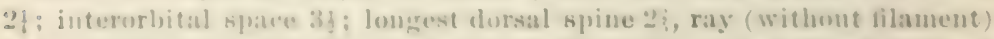
1:; lompent anal spime 2, ras (without tidament) 1!: Jength of pectoral

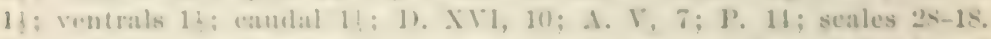

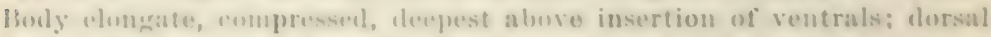
outliue rininer raprilly to urierin of dorsal, fallinge gradually to base of last

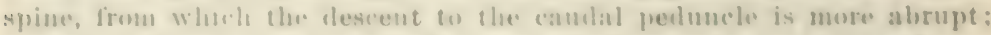
ventral mutline evenly rounded: inferertotal spane convex: eye large,

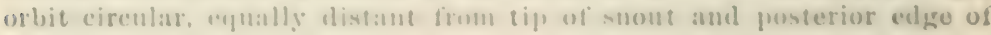


opercle; mouth horizontal, lower jaw projecting; lips thick, the lower without frenum, folding over the upper at their union; premaxillary protractile; maxillary small, nearly vertical in position, and almost hidden by the large preorbital; teeth on both jaws, in 2 series, the outer a single row of 24 canines, largest in front, growing smaller posteriorly, the inner series villiform; all of the teeth with brown-colorerl tips: gill-membranes free from isthmus; branchiostegals 5 ; gill-rakers on first arch 9, short, blunt, far apart. Body covered with largo scales; cheek, opercle, interopercle, subopercle, and occipital portion of head with small seales; ventral part of liead, preorbital area, snont, and anterior part of iuterorbital space naked; one row of seales extending on bases of dorsal and anal fins; scales of body weakly-ctenoid; seales of head smooth; lateral line interrupted on the nineteenth transverse row of scales, begiming again 3 seales lower down and 2 seales in advauce of where it left ofr' and extending to base of caudal; first dorsal spine short and slender, others gradually longer and heavier, posterior spine longest; each spine with a rather stift distal, ray-like attachment, the anterior elge of which projects above the menbrane of in; tips of fourth and fifth rays of dorsal uniting to form a thread-like filament about as long as the diameter of orbit; dorsal, when depressed, extending on caudal one-third its length; first anal spine shortest, others growing gradually longer and hearier, the last $2 \frac{1}{2}$ times as long as the first; spines with distal attachments similar to those of dorsal, third and fourth rays longest, mited at their tips, formiug a slender filament; tip of anal extending a little farther posteriorly than that of dorsal; caudal rounded; tip of pectoral rounded; ventrals located slightly posterior to base of pectoral, extending to rent, outer ray longest, enling in a filament. Color dark, an oblong brownishblack spot at base of each scale on side of borly, the spots growing less distinct abore the pectoral; membranes of dorsal, anal, and caudal with small spots, these more distinct and regularly arranged on soft parts of dorsal and anal; pectoral and ventrals without spots.

Young individuals have a brownish-black spot at base of candal and on side of borly at tip of pectoral; a less distinct spot at upper edge of gillopeuing and also below posterior end of base of dorsal. The darker of these spots are sometimes faintly indicated on the larger individuals. Morelos, Mexico. (Jordan đI Snyeder.)

Known ouly from Rio Ixtla at Puente de Ixtla, Morelos, Mexico. (Type, No. 6150, L. S. Jr. Univ. Mns. Coll. Jordan is snyeler.)

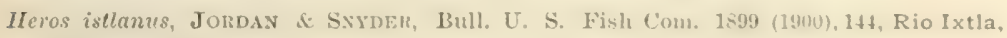
Morelos, Mexico.

Page 1542. After Veetroplus nicaraguensis, Gill d IBranstord, insert:

19.51 (a). NEETHOPIUS CARPIXTS, Jorian \& Suyler.

Ilead 2; ; depth 2; depth of candal pedunclo 6; oyo 5 in head; snout 2! : interorbital space 2: 2 longest dorsal spine 2!; ray 1ł; longest anal spine 2 , ray $1 \frac{1}{3}$; length of pectoral $1 \%$; ventrals $1 \frac{1}{3}$; cautial $\left.1 \frac{1}{1} ; 1\right) . X 1,10 ; \mathrm{A}$. V, 8; P. 15; scales 26-17,7 on candal pedunele. Buly compressed, deepest part above ventrals; dorsal ontline straight from tip of snout to a 
point above anterior edge of orhit, where it is abruptly curved npward and hackwarl to the origin of dorsal tin; from the latter point it oraulually curves duwnward to hase of lirst dorsal ray, from which the descent fo caudal peslumele is athupt; ventral ontline ovenly curved from sumus to cundal perlune le; interorbital space convex, its middle portion dlat tened: orhit cirmlar, nearer to posterior edpe of operclo than to tip of suont, a distane equnal to dianseter of pupil; mouth oblique, lower jas sliglatly projerting; lips thick; lower jaw with a frenum ergal in wislth to ! dianeter of joupil; upper jaw protractile; maxillary covered hy preorbital except at its distil enl; teorls in 2 series on each jaw, the onter seriein a single row, llat or incisor-like, larger in front, growing murh smaller posteriorly, the inner series minute, in marrow bunds, all the teeth loosely attacherl, their tips l,rown-colored; no teeth on vomer or palatines; gillmenloranes forming a fold across the isthmus; gill-rakers on first arch 10, short, far apart. IBolly covered with larre, weakly-ctenoid seales; cheek. opereles, and orcipital portion of head with small, cycloid seales; lower faw, suout, and anterior half of interorbital space naked; hases of dorsal and anal tins with a row of small seales; inter-radial membranes of cumblal witl very small scales on hasal parts; lateral line intermpted on the nineteenth transverse row of seales, begrinning arain 3 seales lower down on the thirl row anterior to where it left off, and extending to base of caudial: 2 short rows of uneons tuhes on inter-radial caudal seales, one above and the other below end of lateral line; first dorsal spine shortest, others pradually longr.r and heavier, each spime with a ray-like attachment projertincr above and posterior to its tip); first aual spino shortest, others gradually longer and heavier, the fourth three times as long an the first; spines with distal attachments similar to those of the dorsal, thirel and fourth rays longest, extending posteriorly an far as those of the dorsal; posterior eilge of caudal somewhat convex; pectoral rounded; ves. trals pointed, the onter ray much the longest, extanding a little leyomd vent. Color in aleohol, Jierht slate; scales with lighter central sputs; posterior parts of dorsal, anal, and candal lighter; in lifo the heal was cor"red with romul and elongate spots of greenish blue on a hacliground of golsten lorown; side of horly with bluish and hownish spots withent zegularity of arrangenent; pectoral and distal part of solt dorsal with a yellowish tinge.

In the ymung of this speeses there ate if or biarli vertical bands, about enual in width to diameter of orloit, on the grosterior part of the loody ; a dark npot sometimes present just beluw latoral line on a vertieal through bases of eleventh dorsal spine.

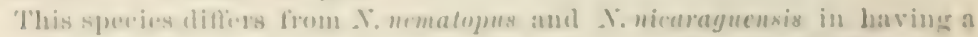
moch deeper looly and fower dorsal and anal spines. Jagma del Carpinte, Mexieo. (fordass at snyeler.)

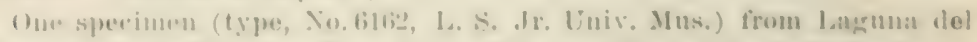

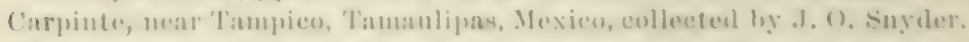

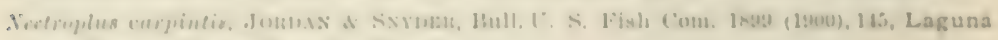
del Carpinte, near Tampico, Mexico.

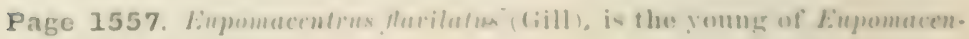
trus rectifirenum (tiifl). 
Page 1612. Before Iyrula, Jordan, insert:

2U22 (a.) MURATOMOTUS DECORIS. Evermann \& Marsh.

Head 2.6 ; depth 3.4 ; eye 4 ; snont 3.5 ; maxillary 4 ; interorbital 4.6 : D. IX, 10; A. III, 9; peetoral 1.6; ventral 2.2; candal 1.6 ; scales $1-26-6$. Body moderately elongate, compressed throughout; the back a little elevated, the caudal peduncle deep and rather long; dorsal and ventral outlines nearly alike, the dorsal somewhat more strongly arehed; auterior profile not trenchant, almost straight from suout to front of dorsal, very slightly convex in front of dorsal and vers slightly concavo between "ye and tip of snout; head pointed, interorbital space broad and flat; cre large, high in position, middle of pupil nearer tip of snont than end of opercle; snout long, somewhat longer than diameter of eye, moderately produced, the lips broad in front, characteristically lahroid; mouth not large, the maxillary not reaching front of orbit, the jaws equal, ammed with strong, sharp teeth, about 4 canines in front of upper jaw, 2 in front of lower; teeth on sides of jaws also canine-like, smaller than those in front, but not distinctly different from them; a fow smaller teeth behind the main row of large ones; vomer and palatines toothless; soft dorsal and aual each with a basal sheath of abont two rows of large scales, that of clorsal extending over half the fin or more, that of anal lower, the fins otherwise naked; lorsal fin continuous, with a shallow noteh, the sprines slevder and pungent, the second longer than the first, tho following ones graduated to the fifth, which is shortest, thence increasing in length to the ninth, which is longest, 2.3 in head; soft dorsal with its middle rays highest, 2.2 in head; anal with three slender, sharp, gradnated spiues, the third longest, 2.2 in head; the soft part similar to soft lorsal, lougest rars 2.3 in head; pectoral large, symmetrical, of 11 rays, the midlle ones longest, reaching past tip of ventral nearly to vent; ventral moderate, pointed, reaching half-way to veut; caudal rounded; seales large, cycloid the lateral line on second row below the dorsal interrupted near the end of dorsal and beginning again on the row below, on canclal peduncle.

Color in lifo: Body chietly green, darker green on back, lighter below; lower parts of head and breast light yellow; a broad white bar from eye obliquely across cheek and opercle, bordered above by an mudulating maroon lime and bolow by a similar but fainter line: a brown bar from oye to snout; t dusky spots near baso of dorsal extending as fainter shades downward and slightly forward to or beyond lateral line, 1 form in front of dorsal, 2 under spinous dorsal, and 1 under soft riss; short paleblue bars or spots on breast and about pectoral; iris blue, a pinkish lorder. surrounding pupil; dorsal greenish, the soft part with yellow shade, a pale-bluo edging to the whole fin, a maroon borkex to the green color pusteriorly just insicle the pale-blue edge, a small dark spot on membraue between seventh and eighth rays aud a blue spot on membrame of tirst spine; anal colored like soft clorsal, the maroon borter exteuding from first spine to last ray insido the pale elging, the dark spot betweon sixth and seventh rays; vontral green near base, pale blue outwardy, the green color bortered by maroon spots; pectoral plain, pale wreen; cambal vers pale transparent blno, a welge-shapee? maroou spot on the a npper rays 


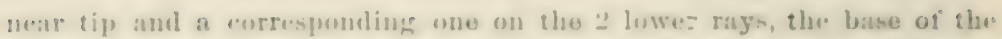
werlere on muter may base of caudal with a pal. molulate vertical har

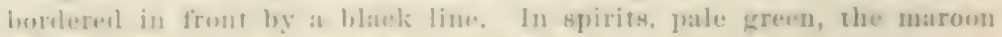
markings faintly persistent, lecoming dusky.

l'uerto kico: lenown anly frum the type, 1.5 inches long, taken in the wine at l'unce, Jannary 30, 1894. Jy tho Cnited states l'ish Commission "xperdition to l'nerto dian. (decoris, besutiful.)

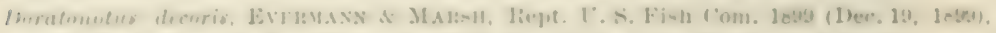

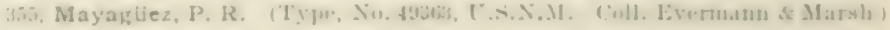

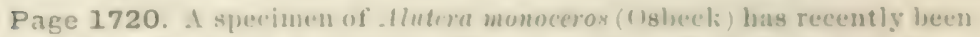

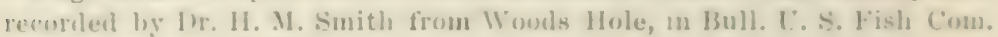
|x:18 (1s!9!), $273,|1| i \mid$, .

Page 1733. Tetrodon nephelus, fionde at Bean, is a good species distinet irum T. spengleri, Bloch, and should stanut as

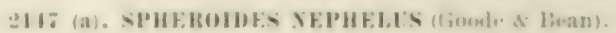

Page 1968. 'T'u the synonymy of Cruniden yrucilis (Herliel), ald:

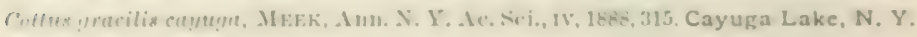

Page 2017. The recent studies of the tide-pond cotruids of the ('alifornia coast by Mr. Arthur Wr. Greeley have resulted in the discovery of sonu. new species and genera and other additional iuformation regarding this group of fishes. The following new species aul genera are to be arlinil:

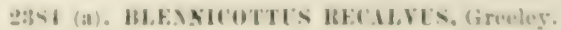

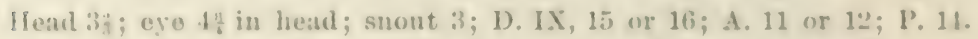
Bumly short, stout, lowal anteriorly; tho lieal very loroad, short and hlumt; -nout olotumo; interorbital space ij fi eye, grooved, the groove leading into a olepressed space behind the ryes; month distinetly terminal, the maxil. lary reaching a vertical helow the anterior algo of orhit, the lower jaw included: minnte conical teeth on jaws, vomer, and palatines: nasal spimes very suall; no preoperoular spiue apparent in alults; edgo of preoperele

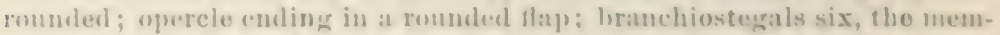
leranes broally united, fren from the isthums; gills 34 , a slit luhimel the last gill. Dorsal fins very long, slightly jouned at the hase, origin of first dor-al directly over tip of opercle, that of soft dorsal in alvanee of origin

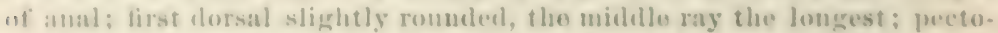
ral rasting origin of anal, membranes of tirst seven mys aleeply emargi. mate: ventrals realehing vent; anal papilla of male very large; anal low. the membrames of all the rays oxerpt the last three derply enarginate. nume of the rays endarged in the male; candal short, slightly romoled.

('irri fiew and smatl, thuse of top of head joined at base in conspionous

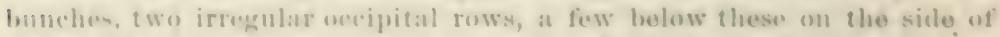
heanl, and on the margins of propperele and operele; a few above origin of pereforal, and a weak row along the anferiofe thild of the lateral line.

Color of boly, light hrows, vermionlated with white, and marked dor-

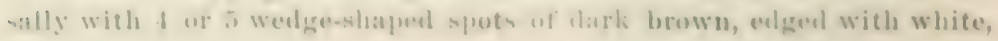


and more distinct posteriorly; 2 pinkish spots on the dorsal side of the caudal peduncle, and a faint shading of the same color on side of head and along anterior fourth of lateral line; entire under surfice dull brown, tinged with olive; fins indistinctly barred with grayish white; tail faintly tinged with pink. In some specimens the color is an almost uniform dull brown while in others the light markings are prominent. Some roung individuals from among the green algae are uniform light green.

The bluntness of the snont and preopercular spines, and the torminal mouth make this species easily distinguishable from all related forms, except 13. globiceps, from which it is separated by the shape and size of it: preopercular and nasal spines, the number of its cirri, 12, and the size of its mouth. The adults of these 2 species can be readily distinguished, but the young of $B$. recalvus is very similar to the young of $B$. globiceps, indicating that 1 . globiceps is probably the ancestral form. Giraril's old description of Oligocottus globiceps has been erroneously associated with this fish, which does not extend so far north as the type locality of 1 . globiceps. B. recalcus is distributed from San Diego to Santa Cruz, whero it is immediately snceeded by B. globiceps on the uorth. No specimens of B. recalrus have been taken north of the region of Santa Cruz. On the other hand, several specimens of $B$. "lobiceps have been collected on the coast of Monterey County south of Monterey Bay, therefore within the range of $B$. recalvus. The relations letween the two species where their ranges overlap is still to be made out. B. recalvus is quite common throughont its range and every where inhabits the deep shaded tide-pools, near low water mark, where a large number will often be found in a single pool. (Greeley.) (recalves, bald in front.)

Centridermichthys globiceps, GüNTnER, Cat., II, 171, 18fio; not of Girard.

Oligocottus globicep8, Jondas \& GiLiBert, Synopsis, 718, 1883.

Blennicottus globiceps, JordaN \& Starks, Proc. Cal. Ac. Sci. 1895, 808; Jordan \& EvERMANs, Fishes of North and Midtlo America, II, 2017, 1898; not Oligocottus globicens Giraril.

Blennicottus recalvus, Greeley, 13ull. U. S. Fish Com. 1899 (Dec. 13, 1899), 9, fig. 1 Pacific Grove, Cal. ('Type, No.6068, L. S.Jr. Univ, MI

\section{6 (a). RUSCICULUS, Greoloy.}

IRusciculus, GREELEY, 1Bull. U. S. Fish Com. 1899 (Dec. 13, 1899), 13 (rimensis

This renus is allied to Oxycoltus, difiering in tho presence of minute prickly scales, which cover dorsal half of body. Preopercular spine simple, sharp. No slit behind the last gill. (ruscum, the buteher's broom, a rough-skiuned plant.)

\section{St (a). BUSCICULCS RMEXSIS, Groeles.}

Head $3 \frac{1}{3}$; eye 4 in head; snont $3 \frac{1}{2}$ in head; D. IX-17 or $18 ; A .14$ P. 14: V. 1,3. Body compressed, very slender, tho euthal pelunclo especially so; heal compressed, flat; suout pointed; interorbital space f eye, grooved; top of hoad flat aud slightly concave; nasal spines larire, blunt, suout abruptly decurved below them. Dorsal half of hody covered with miunte, embedeled, prickly scales partially arranged in obscure

Bull. No. 47, pt. $4-\mathrm{x}$ 
oldipues rows, nones loelow lateral line. Minute prointed touth un jaws, vomer, and palatimes: jaws suluepual, mouth horizuntal, the maxillary reaching a vertical below thes anterior edge of pupil. Margin of pro-

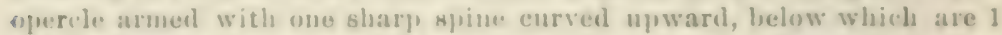
and sometimes 2ै very short blunt proessses; margin of "percle ending dorsally in a pointed 13ap. Branchiostegals fi, the membranes broally united, free from the isthums; no slit behiud the last gill. borsal fius not joined, the soft dorsal very large; first dorsal beginning slightly in atvances of opereular Hap, the upper edge much romuled, the difh spine boing longest; origin of suft dorsal, just in front of origin of the anal in the fenale, directly alsove it in the male, the tin very long: juetoral lagre, reaching a vertical helow ninth ray of soft dorsal; origin of venirals posterior to a point milway hetween anal and base of peetoral in the male, anterior to it in the fomale, the difference causen by the enlargomont of lirst "2 anal rays in the male; anal tin suall, the rays slender. the membrames of all cleeply omarginate; the lirst 2 anal rays of male: grently unlarged, joined by membrang to each other and to the rest of the fin; the posterior euge of tail nearly straight; anal papilla ineunspicuons. C'irri small and searce, alwaye occurring simgly, never in bunches or joined at the lase, with the exeeption of a few pairs along the anterior third of the lateral line; one above each orbit, "2 rows of 3 ench behind these on top of head, 1 eirrus on the insile of each nasal spine; a cirrus on the mul of maxillary, 2 or 3 on the margin of the preopercle below the preoperenlar spine, and a row aloner the anterior lualf of the lateral line. Color, light olive or realdish brown tiaged with lavenuler, marlied dorsally with $f$ or 5 werlpe-shaped, intonted spots of black, a liroken band wi same color alomg the lateral line, sometimes sending hranches loelow it which show a tendeney to inclose romm spots; a more or less distince spot of black on top of the heacl: a faint pustocular line, a spot below the eye, and a preopereular line ruming from eye to esom, all of same color: peetural and caudal inclistinctly barred with lorown, anal finged with it, and the dorsal covered with time brown or black sjoets somotimes very faint; throat and helly jale yollowish white, mnspotted.

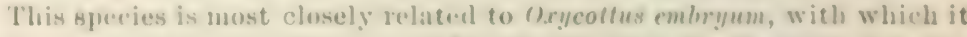

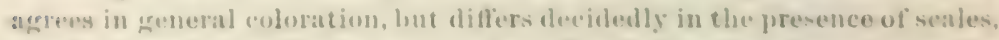
the slemerer louly, the larger mumber of noft dorsal and anal rays, the serrated margin of the prenperele, and the arrangement of the cirri. Rare; only en other speciuens from l'oint Lohos, ('alifornia, are linown t1) us. It inhathite the tide-pouls lined with corallines, and in its conlora-

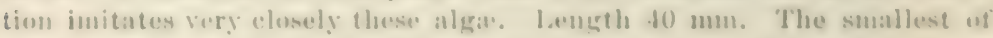

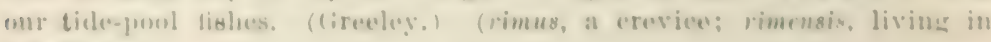
(revions, )

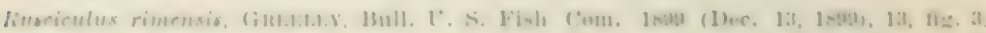

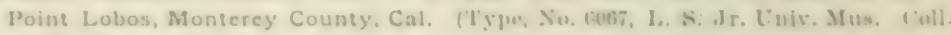
A. W. lireeley.

746 (b). DIALARCHUS, lirveley.

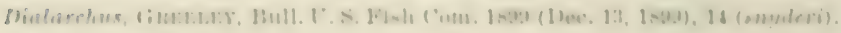

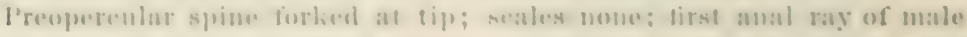

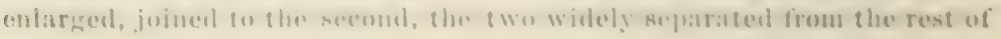


the fin. Closely allied to Oligocottus, differing only in the character of the anal rays of the male.

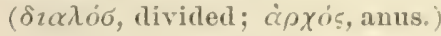

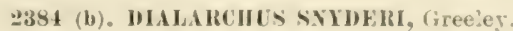

Head $3 \frac{1}{4}$; eye $4 \pm$ in head; snout $3 \frac{1}{2} ;$ D. VIII-18 or $19 ;$ A. 13 to 15 ; I’. 13 to $15 ;$ V. 1, 3. Body elongate, slender, the snont pointed, compressed; minute conical teeth on jaws, vomer, and front of palatines; jaws equal; mouth horizontal, the maxillary 3 in head, reaching a vertical helow anterior edge of pupil. Interorbital space bye, shallowly grooved, the groove leading into a depressed space between the occipital ridges; mas spines large. Margin of preopercle armed with a strong spine, $\frac{1}{2}$ as long as eye, from the npper border of which at base extends a second spine pointing abruptly upward and inward; both spines covered with skin in life; margin of operclo ending in a pointed llap entirely unarmed. Brauchiostegals 6 , the membranes broanly united, free from the isthmus. Gills $3 \frac{1}{2}$, a slit behind the last gill. Dorsal fins laxge, separated by diameter of eye, whole length equaling that from caulal to base of pectoral; first dorsal beginning slightly in aclance of margin of operele, upper edge nearly straight, curving abruptly downward from the sixth spine; origin of soft dorsal in advace of anal; pectoral large, reaching well beyond the origin of the anal; ventrals almost midway between base of pectoral and anal; anal fin small, rays all feelyle in the female, in the male the first ray only greatly enlarged,;oined to the second, the? distiuctly separated from the rest of the fin, the membranes of all except the last 3 or 4 rays deeply emarginate. Anal papilla small, present in the male only. Cirri very numerous, nsually oceurring in bunches of : or 4 , those of head joined at the base, forming a comb; "2 pairs of hunches above orbits, with the rudiments of a thirel bunch in front of these, 3 ou top of head, behind orbits, 2 or 3 bunches just bolow these on side of head, 2 or 3 single cirri on margin of preoperele, a thick bunch above the preoperenlax spines, 4 or 5 on lower margin of opercle, with a thick bunch on its npper margin; a short row above tho baso of the pectoral: a row of bunched cirri along anterior two-thirds of lateral line, another welldefined row along the dorsal fin from the third spine to the sixteenth or seventeenth ray of soft dorsal, this row containing a bunch at the lanse of oach spine and ray, with the occasional exception of the first ray; is or 6 scattered bunches between the dorsal and lateral rows on each side of body; a cirrus at the tip of each dorsal spine.

Color, light reeldish brown, sometimes almost pink, thickly spotterl with fine indistinet white spots; 4 or 5 irregrular dark brown spots along the base of dorsal, a band of same color along lateral line, sometimes very much broken and extenting ventrally, shating into the miform redelish brown below, and including 3 or 1 roumel pinkish spots; a dark hrown post-ocular line, another running forward from the eye, a patch of same color on top of head, another on side of head, and 2 or : 3 on edge of operclo; throat reddish brown with several distinct white spots; lolly bluish green; a silvery white patch hetween the bases of the pectorals: dorsal fins pale reddish brown with black and elear spots; pectoral 
croswel irmegnlarly with whife; anal lin pale pink, crossud with fark brown. There are 2 or :3 perfectly distinct types of coloration, as fullows: simme spereimens from pouls enutaining green alga aro puro lighle green, others from coralline pools are tiuged with lavender, as li. cmerynom. This squecies resembles most closely (), meculosur, which mame has heen erroneonsly applinel to it, but it clithers markedly in its slenderer bouly, more pointed suont, the arrangenunt of the cirri, and the pertecty distinet coleration, alsu in the greater length of the dorsal fins, the culargement of only une anal ray in the male, and the shortness of the maxillary. Coast ut Califurnia.

specimens are at latud from Crescent C'iry, C'al., Bulinas Bay, Mall Mom lhay, Monterey lbay, and Sin Luis Ohispo, Cal. Fomm in all kinds of pools, from sim Francisen to Monterey $13 a y$, lust now hero common. Length (i) man. The most luantilul and artive of the tide-poul tishes, extremely varialileincolor. (Greoleg.)

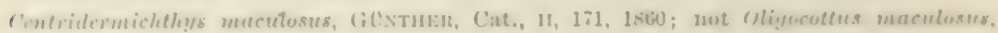
liiraril.

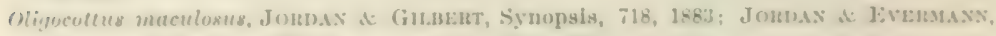
Finlues of Xurth aml Milllle America, 11,2013, is9s.

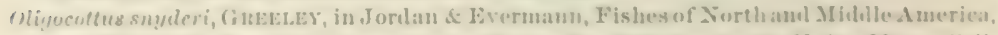

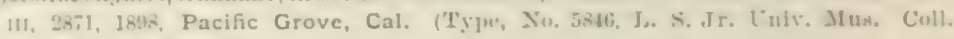
(ireceley A Mtahluren.)

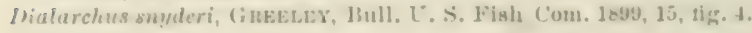

946 (c) EXIMIA, cireeley.

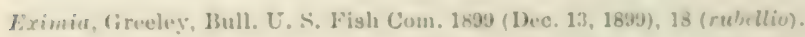

Allied to Oligocottus, but difforing in the presence of a largo threepointed preopercular spiue instead of the simple forked spine of oligocot. lus. Skin smooth. A slit hehiud the last gill.

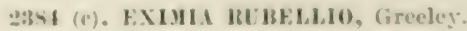

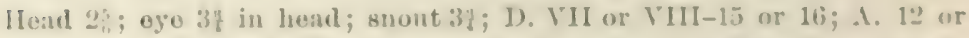
1:3; 1'. 13 or 14; V. 1, 3. Borly compressed, snont pointen amd compresised. leal deep, oceiput narrow, slightly roncavo; inferorlital space narrow, i. the largo eye, shallowly grooved. Nasal spines prominent, very large and pointerl. Teeth small, pointed on jaws, voner and palatimes; jaws cpual, month horizontal, maxillary" 3 in head, reaching a vertical below anterior enlere of pupil. Margin of preppenelo armed with a very strumg spine as long as eye, extending laskwarol and downwarel, bearing on its npprer surface a seeond and thirel spine, both pointing lack and up; all the spines covered with skin in life; operele enting in a rounded tap̧.

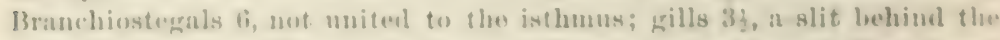
last gill. Aual papilla ineonspienons. Dorsal fins not joined ; dirst dorsal boriuniug in alvanee uf marein of aperele, tirst "2 spisues short, the "1pper margin slightly romoled; soft dlorsal hoginging in advanee of origia of anal, all the rays and spines very slender, pecesal reaching well beyoud thes wrigin of the anal; anal tiu small, thes rays slender. the mem-

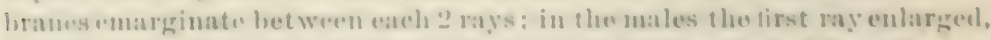

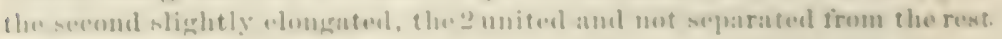


of the fin, as in Dialarchus snyderi; ventrals situated below the upper edge of the base of the pectoral, just reaching the anus. Cirri all distinct, never joined at the base in a coml) as in Dialarchus snyderi; 3 pairs of 2 or 3 cirri each above the orbits, the first directly above the nasal spines, 3 pairs on top of head behind orbits, a few scattered cirri below these on side of head, a bunch of 2 or 3 on end of maxillary, a row on lower margin of preoperele, a large bunch above the preopereular spines, and several seattered eirri on margin of opercle; a row of bunched cirri along the anterior half of lateral line, a row along the base of the dorsal, including a bunch of $3 \mathrm{or}^{\circ} 4$ for each spine and ras, the row bending downward at the last spine of the first lorsal, leaving a space between the cirri and the base of the dorsal spines; a few scattered cirri between the dorsal and lateral rows, and below the lateral line behind the pectoral fin. Color light brown to all shades of light red, pink, or lavender, spotted exerywhere with white, the spots extremely minute on clorsal half of horly, luit more conspicuous ventrally; 5 wedge-shaped spots of dark brown along dorsal side of body; head dark brown, sometimes hotehed with red or green, becoming lighter on side, leaving a dark postocular line extending from eye to the preoperenlar spine, and a dark spot on lower marwin of preopercle, everywhere very finely marked with white and blue; a white spot with a brown center just in front of first dorsal; throit and belly a very light, bluish green, shading into a faint yellow hehind peetoral, and a brownish green on each sicle of anal; all the fins, excepting the rentrals, light brownish green barred with dark brown; candal light reddish brown, anal and tip of peetoral tinged with pinkish. A young individual is lighter and more brilliantly colored. This species is most closely allied to Dialarchus snyderi, from which it differs in the presence of a third preopercular spine, the greater depth ambl comparative length of the head, the larger eye and nabal spines, and the arrangement of the eirri. Many other specimens taken at Monterey Bay, but it is not recorded from any other locality. 'The most brilliantly colored of tho ticle-pool fisheś, inhahiting only the deep pools rich in plant life. (rubellio, a rosy one.)

Eximia rubelliv, Gineeler; Bull. U. S. Fish Com. 1899 (Dec. 13, 1899), 18, fig. 5, Pacific Grove, Cal. (Type, No.G066, I. S.Jr. Unir. Afus. Coll. Greeley it Maluren.)

Page 2027. Gillherlina, Jorlan \& Starks, is preocenpied in Lepidoptera. For its use in ichthyology Dr. Berg las substituted Gilberlidia.

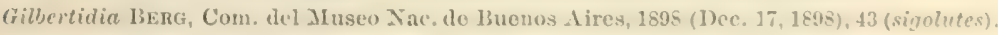

\section{Page 2207. Ater Sicydium vincente, Jordan of Evermann, insert:}

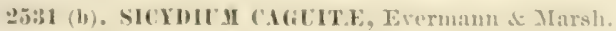

Hearl 4.4; depth 4.8; eye 5.75; snont 2.5; maxillary 2; mandihle 2.75 : interorbital width 3 ; preorbital 3.5 ; D. VI-I, $10 ; \Lambda$. I, 9; seales $83-25$; longest dorsal spine 1.5 in hearl, longest ray 2 ; longést anal spine $2 \mathrm{in}$ head, longest ray 2 ; pechoral 1.1 ; rentral disk 1.75 ; caulal 1.

Body rather stout, heary forward; head large, loroud; mouth large, its width 1.5 in head; lips very thich: maxillary not greatly produced; teeth simple, flexible; a median cleft in upper lip: pectoral somewhat shorter 


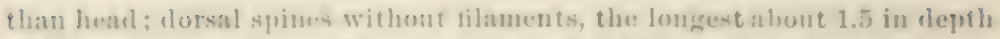
of lusty: space be"tween dorsals ahom erpual to orbit: foft rays of olorsal

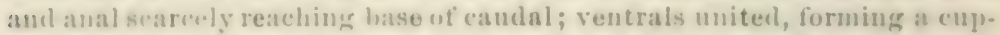
shapeel disk, only aluont two-fifths josturior edgo froe from belly; caudal rumulel. seales very small, retenoid, clensely coveriug entire boily except at lonal strip on loelly ; posturion portion of nape with very line seales; "ntire lue:ul nalient.

C'olon: llark hrown or olivaeeous on heal, side, and back; under parts pale; lins all pale, the anal with a narrow darkish margiu: cauchl someWhat dark: no dark vertical bass on looly and nowe at base of pectoral ; no H-shaped tigure at hase of eambal.

This species is elose to 8 . plumieri, from which it differs ehielly in the color, the sure cumplete squamation, the shorter peretoral, and tho nonfilamentons clatacter of the dorsal spines.

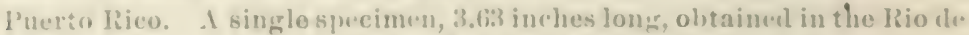
Cagnitas at Caguas, Jamuary !, 15!!), by the li.s. Fish Commission expedition to f'uerto Riro. (Xamed for the Riode C'agnisas, from which the type was obtained.)

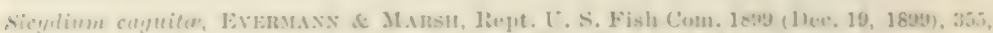

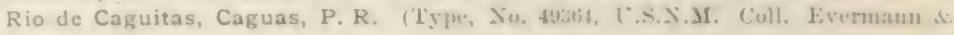
M:arsh.)

Page 2230. Aftr.r liobius ocennicus. P'allas, insert:

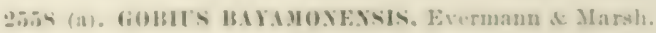

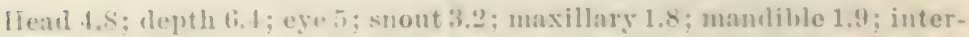

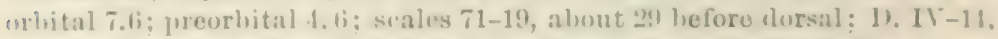
the longest spine alout 0.7 in hionl, the longest ray $1.5 ; .15$, tho longest ray 1.n; peetoral 1.1; ventrals 1.1; caudal very long and pointed. luoty very long and sleneler; bead long; candal pedunclo long; month very large. ohlifue; maxillary loug, reaching past posterior horeler of orbit.

Color as in 6 : occunicus, whirh this squeciey closely resemhles. The smaller

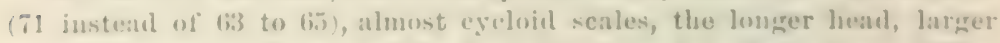
month, lomger maxillary, and tho longer am more slender hody aru ditherenoes which we can not reconcile with the deseriptions of that species or with the mumerous sperimens of it which we have from l'uerto hico.

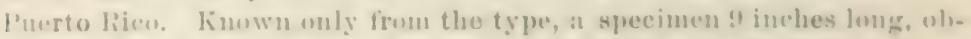

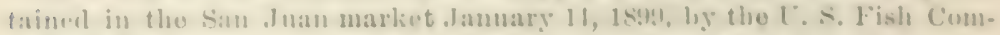
mission expenlition to l'ureto lice. It probahly ("ame from moar the momeh of the Hayanom liver at l'alo Seco, for wlich seremu the species was 11:1111.4.

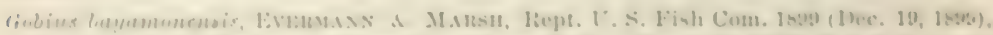

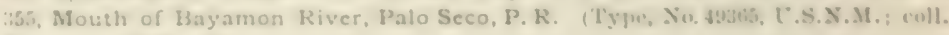
Livermusun \& . Murul,

Page 2218. 'Tu the symunguy uf finbius sopurutur ahl the following:

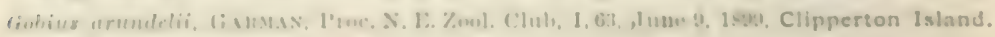
Trye in .I. C.\%. 


\section{Jordan and Evermann.-Fishes of North America. 318ă}

Page 2240. Before, Aboma, Jordan \& Starks, insert:

Q570 (a). BOLLMINYA BOQQUEROXEXSIS, Evermann of Marsh.

Head 4; depth 5.5; eye 3.5 ; snout 4.4 ; maxillary 2.2 ; mandible 2.5 ; interorbital wialth 3 in eye; preorbital 6 ; scales $27-8$; D. VII-13, tho longest spine 1.5 in head, the longest ray $1.2 ; A .12$, the longest ray 1.25 in head; pectoral 1 ; ventrals 1.1 ; caudal 0.4 .

Body long, slender, tapering; head short; snout blunt; month large, oblique; jaws snbequal, maxillary reaching posterior border of pupil; isthmus narrow, the gill-openings renching forward to below preopercle; eyes large, high, close together, the interorbital very narrow and without median keel; no fleshy process on inner edge of shoulder girdle; teoth on iaws in narrow bands, those of outer series somewhat enlarged; operelo short, about 3 in head. Fins moderate; origin of spinous dorsal slightly behind base of pectoral, its spines 7 in number, not filamentons; interspaco between dorsals less than diameter of eje; soft rays of dorsal aul anal reaching, when depressed, beyond base of eaudal; candal long and pointed, as in Gobins oceunicus; pectoral pointed, reaching heyond origin of anal; ventral disk moderate, free from belly, the longest rass barely reaching origin of anal. Seales very large, weakly ctenoid; nape, cheek, and breast scaled, the scales somewhat smaller than on body, about 9 scales before the dorsal.

Color: Pale oliviceous or straw-color, back and upper part of head with profuse tine dark punctulations; under parts pale, breast somewhat dusky; dorsal tius barred with white and dark, a large jet-hlack ocellus on posterior part of spinons dorsal; other fins pale, the ventral disk somewhat dusky in front. The only known Atlantic member of the genus.

Length "2 to 3 inches. Puerto Rico. Knomn only from is specimens dreilged by the U. S. Fish Commission experition to Puerto Rico, of P'nerto lieal, in Ensenada del Boqueron, January 25, 1899. (Named for the type locality.)

Lollmanuia boqueronensis, Evemunx of MARsm, liept. U. S. Fish Com. 1899 (I)ec. 19. 1899), 356, Ensenada del Boqueron, off Puerto Real, in 8.5 fathoms, at l'ish Hawk station 6074. (Type, No. 49366, U.S.N.M. Coll. Evermann d Marsh.)

Page 2245. Beforo Microgobius thalassinus, Jortan of (iilbert, insert:

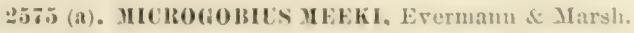

Head 3.75 ; depth 6 ; eye 3.5 ; suout 5.5 ; interorbital 7 ; prenrlital 7 ; maxillary 2; mandible 1.5; scales 55-12; D. VII-17; A. 16.

Body sleuder, greatly compressed, tapering regularly from pectorals to caudal; head moderately heary, interorbital space very narrow; eyo large, high ; mouth large, oblique; maxillary reaching posterior horder of orbit; lower jaw projecting; teeth in bands in each jaw, the outer series greatly enlarged and strongly recurved, those of lower jaw largest; isthmus rather narrow, the grill-openings continue forward.

Body densely scaled, the scales strongly ctenoid, those anteriorly somo. what reduced; nape, breast, and entire head naked. Origin of spinous 
dorsal from snont 3.5 in length: chorsils very close together; npines of firnt dorsal filamentons, exceediug loeal in length; solt dorsal and anal long. their hases aloout eipual, alout 2.5 in body, their last rays reaching past base of candal whes depressmel; cauchal pointed, its longest rays about equal to head; prestoral alont equal to head, reaching origin of anal; ventrals united, aluont reaching origin of anal.

Color: Light alivacentus, dusted wer unitormly with tine dark punctulations: al large datk shoulder spot bet wew the base of pectoral and or igin of spinous florsal; a few indistinct dark areas ou side or beal; lower jaw dark at tip: an olsenre dark bboteh at base of caudal; fins all rather pale except ventrals, which are clark, perhapes bhosh in life; candal somewhat dusky ; anal dark-eilerel. iength 1.5 inches.

This mecies seerns related to M. culepis, Eigenmann of bigenmann, deseribed from Fortress. Monroe, Va., but dillers in the smaller and strungly elonoil scales, freatly eompressed body, and in the coloration.

P'uerto lico; linown only from the type, 1.i) inches loner alrediged by the L. S. Fish Commission expedition to Puertn lieo, Febrnary 8 , 1899. (Xamed for Dr. Seth Lurene Meek, assistant eurator of zoology, Field (olumbian Musemu.)

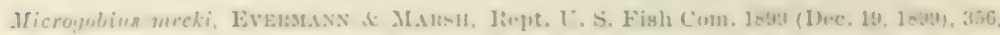
lw.twen Vieques and Culebra islands at Fish llawk station b0s7, in 15.25 lathomy.

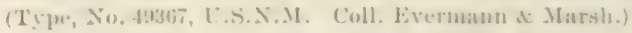

Page 2350. After linnunectes curminulis (Jordan d Gilbert), iusert:

$$
868 \text { (b). GILLIAS, Evermam of Marsh. }
$$

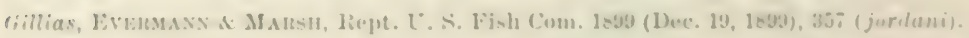

bouly shurt and stont, tapering rapiuly trom tho short, broal hend to the short, emapressed aturlal pechuclo; scales large, rough-cenoid: lateral lino emmplete, or nearly so, broken unler last spines of midelle dorsal; a brual, double-pointed tentarlo ahove eye; dorsal fin diviled into 3 parts, the first of 3 shert spines, the secourl of 11 longer spines, and the third of 7 rays.

This gremus is closely related to Eineanecles, Jorilan a Fverman, from which it dinters in tine presonce of the orbital tentacle, the more complete development of the lateral line, and tho larger seales.

(Xinumel for H). Theolore (iill.

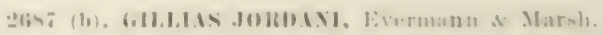

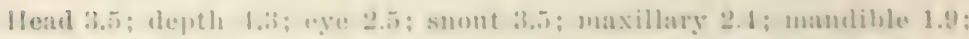

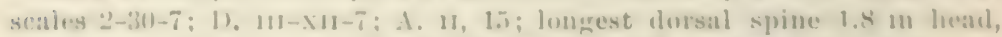

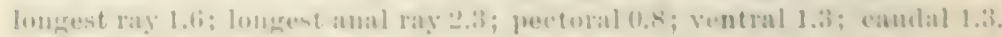

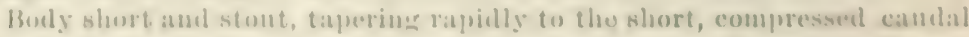

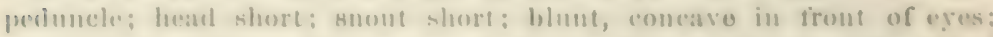

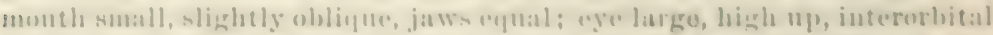

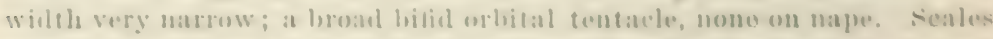

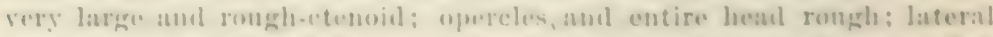

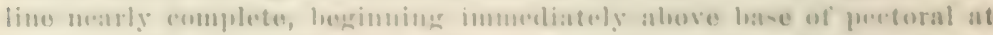

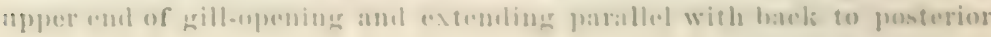


part of middle dorsal fin (or for 12 scales) where there is a break, the line dropping clown 3 seales, then continuing with one or two interruptions to base of eandal; belly and breast sealed; dorsals 3, the first of 3 short, flexible spines, close to the second, which has 12 longer, rather stiffer, spines, separated from the third by a space one-third diameter of eye; anal long and low, the membranes cleeply notcherl between the rays; pectoral of 15 rays, broal and short, reaching posterior end of second dorsal; ventral 2, slender.

Color in alcohol: Brown, body crossed by 4 broad blackish hars, one at the origin of second dorsal, one under last spines of same fin, the third between second and third dorsals, and the fourth under third dorsal; an inky-black bar across candal peduncle at base of caudal tin; head and under parts rusty; fins all barred with light and dark; caudal with a narrow light bar at base, then a black one, then a broader white one, followed by a much broaler dark bar containing some white areas, the fin finally tipped with white. Puerto Rico. 'T'wo specmens of this wellmarked and interesting species were obtained by the U.S. Fish Commission expedition to P'uerto Rico, the type 1.5 inches long (No.49368, U.S.X.M.), taken on the Carlona Light-Honse Reel, at Ponce, February 1, 1899, and another specimen of about the same size taken at the same place the preceding day.

(Named for Dr. David Starr Jordan.)

Gillias jordani, LVEkMañ \& Marsh, Rept. U. S. Fish Com. 1899 (Dec. 19, 1890), 357, Cordona Light-House Reef, Ponce, P. R. (coll. Evermanu \& Jarsh.)

\section{Page 2357. After Malacoctenus lugubris (Poey), insert:}

2696 (a). MALACOCTEILS CULEBHE, Evermann s Margh.

Head 3.35 ; depth 5 ; eye 4.2 ; snont 4.5 ; maxillary 2.2 ; mandiblo 1.8 : interorbital 6.5 ; scales $2-35-11 ; \mathrm{I}$. XXI, 8 ; A. $1 \mathrm{I}, 18$; pectoral 1.3 ; rentral 1.3 ; eaudal 1.4 .

Body slender, compressed; head rather long, pointed, upper profile convex; month large, the maxillary nearly reaching posterior horder of orbit: lips thick, jaws equal; teeth rery small, conical, a single row in each jaw; a single nasal, ocular, and nuchal filament; dorsal fin moderately high, originating above the origin of lateral line, a shallow notch in front of last two dorsal spines, the membrane free from eaudal; anal origin under about tenth clorsal spine; caulal somewhat pointed; pectoral large, reaching anal; rentrals molerate, not reaching anus, of two rays, no spine evident; lateral line rlistinct thronghout, ruming high anteriorly, where it is slightly curved, turning alumptly dowurard over the origin of anal, thence median to base of cambal.

Color in spirits: Body everywhere mottled with dark lorown, in somewhat regularly arranged blotelies, a serios of about nine of these at the base of dorsal, barely extending upon the fin; a similax series of much smaller ones at base of anal, not evident on all specimens: below the series at hase of dorsal are two other series of the same biotches less deep in color and not so well defined, extending the length of hody and sometimes forming, with the upper series, more or less hroken vertical hars: 
hetween the blot:hes a lighteg shate of brown is interwoven with pale strealis of gromucl color: head nearly pale below, save some dark on chin and isthuss; two wide streaks from eye across eheek; opercle dark brown; top of heal with the color of holy; lips with brown and pale tripes; posterior half of maxillary pale; dorsal rather darli; candial uniform gray or fantly barred; anil similar to dorsal in color; the rays with pale tips formiug a white edge; pectoral like eaulal; ventrals palo. Length alout 1.5 inclies.

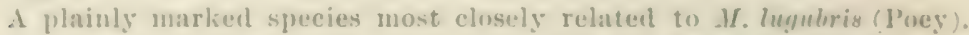
fonerto Rien; only 3 sprecimens linown, all from the coral reefs about ('nlelira Island, Fohruary !), 1s99.

(Ximed for tho typo locality.)

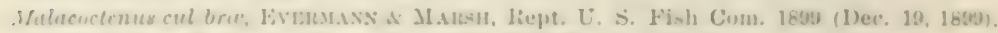

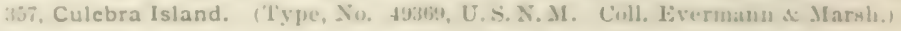

Page 2358. After Malacoctenus gillii (Steindachner), insert:

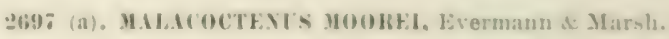

Head 3.6 ; depth 3.7 ; eye 3.5 ; snont 3.4 ; maxillary 4.5 ; mandible 4.5: inferorthital 4 ; scales $3-1.5-5.11 . x+1,11 ; A, n, 20$; pectoral 1 in head: ventral 1.2; candal 1.2; longest dorsal spino 1.5, ray 1.2; longest anal raty 1.5 .

Bohly short, rather stout, compressed; heal short, snont short, but puinted; month rather small, little oblipue, the galpe searcely reaching crloit: tenh in each jaw in a single series; gill membranes broally united across the isthmus; eyo small, interorbital spice widle; ciorsal omline rising abruptly to ahove oye, thence gently curved to origin of dorsal tin. and from there nearly straight to hase of eaudal tin; ventral ontline reegnlarly convex.

Color in aleohol: Light olivaceous, the borly crossed loy ahout 9 or 10 loroul dark vertieal bars, which extend npon dorsal tin, these usnally broalest above, the pale interspaces therefore broalest on lower half of body; the fourth from last is a narrow dark line, the one following it is a fouble spot, the next narrow and indistinct, the last, at hase of candinl, more distinct, followed ly 3 small irregular white spots: top of heal brown; side of heat with fine punetnlations; a dark line muning forward from ex", at dark spot below ey", 2 or 3 dark blotehes on anterior ealge of opercle: muler surfice of head crossed by 3 or 1 irrogular, indintinct dark lines; anulal and anal with tine dusky punctulations; pectoral and ventrals pale.

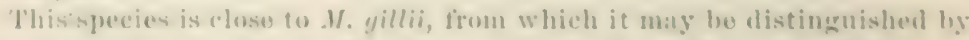
the larger dorsal and anal lins, the greater depth, wider interorbital, and the eroloration.

I'uerto Rieo: linown only from the type, 1.1 inches long, ohtanined lyy

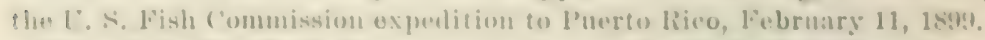

(Xamed for 1). II. F. Moure, natpralist un tho IT. S. Fish Commission stesumer . Illuatrows.)

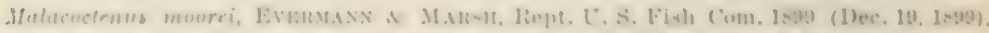

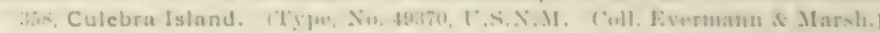


Page 2358. After Malacoctenus bimaculatus (Steindachner), insert:

2697 (b). IALACOCTEXUS IUERTOIRICENSIS, Eremann s Marsh.

Head 3.4 ; depth 3.4 ; eje 4; snout 3.5 ; maxillary 3.4 ; mandible 2.6 ; interorbital 7 ; preorbital 8 ; scales $1-44-8.1 \mathrm{D} . \mathrm{Xx}, 10 ; \mathrm{A} . \mathrm{II}, 19 ; \mathrm{I} .14 ; \mathrm{V}$. 2; C. 13.

Body short, stout, compressed; head rather long, suout long and pointed; month small, little oblique, the maxillary searcely reaching front of orbit; teeth in a single row in each jaw ; gill-membranes broadly united, free from the isthmus; eses high up, interorbital narrow; caudal perluncle short, compressed, its least depth about 3 in heal. Fins rather: large; origin of dorsal over upper end of gill-opening, first spine slightly shorter than second, which is somewhat louger than the third, whose length is about 2.2 in head; no notch behind thircl and fourth spines, all tho spiues from third to fifteenth being about erual in length, the sixteenth and seventeenth being somerhat shorter, tho remaining threo progressively longor; soft dorsal higher, its longest ray about 1.7 in heal; lougest anal ray 1.7 ; pectoral broad, 1.25 in head, reaching anal; ventral barely reaching origin of anal; a pair of sleuder ocular cirri, a small supraocular one, a short, slender, nasal cirrus and a few very slender ones at tho napo; scales large, not crowded anteriorly; lateral line well arched above the pectoral.

Color in alcohol: Brown, much spotted and vermiculated with darker; top of head brown, sides and under parts pale, crossed by about 5 broad, irregular brown bars; side of body with about 5 or 6 broad, dark crossbars, broader than the paler interspaces, broalest and darkest above and oxtending upou dorsal fin; under parts of body paler, moro speckled; spinous dorsal with ummerous small browu specks, a large, black ocellus on base of 3 anterior spines, aud a lirger ono on baso of list 4 dorsal spines, beiug chiefly on boly; soft dorsal, candal, and anal each crossed by several series of small brown spots; pectoral and veutrals pale, the pectoral with a few brown spots at base.

The above description from the type, a female, 2.5 iuclies long, obtainer at IIucares, February 14. 'I'hree female cotypes gotten at Fajardo, February 17, and one at Culobra, February 9, agree closely with the type; 2 of these, however, show faint traces of narrow horizontal lines alone lower part of side.

A male, 2.5 inches lowg, from Culebra, February 11, taken as one of the cotypes, may bo describerl as follows: Head 3.5; depth 3.7; eye 3.8; snout 3.2 ; maxillary 3.1 ; mandible 2.4 ; interorbital 7 ; preorbital 6.2 ; scales 3-15-9; D. xx, 10; A. II, 19; P. 14; V.2; C. 13; longest tlorsal spine 2 in lond, longest raty 1.1 ; longest anal ray 1.5 ; pectoral 1 ; ventral 1.1 ; caudal 1.1. Color in alcohol, tolerably uniform brown; crossbars on side vers faint; longitudiual lines more evilent than in the female; throat and under parts of head mottled with whito and light brown; fins less speckled than in female, the soft dorsal and anal pale, almost without spots.

Another male, 2.25 inches long, from Culebra, February 11, agrees with 
the largo specimen just deserihed, except that the crossibars on hody are more distinet.

This species most closely resembles M. bimaculatus Steindachner, from which it difiers in the litrer heal, greater depth, smaller mouth, nar. rower interorlital, and in the color. The tips of the anal rays are nut white, the Boft dorsal is spotterl like the camblal and anal, and there are no white spots on base of pectoral, as is said to be the case in .1 . bimurulatus.

P'nerto liko; known from the 7 specimens mentionerl above, ail ob. tained ly the l. . . Fish Commissim expention to b'uerto Kico.

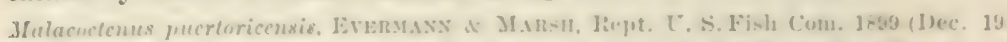

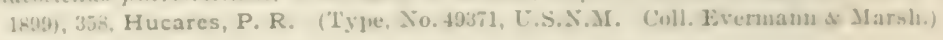

Page 2369. Before Auchenopterus, fiinther, insert :

\section{8 (a). AUCHENISTIUS, Evermann d Marn.}

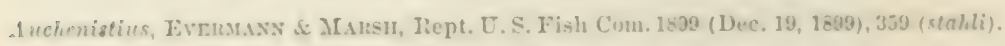

This genus has the form of Auchenopterus and surgests that genus strongly. It differs in the alsenese of a lateral line, in the much sualles seales, in the absence of a notch at the front of the dorsal fin, and in the nnion of the membrano of the anal fin with that of the caudal.

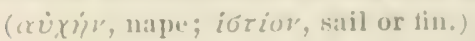

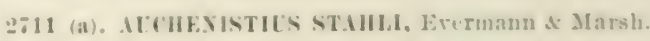

Head $\%$; deptl 6.5 ; eyo 4.8 ; snout 6 ; maxillary 2.8; seales alout is,

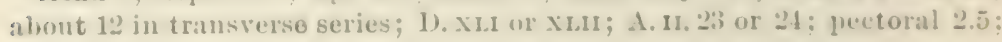
rentral 2.2 ; caludal 1.3 .

Body elongate, somewhat compressed, especially posteriorly, tho dorsal and ventral custlines alilie; heal small, up uer profile straight and descending; snout moderate, pointed; month linge, thos maxillary reaching to or beyond middle of eye; the jaws ofual, heavy and projecting: teeth in lower jaw conical, short and strong, slightly recurved, in one row; teeth in upper jaw similar to those in lower, hut a small prateh of smaller teef h in fromt of jall belsint the main row; teeth on vomer; gill-membrames foincil to the istlunus; nostrils with short tubes, a single thap abose ench oye and one on eateh siden of nape; dorsal tin long, of spines only; last four spinessomer hat lonere thon the preceding, forming a shallow noteh, a feature lacking in the other examples; anal orierin aloont midway between tip of snout and tip of camblal, the fin similar to dersal in shape. lut somewhat lower; membrane of dorsal and anal joined to eamial; caulal suall, pointerl ; pectoral suall, of s rays; ventral small, of 2 latys.

Color in spirits: Bedy ewerywhere with a very slight yellowish tinge, in some specimens a lathed grag̣: one specimen las tracess of 10 or 12 darls erosshars; fins all pale, in one ease with fle dorsal asd anal dark edged.

P'uerto Rieo; linown from the type, a specimen 1.2 inches long, whtained by the U.S. Fish Comminsion expedition to l'uerte licen, Fehruary 1. Is:49, at lence, aud $1: 3$ cotypes, 8 from the coral and algap on the reefs at the month of Culebra luarbor, and sirom l'merto lieal. 
("Named for Dr. A. Stahl, of Bayamon, Puerto Rico, who, under many difficulties put in his way by Spanish anthorities, made important collections of natural history objects of that island.")

Auchenistius stahli, Evenuavy \& JIArsir, Tept. U. S. Fish Com. 1899 (Dec. 19, 1899), 359,

Ponce, P. R. ('Type, No.49372, U.S.N.M. Coll. Evermann \& Marsh.)

Page 2373. Before Auchenopterus fasciatus (Steiudacher), insert:

2717 (a). ACHENopterus MbICIUDLS, Everman of Marsh.

Head 3.2 ; depth 4 ; eye 4 ; snout 4.1; maxillary 2.2; mandible 1.6 ; interorbital $5.3 ; \mathrm{D} . \mathrm{XXX}, 1 ; \Lambda . \mathrm{II}, 17$; pectoral 1.4 ; ventral 1.5 ; cauclal 1.6 ; branchiostegals 6 ; seales $1-31-6$.

Body rather short, compressed; dorsal ontline not elevated; hear moderate, not broad; snout short, pointed; mouth large, ol,lique, maxillary extending to below middle of eye; lips broad, prominent; a loand of conical teeth on each jaw, those on side somewhat enlarged and recurved; a pateh of teeth on voner, none on palatines; gill-membranes broadly united, free from isthmus; eye large, high up; nasal, supraocular, and nuchal regions with fringed tuft-like cirri; a considerable notch between fourth and fifth dorsal spines, but not reaching base of membrane; longest anterior spine scarcely as long as those of the posterior portion; scales large, reduced anteriorly; lateral line anteriorly separated from the dorsal fin by only ono scale; head naked.

Color: Uniform dark brown on head and body, no dark crossbars; dorsal brown, mottled with lighter, narrowly eilged with white; a black spot upon anterior 3 or 4 spines and a large black ocellus upou posterior portion of fin between twenty-second and twenty-fourth spines; anal rather darker, with narrow white edge; candal peduncle black, the fin abruptly white at base, the entire fin being elear white, entirely without specks; pectoral black at base, then barred with white and dark; ventral black at base, tho outer two-thirds barred with black and white.

This species seems to be related to the Pacific-coast species, Auchen. opterus integripinnis, which it closely resembles, but differs from that species in the larger scales, the deeper body, and tho coloration.

Puerto Rico; known only from the type, a specimen 1.5 inches long, obtained by the U. S. Fish Commission expedition to Puerto Rico, February 4, 1899 .

(albus, white; cauda, tail.)

A uchenoptems albicaudus, Evermaxy \& Marsir. Rept. C. S. Fish Com. 1899 (Dee. 19, 189?), 360, Arroyo, P. R. ('Type, No. 49373, U.S.X..I Coll. Evermann \& Marsh.)

9717 (b). AUCHExopteliUs livissckss, Evermann \& Marsh.

Head 34 ; depth 5 ; eyo 5 ; suout 3.8 ; maxillary 2.6 interorbital 5.8; scales $2-32-8$; D. XXX, 1 ; $\Lambda$. II, 18; pectoral 1.5 ; veutral 2; canclal 1.1 .

Body slender and compressed; head moterate, somewhat compressed above; snout pointed; mouth moderate, the jaws equal, the maxillary about reaching front of pupil; lips, especially the upper, prominent; teeth small, conical and sharp, in loth jaws, in a numerous pateh on front of 
npprer jaw, fewer un siles; in lower jaw loss numerons in iront, a long single row of somowhat ktronger tecth on sicles; eye not large; a suall nasal flap, and a 3-or 4-banched tentacle overeye and one at nape; seales rather largo and regularly arranged; dorsal lin with a notch leehind third spine, ancl with ono unhranched soft ray at its cul, the membrano joined to caudal: origin of anal under eleventh dorsal spine; lateral line as usual in Auchenopterus.

('olor in spirits: Everywhere a mearly miform facleal pink, save loreast and lower side of head, which aro paler; a small, inconspienous dark rousul spot on dorsil fin, at twenty-thirel and twenty-fourth spines, a little mearer lases than margin, and male up of very small hlack punctulations; indications of a yellow tinge on front of dorsal and hase of anal in life: tins otlerwise all pale.

Pnerto Rien; lnown only from the type, a specimen 1.3 inches long. ohtained by the linited states Fish ('ommission expedition to l'uerto live January 27,1899 . (rubescens, Jedlening.)

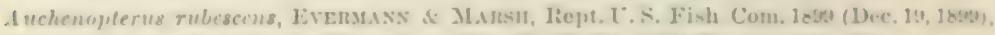
360 , Puerto Real, P. R. (Type, Xu. 4037 , E.S.X.M. Coll. Eve ruanu s Marsh.)

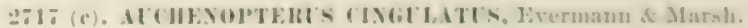

Ilear 3 ; depth 4.4 ; $2-29-7 ; 1) .1 \mathrm{~V}-\mathrm{XXIV}$, the longest spines 3 in heal; $\mathrm{A} .1 \mathrm{I}, 16$, the longest ray 2.25 in head; pectoral 1.3; ventral 1.8; caudal 1.6. Body rather long and slender, strongly compressed; head large, littlo compressed: snont uoderately sharp; mouth lavge, maxillary reaching posterior horder of eye, the lips heary, the jaws sulsernat or tho lower very slightly projecting: tentl conical and sharp. in more than one row in each jaw, most mumeron. in front; a patch on roner; a nasal tilament, a 3-or 4-branched supracocnlar tentacle. and a 4-hrancherl unchal tentacle, the hranches of the latter "ach with a dark dot on their anterior surface. Dorsal originating over edge of preopercle, of spines only, the second slightly longer than first: second, third, and fourth wraduated, the fourth comparatively short, thus forming a noteh partly separating the lirst i spines from rest of tin; dorsal membrane. joined low with caudal; anal free from caudal, about as ligh as dorsal, its thirteenth and fourteenth rays longest; first anal spine under tenth or elevonth dorsal spime: cambal rounded, shorter than head, of alout 13 rays; pectoral large, reaching anal, of 12 rays; ventrals moulerate, of 2 rays, the spune not ovident. Lateral line ruming high te cheventh dursal spine, here abruptly decurved two rows of seales, thence median to hase of eatutal.

('olor in spirits: Burly and heal pale yellow; leoly with theary darkbrown vertical hars, each about a rows of scales wille, extending on the vertical liss; mesubrane of anterior dorsal spines, opuercle, wecipital, and seapular region hoteloed with she same color; a dark har hack ward and

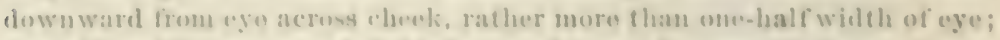

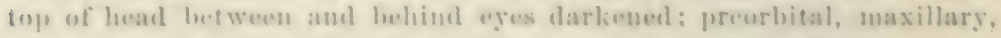
lips, and under part of heat thickly puncetulate with dark; dorsal and anat harred with the entensions of the wide dark boty hass, and with the 
alternating narrower pale interspaces; caudal mottled or irregularly barrer with grayish, its base with the plaiu pale-yellow ground color, which is sharply separated from the rest of the fin by a rurved dark line; posterior half of pectoral barred with dark formed of clots on the rays, the first bar plainest; basal half of pectoral pale; ventral with basal portion dark, the rest barreil like pectoral.

A pretty and strongly marked hlenny, known only from Puerto Rico, where 4 specimens were obtained on the coral reefs at P'once and 1 at Puerto Real by the U. S. Fish Commission expeclition to Puerto Rico.

(cingulatus, banded, from the conspicuons vertical bars.)

Auchenopterus cingulatus, Evermaxx \& MArsh, liept. U. S. Fish Com. 1899 (Dec. 19, 189!), 361, Ponce, P. R. ('T'ype, No. 49375 , U.S.X.MI. Coll. Evermann \& Marsh.)

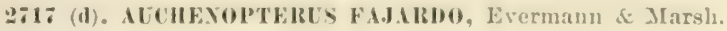

Head 3.25 ; depth 4.8 ; eyo 4.2; snout 4.8; maxillary 1.7; mandible 1.5; interorbital 5.5 ; scales $2-37-8$; $\mathrm{B}$. XXIX, 1 , the longest spine 2.3 in head: A. II, 17; pectoral 1.4; ventral 1.7; caudal 1.4. Body elougate, strongly compressed posteriorly; head moderate, littlo compressed; month large, tho long and slender maxillary reaching beyoud tho posterior horder of orbit; jaws subequal; teeth of upper jaw conical and sharp, in a patch in front, becoming one row posteriorly; teeth in lower jaw similar, but fetrer and weaker; vomerine teeth in two series. Nasal, ocular, and muchal tentacles present, all but the nasal about E-branched. Dorsal origin over edge of preopercle, the first 4 spines graduated, the fourth shortest, thus forming a noteh; dorsal ending with an unbranched soft ray, tho joints visible under a strong lens; membrave of dorsal joined low to candal; anal origin under eleventh dorsal spine and the decurved portion of lateral line; pectoral reaching past front of anal; rentral moderate, of 3 rays, the innermost shorter and slenderer.

Color in spirits: Body and head light reddish, becoming a little paler posteriorly; body with traces of 6 or 8 dark vertienl bars extending on the fins, their margins ill defined; hreast pale, 2 dark moldish bars downward and backward from ese across upper and lower edge of cheek to opercle; maxillary blotched with dark; upper lip and tips of both jaws dark; lower part of head spotted with dark; a row of ahout s small elark spots on edge of preopercle; iris pink: dorsal and anal tins gray, except for the extensions of the dark bars of the body and a few white spots on the dorsal; a distinet ocellus on the twenty-kecond, twenty-third, and twentyfourth dorsal spines and their membranes; hase of candal erray, like the ground color of dorsal and anal; posterior part of caulal with gray mottlings on the rays only, this portion separated from tho luasal part by a space withont pigment on rilys or membrane, making a distinct vertical bar; pectoral and ventrals mottled. Puerto lico.

A handsonels colored blenny, linown only from tho type, 1.63 inches: long, collected February 17, 1899, by the the L. S. Fish Commission expedition to P'nerto Rico. (Named lor the type locality.)

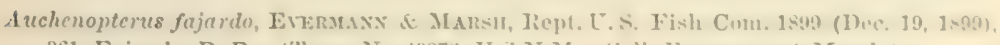
361, Fajardo, P. R. ('I'y je, No.49376, L.S.X.M. Coll. Lvermann A Marsh.) 
Page 2400. Beture (Jphimblemins, (iill, insert:

893 (a). CORALLIOZETUS, Evermamu \& Marsh.

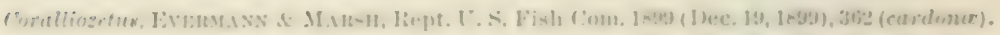

Buly slemeler and strongly compressed, withont scales; head larige, sul). rylimbieal, blunily pointed; mouth large; teeth mot hoolied, about \& enlarmorl conical ones in front of ear jaw, sualler ones behind; vomer with tereth; doisal tin with a noteh letween the rays and spines, the mrubrane connected with camblal; cambal tin romeled; pertoral large, ventrals small and insertul slightly in atrance of pectorals. A stronery marked genus, couspicuons in appearanco log jts heav lueal and thin buly, probaloly related to Ophiohlemins, from which it is technically separated l,y the absence of hoolied eanine teeth, the convex caudal, and the entire alusence of a lateral line.

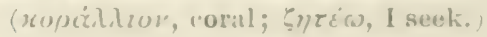

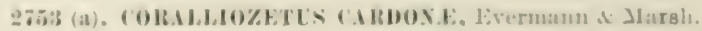

Heal 4; doplth 5.6; "yo 5; snout 4; maxillary 2; 1). XVII, 11; A. 21; pectoral 1.3; rentral 1.8 ; catulal 1.1.

Body scaleless, slender, much compressed ; hud large and heary, not compressed nor depressed; snout very short and blunt; mouth large, lıorizontal, low in position, the maxillary reaching far heyoul the eye; eyes suall, close together, placed high and well forwarl; teoth conical, in a patch on the frout of each jaw, an outer row uf about 8 teeth ( 1 on a side) in each jaw, much enlared; a single row of smaller teeth on sicles of cach faw; teeth on vomer; a small llap at tho nostril and two short filaments abovo eye, ono much the smaller; no appendages at the mape. Dorsal fin long and high, of slender. flexibles spines, and longer solt rays, a notch between the soft and spinous portions; anal longer and lower than soft dorsal; anal and dorsal free from caudal; caudal rouded ; joctoral lare, widle as body, reaching anal or beyond; ventral suall, juserted before pectoral, of 3 rays, the innermost very slomeler.

Color in spirits: Bouly dark rubl, much paler in one specimen; head every. whero iuky, this color dusted upou the body, particularly on the anterior !notion; a palo gray bax downward and hackward across cheok; fias pale, except ventrals and front of dorsal, which havo eolor of head; a row of small rosy sputs aloner lases of anal rays, seeuingly in the tlesh: sometimes a similar fainter row along lase of dorsal. Known only from l'nerto lico, where:3 specimens, each about 1 inch long, wero obtained in Fobruary, 1899, by the C. S. Fish Commission expedition to that island.

(Named for Cardoma, a little islet off J'laỵa de l'once, on the reef of which the type was colloepterl.)

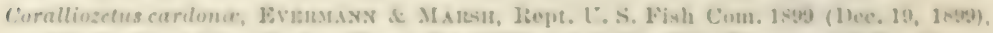

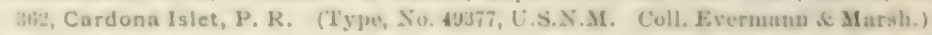


Page 2471. After Lycenchelys paxillus (Goode \& Bean), insert the following:

"S:33 (a). LYCECHELYS MUREVA (Collett).

Head nearly 8 ; depth about 20 ; eye 4.5; D. 112 (including one-half of caudal, 118); .95 (101); P. 13. Body everywhere scaled, head and fins naked; ventrals comparatively close together, 6 times length of pectoral; jaws with teeth in a single series. Lateral line present, but dificult to trace in its entirety; from upper angle of gill-opening a series of rather closely placed, very small pores (connected by a narrow line) descends in a curve to a short distance behind vent; after having reached nearly to base of aual, it runs for a short distance nearly horizontally, whereupou. it ceases, or can not be followed in the same direction with certainty; another lateral line, however, begins about over the vent, and runs horizontally along median line, becoming obsolete toward end of tail; the pores herein are very small and more widely separated, and can be seen only by close observation; the short whitish line which runs through each pore forms here no accompanying line. Length 7 to 11 inches. North Atlantic. Recently taken by tho Ingolf expedition in Dermark Strait.

Lycodes murana, Colletr, Lorh. Selsk. Christ., No. 14, 15, 1878, off Traenen, in Helgeland, Norway; Liitken, Ingolf Expedition, 20, 1898.

Lycenchelys murena, GOODE of BEAN, Oceanic Ichth., 309, 1896.

Page 2578. Chalinura simula, Goode \& Bean, was taken by the Ingolf in Denmark Strait in 912 to 1,236 fathoms.

Page 2587. Before Coclorhynchus, Giorna, insert the following:

2963 (a). MACBOURUS IX(iOLFI, Liitken.

The head is contained about 5 times in the total length. The superior or frontal surfice of the snont is separated from the inferior or more forwardly directed part by a well-cleveloped crest or edge, terminating in 3 spinose osseous tubercles, 1 directly in the middle and 1 on each side, close before the naked spot, where the nostrils have their place, aud continued both above and below the orbits. The broadly triangular snout is prolonged fairly over and before the mouth, which is relatively little, the corners of the mouth falling in a line with the anterior margin of the middle of the orbits. The eyes are large, their diameter surpassing the bread th of the front between the eyes. The teeth form a fine card in both jaws. The head is scaled with the exception of the gill-mombrane, the isthmus, and its foremost superior margin, and almost the whole lower surfice. 'The naked part of the snont is havdsomely embroidered with rows of slime glands. The first dorsal, counting $11+9$ rays, is singularly high and its longest (second) ray is serrate aud as long as the head. 'The second dorsal begins much forward, its foromost rudimentary rays may bo followed until not far from the posterior marein of the first dorsal fin. The pectoral contains 20 rays, and the ventrals, whose external ray tapers to a fine thread and reaches a long stretch beyond the anus, havo 8 rays. It may also be remarked that the tail, as in other Macrurians, is really

Bull. No. 47, pt. $4-\mathrm{XI}$ 
pointed behind, but in several specimens has lost a shorter or longer part ; but the wound has liealed, aud on the thus truncated point of the tail is developed a distinct caudal tin, a plenomenon which is also olsserved in some specimens of the precenting species. The scales show distinct rows of thorns, not lowever so much projecting as in M. goodei. (Liitlen.)

Several spocimens taken hy the Ingolf abont Iceland and in Denmark Strait.

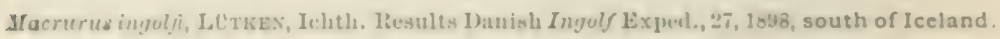

Page 2622. After Hippoglossina bollmani, (iilbert, insert the following:

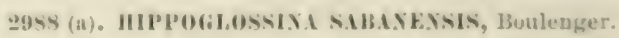

Head 1 ; depth $1 \frac{9}{5}$; eye 5 ; width of mouth 24 in head; maxillary reaching posterior third of eye; teeth small, more strongly developed on right side; eyes moderately large, the lower a little in alvance of the upper: interorbital space tectiform, scarcely balf the width of eve. D. $6 ;$ A. 63 . Gill-rakers very short, tuberculous, 6 ou lower arm of first gill-arch; dorsal commencing above anterior third of upper oye, its longest ray 2 iu head; left pectoral a little longer than right, 1 is in head; left ventral twice as long as right, equaling the head; caudal a little shorter than head, its free edge forming a wide angle, the midhle rays more than half louger than the outer; ilepth of candal peduncle twice it length ; seales 33-110-10, strongly ciliate on both sides; lateral line strongly arched alove the pec. toral. Deop gray brown; 3 large black ocelli bordered with bluish, 2 superposed on midelle of body, equally distant, hetween lateral line and vertical fin, the third on lateral line a little in front of candal peduncle; pectoral yellowish, ventrals blackish. Length $230 \mathrm{~mm}$. One specimen from Rio Sabana, Darion. (Bonlengor.) (sabanensig, from the Rio Sabaua.)

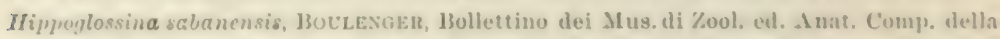
Univ. di Torino, Vol. xIr, Xo. 3\$6, \&, April:29, 1899, Rio Sabana, Darien. (Cull. Dr. Eurieo Festa.)

Page 2789. Villarius, liutter=Haustor, Jorilan of Evermann. All opecies of Imeiurns have villi.

Page 118. 1)r. Albert Giinther has recently eritically studied the Linsaran type specimens of American fishes in the possession of tho linnasu Society " th london, and the results aro published in the proceedings of that suciety for May 24,1 , 899.

These studies of 1)r. (iinther necessitate the following changes from the names adopted ly us in the earlier parts of this work: The Gaff-Top. sail cattish beroment

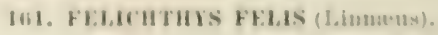

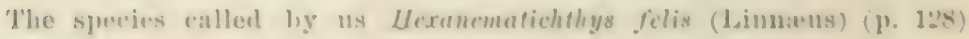
hecounes

146. (i)

'The lied Mind, givers hy us as lipinephelus moculosus (p. 115s), hecomes 
1553. EPIXEPHELUS GLTTATLA (LIMnRU⿻).

The spotted Jewfish, given as I'romicrops guttatus (p. 1162), becomes

155i. PROMIROP's ITALAR (Lichtenstein).

Page 261. After Notropis heterodon (Cope), insert:

406 (a) . Notropis CiLIENTIS Jordan \& Snyder.

Head 3.66 in length; depth 3.33 ; eje 4 in head; snout 4; interorbital space 2.66; depth of caudal perluncle 2.40; height of dorsal 4.66 in length; aual 5.75; length of pectoral 5.66 ; ventral 6 ; caudal 3.83 ; scales in lateral line 33 ; between dorsal and occiput 15 ; between dorsal aud ventral fins $10 ;$ D. $8 ; \Lambda .7$. Bodly deep and wide, deepest part anterior to insertion of dorsal; caudal peduncle long and slender; snout blunt, rounded; mouth oblique; jaws equal; maxillary reaching to a vertical from posterior nostril. T'eeth 4-4, slender, hooked, grinding surface narrow, absent on smallest tooth. Gill-rakers short, blunt; 9 on first areh. Intestinal canal short, with but 1 convolution; peritoneum white, scales large, not notably deeper than long; lateral line incomplete, extending only as far as posterior edge of pectoral. Dorsal fin rounded, the second, third, and fourth rays longest; anal fin similar in shape; ventral rounded, extending to vent, inserted directly under dorsal; pectoral rounded; caudal forked, the lobes ronnded.

Color silvery, an indefinite dark baud extending from tip of snout to end of caudal peduncle; the baud distinct ou snout and caudal peduncle, broader and almost obsolete on middle of body; top of head aud a narrow band on median dorsal part of body dark; ventral parts of body immaculate, except a narrow, dark band extending along base of anal and ventral part of caudal peduncle; chin white; scales on upper parts with dark edges; dorsal and caudal fins slightly dusky; other fins lighter.

This species is of small size, the mature males measuring about 34 mu. in length of body; the females 43 . The males are more slender and have a littlo less dark color on the body. The aftinities of Notropis calientis are with Notropis cayuga, jordani, and others of the subgenus Chriope. The species may be distinguished by its small eye, short, romded snout, deep body, short lateral line, and rounded tins. (Jordan of Surder.)

Lake Chapala basin, Mexico.

Notropis calientis, JoRdan \& Sxrder, Bull. U. S. Fish Com. 1899 (1900), 122, Rio Verde. at Aguas Calientes, Mexico. (Type, No._- L. S. Jr. Ĺniv, Mus. Coll.J.O. Snyler.)

Page 965. 'The generic name lihombus was first used by Humphress in 1797 for a genus of mollusks, and can not therefore be used in fishes. Its place in fishes will be taken by Peprilus Curier (1817). 



\section{INDEX TO NAMES UF FISHEN MEYTIONEII IS THE "ADDITIONAL ADDENDA" IN PART II.}

The following index includes only those names which occur in the "Additional Addenda" found in the present rolume. An index complete for the three other parts is given in Part III.

\begin{tabular}{|c|c|c|c|}
\hline agassizii, Chologaster ............... & $\begin{array}{l}\text { Page. } \\
3156\end{array}$ & Chirostoma album ................. & $\begin{array}{l}\text { I’age. } \\
3165\end{array}$ \\
\hline Agosia klamathensis ............... & 3144 & brere..................... & 3157 \\
\hline bicaudus, Auchenopterus.......... & 3191 & chapala ................ & \\
\hline album, C'hírostoma .................. & 3165 & crystallinum .......... & 3162 \\
\hline Algansea lacustris.................... & 3140 & diazi.................. & \\
\hline Alutera monoceros ................... & 3178 & estor................. & \\
\hline 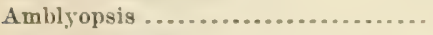 & 3157 & humboldtianum ........ & \\
\hline um.................. & 3158 & lerma ................. & \\
\hline 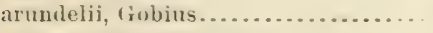 & 3184 & ocotlane ............... & $316: 3$ \\
\hline bei, Etheostoma............. & 3167 & promelas ............. & 3160 \\
\hline tius .......................... & 3190 & Chologaster ...................... & \\
\hline stahli $\ldots . . . . . . . . .$. & 3190 & agassizii.............. & \\
\hline alus ............ & 3191 & cornutus .............. & \\
\hline cingulatus........... & 3192 & papilliferus.............. & 3156 \\
\hline 10.............. & 3193 & Cichlasoma steindachneri ............ & 3173 \\
\hline ns ............. & 3191 & cingulatus, Auchenopterus.......... & 3192 \\
\hline n............... & 3173 & clarkii declirifrons, Salmo.......... & 3147 \\
\hline (................... & 3168 & jordani, Salmo ............... & 3148 \\
\hline . & 3139 & Coralliozetus....................... & 3194 \\
\hline (n.................. & 3149 & cardonæ $\ldots . . .$. & 319 \\
\hline ius............... & 3184 & cornutus, Chologaster.............. & 3156 \\
\hline Blennicottus globiceps ............... & 3179 & Cottus gracilis cayuga.............. & 3178 \\
\hline (2) & 3178 & crystallinum, Chirostoma........... & 3162 \\
\hline ensis ............ & 3185 & culebre, Malacoctenus.............. & 3187 \\
\hline ollmannia.......... & 3185 & Cyclothone megalops ............... & 3150 \\
\hline ................ & 3157 & declivifrons, Salm & 3147 \\
\hline (n. & 3183 & decoris, Doratonotus................. & 317 \\
\hline Calamus kendalli ................. & 3172 & 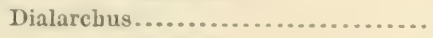 & 3180 \\
\hline um $\ldots \ldots \ldots \ldots . . . . .$. & 3152 & snyderi $\ldots . . . \ldots \ldots \ldots$ & 3181 \\
\hline (2.................... & 3197 & 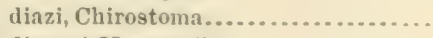 & 3161 \\
\hline tus ............. & 3194 & digueti, Neomugil .................. & 3165 \\
\hline us............... & 3175 & Doratonotus decoris................ & 317 \\
\hline ilis ............... & 3178 & encaustus, Characodon.............. & 3150 \\
\hline Centridermichthys globiceps ........ & 3179 & Epinephelus guttatus............... & 3197 \\
\hline maculosus ...... & 3182 & maculosus............. & \\
\hline $\ldots \ldots \ldots \ldots \ldots \ldots$ & 3166 & Eslopsarum arge $. . . \ldots \ldots \ldots \ldots \ldots . . . . .$. & \\
\hline chapale, $\mathrm{C}$ & 3159 & jordani ............... & \\
\hline . & 3143 & e-tur, Chirostoma................. & 3165 \\
\hline Characodon encaustus............... & 3150 & Etheostoma aubeenaubei .......... & \\
\hline
\end{tabular}




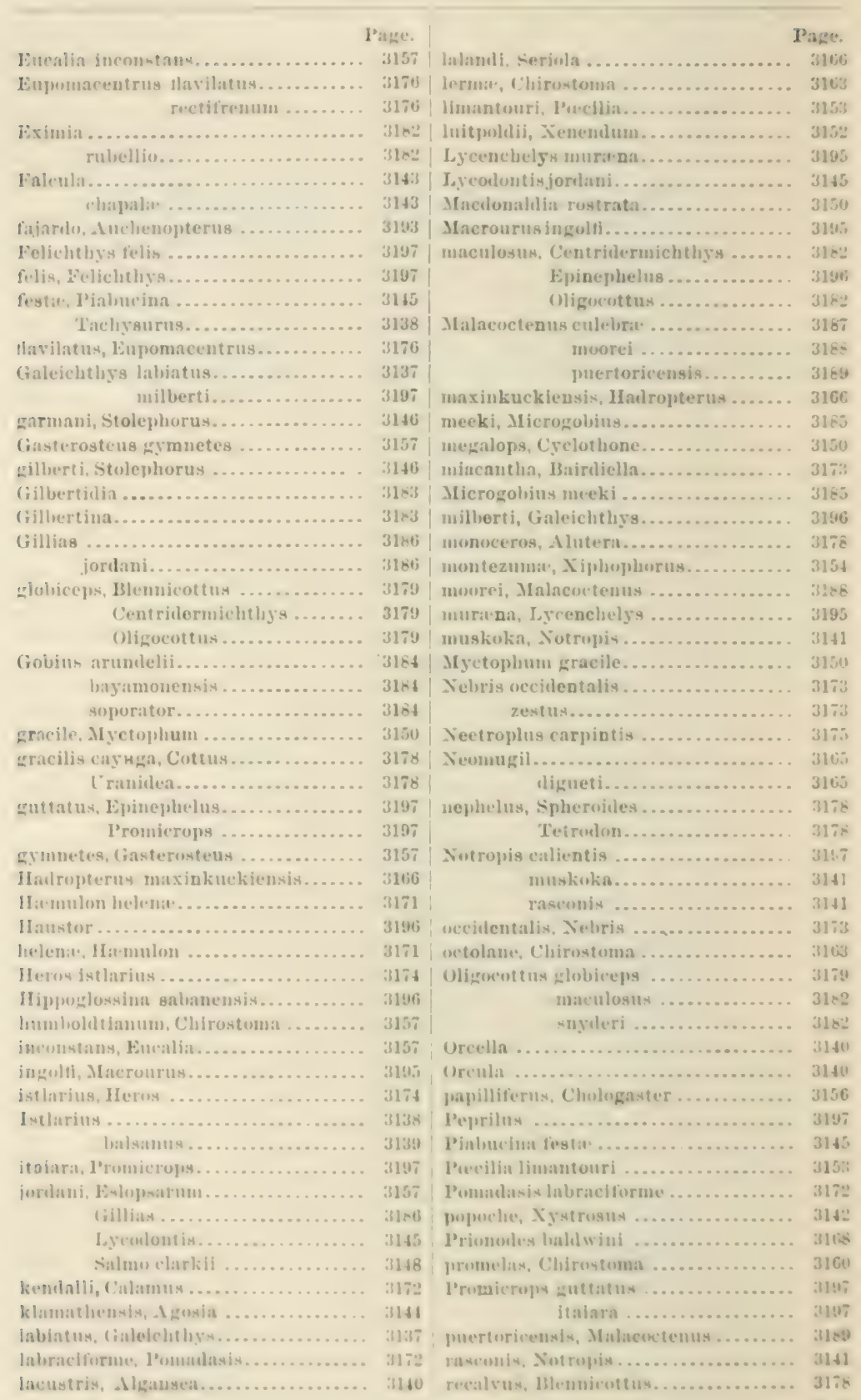




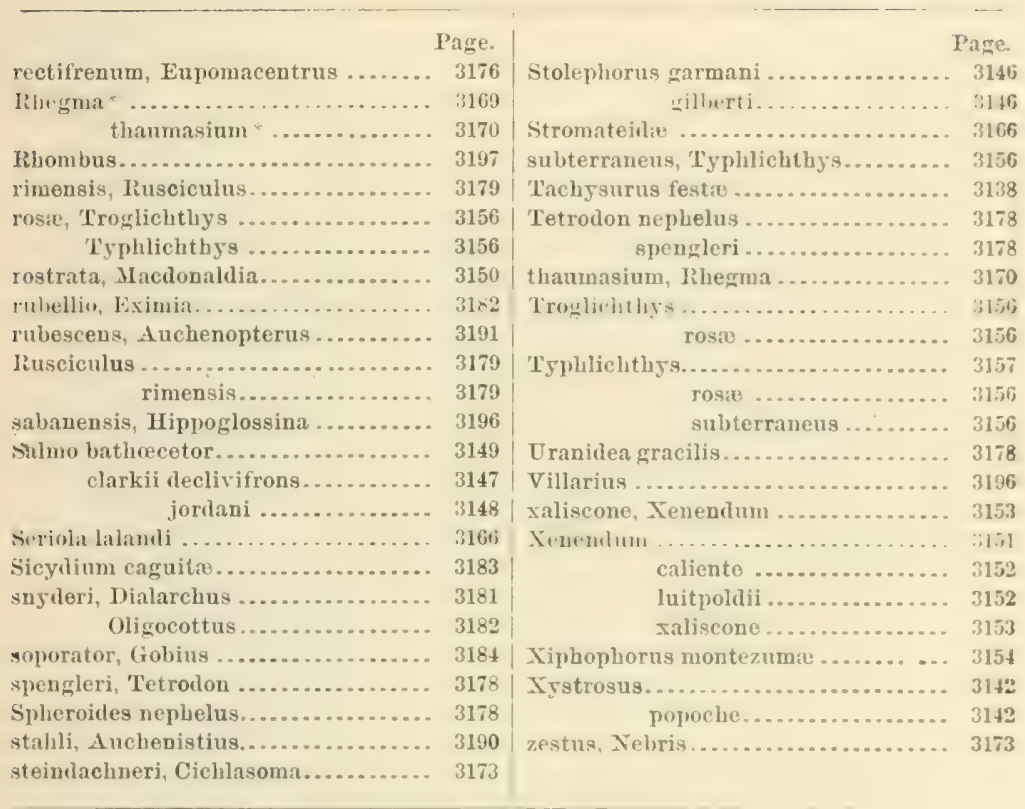

*These names occur as new in this volnme. 



\section{INDEX TO GENERA AND SPECIES ILLUSTRATED.}

Genera and species.

Abenna minima

Ahoma etheostomat.

(2)

然

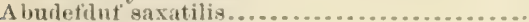

acauthias, Squalus.

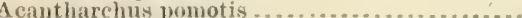

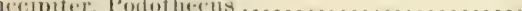

Achirus fasciatus

lineatus. . . . . . . . . . . . . . . . . . . . . .

Acipenser brevirostrum.

ribiculus mos

sturio oxyrhynchus a

trausmontanus.

acipenserinus, Podothecus

ackleyi, Rijia

-

aculeatus, Gasterosteus .................... Stenotimins

acus, 'Iylosurus..

Alinia rimentii

adscensionia, Eninephelu

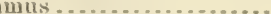

enens, Myosocephílus

asculapitis, dlepisaurus.

Fthourerin lincin:

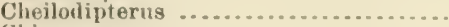

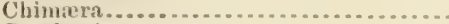

Gambusio .

agassizii, Alepocephalizs

(n)

Micromita

Liparis Sinichthis

aggregatus, Cymatogaster.

A conostomus monticola.

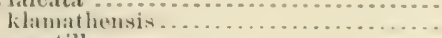
umatilla.

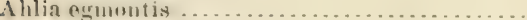

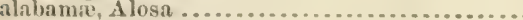
alalunga, Germo.

alafus. P'rionufus.

Kathetostoma.

albolineatus, Fundulus.

Albula vulpes.

all,um. Ilarmulor

Aleiden thoburni

Alepisaurus mesculapius. lerox.

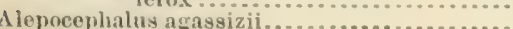

Aleposouи сореі. . . .
Plate and ficrure.

'lext

page:

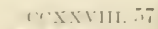

$14 ! 9$

6.9.

XIT, 111

Cстхх1V, $540 \quad 1361$

vi1, 24, 24a

CIV, $\$ 18$

(') (')

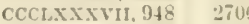

CCCLXXXvi.947 269

$\mathrm{XX1}, 47 \quad 1116$

Xxi, 46 108

$\mathrm{xx}, 45 \quad 10:$

x. $41 \quad 10$

CCCIX. $746 \quad 3061$

$\times 31 \quad 7$

xxxix.97 20

CLI, 408

cxix. $3 \geq 0$ 747

(r.ill it. 1340

Cхพr, $309 \quad 716$

cxvI1, 315 7:-

(vill, $24 \%$ bili

CLXXXI1, 482 11,

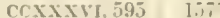

CCCLXi 892.892a 254

CCxev, 715, 715a | $14^{-}$

ХCVI. 258 .it

LIII, 136 - 19

LXXI, 190

xi 11,281

xv, хvт. $3 \overline{7}, 37 u$

Cxxvt, 342 al1

CLXXIX, 474 $111:$

NIx, 40

cxiII, 299. 399

LXXIV. 197 $4:$

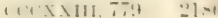

CCCry si4 ․․

(1) $1111.76, \quad 3121$

1. $11, \ldots-7$ 1 $12-7$

Cсххบแ1, 579, 579a $14 ! !$

CXXVII, 347 -

LIII, $135 \quad \$ 1$

1.II, 133 …

LII, 134 :11

SxII. $192,192 a \mid \cdots-11$

CXxxit, 367

11. $11,7,71=1$

сссхххт, $809,809 a \div 1 \%$

cr. 281

IXVII, 179 +11

1.111, 1.28 1:2,

CCLxxxit, 684 1․

хстII, 263 \$14

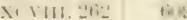

xсv1, 255

$\mathrm{xev}, 257 \quad 50$

L.XIV, 197 4

Lxxy, 199

32013 
Heusengis, I,ycomecten

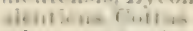

A lexurum armiger.

crext.

(1):4:1!, 711

(rex: $x v^{2}, 7 \in d$

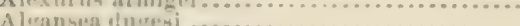

alleterata, (i yonnositrula . .

Alopias vulpes.............................

I lowa alabanas.

$$
\text { apritiona }
$$

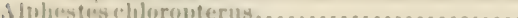

.lpinแa anreolus, Salvelimus. . . . . . . . . . . . .

attivelia, Sieloastolubus.

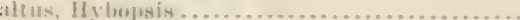

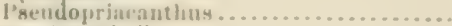

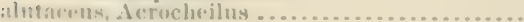

Alutera velingutio.

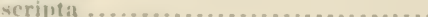

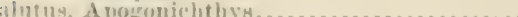

setrastudes.

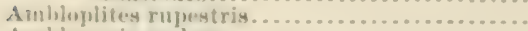

Aublyojusis spelariss

ambly melas . . . . . . . . . . . . . . . . . . . . . . . .

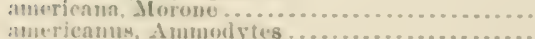
Hemitripterns . . . . . . Menticirrbus.

Polyurion

P'sendoplenrometes................

Imia calia

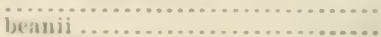

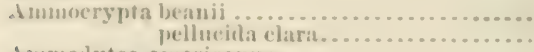

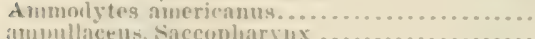

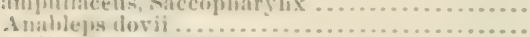

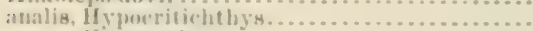

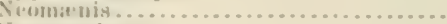

Votacanthus.

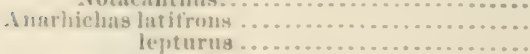

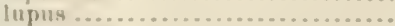

auctolon, Simenichthy

Aucy lopsotta quad rocellata ..................

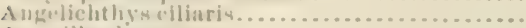

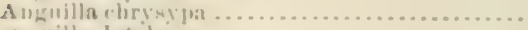

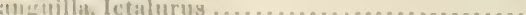

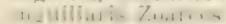

Inimotrenums hilimeatus

stisinan.

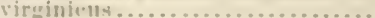

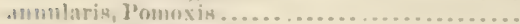

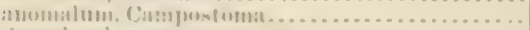

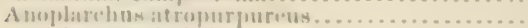

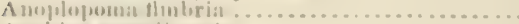

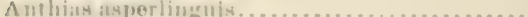

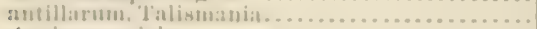

Antimora viola.

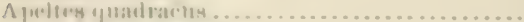

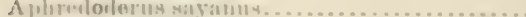

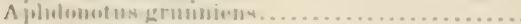

A poulielithye llavidus

aproulus, Divonaruls.

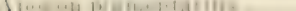

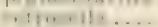

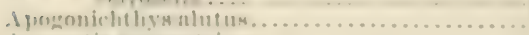

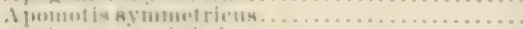

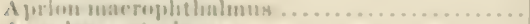

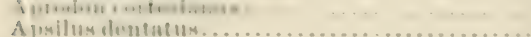

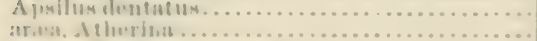

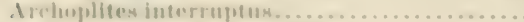

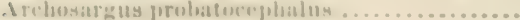


Archosargus nnimaculatus

aretifrons, Calamus.

Aretoscopus japonicus

Aretozenus coriseans.

(n)

arclens, Catostomus..

arenatus, Priacanthus.

argentatus, Gairdropsarus.

argentea, Bathyclupea

Steindachueria . . . . . . . . . . . . . .

argenteus, Larimu

Argentina silus

Argyrovelecusolfersi.

1.

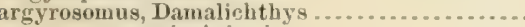
nigripinnis.

armatus, Leptocottus

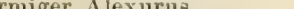

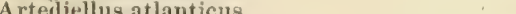

A scelichthys rbodorus ......................

ascensionis, Holocentrus

asper, Exerpes.

(n)

aspera, Limanda

asperlinguis, Anthias

Aspidophoroides gunther.

nonopterygills.

asprella, Crystallaria

asprellus, hadulinus

aspro, Hadropterus.

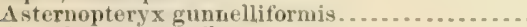

Astrolytes notospilotus .

tatronestlos gernmitor.

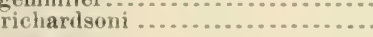

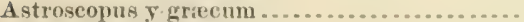

atchafilaye, Signalosa . . . . . . . . . . . . . . . . .

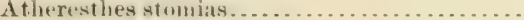

Atlierina arrea.

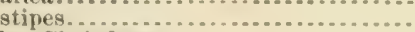

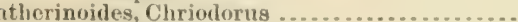

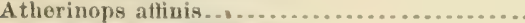

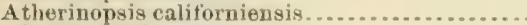

atlantica, Eblemmaria

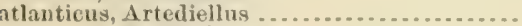

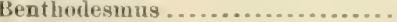

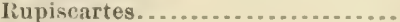

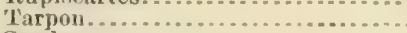

atripinnis, Goodea.

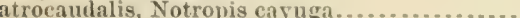

atromaculatis, Semotilus.

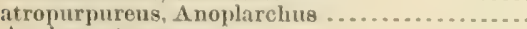

Auchenopterus nox

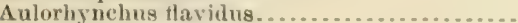

Aulostomus macula us.

aurantiacus, Hypolomus.....

auratus, Millus.

arreolıs, Salvelinug al

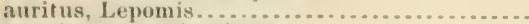

aurofrenatum, Sparisoma . . . . . . . . . . . . . . .

aurolineatum, Bathystoma.

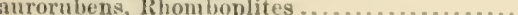

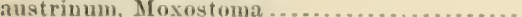

Auxis thazarel.

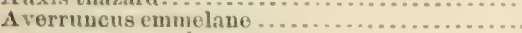

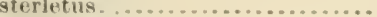

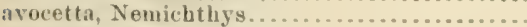

Avocettina gillii

axillaris, Myoxocephalus

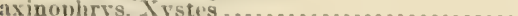

aya, Neomenis.

Azevia panamengig $=.$.

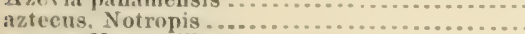

azurea, Hermosilla
CСхvч, 553

F(XIt, 5.)

CCCXXXIII, 807

XCVI, 259

CCLI, 623

XXXIV, 84

('X) 1,51$]$

CCCLxvir, 906

CxxIX, 352

CCCLXVII, 309

CCXXI, 565

Lxxxvir, 232

XCVII, 261, 26!

ccxx

LXXVI, 203

CсCII, 732

ccexxy, 784

CCLXXXY, 689

cCct, 739

CXXX1, 358

CCCxxxvit, 818

CCCLXXVII, 930

CXCIII, 507

CCCx11, 755, $755 a, 7552$

CCCX11, 756, 756

CLXXI, 453

CCLxxit 695

CI.XVI, 438

CCCXLIII, 834

CСLхехIт, 688, 688a XCIII, 251 XeIv, 252

CCCXXXI 5,808 LxIX, 184

(1) $1 \mathrm{xi}^{2}, 417$

CxxII, 333

cxxir, 332

CXVI, 310

CxxvI, 342

Cxxy, 341

CCOXL, 826

CCLXXXV, 68 ?

cxxxv, 374

cсcхxх1x, 825

L.XFII, 177

CXIV, 301

XLI, 114

$\left.\mathrm{XI}_{2}, 101\right)$

CССХนIII, 835

cccxxxv11, 819

cxx, 323

c.x., 324

cLxvill, 4 +2

cxxxII, 36i

II, 5

1xxxut, 220

CL1X $+25,+25 a$

CCXI.II, 6io

CCVII, 5in

CC, $5: 21$

xxxvH. 93

cxxxur, 3fis

cecix. 749, 7490

CCCX, 750, 750

LX, 15i

LIX, 154, 154a, $154 b$

cсхстा1. 721

$\operatorname{ccc} x, 75^{2} .752 \pi$

cхcrit. jif

CCcLxxxIV, 942

Xi. 112

CCxix, $55 s^{\circ}$
1359

135.5

601

601
1679

179

1237

2559

835

2568

1421

526

604
1509

472

3012

2203

1906

2025

$8+8$

2367

1227

2090

2091

1061

1920

103

$2+20$

1899

586

587

2800

790

790

719

807

806

2402

1906

887

2397

409
685

i)

202

2422

2373

iit

654

1040
856

13

$51 !$

1001

16:34

1310

127

81:

2069

?Uil

360

367

2801

1980

2076

1264

$267 \%$

258 


\begin{tabular}{|c|c|c|}
\hline lingera and squecien. & Plate and tigure. & $\begin{array}{l}I, \cdots \\
1=-1\end{array}$ \\
\hline azusens, Galeichthys ... ................ & xxit. 55 & $\because 7$ \\
\hline & ccxxxi1, sins & $1: i$ \\
\hline I: nal, & crevi, cCr.v1, 6aga & i... \\
\hline & ecx $x H_{1}, 000$ & $i:$ \\
\hline M1, & 1.Xv11, 176 & $\therefore:$ \\
\hline 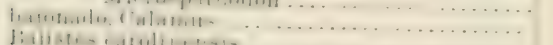 & $\begin{array}{r}\operatorname{cox} x x, 532 \\
\text { cexsit.544 }\end{array}$ & $1=$ \\
\hline 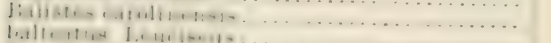 & 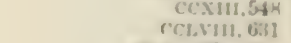 & $1+5$ \\
\hline 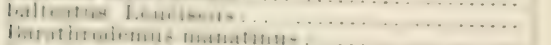 & $x 1.11,105,105 a$ & $:=$ \\
\hline 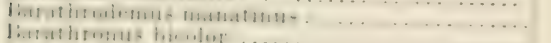 & CCCI,vII, 880 & $\because n$ \\
\hline 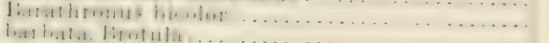 & cectoviti. ins & asi: \\
\hline 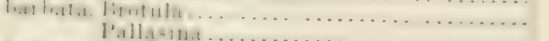 & Cectiv, o: & $2=-1$ \\
\hline & Cccillt, itt & $-1 ;$ \\
\hline 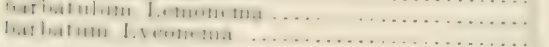 & CCeLXVt, gut & $-\cdots$ \\
\hline 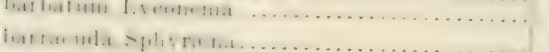 & CCCLI1, 80 3 & 26:: \\
\hline 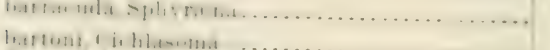 & cxxv11, 349 & $\frac{-3}{-3}$ \\
\hline 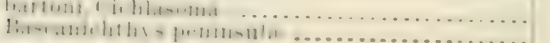 & ссххxи, 5月; & $i=15$ \\
\hline 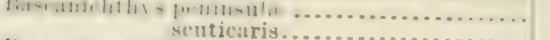 & Lxin, $166,166 n$ & 5 \\
\hline 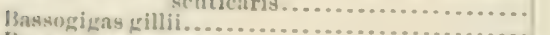 & I.x111, 1655 & $r-$ \\
\hline & 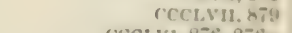 & $284^{-}$ \\
\hline & C'CtV1, 8it, 8iGa & $\therefore:$ \\
\hline 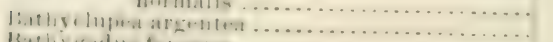 & cecL.vi, 675 & $=;$ \\
\hline & CxxIx, 352 & $-\cdots$ \\
\hline & ceclxvil, gus & 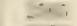 \\
\hline & CCCxI, 753 & -97 \\
\hline & Lxxxvแ1, 234 & $\therefore+$ \\
\hline & cecxxxit, 80: & $\ldots$ \\
\hline & ccexvill, 767 & $\because 1 .-$ \\
\hline & LXXxix, :3e & $\because 4$ \\
\hline & min. & $17:$ \\
\hline & 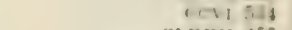 & 1. \\
\hline & ('t.xxit, 45 & $1: 4$ \\
\hline & cсcLxxvנl, (93: & $\because 4$ \\
\hline 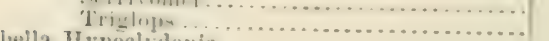 & I.V111, 153 & wi? \\
\hline & CCLXXXVII, 697 & $1,1,-1$ \\
\hline 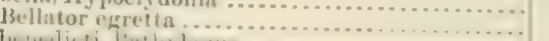 & ('1.xxנx, +75 & 1118 \\
\hline & crexxt. is & 2174 \\
\hline & Lxxxvil, 2:4 & - \\
\hline & cxxxvi,37t & 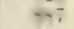 \\
\hline & Lxxxix, 237 & 84 \\
\hline & exxiv.33s & $\because \ldots$ \\
\hline & CCXLIh, 6U: & 112 \\
\hline & CXXX1,35i & -41 \\
\hline & CCLXII, 639, 639a, 638\%, & 172 \\
\hline & 12 & \\
\hline & $17.7 .1111-5 i$ & $\because 24$ \\
\hline & N1:.11: & $\therefore$ \\
\hline & 11, :41 & $\therefore 4$ \\
\hline 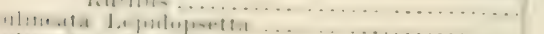 & A.411, 1. & $: 8$ \\
\hline 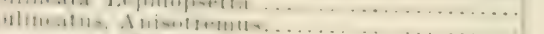 & $\cdots \mid x \times 11.0$ & $\div 4$ \\
\hline & $11111,4=$ & $13 !$ \\
\hline & - $: x, 293$ & $m$ \\
\hline 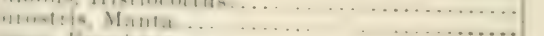 & 12.011 .79 & $: 11-$ \\
\hline & s.11: 0 & w \\
\hline & $\because 41,708$ & $12=$ \\
\hline vithatus lolitin & ExcIv, 5us & 1.14 \\
\hline 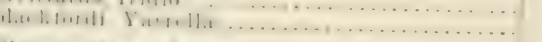 & $\operatorname{ccx} x \times 1 x, 601$ & $1 \div$ \\
\hline (1) & xeIII, 24! & -1 \\
\hline 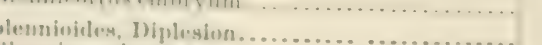 & crett1, 734 & 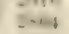 \\
\hline Bennims rintatua $\quad \ldots \ldots \ldots \ldots \ldots \ldots$ & CLxx, tty & 10. \\
\hline & $14 \times 185211-21$ & $\because$ \\
\hline 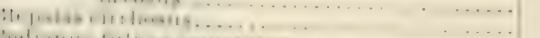 & corxxxvil, n:0 & $=$ \\
\hline 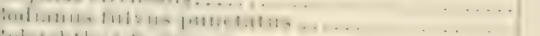 & ccert, 73u, 7ilia, 73th & $=16$ \\
\hline 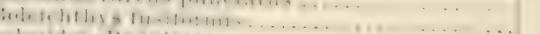 & $1+x \times x+1+-1$ & $1: 15$ \\
\hline 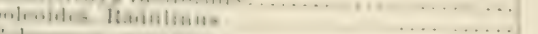 & $61 x \times 118$ ง & Int \\
\hline 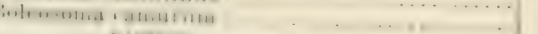 & 1. $1 \times 5 \times 11,64$ & 8515 \\
\hline 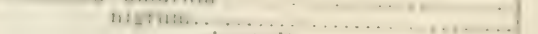 & 6151.4: & $1=1$ \\
\hline blinstedi $\ldots \ldots \ldots \ldots \ldots \ldots \ldots .$. & 6484 & $1.0^{\circ}$ \\
\hline 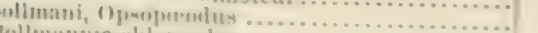 & $11 \times 1 \times 41$ & 1157 \\
\hline 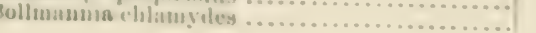 & N11. 110 & 211 \\
\hline & $\cdots \times x \vee 417.4$ & $\ldots 2=$ \\
\hline
\end{tabular}


bonaci, Mycteroperea

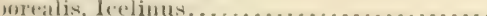

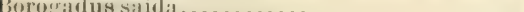

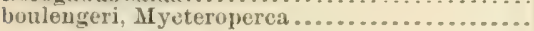
louvitri, silmo clatkii

brachiusculus, Granmicolepis Iirachy istius frenatus

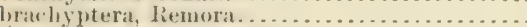

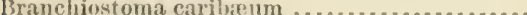

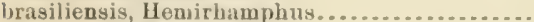
Nareime .......................... Seorluitila.

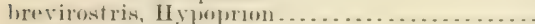
brevirostrum, deipeuser.

brevispinis Sebastole .

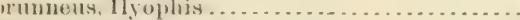

brown dentiex

Brivestema nugator

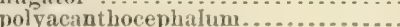

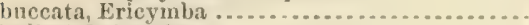

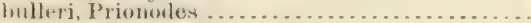

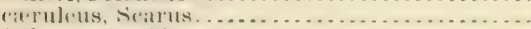
Calamms aret ifrums.

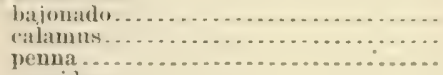

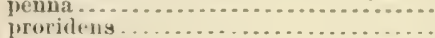

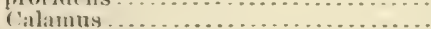
calamms,

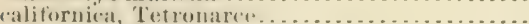

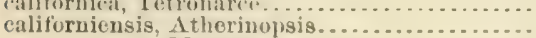
Mulialuna .................... Typlilogrobius ..................

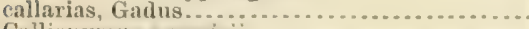
Cillionymus agassizii . . . . . . . . . . . . . . . ('allinris, Inglussin.

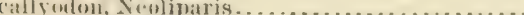

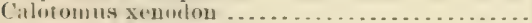

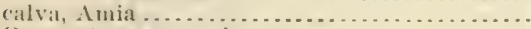
Campostomat anomahum . . . . . . . . . . . . .

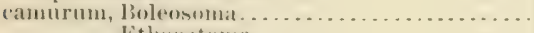
litheostrmiat ....

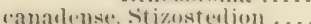
camalus, Rachycentron . . . . . . . . . . . . . . . canaliculatus, Ícolus.

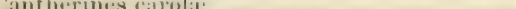
capistratus, f'hat tulun ... . . . . . . . . . . . . . (:)

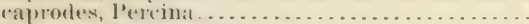
Carand irysos..........................

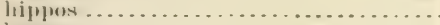
lintiis . . mertusicolit

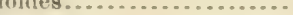

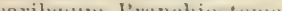

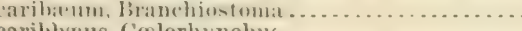
earibbens, Coelorhynchus ................. carinatus, Lainglatbs

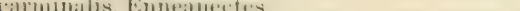

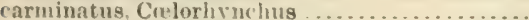

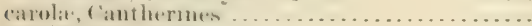

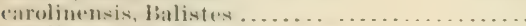

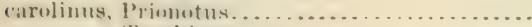

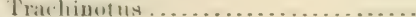
carpio, ('y primulon .... . . . . . . . . . . . . .

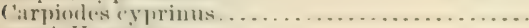

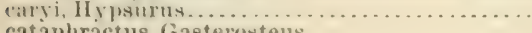

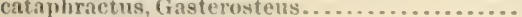

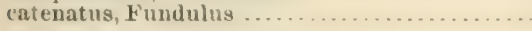

CLXxxyII, 492 CCLXXXIV, 687 CCCI.IX, 885 CLXXXVII, 490

Lxx. 212

CLII, 410 CCVI. 533 $\operatorname{coxxix,580}$ $\operatorname{cccxxx}, 797 ; 797 \alpha$

i. 1

CxVII, 313 XIII, 35, $35 a$ CCLXXVII, 669

, 18

$X \times 1,47$
$\cos$

CCLXXI, 657

CCCLIV, 871

LVI, 145

LV, 141

CCCxL1, 8ะ9

CCCXLI, 828

LI. 129

cxer, 503

CCxLTV, 613

ccxir, 55,

CCXIII, 548

CCxiI, 546

Ccxiv, 549

ccxill, 547

cCxH. 546

Lxi11, 164

ร11. $34,34 a$

CXXY, 34l

ccxx, 560

cecxisx, 795

CCCLXI, 891

CCCXXI11, 779

crcxili, 780

ссCу5 760, 760n ccxlit, 6e9, 609 a

xxir, 51,51त xxxIx, 95

CLXXI, 452

CLXXII, 456

rLXIV, 434

cxLrin, 401

CCLXXXI, 693

CCLYII, 632 CCL, 6:2 crsi. 543

Cr.sv, 43ti, 43 tio

CXLII, 386

cxIr., Bह7

C.XI, 384

CxIIII, 390

CCCLXII, 911

ร. 17

I. 1

ccctxx. 915

L.11, 155

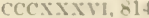

ccclaxix. 914

CCLYIII, $(3: 3)^{\circ}$

ceLvill. 633

состин, jos

cxLII, sด Cxi1, 2!? XII. il

сcхxх1, 385

CXIX, 3을

c15, 278, 278
117

,

117

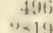

47

1:307

119

$2 \geq 7:$

i:2

i.

1,4

41

175

434

250

: 25

$3: 3$

$2+10$

$\because 41 \%$

: : 11

121.

1 tis.

135

$135 \%$

1:it!

$1: 3.5$

13.io

$1.3+4$

37.

206

$1: 3:$

...26

351

$21 \times$

21:1:

2111

16.1

11::

-115:

1060

107ti

1022

948

1917

1713

$16 \div 7$

1345

11126:

923

(120

9:1

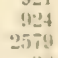

$3 x$

$25 \times 4$

368

'3.30

"yos

1713

17개

$\because 15$ i

944

tii 5

167

$150 \mathrm{~s}$

tif8 


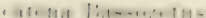

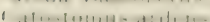

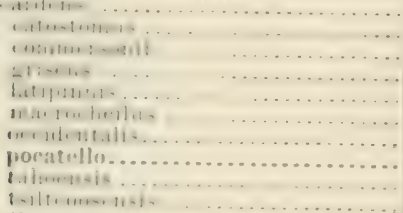

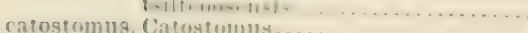

Catulus uter

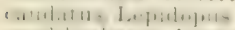

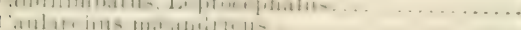

C.

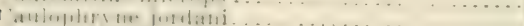

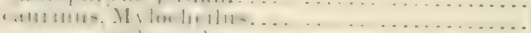
$\rightarrow \mid 1,1-1, \ldots\}, \ldots$

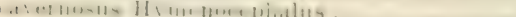

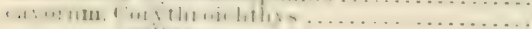
1)

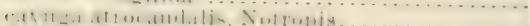

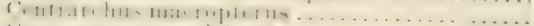

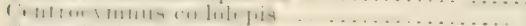

1. Hiruingluss nizer.

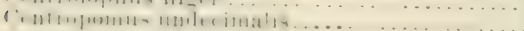

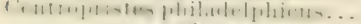

$-1+i a t 11-\ldots \ldots \ldots$.

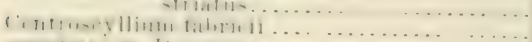

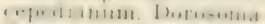

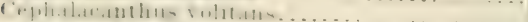

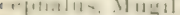

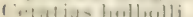

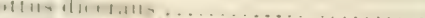

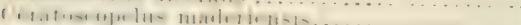

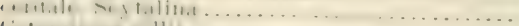

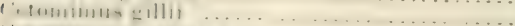

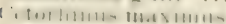

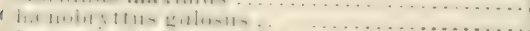

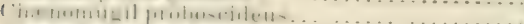

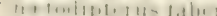

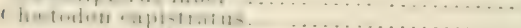

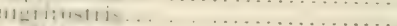
we liatns

chatochon, Jesorenistius .

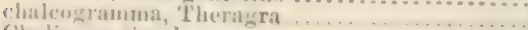

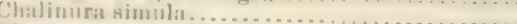

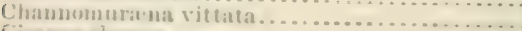

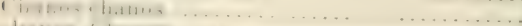

itatis

1 : kariatlls intin-1 Y.1. blowata.

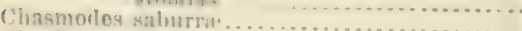

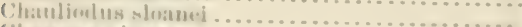

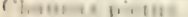

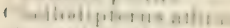

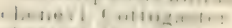

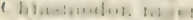

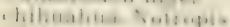

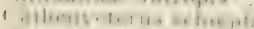

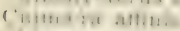

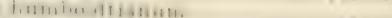

$1310+\backslash 1 p+1,1.9$

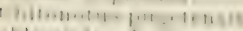

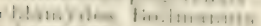

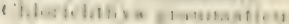

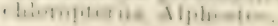

CCCLV1, 876, 876a

$\mathrm{Xx \times IV}, \mathrm{nt}$

$x \times x)_{1}, 77$

$\times \times \times 86,83$

$\times \times x 1,75$

$x \times x 1$, it

xxxu1, 81

$x \times x \mu 1,-9$

xxख11, 7t5

$x \times x ! 1,78$

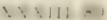

xxxis, \%

III, 12

сxxxv1, 373

1.N11, 145

сcсххх

cec:sxx, 7yd

Cc'Cxcl, $015 \%$ XL. 94

ccr.x. ccelaix, 91"

Cxx, 3:6

CCCL1, 873

XLVI, 114

$\mathrm{CLV}, 417$

V11เ, 25

CXLIX, 403

CLAXIX, 476

cxic, 501 1:01

vili, 20

LXIx, is . 46

cccxxm, 78, 218:

cxiv1, 34: 81

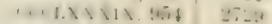

1, Yh

$x C, 242$

CCCXLVII, 849, $849 a$, 단

$2+5$.

$x \mathrm{C}, 2+1 \quad 540$

CLVIT, 4:1 !5!1]

cxxv11,340, 810

ccxlvI, 639 166,

('CI, 62: : 167\%

cexLrH1, 620, 1673

C'CXLIX, 6:1 | 1674

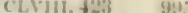

rechx, 887 1 353

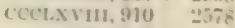

1 111 iी

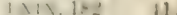

1 $: 19.16-19$ di:

$+18 \div-14$

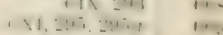

$x \times \times 7,8 ;=07$

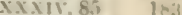

$x \times x y$ at " "ys

cecxxxix, 824 2019

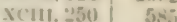

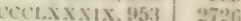

ChXXIX, tif 1113

CLXIX,445 25is]

cecl.xys, t493 | 25itp

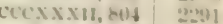

X14?, 116, "26.5

Coxvitis 175

xix, to ai

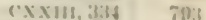

croxulv, 8:37 i ntas

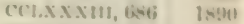

ccoxxvil, int 2:81-

crexte but lib!

CLXXXVI, t6s, to8" 1164

\section{9}

ti

178 itio $\operatorname{lin}$ 175

76

(a)

然象

is

is:

ind

sili

11


Chloroseombrus enrysurus....................

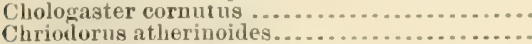

chromis, Pogonias

cluryeargyreus, Brachygent

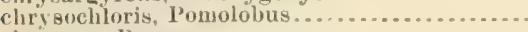
chrysops, Roccus

Stenotomus......-

chrysopterus, Orthopristis.

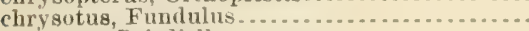

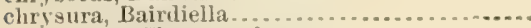

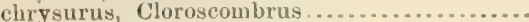

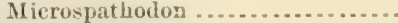

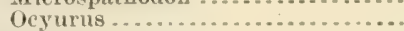

chrysypa, Anguilla

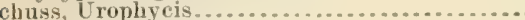

Cichlasoma bartoni.

ciliaris, Angelichth

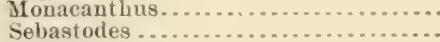

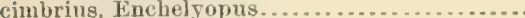

cinereus,

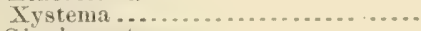

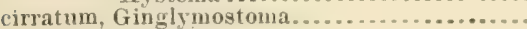

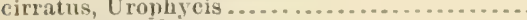

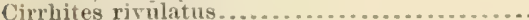

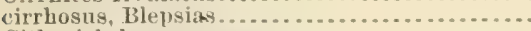

Citharichthys maerops.

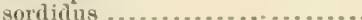

clara, Ammocrypta pellucida .................

clarkii bourieri, salmo..................

\section{henshawi, Salmo}

macionaldi, Salmo.

pleuriticus, Salmo

spilurus, Salmo.

stomias, salno

virginalis, Salmo.

Clepticus parre .............................

Clevelandia ios.

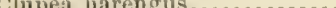

$$
\text { pallasii. }
$$
coccoremis,

centrocymnus.

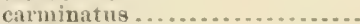
oeca

colian, sicomber ....

colliej, Irydrola rus . . - -

Colmmbia transmontamus. . . . . . . . . . . . . . . .

commersonii, Catostomus ...................

conger, Leptocephalus.

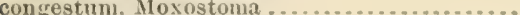

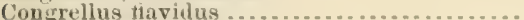

conej Aleposomus

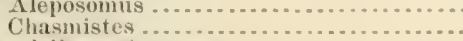

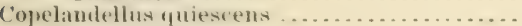

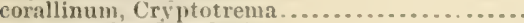

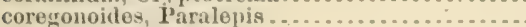

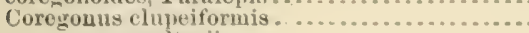
williansoni

cormubica, Lamna.

cornutus, Chologaster.......................... Zanclus.

cortozianus, iprodon

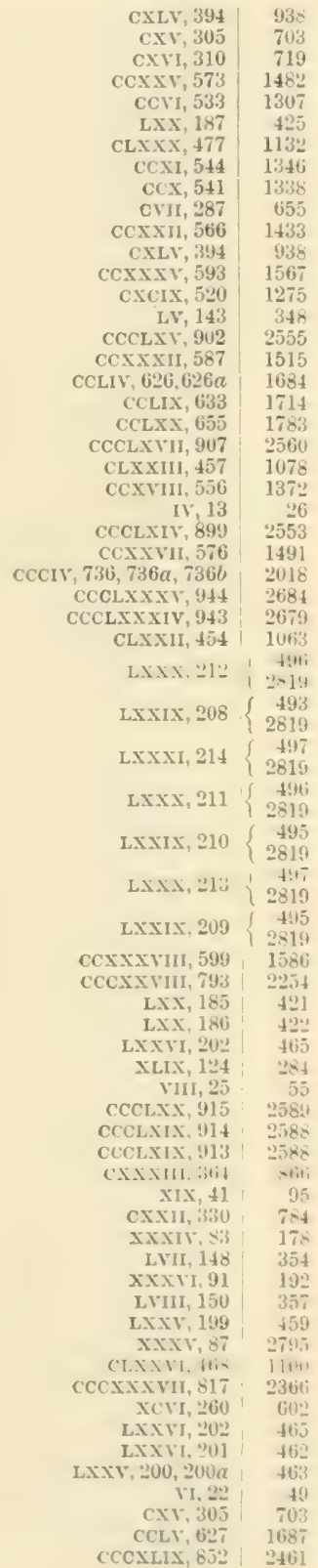


coruscams, Arctozenus

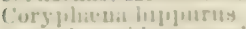

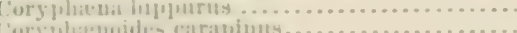

toteusastereliceney

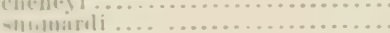

Contus alrutious.

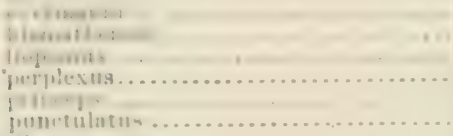

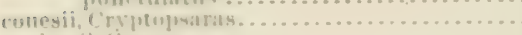

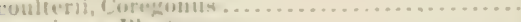

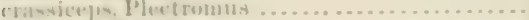

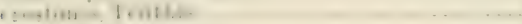

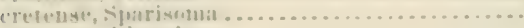

crivtutun, dile nnims.

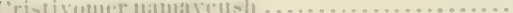

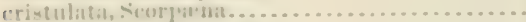

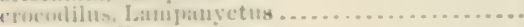

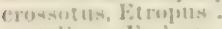

crutalinus, finlory

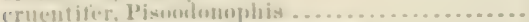

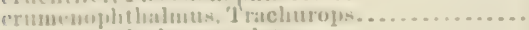

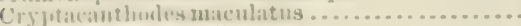

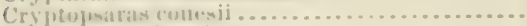

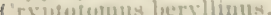

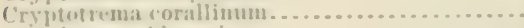

erjomlemsan, - Lliramis..

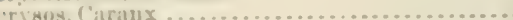

Cristullaria แ⿻

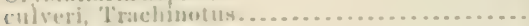

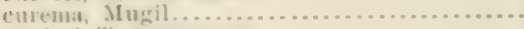

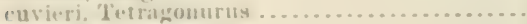

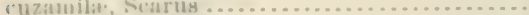

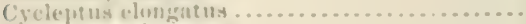

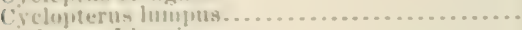

cielupus, Lipario.

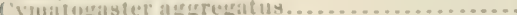

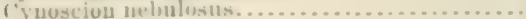
inglohy mothilst.

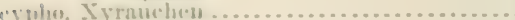

evitriantlin letiolons.

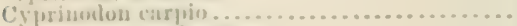

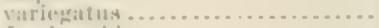

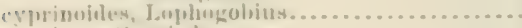

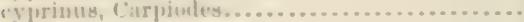

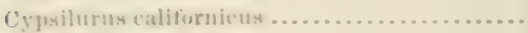

Dobllia peotoralis.

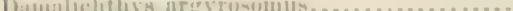

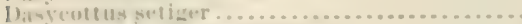

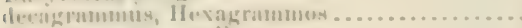

Desatelerus masarullus.

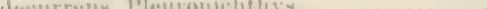

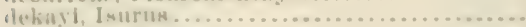

ilounyi, lajparín

duntilus, Aplisus.

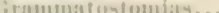

l'arallebethyo

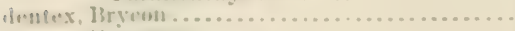
(1)+11\%:r114

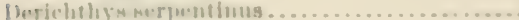

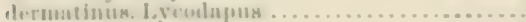

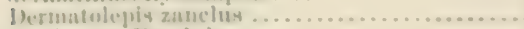

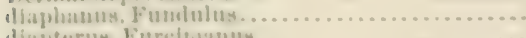

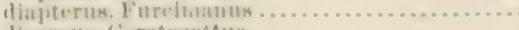

diceraus, ('i.rutoentlus.

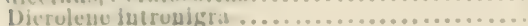

C.41

9::

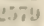

$20 \% 8$

$2=01$

1046

$19: 3$

$1=$

...

$1=$

1055

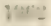

1945

$\because 731$

$+6=$

ris

tent

cxxx. 350

coxis ejleta

c:

[.xxx11, 217

CCLXXI, tibs

$x \mathrm{Cl}, 2+3$

recxixvi, 940

CeCXLVII, e51

$16 \times 11,163$

C.xh1, 385

cocxs? 843

cecre. 950

cexitu, tivg

cecxisil, bl

x1.5, 111

CXLII, JeN

CLXX1, +53

C.LFII, 397

Cxxy, ift CLII, +11

CCXLIV, 61:

xxx. $7=$

cociul, 730

(CеxvI, 764, 764a cсxxv11, 570, 5791

CCXXI, stis

coxx, 561

cexx. 562

$\mathrm{x} x \mathrm{x}, \mathrm{si}$

xix, 70

cxil, 247

cx1, cr11,

cecixv, 7pt

xxx, il

(खix, 310

xcix, 208

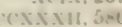

XIV, 30, 3in cec, $7: 7$

CCI.XXx, ti, bing CXL, 38:

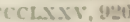

ช่, 3

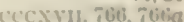

(10, $5: 3: 3$

$\mathrm{x}+1 \mathrm{x}, 254$

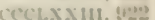
3. $1,1 \$ 1$

1.xxxy1, 2"29

LV, 15:

ccciss, $=80$

(1.

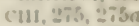
cect. 861

cexer, :me

crelestl, os? $x \rightarrow$

511

In 41

550

stiet

$245=$

374

(9)1

$24+3$

닌

1625

"3isti

$\simeq 51$

[0:3

1061

98

813

$4 \pi$

$10+10$

100

"enati

2118

$14: 5$

itos

If

$1+117$

181

$16: ;$

675

6i1

포

168

ifi

$6 \div 1$

1509 
Dicromita agassizii

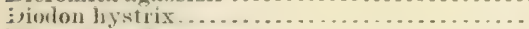

Diploetrum formosum

l)iplesion blemoioinle.s.

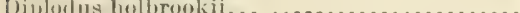

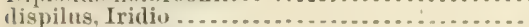

ilorlecatiron, (l)ea.

rolichogaster, Pholis

lolomieu, Micropterus -.......

Dormitator maenlatios. -

dormitor. P'hilymus...

Dorosoma cepédianum -

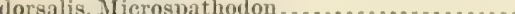

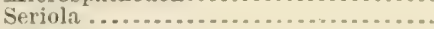

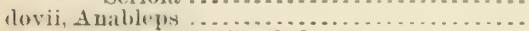

drummond-hayj, Epinephelus . . . . . . . . . . . . .

ductor, Naucrates.........................

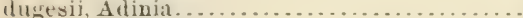

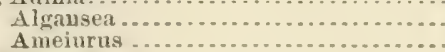

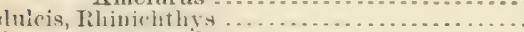

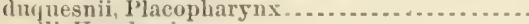

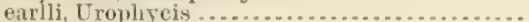

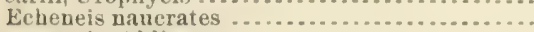

egmontis, Ablia.

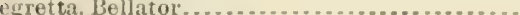

oigenuanni,

Sebastorles .........................

Elanura forficata.

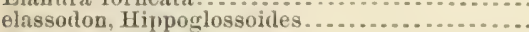

Elassoma evergladei

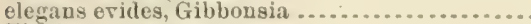

Elegiums navaga ..........................

Elentris pisonis ..........

olongatus, Cycleptus

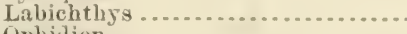

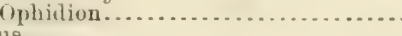

Elops saurus

Ensblemaria atlantica.

Amblematicus, Scarus.

smbryum, Blennicottus

Embryx crotalinus.

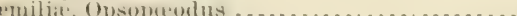

Emmerkia renusta..........................

emmelane, Averiuncus.

Hinpetrichthys merriami.

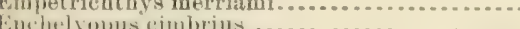

Euneacanthus gloriosus.

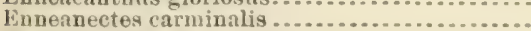

Eumblirys bisun.

Entosplienus tridentatus.

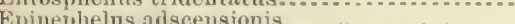

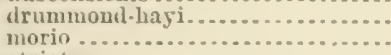
striatus.

Epinnula maristralis

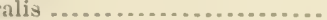

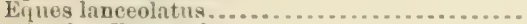

eriarcha, Eurystolo

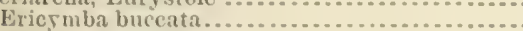

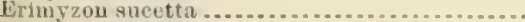

erimacea, Raja..............................

Erotelis smaragdus.

Escolar violaceus.............................

Eslopsarum jorkani

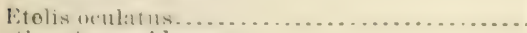

etheostoma, Aboma .........................

Litheostoma camurum ........................

\begin{tabular}{|c|c|}
\hline CCCLT, 874 & 2506 \\
\hline (1.XYl. 6it & 1745 \\
\hline (vi $1 . .80:$ & 12017 \\
\hline ("1.) $\times, 445$ & 1053 \\
\hline 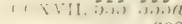 & 1362 \\
\hline CCXL, 603 & 1597 \\
\hline (29.6111. $7 t:$ & 2014 \\
\hline CCCXL11, 831 & 2416 \\
\hline CLAII, $430,430 a$ & 1011 \\
\hline rcexxIy, 783 & 2196 \\
\hline ( $(1 \times x), 7 \& 1$ & 2194 \\
\hline LXIX, 183 & 413 \\
\hline CCxxxivi, 594 & 1568 \\
\hline cxxxIX, 380 & 903 \\
\hline ('xi14, 3v0 & \\
\hline CLXXXIII, 484 & 1159 \\
\hline cxxx1x, 379 & 900 \\
\hline (1.111. 2!*3) & 661 \\
\hline$\times \check{L} L, 98$ & 211 \\
\hline хxvт, 59, 59a, $59 b$ & 138 \\
\hline 1.11. 1:32 & $30 t i$ \\
\hline хxxII, 82 & 198 \\
\hline CCCLXV, 900 & 2534 \\
\hline CCCXXIX, 796 & $\geq 2=69$ \\
\hline LX, 158 & 370 \\
\hline CCCXXI, 773 & 2174 \\
\hline LI, 131 & 304 \\
\hline CCLXX1, 658 & 1789 \\
\hline CCL.XX1X, 701 & $19: 31)$ \\
\hline CCCLXXII, 9:0 & -615 \\
\hline CLIII, 114 & 982 \\
\hline CCCXXXVI, 815 & 2352 \\
\hline CCCLX, 888 & 2537 \\
\hline CCrANT, AE & -200 \\
\hline$x \times x, 72$ & 168 \\
\hline $\operatorname{LIX}_{1} 156$ & 369 \\
\hline 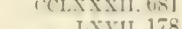 & 1815 \\
\hline LXVII, 178 & $\begin{array}{r}410 \\
.10^{\circ}\end{array}$ \\
\hline $\operatorname{cccsL}, 820$ & $2+02$ \\
\hline $\operatorname{CCXLV}, 614$ & 9004 \\
\hline CCCIII, 734 & 2864 \\
\hline CCCXLVIII, 851 & 2450 \\
\hline X1.15, 11:4 & 218 \\
\hline FYL tillis & 1602 \\
\hline Cсc1х, $749,7 \pm 9 a$ & 2069 \\
\hline $\mathrm{Cx}, 294-294 d$ & 667 \\
\hline 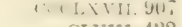 & 2560 \\
\hline CLV111, 4:2 & $9 ! 3$ \\
\hline CCCXXXVI, 814 & 2850 \\
\hline+1.71 .715 & 1938 \\
\hline $\begin{array}{r}1,4 \\
485\end{array}$ & 12 \\
\hline СLXXXI, 482 & 1152 \\
\hline CLXXXIII, 484 & $115 y$ \\
\hline CLXXXIV, 485 & 1160 \\
\hline CLXXXII, 483 & 1157 \\
\hline cxixy, 371 & $\begin{array}{c}580 \\
1150\end{array}$ \\
\hline CCXXVI, 575 & $1+2 \times 9$ \\
\hline (ल..T, 339 & $\begin{array}{ll}803 \\
30 \cdot 3\end{array}$ \\
\hline 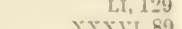 & 302 \\
\hline $\begin{array}{r}x \times 17,89 \\
99\end{array}$ & is \\
\hline 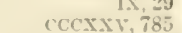 & 2034 \\
\hline & 878 \\
\hline $\operatorname{cssxv}, 370$ & $22+3$ \\
\hline CXX11I, 335 & 793 \\
\hline 1. 1. -1 & $12 \times 2$ \\
\hline CCCXXVII, 792 & 22240 \\
\hline CLXXII, ti6 & 1076 \\
\hline 161016.45 & 1078 \\
\hline CLXXIV, 160 & 108.3 \\
\hline CL.SIIV, 46] & 1084 \\
\hline
\end{tabular}

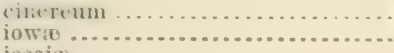

$$
\begin{aligned}
& \text { jessio }
\end{aligned}
$$

Bull. No.47, pt. 1-XI1
1745

12017

1597

1) 4

416

2196

219

568

$30:$

1159

900

661

138

$306 ;$

198

$2-269$

$+174$

304

78 !

615

982

2352

2.146

168

3 ig

410

$40^{\circ}$

1654

2016

2450

218

2069

1667

560

493
2550

1938

15

$115 y$

1160

1157

$5=0$
$4 \times 9$

है०3

185

is

378

$2+43$

384

$12 \times 2$

11) 6

$107 \mathrm{~s}$

10.5 


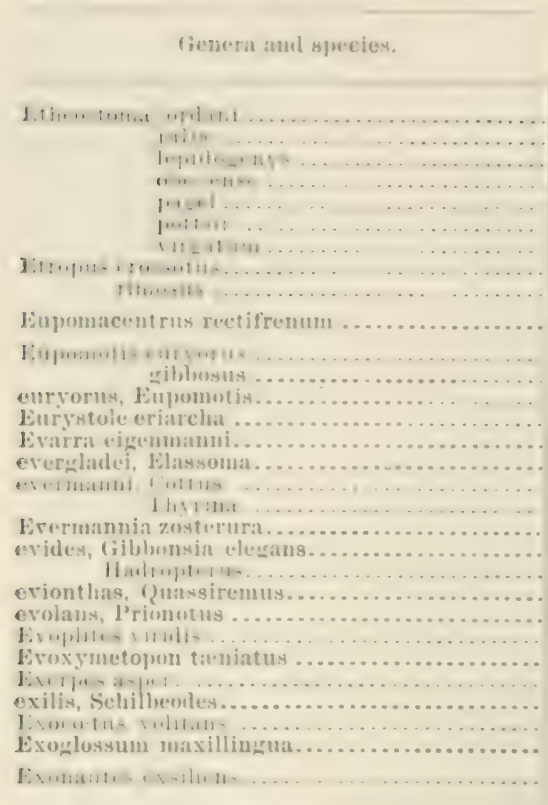

romblelutii

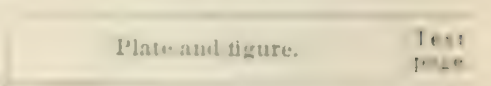

oxsiliens, J:xonamtos.

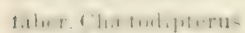

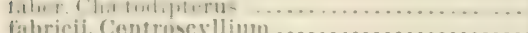

filcata, Agosia plienax, ओ

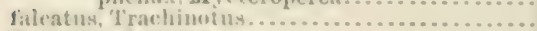

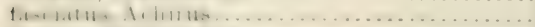

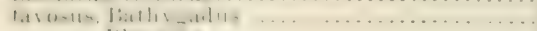

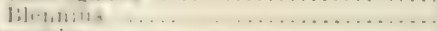

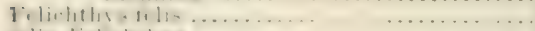

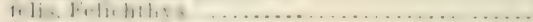

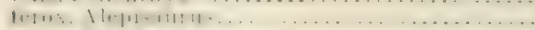
-

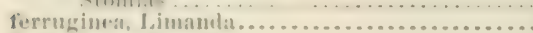

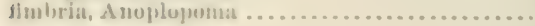

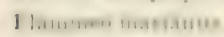

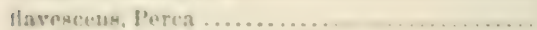

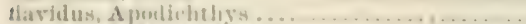

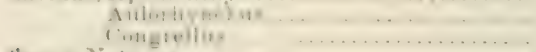

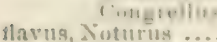

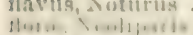

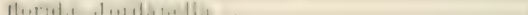

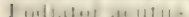

finteras, Symorlus.

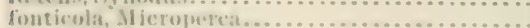

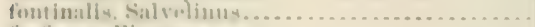

forllowa, Jitumura

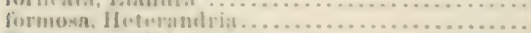

1..

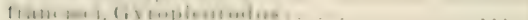

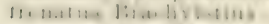

$$
\text { Arinter }
$$

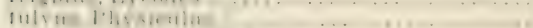

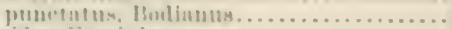

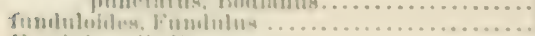

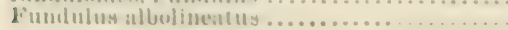


Furdulus catenatus

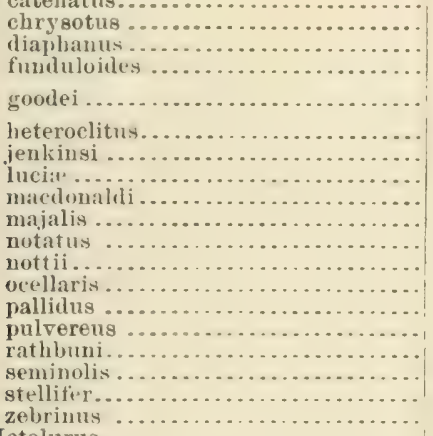

CIV, 278. 278n CVII, 287

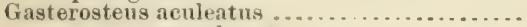
cataphractus $\ldots . . . . . . . . . . . .$. Gastrostomus bairdii. Sichillseoties. Sebastodes

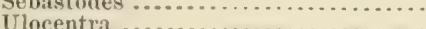

Gilhertidia sigolute. ...................... хxх1х, 497 XCI1, 251 581

cxxxiv, 367 | 871

ccxirI, 557 | 137ti

Ссcхxх1, 815 | 235? CIXI, 439 1015

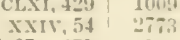

crilli, Avocettina.

\section{Bassogigas}

Cetrmimus .

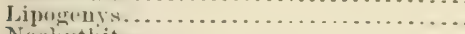

Neolithite.

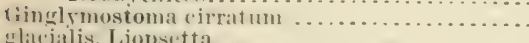

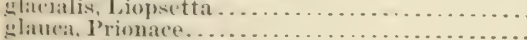

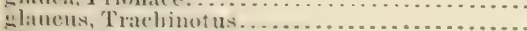

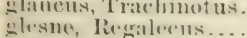

globosa, Lyospliarit.

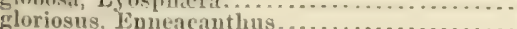

Gnathypops maxillosa - .

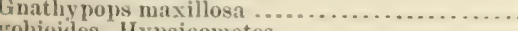
Eobivides, Hy picometes.

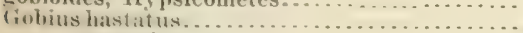

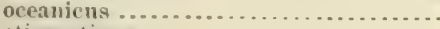

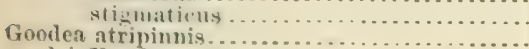
goolei, Fundulus.

LIx, 15t, 15te, 15tb

CCCLVII, 879

IV. $\mathrm{v}, 16,16 \alpha$

CXLN1. 395

CCCLXX, 916

CCLXYI, 649, (i49

CINII, 4"2)

CCCXxxI, 801

cсcxxxil,

CCCXXง 788 сссхxуI, 78!, 789a ссехxт, 787 cxiv, 301 3067
2801 
Euemei, Jilichthys..................... Eurlumatha, Uncorhysuchus.

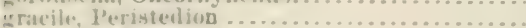

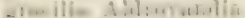

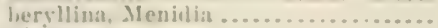

l'hotowectes.........................

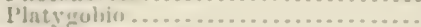

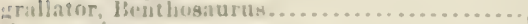

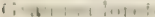

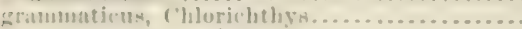

Ciratamatogtemias dentatuy

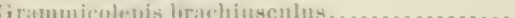

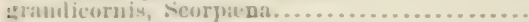

eren-ni, Neoliparis.

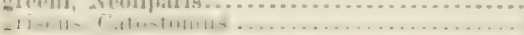
Hexanchus.

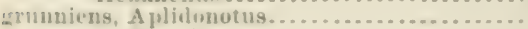

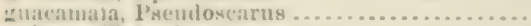

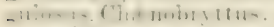

gumbelliturnis, Asternojteryx...............

- i.1.1. !lus, Pi wi

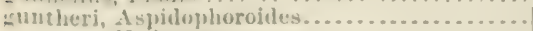

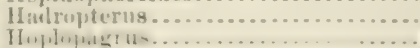

Emtinins, P’ercopsia

Entifer, Uphiclithus.......................

fivmmelis viridis ...........................

fijunveanthus galeatus...

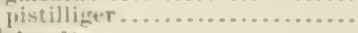

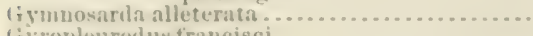

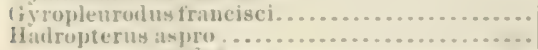

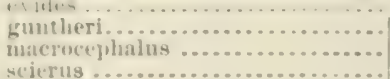

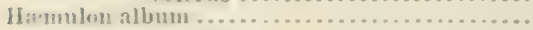

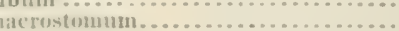

farrat $\ldots \ldots \ldots \ldots \ldots \ldots \ldots \ldots \ldots \ldots \ldots \ldots \ldots$

plumieri ..............................

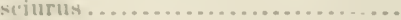

harenens, Clupen . .

llarriota ralejglatna ........................

hasqatus, (iobints.

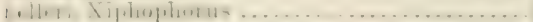

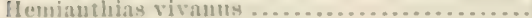

Hemicaranx aublyrlyuchus . . . . . . . . . . . .

Ji-milepidotus, Ilemilepidutus.

Hermilepidutus hernilevilutus

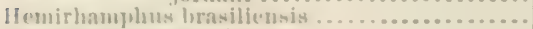

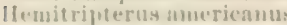

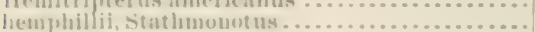

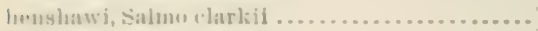

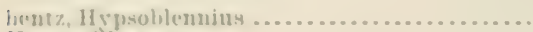

If romosilla a\%ırua...

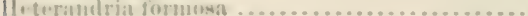

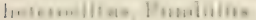

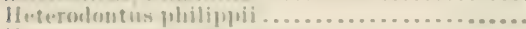

Н

แс

wiving

areitey!

s!

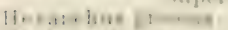

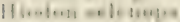

$$
\text { , }
$$

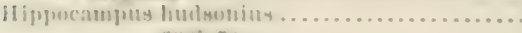
zenteris:

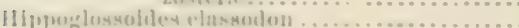
platersulilen.

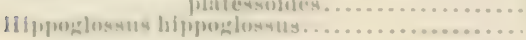

ยCCXLVII, 819

L.xxvu, 205

cer: $x 11,776$

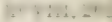

$\operatorname{CXX15,35\% }$

$\mathrm{xc}, 255$

$1.16,139$

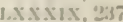

(anili,

(')

X(1), 254

(.2.11, +10

(ल)

recexvi, olis, itila

, t. $2,7,7$

11,8

cextri, 014

CXI.V1, 61 \%

(avilit 1.2)

cCCXIII, 8ith

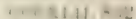

(ссех11, 755, 75, $2,755 \%$

('L.XVII, 43!)

1. $4: 1,31:$

( $x \times 1,329$

1. $115,160^{\circ}$

iCCLII, e $64-28+c$

(C) 730

$\mathrm{cic}, 729,-29 a,-7202$

c.xx15. 366

III, 9

CLXVt, 438

1: $1: 41,441$

('Lxv11, \$39

CLXVI, 437

CL.TVI1, \&11

c'ग11, 528

cटr1, 5Ma

с $(15,530$

(C), 532

CCV. 531

I.x. 185

Nix. 42

( cexxy, Tes

exp ind

c.011, ints

('XI.I, 386

crxc, $704,701 a, 704 b$

cric, 704, 704a, 704b

cexe, 703

cxv1, 313

(сеCу, 7as

croxi, $82 \%$

L.x.x, o

(coxixis, ges

r.xix, 508

(') $10,50 \%$ 7.7! .7. 111, 10

m1.xxx, 6it6, 676n

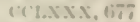

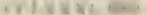

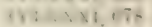

c(1.x. $\times 1,609$

in

19.11, 1-1

85isis it

$1 \times 1 \times 1,8=9$

$6 \times x+2, x \geq 8$

c0(1, $x \times 11,9210$

(20.1. $x+11,919$

crexxt, sts 
hippoglossus, Hippoglossus.................

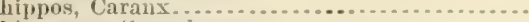

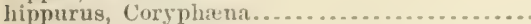

hiriudo, Azurina .......................... Leiocottus

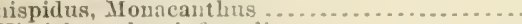

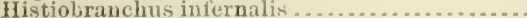

Histiocottus bilobus . . . . . . . . . . . . . . . . . . . . . .

polbolli Ceratias a

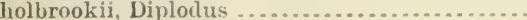

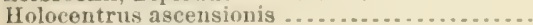

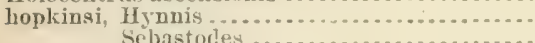

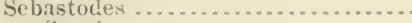

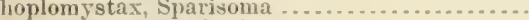

Jioplopagrus gunt heri. . . . . . . . . . . . . . . . . . .

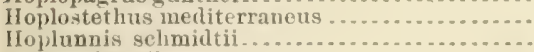

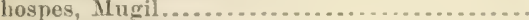

ludsonins, Uippocampus......................

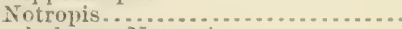
saludinus, Notropis ...............

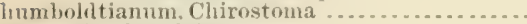

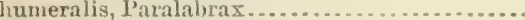

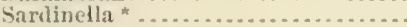

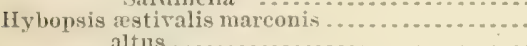
altus .................................. watauga

Hyilrolagus colliei ...........................

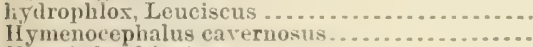

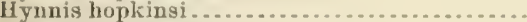

Hypoclydonia bella. . . . . . . . . . . . . . . . . .

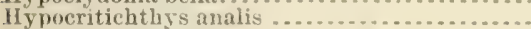
Hypohomus aurantiacus. spilotus.

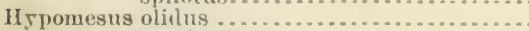
pretiosus ........................

Hypopletrus gemma. . . .

Hyoprion brevirostrig

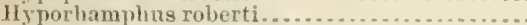
unifasciatus .....................

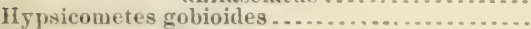

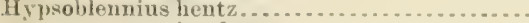
iontluas......................

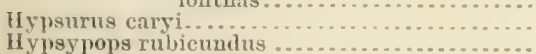

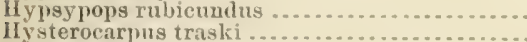

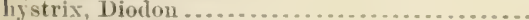

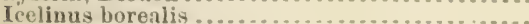

Icelus canaliculat $u s$. . . . . . .

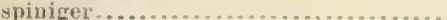

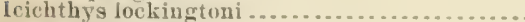

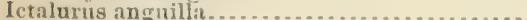

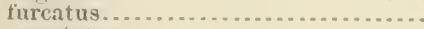
punetatus...........................

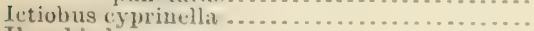

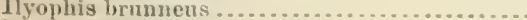

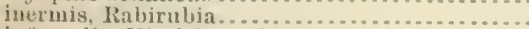

infernatis, Histiohranehus.

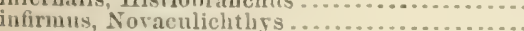

Inopsetta ivcligra

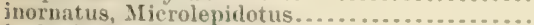

insignis, Sehilhemlen...

intertintus, Arelnoplites.

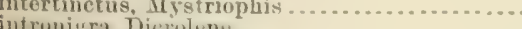

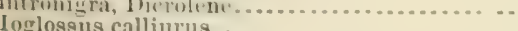

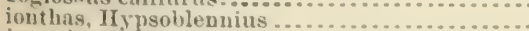

ins, I levelululia ....
CCCLXXI, $918 \mid 2611$

\begin{tabular}{l|l}
$\mathrm{CXL1}, 387$ & 920
\end{tabular}

CXLIX, $402 \quad 952$

CСXхXIII, 588 154t

\begin{tabular}{l|l} 
CCCII. 731 & 2011
\end{tabular}

CCLIX, 634 1715

LVII, 147 35?

CCCIII, 735 | 2018

CCLIII, 625 1684

CCCLXXX1X, 95t 2729

CCXVII, 555, 555a 1362

\begin{tabular}{l|l} 
CXxxI, 358 & 848
\end{tabular}

CxLI1, 391 93:"

CCLXx11.65y 1789

CCXLIT, 611 | 16:2

CхCVI, $513 \quad 1244$

cxxx, $35 \pm \quad 837$

LVII, 151 361

Cxxi11, 345 814

CXXI, 327 77

$\begin{array}{ll}\text { XLFII, 119 } & 269 \\ \text { XLVII, 120 } & 270\end{array}$

xLVII, 120
cxim, 334

Cxc, 493 |1196

\begin{tabular}{l|l} 
LxIII, 193 & 431
\end{tabular}

LIII, 136 | 316

LIV, $138 \quad 321$

I.III, 137 | 319

XIX, $41 \quad 95$

XLII, 104 238

CCCLXIX, $912 \quad 2580$

CXLIII, 391 93?

CLXXIX, $475 \quad 1115$

CCXXx, $582 \quad 1500$

CLXVIII, $413 \quad 1043$

CLXiIII, 4421040

L.XXVII, 231

LXXXI, 230 525

CLXXXIX, 497 1193

CLXXXIX, 496 1193

$\begin{array}{ll}V, 18 & 41\end{array}$

cxvr, $312 \quad 721$

c.5vi 311 721

CсCхxхIII, 805 | 2.94

CССххх1. 823 2391

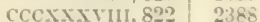

\begin{tabular}{l|l} 
CCXXXI, 585 & 1508
\end{tabular}

CCXXxIr, $591 \quad 1564$

CCXXili, 57 $\vec{i} \quad 1496$

CCLXil, 647 1745

ccr.xxxi, 687 1896

\begin{tabular}{l|l} 
CCLXXXit, 693 & 1917
\end{tabular}

CCLXXXY, $692 \quad 1914$

CLI, $406 \quad 969$

xxv.57 278

\begin{tabular}{l|r} 
xxy, 56 & 134
\end{tabular}

\begin{tabular}{ll|l}
$\mathrm{x} \times$ & 58 & 134
\end{tabular}

\begin{tabular}{l|l} 
Xx. 70 & 163
\end{tabular}

Lx. $145 \quad 350$

Cxcrx, 519 | 1274

LVII, 147 352

CCXLI, 606 1616

CCCLXXVI, 9:7 |

\begin{tabular}{l|l} 
C'CX, 542 & 1341
\end{tabular}

xxriI, 66 147

CLVII, $420 \quad 991$

LXV, 170 $38 \mathrm{G}$

CCCLVII, 882 252\%

UсCXXII, 780 2193

ссехxхуII, 822 2388

CCCXxFII, 793 2254

+ l'late br error labeled sardinella sardina. 


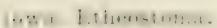

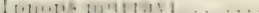

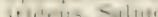
mingas.

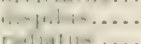

nel hat tio $\ldots$

Intiojuhtorus nigrjeatio

1-115 wis H. Navi

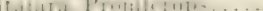

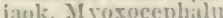

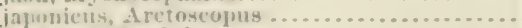

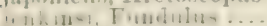

jessiat, Etheostoma

$$
\text { Y. min. }
$$

jorlani, (atulophryo

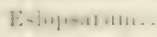

I. Phirmstuma

11. milleplitutin

II vecterunutercat.

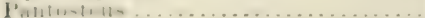

limmulhus.

- Intalanía zonujuo.

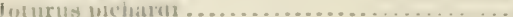

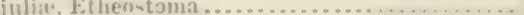

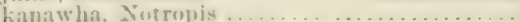

Kathetostoma albigutta $\ldots \ldots \ldots \ldots \ldots \ldots \ldots$

1. inl.1.i. Yi: : 1 in

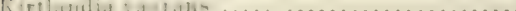

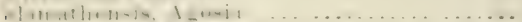

1

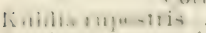

Kiphosus sectatrix.

Labicisthys carinatus y.rinatus

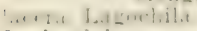

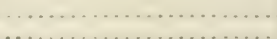

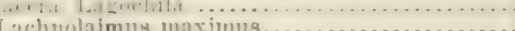

Iartuplurys bieaudalis . -

trigonus

trigueter.

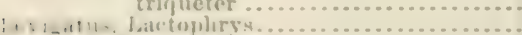

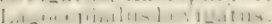

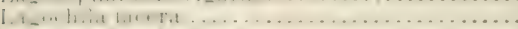

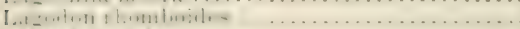

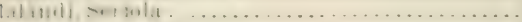

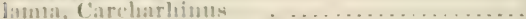

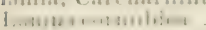

L.

1.

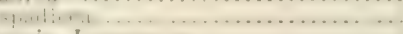

f:mupeeroformis, Lumpenus.

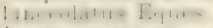

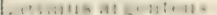

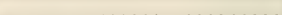

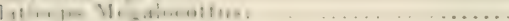

latitruns, Amarliclang

littjulnuis, ('atontomus.

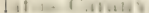

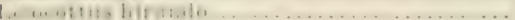

leioponus, Cottus

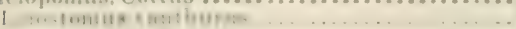

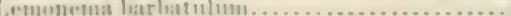

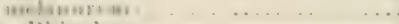

i.ntiuinosus, lihimohatus..................

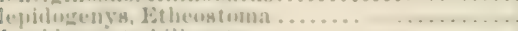

Lephilopentta billinesata ..................

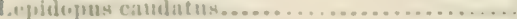

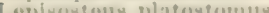

$$
\text { irluturolitis. }
$$

1.0.pounis auritus.
C.I.XXIV. 460

I.XXXix, 239

Lxx1, 216

cr.xxix, 60!

cosen 6urs

('Cxxxix, 600

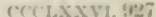

cxxxหน1, 376

VI, 21

m.xxxv, 467, 4hīa, 4870

(rmins: 71

сссхххин, ви Cพ1, 204

(1.XXIV, +61

CC11, 526

cх11, 298

recxe1, 95\%

сххни. 335

cLXX11, 45 ccixe, 70?

('Lxxxim, 493

$x \times x 1,7:$

cCoxixil, 80,

CCLXXX11, 68:

cxxvil, 348

CLXXY) 400

XL1 115

croxxt5, 809, suta

L., $13 !, 1496$

cxxiv, 336

LII, 133

cexcII, 710

CLXIII, 4:"

coxix, 559

LIX, 155 LIX, 156

xxxvm1. $94,94 a$

cCxxxv11, 547

rel $211,639,634 \pi, 6396$ C.LXI, 638

.CLXIII, tiso, bita CCLXI. 6.7

CCLxill, ist

CCLxifl, (ist

xxiult, ys, $4 a$ cexv, 5it:

CXL, del

$$
\text { v. } 17
$$

$\mathrm{xel}, 24 t$

$x \mathrm{Cl}_{2}, 243$

recrist, 84t

crixis, 575

cCxis1.565

(1)

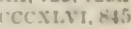

xxxi, it

cxlit, 3ay

cecil, 731

c(x)

cexxi11, 56!

CcCl.xvi, 2(1)

CoCl.Xvi, Mos

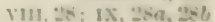

Ct.x.xiv, 40:

ccelaxvi. ges

exxxv, 373

xख11, 40

xxil. 30

1.1. 725,4238 


\begin{tabular}{|c|c|c|}
\hline Genera and species. & Plate and figure. & $\begin{array}{l}\text { Text } \\
\text { page. }\end{array}$ \\
\hline 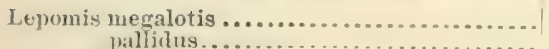 & CLX, 426 & 1002 \\
\hline 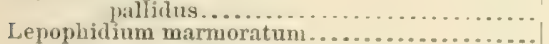 & CLX, 42\% & 1005 \\
\hline & CCCLIII, 866 & 2482 \\
\hline Leptocephalus caudilimbatus $\ldots \ldots \ldots \ldots \ldots \ldots \ldots$ & CCCLIII, 867 & 2484 \\
\hline & LVII, 149 & 355 \\
\hline 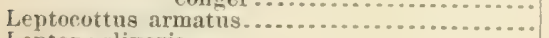 & LVII, 148 & $35 t$ \\
\hline 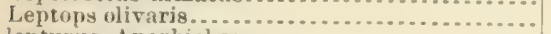 & ССС̈, 732 & 2012 \\
\hline lepturus, Anarbichas. ......................... & $\begin{array}{r}\text { XXY1I, } 62 \\
\text { CCCXLYII, 847 }\end{array}$ & 143 \\
\hline 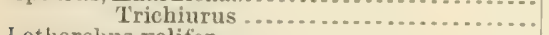 & $\begin{array}{l}\text { CCCXLYII, } 847 \\
\text { CXXXYII, } 375\end{array}$ & 2447 \\
\hline Letharchus velifer ........................... & $\begin{array}{r}\text { CXXXVII, } 375 \\
\text { LXI. } 160\end{array}$ & 889 \\
\hline Lethotremus muticus .......................... & $\begin{array}{r}\text { LXI, } 160 \\
\text { CCCXII, } 758\end{array}$ & 375 \\
\hline 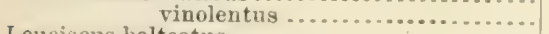 & $\begin{array}{l}\text { CCCXIII, } 758 \\
\text { CCuxIV, } 759\end{array}$ & 2101 \\
\hline 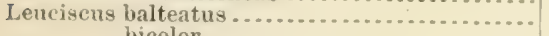 & $\begin{array}{r}\operatorname{CCUTIV}, 759 \\
\times 1, I I, 105,105 a\end{array}$ & 2101 \\
\hline 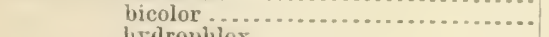 & $\begin{array}{r}\text { XLII, } 105,105 a \\
\text { XLI, } 102\end{array}$ & 238 \\
\hline 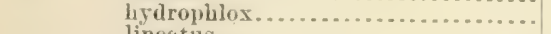 & $\begin{array}{r}\text { XLI, } 102 \\
\text { XLII, } 104\end{array}$ & 232 \\
\hline 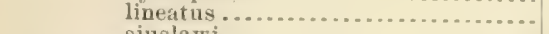 & $\begin{array}{r}\text { XLII, } 104 \\
\text { XII, } 103\end{array}$ & $\begin{array}{l}238 \\
232\end{array}$ \\
\hline 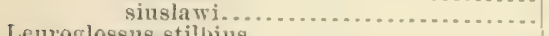 & $\begin{array}{r}\text { XII, } 103 \\
\text { XLIII, } 106\end{array}$ & 2797 \\
\hline Lemoglossus stilbins . . . . . . . . . . . . . . . . . & LxxxบI1, 233 & 527 \\
\hline Limanda aspera $=\ldots \ldots \ldots \ldots \ldots \ldots$ & CCCLXXVII, 930 & 2645 \\
\hline 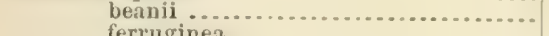 & CCCLXXYIII, 932 & 2616 \\
\hline 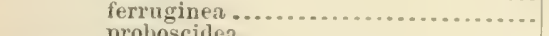 & CCCLXXVII, 929 & $26 \pm 1$ \\
\hline 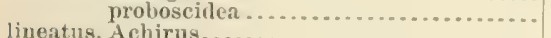 & CCCLXXVin, 931 & 2645 \\
\hline & CCCLXXXTI, 947 & 2697 \\
\hline & XLI, 103 & 232 \\
\hline 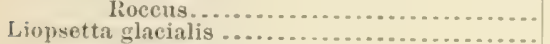 & CLXXX, 478 & 1132 \\
\hline & CCCLXXX, 935 & $26+9$ \\
\hline & CCCLXXX, 936 & 2650 \\
\hline 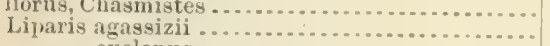 & XXXiv, 85 & 183 \\
\hline 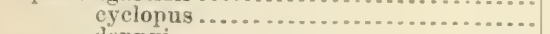 & 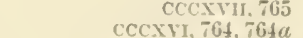 & 2121 \\
\hline 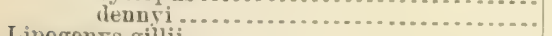 & $\begin{array}{l}\text { сссхуI, } 764,76 \pm a \\
\text { сCсхVII, } 766,760 a\end{array}$ & 2118 \\
\hline 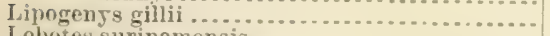 & $\begin{array}{r}\text { CCCXVI, } 766,76 t a \\
\text { ICIx, } 266\end{array}$ & $\begin{array}{r}-124 \\
619\end{array}$ \\
\hline 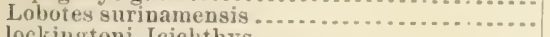 & CxcIV, 510 & 619 \\
\hline 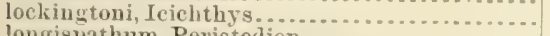 & CLI, 406 & $\begin{array}{r}1235 \\
969\end{array}$ \\
\hline 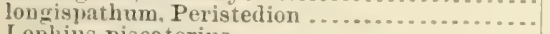 & cecxxi, 775 & $\begin{array}{r}969 \\
2178\end{array}$ \\
\hline 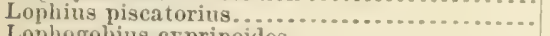 & CCCLXXXYIII, 952 & $\begin{array}{l}2178 \\
2713\end{array}$ \\
\hline 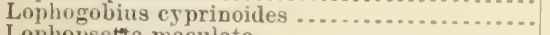 & CCCXXVI, 786 & 2713 \\
\hline 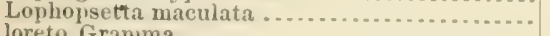 & CCCLXXхи, 938 & $\frac{32}{2660}$ \\
\hline 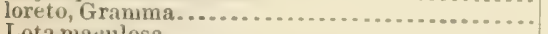 & CXCIII. 508 & 2660 \\
\hline 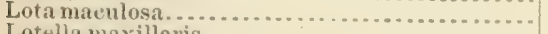 & SCCLXIV, 897 & $12=29$ \\
\hline Lotella maxillaris ............................. & CCCLXIII, 895 & 2550 \\
\hline 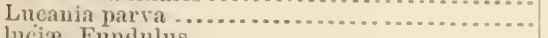 & $\begin{array}{r}\text { CCCLIII, } 895 \\
\operatorname{c1x}, 292\end{array}$ & 2546 \\
\hline lueia, Fundulus & $\begin{array}{l}\text { CIX, } 292 \\
\text { CVII, } 286\end{array}$ & 665 \\
\hline & $\begin{array}{l}\text { CVII, } 286 \\
\text { xeII, } 246\end{array}$ & 654 \\
\hline 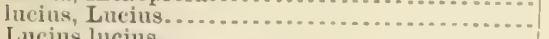 & $\begin{array}{r}\text { XeII, } 246 \\
\text { c, } 269,269 a\end{array}$ & 565 \\
\hline 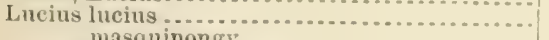 & $\begin{array}{l}c, 269,269 a \\
c, 269,269 a\end{array}$ & $6 \div 8$ \\
\hline 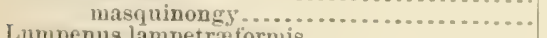 & $\begin{array}{r}\text { C, }-09,-209 a \\
\text { C, } 270\end{array}$ & $\begin{array}{r}6 * 8 \\
6 * 9\end{array}$ \\
\hline 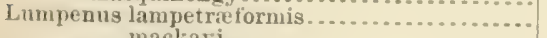 & CCCXLIV, 810 & $\begin{array}{r}6: 9 \\
24: 8\end{array}$ \\
\hline 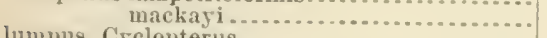 & $\begin{array}{l}\text { CCCXLIT, } 810 \\
\text { CCCXLIS } 839\end{array}$ & $\frac{24: 8}{2436}$ \\
\hline 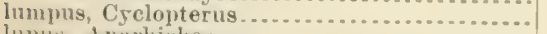 & $\begin{array}{r}\text { CCCXLI5, } 839 \\
\text { CСсхIII, } 757\end{array}$ & 2436 \\
\hline 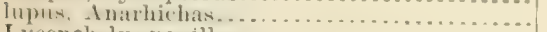 & 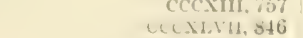 & 2096 \\
\hline 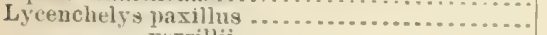 & 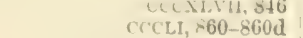 & 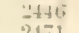 \\
\hline verrillii........................... & $\begin{array}{r}\text { CrCLI, }-60-860 \mathrm{~d} \\
\text { CCCLI. } 859\end{array}$ & 2471 \\
\hline 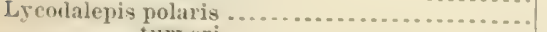 & $\begin{array}{l}\text { CCCLI. } 859 \\
\text { CCCL, } 857\end{array}$ & 2470 \\
\hline 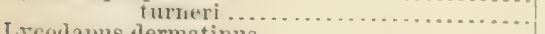 & $\begin{array}{l}\mathrm{CCCl}, 857 \\
\text { l.cLl, sio }\end{array}$ & 2468 \\
\hline 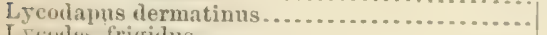 & $\begin{array}{l}\text { L.CLL, sü } \\
\text { CCCLIV, BTU }\end{array}$ & $2+6 i$ \\
\hline 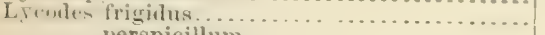 & $\begin{aligned} & \operatorname{CCCLIV} 8 \pi 0 \\
& \quad-C L 1.850^{\circ}\end{aligned}$ & 2492 \\
\hline 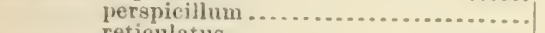 & $\begin{array}{l}-6,1.85 j \\
\text { CCCL, } 855\end{array}$ & 216 \\
\hline 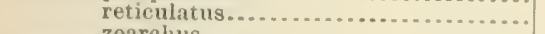 & $\begin{array}{r}\text { CCCL, } 855 \\
\text { CCCXLIX, } 851\end{array}$ & 2465 \\
\hline 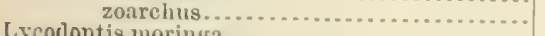 & $\begin{array}{l}\text { CCCXLIX, } 854 \\
\text { CCCXLIX, } 853\end{array}$ & 2465 \\
\hline Lycodont is moringa. . . . . . . . . . . . . . . . . . . . & $\begin{array}{r}\text { CCCXLIX, } 853 \\
\text { LXY. } 171\end{array}$ & 2464 \\
\hline 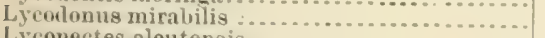 & $\begin{array}{r}\text { L.XV. } 171 \\
\text { CCCLII. } 86^{2}\end{array}$ & 395 \\
\hline 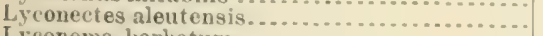 & $\begin{array}{r}\text { CCCLII. } 862 \\
\text { CCCXLVI, } 844\end{array}$ & 2474 \\
\hline 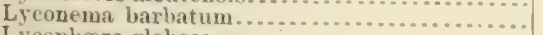 & $\begin{array}{r}\text { CCCXLVI, 8\$4 } \\
\text { CCCLI }, 8633\end{array}$ & 244 \\
\hline Lyosplixera globosa. & $\begin{array}{r}\text { CCCLI, } 863 \\
\text { CCLXVII, } 649,6 \pm 9 \alpha\end{array}$ & 2474 \\
\hline 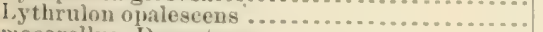 & & 1751 \\
\hline 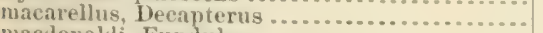 & CCvil, 536 & 1312 \\
\hline 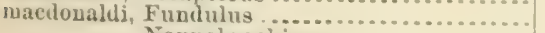 & C.L. 383 & 909 \\
\hline 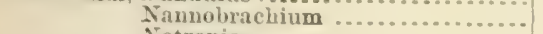 & cVI, 283 & 651 \\
\hline 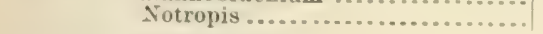 & xCI, 245 & 563 \\
\hline Salmo clarkii ..... & XLIX, 123 & \\
\hline macellus, Prionistius & LXXXI, 214 & 2819 \\
\hline & धст.хxх1. 700 . & 1928 \\
\hline
\end{tabular}


mackayi, Jampenus

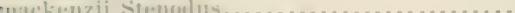

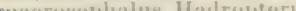
snar row heilus, Catosiomus materoehir, Alilrovantia.

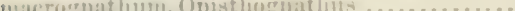

macroplitlsalmus. A jriou......................

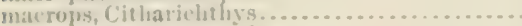

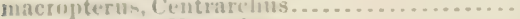

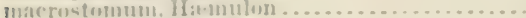
mateulats, I.ophojestta ................ maculitufiasc intus, P'aralahrax . ............... maculatus, . I ulostomus .

Crspravauthudes....................

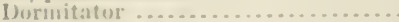

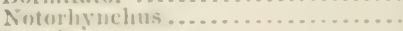

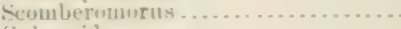

Splieroides........................

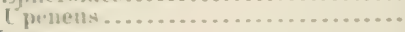

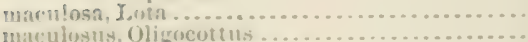

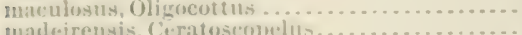

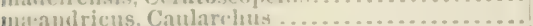

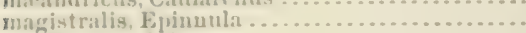

majilik, Fumlulus.

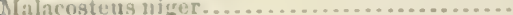

Muglarti, Tralc

Mallotus villosus

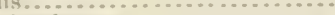

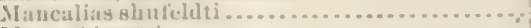

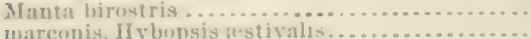

Jusuringta, leissola

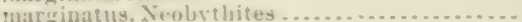

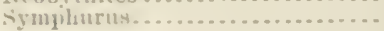

marianus, Filammeo .

marinus, Petromyzon no. selganter.

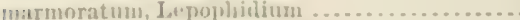

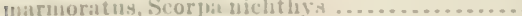

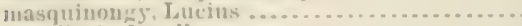

maxillaris, lotella..........................

maxillingua, Exorglossum .....................

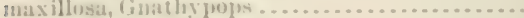

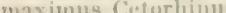

Lachuolaimus ......................

metulis, Liloeentrat.

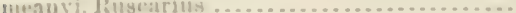

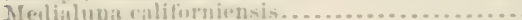

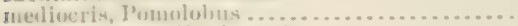

puediterrament, Hogplostethas

mueclusionla. Carans

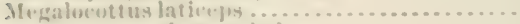
plint yer.jphatlus ..................

garesuletion, Lepormina

Jelasoserammens

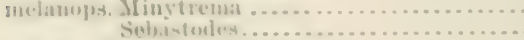

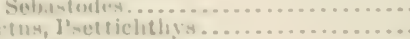

melanost сет thн,

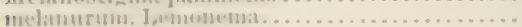

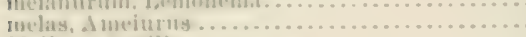

Alelletes panjilio.

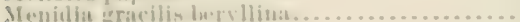

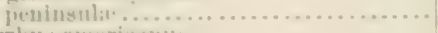

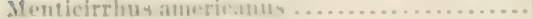

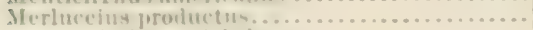

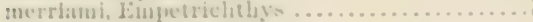

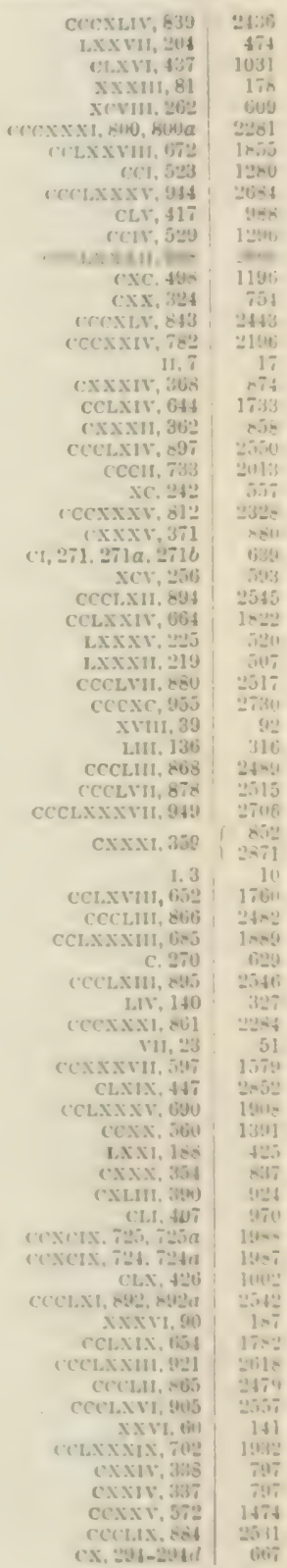

cus

1201

$6=1$

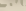

75

19ing

17

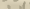

$11:$

320

(i)

80.

(y)

310

aid

10

10

s.:

lnet

48

$3:$ 


\begin{tabular}{|c|c|c|}
\hline Genera and species. & Plate and figure. & $\begin{array}{l}\text { Text } \\
\text { page. }\end{array}$ \\
\hline 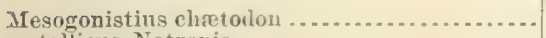 & CLVIII, 423 & $90 \%$ \\
\hline 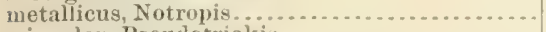 & L. 128 & 297 \\
\hline 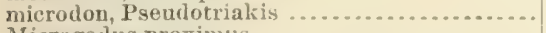 & 15,14 & 37 \\
\hline 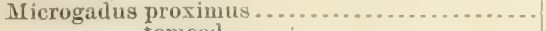 & $\operatorname{ccclx}, 859$ & $253 ! 1$ \\
\hline 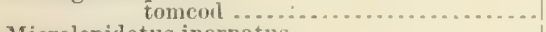 & ccer.x. 890 & $25+11$ \\
\hline 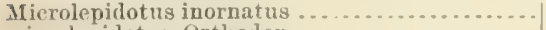 & $\operatorname{ccx}, 542$ & 1341 \\
\hline 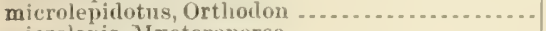 & xxxix, 96 & 207 \\
\hline 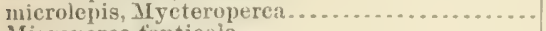 & CLXXXVIII, 494 & 1177 \\
\hline 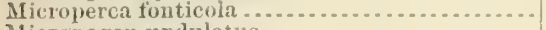 & cLXXVII, 470 & 1104 \\
\hline 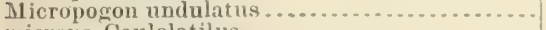 & Cexis. 580 & 1461 \\
\hline micropis, Caulolatilus . . . . . . . . . . . . . . . . . . . . . & сссххх, 799 & $227 \pi$ \\
\hline Micropterus duloujen ...................... & 1.x.11. tiu, 1 ita & {$[111]$} \\
\hline 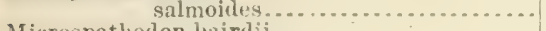 & CLXIII, 431 & 1012 \\
\hline 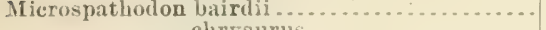 & $\operatorname{cosx} 1 ;, 592$ & 1566 \\
\hline 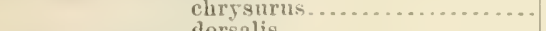 & CCXXXVI, 593 & 1567 \\
\hline dorsalis $\ldots \ldots \ldots \ldots \ldots \ldots \ldots . . . . . . .$. & CCXXxit, 594 & 1568 \\
\hline milberti, Galeichthys . . . . . . . . . . . . . . . . . . . & Xxil1. 53 & 128 \\
\hline miles, Porogadus $\ldots \ldots \ldots \ldots$ & CCCLYIII, 881 & 2520 \\
\hline 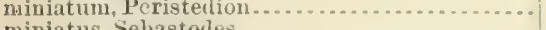 & CCCXXI, 774 & 2178 \\
\hline 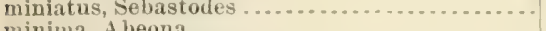 & CCLXX11, 662 & 1794 \\
\hline 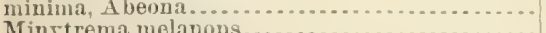 & CCXxili, 578 & $1+97$ \\
\hline 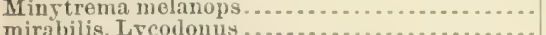 & $\begin{aligned} \text { XIXVI, } 90 \\
\text { CCCLIt } 86^{\circ}\end{aligned}$ & 187 \\
\hline 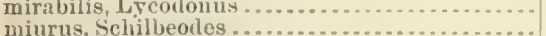 & CCCLII. 862 & $24 \pi 4$ \\
\hline miurus, Schillbeodos $\ldots \ldots \ldots \ldots$ & $\begin{array}{r}\text { XXIX, } 68 \\
\text { CCLXViL, } 650\end{array}$ & 148 \\
\hline Mola mola. . . . . . & $\begin{array}{l}\text { CCLXVII, } 650 \\
\text { CCLXYII, } 650\end{array}$ & $175 \%$ \\
\hline 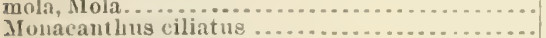 & $\begin{array}{r}\text { CCLXVII, } 650 \\
\text { CCLIX, } 633\end{array}$ & 1753 \\
\hline $\begin{array}{r}\text { Mronaeanthus ciliatus } \ldots \ldots \ldots \ldots \\
\text { hispirlus } \ldots . . .\end{array}$ & $\begin{array}{l}\text { CCLIX, } 633 \\
\text { CCLIX, } 634\end{array}$ & 1714 \\
\hline 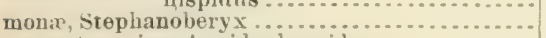 & $\begin{array}{r}\text { CCLIX, } 634 \\
\text { CxגIX, } 353\end{array}$ & 1715 \\
\hline monopterygius, Aspidophoroides.............. & $\begin{array}{r}\text { CXXIX, } 353 \\
\text { CСCXII, 756, 756 }\end{array}$ & 836 \\
\hline Pleurogranmus............... & $\begin{array}{r}\text { CCCXII, } \\
\text { CCLXX1X, } 675\end{array}$ & $\begin{array}{l}2091 \\
1864\end{array}$ \\
\hline montarus, Thymallus tricolor ................ & Lxxxry, 224, 224a & 519 \\
\hline 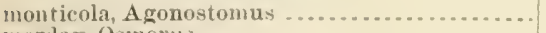 & CXXVI, $3+7$ & $\begin{array}{r}2811 \\
819\end{array}$ \\
\hline 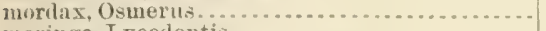 & LXXXVI, 208 & $5 \geq 3$ \\
\hline moringa, Lycodontis... . . . . . . . . . . . . . . . . . . . & LXY, 171 & 395 \\
\hline 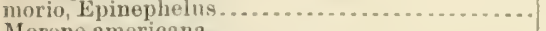 & CLXXXiv, 485 & 1160 \\
\hline 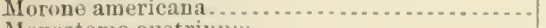 & CLXXXI, 479 & $11: 34$ \\
\hline Moxostoma austrinum $\ldots \ldots \ldots \ldots \ldots$ & хxхтн, 92 & $19 \div$ \\
\hline 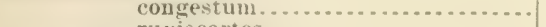 & XxxyI, 91 & 192 \\
\hline 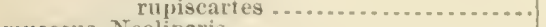 & XxxvII. 93 & 196 \\
\hline 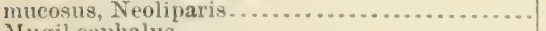 & $\operatorname{cccs} v, 761,761 \alpha$ & 2111 \\
\hline 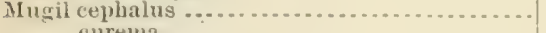 & cxxvi, $3 \$ 3$ & 811 \\
\hline 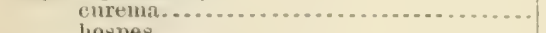 & Cxxy. 344 & 813 \\
\hline 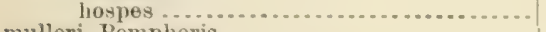 & Cxxill. 345 & 814 \\
\hline 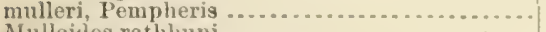 & CLIII, 412 & 978 \\
\hline 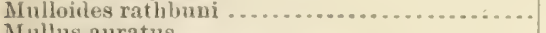 & схxх11, 361 & $85 \overline{7}$ \\
\hline Mullus auratus & Cxxxil, 360 & 856 \\
\hline multifisciatus, Pronotogrammus ............. & C.XC11, 506 & 1226 \\
\hline 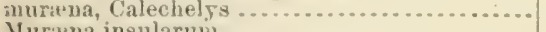 & I.XIII, 164 & 378 \\
\hline 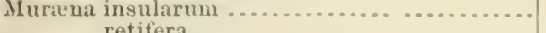 & L.X*1, 17: & 400 \\
\hline 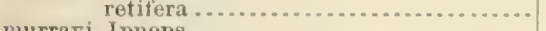 & L.XVI, 173 & 401 \\
\hline 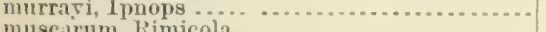 & 1.XXXix, 239 & 547 \\
\hline muscirun, Rimicola $\ldots \ldots \ldots \ldots \ldots \ldots \ldots$ & cсcхххю, 813 & 2338 \\
\hline mutieus, Lethotremus $\ldots \ldots \ldots \ldots \ldots \ldots \ldots$ & Сссхин, 758 & 2101 \\
\hline 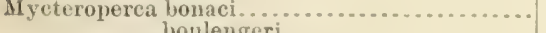 & CLXXXVII, 492 & 1174 \\
\hline 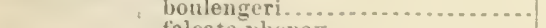 & CLXXXVII, 490 & 1171 \\
\hline 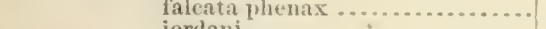 & CLAXXYII, 495 & 1185 \\
\hline 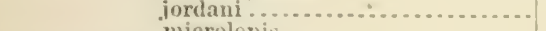 & CLXXXVII, 493 & $117 \sigma_{j}$ \\
\hline rolepis......................... & CLXXXYIII, 494 & $117 \%$ \\
\hline 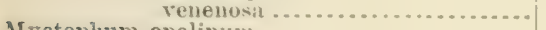 & CLXXXVII 491 & $117: 2$ \\
\hline 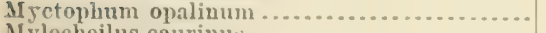 & xeII, 247 & 571 \\
\hline 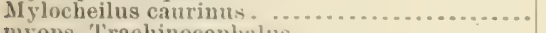 & $\times 2,09$ & 219 \\
\hline 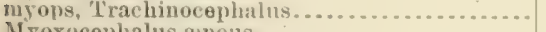 & 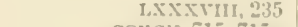 & 533 \\
\hline 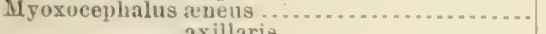 & ссхек: $715,715 \alpha$ & $19 \%:$ \\
\hline axillaris $\ldots \ldots \ldots \ldots \ldots \ldots \ldots . . . . . . . .$. & $\operatorname{coscvix,721}$ & 1980 \\
\hline jaok .................................. & CEXCVI, 719 & 107 \\
\hline 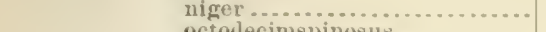 & CекекіII, :23 & 1955 \\
\hline octolecimspinosus ........... & CCXCVI, 717 & 1976 \\
\hline polyacauthocephalus .......... & Cexcri, 718 & $197 \mathrm{i}$ \\
\hline scorpius ...................... & CCAcr, 716; & $10 i t$ \\
\hline verrucosus & Vi1, $722,722 a, 722 \%$ & 1981 \\
\hline verricosus $\ldots . . . . . . . . . . . .$. & CCxev11, 7:0,7:0a & 1979 \\
\hline
\end{tabular}




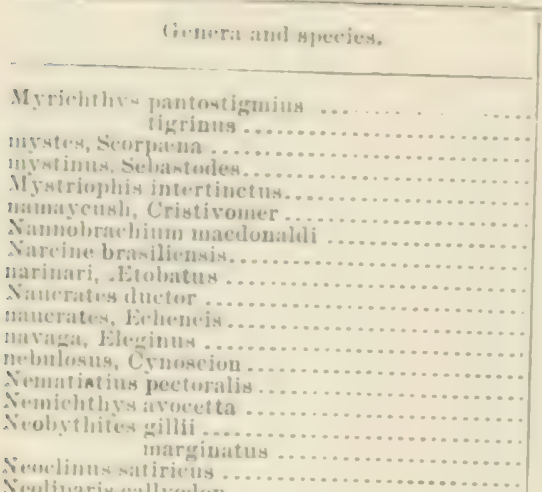

Siculibarisuattyoulon.

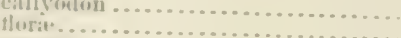

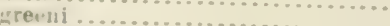

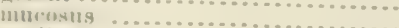

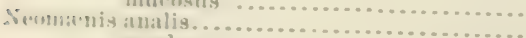

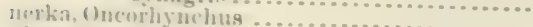

Shasmolon

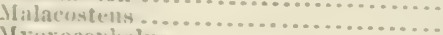

M yuxocephalus

nigrioms, liypoplectrus unicolor.

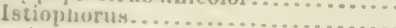

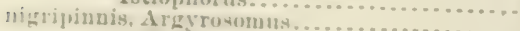
Batliveronus

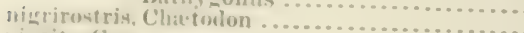
nimpita, Garrupa

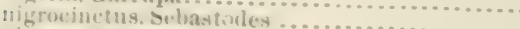

nierrum, Buleusoma .

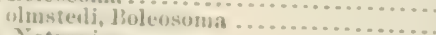

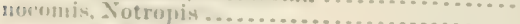
noet nrmus, tichilleodes

nurmalin, 13assozetus.

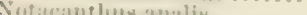

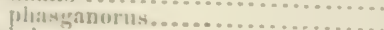

uopatus, Fumdulu世 . Fon

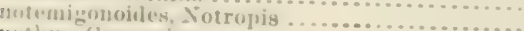

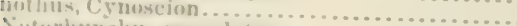

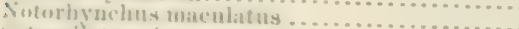
nutuspilotua, Astrulytes.

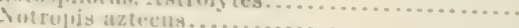
cayuga at rocamblalis. chilumitura

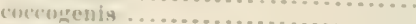

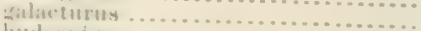

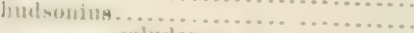

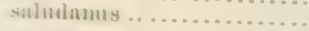

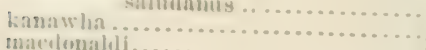
mavedurnalili........................

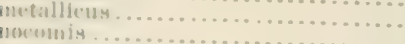

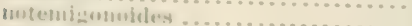

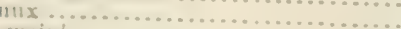

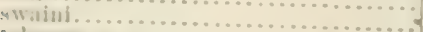

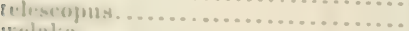

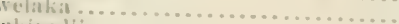

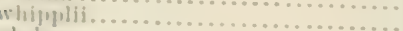

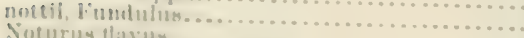

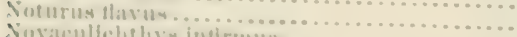

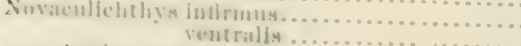

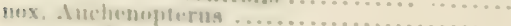

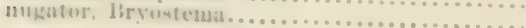

Tiese

biage.

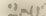

37

1810

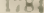

$3 \mathrm{sec}$

501

afis

is

s-

$\sin (x)$

2365

2535

$1+104$

E.05

369

2512

2513

2355

2110

2111

211:

9111

$1: 06$

1258

$1: 04$

1306

til

90

3291

503

1950

1193

xเงแ1, 12!

(')

x.xill, 03

CCX1.1, Gins

rext.I, bus

(19xxv11, 810

coxul, cing 


\begin{tabular}{|c|c|c|}
\hline Genera and species. & Plate and figure. & $\begin{array}{l}\text { lext } \\
\text { paye. }\end{array}$ \\
\hline 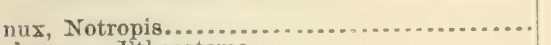 & xI.vir. 117 & 267 \\
\hline 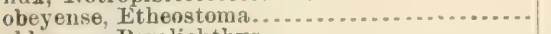 & CLXXV. 463 & 109:2 \\
\hline 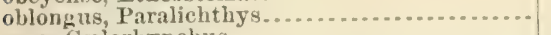 & CCCLXXIV, 924 & $26: 32$ \\
\hline 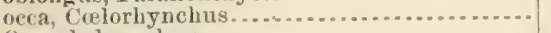 & CCCLX1X, 913 & 2588 \\
\hline 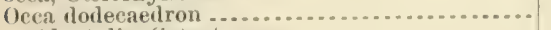 & CCCVIII, 743 & 2044 \\
\hline 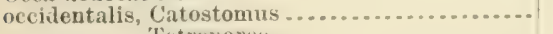 & Xxxir. 79 & 178 \\
\hline 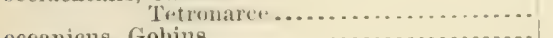 & xi. 33 & 77 \\
\hline 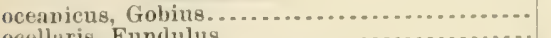 & сссххип, 789, 789a & 2230 \\
\hline 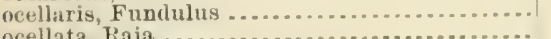 & $\mathrm{CH}, 274$ & 64: \\
\hline 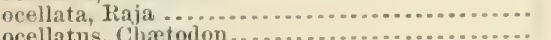 & $\mathrm{x}, 30$ & 68 \\
\hline 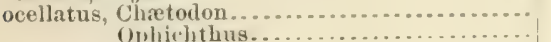 & CCXLIX, 621 & 1674 \\
\hline 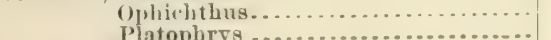 & $\mathrm{CCI}, 524$ & 1282 \\
\hline 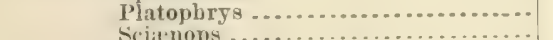 & LXIV, 169 & $38 \%$ \\
\hline 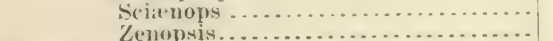 & CCCLхXх11, 939 & $266 ; 3$ \\
\hline 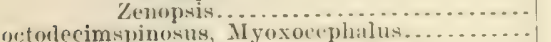 & CсxхI1, 567 & $145: 3$ \\
\hline & CCXLVt, 618 & 1660 \\
\hline 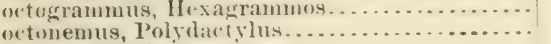 & $\begin{array}{r}\text { CCXCY1, } 717 \\
\text { CCI.XX, } 677\end{array}$ & 1976 \\
\hline & 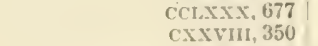 & 1869 \\
\hline 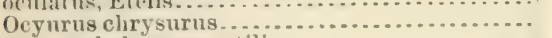 & $\begin{array}{r}\text { Cxxvin, } 350 \\
\text { cxerx, } 520\end{array}$ & 830 \\
\hline 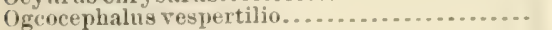 & & 1275 \\
\hline 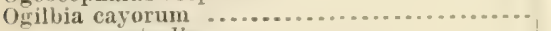 & $\begin{array}{r}\text { CCCXC11, 958, 958a, 958b } \\
\text { CCCI } x, 873\end{array}$ & $\begin{array}{l}2737 \\
2503\end{array}$ \\
\hline 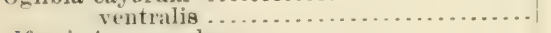 & $\begin{array}{l}\text { CCCLV, } 873 \\
\text { CCCLV, 872 }\end{array}$ & $\begin{array}{l}2503 \\
2503\end{array}$ \\
\hline 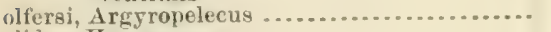 & xст1, $261,261 a$ & $\begin{array}{r}2503 \\
604\end{array}$ \\
\hline olirlus, Hypomesus . . . . . . . . . . . . . . . . . . . . . & LXXXVII, 231 & 525 \\
\hline 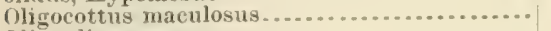 & CCCII, 733 & 2013 \\
\hline 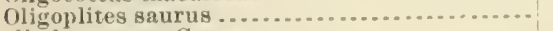 & cxxxvil. 378 & 898 \\
\hline olisthostomus, Gerres ....................... & Cехv⿴囗十, 557 & 1376 \\
\hline 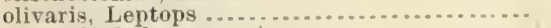 & $\mathrm{xxv11,62}$ & 143 \\
\hline 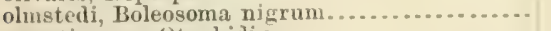 & CI.XXI, 451 & 1057 \\
\hline 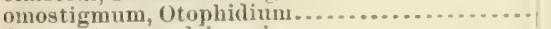 & CCCLIV, 869 & 2490 \\
\hline 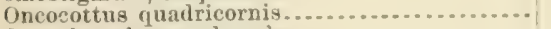 & Ccc, 728 & 2001 \\
\hline 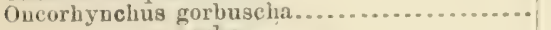 & LxxyH, 205 & 478 \\
\hline nerka $\ldots \ldots \ldots \ldots \ldots \ldots \ldots \ldots . . . . .$. & IXXV11, 207, 207a, $207 b$ & 481 \\
\hline tschawy tscha .................... & LXXV11, 206 & 479 \\
\hline 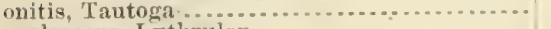 & CCXXXVII, 596 & 1578 \\
\hline 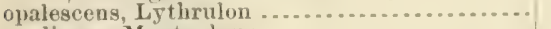 & ccvir, 536 & $1: 312$ \\
\hline 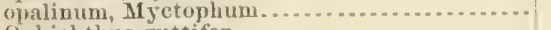 & $\mathrm{xCII}, 247$ & 571 \\
\hline 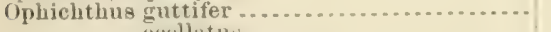 & LXIV, 168 & 383 \\
\hline 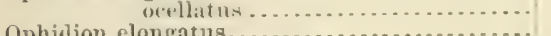 & L.XT, 169 & 383 \\
\hline 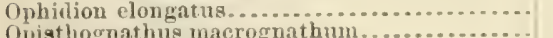 & CCLXXXII, 681 & 1875 \\
\hline 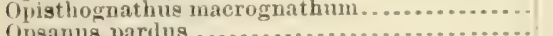 & cccxxx1. 800, 800a & $2: 281$ \\
\hline Opsanus pardus $\ldots \ldots \ldots \ldots \ldots$ & cccxxis 810 & 2316 \\
\hline 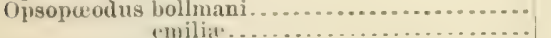 & KLIV, 110 & 248 \\
\hline 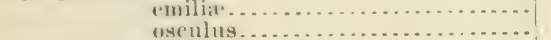 & XIIV, 109 & $2+8$ \\
\hline 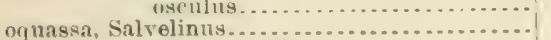 & XLIT, 108 & 348 \\
\hline 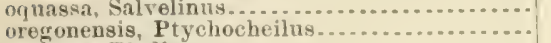 & LXXX11, 221 & 514 \\
\hline 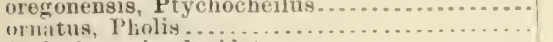 & $\begin{array}{r}\text { XLI, } 101 \\
\text { (CCXII, } 833\end{array}$ & 224 \\
\hline 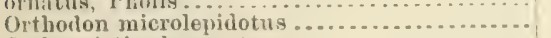 & ('CCXLIT, 8333 & 2419 \\
\hline 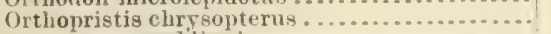 & $\begin{array}{r}\text { Xхxix, } 96 \\
\text { CCX, } 541\end{array}$ & 217 \\
\hline 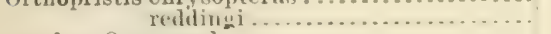 & $\begin{array}{r}\text { CCX. } 541 \\
\text { CCIX, } 5+0\end{array}$ & $1 \cdots i$ \\
\hline 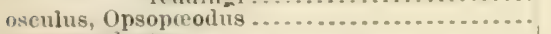 & $\begin{array}{l}\text { CCIX, } 540 \\
\text { XILIV, } 108\end{array}$ & i:3:iti \\
\hline 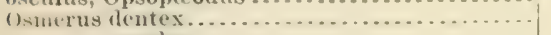 & $\begin{array}{r}\text { XI.IV, } 108 \\
\text { Lxxxi, } 239\end{array}$ & $\because 1 \mathrm{~A}$ \\
\hline 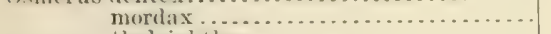 & $\begin{array}{l}\text { LXXXVI, } 229 \\
\text { LXXVI, } 2288\end{array}$ & $\therefore 4$ \\
\hline 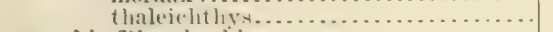 & $\begin{array}{l}\text { LXXXVI, } 228 \\
\text { LXXXv, 207 }\end{array}$ & $\because \because \cdots$ \\
\hline 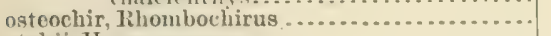 & $\begin{array}{r}\text { Lxxxv, } \frac{247}{798} \\
\text { cсcхxх, }\end{array}$ & $\because-2$ \\
\hline 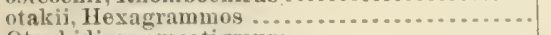 & $\begin{array}{r}\text { CCCXXx, } 798 \\
\text { ('CLXXXI, } 680\end{array}$ & $\because-273$ \\
\hline 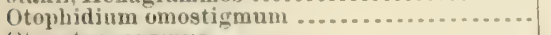 & $\begin{array}{r}\text { ('CLXXXI, } 680 \\
\text { CCCLIV, } 8699\end{array}$ & $1=t i \pi$ \\
\hline 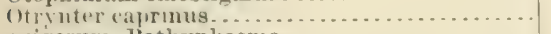 & $\begin{array}{r}\text { CCCLIV, 869 } \\
\text { CCXI, } 543\end{array}$ & $\because 1 ! n$ \\
\hline 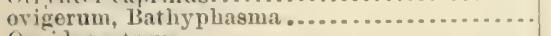 & $\begin{array}{r}\text { CCXI, } 543 \\
\text { CCCXvil, iff? }\end{array}$ & 1,15 \\
\hline 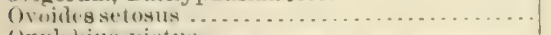 & $\begin{aligned} \text { CCCXTII, ifi? } \\
\text { CCLXV, } 646\end{aligned}$ & $17 \div 0$ \\
\hline 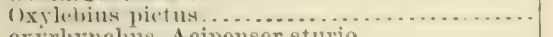 & coLxxxi1, $68 \%$ & isto \\
\hline oxyrliyuchins, Acipenser sturio ............... & $\mathrm{XX}, 45$ & 11. \\
\hline 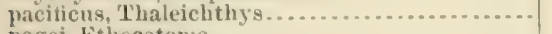 & $\operatorname{Lxx}, 226$ & $\because 21$ \\
\hline 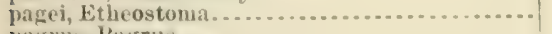 & C1.XXV. 164 & $119:-$ \\
\hline 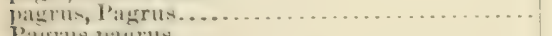 & C'XY, 551 & 1. $\therefore i$ \\
\hline 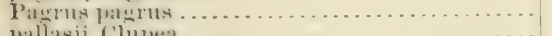 & $(\mathrm{CXY}, 55\}$ & 1:ititi \\
\hline 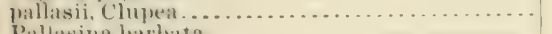 & LXX. 180 & 1:- \\
\hline 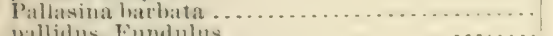 & ccevili, ift & $\because 11: 9$ \\
\hline 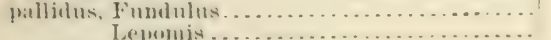 & CI1. $27=$ & i. $\mathrm{s}$ \\
\hline 1. & $\begin{aligned} \text { CLX, } 427 \\
\text { CCCLII, } 865\end{aligned}$ & $\begin{array}{l}1+1,9 \\
\because 193\end{array}$ \\
\hline
\end{tabular}




\begin{tabular}{|c|c|c|}
\hline & \multirow[b]{2}{*}{ Jlate .tud thento. } & \\
\hline & & $\begin{array}{l}\text { Tres: } \\
\text { pugi }\end{array}$ \\
\hline 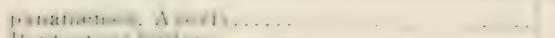 & recisxxiv, 94: & $\therefore 7$ \\
\hline 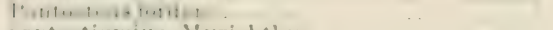 & $\mathrm{x} \times \mathrm{xi}, 73$ & $17 !$ \\
\hline grantontigmins, Myrichthys ................. & 1.:1! it: & $2:$ \\
\hline 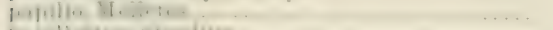 & 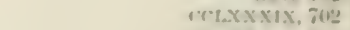 & 12 \\
\hline 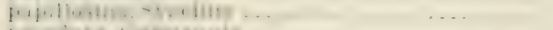 & {$[20: 1, x \times x+11,94]$} & -7 \\
\hline 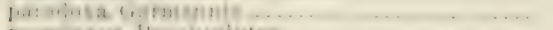 & rexx & $=2$ \\
\hline 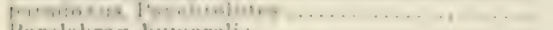 & cecilitu & $2-5$ \\
\hline l'aralabrax linmeralis........................ & CXc, ss! & 160.0 \\
\hline 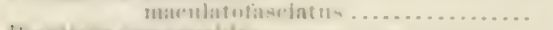 & Cxis: s!in & 118 \\
\hline 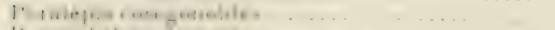 & ani. & 1.2 \\
\hline 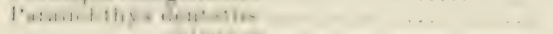 & . $1: 58:: 1=2$ & 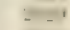 \\
\hline 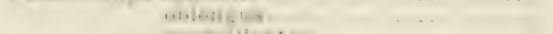 & $1=\quad 8.5,5,25$ & - \\
\hline 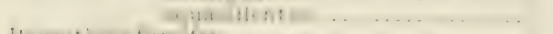 & $\ldots+18004, \%$ & $-1+1$ \\
\hline 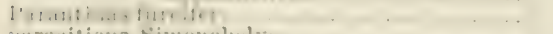 & , s.8., 84 & $1 . .1$ \\
\hline 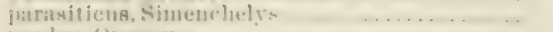 & 1.Ne. Iit & 16 \\
\hline 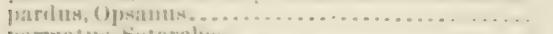 & $\ldots \times \times 8:-113$ & $-i 6$ \\
\hline parmatus, sutarehes ... , . .............. & $\cdot 1: 8: 807$ & the \\
\hline 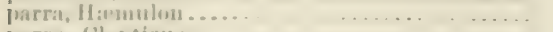 & cr:st, 530 & 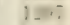 \\
\hline parat, Clopiticus..... & (1.xхxาเแ, $59 y$ & 10. \\
\hline 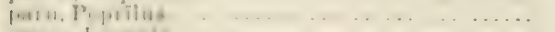 & $(: \ln +144$ & ." \\
\hline 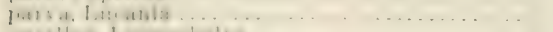 & rix,,$y z$ & $\ldots \cdot$ \\
\hline 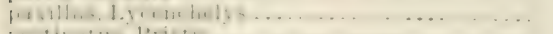 & 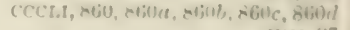 & $-6: 7$ \\
\hline 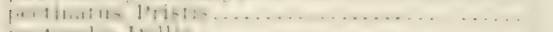 & V111, 27 & 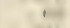 \\
\hline 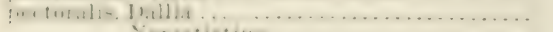 & $x+1 X, 21 ; \pi$ & +14 \\
\hline 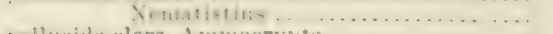 & 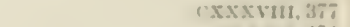 & $\therefore$ \\
\hline 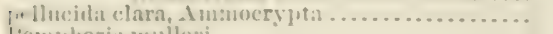 & CLxxแ, 45t & $:=$ \\
\hline 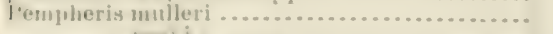 & CLIII, \$1: & i- \\
\hline 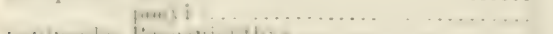 & CLIII, $\$ 13$ & 45 \\
\hline 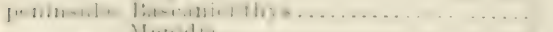 & I.xur, J66, 16\%a & $\overline{3}$ \\
\hline Mendia $\ldots \ldots \ldots \ldots \ldots \ldots \ldots \ldots$ & cxxiv, 33i & $: 17$ \\
\hline 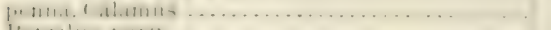 & $1+18.15=0.1$ & 17 \\
\hline 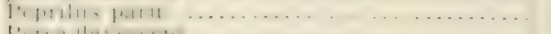 & 12.14 & s.: \\
\hline 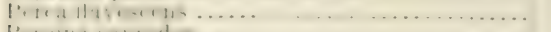 & is: $1: 4$ & $1 \omega_{-}$ \\
\hline 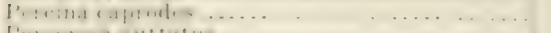 & $1 \therefore 1 \quad \therefore i s i n$ & int \\
\hline 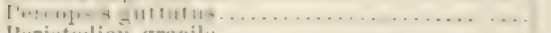 & $\operatorname{cx} \div 3,: 1: 3$ & $\overline{-1}$ \\
\hline 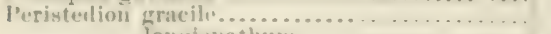 & $\cos x \times 11,750$ & 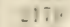 \\
\hline longispathum $\ldots \ldots \ldots \ldots \ldots$ & C(CXX1, 775 & $\because 17$ \\
\hline$n \quad \ldots \ldots \ldots \ldots \ldots \ldots \ldots \ldots$ & ecexxi, it & 1:- \\
\hline 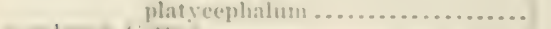 & cecxxil, 77, -7in & $\therefore-$ \\
\hline 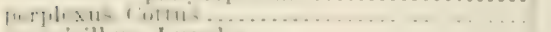 & 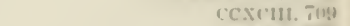 & $\therefore \cdots$ \\
\hline$\ldots \ldots \ldots \ldots \ldots \ldots \ldots \ldots$ & $\because+1, \because$ & 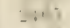 \\
\hline 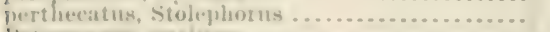 & 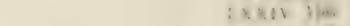 & 43 \\
\hline 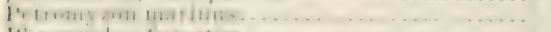 & 13 & iin \\
\hline 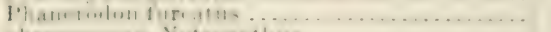 & $(\mathrm{cxx}, 5 \mathrm{~s}: \mathrm{l}$ & $\because \cdots$ \\
\hline 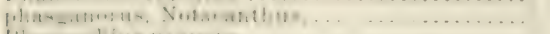 & 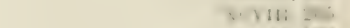 & int \\
\hline 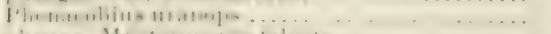 & $1 \therefore, 8.8$ & 4 \\
\hline 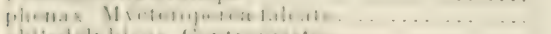 & 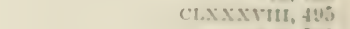 & 110 \\
\hline 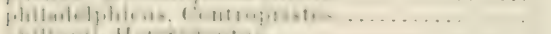 & cxci, 501 & I.W! \\
\hline 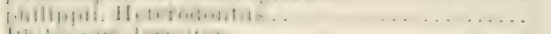 & 114.10 & \\
\hline 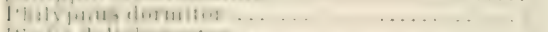 & . $.8 .80=7-1$ & $21 \%$ \\
\hline 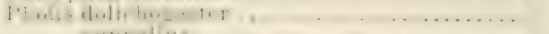 & $\therefore .111,11$ & $\therefore 116$ \\
\hline .... & $1+4.1 .=$ & .611. \\
\hline 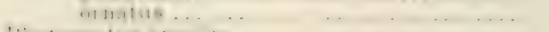 & $1, \because 1: 1,-$ & -41. \\
\hline 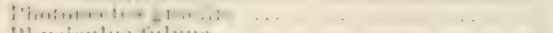 & $\therefore .1 \%$ & $\therefore \because$ \\
\hline 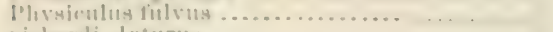 & $\cdots+1$ tht $=\ldots$ & $\pm 1:$ \\
\hline picharali, olnturus ........... ... & 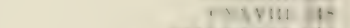 & $\therefore$ \\
\hline 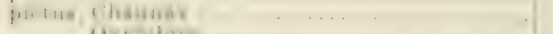 & 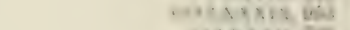 & $\therefore$ \\
\hline rowline & 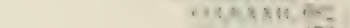 & 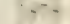 \\
\hline bizmentarius, - 1 pogens. & (1.x. 111, t): & $1:=$ \\
\hline 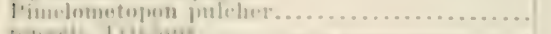 & 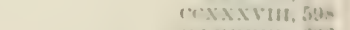 & $i^{-1-}$ \\
\hline 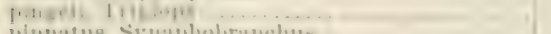 & 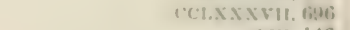 & 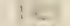 \\
\hline jimmatus, sivn & L.VI, 156 & 118 \\
\hline 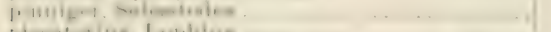 & 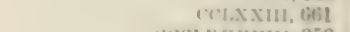 & 7 \\
\hline 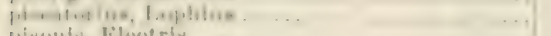 & $\because 70 \times x \times 14,0.72$ & 27 \\
\hline in, Eleotris .............................. & $\cos c x-783$ & $-2=1$ \\
\hline 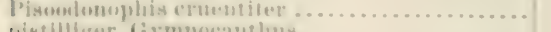 & 1..811, 163 & 7 \\
\hline 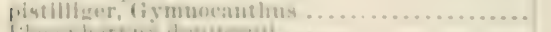 & 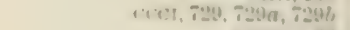 & $\%=$ \\
\hline 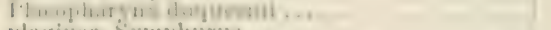 & xxx111, $8:$ & in. \\
\hline 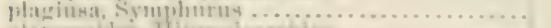 & 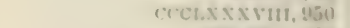 & $2 \pi i n$ \\
\hline 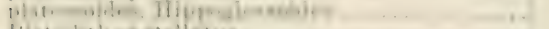 & ('cel.xxแ, 919) & 2.114 \\
\hline us & 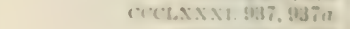 & 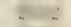 \\
\hline 1111 & cecLxхxแ, u\&! & - \\
\hline 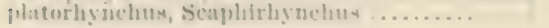 & xxt, 4 & 117 \\
\hline
\end{tabular}


platostomns, Lepisosteus

Platy gobio gracilis.......................... CCICIX, 724,724a 1987

I'lectromus crassiceps.......................

$\operatorname{cxxx}, 356$

suborbitalis.......................

pleuriticus, Salmo clarkii

$\operatorname{Lxx}, 211$

Pleurogrammus monopterygius ...............

I'leuronectes quadrituberculatns .............

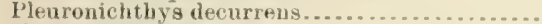

plumieri, Harnulon.

CCLNEIX, 675 2819

$\begin{array}{ll}\text { CCCLAXV, } 926 & 2637 \\ \text { CCV } & 232\end{array}$

$\mathrm{CCY}, 532.130$

XXxil, 76 175

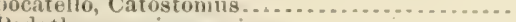
CCCIX, 716

Podothecus acipengerinus........................ accipiter

сссуй, $745,745 a$

vetermus. . .

Pecilia presidionis.

poeyi, P'empheris.

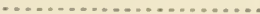

polaris, L codalepis -

Pollachius virens

(1)

picanthocephaium, Bryostema..............

polyacanthocephalus, If yoxocephalus.

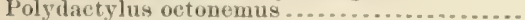

Polyolon spat hula ..... . . . . . . . . . . . . . . . .

l'olyprion americaums.

Polyoteris hichix....

Powacantius arcuatus.

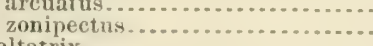

Pomatomus saltatrix.

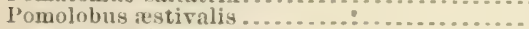
chrysochloris .......................... metiocris . . . . . . pomotis, Acantharchus.

Pomoxis angularis ....................

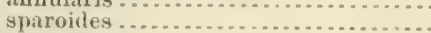

Pontinus macrolepis . . . . . . . . .

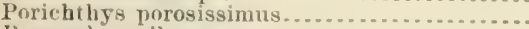

Porogadus miles.

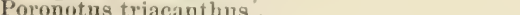

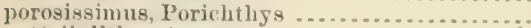

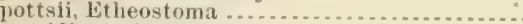

presidionis. P'recilia.

pretiosus, H y pomesug.

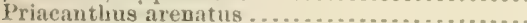

princeps, Cottus ...........................

Prionace glanca................................

Hrionistius macellus . . . . . . . . . . . . . . . . . .

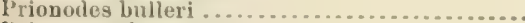

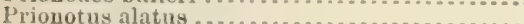

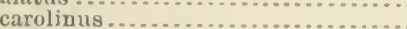
ovolaus ............................

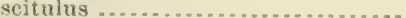

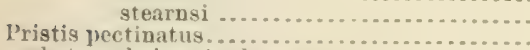
probatocephalus, irchosargus .............. proboscilea, Limanda ......................

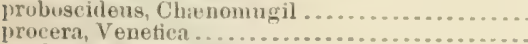

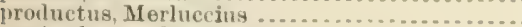
protublorum, Lepophidium

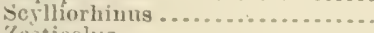

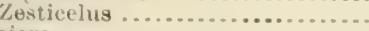

I'romicrops itaiara. - . P'ronotogrammus multifasciatus . . . . . . . . . . .

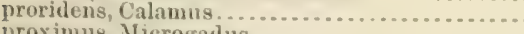

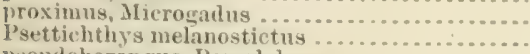

psendoharengus, Pomolobus ................

Pendopleuronoctos smericanus. . . . . . . . . .... CCCIx, 747, 747a CXIY, $303-697$ CLIII, $413 \quad 979$

CCXXY, $573 \quad 1482$ CCCL, $857 \quad 2468$ CCCLIX, $886 \quad 2534$

CCCXLI, 828 2408

CCXCVI, 718 1976

CXXVIII, $350 \quad 830$

$\mathrm{xx} .43,43 a \quad 10$

CLXXxI, $480,480 a \quad 1139$ CCLI, $6 \geq 3 \mid-167$ CCLII, 624 1681 OXLVIII, $400 \quad 946$ LXXI, $190 \quad 420$ LXX, $187 \quad 425$ LXXI. $188 \quad 425$ I.XXI, 189 


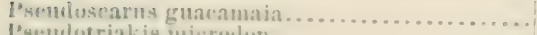

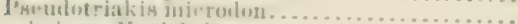

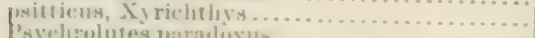

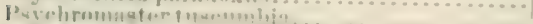

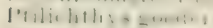

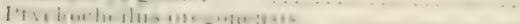

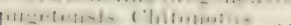

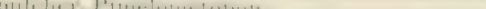

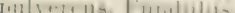

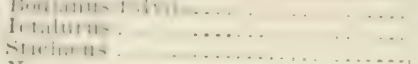

vith

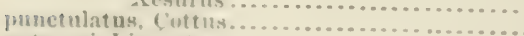

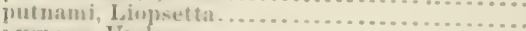

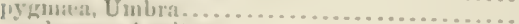

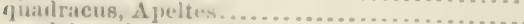

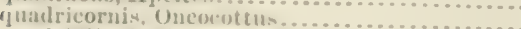

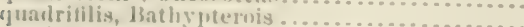

yuadrituberculatus, I'lewronectes...............

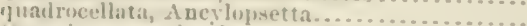

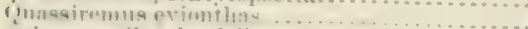

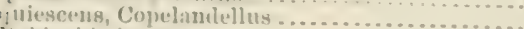

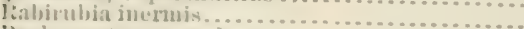

Rachycentron eanalus . ........................

ralliatus, Iriluo.

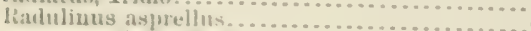

liajia ackleyi.

molenirles

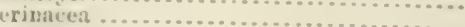

ocellata

stellulatit

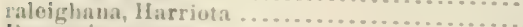

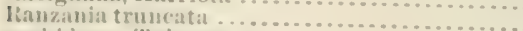

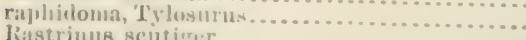

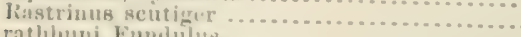

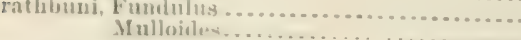

rectifremun, Enponacentrus.................

rolltingi, Orthonristis....

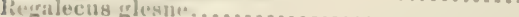

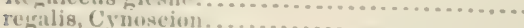

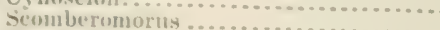

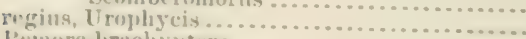

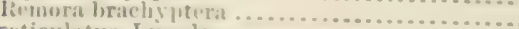

rutiendal114 Livedes

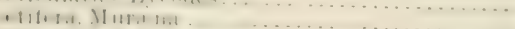

1 : 1 1

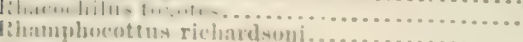

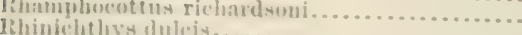

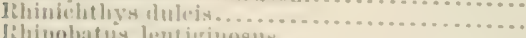

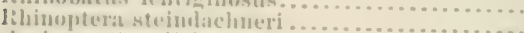

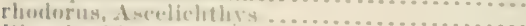

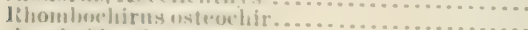

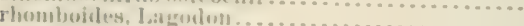

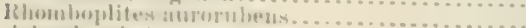

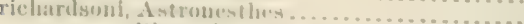

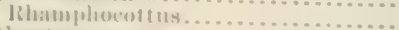

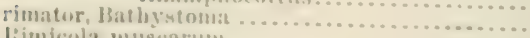

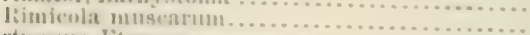

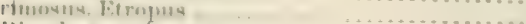

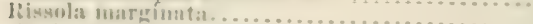

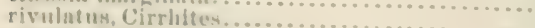

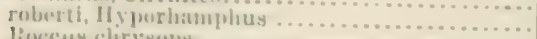

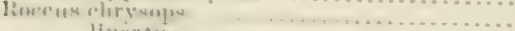

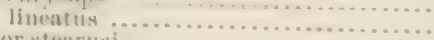

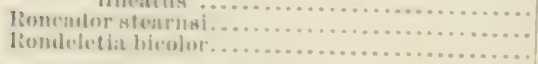

roudeletii, Jisunautes

Limupuilus jorelani.

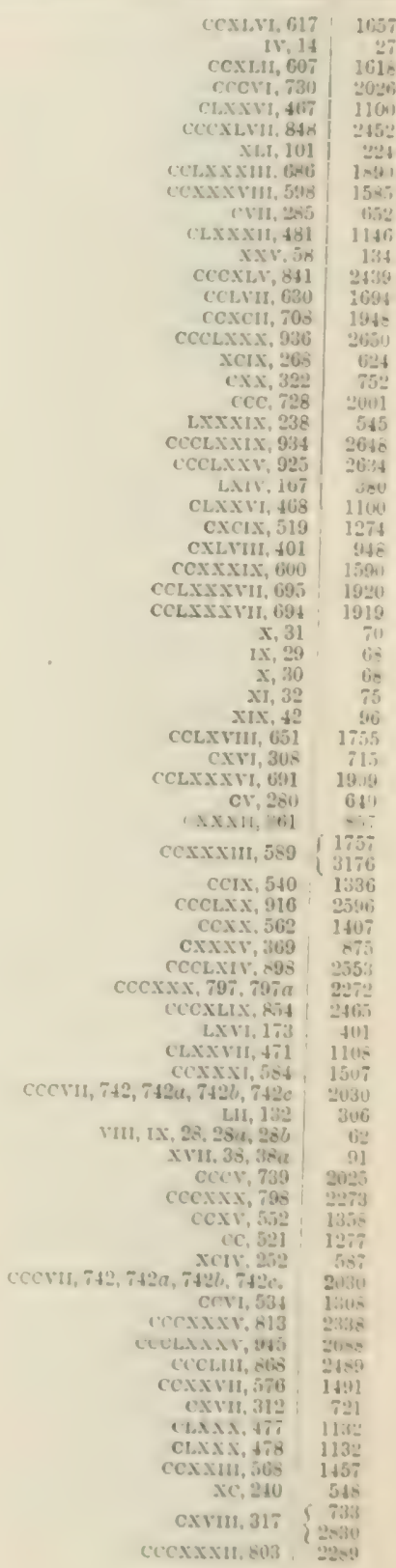


rosaceus, Zalembius.

rubicundus, Acipenser.-.

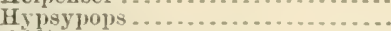
rupestre, Xiphiclion

rupestris, Ambloplites .

$$
\text { Kuhlia. }
$$

Rupiscartes atlanticus

rupiscartes, Moxostoma

Ruscarius meanyi.

Rutilus bicolor.

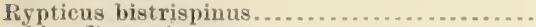

sabina, Dasyatis

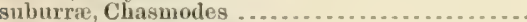

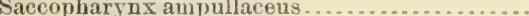

Sagenichtbys ancylodon. . . . . . . . . . . . . . . . .

saida, Borogadus ...........................

Sa!mo clarkii bouvieri.

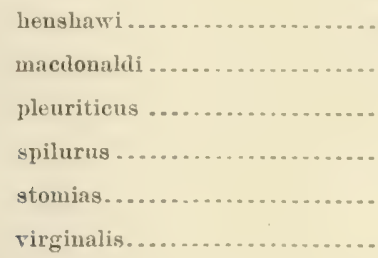

gairdneri

irideus. -

salmoides, Micropterus

saltatrix, Pomatomus.

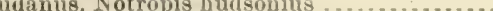

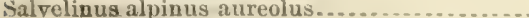

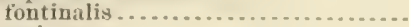

malwa ........................

oquassa.......................

sapidissima, Alosa.

Sardinella lumeralis

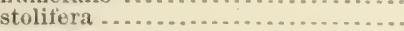

Sarritor frenatus. .

satiricus, Neoclinus.

saurus, Elops

Oligoplites

Scombrosox.

saxatilis, Abudefduf..

sayauns, A phredolerus

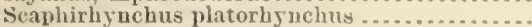

scarus cieruleus.

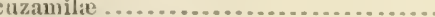

emblematicus.

strongyloceplialug a

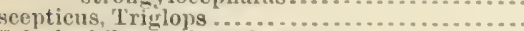

Seliestophilus medusophagus.

Sehilbeodes exilis.

$$
\text { furiosus } \ldots \ldots \ldots \ldots \ldots
$$

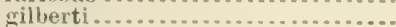

(1)

nocturnus

schmiltii, Hoplunnis.

schopti, Chilonyeterus .....................

M

Scianoj, diutera.

ocellatus

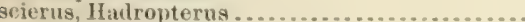

seitulus, Prionotis.

rionotus. .

Scomber wolias $\ldots .$. regalis cсxх1х, 581 1500

$\mathrm{XX} 1,46 \quad 106$

\begin{tabular}{l|l} 
cсxхxIV, 591 & 156
\end{tabular}

CCCXLIY, 838 | 2420

CLT $, 419,419 \alpha, 419 b, 419 c \quad 99$.

CLXi11, $432, \ldots$.

СССххх1 $825 \quad 2397$

XX̌xir, 9319

CCLXXXY, 690 1908

XLIII, $107 \quad 24$

CxCIY, $509 \quad 1233$

хIV, 36, 36a 8

ссCхxхIx, 824 2392

LXYI, $175 \quad 100$

CCXXI, 56t 1416

CCCLIX, 885 253:

Lxxx, 213 if 490

Lxxנx, $208 \mid\left\{\begin{array}{r}493 \\ 2519\end{array}\right.$

Lxxxr, 21t $\left\{\begin{array}{r}457 \\ 2210\end{array}\right.$

Lxx , 211 if 496

2819

LXXIX, $210 \mid\left\{\begin{array}{r}49 . \\ 2810\end{array}\right.$

LXx. $213\left\{\begin{array}{c}49 \\ 48\end{array}\right.$

2819

Lxxix, 209 \{f 495

Lxx

LxxxI, 216

CLXIII, 431 1015

CxLVIII, $400 \quad 910$

XLVIII, 120 270

LXXXIII, 220 511

LXXXI, 218 506

Lхххแ, 219

Lxxxi1, 221 514

LXXII, $191 \quad 427$

LXXII, 193

LXXII, 194 43

CCCx, 751 2073

CCCXXXY, $816 \quad 2355$

LXYII, $178+110$

cxxxviII, 378

Cxvit, 314 705

CCXXxI5, 590 156

\begin{tabular}{l|l} 
CXXII, 331 & 786
\end{tabular}

\begin{tabular}{l}
$\mathrm{x} \times 1,48 \quad 107$ \\
\hline
\end{tabular}

CCXIIY, 613 1652

CCXLIY, 612 1618

CCXLF, 614 3654

$\mathrm{CCuLv}, 61$

CCLAXXVII, 695 CLI, 107

xxyII, 65

149

x.vil, $67,67 a, 67 b$ 140

xxтี11, $66 \quad 147$

Xxix, 68 its

xxvir, 6t 146

LrIII, 151 361

CCLXVI, $648 \quad 1748$

CCLX, 635 1718

CCxxir, 567 1453

CLXVII, 441 1037

Cccxix, 768 2157

\begin{tabular}{l|l} 
cer, 531 & 1303
\end{tabular}

cxxxII, $364 \quad 866$

cxxxiI, 363 | 80,

cxxxi5, 368 87t

CXXXY, 369 875

- Ilate by error labeled Surdinella sardina. 


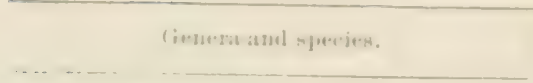

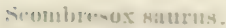

mecumbras, secomber.

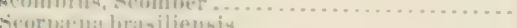

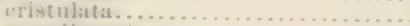

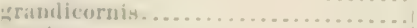

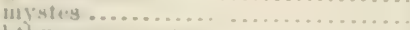

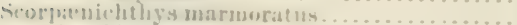

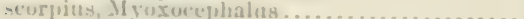

or rijeta, - llitera.

scuticaris, Baseanichiliy. . . . . . . . . . . . . . . . .

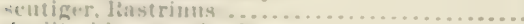

sevllurlámus jorofimulermus

sivetalina ceralale. . . . . . . . . . . . .

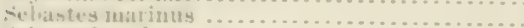

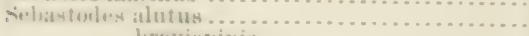

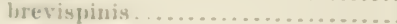

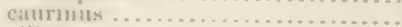

ciliattus. . .....................

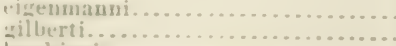

l.m.

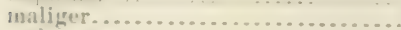

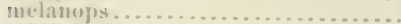

miniatus...

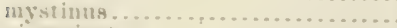

mierucinctus..................

liiuniger.

serriceplas

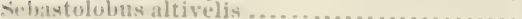

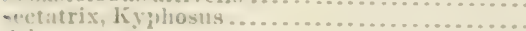

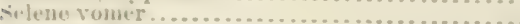

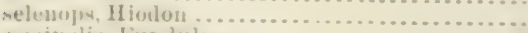

seruiuolis, Fumblulus,

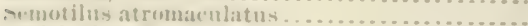

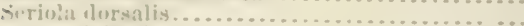

laliumli.............................

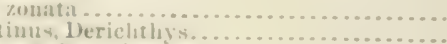

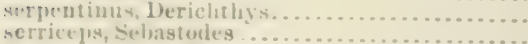

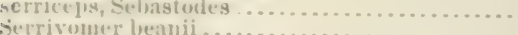

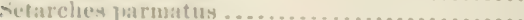

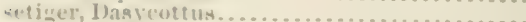

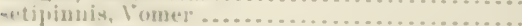

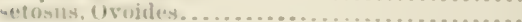

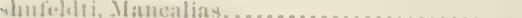

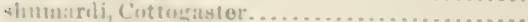

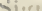

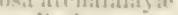

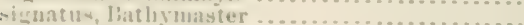

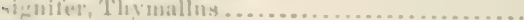

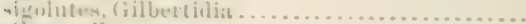

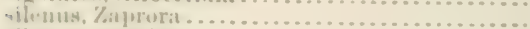

wilum, . Ir:eventina.

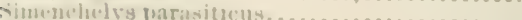

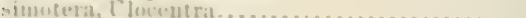

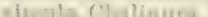

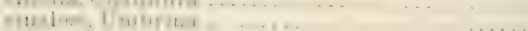

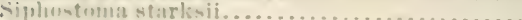

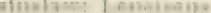

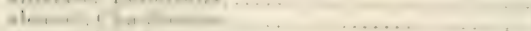

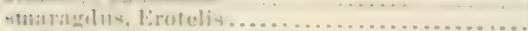

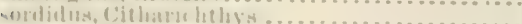

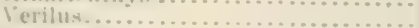

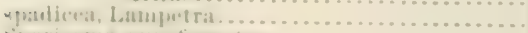

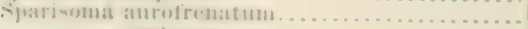

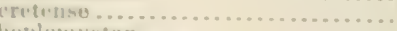

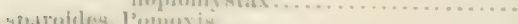

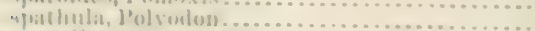

4)

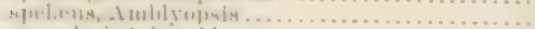

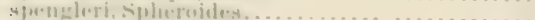

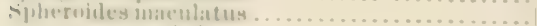
mejolicluy. .

I'late: anel ti:nre.

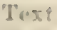

joane.

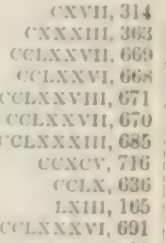

Int?

$1 \times+1$

1850

1080

1603

15 it

17.

378

1909

III. 11

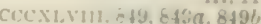

CrLXvH, 650

CCLXX11, 660

CCLXXI, 65i

CCLXXIY, 60

('CLXX, tis.

CcLxx1, 658

CCLXXY, 665

COLXXI, thit

CCLXXIS, Geit

cCLXIx, 65

CCLXxแ1, 66"

CCLXX, $65 t^{\circ}$

(CLXXVI, 667

cos.s.

CCLXXY, 666

CCLXIX, 653

c.cxix, 55y

CXLIV, I'XLY, 393, 3939

L.xงแน, 181

CIV, 270

xis 1010

$1 \times x \times 1 x, 380$

(XI) 382

('xxख1x, 3s!

L., $1+2$

CCLXXV, 666

Lv111, 15:3

CCLXXIX, 67̈

cec, $7 \div 7$

CXLIV, 39:

CCLVY, 646

cecse, 955

CLXvIII, \&4t

L.XIX, 184

C(C)

Lxखा山r,

cccvi, 711

cL.11, 109

L.x.xiा1, 132

L.1. Itt

(1.XX, if

comi

(4. s.s:5, 071

(2xx, 325

$46+11 / 19$

$x \div 1,=0$

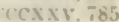

$4014 \times 15,94$

CC.11, 525

11, 6

Coxy.11, Giv

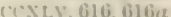

cexs.1\%, 611

(1.15, +16

$x \times,+3,+3 a$

xel. 241

लxi, 307

ret.xiv, 683

भ1.XI5, 644

CELIV (4."
$2 \div \frac{11}{51}$

170

$179 n$

17is

$16=0$

1783

17อ?

in:s

1.:.

182

] 1825

1794

1784

1007

I.5.

18:\%

1763

1350

9ase

414

647

$-2-2$

902

903
$90 \times$

343

1827

$36 \%$

1 (s)

1991

934

1734

2730

1046

coson

as

517

$+028$

2050

$5 \div 6$

$3+9$

$10,5: 3$

‥

A140

$\div 1$

ins

2201

$=0$

1:34

$1: 3$

103

it...

ดล

111]

561

706

$17: 2$

1733

$1=0.9$ 
Sulueroides spengler

testuline 11 .

sphyrana barracula ......................

Sphrma tiburo.

ypilotus, Hypohomus ....................... qpilurus, salmo clarkii . . . . . . . . . . . . . . .

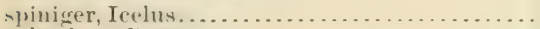

-plenki(ns, beryx . . . . . . . . . . . . . . .

Sifualus acant lias......................

squamilentus, Paralichthys ..................

starksii, Sipliostoma.........................

stathmonotus hemphillii...................

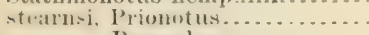

Roneador.

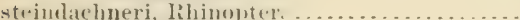

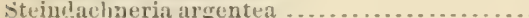

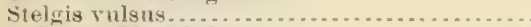

stellatus, Platichithys ..

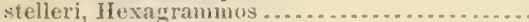

Mroxocephalus......................

stelliter. Fumblulus........................

steilulata, liaja...................................

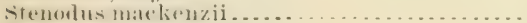

stenotomus atenle:atus.

chrysops..........................

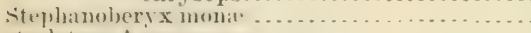

sterletus, Averruncus.

Steruiry depustethes o.

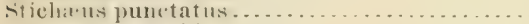

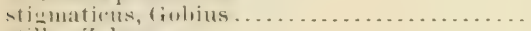

stillie, Zallocys.

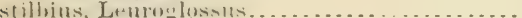

stimes. Itlerillit.

stizostedion canadense .................... vitre'um

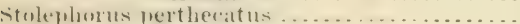

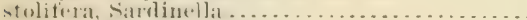

stomias, Itherestles... . . . . . . . . . . . . .

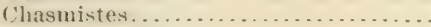

Silme clarlii ....................

Stmuias firox

siriatus, ('intropristes

$$
\text { lipineplatus. }
$$

strouerylocephalus, scarus

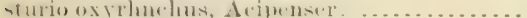

shbhifurcata, I'l variat ......................

suluethitalis, J'lertrumus

subterraneus, Typhliehthys

sllett: Litimy

superciliosns, Hexamrammo

surinamensis, Anisutremus

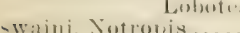

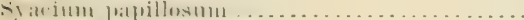

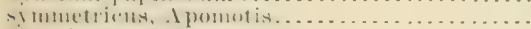

Symplumes margintatus.

plaýusa . .......................

williamsi

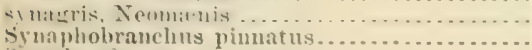

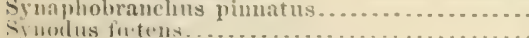

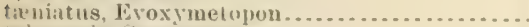

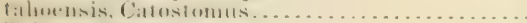

1 alismaniat atutillatmut. . . . . . . . . . . . . . .

'Tarlotonheania temua

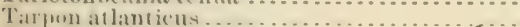

Tilltuma onitis . . . . . . . . . . . . . . . . .

'Iintogolabrus ailspersus

teleseopus, Nintropis.

temul, Fardetombania.

13ull. No. 47, pt. $4-\mathrm{Xi1}$

\author{
CCLXIV, 643
}

cCLXV, 645, 645a 173

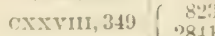

\begin{tabular}{l|l|}
19 & 4
\end{tabular}

(LxViII, 443 : $104^{\circ}$

Lxix, 210 ? 2819

CCIXXYI, 69:3 191

r.ixy, 345

VII, 24, $24 a \quad 54$

CCLAXIN, 92:3 20631

CXx, $325 \quad 7: 1$

CCCXL, 8ะ7 207

$\operatorname{cccxx}, 7 i 1 \quad 2160$

cсxim1, $568 \quad 1457$

xVI, 38, 38 91

CCCLXY1II. 909 2505

cccix, 748, 748e

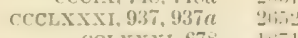

CLXXXi, 678 1si

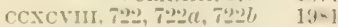

C1: 279 tit

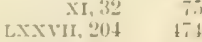

Coxit, 450

ccist, 54t 13:4\%

cxxix, 353

$\operatorname{cccx}, 750,750 a \quad 2-17$

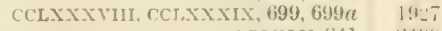

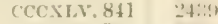

cocxuvi, 78i

CXLYIII, 399 - $=4$

Lxxxvir, 233 -

cxxir, 332

CLXIV, 434 lit:-

CL.XX, $433,433 a \mid$ । $11-1$

Lxxiv, 196

LXXI1, 194

CCCLXXI, 917 "eir!

xखxy.86 2796

Lxxx. $213\{10 \%$

xс15: 253 (

Cxc, 500 11:?

cLxxxu11, 483 11:

CCXLN, 615.615\% - -

CCCXIS. 841

cxxx, 355 |

cxv,306 -i1!

xxxvr.89 19

CCLXxxi, (iT!) 1-:-

cCvil, 533 1:1.

cxcrv.510 1:...

XLIX, 1 25

(CCLXXIII, 941

CLIX, 404

CCCLxxxill, 949:

cCcLxxivII, 950 ㄱ.

CCCLXXIII, 951 ㄱו1

('xсv'I, 518 1:-...

LWT, 146

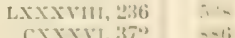

xivili 78 i:

LAXI, 1 198

xerl, 24 s

LXVI, 177

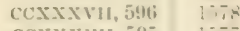

ссхххин, 593 19:

I. 126 Xc: $1,248, \quad \because \because$ 
formis, Lranialeas

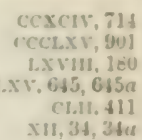

$\operatorname{cosert}$, it

1CCLXY, 901

1.x $13111,1 \pm 0$

C1.11.411

xi1, 34, 34u

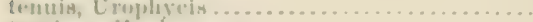

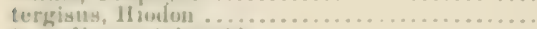

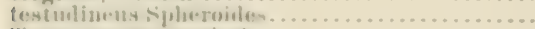

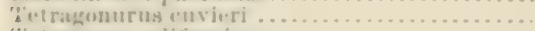

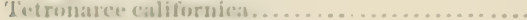

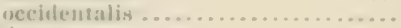

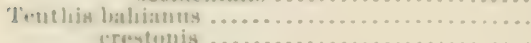

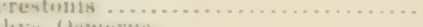

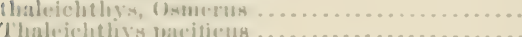

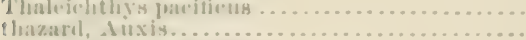

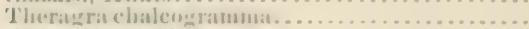

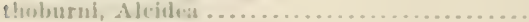

'd'sumallus trieshor.

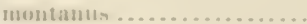

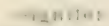

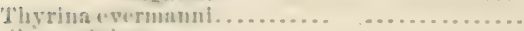

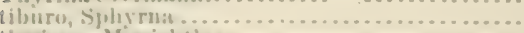

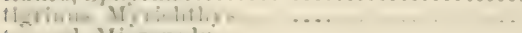

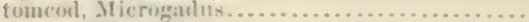

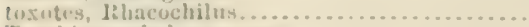

Trachince epladus myojes..................

I'raclsinotus earolinis.

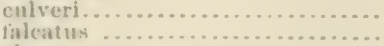

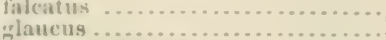

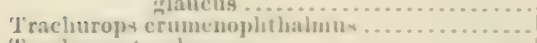

'I'rachurus trachurus. . . . . . . . . . . . . . . . . . . . .

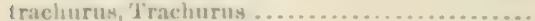

transuontana, Columbia ...................

transmontanus, Acipenser.

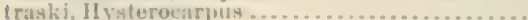

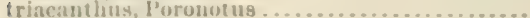

'Triel.iurus lepturus.........................

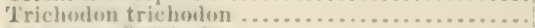

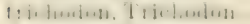

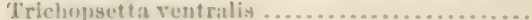

iricolor, Jfolacanthus...

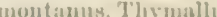

T'hymallus.

iricoruis, Lactoplirys.......................

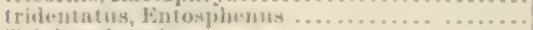

'Trimlops buani

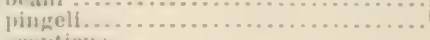

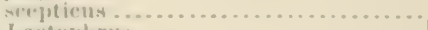

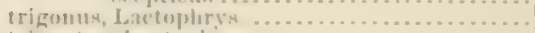

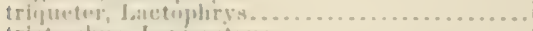

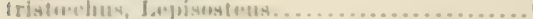

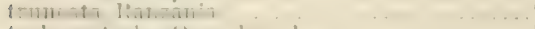

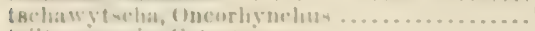

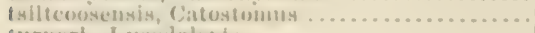

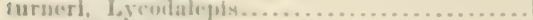

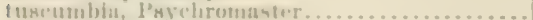

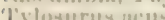

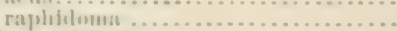

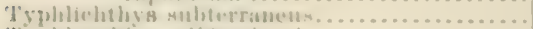

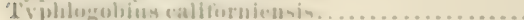

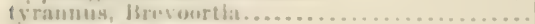

liberentra milloerti.

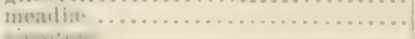

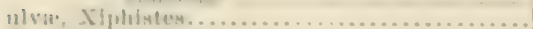

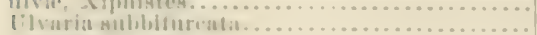

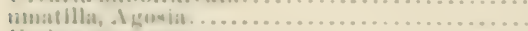

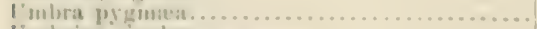

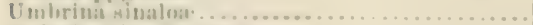

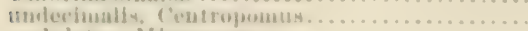

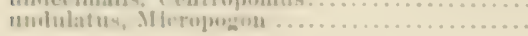

1906

2505

413

1794

9ici

$\times 1,33$

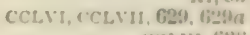

$(\times 1.21,638$

I. $\times \times \times 7,2=1$

LXXXV,

(xхxแ1, 395

crets

crLxxxm, Got

2.xхxแ1, 2:3

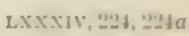

as. von, $2 .-$

$\operatorname{cxx}, 340$

v. $1 !$

C.x. 16]

CrCLX, E90

cсxхxi, 5ni

Lxx $x$ 111, 295

c'xt.

c.xเ.vII, 397

CXL.VI, 390

Cxt.VI, 395

c.xน, $38:$

(X), 384

( $x$ L, 384

(x) $x{ }^{2}, 320$

$x x_{0}+4$

('CXxजम, 57

Cl, 105

сxxxvir, 37

rerxxxu1, 800

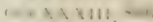

CCCLxxख11, 94 C(LIII. 62:

2.xxilv, 22t, 22te

L.x.xiv, 2033

('). 1,033

cesxxxv!1, 69:

cclxxivi, 090

coldxx+yy, 695 CCLxill, bis, tistas CeL.X1, to57 xi11, 50

xill,
a

L.xงแ1, 206

xxxiा, हो

('ес1., 853

c1xखvt, 460

('xiv, 30s

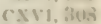

PXv, 31ki

coxxix, igs

I.Xx111, 195

CI.X1X, $4 \pm 6$

c'I.XIX, 417

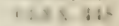

corxum, 8sti

ccrst.

1.11, 138

x०1x, 268

cexivis

ct.xin, 470

exxiv, sivo 
unicolor nigricans, Hypoplectrus

unitasciatus, II rporlamphus ...............

unimaculatus, Arclosargus .

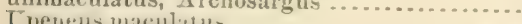

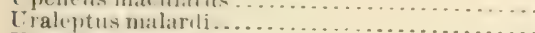

Iraniclea temui-.

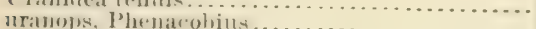

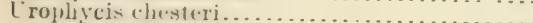

cluss $\ldots \ldots \ldots \ldots$
cirratus $\ldots \ldots \ldots \ldots \ldots$

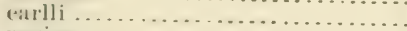

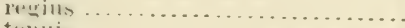

nter, Catulus.

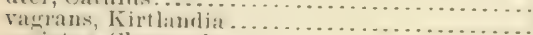

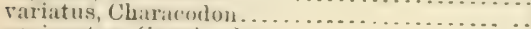

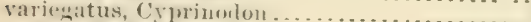

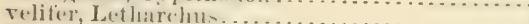

Venefical proceral . . . . . . . . . . . . . . . . .

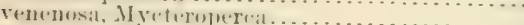

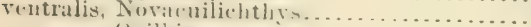

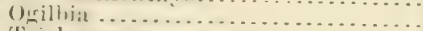

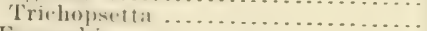

vemista, Eumerelija

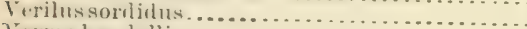

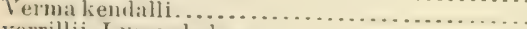

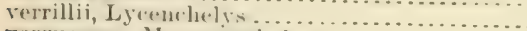

verruensus, Myosiceplatins.

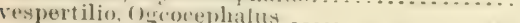

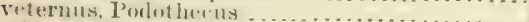

villusus, Vallotms

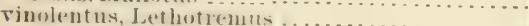

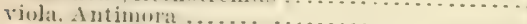

violacrus, Escolar

virens, l’ollatlins.

virgatum, Etlueostumat.

viruninious, Inisutremulu

viridia, Evoplites.

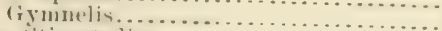

vitremm, Sitizosterlion

vittata, Chammomtritit , . .

vivaltus, I'⿺miantliats

年

Copllatal:autlıus....

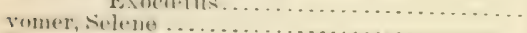

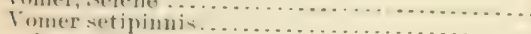

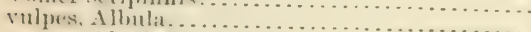
Alopiats.

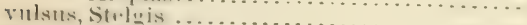

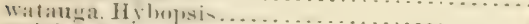

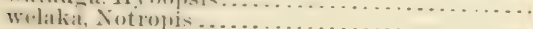

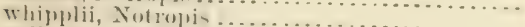

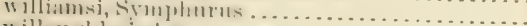

" illumglivi, dirotus . . .

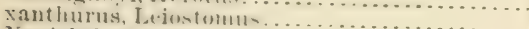

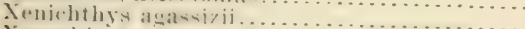

Aemorhirus triatiathio.

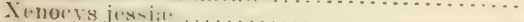

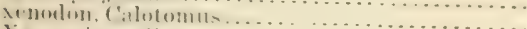

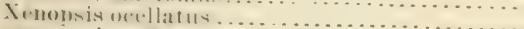

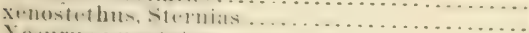

Xesurms punctatus . . . . . . . . . . .

Xiphilinn rupestre . . . . . . . . .

Xiphistes chirus. ulva

Xiphoplanms he]li.i.

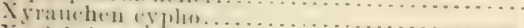

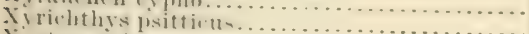

ijoterna cinereum

Iystes axinophrys
C. $V 1,311 \quad 7 \pm 0$

CCXVI, $553 \quad 1359$

CxxxII, $362 \quad 858$

CCCLXI1, 894 2545

CrseIV, 714 1960

L.I, 130

CCCI,

CCCLXY, 90: 2555

CCCLXI6, 8:9 | 255?

CCCLXV,900) 25.54

CCCLXIV, 898 255

CCCLXY, 911 $255 \%$

III, 12 20

Cxxiv, 336

CXI, 295, 295

CXI, CXII, 296,2960

L.XI, 160

LVIII, 152

CLXXXฑT, 49]

CCXLL, 605

CCCLT: 87:

CCCLXXXu, 940

CCXL, 6it3

CC11. 5:5

LX, $159,159 a$

CCCLI, 850

CСхCVII, 720, 720 CCCXCII, $958-95 \times 2$

$\operatorname{CCCIX}, 747,747 \alpha$

LXXXV: 2505

ССCXIV, 759

CCCLXII, 893, 893a

CXxxv, 370

CCcLtx, 886

CLAXV 465

LXxix, 209

Cers, 539

CxerI, ilt

CCCLII, 86t-8tic

CLIIV, $433,433 \alpha$

LXVI, 174

CX゙CII, 505

cCCxxil, T70

Cxख्या, 318

CXLIT, CXL, 393, 393

cillt, 39:

เ.xง111. 17?

เา. $2(1$

CCCIX, it8, 7t:

LIII, 137

XLV, 113

XLVIII, 1:1

CCCLxxxyแI, 951

CL. 408

ссхх11, 509

(C)II, 5$)^{2}=$

(ccxi, 754, 754

CCII 5.',

cсxцII, tiug, 609

CCXLVI, 618

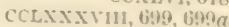

('CLVH, tisu

CCCXLIV. 83.

Co'xhIV, 837

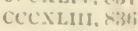

cxv:304

xxivis

cс-1411, 607

сCXVIII, 550

CCCx, 75:, 75: 


\section{EXPLANATION OF PLATES AND FIGURES.}

No'E.-The actual size of the specimen from which each illustration was drawn may, in most instances, be determined by the use of the inch mark beneath the engraving, which, in the photographic reduction of the drawing, is reduced in the same proportion as the drawing itself.

\section{PLATE I.}

1. Branchiostoma caribaum kimeleral

Drawing by A. H. Baldwin from a specimen in the United States National Museum.

2. Polypterus bichir Geoff'. St. Hilaire.

Drawing by Anna L. Brown.

3. Petromyzon marinus Linnxus............................ Drawing by H. L. Todd from No. 10654, U.S.N.M., collected at Woods Hole, Massachusetts, by V. N. Edwards.

4. Entosphenus tridentatus (Gair(ner)

Drawing by A. H. Baldwin from No. 48204, U.S.X.M., collected in Kamchatka by Dr. L. Stejneger.

PLA'TE II.

¿. Lampetra aurea (Bean)

Drawing by H. L. Todd from No. 21524, U.S.N.M., collected by Lucien M. 'T'urner in the Yukon River, Alaska.

(i. Lampetra spadicea liear

Drawing by H. L. Told from the type specimen, No. 38005, U.S.X.M., collected by Prof. A. Duges at Guanajato, Mexico.

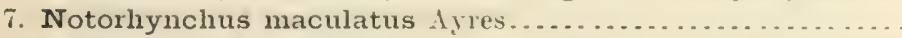
Drawing by H. L. Todd from No. 27191, U.S.X.M., collected in Humboldt Bay, California, by Dr. Jordau.

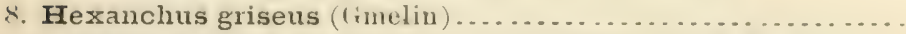

Drawing by H. L. Todd from No. 37790, U.S.N.M., collected in Currituck Inlet, North Carolina, bฐ D. M. Etheridge.

\section{PLA'TE III.}

9. Gyropleurodus francisci (Girard) ....................

Drawing from No. 24977, U.S.N.M., collected at Wilmington, California.

10. Heterodontus philippii

Drawing by Anna L. Brown. 
11. Scylliorhinus profundorum fioole aud Bean.

Drawing by M. M. Suith from the type, No. 35isti, U.S.X.M., collected by the Albatross at $39^{\circ} y^{\prime} \mathrm{N}$. and $72^{\prime} 3^{\prime} 15^{\prime \prime} \mathrm{W}$.

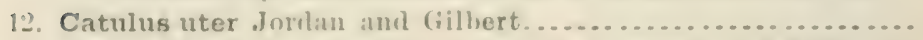

Drawing ly A. II. Maldwin from Yo. 26866i, U.S.X.M., collected at sinta Barbara, California.

\section{PLATE: IV.}

1:3. Ginglymostoma cirratum (Gmelin)....................

Drawing ly H. L. Todd from No.2983, U.S.X.M., collected at Fort Jeflerson, Florida, by Dr. Whitehurst.

14. Pseudotriakis microdon Capello .........................

Drawing by H. L. Todt from No. 32516, U.S.X..M., collected at Amagansett Island by J. B. Edwards.

15. Galeorhinus zyopterus Jordan and Gilbert................

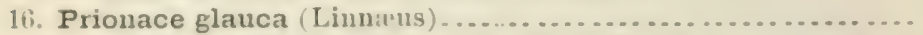

Drawing by H. L. Todd from Yo. 38001, U.S.X.M., collected lyg schooner Grampus.

\section{PLATE: V.}

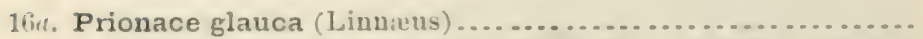

Drawing by II. L. Todd from No. 38001, U.S.N.M., collected by the schooner Girampus.

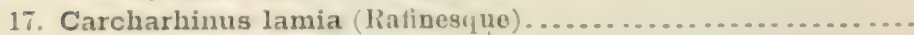

Drawing by 1I. L. Tould.

18. Hypoprion brevirostris P'oey.

Urawing by W. S. Haines from No. 38197 , U.S.X.M., collected by the Albutross at Watlings Island.

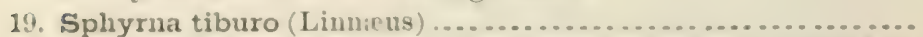

I)riwing by II. L. Todd from No. 26582, U.S.X.M., collected by Silas Stearns at Cedar Keş, Florila.

\section{PLATE VI.}

20. Alopias vulpes (Gmelin)

Drawing hy II. I. Told from No. 25962, U.S.X.M., collected oft Yewport, Rhode Island, by Captain Rocklift:

21. Isurus dekayi ((iill)

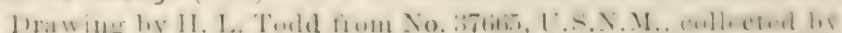
Silas Stearns at P'ensacola, Florida.

22. Lamma cormubica (fumelin).

Drawing ly. II. I. Tould from No. 27368 , U.S. X.M., collecterl at Santa lBarbara, California, lyy Dr. Jorlan.

\section{PIATE: VII.}

… Cetorhinus maximus (Gunner)

Drawing hy S. F. Denton, copied from Annales du Museum d'Historie Naturelle, vol. 18, pl. 6. 


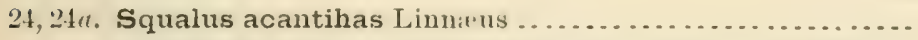

Drawings by S. F. Denton from No. 22316, U.S.N.M., collected at Gloucester, Massachusetts, by the U. S. F. C.

\section{PLATE VIII.}

25. Centroscymnus cœlolepis Bocage and Capello

Drawing by H. L. Todd from No. 26219, U.S.N.M., collected by the Fish Hawk off Newport, Rhode Island.

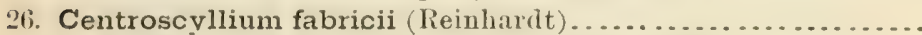

Drawing by H. L. Todd from No. 22879, U.S.N.M., collected by George W. Scott at $44^{\circ} 23^{\prime}$ N., $53^{\circ} 25^{\prime} \mathrm{W}$., in 200 fathoms.

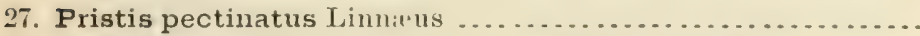

Drawing by H. L. Todd from No. 30678, U.S.N.M., collected at Pensacola, Florida, by Silas Stearus.

28. Rhinobatus lentiginosus Garman; lateral view

Drawing by H. L. Todd from No. 30175, U.S.N.M., collected at Pensacola, Florida, by Silas Stearns.

PLATE IX.

28a, 28b. Rhinobatus lentignosus Garman; dorsal and ventral

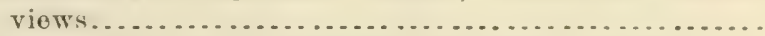

Drawings by H. L. Todd from No. 30175, U.S.N.M., collected at Pensacola, Florida, by Silas Stearns.

29. Raja erinacea Mitchill

Drawing by H. L. Todd from No. 10128, U.S.N.M., collected at

Woods Hole, Massachusetts, by S. F. Baird.

\section{PLATE $\mathrm{X}$.}

30. Raja ocellata Mitchill

Drawing by H. L. Todd from No. 24228 , U.S.N.M., collected at Provincetown, Massachusetts, by U.S.F.C.

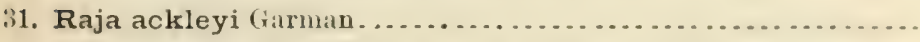

Drawing by H. L. Todd from No. 2684, U.S.N.M., collected by the Albatross at $39^{\circ} 35^{\prime} \mathrm{N}$., and $70^{\circ} 54^{\prime} \mathrm{W}$.

\section{PLATE XI.}

32. Raja stellulata Jordan and Gilbert...................... Drawing by H. L. Todd.

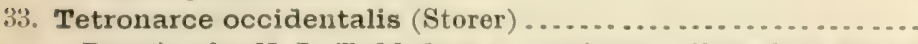
Drawing by H. L. Todd from a specimen collected at Woods Hole, Massachusetts, in the summer of 1881 by the U.S.F.C.

\section{PLATE XII.}

31, 34 4 . Tetronarce californica (Ayres) .....................

Drawings by H. L. Todd from No. 27212, U.S.X.M., collected bS Dr. Jordan at Soqnel, California. 
PILATE XII.

Tiext grage.

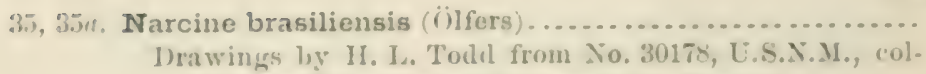
lected hy Silas Stearns at P'ensacola, Florida.

PLATE XIV.

36,36 in. Dasyatis sabina (Le Suenr).

Drawinery by II. I. Todd from No. $3104: 3$, L.S.X.M., collectel at (ialveston, Texas, by Dr. Jordan.

\section{P'ATE XY.}

37. Aetobatus narinari (iuphrasen).

Drawing hy II. L. Toda from No.37196, U.S.X.A., collected by E. F. Wenechaud near Cedar lieys, Florida.

\section{PLATE XVI.}

3\%. Aetobatus narinari (Euphrasen)

Drawing l,y II. L. Todd from Xo. 37196, U.S.X.M., collected by E. F. Denechand near Cedar keys, Florida.

\section{PLATE XVII.}

$38,3 \%$. Rhinoptera steindachneri Evermam and Jenkins........ I rawings log A. If. Baldwin from the trpe, No. 4ise35, U.S.X.M., collected at Gunymas, on the Gulf of California, by Drs. Evermann and Jenkins in 1887.

PLATE XVIII.

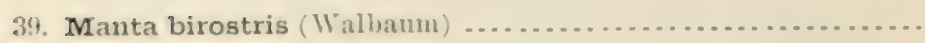

1) rawing by A. 1. 13aldwin.

PIATE XIX.

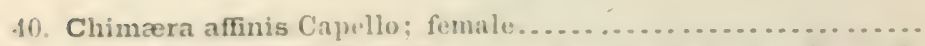

I) rawing ly II. I. Todk from a specinen collected ou the south-

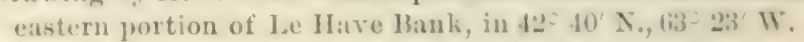

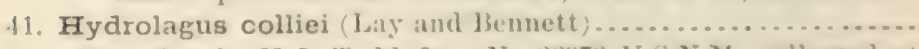
Drawing hy II. I. Twull, from No.2697ti, U.S.X.M., collected at Monteroy, Califoruia, lyy Dr. Jorilan.

12. Harriota raleighama (ioole and 1 Ben ....................

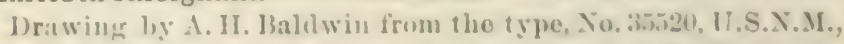

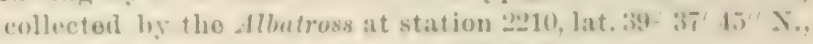
long. $71^{\circ} 18^{\prime} 15^{\prime \prime} \mathrm{W}$, at a depth of 991 fathoms.

\section{PIATE XX.}

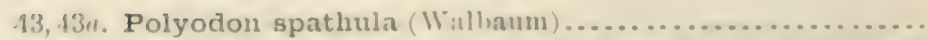

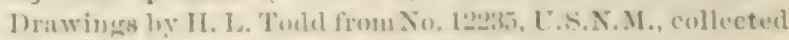
at ''incinnati, (1) 10, ly .J. W. Milner. 
44. Heterocercal tail of Acipenser transmontanus Richardson.. Drawing by Anna L. Brown.

4.). Acipenser sturio oxyrhynchus (Mitchill) .................

Drawing by H. L. Todd from No. 22495, U.S.N.M., collected by William Woltz in the Potomac River.

\section{PLATE XXI.}

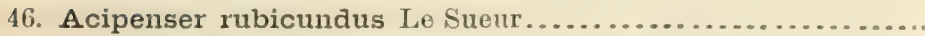

Drawing by H.L. Todd from No. 10252, U.S.N.Mi., collected at Ecorse, Michigan, by J. WT. Milner.

47. Acipenser brevirostrum Lo sueur .....................

Drawing by H. L. Todd from photograph of specimen taken at Woods Hole by the U.S.F.C.

48. Scaphirhynchus platorhynchus (Rafinesque) ............. Drawing by H. L. Todd.

\section{PLA'TE XXII}

49. Lepisosteus platostomus Rafinesque.....................

Drawing by H. L. Todd from No. 3241, U.S.N.M., collected at Cleveland, Ohio, by Spencer r. Baird.

50. Lepisosteus tristcehus (Bloch and Schneider).............

Drawing by A. H. Baldwin from No. 21794, U.S.N.M., collected by Professor Poey in Cuba.

51, 51a. Amia calva Liunieus, female. Drawings by S. F. Denton.

\section{PLA'TE XXIII.}

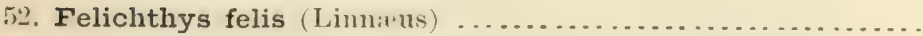

Drawing by H. L. 'Iodd from No. 10122, U.S.N.M., collected at Woods Hole, Massachusetts, loy Spencer F. Baird.

53. Galeichthys milberti (Cuvier and Valenciennes) ............

Drawing by II. L. 'Todd from a specimen collected at Pensacola, Florida, by Silas Stearus.

\section{PLATE XXIV.}

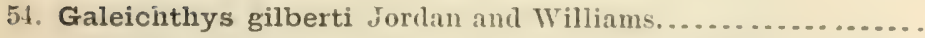

Drawing by Anna L. Brown from the type, No. 1667, L. S. Jr. Univ. Mus., collected by tho Hopkins expedition at Mazatlau, Mexico.

55. Galeichthys azureus Jordan and Williams.................

Drawing by Anna L. Brown fiom the type, No. 1575, L. S. Jr. Unir. Mus., collected by the Hopkins expedition at Mazat. lan, Mexico. 


\section{PIATE XXV.}

iti. Ictalurus furcatus (Le Sueur) ........................ 1::4

Drawing hy M. M. Smith from Yo. 838 , L.S.X.M., typo of l'inelodus uffinis, collected at Brownsville, Texas, by Capt. Van viliet.

57. Ictalurus anguilla Evernann and hésdall...............

Drawing by A. 11. Baldwin from the type, . Jo. 48788 , L.S.X.M. collected by 1)r. Evermann in the Atehatalaya River, Morgan City, Louisiana.

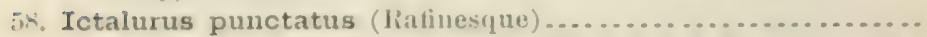

I) rawing l,y II. L. Tohld from No. 27816, U.S.X.M., collected at P'ekin, Illinois, by Dr. S. A. Forbes.

\section{PLATE XXVI.}

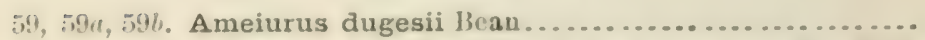
Drawings by 11. L. Todd from the type, No. 23123, U.S.X.M., collecterl by Prot. A. Dugres in Rio Turbio, Mexico.

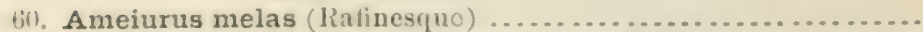

Drawing by H. L. Tould from No. 1497, Li.S.X.M., collected in Aux Plaines River, Illinois, by Robert kenuicott.

\section{PIATE XXVH.}

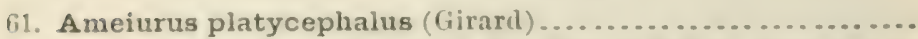
Urawing by H. L. Todd from No. 153!, U.S.X.M., collected by Mrs. E. Daniel at Auderson, South Carolina.

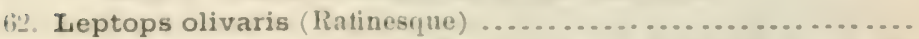
Drawing by II. 1. 'Todd from No. 27873 , U.S.N.M., collected by S. A. Forbes in the lllinois River.

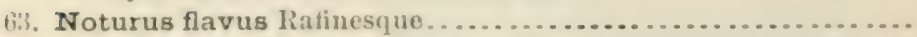

Dr:awing ly II. L. Todl from No. 1478, U.S.N.M., collecterl by Professor Bairl in Lake Champlain, Westport, New York.

6it. Schilbeodes nocturnus (Jordan and Gillhert) ................ Jrawing by II. I. Told from the type, Yo. 36161, U.S.X.M., collected by . Iorlan and Gilbert in the Sabine River uear Benton, Arkansas.

\section{I'IATE: XXVII.}

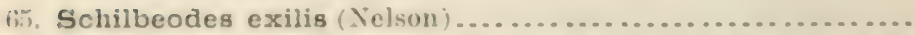

l)rawing ly H. L. Tohll from Yo. 36261, L.S.X.M., collected by Gilhert and swain in tho Ozark liork of the (iasconado River, Marshtielk, Missouri.

66. Schilbeodes insignis (Richardson)

Drawing l,y H. L. Todd from No, 18016, L.S.N.M., collected ly. T. 11. Bean in Consy Creek, Bainbrilge, Penusylvania.

67, 67̈t, 67\%. Schilbeodas gilberti (Jorilau aut Evermaun).......

Drawings ly. \$. F. Denton from the type, No. 39931, U.S.X.M., collected by I). S. . iordan and party in lounoke liver, Salen, Virginia. 


\section{PLATE XXIX.}

Traxt paree.

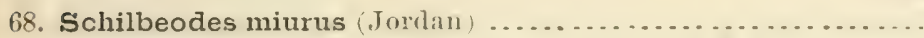

Drawing by H. L. Todd from No. 20926, U.S.N.M.; collected by J. W. Milner in Tar River, North Caroliua.

$69,69 a, 69 b$. Schilbeodes furiosus (Jordan and Meek)..........

Drawings by S. F. Denton from the type, No. 39932, U.S.N.M., collected by D. S. Jordan and party in Nense River, Raleigh, North Carolina.

\section{PLATE XXX.}

70. Ictiobus cyprinella (Cuvier and Valenciennes) ............ 163 Drawing hy H. T. Tokld from No. 20774. U.S.X.M., collected by S. A. Forbes at Normal, Illinois.

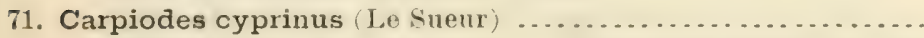
Drawing by H. L. Todd from a specimen collected by Dr. Bean at Havre de Grace, Maryland.

72. Cycleptus elongatus (I. Sueur) ... . . . . . . . . . . . . . . . . Drawing by H. L. Todd from No. 10790, U.S.N.M., collected by J. W. Milner in the Ohio River at Cincinnati, Ohio.

\section{PLATE XXXI.}

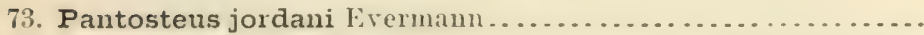

Drawing by A. H. Baldwin from the type, No.43963, U.S.N.M., collected by Evermann and McCormick, in Whitewood Creek, near Deadwood, South Dakota.

74. Catostomus latipimnis Baird and Girard

Drawing by H. L. Todd from No. 20078, U.S.N.MI, collected in the Gila River, Arizona.

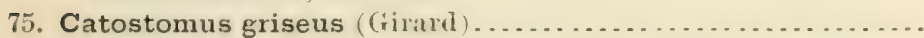

Drawing by S. F. Denton from No. 21197, U.S.N.M., collected by Jordan and Evermann.

\section{PLA'E XXII.}

76. Catostomus pocatello Gilbert and Evermann...............

Drawing by A. H. Baldwin from the type, No. 45385 , U.S.N.M., collected by Evermann and Gilbert in Ross Fork of Snake River, near Pocatello, Idaho.

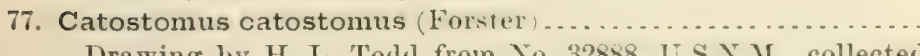

Drawing by H. L. Todel from No. 32888, U.S.N.M., collected by E. W. Nelson, in the Yukon River, Alaska.

78. Catostomus tahoensis Gill aud Jorkan ...................

Drawing by H. L. Todd from No. 5240, U.S.N.M., collected in Lake Tahoe.

\section{PIATE XXXIII.}

79. Catostomus occidentalis Ayres

Drawing by A. H. Baldwin from a specimen collected in the Sacramento River, by Dr. Jordan. 
80. Catostomus tsiltcoosensis Evermann aml Meeli

Text paze.

1)rawing by A. H. Baldwin from the type, Lo. L6179, U.S.X.M., collected by 1)r. Meek in Tsiltcoos Lake, Oregon.

81. Pharymgeal teeth of Catostomus macrocheilus Girarl......

*. Pharyngeal teeth of Placopharynz duquesnii (Le Snenr).... 27943

\section{PLATE XXXIV.}

83. Catostomus commersonii (Lacípiele) ....................

Drawing ly II. I. Todd from No. 10158, U.S.N.M., collected l, J. W. Milner at Ecorse, Michigan.

81. Catostomus ardens Jordin and Gilbert...................

Drawing by II. L. Todd from the type, No. 27363, L.S.N.M., collecterl by Dr. Jordan in Ltah Lake, Ĺtah.

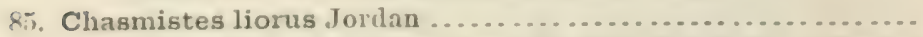

Dratwing by S. F. Denton from No. 3012, L.S.X.M., collected by Dr. Jordau in Utah Lake.

\section{P'IATE XXIV.}

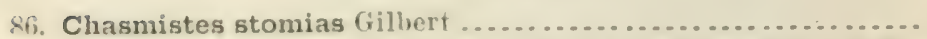

Drawing by A. H. Baldwin from the type, Xo.48223, U.S.X.M... collected hy Gilhert and Cramer in Lpper Klamath Lake, Oregon.

87. Chasmistes copei Erermann and Meole

Drawing by A. II. Baldwin from tho type, No. 482:4, L.S.X.M., collected by Meek and Alexander in Upper Klamath Lake, Oregon.

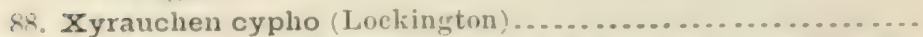

Drawing by S. F. Denton from a specimen collected by Dr. Jordan in Green River, Blake' City, Utah.

\section{PIATE XXXVI.}

89. Erimyzon sucetta Lacépiolo

Iratwing by 11. L. Toild from No, 2T\&tiT, U.S.N.M., collected by Dr. Forlues in Nipisink Lake, Illinois.

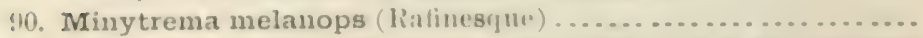
Drawing by H. 1. Todil from No. 17800, C.S.X.M., enllected by Dr. Bean in Round Latke, near Montgomery, Alabama.

91. Moxostoma congestum (Bairul and (iirard)............... Dr:twing hy H. I. Twhl from No. 36510 , U.S.X.M., collected in the lioi Lampasas, Iselton, Texas.

\section{PIATI: XXXII.}

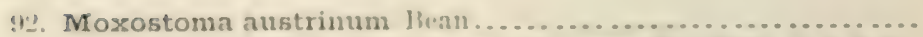

Drawing hy H. L. T'onld from the type, Xo. 23121, U.S.X.M., collecterl at Piedad, in Morelia (Michonean), Mexico, by I'rof. A. Murris. 
Text page.

93. Moxostoma rupiscartes Jordan and Jenkins

Drawing by S. F. Denton from the type, No. 39927, U.S.N.M., collected in the Catawba River, Morganton, North Carolina, by Jordan, Jenkins, and Meek.

\section{PLATE XXXVIII.}

94, 94a. Lagochila lacera Jordan and Brayton ................ Drawing by H. L. Todd from No. 25238, U.S.N.M., collected by J. B. McElwin at Fairview, Teunessee.

\section{PLATE XXXIX,}

95. Campostoma anomalum (Ra finesque) ..................

Drawing by W. S. Haines from No. 36328, U.S.X.M., collected by Jordan and Gilbert in White River, near Eureka Springs, Arkansas.

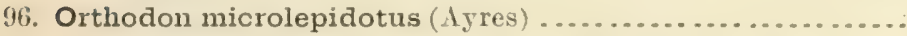

Draming by II. L. Tould from No. 27139, U.S.X.M., collected by Dr. Jordan in the Sacramento River, California.

97. Acrocheilus alutaceus Agassiz and Pickering ...............

Drawing by H. L. 'Todd from No. 30298, U.S.X.M., collected by Capt. Charles Bendire in John Day River, Oregon.

\section{PLATE XL.}

4. Algansea dugesi Bean. . . . . . . . . . . . . . . . . . . . . .

Drawing by A. H. Baldwin from No. 1376 t, U.S.N.M., cullected by Professor Dugès in Mexico.

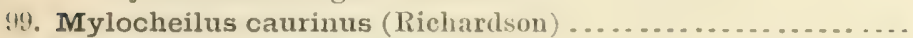
Drawing by A. H. Baldwin.

100. Semotilus atromaculatus (Mitehill) ....................

Drawing by H. L. Todd from No. 19163, U.S.N.M., collected by Robert Kennicott in Anx Plaines River, Illinois.

\section{PLATE XII.}

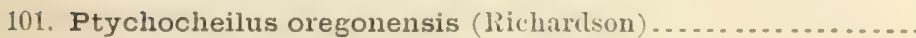
Drawing by $\AA$. H. Baldwin.

102. Leuciscus bicolor ( (iirald) . . . . . . . . . . . . . . . . . . . . . Drawing by Chloe Lesley Starks from a specimen collected in Upper lilamath Lake, Oregon, by Gilbert, Cramer, and Otaki.

103. Leuciscus lineatus ( (iirard) Drawing by S. F. Denton from a specimen collected in Yellowstome Park loy Dr. Jordan.

\section{PLATE ILII.}

104. Leuciscus hydrophlox ('ope) ... . . . . . . . . . . . . . . Drawing loy S. F. Denton from a specimen collected in Yellowstowe Park by Dr. Jordan. 


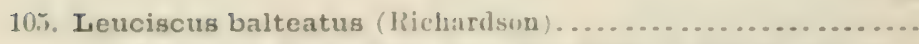

Drawing by H. L. Tochl from No. 36569, U.S.X.M., collected in the Columbia liver, Oregon, l,y J. 11. Clarke.

10\%. Leuciscus balteatus (lichardson) .....................

Drawing ly A. H. Baldwin from No. 43953, U.S.N.M., type of Leuciscus gilli Evermann, collected in Browas Gulch Creek, Silver Bow, Montana, ly Evermann, Jenkins, and Clitpham.

\section{PLATE XIIII.}

106. Lenciscus siuslawi Evermann and Meek

Drawing by A. H. Baldwin from the type, No. 48180 , U.S.X.M., collected lyy Dr. Meek in the Siuslaw River, Mapleton, Oregou.

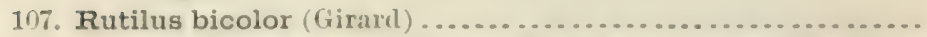

Drawing by Anna L. Brown from a specinen collected by Gilbert, Cramer, and Otaki in Upper Klamath Lake, Oregon.

PLATE XLIY.

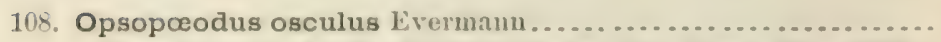
Drawing by A. If. Baldwin from the type, No. 45560 , U.S.X.M., collected by Evermann, Scorell, and Gurley in Buflalo Bayou, near Houston, 'Texas.

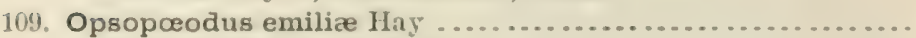
Drawing ly A. II. Baldwiu.

110. Opsopœodus bollmani Gilbert ....................... Drawing by S. F. Denton from the type, collected by Bollman and Fesler in Buckland Creek, Millen, Georgia.

\section{PIATE XIS.}

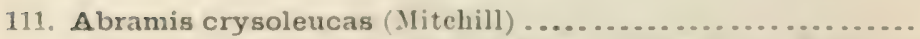

Drawing from No. 20243, U.S.X.M., collected br Professor Bairl in the IIackensack liver, New Jersey.

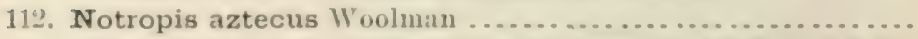
Drawing by A. I. Baldwin from the trpe, No.45569, L.S.X.M. collected by A. J. Woolman at the City of Mexico.

113. Notropis welaka Lvermann and Kendall ................. 1)rawing by A. 11. Baldwin from the type, No. 48786 , L.S.X.M., colleetrol by Dr. Kendall in St. Johns River, Welaka, Floridi.

\section{PLATE XINI.}

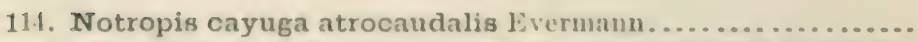
Drawing by A. H. Baldwin from the type, No.45is.7, U.S.X.M., collecterl hy Evermann, scovoll, and (imrley in tho Neches River, mar l'alestine, T'exas. 
115. Notropis kanawha 'Text page

Drawin. $\mathrm{S} . \mathrm{F}$. J collectud by Jolutan, Evermam the type, No. 39!12x, I.S.A.I., Wy theville, Virginia.

116. Notropis chihuahua IVoolmall

Drawing by A. II. Baldwin from the type, Jo. 1.151, C........... collecterl by Iroolman and Cox in tho lio de las Conchus, Chihualua, Mexico.

\section{PLATE XLVII.}

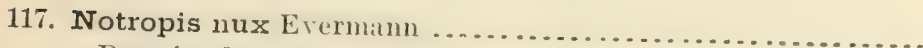
Drawing by A. H. Baldwin from the type, No. 45555 , U.S.N.M. collected by Evermann, Scovell, and Gurley, in Trinity River, near Palestine, Texas.

118. Notropis nocomis Evermann.

Drawing by A. H. Baldwin from the type, No. 45556, U.S. N.M. collected, by Evermann, Scovell, and Gurley in Trinity River, Magnolia Point, Texas.

119. Notropis hudsonius (Clinton)

Drawing by A. H. Baldwin from a specimen collected by Geo. D. Head in Ki]patrick Lake, Minnesota.

\section{PLATE XLTIII.}

120. Notropis hudsonius saludanus (Jordan and Braston)...... Drawing by H.L. Todd from No.23513, U.S.N.M., the type of Hudsonius euryopa Bean, collected by A. Grares in McBean Creok, Georgia.

121. Notropis whipplii (Girard)

Drawing by H. L. Todd from No. 36748 , U.S.N.M., collected in White River, Indiana, by Dr. Gilbert.

122. Notropis galacturus (Cope)

Drawing by A. H. Baldwin from a specimen collected ... Evermann, Scovell, and Gurler in Tellico River, Plains, Tenuessee.

\section{PLATE ILIX.}

123. Notropis macdonaldi Jordan and Jenkins.

Drawing by S. F'. Denton from the collected by Jordan and. Jenkins Luray, Virginia.

124. Notropis coccogenis (Cope)

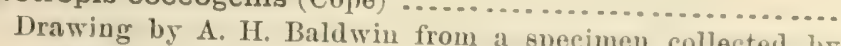
Erermanu, Scovell, and Gurley a specimen collected by Mount Vord, Teunessee.

125. Notropis swaini Jorilin

Drawing by A. H. Baldwin from a specimen collected bF Evermann, Scovell, and Gurley in San Marcos River. San
Marcos, 'Texas. 
PIATE: L.

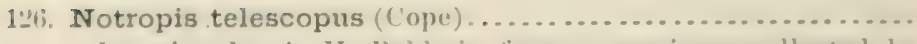

Irawing by A. H. Baldwin from a specimen collected by Evermann, seovell, and (inrley in Tellico River, Tellico I'lains, 'l'ennessee.

127. Notiopis notemigonoides Lvermann ....................

Drawing by A. H. Ballewin from the type, No. 45559 , U.S.X.MI., collected ly Evermann, Scovell, and Gurley in Veches liver, near l'alestine, T'exas.

128. Notropis metallicus Jorlan and Meek

Drawing by $A$. II. Baldwin from a specimen collecterl by A.J. Woolman in the Withlacoochee River, near Richland, Filoridla.

PLATTE LI.

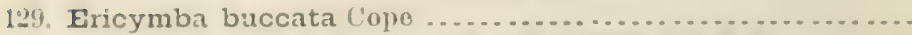

Drawing by A. H. lBalılwin from No. 36803, U.S.X.M., collected by Dr. Meek in Lost Creek, near Defiance, Ohio.

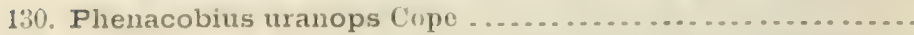

Drawing by $\Lambda$. I. Baldwin from a specimen collected by Evermanu, Scovell, and Gurley in Chickanatuga Creek, Lee s Gordon's mill, Georgia.

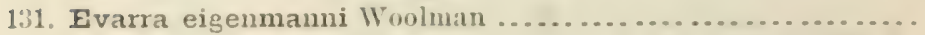

Drawing by A. H. Baldwin from the type, No. 45571 , L.S.N.M., collected hy Woolman aul Cox at the City of Mexico.

PLATE IAI.

192. Rhinichthys dulcis (Girard)

Drawing by S. F. Denton from a specimen collected by Dr. Forilan in Yellowstone P'ark.

193. Agosia klamathensis Lvermann and Mleek .................

Drawing by A. II. Baldwin from the type, No. 482.5 , U.S.N.M., collected by ifeek and Alexander in Upper Klamath Lake, Oregon.

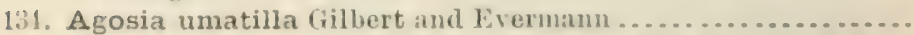

Drawing by A. II. Baldwin from the type, No.45390, U.S.X.M., collected hy Gilbert and Rutter in the Columbia River at Umatilla, Uregon.

\section{PLATE HII.}

1:5. Agosia falcata Eigenu:mu and Eigenmann ...............

Drawing by A. H. Baldwin from the typo collected by C. H. Eigrenam in looise River near Calelwell, Itaho.

136. Hybopsis astivalis marconis Jordan and Gillert .......... Irawing ly $\Lambda$. 11. Baldwin from at specimen collected in the San Marcos River, San Maregs, Texas, by Evermann, seovell, and ciurloy.

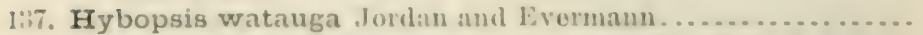

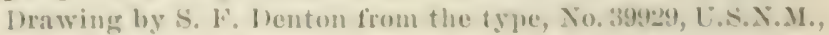
collectul ly durlan and Evermann in the Watanga liver near Elizabethtown, Tennessee. 
PLATE LIV.

Text page.

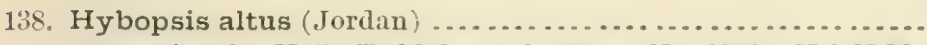

321

Drawing by H. L. 'Todd from the trpe, No. 23125, U.S.N.M., collected in Lake Tupataro, Guanajuato, Mexico, by Prof. A. Dugès.

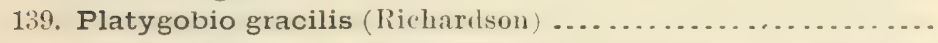

Drawing by A. H. Baldwin from a specimen collected by Evermann, Cox, and Rutter in White River near Chamberlain, South Dakota.

110. Exoglossum maxillingua (Le Sueur)

Drawing by Anna L. Brown.

PLATE LV.

141. Brycon dentex (riinther . . . . . . . . . . . . . . . . . . . . . .

Drawing by A. H. Baldwin from No.39909, U.S.N.M., collected by Dr. L. E. H. Best in Nicaragua.

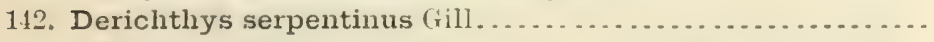

Drawing by H. L. Todd from the type collected by the Albctross in the Gulf Stream at Station 2094 in 1,022 fathons.

113. Anguilla chrysypa Ratinesrue . . . . . . . . . . . . . . . . . . .

Drawing by H. L. Todd from No. 20170, U.S.N.M., collected at Holjoke, Maseachusetts.

\section{PLATE LVI.}

14. Simenchelys parasiticus (iill . . . . . . . . . . . . . . . . . . Drawing by H. L. Todd from No. 21673, U.S.N.M., collected by Capt. N. MePhee uear Sable Island Bank.

115. Ilyophis brumneus Gilbert. . . . . . . . . . . . . . . . . . . .

Drawing by A. H. Baldwin from the type collected by the Albatross at Chatham Islaud.

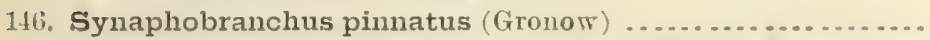

I) rawing by H. L. Tork from No. 216k1. U.S.N.M., rollecterl by Captain Olsen on Le Have Bank.

\section{PLATE LVII.}

147. Histiobranchus infernalis Gill

Drawiug by H. L. Todd from No. 38205, U.S.N.M., collected by the Albatross in lat. $36^{\circ} 35^{\prime} \mathrm{N}$, long. $74^{\circ} 03^{\prime} 30^{\prime \prime} \mathrm{W}$.

11. Leptocephalus conger (Limuirus) . . . . . . . . . . . . . . . . . Drawing by H. L. Todd from No. 16027, U.S.N.M., collected at Noank, Comnecticut, by J. IV. Latham.

149. Leptocephalus caudilimbatus (Poøy) ................... Drawing by H. L. 'Todd from the type of Conger caudicula Bean, No. 30709, U.S.X.M., collected by Silas Stearus at Pensacola, Florida.

Bull. No. 47, pt. $4-\mathrm{xIV}$ 
PIATE: LIII.

1:0. Congrellus flavus (Goole and Bean) 357

Drawing by H. I. Todd from the type, No. Htive, U.S.X.M. collecterl hy the Mllotross in the Ginlt stream.

151. Hoplumis schmidtii Kat1p .......................

Drawing lig J. C. Tan Honk from No, 44240, L.S.N.M., collocted by the Allatross at Station 2102.

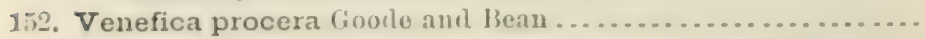

Drawing ly H. L. 'Todh from a specimen collected in the Gult stream.

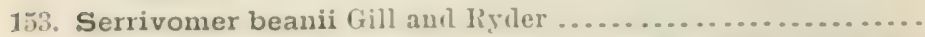

Drawiner by $\AA$. Il. Balkwin from the type, No.33383, U.S.X.M. collecterl hy the Albatross in the Gulf Stream.

PIATE LIA.

154. Avocettina gillii Bean ............................ 367 ;
Drawiug by A. M. Baldwin from the trpe, No. 4t239, U.S.X.M., collected by the Albutross east of Prince of Wales Island, Alaska, at Station 2859.

154a, 15.6. Avocettina gillii Bean,$\ldots . . . \ldots \ldots . . . . . . . . . .$.

Drawings by Anna L. Brown from No. 679, L. S. or. Univ. Mus., collected by the Albatross at Station 2860.

155. Labichthys carinatus Gill and Irycler.

Drawing by A. H. Bald win from the type, No. 33369, U.S.X.M. collected by the Albutross at Station 2076 in the Gulf Stream.

156. Labichthys elongatus Gill and Ryder

Drawing by A. H. Baldwin from the type, No. 33755, U.S.N.M., collected by the Alluatross at Station 2100 in the Gult stream.

\section{PLATE LA.}

157, 157 a, 157 h. Nemichthys avocetta Jordan and Gilbert...... Drawines ly Anna L. Brown from a specimen in the l'rovineial Museum at Victoria, British Columbia, collected hy II. W. Norgate, at Beacon Hill, near l'ictoria.

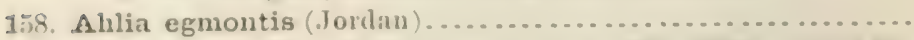
Drawing ly II. I. Told from the type, No. 35086, U.S.X.M., cellected lyg Dr. Jordan at Lirmont Koy, Florila.

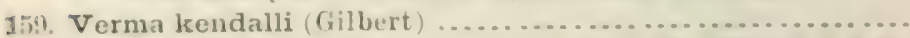

Drawing by A. 11. Bald win from the type, No.4301, U.S.X.M., collected by W. C. Kontall oft west coast of Florida.

\section{PLATE IAI.}

160). Letharchus velifer fioole and liean $\ldots . . . \ldots \ldots \ldots \ldots \ldots . . . . .$. Drawing by II. I. Todd from the type, fo. 314.8, l.s.X.... collected lyg Kaiser and Martin, on west const of Florida.

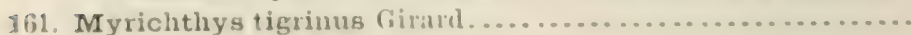

brawing ly A. 11. Ralelwin. 
PLA'IE LXII.

Text page.

162. Myrichthys pantostigmius Jordan and McGregor ......... 2802

Drawing by W. S. Atkinsou from the type, No. 5710, L. S. Jr.

Univ. Mus., collected by R. C. McGregor, at Clarion Island.

163. Pisoodonophis cruentifer Goode and Bean ................

Drawing by A. H. Baldwin from the type, No. 28938, U.S.N.M., collected by the Fish Hawk.

\section{PLA'TE LXIII.}

161. Calechelys muræna Jordan and Evermann ...............

Drawing by A. H. Baldwin from the type, No. 37996, U.S.N.M., collected by Silas Stearns at the Pensacola Suapper Banks.

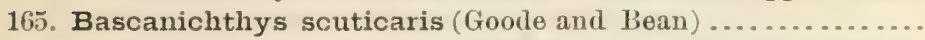

Drawing by H. L. Todd from the type of Sphagebranchus teres Goode and Bean, No. 31457, U.S.N.M., collected by Kaiser and Martin on west coast of Florida.

$166,166 a$. Bascanichthys peninsulæ (Gilbert) ................

Drawings by A. H. Baldwin from the type, No.44297, U.S.N.M., collected by the Albatross in La Paz Bay, Lower California.

\section{PLATE LXIV.}

167. Quassiremus evionthas (Jordan and Bollman) .............

Drawing by A. H. Baldwin.

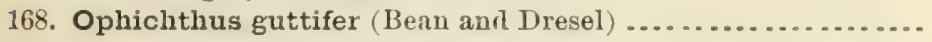

Drawing by H. L. Todd from the type, No, 32617, U.S.N.M., collected by Silas Stearns ou the Pensacola Snapper Banks.

169. Ophichthus ocellatus (Le Sueur) ......................

Drawing by H. L. Todd from No. 22289, U.S.N.M., collected by Silas Stearns at Pensacola, Florida.

PLATE LXV.

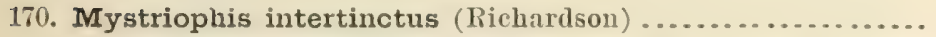

Drawing by H. L. Todd from No. 22865 , U.S.N.M., collected by Kaiser and Martin on west coast of Florida.

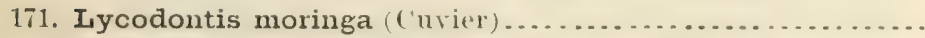

Drawing by H. L. Todd from No. 6994, U.S.N.MI., collected at Garden Key, Florida.

172. Muræena insularum Jordm and Davis...................

Drawing by A. H. Baldwin.

\section{PLATE LXVI.}

173. Muræna retifera Goode and Bean

Drawing by H. L. Fodd from the type, No. 31393, U.S.N.M., collected by C. C. Leslie, at Charleston, South Carolina.

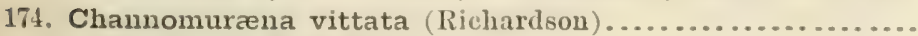
Drawing by A. H. Baldwin.

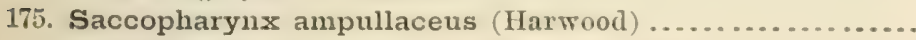
Drawing from Günther, Challenger Report, Vol. xxi, Pl. LxTI. 


\section{PLATE LAVII.}

Text page,

17ti. Gastrostomus baixdii Gill and Ryder 106

Drawing ly II. L. Todd from No.333s6, U.S.X.M. , collected ly the Albatross at Station 207.4 , in N. lat. $41^{\mathrm{C}} 43^{\prime}$, W. long. $6.5^{2} 21^{\prime} 50^{\prime \prime}$, at a ilepth of 1,309 fathoms.

17\%. Tarpon atlanticus (Cuvior and Valencienues)

Drawing by H. L. Todd.

17. Elops saurus Limmants.

Drawing lyg II. L. Todd.

\section{PLATE IAXVIII.}

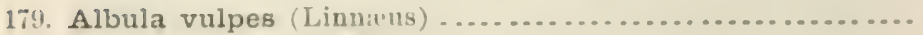

Drawing liy II. L. Todd.

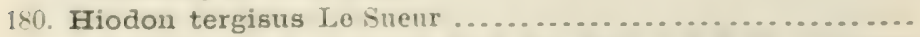

Drawing by II. L. 'Iodd from No. 8710, U.S.N.M., collected by Georgo Clark, at Ecorso, Michigan.

181. Hiodon selenops Jordan and Bean .....................

Drawing ly II. L. Told from the type, No. 19814, U.S.X.M., collected hy Daniel J. Duffy, at Chattanooga, 'T'ennessoe.

I'LATE LAIX.

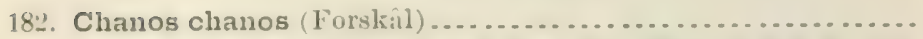

Drawing by A. H. Balelwin from Yo. 2S.240, U.S.X.M., collecter by Dr. Gilbert at Mazatlan, Mexico.

18:3. Dorosoma cepedianum (Le Sueur) ..................... Drawing by H. L. Torld.

184. Signalosa atchafalaya Evermann and Kenclall ............ Drawing by d. H. Baldwin from tho type, No. 18790 , U.S.X.M., collected hy Fred M. Chamberlain in the Atchafiaya River at Melville, Louisiana.

\section{PIATH IXX.}

$1-\therefore$. Clupea harengus Linnirus

I) rawing by H. L. T'odel.

Iri. Clupea pallasii Cuvier and Valenciennes................. Driwing by H. I. Todtl from No.27T18. U.S.X.M., collected by L. Bailey at Unalaska.

187. Pomolobus chrysochloris Rafinesque ..................

Drawing ly, H. L. Todll from No.3015!, L.S.Y.M., collected by Silas Stenrns at l'ensucola, Florida.

\section{PLI'L LXX.}

18\%. Pomolobus mediocris (Mitrhill) ........................

J)ring hy M. L. 'Tuld from No. 25132, U.S.X.M., cullected at Washington marliot by l)r. Bean. 
189. Pomolobus pseudoharengus (Wilson)

Drawing by H. L. Todd from No. 25197, U.S.N.M., collected at Washington market.

190. Pomolobus æestivalis (Mitchill)

Drawing by H. L. Todd from No. 32639, U.S.N.M., collected at Washington market.

\section{PLATE LXXII.}

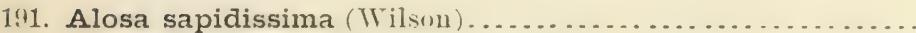

Drawing by H. L. Todd from No. 25099, U.S.N.M., collected b5 U. S. F. C. at Norfolk, Virginia.

192, 192 . Alosa alabamæ Jordan and Evermann ..................

Drawings by A. H. Baldwin from the types, Nos. 47690 male, and 47689, female, U.S.N.M., collected by J. N. Fitts in Black Warrior River, at Tuscaloosa, Alabama.

\section{PLATE LXXII.}

193. Sardinella humeralis (Cuvier \& Valenciennes)

Drawing by H. L. Todd from the type of Harengula pensacole Goode and Bean, No. 22831, U.S.N.M., collected ly Silas Stearns at Pensacola, Fiorida.

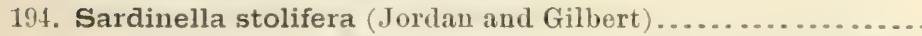

Drawing by Anna L. Brown from No. 2693, L. S. Jr. Univ. MIus., collected by the Hopkins Expedition at Mazatlan, Mexico.

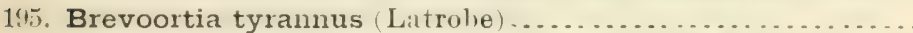

Drawing bo H. L. Todd from No. 20666, U.S.N.M., collected at Woods Hole, Massachusetts.

\section{PLATE LXXIY}

196. Stolephorus perthecatus Goode and Bean .................

Drawing by H. L. Todd from No. 30483, U.S.N.M., collected by Silas Stearns in south Florida.

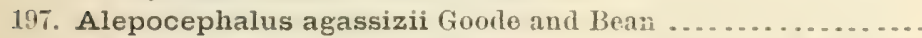

Drawing by H. L. Torld from No, CCCXXXVIII, M. C. Z. collected by A. Agassiz in N. lat. $38^{\circ} 18^{\prime} 40^{\prime \prime}$, W. long. 73 $18^{\prime} 10^{\prime \prime}$, at a depth of 922 fathoms.

198. Talismania antillarum Goode and Bean ....................

Drawing by M. M. Smith from the trpe, No. 43739, L.S.X.M., collected by the Albatross at Station 2394, in N. Jat. 28 38 $30^{\prime \prime}, \mathrm{W}$. long. $87^{\circ} 02^{\prime}$, at a depth of 420 fathoms.

\section{PLATE IXXY.}

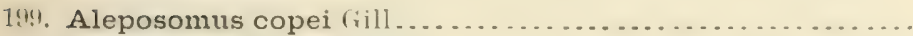

Drawing by H. L. Todd from the trype, No. 33551, U.S.N.M.

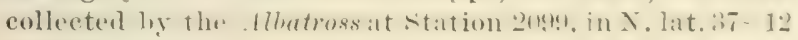
$20^{\prime \prime}$, W. long. $69039^{\prime}$, at a depth of 2,949 fathoms. 
2(1). Coregonus williamsoni (iirarel

Drawing by A. II. Baldwin from a loreeding male collecterl by 13. A. Bean in Little Spokane liver, near Clarlis Spring, IVashington.

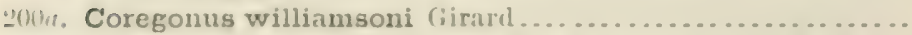
1) rawing ly d. H. Baldwin from a youner individual colo lected by 1 r. Kermann in Montanit.

\section{PIATE I.XXI.}

201. Coregonus coulterii Ligenzanu and Ki renuann. . . . . . . . . Drawing by A. II. Ballwin from the type, No. 11875 , L.S. X.M., collected hy Dr. Eigenmann in the Kicking IJorse River at lielıl, British Columbia.

202. Coregonus clupeiformis (Mitchill) ..................... Drawing l,y II. L. Tould from No. 1030(1), L.S.X.M., collecterl by (ieorge Clark at Eeorse, Michigan.

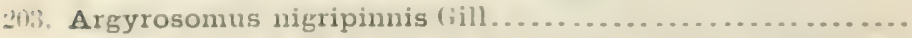
l)rawing by $А$. H. Baldwin from a neary rije male collected in Lako Miehigan, oft Sheborgan, Wiseousin, in 90 fathous.

\section{PLATE IXXVII.}

201. Stenodus mackenzii (Richardson)

1)rawing from No. 29889, I.S.X.M., collected at Nulato, Alaska, by L. W. Nelson.

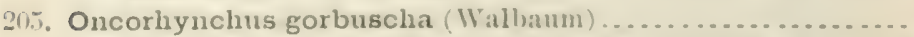

brawing by II. L. Todel from No, 27743, L.S.X.M., collected by Dr. Bean in C'ooks Inlet, Alaskiı.

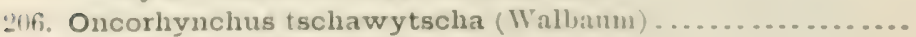
1) rawing by H. L. 'Todk.

\section{PIATE IXXYIII.}

201. Oncorhynclus nerka (Wallsam)

lrawing by A. II. Baldwin from a male specinen of the latge form collected by T. M. Willians at Bigr Payetto Lake, itlaho.

20iu. Oncorhynchus nerka (Wallhaum)

Drawing ly $A$. li. labldwin from a lemale specimen of the -mall form collected by Dr. Evermann at Alturas Lake, litiluo.

2(1\%. Oncorhynchus nerka (W:albaum) . 
209. Salmo clarkii virginalis (Girard)

Drawing by S. F. Denton from a specimen collected by Peter Madsen in Utah Lake, Utah.

210. Salmo clarkii spilurus (Cope) $495 ; 2819$

Drawing by S. F. Denton from a specimen collected by Jordan and Evermann at Del Norte, Colorado.

\section{PLA'TE LXIX.}

211. Salmo clarkii pleuriticus (Cope) $196 ; 2819$

Draming by S. F. Denton from specimen collected by Jordan aud Evermann at 'Trappers Lake, Colorado.

212. Salmo clarkii bouvieri (Beudire) . . . . . . . . . . . . . . . 496; 2819 Drawing by S. F. Denton from the type, collected by Capt. Charles Bendire at Waha Lake, Itaho.

21i. Salmo clarkii stomias (Cope) ..................... 497; 2819 Drawing by S. F. Denton from a specimen collected by Jordan and Evermann at Twin Lakes, Colorarlo.

\section{PLATE IAXXI.}

214. Salmo clarkii macdonaldi Jorkan and Evermanu ....... 497; 2819 Drawing by S. F. Denton from the type, No. 41730 , U.S.N.M., collected by George Fisher in ' $\mathrm{T}$ in Lakes, Colorado.

215. Salmo gairdneri Richardson . . . . . . . . . . . . . . . . . .

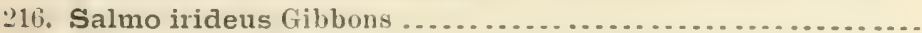

Drawing by S. li. Denton from No. 37782, U.S.N.M., a male esample, collected by William Montgomers at Verona, Missouri, where the species had been introduced.

\section{PLATE LXXXII.}

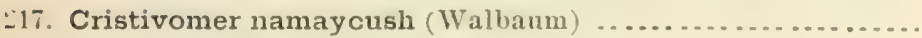
Drawing by $\mathrm{S}$. F. Denton.

21\%. Salvelinus fontinalis (Mitchill) . . . . . . . . . . . . . . . .

Drawing by H. L. Todd froin No. 28651, U.S.N.M., collected by E. G. Blackford.

219. Salvelinus malma (Walhaum) ...................

Drawing by H. L. Toda from No. 27740, U.S.N.M., collected by 'T'. H. Bean in Cooks Inlet.

\section{PLA'TE LXXXIII.}

2.? (). Salvelinus alpinus aureolus (Bean) ..................... Drawing by H. L. Todd from No. 39899 , U.S.N.M., a female, collected by E. 13. Hodge at Sumapee Lake, New Hampshire.

$2: 21$. Salvelinus oquassa (Girard)

Drawing by H. L. Tohl from No. 2068s, U.S.N.M., collected by E. G. Blackford at Oguassa Lake, Maine.

2.2.). Thymallus signifer (Richardson)

Drawing by H, r. Todd from a specinem collected by th. Nelson at Nulato, Alaska. 


\section{PIATE LXXXIV.}

'ext jianen.

223. Thymallus tricolor Cope ....................... $518 ; 2811$

I)rawing l, H. 1. Torbl firom No. 11115, U.S.X.M., collected by .T. W. Milner in Ansable liver, Michigan.

221. Thymallus tricolor montanus (Milner) .............. $519 ; 2871$

Drawing hy S. F. Jenton from an specimen collected loy Dr. Jorelan at Horsethief Springs, Montana.

2.'1\%. Thymallus tricolor montanus (Milner) $519 ; 2 \times 71$

1)awing by A. Il. Baldwin from a young example collecterl in the Yellowstone l'ark by Jr. Evermann.

\section{PLATE IAXXY.}

225. Mallotus villosus (Miiller)

Drawing ly II. L. Todk from No. 3418, U.S.N.M., collected by Dr. L. Coues in Grosswater Bay.

2.6. Thaleichthys pacificus (Richardson)

Drawing ly 11. L. 'Todd from No. 28001, U.S.X.M., collected by Marcus Baker in Chilkat kiver, Alaska.

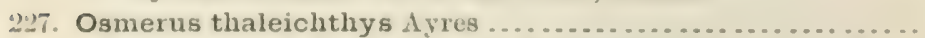

Drawing by H. L. Todd from Yo. 2805i, U.S.N.M., collected by Dr. Bean at San Francisco, California.

\section{P'LA'TE LAXXVI,}

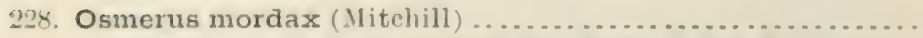

Jrawing by H. L. Tould from No. 21435, U.S.X.M., collected by V. N. Edwards at Woods Hole, Massachusetts.

229. Osmerus dentex Steinulachner . . . . . . . . . . . . . . . . . .

I) rawing ly II. 1. Todel from No. 27914, U.S.X.M., collected at l'ort Clarence, Alaska, by Dr. Bean.

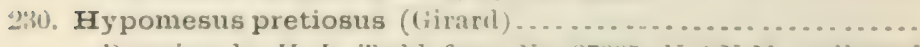

Drawing by H. L. Todd from lo. 27995, U.S.X.M., collected in lakutat Bay, Alaska, by br. Bean.

PIATE IXXXYII.

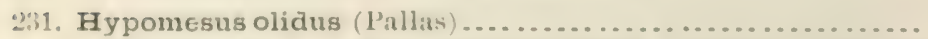

Drawing by II. L. Todd from No. 2397:3, V.S.X.M., collecterl by I. M. 'T'urner at .it. Michateld, Mlaska.

232. Argentina silus $\Lambda$ semins . . . . . . . . . . . . . . . . . . .

Drawing hy H. 1. Tould from No. 37801, U.S.N.M., collected by E. H. Bunker at Bidlloford l'ool, Mano.

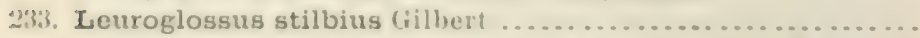

1)rawing loy A. H. Bald win from the type, No. $112 \times 3,1$.S. N. M. .

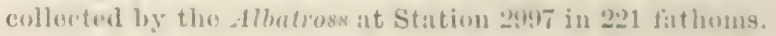

\section{PATI: I.XXXIII.}

231. Bathylagus benedicti (iomle and Ben .

1.)rawing by A. H. Baldwin from a specimen collected be the Allutross at station 2711 , in X. lat. 38 is , W. long. To $0 \bar{T}^{\prime}$, in 1,384 tithoms. 
235. Trachinocephalus myops (Forster) .........................

Drawing by H. L. Told.

236. Synodus føtens (Linnirus) . . . . . . . . . . . . . . . . . . . . .

Drawing by H. L. Torld from No. 25998, U.S.N.M., collected by C. C. Leslie at Charleston, South Carolina.

\section{PLATE LXXXIX.}

237. Benthosaurus grallator Goode and Bean ................

Drawing by H. L. Todd from a specimen collected by the Blake at Station CLXXIr, in N. lat. $24^{\circ} 23$, W. long. $84^{\circ}$ $23^{\prime}$, in 1,850 fathoms.

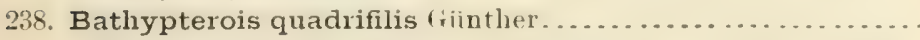

Drawing by A. H. Baldwin from a specimen collected by the Blake at Station XCrin, oft St. Vincent.

239. Ipnops murrayi (iinther . . . . . . . . . . . . . . . . . . . i); $; x$

Drawing by H. L. Todd from a specimen collected by the Blake at Station CCxxxm, in N. lat. 21036 , W. long. $81^{\circ}$ $5^{\prime}$, in 955 fathoms.

\section{PLATE XC.}

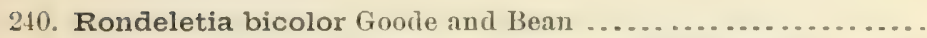

Drawing by H.L. Todd from the type, No. 38202, U.S.N.M. collected by the Albatross at Station 2206 , in N. lat. $36^{\circ} 47^{\prime}$, W. loug. $73^{\circ} 25^{\prime}$, in 1,641 fathoms.

241. Cetomimus gillii foole and Bean . . . . . . . . . . . . . . . .

Drawing by M. M. Smith from the tspe, No. 35529, U.S.N.M., collected by the Albatross at Station 2206 , in N. lat. $39^{\circ} 35^{\prime}$, W. long. $71^{\circ} 24^{\prime} 30^{\prime \prime}$, in 1,043 fathoms.

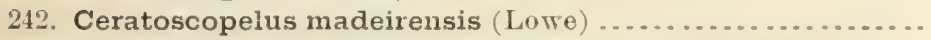

Drawing by J.C. Tan Hook from No. 43776, U.S.N.M., collected by the Albatross at Station 2528 , in N. lat. $41^{\circ} 47$ W. long. $65^{\circ} 37^{\prime} 30^{\prime \prime}$, in 677 fathoms.

\section{PLATE XCI.}

243. Lampanyctus crocodilus ( Risso ). . . . . . . . . . . . . . . . Drawing by A. H. Baldwin from a specimeu collected at Nico, and obtained by the U.S.N.M. through the lioyal Zoological Musenm at Florence, Italy.

244. Lampadena speculigera Goode and Bean ................

Drawing by J. C. Van Hook from the type, No.43797, U.S.N.M., collected by the Fish Mawh at Station 797, oth' Nomport, Rhode Island, in 16.5 fathoms.

21.5. Nannobrachíum macdonaldi Goodo and Bean .............

Drawing by S. F. Dentou from No.35145, U.S.N.M., collected ly the Albatross at Station 2182, in N. lat. $399^{2} 25^{\prime} 30^{\prime \prime}, W$. long. $71^{\circ} 44^{\prime}$, in 861 fathoms. 


\section{PI.ATE: ICII.}

216. Athoprora lucida (ioode and ise:n ...................... isis

Drawing ly A. H. Baldwin from Xo HosI, U.S.X.A., collected by the .fllatross at station 2127 , in X. Int. 19- $45^{\prime}, W$. long. 7.;-04, in 1639 fatlioms.

217. Myctophum opalinum Gomle :tud Benn ................. I) rawing ly J. C. Yan llook from Yo. 437.98, U.S.X.M., collected by the .llbutross at Station 2585, in X. lat.390 0s' $30^{\prime \prime}$, IV. long. $72-1 \tau^{\prime}$, in jole fathoms.

24. Tarletonbeania tenua Ligenman and Eigenmanı.......... I) rawing lyy A. II. Baldwin from No. 41882, U.S.X.A., collerted by C. H. Eigenmann off P'oint Loma, near San Diegro, Cnliforniat.

\section{PLATE XCII.}

219. Yarrella blackfordii (ioode aud Bean ...................

Drawing by A. II. Baldwin from the type, No.4424:, U.S.X.M., collecterl by the Alhatross at Station 2376 , in N. Lat. $29=03$ $15^{\prime \prime}, \mathrm{IV}$. long. $88^{\circ} 16^{\prime}$, in 321 fithoms.

250. Chanliodus sloanei Bloch and schneider................

Drawing by H. L. Todd from No. 23120, U.S.N.A., collected by Capt. Charles Anderson and erew, of the Giloncester tishing fleet, in X. lat. 42-08', W. long. 65-35', in 185 fat homs.

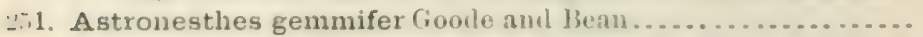
1) rawing los A. M. Baldwin from the type, No, 2-1645, U.S.N.M., collecterl hy the schomer Pola. Hire from the stomach of a halibut, in N. lat. $41 \cdot 25^{\prime}, \mathrm{W}$. lone. $53-12$, in 300 fathoms.

\section{PIATE XCIV.}

252. Astronesthes richardsoni l'oey.

Drawing by M. M. Smith from No.35.10, L.S.X.M., collected

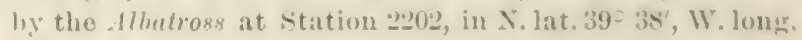
71. $34^{\prime} 45^{\prime \prime}$, in 515 fithoms,

2..... Stomias ferox Reinharilt

1)rawing by H. L. Todel from No. 23360, U.S.X.M., collected by Cipt. Invid Cammel and erew, of the (iloucester fishing lleet, at East Banyuerenx.

$2:$. Grammatostomias dentatus fionde and Bean .............. I) rawing by II. L. Todd from the type, Xo. 37370, U.S.X.M.,

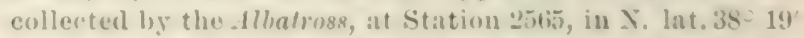

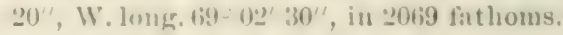

\section{PIATE XI:}

…. Photonectes graciles fioode and liean

l)rawing loy M. M. Smith from the type, collected by the Bletic at station X1., off Martinipne, in 172 tathoms. 
256. Malacosteus niger Ayres.

Drawing by H. L. Tould, from No. 32169, U.S.N.M., collected by Capt. Charles Anderson and crew, of the schoomer Alice G. Wonson, on the northeastern edge of Georges Bank, in 125 fathoms.

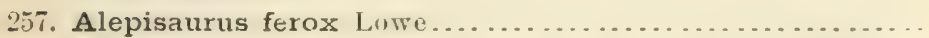

Drawing by H. L. 'Todd from No. 20593, U.S.N.M., ol. tained in the New York market by E. G. Blackford.

\section{PLA'TE XCVI.}

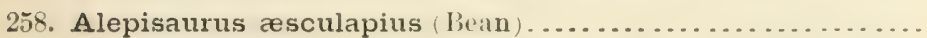

Drawing by H. L. Todd from the type, No. 27705, U.S.N.M., collected by Robert King at Unalaska, Alaska.

259. Arctozenus coruscans (Jordan \& Gilbert) ................ . .

Drawing by A. H. Baldwin from the type, No. 27171, U.S.N.M., collected by Mr. Brown, at Port 'Townsend, Washingtou.

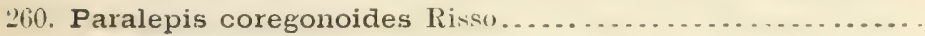

Drawing by H. L. Todd from a specimen in the Acalemy of Natural Sciences of Montreal.

\section{PLATE ICVII}

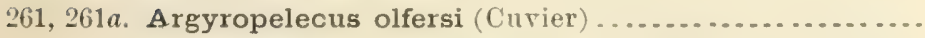
Drawings b5 H. L. Todd from No. 33393, U. S. N. M., collected by the Albatross in N. lat. $41^{\circ} 40^{\prime} 30^{\prime \prime}, \mathrm{W}$. long. $65^{\circ} 35^{\prime}$, in 855 fathoms.

\section{PLATE XCVIII.}

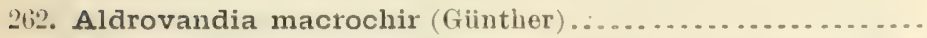

Drawing by H. L. 'Todd from a specimen collected by the Blake at Station Lrir, off Havana, in 2.12 fithoms.

263. Aldrovandia gracilis Goode and Bean.

Drawing by S. F. Denton, from a specimen collected by the Blake at Station Lxx, oft Guadaloupe, in 769 fathoms.

264. Notacanthus amalis (iill ... . . . . . . . . . . . . . . . .

Drawing by H. L. Todd from the type, No. 37856, U.S.X.M., collected by the Albatross at Station 2677 , in N. lat. 32039 , W. $\operatorname{long} .76^{\circ} 50^{\prime} 30^{\prime \prime}$, in 478 fathoms.

26.). Notacanthus phasganorus Goode.

Drawing by H. L. Tokd from the type, No. 25972, U.S.N.M., collected by Capt. Briggs Gilpatrick, of the schooner Gutherer, from the stomach of a ground shark, on the Grand Bank.

\section{PLATE XCIX.}

266. Lipogenys gillii Goode and Beau . . . . . . . . . . . . . . . . . . .

Drawing by H. L. Todd from the type, No. 39212, U.S.N.MI.. collected by the dlbatross at Station 2742 , in N. lat. 37046 $30^{\prime \prime}$, W. long. $73^{\circ} 56^{\prime} 30^{\prime \prime}$, in 865 fathoms. 
Ti.re grome.

27i. Dallia pectoralis Bean......................... ti21

Drawing ly II. I. Tould from the type, No. 234!s, I .S.X.MI., collected by 1.. M. Turur. at s.t. Michaels, . Ilaska.

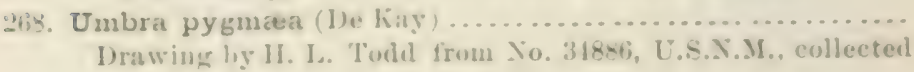
in New Jersey.

PISTE C:

269,2698 . Lucius lucius (Iinn:11s) .......................

I)rawings by II. I. Tould from Xo. 9389, U.S.X.M., collected at Erorse, Michigan.

170. Lucius masquinomgy (Mitrhill) ......................... J)awing ly II. I. Torld from \$o. 10607, L.S.X.M., cullected hy (ieo. Clark at Eicorse, Michigan.

\section{PIATE: CI.}

271,271 . Fundulus majalis (Walhatmu) ..................

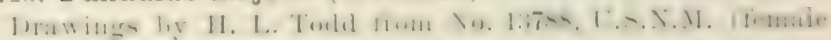
and malo), collected hy the U. S. Fish Commission at II wods Hol', Massachusetts.

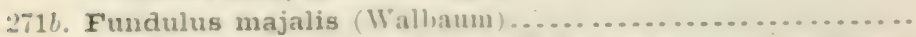

Drawing by A. 11. Baldwin from a young examplo collected by II. M. Suith at st. (ieorge Island, Marylaud.

I'I.A'T: CII.

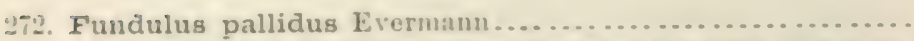

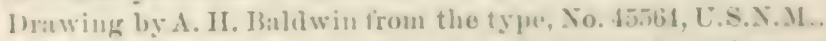
collectml ly Evermaun, scovell, amel firley in falreston Bay, near Swan Lake, finlveston, Texas.

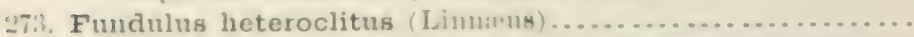
1)rawing by $\mathrm{A}$. 11. Baldwin from a male example collecteal by II. M. Suith at st. fieorgo Islami, Marylamel.

27. Fundulus ocellaris . Iorlan and Gilhert..................

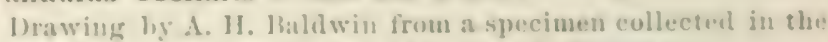
Withlacooclses Siver, Florida, lyy A. .J. Wooluan.

\section{I'. ATK (:III.}

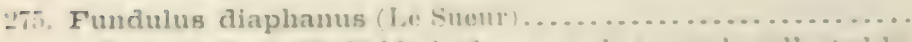
l)awing by 1. H. Bahldwin from a male example collected by II. M. Suith at St. fieorge Islami, Marylaul.

27v. Fundulus diaphanus (1.e surur) ................... Drawing loy 1. 11. Balilwin from a fenale oxample collecterl

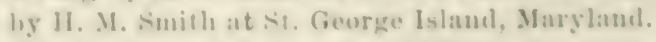

27i, Fundulus zebrinus .Julan and filluert .................

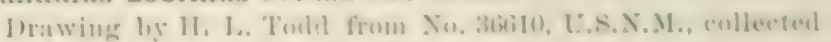
at Ellis, Kausas, l,y I. W. C'ragin. 
PLATE CIV.

277. Fundulus seminolis Girard

Drawing by A. H. Baldwin from a specimen collected in Charlie Apopka Creek, Florida, by A. J. Woolman.

$27 \times$. Fundulus catenatus (Ntorer

Drawing by A. H. Baldwin from a malo example collected by Dr. Gurley in Ball Creek, near 'Tazerrell, Teunessee.

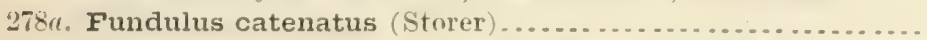

Drawing by H. L. Todd from Yo. 36456 , U.S.N.M., collected by Jordan and Gilbert in Saline River, Benton, Arkansas.

\section{P'LA'TE CV.}

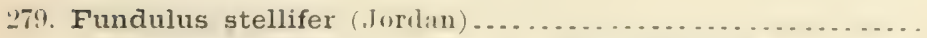

Drawing by H. L. Todd from No. 17888, U.S.N.M., collected by Dr. Jordan in Etowa River, Rome, Georgia.

280. Fundulus rathbuni Jordan and Meek...................

Drawing by S. F. Denton from the type, No. 39860, U.S.X.M., collected in Allemance Creek, Greenshoro, North Carolina. by Jordan, Jenkins, and Meek.

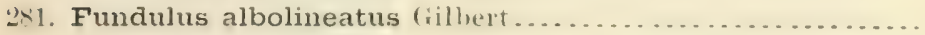

Drawing by S. F. Denton from the trpe, collected by P. H. Kirsch in Spring Creek, Huntsville, Alabama.

\section{PLATE CVI.}

282. Fundulus funduloides (Evermanu)

Drawing by A. II. Baldwin from the trpe, No. 45563 , U.S.N.M., collected by Evermann, Scovell, and Gurley in Dickinson Bayon, near Galveston, Texas.

283. Fundulus macdonaldi (Meek).

Drawing by S. F. Denton from the type collected by Meek Drew, and Rettger in Jones Creek, Dixon, Missouri.

2xi. Fundulus jenkinsi Fvermann ....................... Drawing by A. H. Baldwin from the type, Yo. 45562 , U.S.N.M. collected by Evermann, Scovell, and Gurley in Dickinson Bayou, near Galveston, 'Texas.

\section{PLATE CVII.}

285. Fundulus pulvereus (Evermann

Drawing by A. 1I. Baldwin from the trpe, No. t5561, U.S.X.M. collected it Dickinson Bayou, near Galveston, Texas, in Evermann, Scovell, and Gurley.

2ab. Fundulus lucia (Baird Drawing by $A$. H. IBaldwin from a specimen collected hy 11. M. Smith at St. George Islame, Maryland.

287. Fundulus chrysotus Ilolhrook

Drawing by A. II. Baldwin from a specimen collected lyy A.J. Woolman in Floriela. 
ILATE: CIII.

Test page.

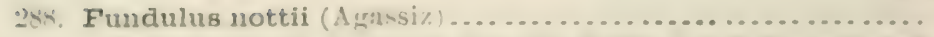
6.56

l)atwing ly. II. L. Tould from Xo.31139, L.S.N.M., collected in Elbow Creek, Florida, by J.A. Henshall.

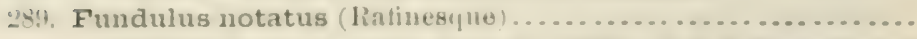

1)rawing by H. I. T'orke from No, 36321, U.S.N.M., collected in White liver at Eureka springs, Arkansas, lỵ Jordan anci filliert.

23i1). Adinia dugesii (Bean)

Drawing ly H. L. Todel from the type, No.37×31, U.S.X.y. collected by Prof. A. Dugis, iu Ginanajuato, Mexico.

\section{PIAATE CIX.}

291. Fundulus goodei (Jordan) $661 ; 2831$

Drawing by A. II. Baldwin, from a specimen collected lyy A. .r. Woohnan in Forida.

292. Lucania parva (Baird and (iirard).

Drawing hy $\mathrm{W}$. \$. Haines from a specinen collected by Dr. Be:m in New Jersey.

29.3. Characodon bilineatus Bean

Drawing by W. S. Haines from the type, No. 37832, U.S.X.M., collected ly Profossor Dugr's in Guanajuato, Mexico.

PLATE CX.

294, 294a, 291b, 294c, 294d. Empetrichthys merriami Gilbert ..... I) rawing from the trpe, No. 46101, U.S.N.M., collected by Merriam and Batiley in Ash Meadows, Amargosa Desert, on the boundary between California and Nevada.

\section{PIATE CXI.}

29.5. 29.3a. Characodon variatus Bean 669

1) Matwing ly. H. L. Todll from Yo. 37810, U.S.N.M., the type of C ferrufinens Bean, collectel hy Professor Dugis in (illamajuato, Mexico.

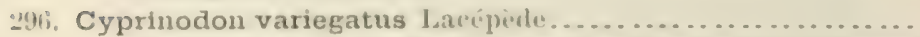

Drawing ly $\Lambda$. If. Maldwin from a male oxample collected by II. M. smith at St. George lsland, Marỵlaud.

\section{PLATE C.TII.}

othich. Cyprinodon variegatus lacepriele

lrawing by $A$. H. Baldwin from a young example collected by II. M. Suith at st. (ieorgo Island, Marylaud.

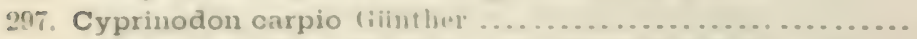
lowwing ly H. L. Todd from Xio. 32151, U.S.X.M.. collected by I)r. J. W. Velie at Buea ('iega Bay, k'Jurida.

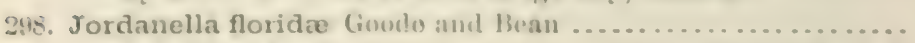

J) rawing by A. H. Baldwin from a specimen collected by A. J. Wouluan in Florida. 


\section{PLATE CXIII.}

299. Gambusia affinis (Baird and Girard) . . . . . . . . . . . . . . .

Drawing bs A. H. Baldwin from No.37839, U.S.N.M. a male, collected by Professor Dugies in Mexico.

299a. Gambusia affinis (Baird and Girard) ......... . . . . . . . . .

Drawing by A. H. Baldwin from a female example collected by H. M. Smith at St. George Island, Marsland.

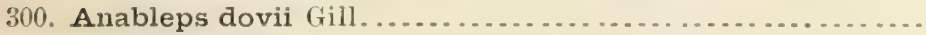

Drawing by A. H. Baldwin from No.48214, U.S.N.M., collected by E. W. Nelson in Tehuautepec, Mexico.

\section{PLATE CXIV.}

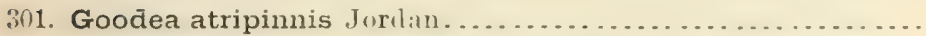

Drawing from the type, No. 23137, U.S.N.M., collected by Professor Dugies at Leon in Guanajuato, Mexico.

302. Heterandria formosa A gassiz . . . . . . . . . . . . . . . . .

Drawing by A. H. Balkwin.

303. Pœcilia presidionis Jordan and Culver . . . . . . . . . . . . . . .

Drawing by Anna L. Brown from the type, a female, No. 2687, L. S. Jr. Univ. Mus., collected by the Hopkins Expedition at Mazatlan, Mexico, in the Rio Presidio.

\section{PLATE CXV.}

301. Xiphophorus helleri Heckel.........................

Drawing by A. H. Baldwin from No. 44918, U.S.N.M., collected by A. L. Herrera at Vera Cruz, Mexico.

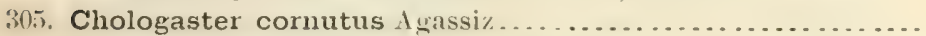

Drawing by S. F. Denton from the type of $C$. avitus Jordan and Jenkins, No. 39861, U.S.N.M., collected by Jenkins and Meek in the outlet of the Dismal Swanp, Virginia.

306. Typhlichthys subterraneus Girard . . . . . . . . . . . . . . . .

Drawing by H.L. Torld from No. 36806, U.S.N.M., collected in Mammoth Care, Kentucky.

307. Amblyopsis spelzus DeKay ..

Drawing by H. L. Todd from No. 5863, U.S.N.M., collected in Mammoth Cave, Kentuclí.

\section{PLATE CXVI.}

308. Tylosurus raphidoma (Ranzaui)

Drawing by H. L. 'Todd.

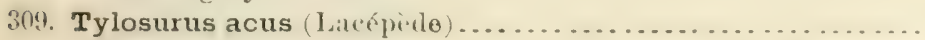

Drawing by H. L. Todd from No. 21422, U.S.N.M., received from E. G. Black ford.

310. Chriodorus atherinoides Goode and Beau .................

Drawing loy H. L. Todd from the type, No. 26593, U.S.N.M., collected by Silas Stearus at Key IVest, Florida.

311. Hyporhamphus unifasciatus (Rauzani) ..................

(ix.)

Drawing by II. L. Todd from No. 16944, U.S.X.M.. collected by Otto Lugger in Chesapeake Bay. 


\section{PI.ATE C.XVH.}

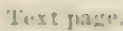

:il:. Hyporhamphus roberti (C'uvir r and Valenciennes) .........

Drawing by W. . Haines from at specinen colleced at Lougport, Xew Jursey, ly Dr. Bean.

:313. Hemirhamphus brasiliensis (Linnitus),

Drawing hy H. L. 'loold from No. 26tok, I.S.X.M., collected at Hungers Wharf; V'irginia, by .J.'T. Wilkins.

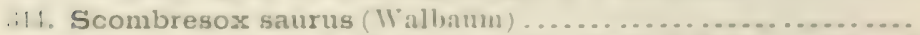

Drawine by II. L. I'odd from Yo. 19853, L.S.X.M., collected by V. N. Eilwards at Wools Hule.

315. Fodiator acutus (C'uvier and V'alentiennes)...............

l)rawing ly A. H. Balelwin from No. 43127, U.S.X.M., collected ly the Albatross at l'anama.

\section{PLATE CXYH.}

:31i. Exonautes exsiliens (Miiller) $732 ; 2: 301$

l)rawing by 1I. L. Todd from Yo. 25187, U.S.X.M., collected by l.. f. Blackford.

:317. Exonautes rondelettii (Cnvier and V"alenciennes) ...... 733; ; 2 -31)

Drawing by II. L. Todll from Yo. 21871. U.S.N.M., collected

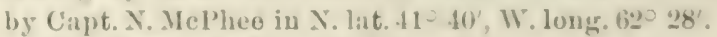

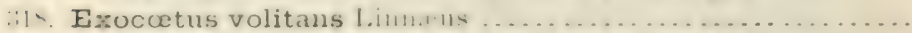

Drawing ly H. L. Todel from Xo, 20636, L.S.X.I., collected by V.N. Edwarls at Wools Ilole.

\section{PLATE CXIX,}

319. Cypsilurus californicus (Cooper) 740: :2ख30)

Jrawing by IT. L. Todd from No. 26907, L.S.N.A., collected by Dr. Jorkan at santa Barbara, C'alifornia.

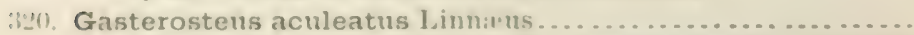

Drawing by 11. L. Tould from No. 2087., L.S.X.M., collected at Woods Hole, Massachusetts, by V. X. Edwaris.

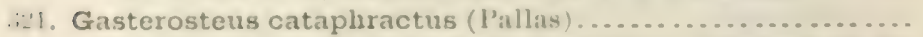
Drawing by II. L. Tould from a specinen collected by Dr. lienn at St. P'anl Island, Alaskin.

\section{PLATE CXX.}

...2. Apeites quadracus (Miteliill)

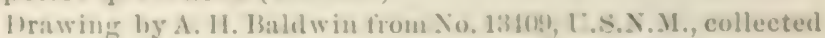
by V.X. Vilwards at Womls Ilole, Massachusetts.

:2:3. Aulorhyuchus flavidus (iill

Drawing hy A. 11. Bahlwin from Xo, 21585, U.S.X.M., collected by . l. (i. Swan in l’nget somml.

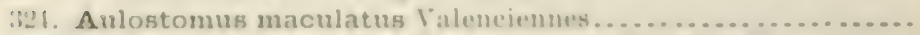
brawing ly 11. L. Tould tron Xo. Blsis, C.S.X.M., collected by U.s. Fish C'munis-ion, Potomac River, Washington. 
325. Siphostoma starksii Jordan \& Culver...................

Drawing by Anna L. Brown from the type, No. 2686, L. S. Jr. Univ. Mus., eollected by the Hopkins Expedition in the Rio Presidio, Mazatlan, Mexico.

326. Corythroichthys cayorum Evermann aud Kendall.......... Drawing by A. H. Baldwin from the trpe, No. 48784, U S.N.M., a male, collected by Evermann and Kentall at Key West, Florida.

\section{PLATE CXXI.}

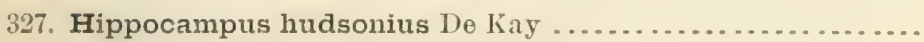
Drawing by H.L. Todd.

328. Hippocampus zosteræ Jordan and Gilbert Dratwing by W. S. Atkinson from a specimen collected by Dr. Jordan at Pensacola, Florida.

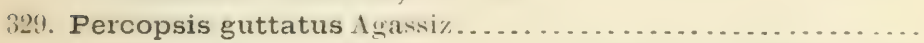
Drawing by A. H. Baldwin from a specimen collected by Dr. Evermann in East Okoboji Lake, Iowa.

\section{PLA'TE CXXII.}

330. Columbia transmontana Eigenmann and Eigenmann ........ Drawing by A. H. Baldwin from the type, collected at the month of the Umatilla River, Oregon, by Dr. Eigenmann.

331. Aphredoderus sayanus (Gilliams) ..................... Drawing from No. 34401, U.S.N.M., collected by William MeAdams in the Illinois River.

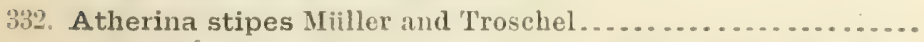

Drawing by A. H. Baldwin from No. 38456 , U.S.N.M., collected by the Albatross in the Bahamas.

\section{PLATE CXXIII.}

333. Atherina aræa Jordan and Gilbert.

Drawing by W. S. Haines from the type, No. 34967, U.S.N.M., collected by Dr. Jordan at Key West, Florida.

334. Chirostoma humbolditanum (Cuvier and Valenciennes)..... Drawing by A. H. Baldwin from Yo. 44156, U.S.N.M., collected by Scovell and Woolman in Mexico.

335. Eslopsarum jordani (Woolmau) .................. $793 ; 2810$ Drawing by A. H. Baldwin from the type, collected by A. J. Woolman in the canals at Salamanca, Mexico.

\section{PLATE CXXIY.}

336. Kirklandia vagrans (Goode and Bean) ...................

Drawing by H. L. Todd from No. 2286t, U.S.N.M., collected by Silas Stearns at Pensacola, Floricla.

Bull. No. 47, pt. $4-\mathrm{XV}$ 


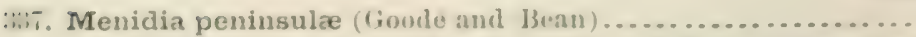

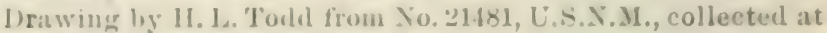
Pensacula, Forida., by silas stearus.

338. Menidia gracilis beryllina (Cope) . . . . . . . . . . . . . . . . Drawing by A. H. Balıwin from is specinen cullectod by $\mathbf{H}$. M. Suith in the Potumac River.

\section{PIATE CXXV.}

339. Eurystole eriarcha (Jordin and Gilbert).................. Drawing by Anna L. Brown from No. 2689, I. S. Jr. Cuis. Mus, collected by the Hopkins expectition at Mazatlan, Mexico.

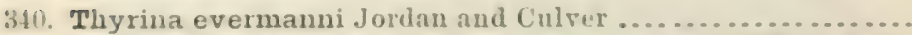
Drawing by Anma L. Brown from the type, No.2688, L. S. Jr. Luiv. Mus., collected by the Hopkius Expedition at Mazat. lan, Mexico.

i11. Atherinopsis californiensis Girard ...................... Drawing from No. 26761, U.S.N.M., collected by Dr. Jordau at San Viego, California.

\section{PLATE CXXVI.}

312. Atherinops affinis (Ayres)

Drawing by Anna L. Brown.

313. Mugil cephalus Linn:us

Drawing by H.L. 'Todd from No. 2.456, U.S.X.M., collected at Woods Hole, Massachusetts, by V. N. Edwarels.

31. Mugil curema C'uvior and Valeucienues................... Drawing by H. L. Todel.

\section{PLATE CXXVII.}

315. Mugil hospes Jordan and Culver

1) rawing by Anna L. Brown from the type, No.2s@o, L. S. Jr. Unv. Mus., collected by the Hopkins Expedition at Mazatlau, Mexico.

:11i. Chaenomugil proboscideus (iiinther) ...................

Drawing by A. 11. Baldwin from No. 4ti5t3, U.S.X.M., collected by tho Albatross at Clarion Island.

: 17. Agonostomus monticola (Bancruft) ................... Drawing by A. H. Baluwin from No. 15182, L:S.X.M., collected by 1: W. Nelson in Mexico.

\section{PLATE CXXYII.}

:38. Joturus pichardi l'noy

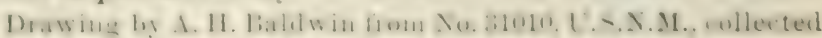
by Captain Dow at l'anama. 
:14. Sphyraena barracuda (Walbaum)

Drawing by II. L. 'Todd from No. 14978, U.S.N.M., receired from E. G. Black ford, collected in Florida.

350. Polydactylus octonemus ( (iirard) . . . . . . . . . . . . . . . . .

Drawing by H. L. 'Todd from No. 22821, U.S.N.M., collected b5 Silas Stearns at P'ensacola, Florida.

\section{PLA'TE CXXIX.}

:i1. Ammodytes americanus Do Kay

Drawing by H. L. Todd from No. 16500, U.S.N.M., collected by the U.S. Fish Commission at Nantucket.

3.,2. Bathyclupea argentea Goode and Bean..................

Drawing by A. H. Baldwin from the type collected by the Blake, of Neris.

3.i.). Stephanoberyx monze Gill

Drawing by H. L. 'Todd from the type, No.33445, U.S.N.M., collected by the Albatross at Station 2077, in the Gulf Stream.

\section{PLATE CXXX.}

ii.t. Hoplostethus mediterraneus Cuvier and Valenciennes..... Drawing by A. H. Baldwin from No. 43624, U.S.N.M., collected by the Albatross at Station 2059.

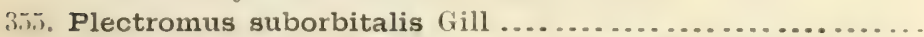

Drawing ly H. L. Told from the type, No. 3:3271, U.S.X.M., collected by the Albatross at Station 2036 , in N. lat. $38^{\circ} 52^{\prime} 40^{\prime \prime}$, IV. long. $69^{\circ} 24^{\prime} 40^{\prime \prime}$, in 1,735 fathoms.

356. Plectromus crassiceps Giinther

Drawing by S. F. Denton from No. 33378, U.S. Y.M., collected by the Albatross in N. lat. $41^{\circ} 40^{\prime} 30^{\prime \prime}$, W. long. $65^{\circ} 35^{\prime}$, in 855 fathoms.

\section{PLA'IE CXXXI.}

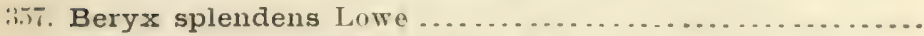

Drawing by M. M. Smith from a specimen collected by the Albatross at Station 2415 , in N. lat. $30^{\circ} 44^{\prime}$, W. $\operatorname{long} .79^{\circ} 26^{\prime}$, in 4.40 fathoms.

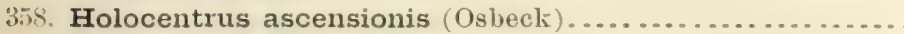
Drawing by H. L. 'Todd.

359. Flammeo marianus (Curier and Valenciennos) ......... 852; 2871

Drawing by $\mathrm{W}^{\mathrm{T}}$. S. Atkinson from specimen No. 4972, L. S. J . Univ. Mus., collocted by Rev. J. S. Roberts at Kingstou, Jamaica.

\section{PLATE CXXXII}

360. Mullus auratus Jordan and Gilbert

3ti1. Mulloides rathbuni (Evormann and Jenkins) .............

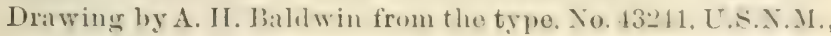
collected by Evermann and Jeukins at Guaymas, Mexico. 
Thert page

iti2. Upeneus maculatus (Bloch) ........................ 858

Drawing hy II. L. Todul from No. 21910, I.S.X.M., collected log V.X. Edwards at Woods Ilole, Massichusetts.

\section{IPATE CXXXII.}

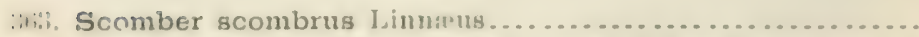

Drawing by 11. L. Tod,l from No. 25256, U.S.X.M., obtained in the Washington marliet.

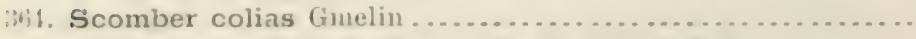

Drawing by II. L. T'orld from Yo. 23180, L.S.X.M., collected by tho U.S. Fish Commission at Provincetown, Massachusetts.

:35. Auxis thazard (Lacépiedo) ..........................

I) ratwing by II. L. Tould from No. 3.3136. U.S. . . M., collected by tho U.S. Fish Commission at Wools Hole, Massachusetts.

\section{PLATE CXXXIV.}

:6it, Gymnosarda alleterata (Ratinesıue) ................... Urawing by II. L. 'Tould from No. 10136 . U.S.N.M., collected ly S. F. Baird at Woods Hole, Massachusetts.

:6i7. Germo alalunga (Gmelin) ............................

Drawing l,y H. L. Tould from Xo. 21814, U.S. X.M. collected by Capt. William Thompson of the schooner Magic, in 300 fathoms.

3ix. Scomberomorus maculatus (Mitehill) ..................

Drawing ly H. L. Tould from No. 1055.), U.S.X.M., obtained ly E. (r. Blackford in the Now York market.

\section{PLATE CXXXV.}

:hil. Scomberomorus regalis (Bloch) ........................ Drawing hy 1I. I. Tould from No. 12527, U.S.X.M. collected by L. (i. Jlackford at liey West.

:371). Escolar violaceus (Bean) $878 ; 2813$

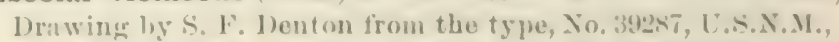
collected hy Capt. Thomas 'hompson on L.e Have Bamle in 125 fitloums.

:i, Epinumla magistralls Poey .........................

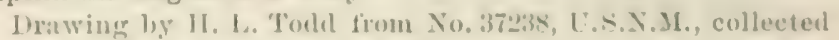
ly the dibutrose in the Caribbean sea.

\section{HIATI: C'XXXI.}

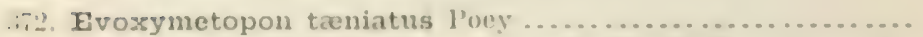
1) xawing l,y II. I. T'udil from No. 57:35, U.S.X.Al, collected lig P'rofessor l'ouy in C'uba.

:373. Lepoidopus cauchatus i.uphraset.)

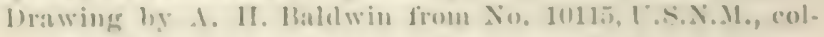
lected loy Johu Xintus at C'apo sin Lancas, Lower C'alifurnia. 
374. Benthodesmus atlanticus Goode and Bean ............... 887

Drawing by H. L. Tould from the type, No. 29116, U.S.N.M., taken from the stomach of a halibut by Capt. R. Morrison, of the schooner Laura Nelson, on the west edge of the Grand Bank of New foundland, in 80 fathoms.

\section{PLATE CXXXYII.}

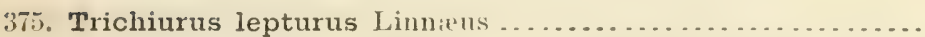

Drawing by H. L. Todd from No. 18028, U.S.N.M., collected by Dr. Janeway at St. Augustine, Florida.

376. Istiophorus nigricans (Lacépìde)

Drawing by H. L. Todd from No. 37923 , U.S.N.M., collected by U. S. Fish Commission at Woods Hole, Massachusetts.

\section{PLATE CXXYVIII.}

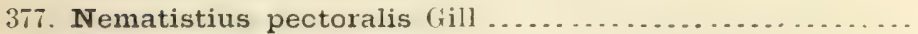

Drawing by A. H. Baldwin.

378. Oligoplites saurus (Bloch and Schneider).

Drawing by H. L. Todd from No. 16354, U.S.N.M., collected by the U.S. Fish Commission at Memensha Bight.

\section{PLATE CXXXIX.}

379. Naucrates ductor Linnreus

Drawing by H. L. Todd from No. 23197, U.S.N.M., collected by William A. Bansett at New Bedford, Massachusetts.

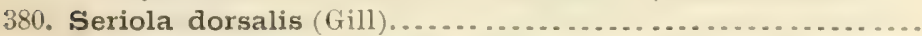

Drawing by H. L. Todd from a specimen collected by Jordan and Gilbert at San Pedro, California.

$3 \times 1$. Seriola zonata (Mitchill) ... . . . . . . . . . . . . . . . . . . .

Drawing by H. L. Todd from No. 18720, U.S.N.M., collected by V. N. Edwards at Woods Hole, Massachusetts.

\section{PLATE CXI.}

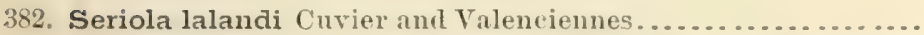

Drawing by H. L. 'Todd from No.37918, U.S.X.M., collected by the U.S. Fish Commission at Woods Hole, Massachusetts.

383. Decapterus macarellus (Cuvier and $\mathrm{V}$ alenciennes) .......... Drawing by H. L. Todd.

384. Trachurus trachurus (Limniens) ......................... Drawing by H. L. 'Todid from No. 23421, L.S.N.M., collected at Nerport, Rhode Island.

\section{PLA'I'E CXII.}

385. Trachurops crumenophthalmus (Bloch) ................ Drawing by H. L. Todd from No. 20681, U.S.N.M., collected by V. N. Edwards, at Wools Hole, Massachusetts. 
:ix6. Hemicaranx amblyrhynchus (Cuvier anul Valencionues)....

Drawiug ly II. L. T'uell from Xio.3017\%, U.S.X.d., collected ly Silas Stearns, at P'ensacola, Floricla.

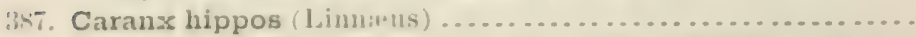

I) rawing by II. L. Todel from No. 10131, L.S.X.M., collected by V. N. Eilwards, at Woouls Hole, Massachusetts.

\section{PIATE CXIAI.}

3kr. Caranx latus Agrasiz

Drawing by II. L. Tould from Yo. 20247, I.S.X.M., collectenl by V. N. Fitwards, at Woods Hole, Massachusotts.

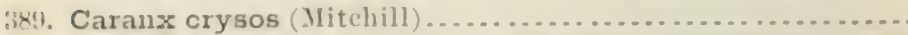

Drawing by II. L. Torld from No. 16512, L.S.X.M., collected by the L.S. Fish Commission at Woots Hole, Massachusetts.

\section{PIAT'E CXIIII.}

890. Caranx medusicola Jorlan and Starks . . . . . . . . . . . . . .

Drawing by Anna L. Brown from the type, So. 26iti, L. S. Jr. Lniv. Mus, collected by tho Hopkins Expedition at Mazatlan, Mexico.

391. Hynnis hopkinsi Jordan and Starks..................... Drawing by Anna L. Brown from the type, No. 1563\%, L. s. Jr. Univ. Mus., collected by the Hopkins Expedition at Macatlau, Mexico.

\section{I'LATE: CXIIV.}

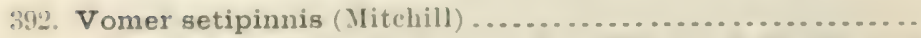
brawing by H. I. Todlel from Yo. 16252, U.S.X.M, obtained by E. G. Black ford in the Fulton Market, New York.

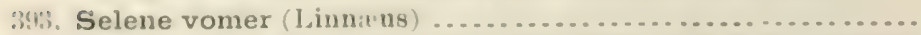

Drawing loy H. L. Tould from No. 2007, L.S.X.M., at 5oung examplo, collected by C. J. Coploy at Stapleton, Staten Island.

\section{I'LATH: CXI,}

:143ı. Selene vomer (Linnitus) ........................... Drawing by 1I. I. Tould from No. 20.79, L.S.X.M., an adult.

341. Chloroscombrus chrysurus (Limuitus) . . . . . . . . . . . . .

J)awing hy I. I. Tould from No. 21256, L.X.X.M., collected by Dr. Goule in the St. Johns River, l'lorida.

\section{PI. ITE CXI,VI.}

:95. Trachinotus glaucus (13loch)

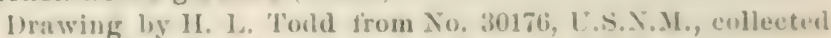
by Silas stearus at l'enqucola, Florida.

:biti. Trachinotus falcatus (Linnitus) ......................

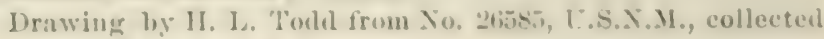
ly Silas stearms in Florida. 


\section{PLA'TE CXLVII.}

397. Trachinotus culveri Jordan and Starks.

Drawing by Auna L. Brown from the type, No. 2691, L. S. Jr. Univ. Mus., collected by the Hopkins Expedition at Mazatlan, Mexico.

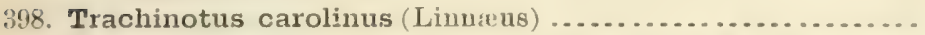

Drawing by H. L. Todd from No. 10427, U.S.N.M., collected by S. F. Baird at Woods Hole, Massachusetts.

\section{PLA'TE CXLVIII}

399. Zalocy's stilbe Jordan and MeGregor.

Drawing by Auna L. Brown from the type, No. 11996, L. S. Jx. Univ. Mus., collected by R. C. McGregor, at Clarion Island.

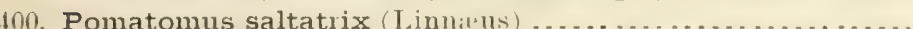
Drawing by H. L. Torld, from a east.

401. Rachycentron canadus (Linueus)

Drawing by. H. L. Tocld from No. 18563, U.S.N.M.

\section{PLATE CXLIX}

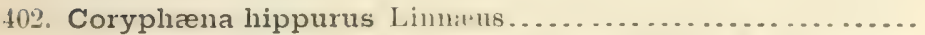

Drawing by H.L. Todd from No. 16482, U.S.N.M., obtained by E. G. Blackford in Fulton Market.

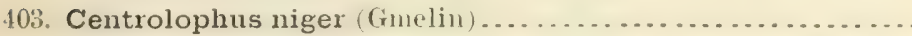

Drawing by S. F. Denton from a specimen obtained at Dennis, Massachusetts.

\section{PLATE CL.}

404. Peprilus paru (Limin'Is)

Drawing by H. L. Todd from No. 15234, U.S.N.M.

105. Poronotus triacanthus (Peck)

Drawing by H. L. Told from No. 787, U.S.N.M., collected by S. F. Baird at Beesleys Point, New Jersey.

\section{PLATE CLI.}

406. Icichthys lockingtoni Jordan aud Gilbert

Drawing by A. H. Baldwin from No. 27397, U.S.N.M., collected at Point Reyes, Califoruia.

407. Schedophilus medosophagus Coceo.

Drawing from H. L. Touk from a specimen collected by the Allutross in lat. $27^{\circ} 49^{\prime} \mathrm{N}$., $76^{\circ} 12^{\prime} \mathrm{W}$.

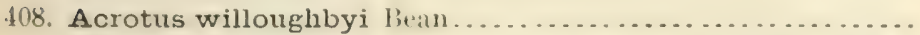

Drawing by H. L. Todd from the type, No.39340, U.S.N.M., collected by Chas. Willoughloy at the Quinault Agrency, west coast of Washington. 


\section{PIATE CLII.}

109. Zaprora silenus Jorlan ... . . . . . . . . . . . . . . . . . . . . . .
J)rawing ly Anna L. Brown from the type, in the J'lovincial 2850 Musen at Vietoria, Britioh Colmubia, collected ly $11 . '$. Stainton, at Nanaiuso, Vancouver Island.

110. Grammicolepis brachiuscu.us l’oey ...................... Drawing by A. II. Baldwin from a drawing by R. W. Sluteldt. Jour. Morplı, Vol. 11.

11. Tetragomuxus cuvieri lisso 476

Drawing by Mary Hilheluandt from a speciuen collected log I. X. Eilwards at Woods Hole.

\section{VITE CLII.}

112. Pempheris mulleri Poer

Drawing by M. M. Smith from No. 37111, U.S.N.M., collected by tho diluatross at Coznmel.

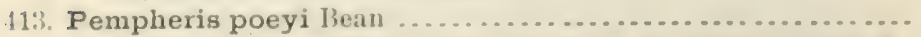
Drawing by M. M. Smitle from the type, No. 37184, U.S.X. M.. collected by Prof. l'oey in Cuba.

414. Elassoma evergladei Jorklan ..........................

Drawing by A. H. Ballwin from a specimen collected by A. J. Woolman in Floricla.

\section{PLATE CLIV.}

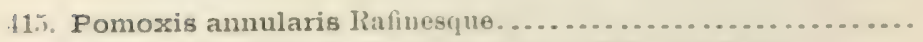
I) rawing by H. L. Todd from No. 10387, U.S.N.M.

116. Pomoxis sparoides (Lacépiele) . . . . . . . . . . . . . . . . I) rawing by II. L. Todd from No. 10077, U.S.N.M.

P'IATE CLV.

117. Centrarchus macropterus (Lacépiele) ................... Hrawing l,y II. L. 'lodel from Xo. 20397, U.S.A.M., collected l,y II. S. Hyatt at lingstom, North Carolina.

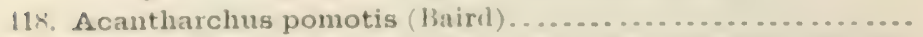

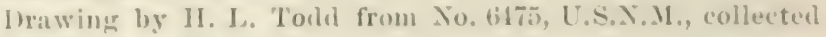
at 'Tarboro, North C'arolina.

\section{I'LAT: CI,I.}

119. Ambloplites rupestris (liafinespue) ................... Drawing hy II. L. Tould from No. 9lo1, LAS.X.M., collected lyy (ieorge C'lark at Keorse, Michigan.

119, A, 13, ( . Skull of Ambloplites rupestris (lialinesifue) ......

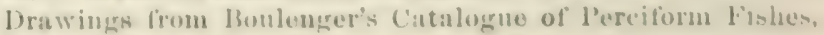
Vol, เ, p. 3. 


\section{PLA'TE CLVII.}

420. Archoplites interruptus (Girard)

Urawing by H. L. Todd from No. 27137, U.S.N.M., collected by Dr. Jordan at San Francisco.

421. Chrenobryttus gulosus (Cuvier and V'alenciennes) ..........

Drawing by H. L. 'Todd from No. 17803, U.S.X.M., collected by Kumlien and Bean in Jackson Lake, near Montgomery, Alabama.

\section{PLATE CLVIII.}

122. Enneacanthus gloriosus (Holbrook) ..................... Drawing by H. L. Todd from No. 20356, U.S.N.M., collected by C. C. Abbott at Trenton, New Jersey.

423. Mesogonistius chætodon (Baird) ..................... Drawing from No. 20354, U.S.N.M., collected bs C. C. Nbbott at Trenton, New Jersey.

\section{PLA'TE CLIX.}

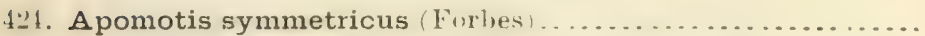

Drawing by A. H. Baldwin from a specimen collected by Evermann, Scovell, and Gurley in Kilpers Pond, Houston, Texas.

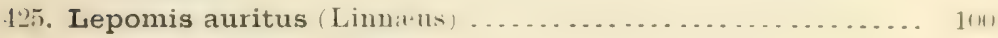
Drawing by H. L. Todd.

425a. Skull of Lepomis auritus (Linneus) ................... . . . Drawing from Boulenger's Catalogue of Perciform Fishes, Vol. I, p. 24.

\section{PLA'TE CLX.}

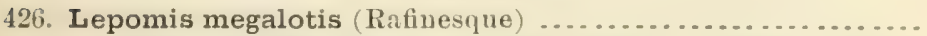

Drawing by H. L. 'Todd from No. 36465, U.S.N.M., collected by Jordan and Gilbert in Saline River, Benton, Arkansas.

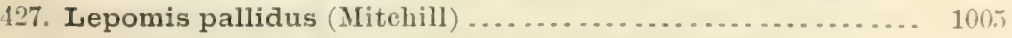

Drawing by H. L. Todd from No. 84t8, U.S.N.M.

\section{PLA'TE CLXI.}

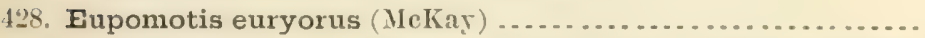

Drawing by H. L. Todd from the type No. 4109 , U.S.N.M., collected in Lake Huron, at Fort Gratiot, Michigan.

429. Eupomotis gibbosus (Linnivus) ........................

Drawing by H. L. Todd from No. 4163, U.S.N.M., collected by Professor Baird in Root River, Wiscousin.

\section{PLATE CLXII.}

430. Micropterus dolomieu Lacépède ... . . . . . . . . . . . . . . . . .

Drawing by H. L. Tord from No. 14143, U.S.X.M., obtained in the Washington market.

430 a. Vertebræ of Micropterus dolomieu Lacépècle

From Boulenger's Catalogue Perciform Fishes, Vol. I. 1). 4. 


\section{PIATE (I.XHI.}

4:1. Micropterus salmoides (1.ikeipinte) .................. 1012 I) rawing ly H. L. I'onlel.

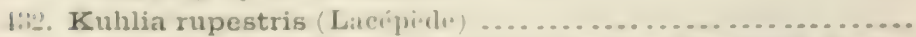
Drawings from l3onlenger's Catalonte of l'erciform Fishes, Vol. 1, tig. 1, 1. 35.

\section{PLA'TE CISIV.}

l:i:3. Stizostedion vitreum (Mitchill) ........................ Jrawing by II. L. Todel from Yo. 1007:, U.S.X...1., collected by 1. W. Milner at liochester, New lork.

lit:in. Tail of Stizostedion vitreum (Mitchill) .................. 1) rawing ly Anna L. Brown.

fir. Stizostedion canadense (Smith) ....................... Drawing by H. L. Tould from No.10555, U.S.N.M. collected by J. W. Milner at Eeorse, Michigan.

\section{PLATE C'LAV.}

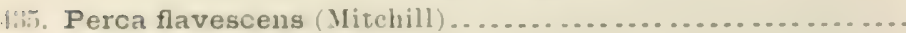
Drawing hy H. L. 'T'odd.

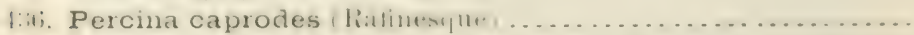
Drawing loy II. L. Todıl from No.34744, U.S. Y..M., collected by II. G. Dresel in Licking County reservoir, Ohio.

listin. Skull of Percina caprodes (Ratinesque) .................. Drawing from Boulenerer's Catalogue of Perciform Fishes, Vol. I, tig. 7, P. 5\%.

\section{PIATE: C'LAVI.}

1:37. Hadropterus macrocephalus (Cope)

Irawine by 1. H. Baldwin from a specimen collected hg lur. Cimpley in Indian Creak, near C'umberland Gap, 'enuessee.

13x. Hadropterus aspro (C'ope amd Jorkau) . . . . . . . . . . . . . . Drawing ly A. II. Ballwin from a specinen collected low Evermanu, Scovell, and Gurley in Chickamauga Creok, at L.e. and (iordon's mill, (ieoreria.

\section{I'LATE (:ISTII.}

139. Hadropterus gruntheri Eigemmann and ligenmann ....... J)awing by A. 11. Baldwin from a speeimen collected ly 1. J. Woolman in the lien River of the North. Moorehear, Minnesotit.

150. Hadropterus evides (.Jordan and ('opelamb)

Jrawing ly d. M. Baldwin from a squeimen collected by Evermann, soovell, and (iurloy in Clinch River, at Walkers Forl, Tennessece.

11. Hadropterus scierus swain

1) rawing by . 1. 11. Baldwin from a specimen collected by Lveruann, somvell, and (iuley in Climeh liver, at llalkers Ford, Tounessev. 


\section{PLATE CLXVIII.}

44.2. Hypohomus aurantiacus (Cope) ...........................................
Drawing by A. H. Baldwin from a specimen collected by Evermann, Scovell, and Gurley in Clinch River, at Walkers Ford, Tennessee.

41:. Hypohomus spilotus (Gilbert)

Drawing bs A. H. Baldwin.

444. Cottogaster shumardi (Girard) ........................................... collected
Drawing by H. L. Todd from No. 17852, U.S.N.M. by Dr. Jordan in the Wabash River, Indiana.

\section{PLATE CLXIX.}

145. Cottogaster cheneyi Evermann and Kendall ............... 2851

Drawing by A. H. Baldwin from the type Yo. 48781 , U.S.N.M., collected by Evermann and Bean in Racket River, Norfolk, New York.

46. Ulocentra gilberti Erermann and 'l'hobnrn.................

Drawing by A. H. Baldwin from the type No. 47531 , U.S.N.M. collected by Erermann, Seovell, and Gurley in Clinch River, Walkers Ford, Tennessee.

47. Ulocentra meadize Jordan and Evermann ....... . . . . . . . . .

Drawing by A. H. Baldwin from the type No. 48903, U.S.N.M., collected by Dr. Gurley in Indian Creek, Cumberland Gap, Tennessee.

\section{PLA'TE CLXX.}

448. Ulocentra simotera (Cope)

Drawing by A. H. Baldwin from a specimen collected by Evermann, Scovell, and Gurley in Arnwine spring, Mount Veril, Teunesseo.

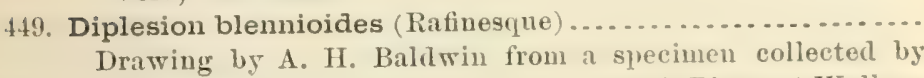
Evermann, Scovell, and Gurley in Cliuch River, at Walkers Ford, Tennessee.

450. Boleosoma nigrum (Rafinesque) .......................

Drawing by A. H. Baldwin from a specimen collected by R. R. Gurley in Ball Creek, uear Tazewell, 'I'ennesseo.

\section{PLA'TE CLXXI.}

4i). Boleosoma nigrum olmsterli (Storer) ................... . . Drawing by H. L. Todd from No. 30243, U.S.N.M., collected by Col. Marshall MeDonald in the Potomac Rirer, Gunston, Virginia.

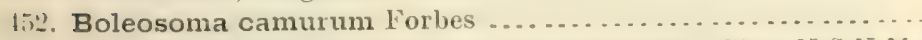

Drawing by H. L. Todd from the type No. 23455, U.S.N.M. collected by Dr. Jordan in the Illinois liver.

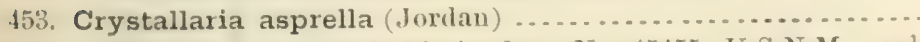

Drawing by A. H. Baldwin from No. 4545.), U.S.N.M., collected by Dr. Evermann in the Wabash River, New Harmons, Indiaua. 


\section{I'LATE: CLXXII.}

l'ext yasen.

151. Ammocrypta pellucida clara (Jorilan and Meek).......... 106is

lorawing by S. F. Denton from the type No.35828, IT.S.N.M., collected by Jorkan and Meek in the Des Moines River. Ottumwa, lowa.

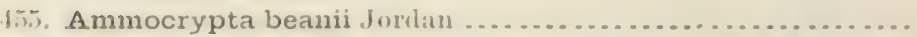

1) rawing ly A. H. Baldwin from No. 1783:3, C.S. N.M., col. lected by Bean and Maxson in Notalbany River, Lonisiana.

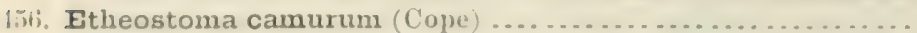

Drawing by A. II. Baldwin from a specimen collerted liy R. R. Gurley in Indian Creek, near Cumberland Gap, Tennessee.

\section{PLATE CLXXIII.}

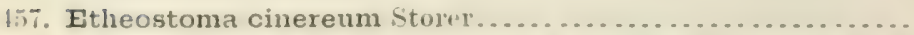

Dratring by A. I. Baldwin from a specimen collected hy $P$. 11. Kirsch in Wolf liver, Brrilstown, T'ennessee.

158. Etheostoma jordani Gilbert

Drawing by S. F. leenton.

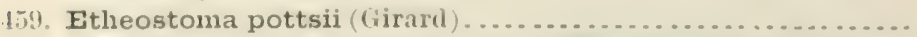

Drawing by II. L. Told from No. 38245, L.S.X.M., the type of Etheostoma micropterns Gilbert, colloeted by E. Wilkinson in Chihualua, Mexico.

\section{P'LATE CLAXIY.}

U60. Etheostoma iowa Jorlan and Meek

Irawing by 1 . 11. Baldwin from a specimen collected by A. J. Woolman in Pomme de Terre River, Appleton, Wisconsin.

161. Etheostoma jessize (Jordan and Jraytou) ................ Drawing by H. L. Tould from No. 27896, U.S.X.M., collecterl by Dr. l'orbes, in Lake P'euria, Illinois.

16:2. Etheostoma lepidogenys Lvermam and lienilall ........... l)awing by A. H. Bahlwin from the type, No. 4840, L.S.X.M., collected by Lvormann, scovell, and Gurley, in Comal Spring, New Branniels, Texas.

\section{PLATE CLXXY.}

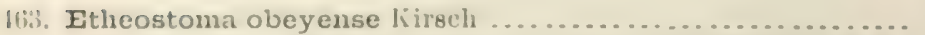

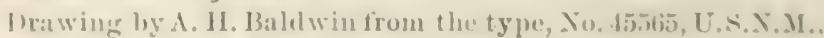
collented by In. Kirsch, in tributary of Oheys River, Clinton county, lientucky.

ftil. Etheostoma pagei Merek . .

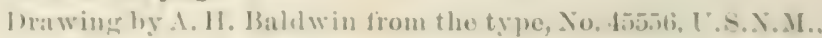
collected by 1)r. Meek in Spring Branchat Nensho, Missouri.

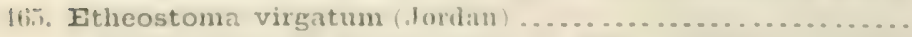

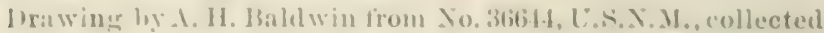
by dillert and swain in liock Cantlo liver, livingrston. kentueky. 


\section{PLATE CLXXVI.}

466. Etheostoma julize Meek 1093

Drawing by S. F. Denton from the type collected by Meek, Drew, and Rettger in James River, near Springfield, Missouri.

467. Psychromaster tuscumbia (Gilbert and Swain) ............

Drawing by A. H. Baldwin from the type, No.36154, U.S.N.M., collected by Gilbert and Swain in Spring Creek, Tuscumbia, Alabama.

41ix. Copelandellus quiescens (Jordan) .................... Drawing by A. H. Baldwin.

\section{PLATE CLIXVII.}

469. Boleichthys fusiformis (Girard)

Drawing by H. L. Todd from No.36t15, U.S.N.M., collected by Jordan and Gilbert in Washita River, Arkadelphia, Ar. kansas.

470. Microperca fonticola (Jordan aud Gilbert) ................ Drawing by Ernest Copeland from the type, No. 36523, U.S.N.M., collected by Jordan and Gilbert in San Mareos River, San Mareos, Texas.

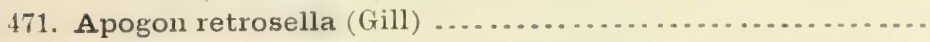

Drawing by Anna L. Brown from To. 2917. L. S. Jr. Univ. Mus, collected hy the Hopkins expedition at Mazatlan, Mexico.

\section{PLA'TE CLXXVIII.}

472. Apogon pigmentarius (Poø

1109

473. Apogonichthys alutus (Jordan \& Gilbert) . .................

Drawing by A. H. Baldwin from No.30874, U.S.N.M., collected by Jordan and Stearms at Pensacola, Florida.

\section{PLATE CLIXIX.}

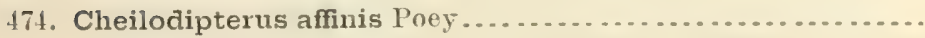

Drawing by A. H. Baldwin from No. 37416 , U.S.N.M., collected by Professor Poey in Cuba.

475. Hypoclydonia bella Goode and Beau ....................

Drawing by S. Is. Denton from No.39383, U.S.N.M., collected by the Albatross at Station 2426, in N. lat. $36^{\circ} 01^{\prime} 30^{\prime \prime}, \mathrm{W}^{\circ}$. long. $74^{\circ} 47^{\prime} 30^{\prime}$, in 93 fathons.

176. Centropomus undecimalis (13loch) ..................... 1101

Drawing by H. L. Todd from No. 19907, U.S.N.M.

\section{PLATE CLXXI.}

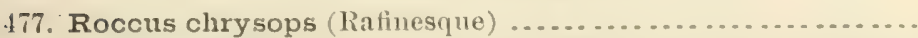

Drawing by H. L. Todd from No. 10326, U.S.X.M., collected by J. IV. Milner at Sandusky, Ohio.

478. Roccus lineatus (Bloch

Drawing by H. L. Todel from No. 25219, U.S.N.M., obtained by Professor Baird in the WTashington market. 
PLATE CLXXXI.

'Text page.

479. Morore americana (Gmolin

1134

Drawing by II. L. Todd from No. 15861, U.S.N.M., received from the Now York market.

$480,480 a$. Polyprion americanus (Bloch ot Schneiler)

Drawings from IBoulenger's Catalogne of Perciform Fishes Vol. I, tig. 12, p. 1.t9.

\section{PLATF: CLXXXII.}

181. Bodianus fulvus punctatus (Linnieus)

Drawing by A. H. Baldwin from No.33717, U.S.N.M., collected by J.C. Brevoort in the West Indies.

18:. Epinephelus adscensionis (Osbeck)

Drawing lyy H. L. Todd from No. 26574, U.S.N.M., collected by Silas Stearus, at Key West, Florida.

\section{PLATE CLXXXIII.}

183. Epinephelus striatus (Bloch)

$115 \%$

Drawing by H. L. Torld from No. 31910, U.S.N.M., collected by Silas Stearns at Pensacola, Florida.

181. Epinephelus drummond-hayi Goode and Bean .............

Drawing by II. L. Todd from No. 31719, U.S.N.M., collected by Silas Stearns at Pensacola, Florida.

\section{PIATE, CLXXXIV.}

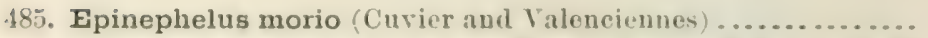

Drawing by 11 . L. Tould from No. 22129, U.S.X.M. I., collected in Florida and oltained in the Washington market by Dr. (ioode.

486. Garrupa nigrita (Holbrook)

I)rawing l,y H. L. Tould from Yo, 37207, U.S.N.M., collected by the Allutross.

\section{PLATE CLXXXY.}

487. Promicrops itaiara (Lichtenstoin)

Drawing by II, L. Tudd from No. 22306, U.S.X.M., collected at mouth of st. fohns River, Floricla.

$487 \pi, 487 h$. Promicrops itaiara (Lichtenstein).................

Drawings trom Boulenger's Catalogne of Perciform Fishes, Vol. 1, fig. 1:3, p. 164.

\section{PIATF CIAXXY}

188. Alphestes chloropterus (C'uvier and Valencisnnes)......... Drawing by. A. Baddwin from Xo. 98:1, 1.3.N.M., collected by l'rofessor l'oey in C'ubat.

18\%a. Alphestes chloropterus (C'uvier and Valenuienues)........ Drawing irom Boulenger's Catalogue of l'ereiform lishes, Vol, 1, tig. 15. 1). ํ.5. 
489. Dermatolepis zanclus Evermann aud Kendall ........... 28.4

Drawing by A. H. Baldwin from the type, No. 48843, U.S.N.M., collected by Evermaun aud Kendall at Key West, Florida.

\section{PLATE CLXXXYI.}

490. Mycteroperca boulengeri Jordan and Starks ..............

Drawing by Anua L. Brown from the type, No. 1691, L. S. Jr. Unir. Mns., collected by the Hopkins expedition to Mazatlan, Mexico.

491. Mycteroperca venenosa (Limnieus) .......................

Drawing by H.L. Todd from No.35103, U.S.N.M., collected by Dr. Jordan at Havana, Cuba.

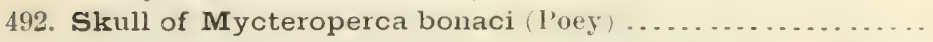

Drawing from Boulenger's Catalogue of Perciform Fishes, Vol. I, fig. 19, p. 258.

\section{PLATE CLXXXVIII.}

493. Mycteroperca jordani (Jenkins and Evermann)........... Drawing by A. H. Baldwin from the type, No. 39628 , U.S.N.M., collected at Guaymas, Mexico, by Jenkins and Evermann.

494. Mycteroperca microlepis (Goode and Bean) ..............

Drawing by H. L. Todd from No. 26587, U.S.N.M., collected by Silas Stearus at Key West, Florida.

495. Mycteroperca falcata phenax Jordan and Swain ..........

Drawing by H. L. Todd from No. 34992, U.S.N.M., collected at Key West, Florida, by Dr. Jordan.

\section{PLATE CLXYXIX.}

496. Hypoplectrus unicolor nígricans $(\mathrm{Poey})$.................

Drawing by H. L. Todd from No. 3423, U.S.N.M., collecter? at Garden Kes, Florida.

497. Hypoplectrus gemma Goode aud Beau ..................

Drawing by H. L. Todd from the type, No. 3122, U.S.N.M., collected at Garden Kes, Florida.

\section{PLATE CXC.}

498. Paralabrax maculatofasciatus (Steindachner) .............

Drawing by A. H. Baldwin frogn No. 34754, U.S.N.M., col. lected br Rosa Smith at San Diego, California.

499. Skull of Paralabrax humeralis (Cuvier and Valenciennes)...

Drawiug from Boulenger's Catalogue of Perciform Kishes, Vol. I, fig. 20, p. 275.

500. Centropristes striatus (I.inni 11 ) . . . . . . . . . . . . . . .

Drawing by H. L. Todd from No. 14838, U.S.N.M., collected by the U. S. Fish Commission at Noank, Counecticut. 


\section{P'LATE CXCI.}

ill1. Centropristes pliladelphicus (Linntrus) . . . . . . . . . . . . 1201

Drawing by A. H. Baldwin from No. 33161, U.S.N.M., collected by Dr. Gilbert at C'harleston, Sonth C'arolina.

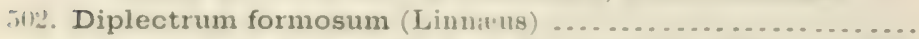
Urawing by H. L. Todk from No. 215.3, U.S.X.M., collected by C. C. Leslio at Cliarleston, Sonth Carolina.

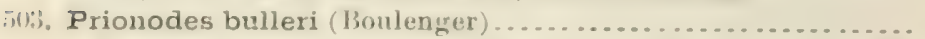
Drawing from lBoulenger's Catalogrue of P'erciforn Fishes, Vol. I, pl, x.

\section{PLA'IE CXCII.}

iol. Paxanthias furcifer (Cuvier and Valencienueg) ............. Drawing by $\Lambda$. H. Baldwin form No. 12510, U.S.N.M., collectud by Professor Poey in Cuba.

515. Hemianthias vivanus (Jordan and swain) ................ Drawing by 11 . S. Atkinson from specimen No. 1655, L. S. Jr. Univ. Mus., collected ly Dr. Jordau at Pensacola, Floricla.

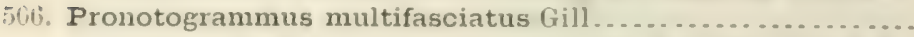

J)atwing by H. L. 'Iodld from the type, No. 2762, U.S.N.M. collecterl by John Xintus at C'ape Sau Lucas, Lower Californis.

\section{PLAT'E CXCIII.}

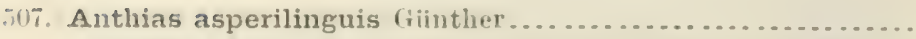

Drawing from 13oulonger's Catalogue of Pereiform Fishes. lol. 1. 1.1. Ni11.

i). Gramma loreto l'ooy

Drawing hy .J. W. Folsom from the type, No. 10031, M.C.Z., collected by Professir loey at Matanzas, C'uba.

\section{PL.I'IF CXCIV.}

.1) Rypticus bistrispinus (Mitchill) 1233 Drawing by H. L. 'Todel.

i11), Lobotes surimamensis (Bloch)... 123.1 Drawing loy 11 , L. 'Todd.

\section{PIATE CIET,}

i11. Priacanthus arenatus Cuvier anl Valeneiemes.

Drawing by II. I. Tould from No. 20680, L.S.X.M., collecterl by V. X. Vilwards at Wouds Hole, Massachusetts.

il:. Pseudopriacanthus altus (iill

1)rawing loy 11. I. "Toull from Xo, 2317, Ci.s. X. M. collected by the Albulross ofl liey Wiest. Forielit. 


\section{PLATE CXCVI.}

i13. Hoplopagrus guntheri Gill

Drawing by A. H. Baldwin from No. 28367, U.S.N.M., collected by Dr. Gilbert at Mazatlan, Mexico.

514. Evoplites viridis (Valenciennes)

Drawing by W. S. Atkinson from a specimen collected by Alfonce Forrer at Tres Marias Islands, Mexico.

\section{PLATE CXCVII.}

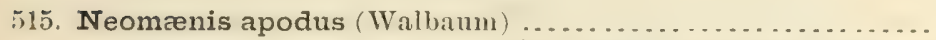

Drawing by H. L. Todd from No. 332.20, U.S.X.M., collected by Dr. Henshall at Indian River Inlet, Florida.

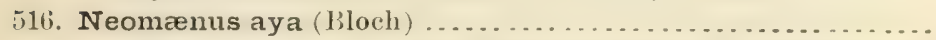

Drawing by A. H. Baldwin from a specimen obtained in the Washington Market.

\section{PLATE CXCVIII.}

517. Neomænis analis (Cuvier and Valenciennes).

Drawing by H. L.'Todd from No.35030, U.S.N.M.

518. Neomæenis synagris (Linn: ‘ns)

Drawing by H. L. Todd from No. 36922, U.S.N.M., collected by the U. S. Fish Commission at Key IVest, Florida.

\section{PLATE CXCIX.}

519. Rabirubia inermis (Peters)

Drawing by Auna L. Brown from No.4983, L. S. Jr. Univ. Mus., collected by the Albatross at Panama.

520. Ocyurus chrysurus (Bloch)

Drawing by S. F. Denton from No. 26583, U.S.N.M., collected by Silas Stearus at Key West, Florida.

\section{PLATE CC.}

5.) Rhomboplites aurorubens (Curier and Valenciennes) ....... Drawing by H. L. 'Todd from No. 21338, U.S.N.M., collected by Silas Stearns at Pensacola, Floricla.

5.2. Apsilus dentatus (inichenot

Drawing by A. H. Baldwin from No. 9809, U.S.N.M., collected by Professor Poey in Cuba.

\section{PLATE CCI.}

i:?. Aprion macrophthalmus (Miiller and Troschel)............ Drawing by M. M. Smith from a specimen collected by the Blake in N. lat. $23^{\circ} 13^{\prime}, \mathrm{W}^{\prime}$. long. $89^{\circ} 10^{\prime}$, in 84 fathoms.

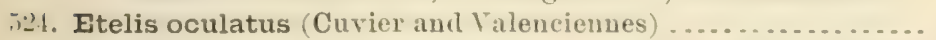
Drawing by $\Lambda$. H. Baldwin from No. 35049, U.S.Y.M , collected by Dr. Jordan at Haraua, Cuba.

Bull. No. 47 , pt. $4-\mathrm{x}$ - 
PIATE CCII.

Text page.

i-s. Verilus sordidus Poey

1281

Drawing l,y 1 . II. Bald win from No.12565, L.S.X.M., colleeted by Profensor l'oey in C'ula.

5'-4i. Xenocys jessize Jorlan and Bollman ...................

Drawing hy A. II. Baldwin from the type, No.41166, U.S.N.M.,

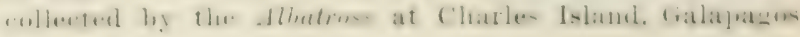
Group.

\section{PIAATE CCLII.}

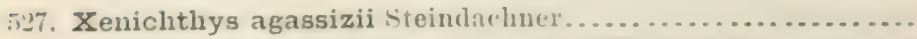

Jrawing by $\Lambda$. II. Baldwin from No. 26671, U.S.N.M., collected in the Galapagos Islands.

i.- Hremulon album C'urier and Valencienues.................

Drawing by II. L. Tould from No. 35035, U.S.X.M., collected at Ley West, Florida, by Dr, Jordan.

PLATE CCIV.

529. Hæemulon macrostomum Giinther

1296

Drawing by 11. L. Toeld from No.30057, U.S.N.M., received from the Kingston P'ublic Museum, Jamaica.

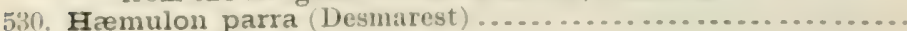
Drawing by H. L. 'Todd.

PLA'TE C C Y

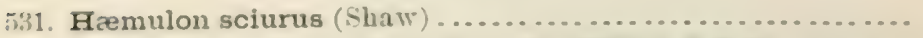
Drawing by II. L. Tould from No.32603, U.S.X.M., collected at Key West, Florida, by .J. A. Henshall.

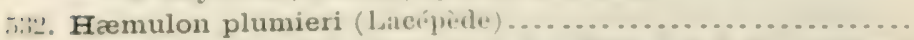

Drawing by H. L. 'Todel from No. 20980, L.S.X.M., collecked by Dr. Goode at Charleston, Sonth Carolinat.

\section{PLATE CCVI.}

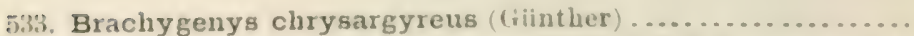
Drawing ly M. L. 'Toda from No. 3.5150, L.S.X.M., collected at Havana, Cula, by 1)r. Jordan.

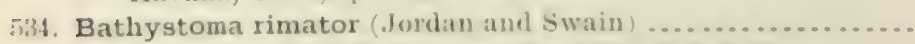
Drawing by II.L. Todel from No. 31957. L.S.N.M., collected by the Albatross at st. Thomas, West Indies.

\section{PIATIE, CCVII.}

535. Bathystoma aurolineatum (' 'uvier and Vnleneiennes) ....... Drawing hy H. L. T'odal from No. 26i67, L.S.N.A., collected lyy Silats Stearns at Key West, Jlorida.

i336. Lythruion opalescens I ordan aud starks.................

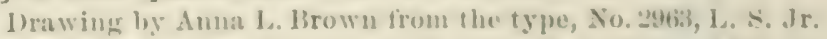
Lniv. Mus., collected by the Hopkins Expedition at Mazatlan, Mexien. 


\section{PLATE CCVIII.}

537. Anisotremus surinamensis (Bloch)

Drawing by A. H. Baldwin from a specimen collected by Evermann and Bean in Indian River at Fort Pierce, Florida.

538. Anisotremus bilineatus (Cuvier and Valencienues).........

Drawing by H. L. Todd from No. 30878, U.S.I.M., collected by Dr. J. A. Henshall in Indian River Inlet, Florida.

\section{PLATE CCIX.}

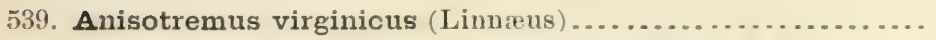

Drawing by H. L. Todd from No.33189, U.S.N.M., collected by Dr. J. A. Henshall at Key West, Florida.

540. Orthopristis reddingi Jordan and Richardson.............

Drawing by Anua L. Brown from the type, No. 3458, L. S. Jr. Univ. Mus., collected bJ J. A. Richardson in La Paz Harbor, Lower California.

\section{PLATE CCX.}

541. Orthopristis chrysopterus (Linneus) ..................

Drawing by H. L. Todd from No. 3113, U.S.N.M., collected at Pensacola, Florida.

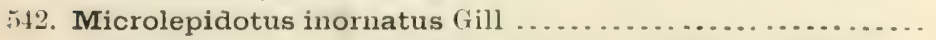

Drawing by A. H. Baldwin, from No. 43267, U.S.N.MI, collected by Evermann and Jenkins at Guaymas, Mexico.

\section{PLATE CCXI.}

543. Otrynter caprinus (Bean)

Drawing by H. L. Todd from No. 32683, U.S.N.M., collected by Silas Stearns at Pensacola, Florida.

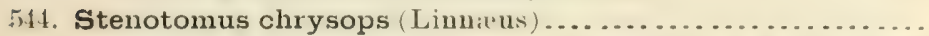

Drawing by H. L. Todd from No. 10425, U.S.X.M., collected by Professor Baird at Woods Hole, Massachusetts.

\section{PLATE CCXII.}

515. Stenotomus aculeatus (Cuvier and Valenciennes) .............. Drawing by H. L. Told from No. 24694, U.S.N.M., collected by R. E. Earll near Charleston, South Carolina.

546. Calamus calamus (Cuvier and Valenciennes) ..............

Drawing by H. L. Todd from No. 35040, U.S.N.M., collected by Dr. Jordan at Key West, Florida.

\section{PLATE CCXIII.}

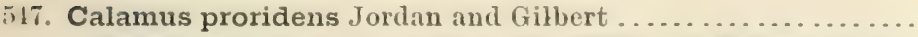

Irawing by H. L. Todh from No. 35056, L'S.X.M., collected by Dr. Jordan at Key West, Florida.

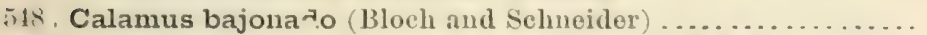
$1: 33 x$

Drawing by H. L. Todd from No. 35042, U.S.N.Mr, collected by Dr. Jordan at Key West, Florida. 


\section{PIATE CCAIV.}

519. Calamus penna (Cuvier and Valenciennes)

Drawing by H. L. Todd from Jo. 6131, L.S.X.M. collected in Charlotte Harbor, Florila.

ninc. Calamus arctifrons Goode and bean.

Drawing by H. L. 'Torld from the type, No. 30163, U.S.N.M., collected by Silas Stearns at P'ensacola, Florida.

\section{P'LATE CCXV.}

551. Pagrus pagrus (Linniens)

Drawing ly II. L. Todd from a specimen collected by Silas Stearns at Pensacola, Floricla.

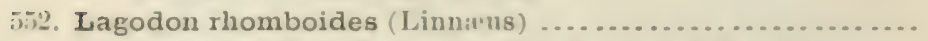

Urawing by II. L. Todd from No. 21:80, U.S.X.M., collected by Dr. Goode in St. Johns River, Florida.

\section{PLATE CCXVI.}

553. Archosargus unimaculatus (Bloch)

1)rawing ly H. L. T'odd from Yo. 35031, L.S.X...., collected by Dr. Jordin at liey West, Florida.

551. Archosargus probatocephalus (Walbaum)

Dr:Lwing by II. L. Todd from Yo. 196t U S y y. collected by Dr. II. C. Yarrow at Beaufort, North Carolina.

\section{PLATE CCXVII.}

5.i. Diplodus holbrookii (Bean)

Drawing by II. I. Todel from No. 32632, C........., collected. at I'ensacola, Floricla.

5... Diplodus holbrookii (Bean)

Drawing by II. L. Todll from a young example, No. 1693 , L.S.X.M.

\section{I'LATE CCXVII.}

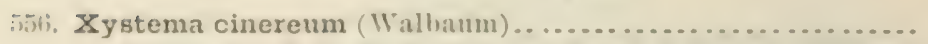

I):awing ly 11. L. Torkl from No. 35051, U.S.S.M., collected ly Inr. Jordan at liey West, florida.

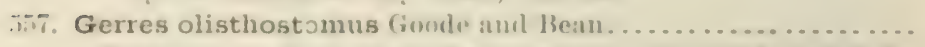

brawing lo H. L. Todt from the type, No, 2.5118, U.S.X.M., rollected ly, R. E. Earll in Indian River, Florida.

\section{PLATE C'XIX.}

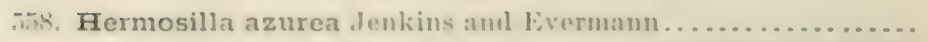

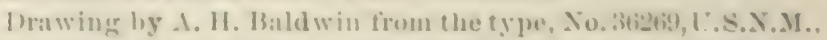
collected by denkins and liverman at (inaymas, Jexico.

5is. Kyphosus sectatrix (1,inn: us) . . . . . . . . . . . . . . .

Drawiner ly II. T. Tould frum Xo. 2(Mi35, U.S.X.M., collected at Woods Hole, Massachusetls. 


\section{PLATE CCXX.}

560. Medialuna californiensís (Steindachner)

Drawing by H. L. Todd.

561. Cynoscion nothus (Holbrook)

Drawing by H. L. Todd from No. 34921, U.S.N.M., collected by the Albatross at Trinidad, West Indies.

5i:. Cynoscion regalis (Bloch and Schneider)

\section{PLATE CCXXI.}

5i3. Cynoscion nebulosus (Cuvier and Valenciennes) ...........

Drawing by H. L. Todd.

5it. Sagenichthys ancylodon (Bloch and Schneider).............

Drawing by A. H. Baldwin from No. 29722, U.S.N.M., collected by Dr. Gilbert at Panama.

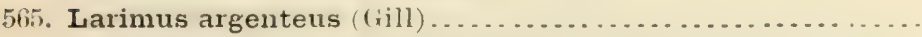

Drawing by Auna L. Brown from a specimen in L. S. Jr. Univ. Mus.

\section{PLATE CCXXII.}

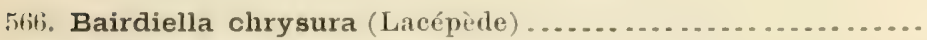

Drawing by H. L. Todd.

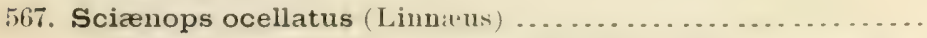

Drawing by H.L. Todd from No.622, U.S.N.M., collected by Dr. Kennerly at Indianola, Texas.

\section{PLATE CCXXIII.}

568. Roncador stearnsi (Steindachner)

Drawing by H. L. Todd from No. 26864, U.S.N.M. . collected bs Dr. Jordan at Santa Barbara, California.

569. Leiostomus xanthurus Lacépède

Drawing by H. L. Todd from No. 20222, U.S.N.M., collected by Samuel Powell at Newport, Rhode Island.

\section{PLATE CCXXIV.}

570. Micropogon undulatus (Linnirns)

Drawing by H. L. Todd from No.20742, L.S.N.M., collected by Samuel Powell at Newport, Rhode Island.

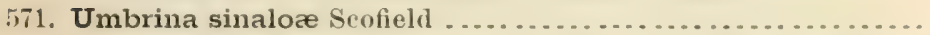

Drawing by Anua L. Brown from the type, No.1632, L.S. Jr. Unir. Mus,, collected by the Hopkms Expedition at Mazatlan, Mexico.

\section{PLATE CCXXY}

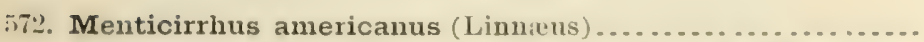

Drawing by H. L. Todd from No. 22832, U.S.N.M., collected by Silas Stearns at Pensacola, Florida.

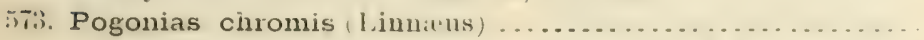
J. C. Willetts at Matanzas River Inlet. Florida. 


\section{PITE: CCXXVI.}

57t. Aplidonotus grumniens liatinesque

$1.1 \times 1$

Drawing l,y H. L. Todd from No.105t2, U.S.N.M., collected l.y J. W. Milner at Ecorse, Michigan.

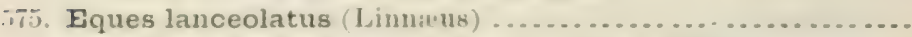

Drawiug by H. L. Todel from No.32097, U.S.X.M., collected by Professor Poey in Cuba.

\section{PLATE CCATVII.}

iir. Cirrhites rivulatus Valenciennes

Drawing by A. H. Baldwin from a specimen collected by the Alliatross in the Pacitic.

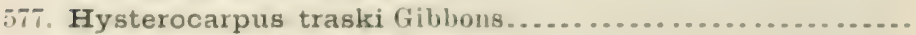
Drawing by J. C. Van Ilook.

\section{PIATE CCXXVIII.}

5it. Abeona minima (Gibbous)

Drawing by A. H. Baldwin from Yo. 26913, U.S.X.M., collected by Dr. Jordan at Sauta Barbara, California.

579. Cymatogaster aggregatus Gibbons................... Drawing by M. M. Smith from No."31971, U.S.X.M., a male, collected at Fort Wrangell, Alaska, by Capt. H. E. Nichols.

$\therefore$. Cymatogaster aggregatus Gibbons...................... Drawing by W. S. Atkinson from a specimen in I. S. Jr. Unir. Mus.

\section{PIATE CCXXIX.}

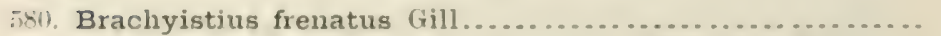

Drawing by A. H. Baldwin from No. 26940, U.S.N.MI., col= lected by Dr. Jordan in Monterey Bay, California.

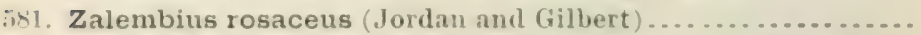
Dratwing ly W. S. Atkinson from a specimen in L. S. Jr. Univ. Mus.

\section{PLATE CCXXX.}

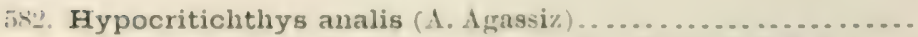
l)rawing by A. H. Baldwin trom No. 27075, U.S.X.M., col. leeted by Dr. Jordan in Monterey Bay, California.

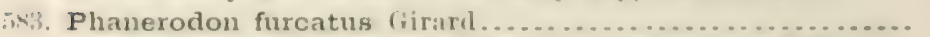

Drawing by A. H. Baldwin from No. 21905 , U.S.X.M., col. lected ly Dr. Jordan at San Diego, California.

\section{PLATE CEXXXI.}

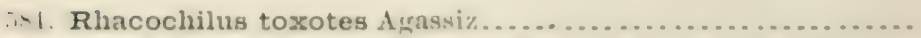

Drawing from No. 27015 , U.S.N.M., collected by Dr. Jordan at Monterey, California.

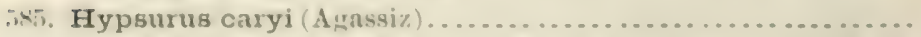

lrawing by $\mathbb{W}$. S. Atkinson from a specimen in L. S. or. Univ. Mus. 


\section{PLATE CCXXXII.}

586. Damalichthys argyrosomus (Girard)

Drawing by H. L. Told from No. 29811, L.S.X.M., collecert by Capt. H. E. Nichols in Friendly ('ove, British ('olumbia.

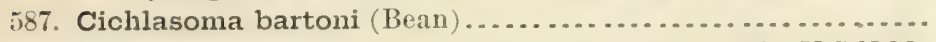

Drawing by A. H. Baldwin from the type, No. 43765 , U.S.N.M., collected by Professor Dugès at Huazteca Potosina, San Luis Potosi, Mexico.

\section{PLATE CCXXXIII.}

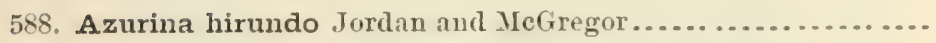
1544

Drawing by Anna L. Brown from the type, collected by R. C. McGregor at Guadalupe Island.

589. Eupomacentrus rectifrenum (Gill) ............... 1557; 3176

Drawing by Anna L. Brown from No. 3460, L. S. Jr. Univ. Mus., collected by the Hopkins Expedition at Mazatlan, Mexico.

\section{PLATE CCXXXIV.}

590. Abudefduf saxatilis (Linn: $u$ ).

Drawing by A. H. Baldwin from No. 33688, U.S.N.M, collected by J. C. Brevoort in the West Indies.

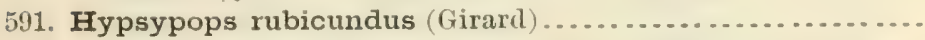

Drawing by W. S. Atkinson from No. 43080, U.S.N.M., collected by Rosa Smith at San Diego, California.

\section{PLATE CCXXXY.}

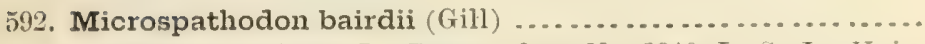
Drawing by Anna L. Brown from No. 2940, L. S. Jr. Unir. Mus., collected by the Hopkins Expedition at Mazatlan, Mexico.

593. Microspathodon chrysurus (Curier and Valenciennes) .....

Drawing by A. H. Baldwin from No. 13042, U.S.X.M., collected by Professor Poey in Cuba.

\section{PLATE CCXXXVI.}

594. Microspathodon dorsalis (Gill)

Drawing by Anna L. Brown from No. 2895, L. S. Jr. Unir, Mus., type of M. azurissimus Jordan and Starks, collected by the Hopkins Expedition at Venados Island, Mazatlan, Mexico.

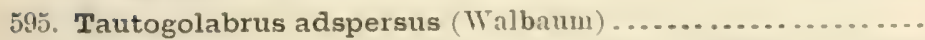

Drawing by H. L. Todd from No. 17745, U.S.X.M., collected by V.N. Edwards at Woods Hole, Massachusetts. 


\section{PIATE CCXXXII.}

Text jate

5\%: Tautoga onitis (Limninus) 1578

Drawing by H. L. Todk from No. 17738, U.S.X.M., collected by

V.N. Eilwards at Wools Hole. Massachusetts.

597. Lachnolaimus maximus (Walbaumm)

Jrawing ly 11. L. Todel.

\section{PLATE CCXXXVIII.}

5is. Pimelometopon pulcher (Ayres)

1) rawing by H. L. Toold from No. 24890, U.S.N.M., collectedl ly. Dr. Jordan at San Diego, California.

5:4. Clepticus parra (Bloch and Schneider)

Drawing lyg A. H. Ballwin from No. 9797, U.S.X.M., collected by P'rofessor Poes in Cuba.

\section{PLATE CCXXXIA}

601). Iridio radiatus (Linnetus)

Drawing by 1I. L. Todd from No. 31168, U.S.X.M., collected at Key West, Florida.

601. Irido bivittatus (Bloch)

Drawing by A. H. Baldwin from No. 35168 , U.S.N.M., collected at key West, klorida.

\section{PIATE CCXI.}

602. Iridio dispilus (Giinther)

Drawing by Anna L. Brown from No. 2904, L. S. Jr. Univ. Dus, collected by the Hopkins Expedition in the Astillero at Mazatlin, Mexico.

60)3. Emmeekia venusta (Jenkins \& Kvormaun)

Drawing by A. II. Baldwin from the trpe, No. 39631, U.S. Y.M. collected by Jenkins \& Evermann at Guaymas, Mexico.

\section{I'LATE CCXLI.}

bi1. Chlorichthys grammaticus (Gillert)

1) rawing by $\Lambda$. H. Balclwin from Mo. 46934 , U.S.X.M., collecterl by the Albatross at Socorro Isiand.

(io). Novaculichthys ventralis (Bean)

Jawine by S. F. Denton from the type, No. 37077 , U.S.X.M. collected lyg Dr. Bean at Cozunel Island.

(iok, Novaculichthys infirmus (Be:an)

Drawing by 11. L. 'Todd from the type, No. 37076, U.S.N.M.. collected lyy Dr. Bean at Cozumel Island.

\section{PLATE COXLH.}

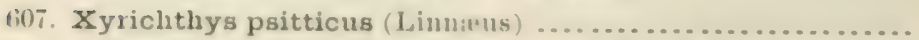

Drawing l,g. II. L. Todd from Xu, ist.5, U.S.X.M., collectedl hy 1)r. Whitelusst at Giarden key, Horidn.

bils. Crytotomus beryllinus (Jordan and Swain)...............

Irawing ly $11 . \mathrm{L}$. Told from a specimen collocted by the .lllutrons. 


\section{PLATE CCXLIII.}

609, 609a. Calotomus xenodon Gilbert . 1626

Drawiugs by A. H. Ballwin from No.46993, C........, collected by the Albatross at Socorro Island.

610. Sparisoma aurofrenatum (Cuvier and Valencienues) ........

Drawing by Anua L. Brown from a specimen in L. S. Jr. Univ. Mus.

\section{PLATE CCXLIV.}

611. Sparisoma hoplomystax (Cope)

Drawing loy H. J. Todel trom No. 3.5173, L.S.X.M., type of S. cyanolene Jordan and Swain, collected by Jordan and Dse at Key West, Florida.

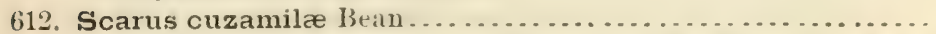
1634

Drawing by S. F. Denton from the type, No.37128, U.S.N.M., collected by Dr. Bean at Cozumel Islaud.

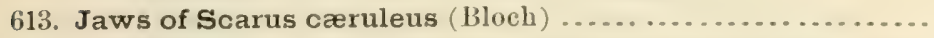

Drawing by Anna L. Brown from a speeimen in L. S.Jr. Univ. Mus.

\section{PLATE CCXLY.}

614. Scarus emblematicus Jordan and Rutter 165

Drawing by W. S. Atkinson from the type in L. S. Jr. Unir. Mus., collected by Rev. J. S. Roberts in Jamaica.

615, 615a. Scarus strongylocephalus

Lower and upper pharyngeal bones. Drawings by Anna L. Brown.

616, 616a. Sparisoma cretense.

Lower and upper pharyngeal bones. Drawings by Anna L. Brown.

\section{PLATE CCXLVI}

617. Pseudoscarus guacamaia (Cuvier)

Drawing by H. L. 'Told.

618. Zenopsis ocellatus (Storer)

Drawing from a specimen in the Boston Society of Natural History.

\section{PLATE CCXLVII.}

619. Chætodipterus faber (Broussonet)

Drawing by H. L. 'Todd from No. 22921, U.S.N.M., from east coast of United States.

\section{PLATE CCXLVIII.}

620. Chatodon nigrirostris ((iill

Drawing los WV. S. Atkinson from No. 5981, L. S. Jr. Univ. Mus., collecterl by R. C. MeGregor at Clarion Island. 
PLATE CCXIAI.

Text page.

(i:)1. Chatodon ocellatus (IJloch)

Drawing by W. \&. Haines. Specimen collected by Dr. Bean at Beesley's P'oint, New Jersey.

\section{PLATE C'L.}

1i:-2. Chatodon capistratus Iinnerus .

Drawing by M. M. Smith from No. 30143, L.S.N.M., received from the P'ublic Museum of Kingston, Janaica.

\section{PLATE CCLI.}

1023. Pomacantluus arcuatus (Linn:eus)

Drawing by H. L. Todd from No. 33197, U.S.N.M., collected by Lewis (i. Mitchell at Barnegat, New Jersey.

\section{PLATE C'CLII.}

1.21. Pomacanthus zonipectus (Gill) .......................

Drawing from No. 31482, U.S.N.M., collected by Kaiser and Martin in West Florida.

\section{PLATE CCLIIL}

(i25. Holacanthus tricolor (Bloch)

brawing by A. I. Baldwin from a specimen collected by Dr. Evermann at Arrogo, P'uerto Rico.

\section{PLATE CCLIY.}

1i26. Angelichthys ciliaris (Linu:eus)

l)awing by A. H. Baldwin from No.300:3, U.S.N.M., received from the P'ublic Musem at Kingston, Jamaica.

(i26u. Angelichthys ciliaris (Linnens), slieleton showing rertebrit rednced in number and enlaryed .......................

Drawing by W. S. Atkinson from a specimen in L. S. Jr. lniv. Mus.

\section{PLATE CCLV.}

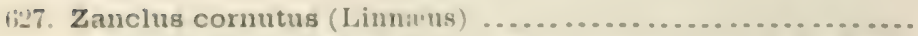

Drawing by W. S. Atkinson from at specimen in L. S. Jr. linis. Mns.

$$
\text { PLATE CCLVI. }
$$

6i2x. Tenthis crestonis Jordan and Starks.

Drawing ly Anna 1. Brown from the type, No. 2sing, 1. S. Ir. Viniv. Mus., collected hy the Hoplins Expectition in the Astillero at Mazatlan, Mexico.

6)

Drawing hy H. L. Todd from No. 3124, U.S.X.M., collected at (iarden lieg, Florida. 


\section{PLATE CCLVII.}

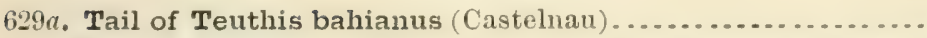
1693

630. Xesurus punctatus (Gill) . . . . . . . . . . . . . . . . . . . . 16.91

Drawing by Anna L. Brown from No. 2911, U.S.N.M., collected by the Hopkins Expedition at Mazatlan, Mexico.

\section{PLATE CCLVIII.}

631. Balistes carolinensis Gmelin

Drawing by H. L. Todd from No. 15233, U.S.N.M., collected at New York.

632. Cantherines carolæ Jordan and MeGregor . . . . . . . . . . . . . .

Drawing by Anna L. Brown from the type, No. 11995, L. S. Jr. Univ. Mus., collected by R. C. MeGregor at Socorro Island.

\section{PLATE CCLIX.}

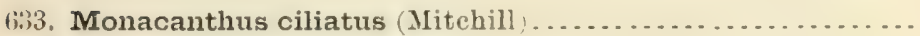

Drawing by W. S. Haines.

6i34. Monacanthus hispidus (Linuitus)

Drawing by W. S. Haines.

\section{PLATE CCLX}

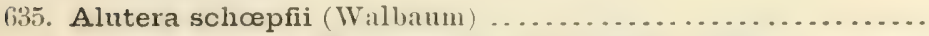

Drawing by IV. S. Haines from No. 6068, U.S.N.M., collected by Judge Steele at Cedar Keys, Florida.

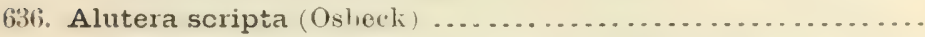

Drawing by W.S. Haines from No. 34397, U.S.N.M., collected by V. N. Edwards at IVoods Hole, Massachusetts.

\section{PLATE CCLXI.}

637. Lactophrys triqueter (Limnitus) . . . . . . . . . . . . . . . . .

Drawiug by W. S. Haines from No. 5989, U.S.N.M., collected at Garden Key, Florida, by Dr. Whitehurst.

638. Lactoplurys tricornis (Linuitus)

Drawing by W.S. Haines from No. 21518, U.S.N.M., collected by C. C. Leslie at Charleston, South Carolina.

\section{PLATE CCLXII.}

$639,639 a, 639 b$. Lactophrys bicaudalis (Limnens) .............. Drawings by H. L. Toda from No.37130, U.S.N.M., collected at Cozumel Island by the Albatross; $640 a$, ventral riew; 610b, front view.

\section{PLATE CCLXIII.}

$640,610 a$. Lactophrys trigonus (Limuens) ................... Drawing by H. L. Todd from No. 7987, U.S.X.M., collected at Garden Key, Florida, by Dr. Whitelurst.

641. Lagocephalus levigatus (Linn:eus) . . . . . . . . . . . . . . .

Drawing by H. L. Todd from No. 35135, U.S.N.M. 


\section{PIAT: CCISIV.}

612. Spheroides mephelus Gookle and Bean ....................

Drawing by H. L. Todel from Yo. 3112x, U.S.X.M., the type. collected ly R. E. Larll in Indian River, Florida.

(il3. Spheroides spengleri (Bloch) ........................

Drawing from No. 20608, C.S.X.M., collected by V. X. Eilwards at Woods Hole, Massachusetts.

611. Spheroides maculatus (Bloch and Schneider) ..............

Drawing by W. S. Haines from No. 14827, U.S.N.M., collected by the L. S. Fish Commission at Joank, Connecticut.

\section{PIATE CCIXY.}

615, 615a. Spheroides testudineus (Linniens) .................

Drawings by H. L. Fould from No. 30060, U.S.X.M., receivel from the museum at Kingston, Jamaica.

6il6. Ovoides setosus (Rosa smith) ..........................

Drawing by W. S. Atkinson from No.319, L. S. Jr. Univ. Mus., collected bs the Albutross at Clarion Island.

\section{PLA'TE CCIXIVI.}

617. Diodon hystrix Linnat18.

Drawing by 1H. L. Todel from No.5985, U.S.X.M., collected by II. E. Wright at 'Tortugas Islands.

6is. Chilomycterus schœpfi (Walbaum)

Drawing from a specimen collected at Joank, Connecticut.

\section{PIALE CCLAVII.}

619. Lyosplıara globosa Evermamn and Kendall.

Drawing he A. H. Jaldwin from the type, No. 48794 , U.S.N. M. collected by W. C. Kendall in the liappahanuock Rirer. Jirginia.

6i!t!. Lyosphara globosa livermann and liendall.

1)rawing by A. II. Baldwin from the cotype, a younce example collected ly Evermann and Kondall at Cape Florida. Finricla.

(ii) (?. Mola mola (limuinus)

Jrawing taken from a washed drawisg taken from a cast.

P'ATE: CCIXVIII.

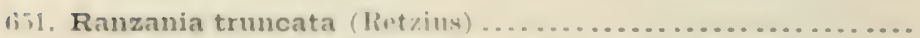

1)rawing from a specimon collected in the Bermulas.

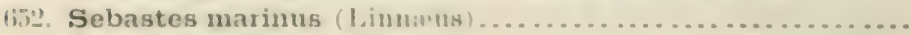

1) rawing loy 11. L. Tould. 


\section{PLATE CCLAIX.}

(i.).3. Sebastolobus altivelis Gilbert

Drawing by A. H. Baldwin from the t5pe collected by the Albatross at Station 3338, south of the Alaskan Peninsula, in 625 fathoms.

654. Sebastodes melanops (Girari) . . . . . . . . . . . . . . . . . . .

Drawing by H. L. Torld from No. 27628, U.S.N.M., collected by. Dr. Bean at Sitka, Alaska.

\section{PLATE CCLXX.}

(6.). Sebastodes ciliatus (Tilesins)

Drawing by H.L. Todd from No. 32014, U.S.N.M., collected in Tolstoi Bay, Alaska, by Capt. H. E. Nichols.

(is). Sebastodes mystinus (Jorlan and Gilbert) .................

Drawing by H. L. Todd from No. 27031, U.S.N.M., collected by Dr. Jordan at Monterey, Califoruia.

\section{PLATE CCLXXI.}

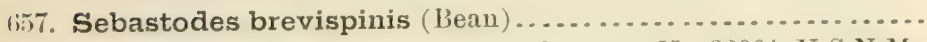
Drawing by W. S. Haines from the type, No. 32001, U.S.N.M., collected by Capt. H. E. Nichols in Hassler Harbor, Alaska.

658. Sebastodes eigenmanni Cramer ... . . . . . . . . . . . . . . . .

Drawing by Anna L. Brown from the type, No.4016, L. S. Jr. Univ. Mus., collected by Dr. W. W. Thoburn at Pacific Grove, California.

\section{PLATE CCLXXII}

(15)!. Sebastodes hopkinsi Cramer.........................

Drawing by Anna L. Brown from the trpe, No. 2282, L. S. Jr. Unir. Mus., collected by Gilbert and Starks at Pacific Grove, Califoruia.

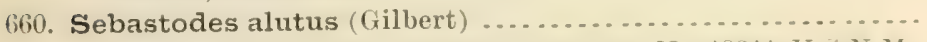
Drawing by A. H. Baldwin from the type, No.48244, U.S.X.M., collected by the Albutross at Station 3339, Sauta Barbara Islands.

\section{PLATE CCLNXIII.}

661. Sebastodes pinniger (Gill)

Drawing by II. L. Todd from No, 27488, U.S.X.M., collected by J.G. Swan at Teah Bay, Washington.

(iti:. Sebastodes miniatus (Jordan and Gilbert)

Drawiug by W. S. Atkinson from a specimen in L. S. Jr. Univ. Mus., showing vertebre in moderate number, relatively increased in size. 
PI.ITE CCLXXIN.

Text patere. $1 \times 211$

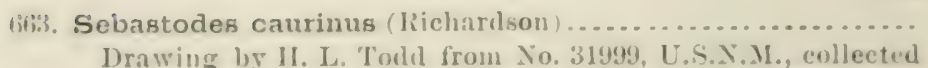
by Cayt. H. E. Nichols in Departure Bay, British Columbia.

fiti. Sebastodes maliger (Jortan and Gilhert) ............... 18:2

Drawing by II. L. Todd from No. 27T13. U.S.N.M., collected liy Dr. Bean at Sitka, Aliska.

\section{PLATE CCLXXY}

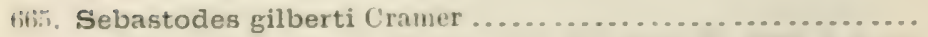

Drawing by Anna L. Brown from the trpe, ㅅo. 3893, L. S.Jr. Luiv. Mus, collected by Ir. Jorlan in tho San Francisco market.

fik6. Sebastodes serriceps (Jordan and (iilbert)................

Drawing ly 11. L. Todd from No. 27041, U.S.N.M., collected by Dr. Jordan at Monterey, California.

PLATE CCLXXVI.

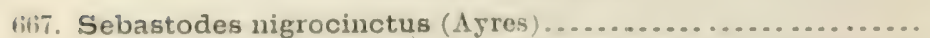
Drawing by I. L. Todd from No. 27285 , U.S.X.M., collected ly. Dr. Jordan in Puget Sound.

668. Scorpæna cristulata Goodle and Bean...................

Drawing by H. L. Todd from the type, Ao. 39326, U.S.N.M., collected by the Albatross at Station 2415 , in X. lat. $30^{2} 44^{\prime}$, IV. loug. $79^{\circ} 26^{\prime}$, off Georgia, in 440 fathoms.

\section{PLATE CCLXXYII.}

669. Scorpæena brasiliensig Cuvier and Valenciennes ............ Drawing by H. L. Todel from No. 30169, U.S.N.M., collected by Silas Stearns at P'ensacola, Floricla.

670. Scorpæena mystes Jordan and Starki...................

Drawing by Anna L. Brown from the type, No. 2919, L. S. Jr. Iniv. Mns., collected hy the Hopkins Expedition at Mazat. lan, Mexico.

\section{PLATE, CCIAXYIII.}

171. Scorpana grandicornis Curier and $\mathrm{V}$ alenciennes.......... Drawing by II. L. Todd from Xo. 35101, U.S.X.M., collected Wy Dr. Jordan at Koy West, Floricla.

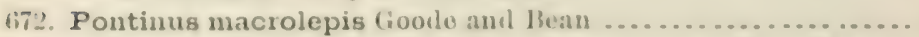

Drawing by A. H. Ballwin from a specimen collected by the Bluke off IBarbalos.

\section{PIATE C'IAXIX.}

673. Setarches parmatus Goode

l)rawimg by H. L. Toth from a specimen collected by the Allatrose at Station 23:34. 
674. Anoplopoma fimbria (Pallas)

Drawing by H. L. Todd.

675. Pleurogrammus monopterygius (Pallas)

Drawing by A. H. Baldwin from a specimen collented by Dr. Evermann at Atka Island, Alaska.

\section{PLATE CCLAXX.}

676. Hexagrammos decagrammus (Pallas) .................. . .

Drawing by W. S. Haines from No.27710, U.S.N.M., collected at Old Sitka by L. A. Beardslee.

676a. Skeleton of Hexagrammos decagrammus

A mail-cheeked fish from northern waters, showing vertebrie increased in number and diminisled in size. Drawing by W. S. Atkinson from a specimen in L. S. Jr. Univ. Mus.

677. Hexagrammos octogrammus (Pallas) 1869

Drawing by W. S. Haines from No. 27975 , U.S.N.M., collected at Unalaska by Dall and Bean.

\section{PLATI CCLXXXI.}

678. Hexagrammos stelleri Tilesius .........................

Drawing by H. L. Todd from No. 21530, U.S.N.M., collected at St. Michaels, Alaska, by L. M. Turner.

679. Hexagrammos superciliosus (Pallas)

Drawing by M. M. Smith from No.2793t, U.S.N.M., collected at Chernoff'sky, Unalaska Island, by ' $\mathrm{T}$. H. Bean.

680. Hexagrammos otakii Jordan and Starks ..... . . . . . . . . . . Drawing by Anna L. Brown from the type, No. 3704, L. S. Jr. Univ. Mus, collected by Keinosuke Otaki at Tokio, Japan.

PLATE CCLXXXII.

681. Ophidion elongatus Girard

1875

. Drawing by H. L. Todd from No. 27657, U.S.N.M., collected by Dr. Bean at Sitka, Alaska.

68:. Oxylebius pictus Gill . . . . . . . . . . . . . . . . . . . . .

Drawing by Anua L. Brown from No. 2381, L. S. Jr. Unir. Mus, collected by the California Fish Commission at san Franciseo, Califoruia.

683. Jordania zonope Starks

Drawing by Anna L. Brown from the type, No. 3124, L. S. Jr. Univ. Mus, collected by Miss Maudo Parker and Adam Hubbert at Point Orchard, Puget Sound, Washiugton.

\section{PLATE CCLXXXIII.}

684. Alcidea thoburmi (Gilbert) .........................

Drawing by A. H. Baldwin from a specimen collected by the Albatross at Station 3350.

685. Skull of Scorpæenichthys marmoratus (Ayres) 
i-1; Chitonotus pugetensis (Steiudachuer).

$18(4)$

1) rawing ly A. II. Baldwin from No. 27238. U.S.N.M. coulleetod loy lor. Jordan in the strats of Juan de Fuea, Washiugton.

\section{PIATE CCIAXXIV}

ii- Icelintus borealis (iilhert

Drawing hy A. H. Baldwin from the type collected by the Allulrows at station :322:3.

likx. Astrolytes motospilotus (Girard).

1899

Drawing hy A. II. Baldwin from No. 27146, I.S.X.M., collected b.5 Dr. Jordan in l'uget Sound.

lis8a. Astrolytes notospilotus (Girarl) . . . . . . . . . . . . . . . .

Drawing from No. 23936 , L.S.X.11., a young example, collected l,y W. II. Dall in the Shumagin Islauds.

\section{PIATE CCIAXXV.}

livis. Artediellus atlanticus Jorkan and Evermann .............. Drawing by H. L. Todk from tho type, No. 21069, U.S.X.M., collected in Massachusetts Bay in 90 tathous.

(i) Ruscarius meanyi Jorkan and Starks ... . . . . . . . . . . . . .

1)rawing ly Annat L. Brown from the trpe, No.3127, L. S. Jr. Univ. Mus., collected ly E. C. Starks at Point Orchard, Puget Sound, Washington.

PLATE CCLXXXVI.

(i!). Rastrinus scutigex (Bean).

Drawing by W.S. Atkinson from a specimen in 1.. S. Jr. Univ. IIus.

1i!12. Icelus spiniger Gilbert

I) rawing by A. II. Bablwin from the rype, collected by the Albatross at Station:3:78.

(ii). Icelus canaliculatus filliert

I) rawing hy A. H. Baldwin from the type, collected by the Albutrose at station 3309, ofl' L'nalaska.

\section{PLATE CCISXXXII.}

(is!). Radulinus boleoides (ijlbert

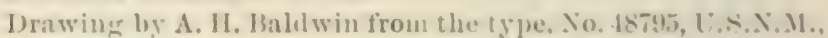
colloctol hy the Albatross at station 36ill, ofl santa C'atalina [sland, Calitornia, in in! fathous.

1i!.i. Raduliums asprellus (iilbert

Drawing ly Anua 1. Brown frou No. :37s1, L. s. Ir. Univ. Mus., collected hy E. ( . Starlss in l'uret Somud, near Seattle. IIashington.

1i!ti. Triglops pingeli lieiuharalt .......................... Drawing hy 11. I. Tould from a specismu taken in trawl \&

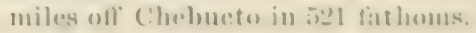




\section{PLATE CCLXXXVII.}

697. Triglops beani Gilhert.

Drawing by H. L. Todd from the type, collected lyg the Albutross at Station 3220.

4i:8. Triglops scepticus (iilhert

Drawing by A. H. Baldwin from the type, collecterl by the Albatross at Station 3339.

699. Sternias xenostethus (Gilbert)

Drawing by A. H. Baldwin from the type, collected by the Albatross at Station 3220 , north of Unalaska, in $3 \pm$ fathoms.

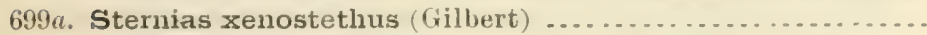

Drawing by $\mathrm{A}$. H. Baldwin from the type. co lected lyy the Albatross at Station 3220 , north of Unalaska, in 3 f fathoms.

\section{PLATE CCLAXXIX.}

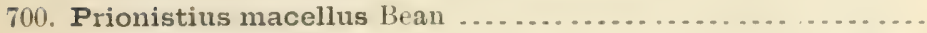

Drawing by H. L. Torld from the type, No. 31958, U.S.N.M., collected by Capt. H. E. Nichols in Carter Bay, British Columbia.

701. Elanura forficata Gilbert

Drawing br Anna L. Brown from the type, collected by the Albatross at Station 3214 , in the Aleutian Islauds.

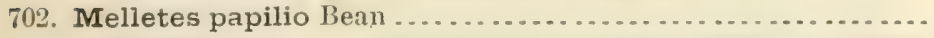

Drawing by H. L. Todd from the type, No. 23751, U.S.N.M., collected by H. W. Elliott at St. Paul Island, Pribilof Group, Bering Sea.

\section{PLATE CCXC.}

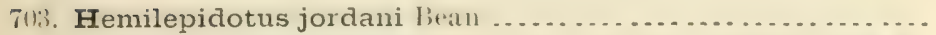

Drawing by H. L. Todd from the type, No. 27598, U.S.N.M., collected by Sylvanus Bailey at Unalaska.

701, 704a, 704b. Hemilepidotus hemilepidotus (T'ilesins) .......

Drawings by S. F. Denton from No. 27609, U.S.X.M., collected at Sitka by T. H. Bean.

\section{PLATE CCICI}

705. Enophrys bison (Girard

Drawing by H. L. Todd from a specimen collected by Dr. Jordan in Puget Sound.

706. Ceratocottus diceraus (Pallas)..

Drawing by W.S. Haines, from No. 32007, U.S.N.M., collected by Capt. H. E. Nichols iu 'Tolstoi Bay, Alaska.

\section{PLATE CCXCII.}

7)7. Cottus evermanni (illbert

Drawing by Chloe Lesley Starks from the trpe, No. 48228 , U.S.N.M., collected by Gilbert, Cramer, and Otaki in Lost River, near Lostine, Oregon.

Bull. No. 47, pt. $4-$ XVII 
708. Cottıs punctulatus $($ Gill ) ........................... 1948

Drawing by S. F. Donton from a specinets collected in Yel. lowstone Park by 11. Jordau.

\section{PLATE CCXCIII.}

709. Cottus perplexus Gilliert and Evirmann ..................

Drawing liy A. H. Baldwin from the type, No. 45387, L.S.X.M., collected by 10rs. Gilbert and Jenkins in tho Skvokumchuck River, near Chehulis, Washington.

710. Cottus klamathensis Gilhert ........................

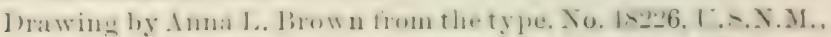

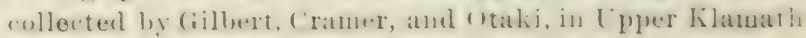
Lake, Oregon.

711. Cottus aleuticus Gilbert

Drawing from No. 2692.), U.S.X.M., the tspe of Craniden microstoma Lockingtou, collecterl by $\mathrm{W}$. J. Fischer at st. Paul, Kadiak Islaud, Alaska.

\section{PIATE CCXCIV.}

712. Cottus leiopomus Gilbert and Evermann .................

Drawing ly A. II. Baldwin from the type, Xo. 45389, U.S.X.M., collected l,s H. II. Kinsey in Little Wood River, near Sho. shone, Idiho.

713. Cottus princeps Gilbert............................

Drawing by Anna L. Brown from the type, No. 48227, L.S.X.M., collected by Gilbert, Cramer, and Otaki in Upper Klanath Lake, Oregon.

714. Uranidea temuis Evormann and Ileek....................

Drawing l,y $\mathbf{A}$. H. Baldwin from the type, No. 182.29, U.S. I.M., collected by Meeli and Aloxander in Upper Kilamath Latie, Oreiron.

\section{PLATE CCACV.}

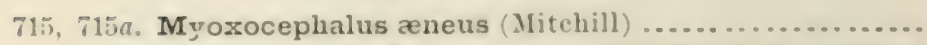

1)rawings by H. L. Toll from No. 15093, L.S.X.M., collected by Mr. Copley at 'lompkinsville, Jew York.

716. Myoxocephalus scorpius (Linnitus) ....................

Drawing l.y II. L. 'Todd from No. 21989, U.S.N.M., collected by 1. Kumlein in C'muluerland cinlf.

\section{PLATE CCXCVI.}

717. Myoxocephalus octodecimspinosus (Mitehill)

Drawing hy II. 1. Tould from No. 1552, L.S.X.M., collecterl hy Professor liaird at Beesley l'oint, Now Jorsey.

718. Myoxocephalus polyacanthocephalus l'allas ................ Drawing loy H. I. Tould from Xo, 23499, L.S.X.M., collected by W. H. Dall at lualaska, Alaska.

719. Myoxocephalus jaok (Cuvier and Valeneiennes)...........

D):แwing by II. L. Tould from No. 21522.2, U.S.X.M., collected loy L. M. Turnor at St. Michaels, Alaska. 


\section{PLATE CCXCVII.}

720, 720a. Myoxocephalus verrucosus (Bean)

Text page.

Drawing by H. L. Todd from No. 27847, U.S.N.M., collected by Dall and Bean in Plover Bay, Alaska.

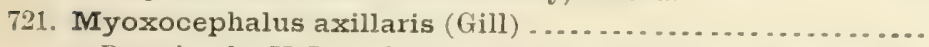

Drawing by H. L. Todd from No. 32442, U.S.X.M., collected by U. S. Signal Service Point Barrow Expedition at Bering Straits, Alaska.

\section{PLATE CCXCVIII.}

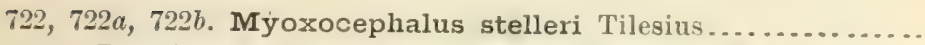
Drawings by H. L. Todd from No. 32442, U.S.N.M., collected by U. S. Signal Service at Bering Straits, Alaska.

723. Myozocephalus niger (Bean ) . . . . . . . . . . . . . . . . . . .

Drawing by H. L. Todd from the type, No. 27971, U.S.N.If., collected by Dr. Bean at St. Pail Islaud, Bering Sea.

\section{PLATE CCXCIX.}

724, 724a. Megalocottus platycephalus (Pallas)..............

Drawings by A. H. Baldwin from No. 21522, U.S.N.M., collected at St. Michaels, Alaska, by L. M. Turner.

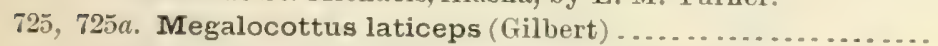

Drawings by $\mathrm{A}$. H. Baldwin from the type, collected by the Albatross in the Nushagak River, Alaska.

\section{PLATE CCC.}

726. Zesticelus profundorum (Gilbert)

Drawing by A. H. Baldwin from the type, collecterl by the Albatross at Station 3329 in Bering Sea, north of Unalaska.

727. Dasycottus setiger Beau.

Drawing by Anna L. Brown from No. 3761, L. S. Jr. Univ. Mus, collected by E. C. Starks in Puget Sound, near Seattle, Washingtou.

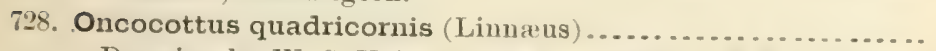

Drawing by W. S. Haines from No. 32962, U.S.N.M. collected by E. W. Nelson at St. Michaels, Alaska.

\section{PLATE CCCI.}

729, 729a, 729b. Gymmocanthus pistilliger (Pallas) ................

Drawings by H. L. Todd from No. 21743, U.S.N.M. collected at Niantilik Harbor, Cumberland Gulf, by Ludwig Kumlien.

730. Gymmocanthus galeatus Bean

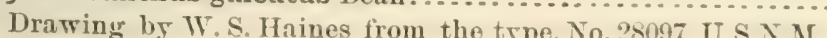
1990 collected by Dr. Bean at Unalaska, Mlaska. 


\section{PLATE CCCII.}

731. Leiocottus hirundo Girard

Drawing by A. H. Baldwin from No. 313, U.S.X.M., collected by IV. P. 'Trowbrillge at San Miguel, California.

732. Leptocottus armatus (iraral

Drawing ly H. L. Todd from No. 27968 , U.S.X.M., collected at Sitka, Alaska, by Dr. Bean.

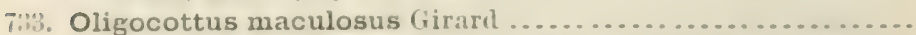

Drawing by H. L. Todd from No. 227514, U.N.X.M., collected hy Dr. Boan at Sitka, Alaska.

\section{PLATE CCCII}

734. Blennicottus embryum (Jorlan and Starks) $2016 ; 2861$.

Drawing by Anma I. Brown from the type, No.3128, L. S. Jr. Univ, Mus., collected by E. C. Starks, Jeah Bay, Washington.

73\%. Histiocottus bilobus (Cuvier and Valenciennes) ............ Drawing by W. S. Haines from No. 30307, U.S.N.M., collected by W.J. Fischer at St. Panl, Kadiak Island, Alaska.

\section{PLATE CCCIV.}

736. Blepsias cirrhosus (Pallas)

Drawing by A. H. Baldwin from No. 27118 , L.S.N.M., collected hy Dr. Jordan 11 the Straits of Fuca.

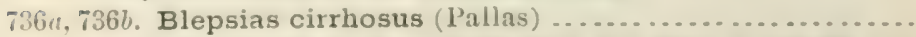

Drawings by H. L. Todd from No. 30307 , U.S.S.M., collected by W. J. Fischer at st. Paul, Katiak Island, Alaskat.

\section{PIATE CCCY.}

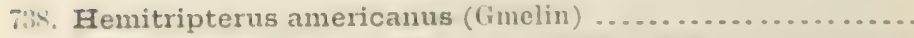
Drawing by II. L. Todd from Xo. 23199, U.S.N.M., collected by the U. S. Fish Commission at Halifax, Nova seotia, in 16 titthoms.

739. Ascelichthys rhodorus Jordan and (iilhert................

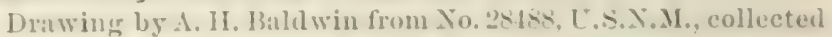
by J.G. Swan at Neah Bay, Washingtnu.

\section{PLATE CCCVI.}

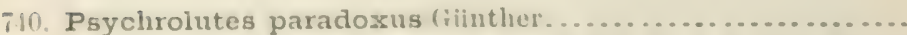

Drawing loy Inna 1. Brown from No. 3337, L. s. Jr. Univ. Mus., collected lig l:. (C. Starks in l'uget somud near Point ()rchard, Washingtun.

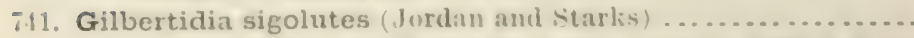
1) rawing by Anna I. Brown from the type. Xo, 3129, L. S. J

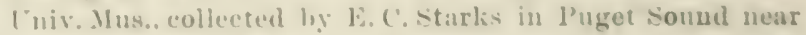
P'oint Grelaral, II:ablington. 


\section{PLATE CCCVII.}

742, 742a. Rhamphocottus richardsoni Giinther

Drawings by H. L. Todd from a specimen collected by

W. N. Lockington, in California.

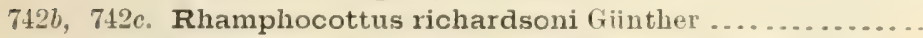

Drawings by Anna L. B̈rown from No. 3385, L. S. Jr. Unir. Mus., collected by E. C. Starks in Puget Sound near Point Orchard, Washington.

\section{PLATE CCCVIII.}

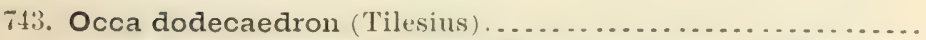

Drawing by Arina L. Brown from No. 14936, U.S.N.M., collected by Gordon Prince in Kamchatka.

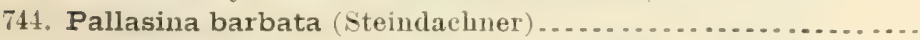

Drawing by H. L. Todd from No. 28052, U.S.N.M., collected at Port Mulgrare, Alaska, by Dr. Bean.

$745,745 a$. Podothecus accipiter Jordan and Starks .............

Drawing by Anna L. Brown from the type, No. 3835,

L. S. Jr. Univ. Mus, collected by Capt. J. C. Blair at Robben Island.

\section{PLATE CCCIX.}

746. Podothecus acipenserinus (Tilesius) ....................

Drawing by M. M. Smith from No. 32481, U.S.N.M., collected by W. J. Fischer at Kadiak Island, Alaska.

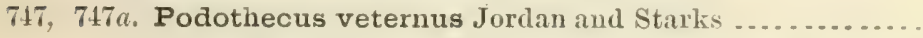

Drawing by Anna L. Brown from the type, No. 4823, L. S. Jr. Unir. Mus., collected by Capt. J. C. Blair at Robben Island.

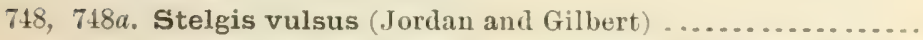

Drawing by Auna L. Brown from the type in the U.S.N.M., collected by Jordan and Gilbert at Point Reyes, California.

$749,749 a$. Averruncus emmelane Jordan and Starks..........

Drawing by Anna L. Brown from the type, No. 3135, L. S. Jr. Univ. Mus, collected by E. C. Starks in Puget Sound near Point Orchard, Washington.

\section{PLATE CCCX.}

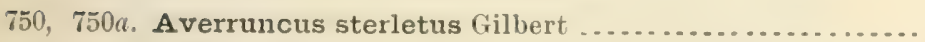

Drawings by Auna L. Brown from the trpe in the U.S.N.M., collected by the Albatross at Station 3662 , oft' Avalon, Coronado Island, California, in 47 fathoms.

751. Sarritor frenatus (Crilbert

Drawing by A. I. Baldwin from the type collected by the Albatross at Station 3229. 
752, 752a. Xystes axinophrys Jordan and starlis ...

Drawings by Anua L. Brown from the type, No. 3130, L. S. Jr. Lniv. Mus., collected l,y E. C. Starks in Puget Sound near seattle, Washington.

\section{PLATE CCCXI.}

753. Bathygonus nigripinnis Gilbert

Drawing by A. II. Baldwin from Lo. 4h614, C.S.X.M., col. lected hy the Albutross in the Aleutian Islands.

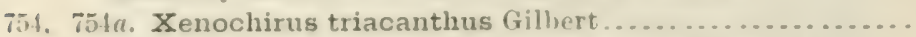

Drawings by Anna L. Brown frou No. 3760, L. S. Jr.

Univ. Mus,, collected l,y E. C. Starks in Puget

Sound near Seattle, Washington.

PLATE CCCXII.

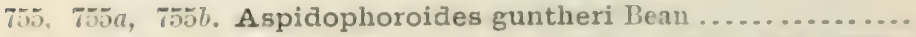

Drawings by W. S. Haines from Yo. 3703:, U.S.N.M., collected by G. M. stoney in Alaska.

756, 756a. Aspidophoroides monopterygius (Bloch) ...........

Drawings by H. L. Todd from No. 21761, U.S.X.M., collected hy the U.S. Fish Commission at Sandwich Point, Halifax, Nova Scotia, in 18 fathoms.

\section{PLATE CCCXIII.}

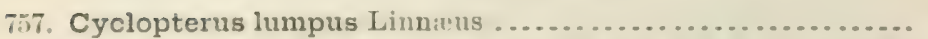

Draming by H. L. Tod, from No. 14795, U.S.X.M., collected by the U.S. Fish Commission at Eastport, Matiue.

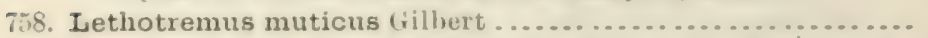

Drawing by A. H. Baldwin from the trpe, collected by the Albatross at station 3223, in Unimak l'ass, Alaskat.

\section{PLATE CCCXIY.}

759. Lethotremus vinolentus Jordan and Starks...............

Drawing by Anna L. Brown from the tỵe, No.3131, L. S.Jr. Univ. Mus, collected by L.C. Starks in P'uget somul, near Seattle, Washington.

760, 760a. Neoliparis callyodon (l'allas)

2096

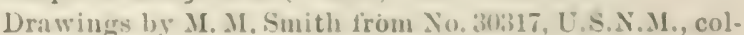
leeted by W. J. Fiseher at St. P'aul, Katiak Island, Alaska.

\section{PLATE CCCAV.}

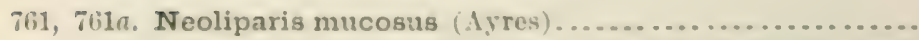

Drawings loy Anna L. Brown fron No, : sci., collector by H. D. 1)unn ofl san Francisco, Califurnia. 
762. Neoliparis florae Jordan and Starks

Drawing by Anua L. Brown from the type, No.3126, L. S. Jx. Univ. Mus., collected by E. C. Starks at Waadda Island, Neah Bay, Washiugton.

\section{PLATE CCCXVI.}

763, 763 . Neoliparis greeni Jordan and Starks. . . . . . . . . . . . .

Drawings by Anna L. Brown from the type, No. 3019, L. S. Jr. Univ. Mus., collected by A. N. Green in Victoria Harbor, Victoria, British Columbia.

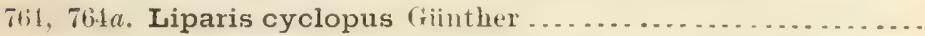
Drawings by H. L. Told from No. 24007, U.S.N.M., collected at Port Muller, Alaska, by W. H. Dall.

PLATE CCCXVII.

765. Liparis agassizii Putnam

Drawing by H. L. Todd from one of the types of $L$. gibbus Bean, No. 24047, U.S.N.M., collected at St. Paul Island, Bering Sea, by H. W. Elliott.

766, 766

Drawings by Anna L. Brown from the type, No.3703, L. S. Jr. Univ, Mus., collected by E. C. Starks in Admiralty Inlet, near Seattle, Washington.

\section{PLATE CCCXVIII}

767. Bathyphasma ovigerum Gilbert

Drawing by A. H. Baldwin from the type, No. 48622 , U.S.N.M., collected by the Albatross at Station 3342, off Queen Charlotte Island, in 1,588 fathous.

Tix. Prionotus carolinus (Limirus) . . . . . . . . . . . . . . . . . . .

Drawing by H. L. Todd from No. 774, U.S.N.M., collected by Professor Baird at Beesleys Point, New Jersey.

\section{PLATE CCCXIX}

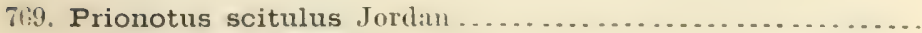

Draming by H. L. Todd from a specimen collected by G. Wiuxdemann in Florida.

770. Prionotus alatus Goode and Bean

Drawing from the type, collected in deep water oft Charleston, South Caroliua.

\section{PLA'TE CCCXX.}

771. Prionotus stearnsi Jordan and Swain

Drawing from a specimon obtained on the Pensacola Snapper Banks.

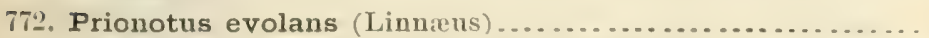

Drawing by H. L. Todel from No. 3556 , L.S.N.M., collected h.y Professor Baird at Wools Hole, Massachusetts. 


\section{PLATE CCCXXI}

773. Bellator egretta (Goorle and Beau)

I)rawing ly M. M. Suith from the type in the M. C. Z., collected by the Blake at Station Lxr, oft Barbados.

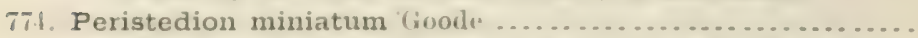

Drawing ly II. I. Todd from the ty pe, No. 26023, U.S.N. M., collected by the Fish II anck at Station 869 , in N. lat. $10^{\circ} 02^{\prime \prime} 18^{\prime \prime}$, W. loug. $70^{\circ}-23^{\prime} 06^{\prime \prime}$, in 192 fathons.

755. Peristedion longispathum Goole and bean ...............

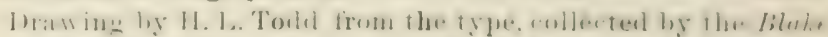
at Station Lvin, oft Havana, in 212 fathoms.

\section{PIATE CCCXXII.}

776. Peristedion gracile Goode and Beas

Drawing by II. L. Todd from the type, collecterl by the Albatross at Station 2401, in N. lat. $28^{\circ} 38^{\prime} 30^{\prime \prime}$, W. loner. $85^{\circ} 52^{\prime} 30^{\prime \prime}$, in 142 fathoms.

777. 777a. Peristedion platycephalum Goode and Bean......... Drawings by H. L. T'odd from the type, collected by the Blake at Station Lx, oft' Barbalos, in 123 fiathoms.

\section{PLATE CCCXXIII.}

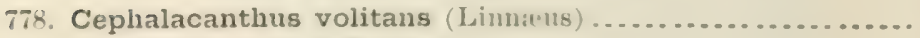
Drawing ly W. S. Haines.

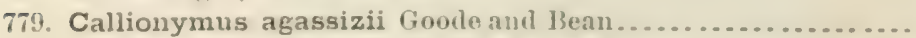
Drawing by H. L. Todd from a specimen collected br the Blake at Station $\mathrm{x} x \mathrm{x}$, off Jarbados, in 209 fathoms.

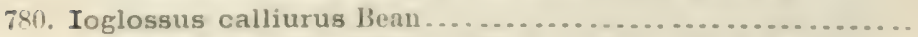
Drawing from No.30797, U.S.X.M., collerted by Silas Stearns at the Pensacola Snapper Baulss.

\section{I'LATE CCCXXIV.}

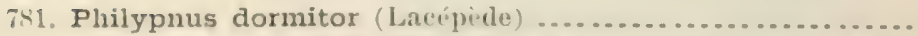
Drawing ly A. Il. Baldwin from a specimen collected by Dr. Evermann at Palo Seco, Puerto Rico.

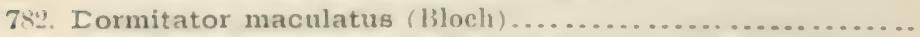
lorawing ly A. H. Baldwin from a speeimen collected by Dr. Evermann at Hucares, Puerto lico.

PLATE CCCXXY.

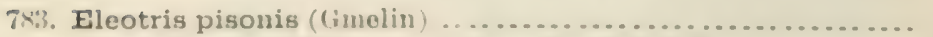

I) rawing loy A. H. Baldwin from Xo, 5757. U.S.N.M., collected hy Dr. Whitehurst at Garten kiey, Florida.

781. Alexurus armiger Jurdau and lichardson................. Drawing by Anna 1. Brown from the type, Xo. 3155, L. S. Ir.

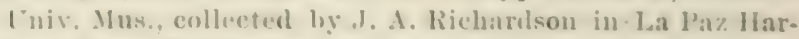
bur, lower ('alifornia.

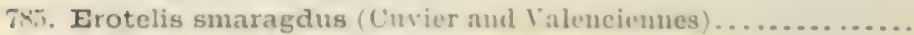
$215: 3$ $\because 186 ;$ (1) Drawing ly A. H. Baldwin. 


\section{PLATE CCCXXVI}

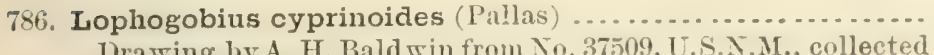

Orawing by A. H. Bald win from No. 37509, U.S.X.M., collected by Professor Poey in Cuba.

787. Gobius stigmaticus (Poeg) ............................................. collected
Drawing by W. S. Haines from No. 30469, U.S.N.M. by Silas Stearns in south Florida.

788. Gobius hastatus Girard

Drawing b5 H. L. Todd from No.35155, U.S.N.M., collected by Dr. Jordan at Ḱey West, Florida.

\section{PLATE CCCXXVII.}

789. Gobius oceamicus Pallas

Drawing by A. H. Bald win from No. 35155, U.S.N.M., collected by Dr. Jordan at Key West, Florida.

7x!! G. Gobius oceanicus Pallas

Drawing by A. H. Baldwin from a specimen collecterl by Dr. Evermaun at Palo Seco, Puerto Rico.

790. Garmannia paradoxa (Giinther)

Drawing by Anua L. Brown from No. 3765, L. S. Jr. Unir Mus., collected by the Hopkins Expedition at Mazatlan, Mexico.

\section{PLATE CCCXIVIII.}

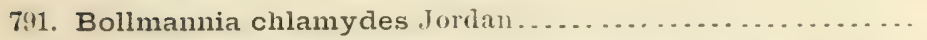

Drawing by W. S. Atkinsou from a specimen in L. S. Jr. Univ. Mus., collected by the Albatross oft the coast of Colombia.

792. Aboma etheostoma Jordan and Starks .................. . .

Drawing by Anna L. Brown from the type, No. 3459 , L. S. Jr. Univ. Mus., collected by the Hopkins Experlition in the Astillero at Mazatlan, Mexico.

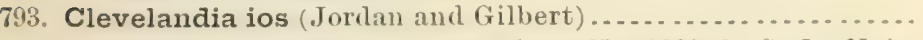

Drawing by Anna L. Brown from No. 3666, L. S. Jr. Unir. Mus., collected by E. C. Starks in Elliot Bay, near Seattle, W ashingtou.

\section{PLATE CCCXXIX}

794. Evermannia zosterura (Jordan and Gilbert)...............

Drawing by Amna L. Brown from No. 2927, L. S. Jr. Unir. Mus., collected by the Hopkins Expedition at Mazatlan, Mexico.

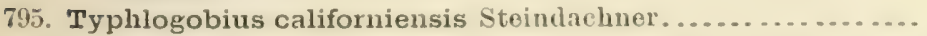

Drawiug by.A. H. Baldwin from No. 34747, U.S.N.M., collected by Rosa Smith at San Diego, California.

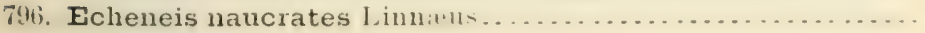

Drawing by H. L. Todd. 


\section{PLATL CCCXXX.}

$797,7 ! 7 \iota$. Remora brachyptera (Lowe) . . . . . . . . . . . . . . . . 2272

Drawing hy IL. L. Todd from No. 23374, U.S.X.M., collected by Daniel MeEuchran in X. lat. $42^{\circ} 10^{\prime}$, W. long. 63-6', in 250 fiatlioms.

798. Rhombochirus osteochir (Cuvier) ..................... l)rawing by H. L. T'odd from Vo. 19022, U.S.X.M., collected by the L. . F Fish Commission at Woods Hole, Massachusetts.

799. Caulolatilus microps Gookle and Bean.

Jrawing by 1I. L. Todd from No. 20971, U.S. N.M., collected by Silas stearns at the P'ensacola Snapper l3anks.

\section{PLA'E CCCXXXI.}

800. Opisthognathus macrognathum Yoes

Drawing by II. L. Torld from No. 5936 , L.S.N.M., the type of O. $8 \mathrm{cuphim}$ ho Goode and Bean, collected by Dr. Whitehurst at Ciarden Key, Filorida.

800 a. Opisthognathus macrognathum l'oey.................. Drawing by A. H. Baldwin from Yo. 5936, U.S.N.M., the type of 0 . scuphiurus Goode and Bean, collected by Dr. Whitehurst at Garden Koy, Floriela.

8(11. Guathypops maxillosa l'oey .........................

Drawing ly 1I. L. 'Todd from No. 5866, U.S.N.M., collected at Garden liey, Florida.

\section{PLATE CCCXXXII.}

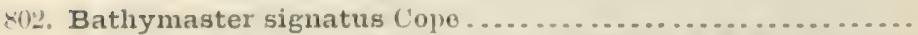

Dratring by II. L. Todd from No. 27646, L.S.X.M., collected by Capt. E. P. Hereudeen at the Shumagin Islauds, Mlaska.

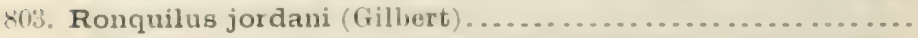

Drawing log Anna L. Brown from No. 3.10, L. S. Jr. Univ. Mus., collected by the Young Naturalist Society of Seattle in Elliot Bay, near soattle, Washington.

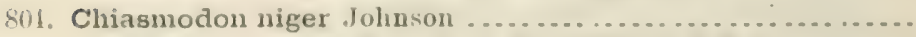
Drawing lsy 1I. L. 'Todd from No. 256333, U.S.X.M., collected by Capt. Thomas $\mathrm{F}$. Hodgilon at the surtace on Le Have Bank.

\section{PLATE CCCXXXIH.}

80.). Hypsicometes gobioides Goole . . . . . . . . . . . . . . . . . . Drawing ly A. I. Baldwin from a specimen collected by the Allatrose at Station :377\%.

Soti. Trichodon trichodon ('Tilesius) ........................ 1)rawing by M. M. Smith from Yo.27980, U.S.X.M., collected hy Mareus Baker at the Shmmagin Islands.

807. Arctoscopus japonicus (Steindachner) ..................

Drawing hy W. S. Atkinson from Jo. 5tisti, L. S. Jr. Univ. Mus., collected by tho flbatros8 at sitation 3652 . 


\section{PLATE CCCXXXIV.}

808. Astroscopus y-græcum (Cuvier and Valenciennes)

Drawing by M. M. Smith from No. 18029, U.S.N.M., collected by J.C. Willets at Matanzas River Inlet, Florida.

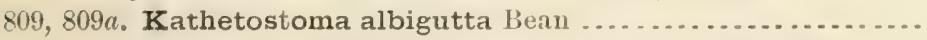

Drawings by M. M. Smith from No. 2403, U.S.N.M., collected by the dibatross.

PLATE CCCXXXV.

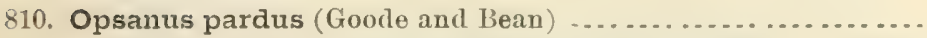

Drawing by H. L. Todd from the type, No. 22237, U.S.X.M., collected by Silas Stearns at the Pensacola Suapper Banks, Florida.

811. Porichthys porosissimus (Cuvier aud Valencienues).........

Drawing by A.H. Baldwin from a specimen collected by the Albatross at Station 2121.

*12. Caularchus mæandricus (Girard)

Drawing by H. L. Todd.

81:). Rimicola muscarum (Meek and Pierson) ..................

Drawing by A. H. Baldwin from the type in L. S. Jr. Univ. Mus., collected by Meek and Pierson in Monterey Bay, California.

\section{PLATE CCCXXXYI.}

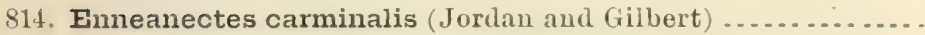

Drawing by Anna L. Brown from Y̌o. 385t, L. S. Jr. Univ. Mus. collected by the Hopkins Expedition at Mazatlan, Mexico.

815. Gibbonsia elegans evides (Jordau and Gilbert) .............

Drawing by A. H. Baldwin from No. 34781, U.S.N.M., collected by Rosa Smith at San Diego, California.

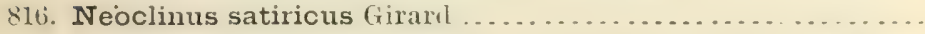

Drawing by W. S. Atkinson from No. 2208, L. S. Jr. Univ. Mus, collected by Dr. Gilbert at Pacific Grove, California.

\section{PLATE CCCXXYYII.}

817. Cryptotrema corallinum Gilbert

Drawing by W. S. Atkinson from a specimen in L. S. Jr. Univ. Mus.

818. Exerpes asper (Jenkins and Èvermanu)

Drawing by A. H. Baldwin from the type, No. 39613, U.S.X.X., collected by Jenkins and Erermanu at Guaymas, Mexico.

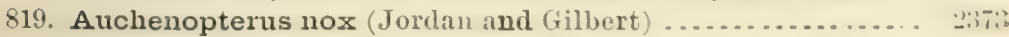
Drawing by Anna L. Brown trom the type collected by Dr. Jordan at Key West, Florida. 


\section{PIATE CCCXXXVII.}

Text paize.

x̌2). Blenuius favosus (ioodi. and Jean $23 \times 11$ 1)rewing from tho type, No. 2629, U.S.X..., collected by G. $1 V^{*}$ iirlemann at Garlen Key, Florida.

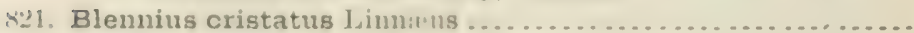

8.:. Hypsoblennius ionthas (Jordan and (illhert)

\section{PIAATE CCCXXXIX.}

8.3. Hypsoblennius hentz (Lo Sueur) ......................

Drawiug by M. M. Smith from So. 26308, U.S.Y.M. collected at Charleston, South Carolina, by C.C. Leslic.

824. Chasmodes saburrae Jorlatu aul Gilbert

Urawing by H. L. T'odd from the trpe, No. 30824, U.S.X.M. collected by Jordan aud Stearus at l'ensacola, Florida.

\$ั5. Rupiscartes atlanticus (C'uvier and Valenciemues) . . . . . . . . Drawing b5 A. 11. Maldwin from No.36946, L.S. X. M., collectod by C. H. 'Townsend at San Cristobal, Lower California.

\section{PIATE CCCXI.}

४26. Emblemaria atlantica .Jordan and Evermann ............. Drawing by A. II. Baldwin from the type, No.33915, U.S.N.M., collected hy Silas Stearns at tho Pensacola suapper Banks, Florida.

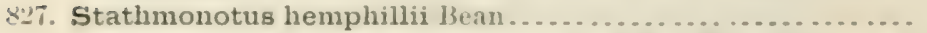

Drawing by H. L. Tokll from the tspe, No. 37193, U.S.N.M. collected hy Henry Hemphill at Key West, Florida.

\section{PLA'T'E CCCXII}

828. Bryostema polyactocephalum (I'allas) .................. Drawing by 11. L. 'Todd from No.36859, I.S. N. M., collected by J. Ci. Swan in F'uret sound, 11 ashington.

8.9. Bryostema nugator Jordan and Williams................. 1) rawiug l,y Anua 1. Jrown from the type, No.3134, L. S. Jr. Unir. Mus., rollectud by the Inung Xat. Soc of Seattlo in Elliot lbay, near Soattle, Washingron.

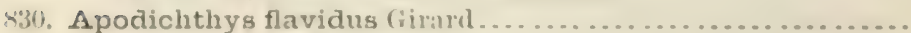

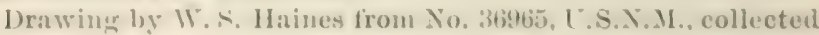
by J. (i, Swan at I'ort T'ownsenu, Washington.

\section{PLATE (:CXIII.}

\$31. Fholis dolichogaster (1'allas)

I)awing by H. L. Tould from No.33834, L.S.N.M., collected by In. 1. Stojuegrer at Buringe Islank.

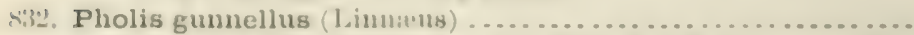

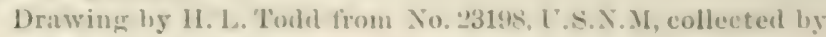
Ii. E. Earll at Ciloucenter, Massachusets. 
833. Pholis ormatus (Girard) 2419

Drawing by M. M. Smith from No. 27996, U.S.X.M., collected by Dr. Bean at Sitka, Alaska.

\section{PLATE CCCXLIII}

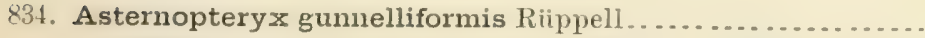

Drawing by A. H. Baldwin from No. 48153 , U.S.X.MI., collected by Schuchert and White in Omanak Fjort, Karsak, Noursook Peninsula, Greenland.

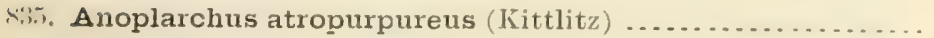

Drawing by H. L. Todd from No. 29820, U.S.N.II., collected by L. M. Turner at Atka Island, Alaska.

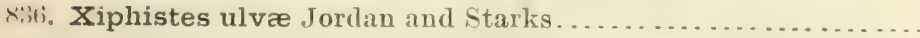

Drawing by Auna L. Brown from the t5pe, No. 3132 L. S. Jr. Univ. Mus., collected bý E. C. Starks at Waadda Island. Neah Bay, Washington.

\section{PLATE CCCXLIV.}

837. Xiphistes chirus (Jordan and Gilbert) 2424

Drawing by H. L. Todd from No. 2396t, U.S.N.M, collected by W. H. Dall at Amchitka Island, Alaska.

838. Xiphidion rupestre (Jordan and Gilbert).................. Drawing by H. L. Todd from a specimen collected br Srlvanus Bailer at Sitka, Alaska.

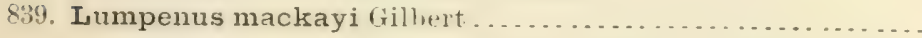
Drawing by A. H. Baldwin from the type collected in the Nusbagak River, Alaska, by the Albatross.

840. Lumpenus lampetræformis (Walbaum) .................. Drawing by H. L. Todd from No. 13852, U.S.N.MI.

\section{PLATE CCCALY.}

841. Stichæus punctatus (Fabricius)

Drawing by H. L. Todl from No. 21718, U.3.X.M., collected by L. M. 'Turner at St. Michaels, Alaska.

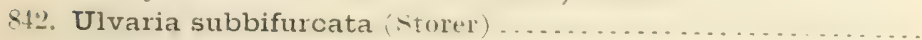

Drawing loy H. L. Todd from a specimen from the Academy of Natural Sciences of Montreal.

813. Cryptacanthodes maculatus Storer . ....................

Draning lo5 H. L. Todd from No. 22309, U.S.N.M. ohtained from E. G. Blackford.

\section{PLATE CCCALTI.}

814. Lyconectes aleutensis Gilbert...

Drawing by A. H. Baldwin from the type, collected by the Albatross at Station 3312, north of Lualaska, in 45 fathoms.

… Anarhichas latifrons Steenstrup and IIallgrimsson. ........

Drawing by H. I. Todel from Yo. 21373, U.S.X.M., collected by Capt. J. W. Collins in N. lat. $43^{\circ} 56^{\prime}$, W. long, $59^{\circ} 04^{\prime}$. 
PLATE: CCCALNII

licxt pange.

811. Anarhichas lupus Linnirus 2416

Drawing by H. L. Todel from Yo. 21846, L.S.N.M., collected by Capt. John Gourrille at Georges Bank.

817. Anarhichas lepturus Bean.

Drawing from the type, collected ly L. M. Turner at St. Michaels, Alaska.

818. Ptilichthys goodei liear

Drawing by II. L. Torld from the type, No. 26619, U.S.N.M.. collected at Iliulink (Unalaska), Alaska.

\section{PLATE CCCXLYIII.}

$849,819 a, 849 b$. Scytalina cerdale Jordan and Gilbert ...........

Drawings by Anua L. Brown from No.3389. L. S. Jr. Unir, Mus., collecterl by E. C. Starks at Waadda Island, Neah Bay, Washington.

850. Zoarces anguillaris (Peck)

Drawing by H.L. Todd from No. 10.138, U.S.N.M., collected by the U.S. Fish Commission at Eastport, Maine.

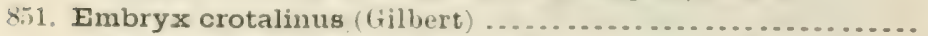

Drawing by A. H. Balewin from the type, collected by the Albatross at Station 2980, ofì Sauta Barbara Islauds.

\section{PLATE CCCXLIX.}

852. Aprodon cortezianus Gilbert

Drawing by A. H. Baldwin from the ty pe, No. $4615 \pi$, U.S.N.M., collected by the Albatross on Cortez Banks, off San Diego, California.

853. Lycodes zoarchus Goode and Bean.

Drawing by 11. L. Todd from the type, No.39298, U.S.X.M. collected by the Albatross in X. lat. $44^{\circ} 46^{\prime} 30^{\prime \prime}$, W. long. $59^{2}$ $55^{\prime} 45^{\prime \prime}$, oft Nova Scotia, in 130 fathoms.

851. Lycodes reticulatus Reinharit

Drawing by II. L. Todel from a specinen collected by Capt. R. Markuson at S. W. Banqquereau, in 300 fathoms.

\section{PLATE CCCL.}

855. Lycodes perspicillum krị̈yer

Drawing by II. L. Tould from No. 39336 , U.S.X.M., collected by the Allatross at station 2456 , in X. lat. $4729, W$. long. 52018 , in xif fathoms.

sirt. Lycodes frigidus Collett.

Drawing by II. L. Todd from No. 32995, U.S.N.M., collected

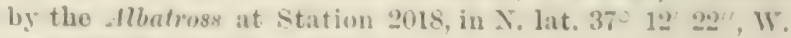

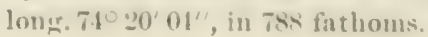


857. Lycodalepis polaris (Sabine)

Drawing by H. L. Todd from No. 27748, U.S.N.M., the type of Lycodes soccineus Bean, collected by Dr. Bean at Big Diomede Island, Bering Strait.

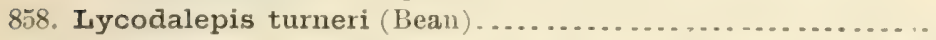
2468

Drawing by H. L. Todd from the type, No.21529, U.S.N.M., collected by L. M. Turner at St. Michaels, Alaska.

\section{PLATE CCCLI.}

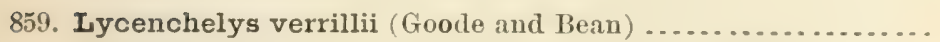

Drawing by H. L. Todd from No. 21015, U.S.N.M., an old male, collected by the U. S. Fish Commission, 27 miles southwest of Chebucto, Nova Scotia.

860), $860 a, 860 h, 860 c, 860$. Lycenchelys paxillus (Goorle and bean).

Drawings by H. L. Todd from the trpe, No. 22177.

U.S.N.M., collected by Capt. J. W. Collins in N. lat. $42^{\circ} 48^{\prime}$, W. long. $63^{\circ} 07^{\prime}$.

861. Furcimanus diapterus (Gilbert)

Drawing by W. S. Atkinson from a specimen in L. S. Jr. Univ. Mus.

\section{PLATE CCCLII.}

862. Lycodonus mirabilis (Goode and Bean) ..................

Drawing by S.F. Denton from No. 39207, U.S.N.M., collected by the Albatross at Station 2742 , in N. lat. $377^{\circ} 46^{\prime} 30^{\prime \prime}$, W. long. $73^{\circ} 56^{\prime} 30^{\prime \prime}$.

863. Lyconema barbatum Gilbert.

Drawing by A. H. Baldwin from the type, collected by the Albatross at Station 3129, in 204 fathoms.

$861,864 a, 861 b, 864 c$. Gymmelis viridis (Fabricius).............

Drawing by H. L. Todd from No. 21999a, U.S.N.M., collected at Niantilik Harbor, Cumberland Gulf, by W. A. Mintzer.

865. Melanostigma pammelas Gilbert

Drawing by A. H. Baldwin from the type, collected by the Albatross at Station 3202 , on coast of California.

\section{PLATE CCCLIII.}

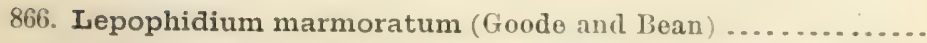
Drawing by M. M. Hildelorant from the trpe, No. 37237, U.S.N.M., collected by the Albatross in X. lat. $23^{\circ} 10^{\prime} 39^{\prime \prime}$, W. long. $82^{\circ} 20^{\prime} 21^{\prime \prime}$, in 213 fathoms.

867. Lepophidium profundorum (Gill) . . . . . . . . . . . . . . . . .

Drawing by A. H. Baldwin from a specimen collected by the Albatross at Station 2012.

868. Rissola marginata (De Kay) 
I'IATE: C C CI,IV.

T'ext jage.

8ii!. Otophidium omostigmum (Jurlan and (Bilbert). 2490

llrawing hy 11. L. Todld from the type, Xo, 296670, U.S.X.M., collected by Jordan and stearns at the P'ensacola Suapper Banks.

87i. Lycodapus dermatinus Gilbert

Drawing by A. H. Baldwin from the type, collected by the Albatross at Station 3162, ofl' Lower California.

871. Brotula barbata (Bloch and Schueiter) ...................

Drawing hy A. II. Baldwin from No. 5337, U.S.N.M., collectell by Professor i'oey in C'ubit.

P'LATE: CCCLN.

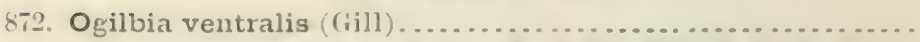

Urawing by Annil L. Brown from No. $2903, \mathrm{~L}$. S. Jr. Unir. Mus., collected by the Hopkins Expedition at Mazatlan. Meximo.

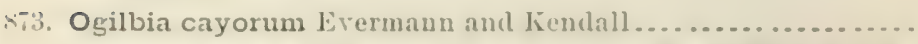
Bawing l,y A. H. Baled win from the trpe, No.487t2, U.S.X.M.. collected by Evermann and Keudall at hey West, Florida.

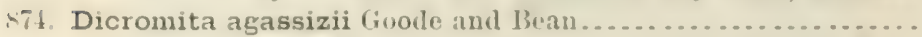

brawing by II. L. 'Todd from the type, collected hy tho Bluke oft' (irenata.

\section{P'LATE CCCLVI.}

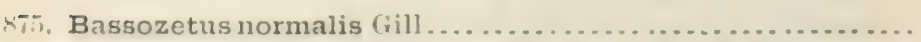

Drawing by H. L. Todd from No. 39116, U.S.X.M., collected by the flbutross in X. Iat. $28^{\circ} 02^{\prime} 30^{\prime \prime}, W^{\prime}$. Iong. $87^{\circ} 43^{\prime} 45^{\prime \prime}$.

4iti. Bassozetus catena fioole and Bean...................... Drawn by A. H. Baldwin from the trpe, No. 373!1, U.S.X.M., collected by the Albutross in X. lat. 2800 $15^{\prime \prime}, \mathrm{W}$. long. si- 12', in 1,467 fathoms.

sirir. Bassozetus catena (ioole and Bean

Drawn by 1I. L. Todd from same specimen.

\section{PLATE CCCLNII.}

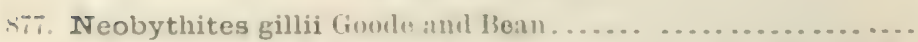

Drawing by A. 11. Baldwin from the type, No.:37:H0, U.S.X.M.

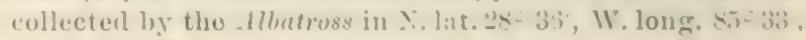
in 111 fithoms.

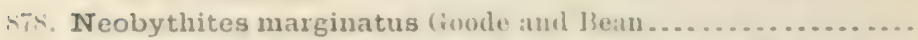

Dratwing hy H. L. Tould from the typo collected by the Blake ofl Barlatos, in 20! ditlsoms.

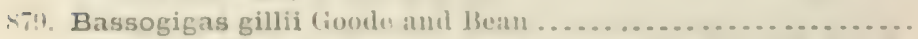

Drawing hy 1. H. Baldwin from the type, No, 3an1, U.S.X.M., collected by the Albutross ofl' C'ape Henlopen, Delaware, in X. lat. $3 ! 1=35)^{\prime}, 11$, long. 70 (5), in 1, 106 fothoms.

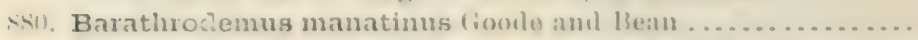

1) rawing hy H. I. Tould from the typo in M. ('. \%, collected hy

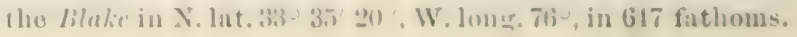




\section{PLATE CCCLVIII.}

881. Porogadus miles Goode and Bean 2520

Drawing by A. H. Baldwin from the type, No. 35625, U.S.N.M., collected by the Albatross at Station 2230 , in N. lat. $38^{\circ} 27^{\prime}$, W. long. $73^{\circ} 02^{\prime}$, in 1,168 fathoms.

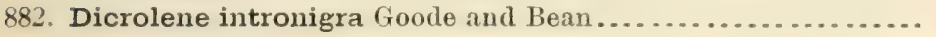

Drawing by H. L. Todd from a specimen collected by the Blake in the Gulf Stream.

883. Barathronus bicolor Goode and Bean.

Drawing by M. M. Smith from the type collected by the Blake at Station Lxxi, off Guadeloupe, in 769 fathoms.

\section{PLATE CCCLIX.}

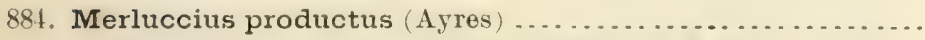

Drawing by H. L. Todd from No. 26638, U.S.N.M., collected by W. F. Prosser at Seattle, Washington.

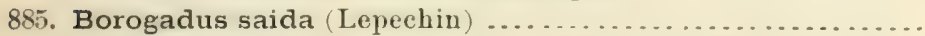

Drawing by H. L. Todd from No. 24031, U.S.N.M., collected by L. M. 'Turner at St. Michaels, Alaska.

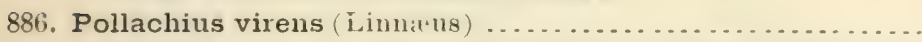

Drawing by H. L. Todd from No. 10443, U.S.N.M., collected by Professor Baird at Eastport, Maine.

PLATE CCCLX.

887. Theragra chalcogramma (Pillas)

Drawing by H. L. Todd from No. 27637, U.S.N.M., collected by W. H. Dall in Pirate Cove, Shumagin Island, Alaska.

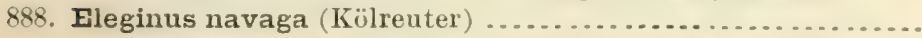
Drawing by H. L. Todld from No. 9286 , U.S.N.M., collected by H. Bannister at St. Michaels, Alaska.

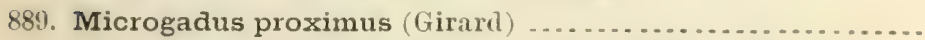

Drawing by H. L. Todd from No. 27982, U.S.N.M., collected at Yakutat Bay, Alaska, by Dr. T. H. Bean.

890. Microgadus tomcod (Walbaum) ....................... Drawing by H. L. Todd from Yo. 17733, U.S.N.M., collected by V. N. Edwarls at Woods Hole. Massachusetts.

\section{PLATE CCCLXI.}

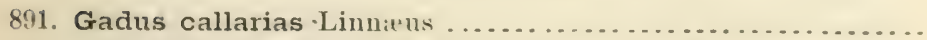
Drawing by H. L. Tould from No. 10444, U.S.N.M., collected by Professor Baird at Eastport, Maine.

892. Melanogrammus æeglefinus Linn: 1 . ..................... Drawing by H. L. Todd from No. 10440, U.S.N.M., collected by Professor Baird at Eastport, Maine.

892a. Melanogrammus æeglefinus Linnieus. Drawing of skull by H. L. Todd.

Bull. No. 47, pt. 4-XviI 


\section{HL.TTE CCCLIII.}

893.893a. Antimora viola (Goode and Jean) ................ 2. . 44

Drawings ly H. I. 'T'od, from the trpe, No. 21837, U.S.X.M., collected by Capt.J. W. Collius on be Have Bauk, in 100 to : 500 fathous.

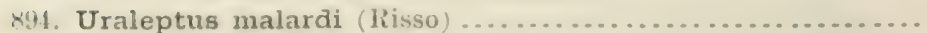

Jrawing by II. L. Toold from a specimen collected by the Bluke at Station Ixxxi, ott Neris.

\section{PLA'E ( CCLXIII.}

845, Lotella maxillaris Bean

2isii

Drawing by 11. L. Tolle from the type, Jo. 24832, U.S.X.M., collected by the Fish IIauk in X. lat. $39 \div 55,11$. long. $70 \div 28$, in 396 fathoms.

\$\$6). Physiculus fulvus Bean ..........................

Dratwing ly H. L. Told from the type, No.28t66, U.S.X.M., collected by the Fish Haw in X. lat. $40^{\circ} 01^{\prime}$. W'. loug. $69^{\circ} 56^{\prime}$, in 79 fathoms.

\section{PLATL CCCLAIV.}

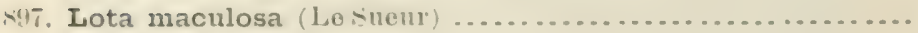
Jrawing los 11. 1. 'I'odel.

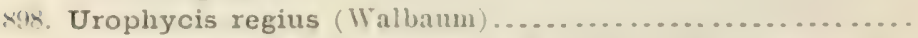

Drawing by II. L. Tould from Yo. 20923, L.S.N.M., obtained by L. G. Blackford from the New York Ayuarium.

3!19. Urophycis cirratus (Goole and Bo:tu) ................... Drawing by II.L. Todel from the type, No. 34059, L.S.N.M.. collected ly the Albulross at Station "376, in X. lat. $29=03$ ' 15 ". II. long. $88 \div 16$.

PIATE CCCLAV.

()). Urophycis earlli (Bean)

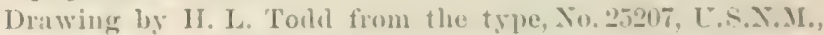
collected by li. E. Earll at Charleston, south Caroliua.

(M1. Urophycis temuis (Mitchill) ........................ I) Yawing ly II. L. Tohlel from No. 21029, U.S.X.M., cullocted by the sipeduell in Halifax Harbor.

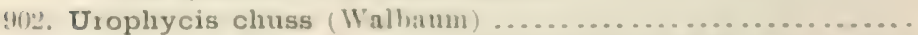

Dratwing by II. L. Fode from No. 28707 , L.S.N.M., collected by

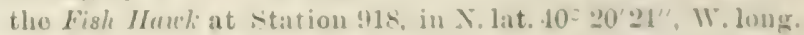
70) $41^{\prime} 3\left(0^{\prime \prime}\right.$, in 215 fithoms.

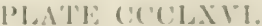

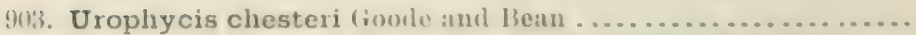

Drawing by 11. 1. 'Tohlel from No, 21810, L.s.X.M., collected loy the siperluell at Station 17, oft Cape Am, in 140 finthoms. 
904. Lemonema barbatulum Goode aud Bean...................... 2556

Drawing by W. S. Haines from No. 38331, U.S.N.M., collected by the Albatross at Station 2397 in N. lat. $28^{\circ} 42^{\prime}$, W. Iong. $86^{\circ} 36^{\prime}$, in 280 fathoms.

905. Lemonema melanurum Goode and Bean ..................

Drawing by W. S. Haines from the type, No. 38270, U.S.N.M., collected by the Albatross at Station 2415 in N. lat. $30^{\circ} 44^{\prime}$, W. long. $79^{\circ} 26^{\prime}$, in 440 fathoms.

\section{PLATE CCCLXVII.}

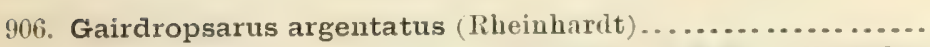
Drawing by H. L. Todd from No. 7212, U.S.N.M., collected at Nahaut, Massachusetts, by F. IV. Putnam.

907. Euchelyopus cimbrius (Linmeus) ...................... Drawing by H. L. Todd from No. 21721, U.S.N.M., collected by Elward Brown in Bay Chaleur.

908. Bathygadus favosus Goode and Bean .................... . .

Drawing by H. L. Todal from the type collected by the blatie off Martinique, in 472 fathoms.

\section{PLATE CCCLXVIII.}

909. Steindachneria argentea Goole and Bean ................ Drawing by H. L. Todd from the type, No. 37350 , U.S.N.M. collected by the Albatross in N. lat. $39^{\circ} 14^{\prime} 30^{\prime \prime}$, W. long. $88^{\circ} 9^{\prime} 30^{\prime \prime}$, in 68 fathoms.

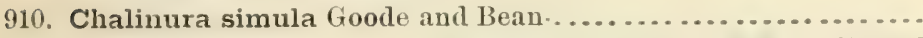

Drawing by H. L. Todd from No. 39152, U.S.N.M., collected by the Albatross at Station 2095 in N. lat. $39^{\circ} 29^{\prime}$, W. long. $70^{\circ} 58^{\prime} 40^{\prime \prime}$, in 1,342 fathoms.

911. Coryphænoides carapinus Goode and Bean

Drawing by H. L. Todd from a specimeu collected by the Blake in N. lat. $39^{\circ} 43^{\prime}$, W. long. $70^{\circ} 55^{\prime} 25^{\prime \prime}$.

\section{PLATE CCCLXIX.}

912. Hymenocephalus cavernosus (Goode and Bean) ........... Drawing by S. F. Denton from the type, No. 37337, U.S.N.M. collected by the Albatross at Station 2398 in N. lat. $28^{\circ} 15^{\prime}$ IV. long. $86^{\circ} 26^{\prime}$, in 227 fathoms.

913. Cœlorhynchus occa (Goode and Bean) ....................

Drawing by H. L. Todd from the type No. 37334 , U.S.N.M., collected by the Albatross at Station 2396 in N. lat. $28^{\circ} 34^{\prime}$, IV. loug. $86^{\circ} 48^{\prime}$, in 335 fathoms.

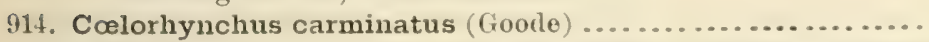

Drawing by H. L. Todd from No. 26187, U.S.N.M., collected ly the Fish Hank at Stations s\$3, off Marthas Vineyard, in 372 fiathoms. 


\section{P'LATE CCCLAX.}

Text pace.

915. Colorhynchus caribbaus (Goode and Bean)

2589

Drawing by H. L. Todel from the type, No. 37333, U.S.X.M.. collected by the Albatross at Station 2377 , in the northern part of the Gulf of Mexico, in N. lat. $299^{\circ} 7^{\prime} 30^{\prime \prime}$, W. loug. $88^{\circ} \gamma^{\prime}$, in 210 fathoms.

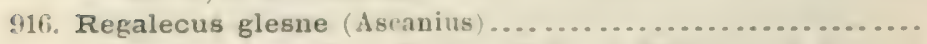

Drawing from Day, Fishes of Great Britain and Ireland, 1). 61 .

\section{PLATE CCCLXXI.}

117. Atheresthes stomias (Jordan and Gilbert) ................ 1) rawing by A. H. 13aldwin from No. 27186, U.S.N.M., col. leeted by Dr. Jordan at Point Reyes, Califoruia.

918. Hinpoglossus hippoglossus (Linnitus) ..................

Jrawing ly II. L. Todd from No. 27605 , U.S.N.M., collected at Marmot Island, Alaska, by Dr. Bean.

\section{PIAATE CCCLXXII.}

919. Hippoglossoides platessoides (Fabricius) ............... Drawing by II. L. Todd from No. 21002, U.S.X.M.M, collected at I,e Have Bank.

920. Hippoglossoides elassodon Jorlan and Gilbert............

\section{PIAATE CCCLXXIII.}

921. Psettichthys melanostictus Girard.

Drawing by H. L. 'lodd frou No. 24167 , U.S.N.M., collected at San Francisco, Califoruia, by Dr. Jorkau.

924. Paralichthys dentatus (Linutens) ....................... Drawing by A. 11. Baldwin from a specimen collected by Dr. 1I. M. Suith at st. (ieorge Jsland, Maryland.

\section{PLATE CCCIXAIV.}

923. Paralichtlys squamilentus Jorkan and Gilbert............. Drawiug by 11. 1. 'Todel trom No.30562, U.S.X..M., collected at I'ensacola, Floricla, hy Jurdan and stearus.

924. Paralichthys oblongus (Mitchill) .................... 1) raving by H. L. Toxld.

\section{PLAT: CCCLAXY.}

925. Ancylopsetta quadrocellata Gill................... 2634

526. Pleuronichthys decurrens Jordan and filliert.............

Drawing by A. H. Ballwin from Yo. 27115, U.S.X.M., col. lected by Dr. Jordan at san Francisco, California. 


\section{PLATE CCCLXXVI.}

927. Inopsetta ischyra (Jordan and Gilbert)...................

Drawing by H. L. Todd from No. 32913, U.S.N.M., collected at Unalaska by E. W. Nelson.

928. Lepidopsetta bilineata (I sres)

Drawing from No. 27602, U.S.N.M., collected by Dr. Bean at St. Paul, Kadiak Islaud, Alaska.

\section{PLATE CCCLXXVII.}

929. Limanda ferruginea (Storer)

Drawing by H. L. Todd from No. 21903, U.S.N.M., collecter at Gloncester, Massachusetts, by the U. S. Fish Commission.

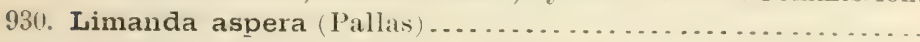
Drawing from No. 27944, U.S.N.M., collected by Dr. Bean at Sitka, Alaska.

\section{PLATE CCCLXXVIII.}

931. Limanda proboscidea Gilbert.

Drawing by A. H. Baldwin from the trpe, collected by the Albatross in Bristol Bas, Alaska.

932. Limanda beanii (roole

Drawiug by H, L Todd from the type, No. collected by the Fish Hawh at Station 875, off the south coast of New Enigland.

\section{PLATE CCCLXXIX.}

933. Pseudopleuronectes americanus (Walbaum) ............... Drawing by H. L. 'Todd from a specimen in the U.S.N.M.

934. Pleuronectes quadrituberculatus Pallas

Drawing by H. L. Todd from No. 28025, U.S.N.M., collected at St. Paul, Kadiak, b5 IV.J. Fischer.

\section{PLATE CCCLAXX.}

(3). Liopsetta glacialis (Pallas)

Drawing by H. L. Todd from No. 27947, U.S.N.M., collected in Kotzebue Sound by Dall and Bean.

936. Liopsetta putnami (Gill) . . . . . . . . . . . . . . . . . . . . . .

Drawing by H. L. Todd from No. 5368, U.S.N.M., collected at Salem, Massachusetts, b5 F. W. Putuam.

\section{PLATE CCCLXXXI.}

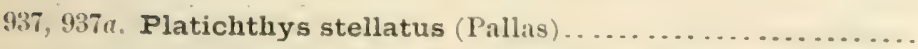
Drawing by H. L. Todd from No. 27610, U.S.N.M.. col. lected log Dr. Bean in Alaska. 


\section{PLATE CCCLXXXII.}

15. Lophopsetta maculata (Mitchil1).................... 2660

Drawing by H. L. Todk.

(4:4). Platophrys ocellatus (Agassi\%)..................... g6ti3

Drawing by H. L. Todd from Xo. 34972 , U.S.N.M., type of P. nebularis Jorian and Gilbert, collected by Dr. Jorian at Key West, Florida.

\section{PLATE CCCLXXXIII.}

940. Trichopsetta ventralis (Goodo and Bean)................

Dratwing by H. L. Todd from No. 37372, L.S.N.M., collected by the Albatross at Station 2378 , in x. lat. $29=14^{\prime} 30^{\prime \prime}, \mathrm{W}$. long. $88^{\circ} 09^{\prime} 30^{\prime \prime}$, in $68^{\text {fathoms. }}$

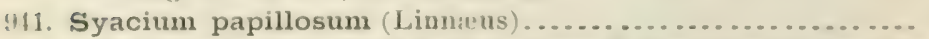

Drawiug by H. L. Told from the type of lleminombus potulus Bean, No. 30180, U.S.N.M., collected by silas Stearns at Pensacola, Florida.

\section{PLATE CCCLAXXIS.}

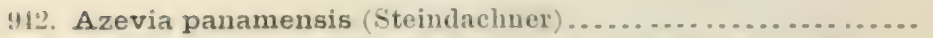

Drawing by A. II. Baldwin from No. 15, Mns. Comp. Zool.

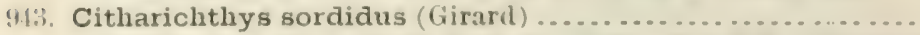

Drawing by H. L. Todd from No. 31991, U.S.N.M., collected by Capt. H. E. Nichols in Johustons Straits, British Columbia.

\section{PLATE CCCLXXXV.}

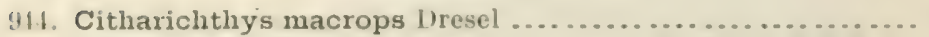

Drawing l,y H. L. Todd from the type, No. 21500, U.S.X.M., collected by Silas Stearns at Pensacola, Florida.

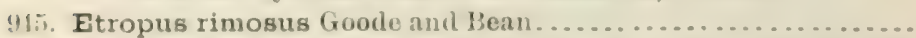

1)rawing by II. L. Todd from the type. collected hy the

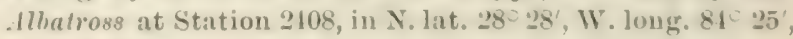
in 21 fathoms.

\section{PLAT' CCCISXXVI.}

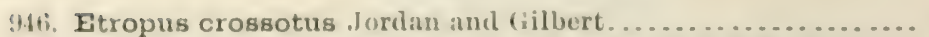
Irawing by II. L. Tould from Yo. 26.71, U.S.Y.M., collected by Silas Stearns at Cedar keys, l'lorida.

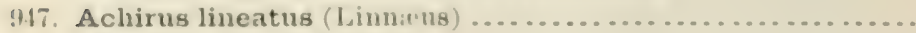
Drawing by 11. L. 'Todd.

\section{PIATF CCCLXXXVII.}

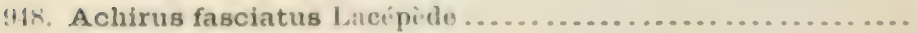
1)rawing loy II. 1. T'udel.

449. Symphurus marginatus (Goode and Be:an)

Drawing by 11. 1. Todd from a specimen collected by the Allutross at station 23767 , in X. lat $29-0.3^{\prime} 15^{\prime \prime}, 11$. long. $88^{\circ}$ $16 i^{\prime}$, in 321 fathoms. 


\section{PLA'TE CCCLXXXYIII.}

Test page.

950. Symphurus plagiusa (Linnseus) 2710

Drawing by H. L. Todd from No. 15017, U.S.N.M., collected by Dr. H. C. Yarrow at Beanfort, North Carolina.

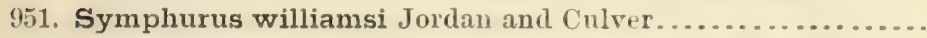

Drawing by Anna L. Brown from the type, No. 2943, L. S. Jr. Unir. Mns., collected by the Hopkins Expedition at Mazatlan, Mexico.

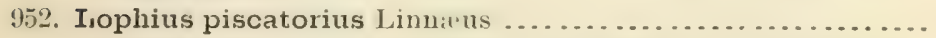
Drawing by H. L. Todd from a specimen in the U.S.N.M.

PLATE CCCLXXXIX.

953. Chaumax pictus Lowe 2724

Drawing by H. L. Todd from No. 26021, U.S.N.M., collected by the Albatross at Station 869.

954. Ceratias holbolli Kröver

Drawing by A. H. Baldwin from Gaimard, Voy. Skand., Poissous, pl. Ix.

\section{PLATE CCCXC.}

95\%. Mancalias shufeldti (Gill)

Drawing by H. L. Todd from the type, No. 33552, U.S.N.M., collected by the Albatross at Station 2099, in N. lat. $37^{\circ} 12$ $20^{\prime \prime}$, W. long. 69 $39^{\prime}$, in 2,949 lathoms.

956. Cryptopsaras couesii Gill

Drawing by H. L. Todd from No. 33558 , U.S.N.M. collected by the Albatross at Station 2101 , in N. lat. $38^{\circ} 13^{\prime} 30^{\prime \prime}$, W. long. $68^{\circ} 24^{\prime}$, in 1,686 fathoms.

\section{PLATE CCCXCI.}

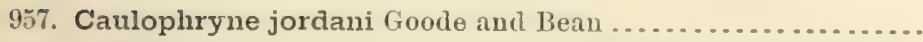

Drawing by S. F. Denton from the type, No. 39265, U.S.N.M., collected by the Albatross in N. lat. $39^{\circ} 27^{\prime}, W$. loug. $71^{\circ}$ $15^{\prime}$, in 1,276 fathoms.

\section{PLATE CCCACII.}

$958,958 a, 958 \bar{b}$. Ogcocephalus vespertilio (Linnæus) ........... collecter by the Albatross in N. lat. $24^{\circ} 25^{\prime} 30^{\prime \prime}$, W. long. $81^{\circ} 47^{\prime} 45^{\prime \prime}$ 。 



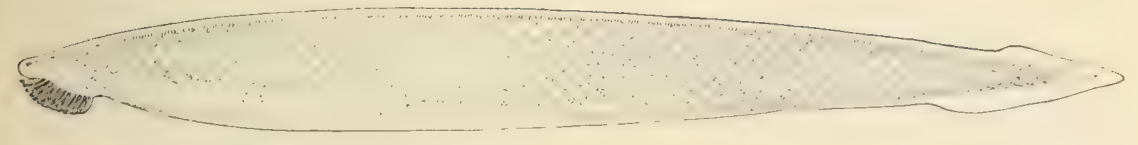

1
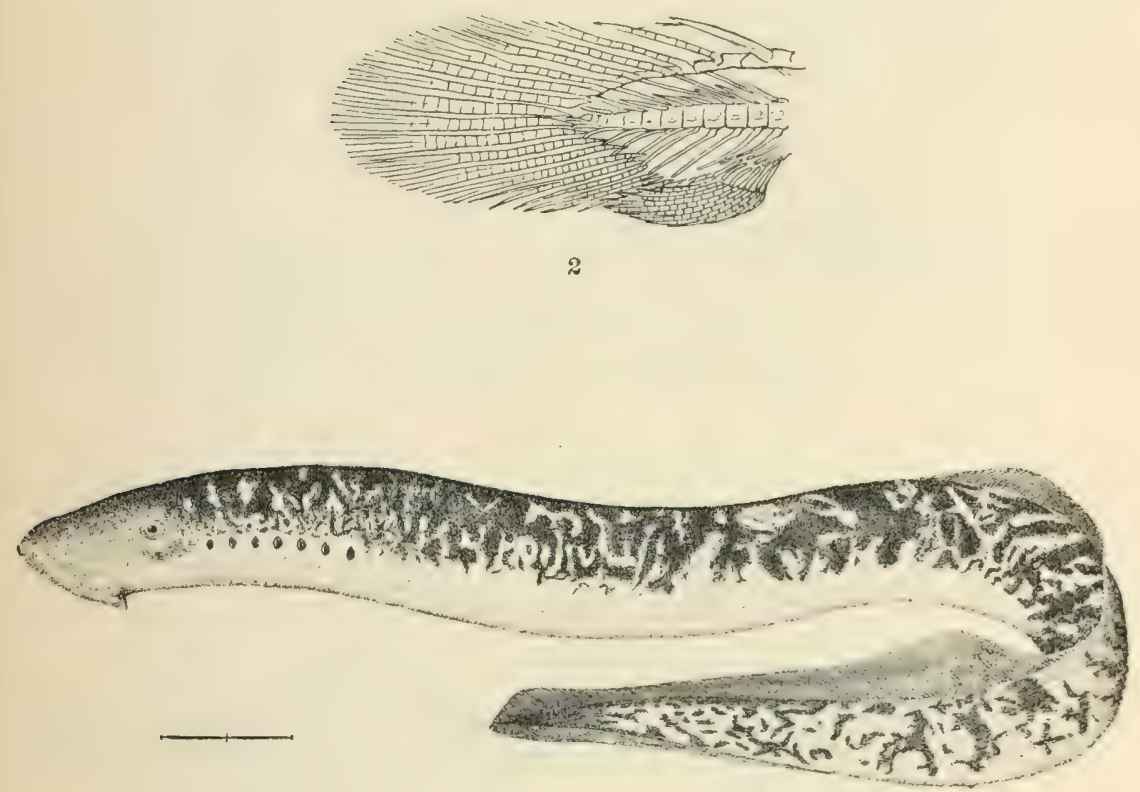

3

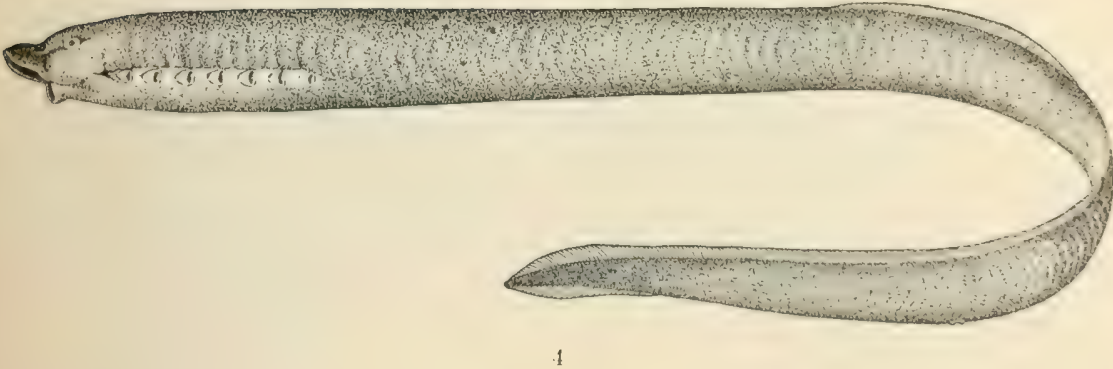

1. BHANCHYOSTOMA CARIB.EUM, (1) 3.)

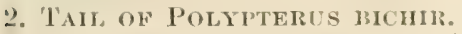

3. P'ETROMYZON MARINES. (I', 10.)

4. ENTOSIIENUS TRIDENTATIS. (1'.12.) 



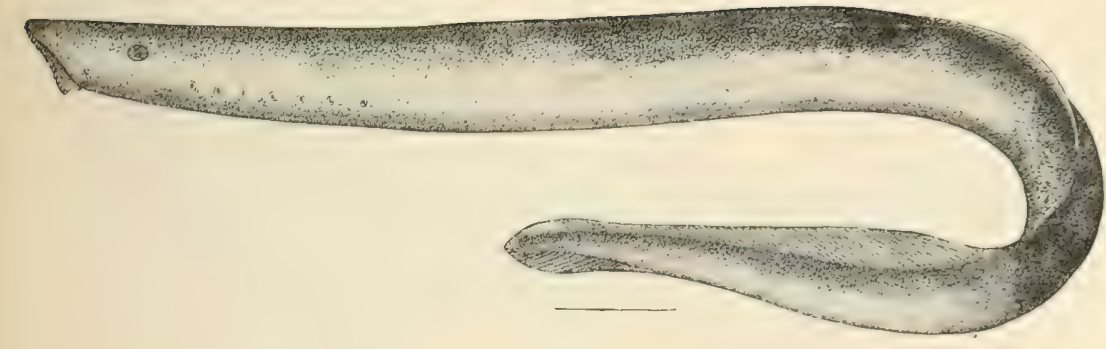

5
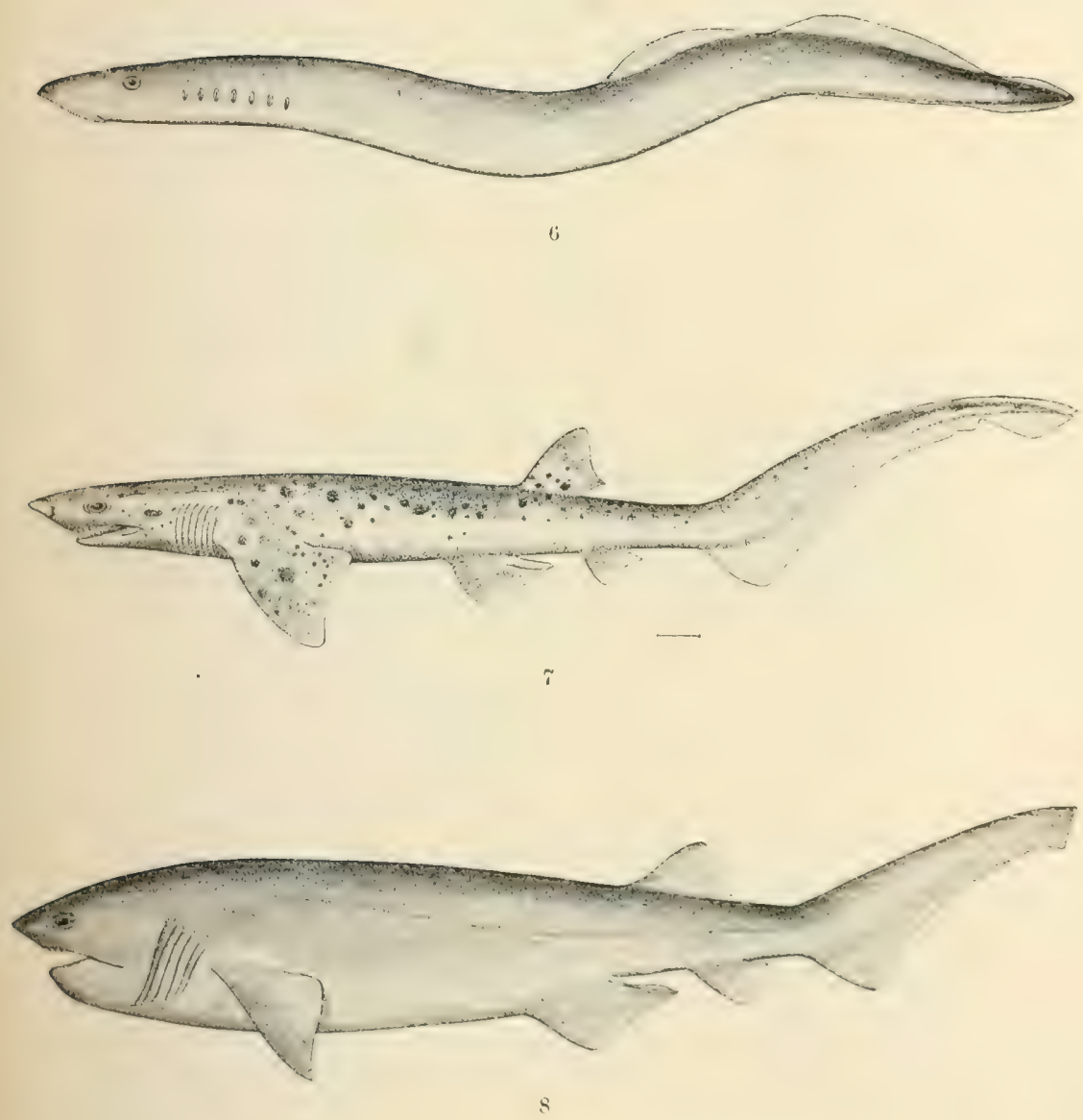

5. Lampetra AUliea. (P. 13.)

6. LAMI'ETIRA SIADICEA. (P. 13.)

7. Notorhy NCHUS Maculatus. (P. 17.)

8. Hexanchus giseus. (P, 19.) 


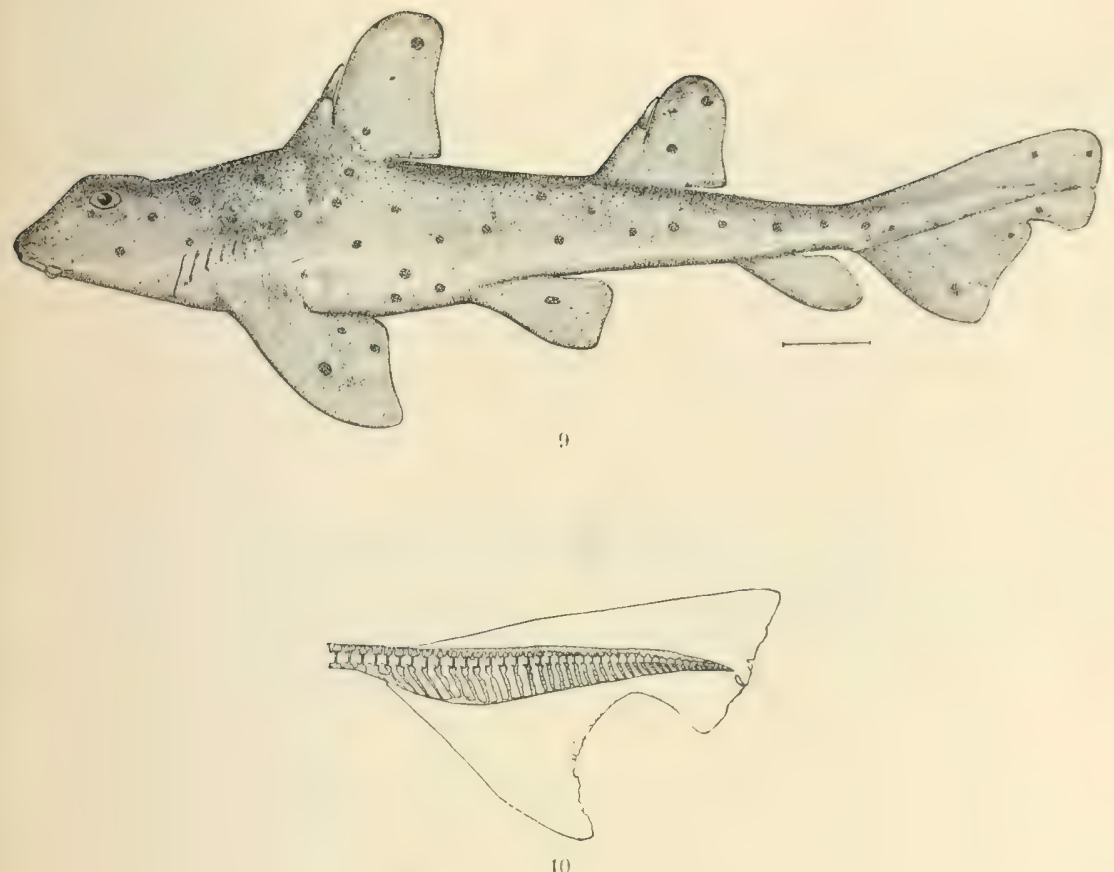

10
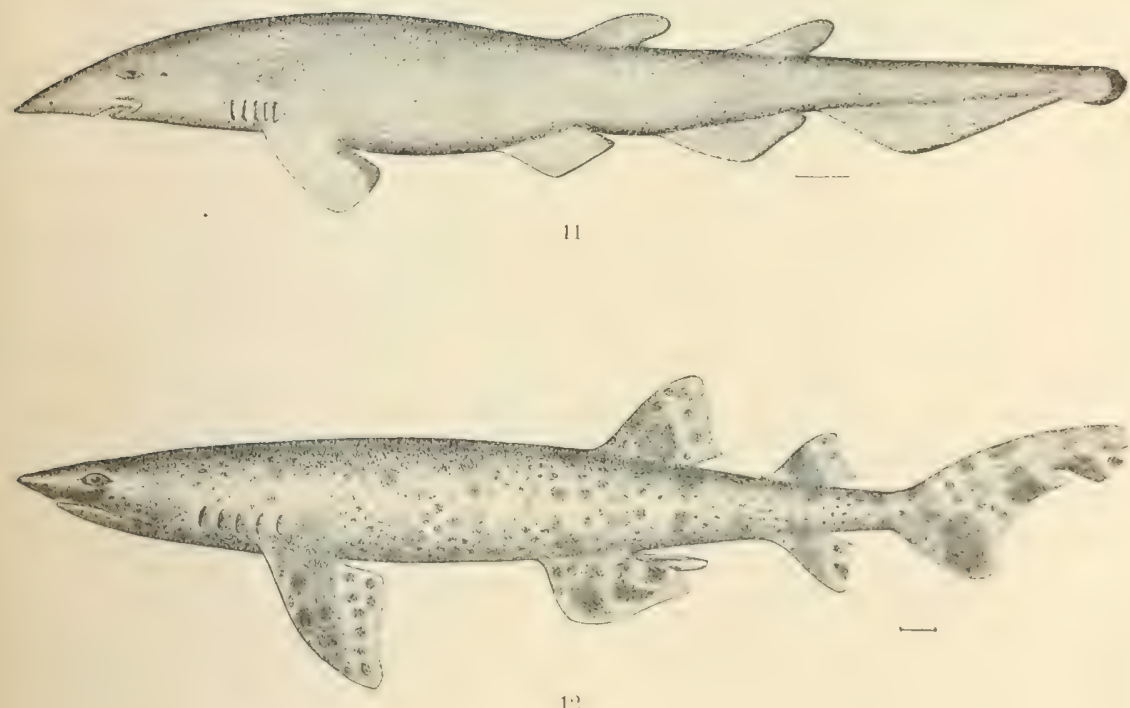

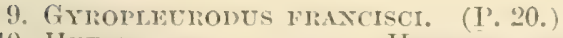

10. HETEROCEICAL TAI, OF IIETERODONTUS NIIIIPPII

11. SCYLLIORIIXUE PlOFCNDORUM. (I'.22.)

12. CATLLLS VTER. (1'.25.) 



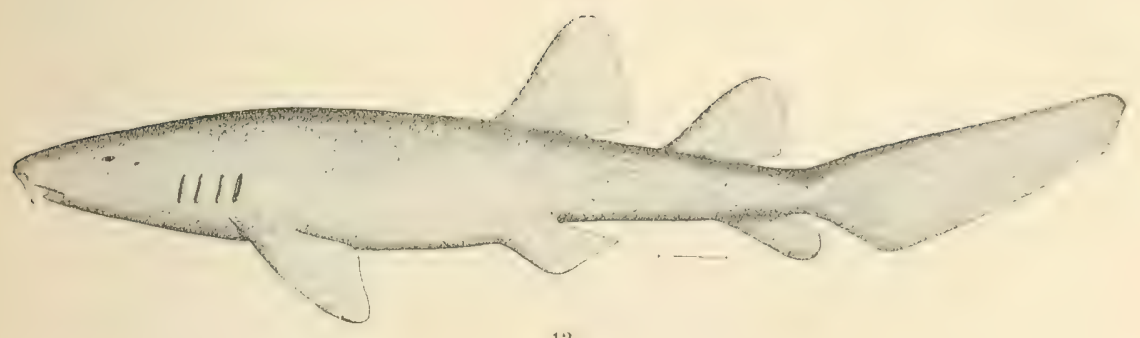

$1: 3$

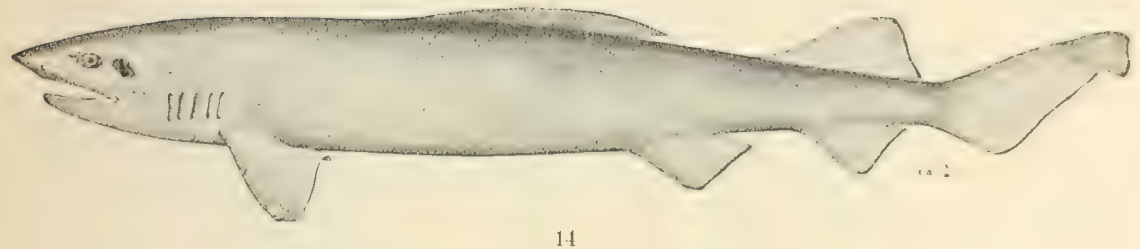

14

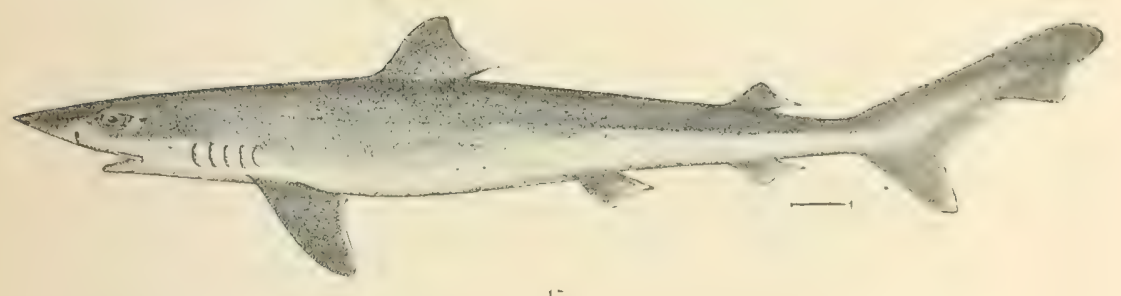

is

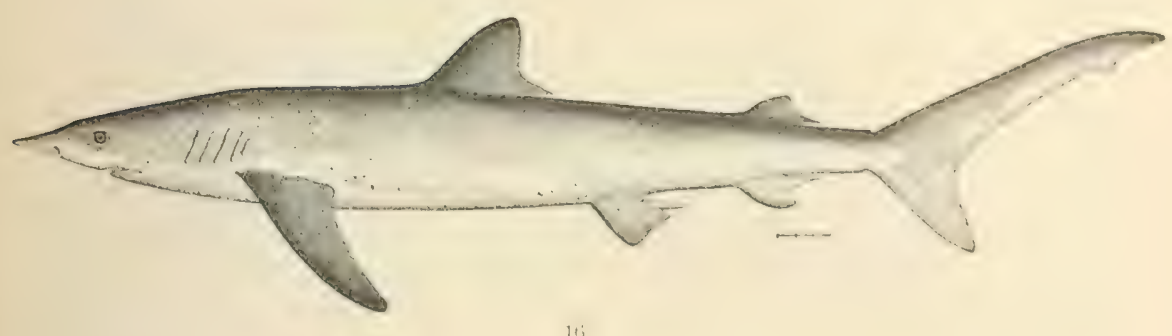

13. Gixglymostoma cirratem. (1.20)

14. PSEUDOTHAKIS MCROION. (P. 27.)

15. G.MLORHIT'S \%OPTERCS, (P.32.

16. P'IIIOXACE: GLIC'CA. (1'.33.) 


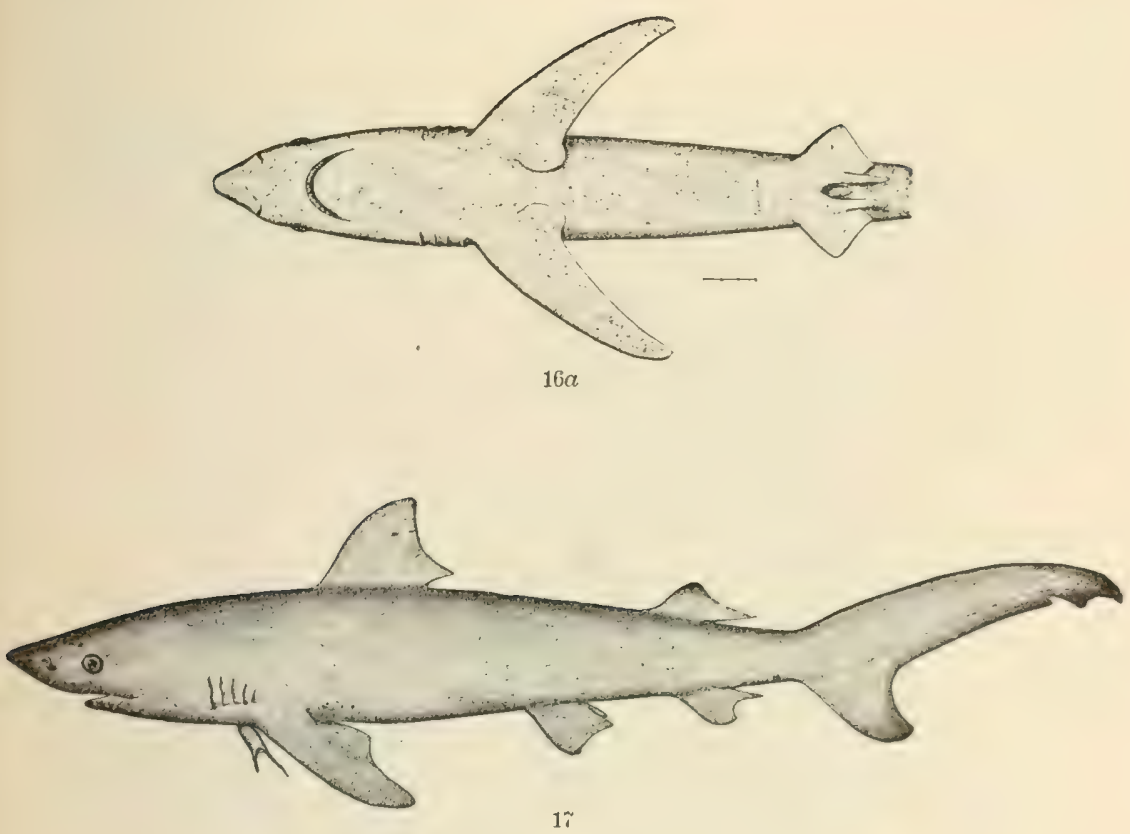

17
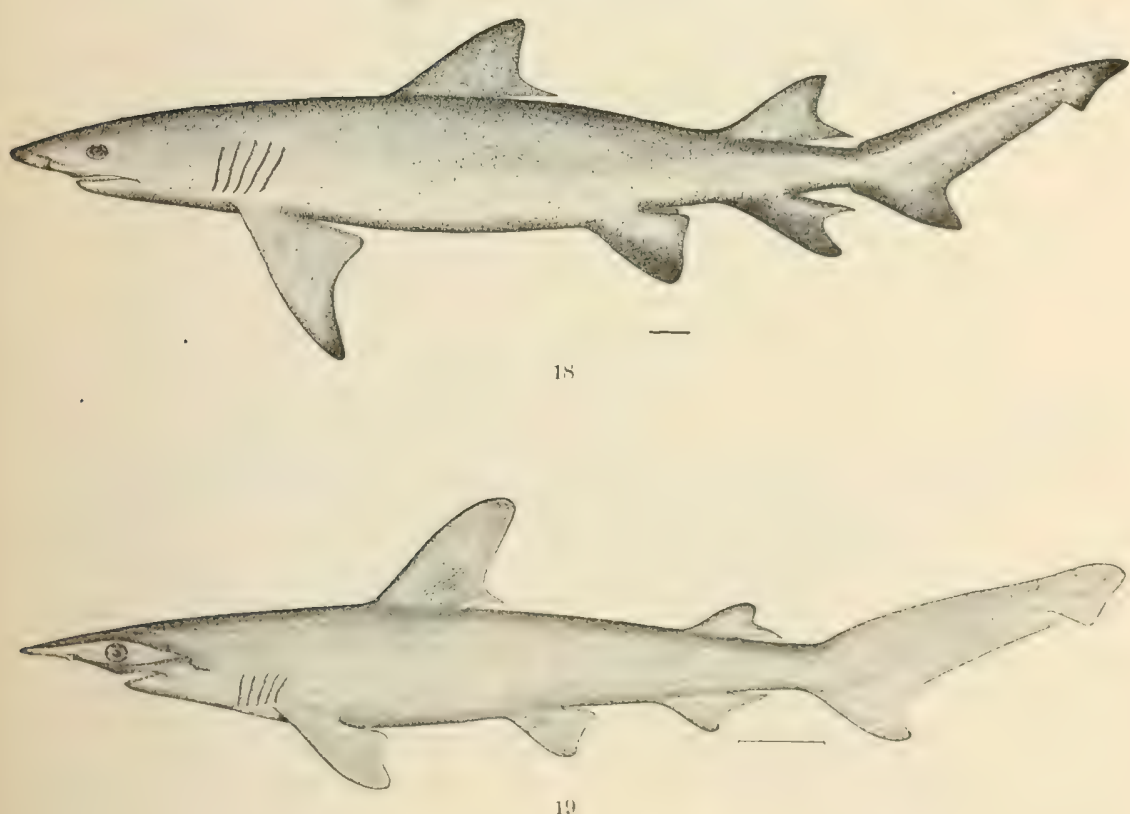

16a. Prionace glavca. (P. 33.)

17. CalichariINus lamia, (P. 38.)

18. HYPOIIIOX MREVIROSTRIS. (P.41.)

19. SPHLRA THBURo, (P. 4.) 


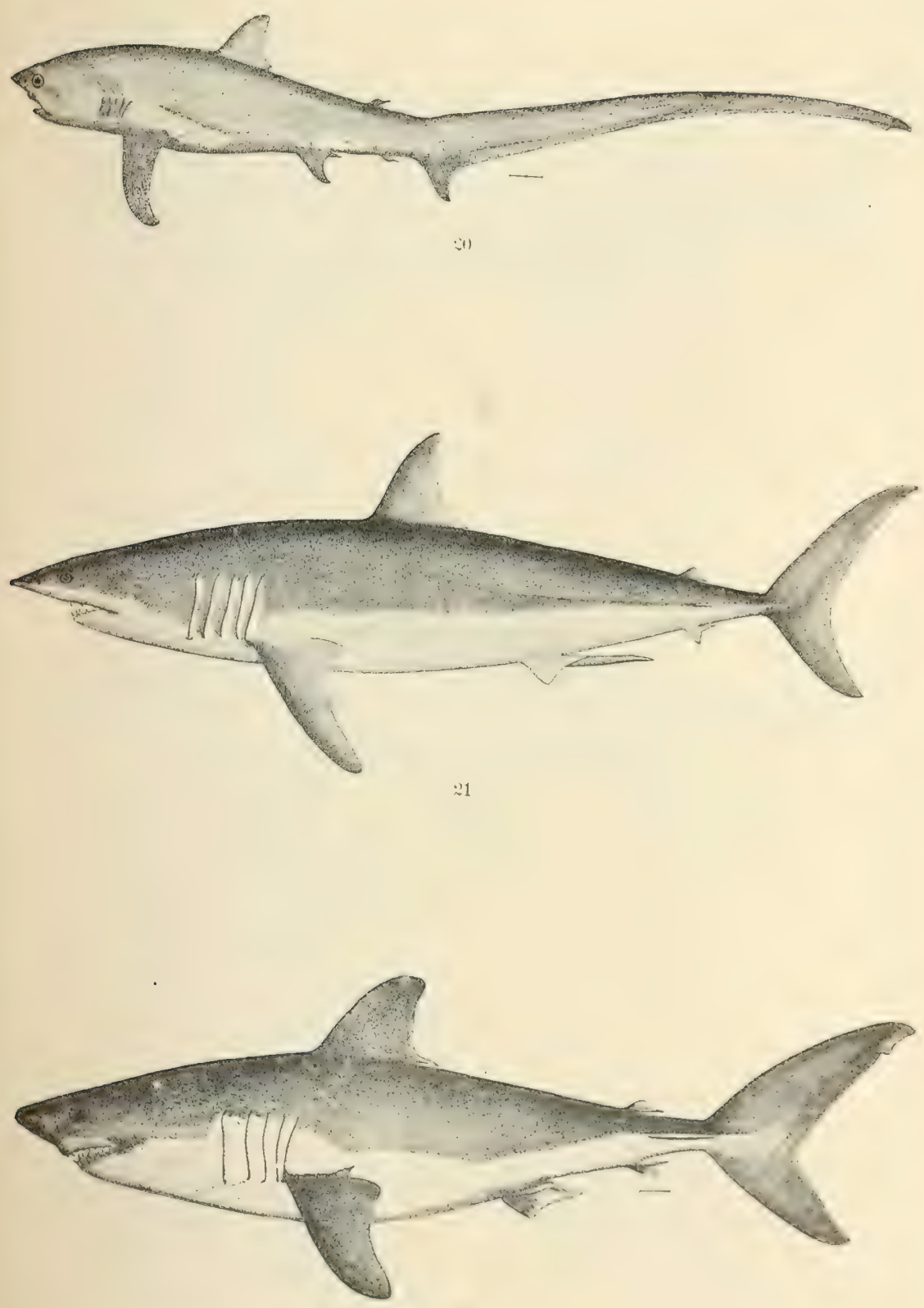

(3)

20. Atopias vulpes. (P.45.)

21. IsUnes DHKay. (1', 48.)

2.2. LaMNA CORNUBica. $(1,49$. 



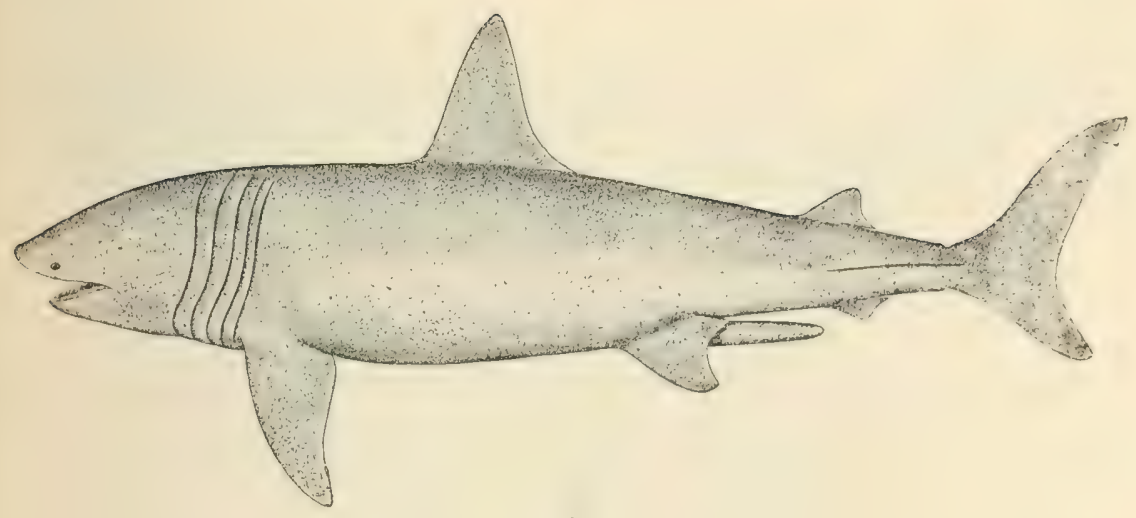

23

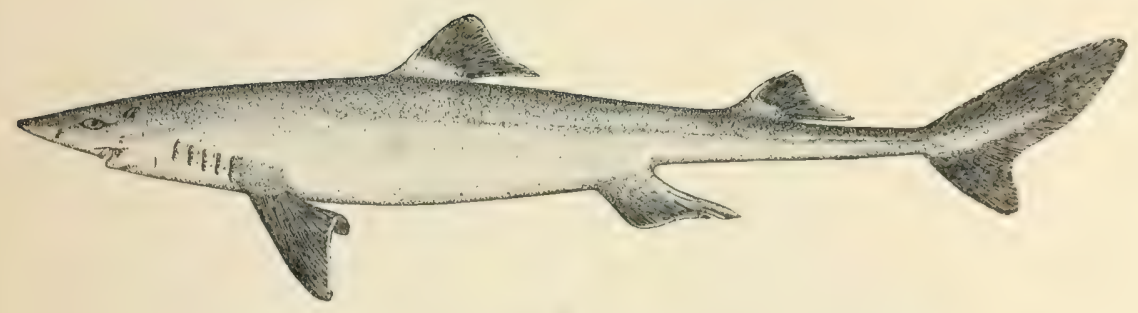

24

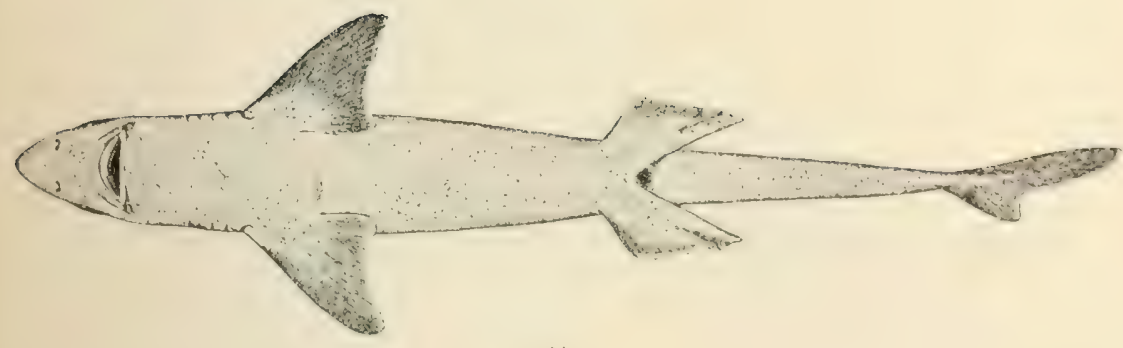

$\because 4 a$

23. Chtominus maximus. ( $P$, 51.)

24, 24a. Sevalus acaxtilas. ('’ 54 .) 



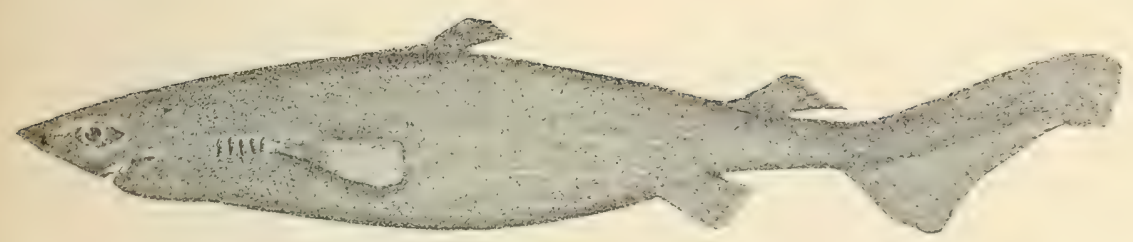

:5)

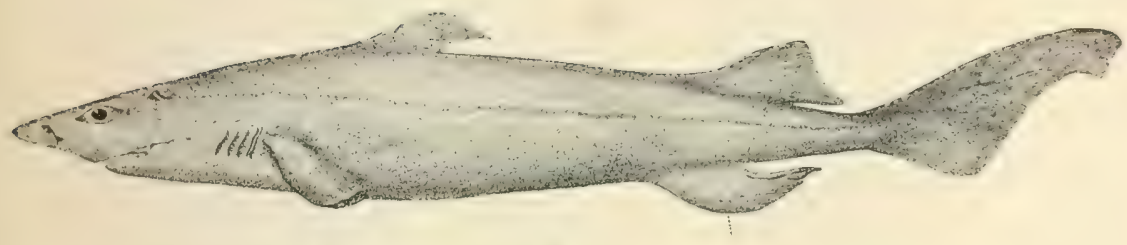

26

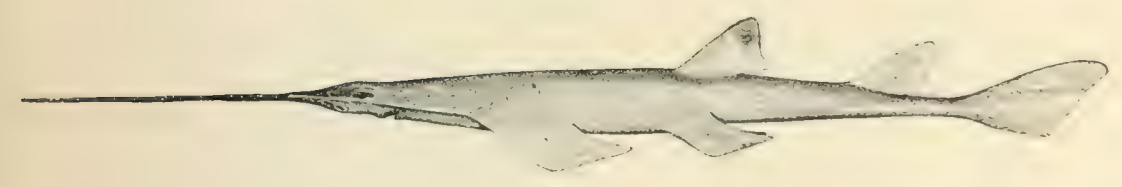

$2 \pi$

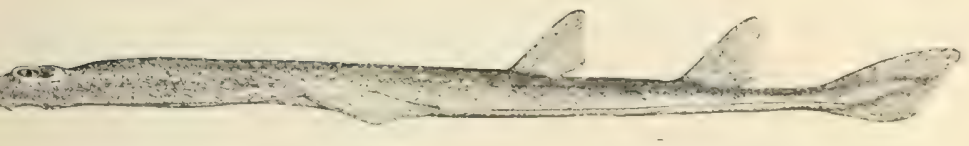

$\therefore$

25. Centroscymas calolepis. (P.55.)

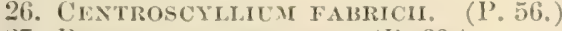

27. Pristis PJeTINATUS. (l’.60.)

28. RHINOBATU LENTIGINOSUS. ( $\left(l^{\prime} \cdot 62.\right)$ 


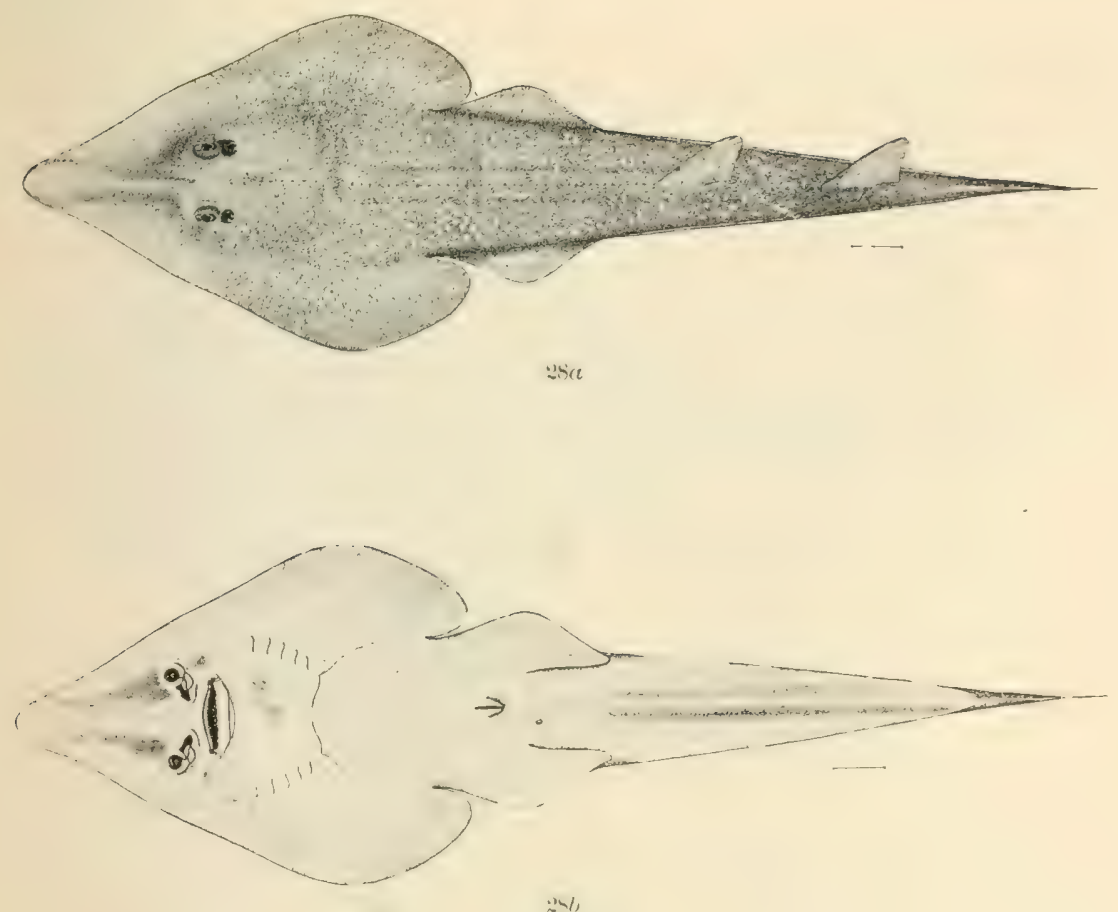

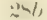

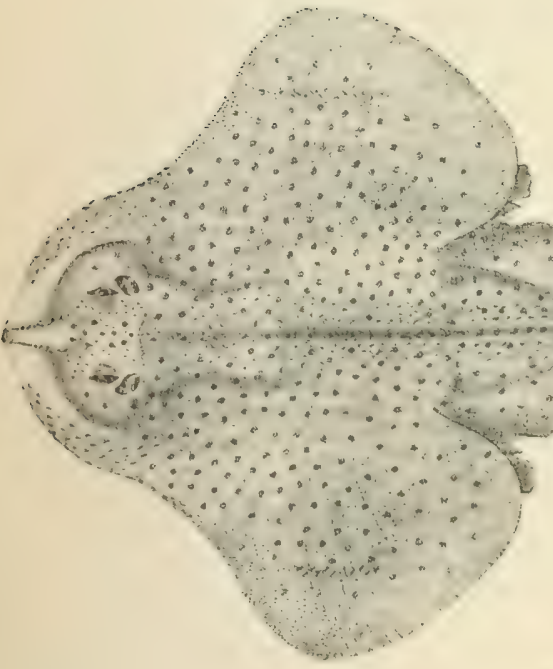

$\because ?$

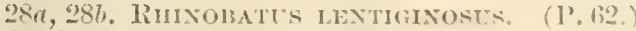

29. RAAL ERINACEA. (P.68.) 



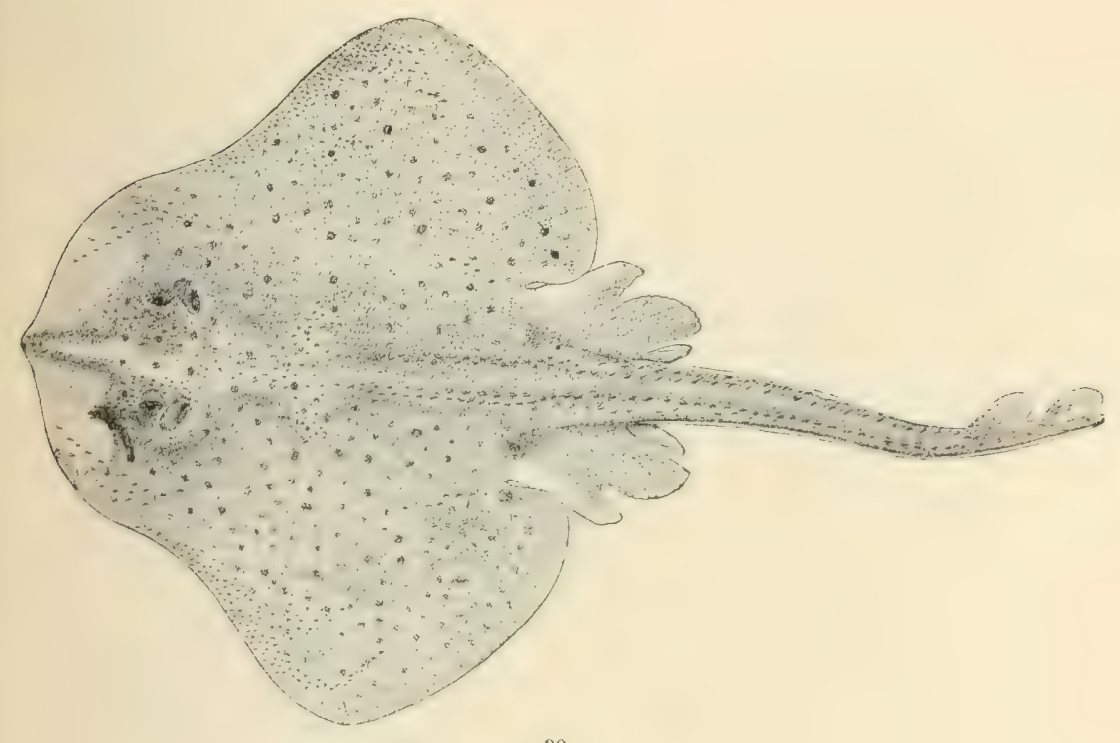

30

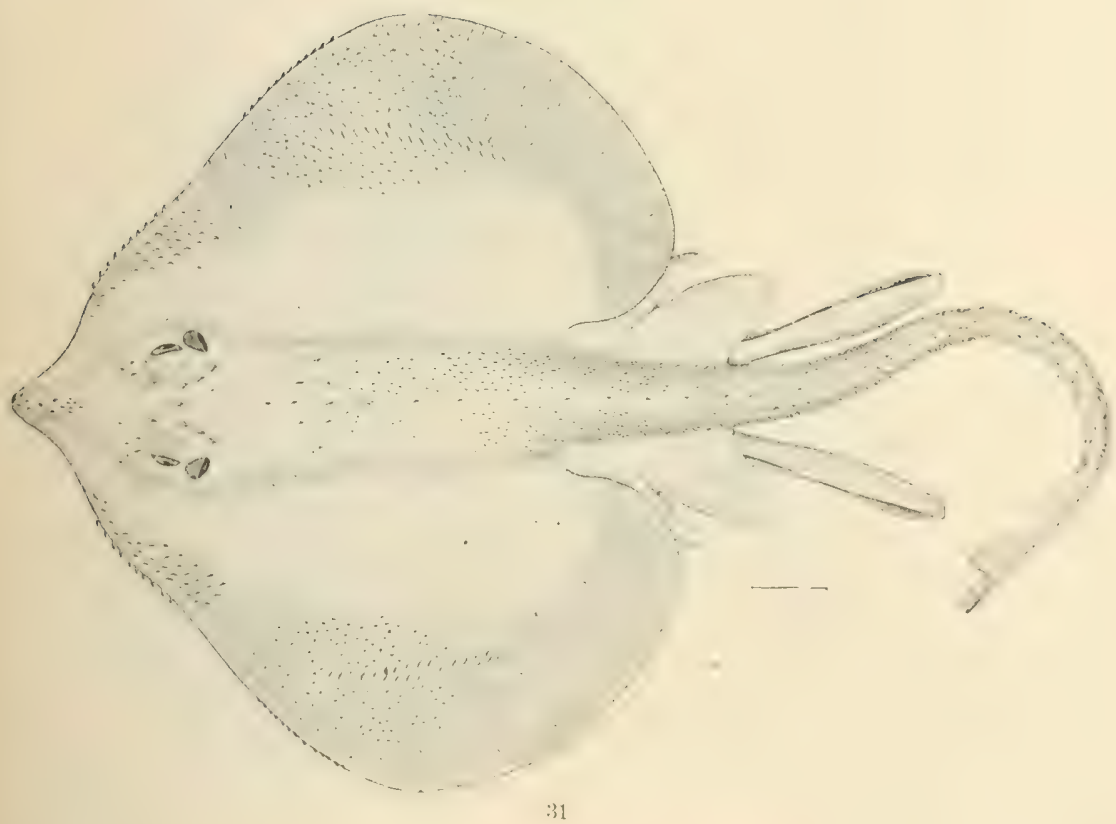

30. RAIA OCELLATA. (P, 68.)

31. RAJA ACKLIYI. (I', 70.) 



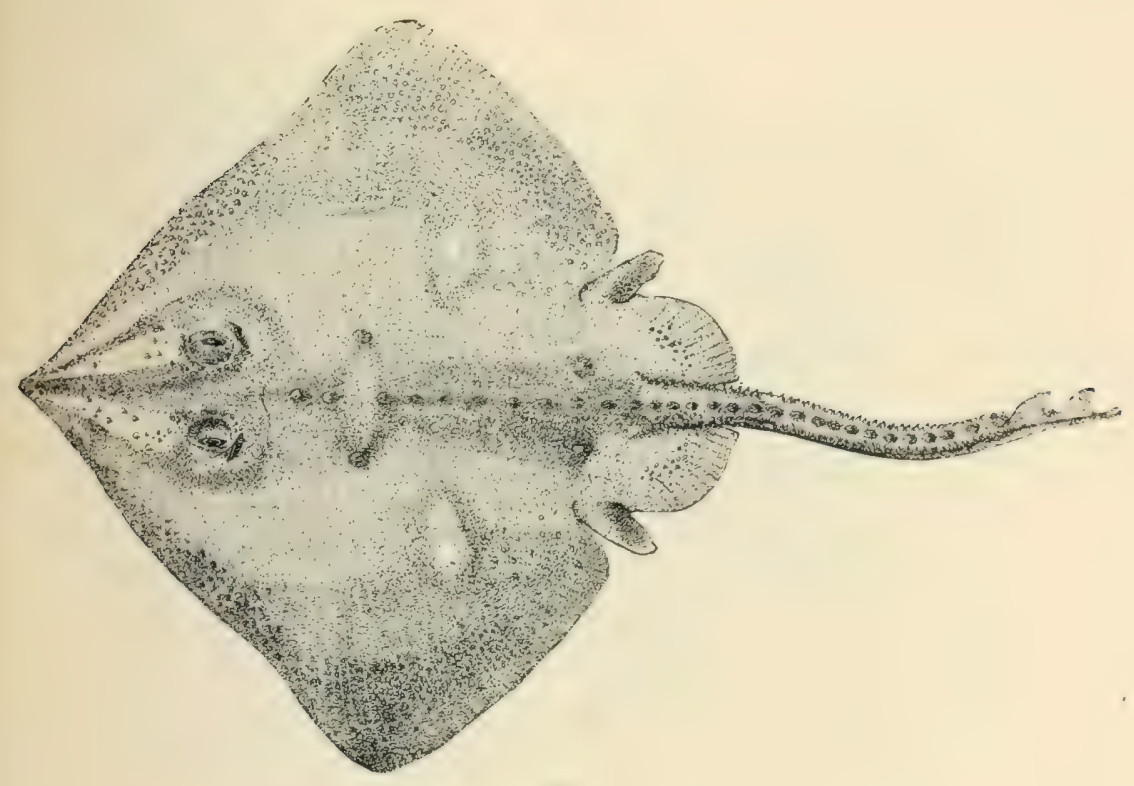

32

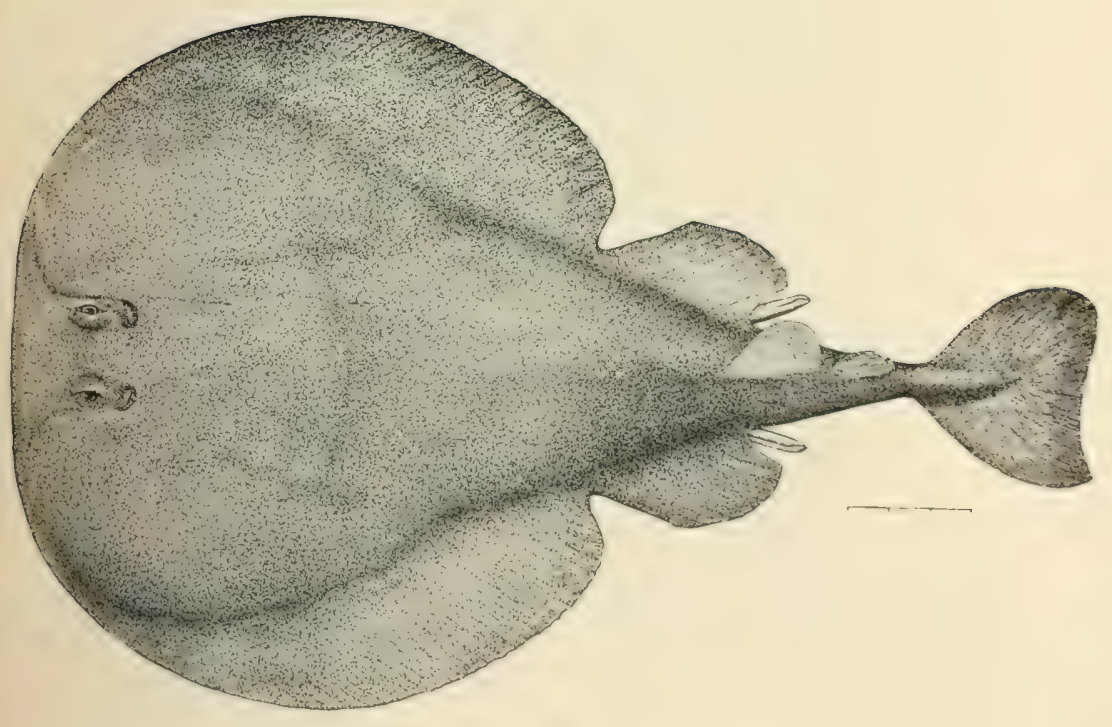

33

32. Ra.tA STELI,ULATA. (P. 75.)

33. Tetronalace ofchintais, (P.77.) 



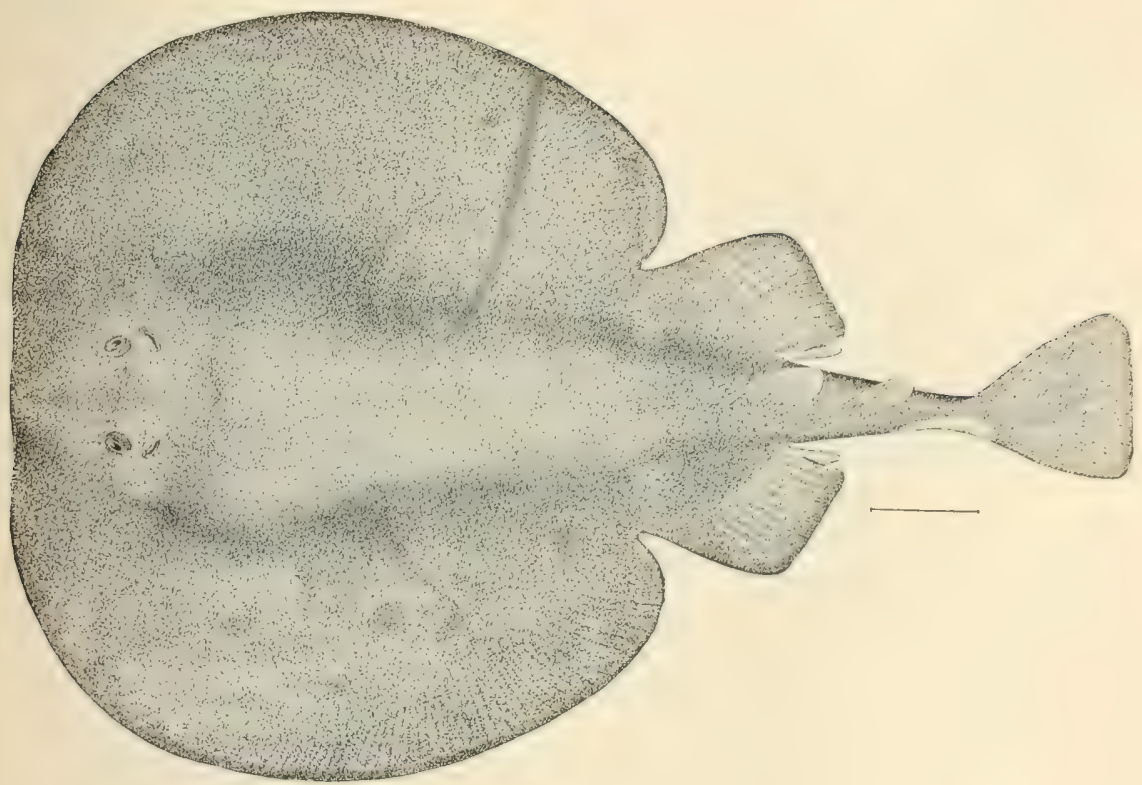

31

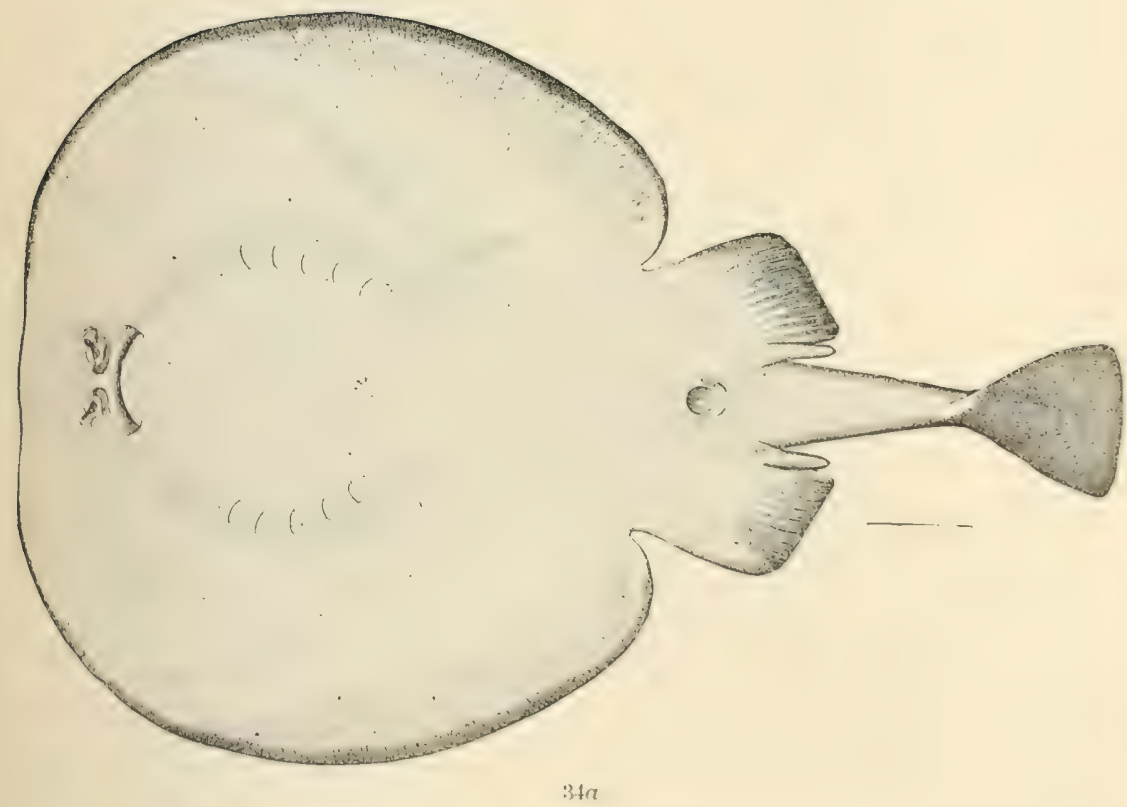

34, 34a. Tetronaren calmomica. (1'.77.) 



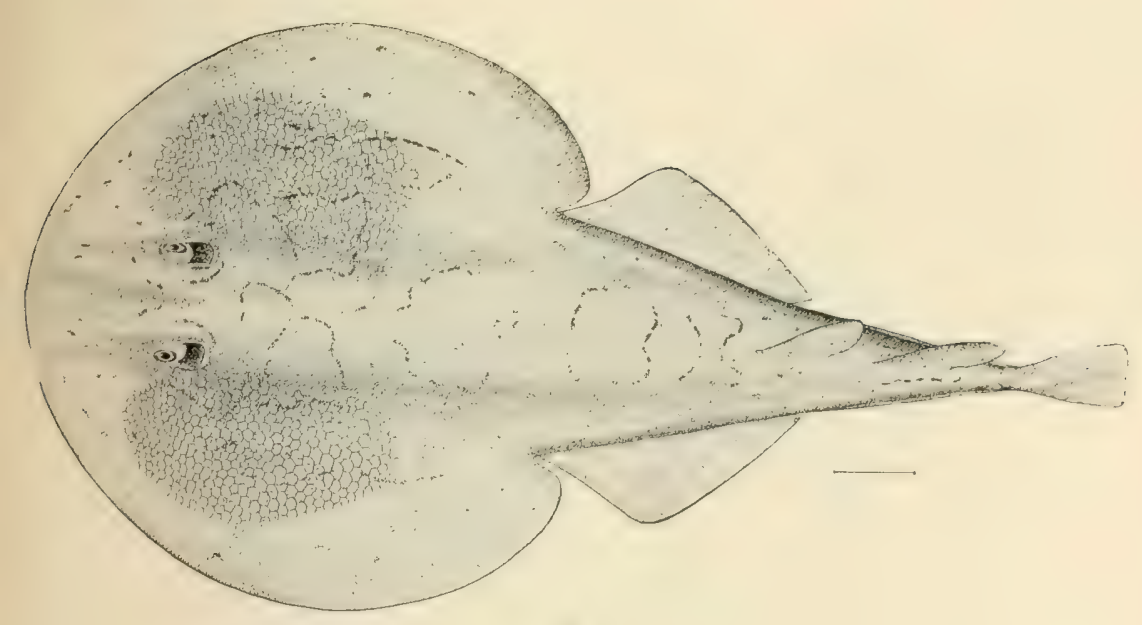

3.)

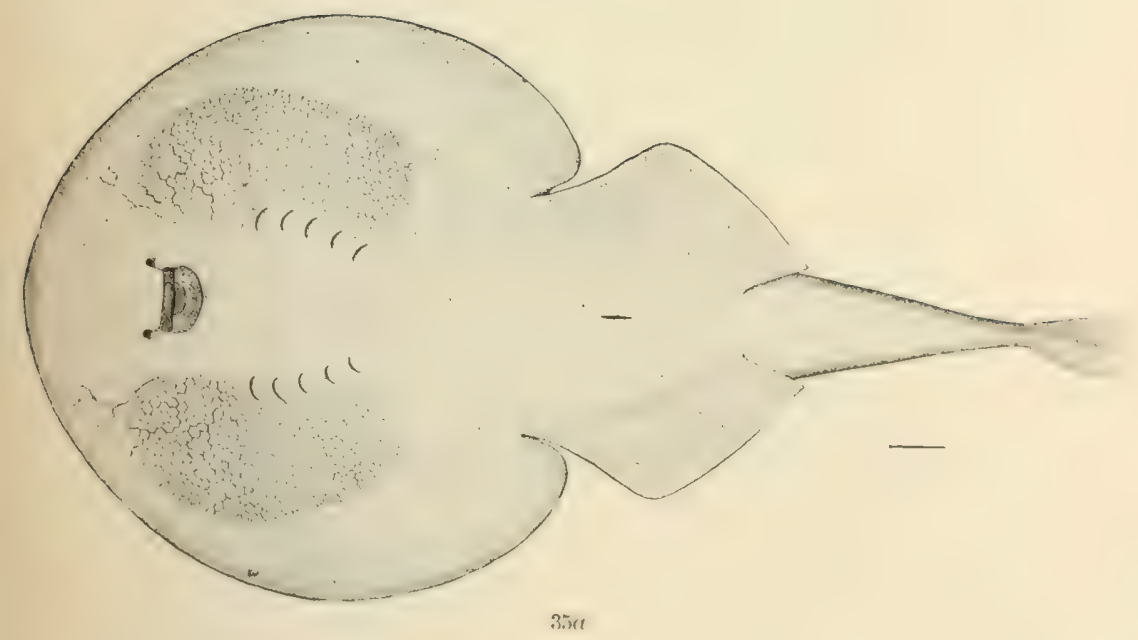

35, Зรа. Narcine mRasiliensis. (1'.78.) 


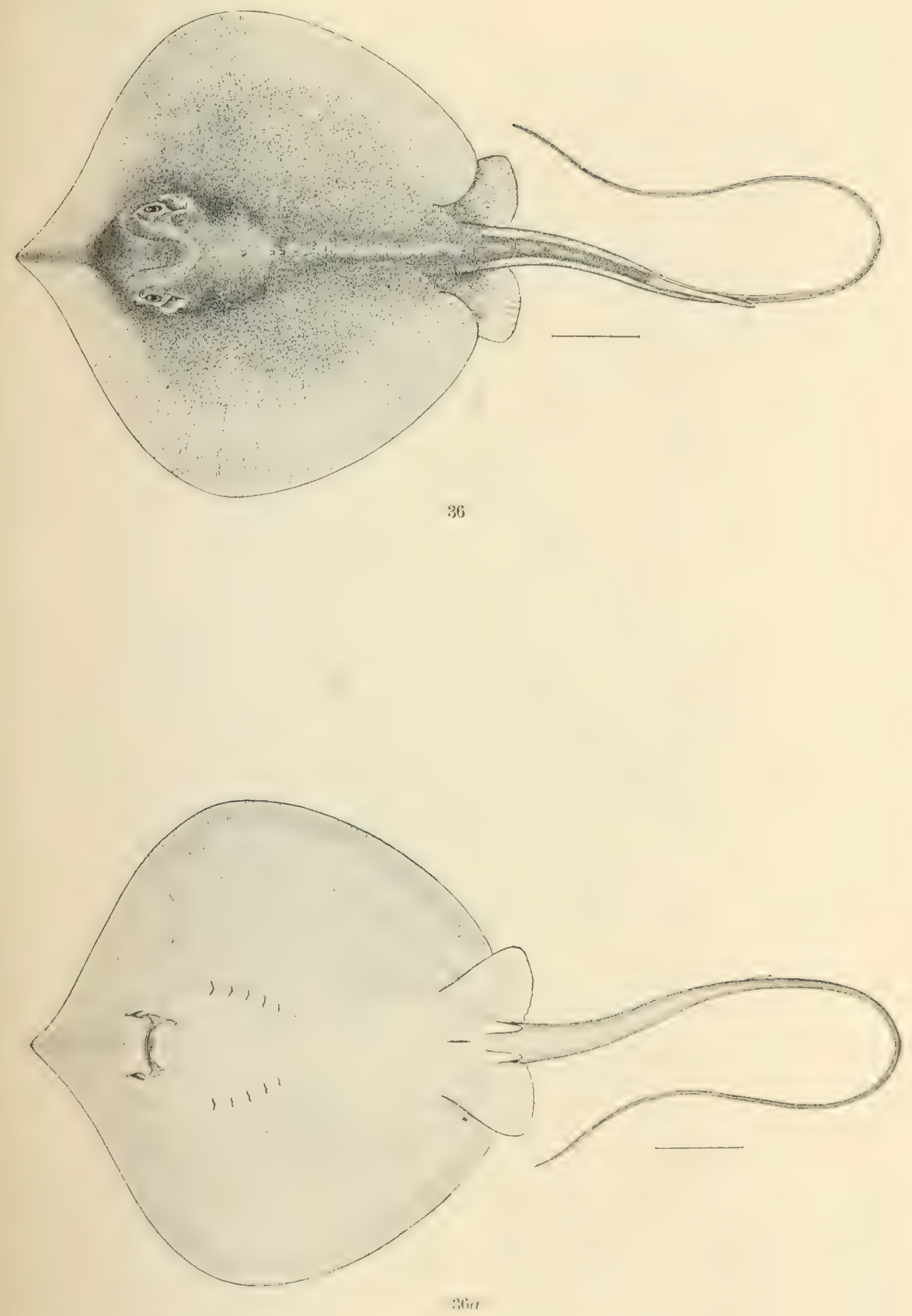

36, 36a. DAsyatis SAMANa. (1',84.) 



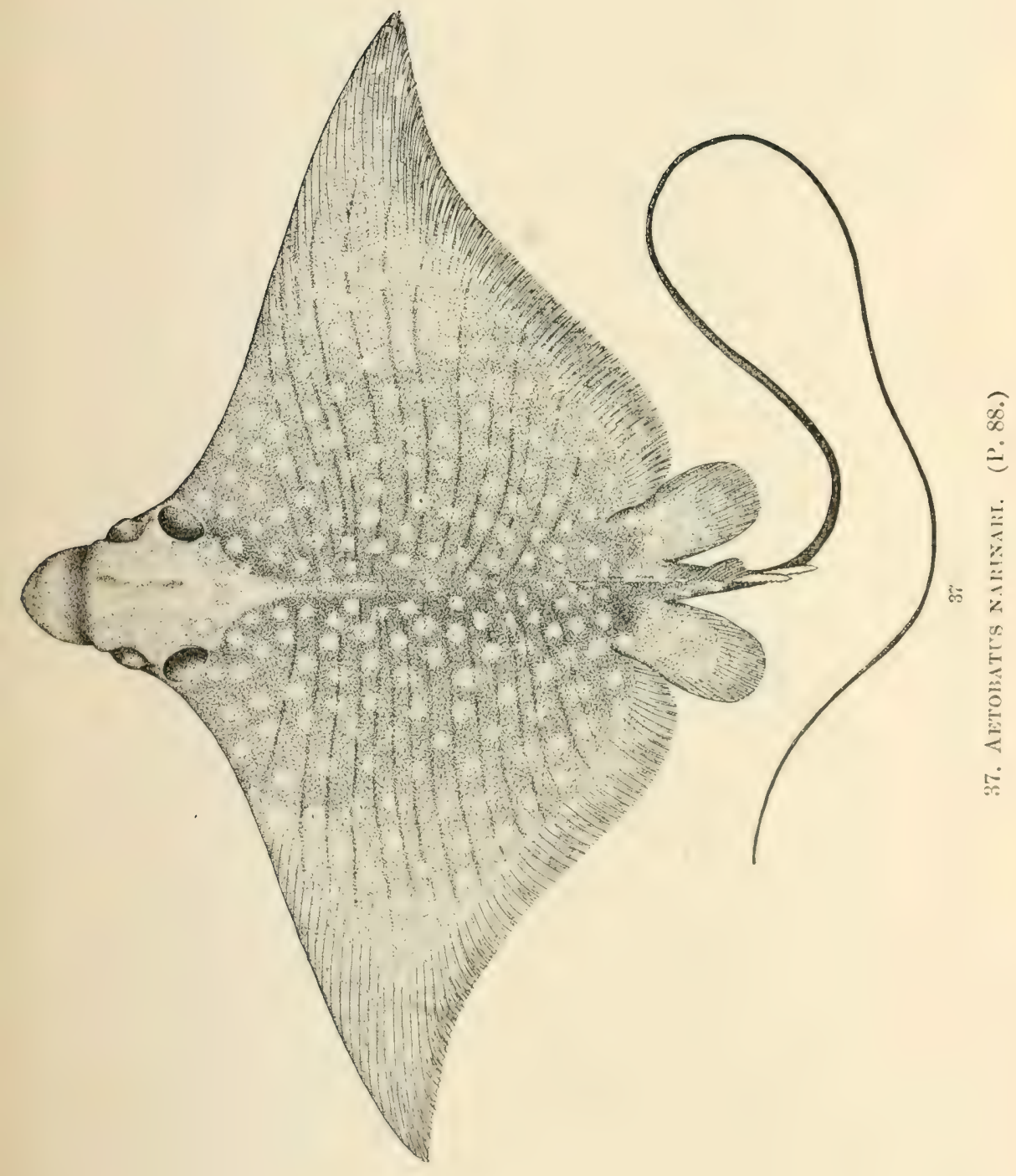





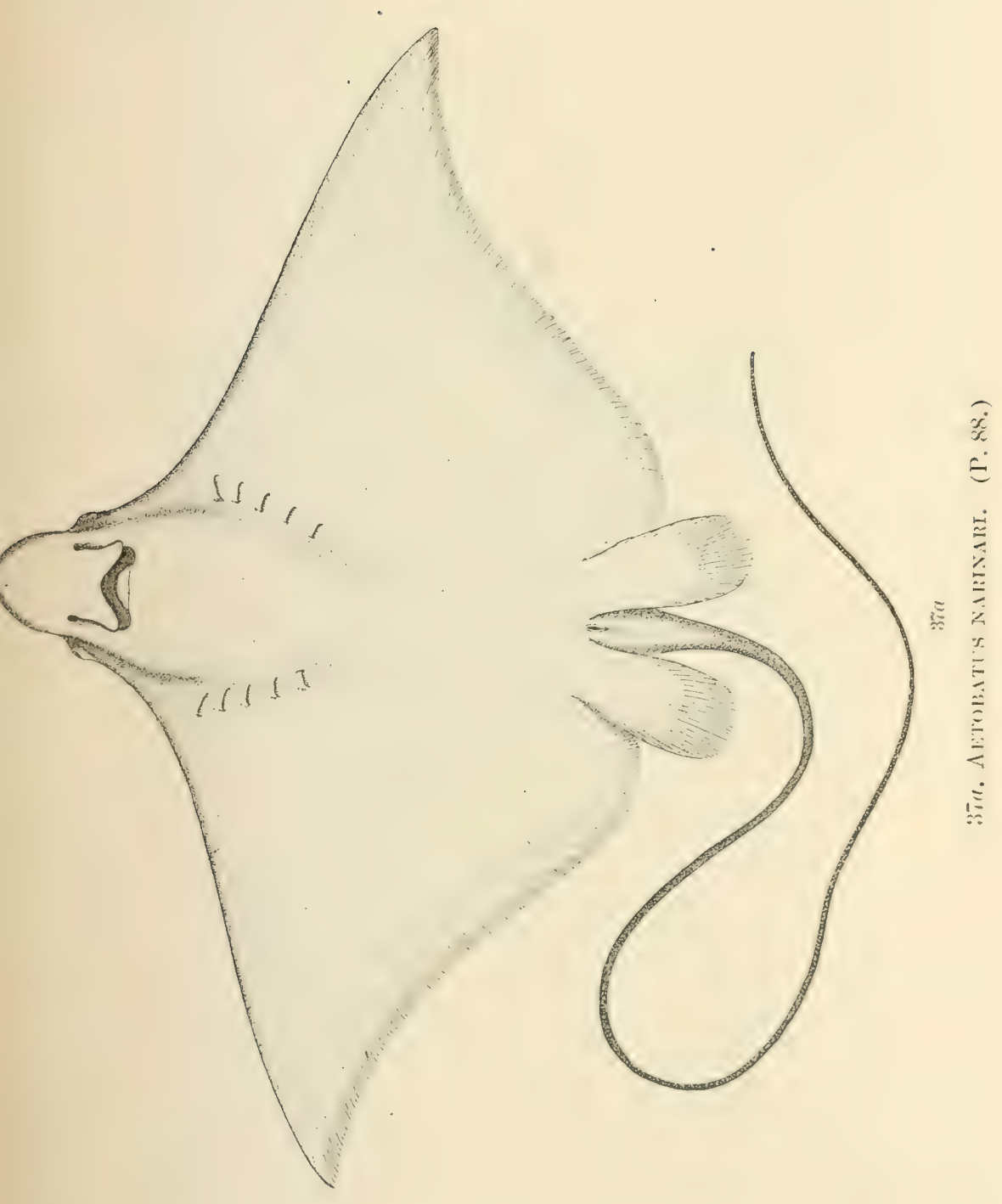





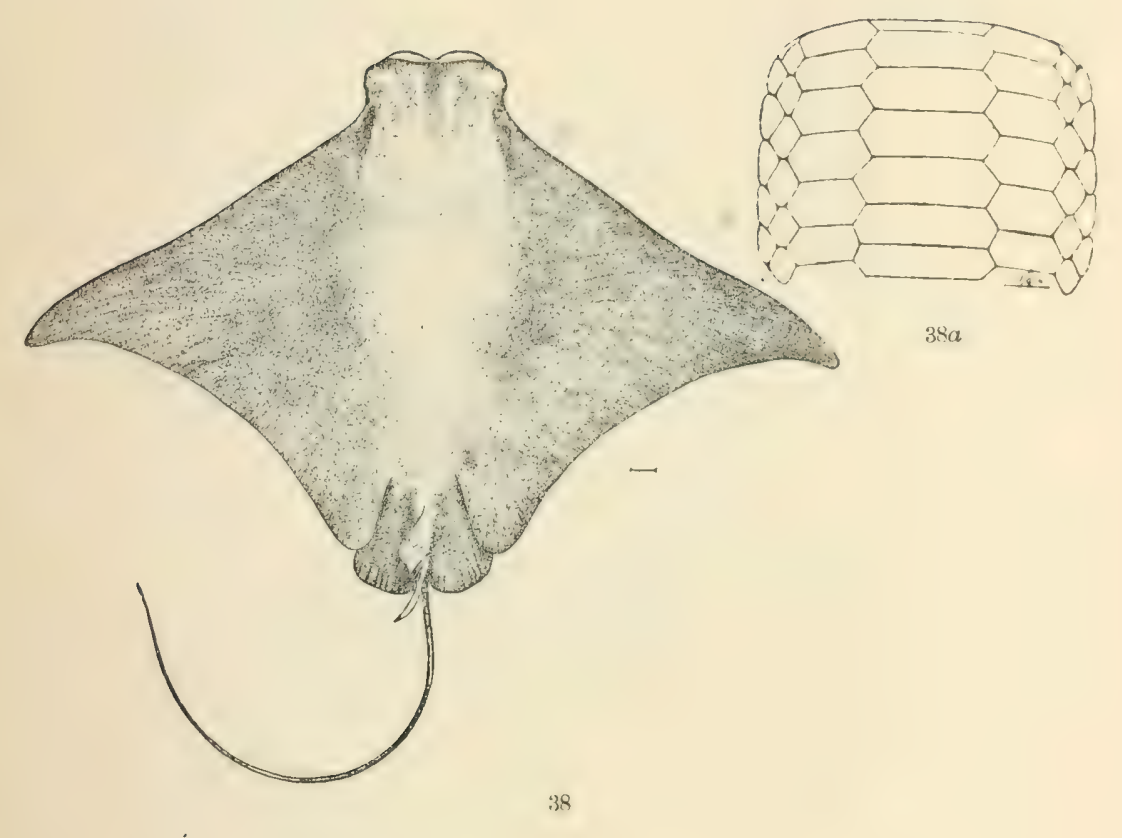

38. RHinoptera steindaCHNERI. (P. 91.)

38a. Paved teeth of Rirnoptera steindachineri. (P. 91.) 



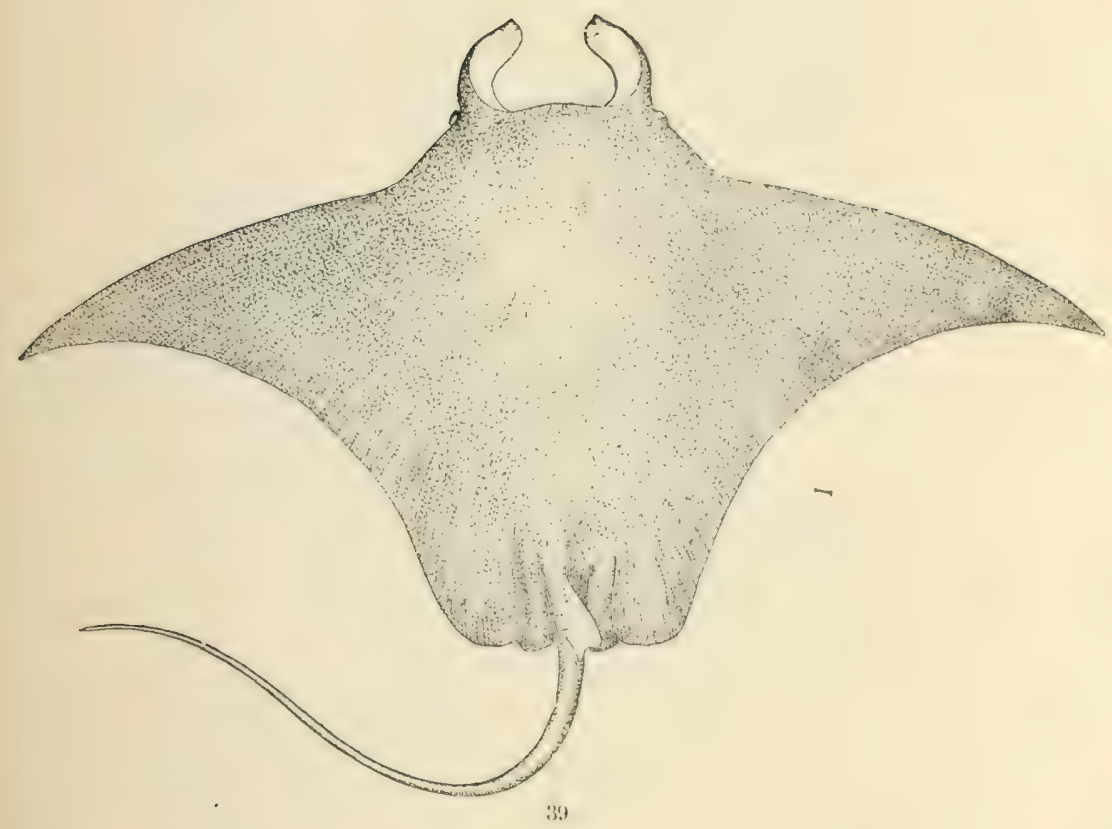

39. Manta bhostris. (P.92.) 


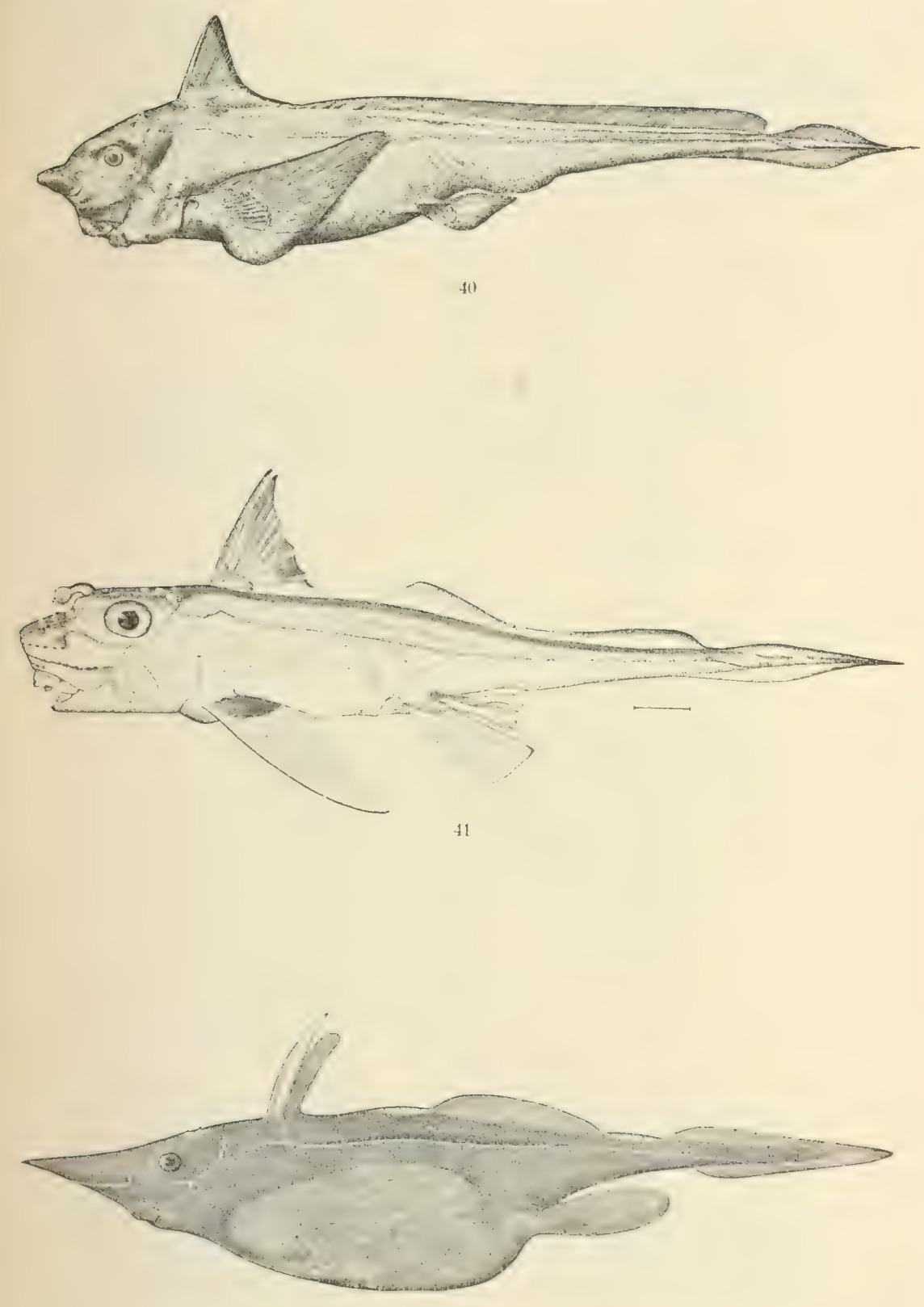

1:

40. Chinera arminis. (P. 95.)

41. Hydrolagus Colirier. (P.95.)

42. HakmotTa malemgihana. (P. 96.) 


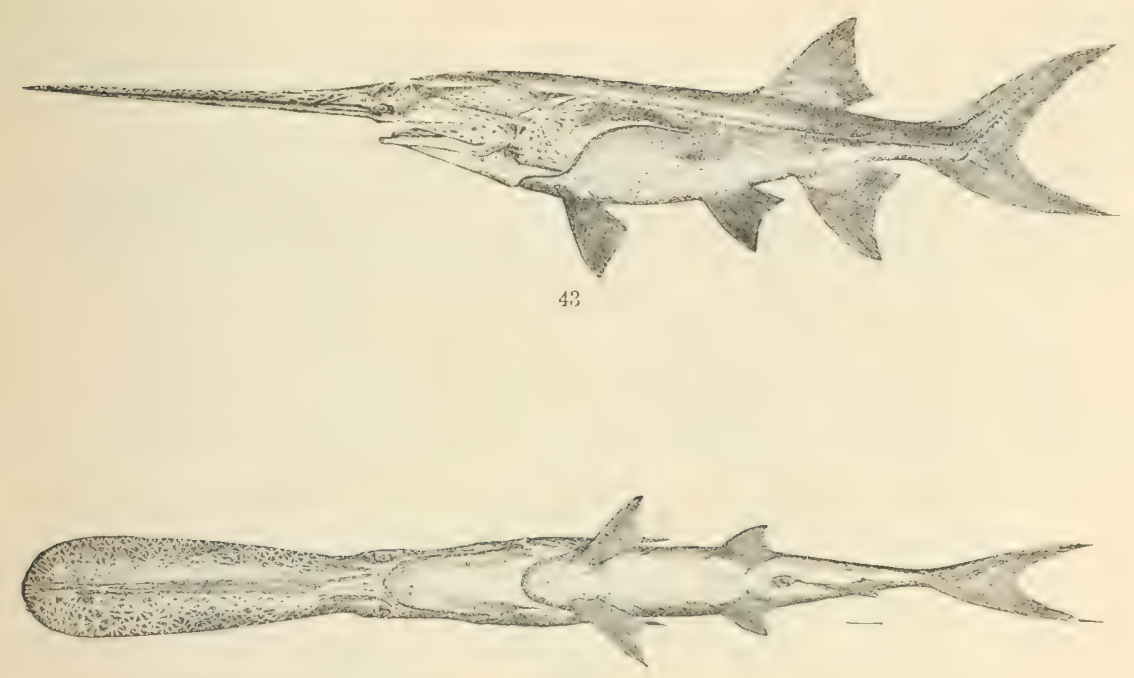

4:ie

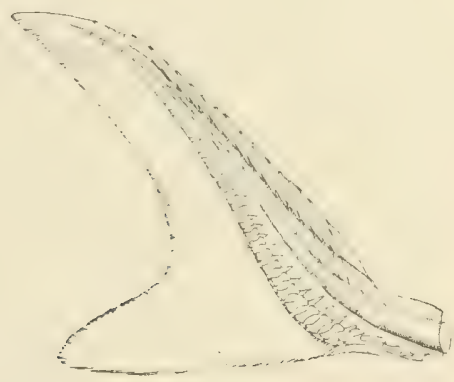

41

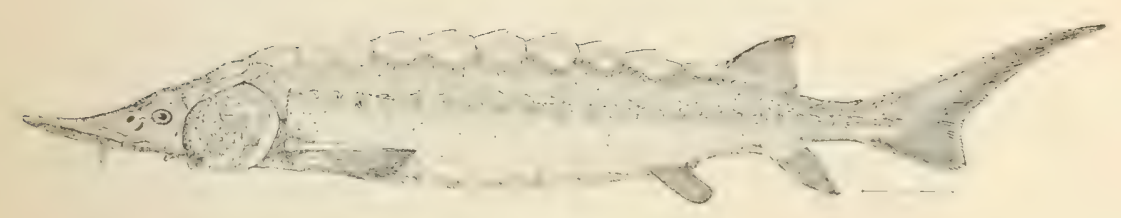

43, 43a. Polyodon spatnuta. (P. 101.)

44. Heterocercal tall of Acirenser transmontanus. (P. 104.)

45. ACIPENSER Sturio) OXYrin'NChUS. ( $P, 105$. 



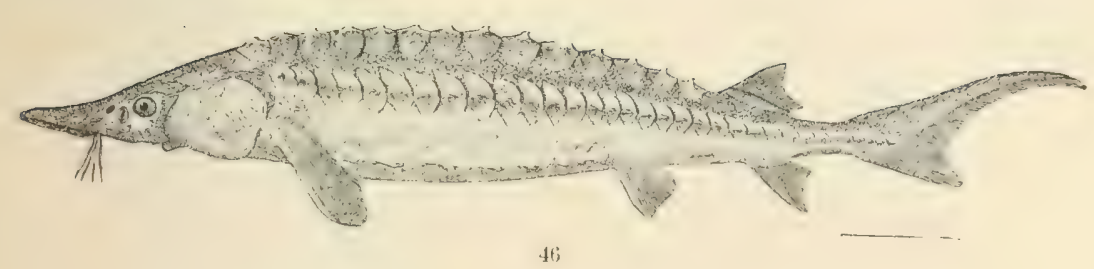

$4 i$

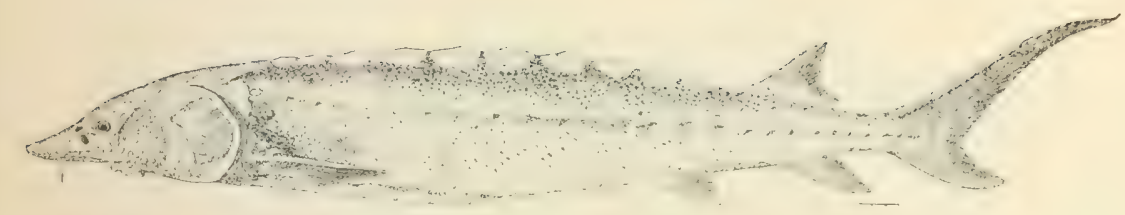

$4 i$

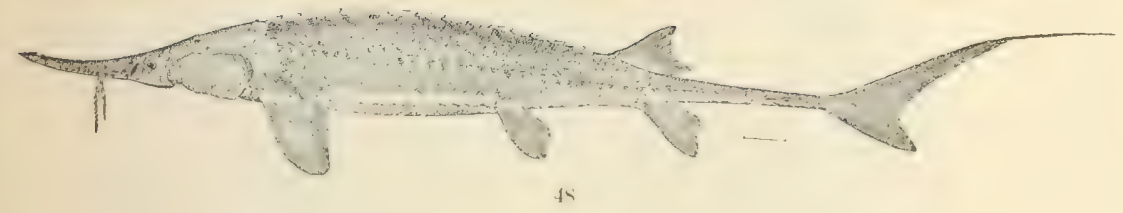

46. Acipenser rubicundus. (P. 106.)

47. Achinser breyrostrum. (P. 106.)

48. SCAPHIRHYCHUS PLATORYNCHUS. (P. 107.) 



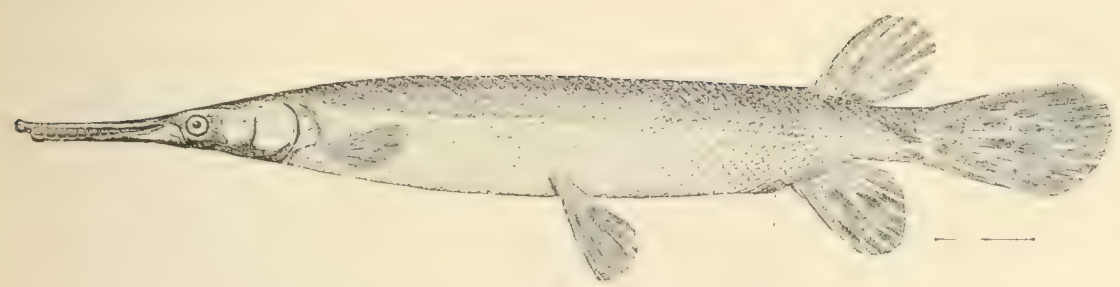

49

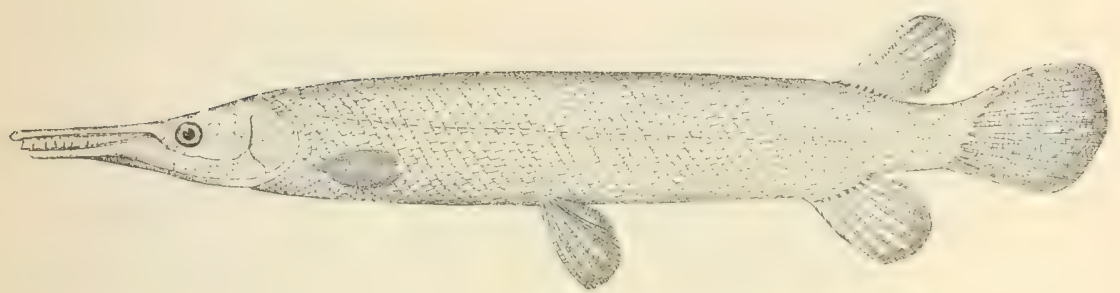

j)

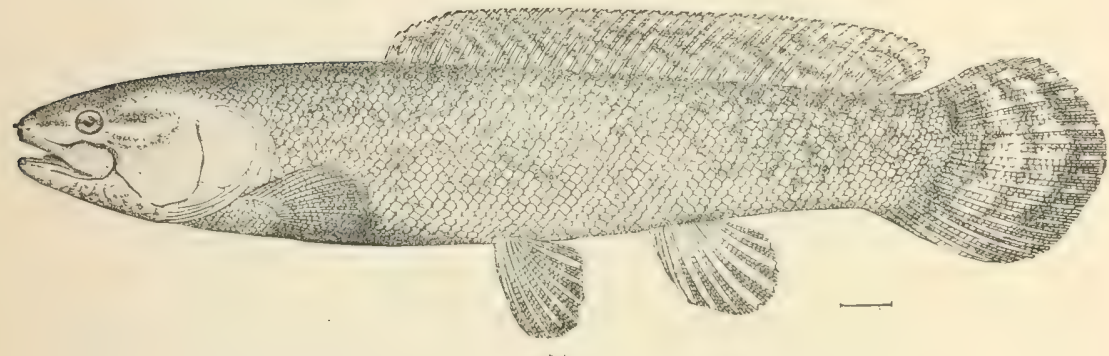

51

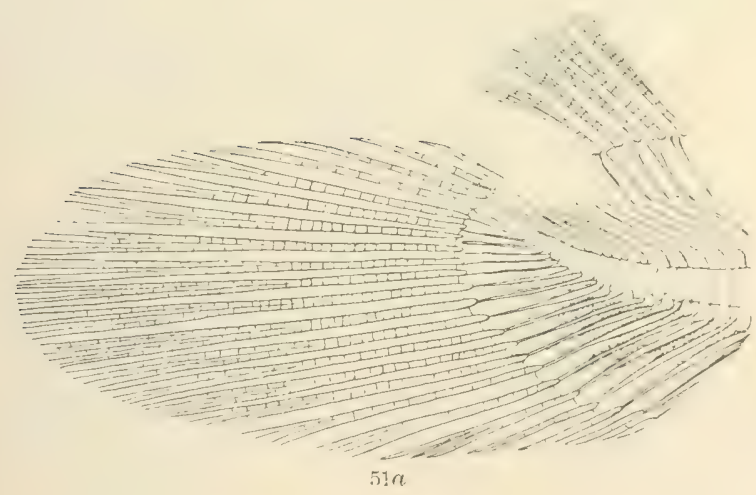

49. IEPISOSTELS PIATOSTOMIS. (P. 110.)

50. LEPISOSTEI'S TRISTCECIUN. (l', 111.)

วั1. ANIA (ALVA; female. (I'. 113.)

5 la. HeTElOOCERCAL TAIT, OH AMIA CAIVA. P. 11:3.) 


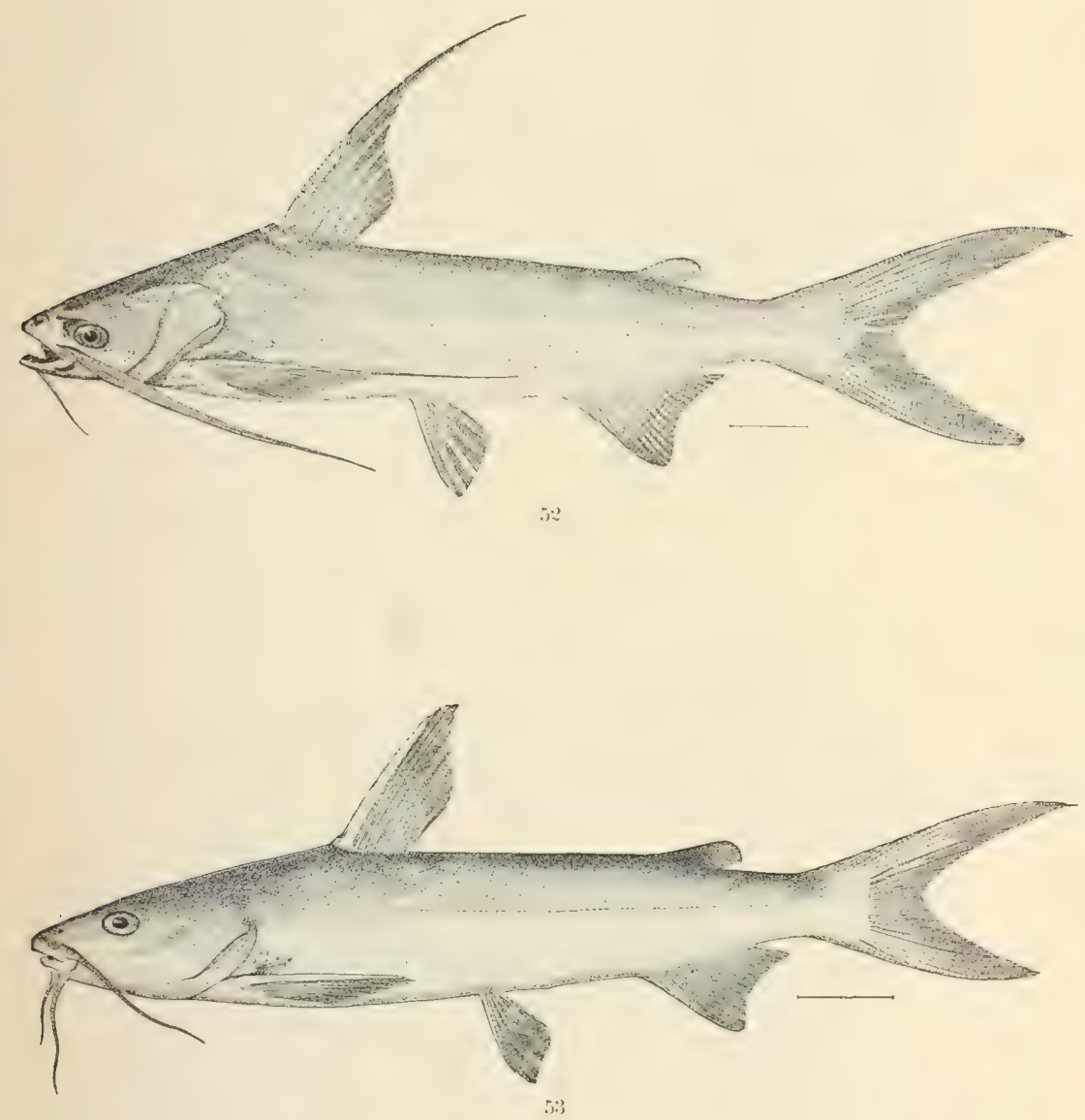

52. Felichthys marinus. (P. 118.)

53. Galeichtiys Felis. (P.128.) 



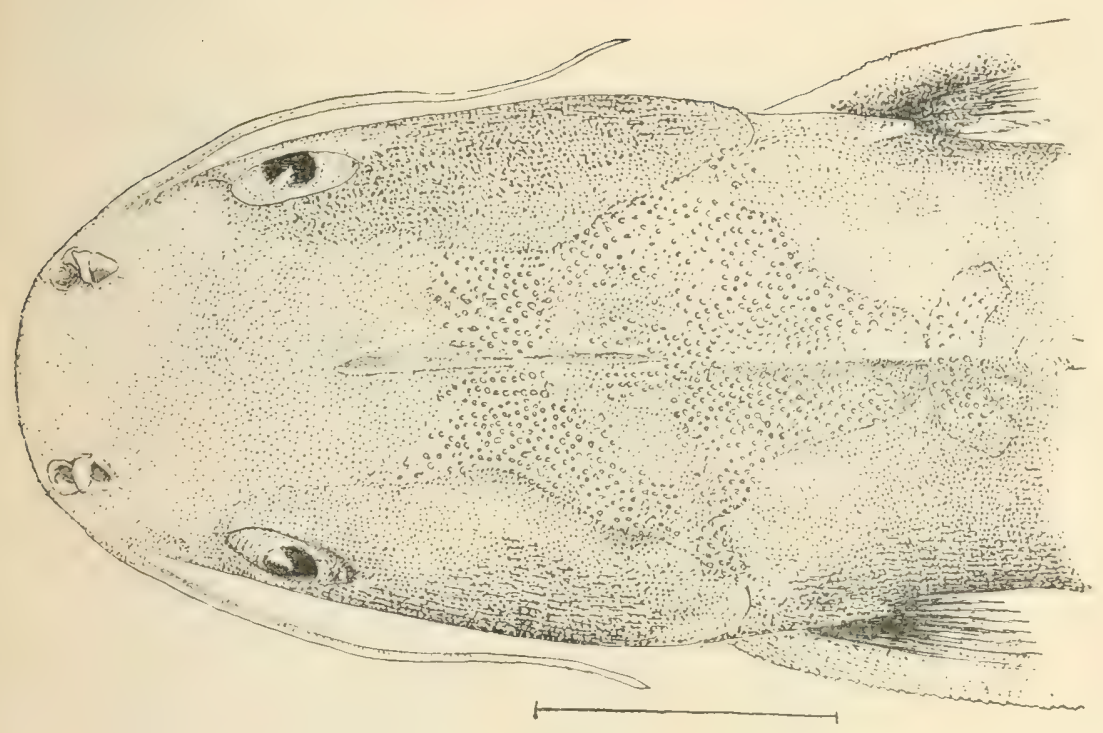

54

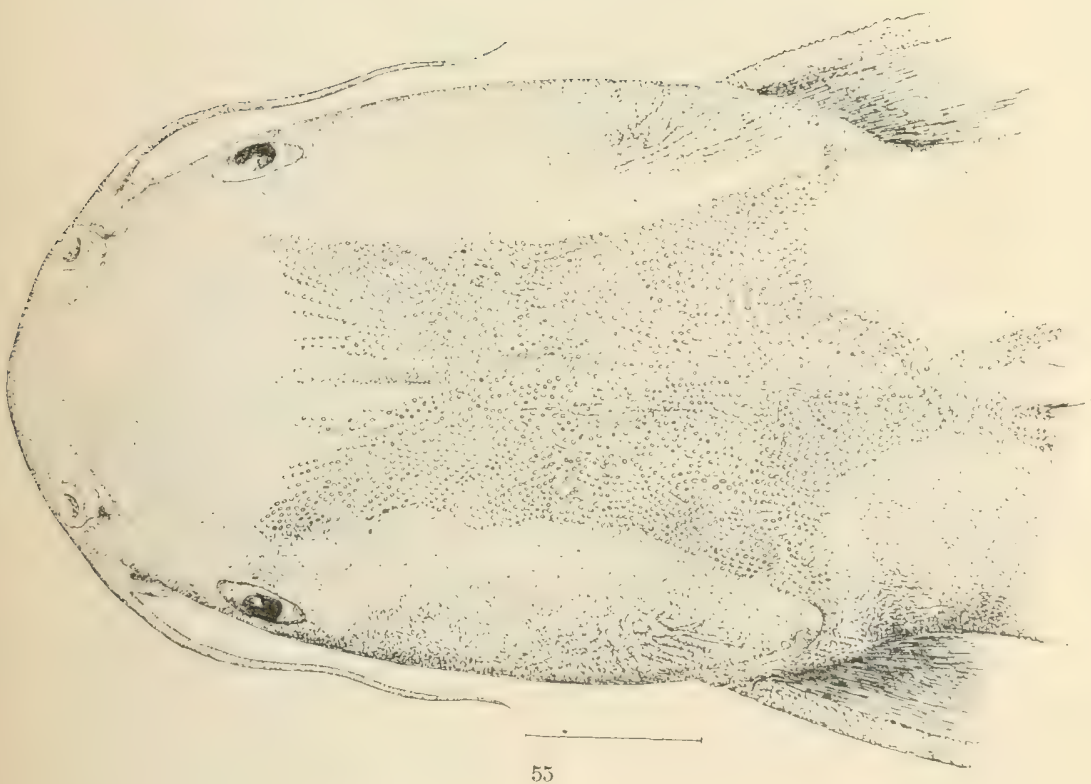

51. ('ALEICHTHY GILBERTI. (1'. 2773.)

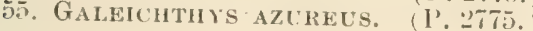




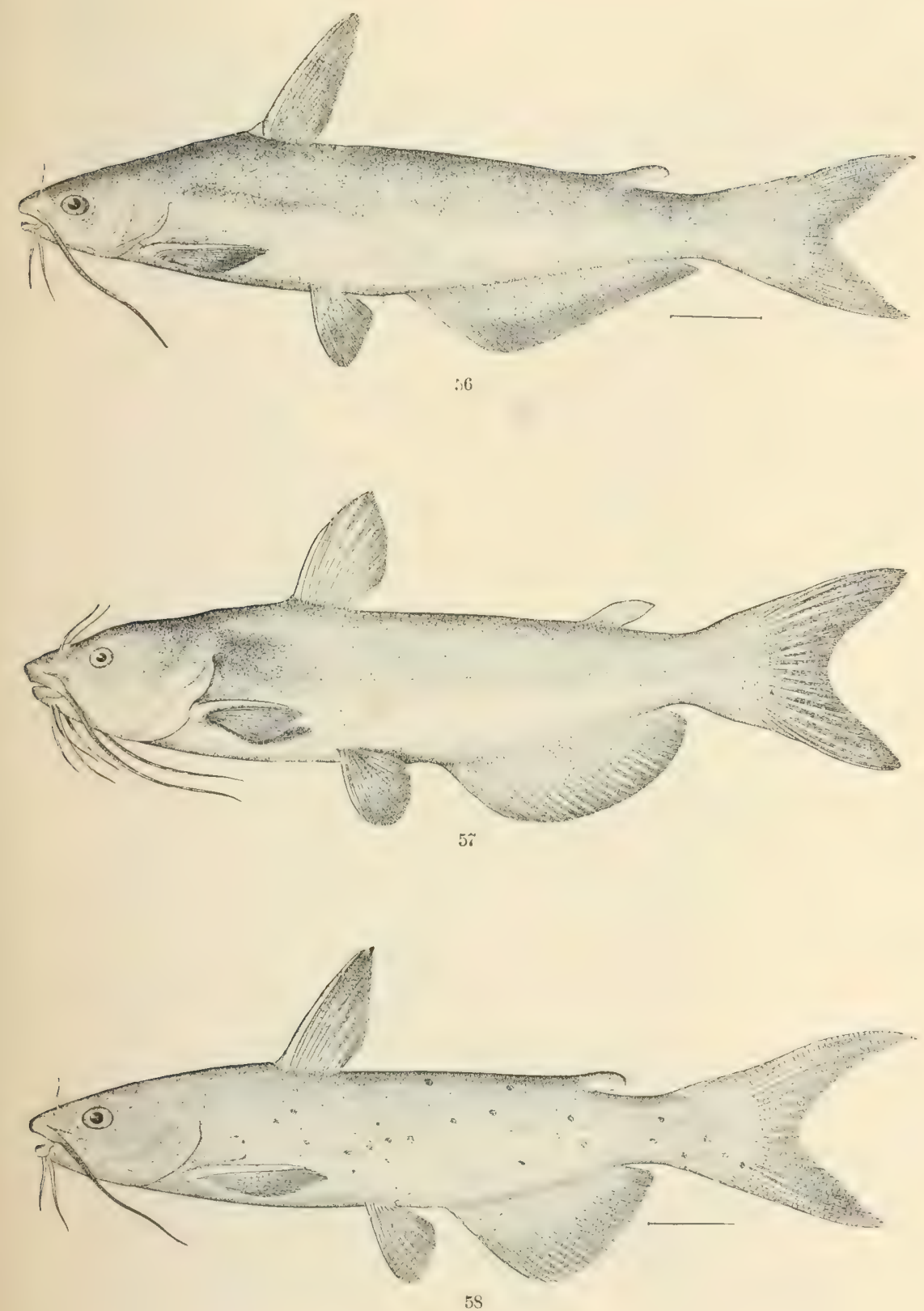

56. Ictalurus furcatus. (P. 134.)

57. ICtalurus aNGUilla. (P. 2788.)

58. ICtalurus punctatus. (P. 134.) 



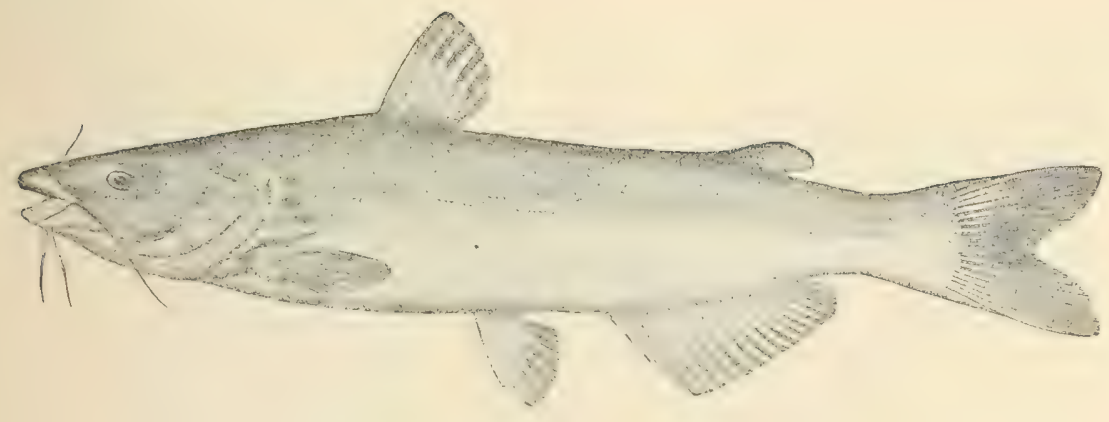

59
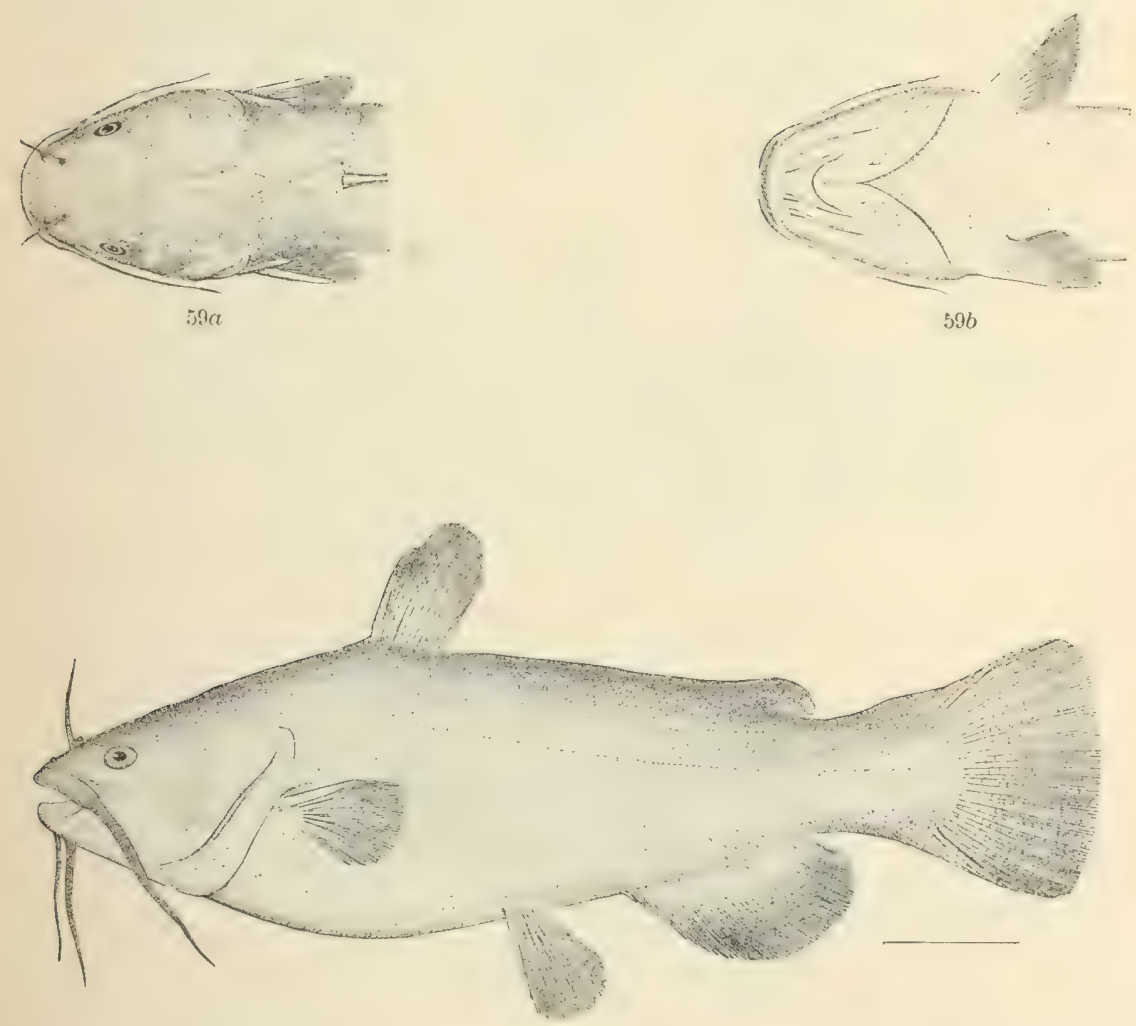

60

59, 59a, 59b. Ameiurus DUGESI. (P. 138.)

60. Aimiures melas. (P. 141.) 


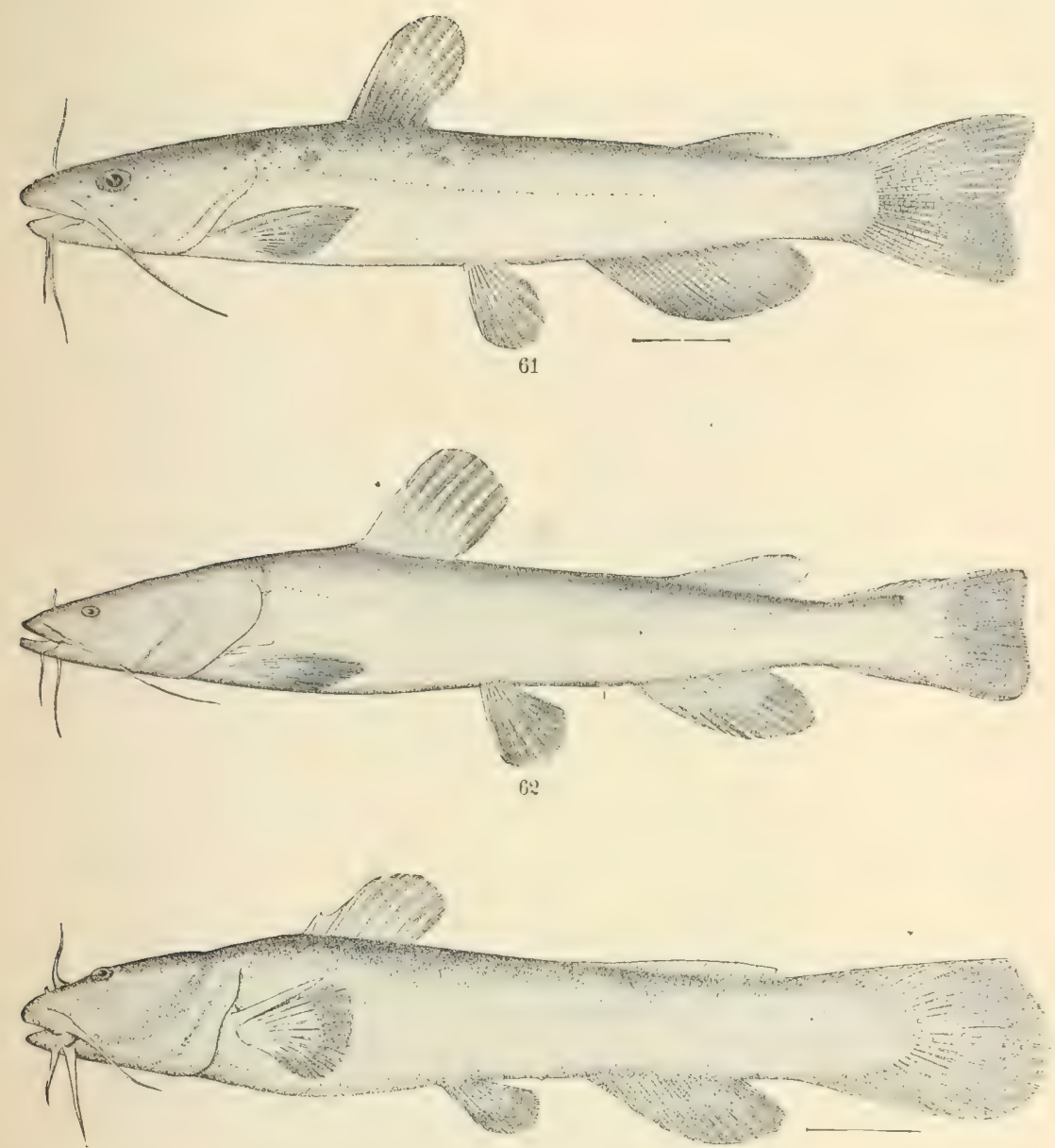

63

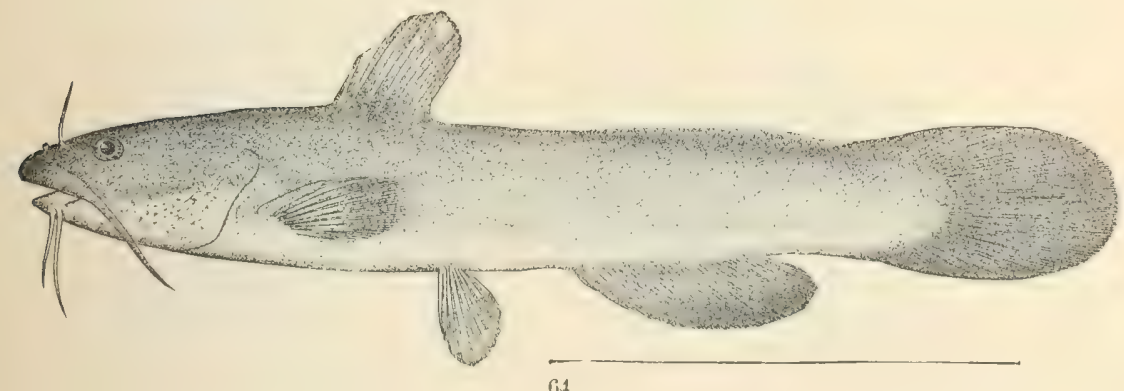

61

61. Ameivrus platycephalus. (P. 142.)

62. LEPTOPS OLIVARIS. (P. 143.)

63. Noturus Flavus. (P. 144.)

64. SCHIlbeodes Nocturnus. (P. 146.) 



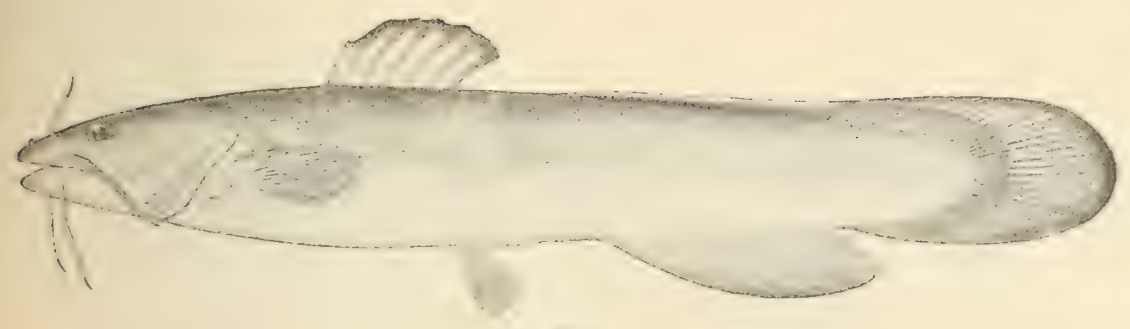

(i.)

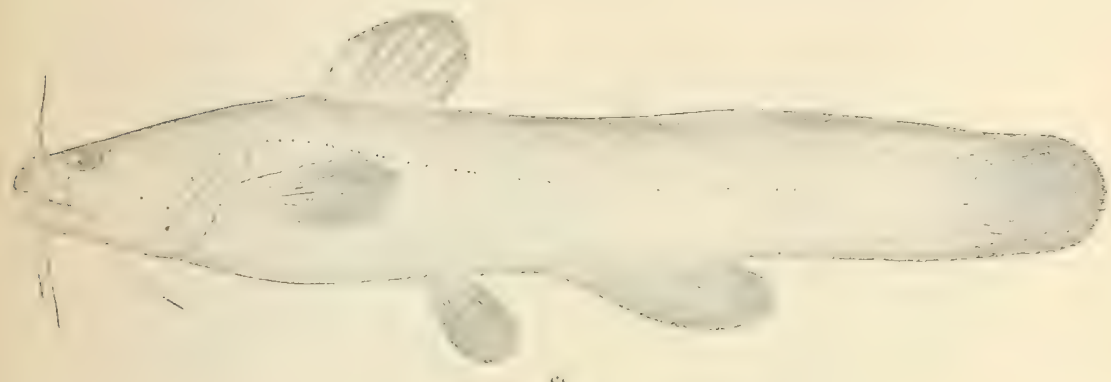

ii,

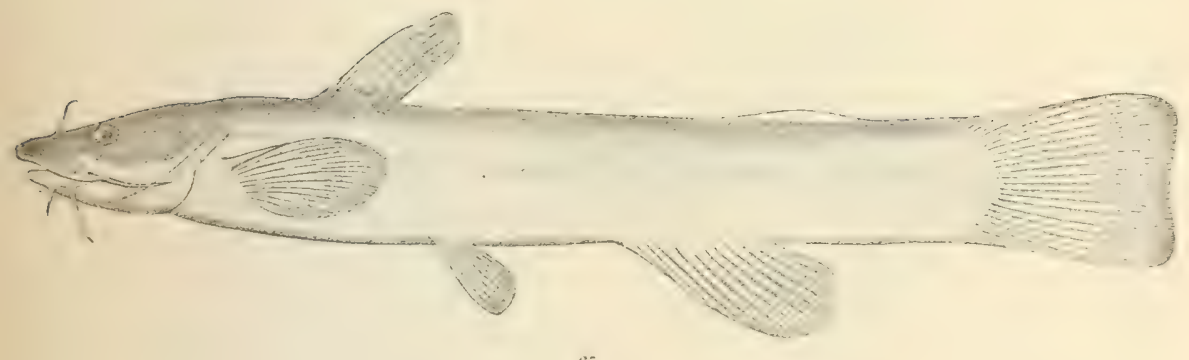

5i:
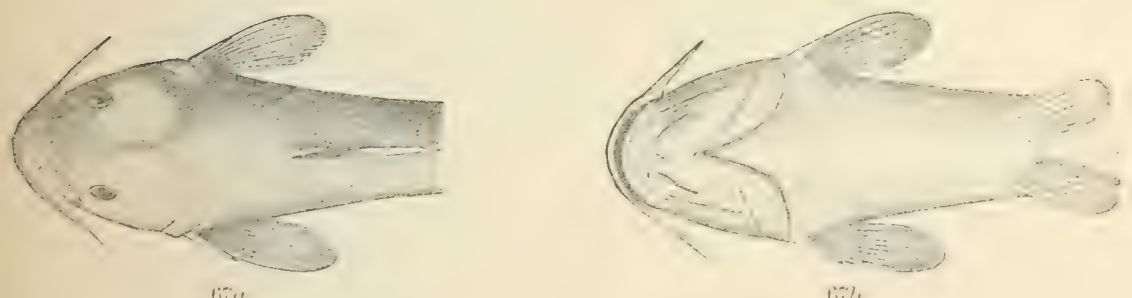

tit?

65. Schilbeones exilis. (P. 147.)

66. SCHIIBEODES XNAIGNIS. (I'. 147.)

$67,67 a, 67 b$. SCHILlieodes GILberti. (P. 148.) 



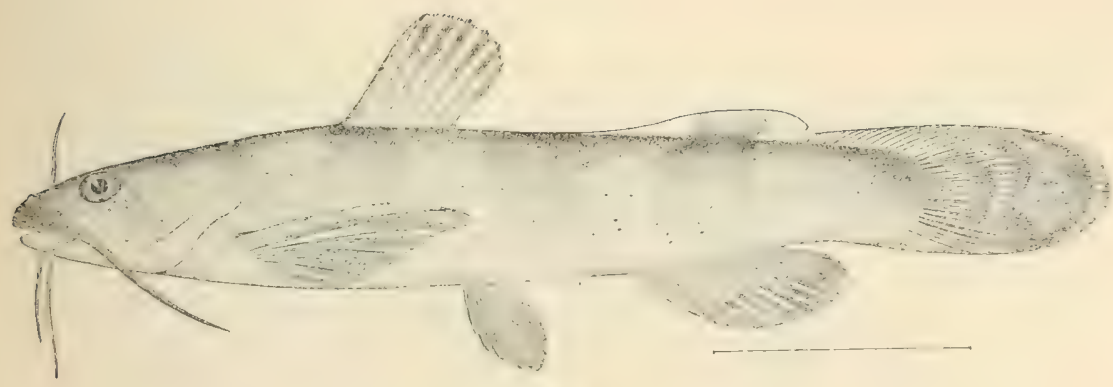

bis

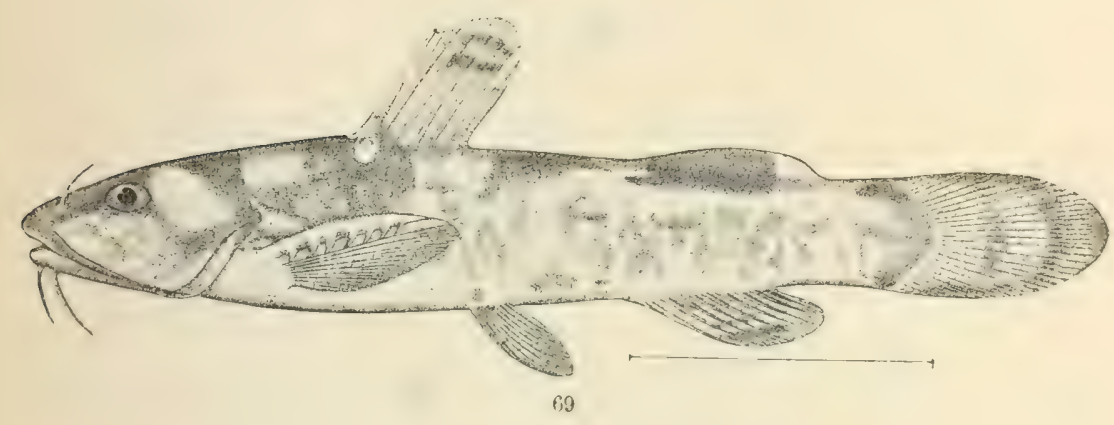

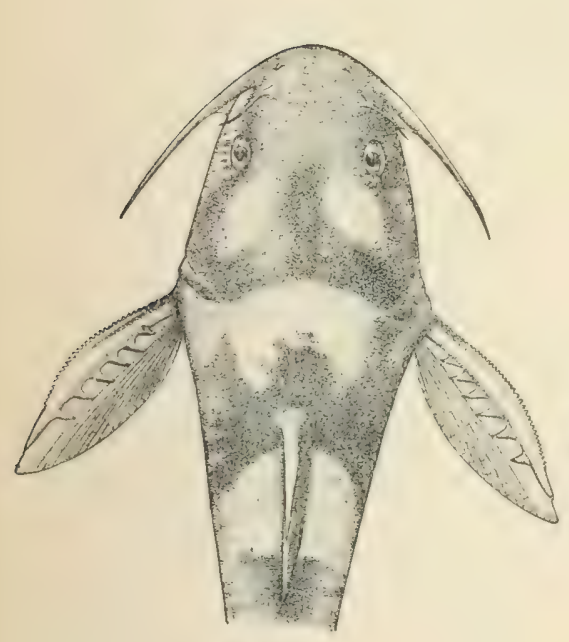

(i)!c,

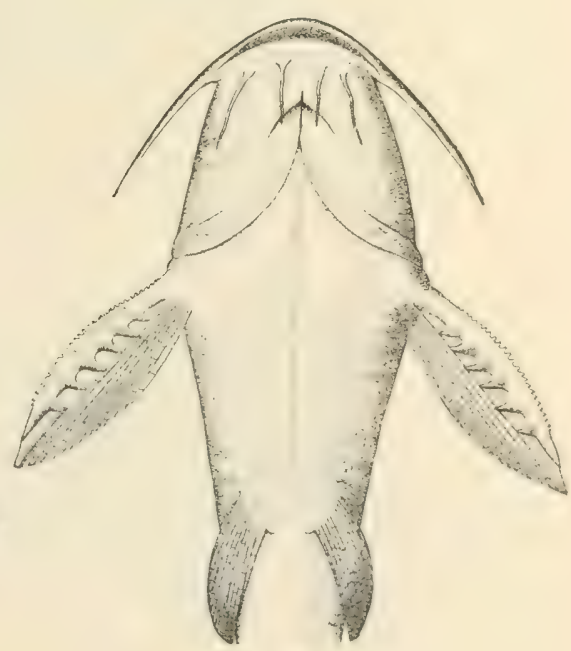

$6: 37$

68. Schllbeodes mirrus, (P. 148.)

$69,69 a, 69 b$. SCHILEODES FURIOSUS. (P.149.) 


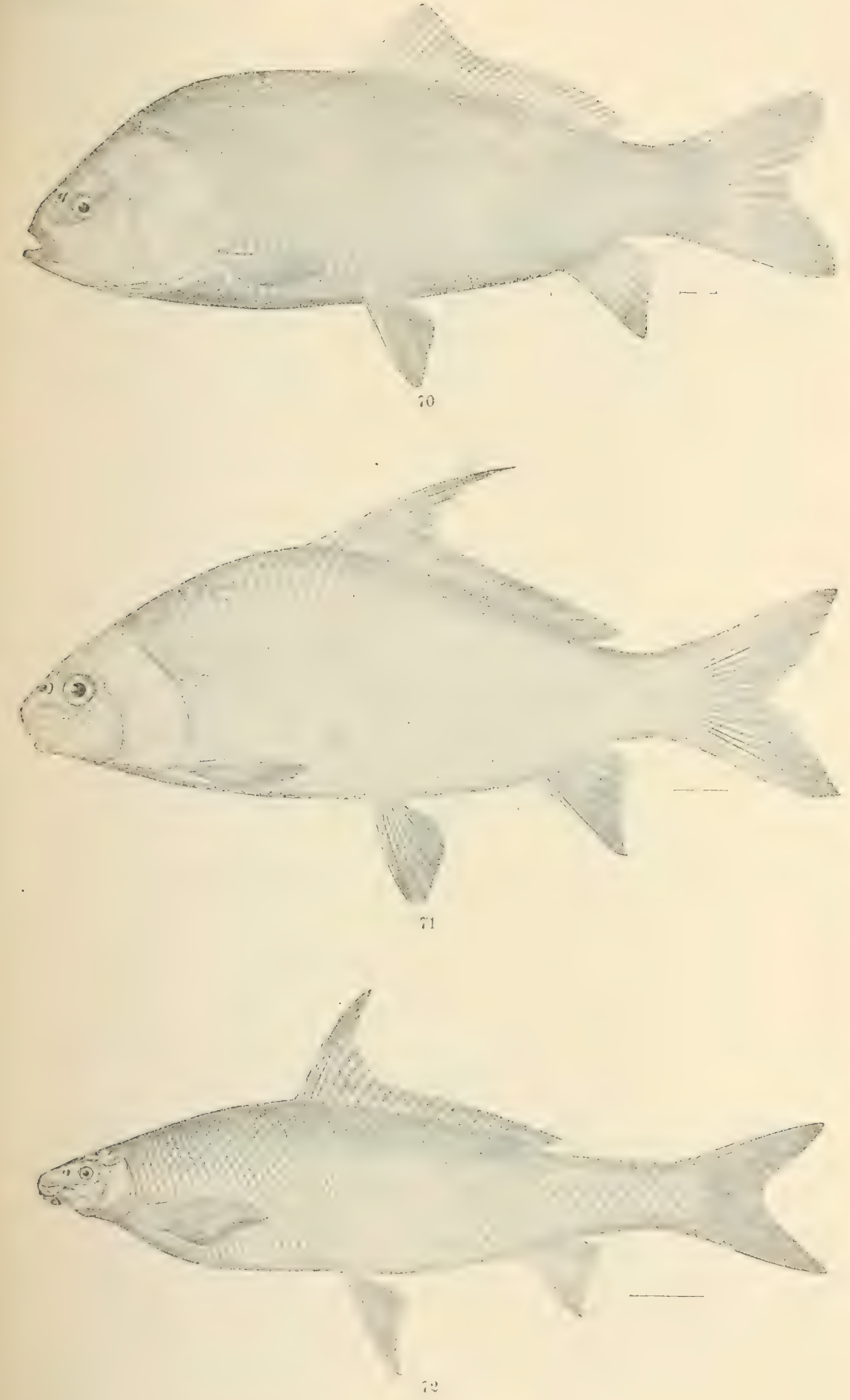

70. ICtoben cyptinetala. (P, 163.)

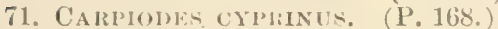

72. CyClempl's houngatus. (P. 168. 



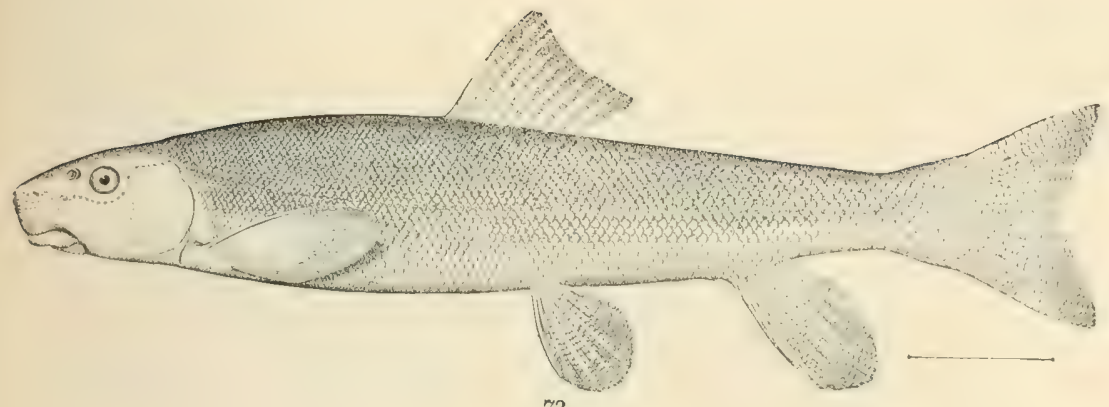

73
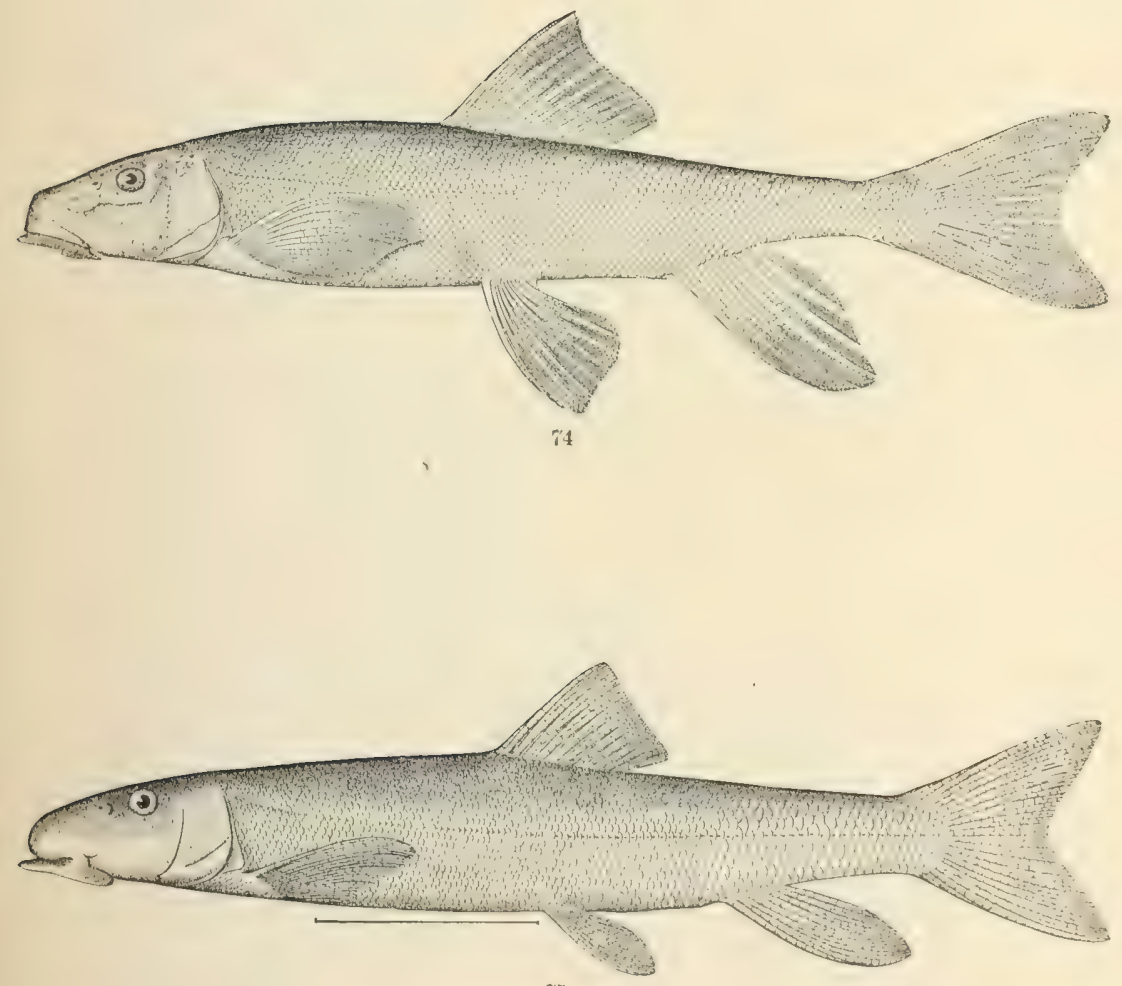

75

73. Pantosteus JoRDani. (P. 171.)

74. Catostomus latipinnis. (P. 17..)

75. Catostomus Girisés. (I', 175.) 



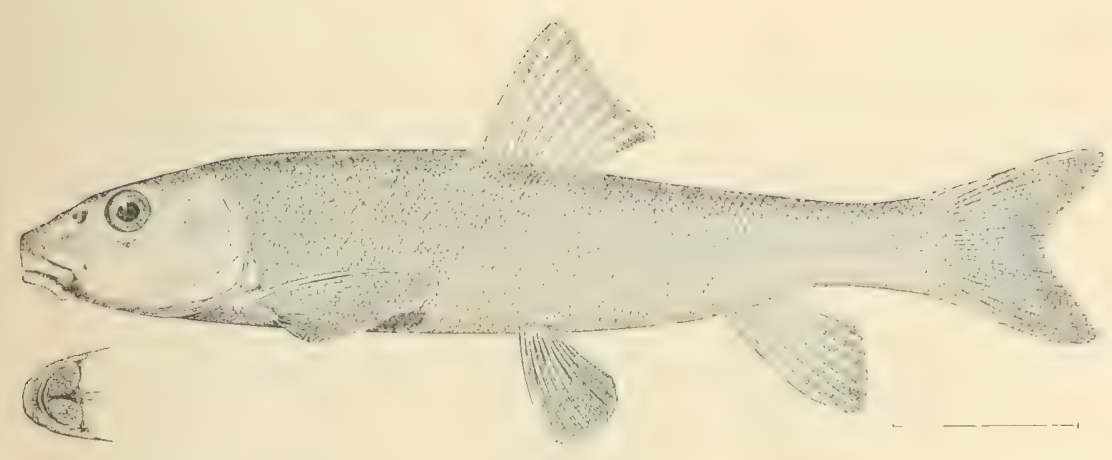

76
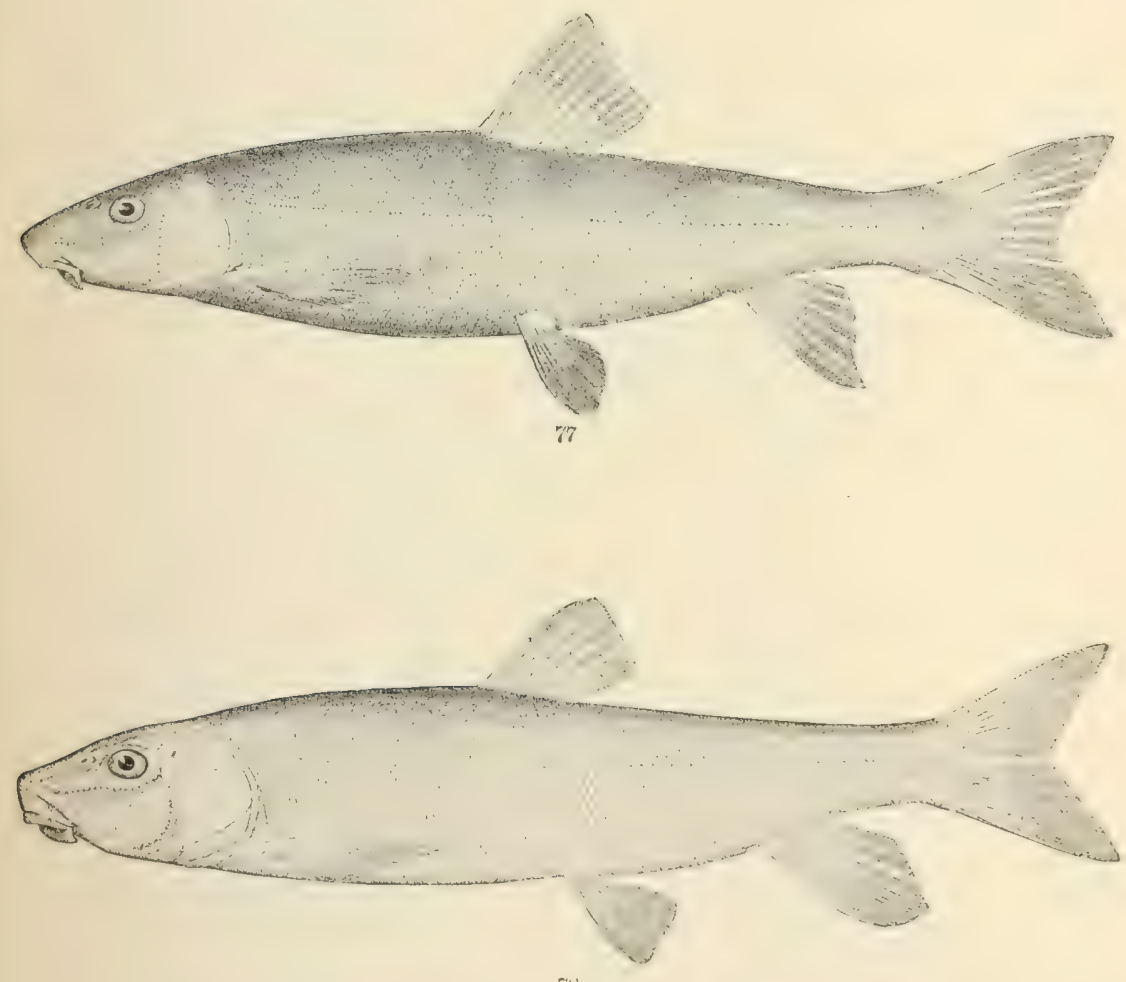

is

76. Catostomus mocatello. (P. 175.)

77. Catostomus CatostomLs. (1'.176.)

78. Catostonus taronnsis. (P.177.) 


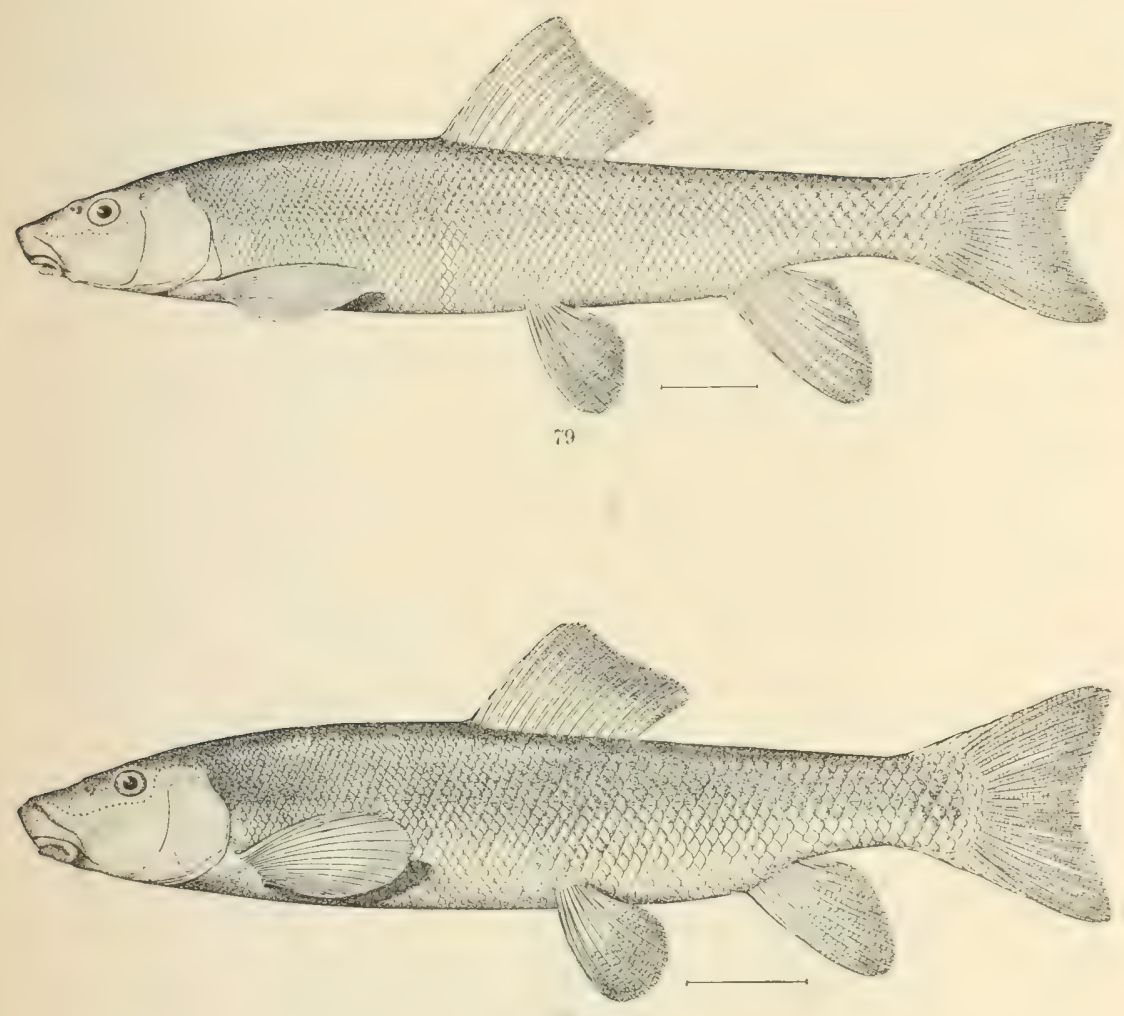

so

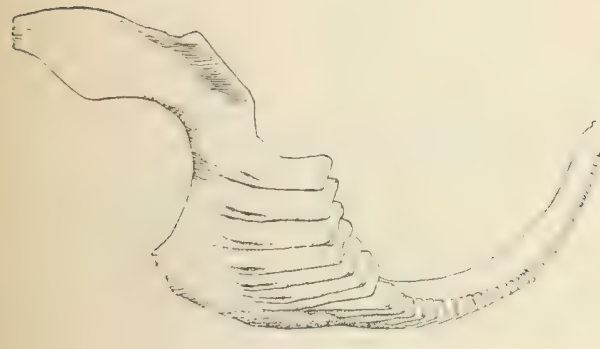

81

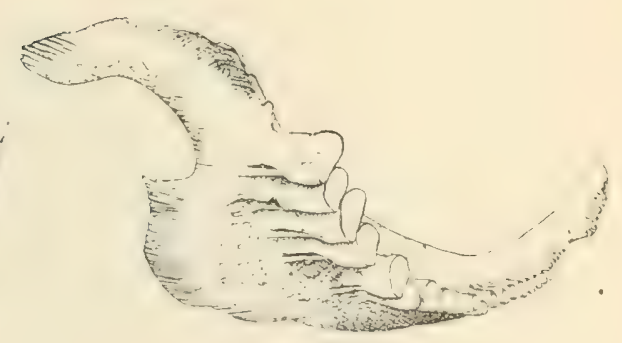

S:

79. Catostomus occmentalis. (P. 178.)

80. Catostomus tsiltcoosensis, (P. 2793.)

81. Pharyvgeal teeth of Catostomus maCrochetius. (P. 178.$)$

8:. Pharyngeal TElith of Pracopilaryal duquesnil. (P. 198.) 



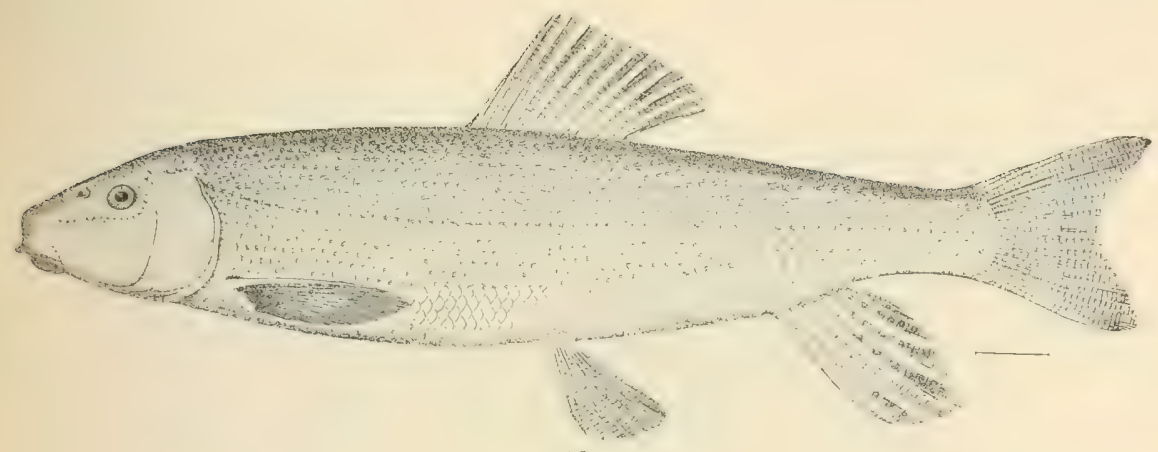

s.3
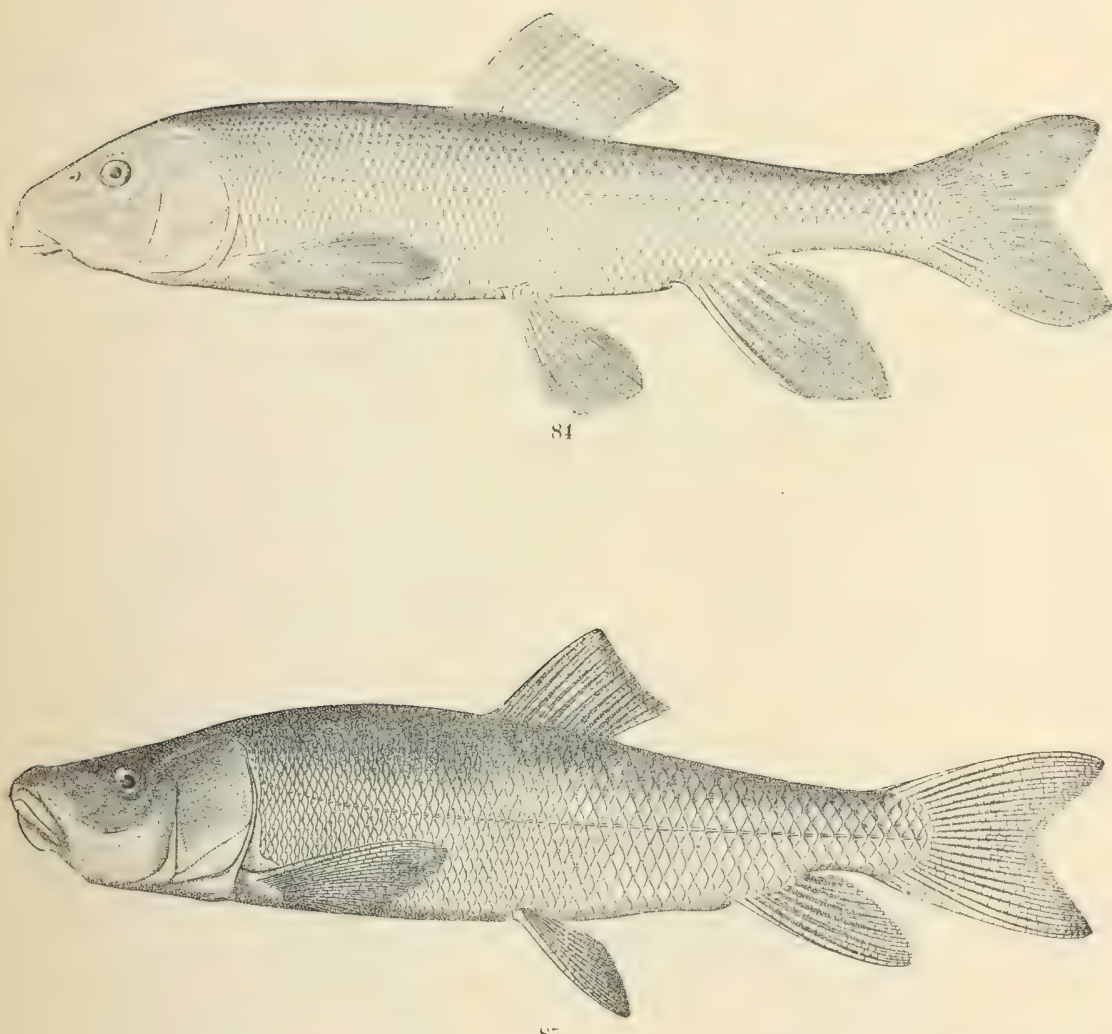

8.i.

83. Catostomus commersonit. (P. 178.

84. Catostomus ardens. (P. 179.)

85. Chasmisteis liorts. (P. 183.) 



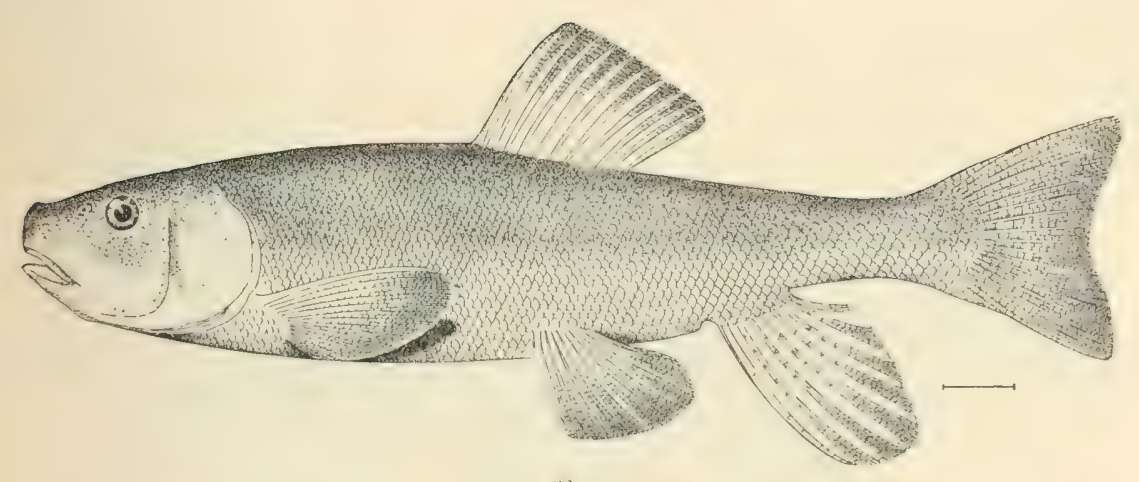

St)

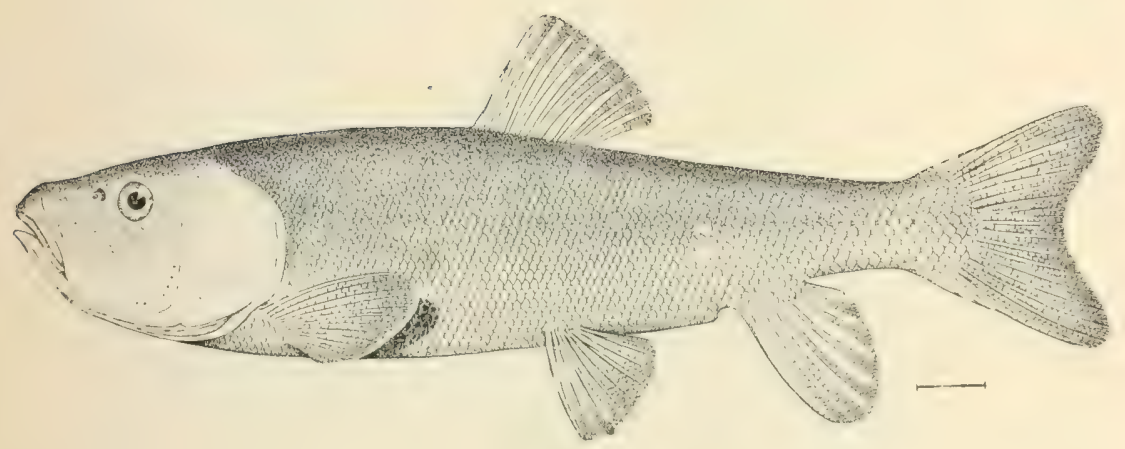

$87^{7}$

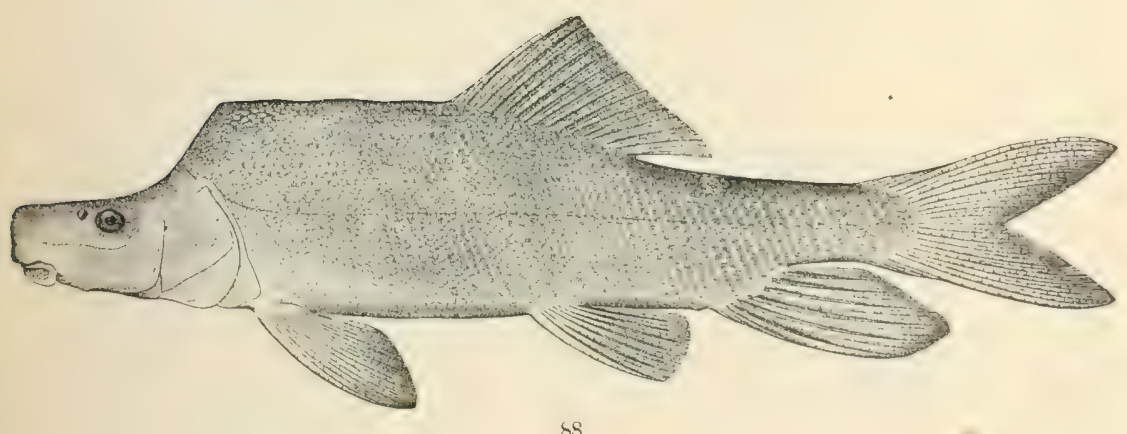

86. Chasmistes stomias. (P. 2794.)

87. Chasmistes COPEI. ( $\mathrm{P} .2795$.

88. XYRAUCHEN CYPHO. (P. 184.) 


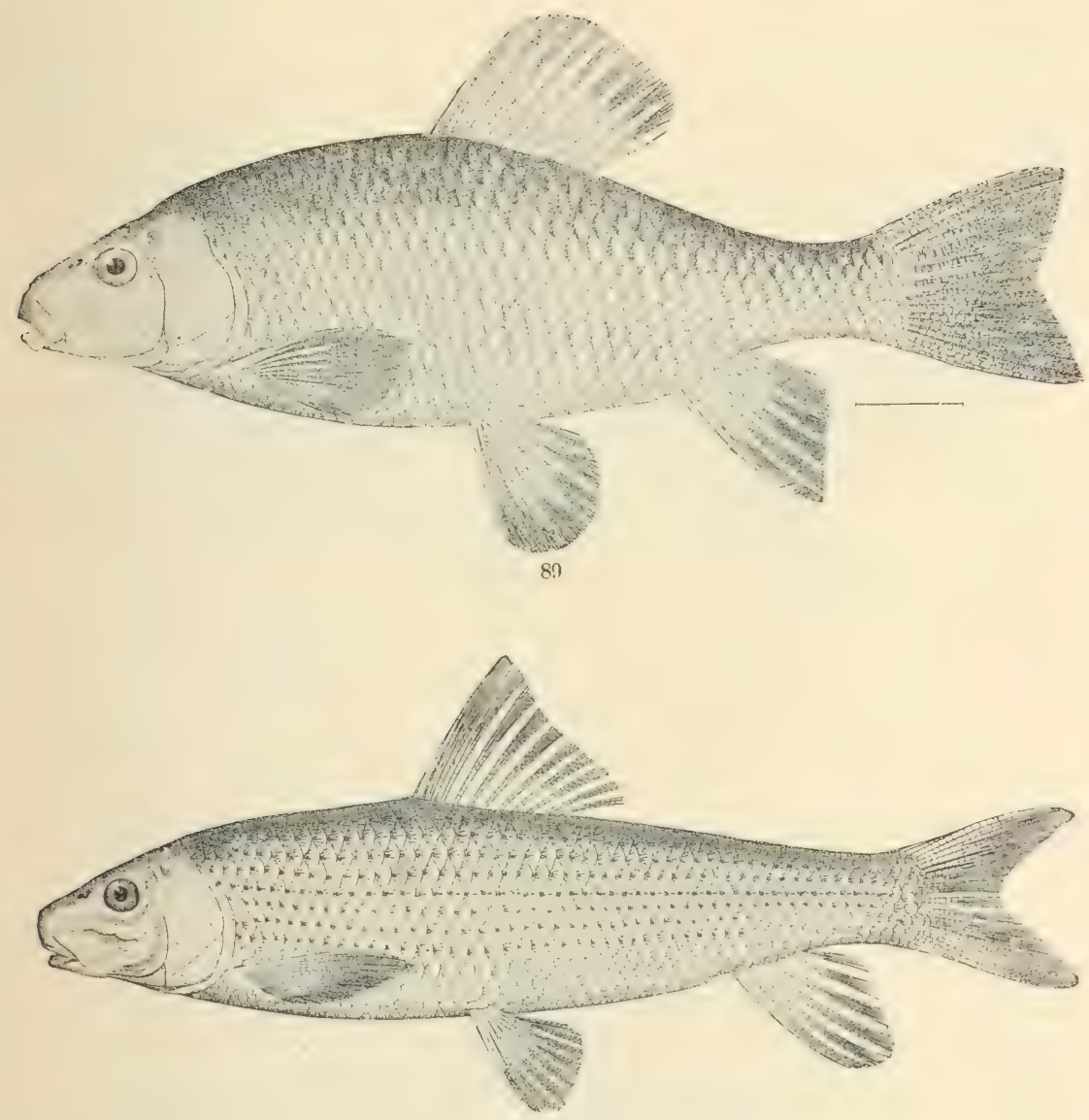

90

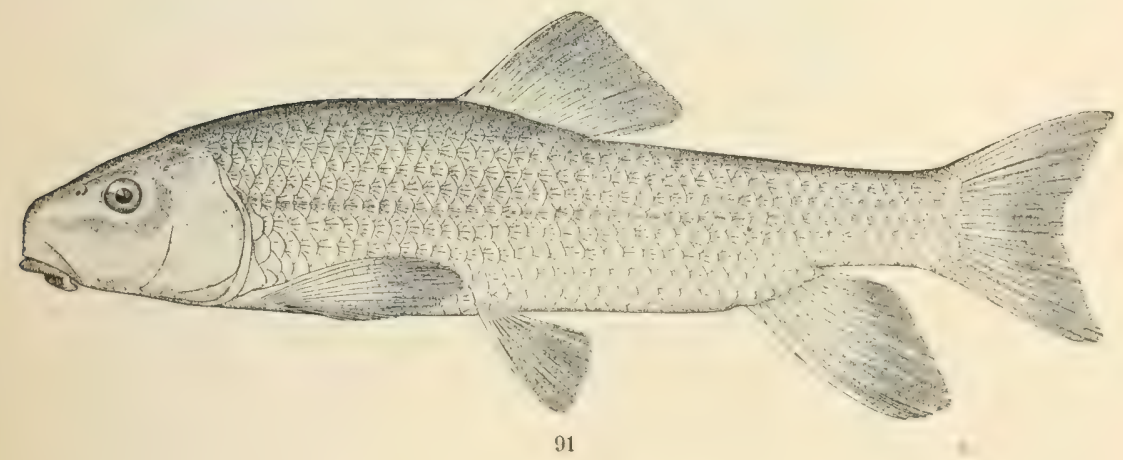

89. ERIMYZoN sUCETTA. (P. 185.)

90. MinYtREMA MELANops. (T', 187.)

91. Моxostoma coniestum. (P. 192.) 



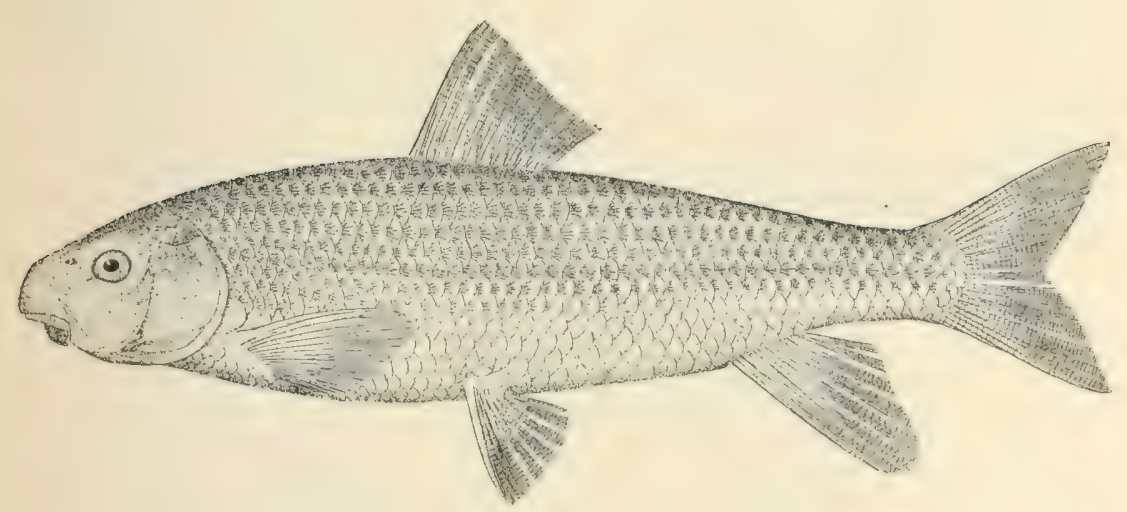

92

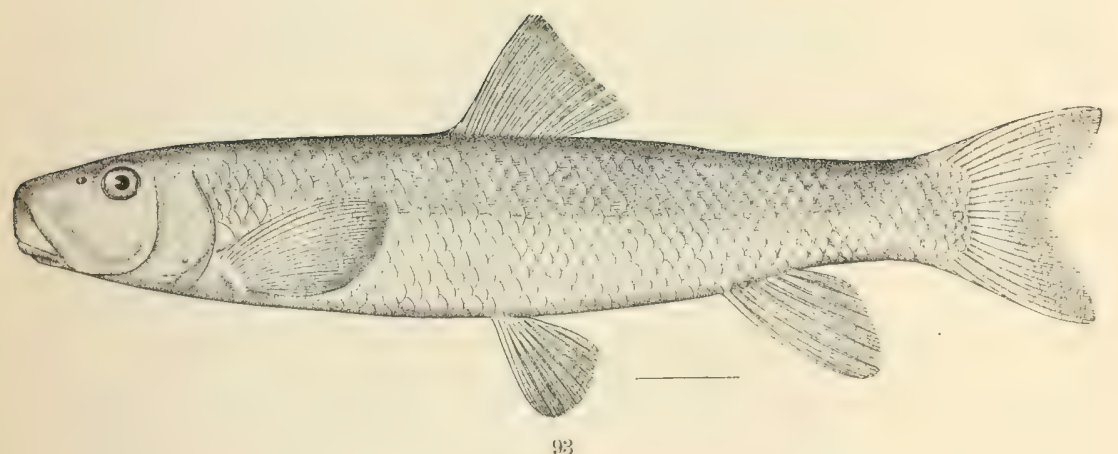

92. Moxostoma a Ustrinum. (I', 192.)

93. Moxostoma izUpiscartes. (P. 196.) 


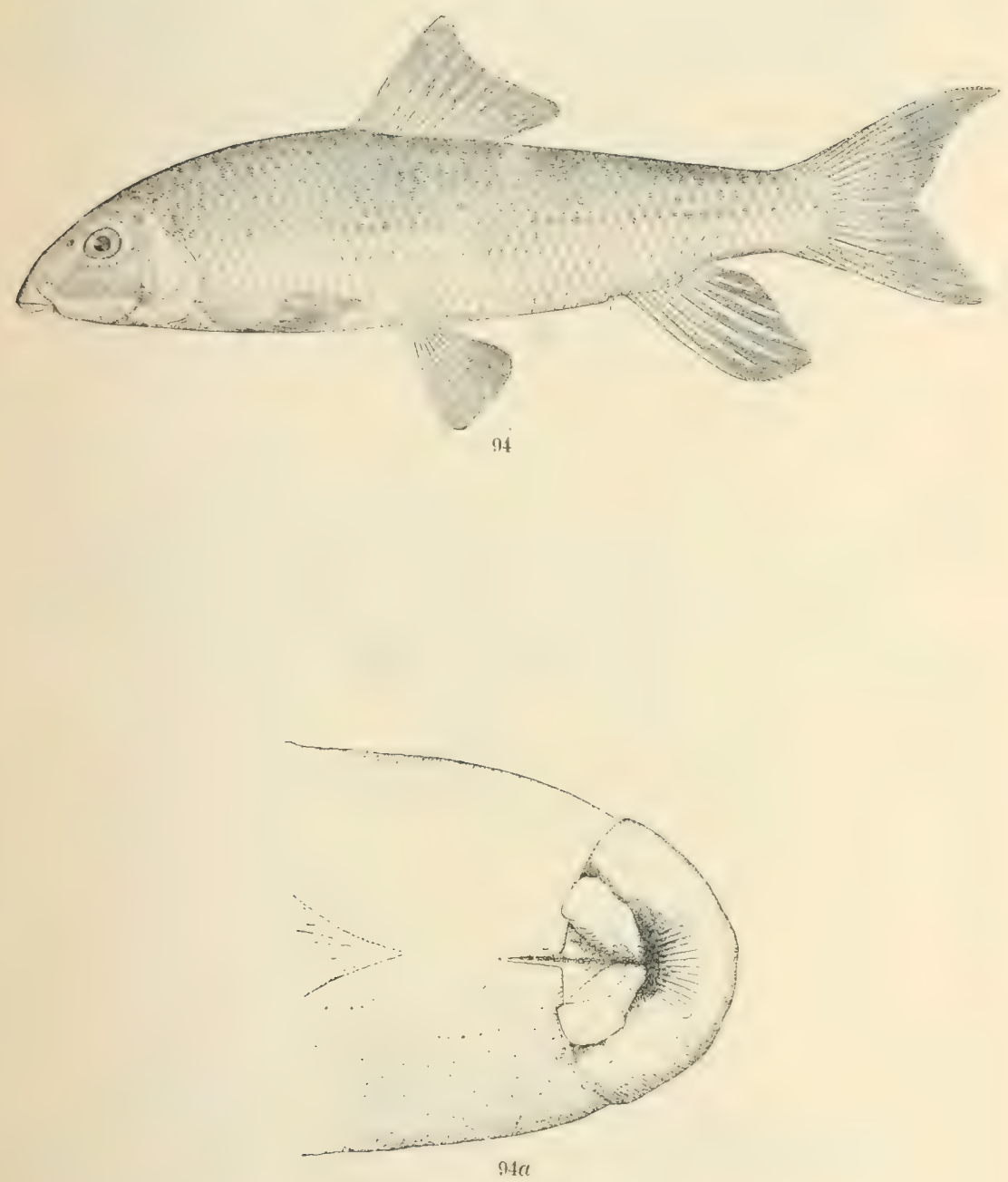

94, 94a. Lagochila lackma. (P. 199.) 



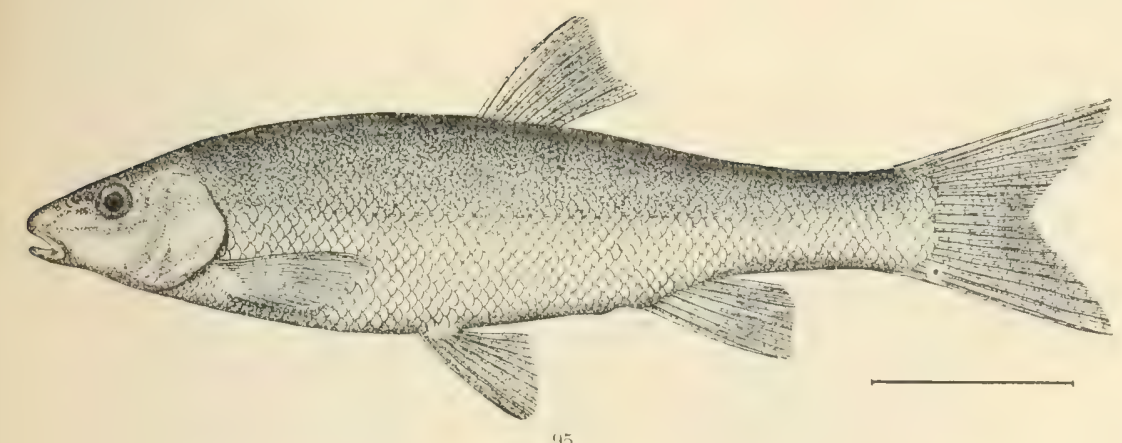

!5

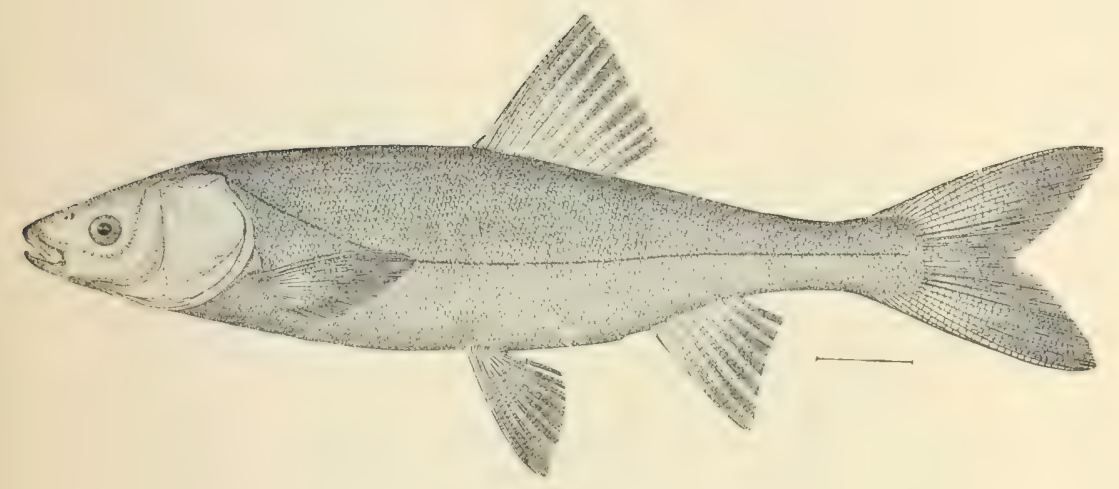

96

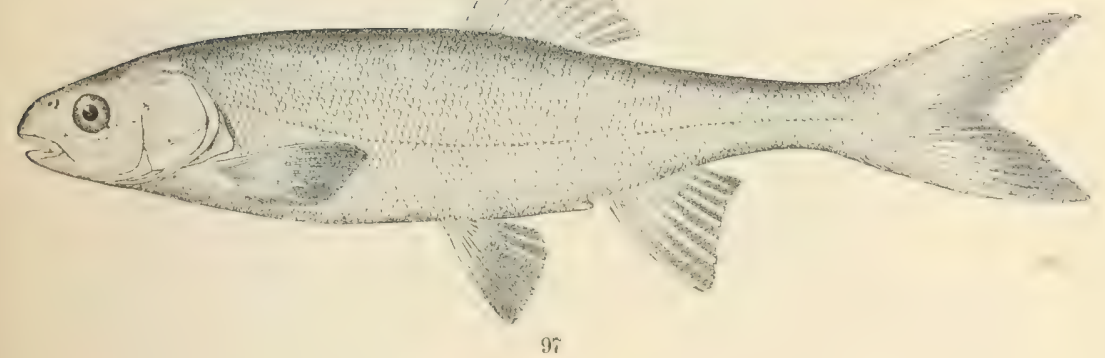

95. Campostoma anomalum. (P. 205.)

96. ORTHODON MICROLEPIDOTYS. (P. 207.)

97. Ailionerims aldTACEUs. (P. 208.) 



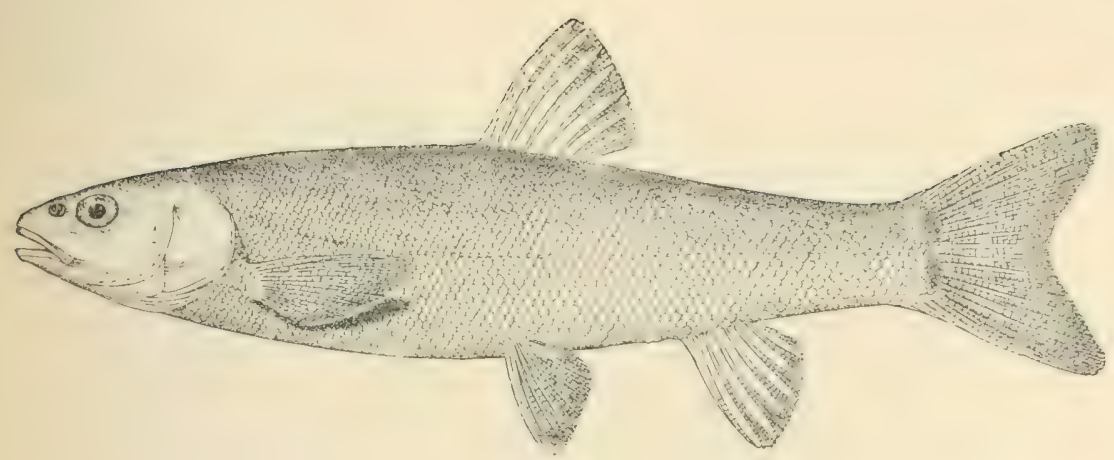

98

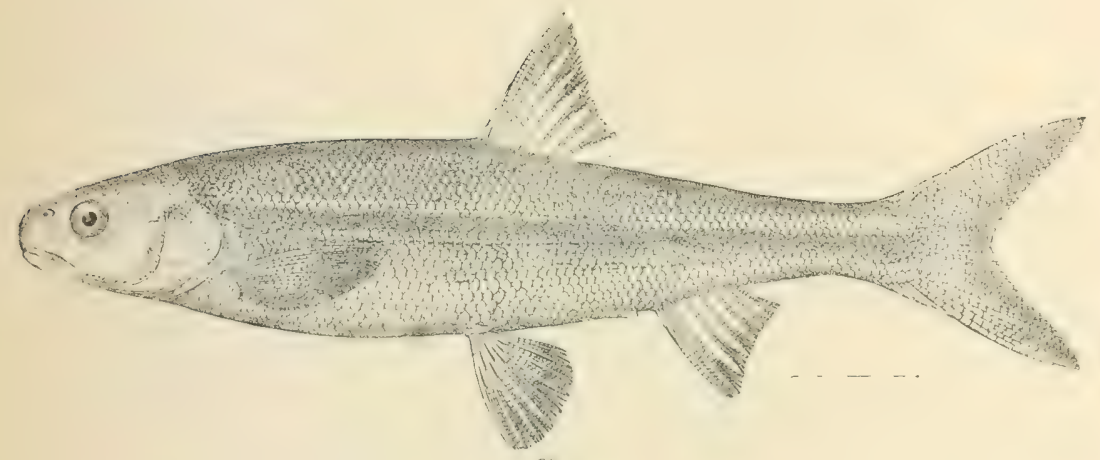

99

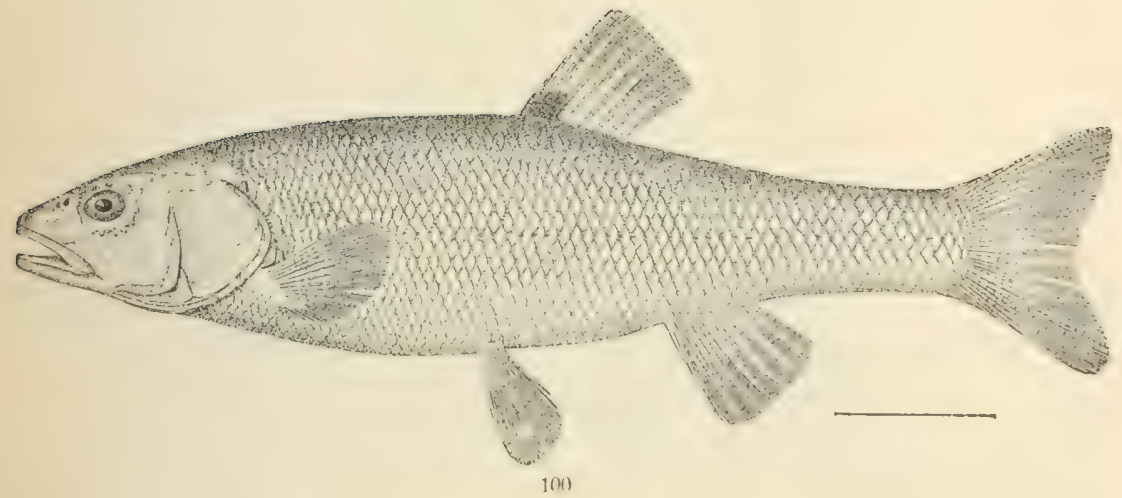

98. Algansea dugesi. (P. 211.)

99. MYLOCHEHLUS CAURINUS. (P', 219.

100. SEMOTILUS atromaculates. (P. 222.) 



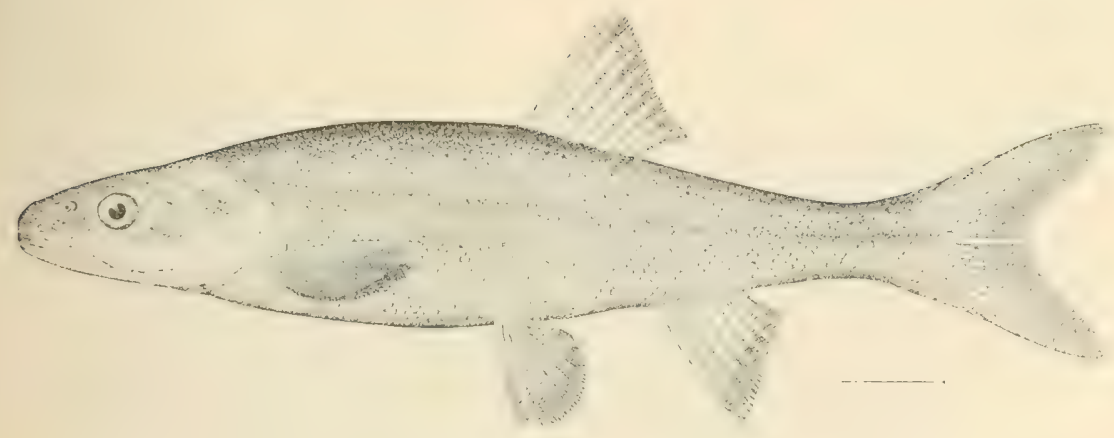

101
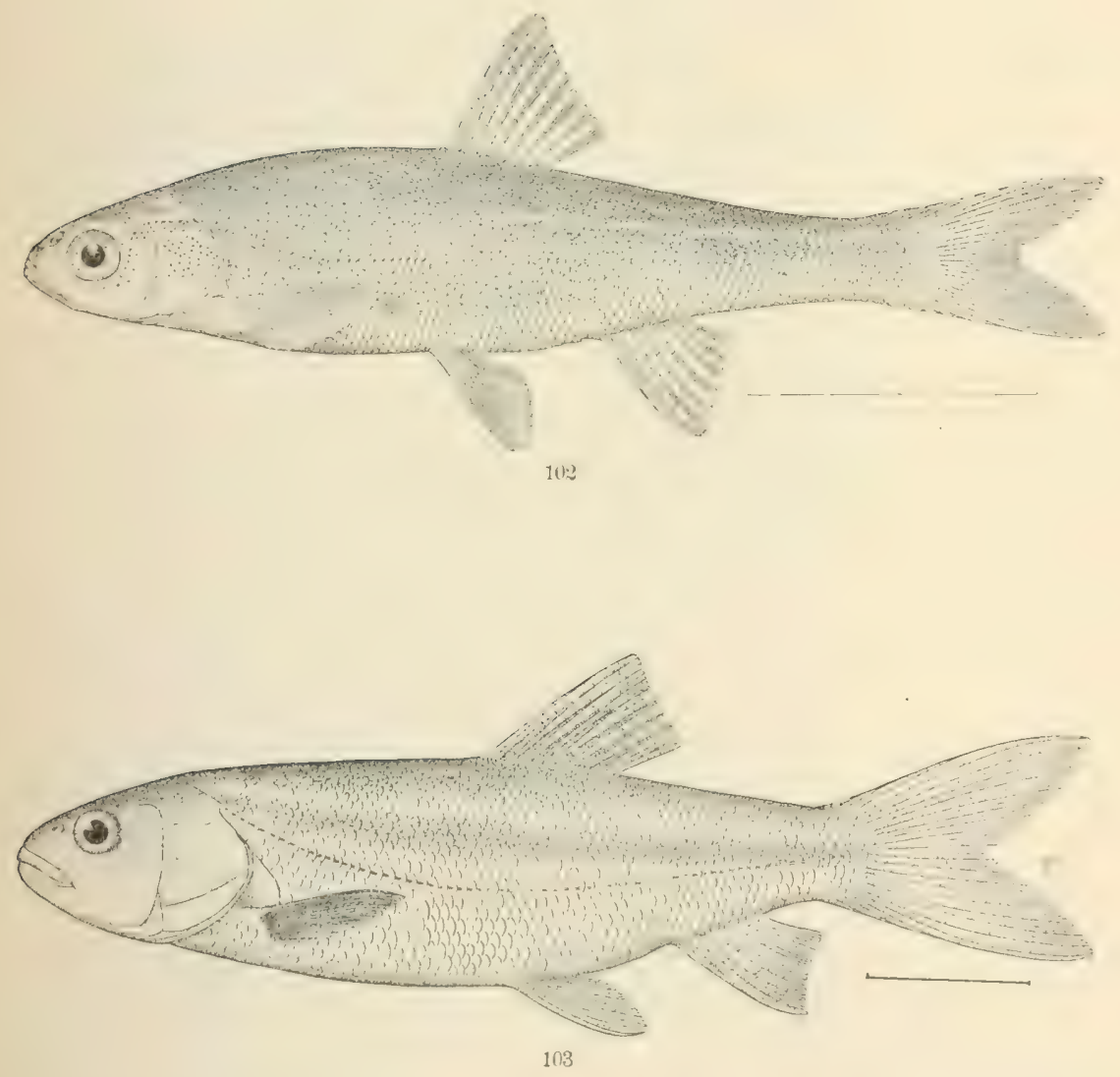

101. Ptychocheilus oregonensis. (P. 224.)

102. LEUCIACUA 13ICOLOR. (P. 232.)

103. LEUCIACUS LINEATUS. (P. 232.) 



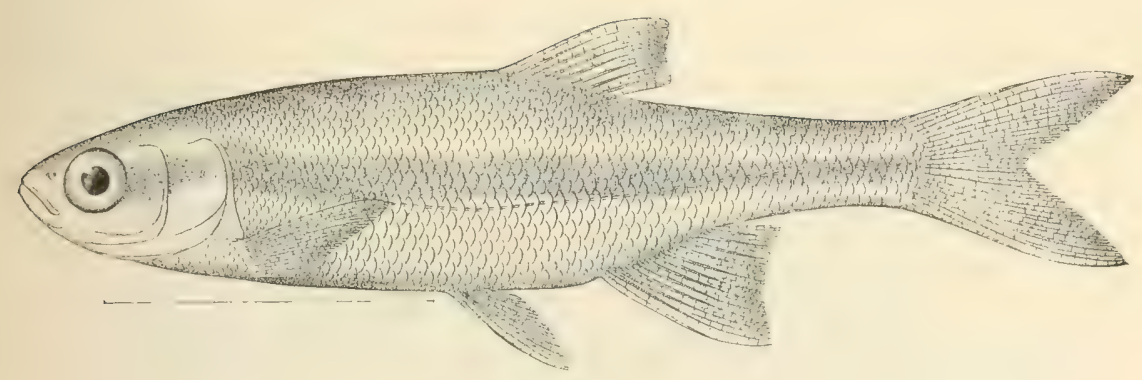

101

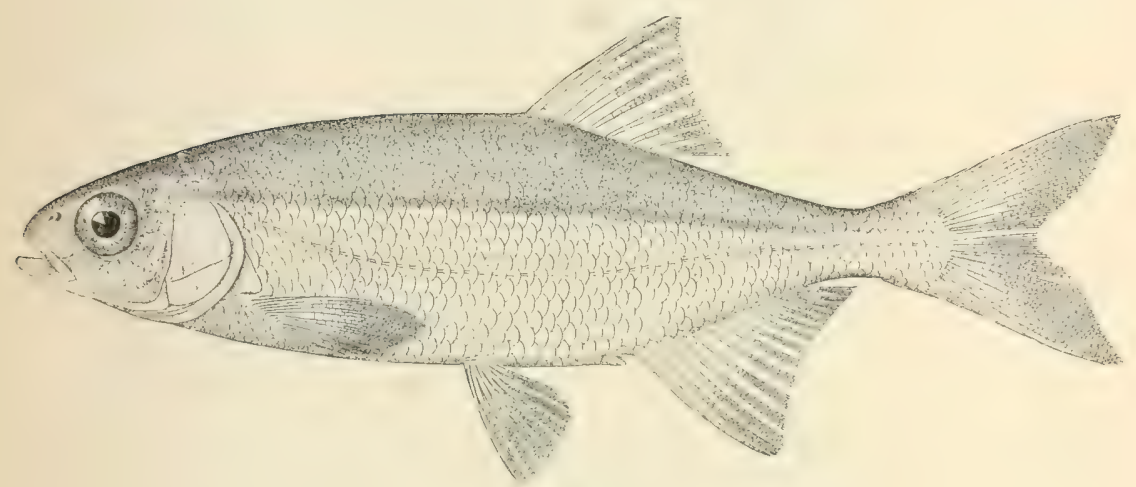

105

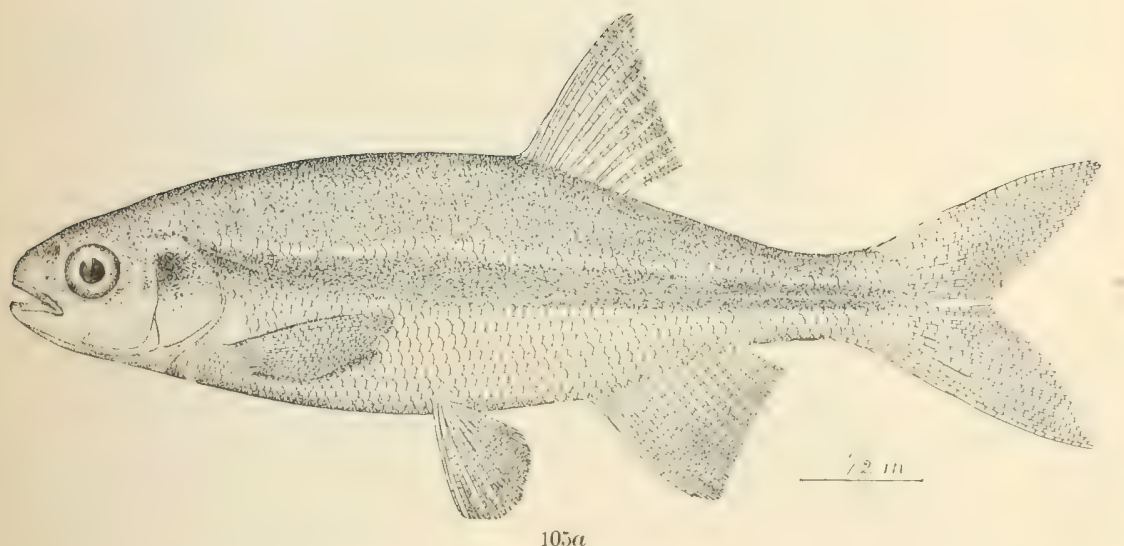

104. LEUCISCE's MYIROPIILOX. (P. 238.)

10:. LET'ISCTS BALTEATC: (P. 238.)

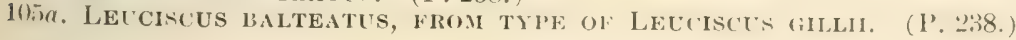





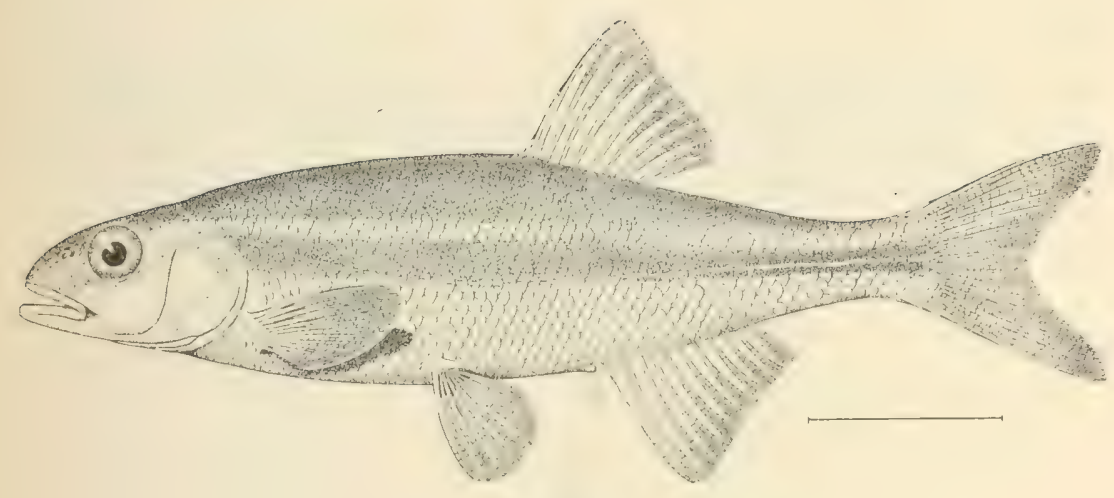

106

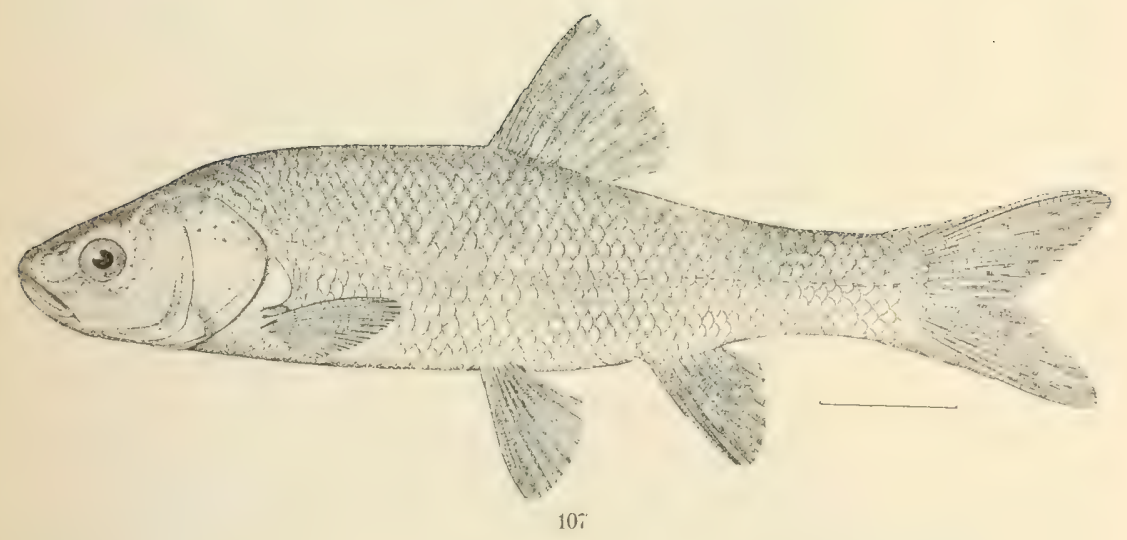

106. LeUCISCUS SIr'SLAWI. (I', 2797.)

107. Ritill's BICOLOR. (P. 244.) 


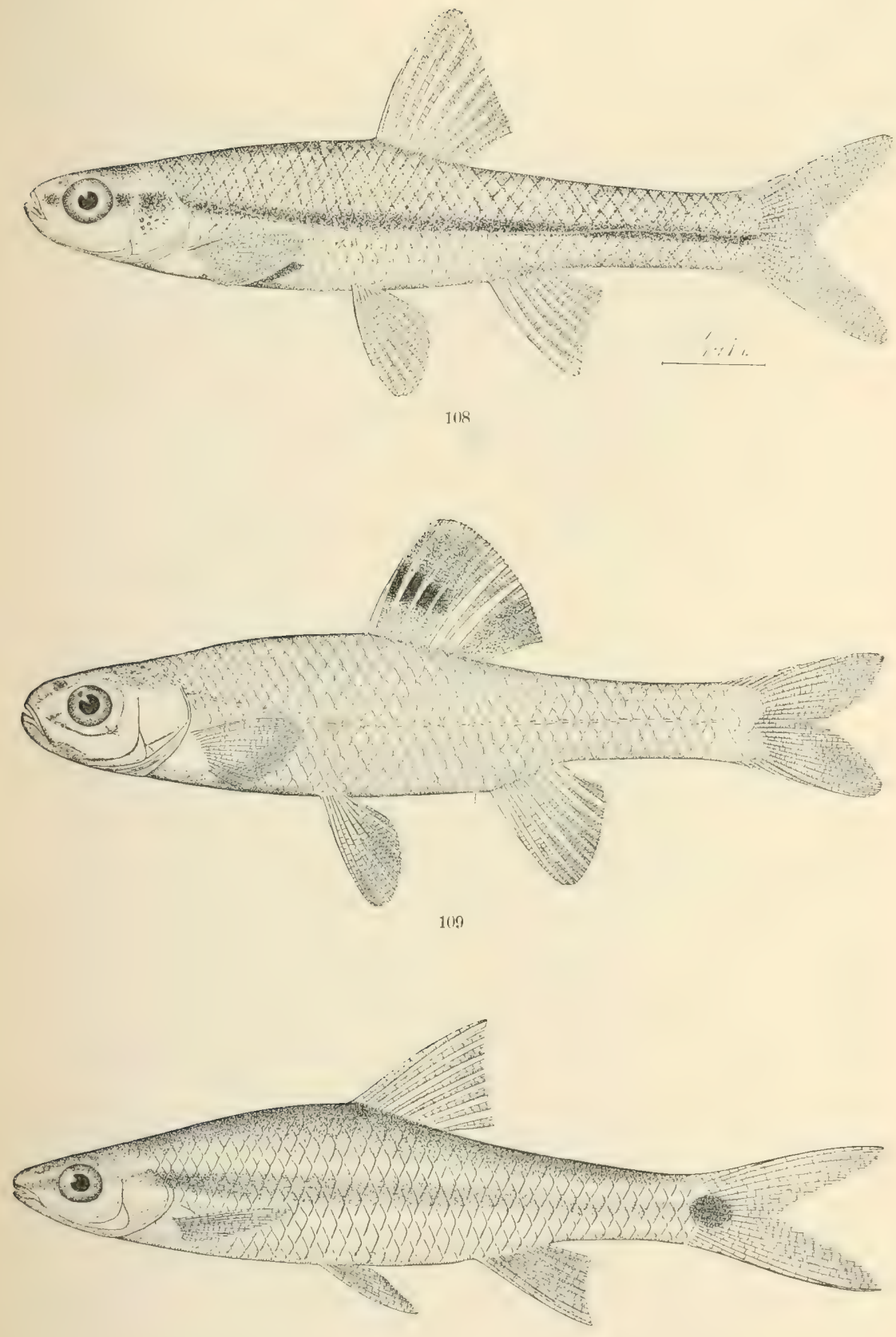

110

108. OPSOPEODUS OSCULUS. (P. 248.)

109. OPSOPQODUS EMILYA. (P. 248.)

110. Opsopelodus BolliaNi. (P. 249.) 


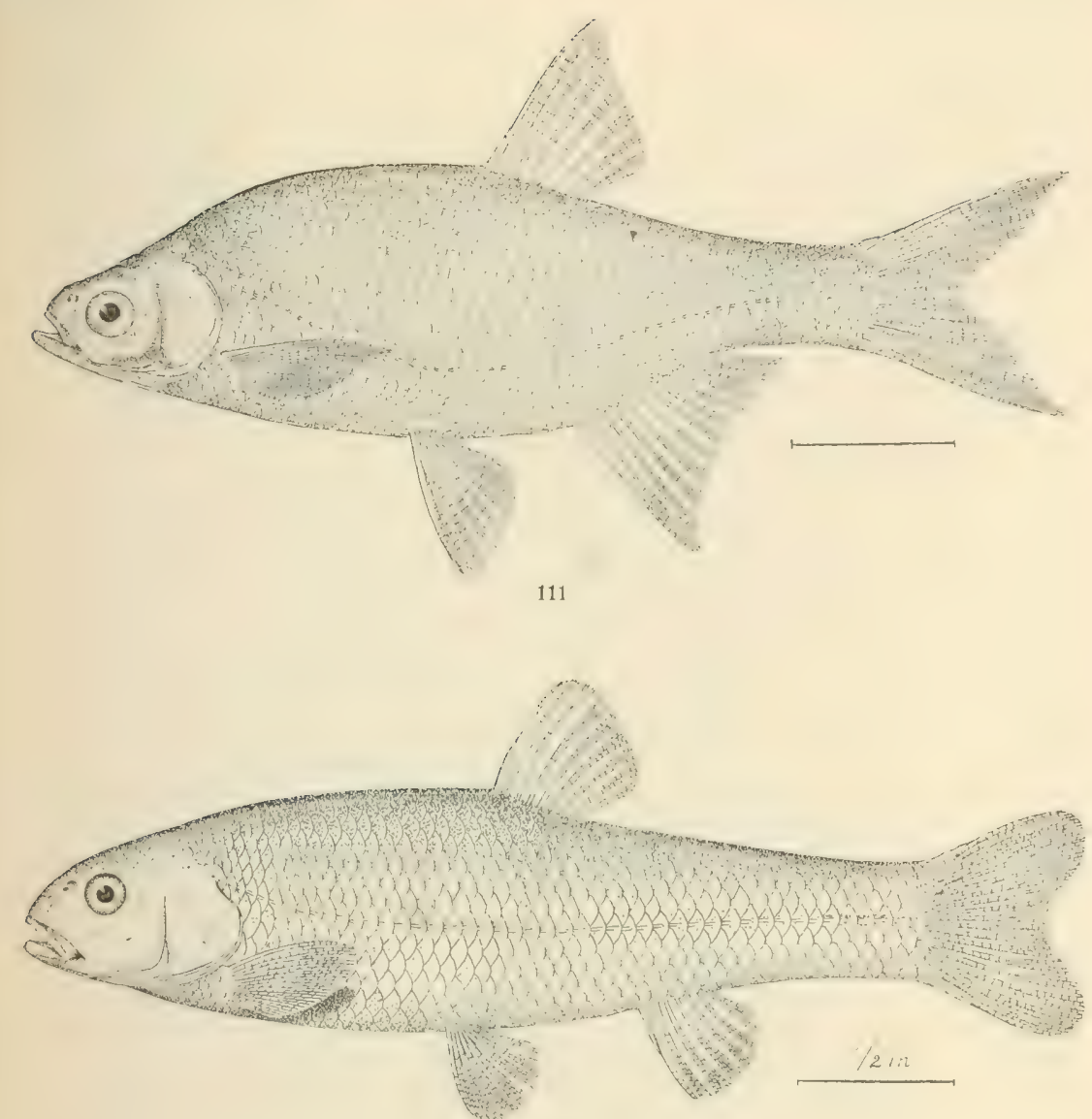

$11 \%$

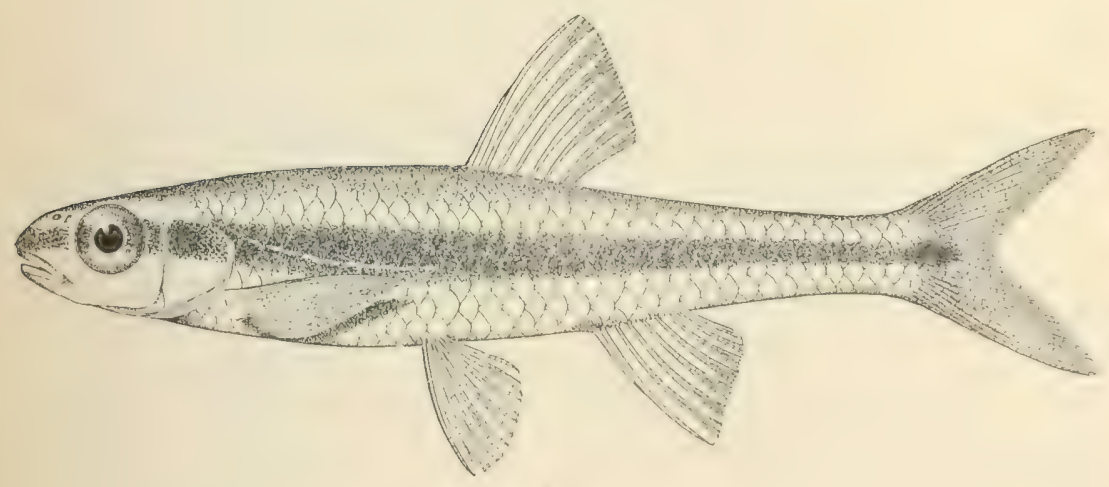

113

111. Aibranis crysoleucas. (1', 250.)

112. Notropis aztreUs. (P. 258.)

113. Notrol'ts Welaka, (P. 2799.) 



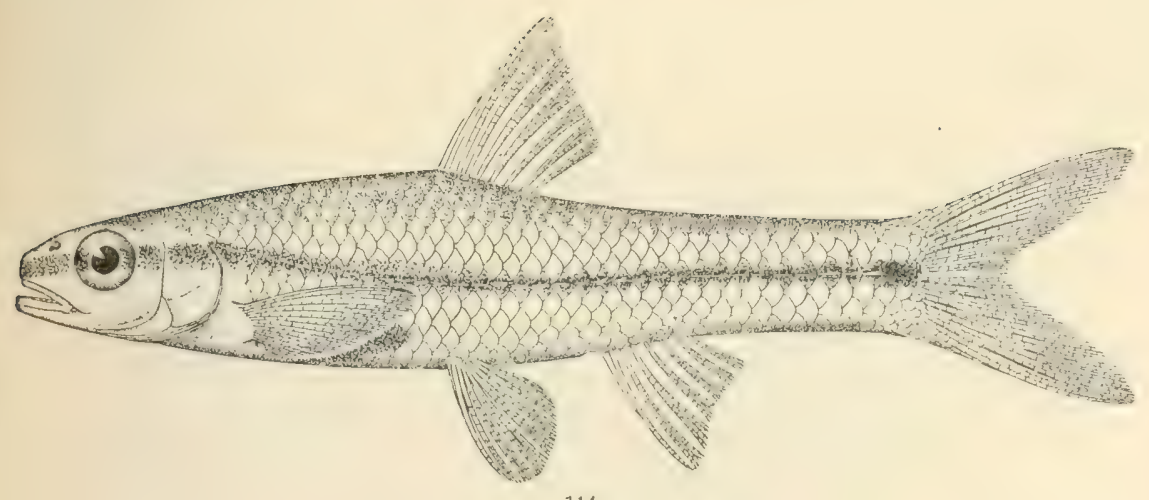

114

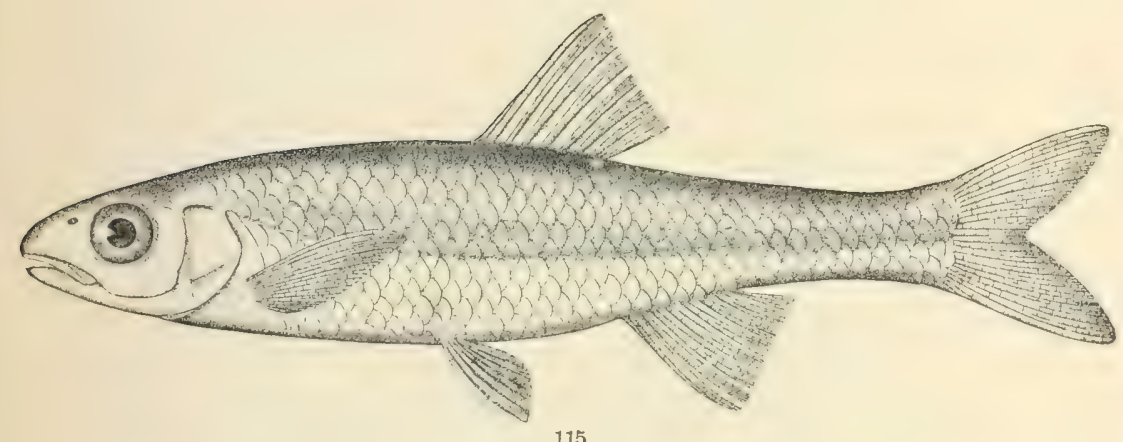

115

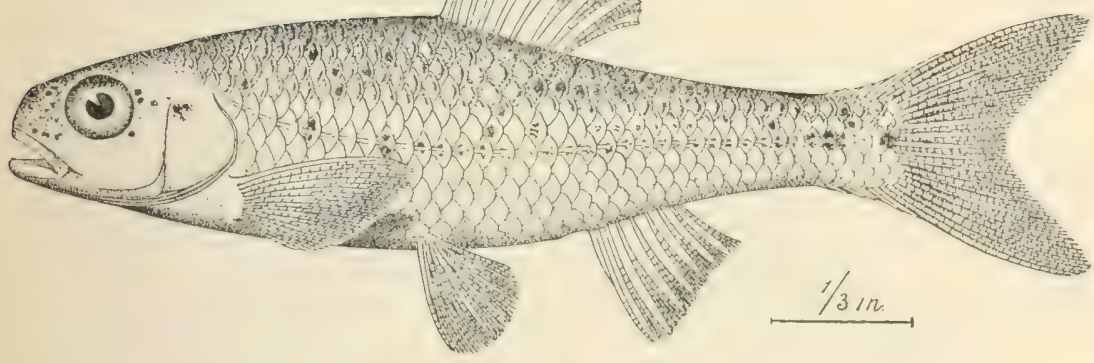

116

114. Notrolis CaYtga atrocaudalis. (P. 260.)

115. NOTROPIS KANAWHa. (P. 264.)

116. NOTROPIs CHIHUAHUA. (P. 265.) 



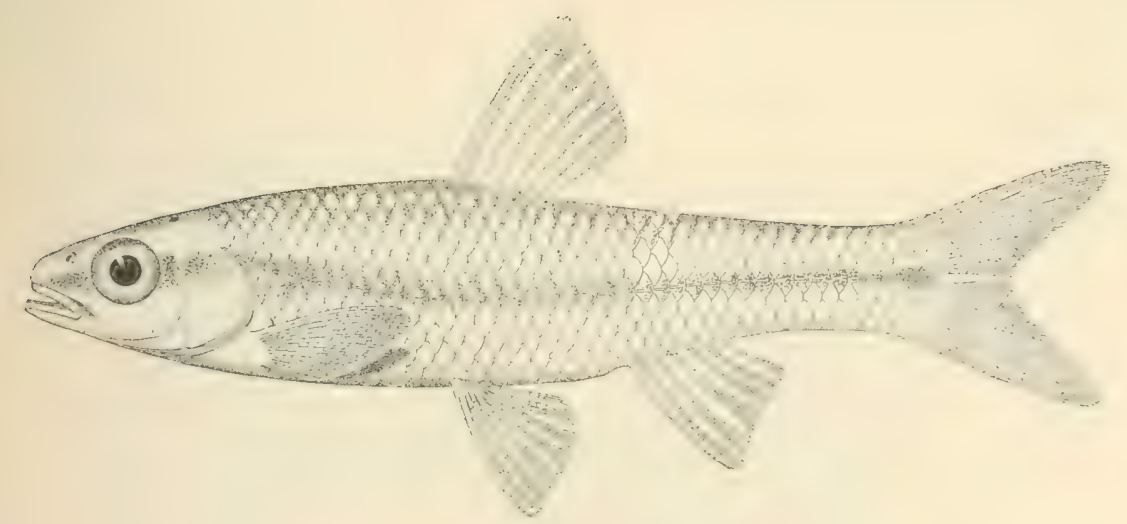

$11 \%$

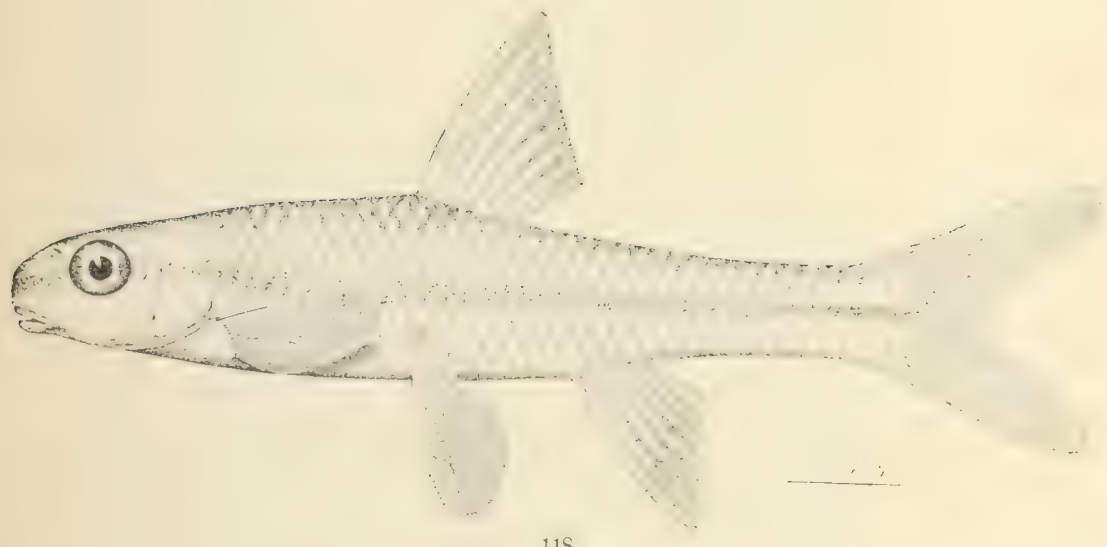

11 S

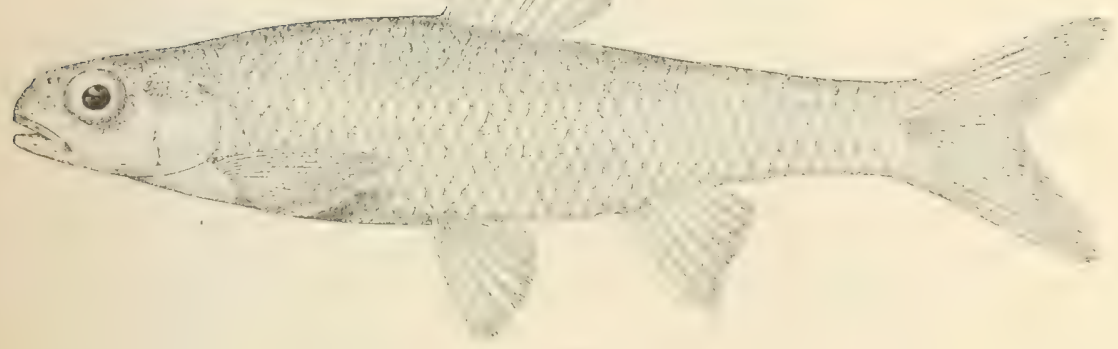

$11 ! 1$

117. Notroris vux. (I', 267.)

118. NoTROPIS NoCOMIS. (P.268.)

119. NotropIs HuDsoxics. (P. 269.) 




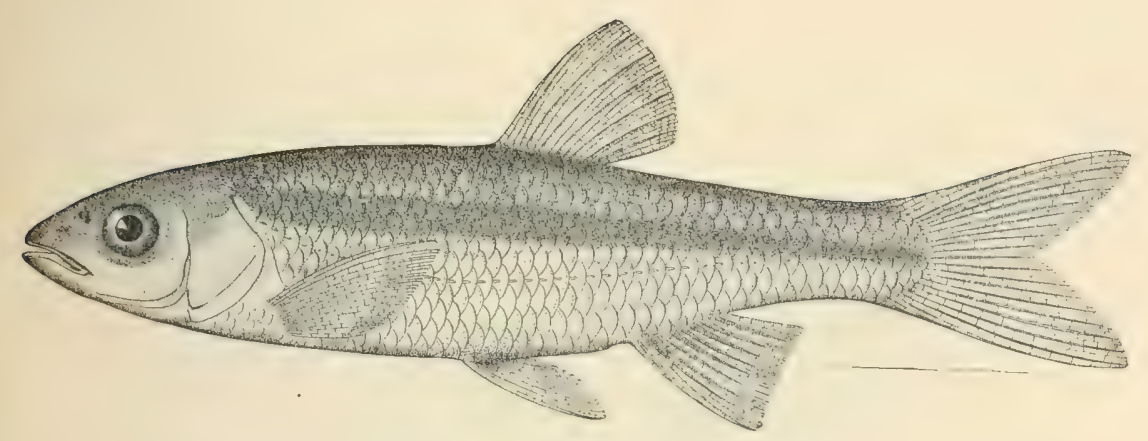

$1: 3$

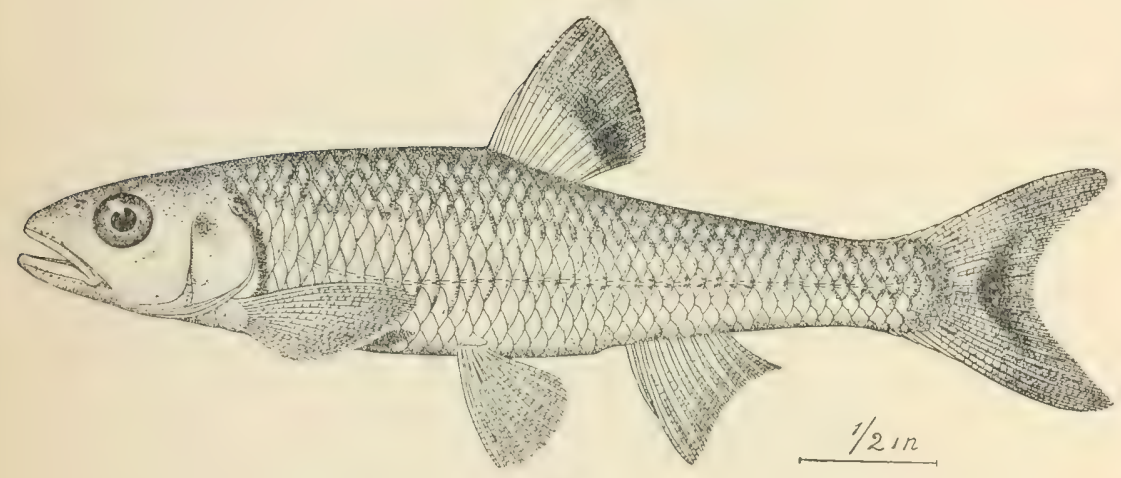

124

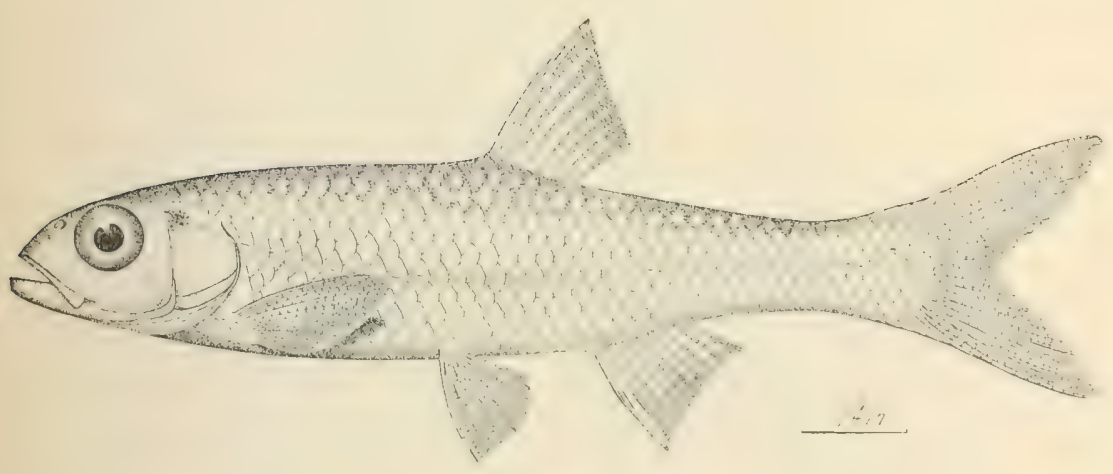

125

123. Notroris MACDONALDI. (P. 284.)

124. NOTROPIS COCCOGENIS. (P.284.)

125. Notropis SWAINI. (P. 290.) 



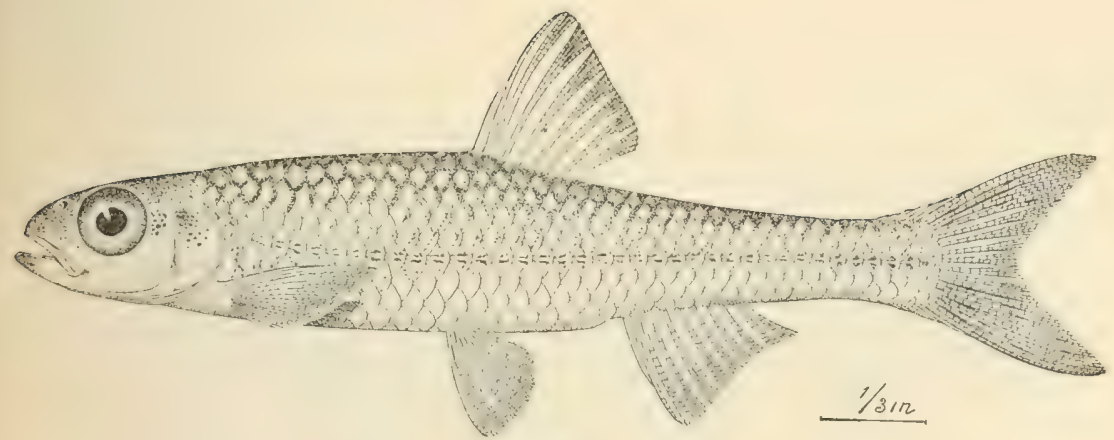

120

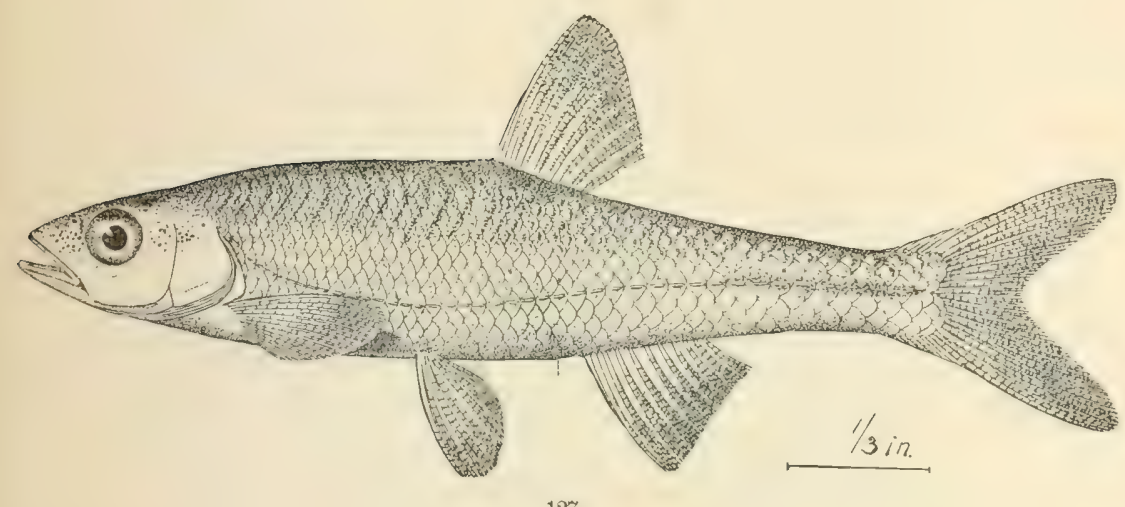

127

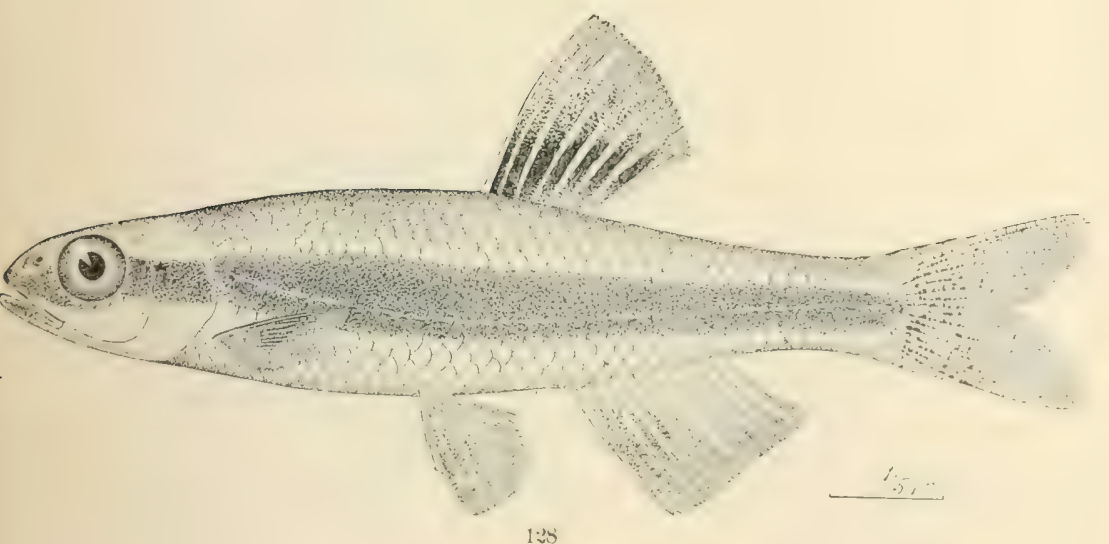

126. Notropis teiescopus. (P. 292.)

127. Notropis NoteMIGONOIDES. (1'.292.

128. Notrop'is Metallicus. (P. 297.) 


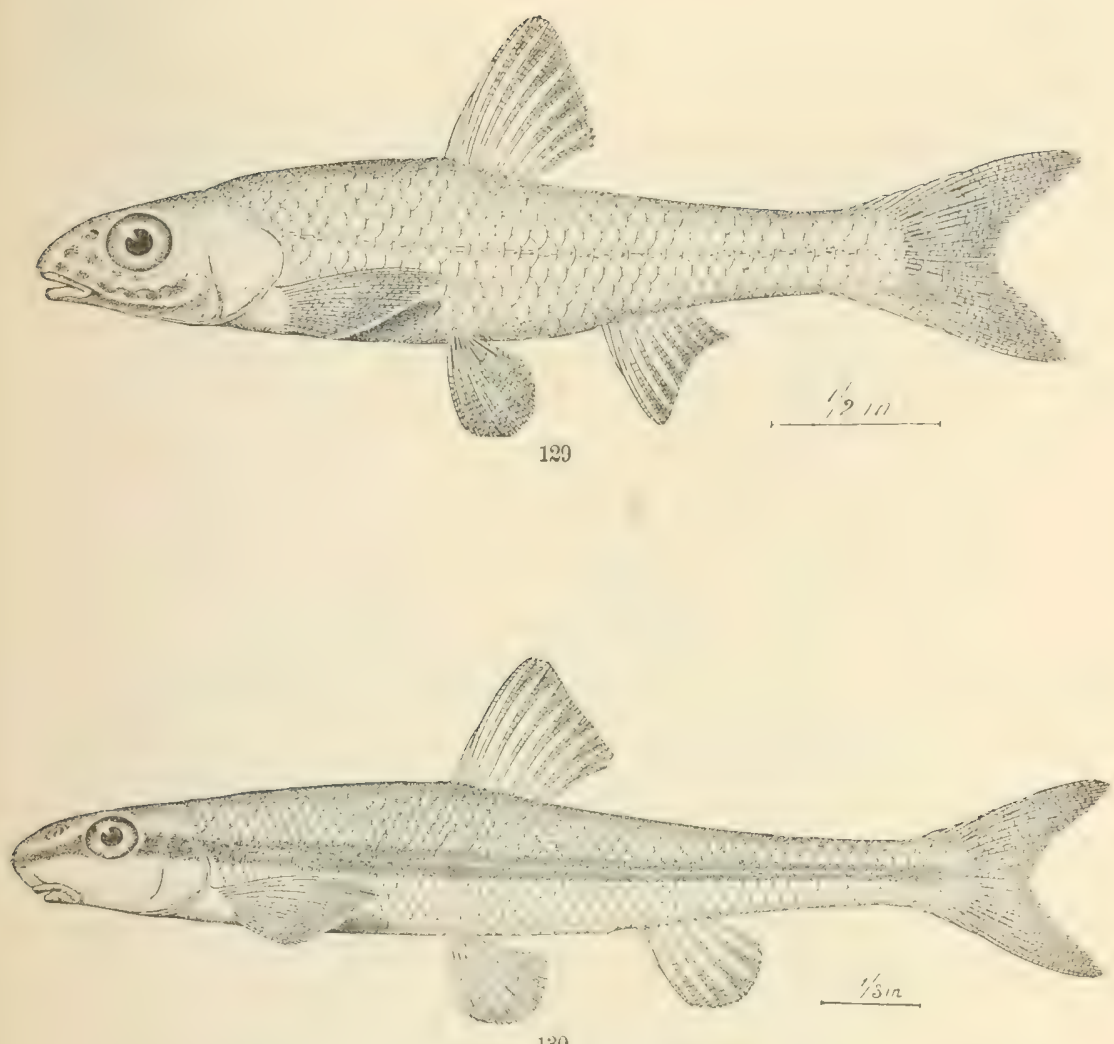

130

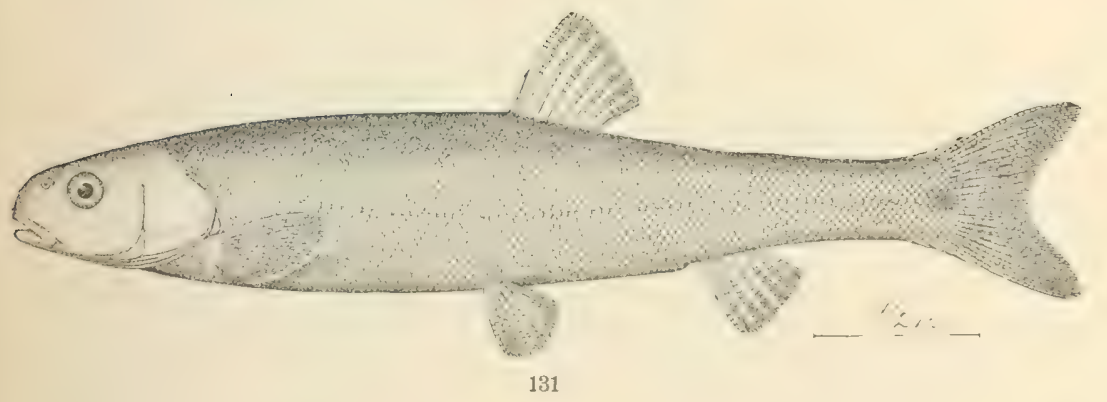

129. Erictima buccata. (P. 302.)

130. Phenacolics uraxops. (P. 304.)

131. EVARRA EIGENMANYI, (P.304.) 


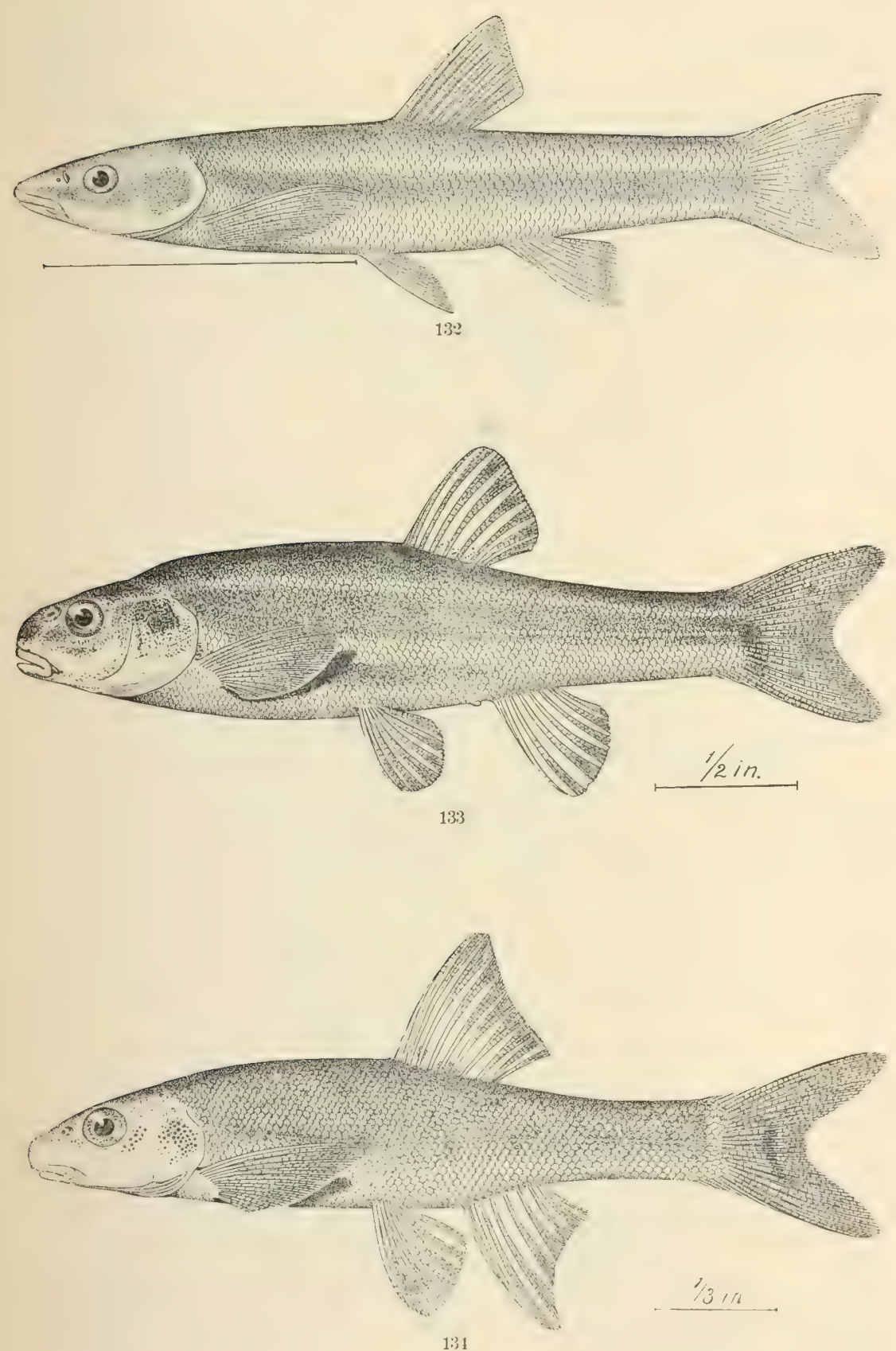

132. RHinichtirs Dulcis. (P. 306.)

133. Agosia klamathlisis. ( $\mathrm{P} . \mathrm{C}^{-}$)

134. Agosia viratilla. (P. 313.) 


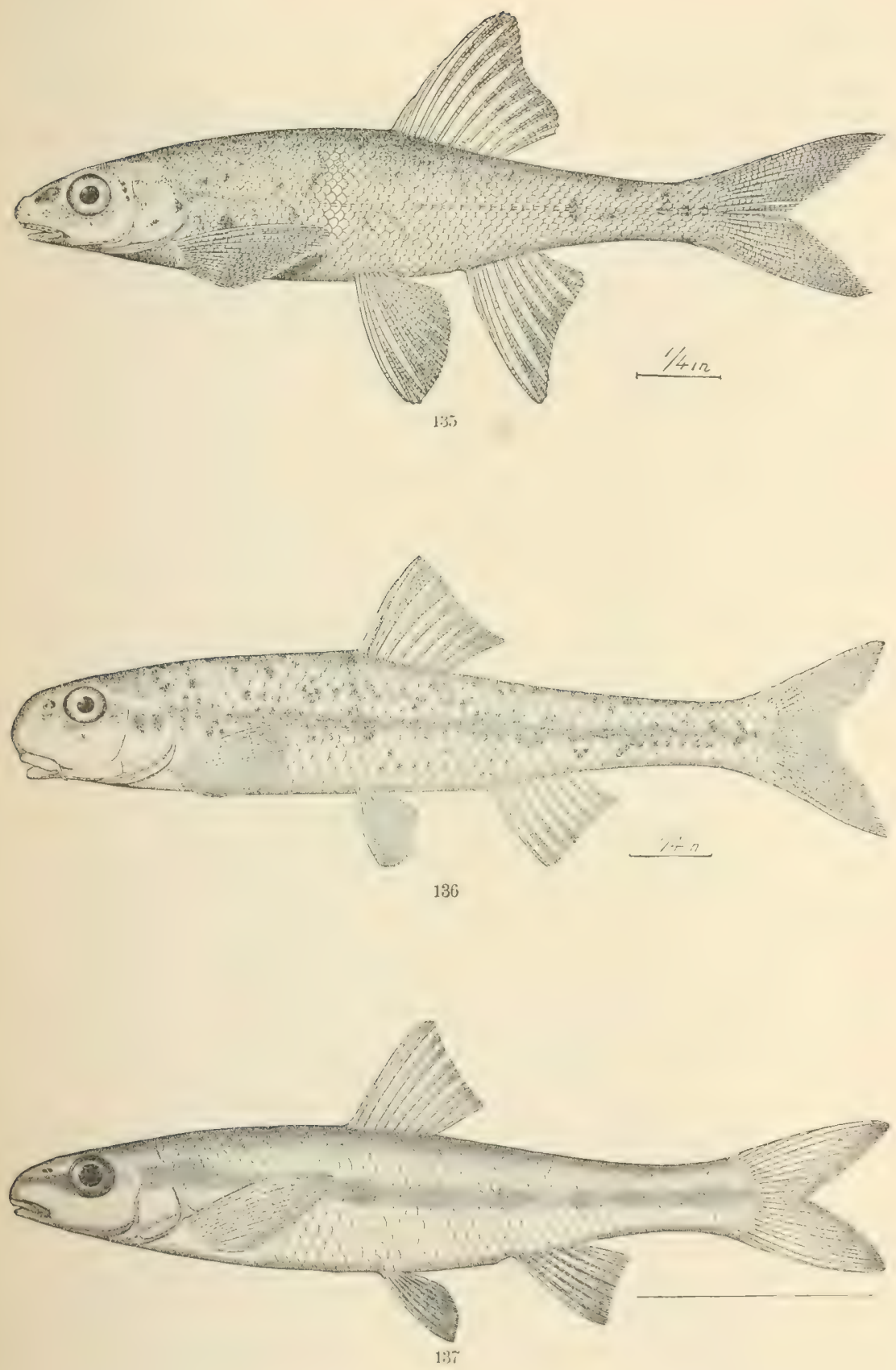

135. Agosia falcata. (P. 313.)

136. HYBOPSIS ESTIVALIS MARCONIS. (P. 316.)

137. Hyiopsis watauga. (P.319.) 



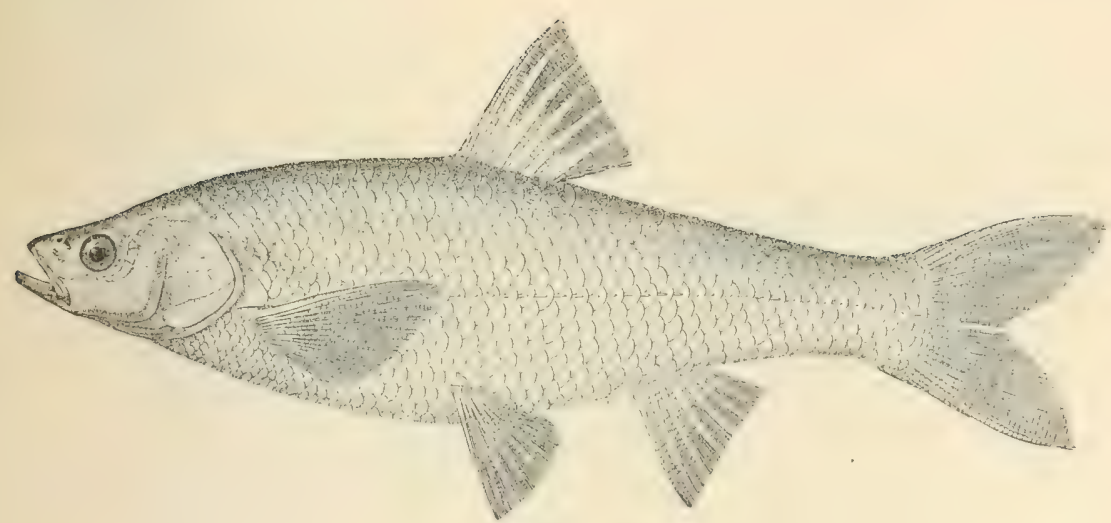

134

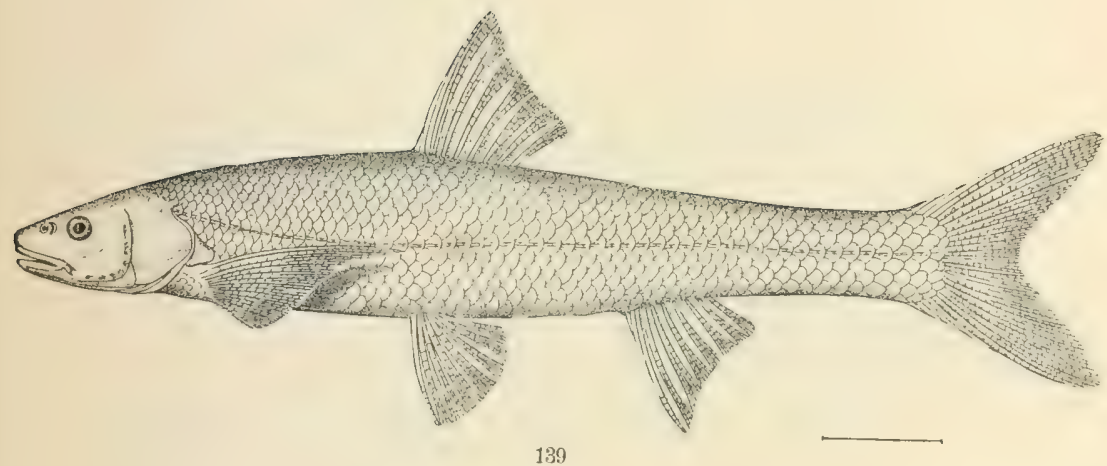

139

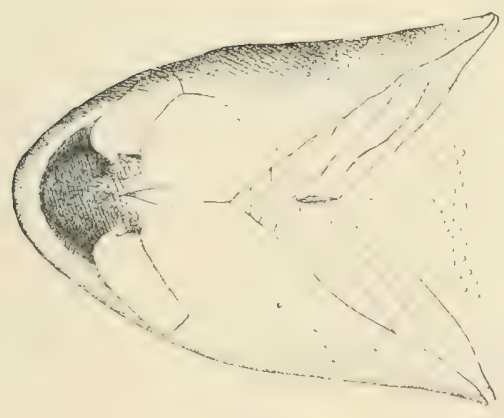

1.40

138. HYropsis altis. (P. 321.)

139. Pr.itygobio gracilis. (P. 326.)

140. Exuglosisu Maxillingua. (P. 327.) 


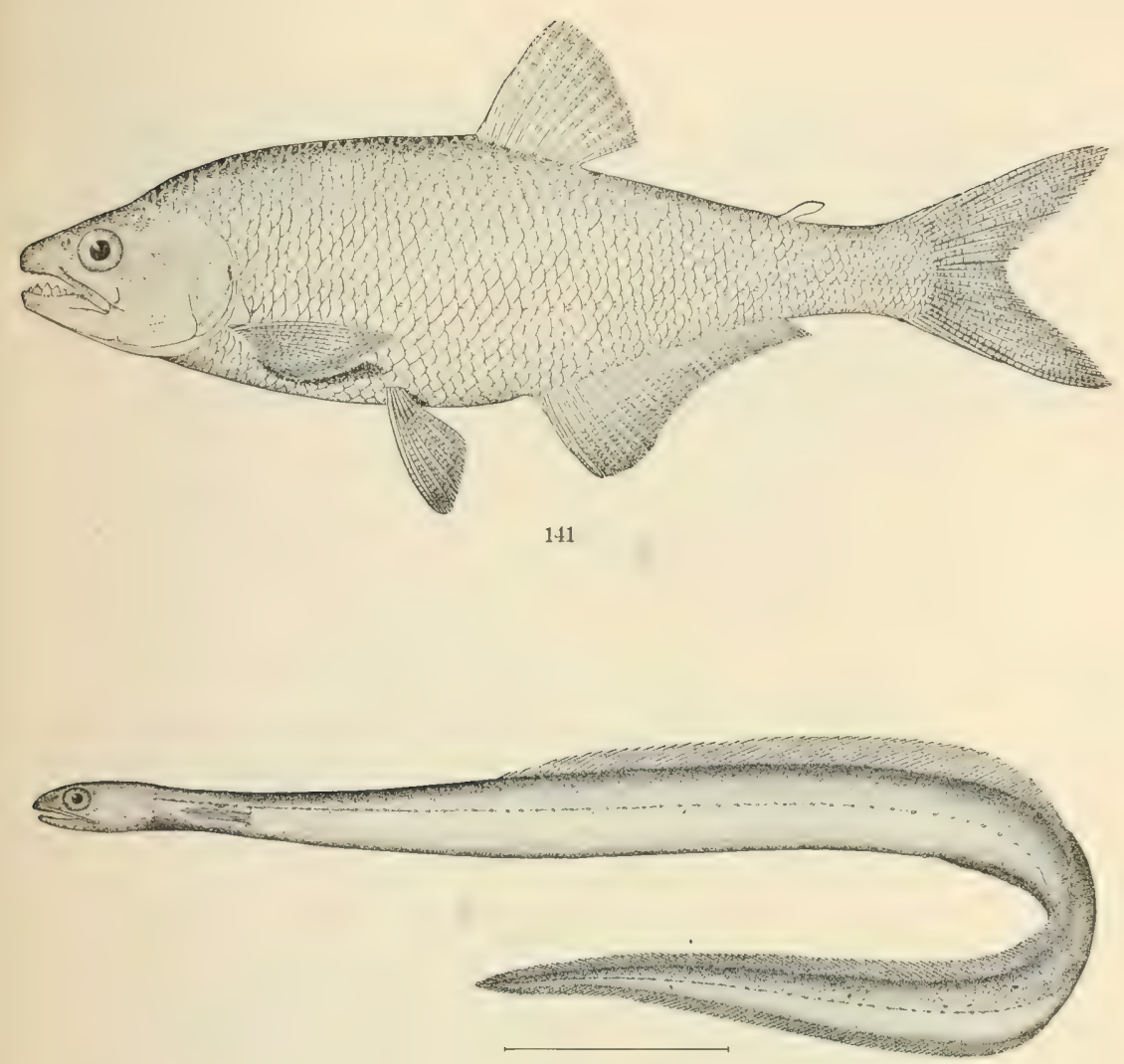

142

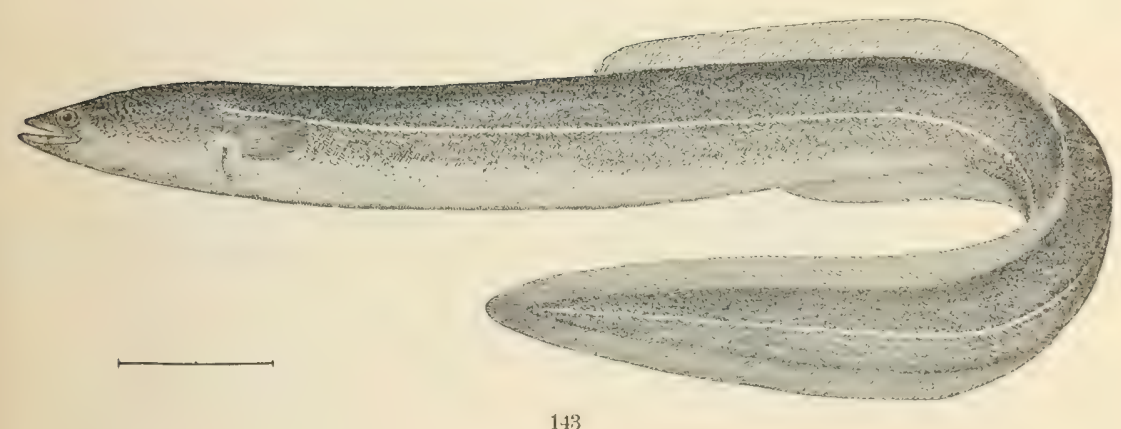

141. Bry CON DENTEx. (I'. 337.)

142. DERICITTHYS SERPENTINUS. (P. :313.)

143. ANGUILIA CIIRYSYIA. (P'. :348.) 



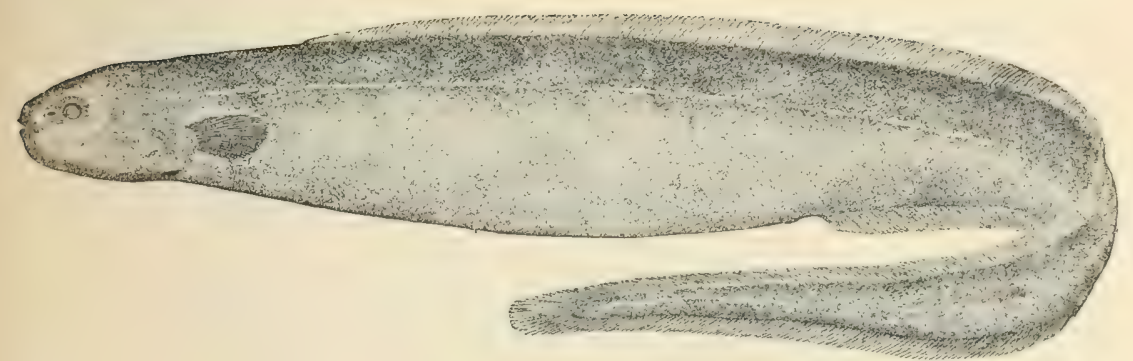

144

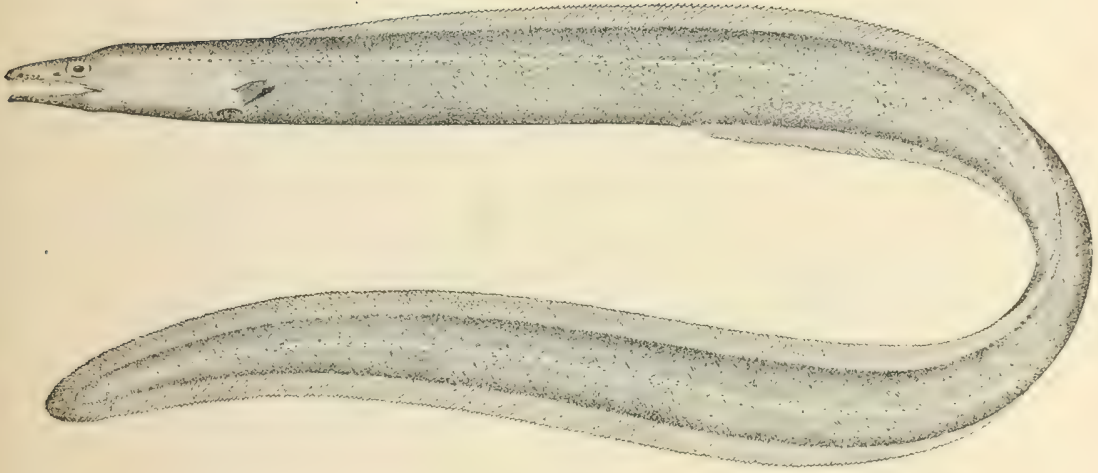

145

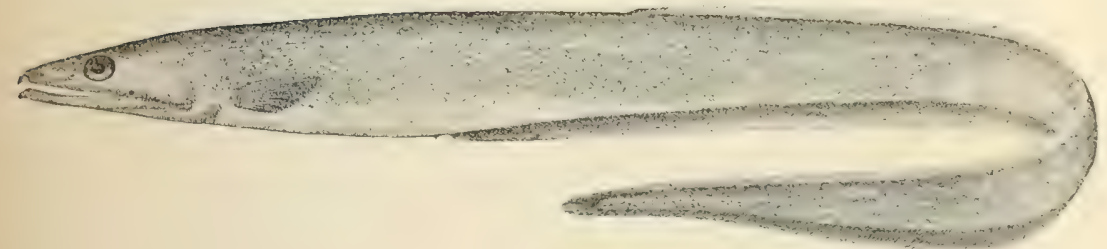

116

144. Simenchelys parasiticus. (I’349.)

145. IlYOPHIS BRUNNEUS. (P. 350 .)

146. Sinaphobrancius pinNatus. (P. 331.) 


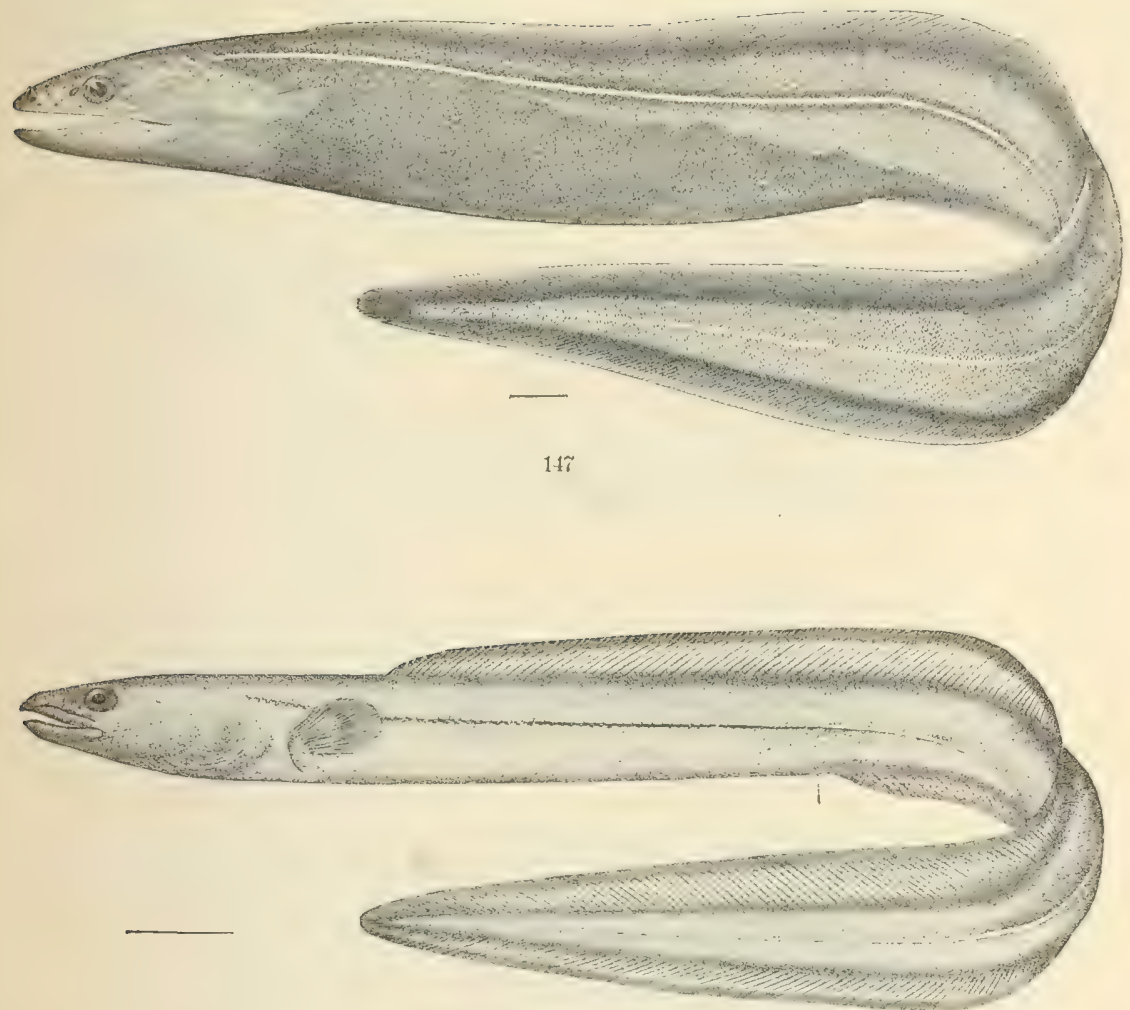

1.18

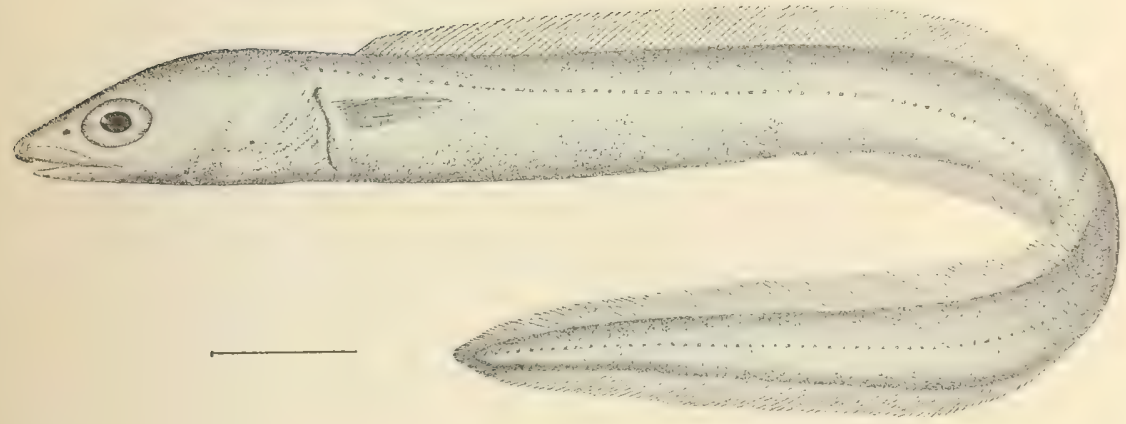

$1+4$

147. Histiolbanchus infeinalis. (P. 352.)

148. LEPTOCEPIALUS CONGER. (P. 354.)

149. Leptocephalus caudilmbatus. (P. 355.) 



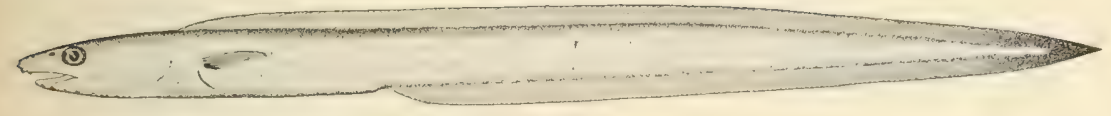

150

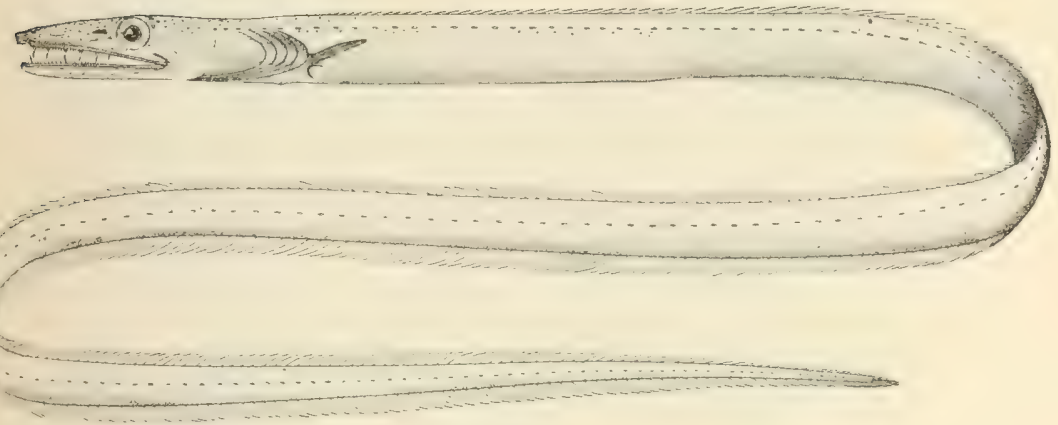

1.51

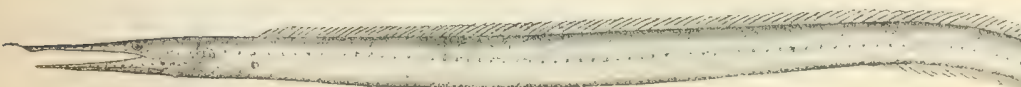

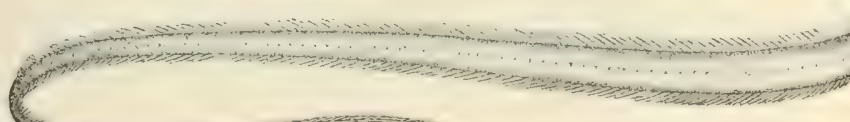

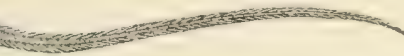

172

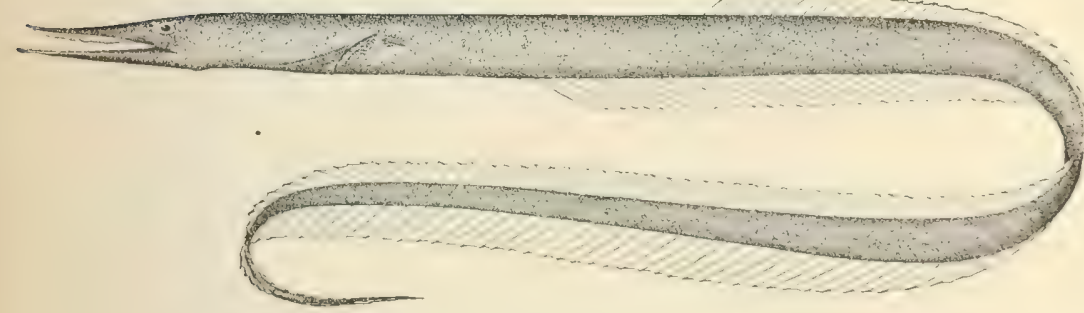

150. Congrellus Flavus. (P. 357.)

151. HoplunNis sCHMIITII. (P. 361.$)$

152. VENEFICA PROCERA. (P. 365.)

153. SERRIVOMER BLANI. (P.367.) 


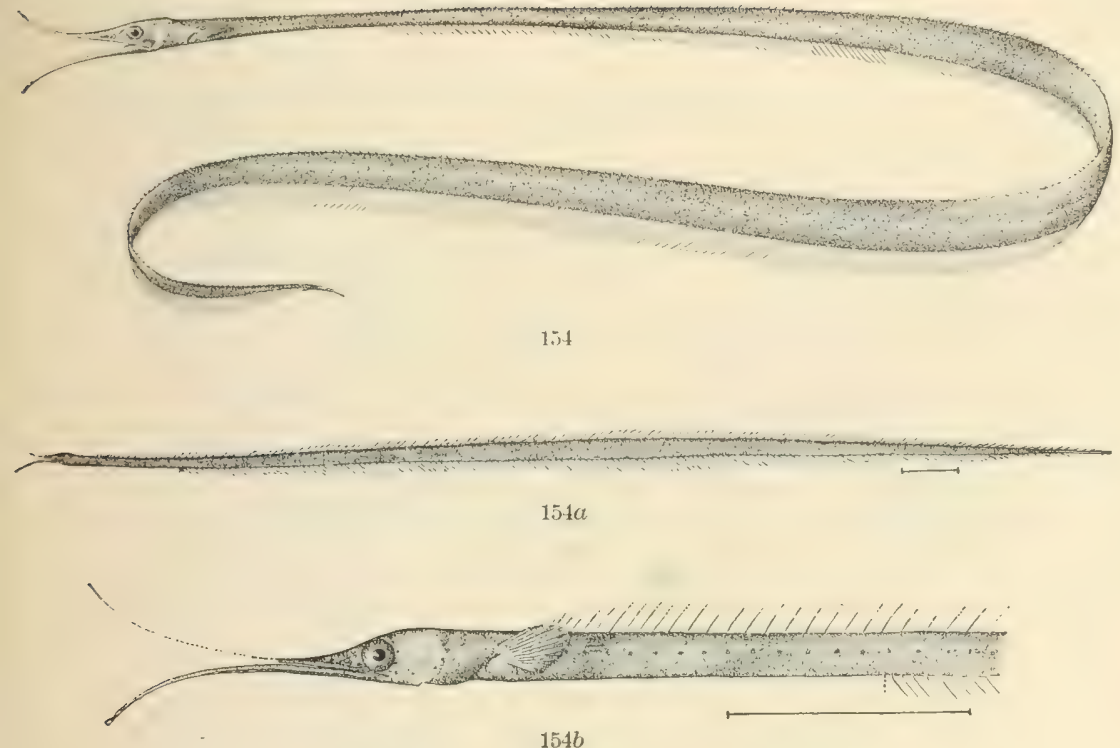

$154 b$
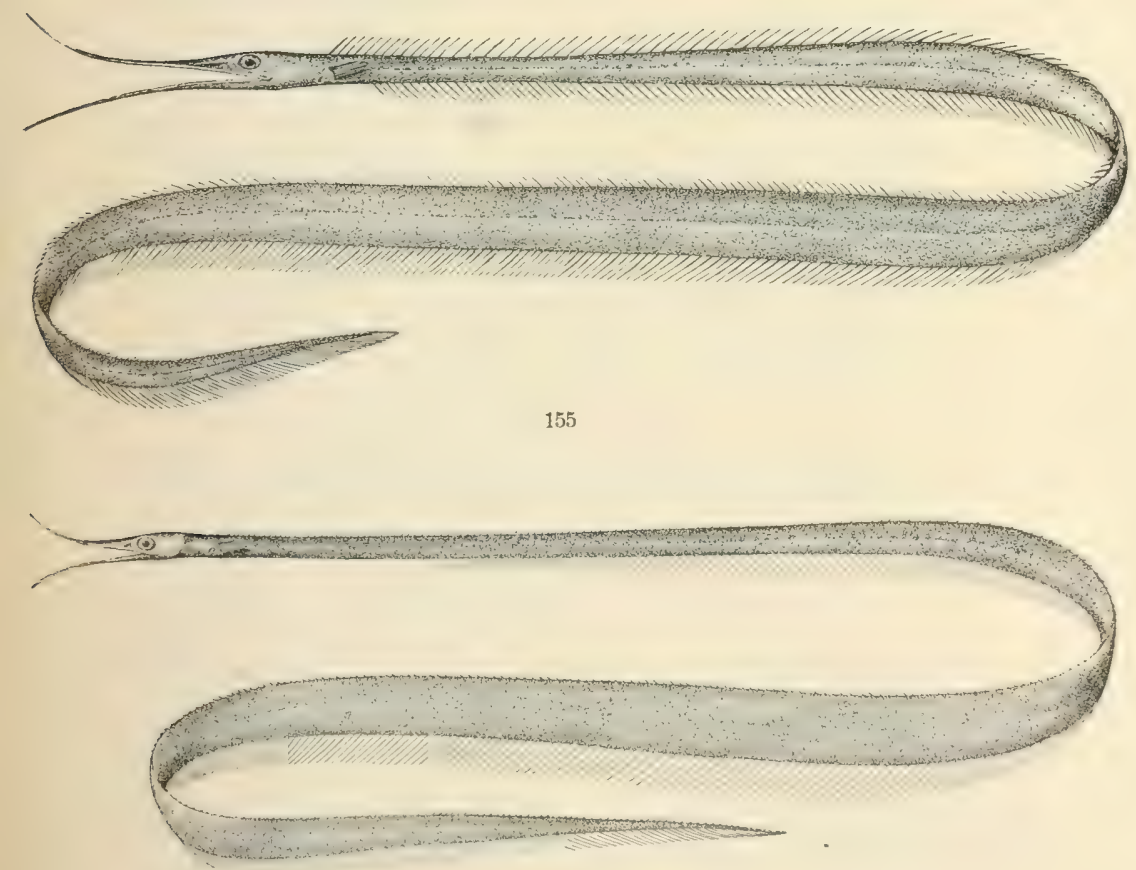

$151 ;$

154. Avocettina gilli. (Pp. 367, 2801.

154a, 144b. AVOCETTINA FILLI. (P. 2801.)

155. LabicituY's carinatus. (P. 368.)

156. LABICHTHY'S ELONGATUS. (1'369.) 



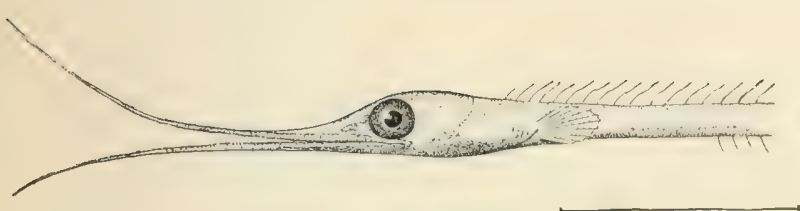

$15 \tilde{\pi} \alpha$
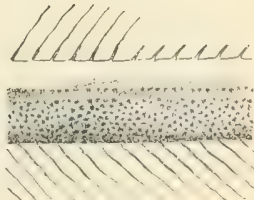

$15 \%$

C.

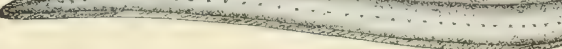

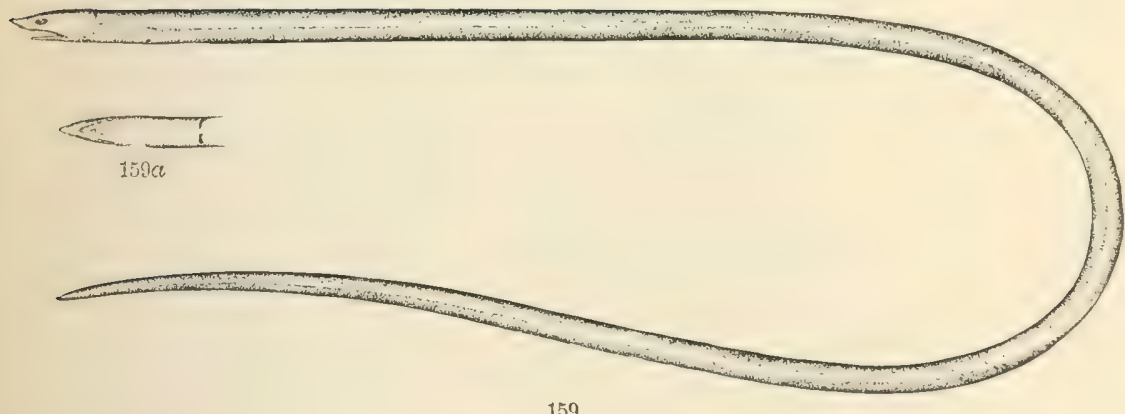

159

157, $157 a, 157 b$. Nemichthys Avocetta. (P. 369.)

158. AHLiA Egmontis. (P. 370.$)$

159, 159a. VERMA KENDALLI. (P. 375.) 



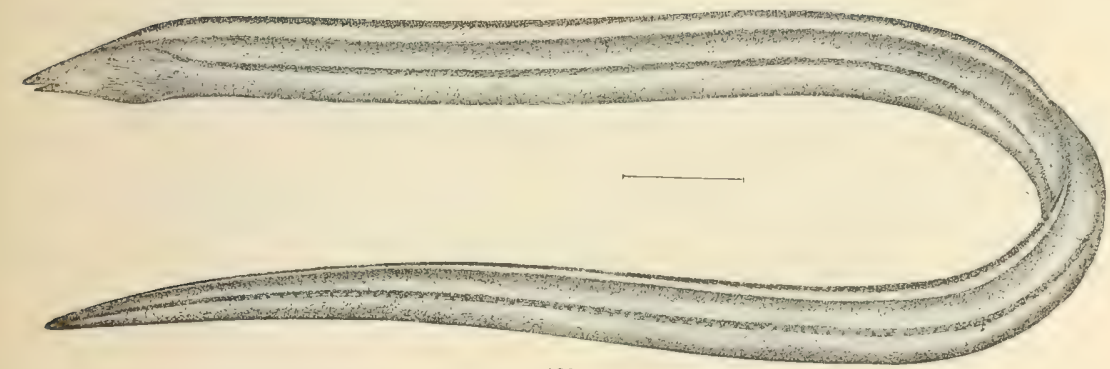

160

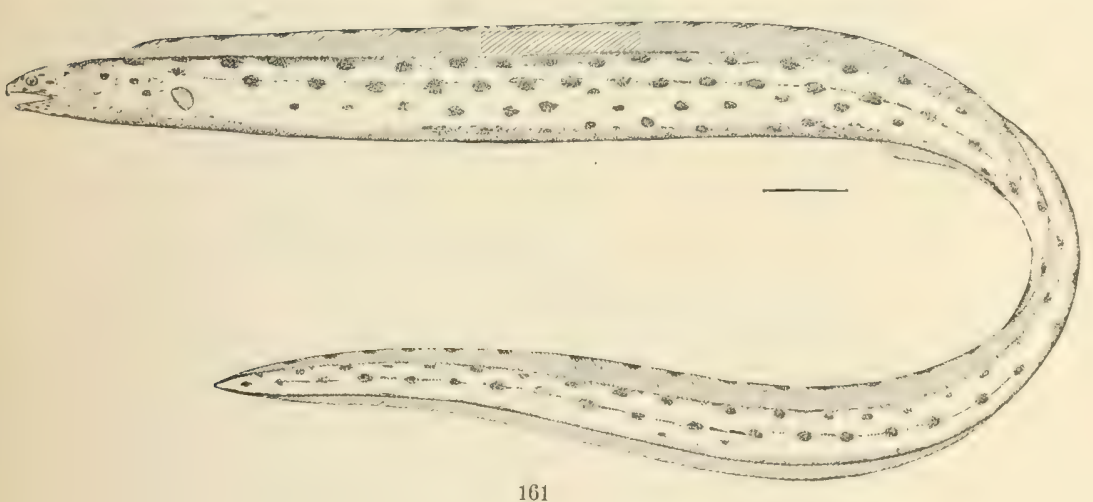

160. LETHARCHUS VELIFER. (P. 375.)

161. Myrichtiys tigrinus. (P. 376. 


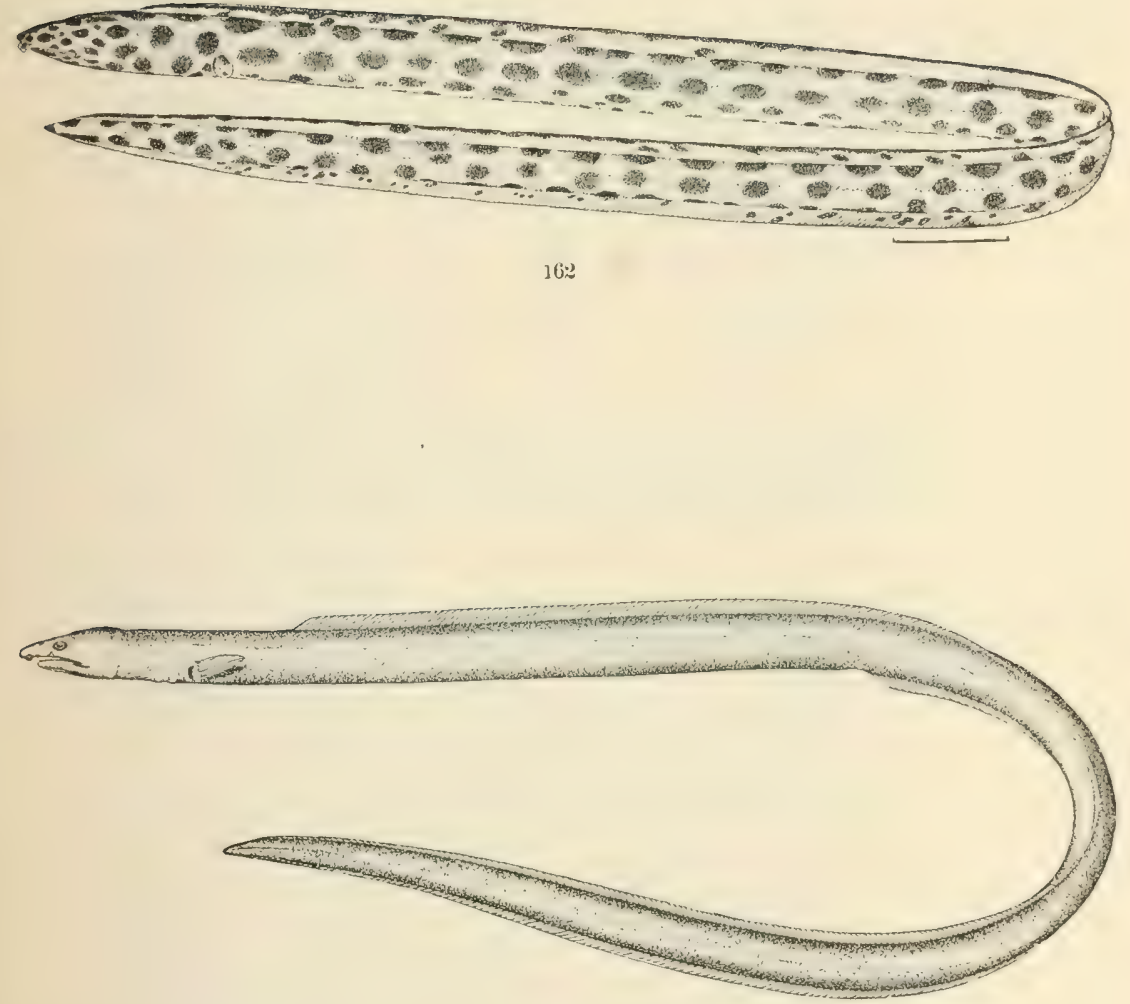

163

162. Myrichthys pantostigmius, (P. 2802.)

163. PISOODONOPHIS CRUENTIFER. (P. 377.) 



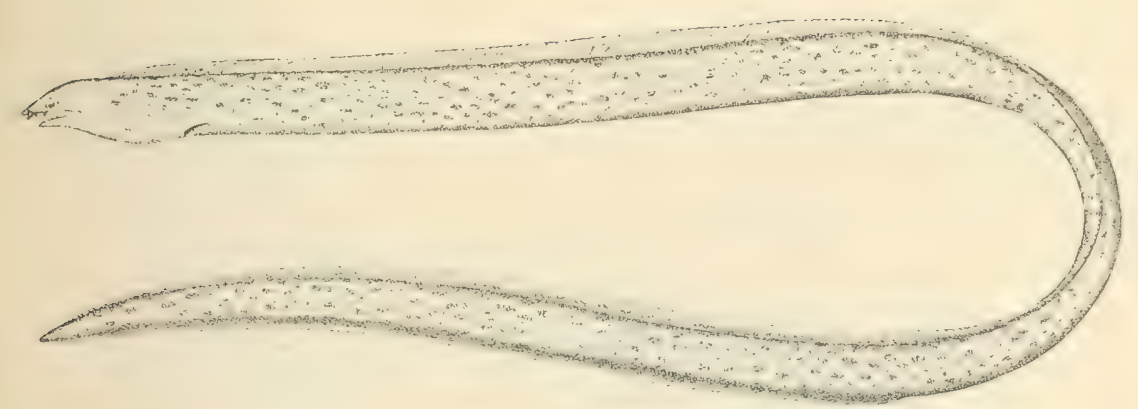

164

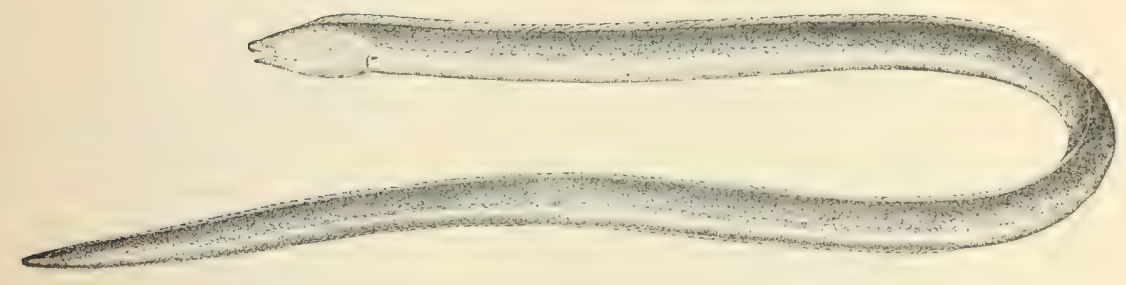

165

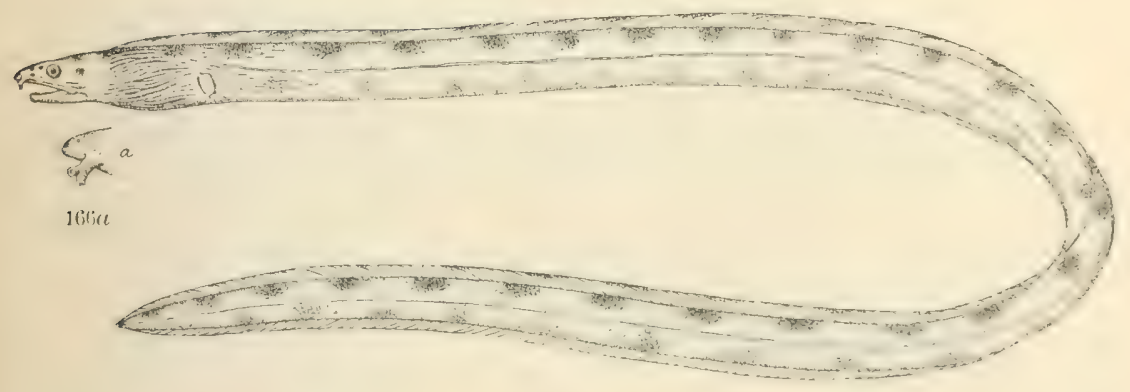

teiti

164. Calecinelys muliena. (P. 378.)

165. BASCANICHTHYS SCUTICARIS. (P. 378.)

166, 166a. BASCANICHTHYS PENINSULA. (P. 379.) 


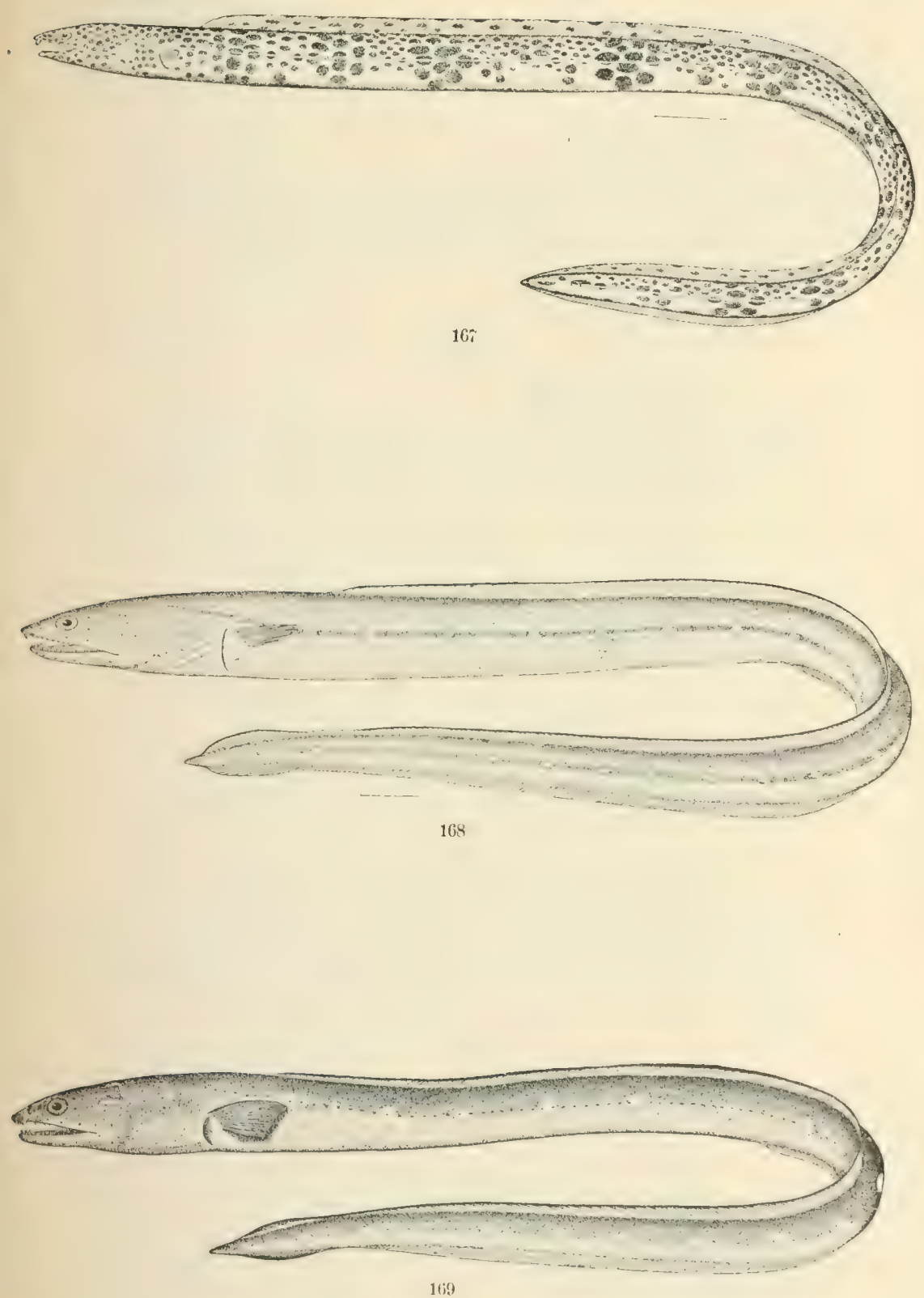

167. QAssiremes eviontias. (P. 380. )

168. OPHichtius GUtTIFER, (P. 383.)

169. Ophichthus ocellatus. (P.383.) 



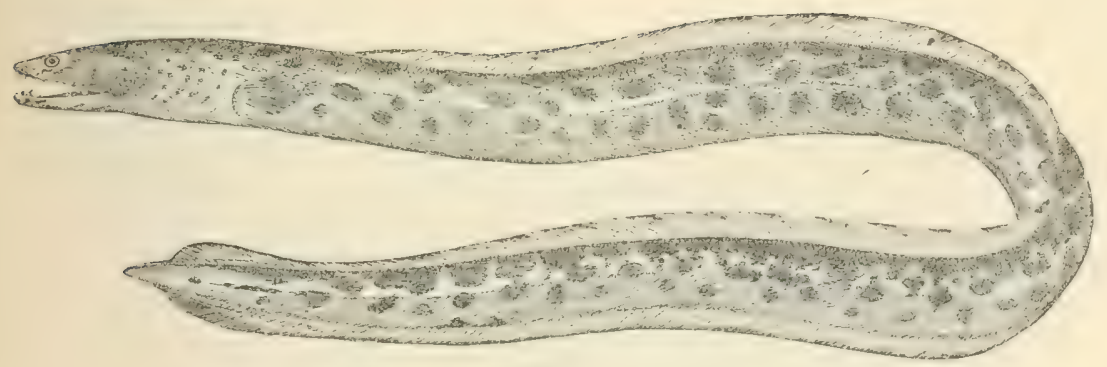

1ii)

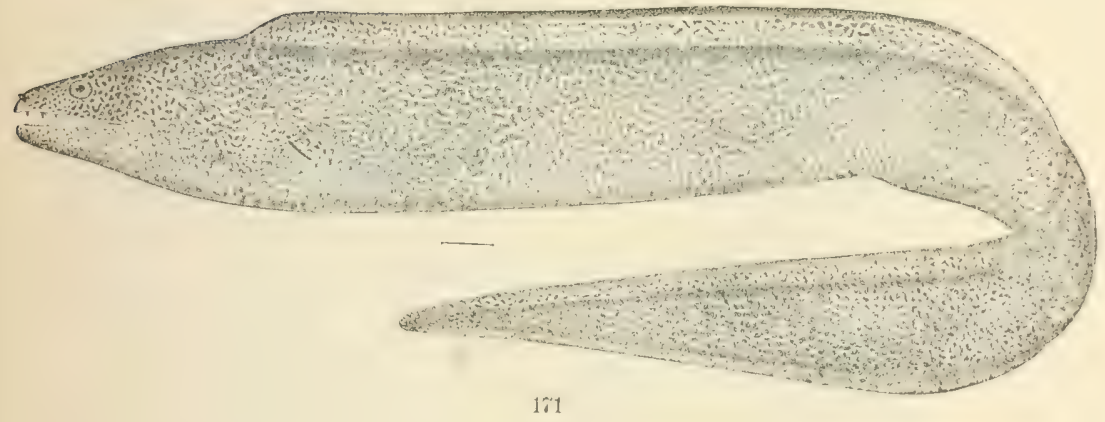

101

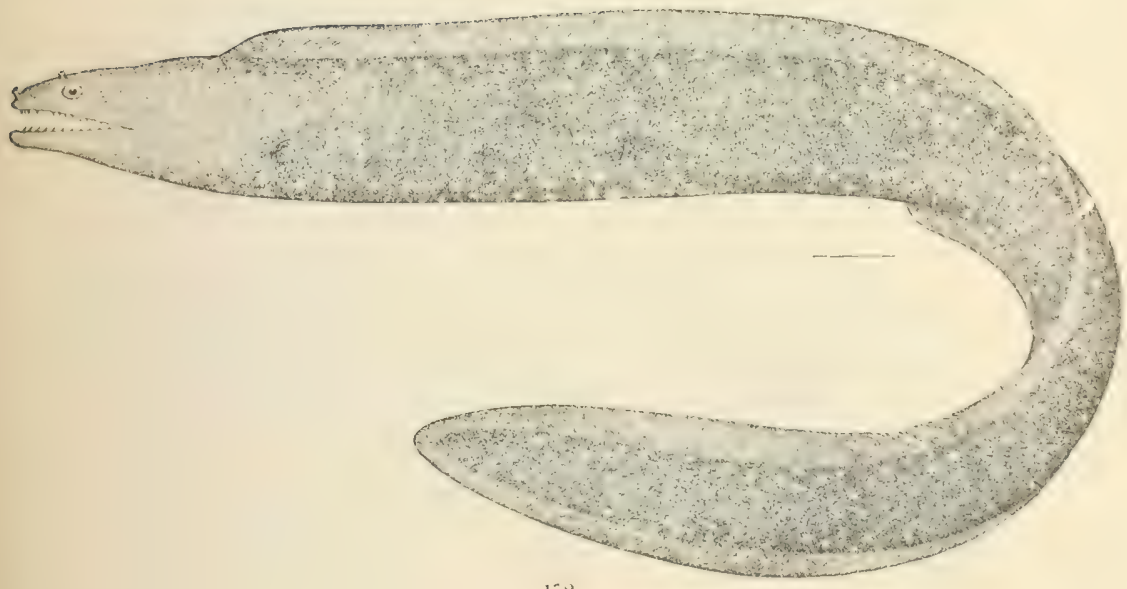

$17:$

170. MYstunopHIs Ixtentinctus. (P. 386.)

171. LYCODONTIS MORINGA. (1).395.)

172. MLrina iNsulatua. (P. 400.) 


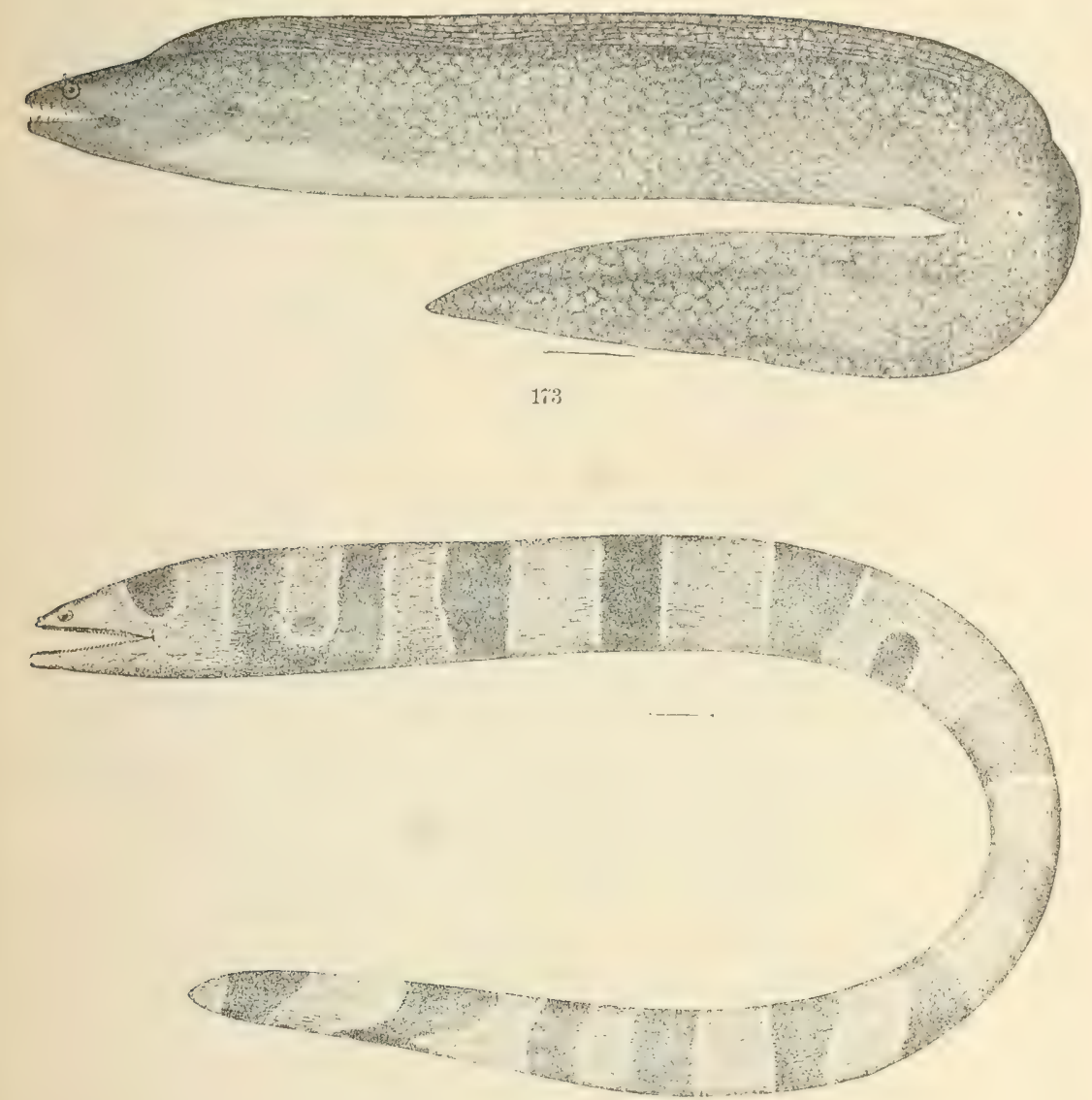

171

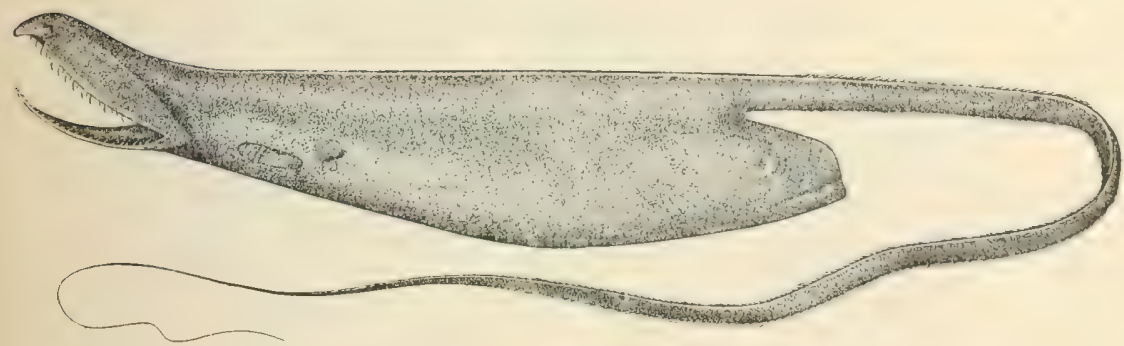

17.)

173. MUR.eNA Retifkra. (P. 401.)

174. Channoierena vittata. (P. 404.)

175. Saccopharyax ampullaceus. (I'.406.) 


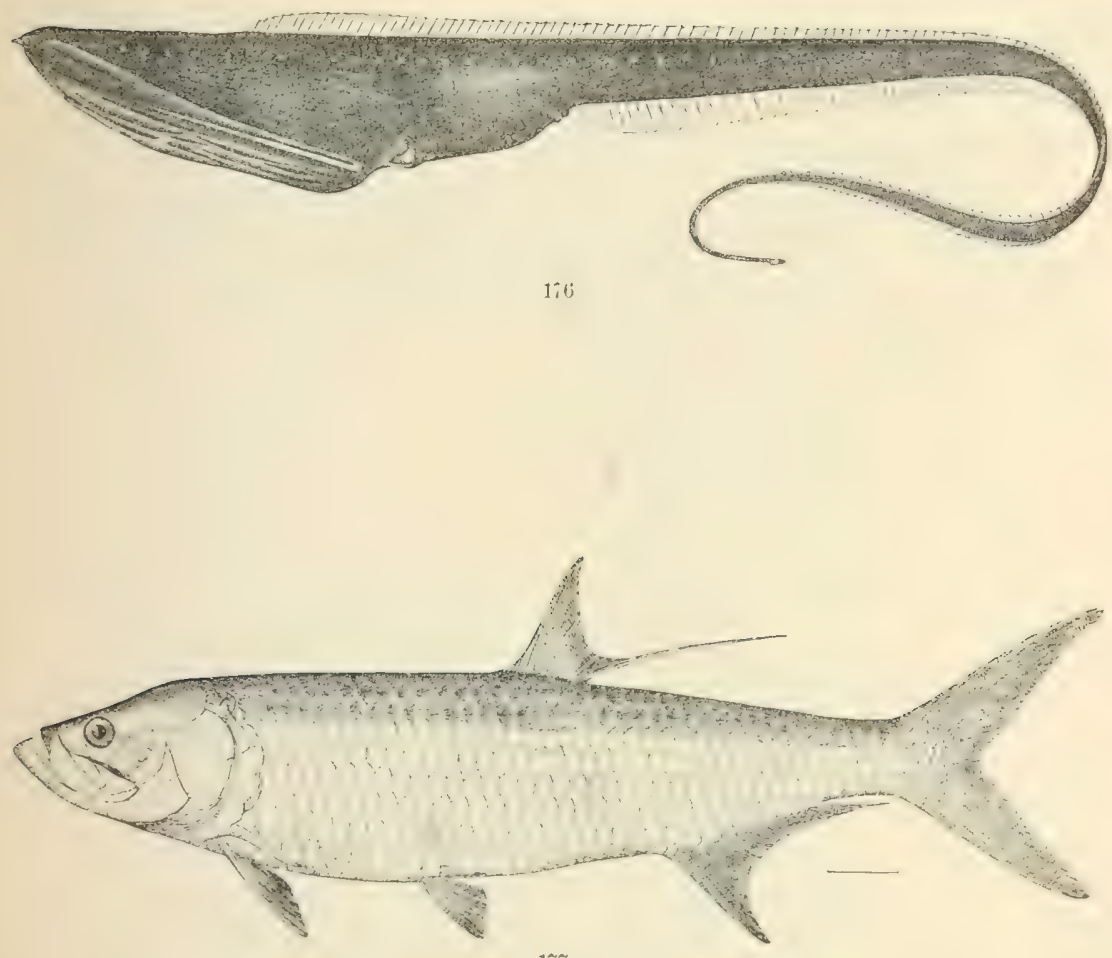

$1 \pi \tilde{1}$

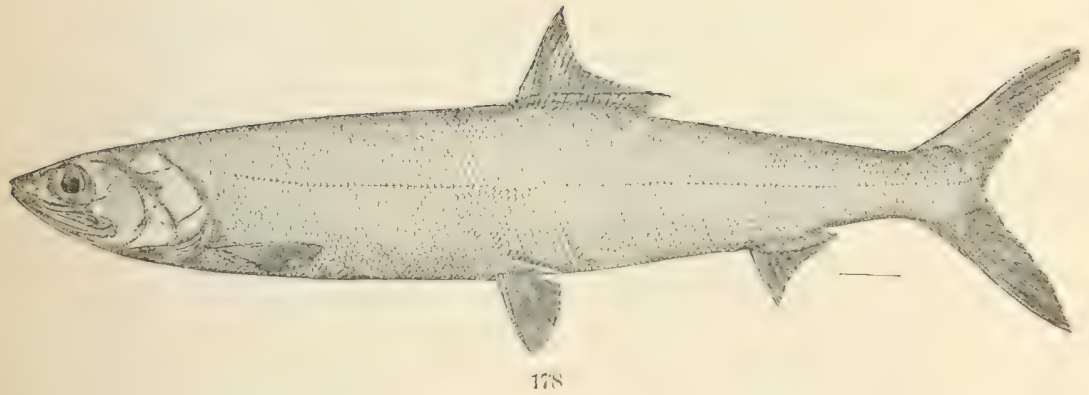

176. Gastiostomus Bandir. (P.406.)

177. 'IARPON ATLANTICUS. (P. 409.)

178. Lilops satrus. (P.410.) 



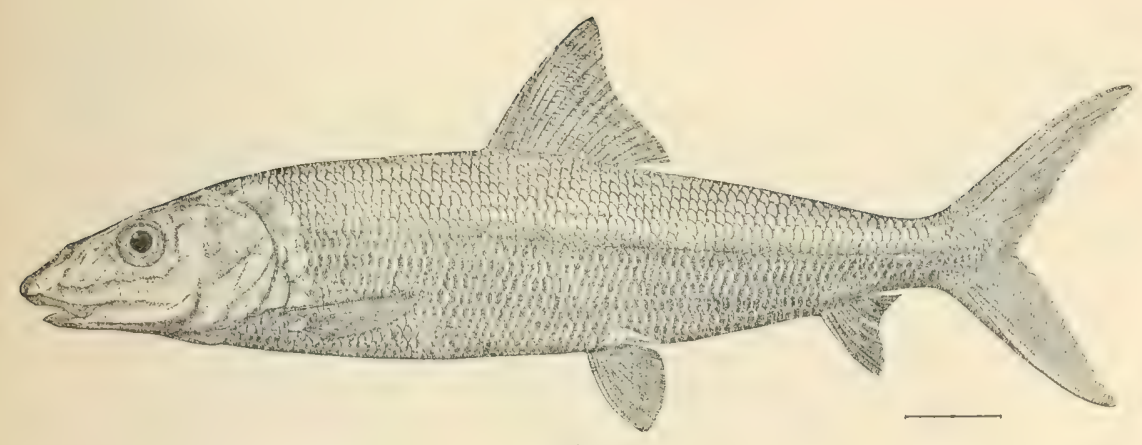

179

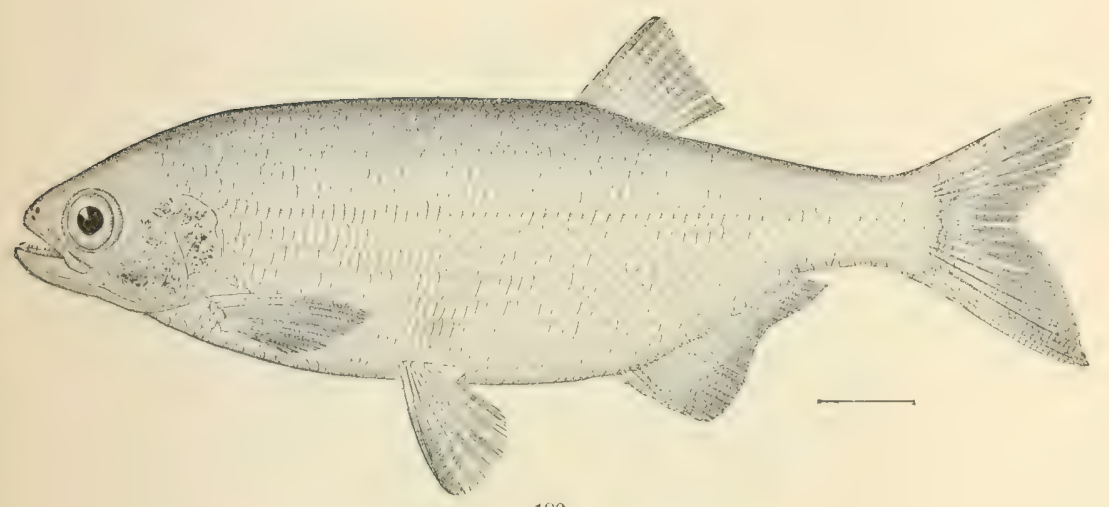

180

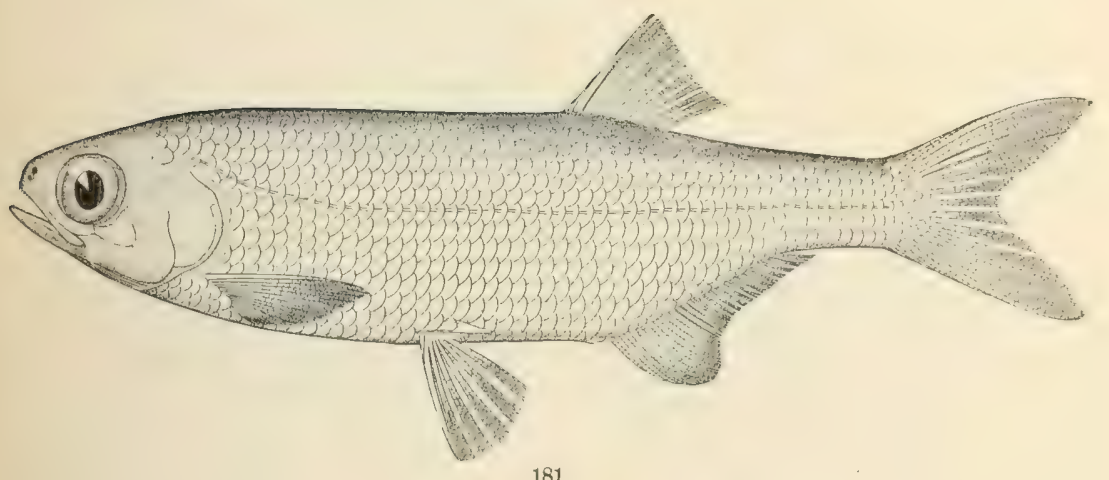

181

179. Albula vulpes. (P, 411.)

180. HIODON TERGISUS. (P. 413.)

181. HIODON SELENOPS. (P. 414.) 


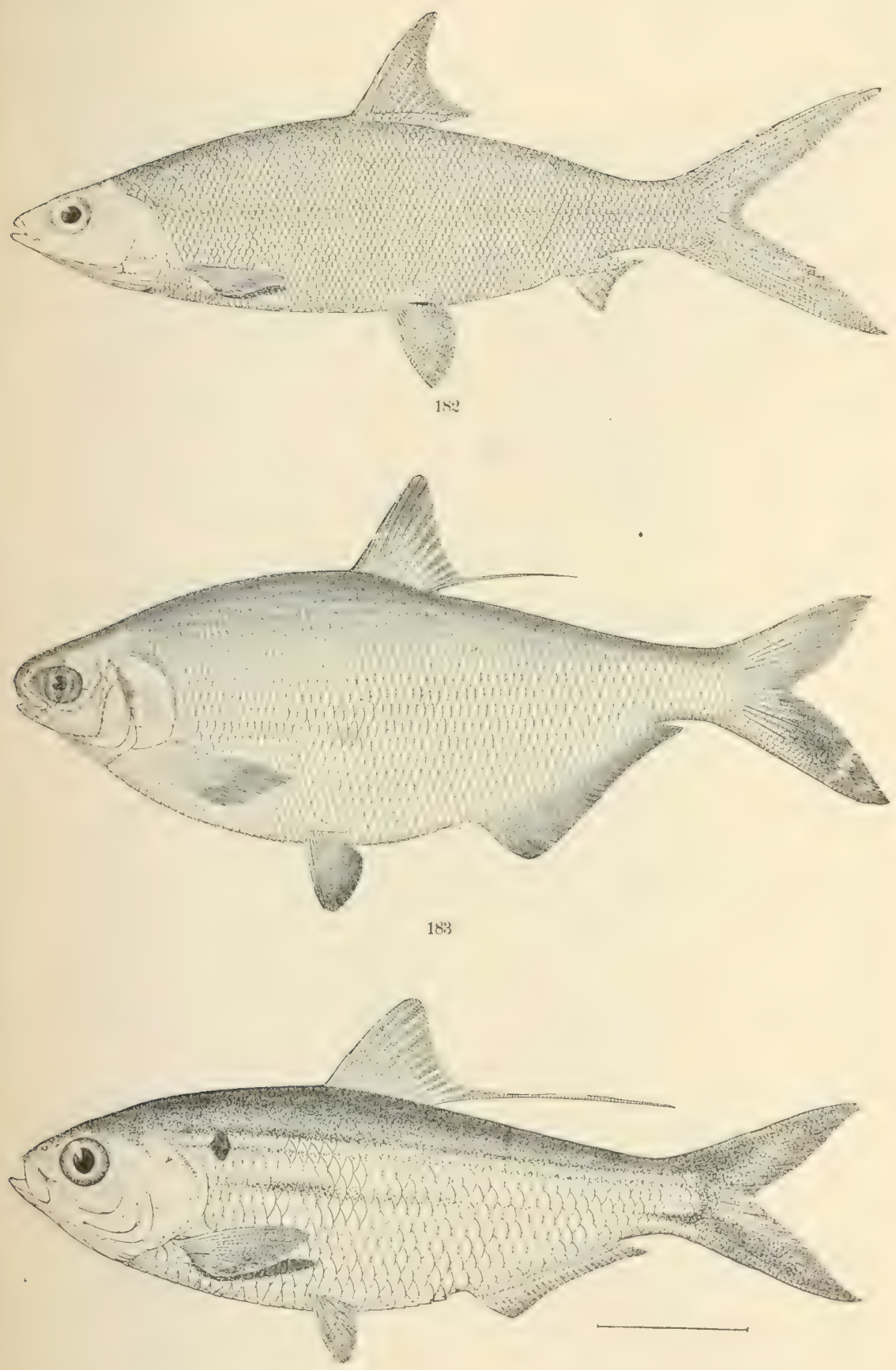

184

- 182. Chanos chajos. (

183. DOROSOMA CEPEDANUM. (I’. 416.)

184. SIGNAIOSA ATCHAFALAYA. (P. 2809.) 


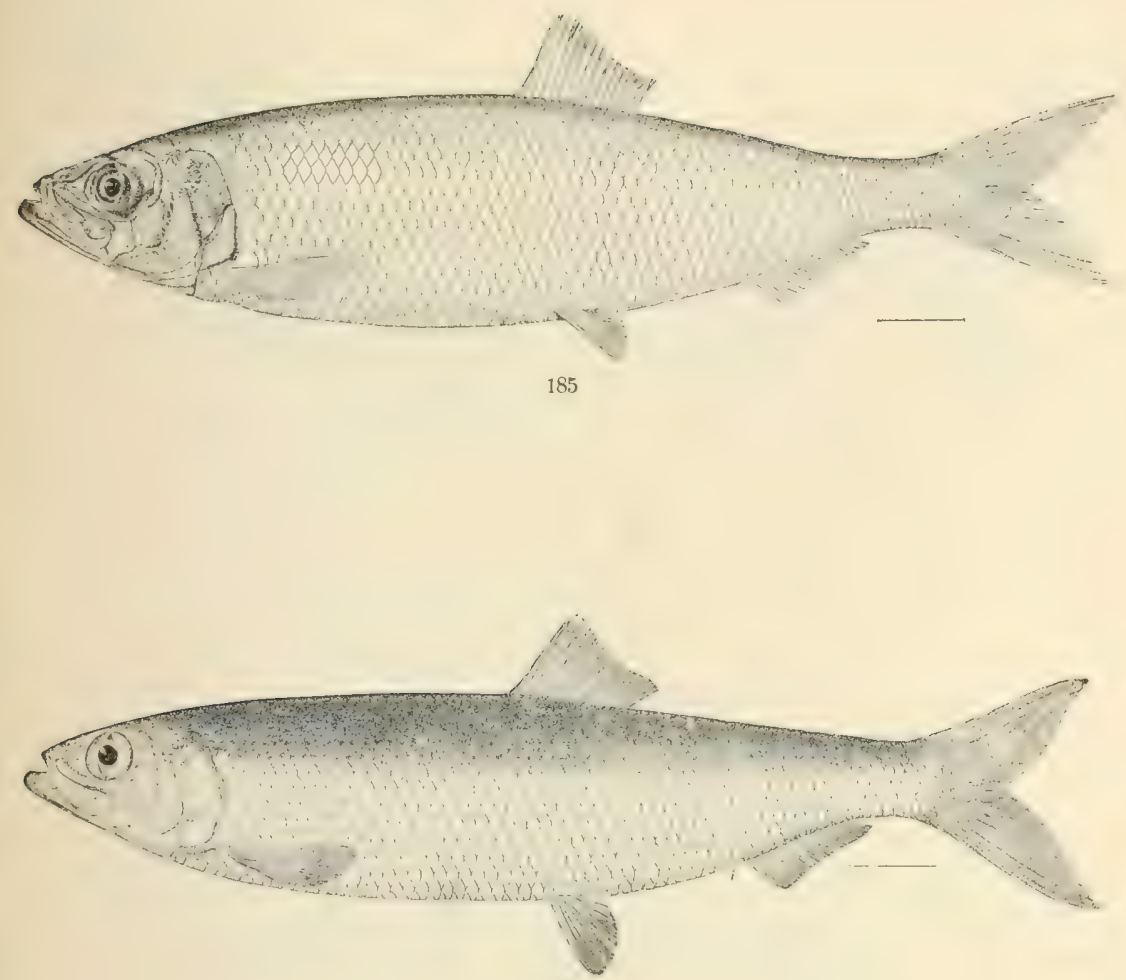

186

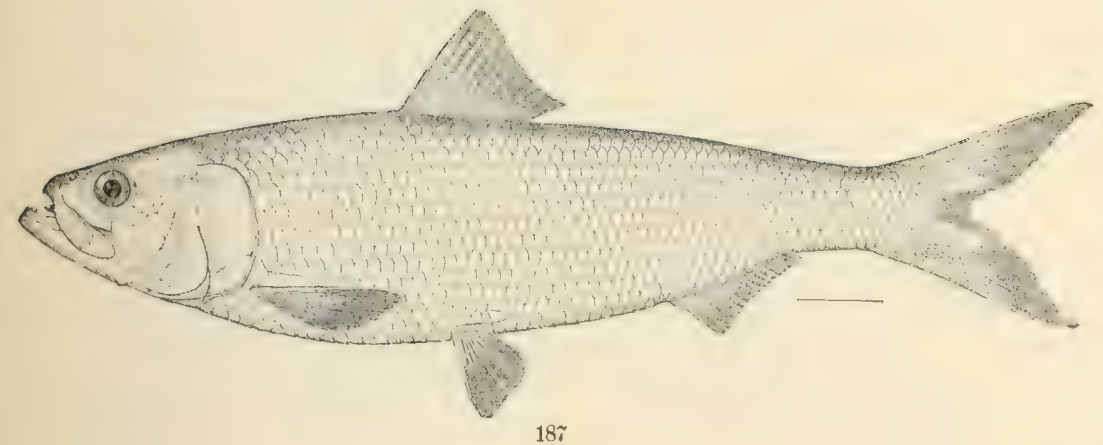

185. Clupea harejgus, (P. 421.)

186. Clupea Pallasit. (P. 422.)

187. POMOLOBUS CHRYSOCHLORIS. (P. 425.) 


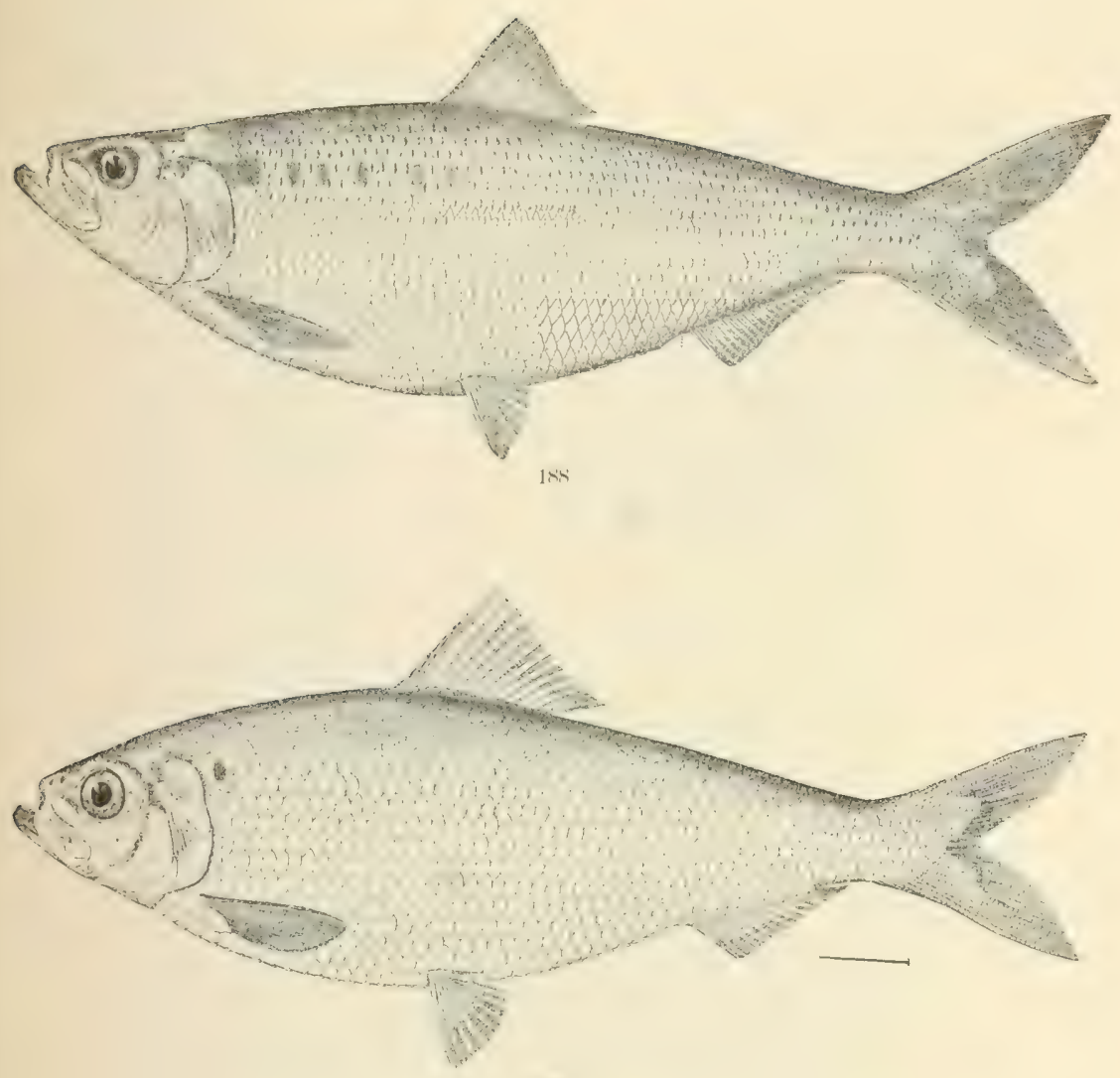

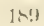

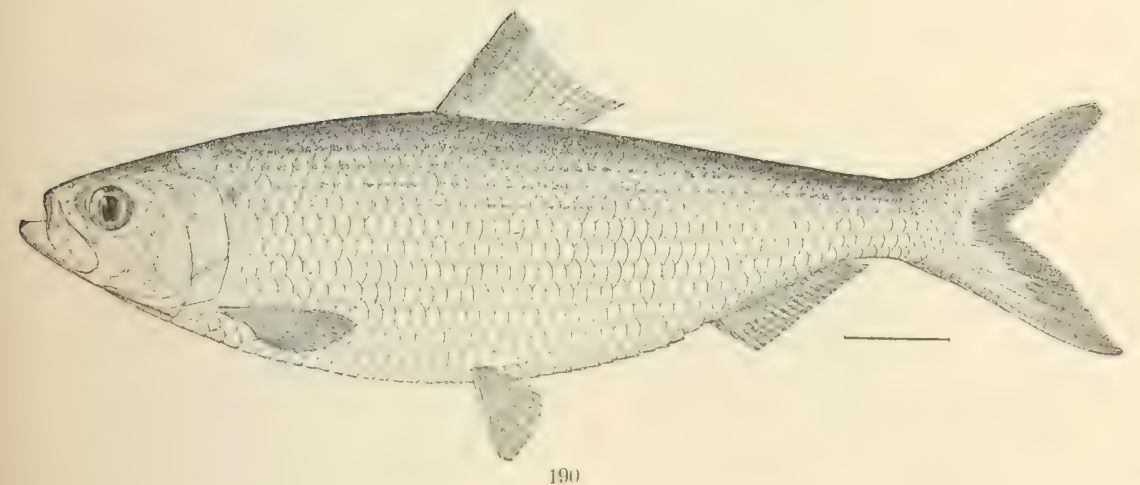

188. POMOLOBUS MEDIOCRIS. (P.425.)

189. Pomolobus Pseudoharengus. (P. 426.)

190. Pomolonus Nistivalis. (P. 426.) 



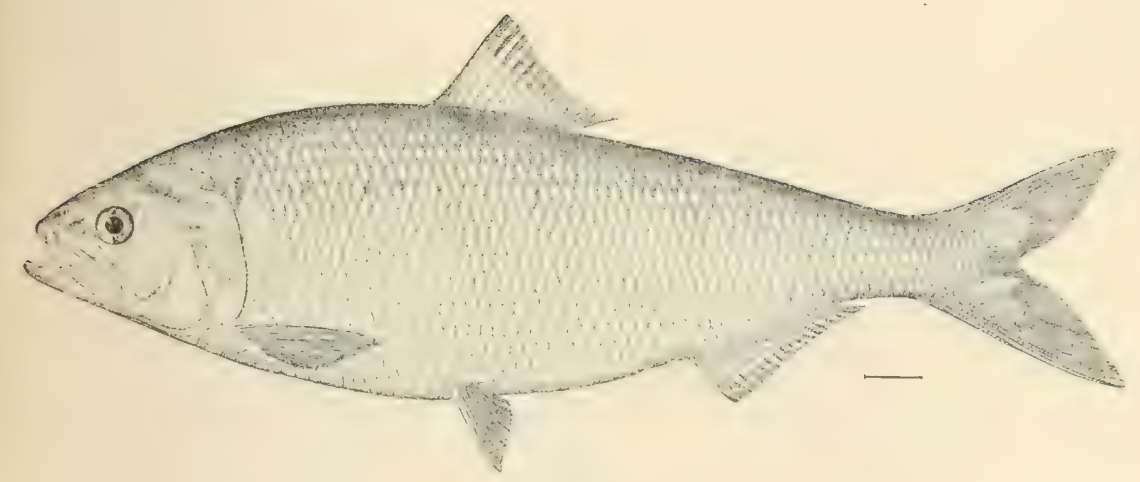

191

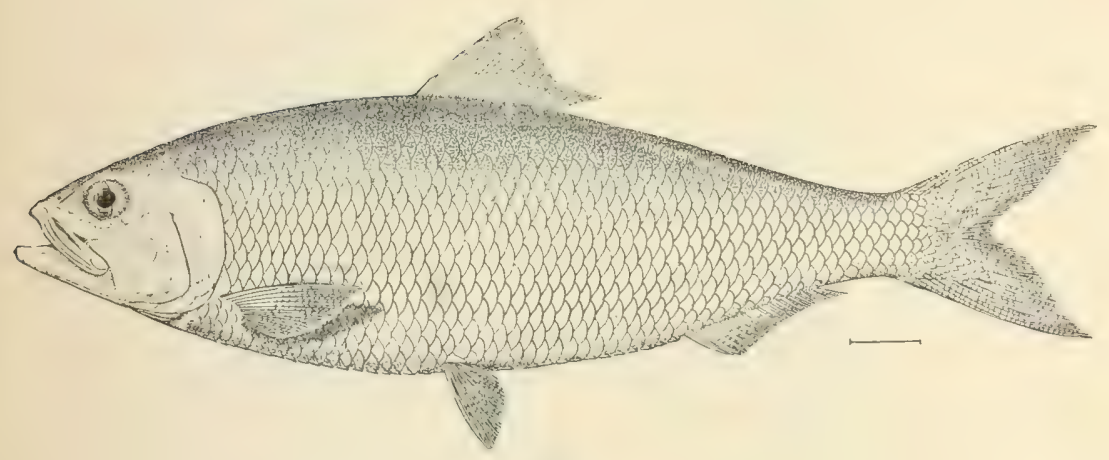

19:

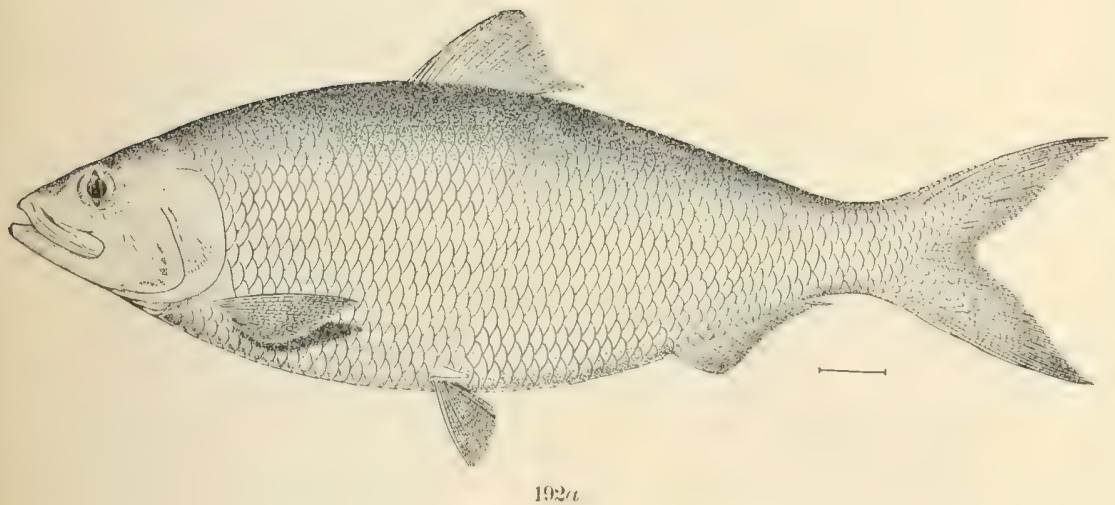

191. Alosa sapidissima. (P.427.)

192. Alosa alabame; male. (P. 2810.)

192a. Alosa alabama; female. (P.2810.) 



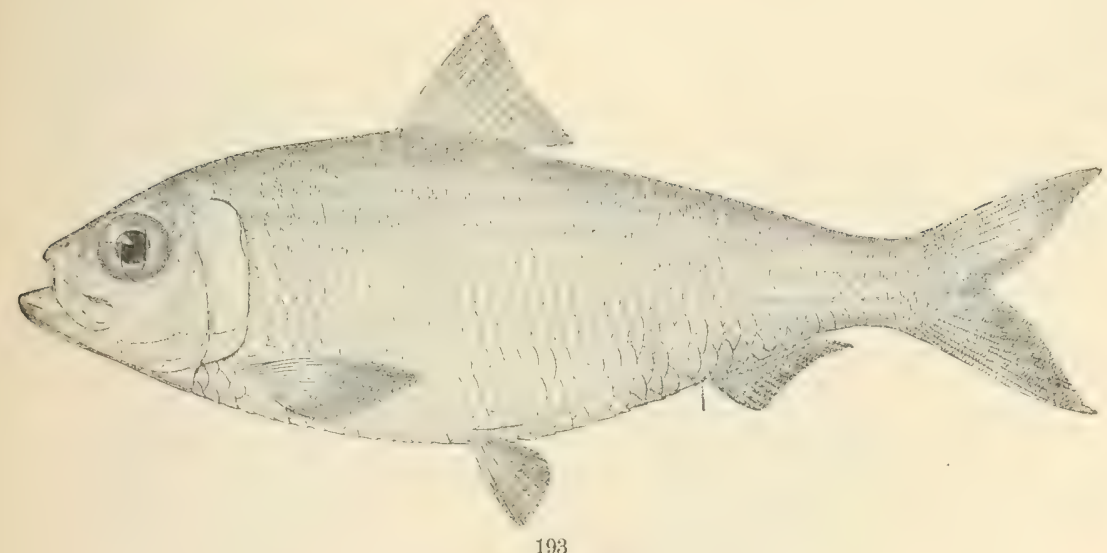

193

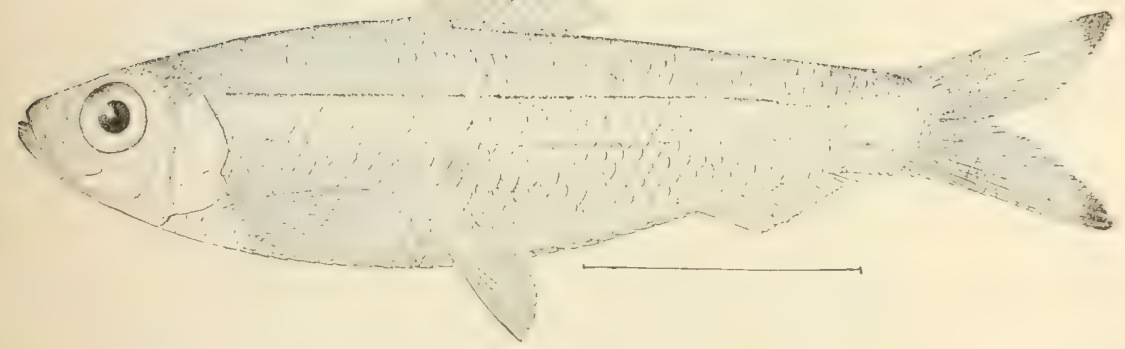

194

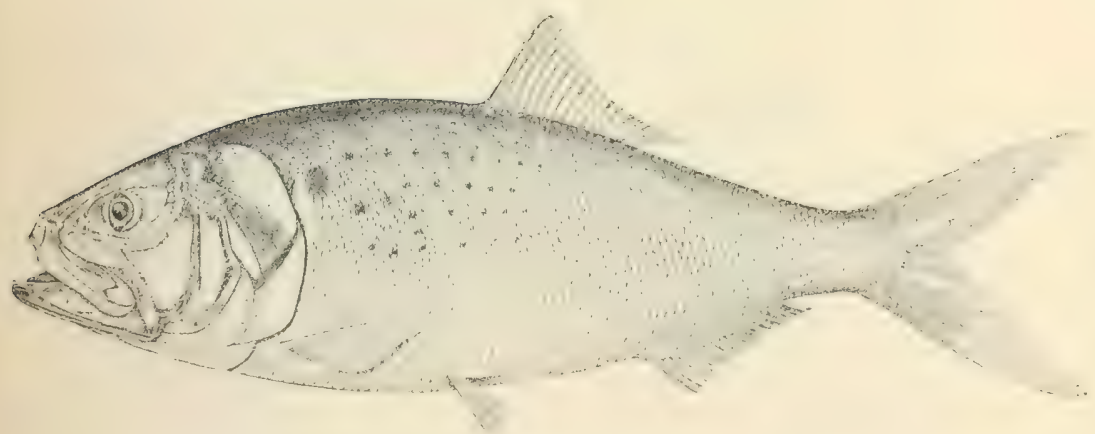

195

193. SARDINELLA SARIINA. (P.430.)

194. SARDINELLA STOLIFERA.' ( $\left.\mathrm{P}^{\prime}, 431.\right)$

195. BireYoortia TYRANNUS. (P. 433. ) 



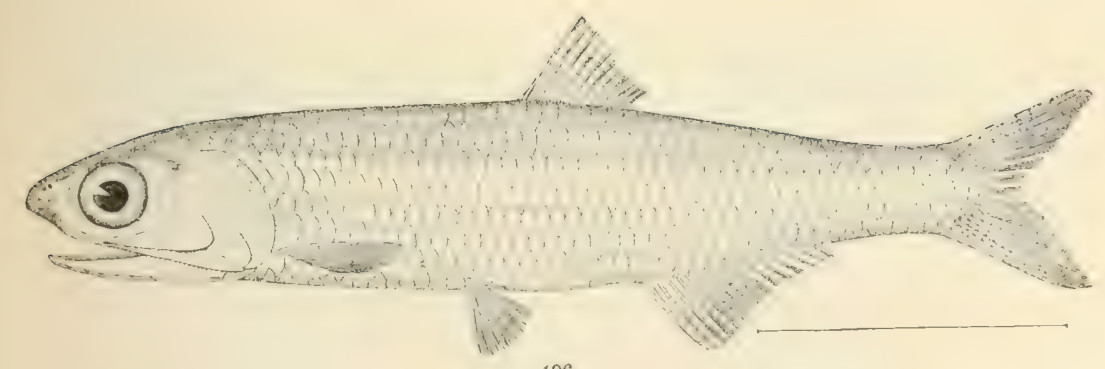

196

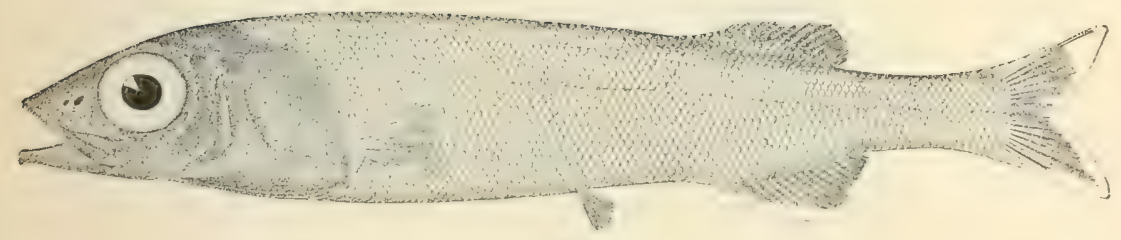

$19 \pi$

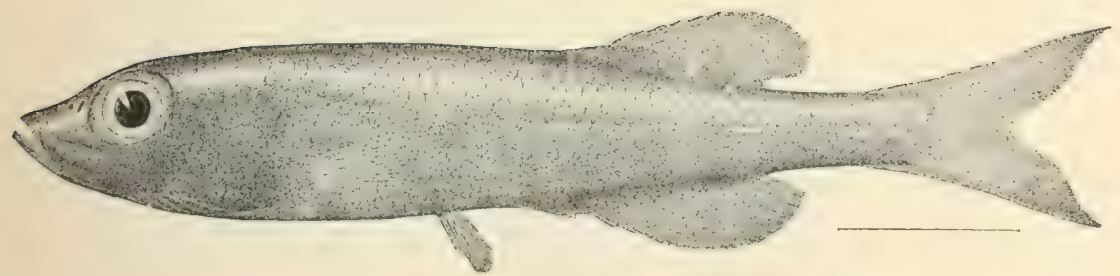

198

196. Stolephorus perthecatus. (P. 442.)

197. AlepoCephalus agassiziI. (P.453.)

198. Talismania aNtillarum. ( $\mathrm{P}, 455$. 



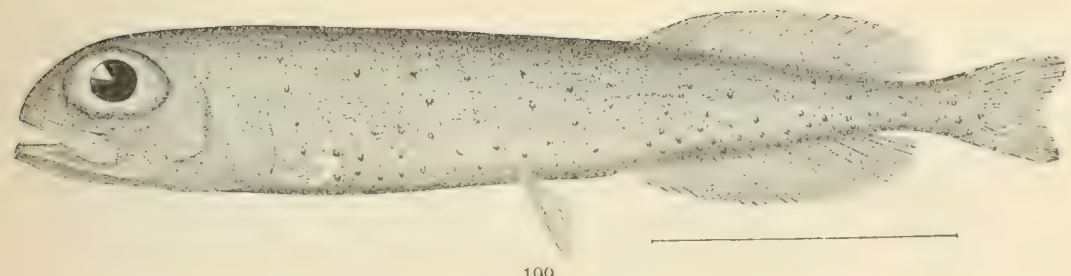

193

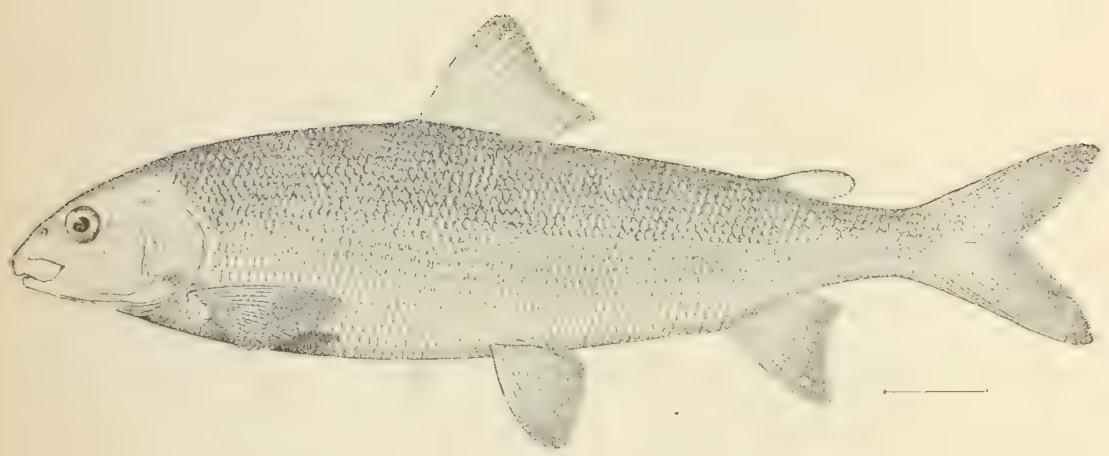

200

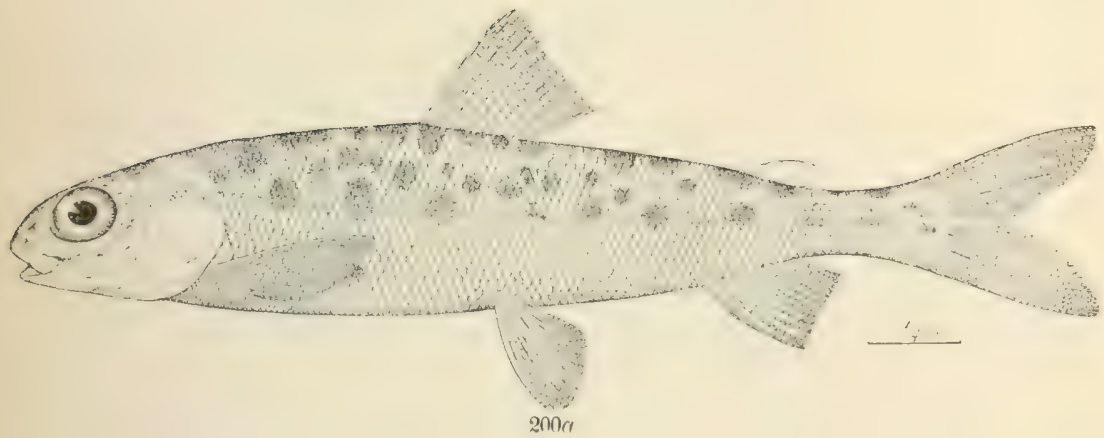

199. Alwosonus COPEI. (P. 459.)

200. Coregonus Wili.iamsoni. (1'.463.)

200a. Corkgoni's WIlliamsoxi; yommg. (P. 463.) 


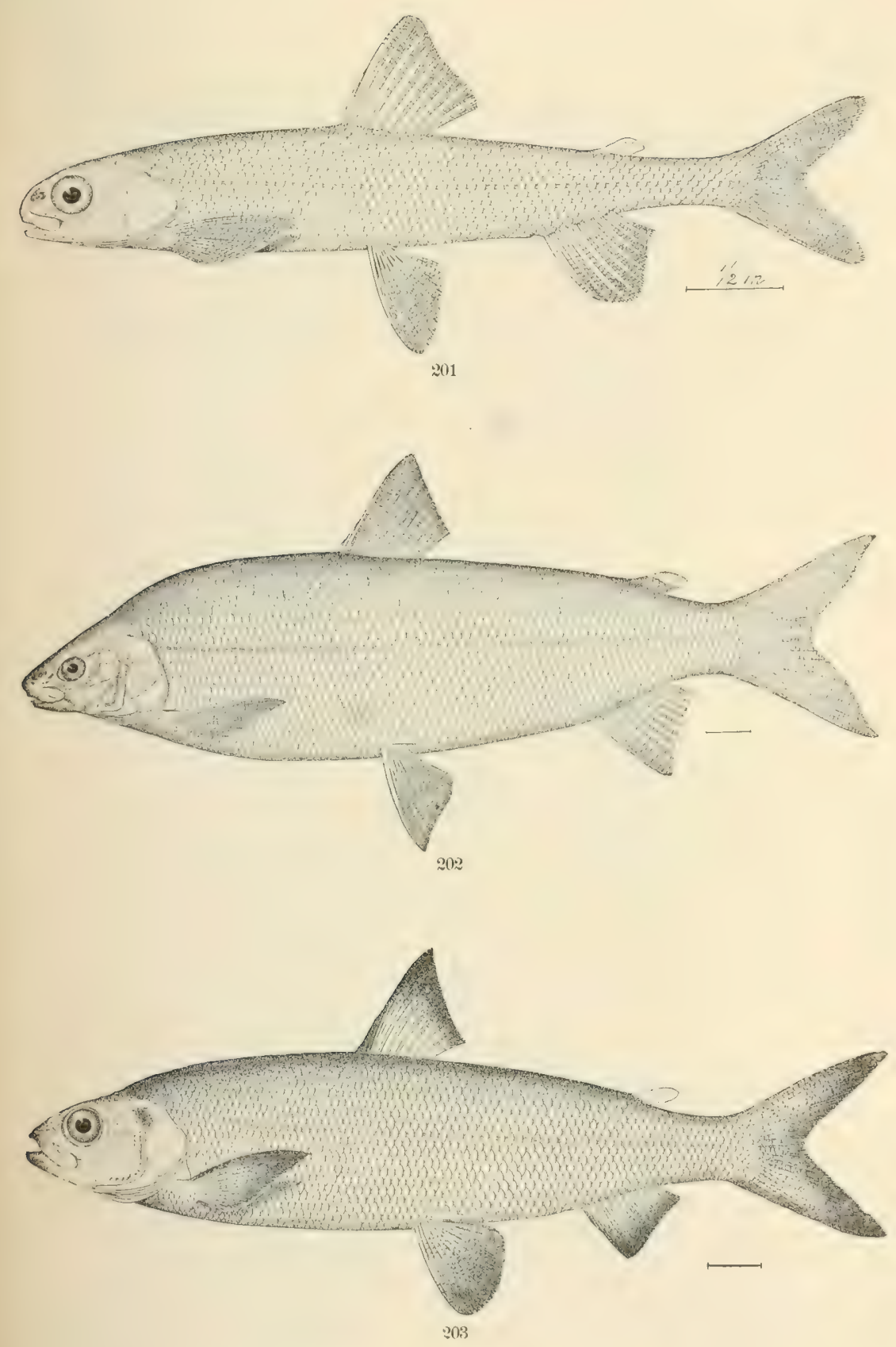

201. Corfenus coulterif. (P. 462.)

202. Coregonus ClUPEIFORMis. (P. 465.)

203. Argyrosomus NigripinNis. (P. 472.) 


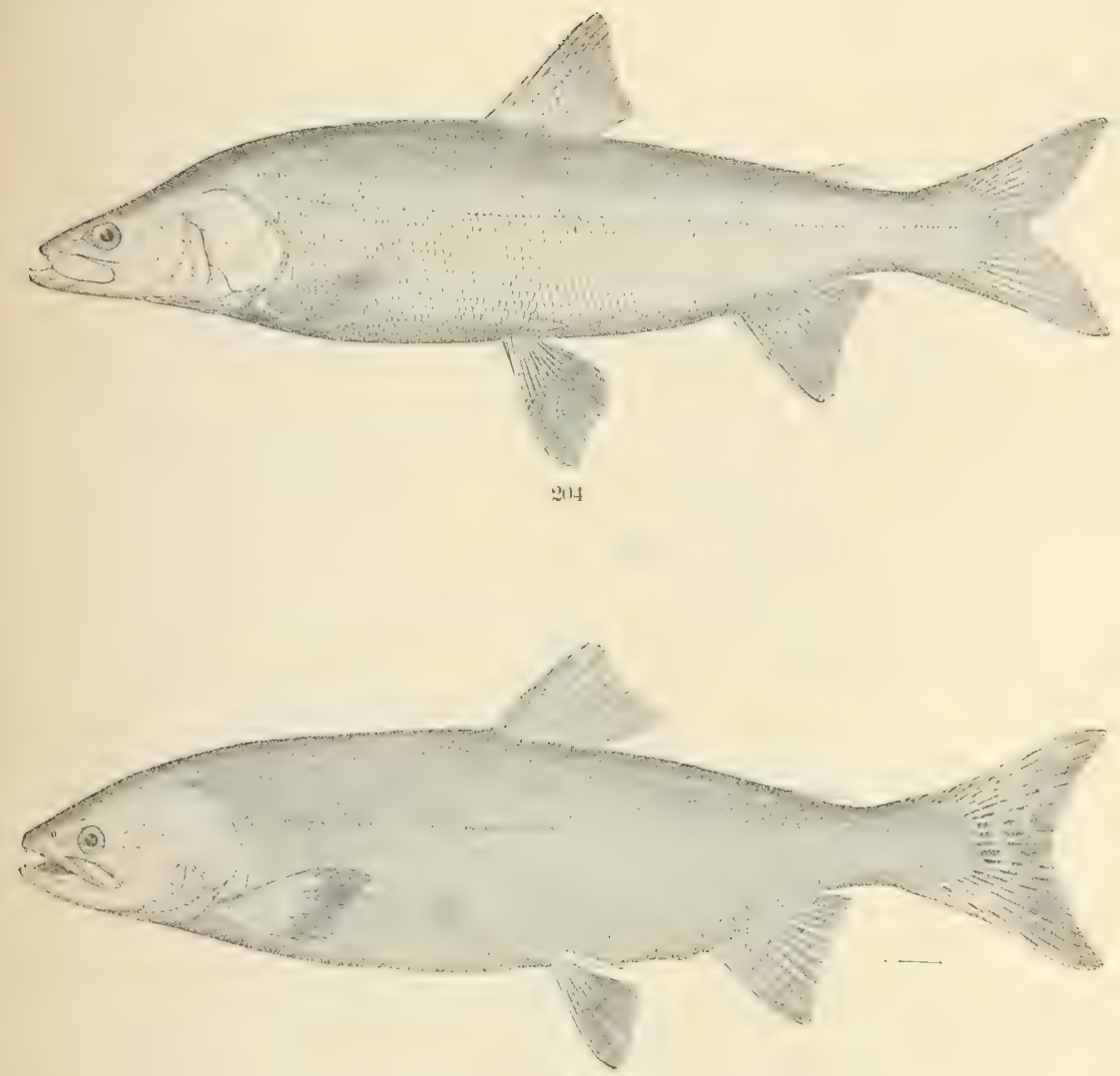

205

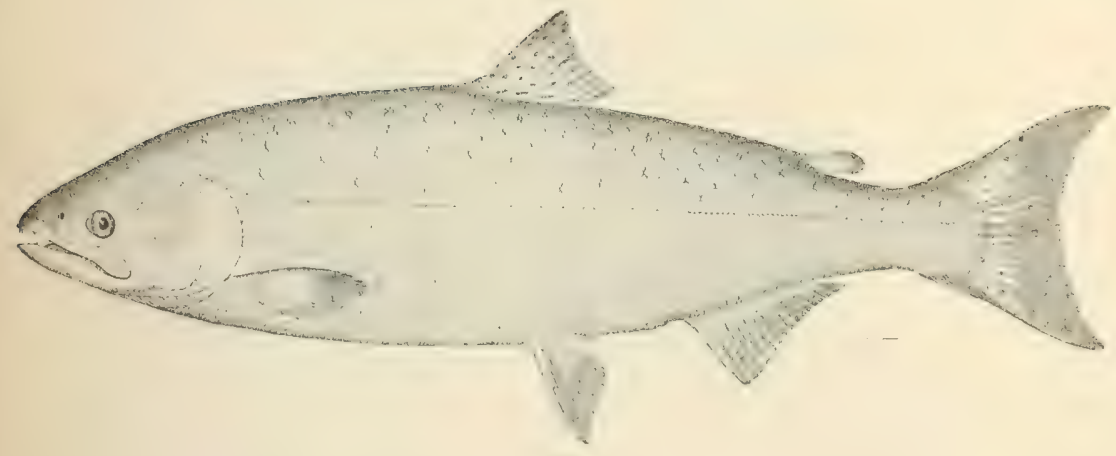

301

204. STENODUS MACKEN\%11. (P. 474.)

205. ONCORIYNCHCS GORBUSCHA. (P. 478.)

206. ONCORHYNCHUS TACHAWYTSCHA. (P.479.) 


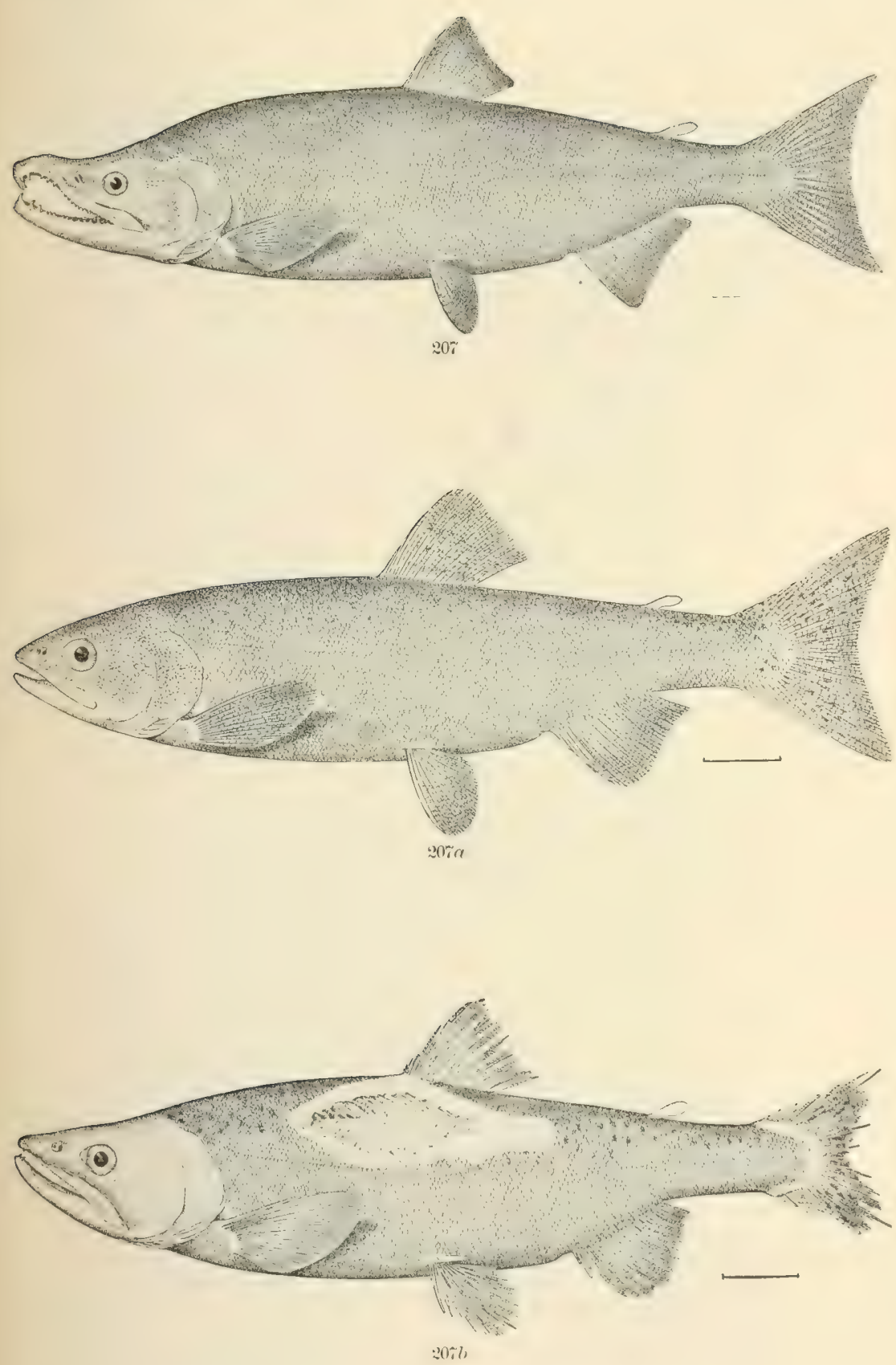

207. ONCORHYNCHUS NFiki; adult male, large form. (P. 481.)

207a. ONCORIYNCHUS NERKA; adult female, small form. (P. 481.)

207b. ONCORIYNCHUS NERKA; adnlt mutilated male, small form. (P. 481.) 


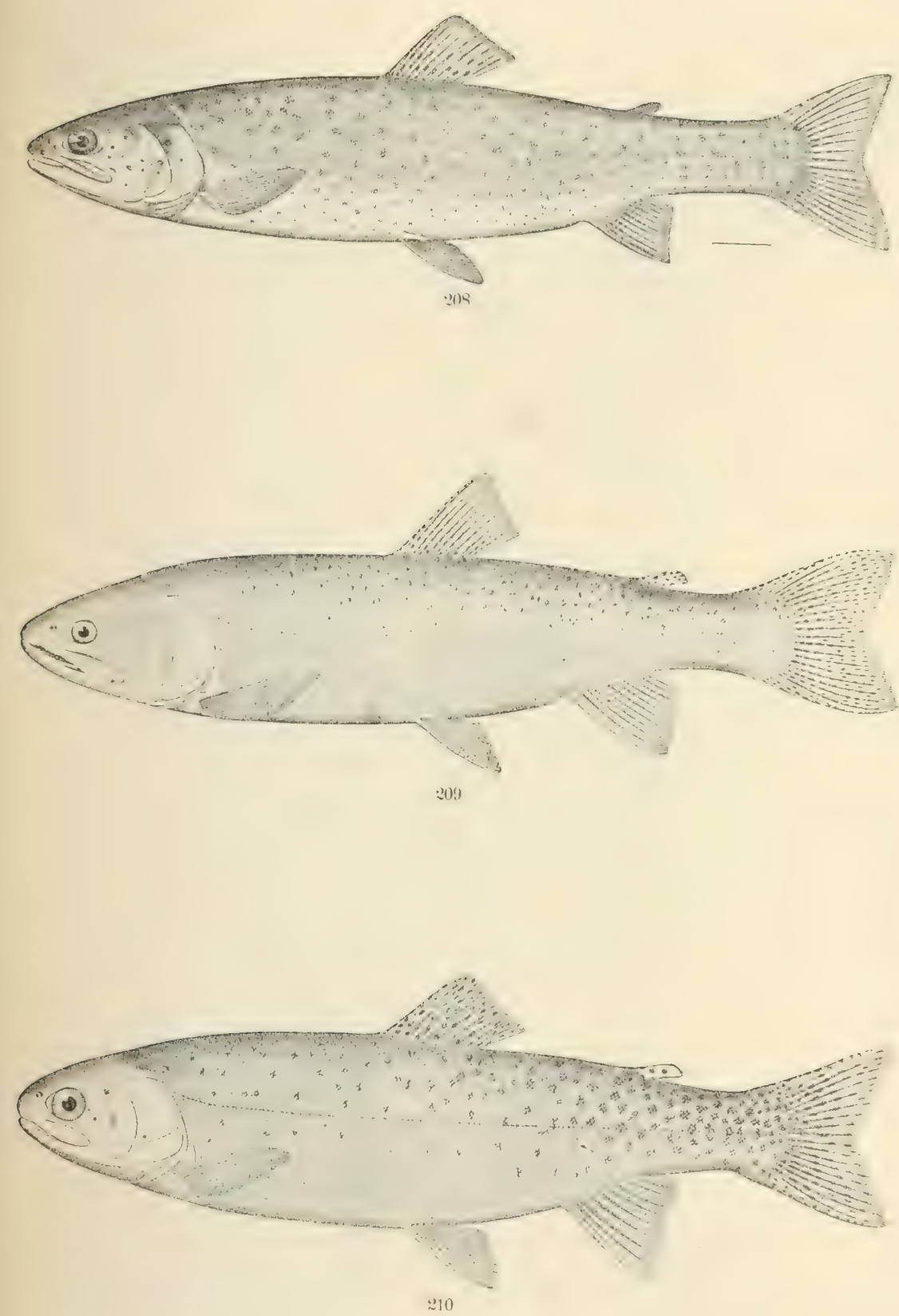

208. SALMO) CLARKII IIENSHAWт. (Pp. 493, 2819.)

209. SALMO CLARKII VIRGINALIS. (I'p, 495, 2819.

210. Salmo clarkil spilurus. (Pp. 495, 2819.) 


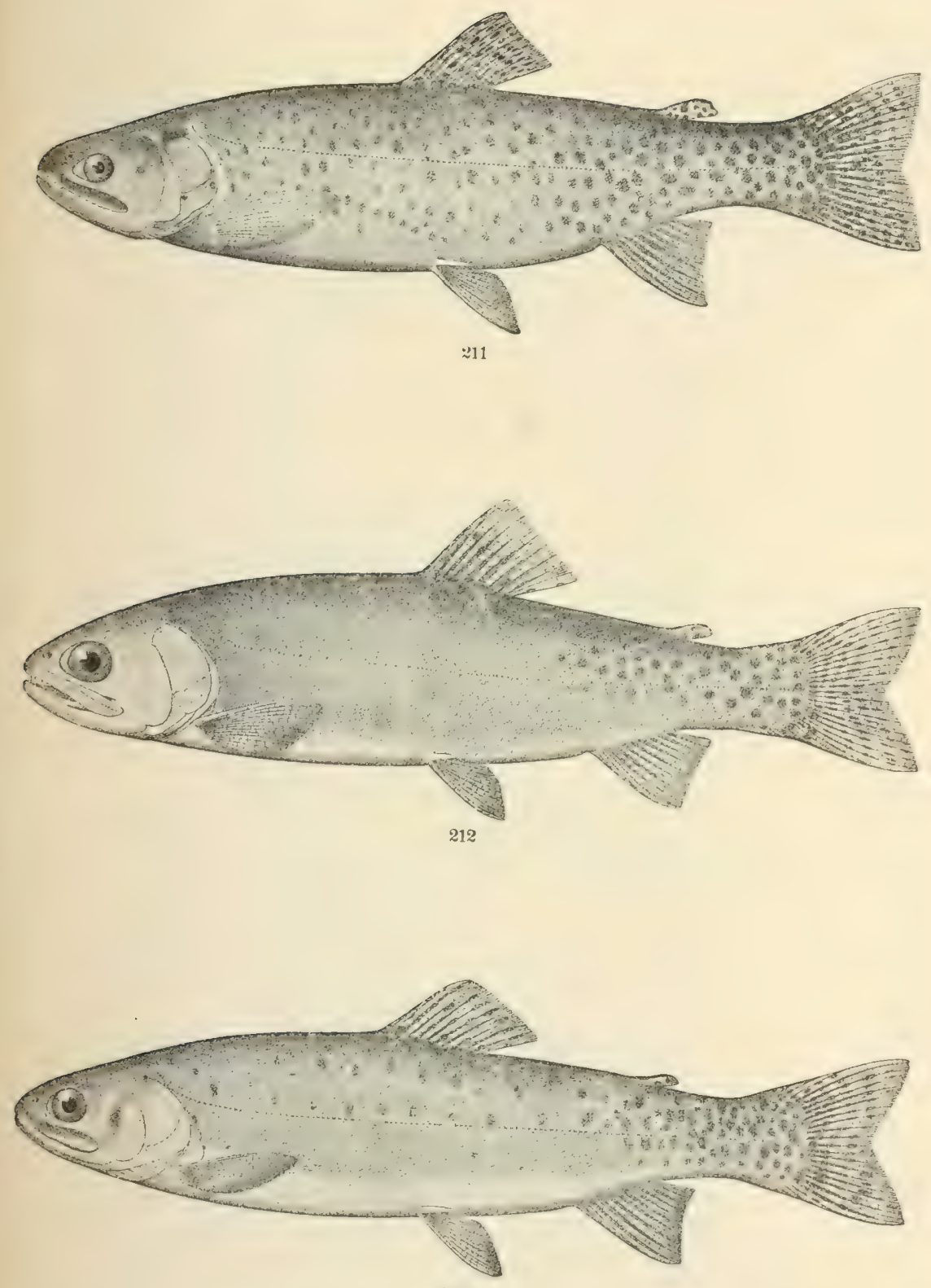

213

211. Salio Clarkil Pleuriticus. (Pp. 496, 2819.)

213. SALMO Clarki BOUVIERI. (Pp. 496, 2819.)

213. Saljo CLARKII STOMIAS. (Pp.497, 2819.) 



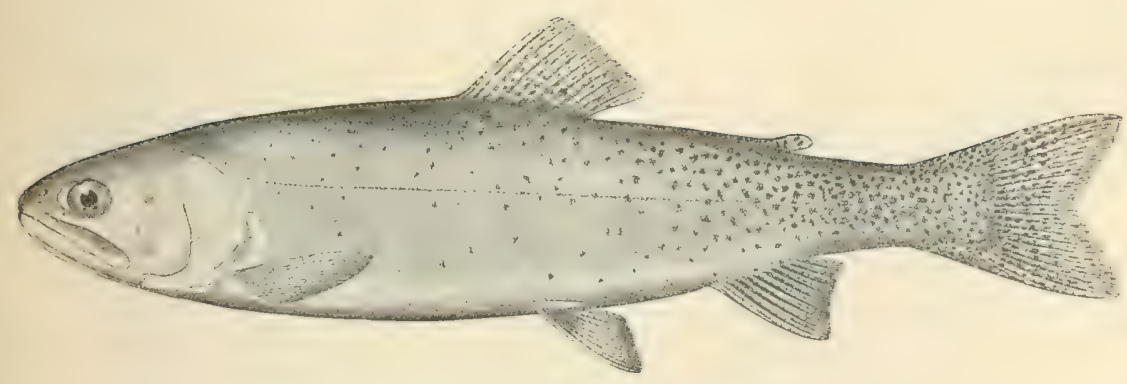

214

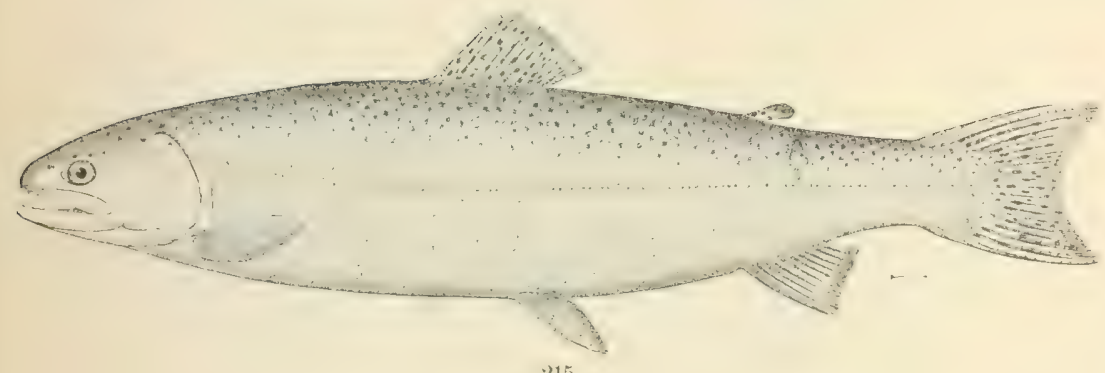

15

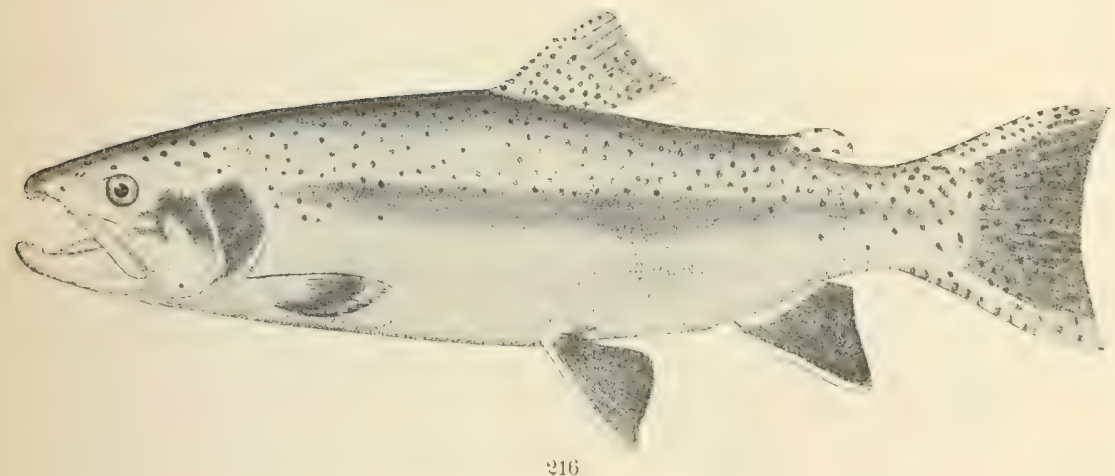

214. Salmo Clarkit maCdonatdi. (Pp. 497, 2819.)

215. SALMO GAIRDNERI. (P.497.)

216. SALMO IRIDEUS. (P.500.) 




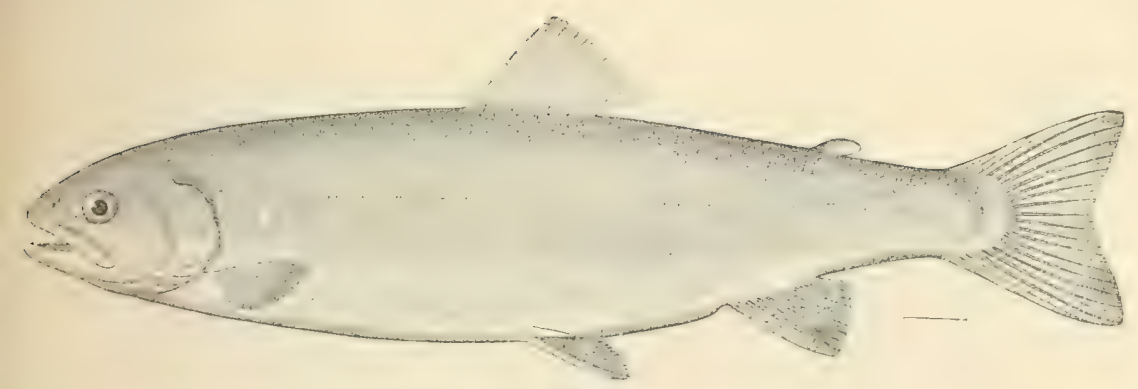

220

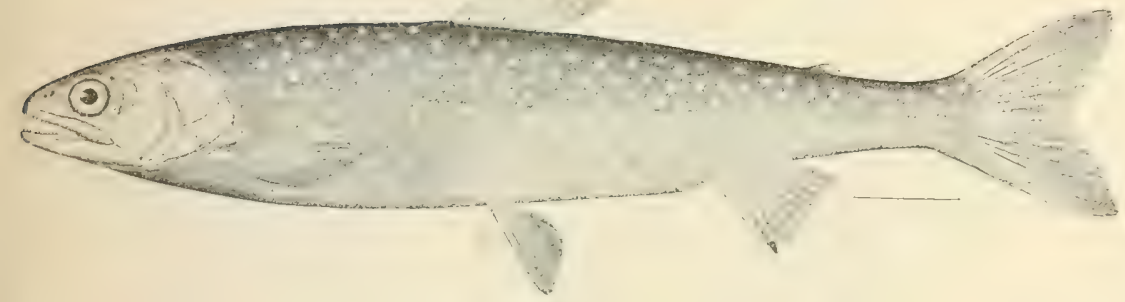

221

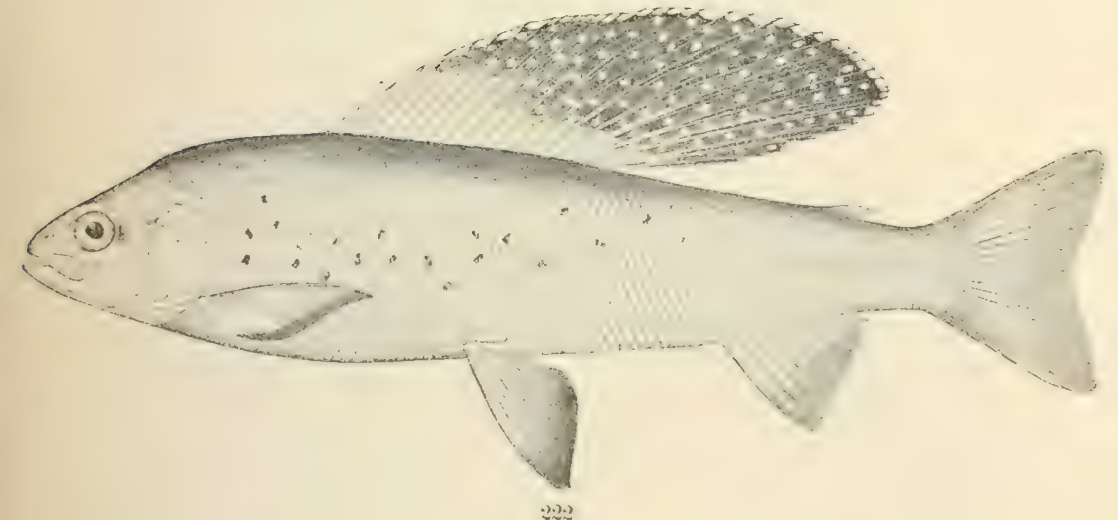

220. Salvelinus AlPLNUS AUREOLUS. (P. 511.)

221. SALVELINUS OQUASSA. (P. 514.)

222. 'THYMALLUS SIGNIEER. (P. 517.) 



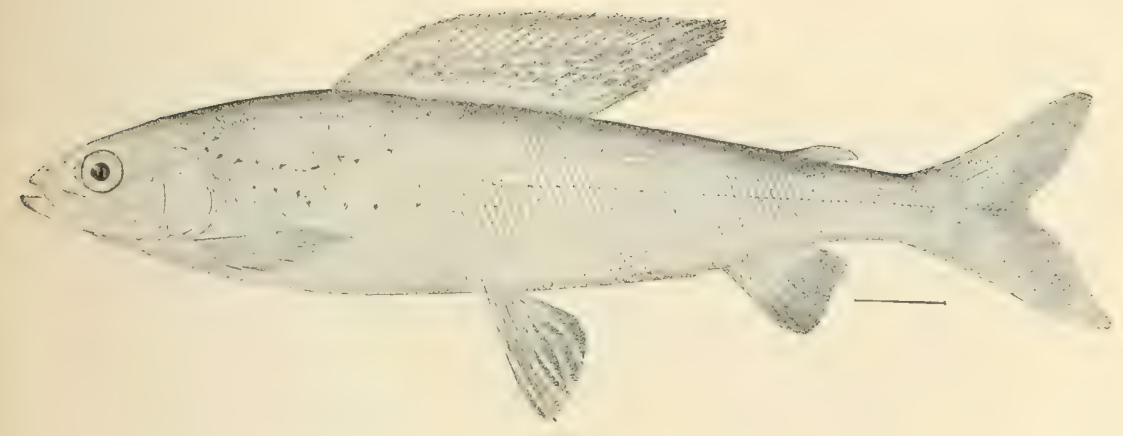

223

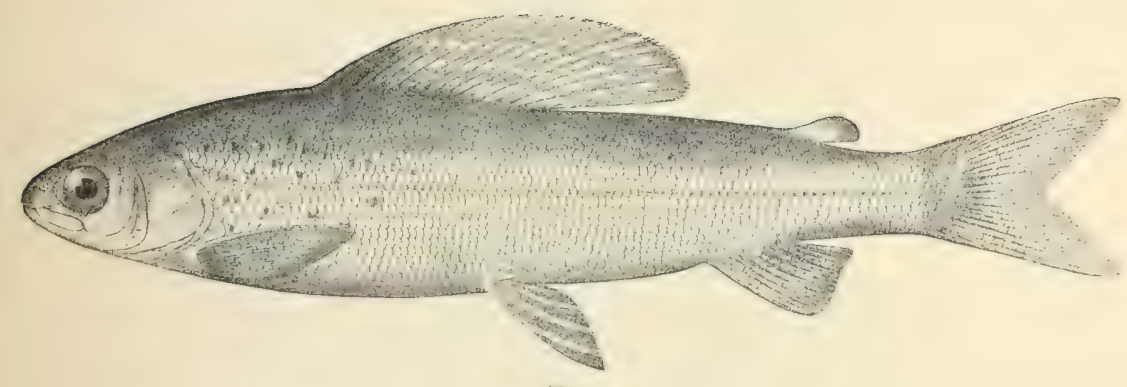

221

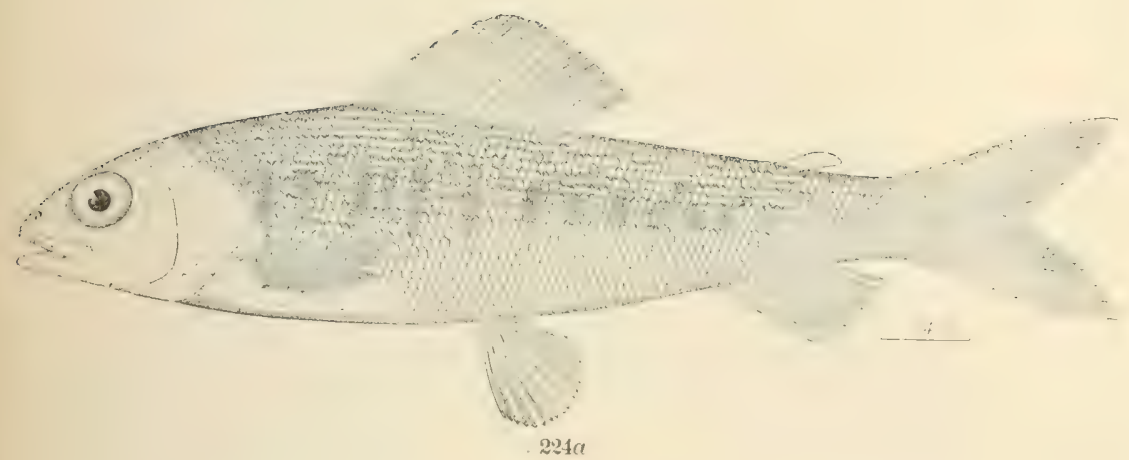

223. 'Thyallus Tricolor. (Pp. 518, 2871.)

224. Thymallus tricolor montanus. (Pp. 519, 2871.)

224a. THYMallus Tricolor Montanus; young. (Pp.519, 2871.) 



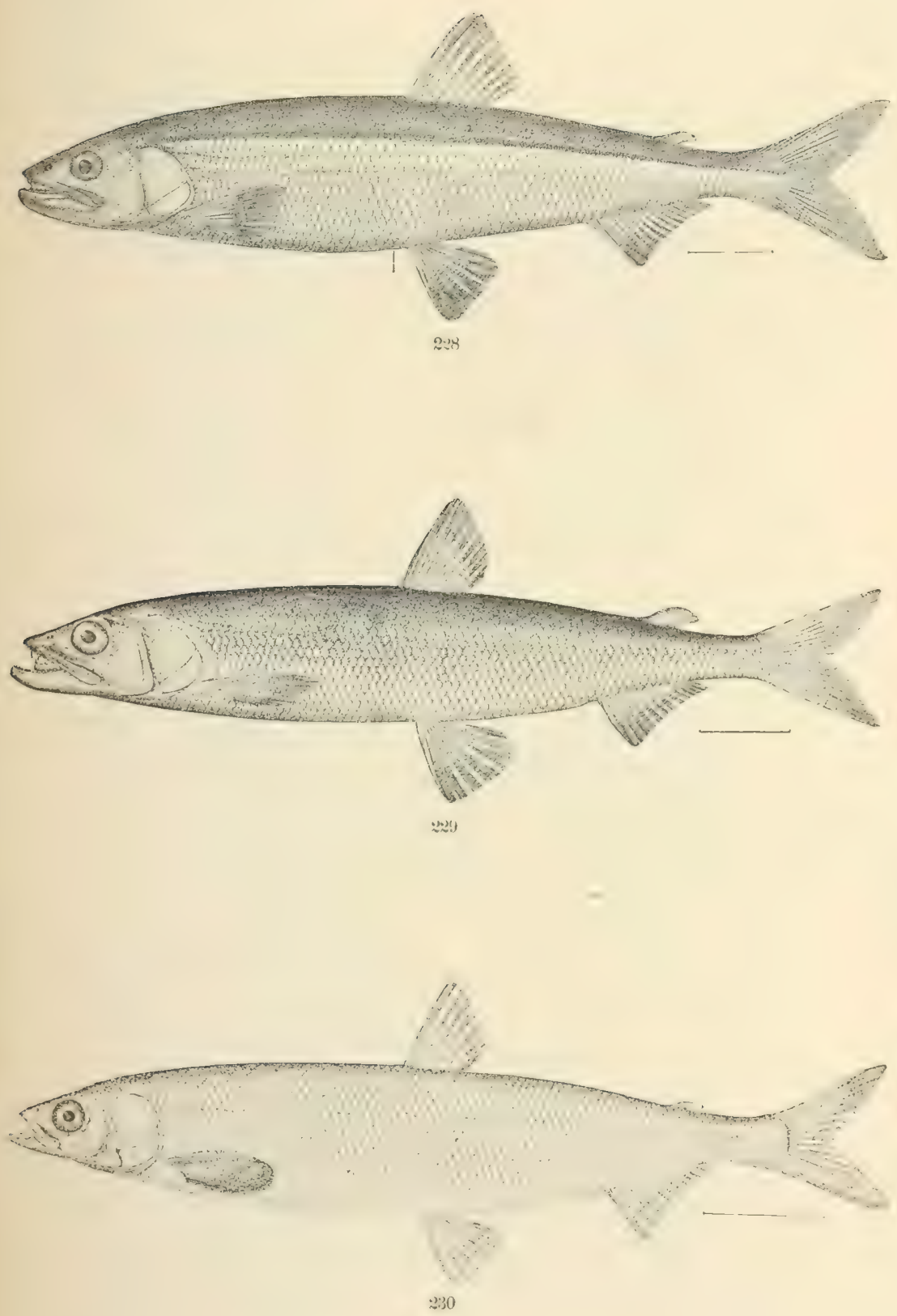

22x. OsMEILS Molidax, (P. 523.)

229. OSMERUA DENTEX. (P. 524.)

230. HYPOMESUS PRETIOSUS. (P’. J', 


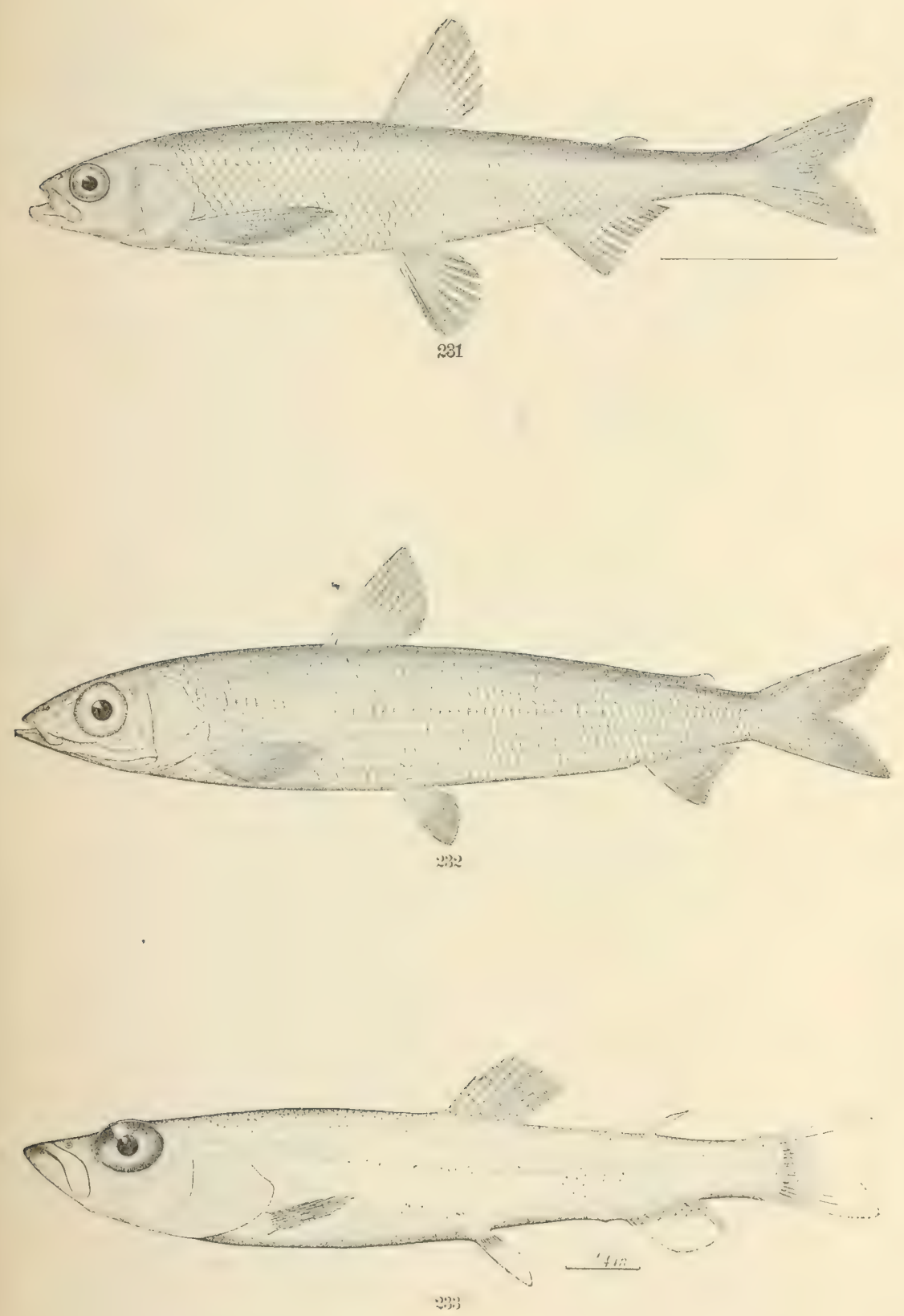

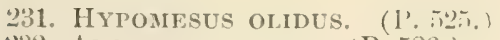

232. Argentina silus. (P. 526.)

233. Levioglossus STILiBIUS. (P. 527.) 




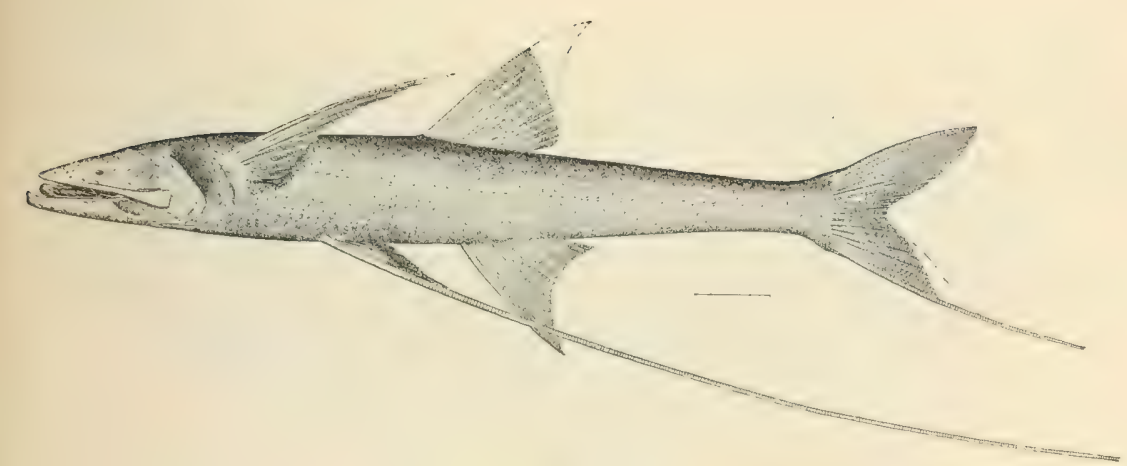

237

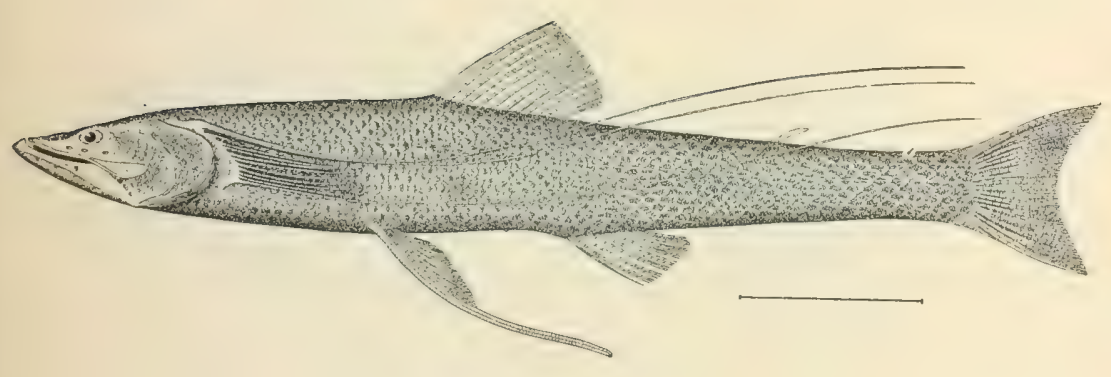

238

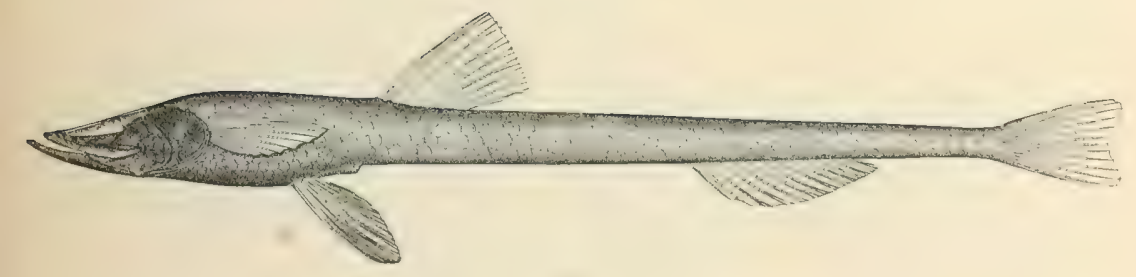

237. Benthosaurus grallator. (P. 543.)

238. Bathypterors QUadrifilis. (P. 545.)

239. IPNOPS MURRAYI. (P. 547.) 


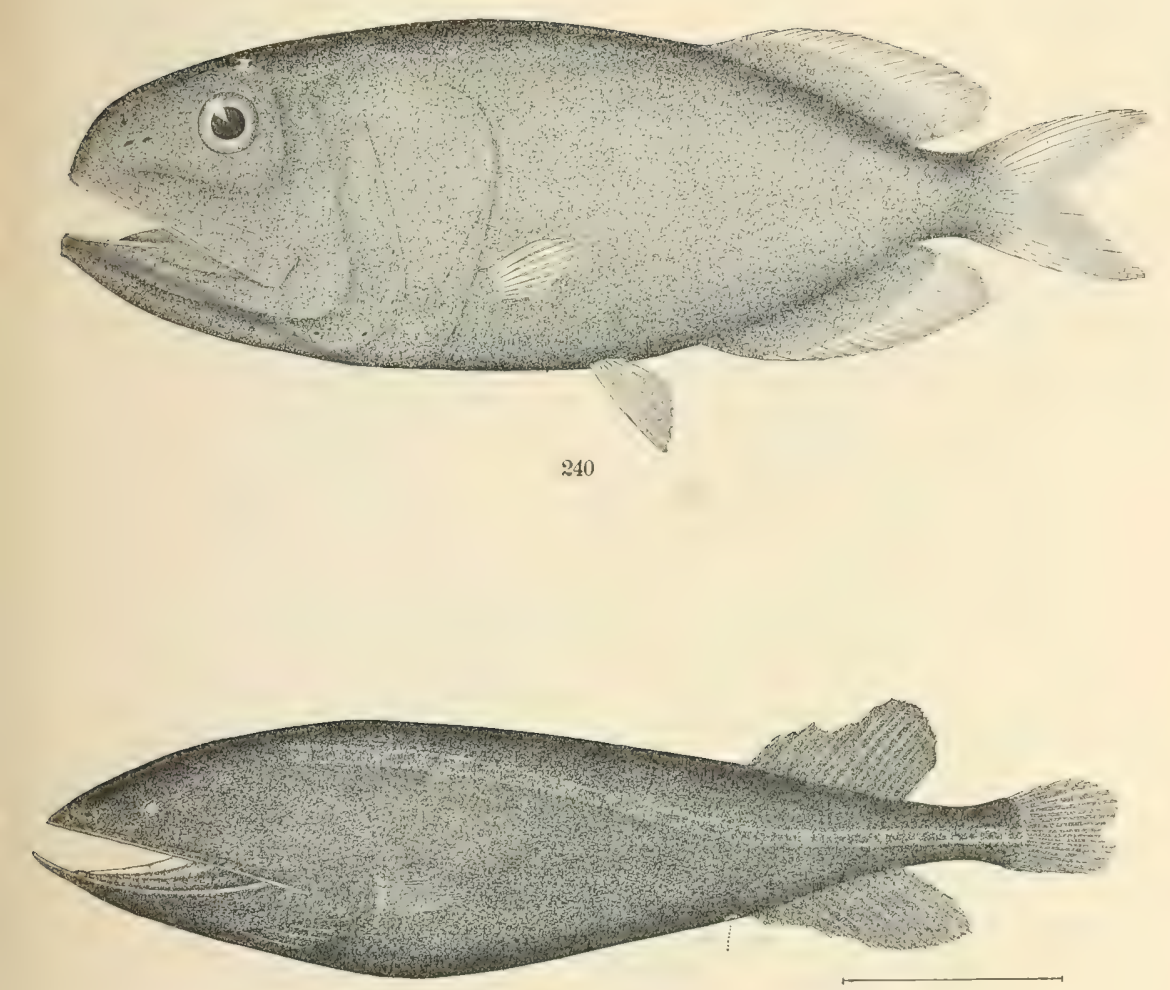

241

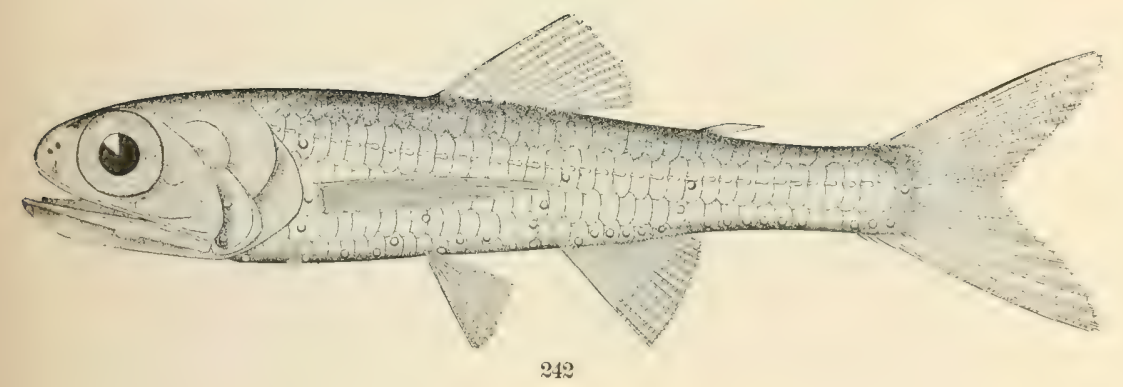

240. RONDELETIA BICOLOR. (P. 548.)

241. Cetomimus Gil.Lit. (P. 549.)

242. Ceratoscopelus iadeirensis. (P. 557.) 



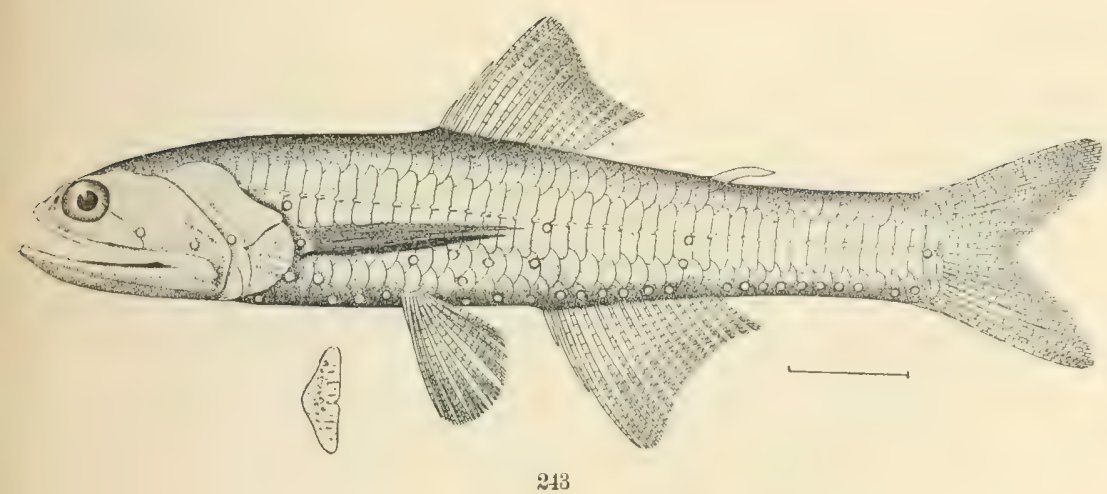

243

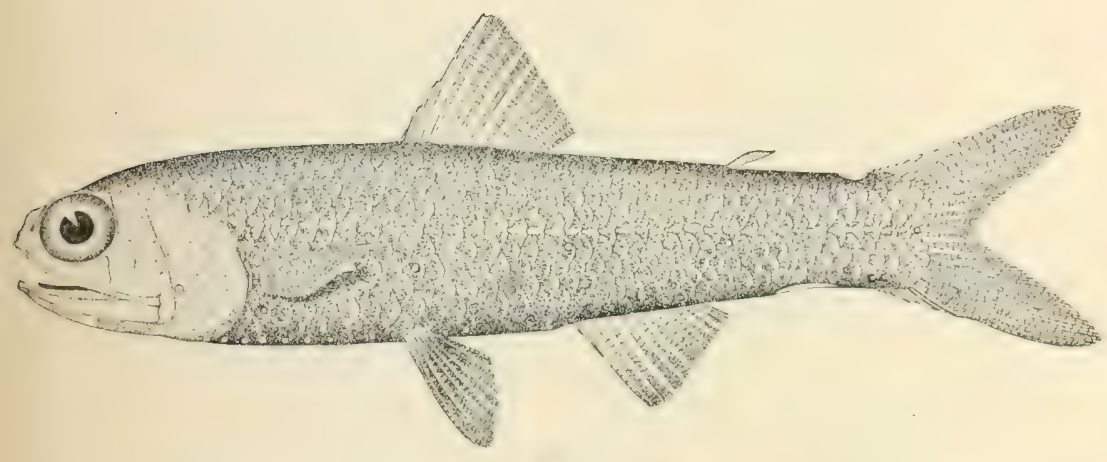

$\approx 14$

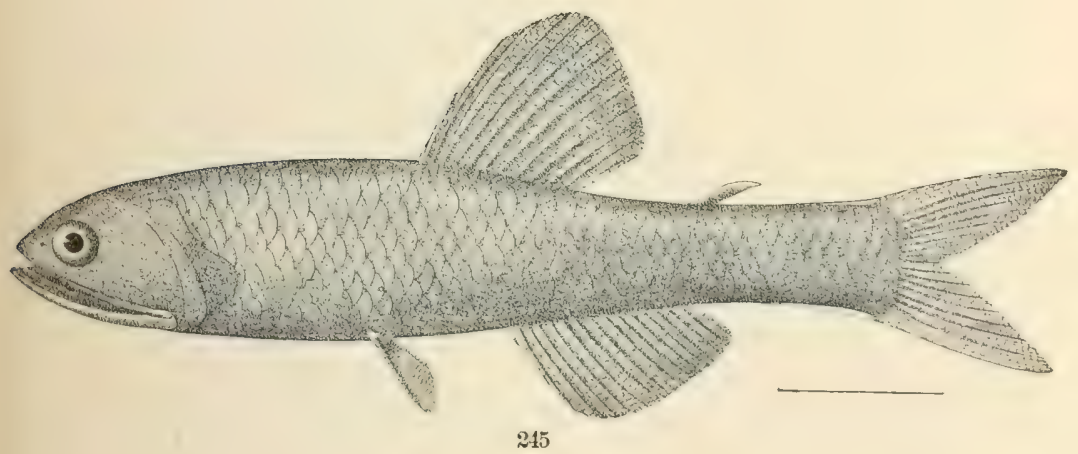

243. LAMPANYCTUS CROCODILUS. (P. 558.)

244. LAMPADENA SPECULIGERA. (P. 561.)

245. Nannobrachium MaCdonaldi. (P.563.) 



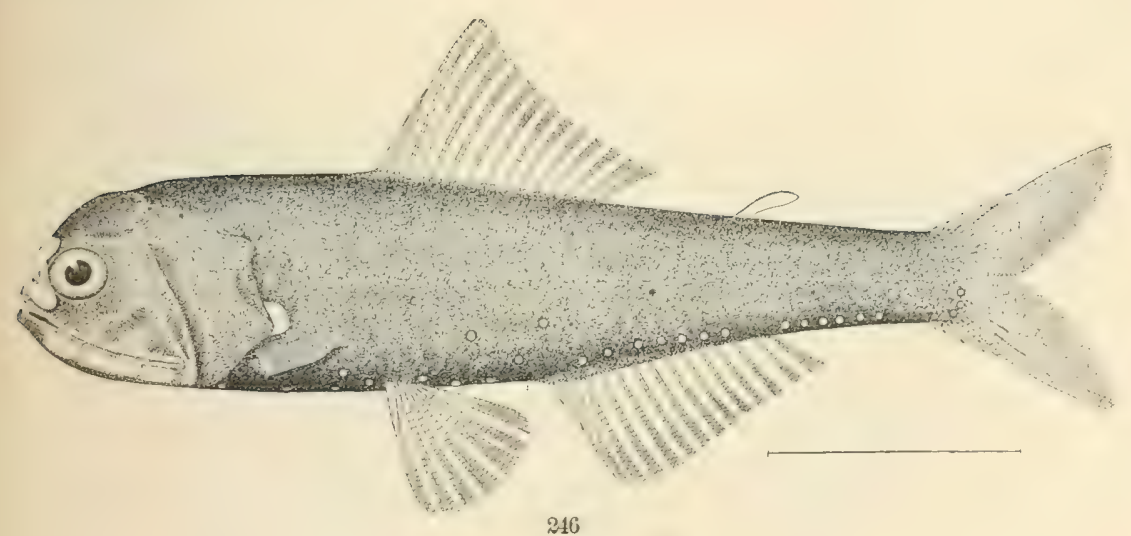

216

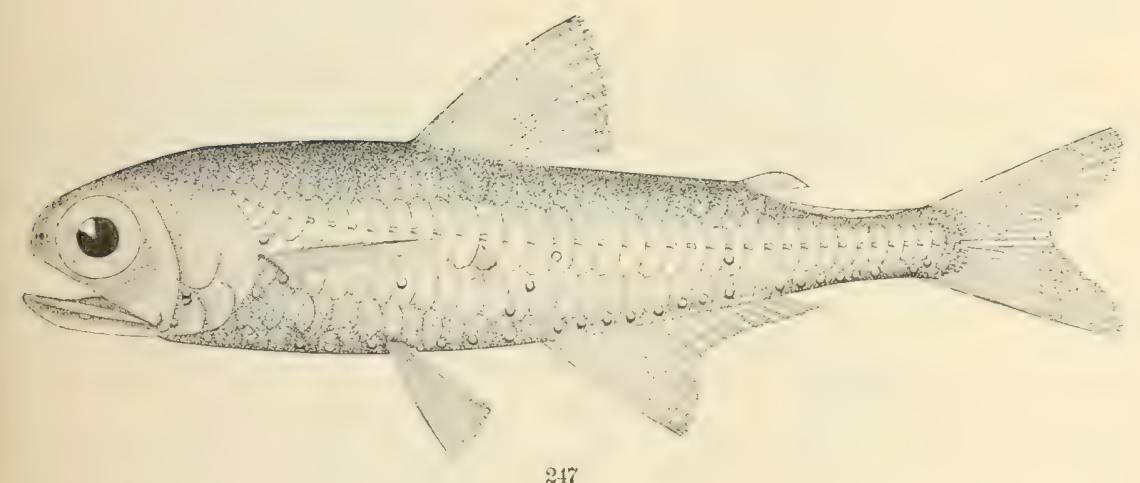

247

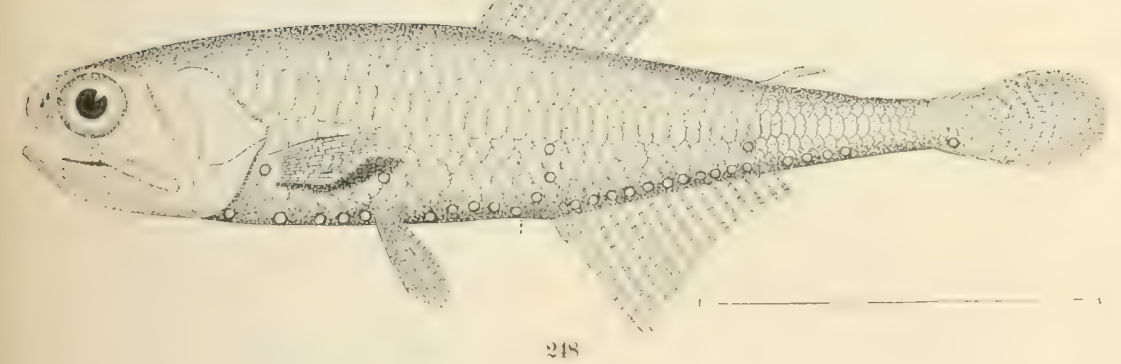

246. ETHOPIRORA LUCIDA. (P. 565.)

217. MYCTOPHUM OPALINUM. (P.571.)

248. 'Tarletonibania TeNuA. (P.575.) 


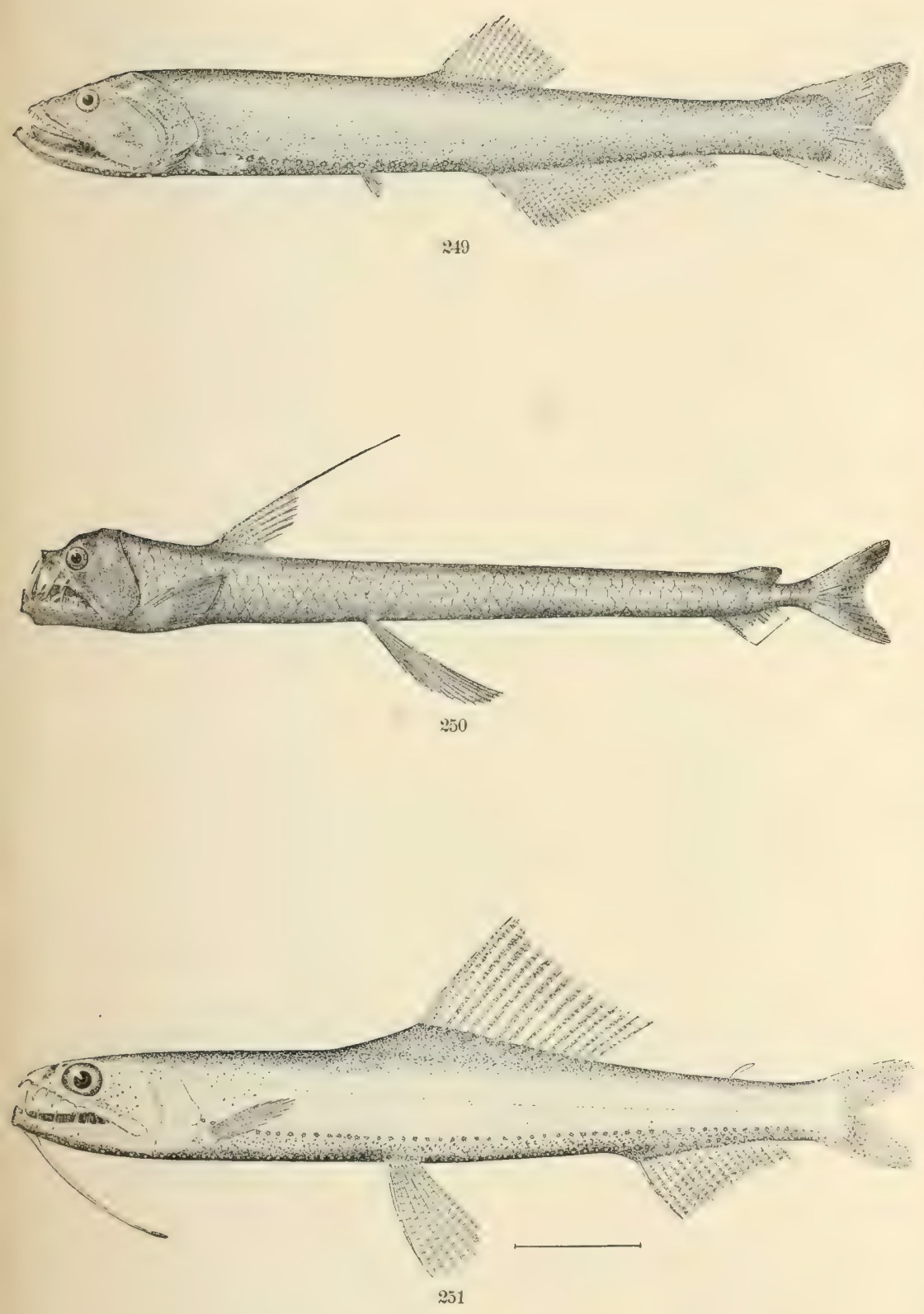

249. YarRella BLACKFORdi. (P. 584.)

250. Chauliodus sloanfi. (P. 585.)

251. Astronesthes GEMMIFER. (P. 586.) 



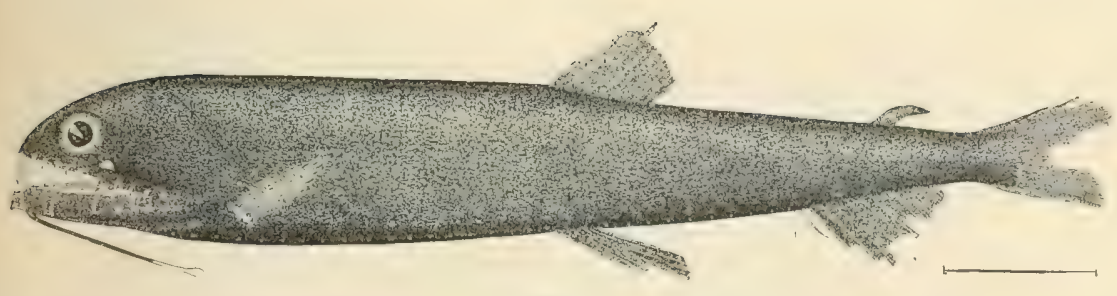

252
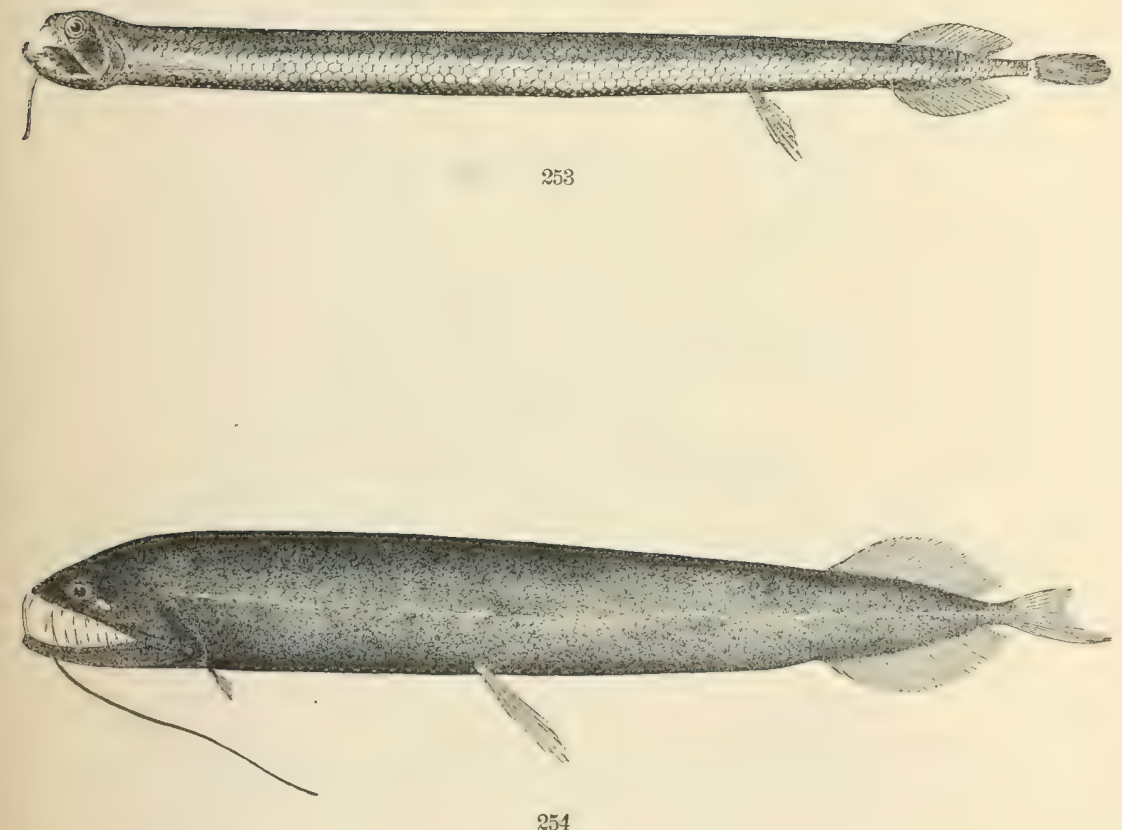

252. Astronesthes RICHARDSONI. (P. 587.$)$

253. STOMIAS FEROX. (P. 588.)

254. Gramiatostomas dentatcs. (P.590.) 


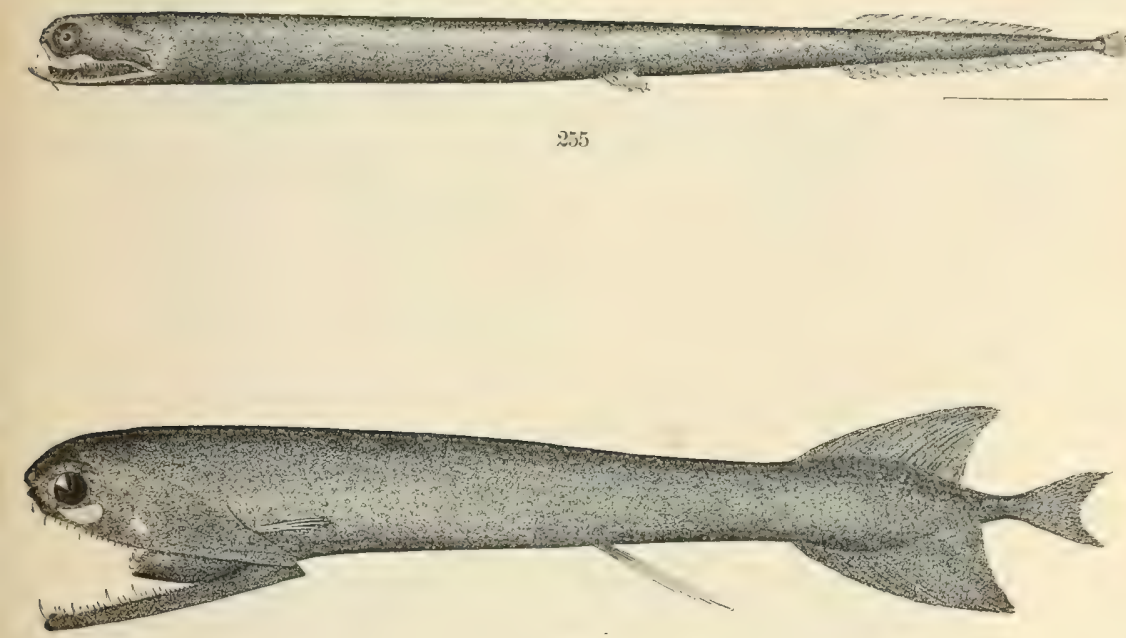

256

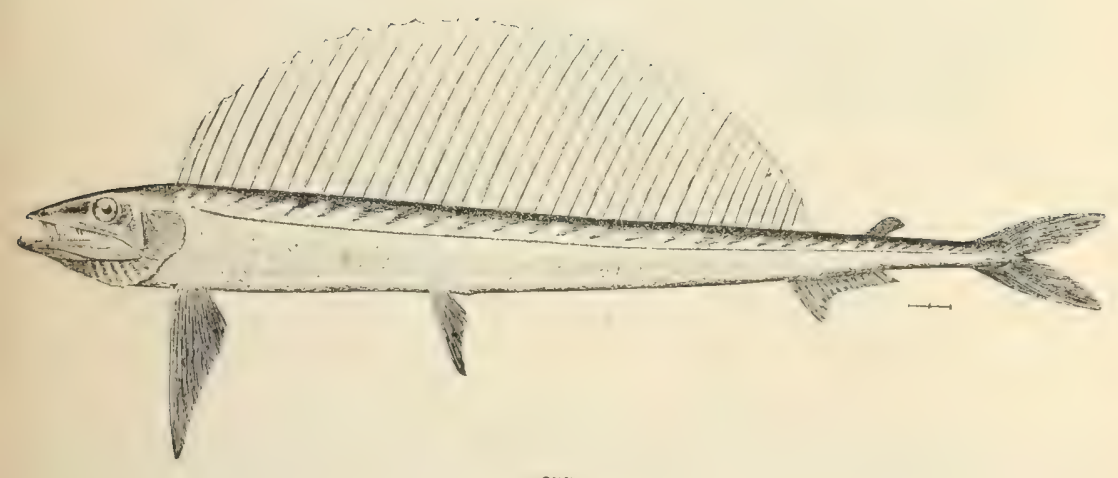

257

255. Photonectes gracilis. (P. 591.)

256. Malacosteus Niger. (P. 593.)

257. Alepisaurus ferox. (P. 595.) 




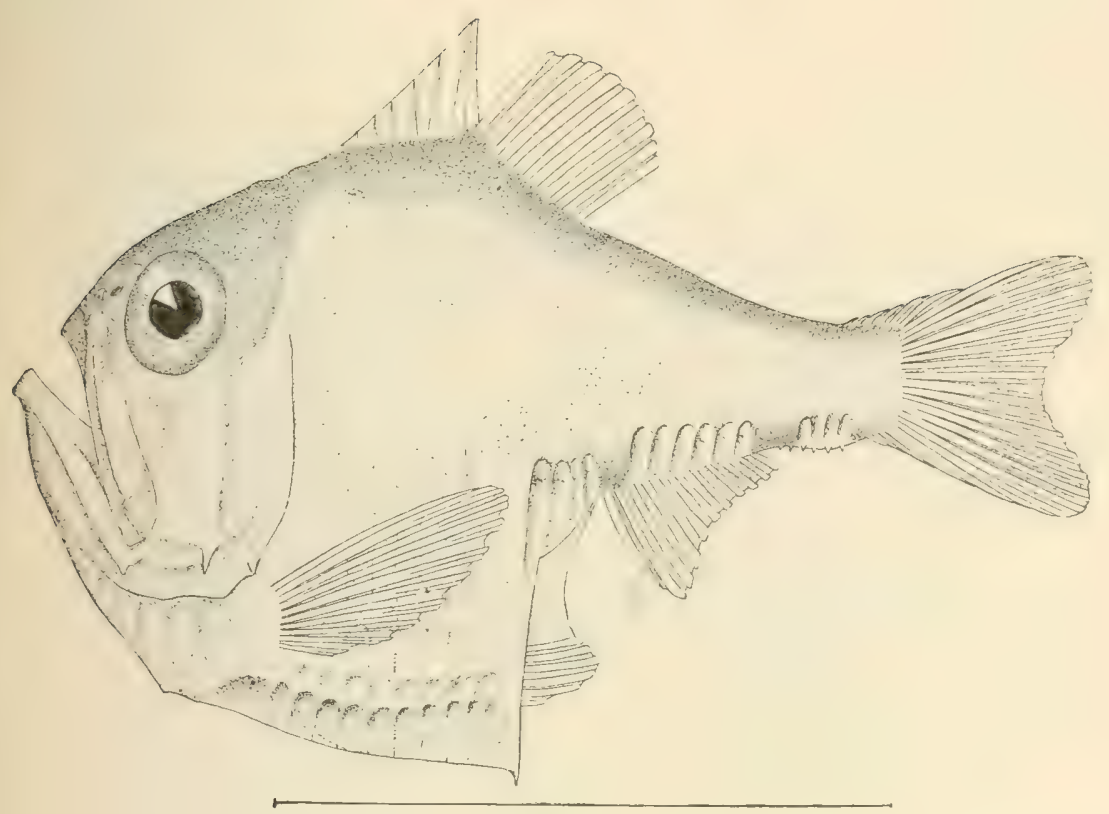

$: 61$

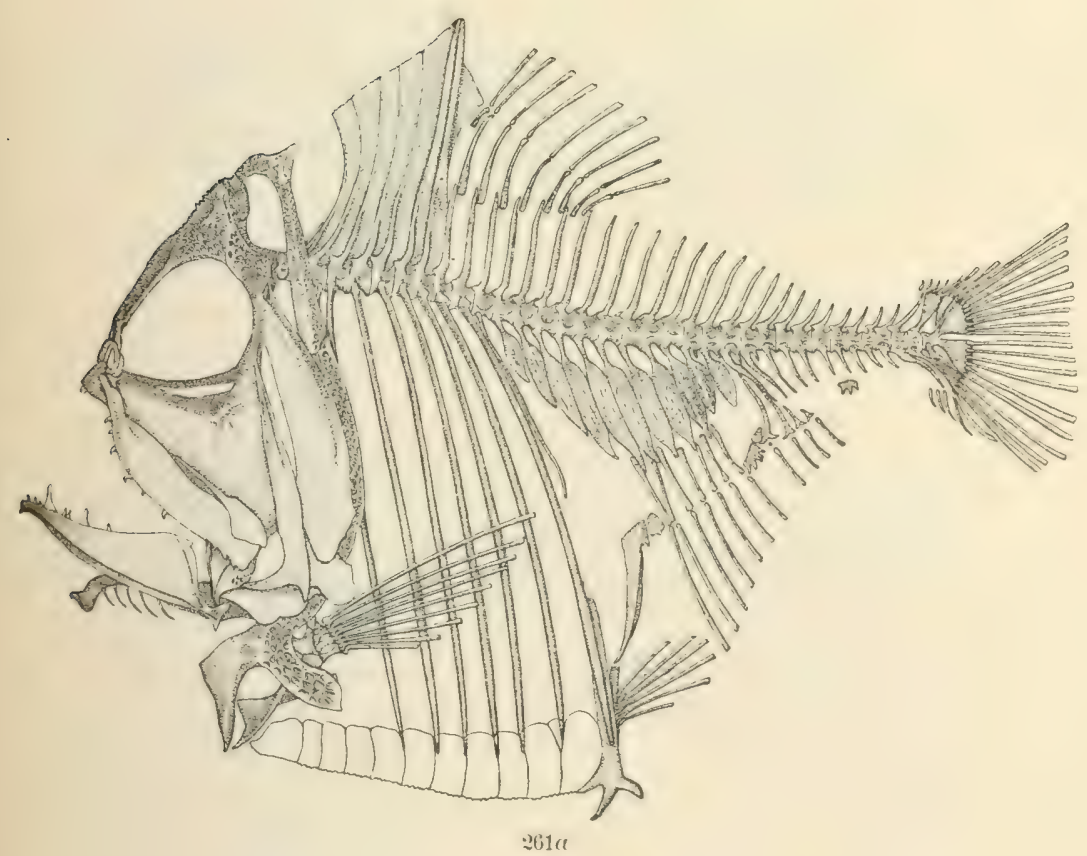

261, 261a. ARGYROPELECUS OLFERS. (P. 604.) 


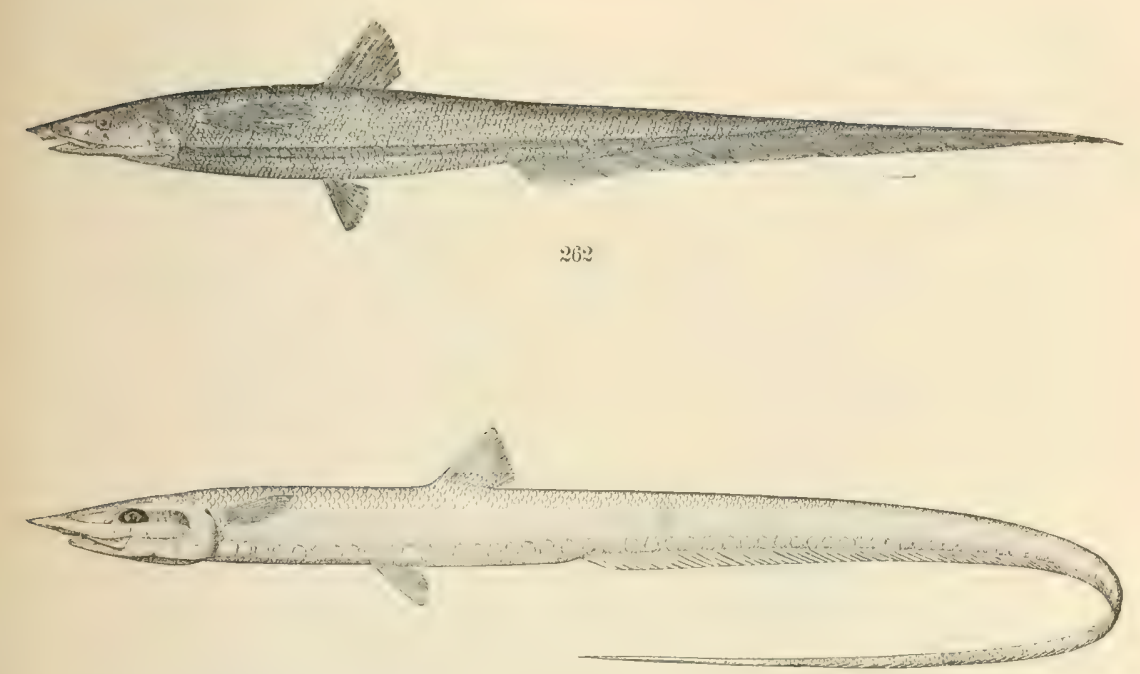

263.3
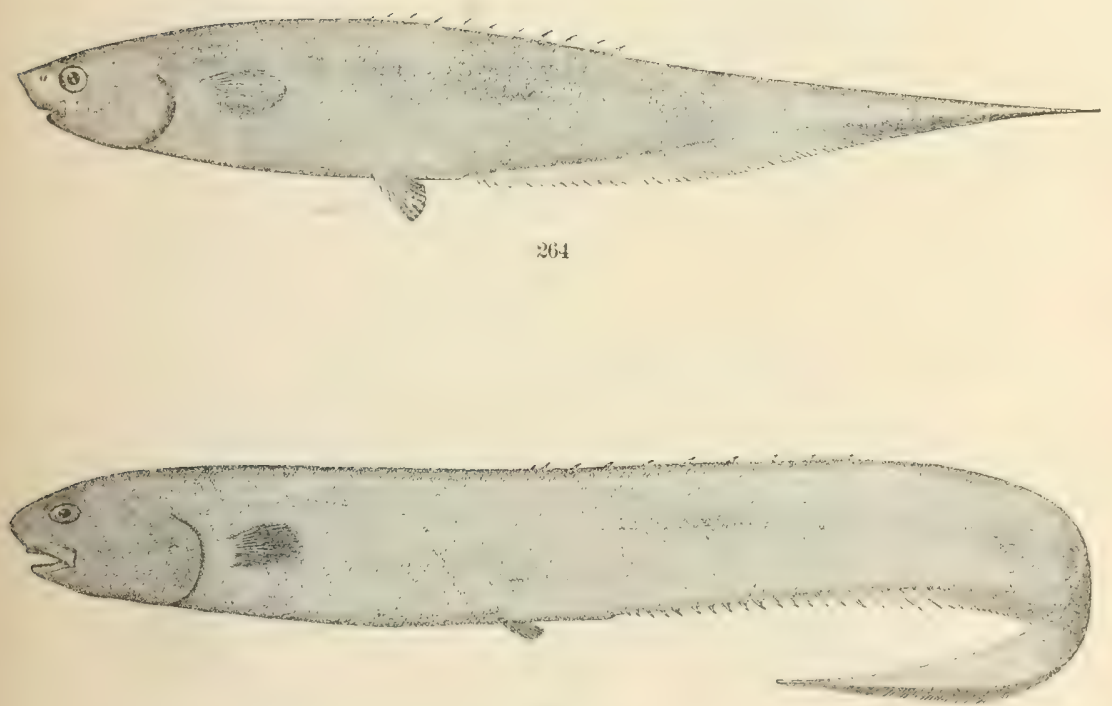

26.5

262. Aldrovandia macrochir. (P. 609.)

263. Aldrovandia gracilis. (P. 610.)

264. NotacantuUs analis. ( $P .615$.

265. Notacanthus phasganorus. (P. 616.) 




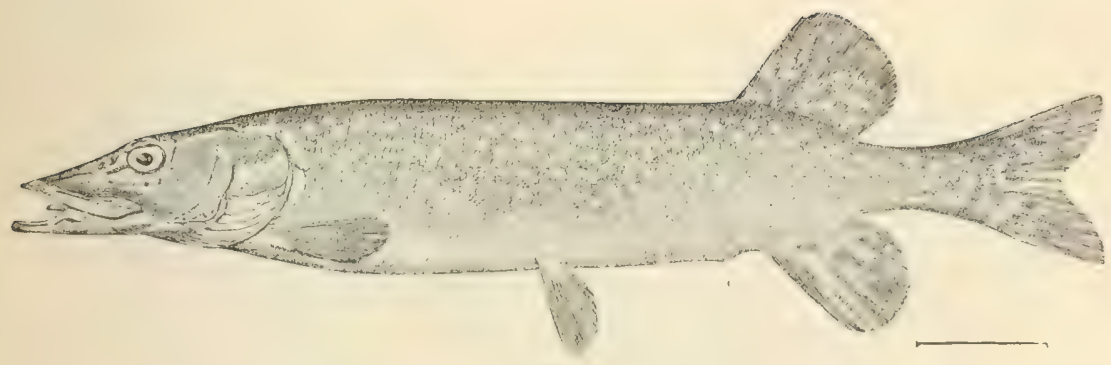

269
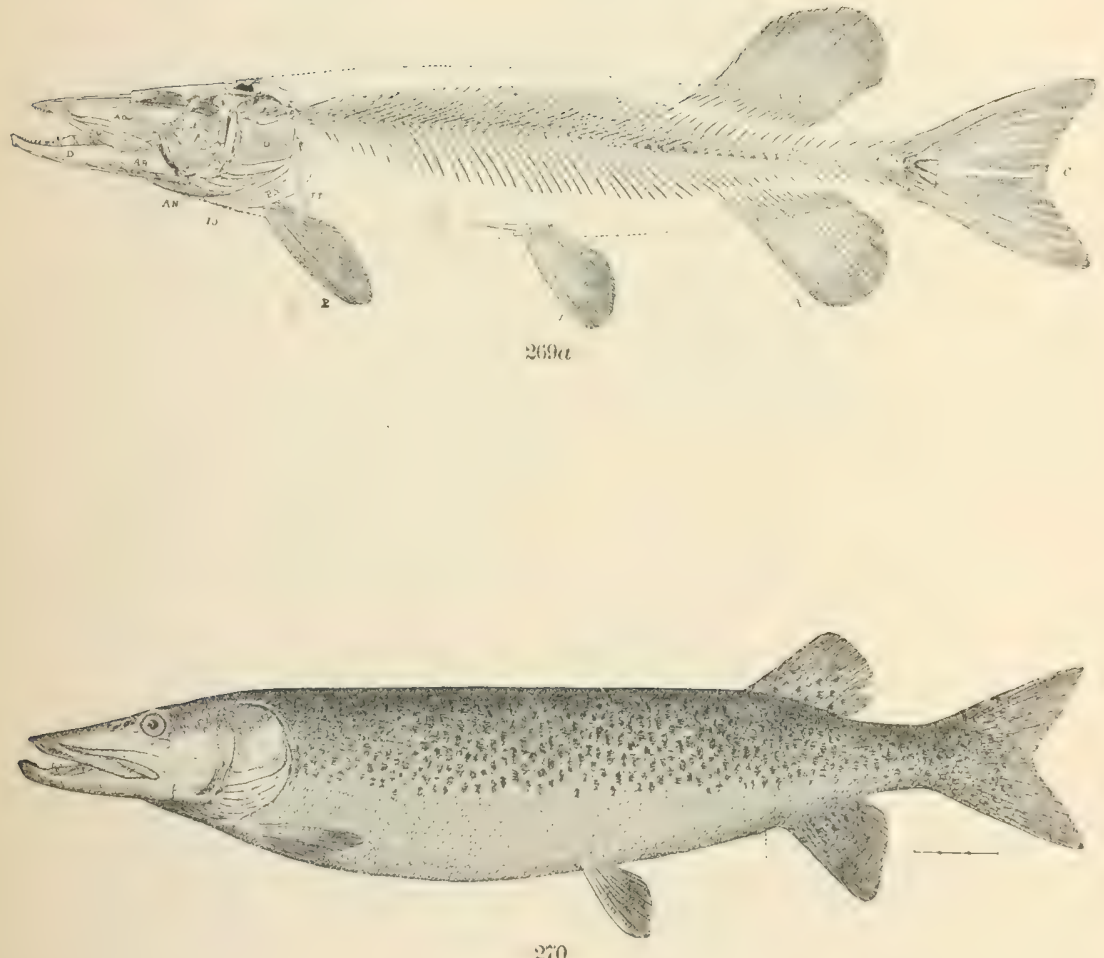

269, 269a. LuCIUS LUCIUS. (P. 628.)

270. LUCIUS MASQUINONGY. (P. 629.) 


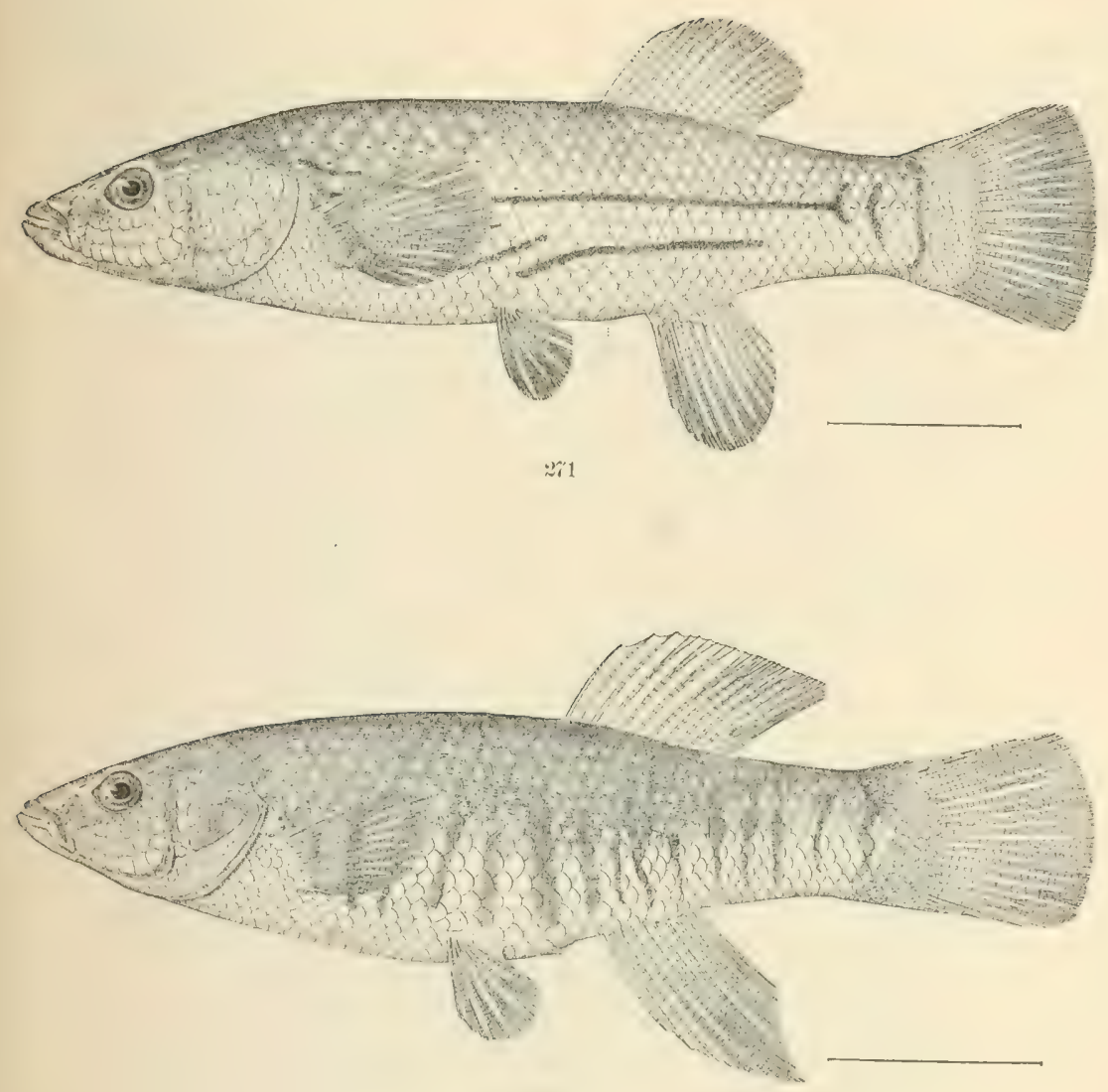

2i1 $1 \alpha$

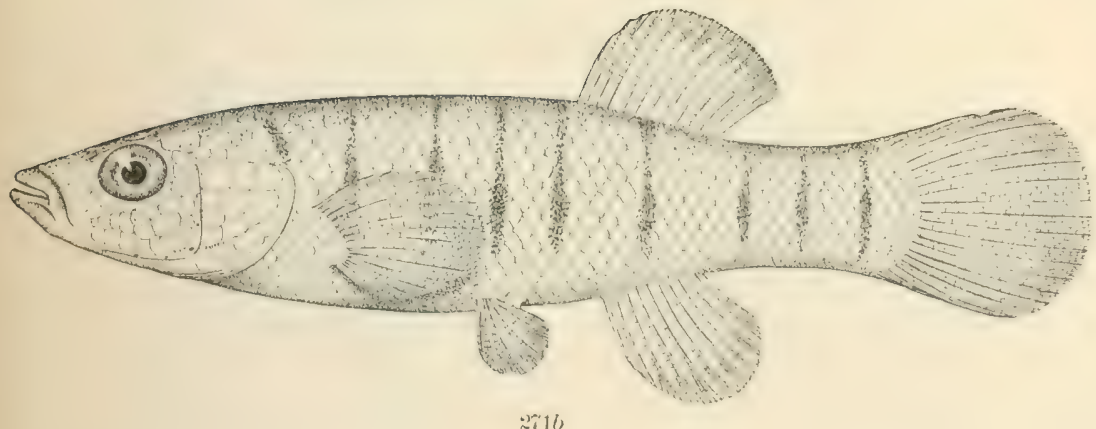

271. FuNiulus MaJAlis; female. (P. 639.)

271a. Fundules MaJAlis; male. (P. 639.)

271b. FUNDULUS MAJALIS; young. (P.639.) 



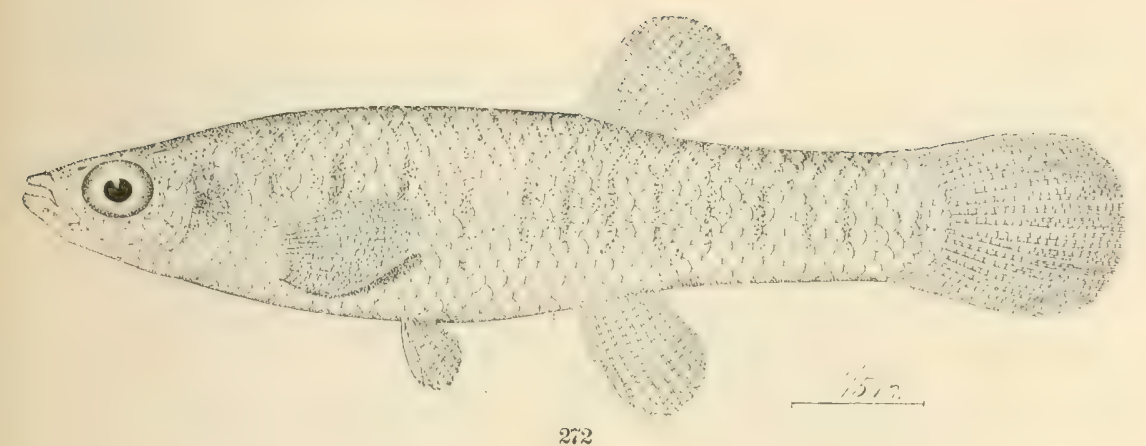

$2 \% 2$
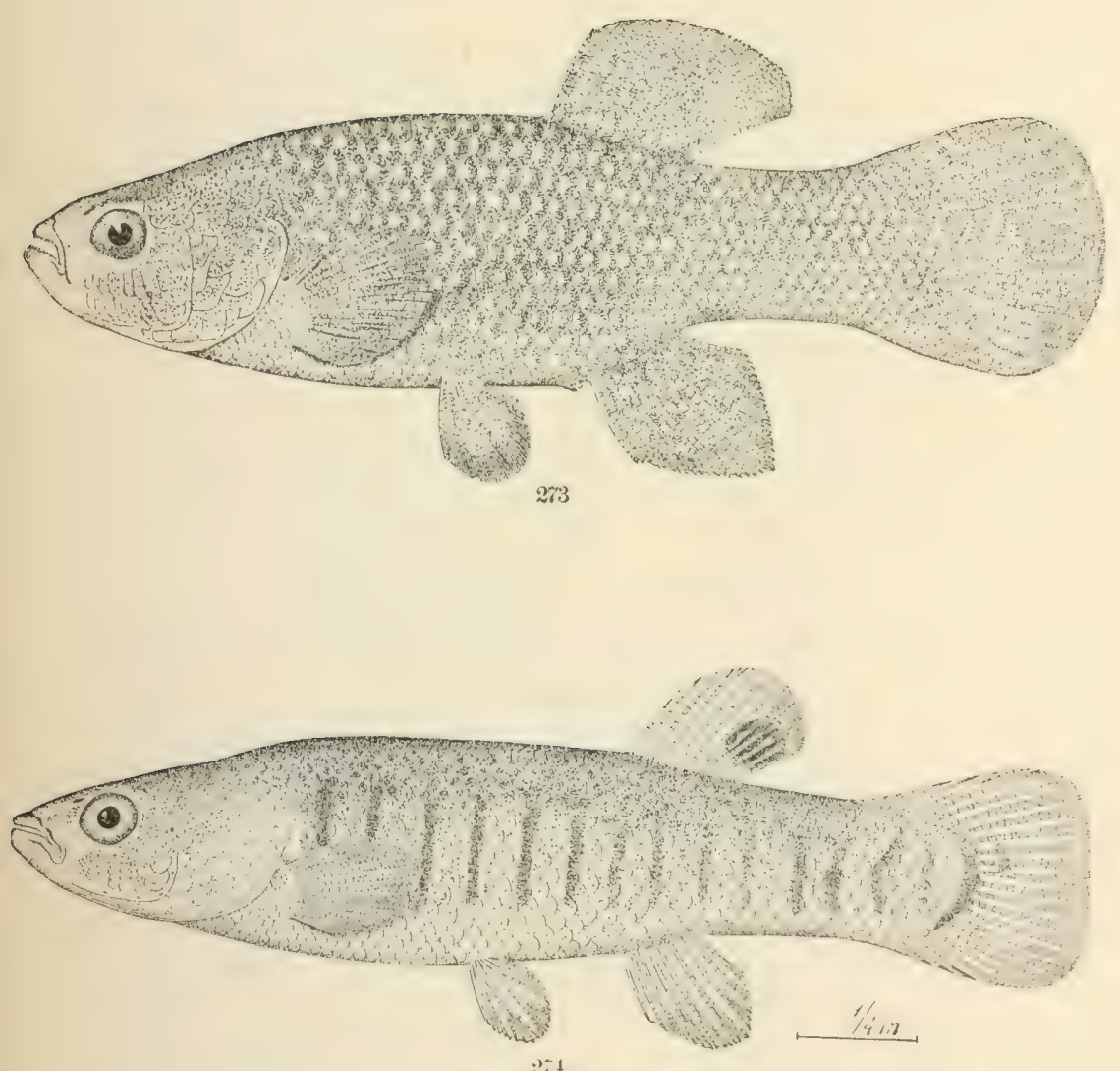

272. Fundulus pallidus. (P. 638.)

273. Fundulus Heteroclitus; male. ( $P$, 640.)

274. Fundulus OCELlaris. ( $\mathrm{P}, 642$.) 



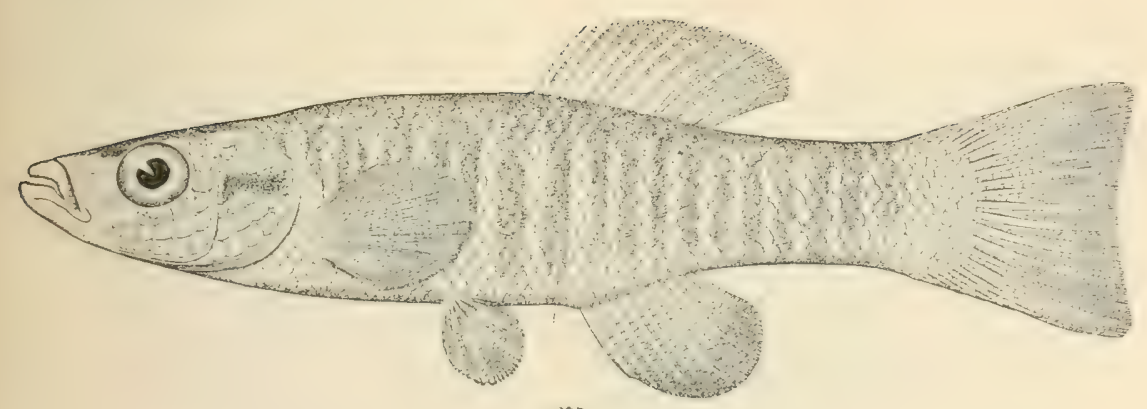

205
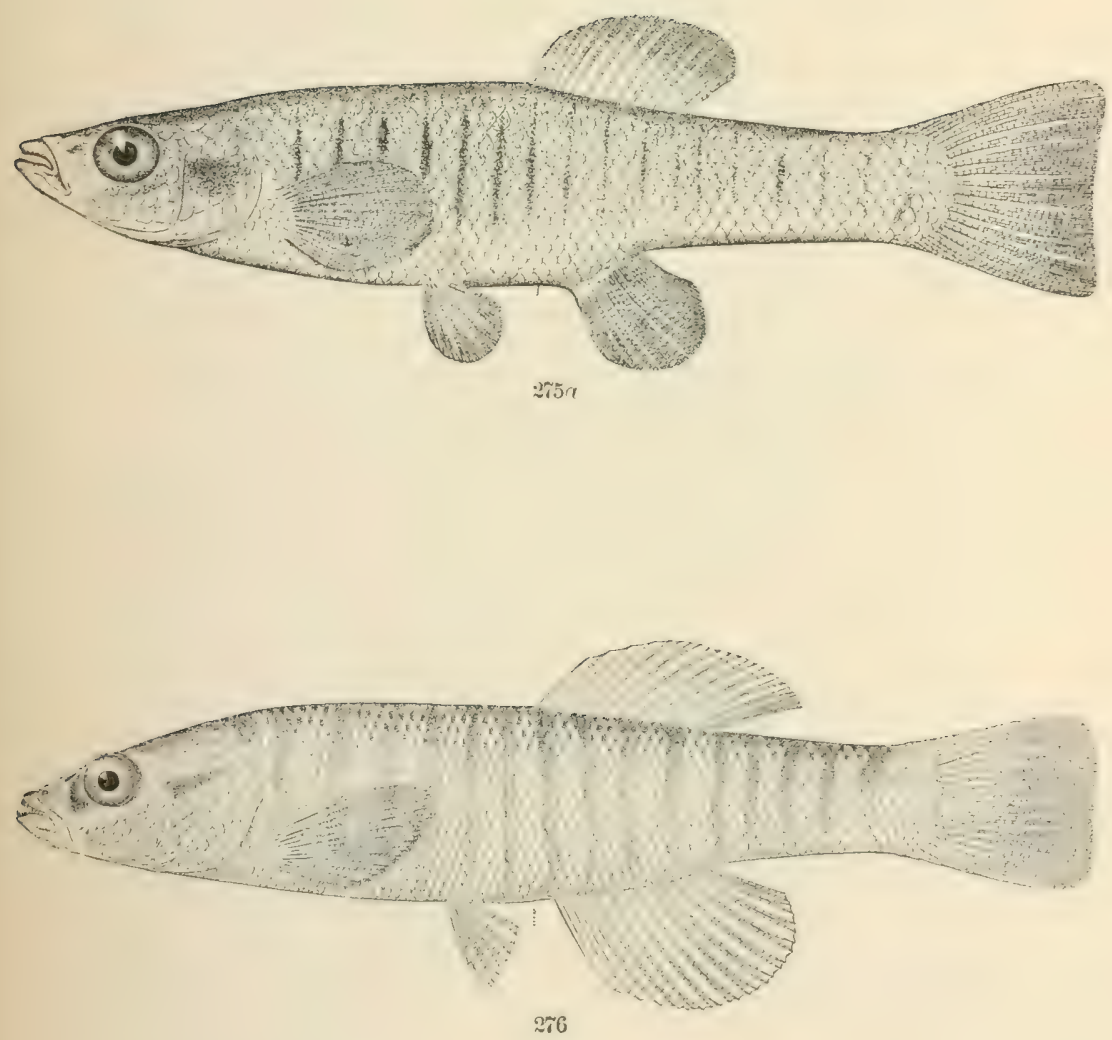

275. Fendulus diaphanus; male. (P. 645.)

275a. FUNDULUS DIAPHANUS; female. (P. 645.)

276. Fundulus zeBrinus. (P. 646.) 



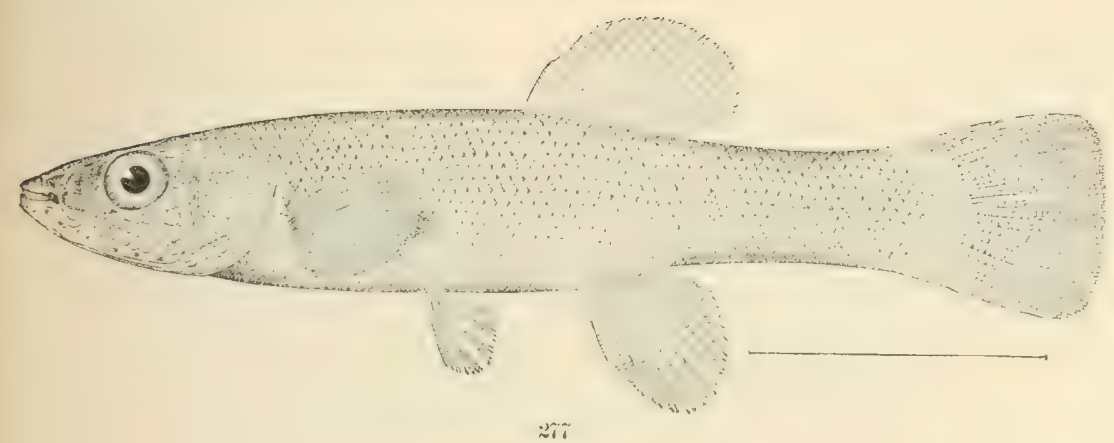

2กิ

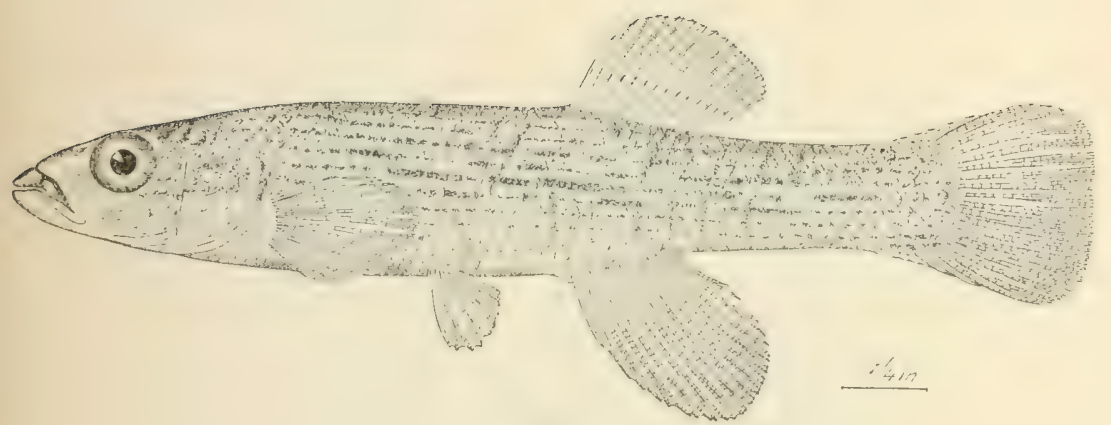

2ร

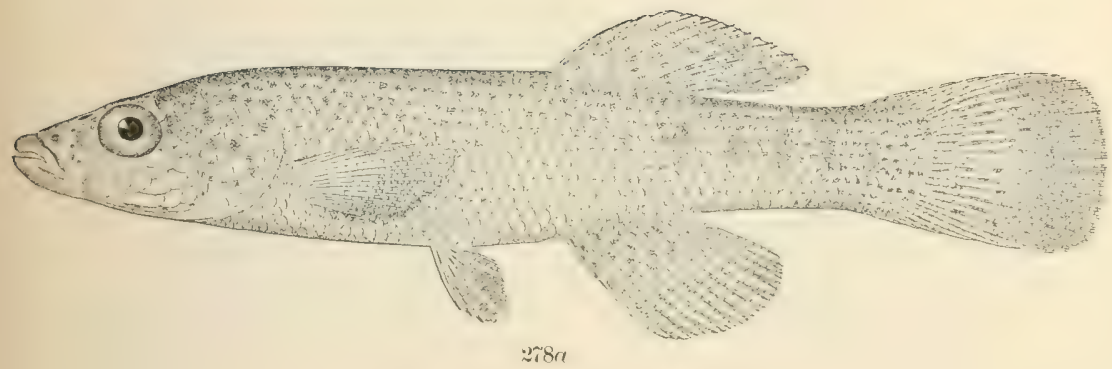

277. Fundulus seminolis. (P. 647.)

278. Fundelus Catenatus; male. (P. 648.)

278a. Fundulus catenatus; female? (P. 648.) 



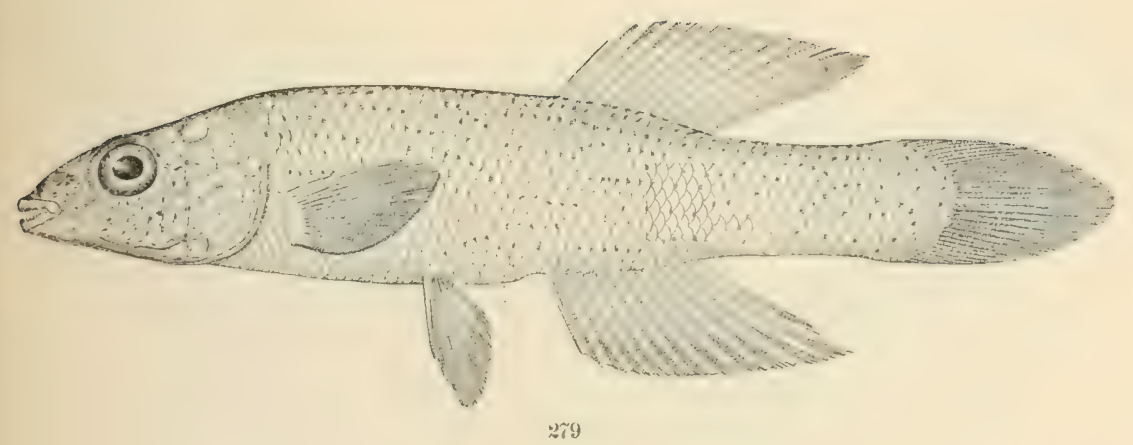

ะช9

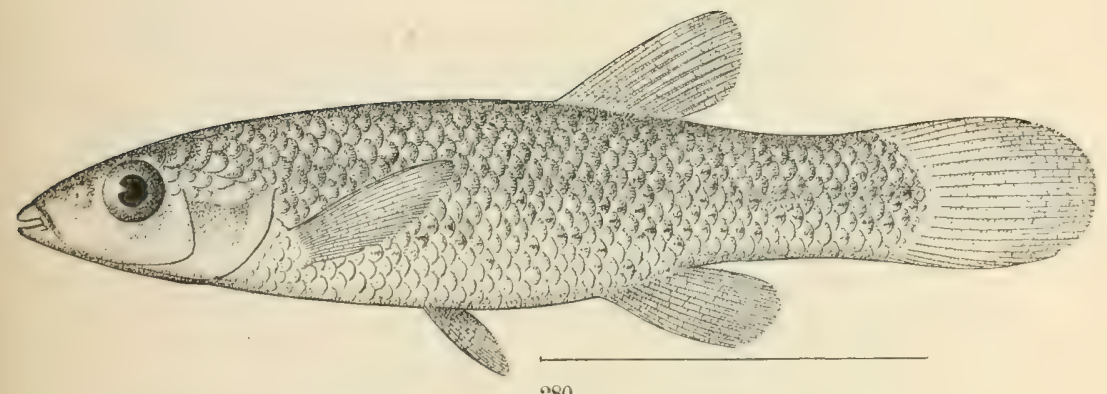

280

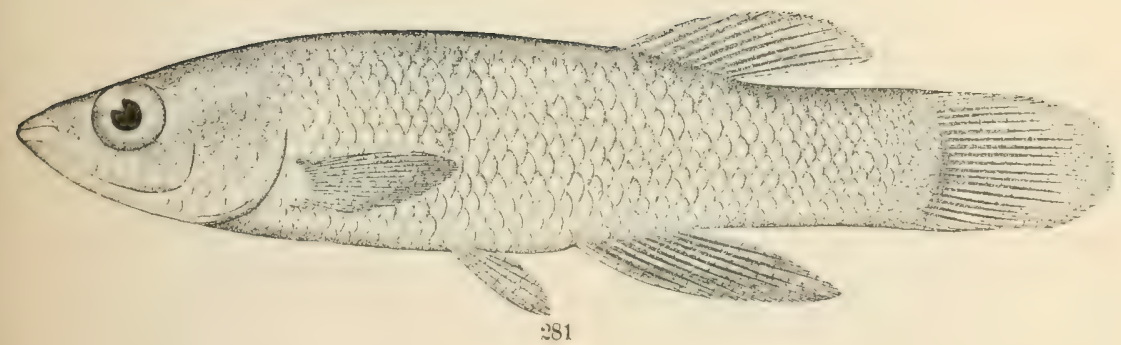

279. Fundulus stellifer. (P. 618.)

280. Fundulus RATHiUNi. (P. 649.)

281. Fundulus albolineatus. (P. 619.) 



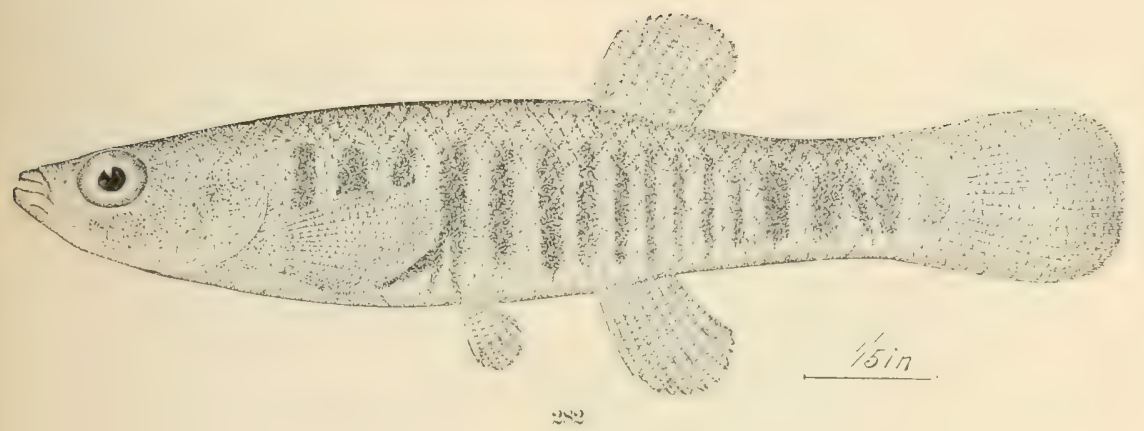

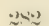

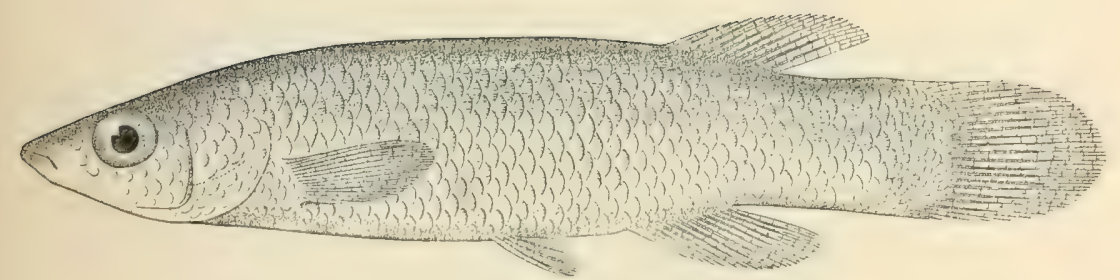

283

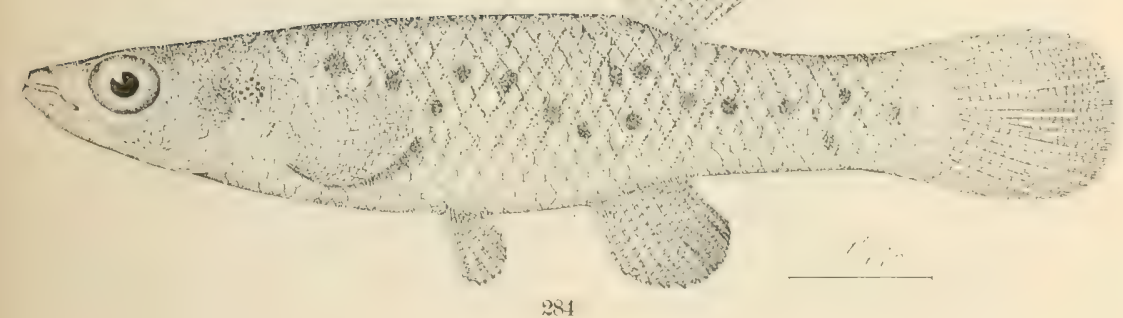

282. FUNDULUS FUNDULOIDES. (P. 650.)

283. FUNDULUS MACDONALDI. (P. 651.)

284. FuNDULUS JENKINSI. (P. 651.) 


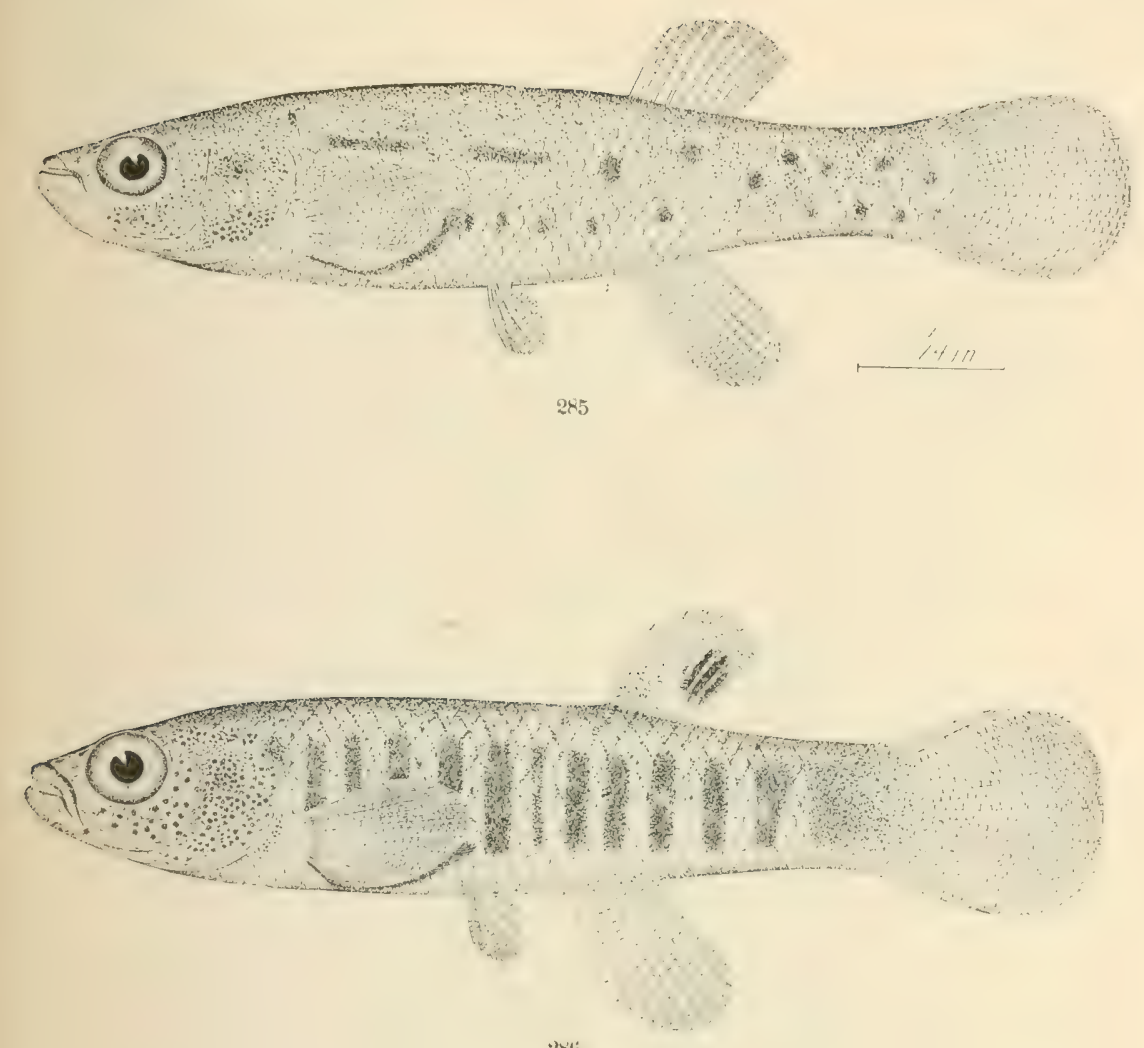

: 2 (i)

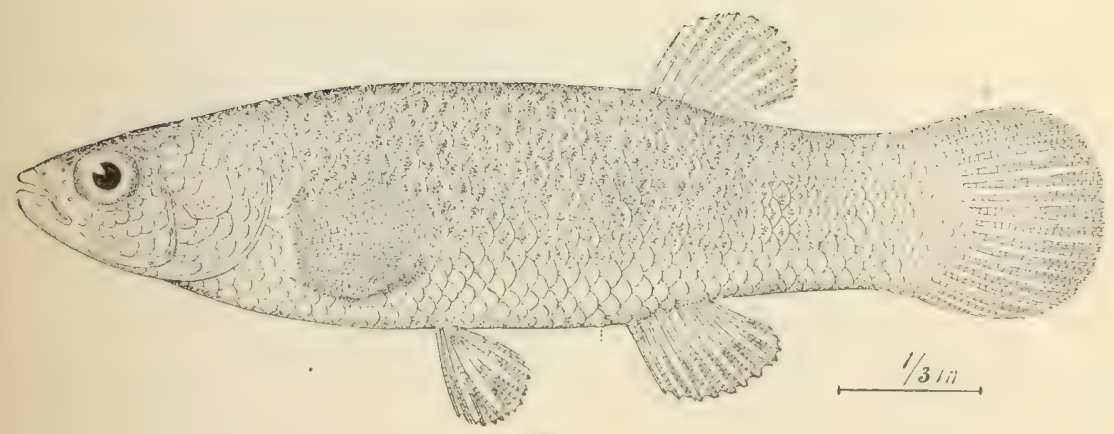

$28 i$

285. Fundilus pulyereus. (P, 652.)

286. FUNDULUS LUCIE. ( $P$. 654.)

287. Fundulus chrysotus. (P. 655.) 



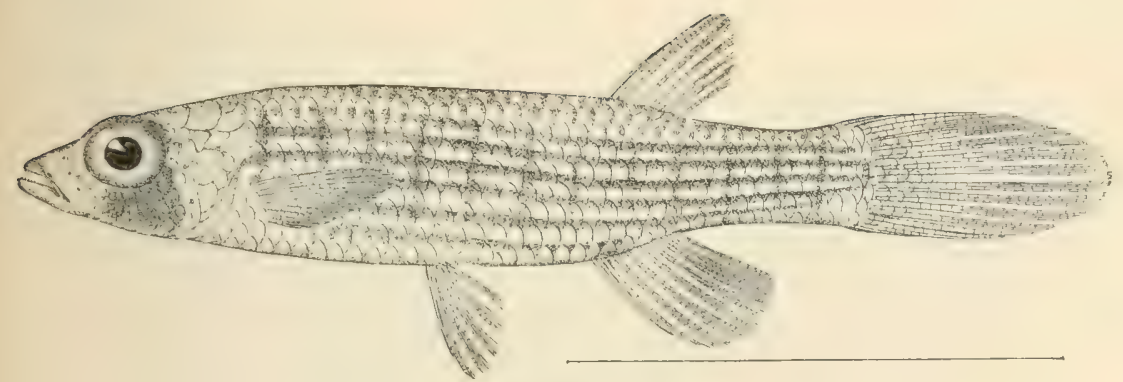

288

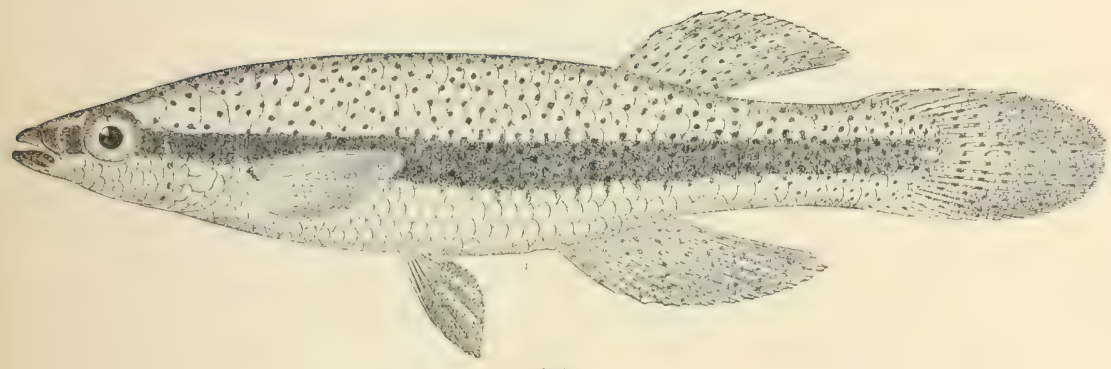

289

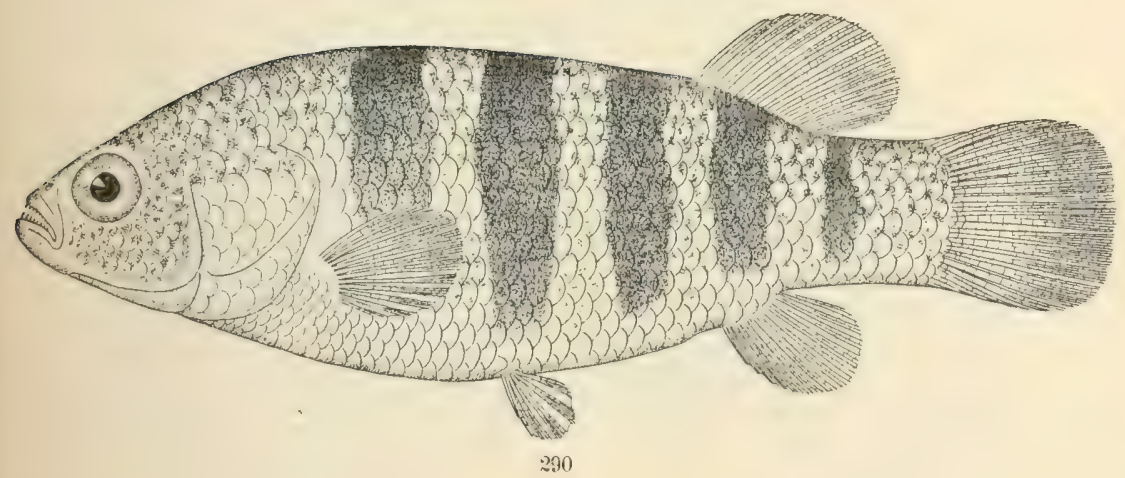

288. Fundulus Notti. ( $\mathrm{P}, 656$.

289. Fundulus notatus. (P. 659.)

290. Adinia DUGESII. (P. 661.) 


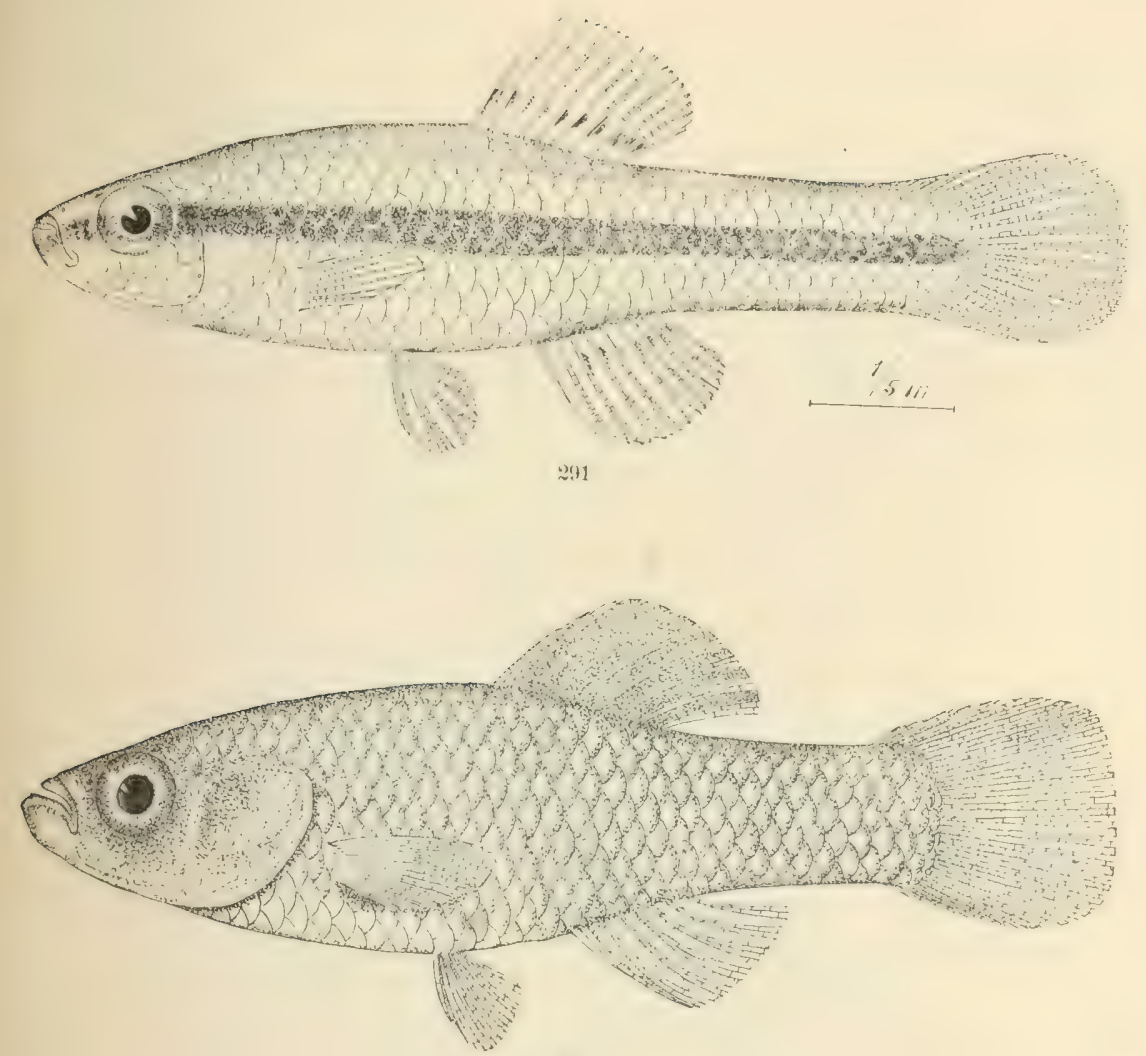

29

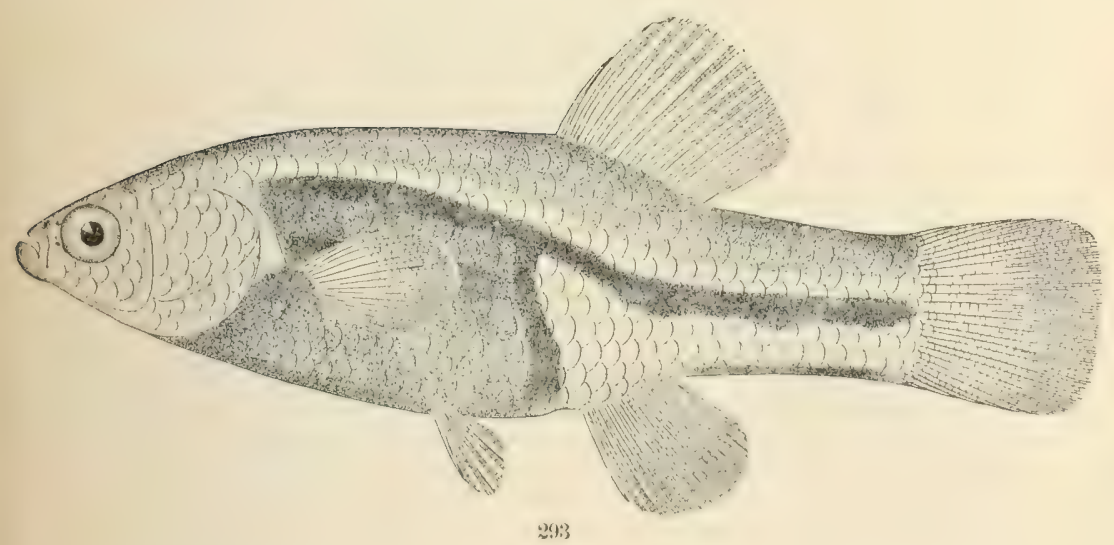

291. Fundulus gooder. (Pp. 664, 2831.)

292. LuCania parva, (P. 665.)

293. Characodon milineatus. ( $\mathrm{P}, 668$. ) 



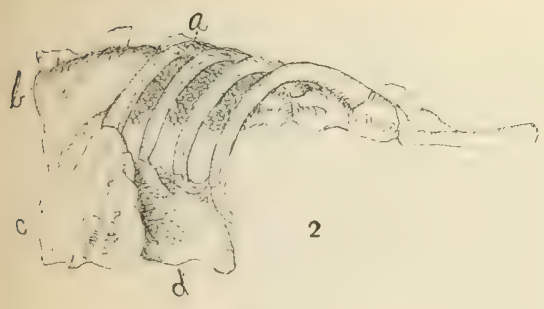

$29.4 a$

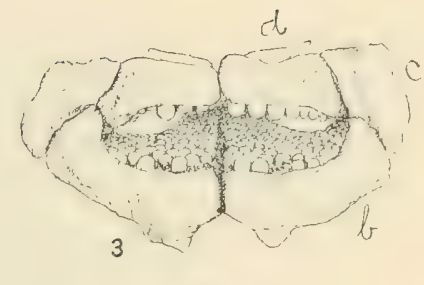

$: 3916$

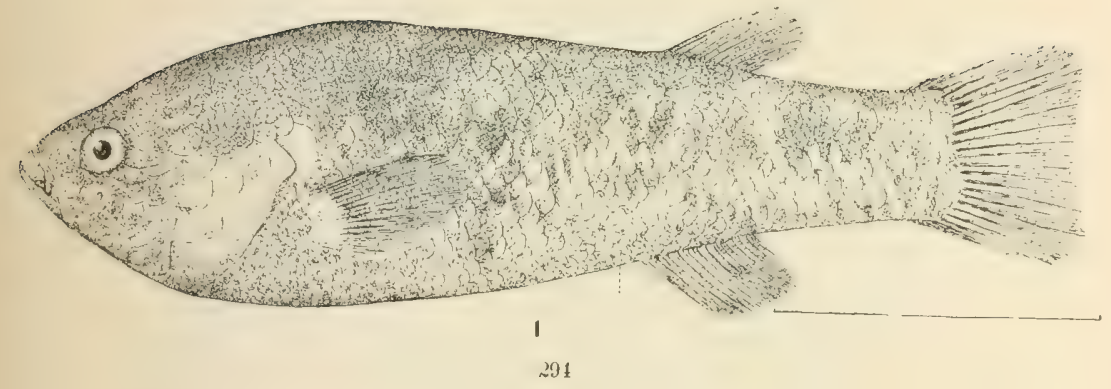

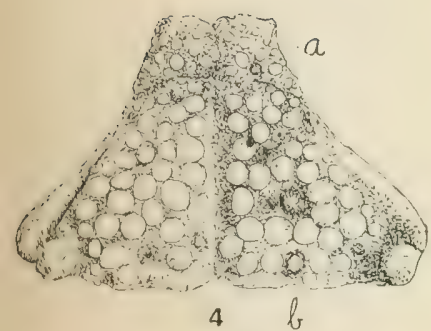

$294 c$

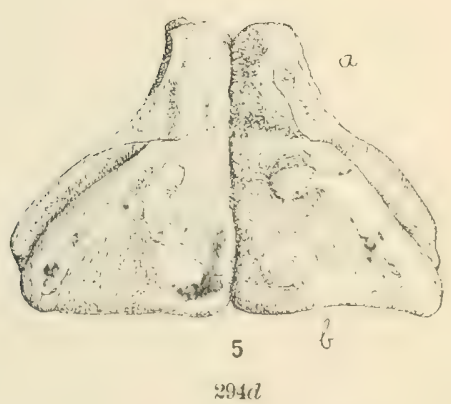

294, 294a, 294b, 294c, 294d. EMPetrichthy's MERmiм. (Р. 667.) 


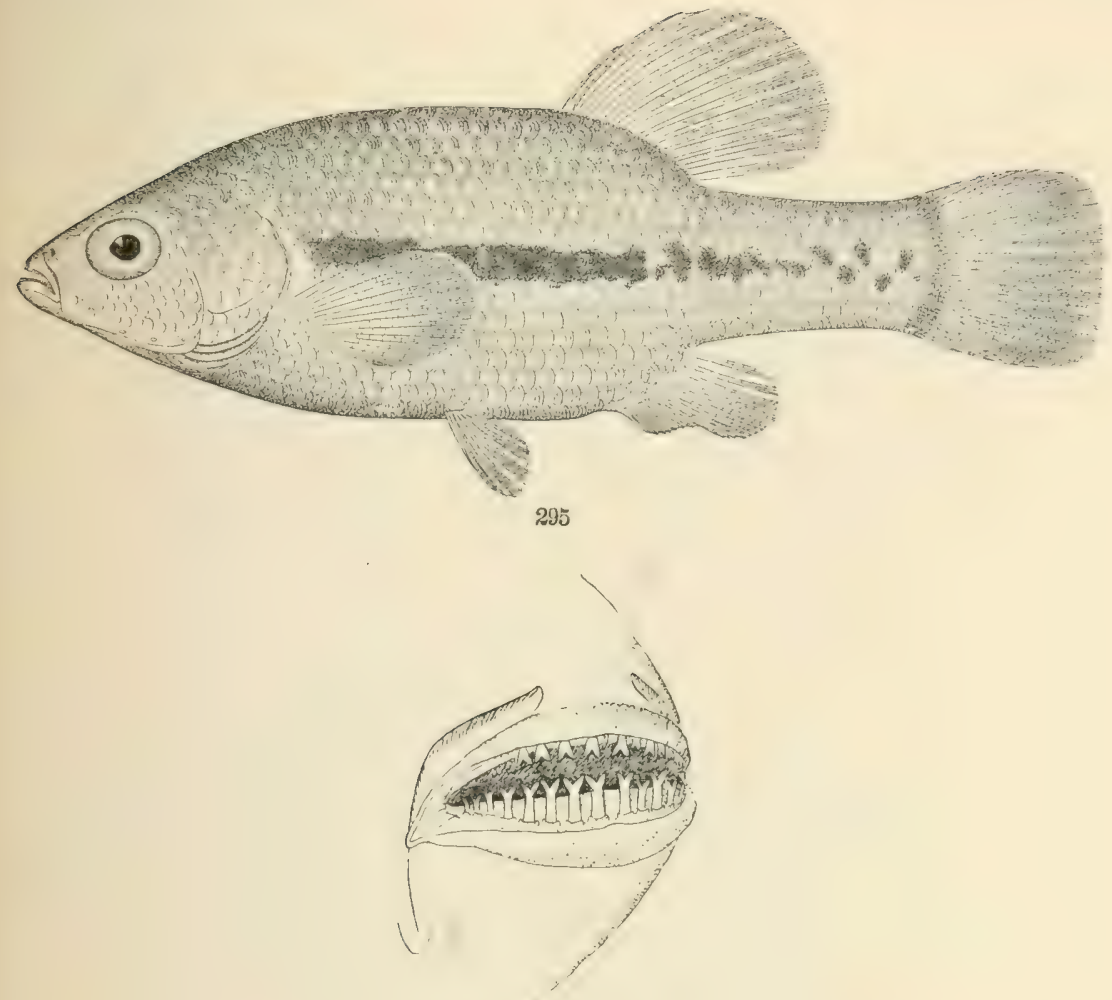

2905

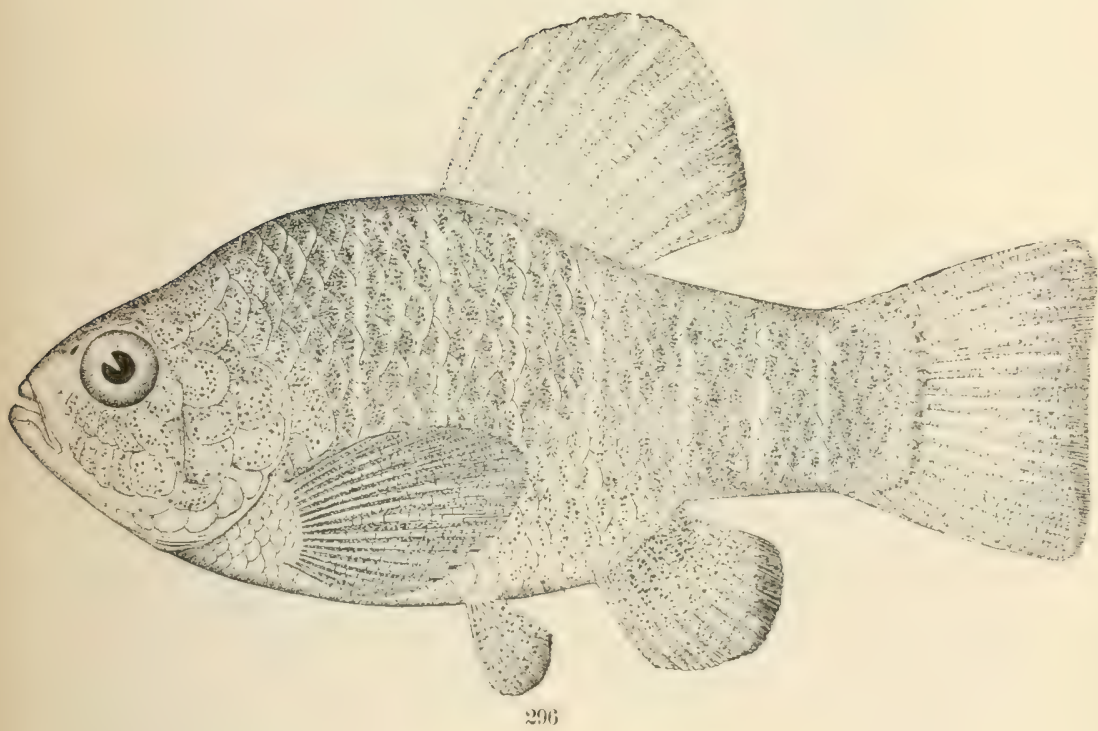

295. Characodon valiatus. (P. 669.)

295a. TEETH OF CHARACODON VARIATUS. (P. 669.)

296. Cyprinoton variegatus. (P. 671.) 



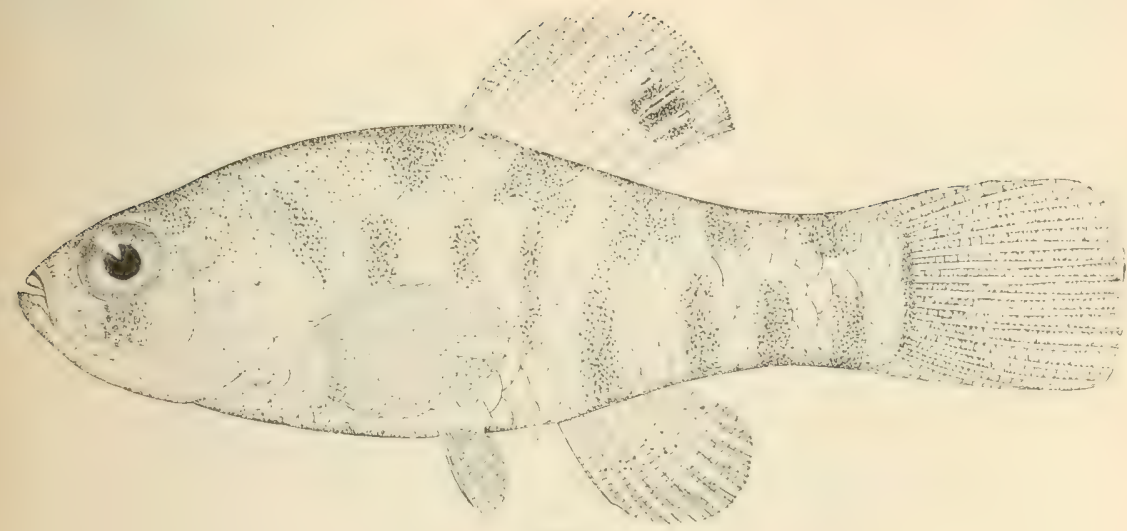

296
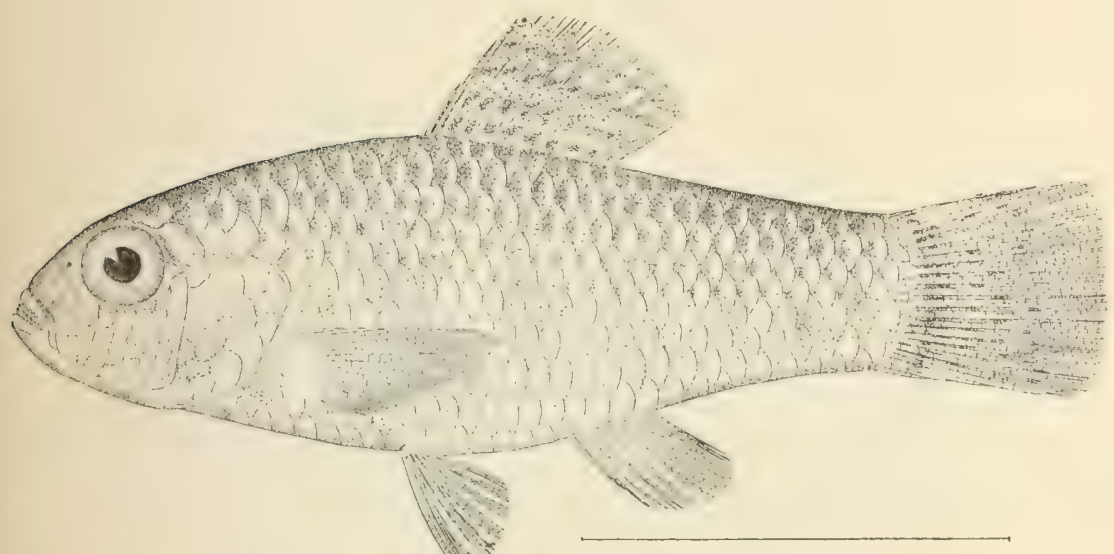

297

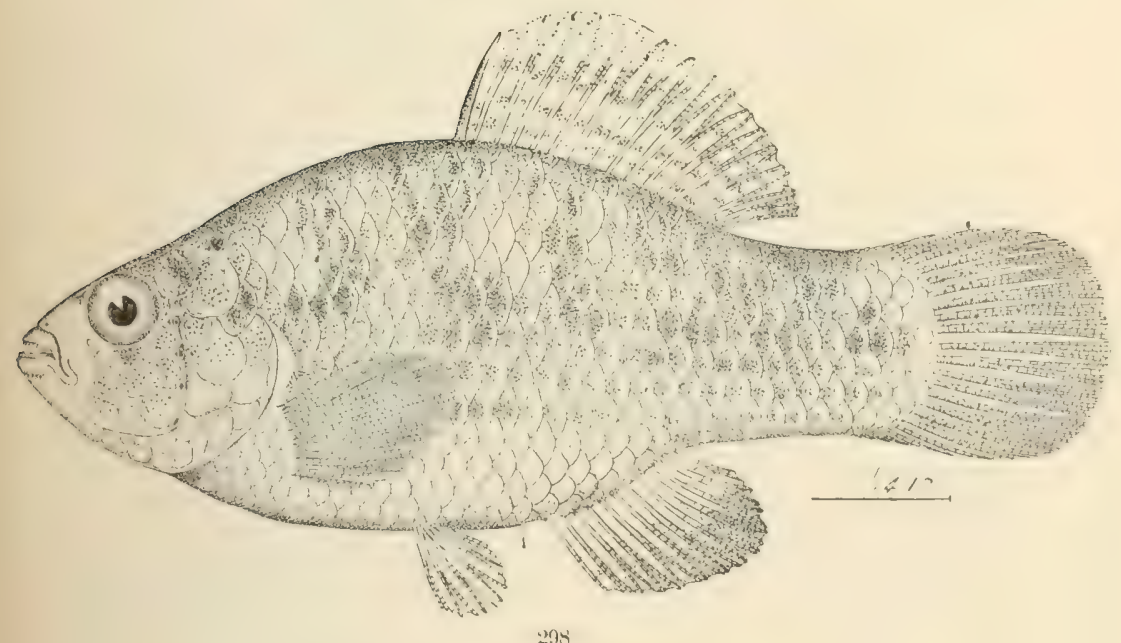

m9s

296a. Cýpinonon variegatus; young. (P. 671.)

297. CYPRINODON CARPIO. (P. 675.

298. JORDANELLA FLORIDA. (P. 677.) 



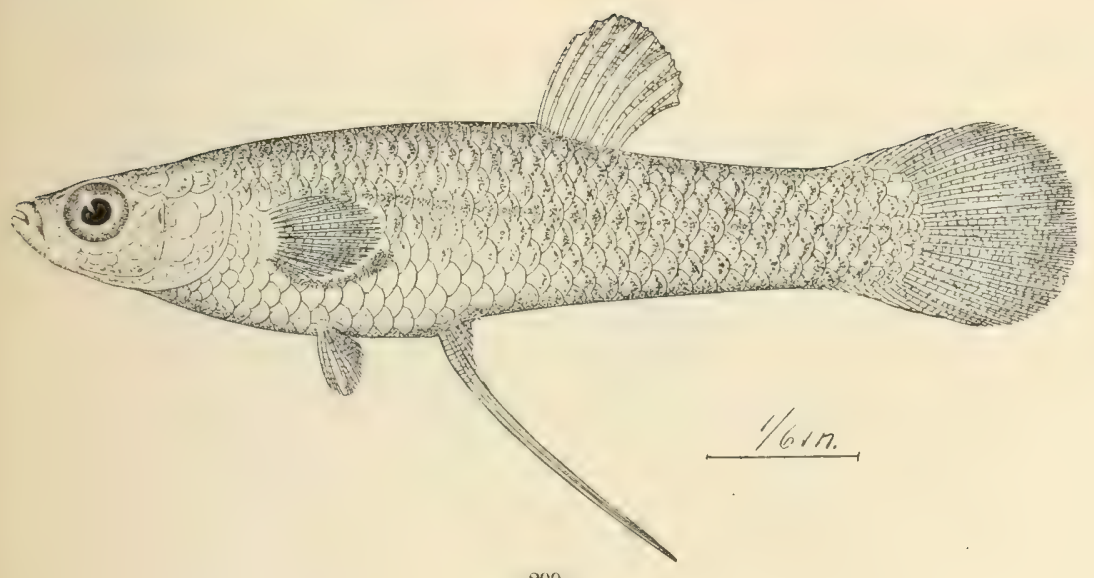

299
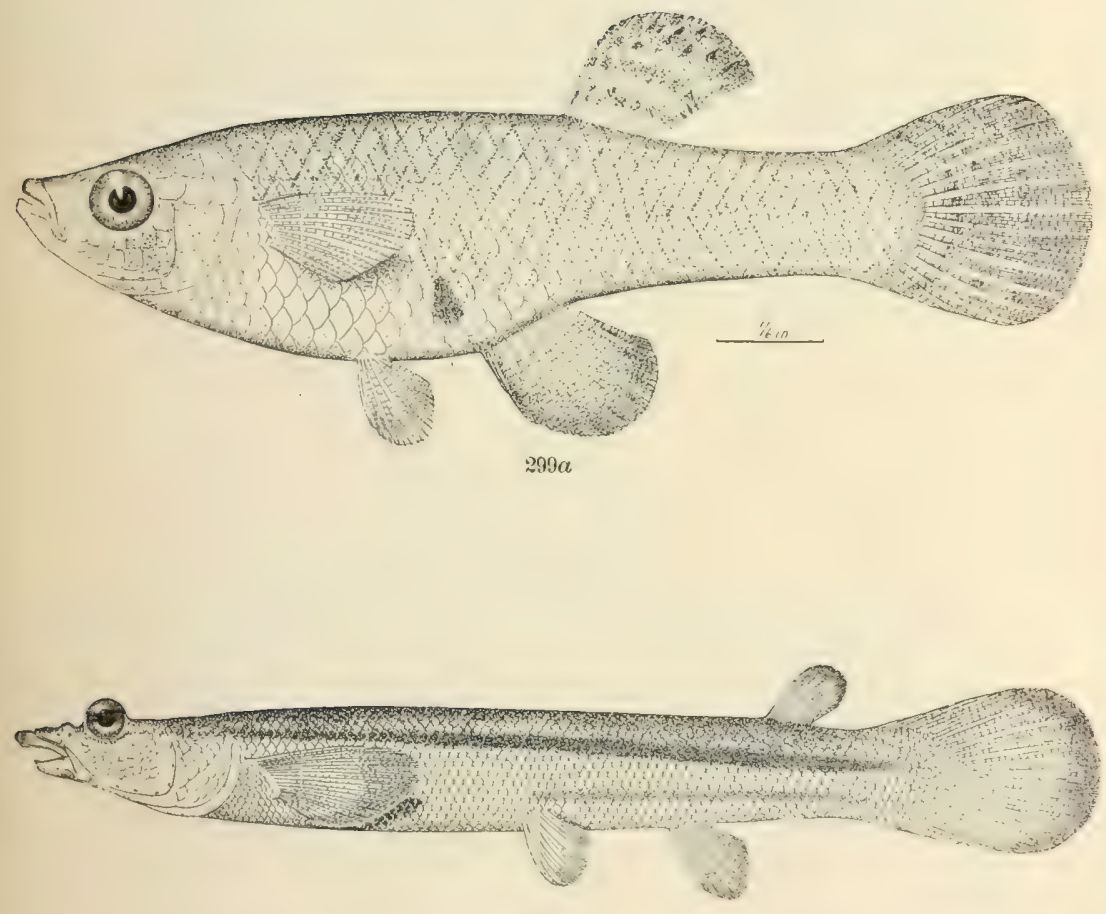

300

299. Gambusia affinis; male. ( $\mathrm{P}, 680$. )

299a. Gamizosia affinis: female. (P. 680.)

300. ANABLEPS DOVII, ( $\left.\mathrm{H}^{\prime} \cdot 685.\right)$ 





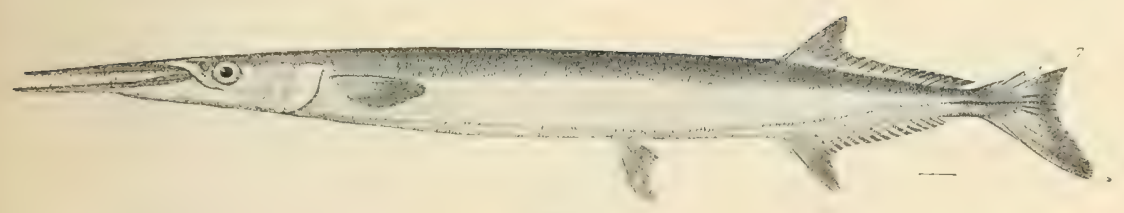

308
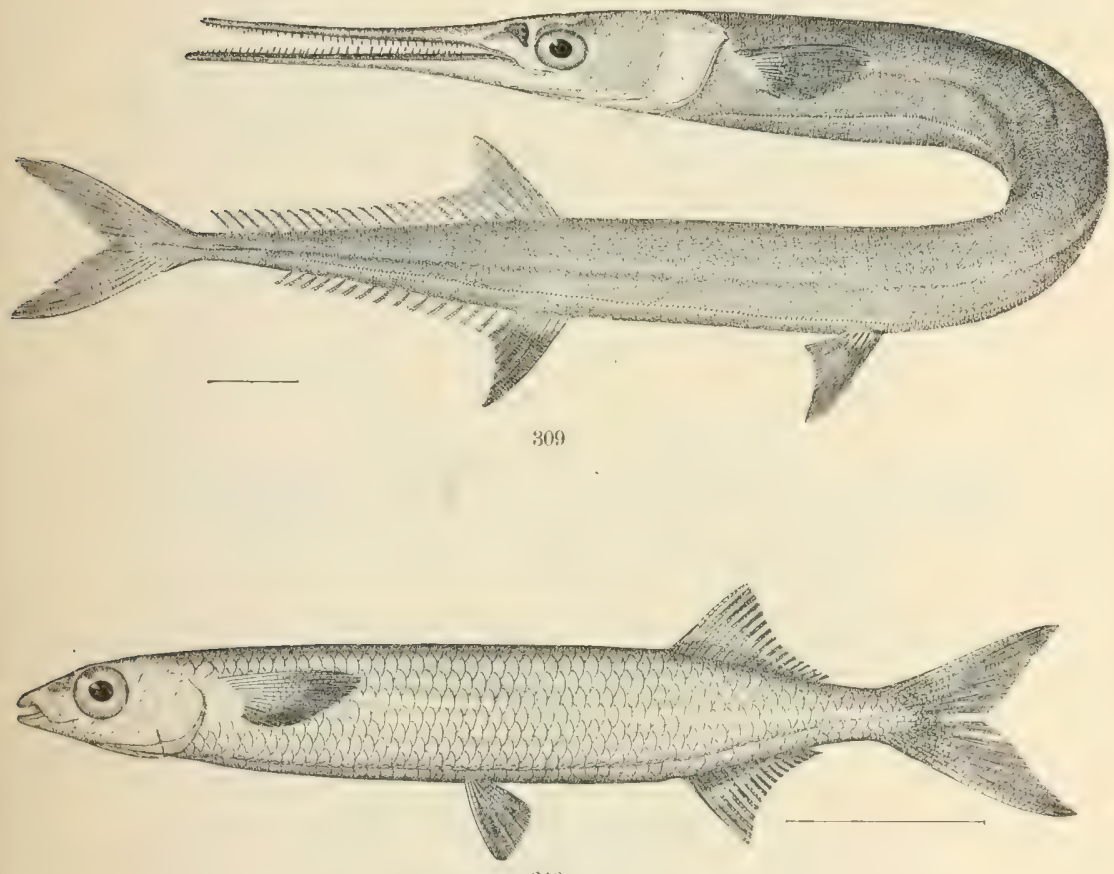

310

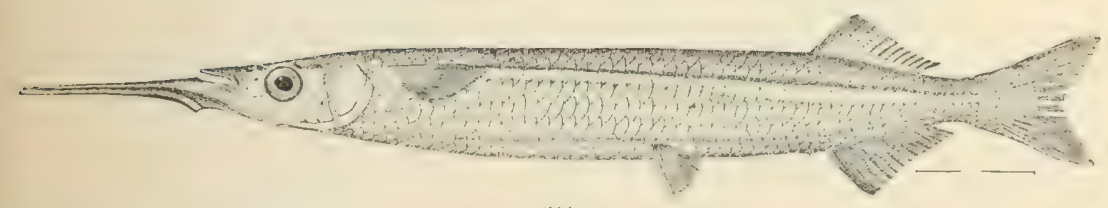

311

31)8. Tylosurus raphidoma. (P. 715.)

309. Trlosurus aCUS, (P. 716.)

310. Cirriodorus atherinoIdes. (P. 719.)

311. Hyporhamplus tinifasciatus. (P.720.) 



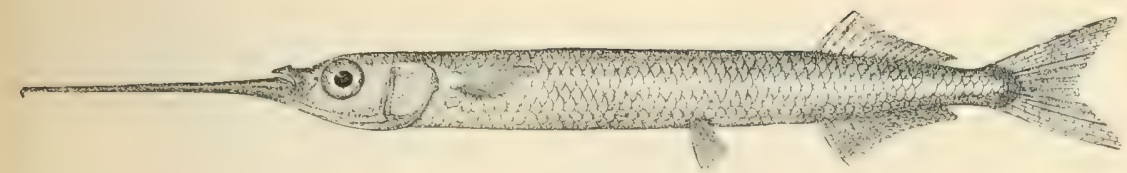

312
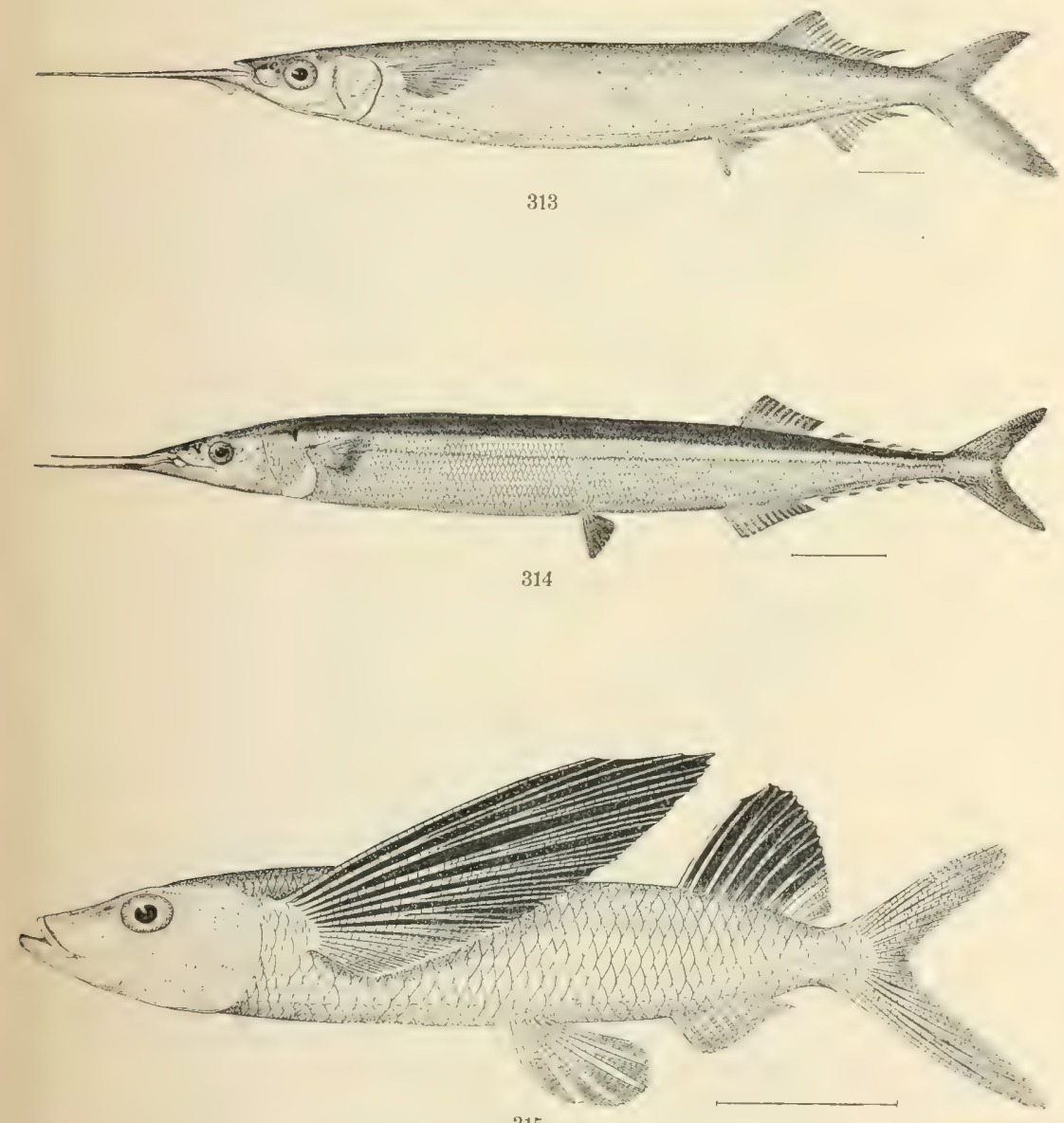

315

312. HYPORHAMPHUS RoBERTI. (P. 721.)

313. Hemiramilus BRasilikNsis. (P. 722.)

314. SCOMBRESOX SAURUS. (P. 725.)

315. Foditor acutus, (P. 728.) 



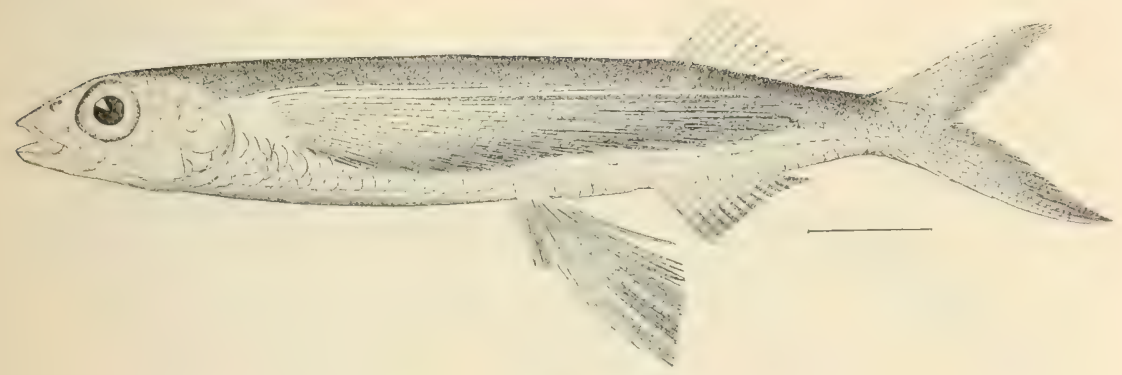

316
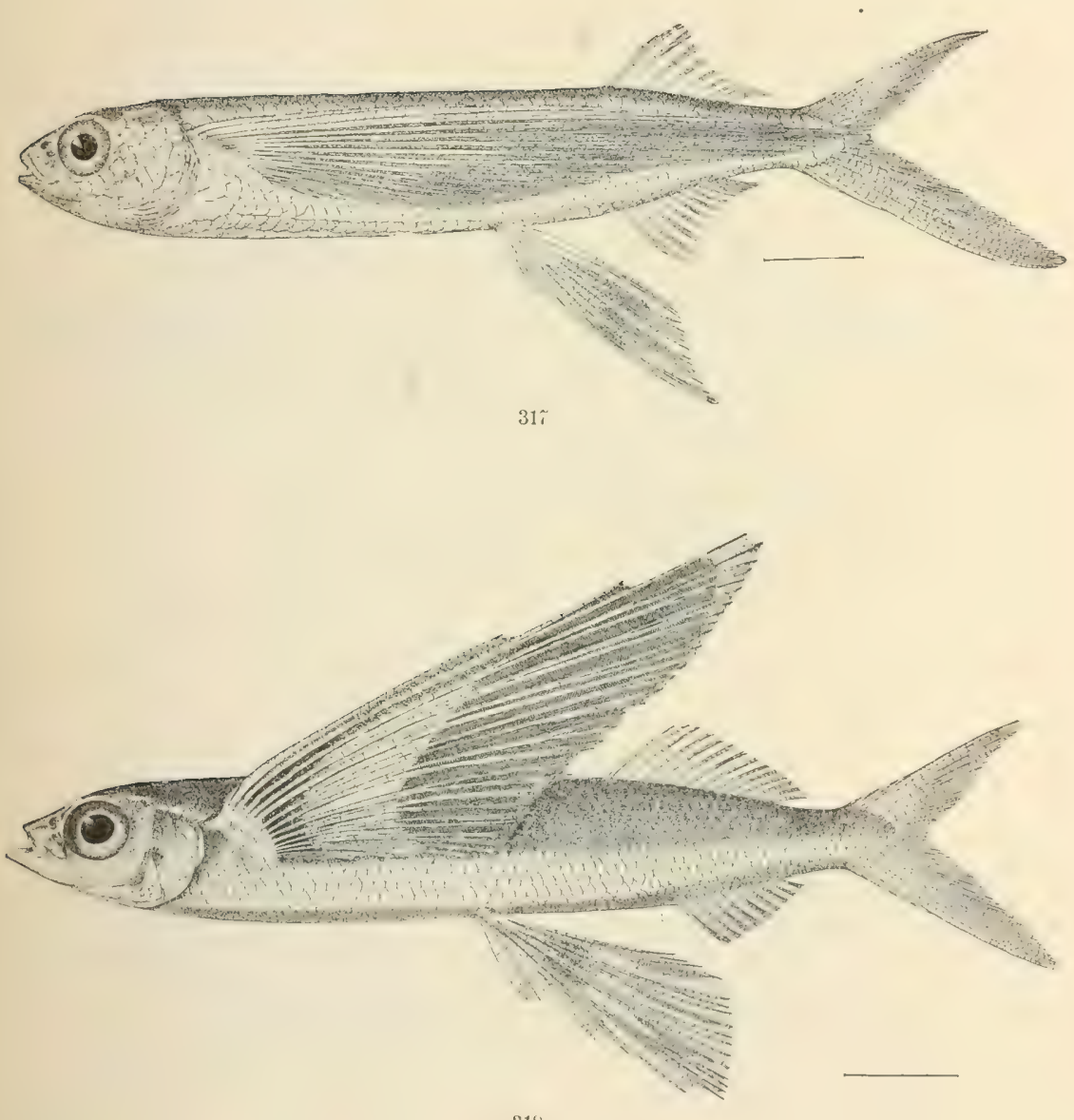

$31 \mathrm{~s}$

316. Exonautes exsimens. (Pp. 732, 2830.)

317. Exonautes RoNdeletil. (Pp. 733, 2830.)

318. Exoceitus volitans. (P. 734.) 


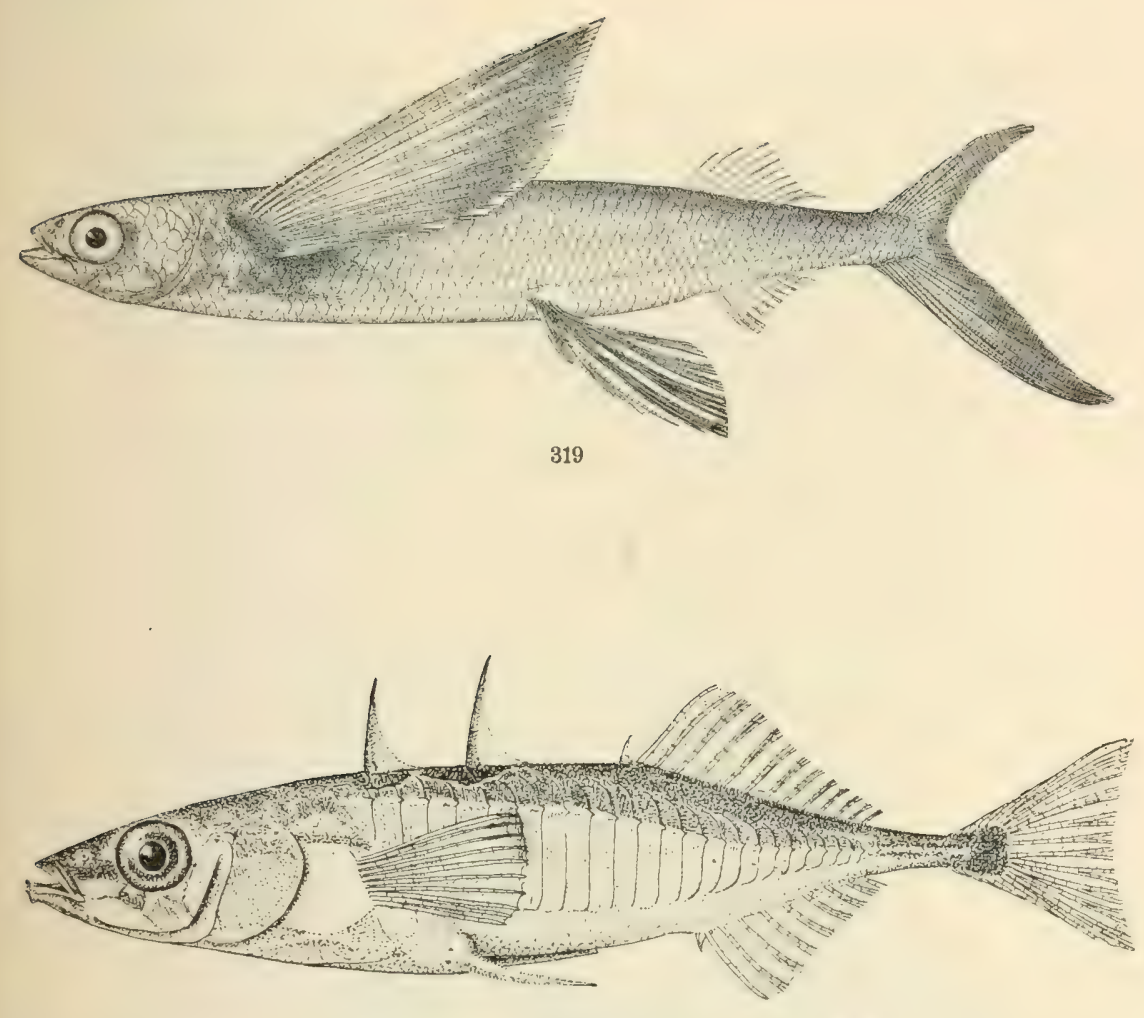

$3: 0$

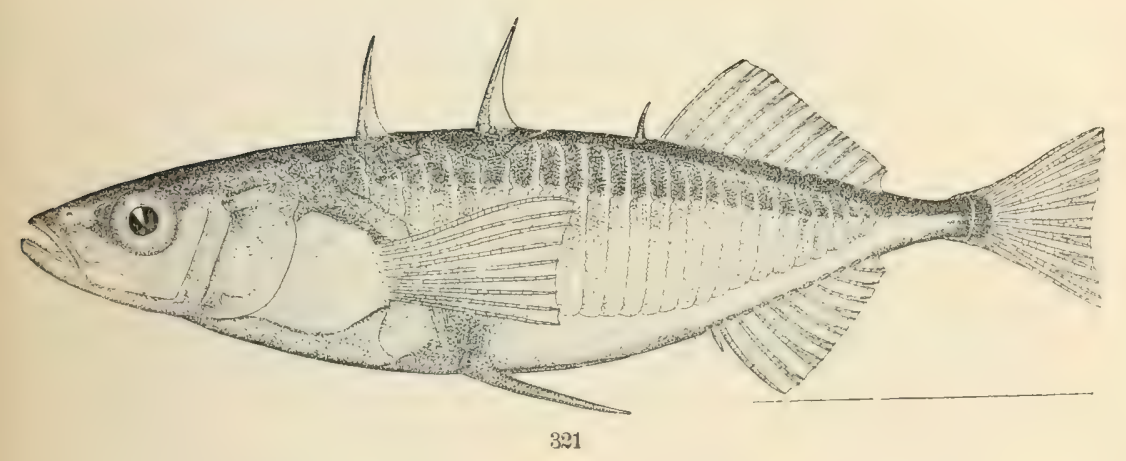

319. Cypsilurus californicus. (Pp. 740, 2830.)

320. Gasterosteus aculeatus. (P. 747.)

321. Gasterostevs Catapiractus. (P. 749.) 



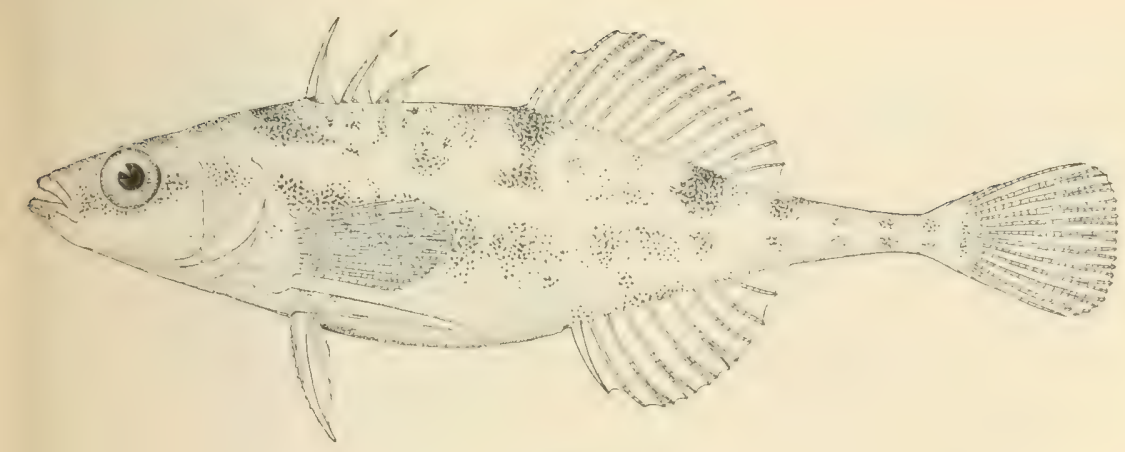

$32: 3$

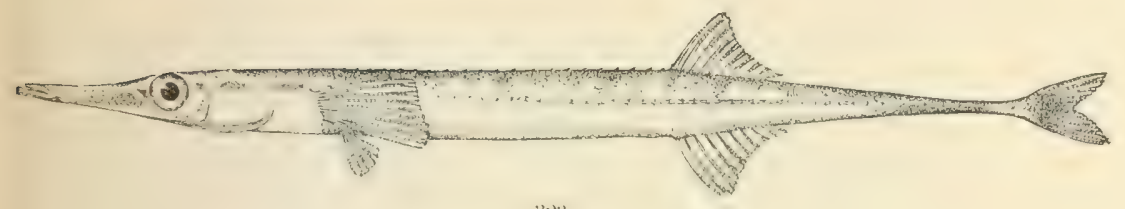

:F:

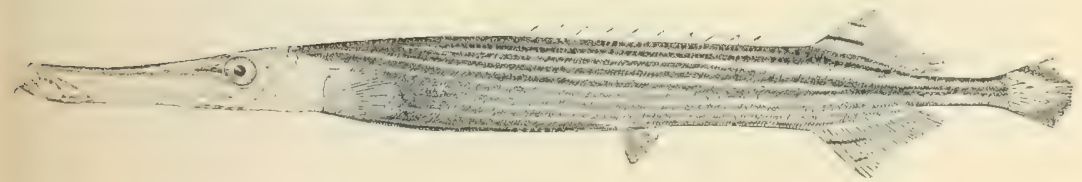

$3 \div 1$

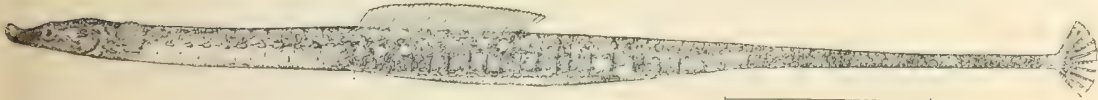

$3 \%$

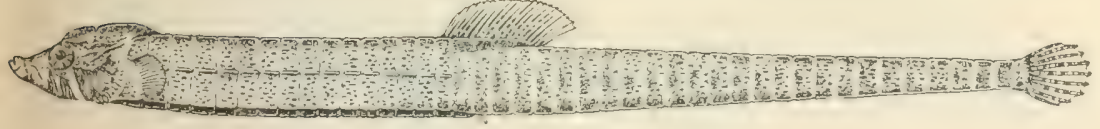

$3: 26$

322. APELTES QUADRACUS. (P. 752.)

323. Auloriy NCHUS Flavidus. (P. 754.)

324. Aulostomus maculatus. (P. 751.)

325. SIPHOSTOMA STARKSI. (P. 771.)

326. Corythioichthys CaYoruir. (P. 2838.) 


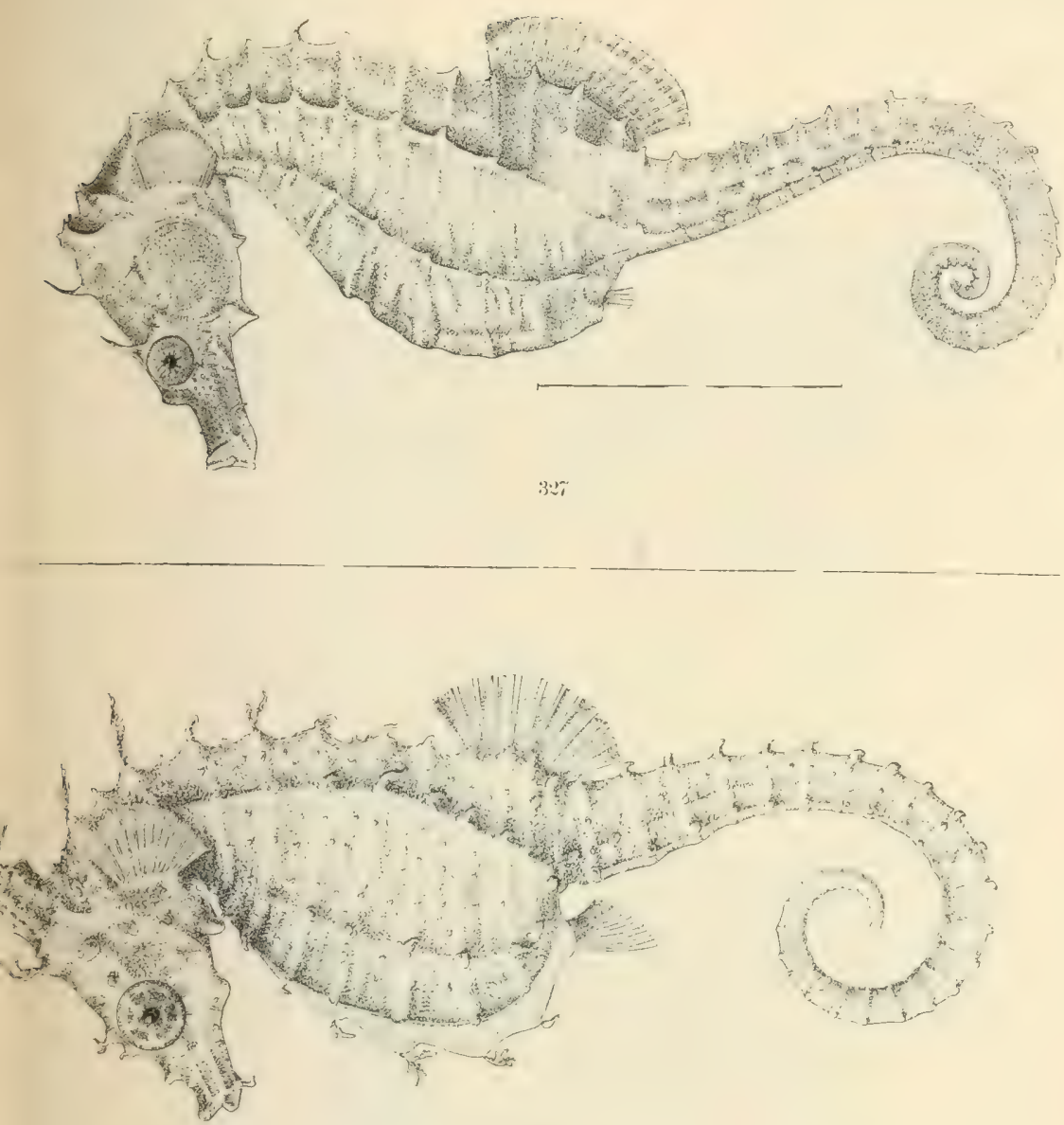

$3: N$

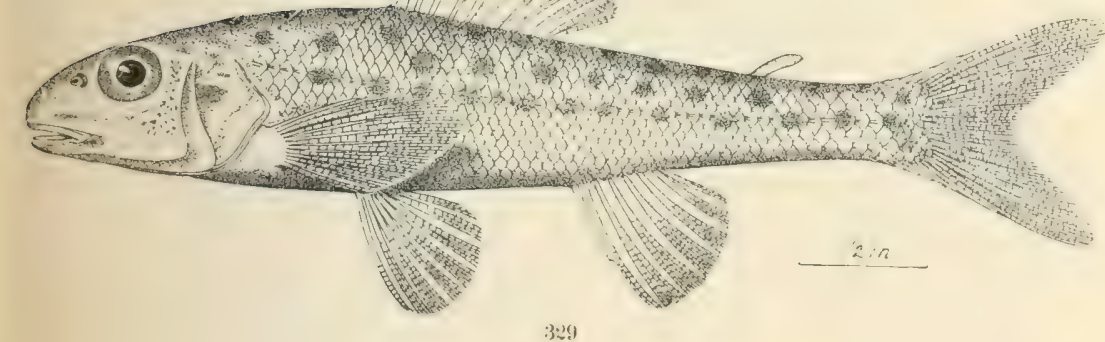

327. Hiptocample mudonius. (P. 77 .

328. HiPPOCAMPUS ZOSTER.1. (P.7\%,

329. Pelicol'sis guttatus. (ए'.784.) 




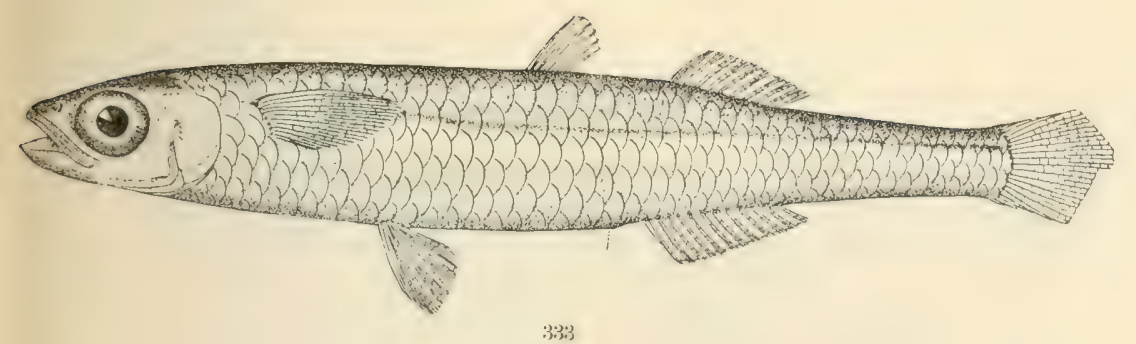

$3: 303$
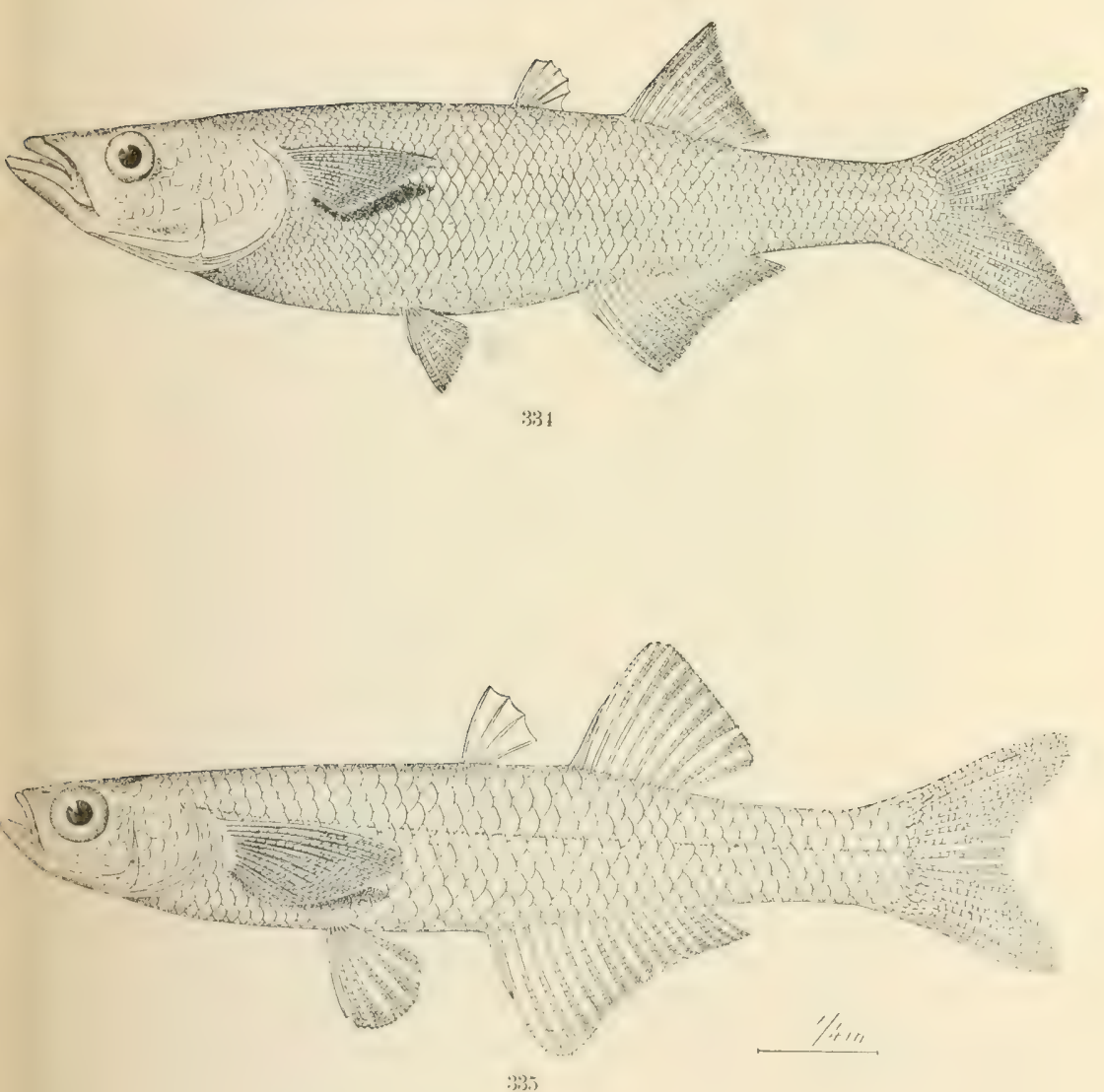

333. Atmerina arrea. (P. 790.)

334. Chmostoma humbolianum. (P. 793.)

335. Eslopsarua jolbdan. (Pp. 793, 2840.) 



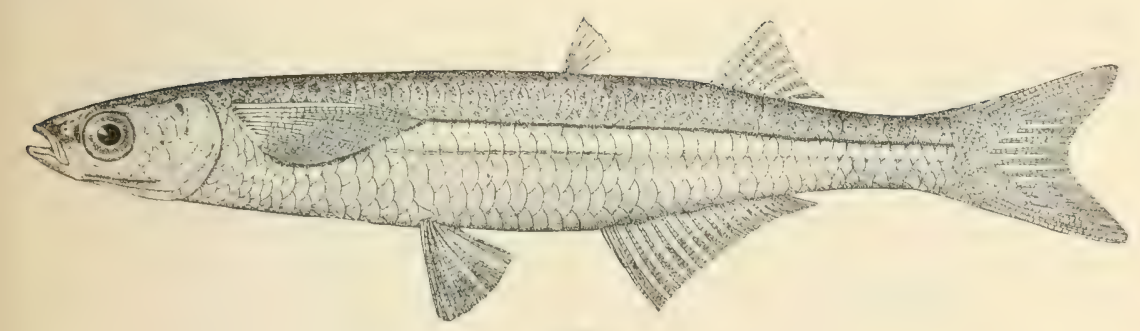

336
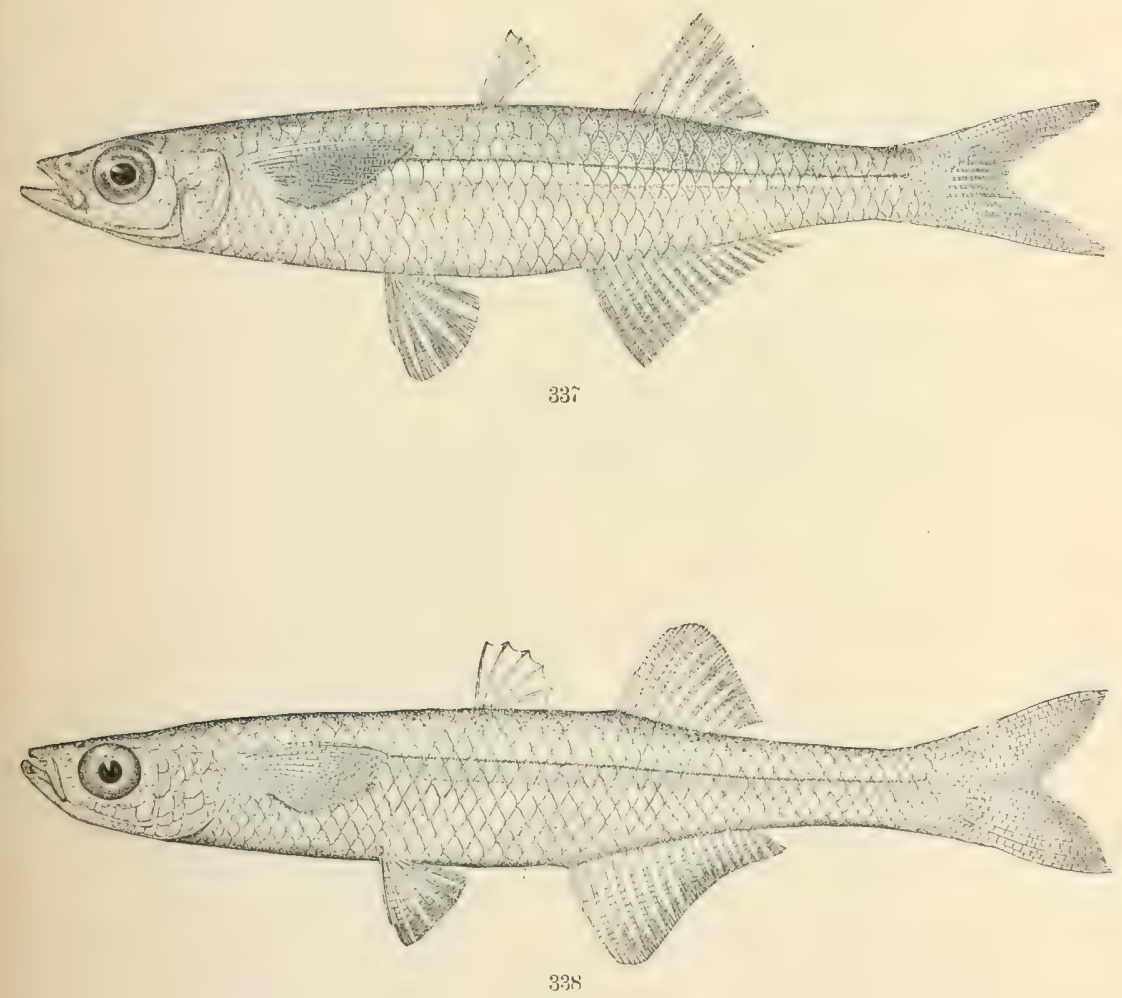

336. Kirtlandia vagrans. (P. 794.)

337. Menidia peninsula. (P. 797.)

338. MENIDIA GRACILIS BERYLLINA. (P. 797.) 


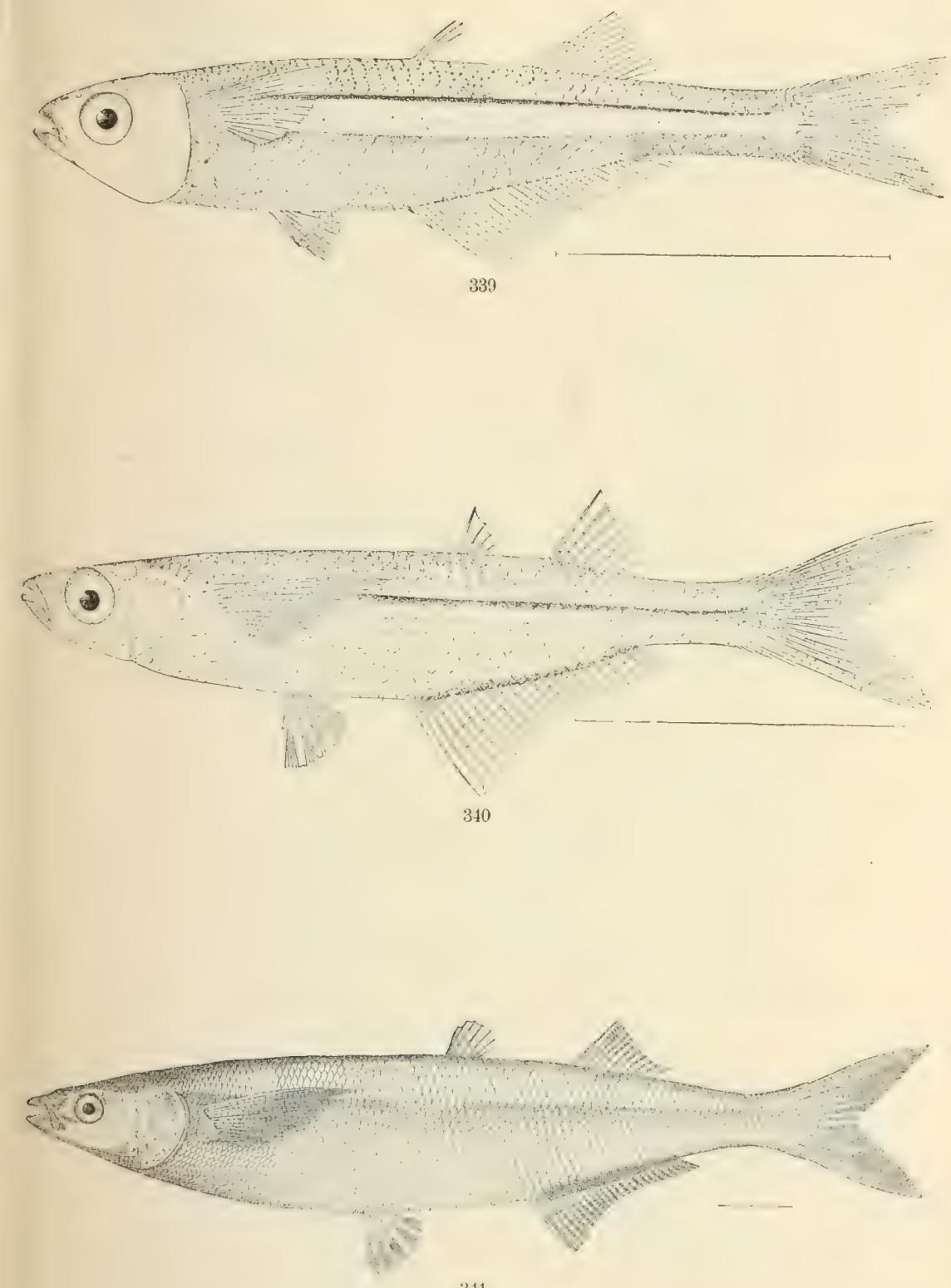

339. Eurytole eriarcia. (P. 803.)

310. Thyrina evermanit. (P. 804.)

311. Atherinopsis californiensis, (P. 806.) 



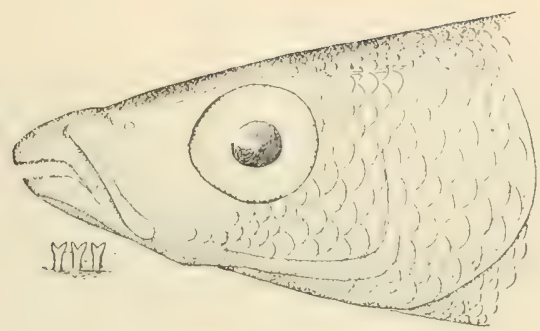

342

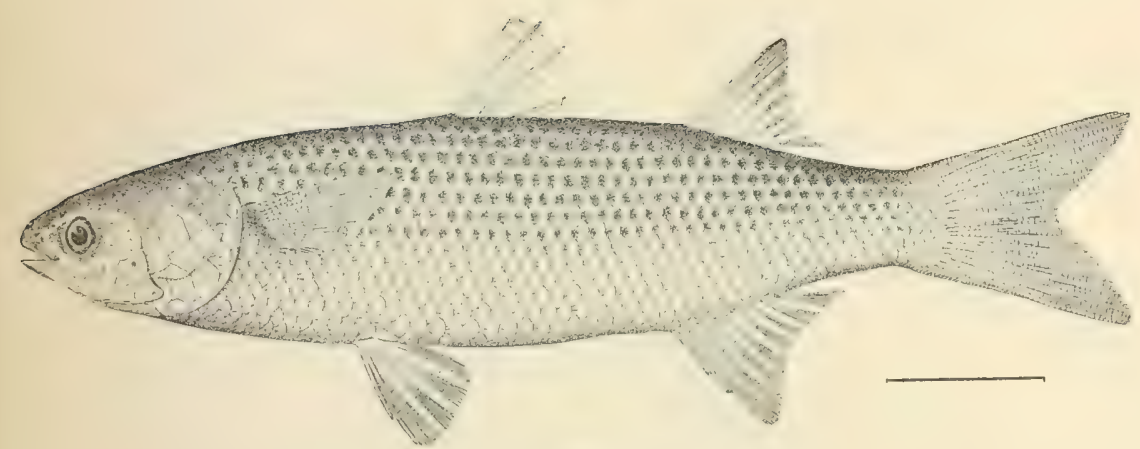

343

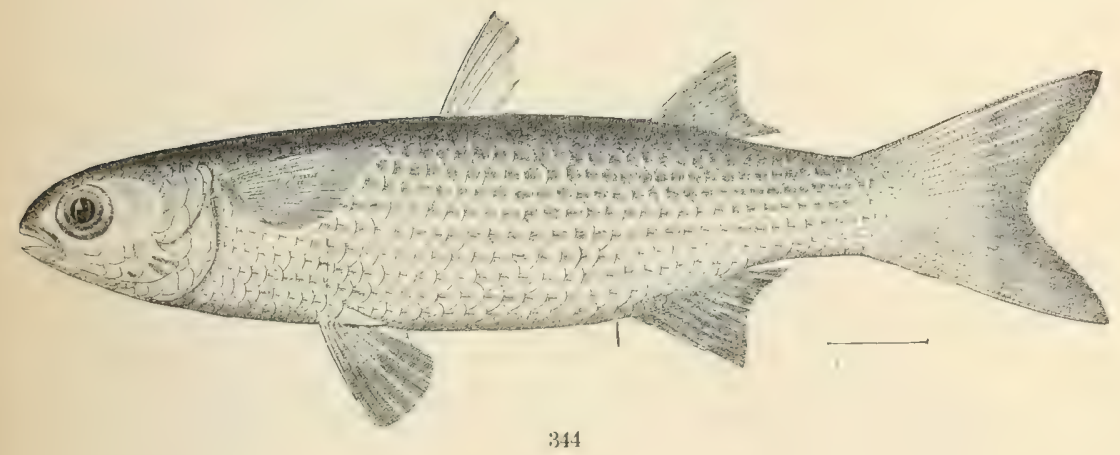

342. ATHERINOPS AFFINIS. (P. 807.)

343. Mugil cephalus. (P. 811.)

344. Mugil cureia. (P.813.) 



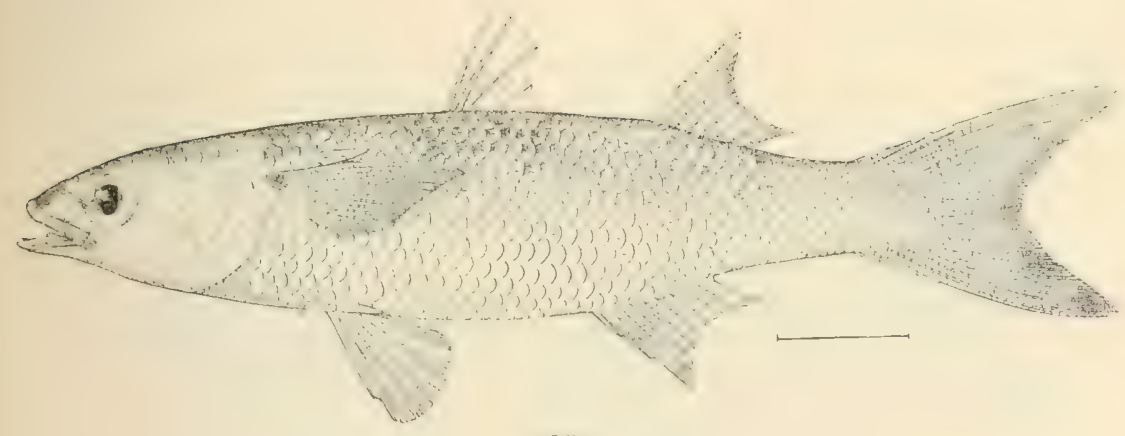

345

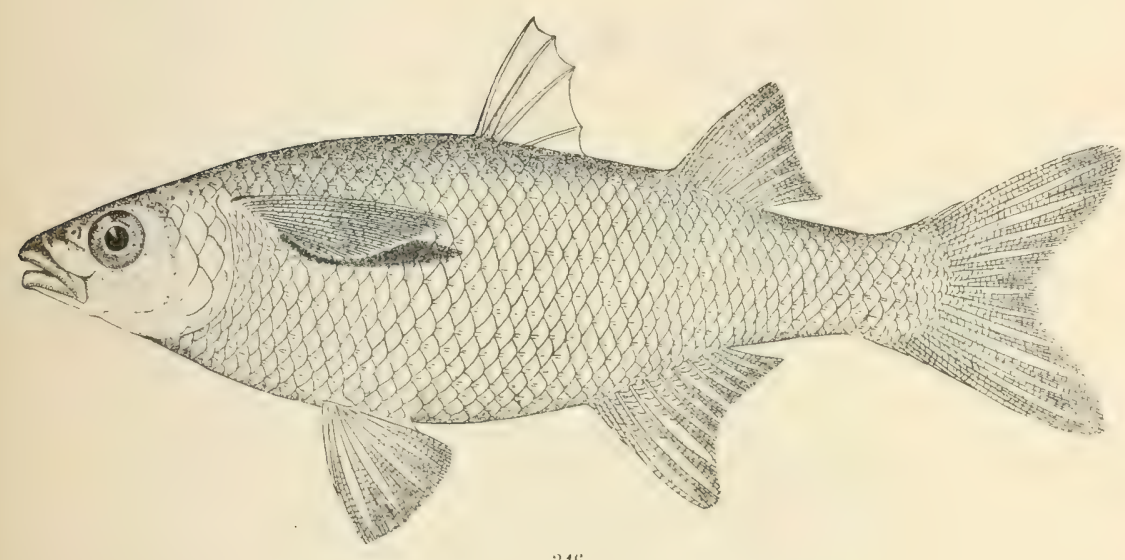

341i

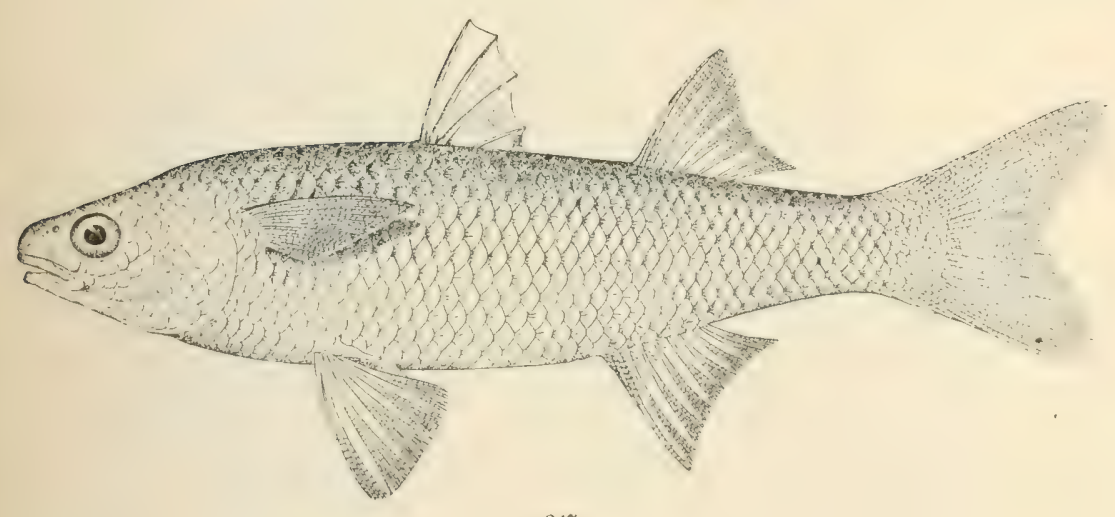

347

345. MUGIL Hospes. (P. 814.)

346. CHeNOMUGIL pROBOSCIDEUS. (P. 816.)

347. AgONOSTOMUS MONTICOLA. (P. 819.) 



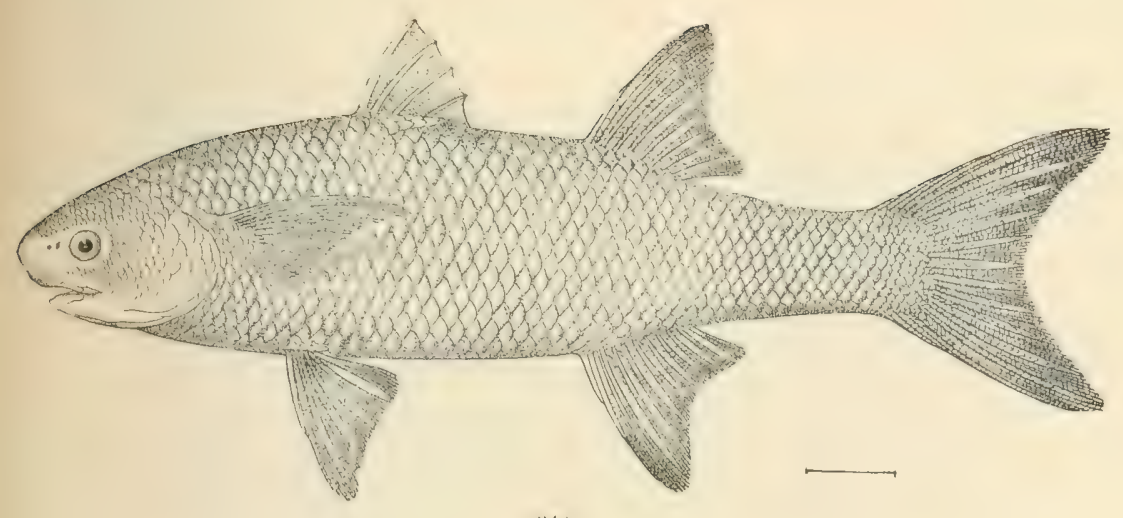

342

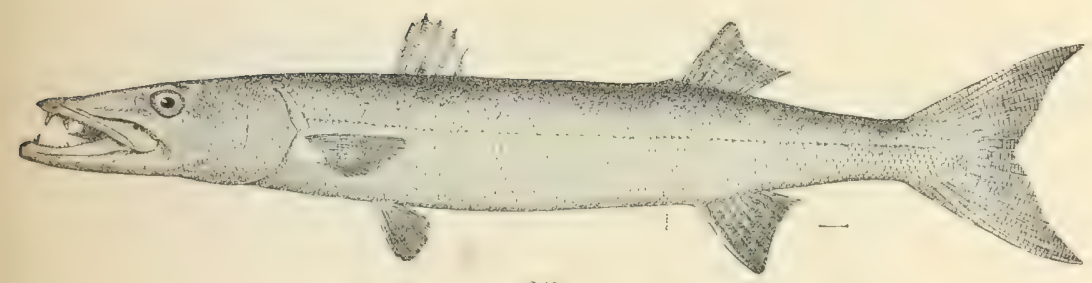

319

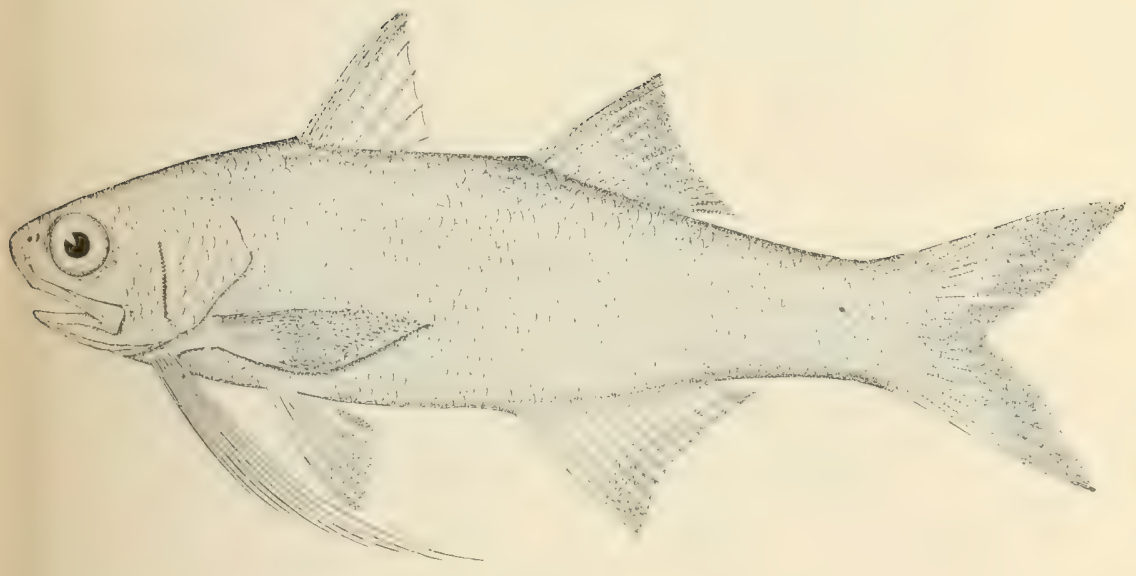

350

348. JotURUS PICHARDI. (P. 821.)

349. SPHYRENA BARRACUDA. (Pp. 823, 2841.)

350. Polydactylus octonemus. (P. 830.) 



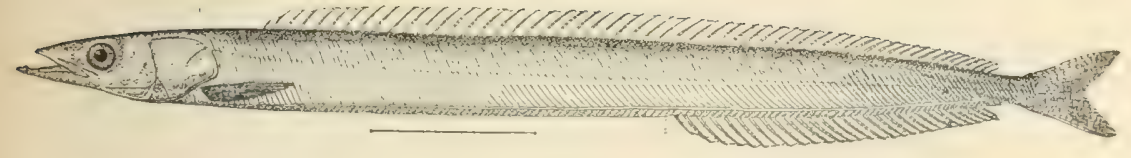

351
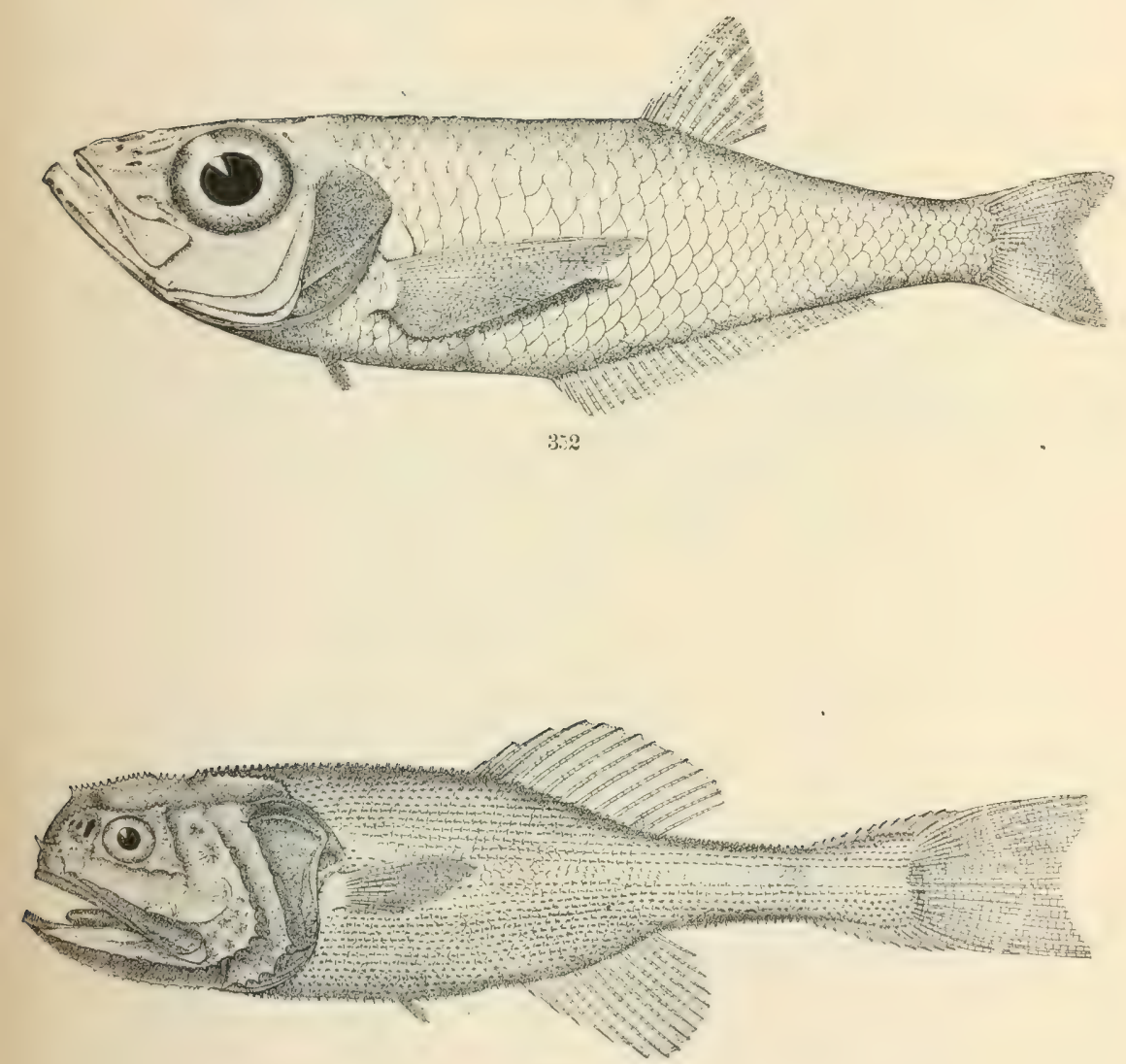

353

351. Ammonytes americanus. (P. 833.)

352. BATHYClUPEA ARGENTEA. (P. 835.)

353. STEPHANOBERY MONE。 (P. 836.) 


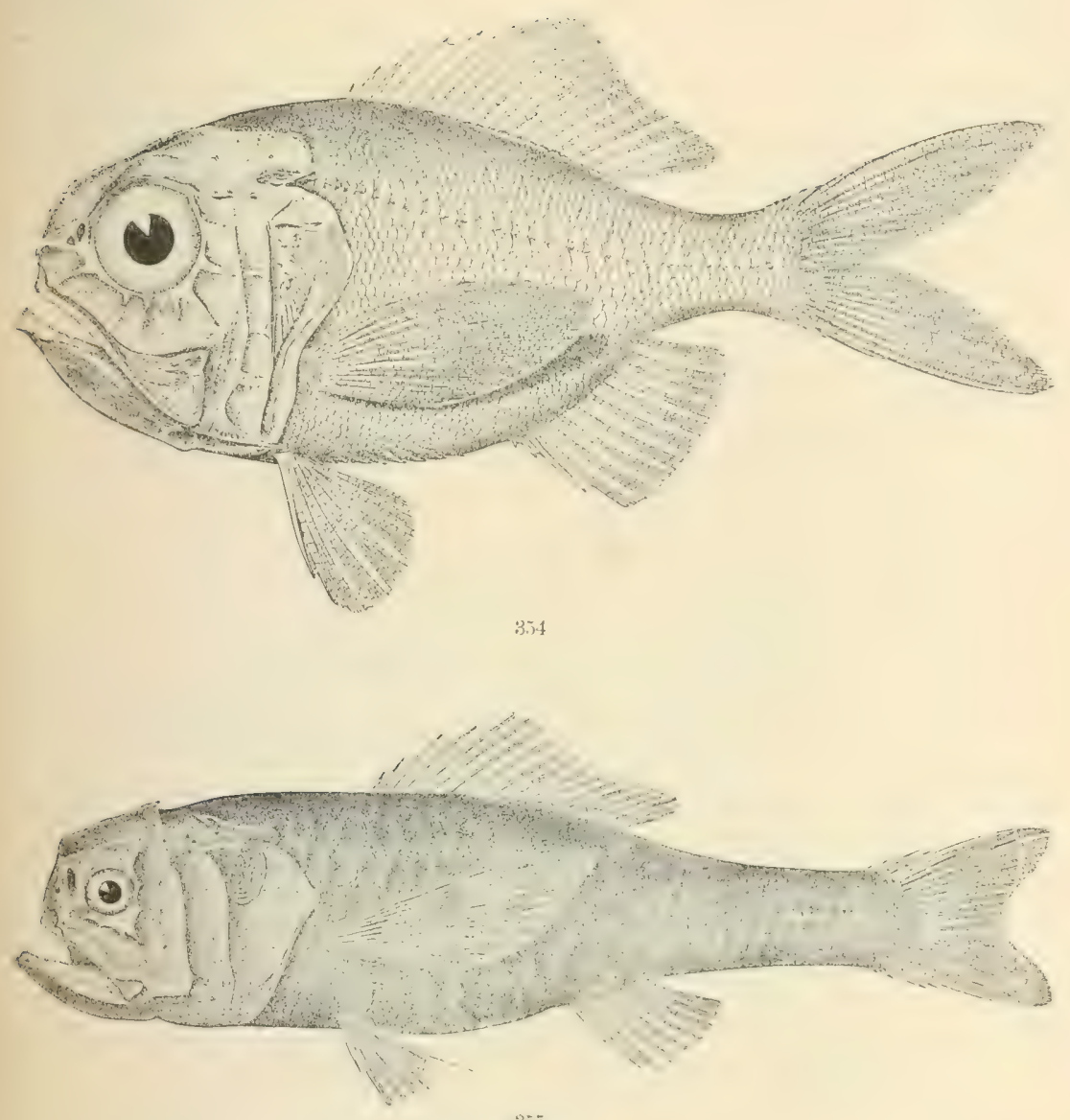

235

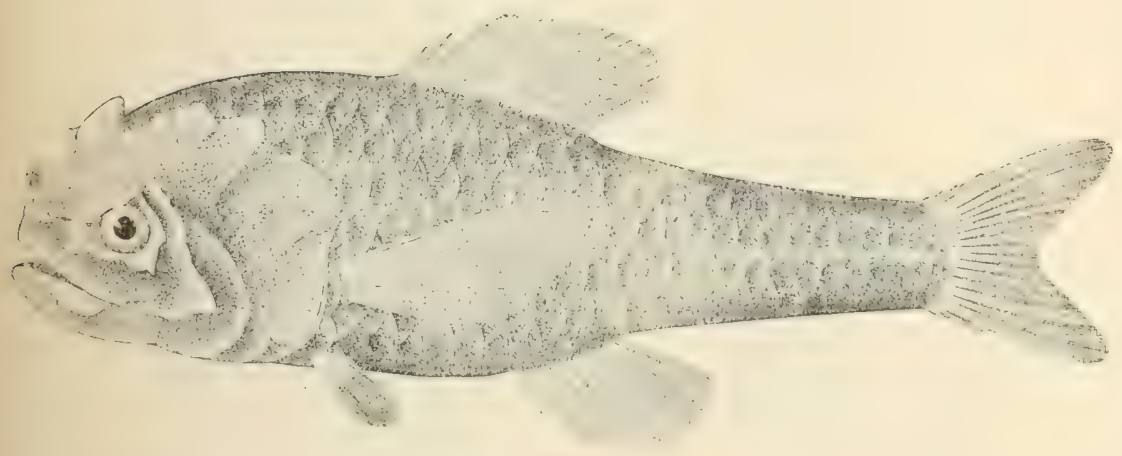

$\therefore$ ini

354. Hollostetius meIterraxeus. (1) 837.)

3i5. Phectionius subolilitilis. (

356. Plectionus crassicel's. (P. 843.) 



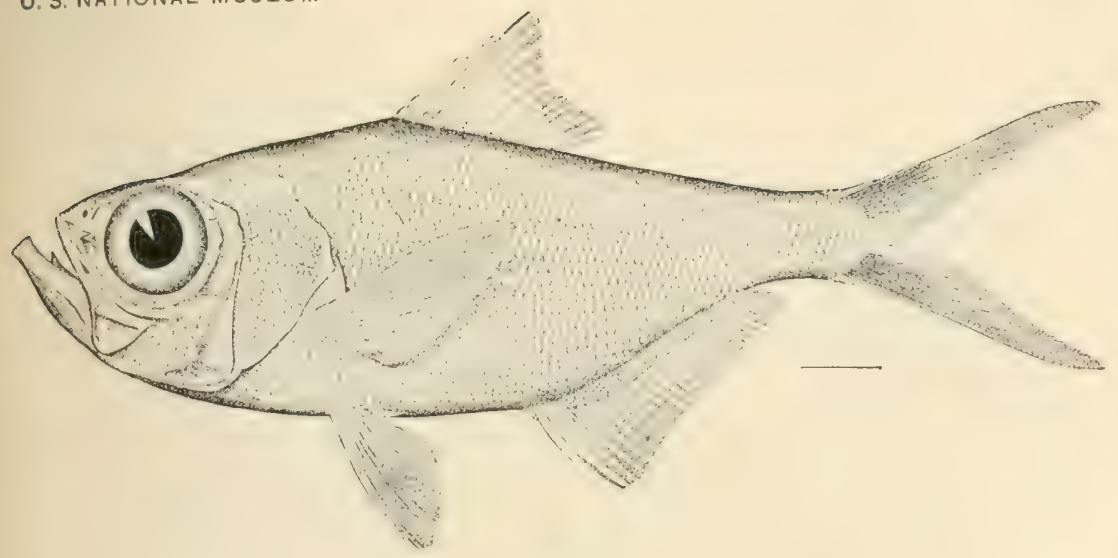

$35 \pi$

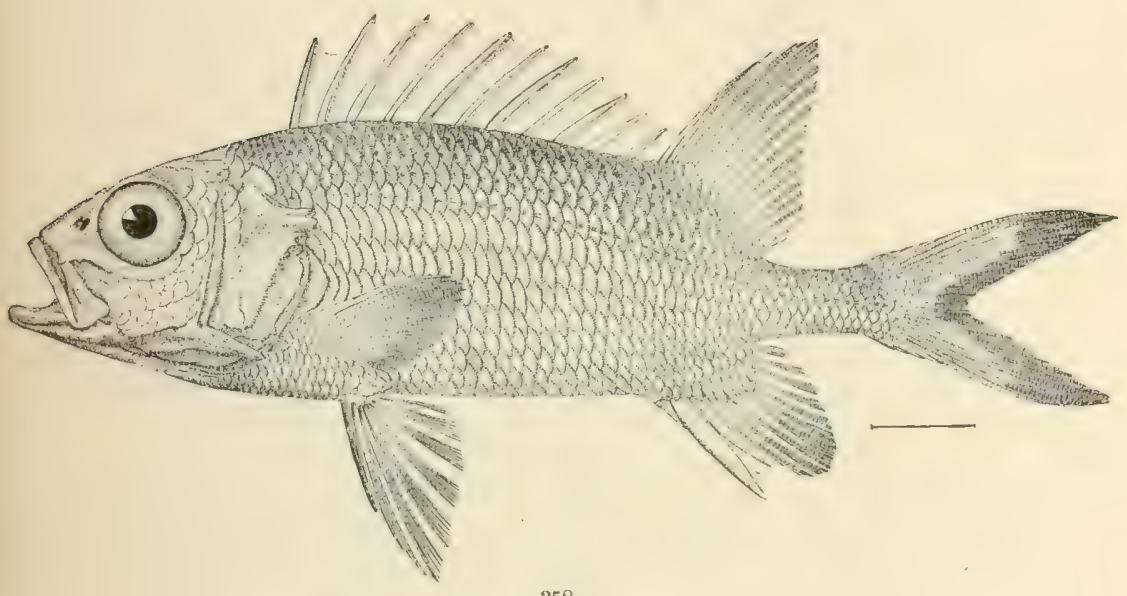

358

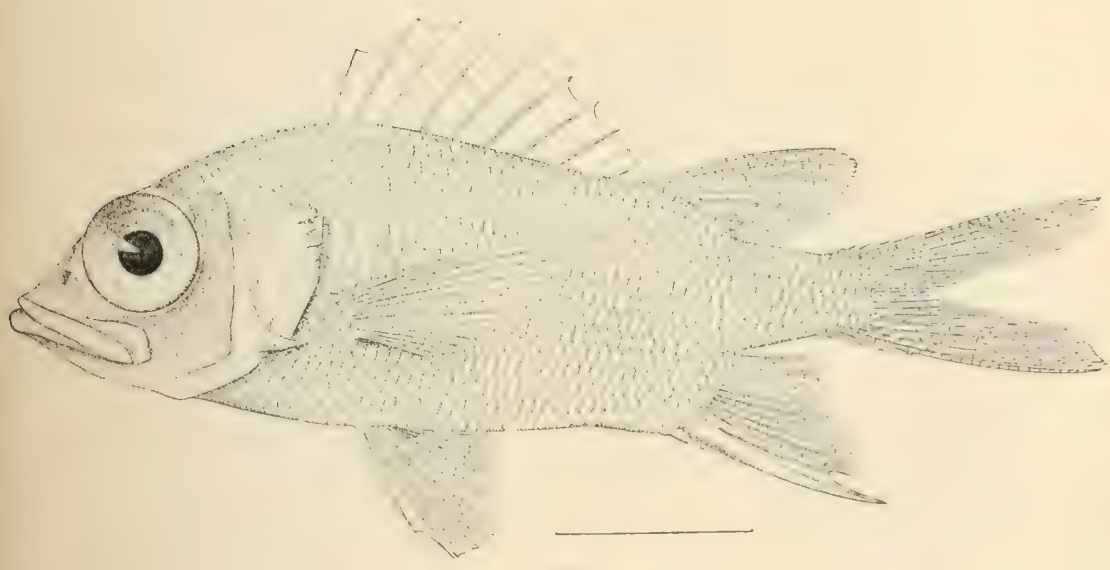

3,59

357. BerYX SPLENDENS. (P. 844.)

358. Holocentrus ascensionis. (P. 848.)

359. Flamieo marianes. (Pp. 852, 2871.) 


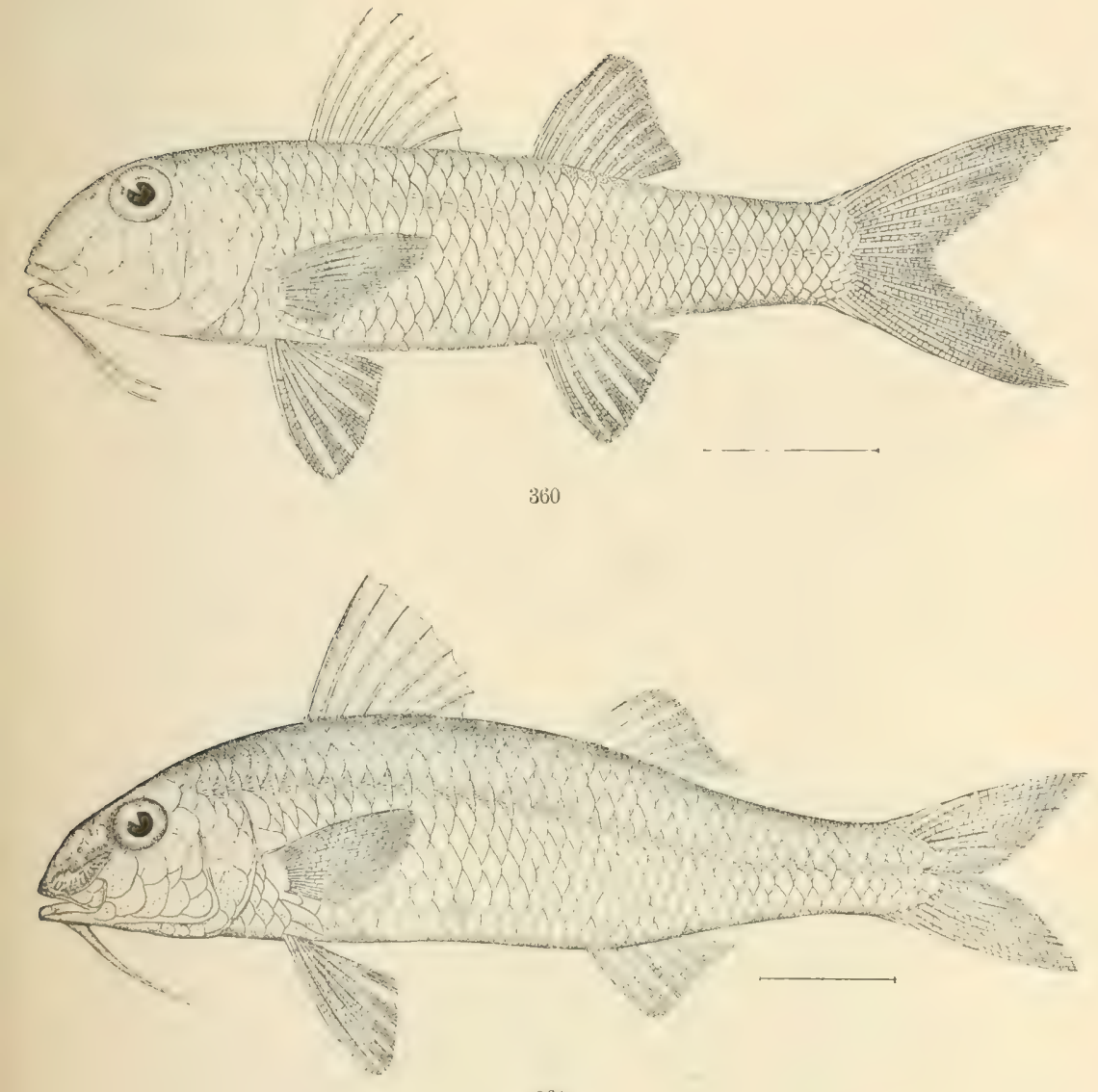

361

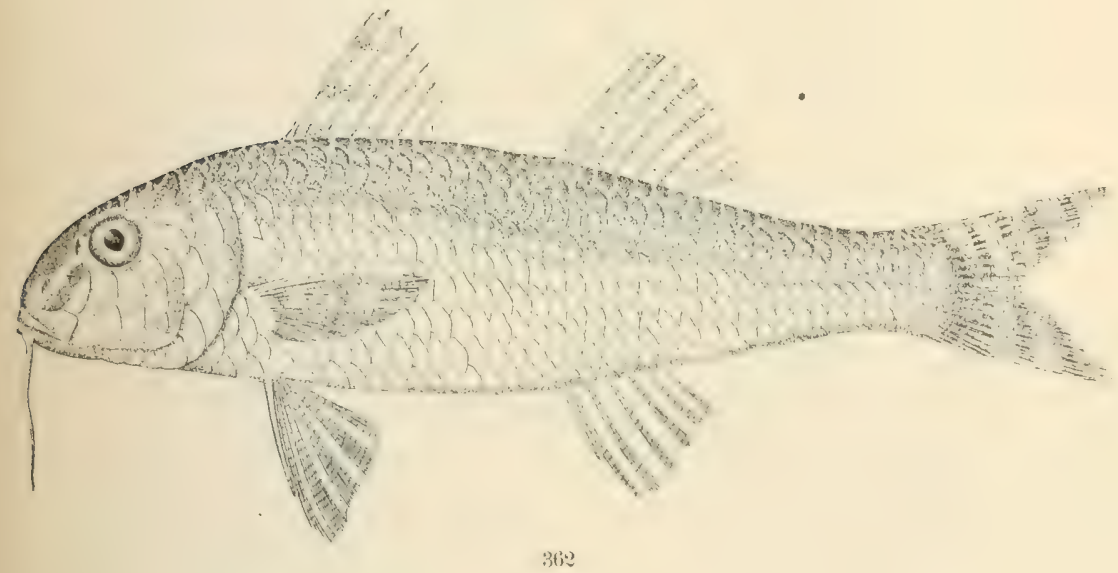

360. Mullus auratus. (P. 856.)

361. Mulloides ratibuni. (P. 857.)

362. UPFNEUS MACULATUS. (P. 858.) 



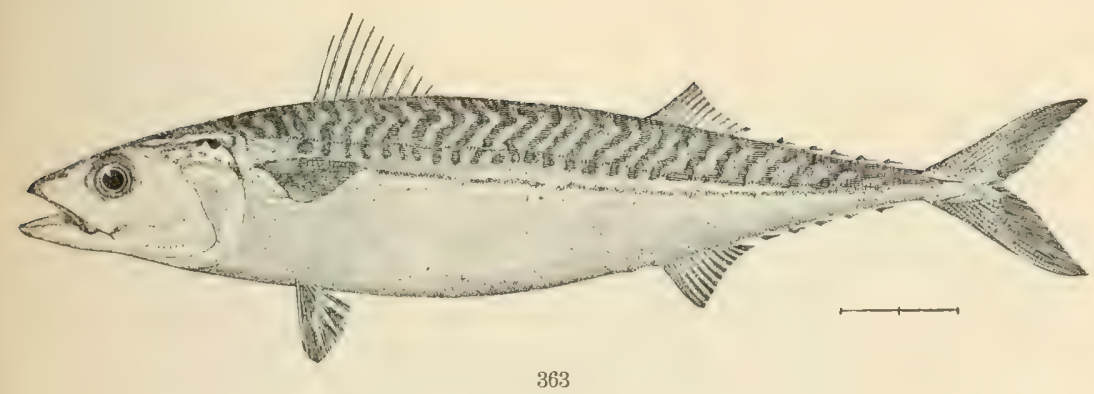

363
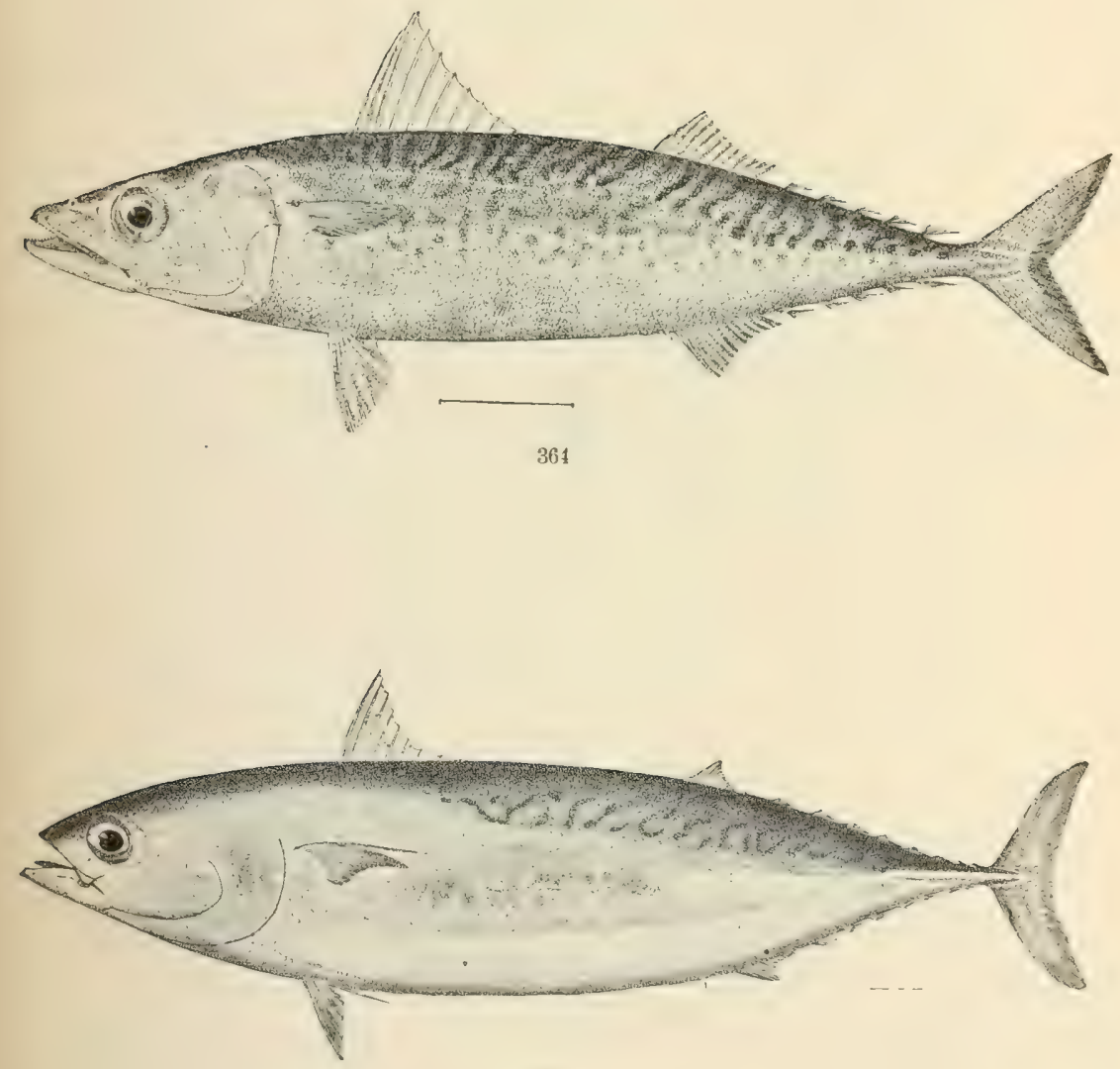

36.;

363. Scomber scombrus. (P. 865.)

364. Scomber Colias. (P. 866.)

365. Auxis thazard. (P. 867.) 


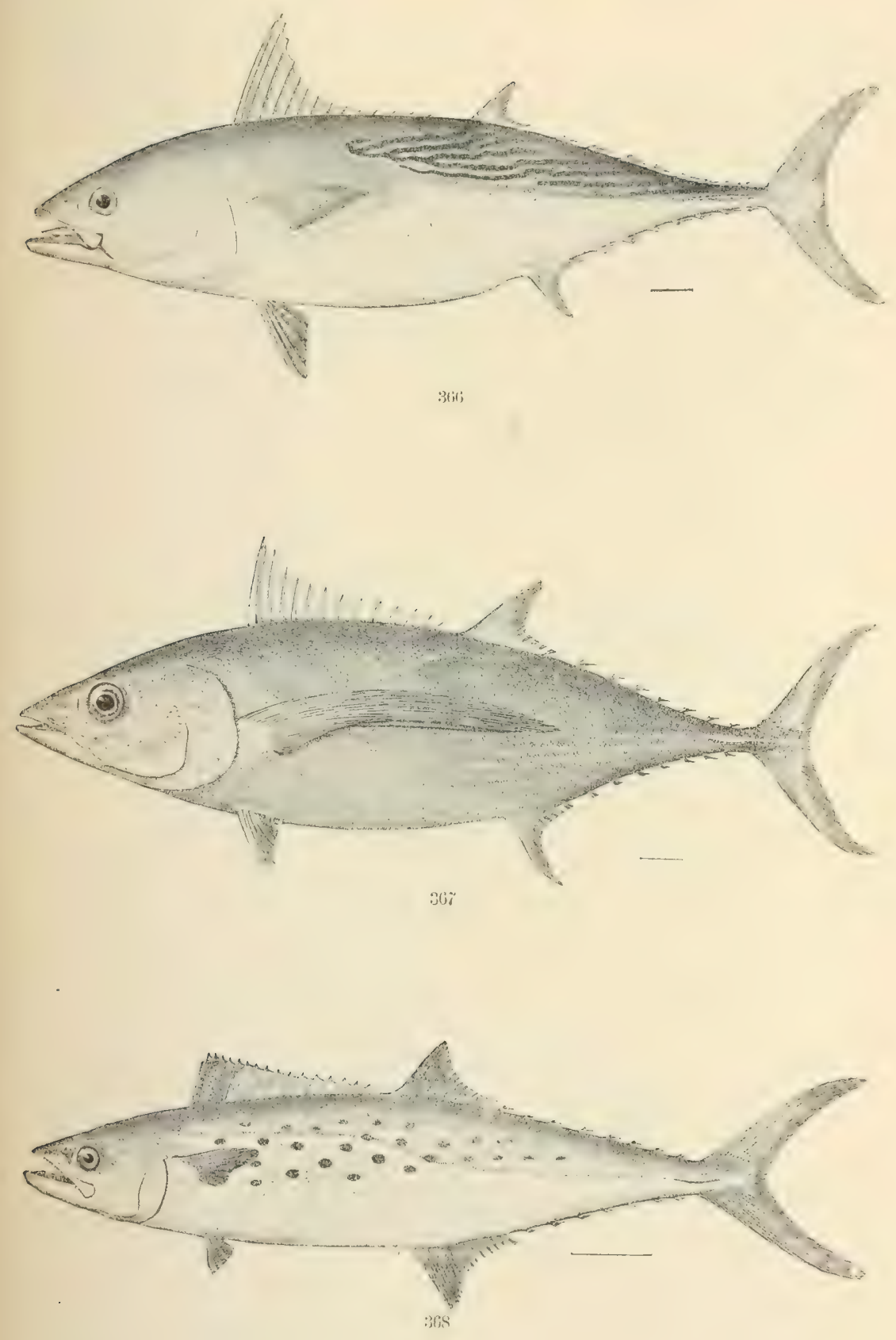

366. Gyminosaida aldeterata. (P. 869.)

367. GERMO ALALENGa. (P. 871.)

368. SCOMlizomorus MaCULATUS. (P. 874.) 



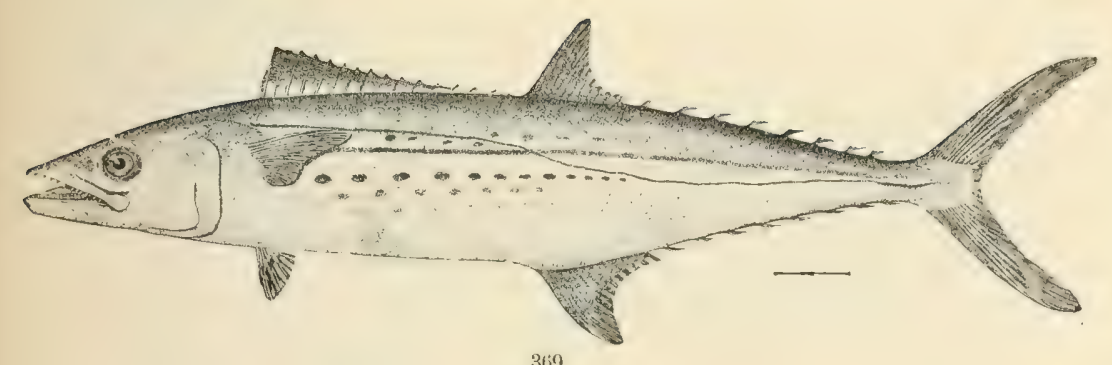

369

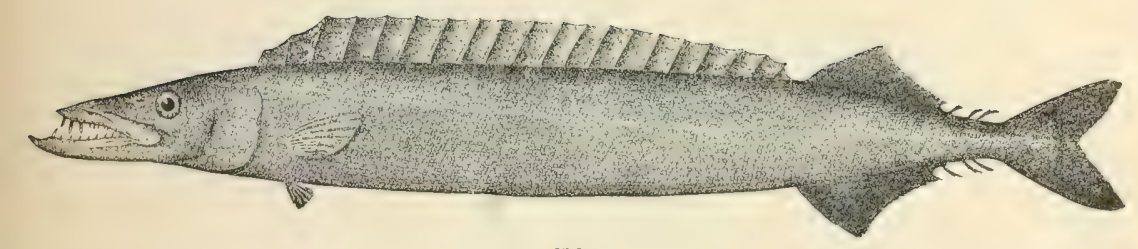

$3 r 0$

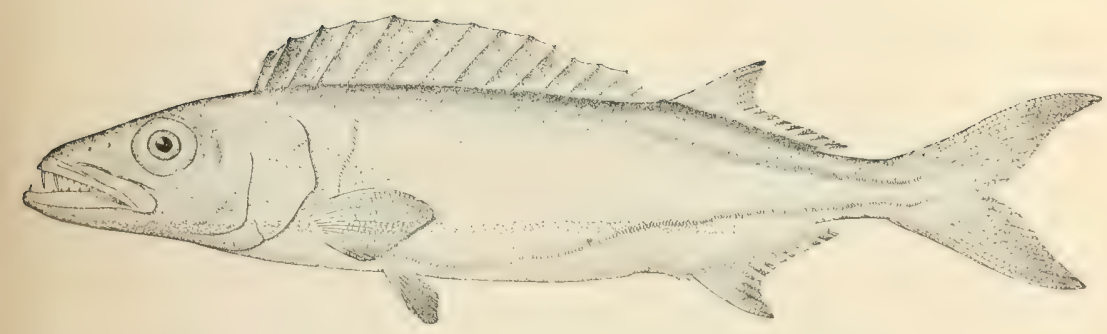

371

369. SCOMBERoMORUS REGALIS. (P. 875.)

370. Escolar violaceUs. (Pp. 878, 2843.)

371. Epinnula magistralis。(P.880.) 



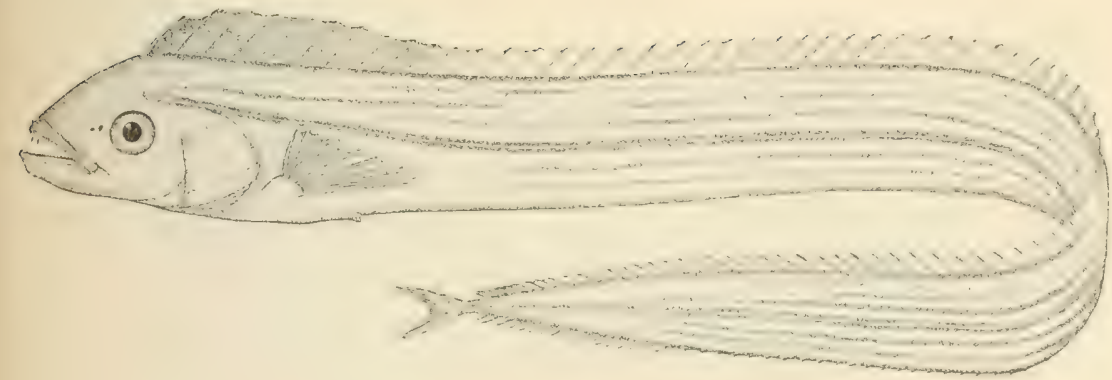

$3 \%:$

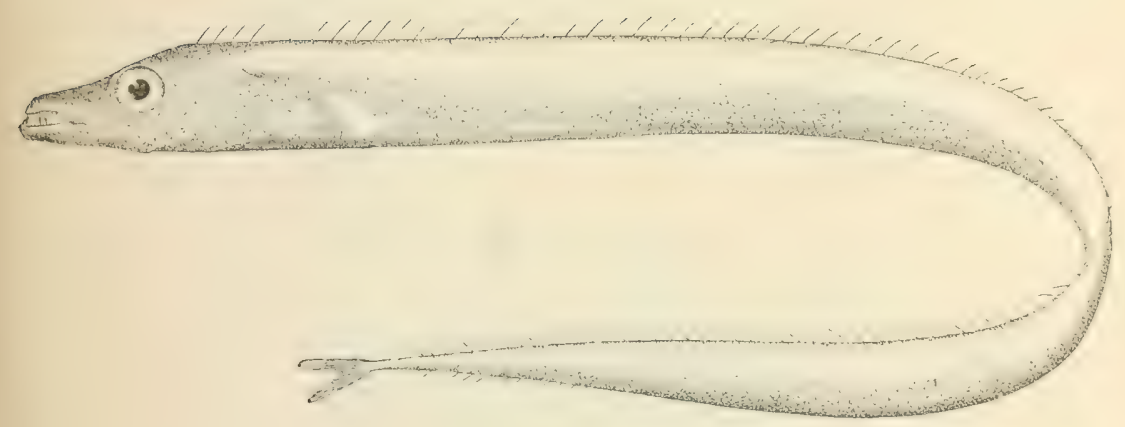

$\because: \cdots: 3$

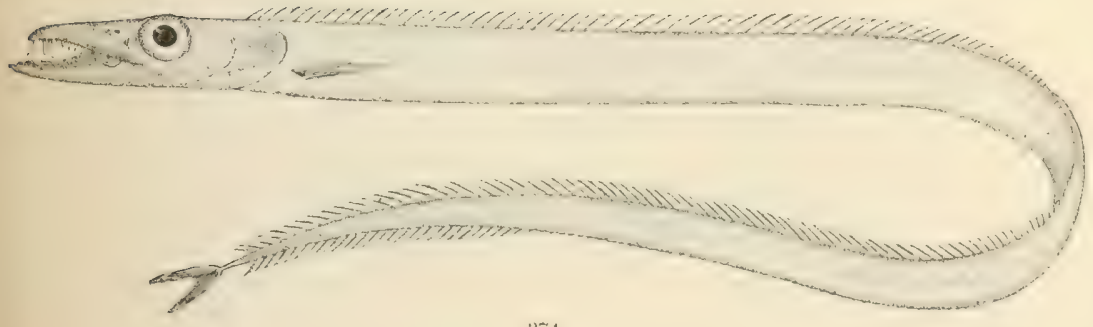

3it

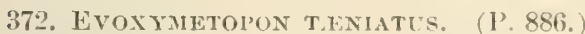

373. LePIDOPUS CAUDATUS. (P.886.)

374. BENTHODESII'S ATIANTICL' (P. 887.) 



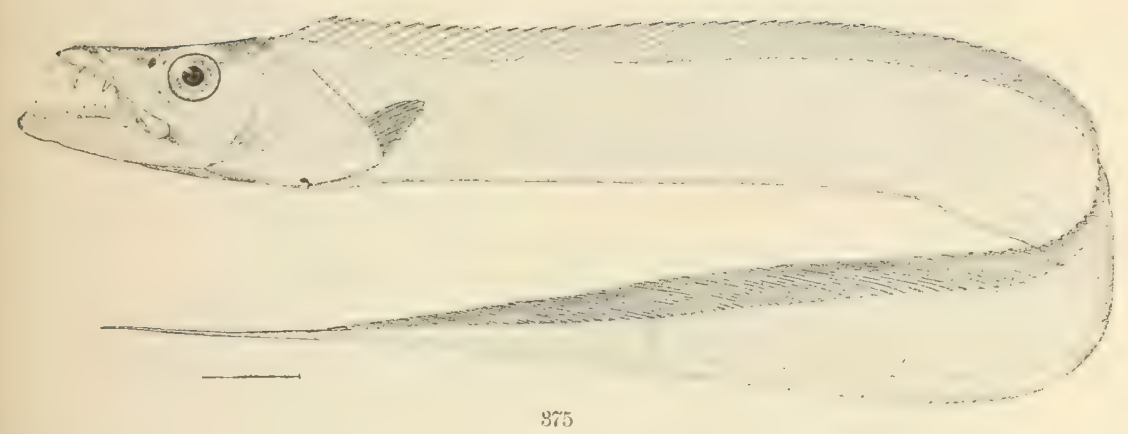

$8 \% 5$

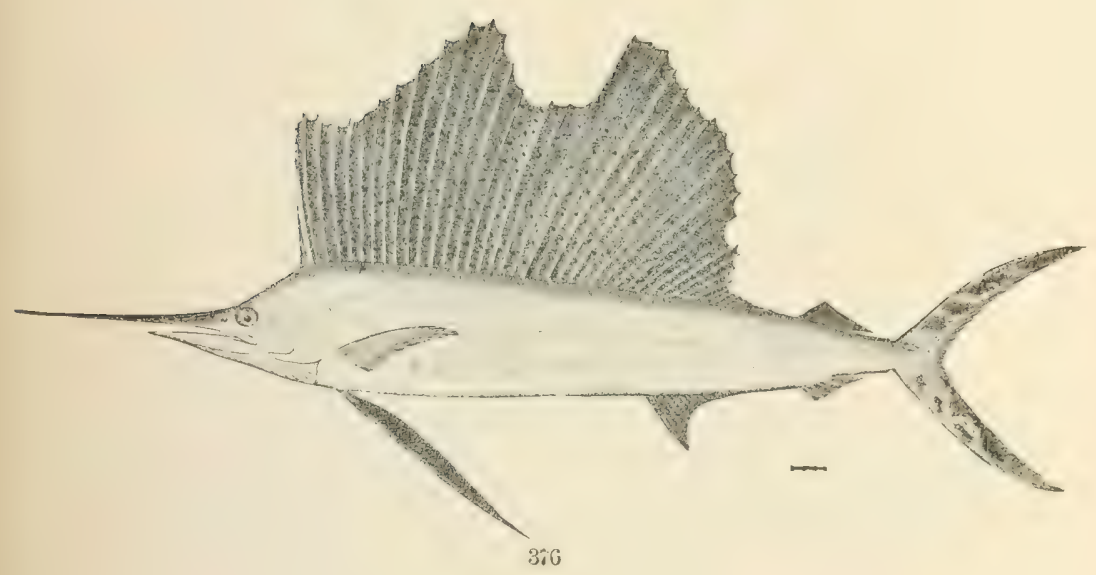

375. Trichurus lepturus. (P. 889.)

376. ISTIOPHORUS NIGRICANS. (P. 891.) 



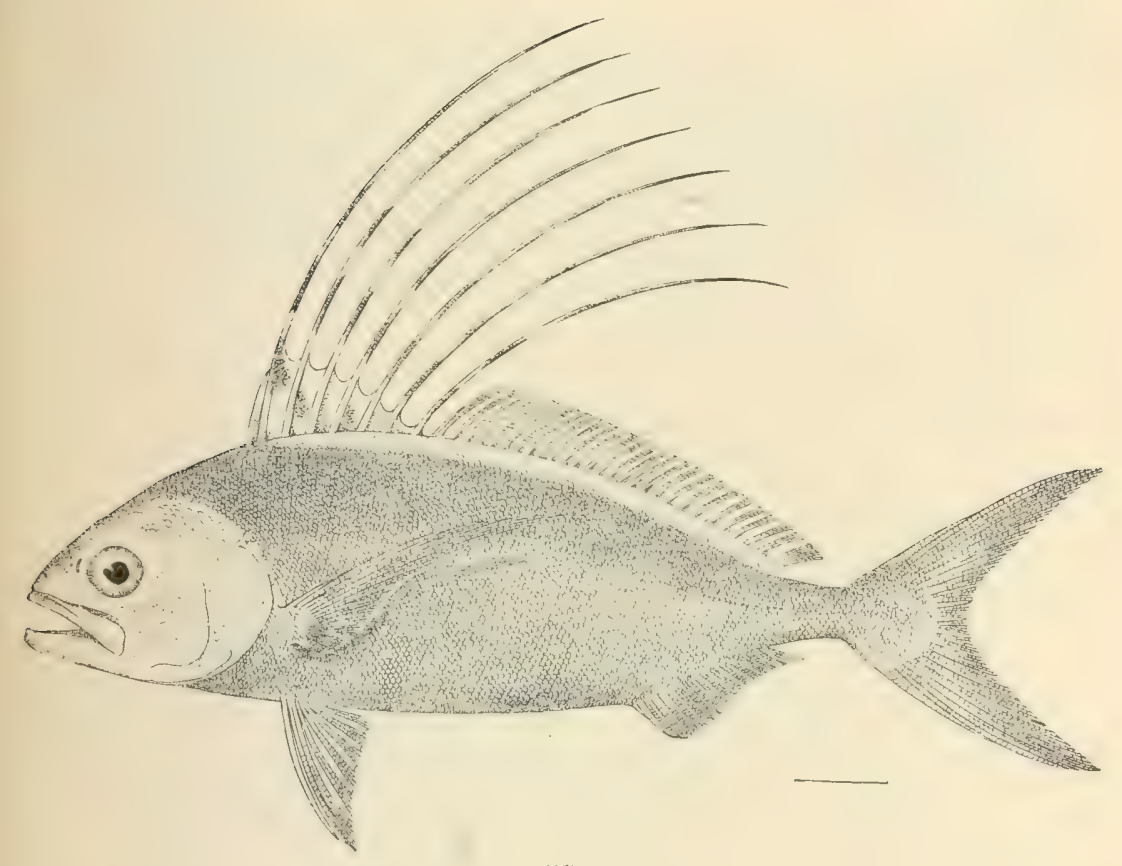

$3 i \overline{1}$

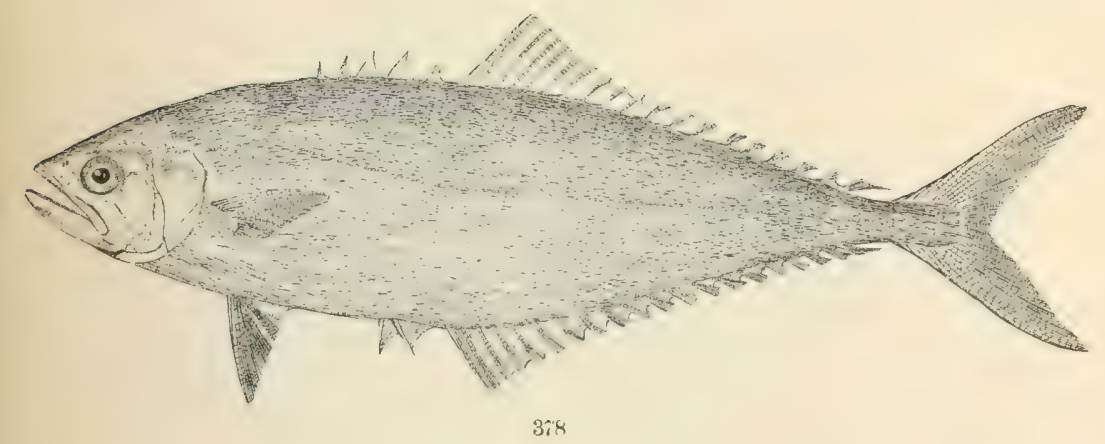

377. Nematistius Pectoralis. (P. 895.)

378. Oligoplites saurus. (P. 898.) 





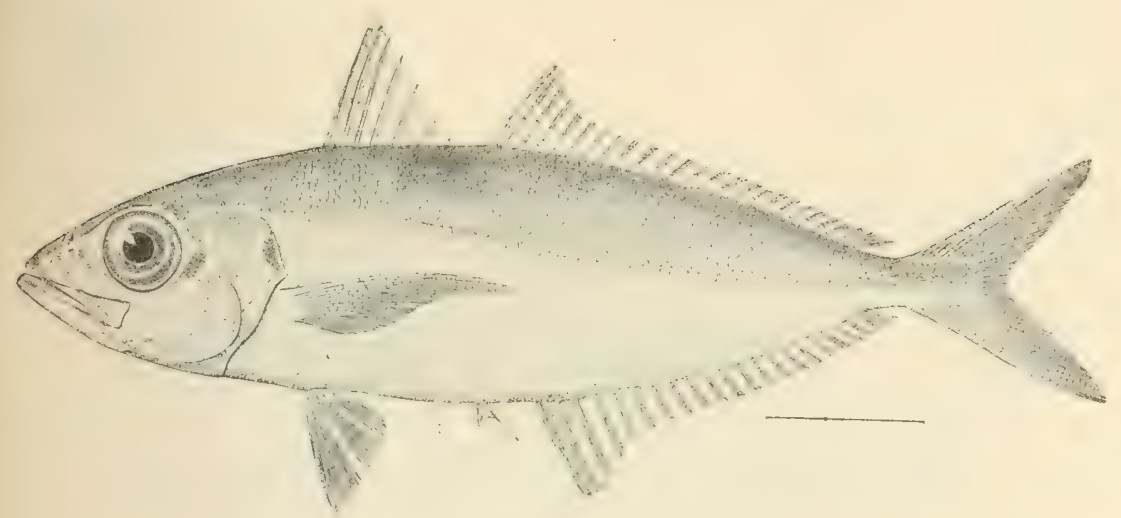

$3 \cdot 5$

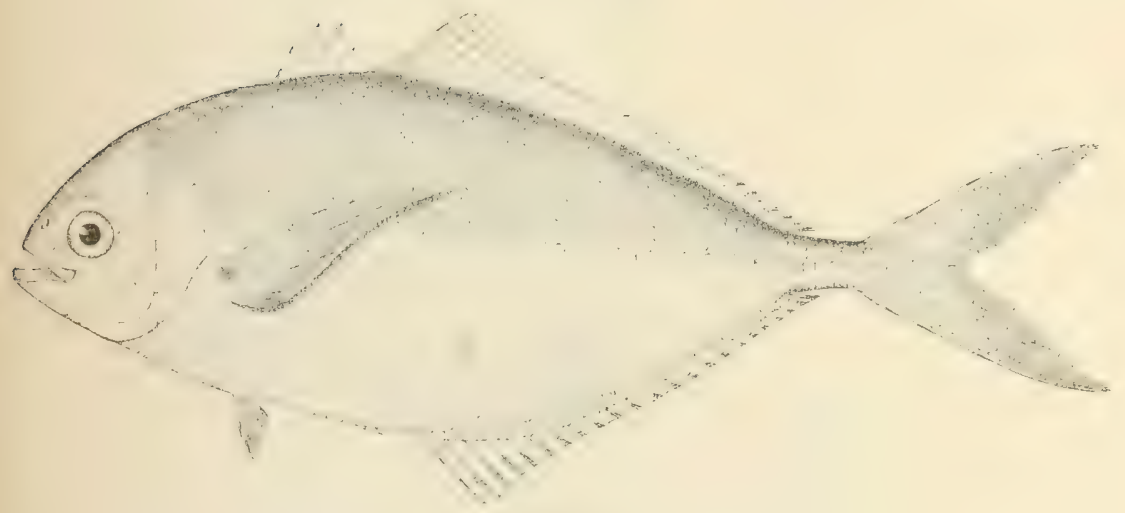

$3 \times 6$

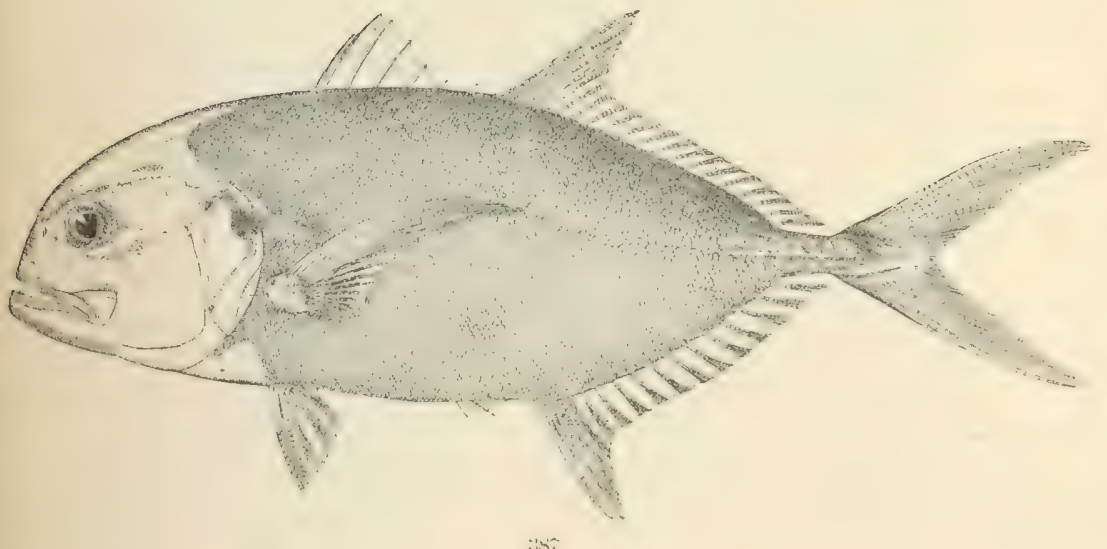

385. Trachirols Clemenom HTHAMUS. (P. 911.)

386. HWMICARANX AMBLTRHNCHUS. (P. 912.)

387. Carani iliplos. (I. 920.) 


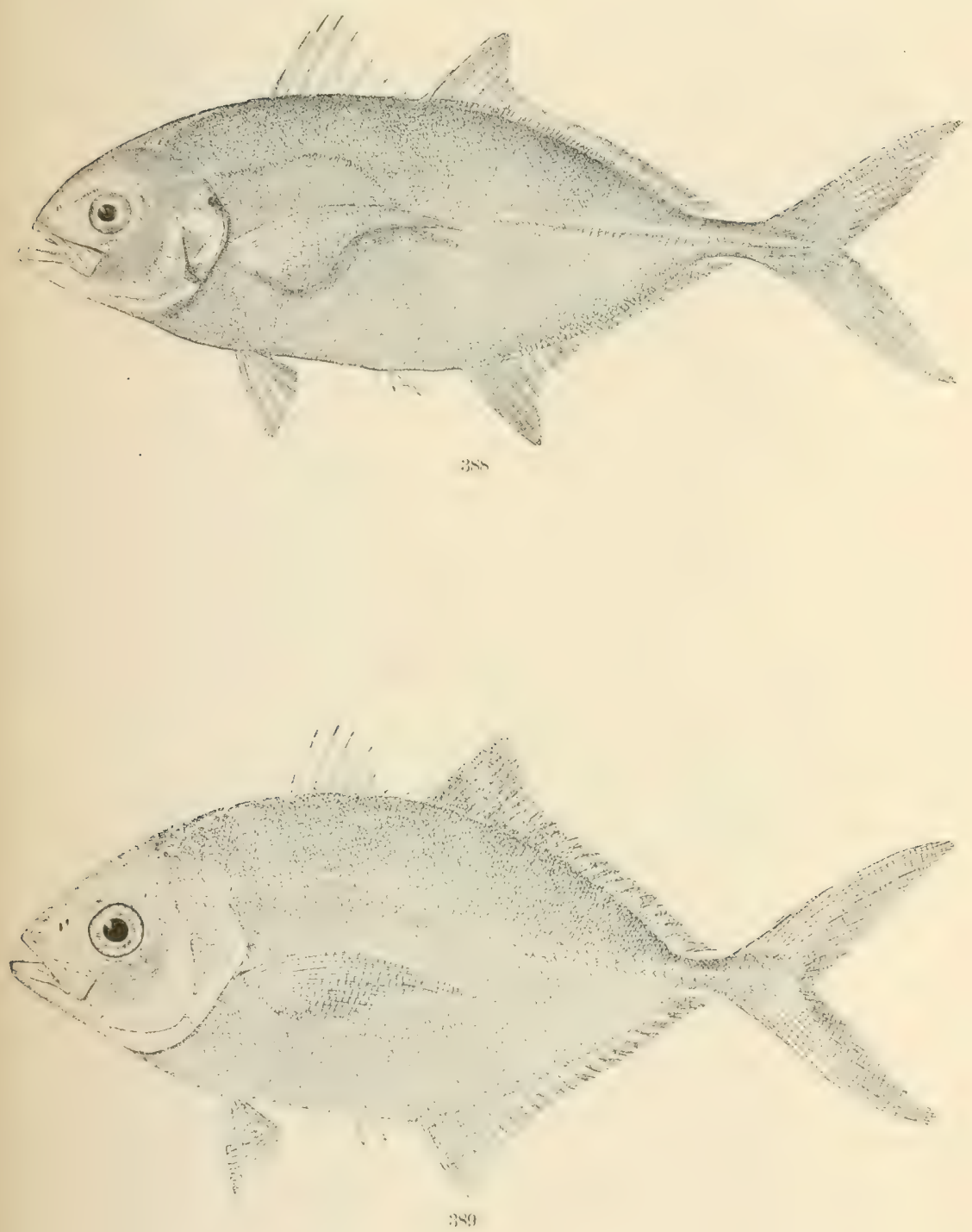

388. Calianx crysos. (P. 921.)

889. Carand late's, (P. 923.) 



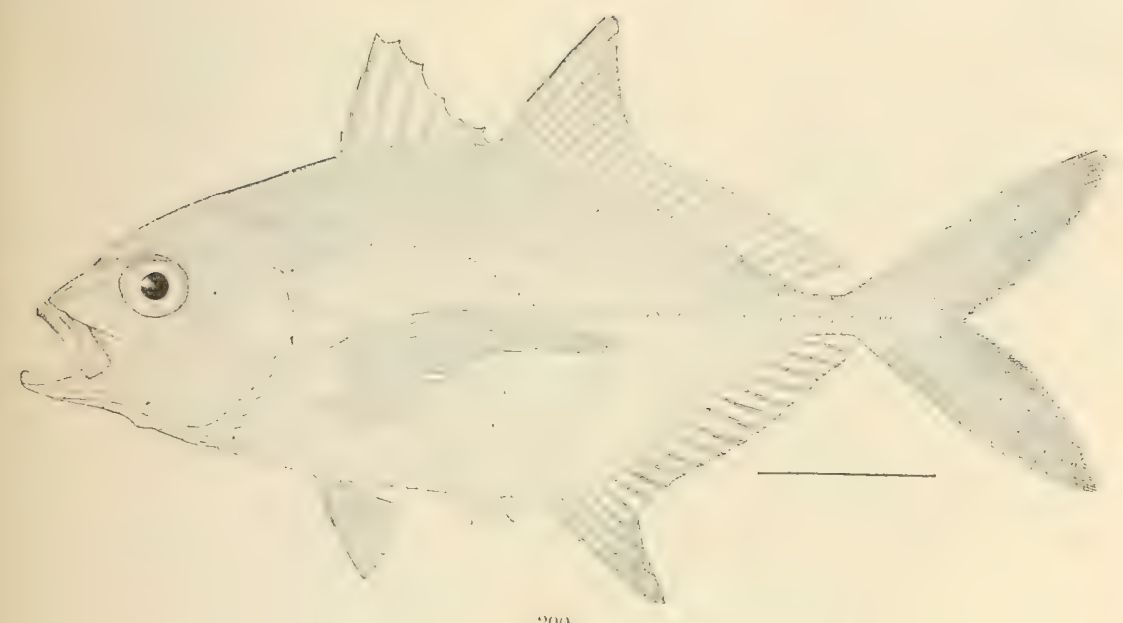

;) (I)

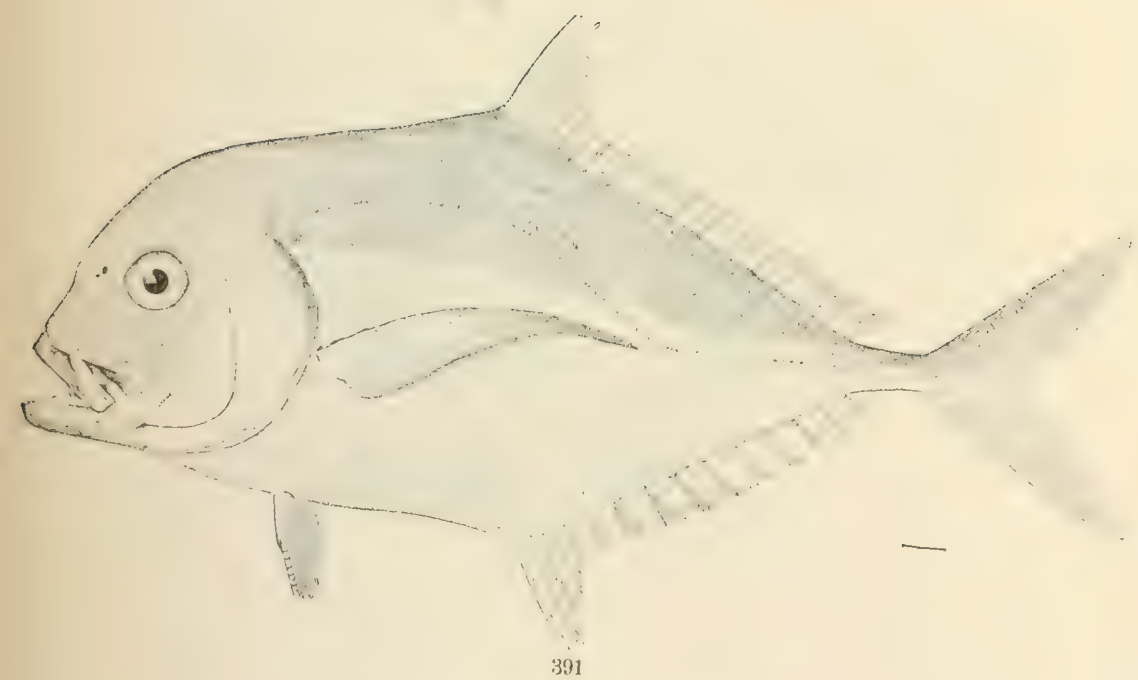

390. Caranx medusicola. (P. 924.)

391. HYNAIS HOPKINSI. (P. 933.) 



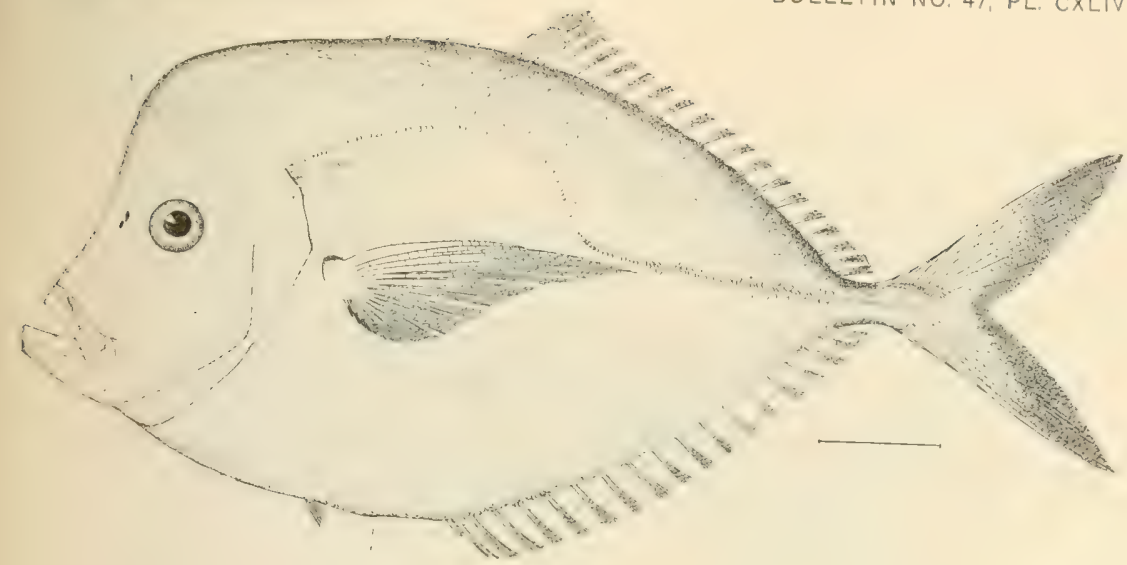

392

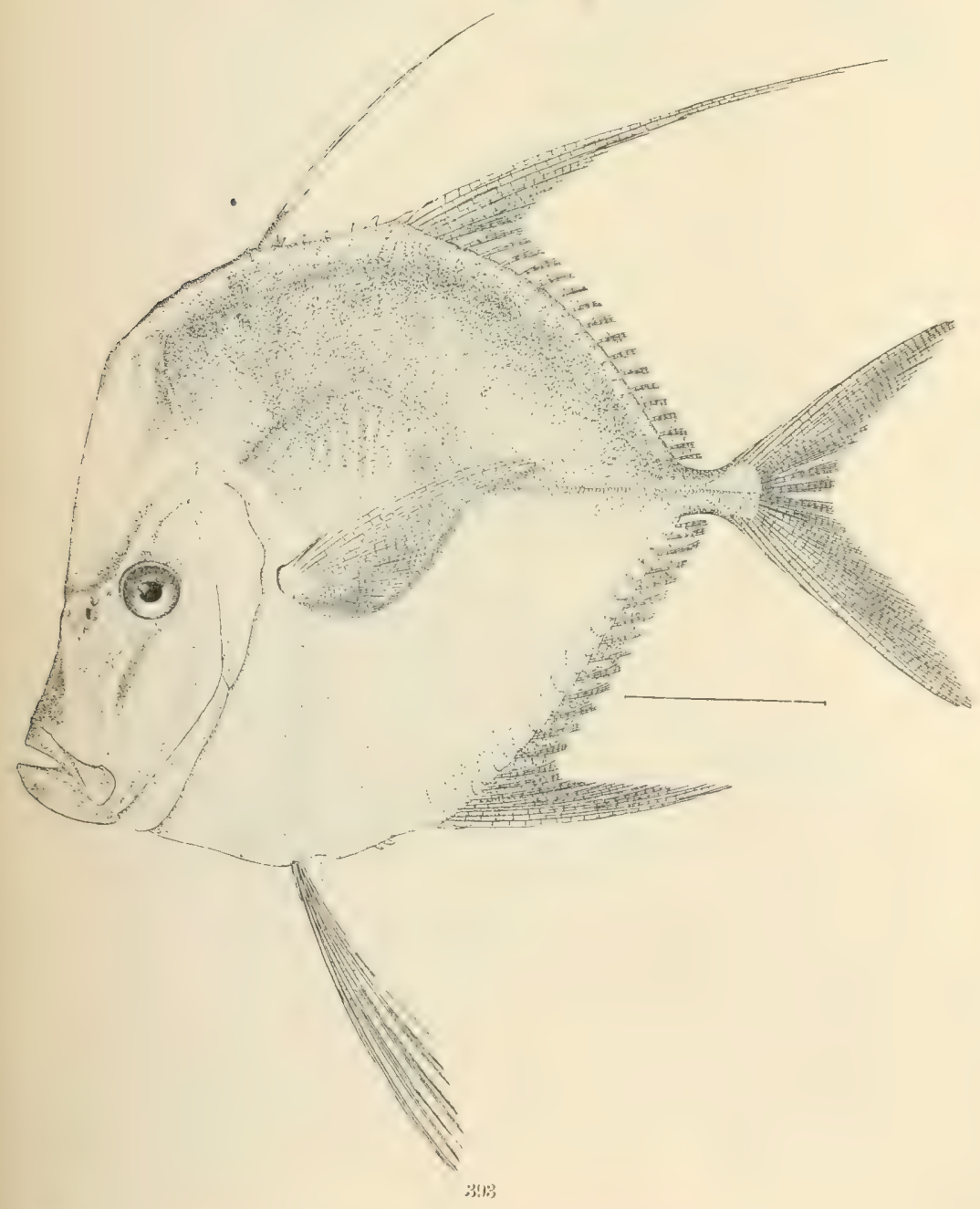

392. Vomer sethrixis. (I'. 934.)

393. SELENE VOMER; young. (1',936.) 


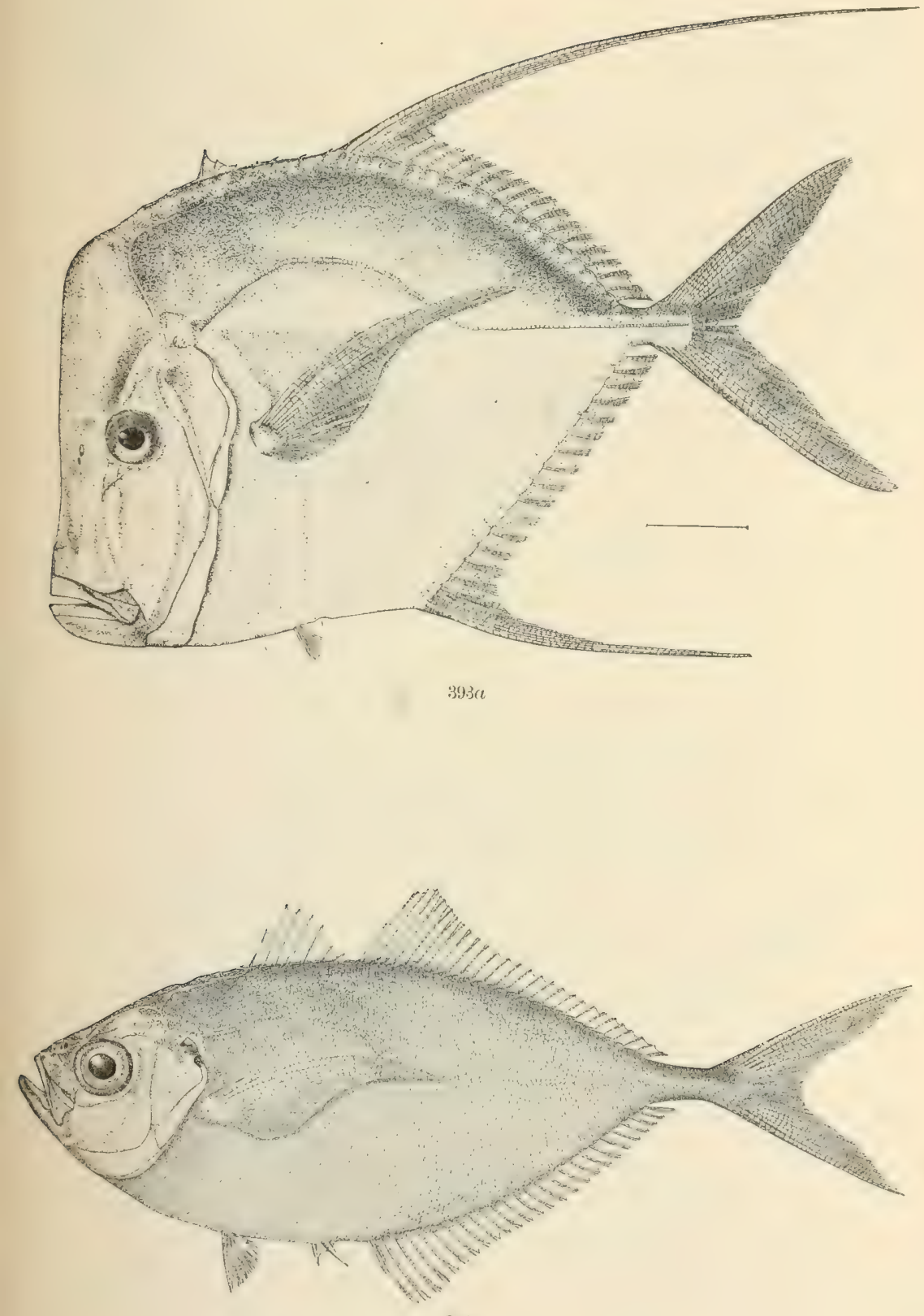

391

393a. Selene vomer; adult. (P. 936.)

394. Chroroscombus chrysurus. (P. 938.) 


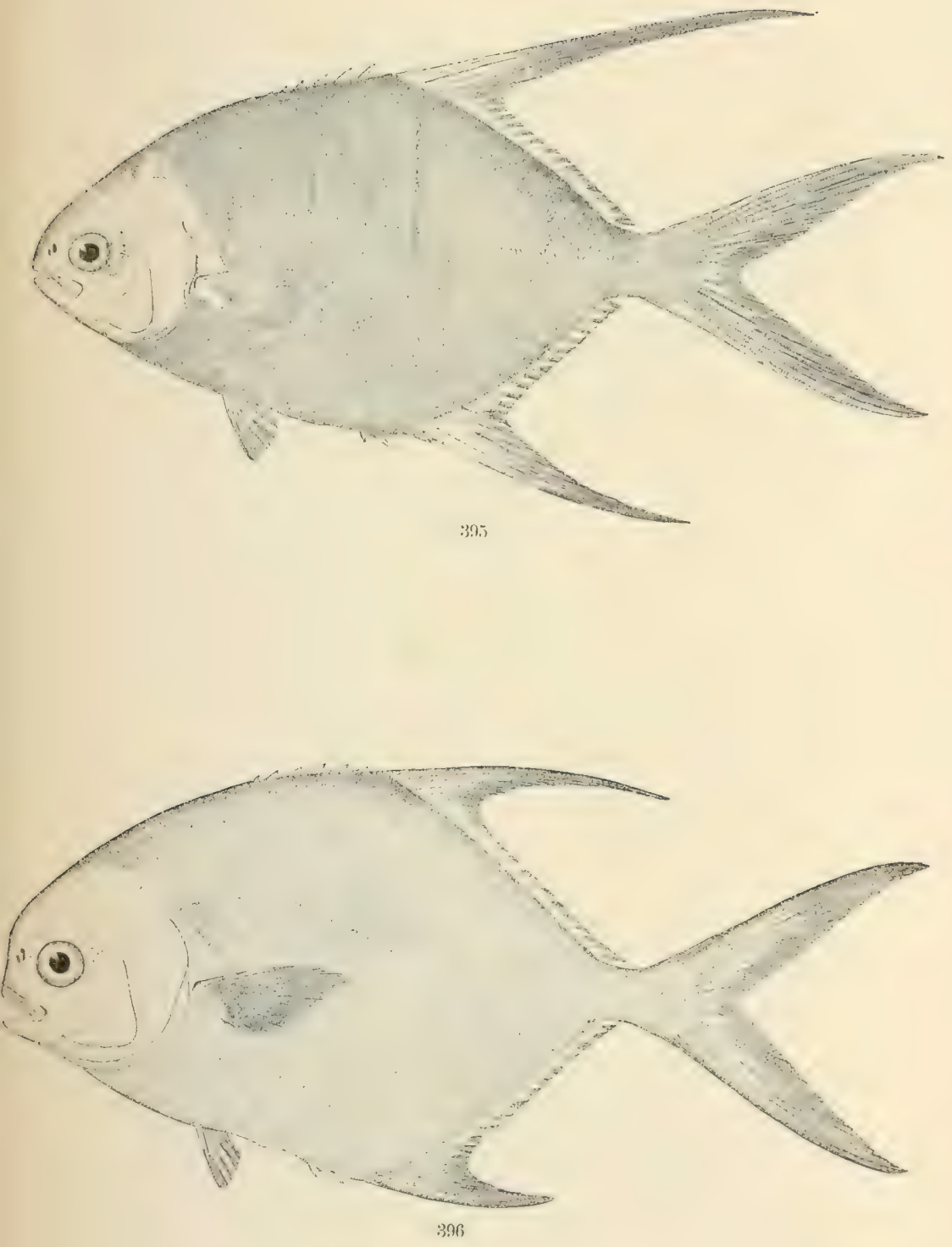

395. Trachicotus graucus. (P. 940.)

396. 'Trachinotus Falcatus. (P. 911 .) 


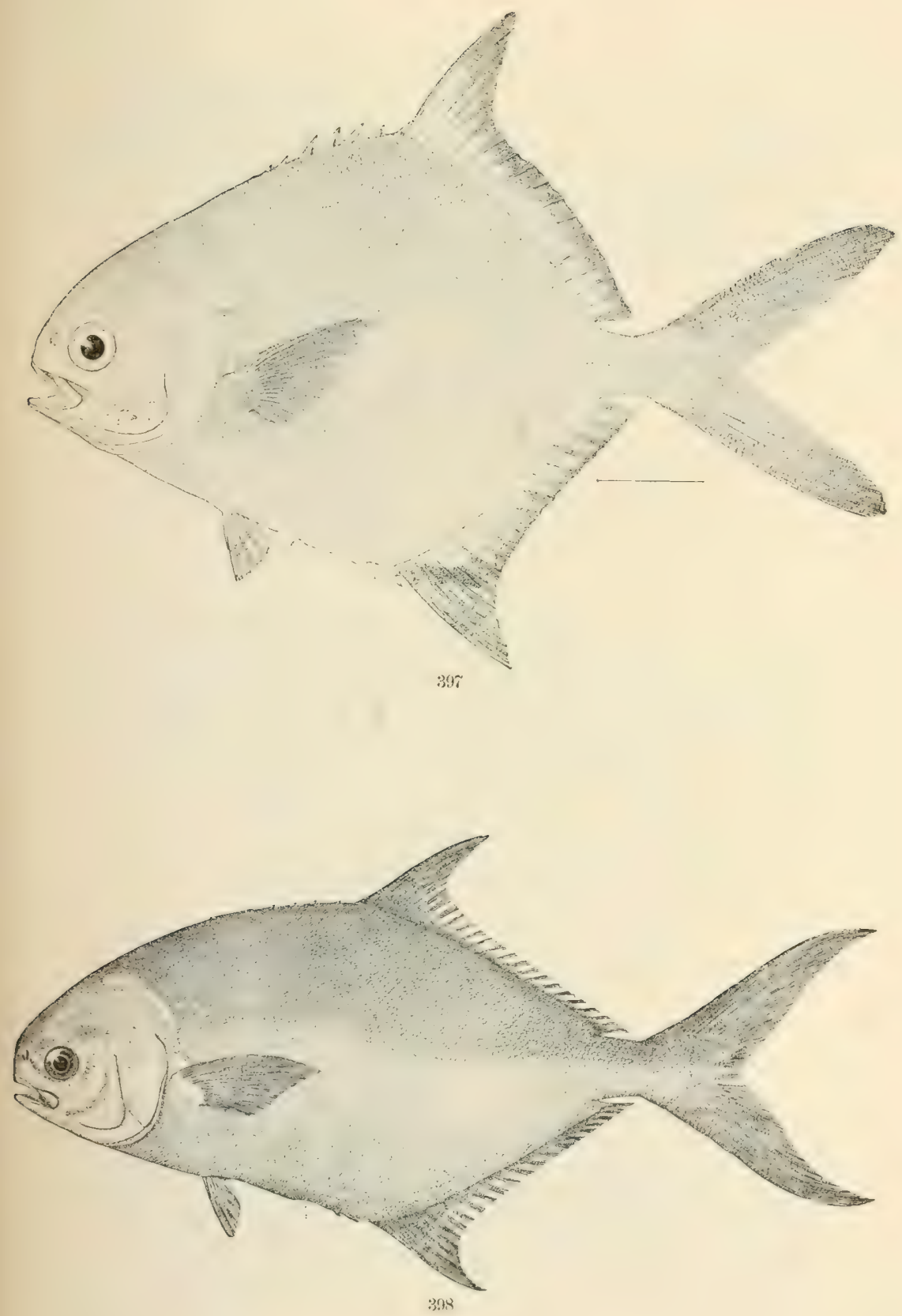

397. 'Trachixotus culveli. (P. 942.)

398. 'Trachinotus carolinus. (P. 944.) 


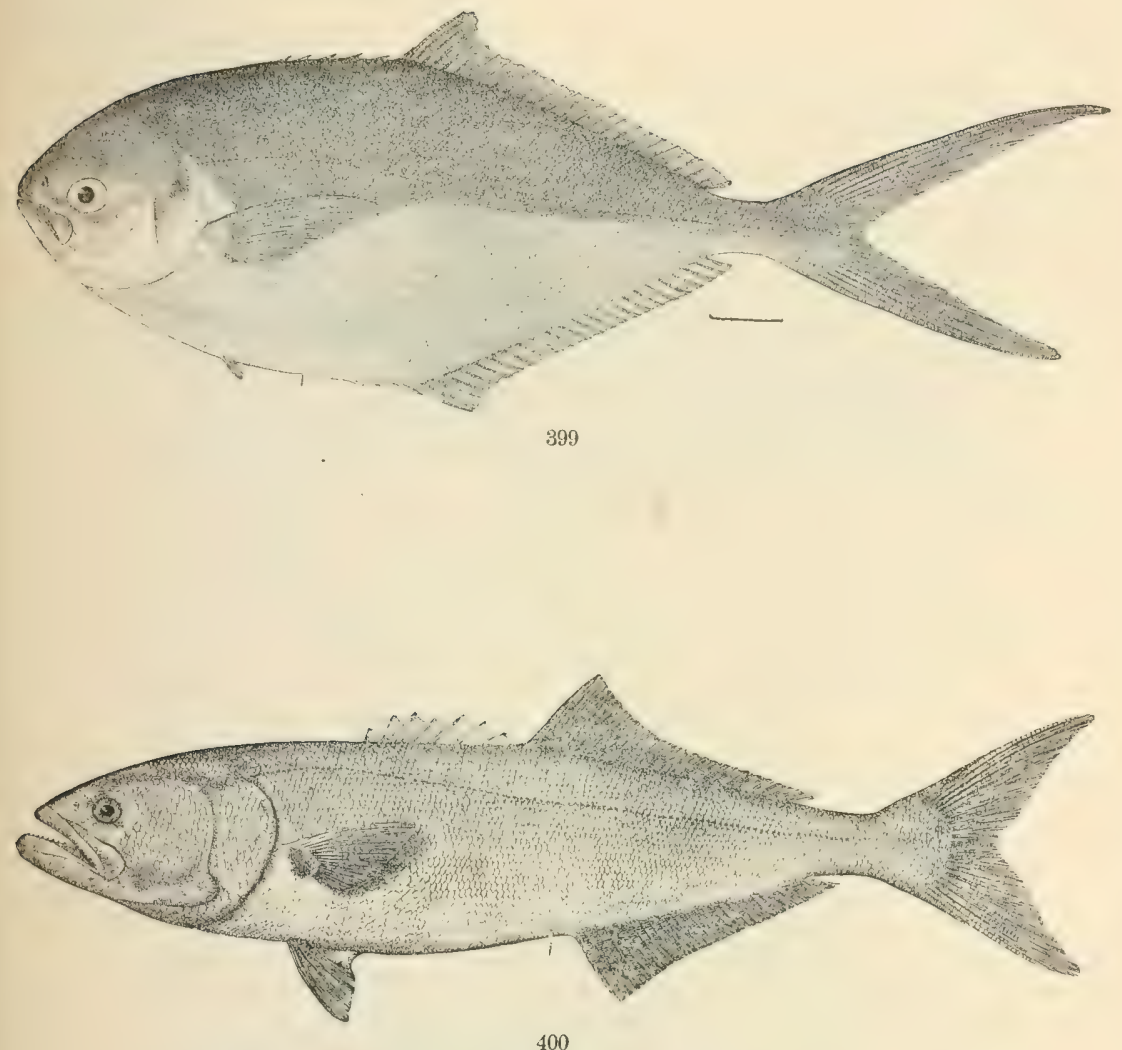

400

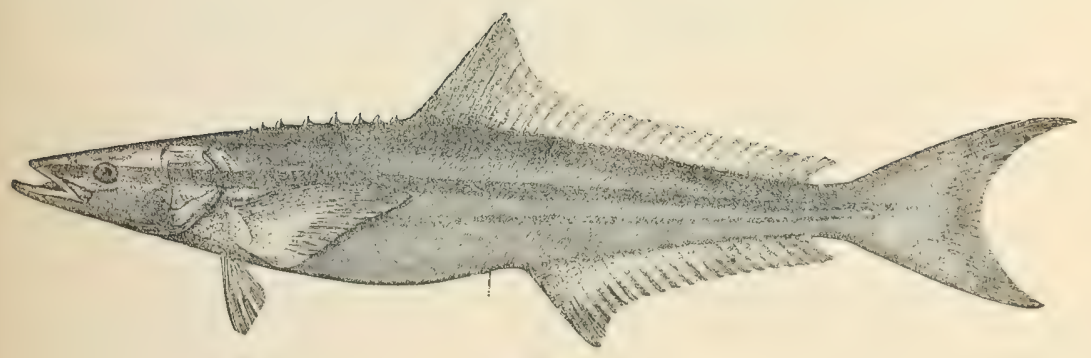

401

399. ZALOCYS STILRE. (P. 2848.)

400. Pomatomus saltatrix. (P. 946.)

401. RaChYCENTRON CANADUS. (P.948.) 


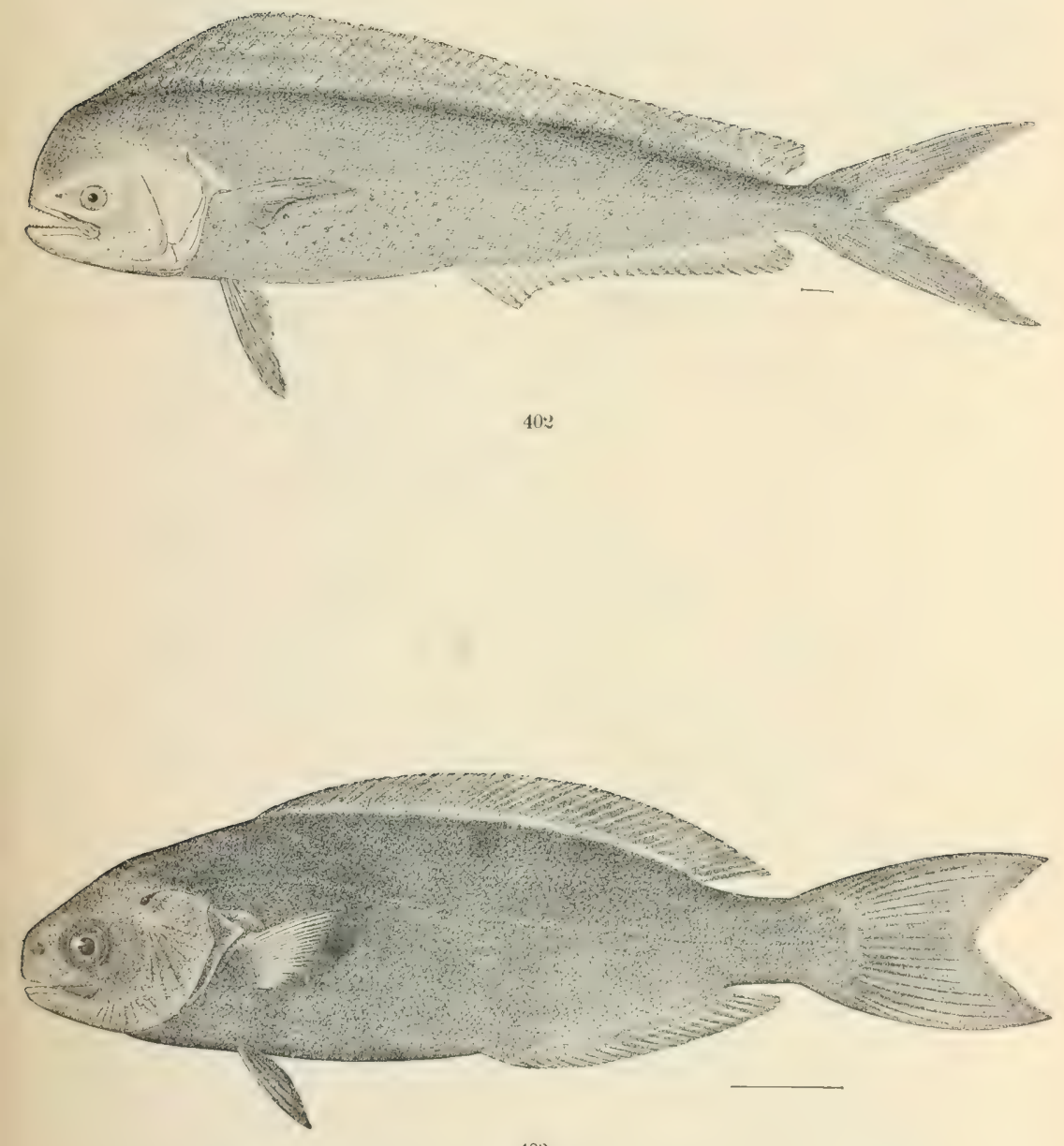

403

402. Coryphend hippurus. (P. 952.)

403. Centrolopilus niger. (P.963.) 


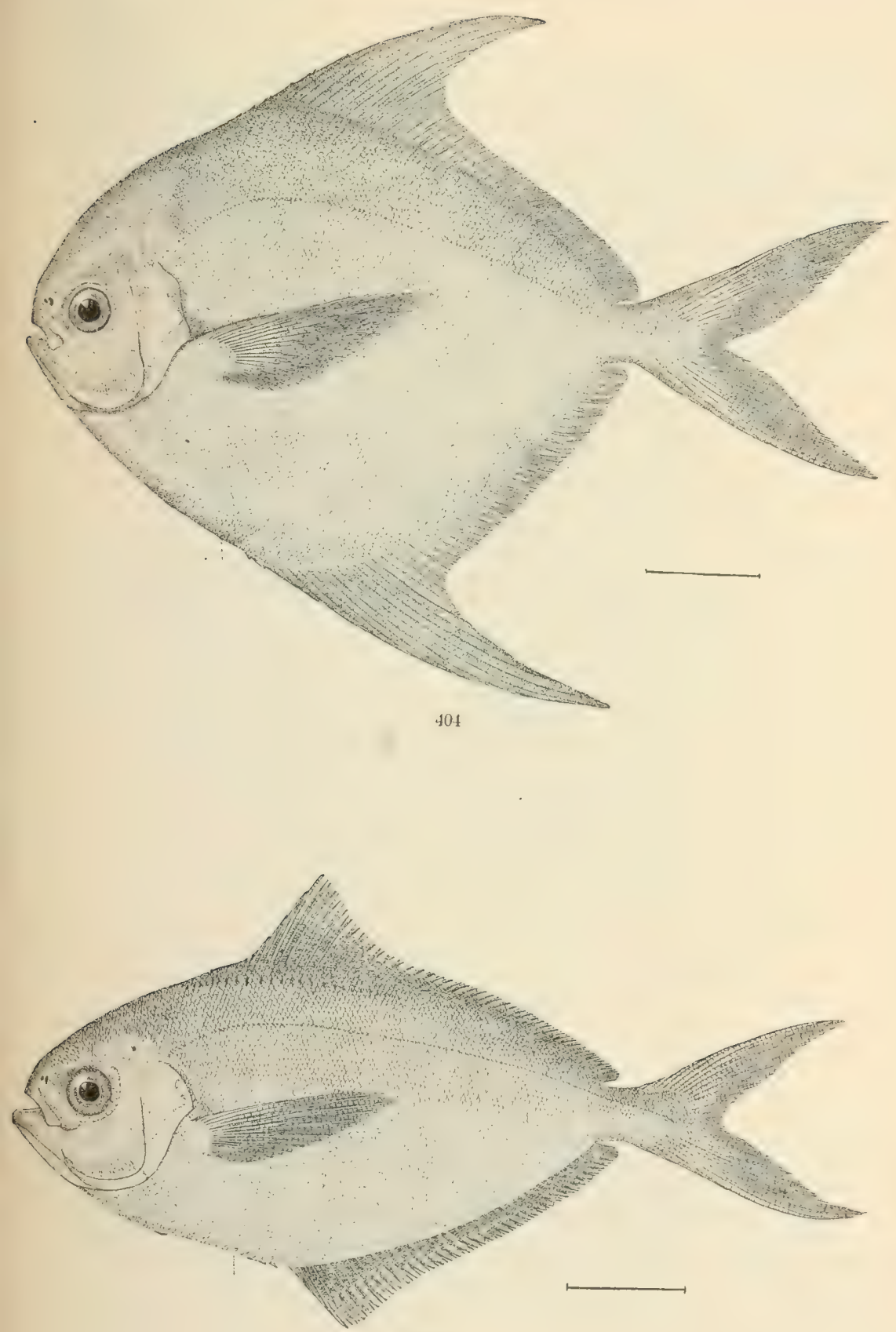

405

404. Rhomizus PARU. (P. 965.)

405. Poronotes triacantuls. (Pp. 967, 2819.) 



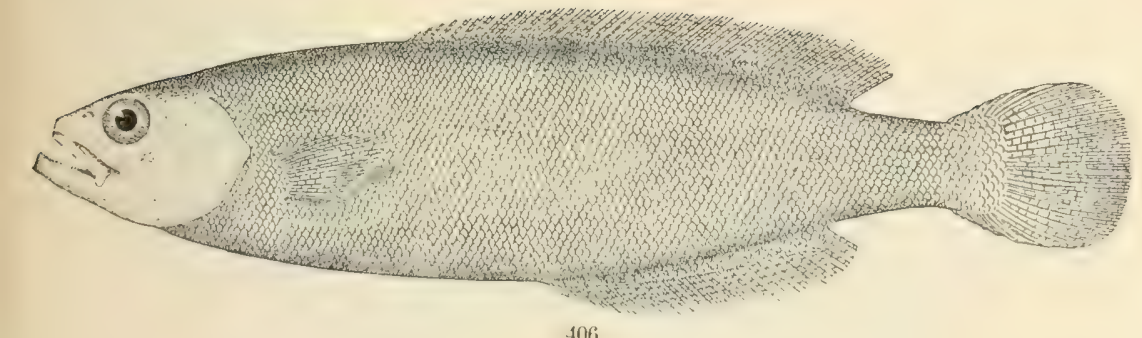

406

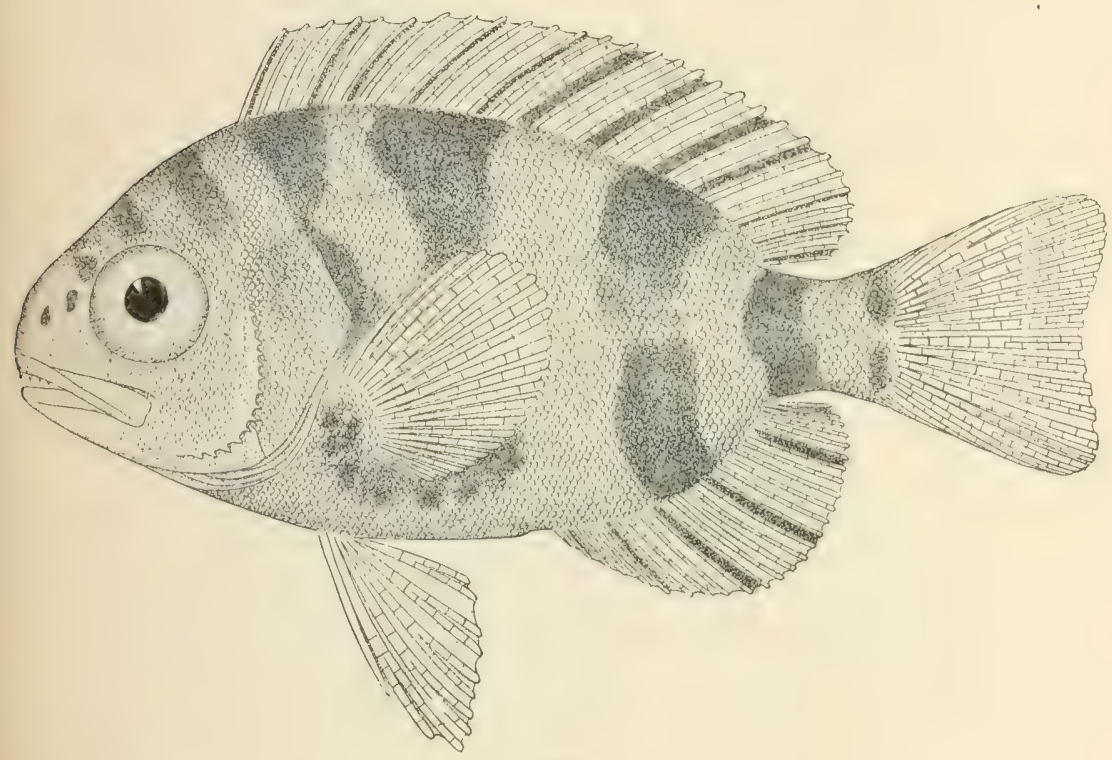

$40 \%$

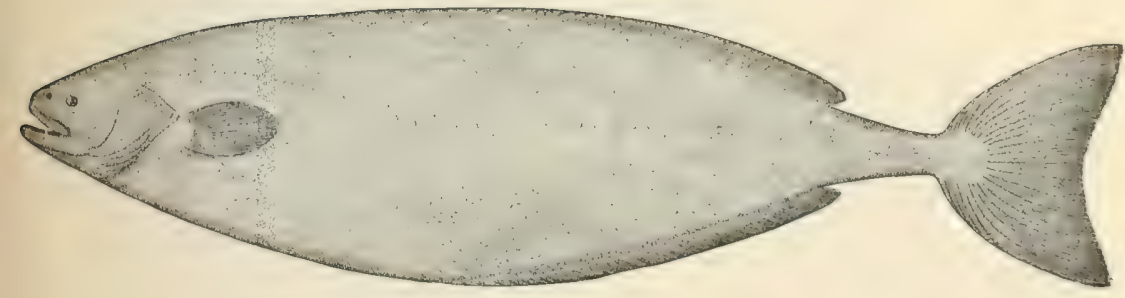

408

406. ICICHTHYA LOCKINGTONI. (P. 969.)

407. SCHEDOPIILUS MHDUsophaGC's. (P. 970.)

408. ACRotes WillouginY. (P. 973.) 




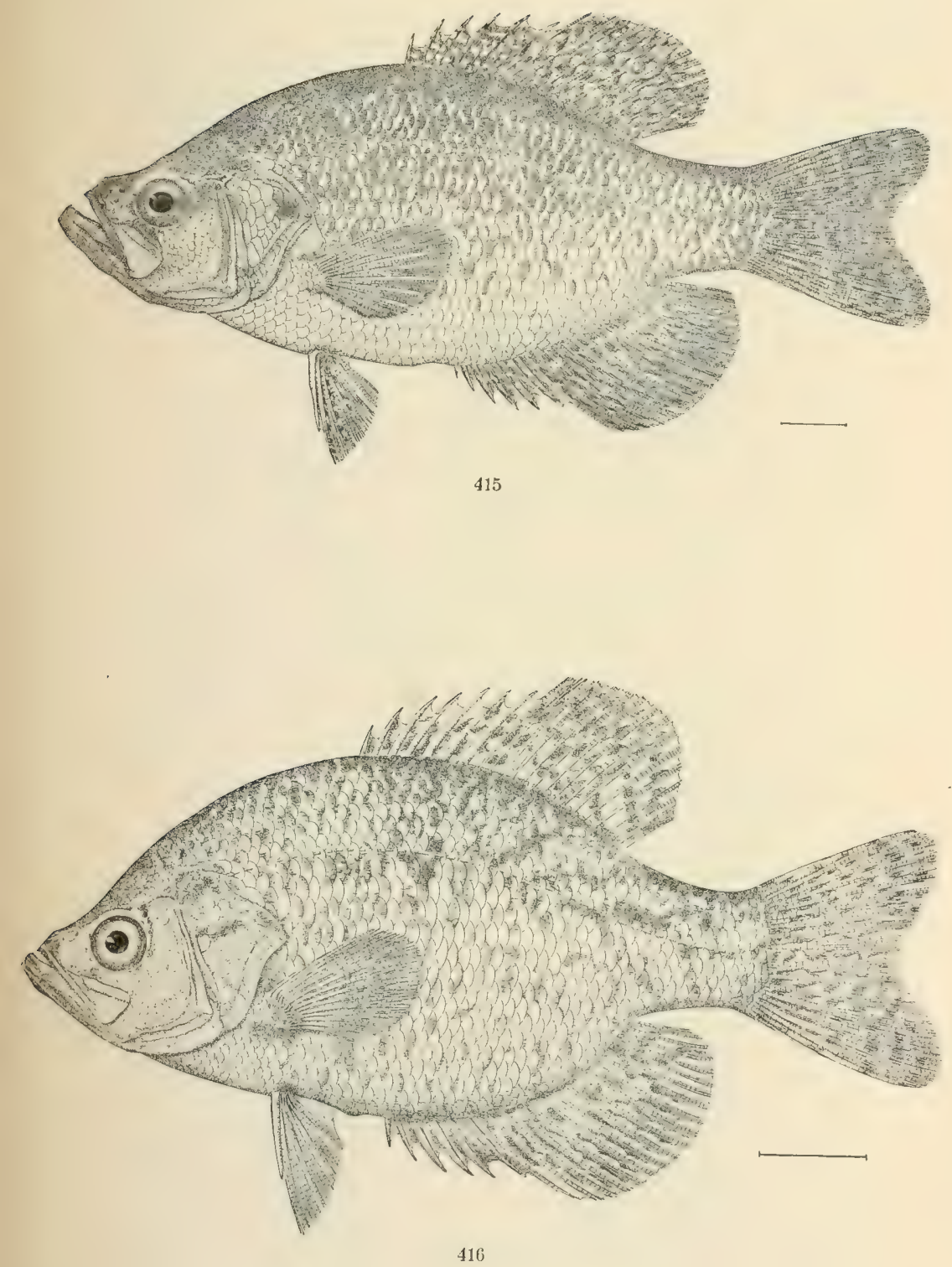

415. Pomoxis annularis. (P. 987.)

416. Pomoxis SParoides, (P.987.) 



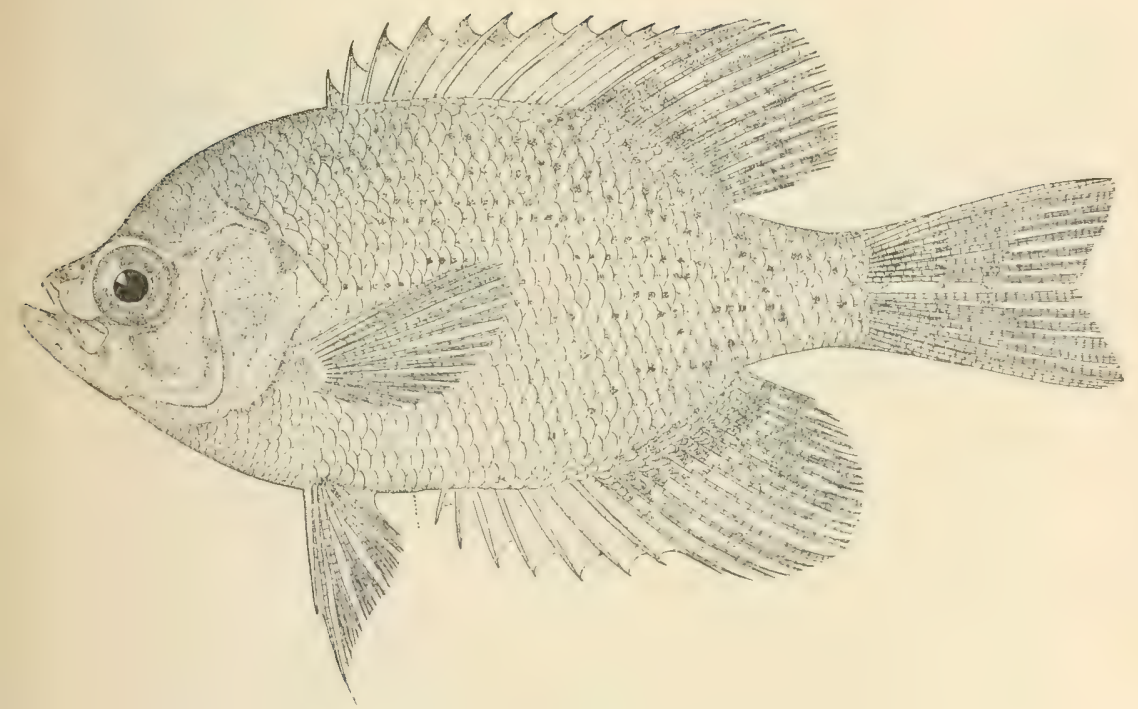

417

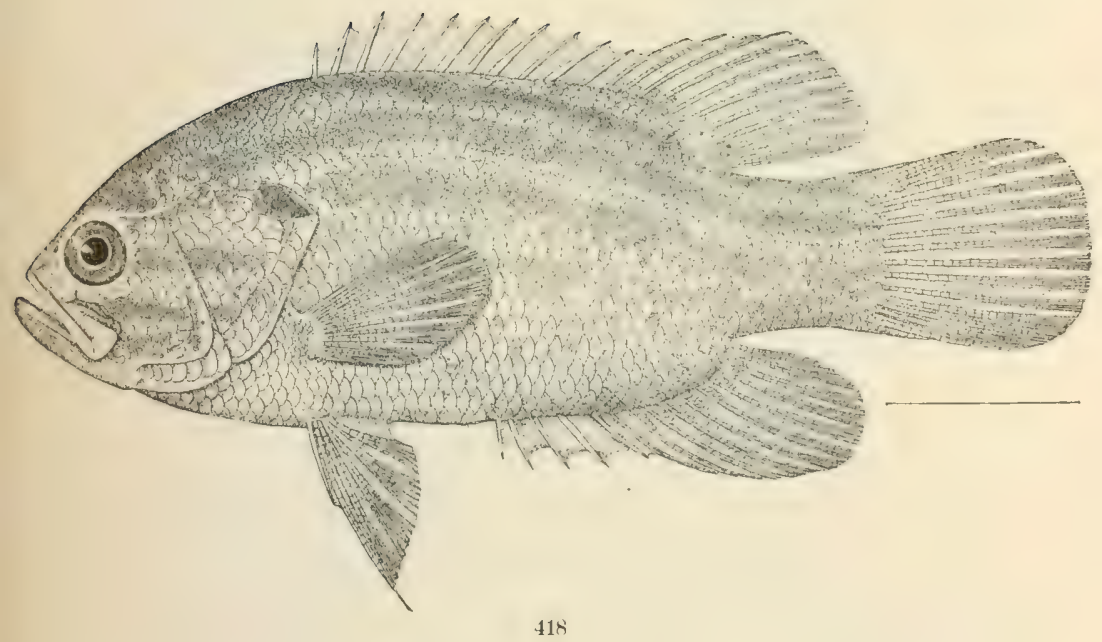

417. Centrarchus macropterus. (P. 988.)

418. Acantilarchus pomotis. (P.989.) 



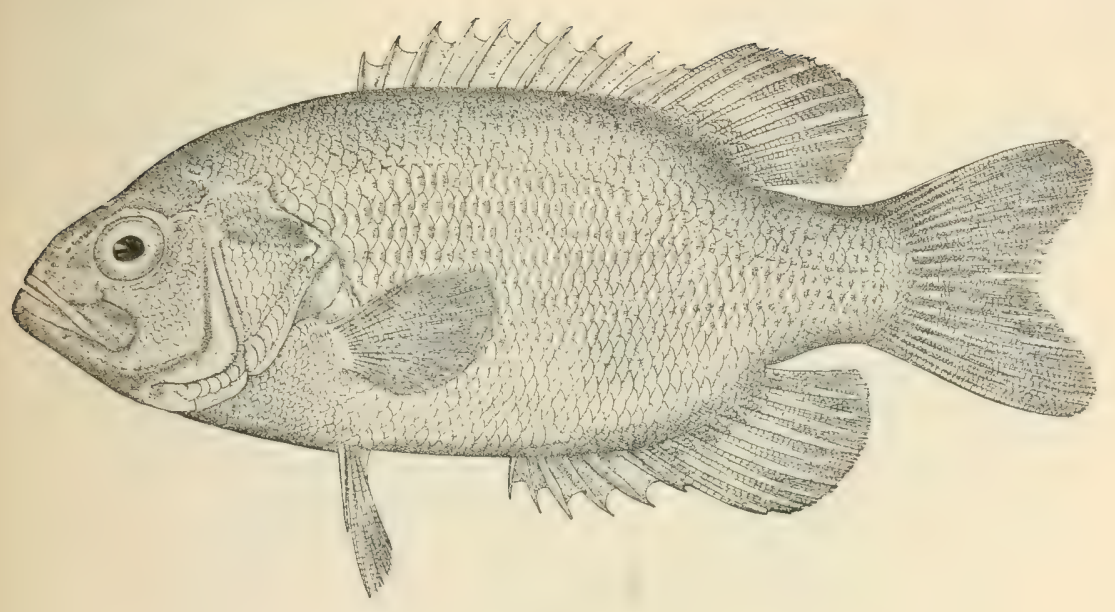

419

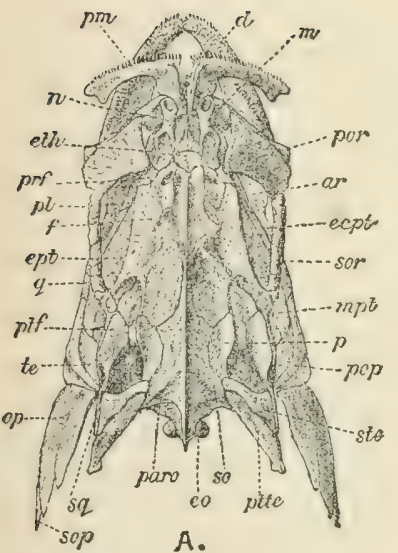

A.
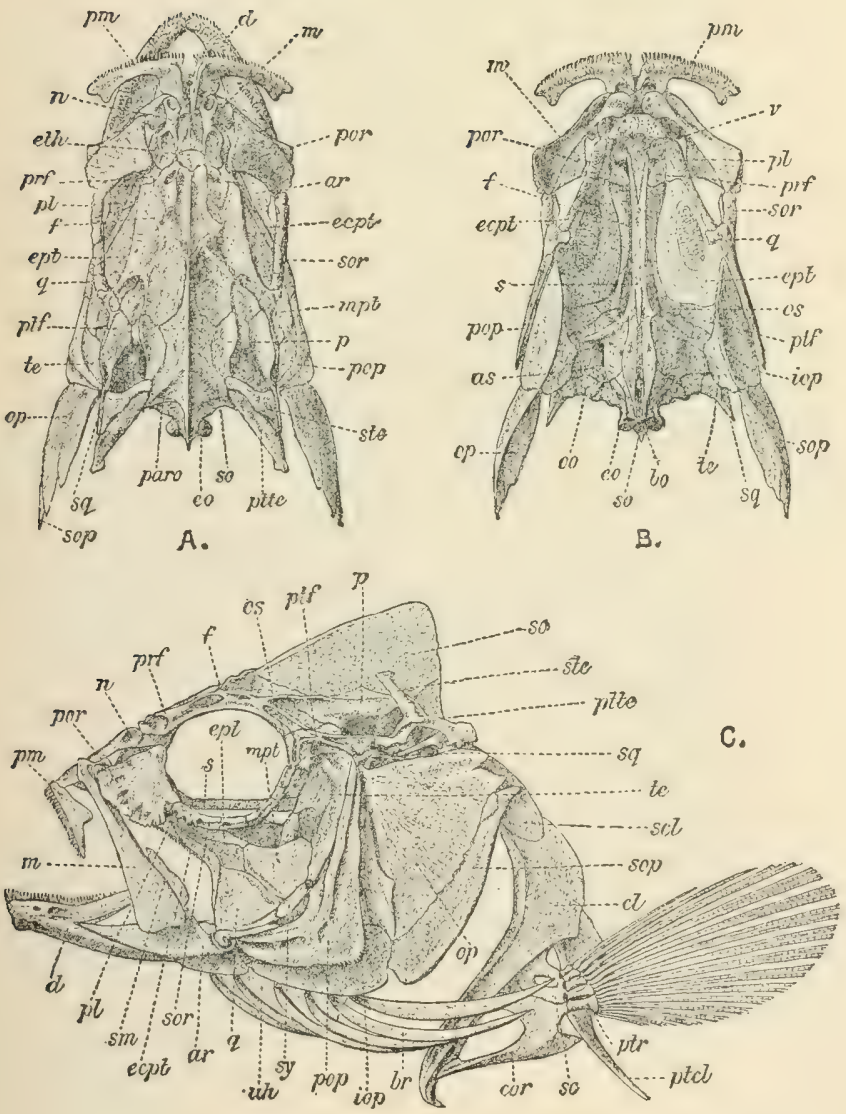

419. Ambloplites rupestris. (P. 990.)

A, $B$, C. SKull OF AMbloplites RUPestris. (P. 990.) 


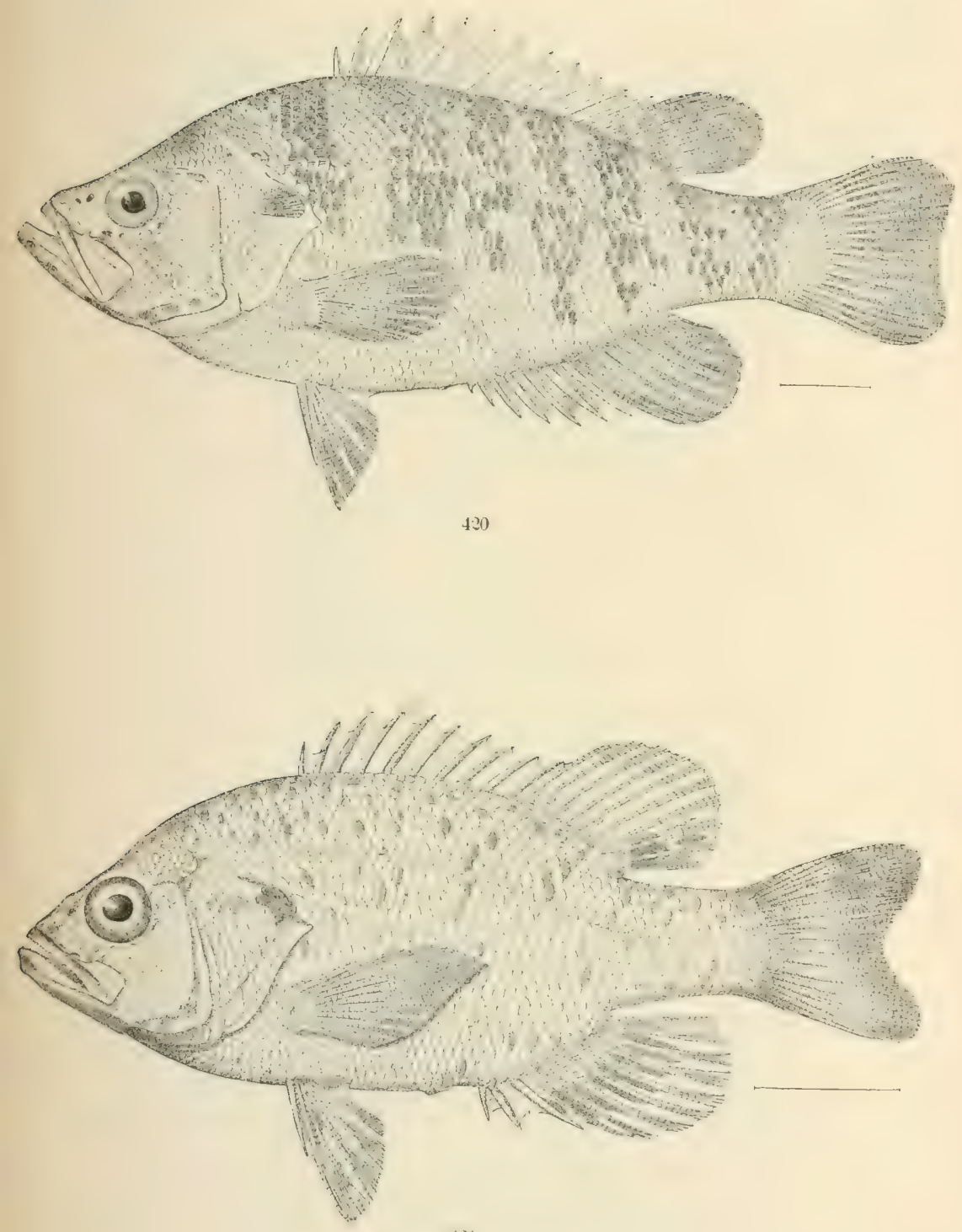

$4 \approx 1$

420. Archoplites interiuptus. (P. 991.)

421. Cin enobrtttus filosus. (P. 992.) 



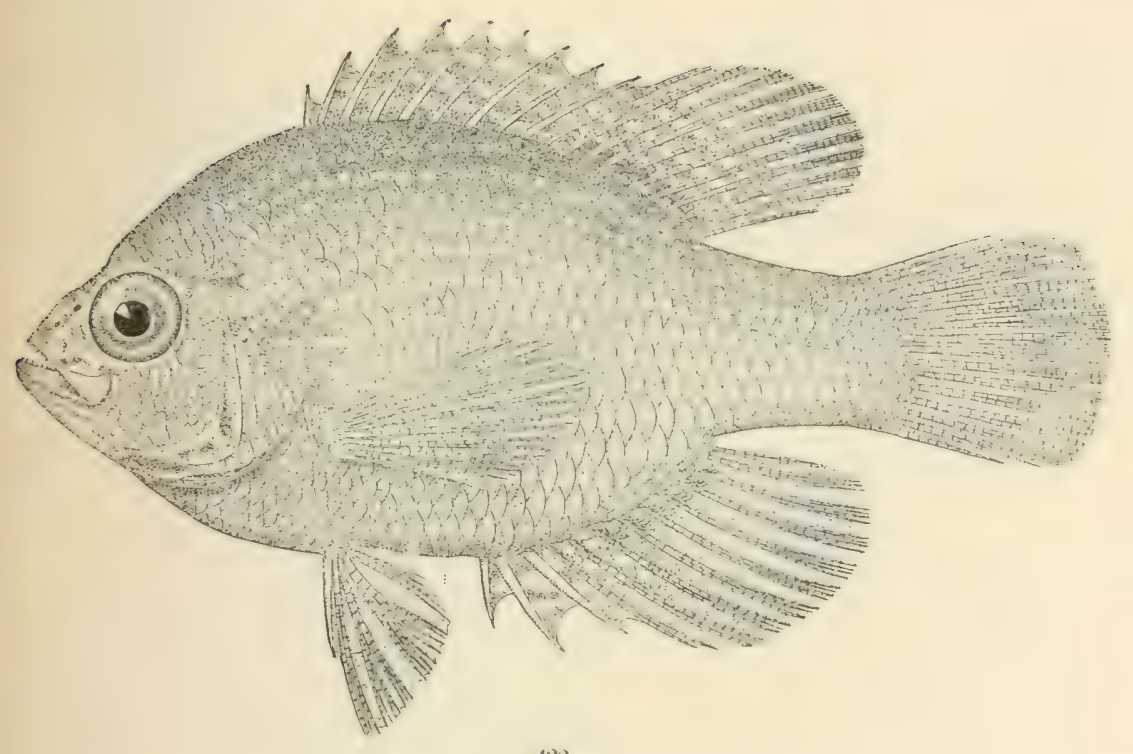

$42:$

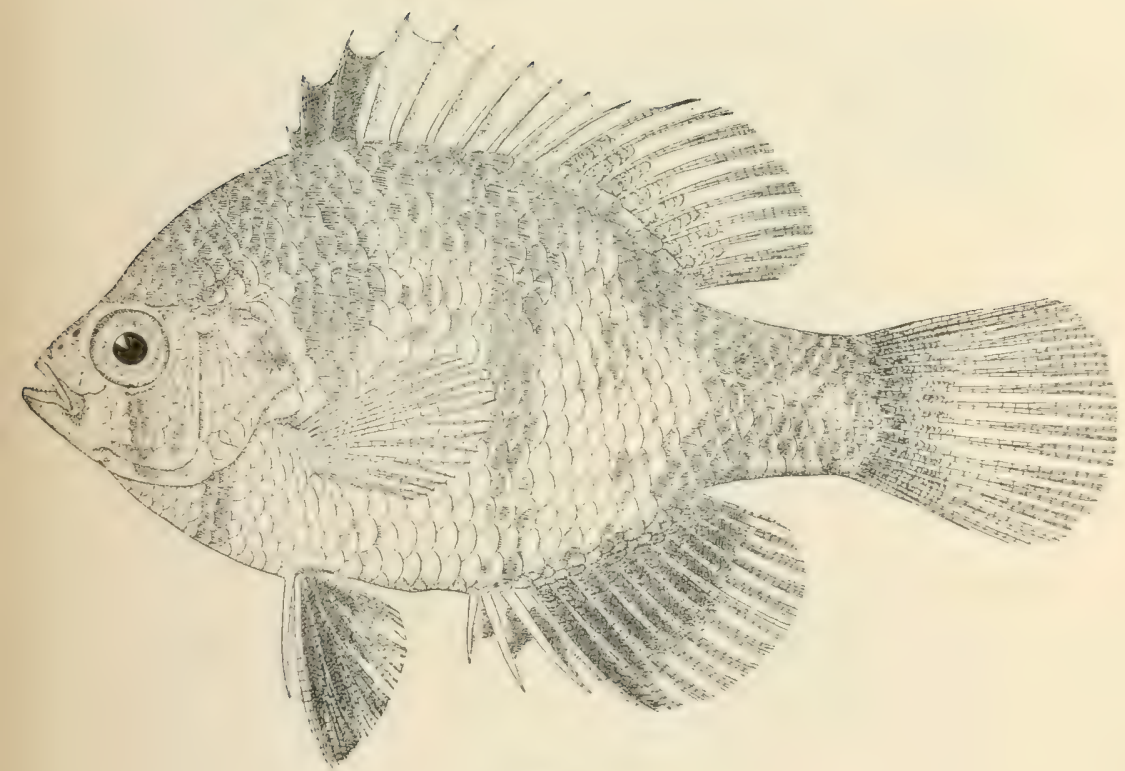

493

422. ENNEACANTHUS GLORIOSUS. (P. 993.)

423. MesogonistiUs CHETODON. (P. 995.) 



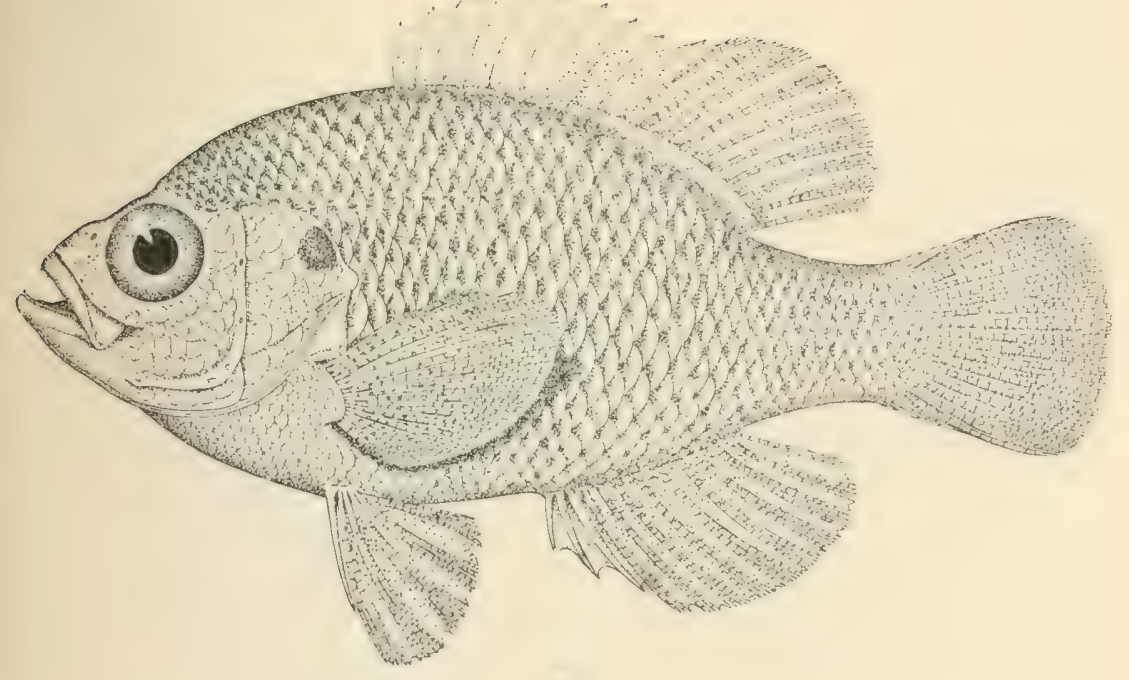

421

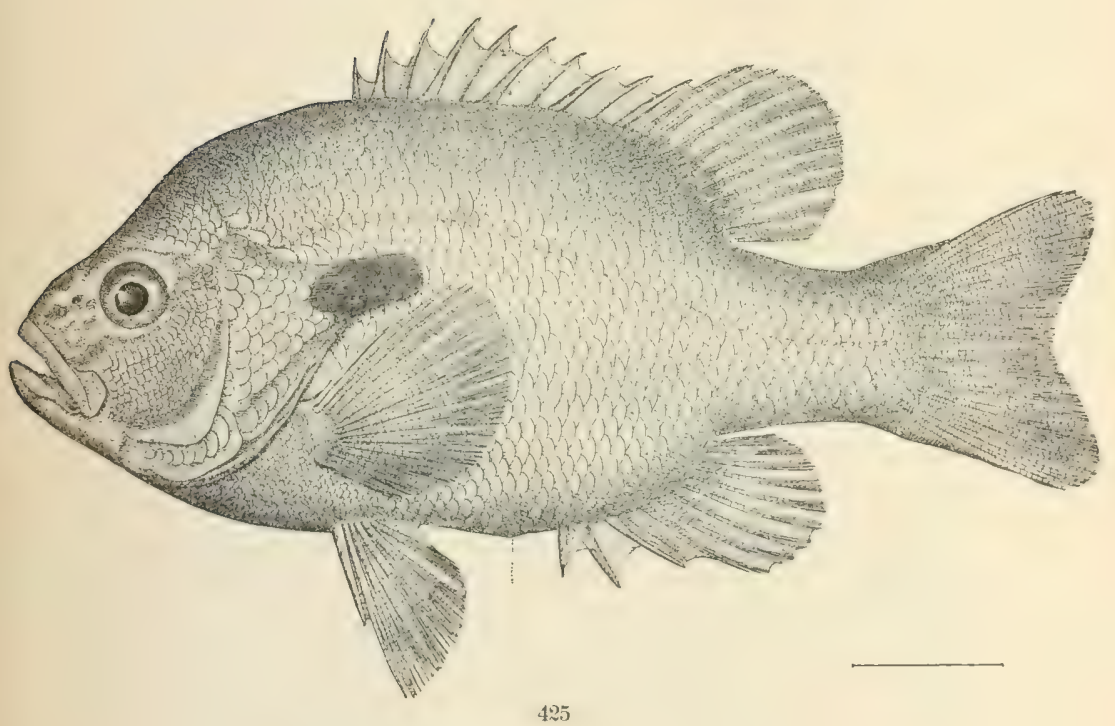

425

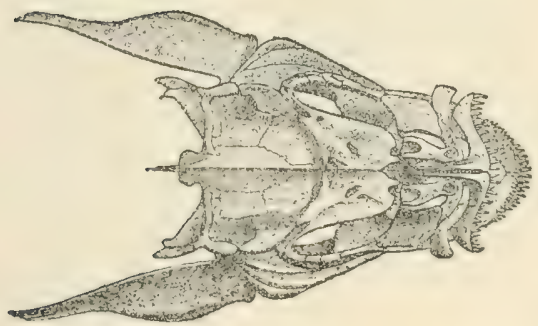

4:2ง

424. Apomotis smunetricus. (P. 998.)

425. Leiromis alditus. (P. 1001.)

425a. SKUlL OF Lepoins aURitus. (P. 1001.) 




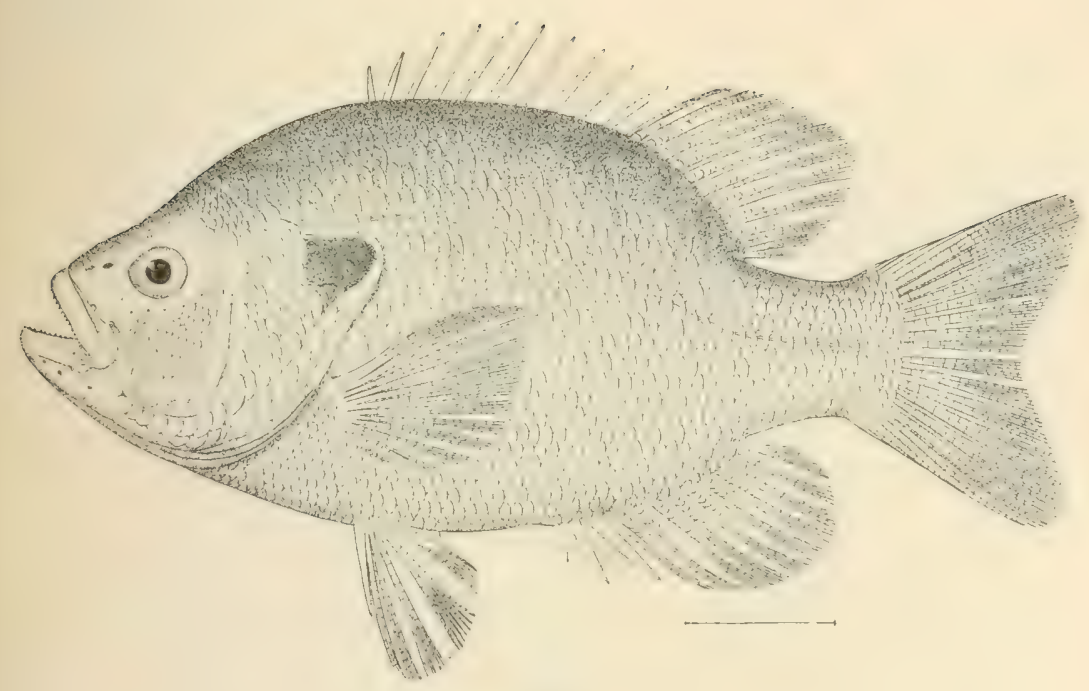

$4: 3 x$

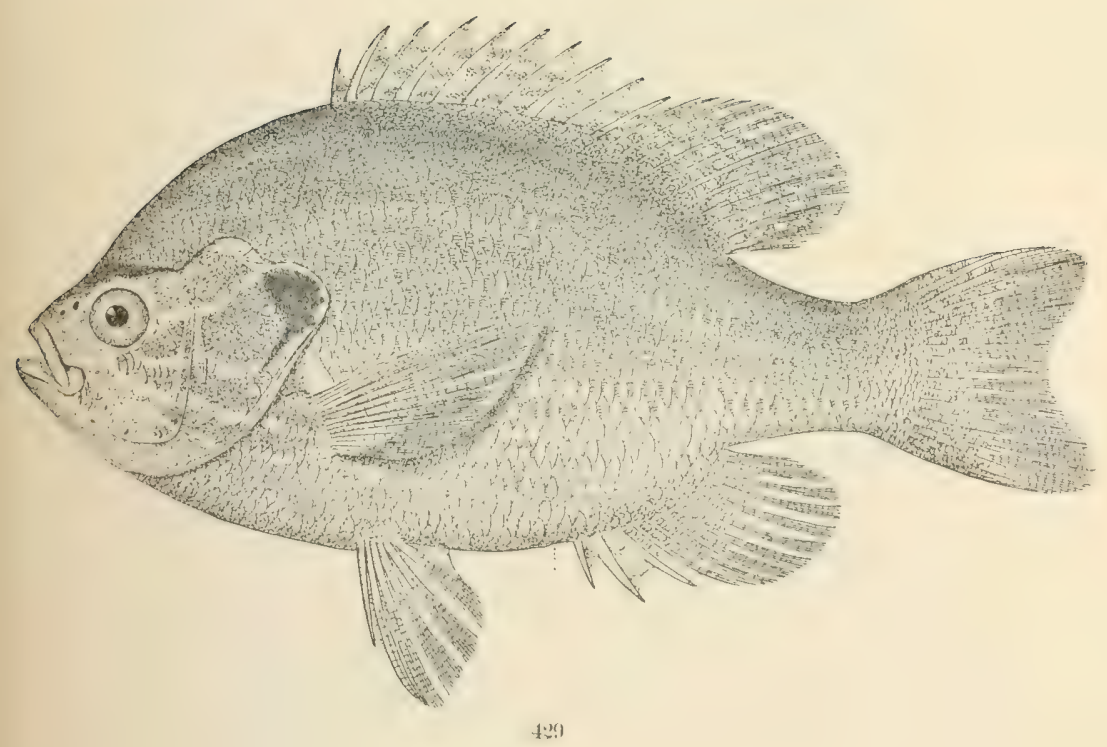

428. Eupomotis euryorus. (P, 1008.)

429. Eupomotis gibBosus. (P. 1009.) 


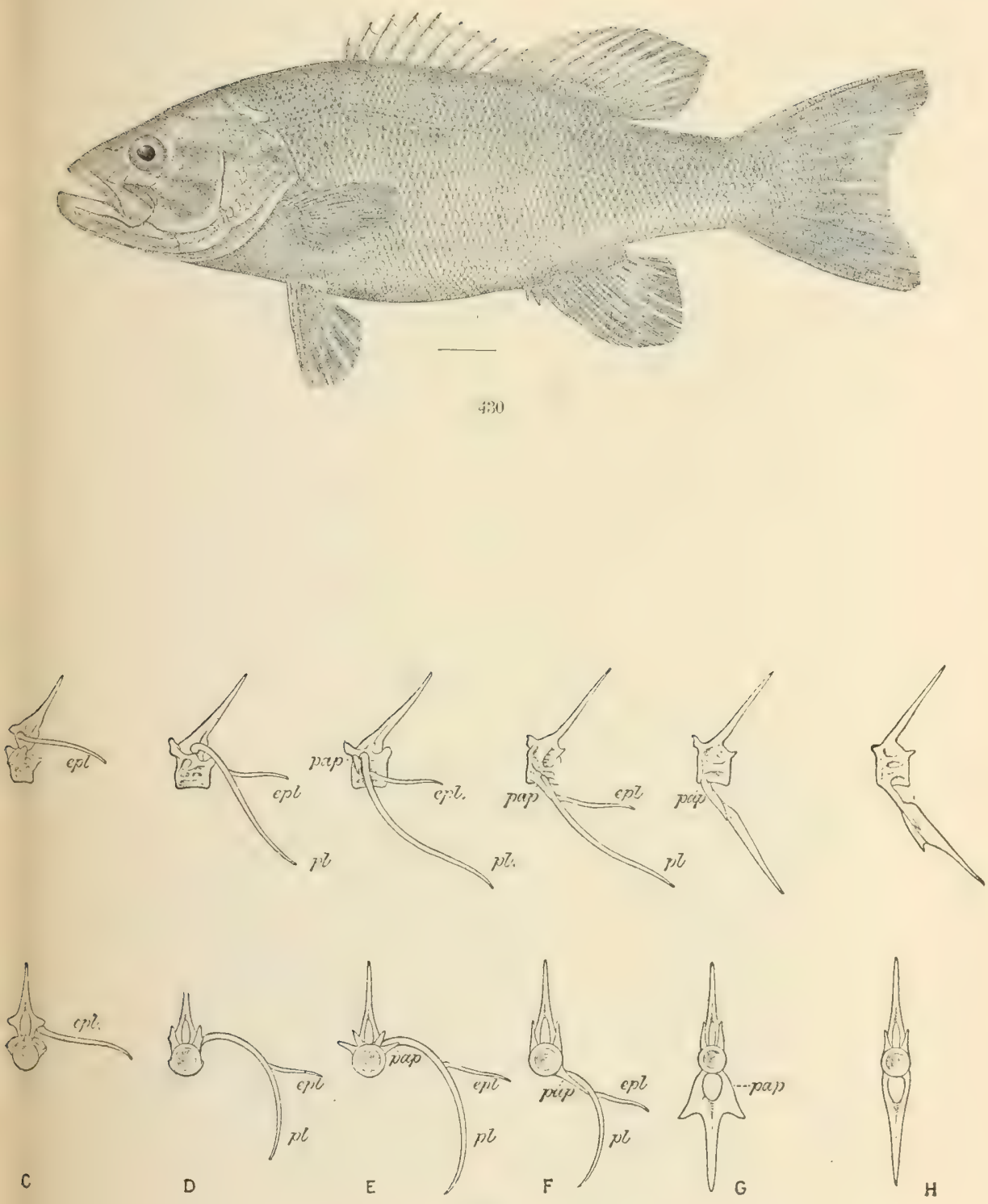

430. Micropterus dolomiet. (P. 1011.)

430a. VERTEBRE OF MICROPThRUS DOLOMIEU. (P. 1011.) 


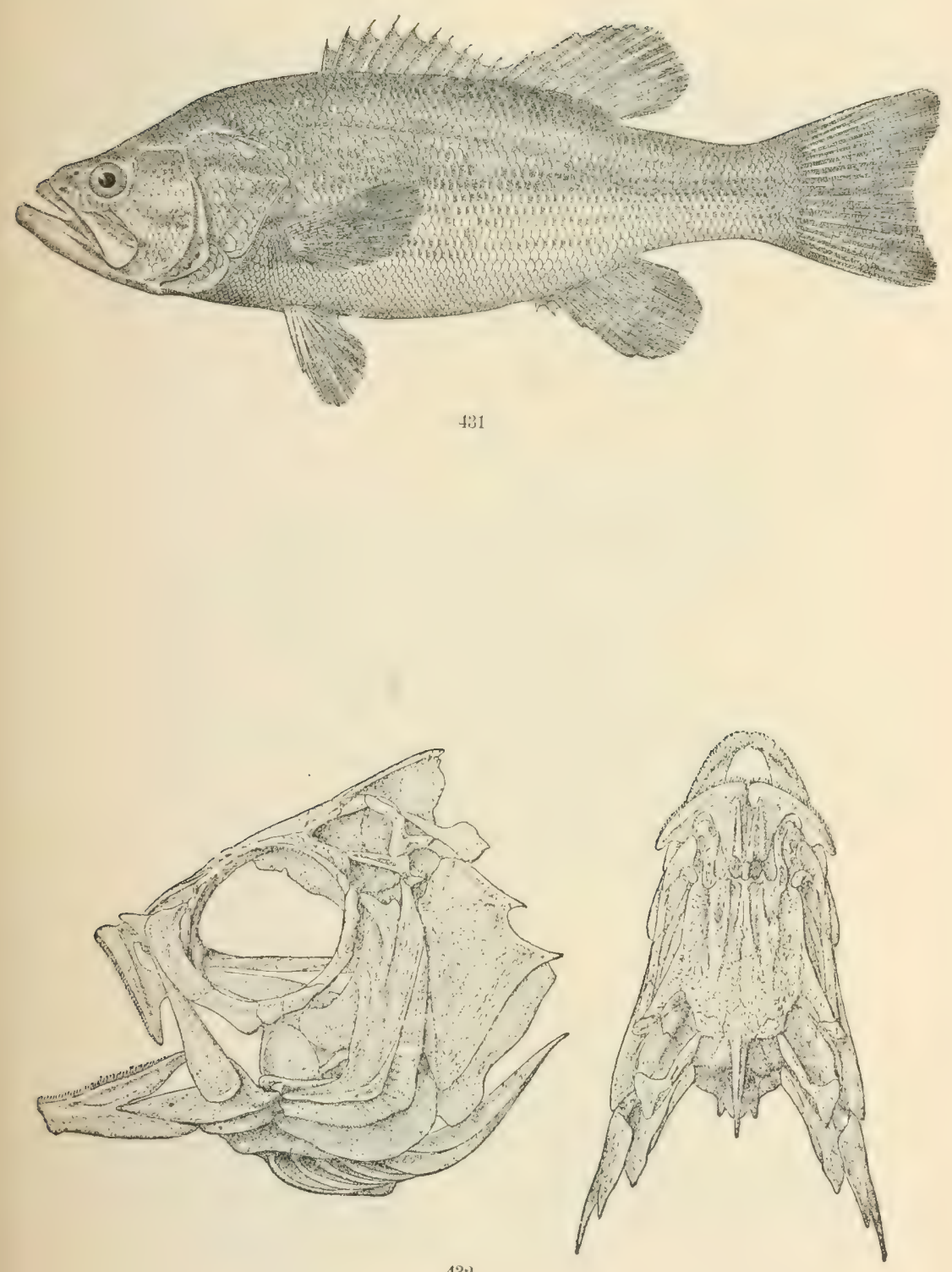

43:

431. MICROPTERUS SALMOIDES. (P. 1012.)

432. SKull OF Kuirla irUpestris. 




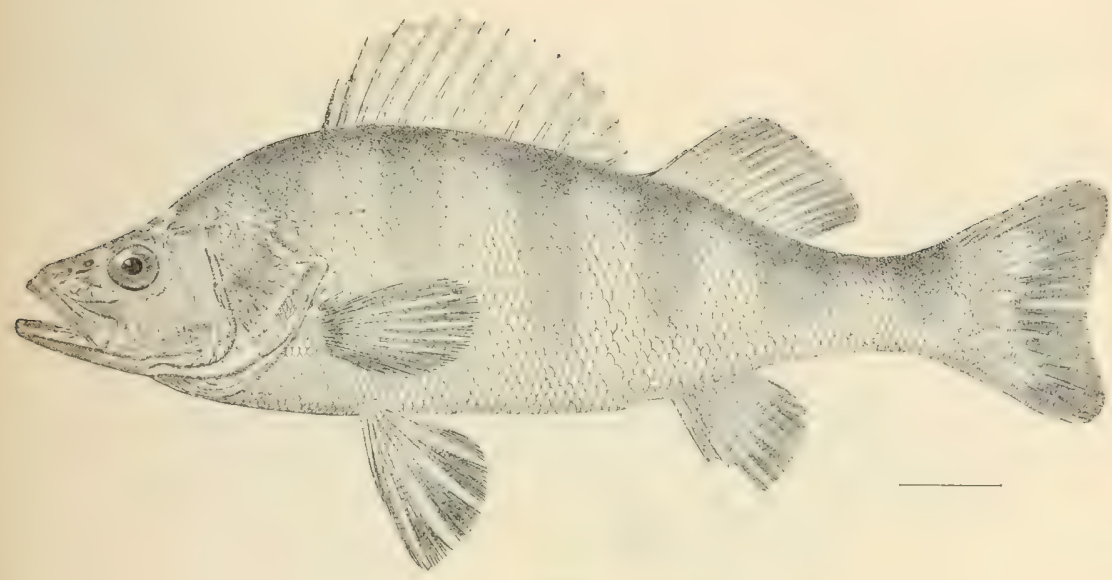

$1: 35$

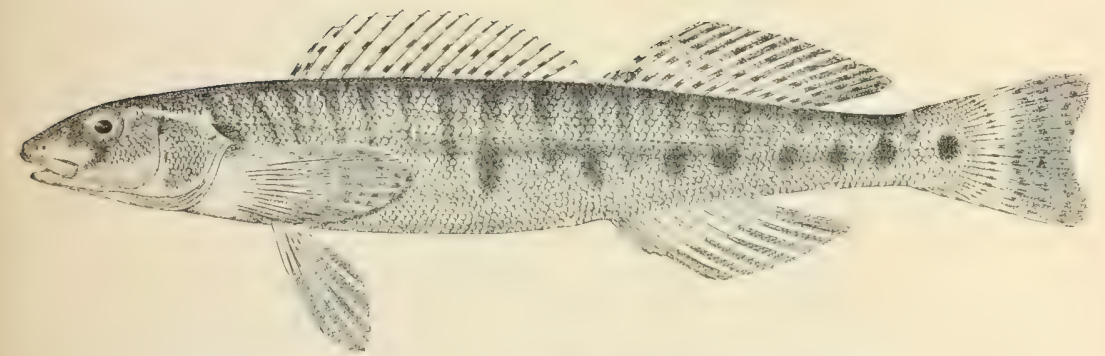

130

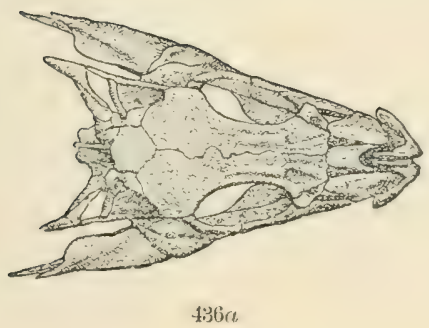

435. Pelica flatescens. (P. 1023.)

436. Percina caprodes. (P. 1026.)

436a. Skull of Percina caprodes. (P. 1026.) 



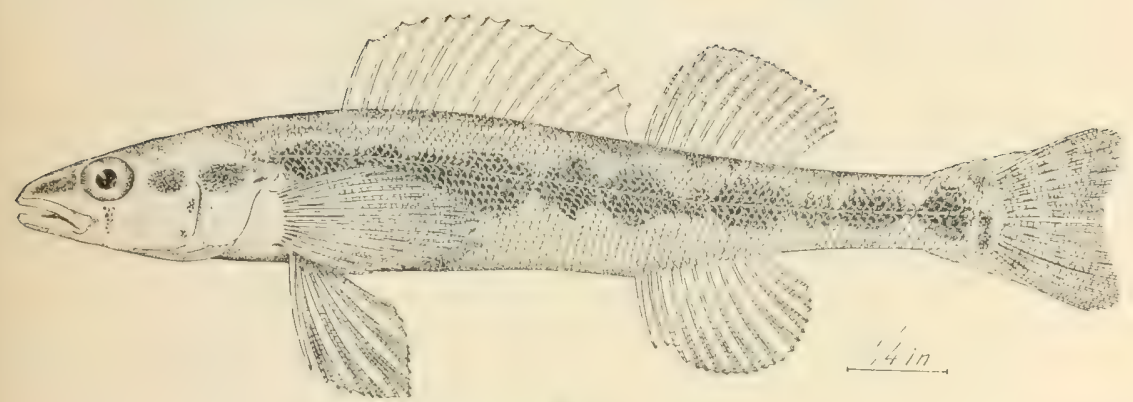

433

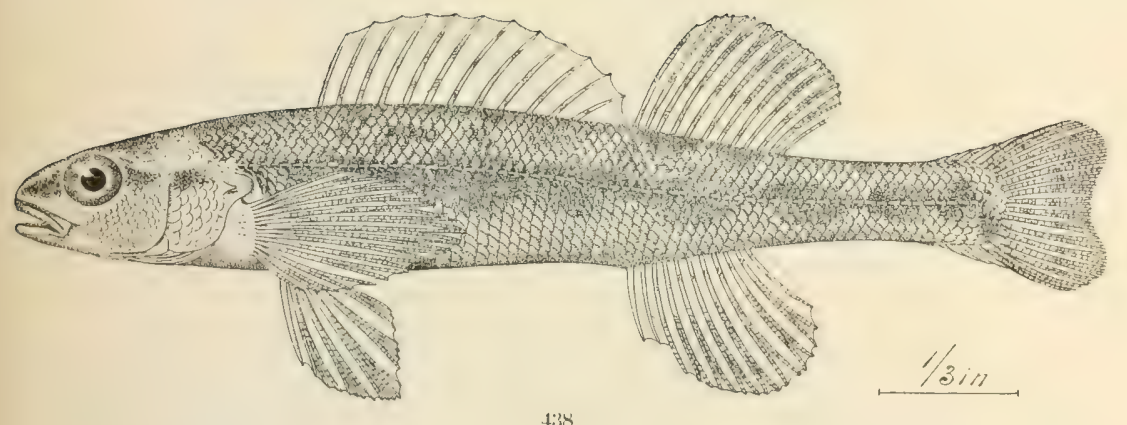

437. Hadropterus macrocephalUs. (P. 1031.)

438. Hadropterus aspro. (P. 1032.) 



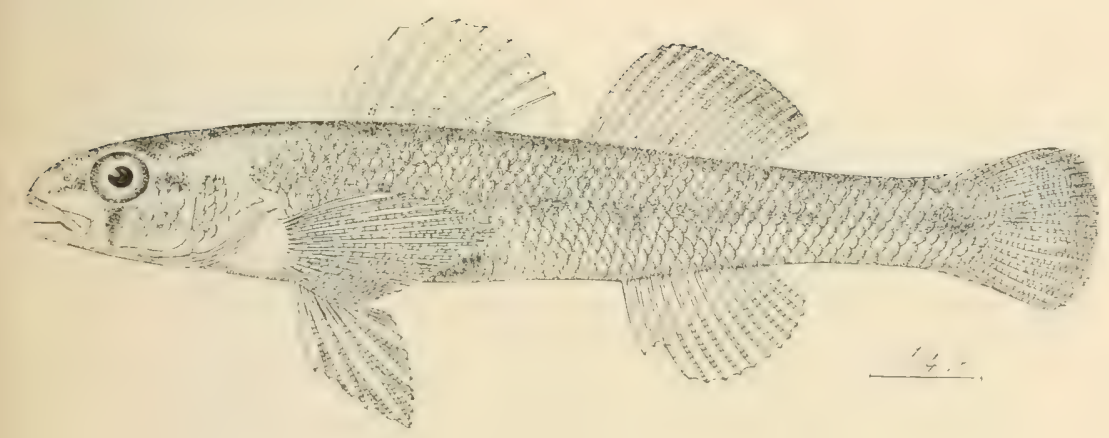

439)

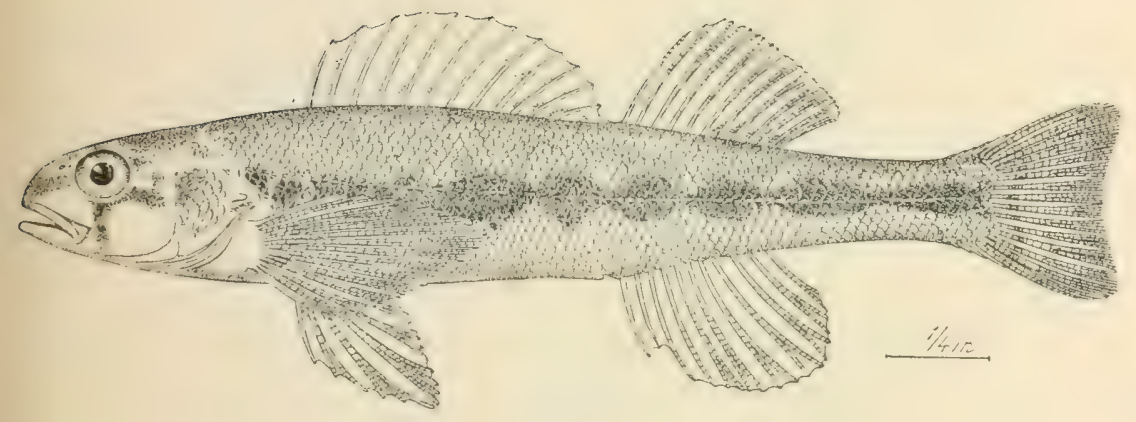

$4+1$

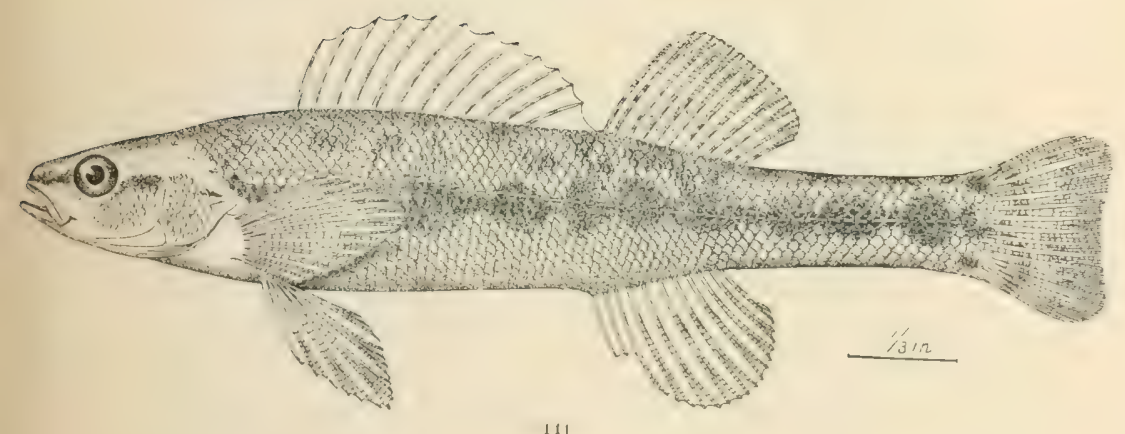

439. HADROPTERTS GUNTHERI. (P. 1033.)

440. Hadroptercs ENIDES. (P. 1036.)

441. Hadroltert's sciekUS. (P. 10:it.) 




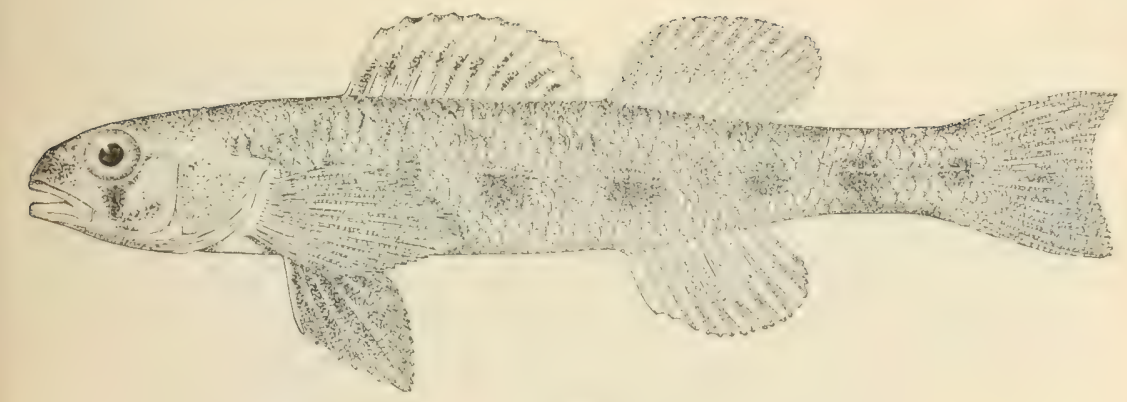

415
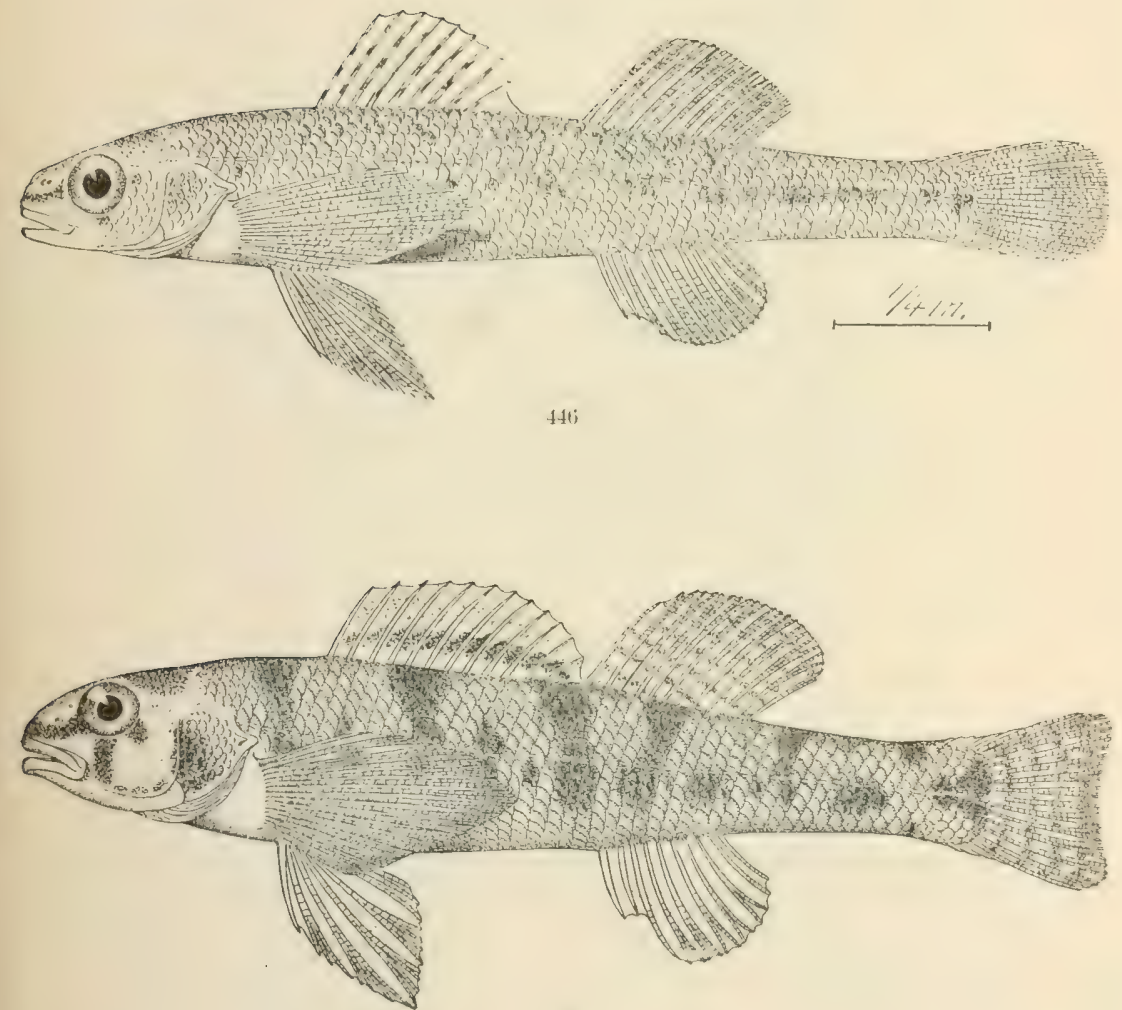

$44 \pi$

445. COTTOGASTER CHFNEYI. (P. 2851.)

446. Ulocentra GILIBERT. (P. 1049.)

447. Ulocentra MEAdid. (P. 2852.) 




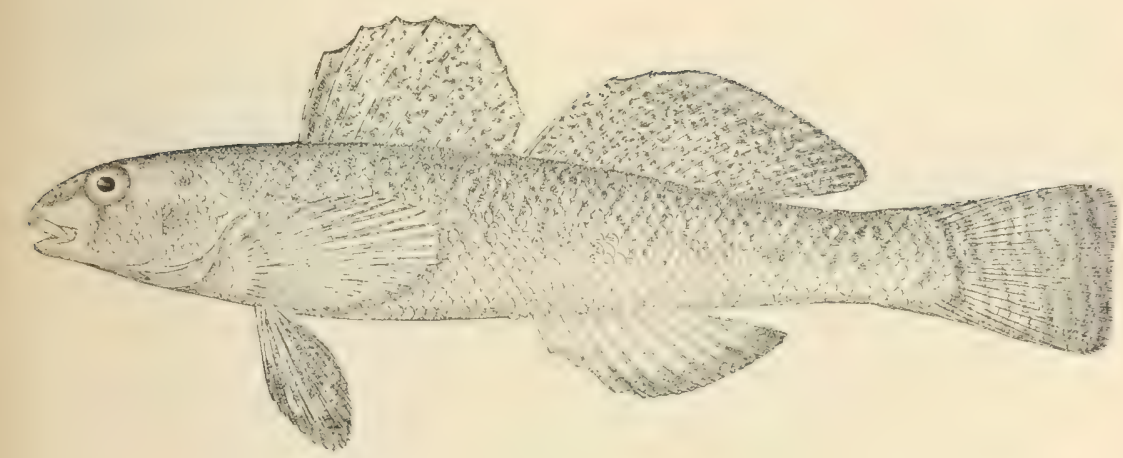

451

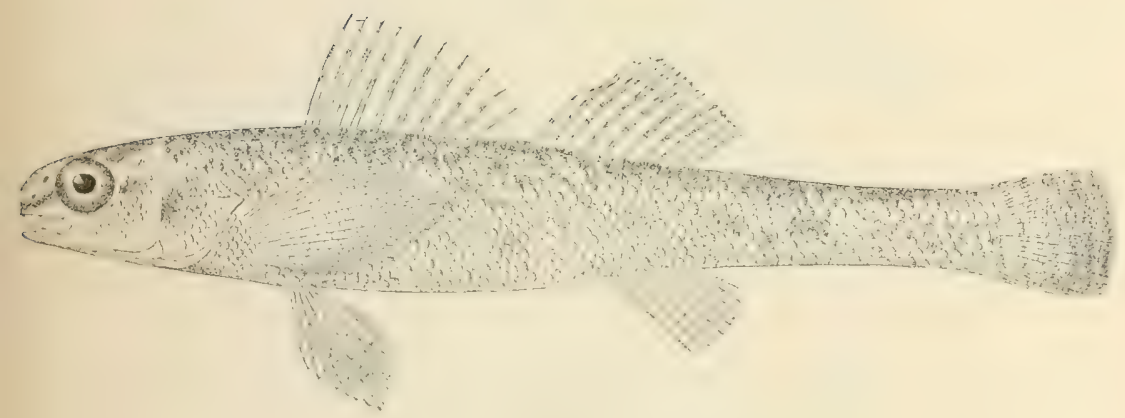

$4: 0$

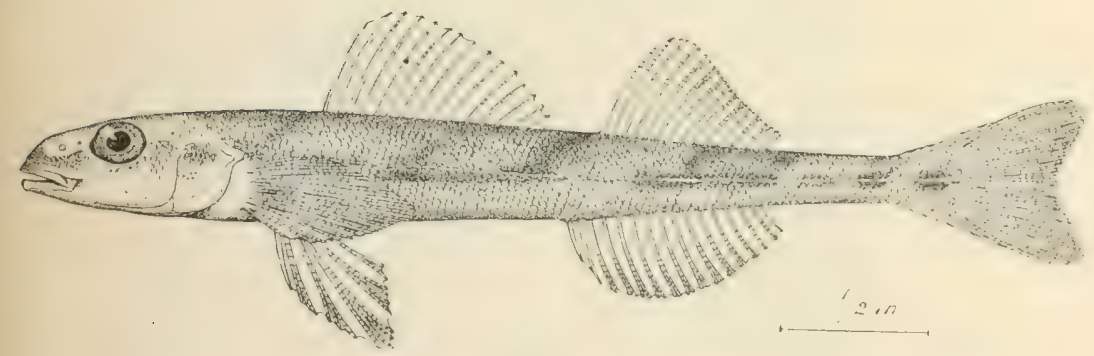

$45: 3$

451. Boleosoma NigRUM OLMSTEDI. (P. 1057.)

452. Boleosoma CaMUTum. (P, 1060.)

453. Crystallaria asprella. (P. 1061.) 



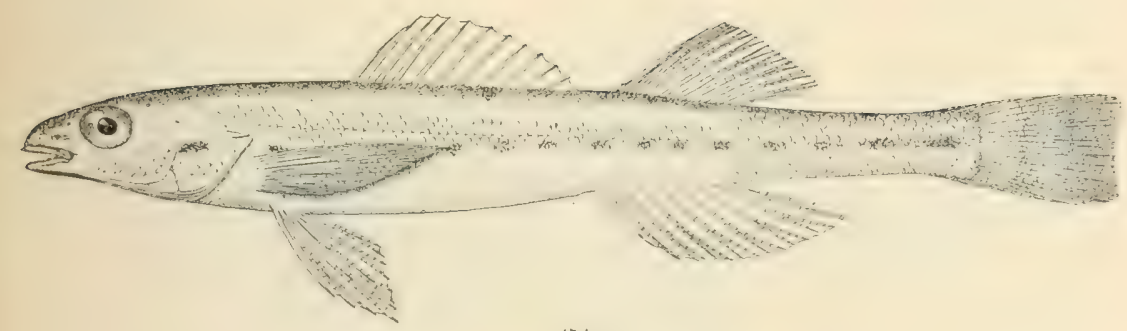

451

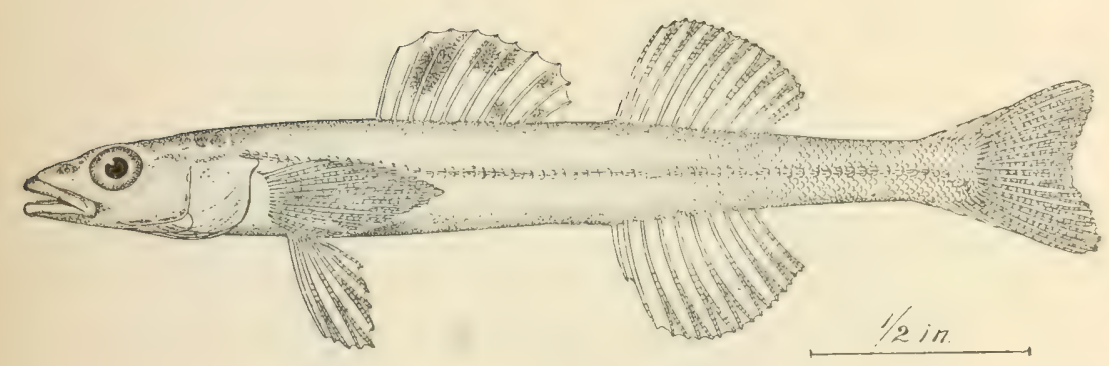

455

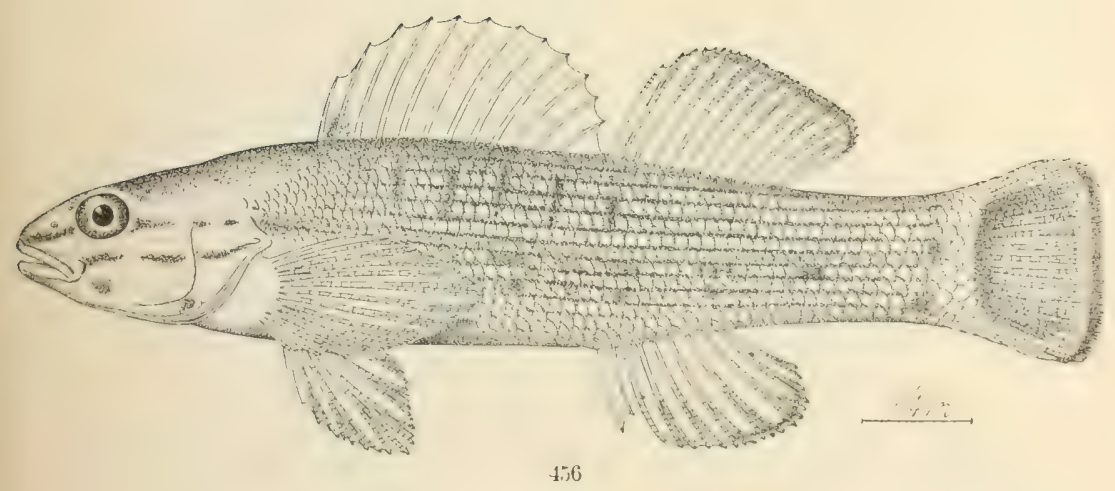

45t. AMHOCRYPTA PELLUCIDA CIAAR. (P. 1063.)

455. AMMOCRYPTA BEANII. (P. 1064.)

456. ETHEOSTOMA CAMURUM. (P. 1076.) 



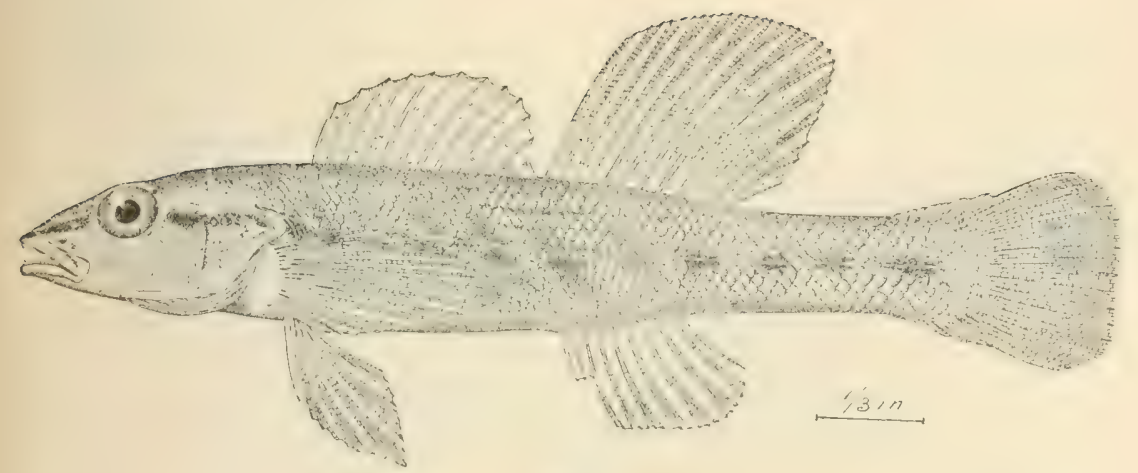

$4 \%$

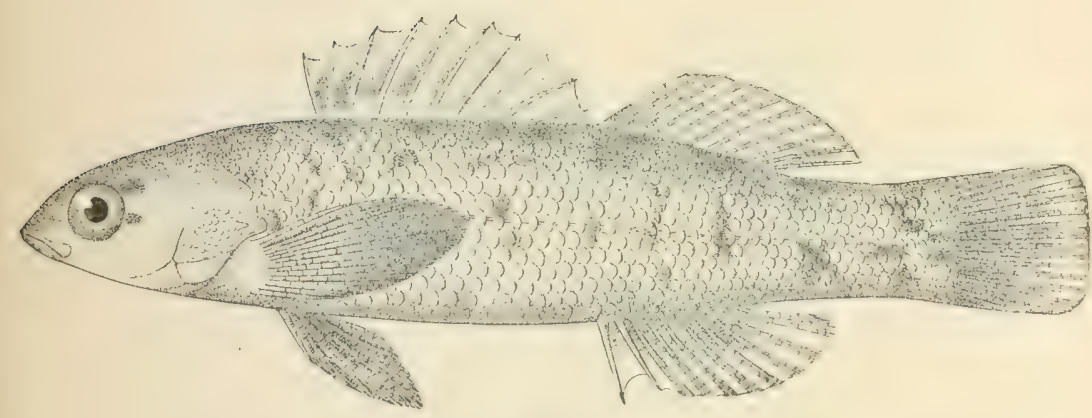

45

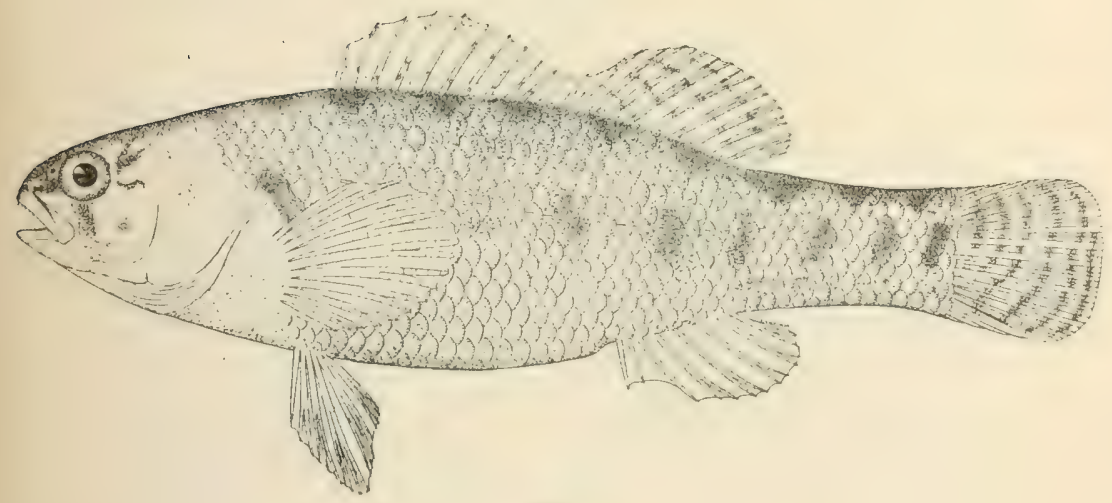

459

457. Ethlostoma cinelzeum. (P. 1078

458. Etheostoma mormani. (P. 1079.)

459. EThlostoma potrsil. (P. 1082.) 



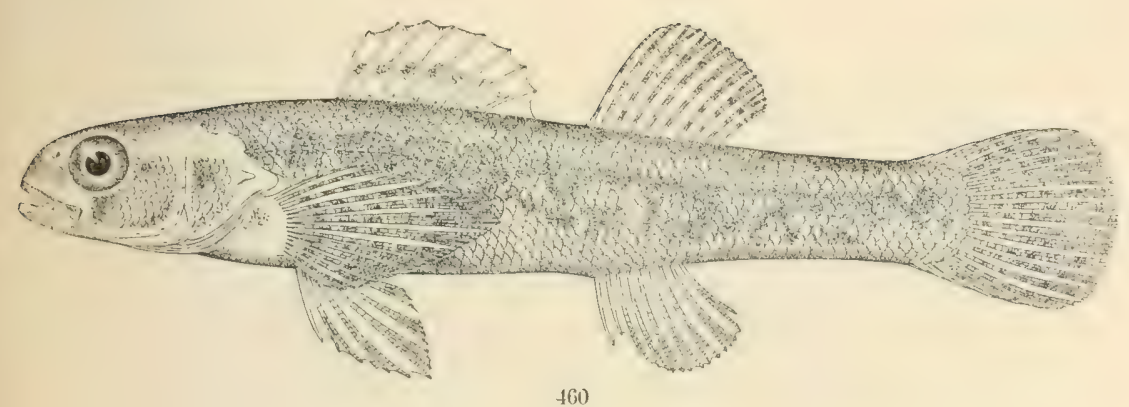

460

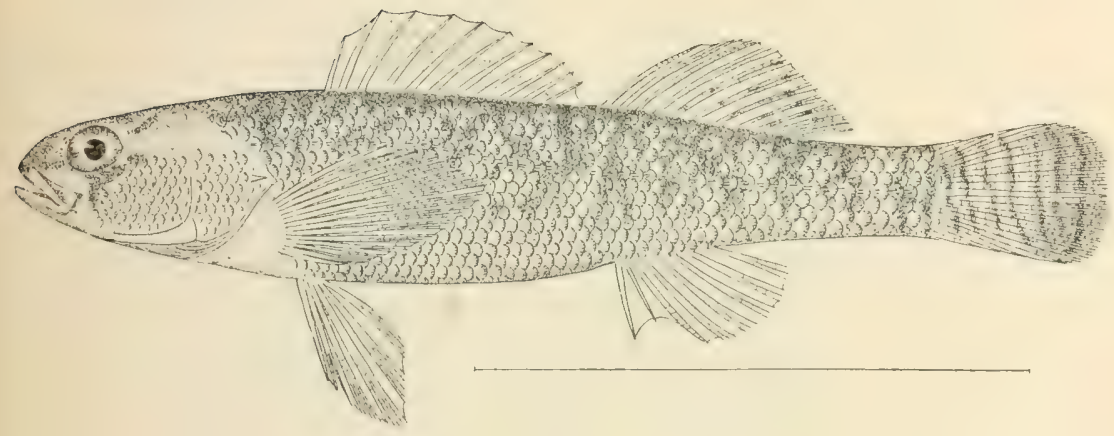

461

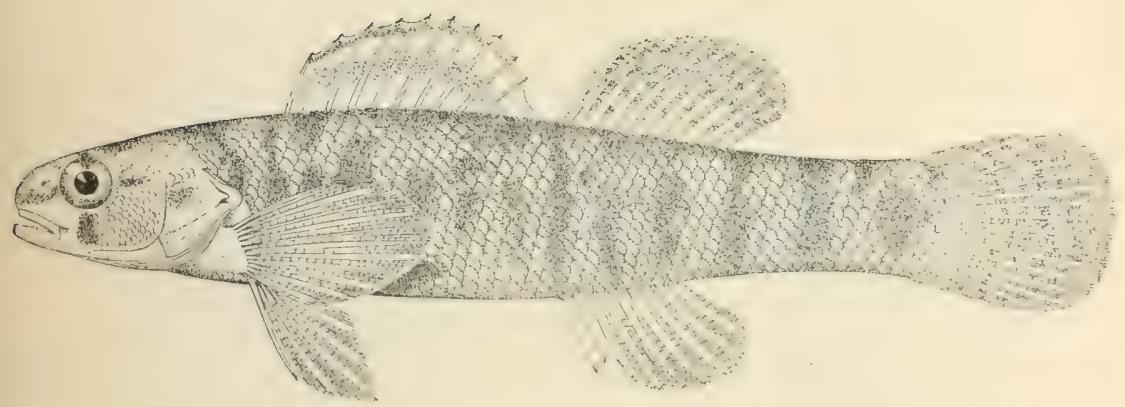

462

460. Етнеовтома 10\%.玉. (P'. 1083.)

461. Etheostoma Jesise. (P. 1081.)

462. Eтheostona leptoggenys. (P. 1087.) 


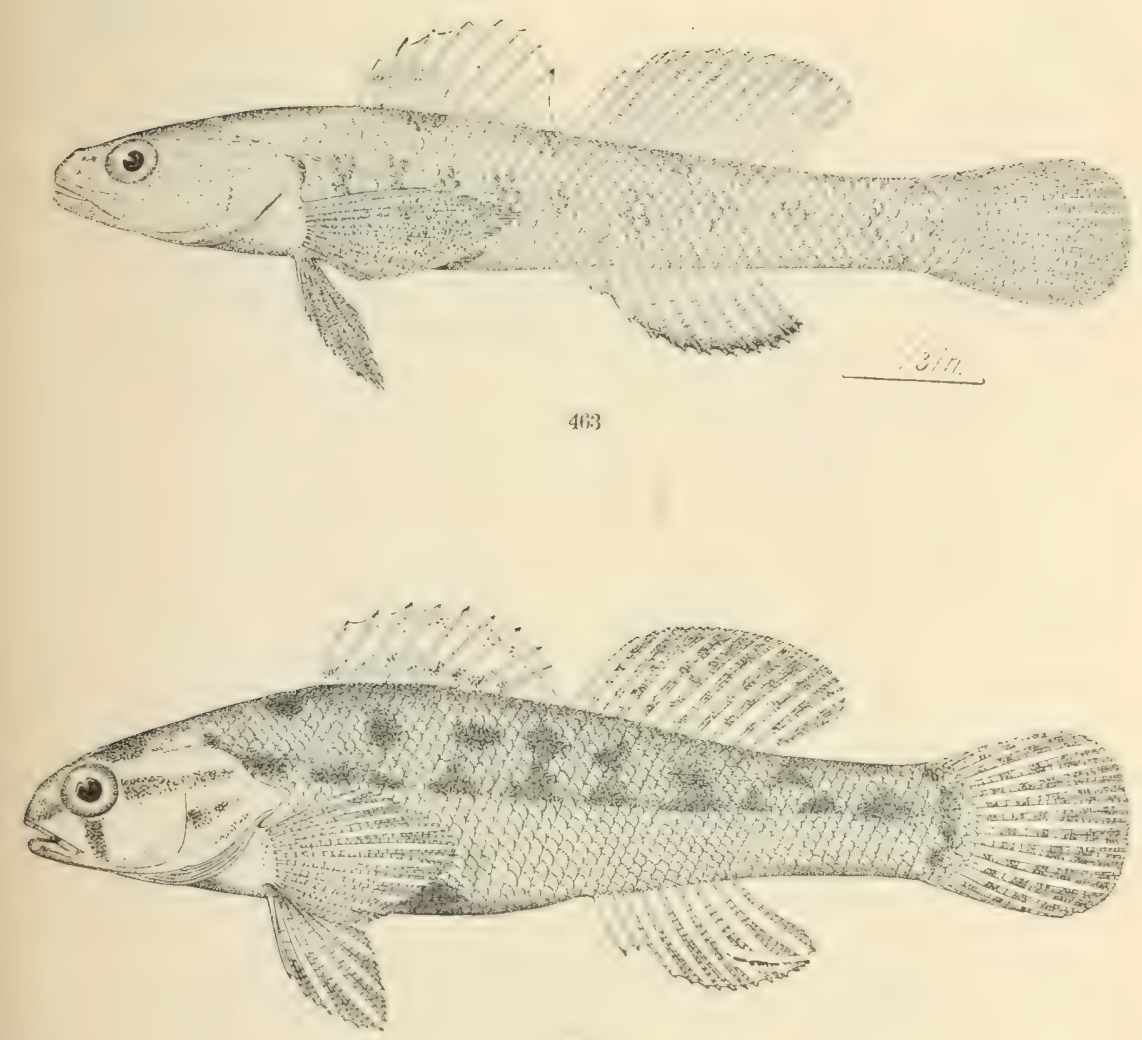

464

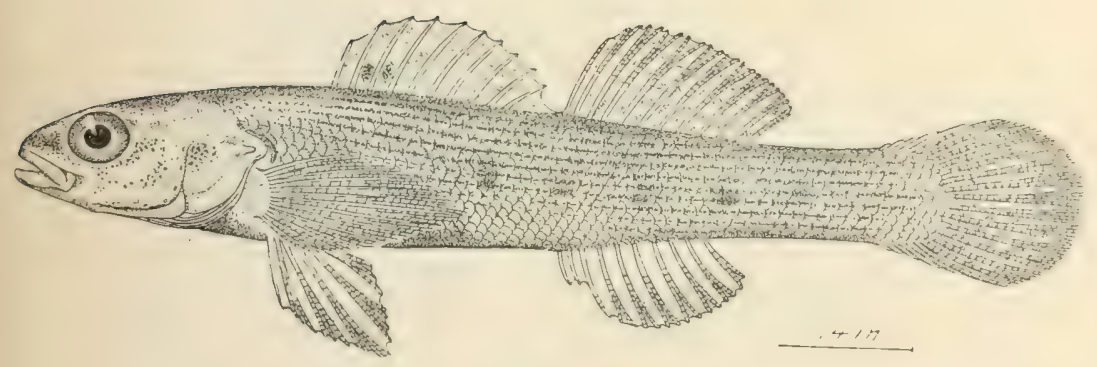

465

463. Etheostoma obfyense. (1'. 1092.)

464. Etheostoma PAGei. (P. 1092.)

465. Etheostoda virgatum. (P. 1093.) 



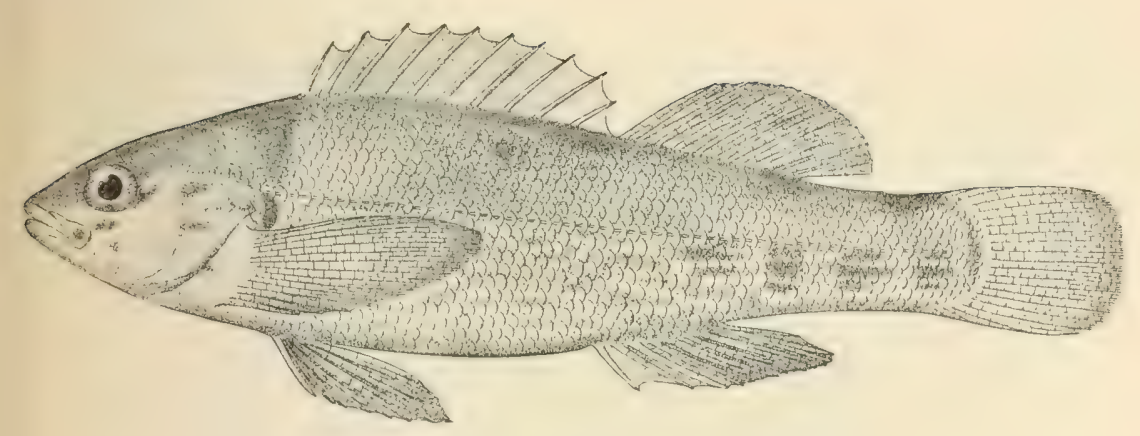

466

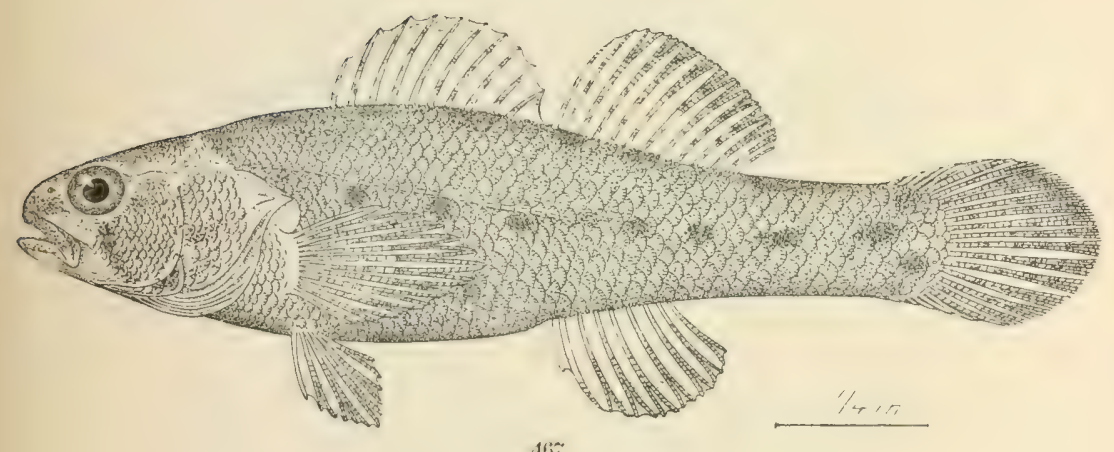

$466^{\circ}$

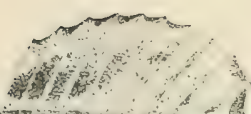

पारुका
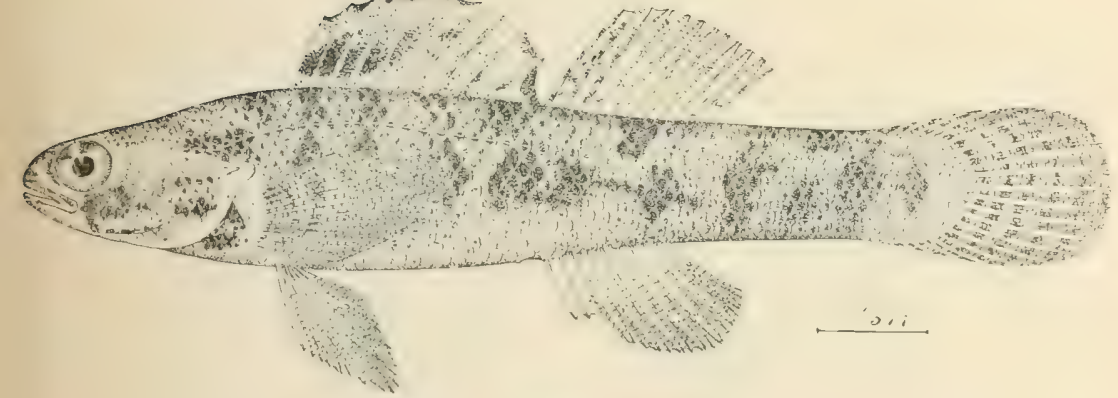

465

466. ETHEOSTOMA JULIE. (P. 1093.

467. PSYChromaster TUSCUMBIA. (P. 1100.)

468. Copelandellus Quiescens. (P. 1100.) 



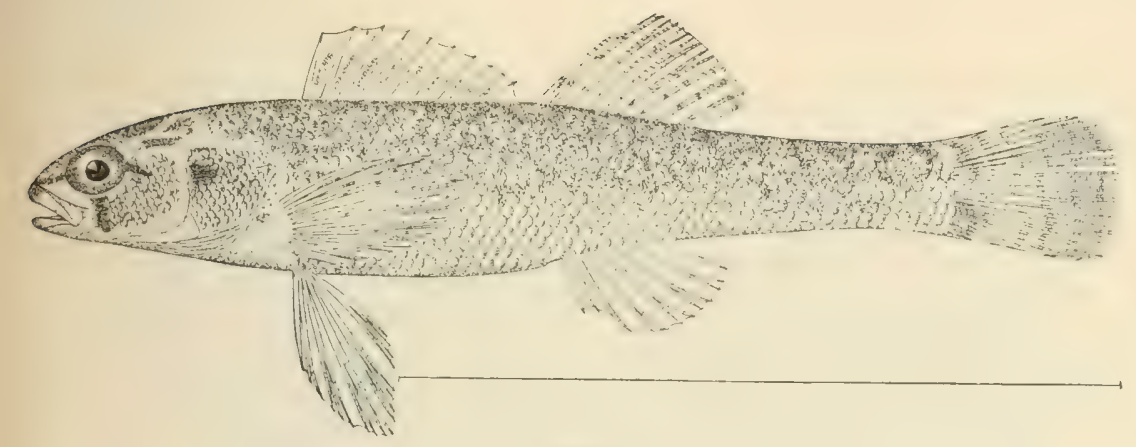

469

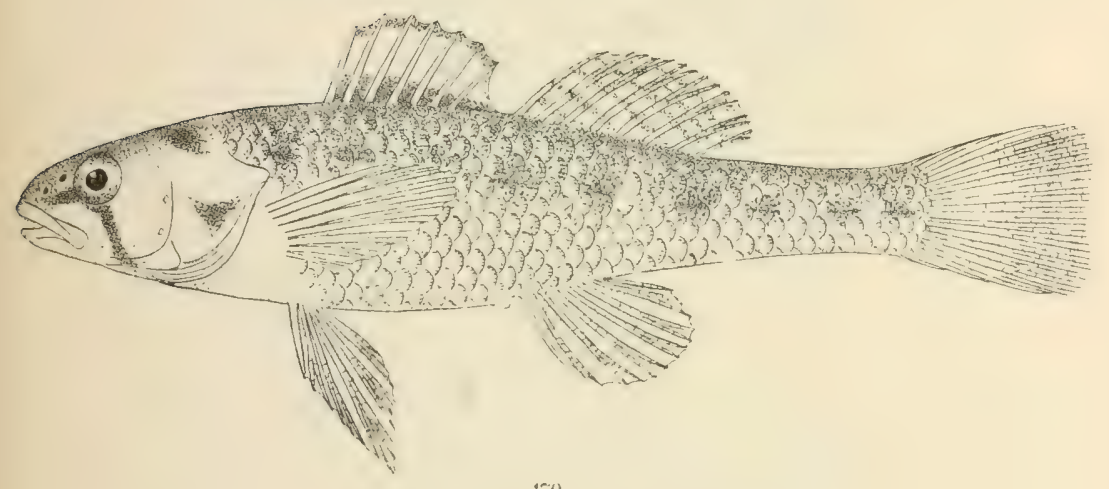

40

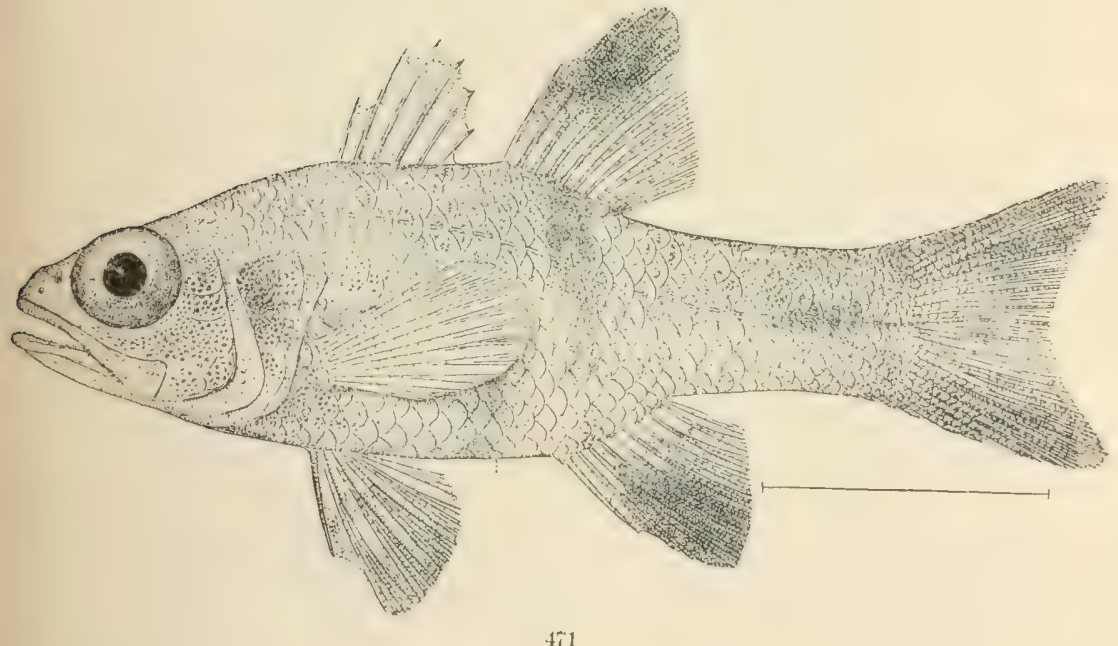

469. Boleichthys FUSIFormis. (P. 1101.)

470. Microphrea Fonticola. (P. 1104.)

471. Apogon retrosella. (P. 1108.) 


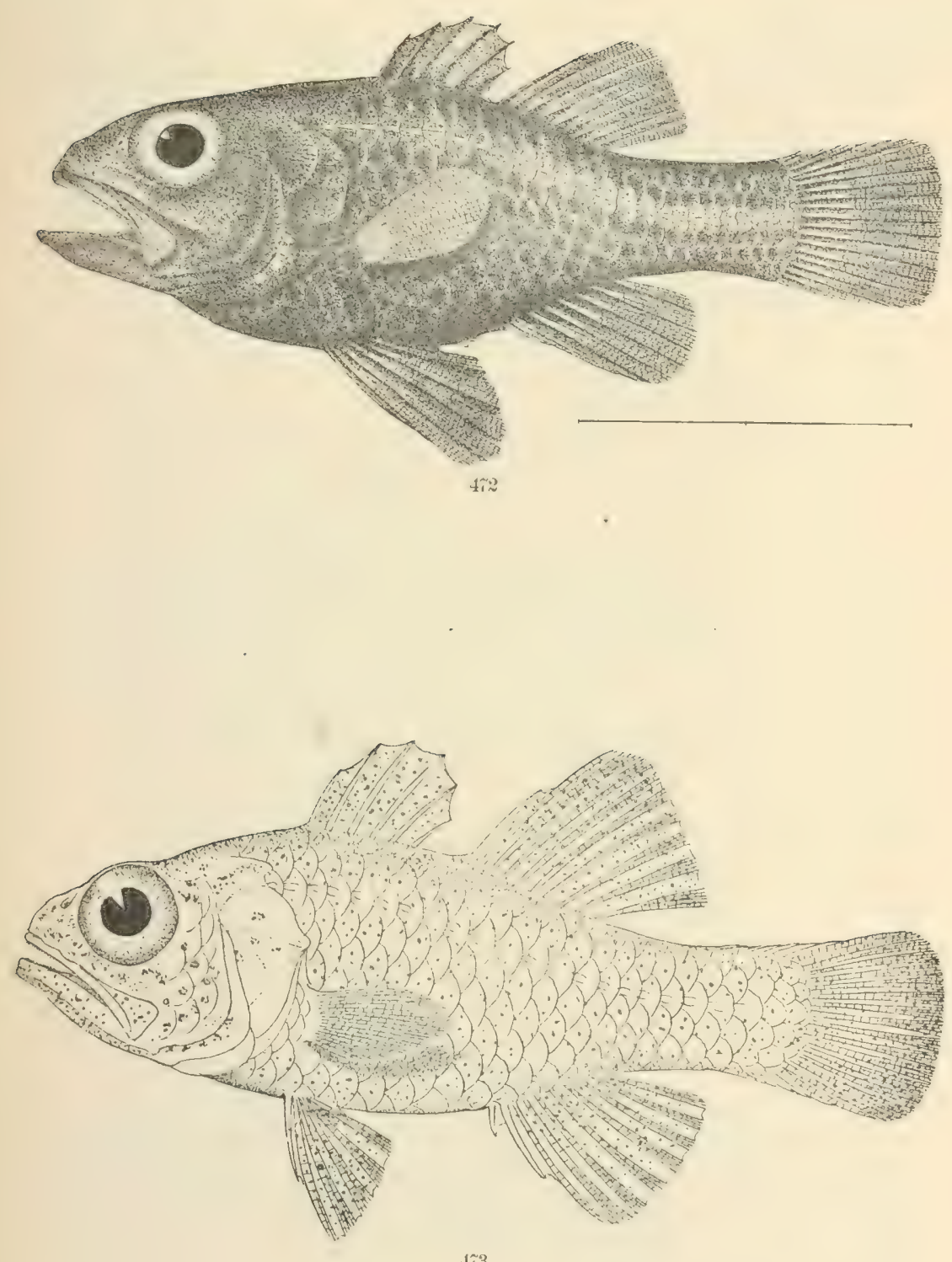

$4 \pi 3$

472. APOgON pigmentakiUs. (P. 1109.)

473. Apogonichtims alUtus. (P. 1110.) 



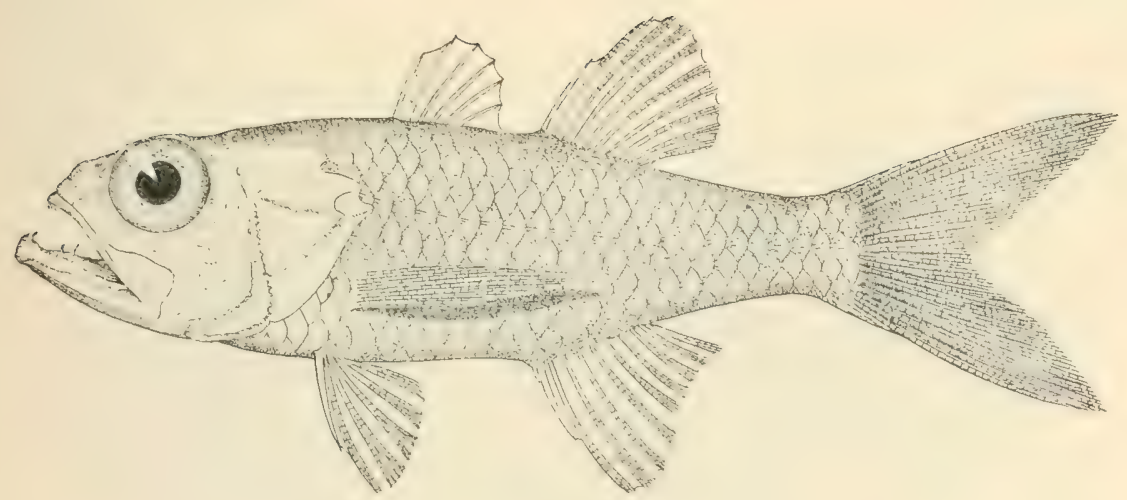

$4 \pi 4$

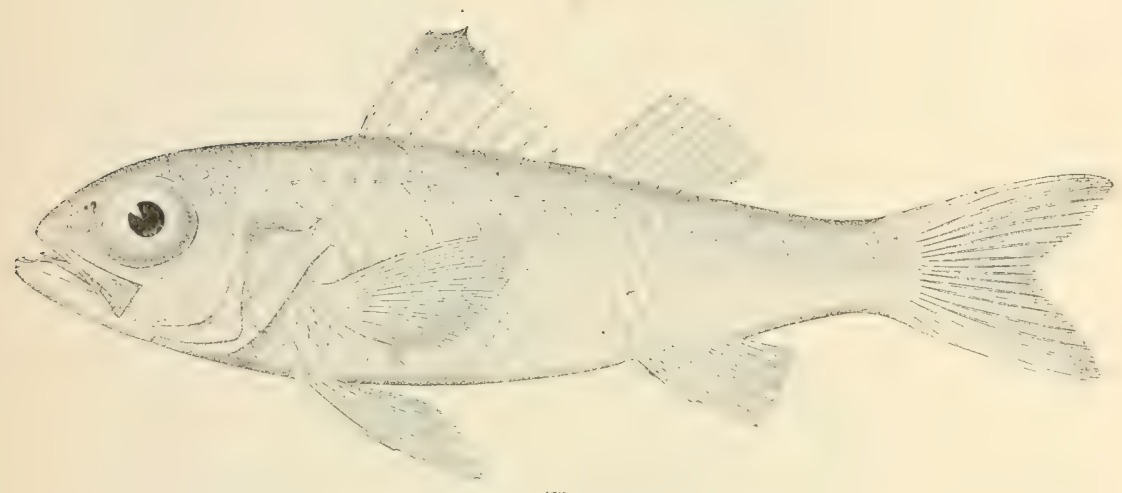

$4 \pi 5$

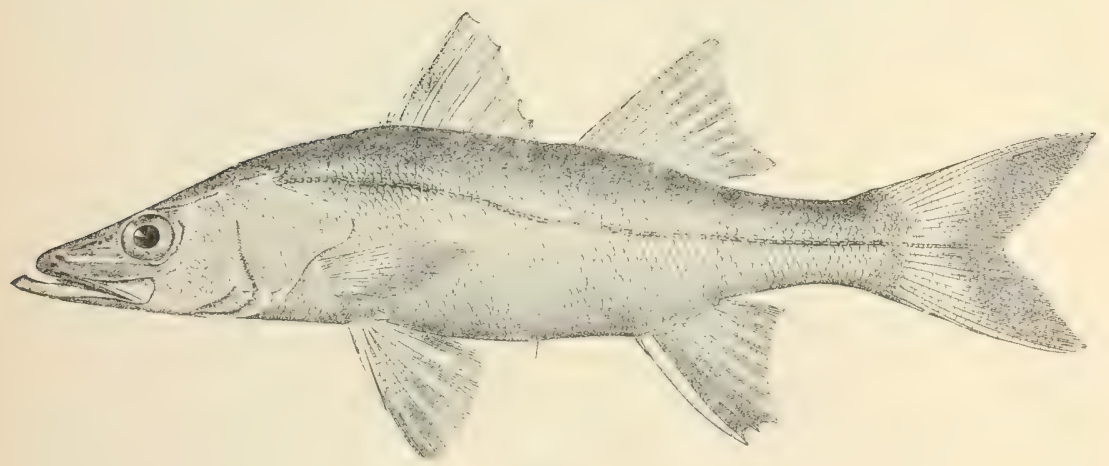

$4 \%$

474. Chemonipterus afinis. ( $P .1113$. )

475. HYPOCLIDONIA BELLA. (P. 1115.)

476. Centropomes undechials. (P. 1118.) 



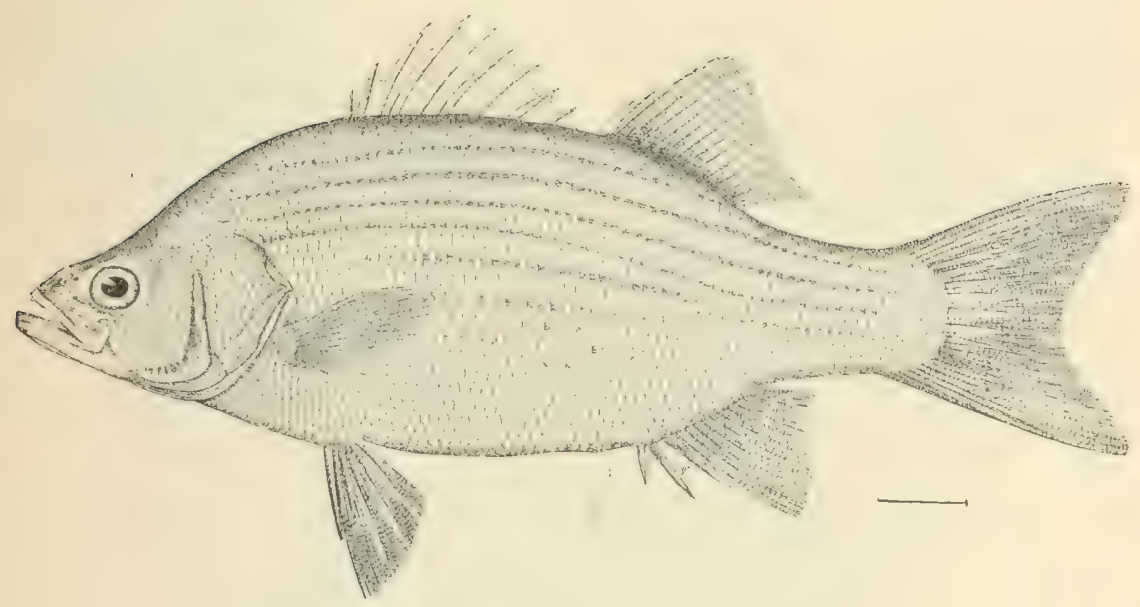

$4 \pi \%$

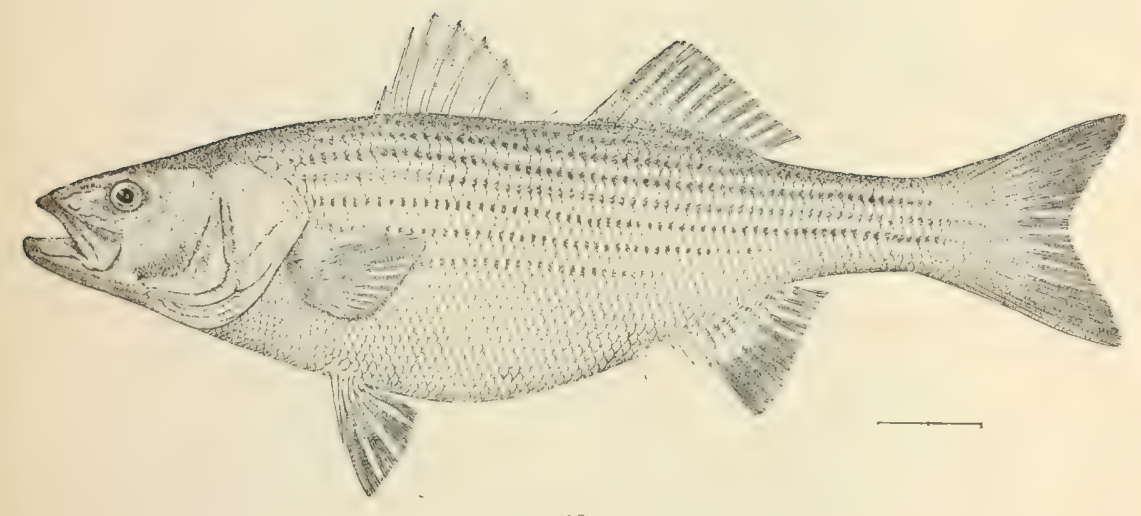

477. Roccus CHRYsops. (P. 1132.)

478. Roccus lineatus. (P. 1132.) 


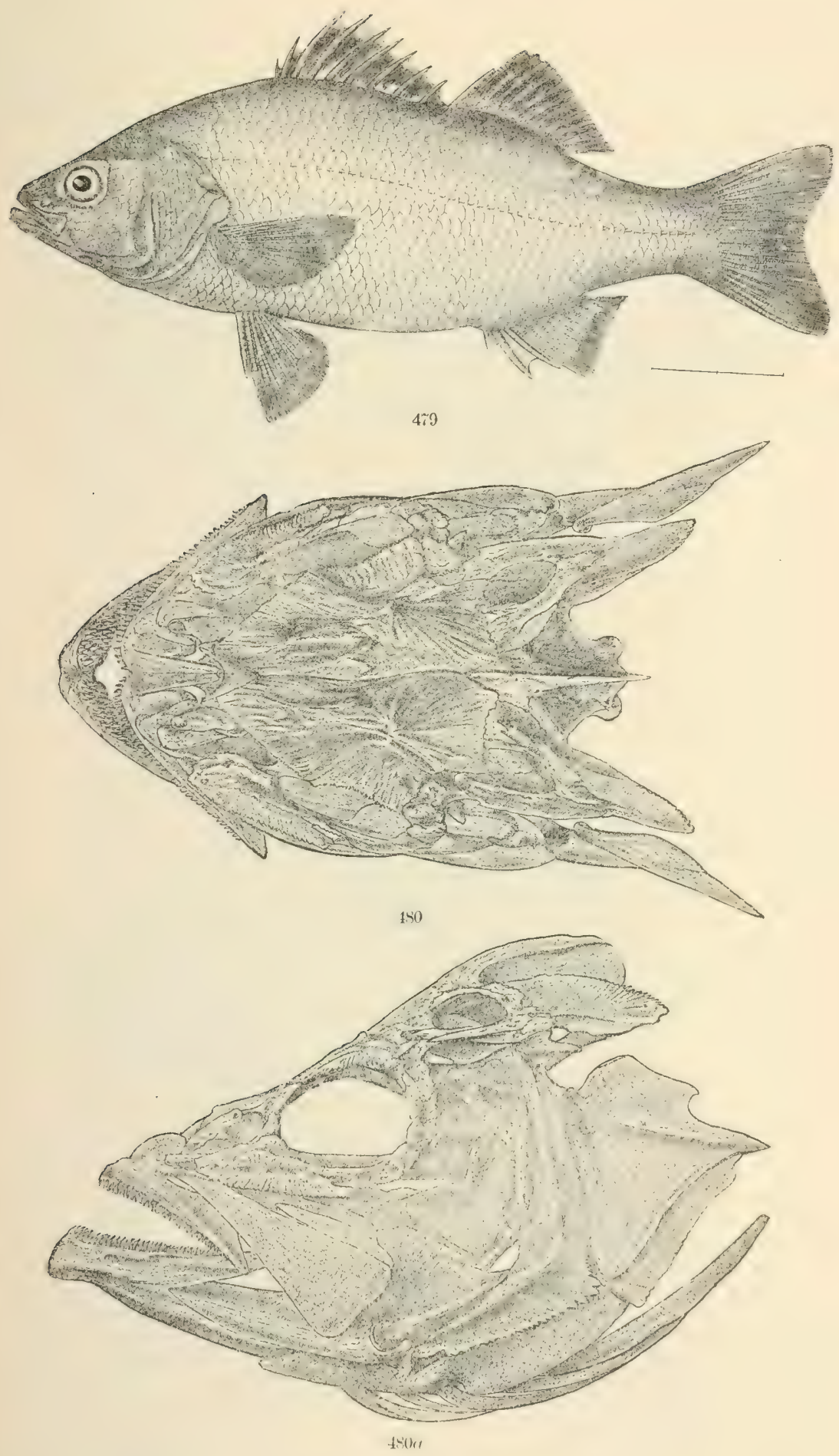

479. MORONE AMERICANA. (I'. 1134.)

480, 480a. Skull OF Polyprion americanus. (P. 1139.) 



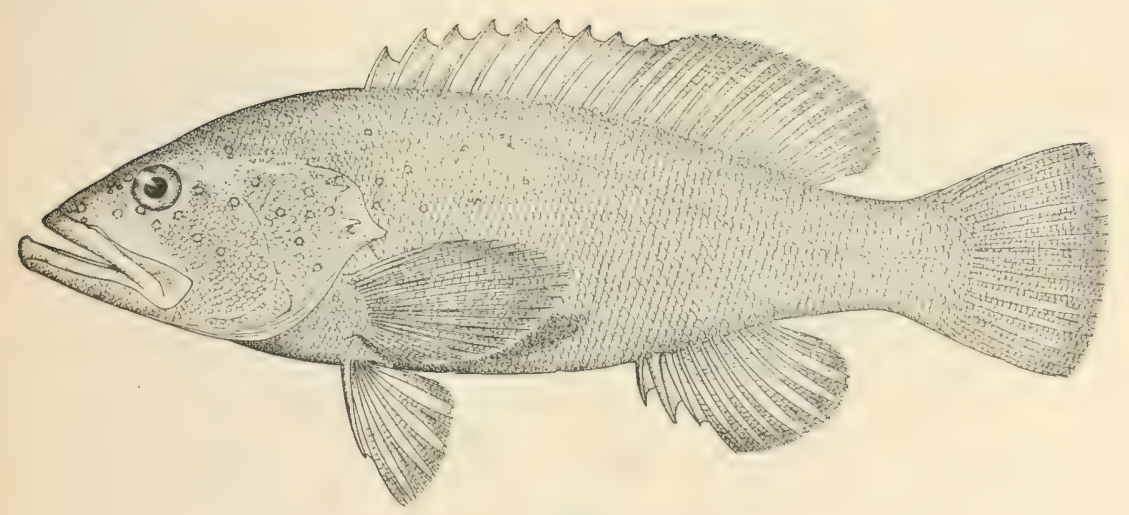

481

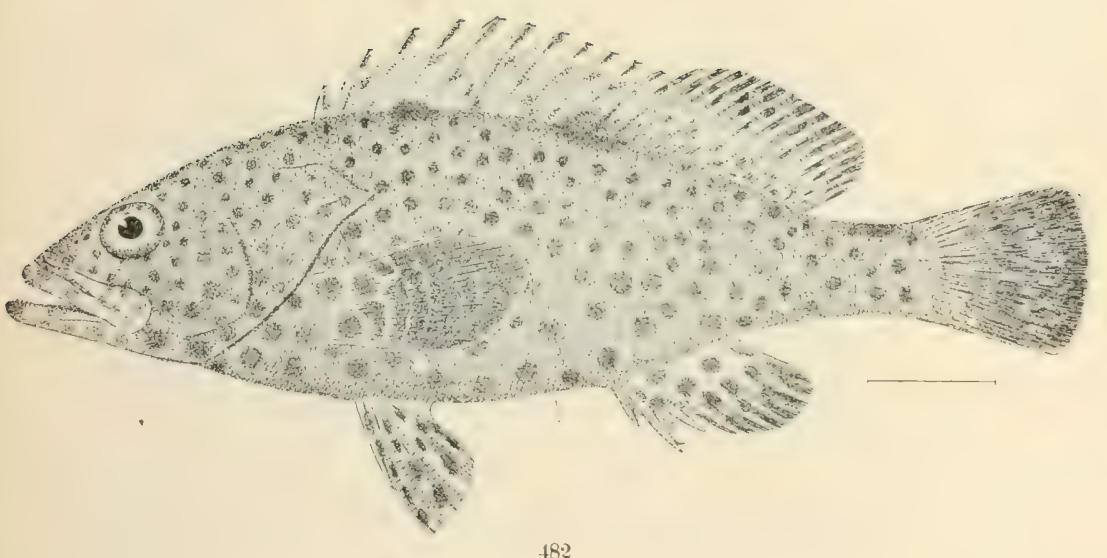

482

481. Bodianus fulvus punctatus. (P. 1146.)

482. EPINEPHELUS ADSCENSIONIS. (P. 1152.) 


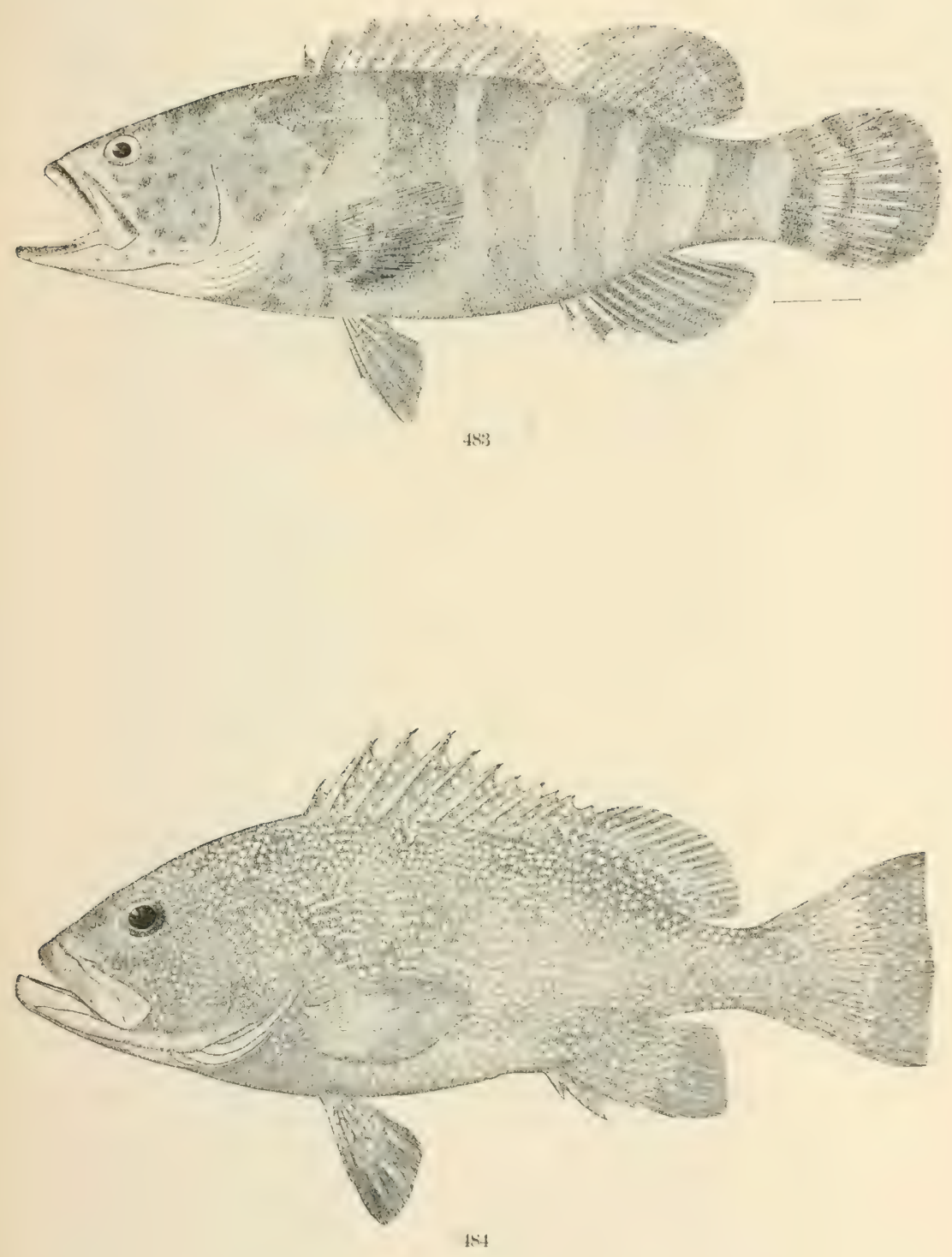

483. Einemielus striatus. (P. 1157.)

484. EPINEPHELUS DRUMMOND-IAYY. (P. 1159.) 


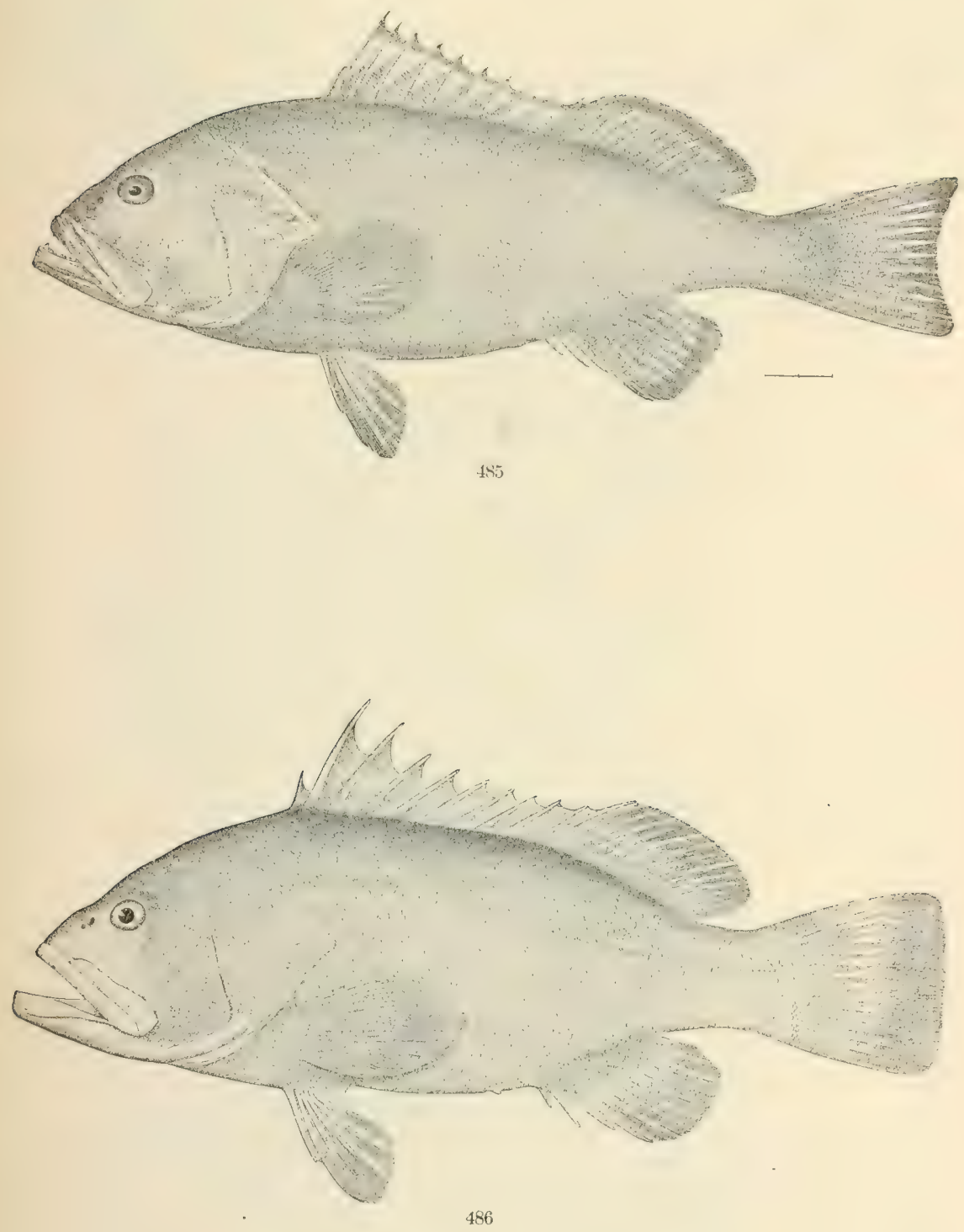

485. Epinephelus morio. (P. 1160.)

486. Garrupa nigitita. (P. 1161.) 


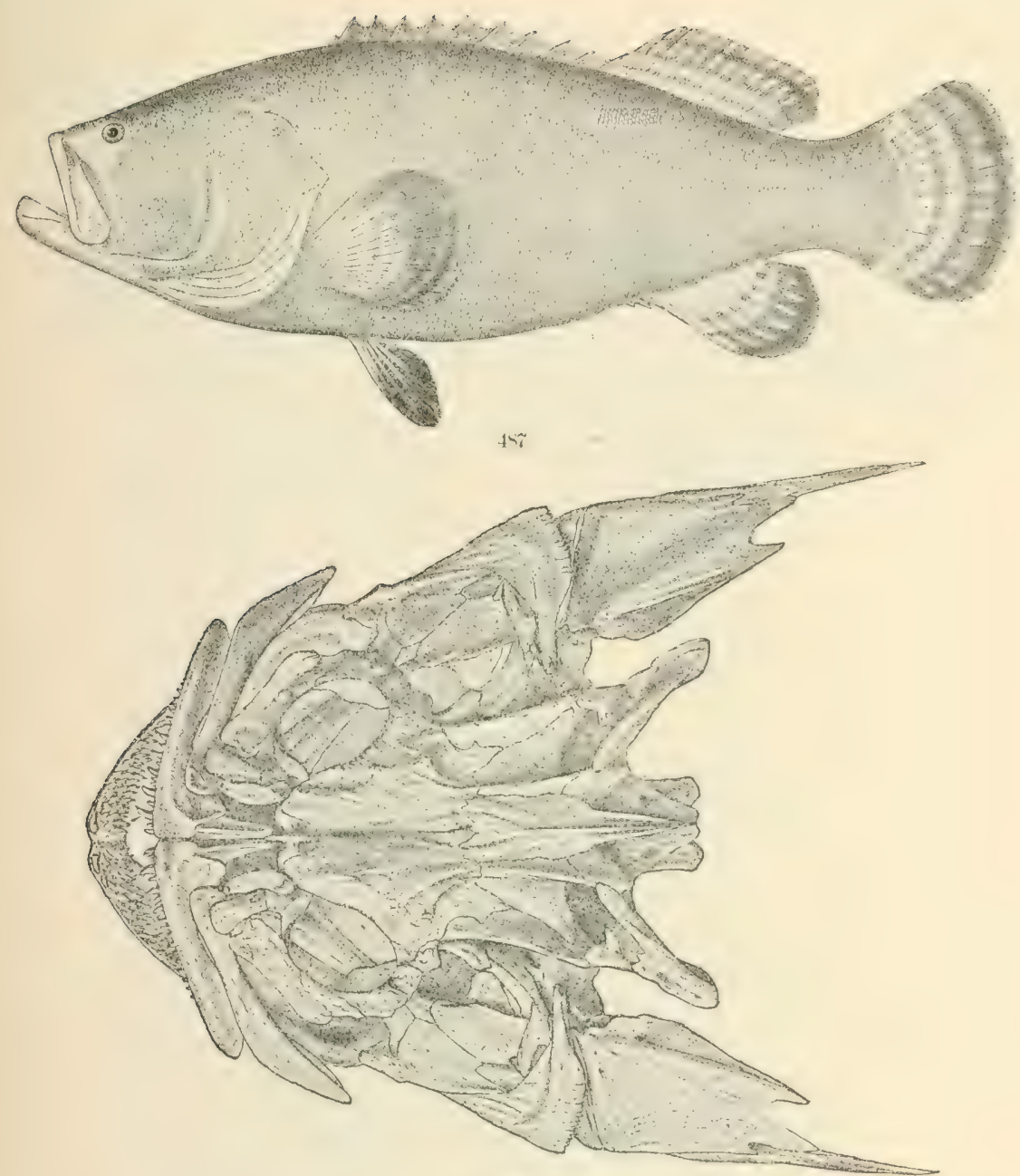

4 Sĩa

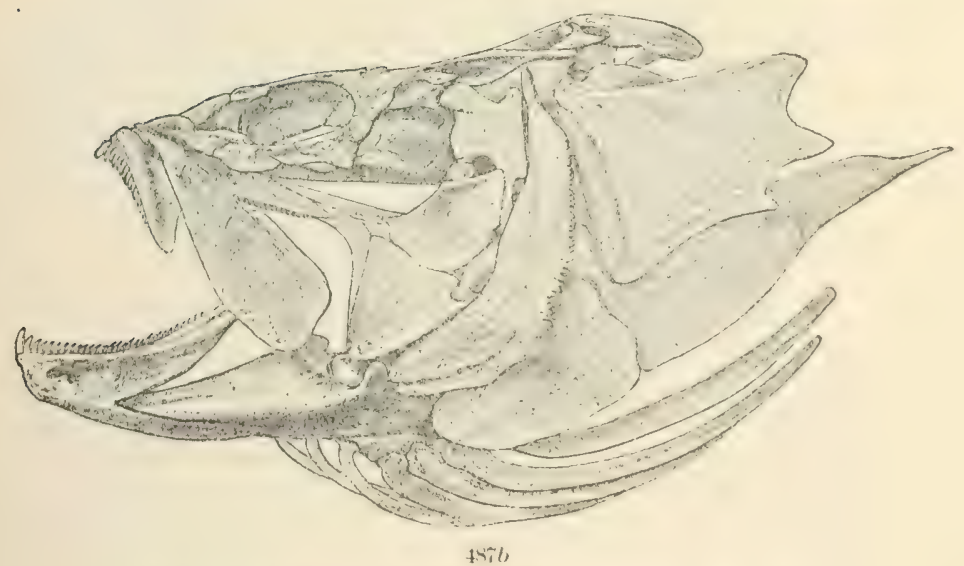

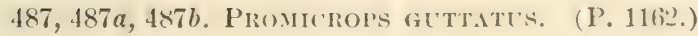





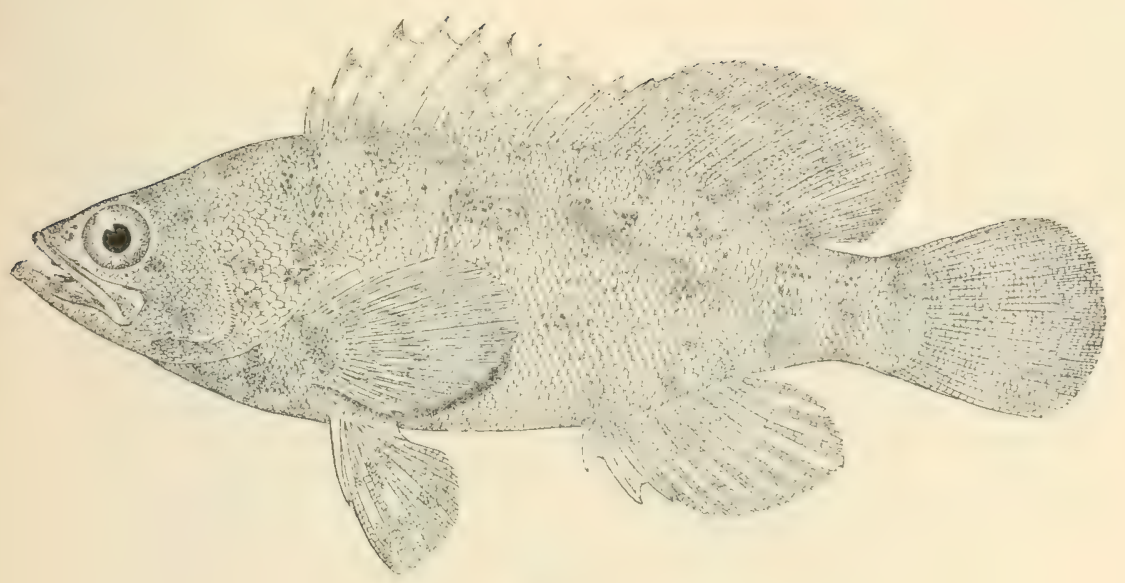

488
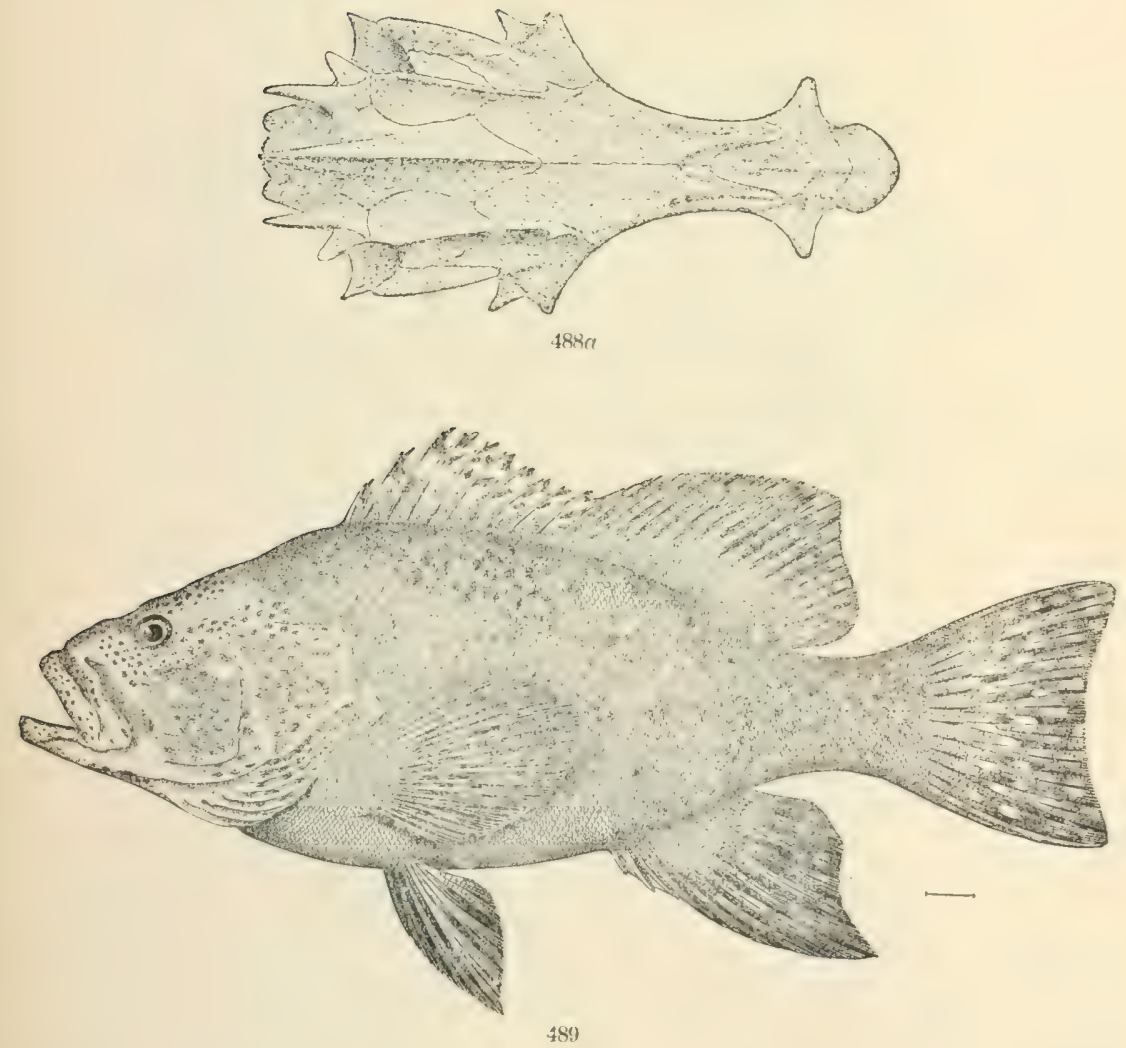

488. Alphestes afer. (P, 1164.)

488a. Shell of Alphestes AFER. (P. 1164.)

489. DERMATOLEPIS ZaNCLUS. (P. 2854.) 



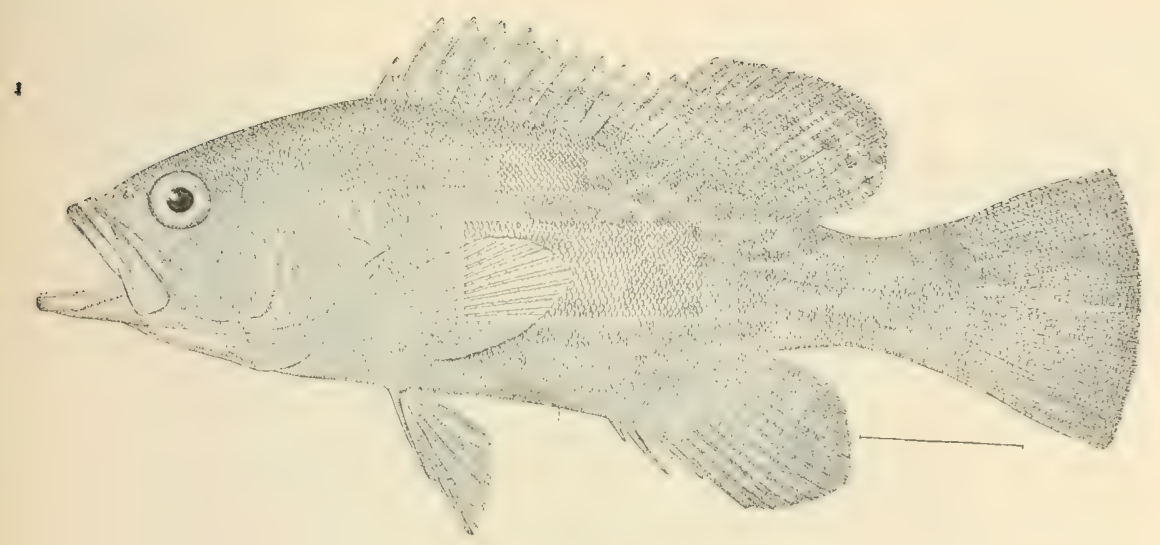

490

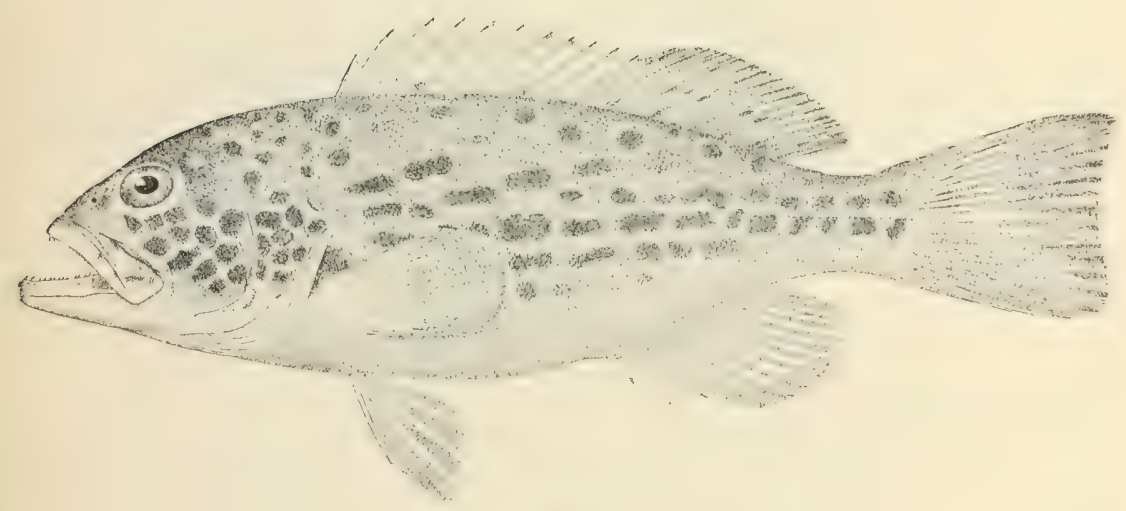

491

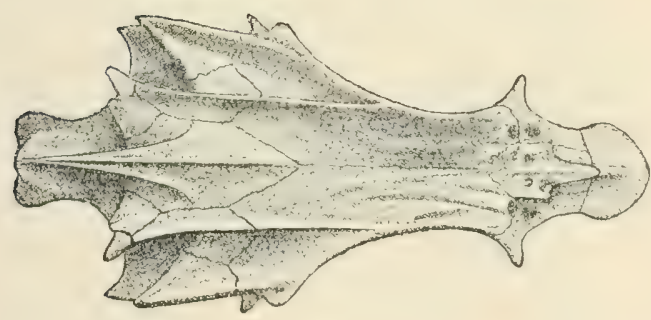

492

490. MYCTEROPERCA BOULENGERI. (P. 1171.)

491. Mycteroperca Venenosa. (P. 1172.)

492. SkUll of MYCTERoperca Bonaci. (P. 1174.) 


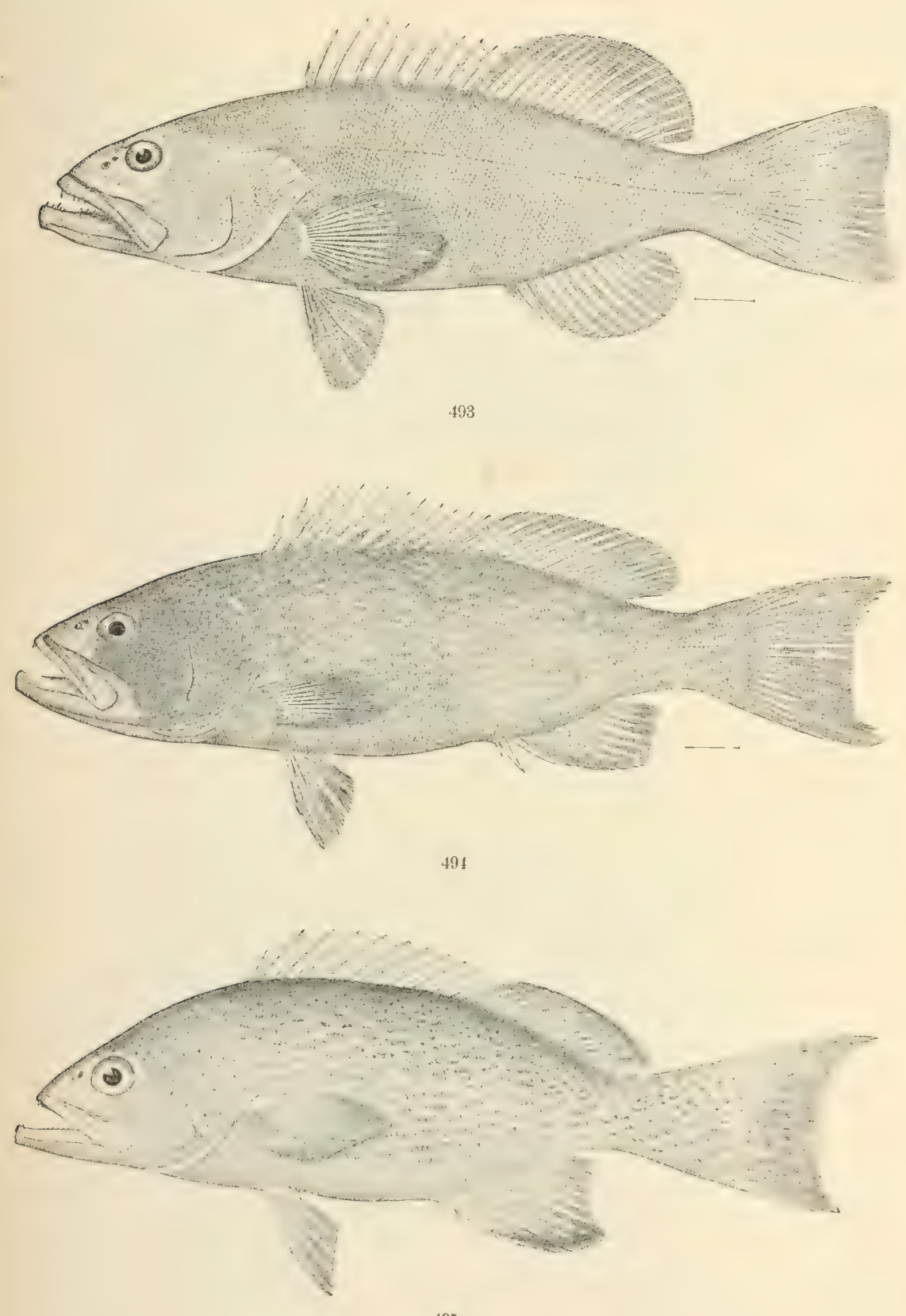

195

493. MYCTEROPERCA JORDANI. (P. 1176.)

494. MYCTEROPERCA MICROLEPIS. (P, 1177.)

495. MYCTEROPERCA FALCATA PHENAX. (P. 11N, ) 



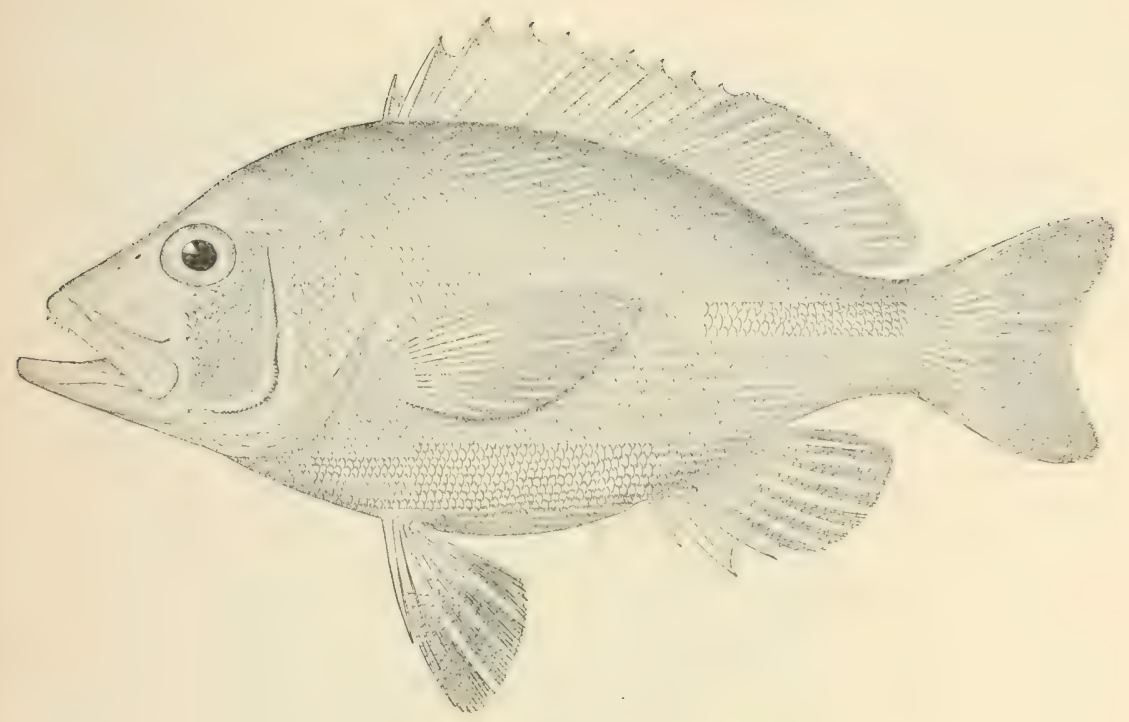

$496^{\circ}$

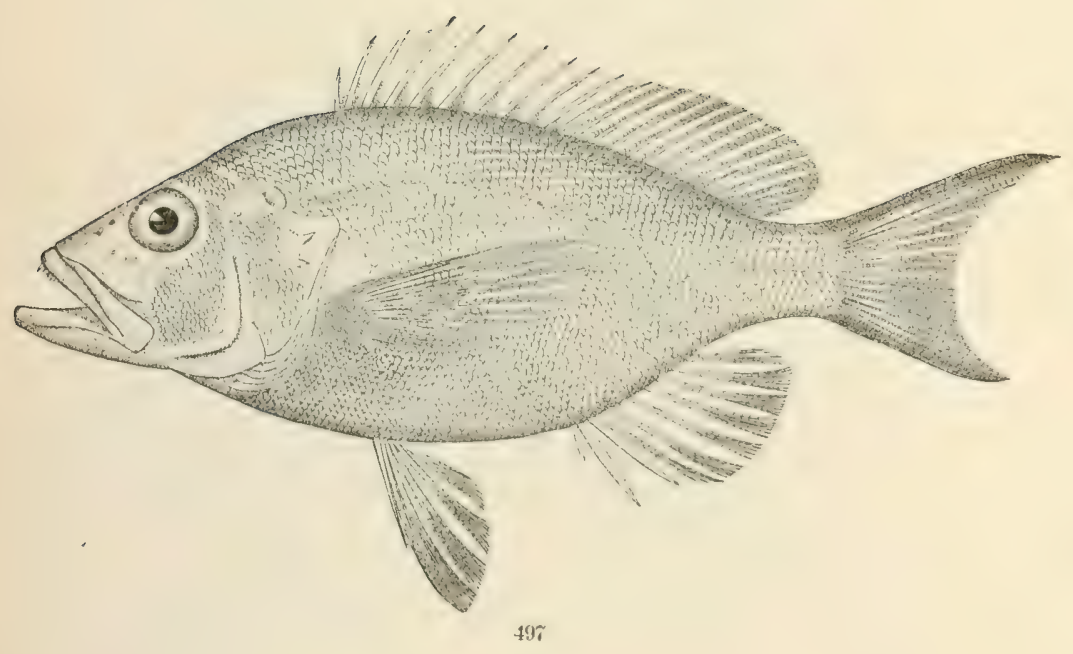

496. HYPOPLECTRUS UNICOLOR NIGIICANS. (P. 1193.)

497. Hy roplectrus GeMma. (P. 1193.) 



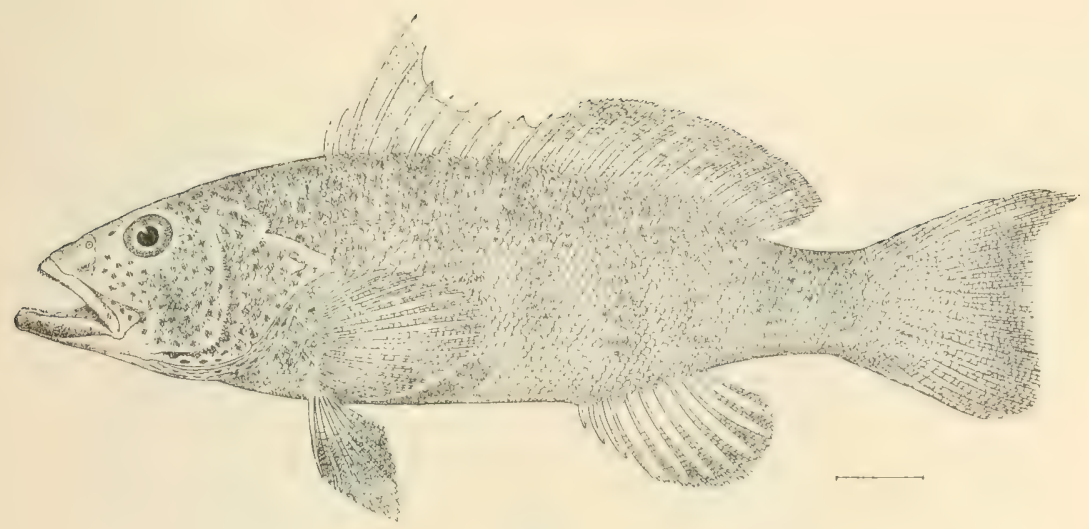

498

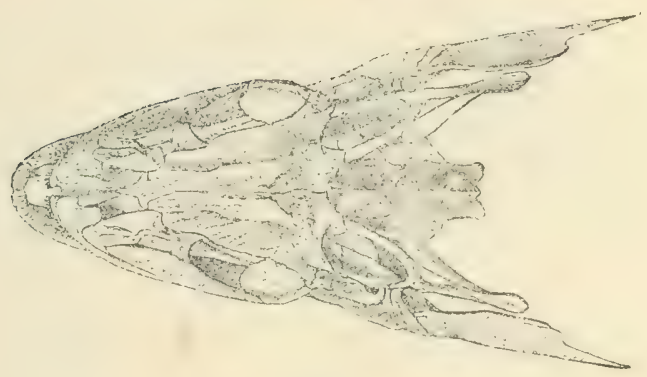

199

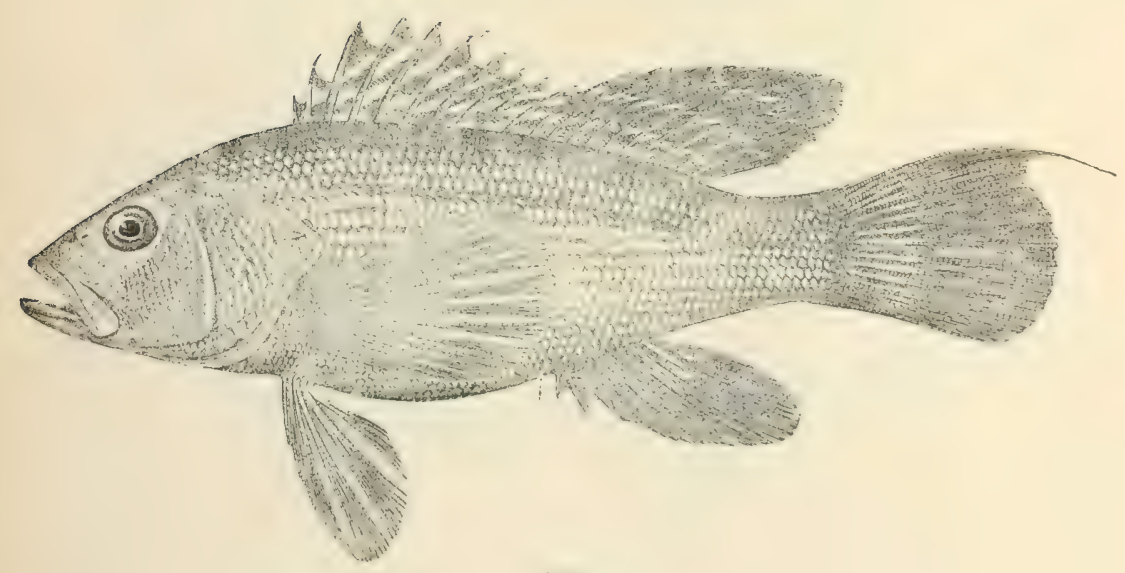

i(k)

498. Paralabrax maculatofasciatus. (P. 1196.)

499. PaliaAirax huMeralis. (P. 1196.)

500. Centropristes striatus. (P. 1199.) 



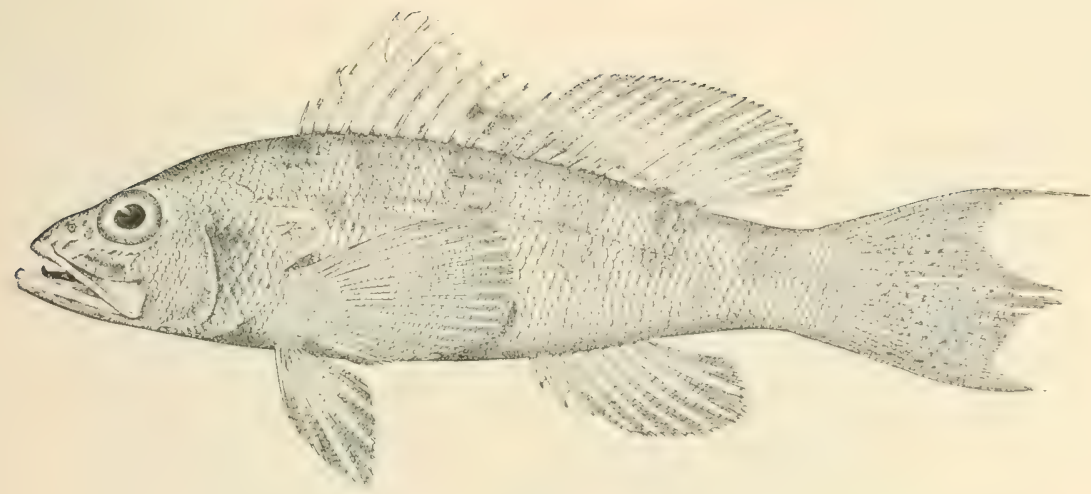

501

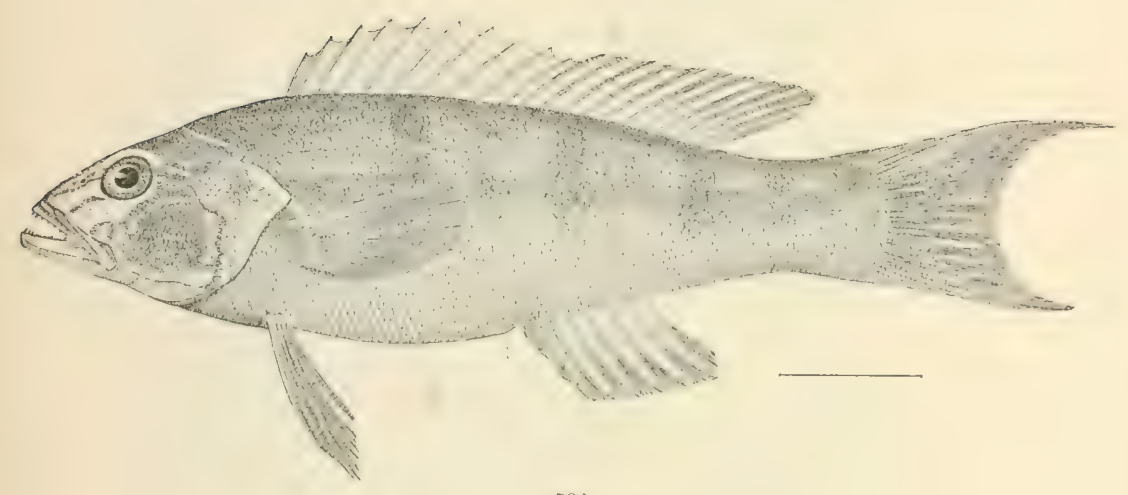

502

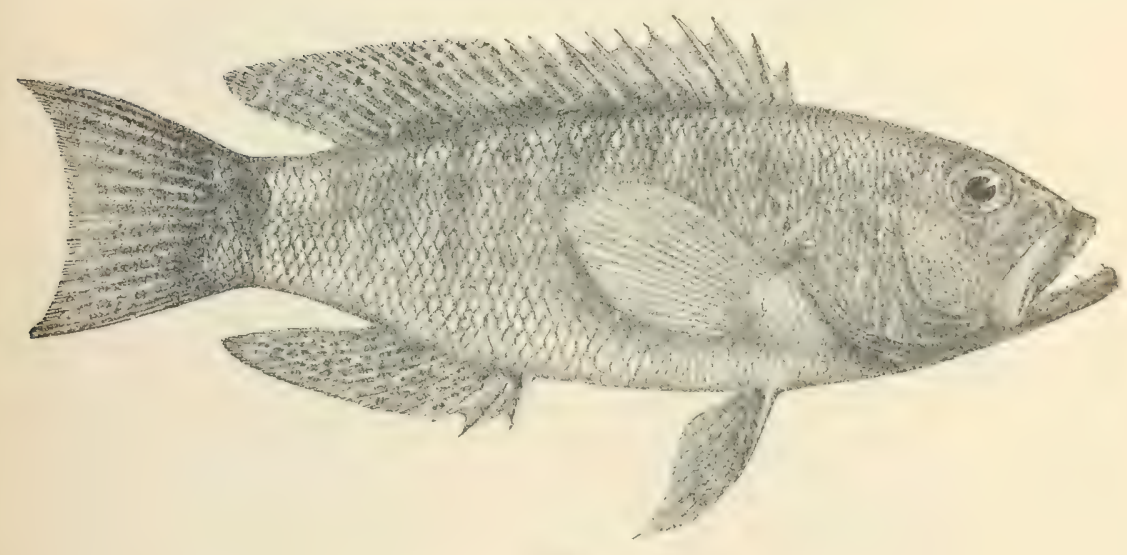

501. Centropristes PHLAdelphicus. (P. 1201.)

502. DIPLECTRUM FORMOSUM. (P. 1207.)

503. PRIONODES BLLLERI. (P. 1213.) 


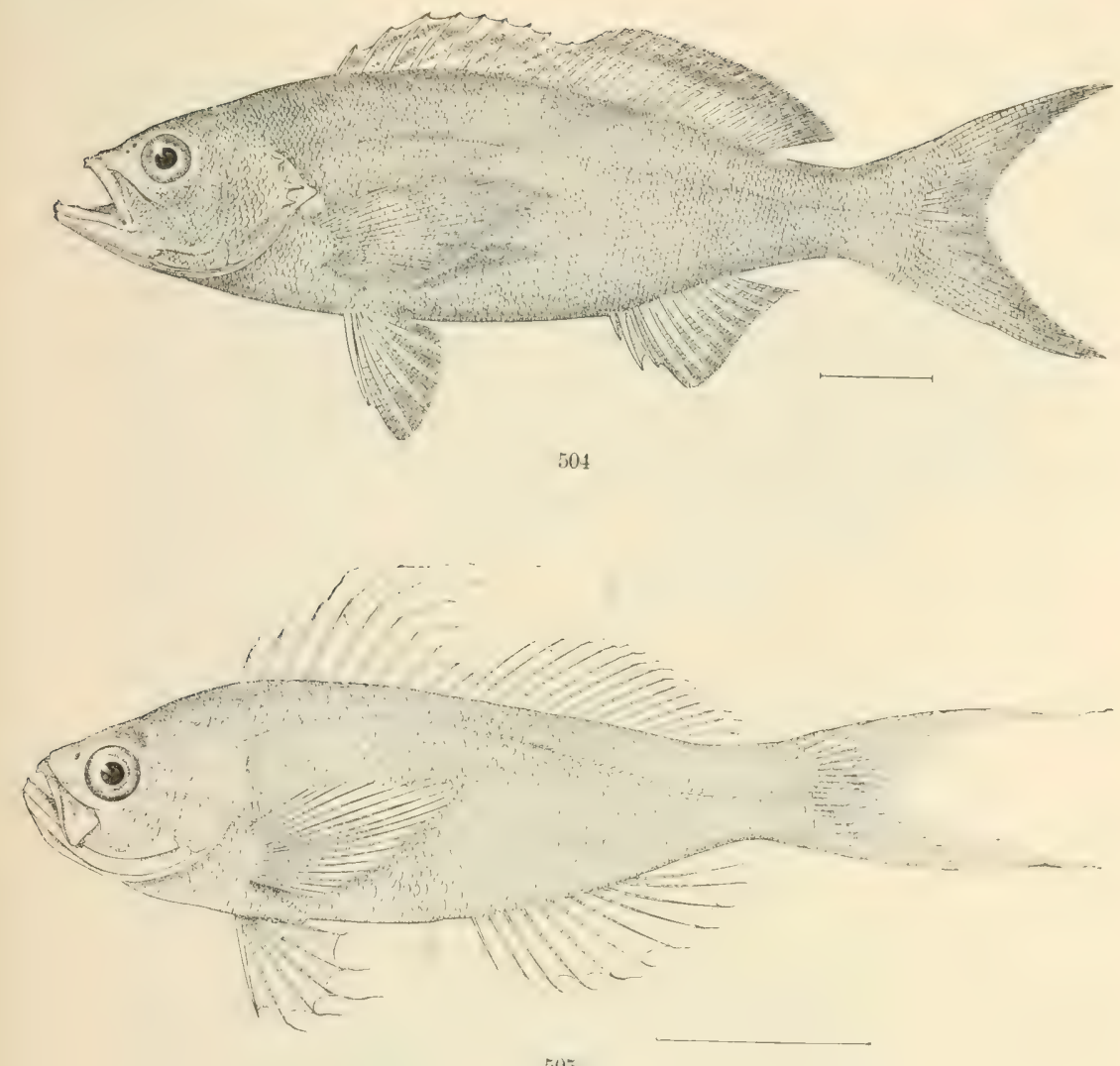

50.5

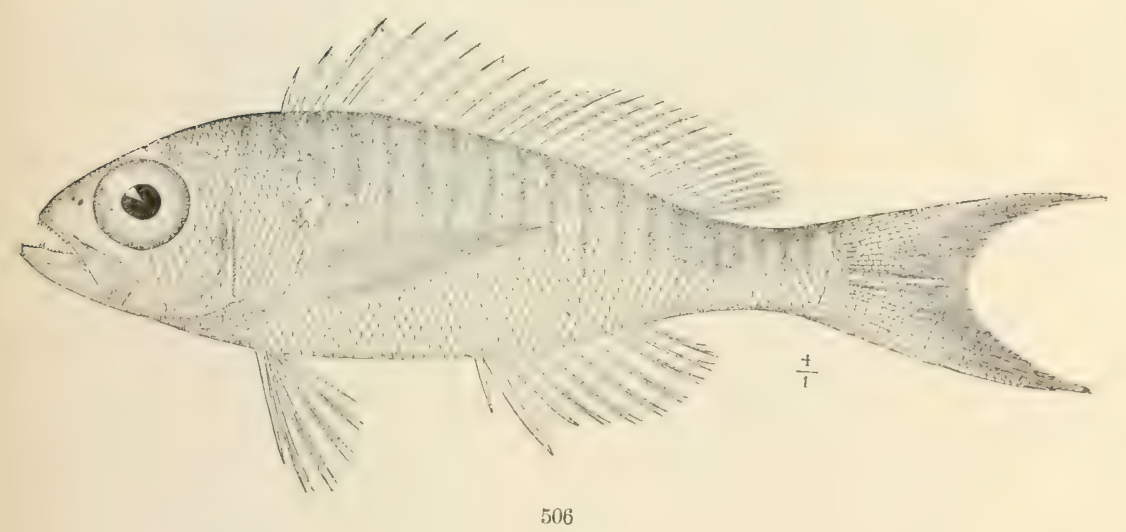

504. Parantilias furcifer. (P. 1221.)

505. HeMintinias Vivanus (P. 1223.)

506. Pronotogramals multifasciatus. (P. 1226.) 



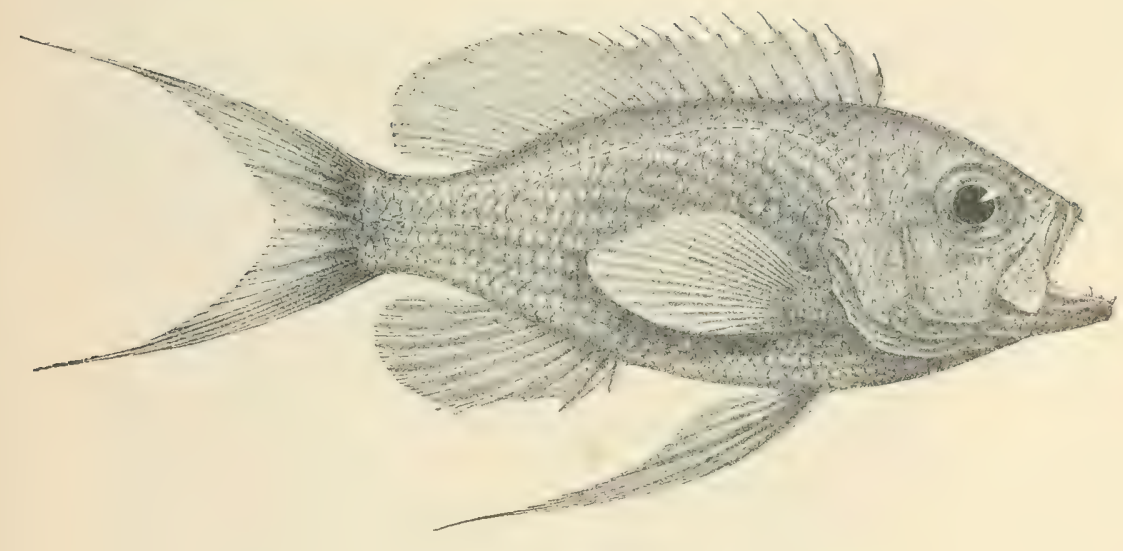

$j 0 \tilde{6}$

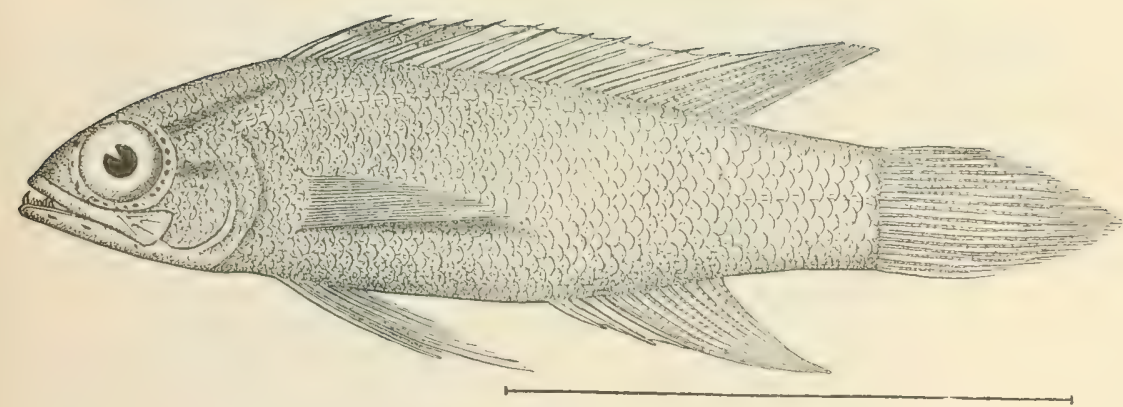

508

507. Anthias asperilinguis. (P. 1227.)

508. Gramia loreto. (P. 1229.) 



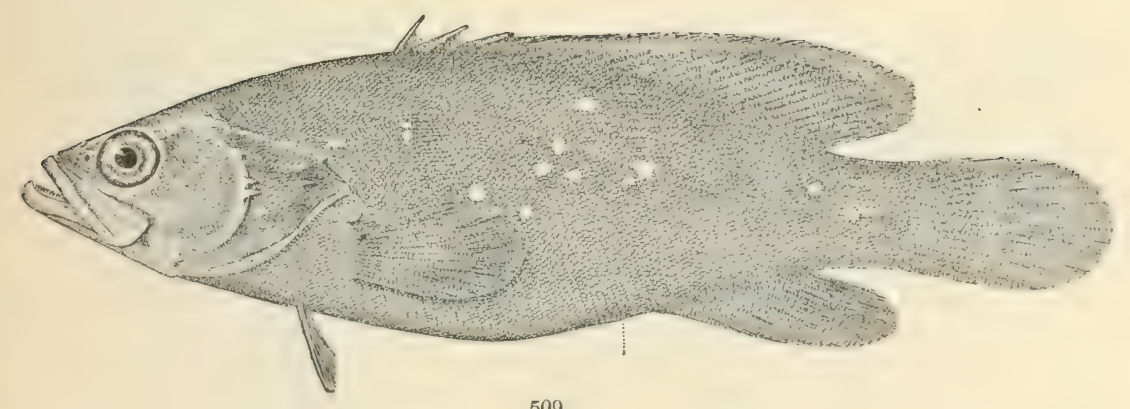

509

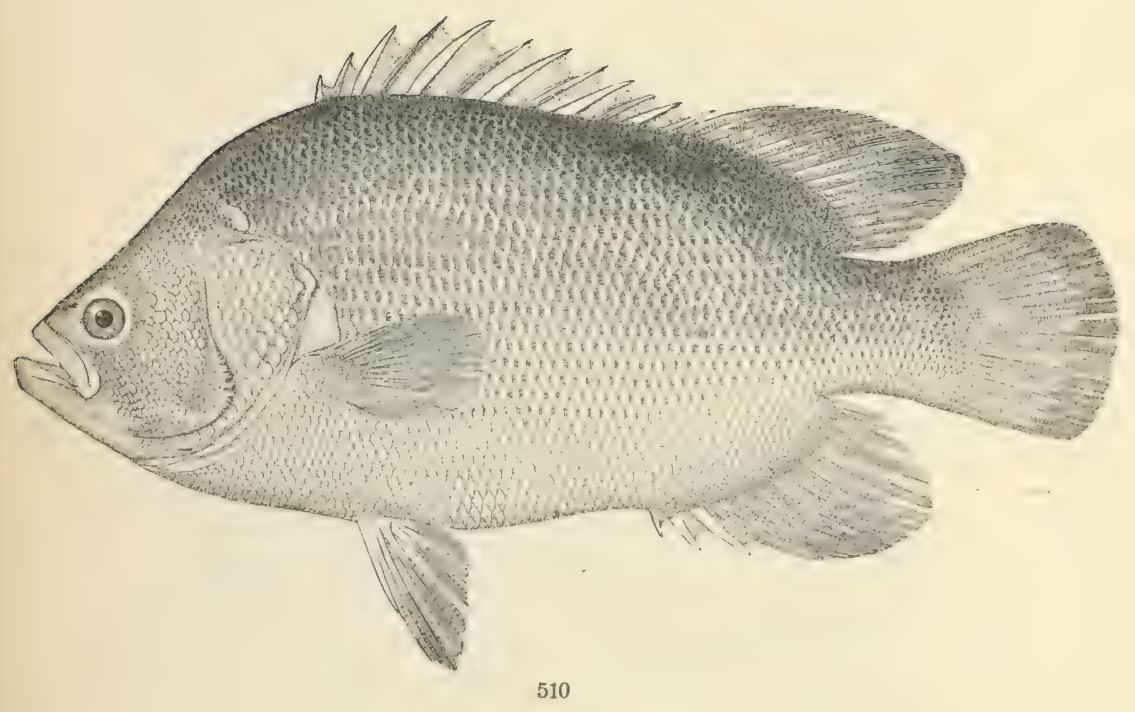

509. RYPTICUS BISTRISPINUS. (P. 1233.)

510. LOBOTES SURINAMENSIS. (P. 1235.) 


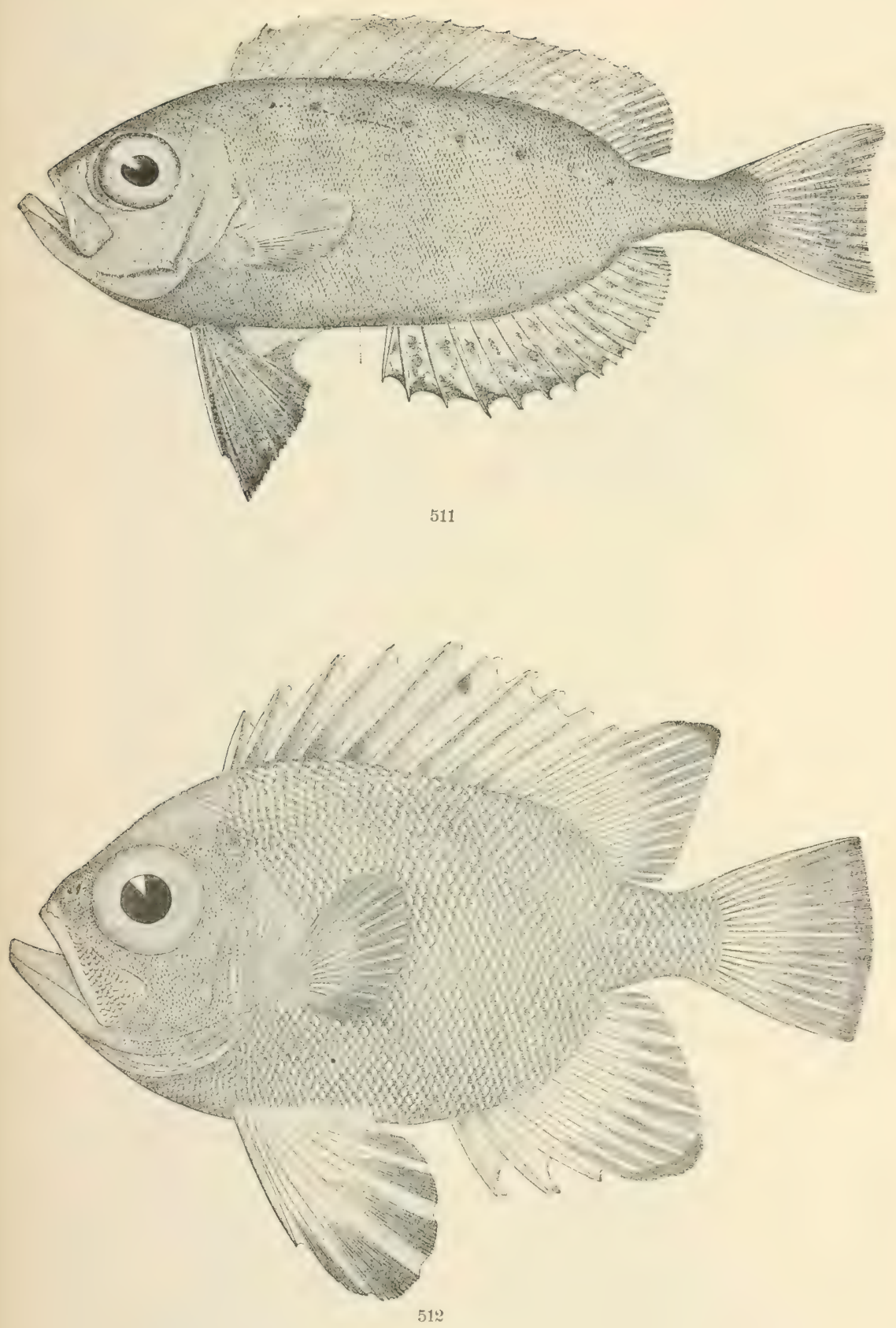

511. Priacanthus arenatus. (P. 1237.)

512. Pseudopriacanthis altus. (P. 1239.) 


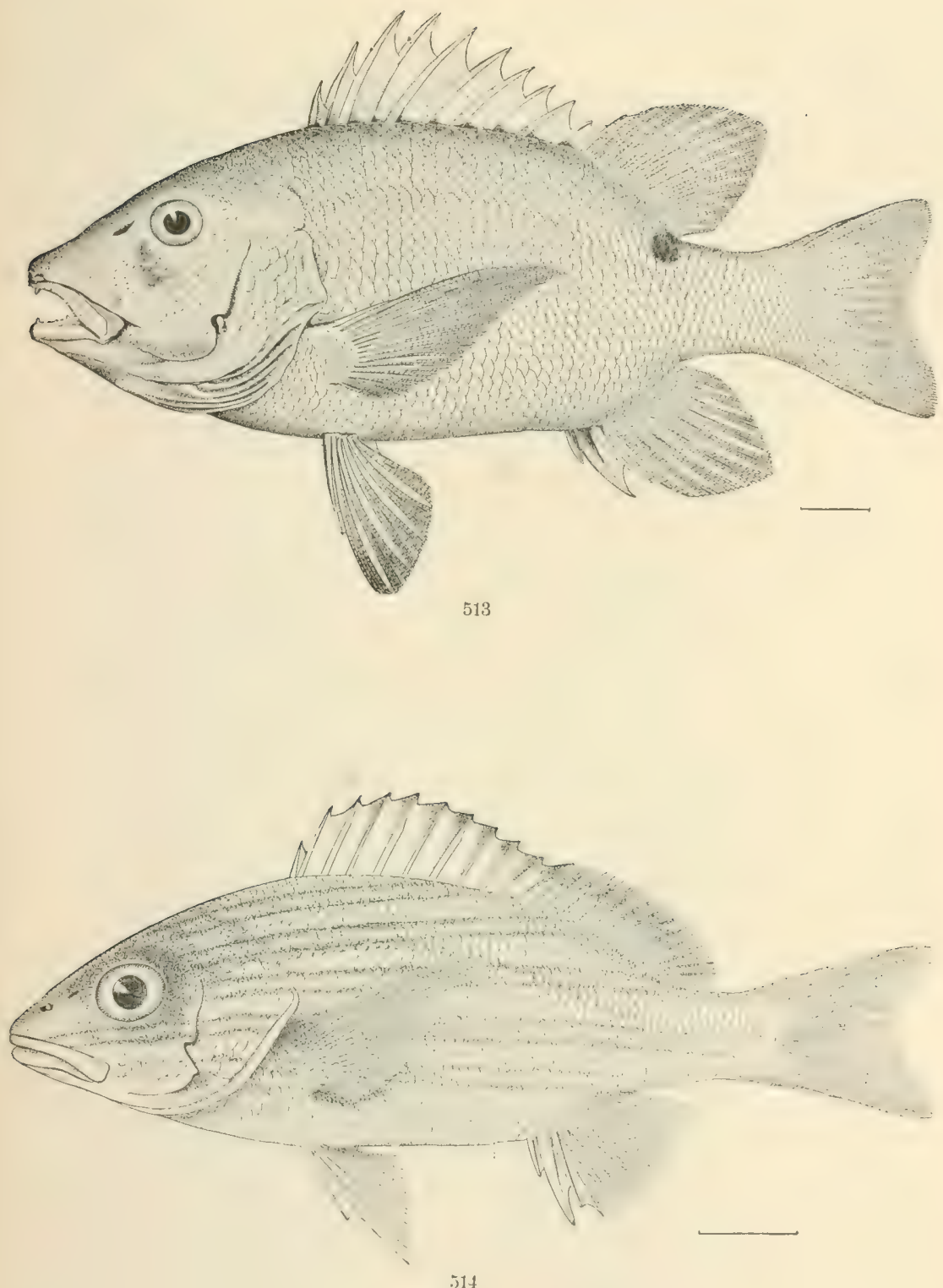

513. Hoplopagrus guntheri. (P. 1244.)

514. EvorLITES VIRIDIS. ( $1,1246$. 



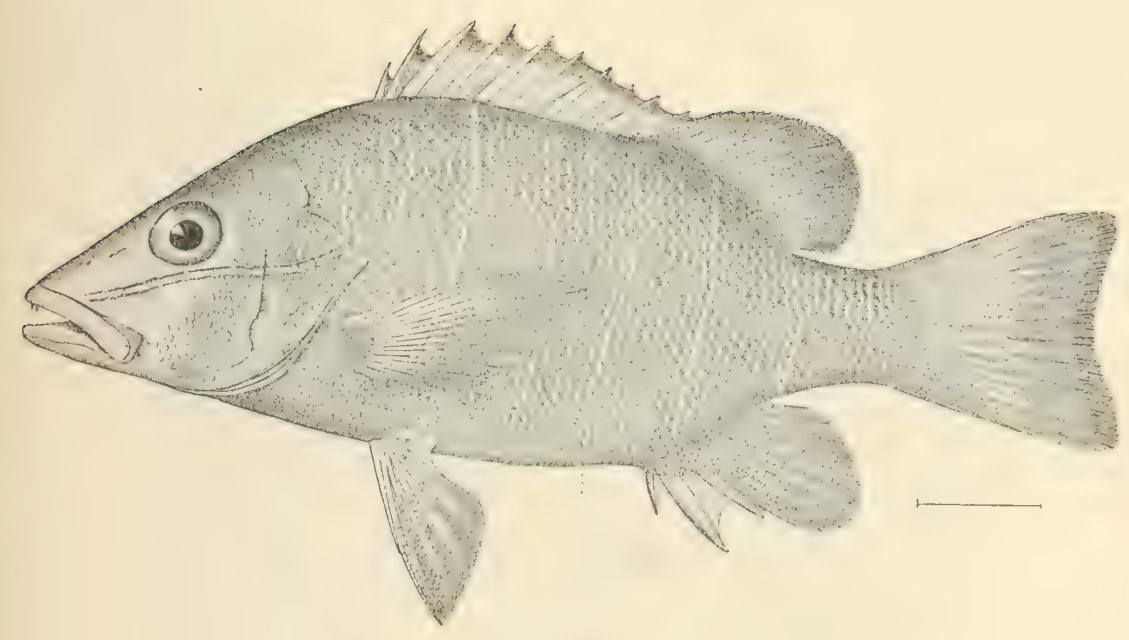

515

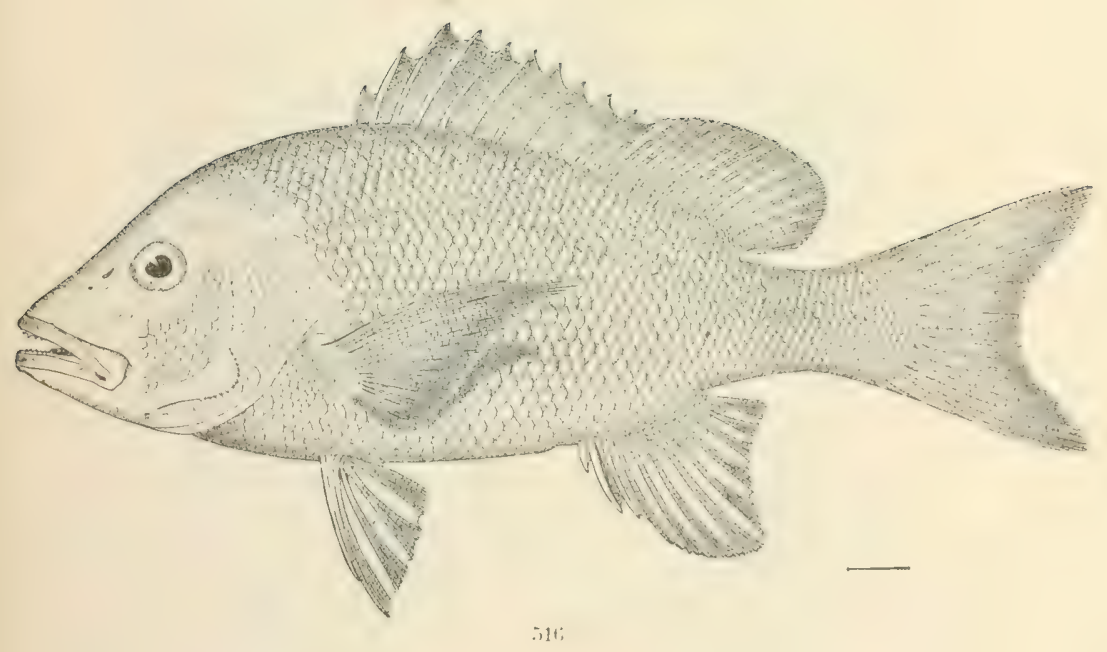

515. NEOMENIS APODUS. (

516. NEOMENIS AYA. (P. 1261.) 



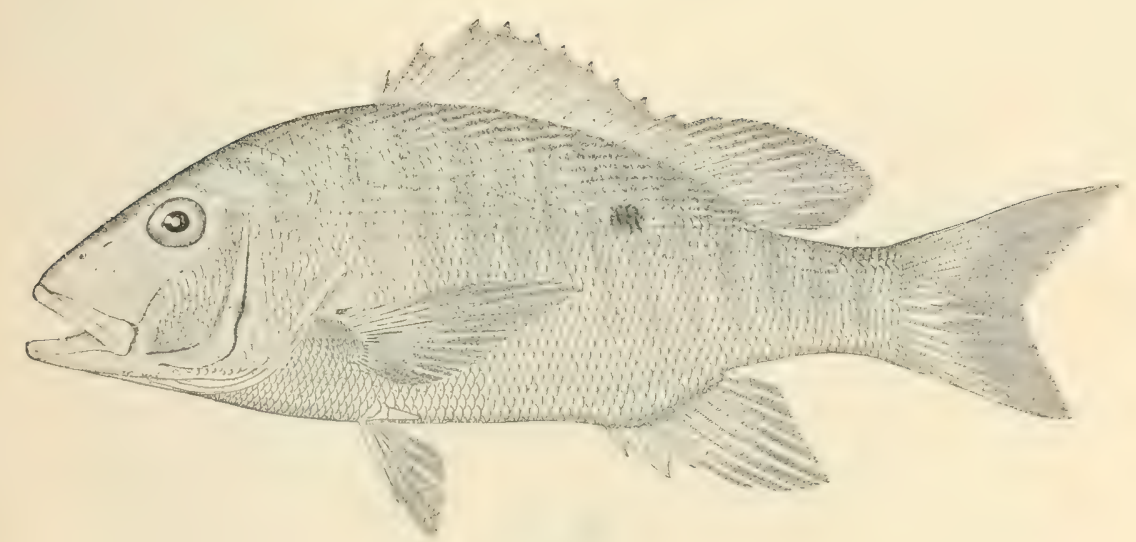

$51 \%$

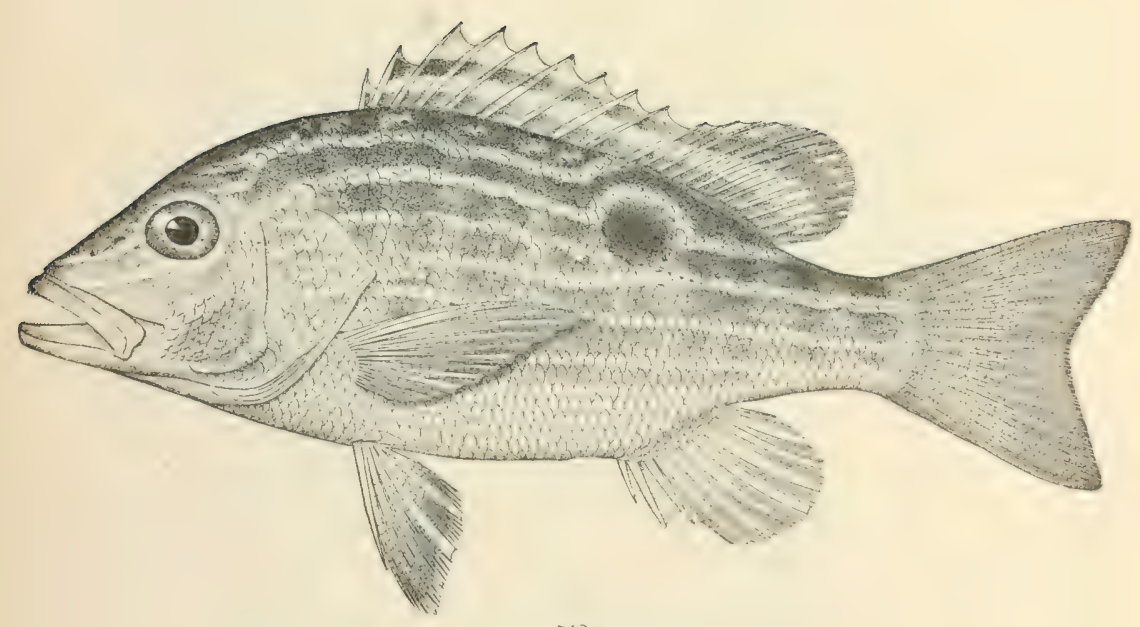

$\$ 18$

517. Neom enis analis. (P. 1265.)

518. NEOMENIS SYNAGRIS. (P. 1270.) 



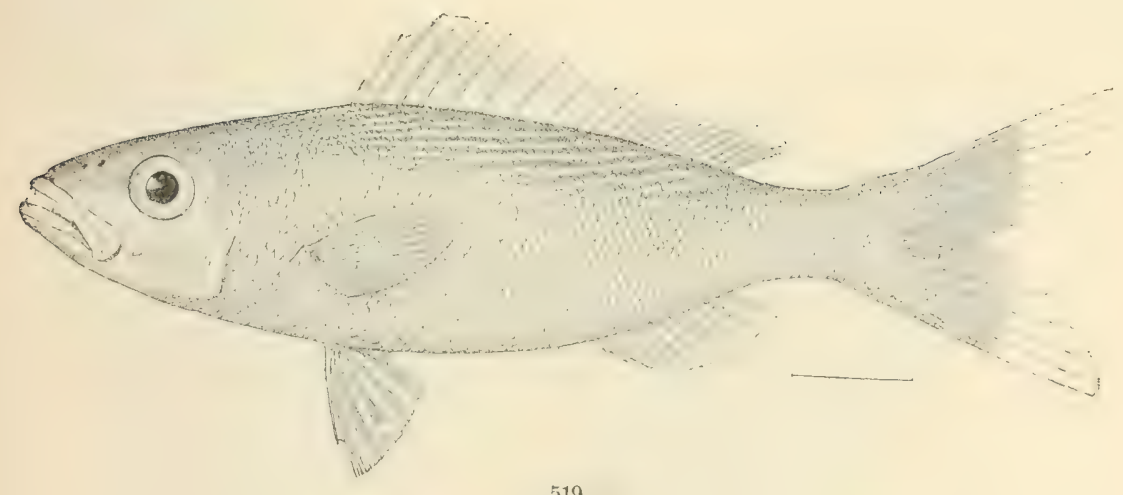

519

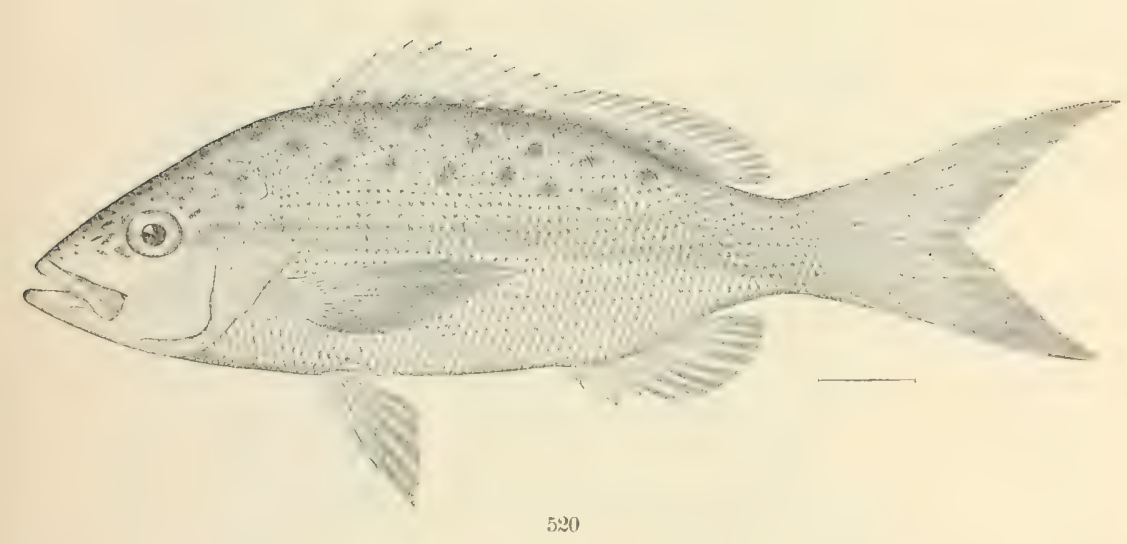

519. Ramirubia inerists. (P. 1:74.)

520. OCYURUS CIRYSURUS. (P.1275.) 

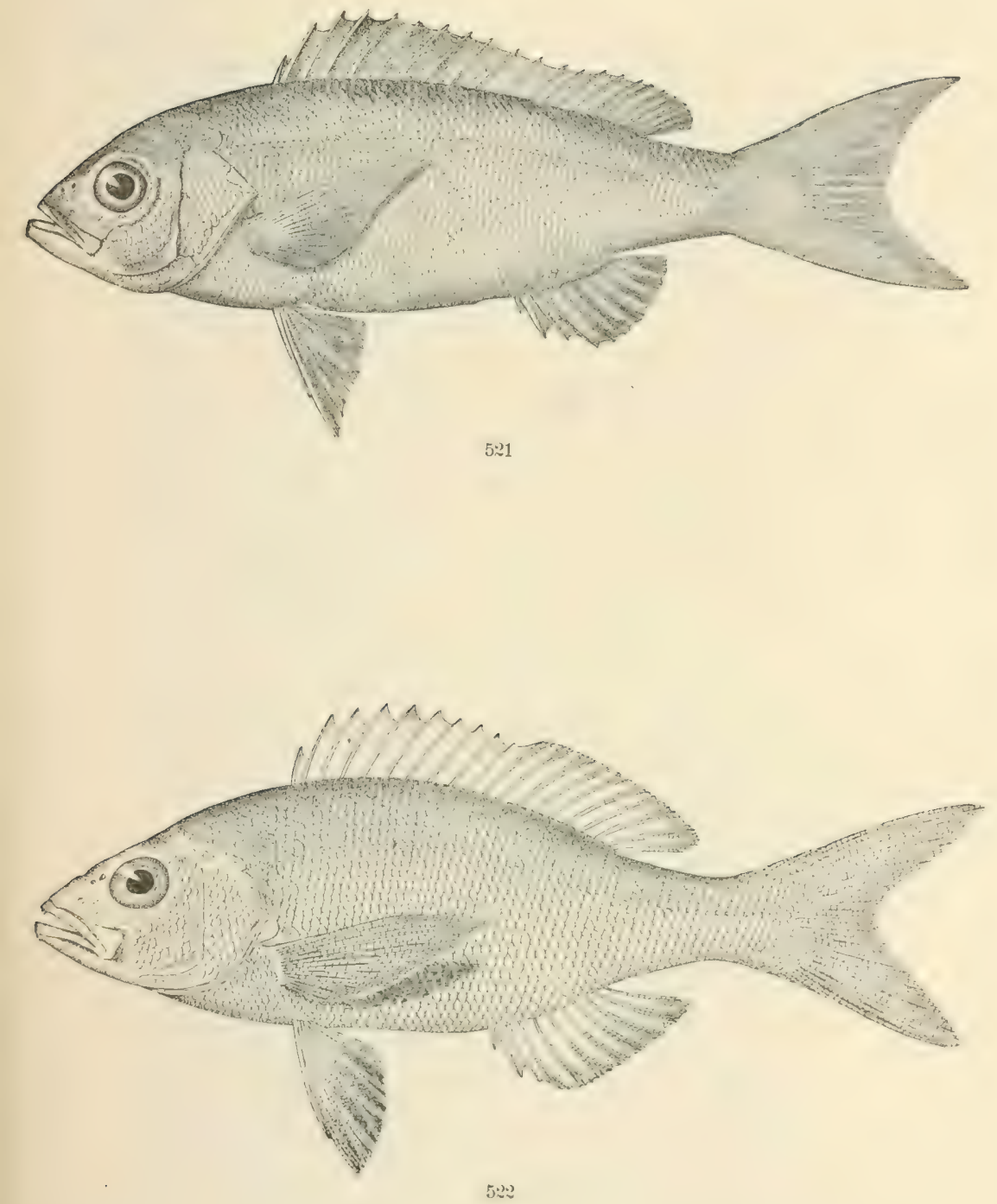

521. RHomborlites aURorUbens, (P, 1277.)

522. Apsilus Dentatus. (P. 1278.) 



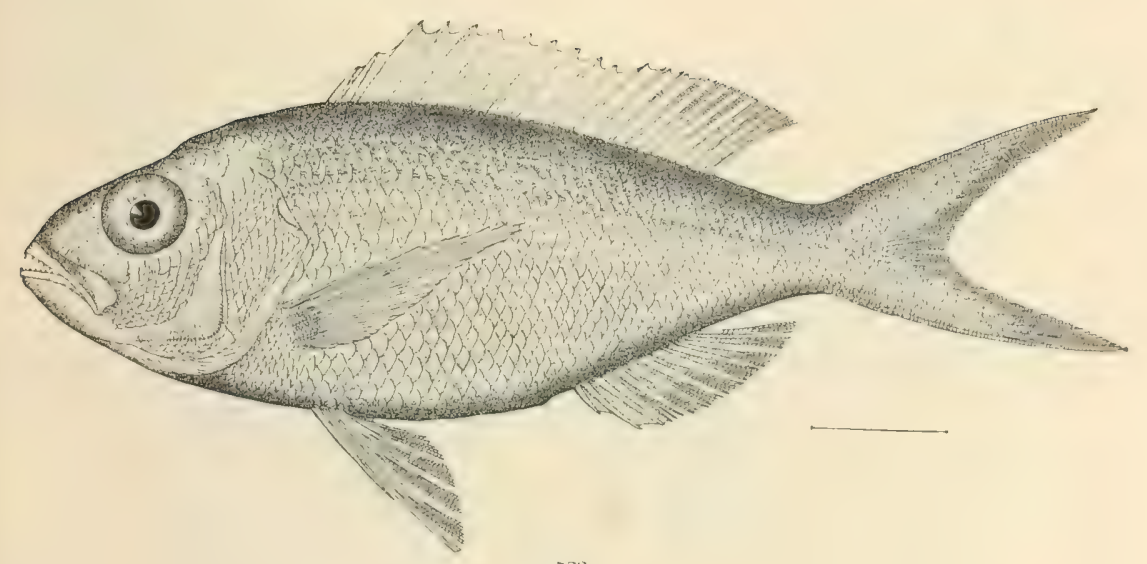

523

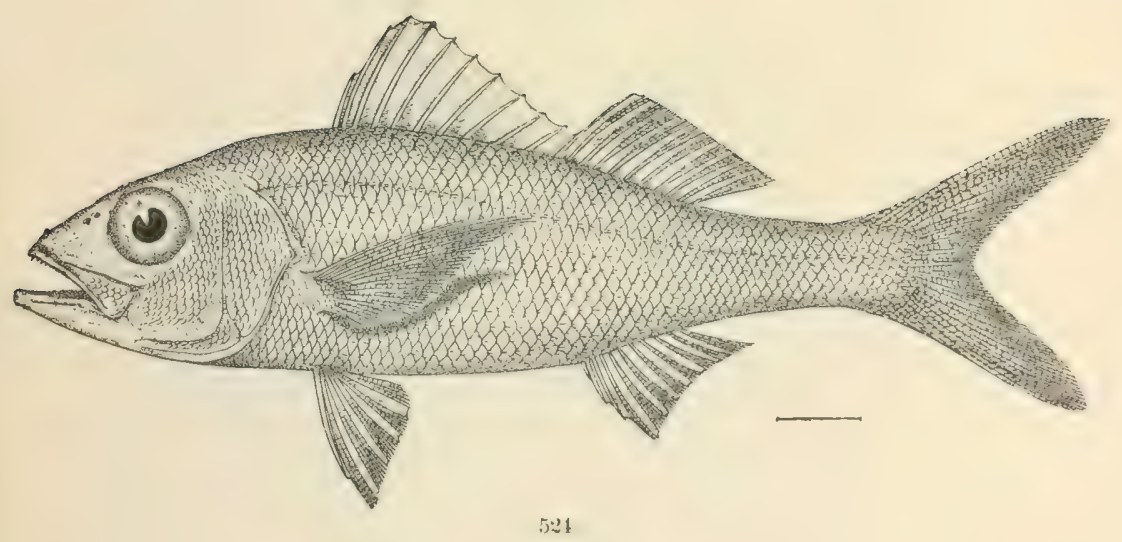

523. Aprion mackophrhalidus. (I’, 1280.

524. Etelis oculatus. ( 


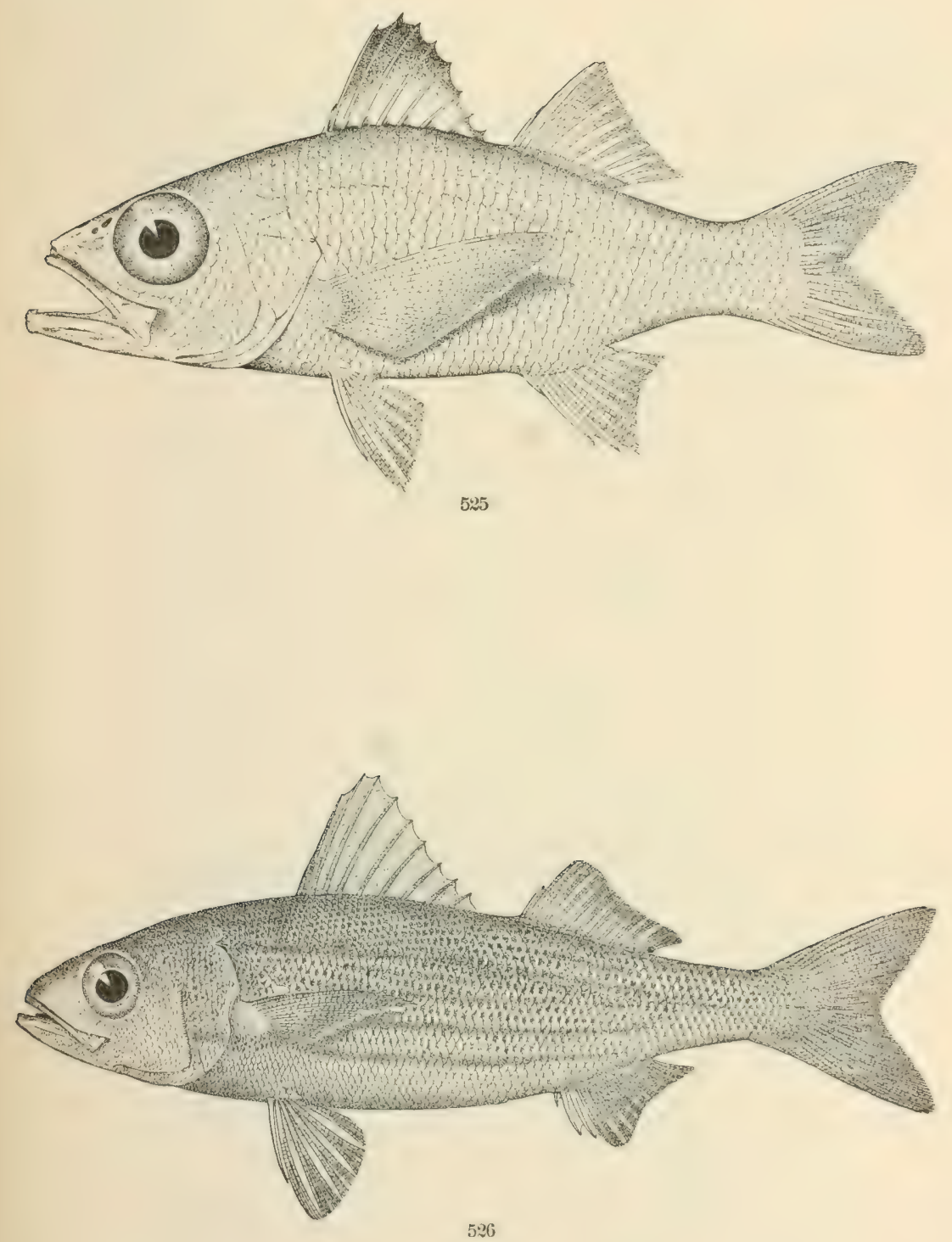

525. Verilus SORDIDUS. (P. 1284.)

526. XENOCYS JESSIA. (P. 1285.) 



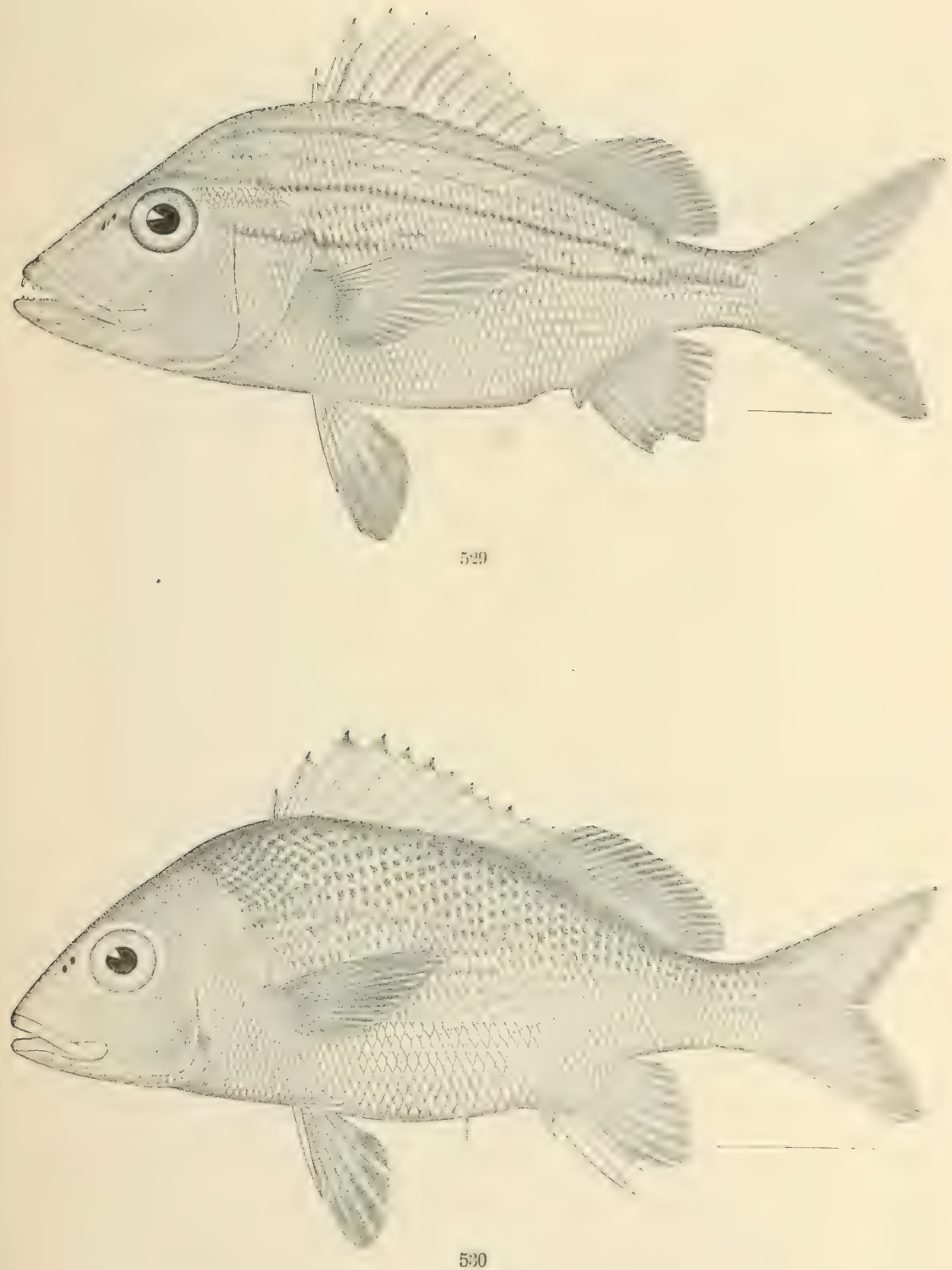

529. H EMULON MACROSTOMUM. (P. 1296.)

530. HEMULON PARRA. (P. 1297.) 



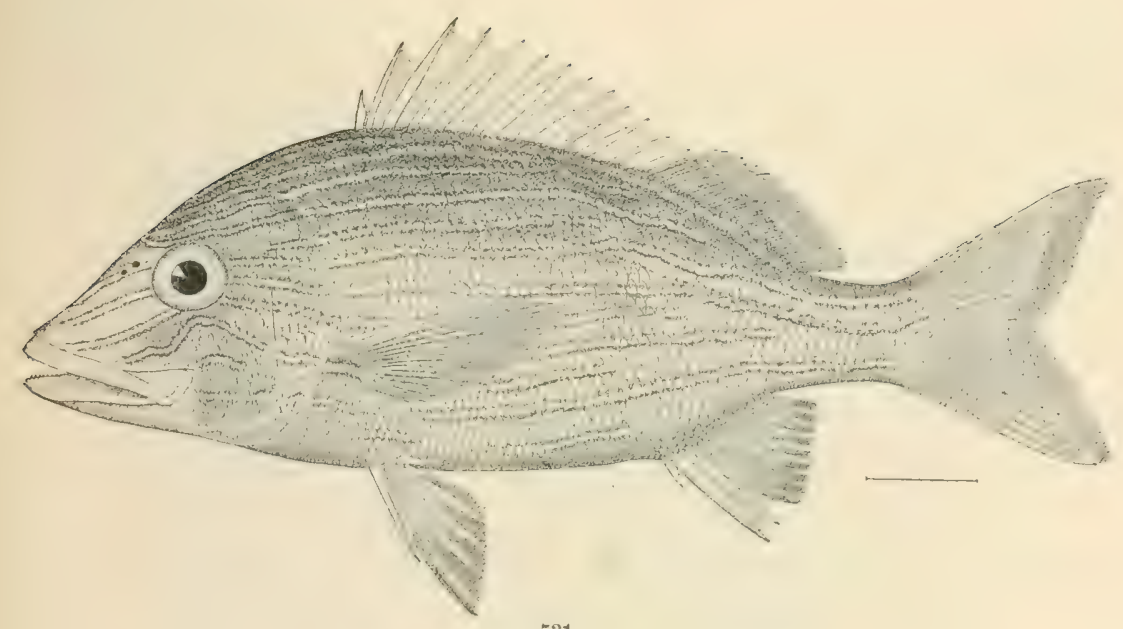

531.

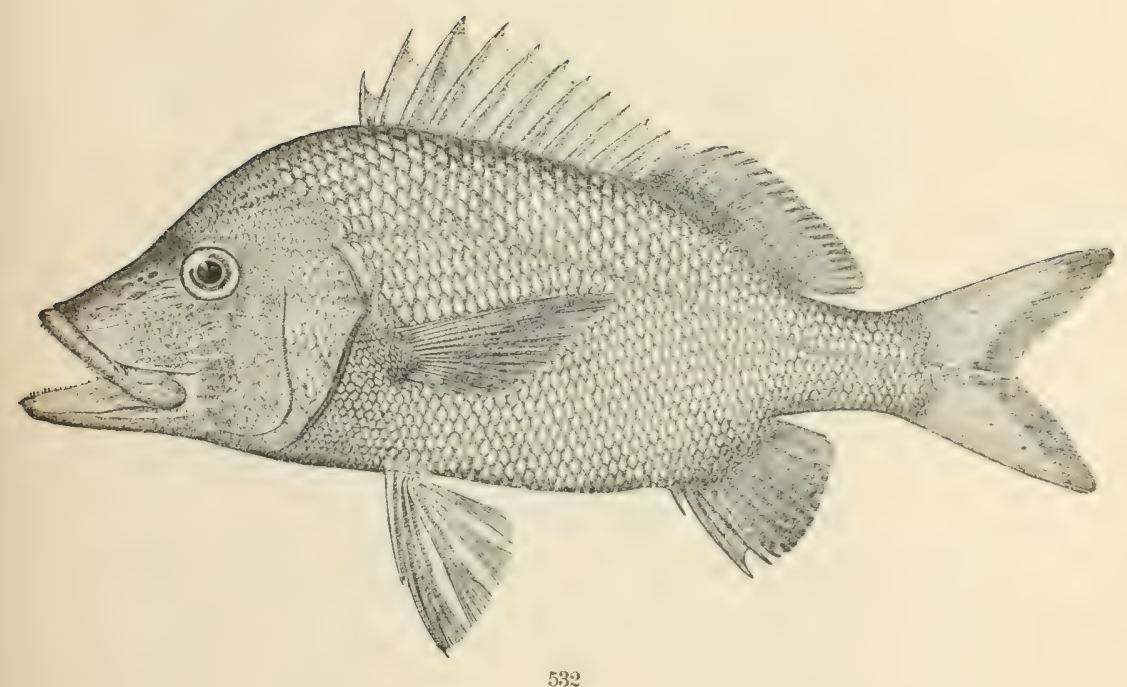

531. HEMULON SCIURYS. (P. 1303.)

532. HEMULON PLUMIERI. (P. 1304.) 


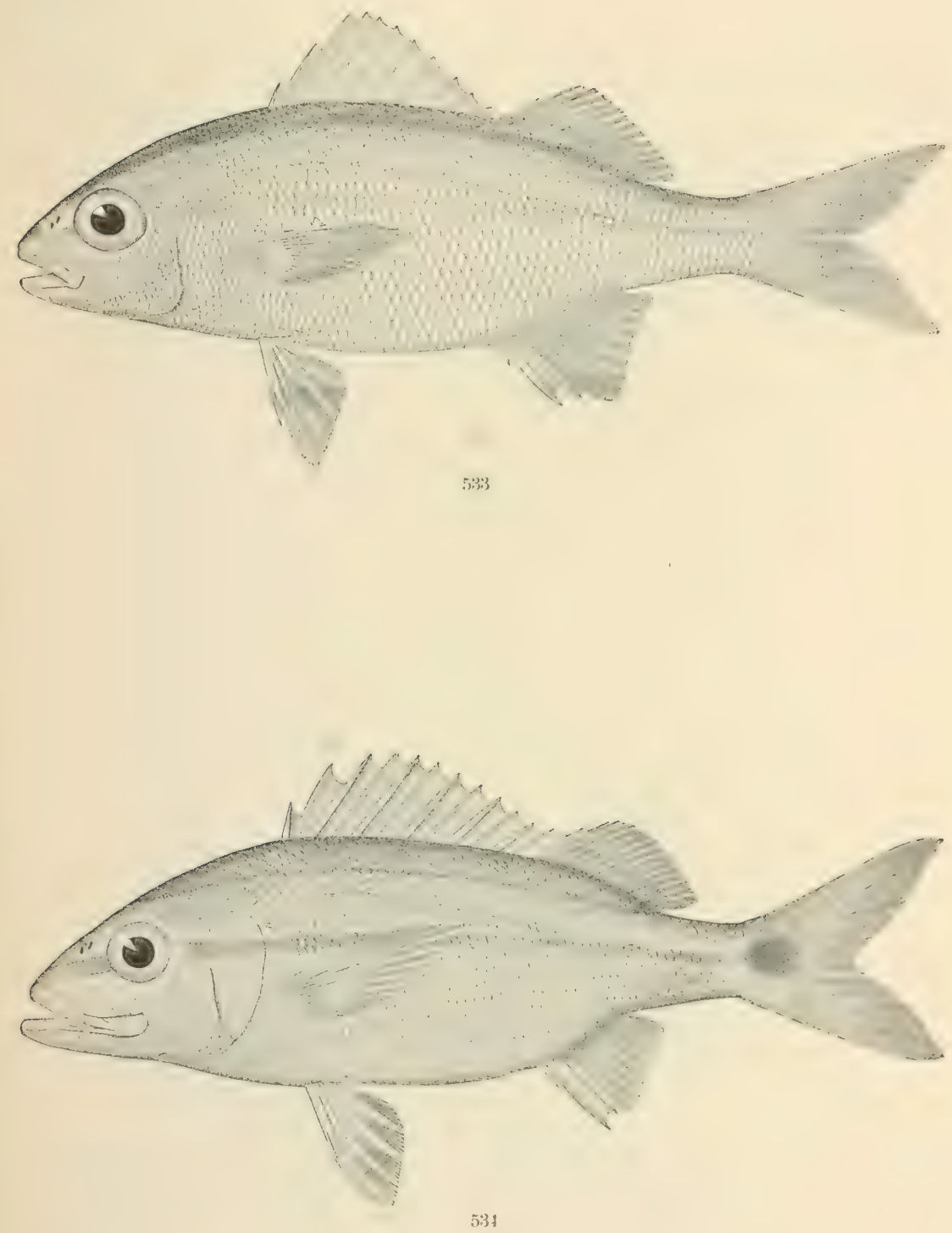

533. Brachygenys Chrysargyreus. (P. 1307.)

534. Batiystoma minatol. (P. 1308.) 


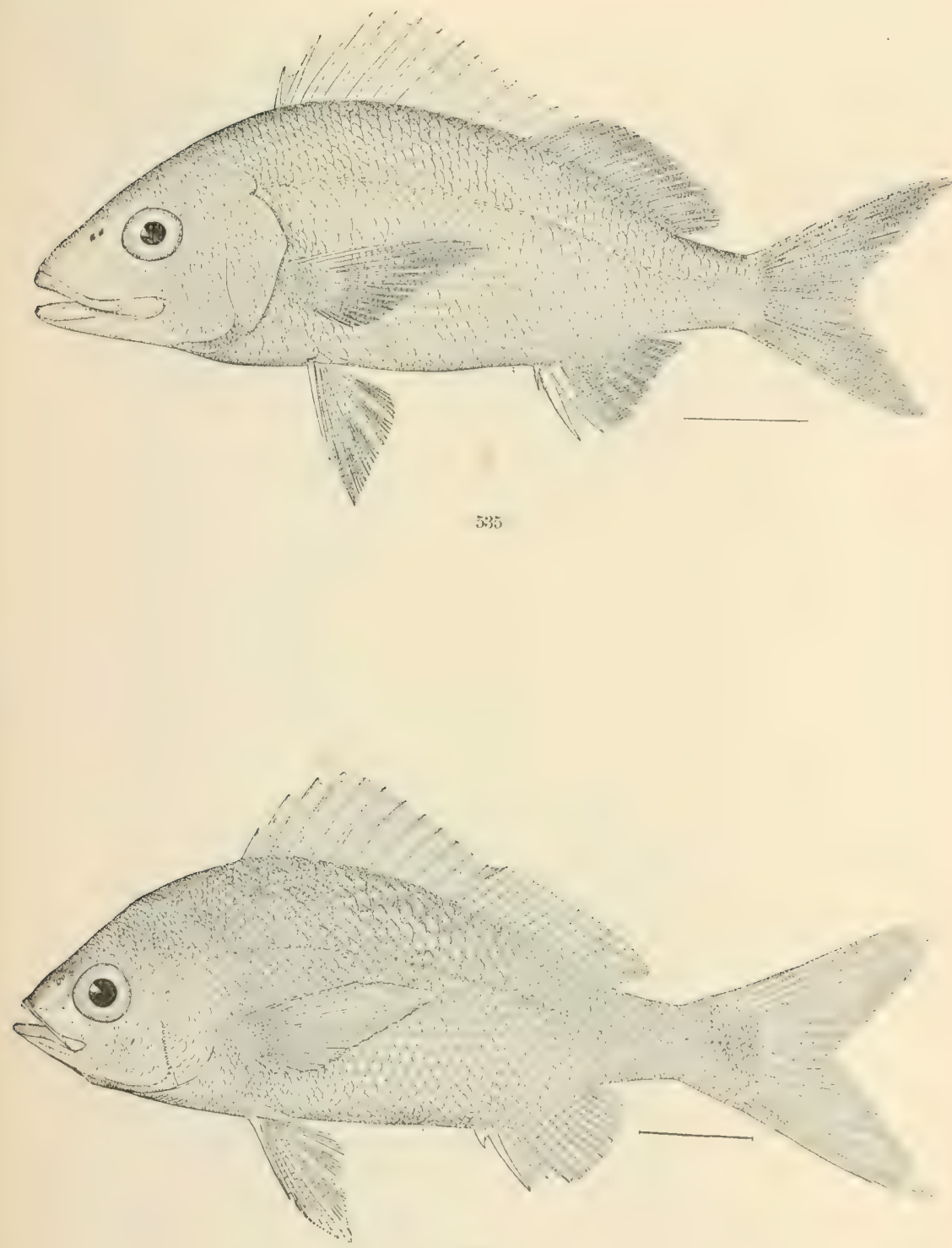

$536^{3}$

535. Batiystoma aurolineatum. (P. 1310.)

536. LYTHRULON OPALESCENS. (P. 1312.) 


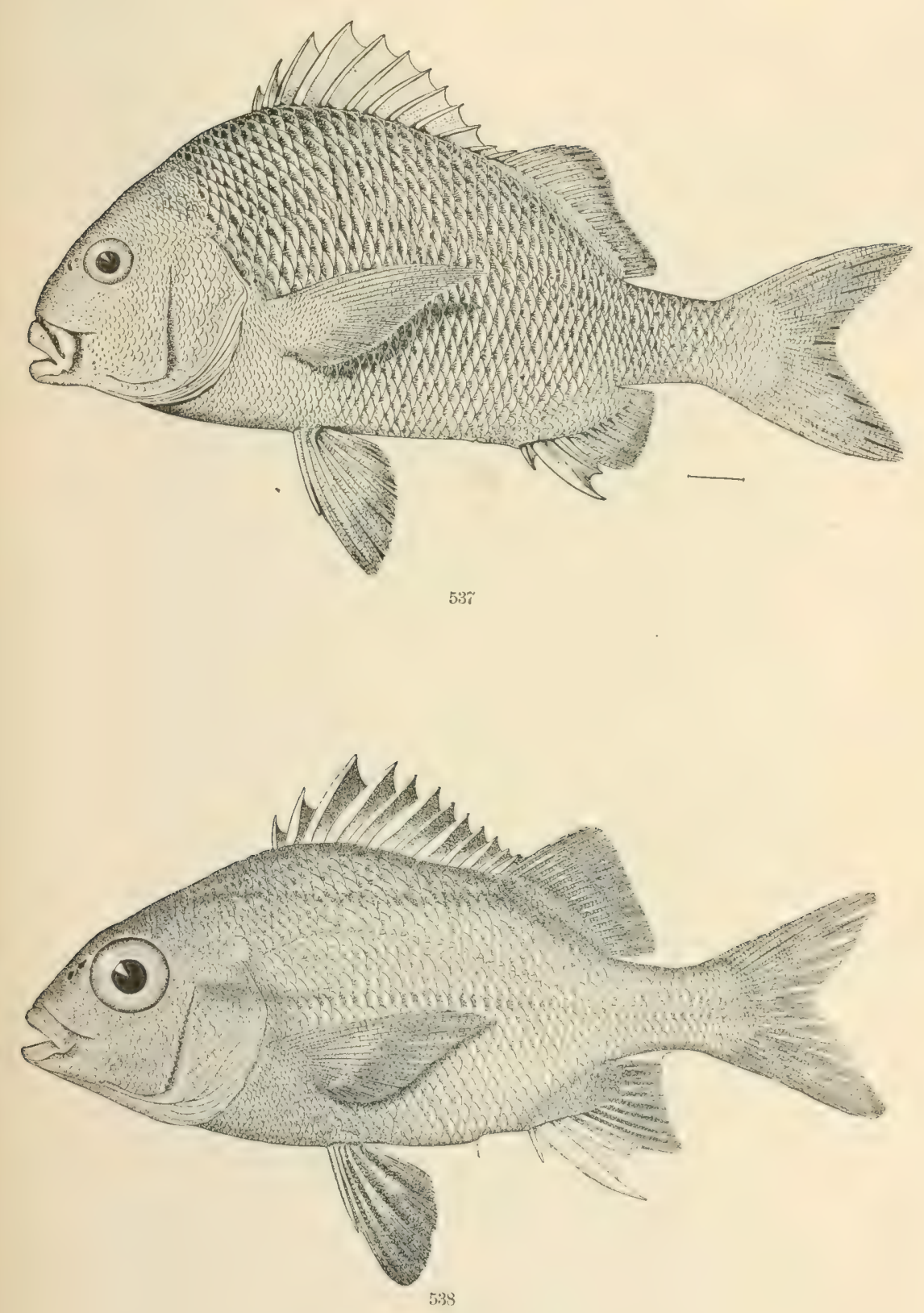

537. ANisotrenus surinamensis. (P. 1318.)

538. Anisotremus illineatus. (P. 1319.) 



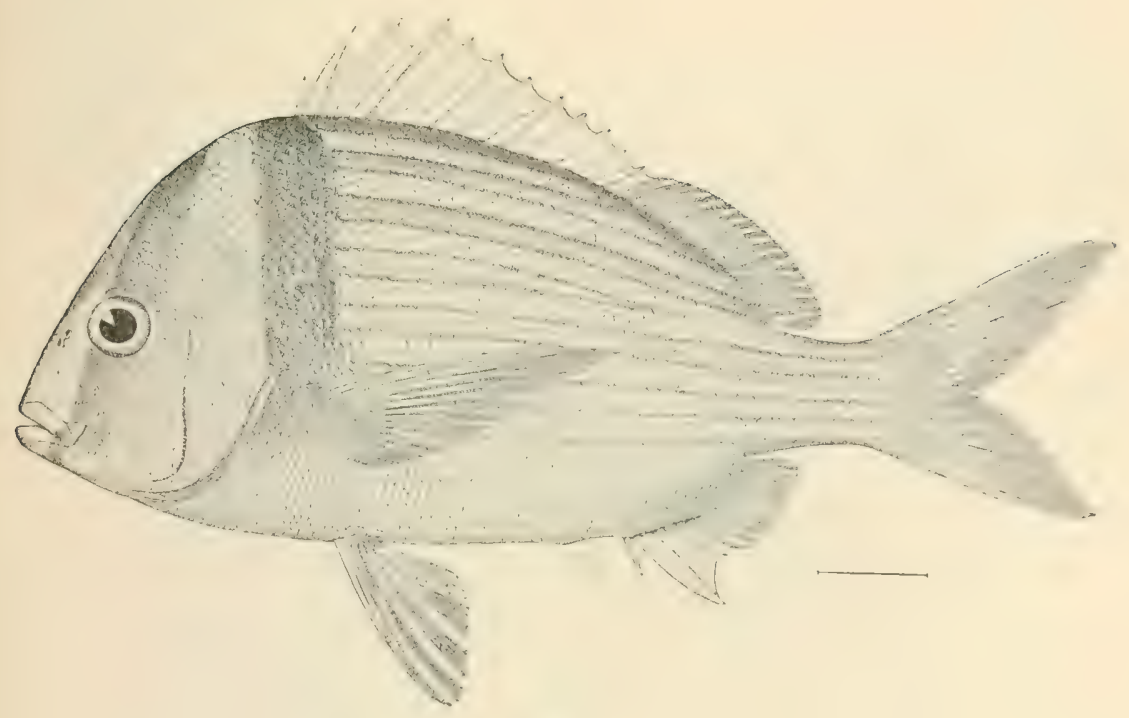

$5: 39$

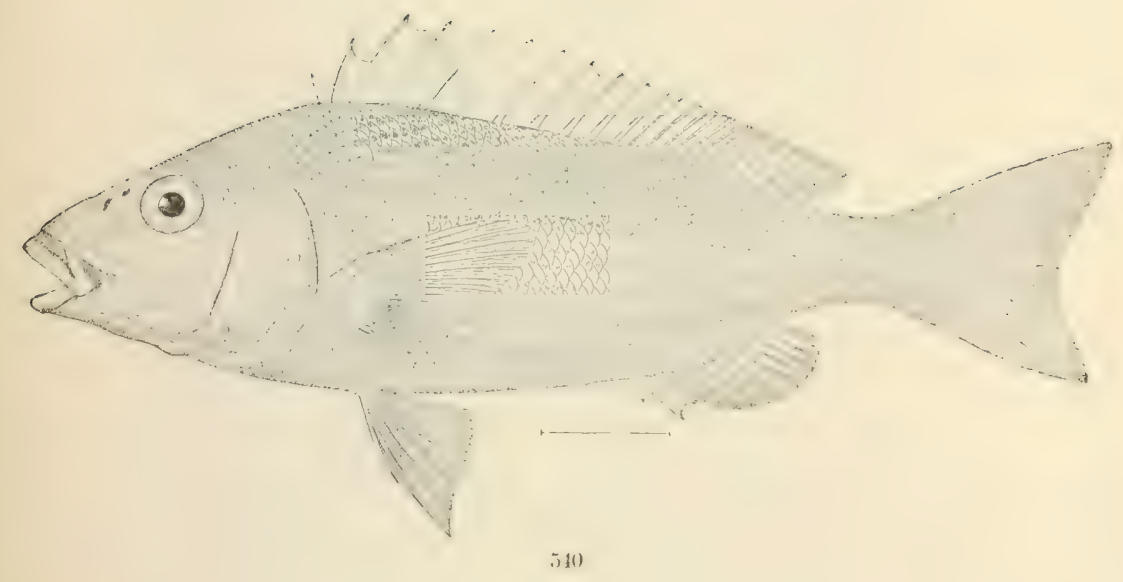

539. Anisotrenus virginicus. (P. 1322.)

540. ORTHOPRISTIS REDDINGI. (P. 1336.) 


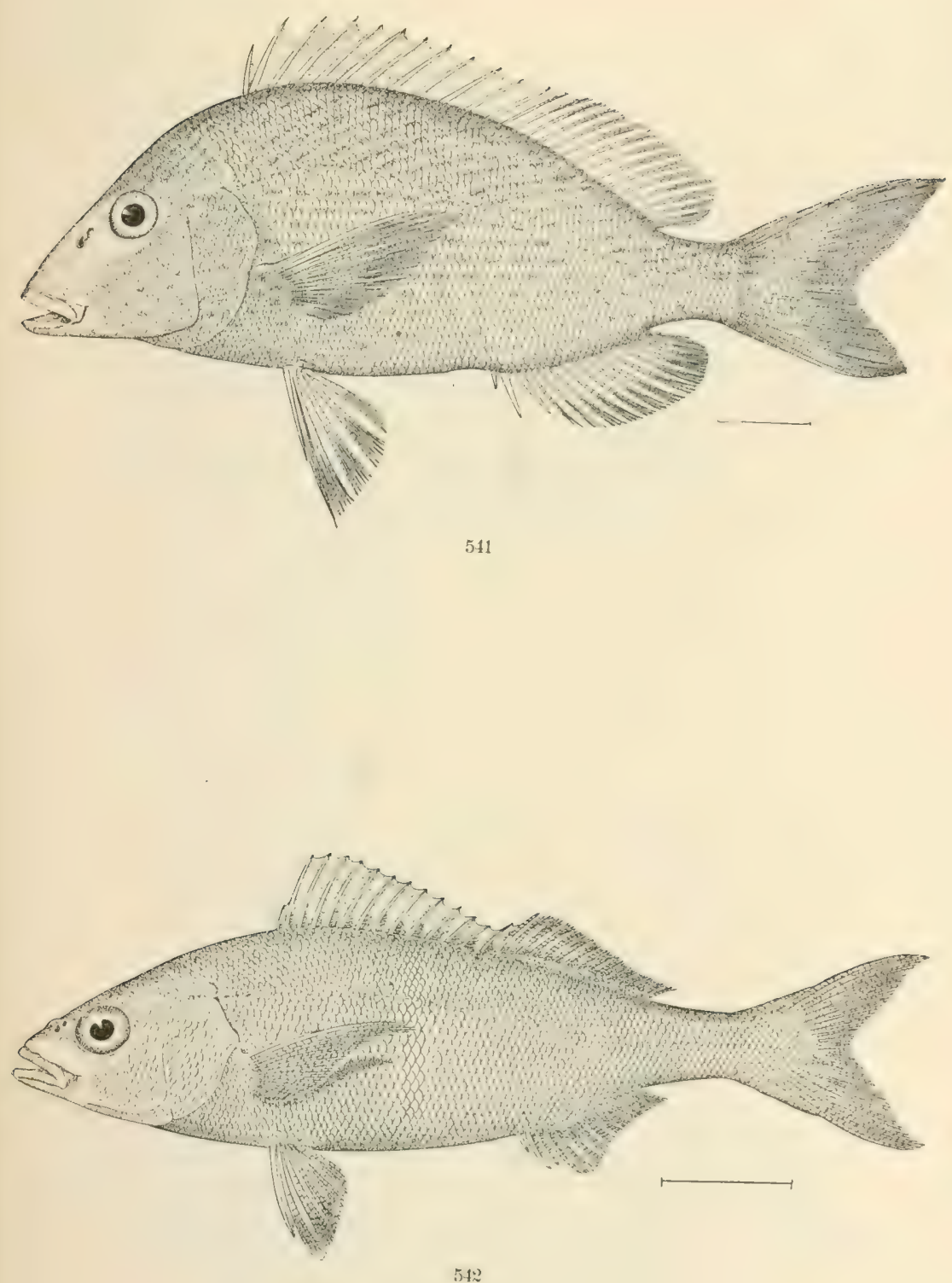

541. OrThoplistis CHRYSOPTERUS. (P. 1338.)

5.12. Microlepidotus ixorvatus. ( $\mathrm{P} .1341$. 



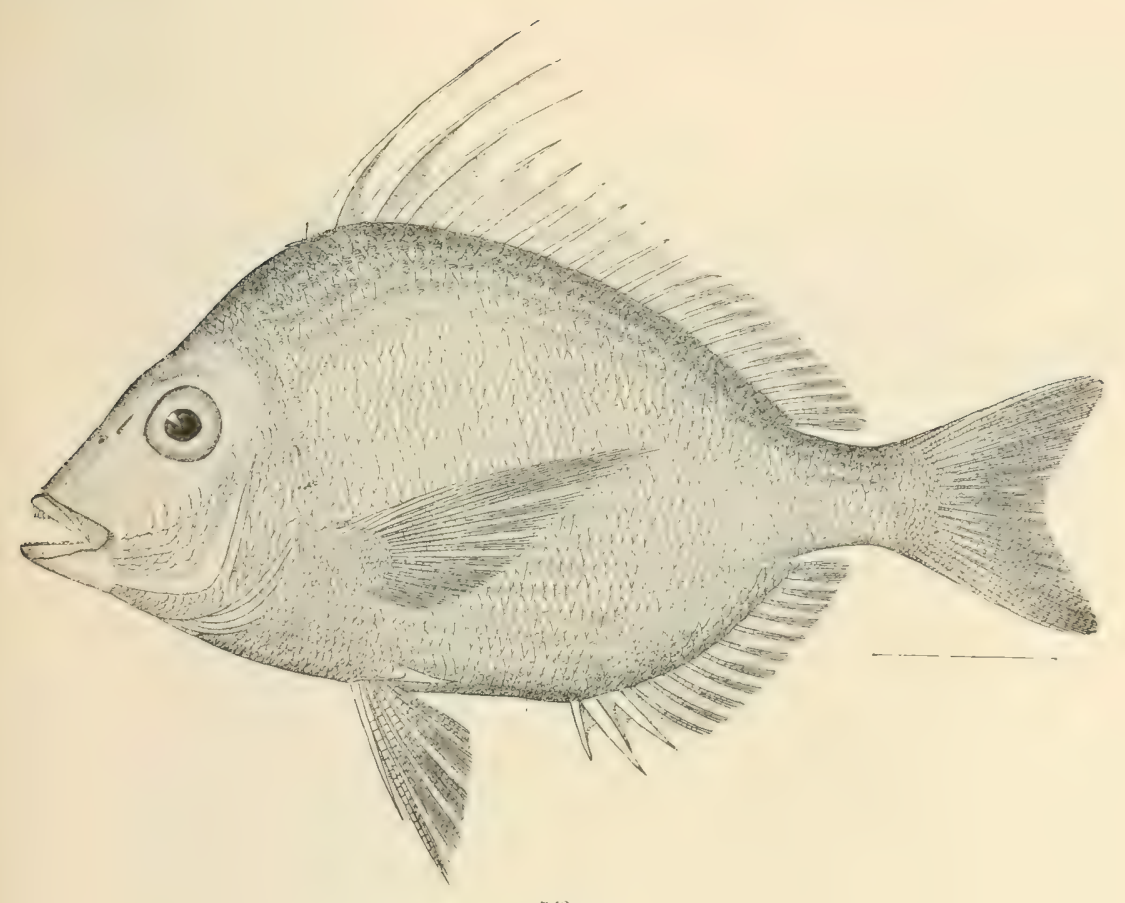

543

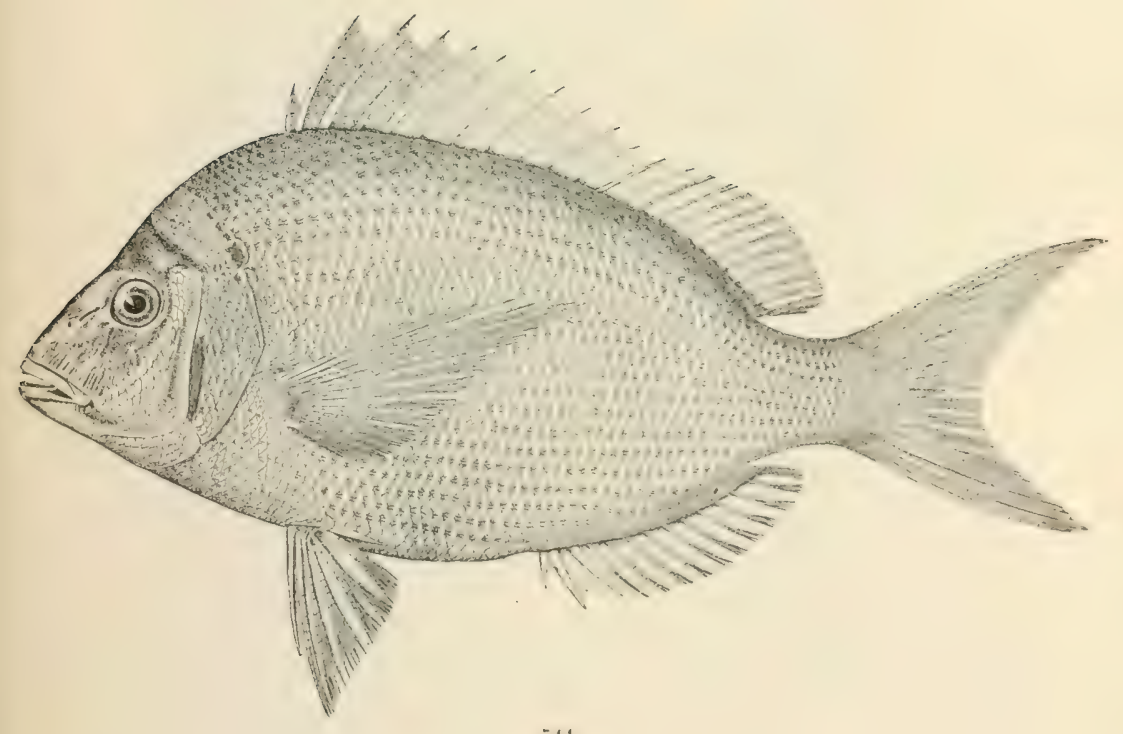

514

543. Otrynter caplinus. (P. 1345.)

544. Stenotomes chrysops. (P. 1316.) 


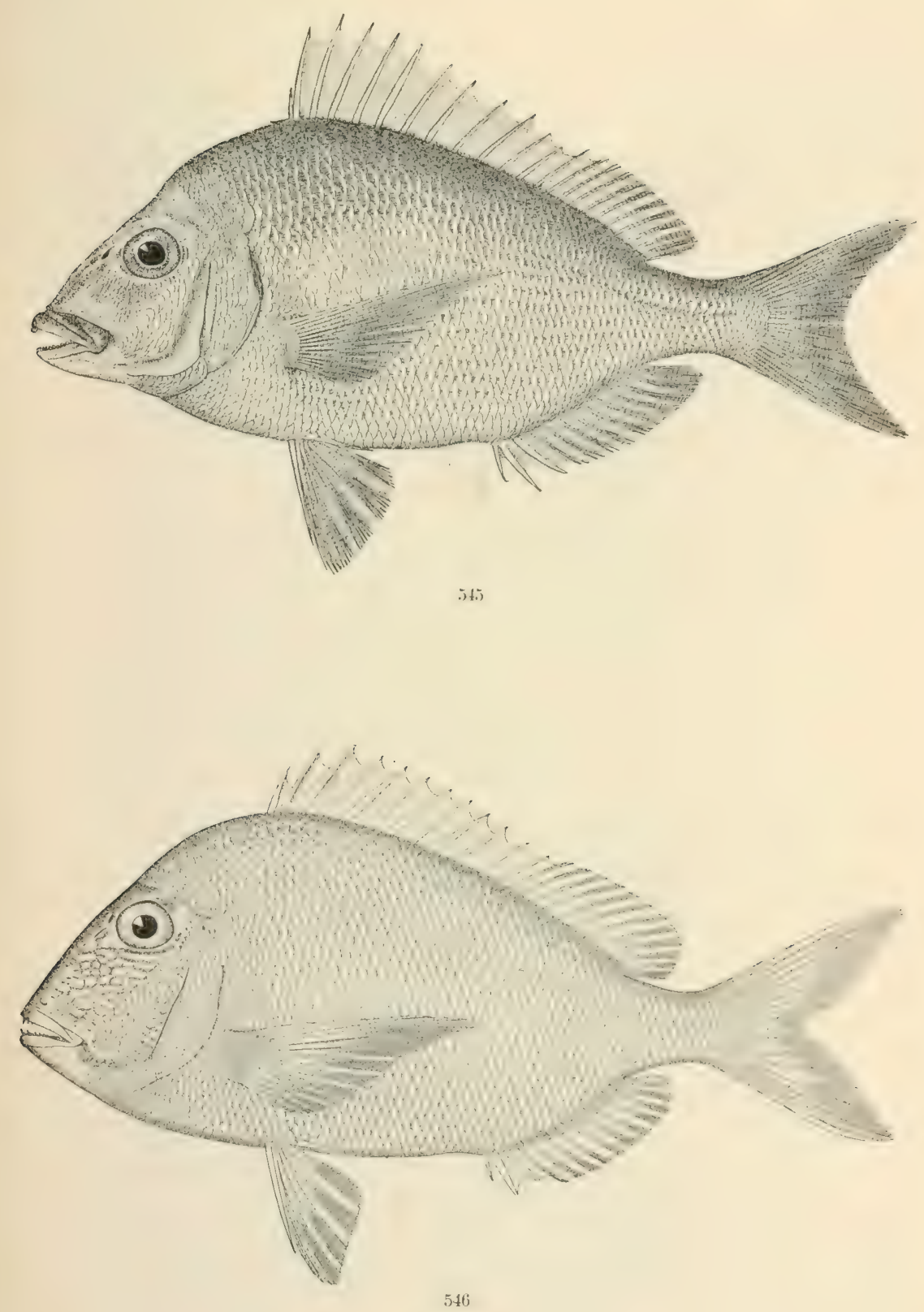

545. STenotonus aculeates. (P. 1346.)

546. Calames calamus. (P. 1349.) 



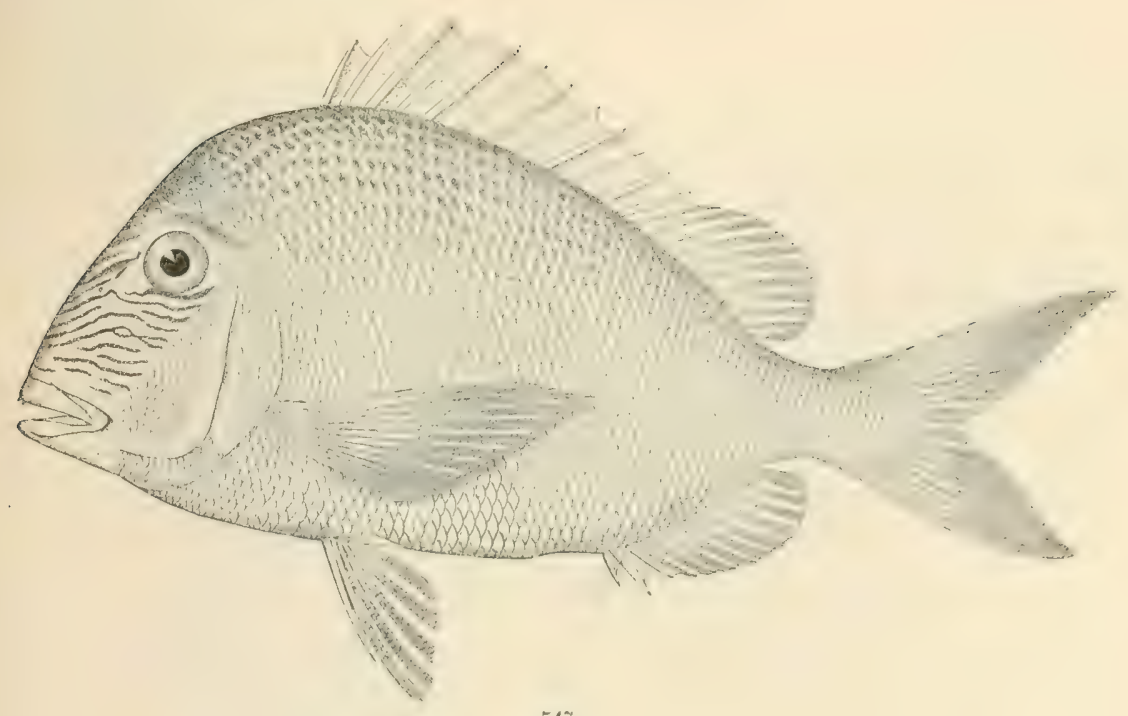

$54 i$

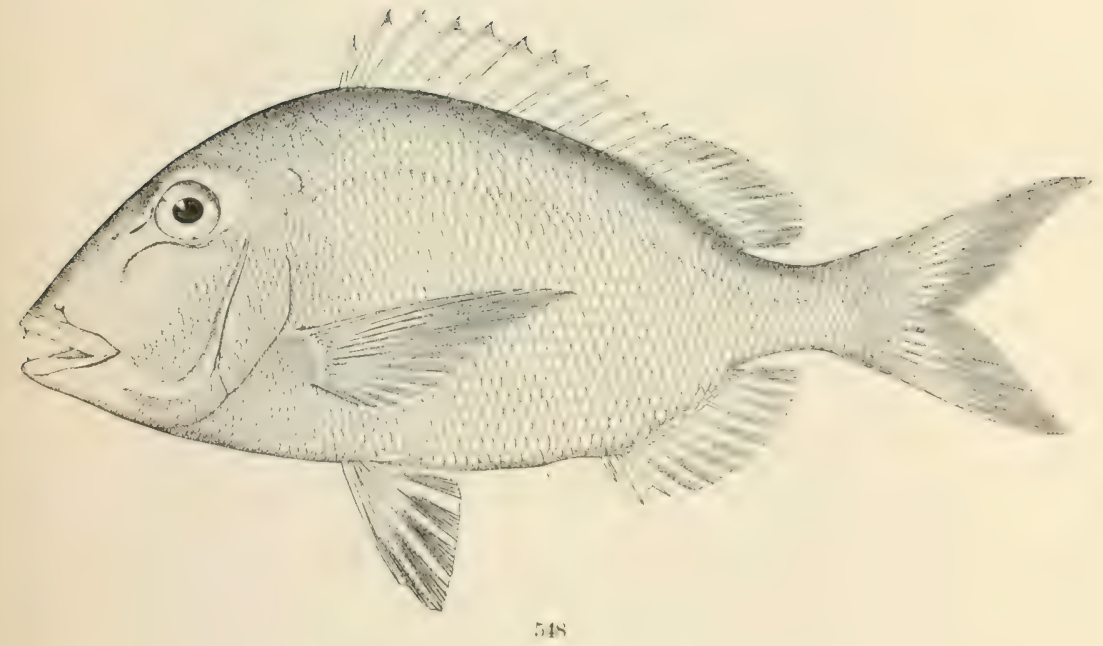

547. Calamus prorinexs. (P. 1350.)

518. Calamus bajonado. (P. 135\%.) 


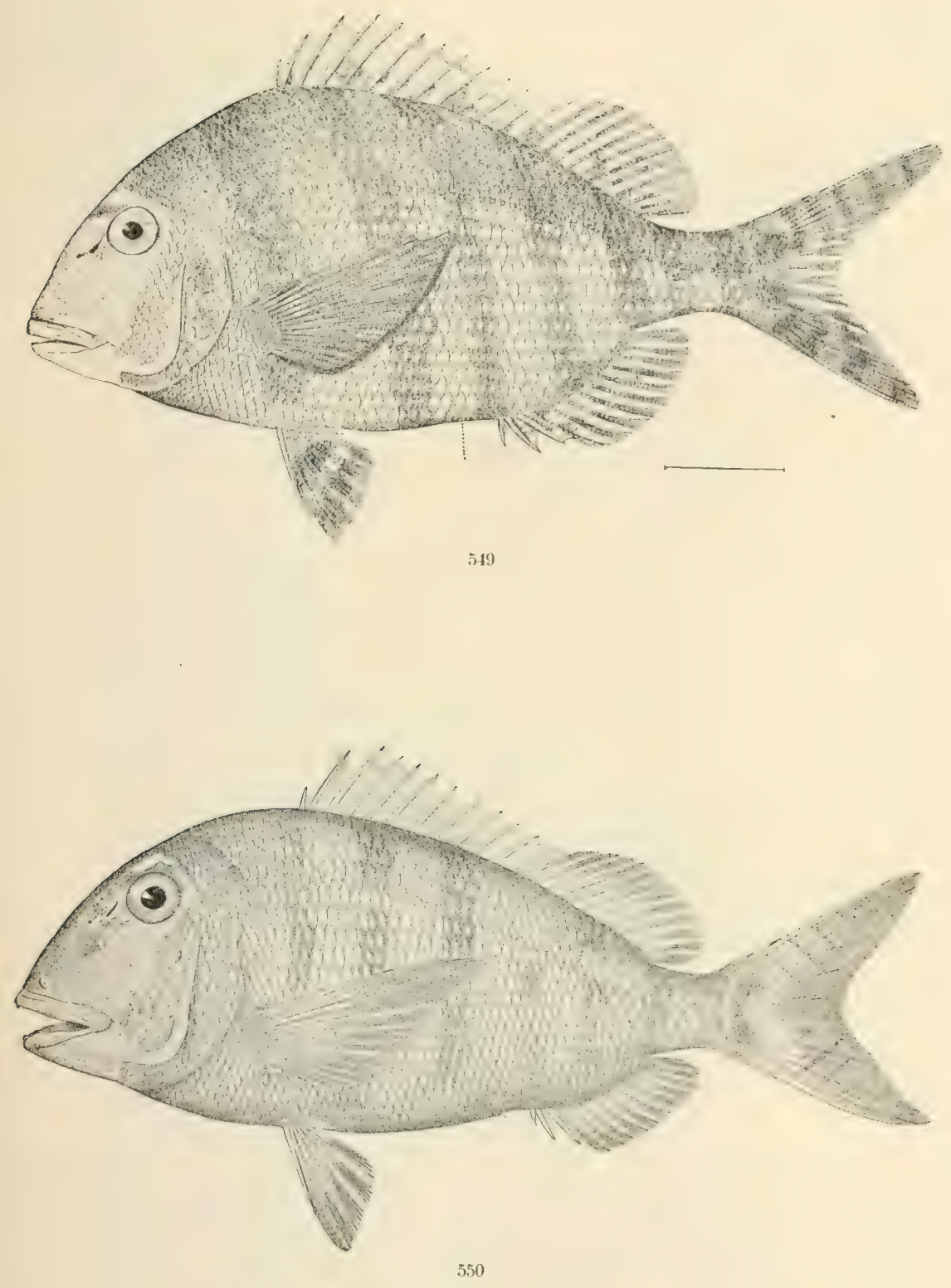

549. Calajus penna. (P. 1354.)

550. Caladius arctifrons. (P. 1355.) 


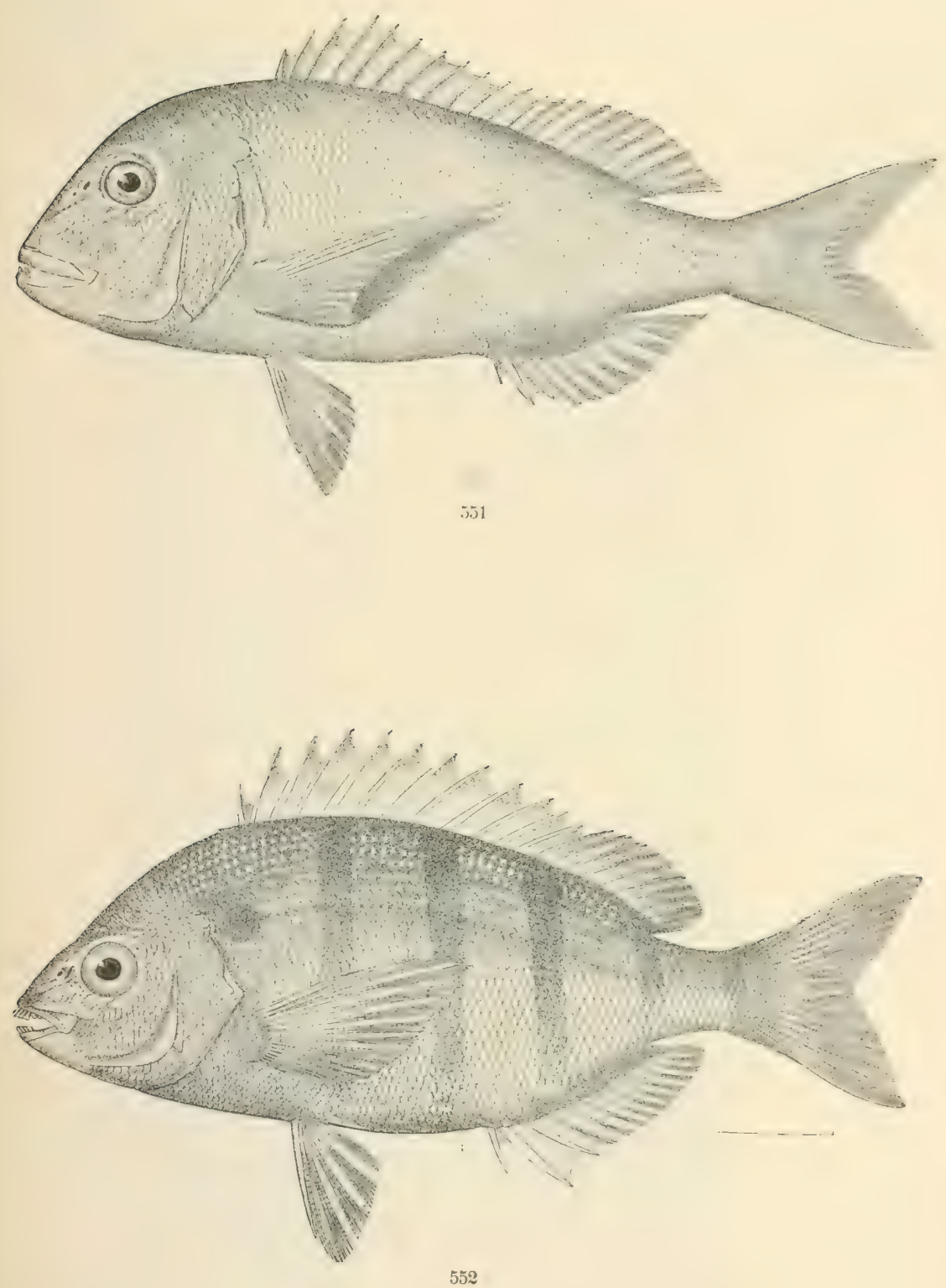

551. Pagrcs Pagrus. (P. 1356.)

552. LAGODON RIOMBOIDES. (P. 1358.) 



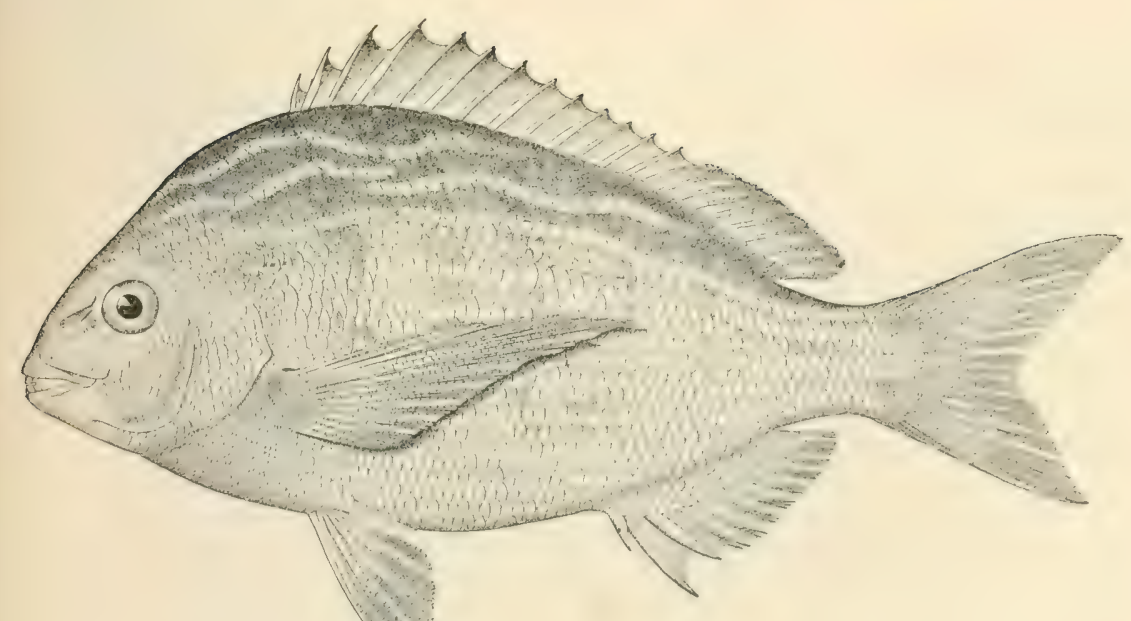

553

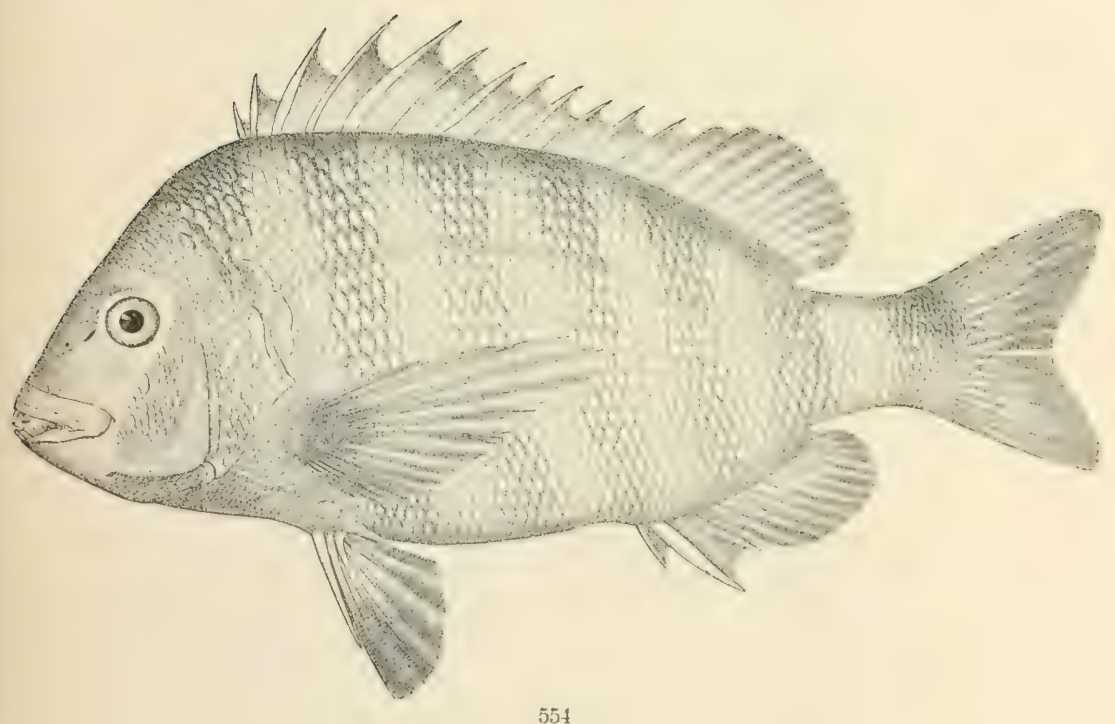

553. Archosargus UNimaculatus. (P. 1359.)

554. Alichosaligus plobatocephalus, (P. 1361.) 


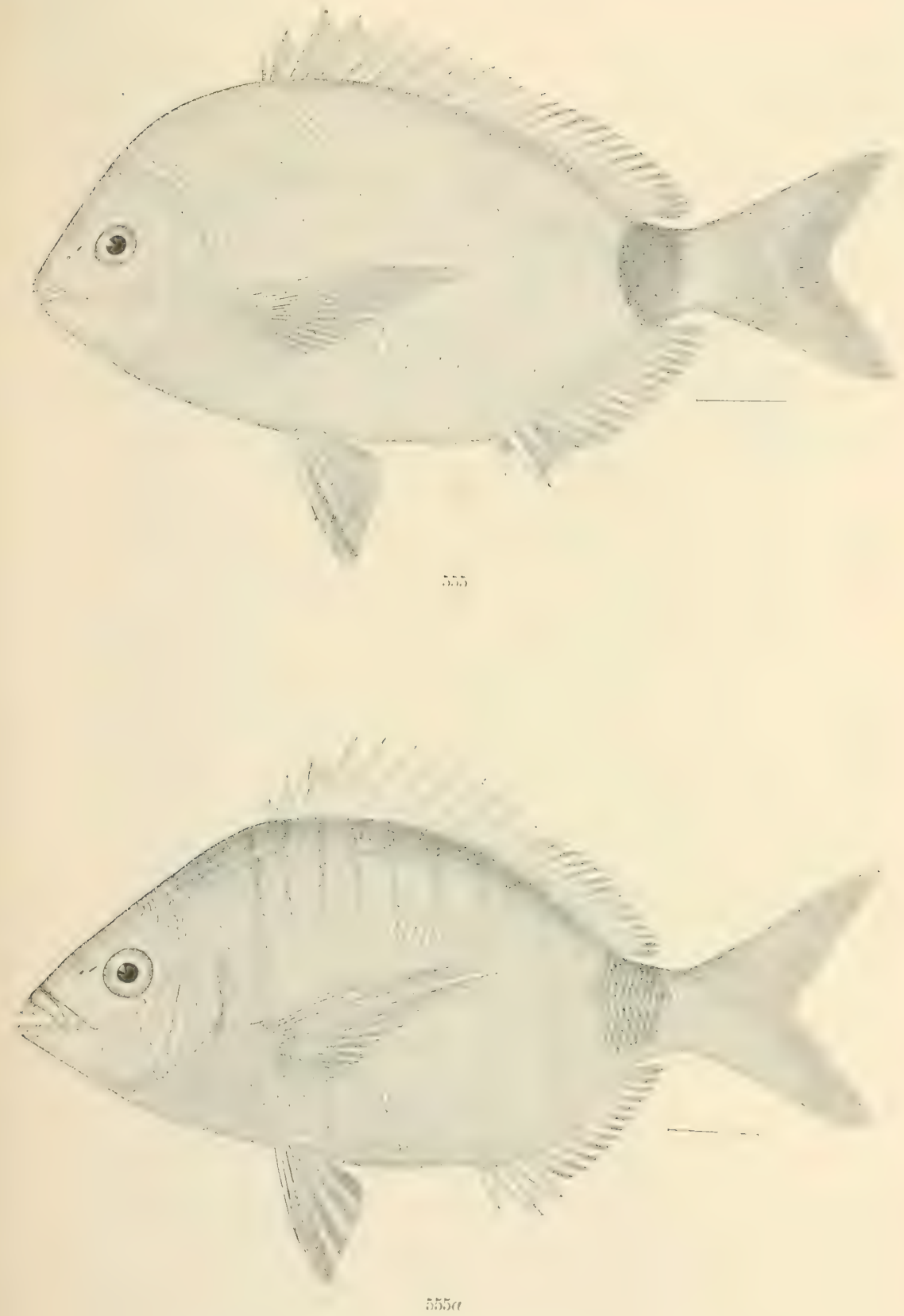

55. DHיLODE HOL.13ROOKII. (P. 1362.)

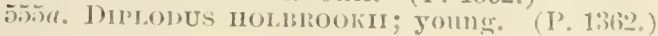




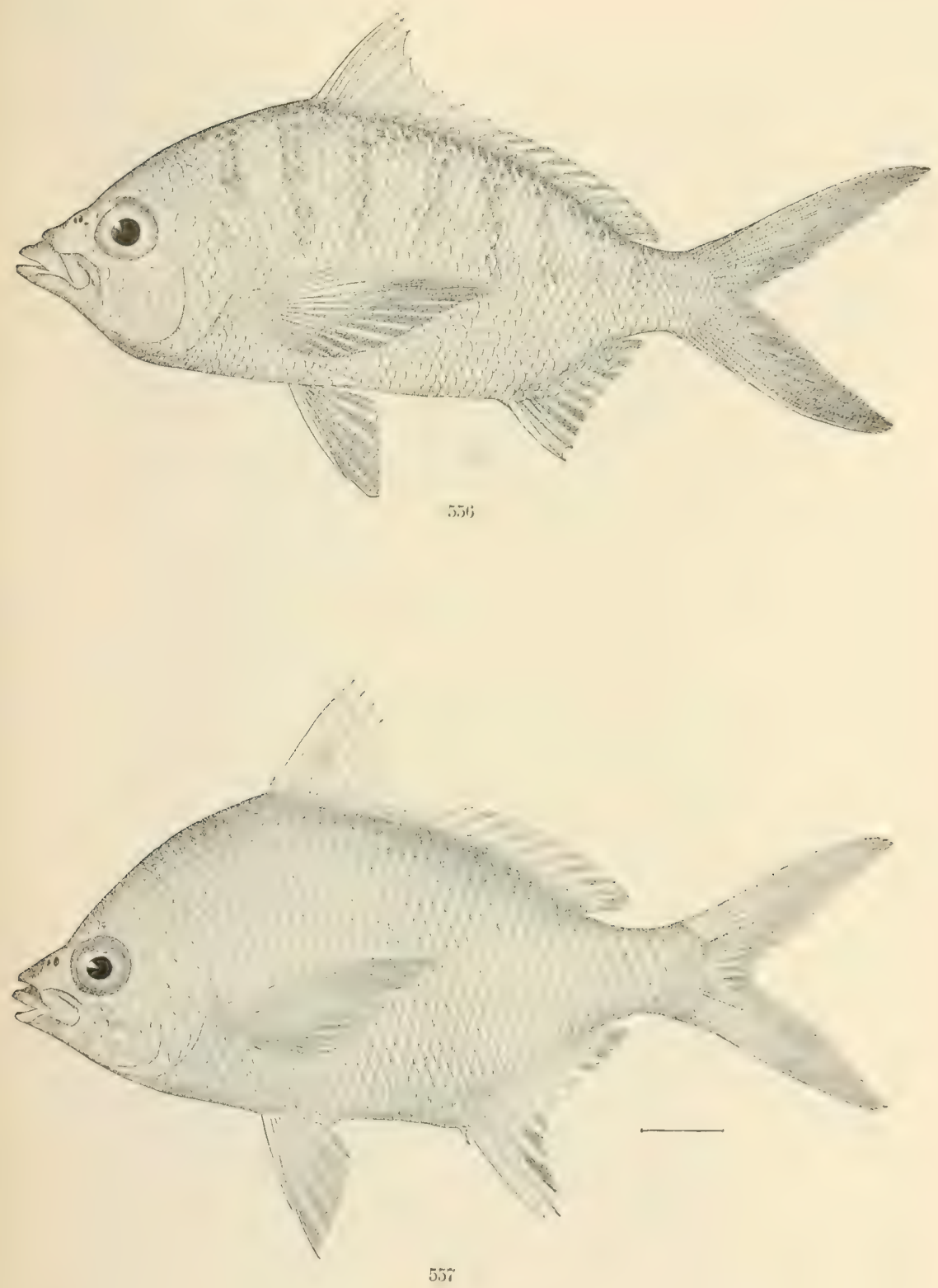

556. Xystema cinereum. (P. 1372.)

557. Gerres olisthostomus. ( $\mathrm{P}, 1376$. 


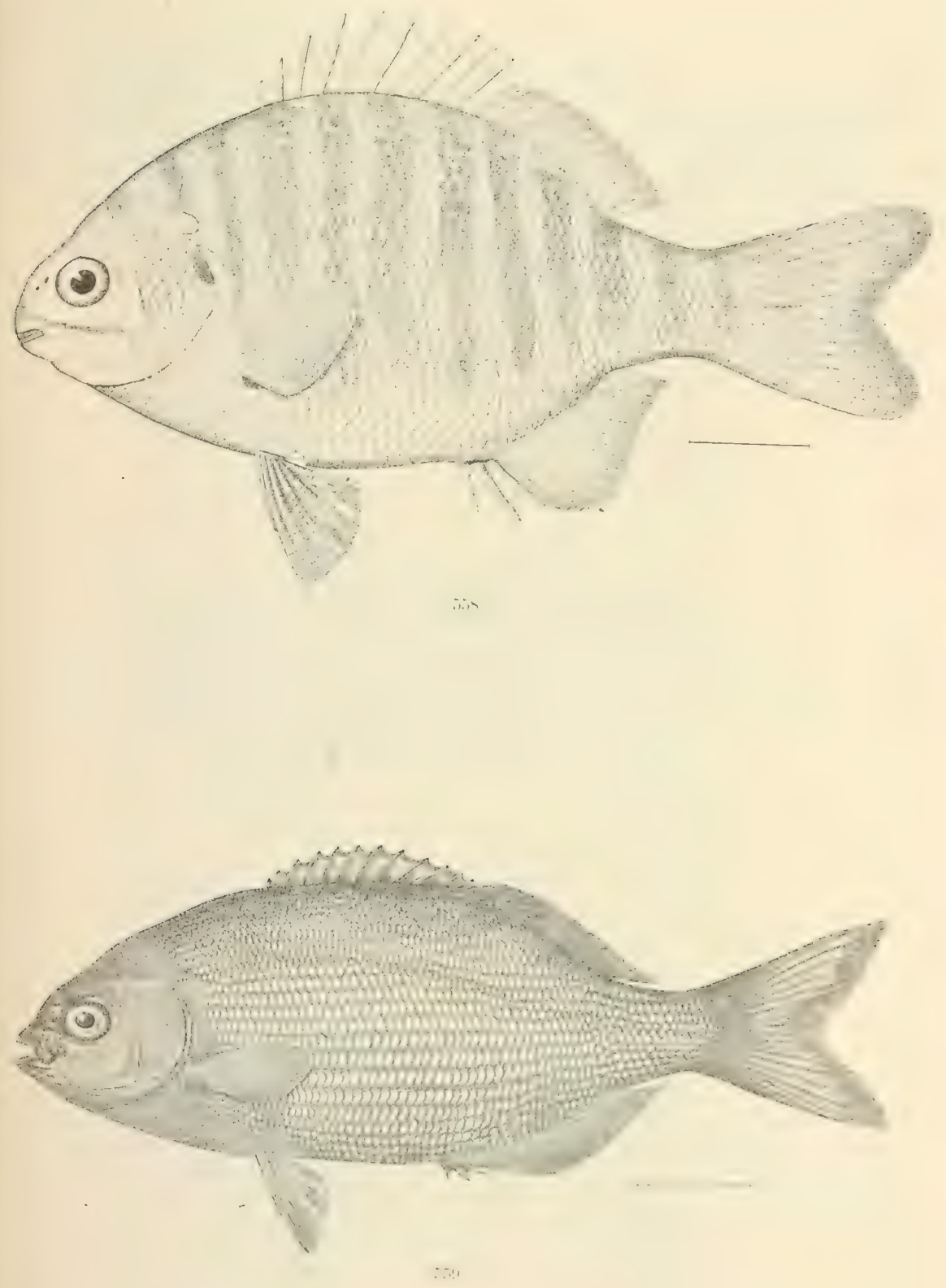

5.'. MEIRTOSILLA A\%UREA. (I', 1383.)

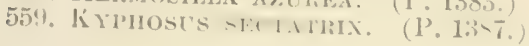




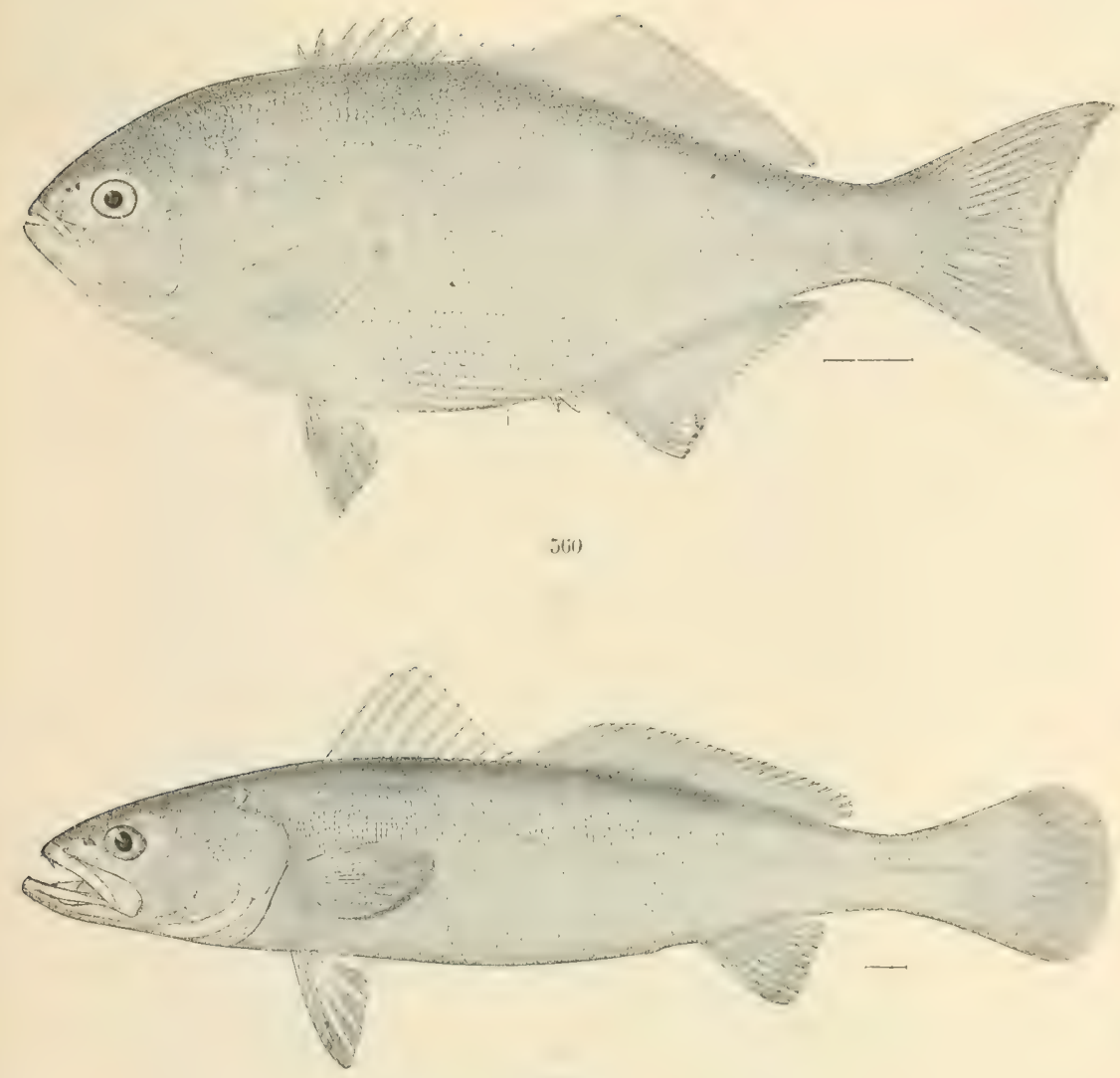

561

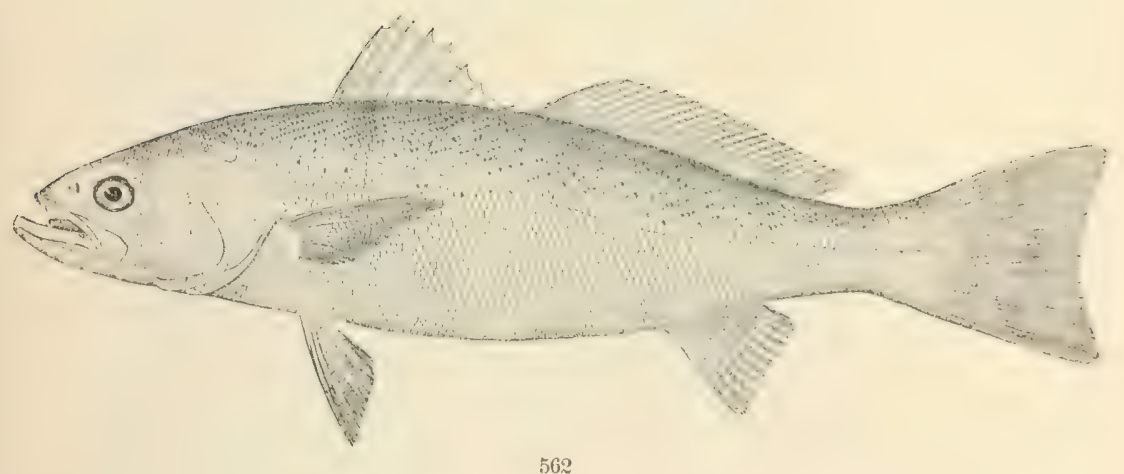

560. Medialuna californiensis. (I'. 1391.)

561. CyNoscion nothus. (

562. CYNUSCION REGAI.IS. (I', 1407.) 


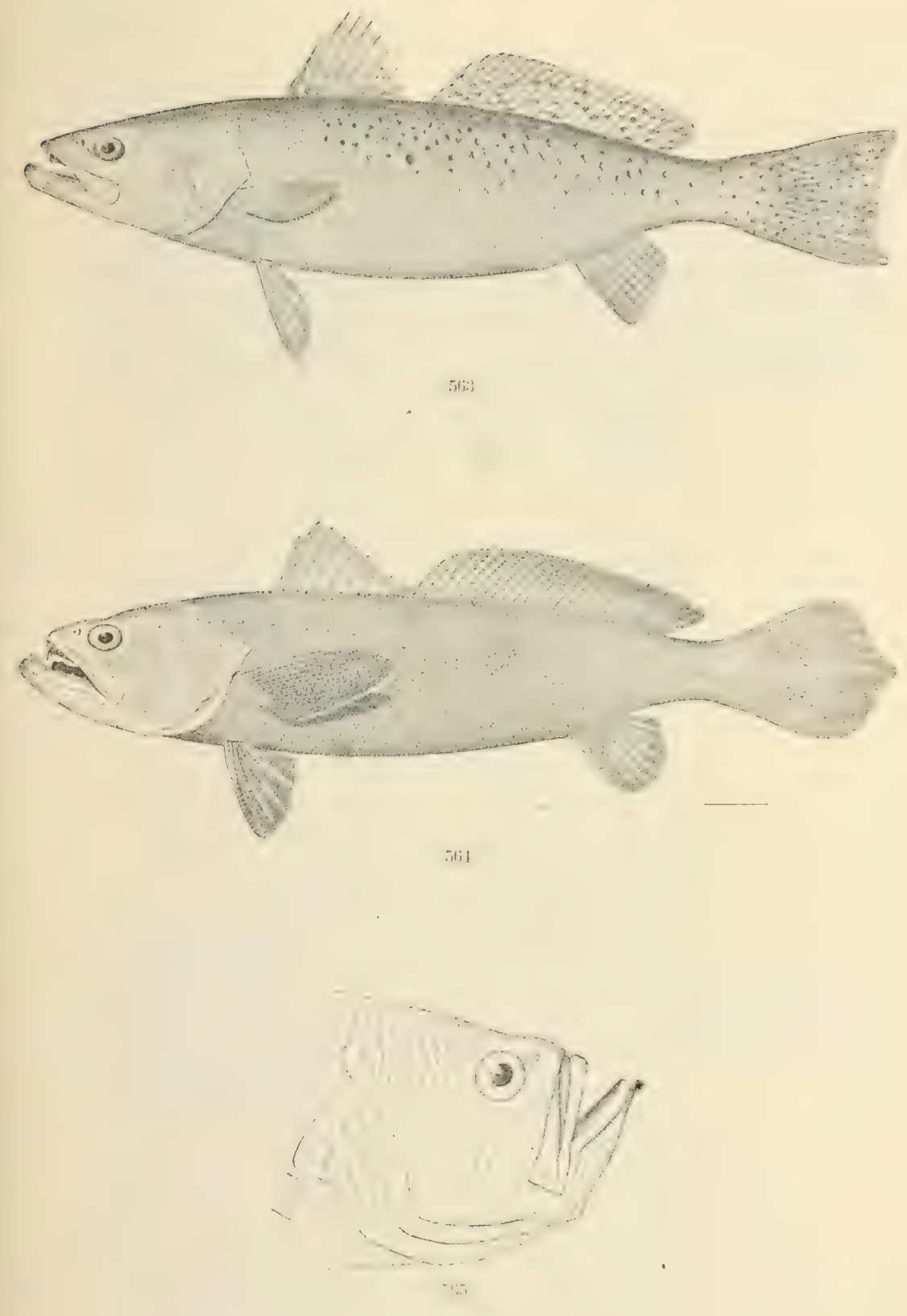

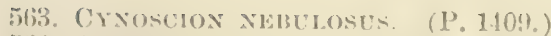

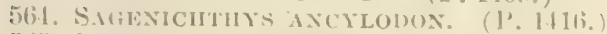

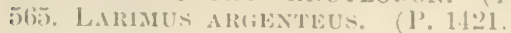




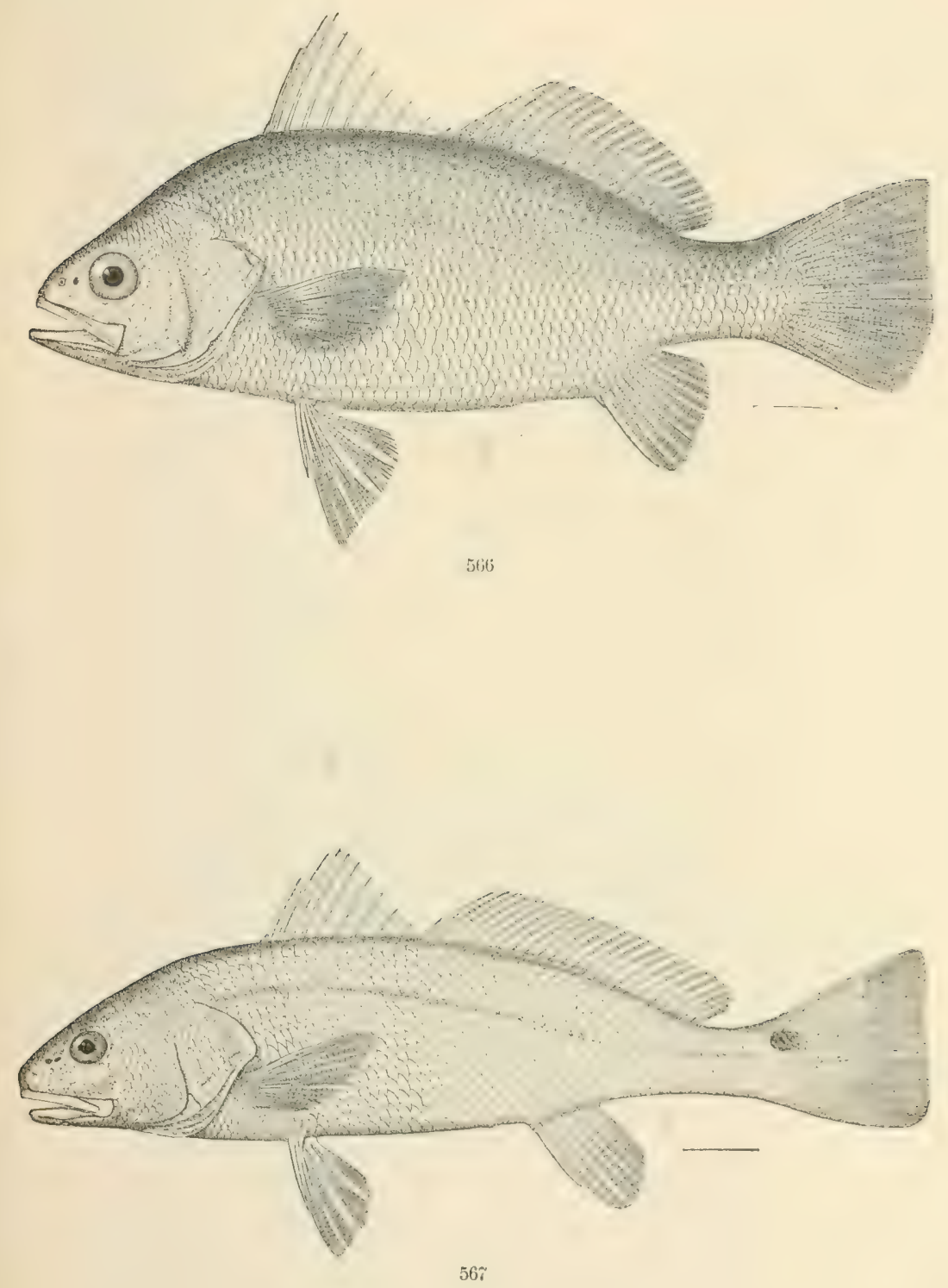

566. Bairdiella chrysura. (P. 1433.)

567. SCleNOPS OCELlatus. (P. 1453.) 


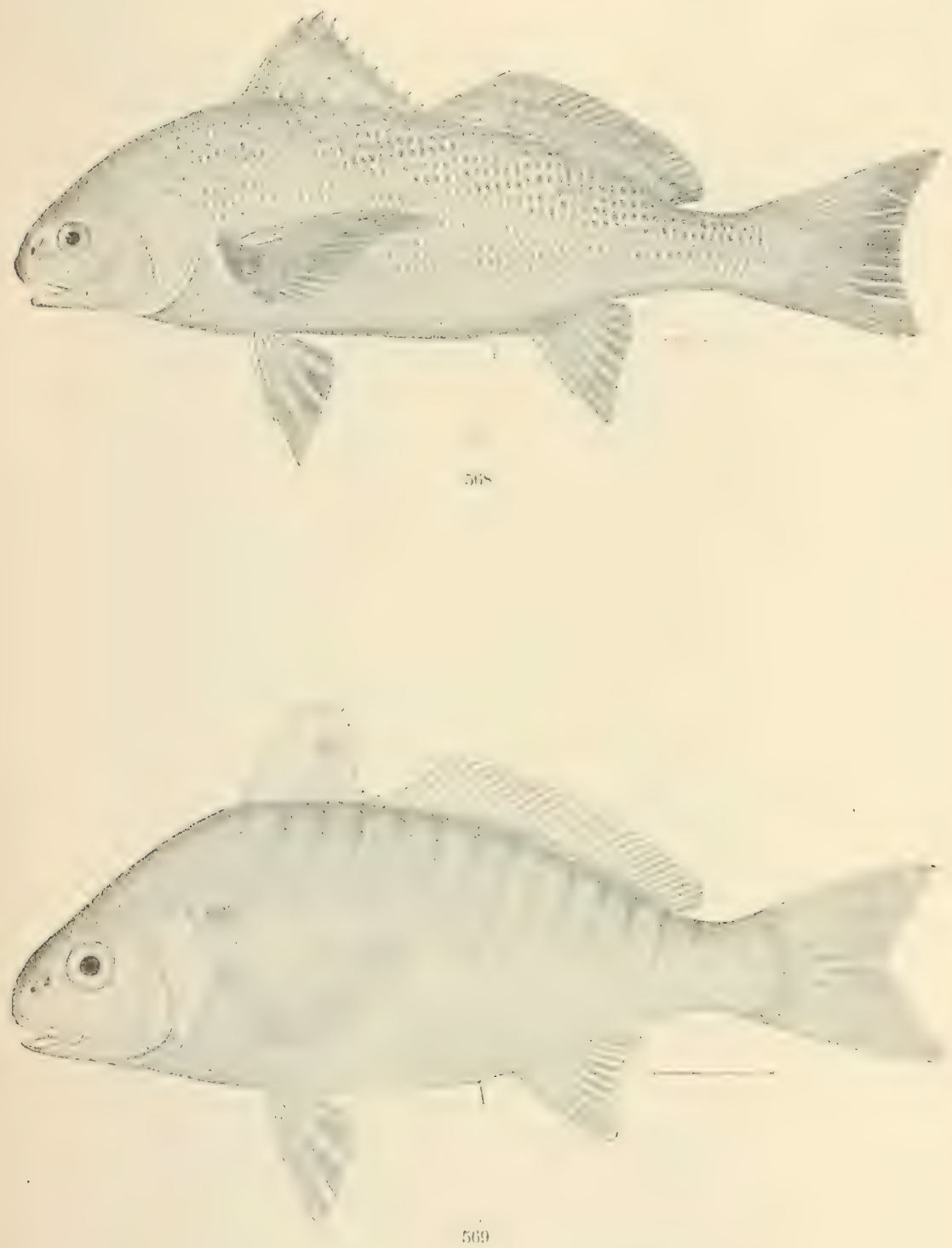

568. RoNCADOR STEARNAr. ( 1 '. 1457.)

569. Lemostoncs xaxthures. (1', 1458.) 



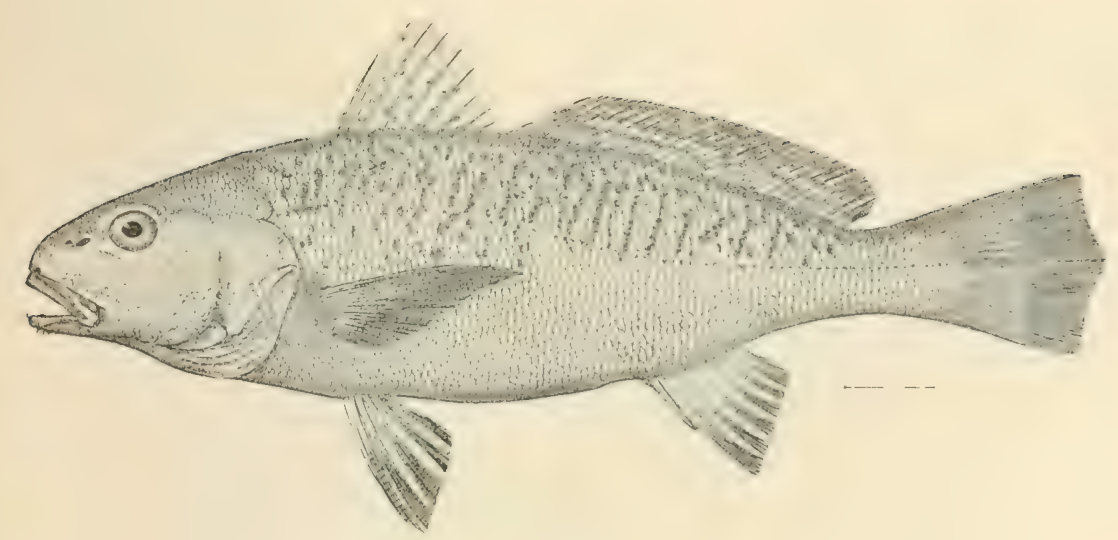

5 ro

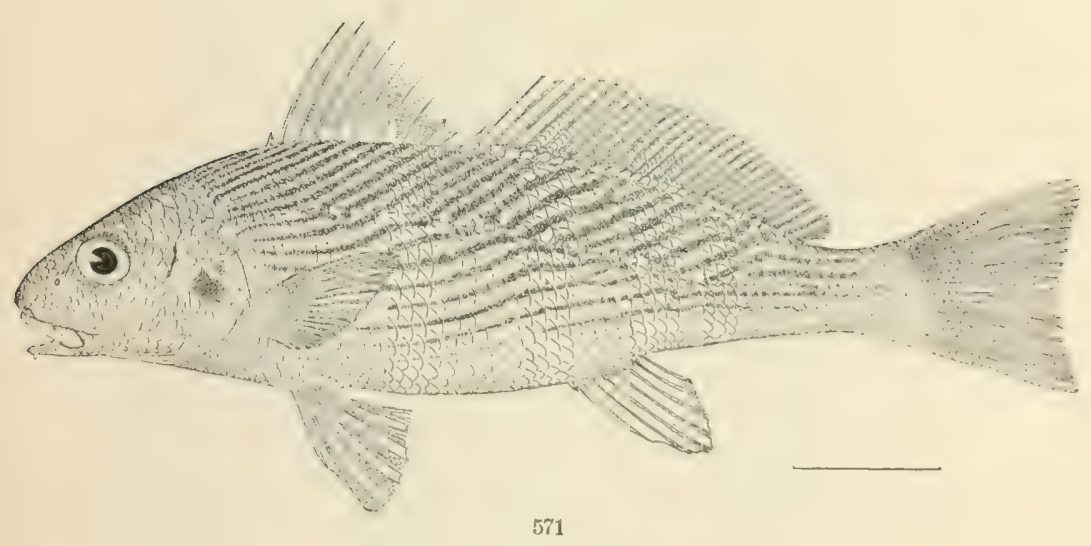

570. Michopogor UNDLLATUS. (P. 1461.)

571. UMIBRINA SINALOF. (P. 14ti8.) 


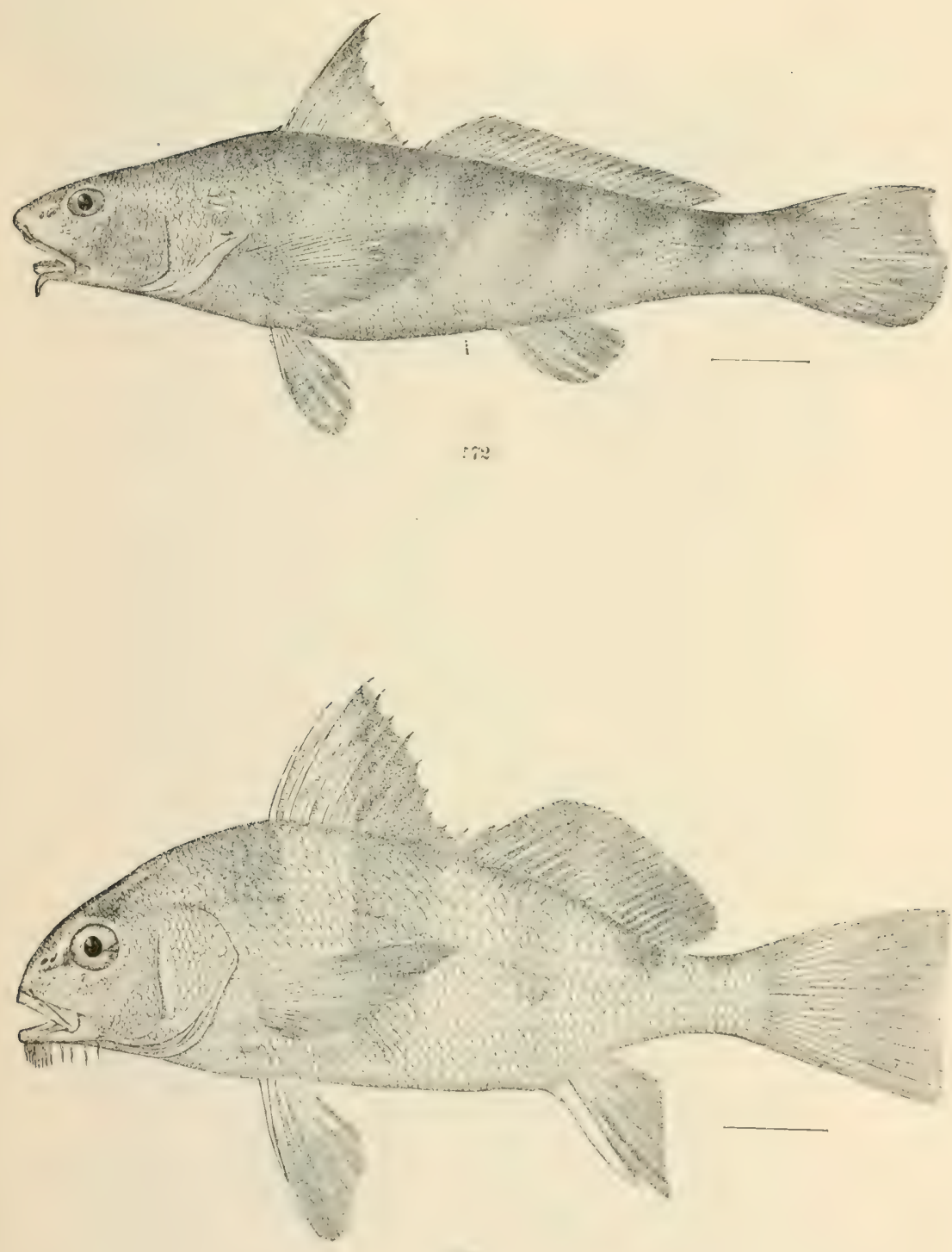

572. Menticirlinus americanus. (P, 1474.)

573. Pogonias CHROMIS, (P. 1482.) 



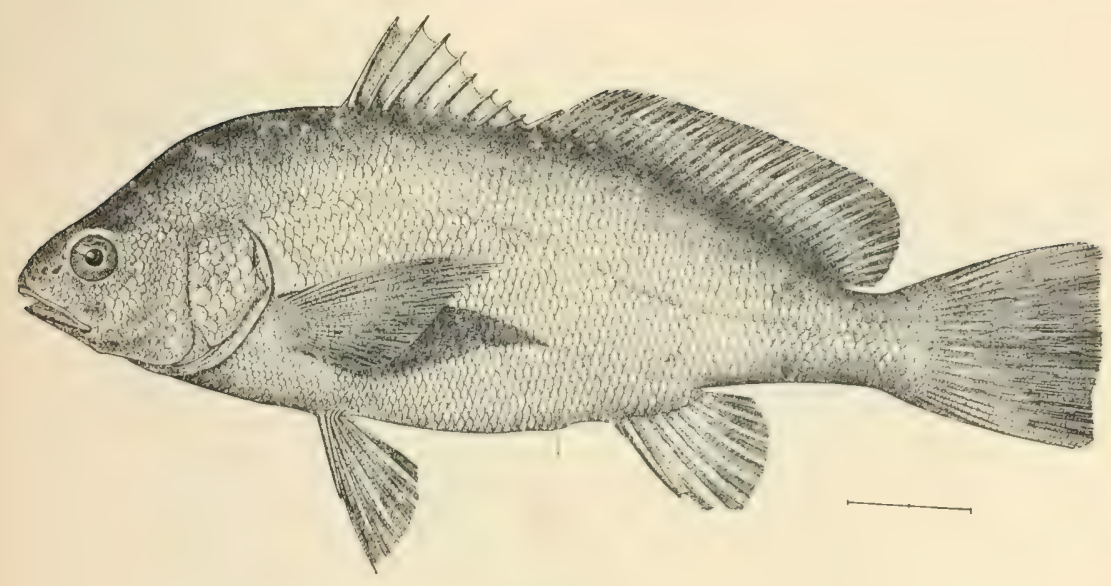

574

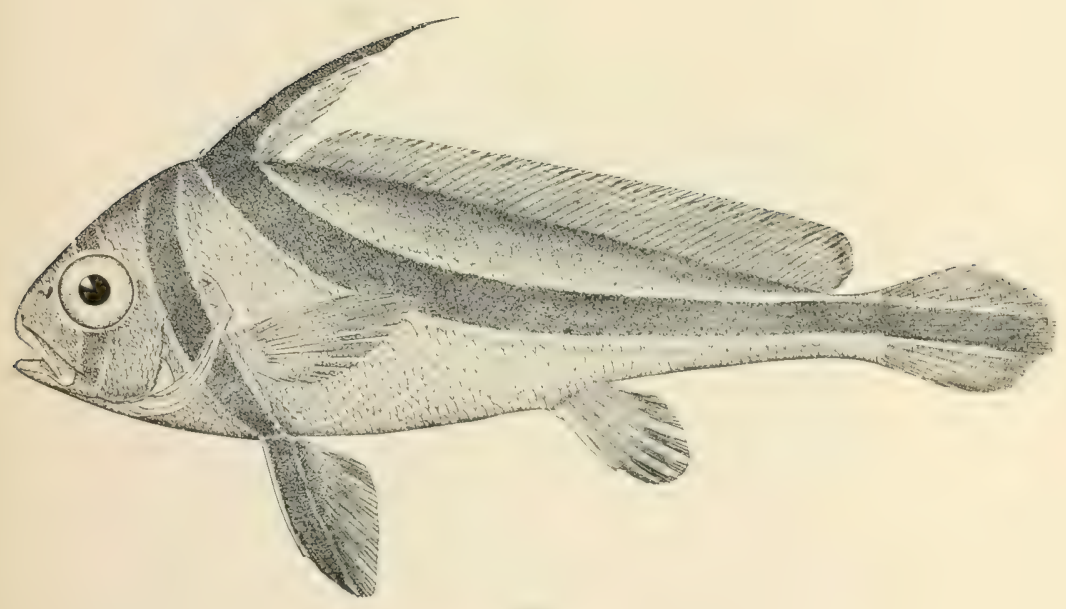

575

574. Aplodinotug grunniens. (P. 1484.)

575. Eques laNCeolatus. (P. 1489.) 


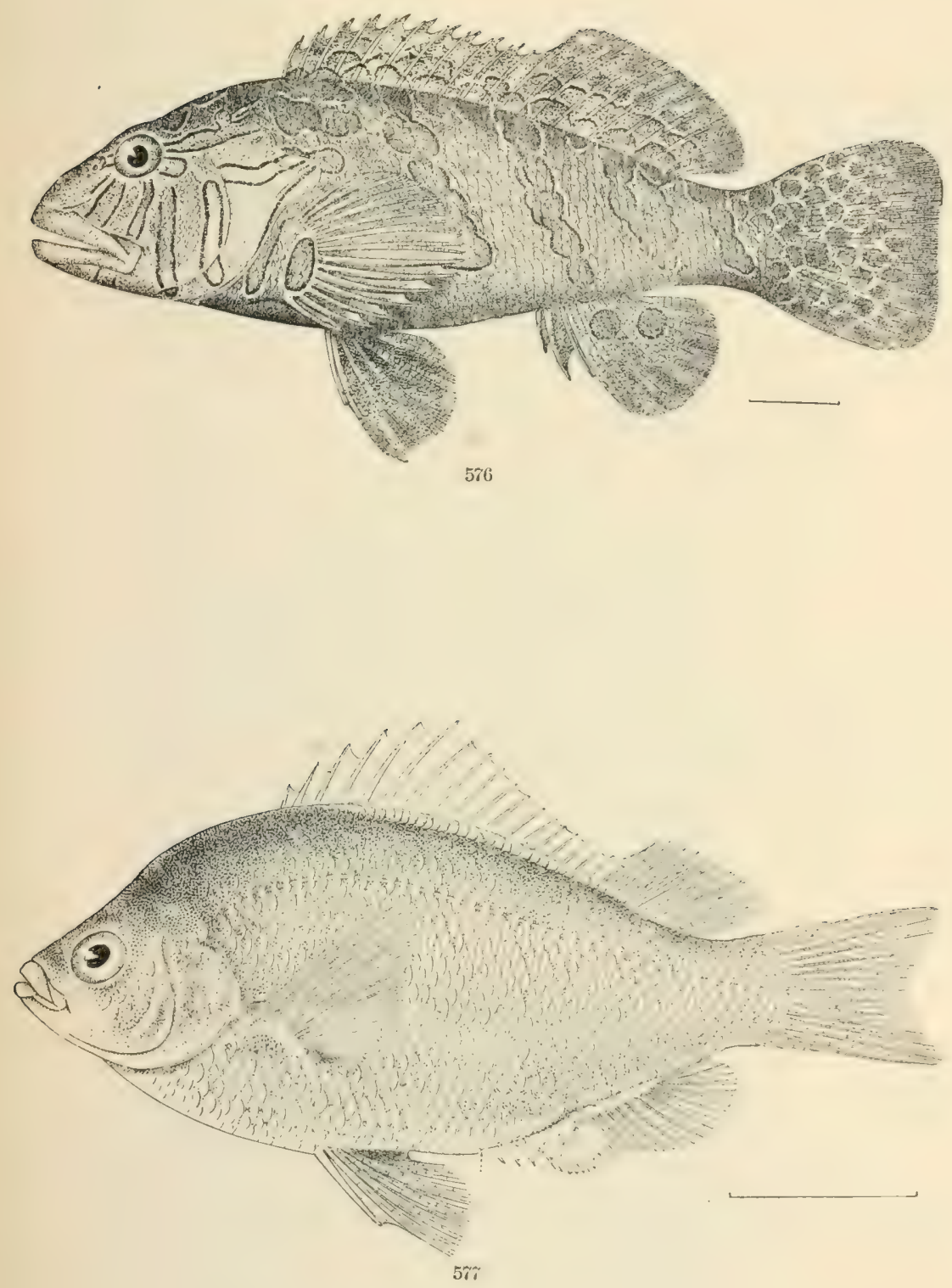

576. Cirruites rivelates. (P. 1491.)

577. HYSTEROCARPUS THASII. (P. 1496.) 


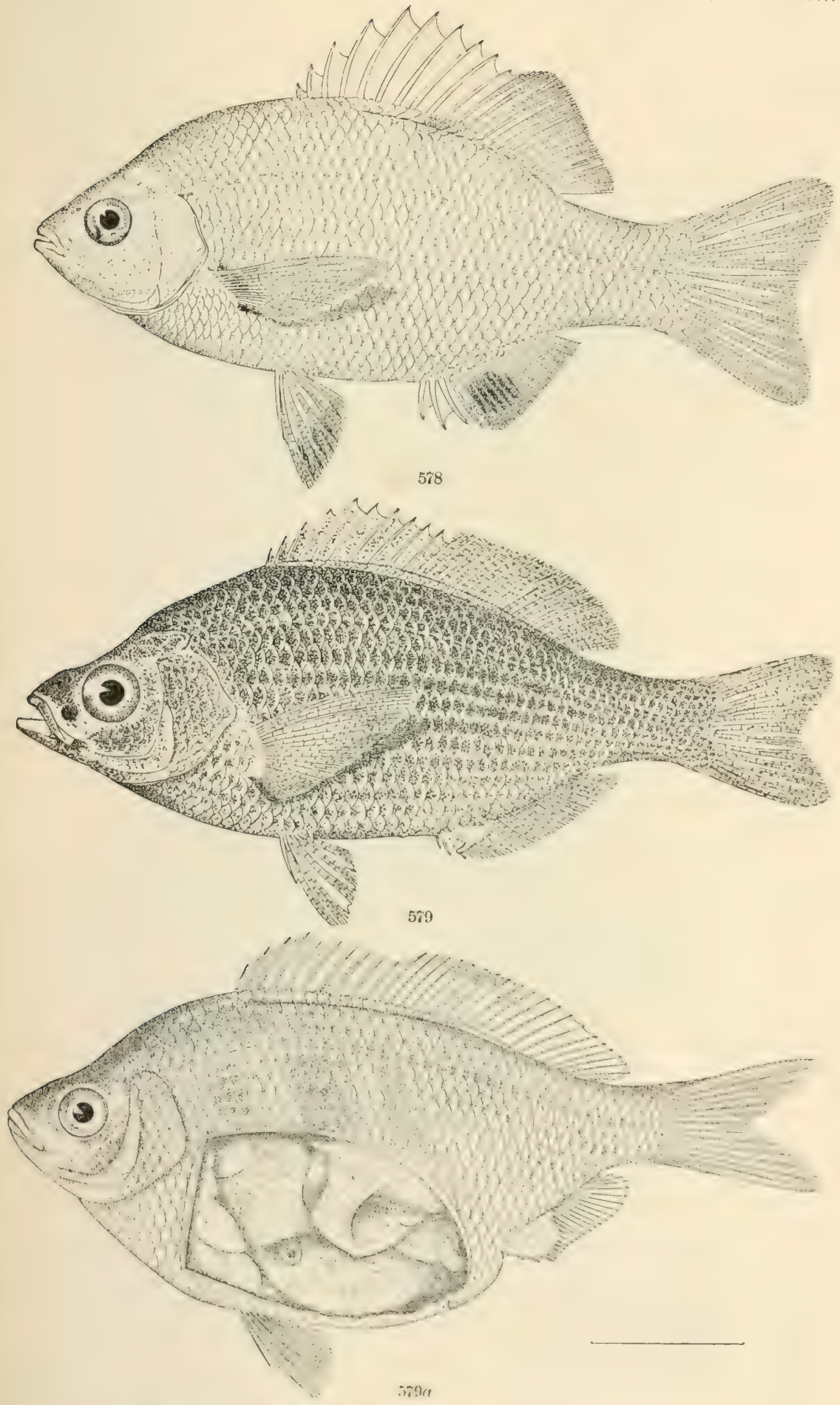

578. ABEONA MINIMA. (P. 1497.)

579. Crmatogaster aggliegatus. (P. 1498.)

579a. Cymatogaster aggregatus; femalo. (P. 1.198.) 


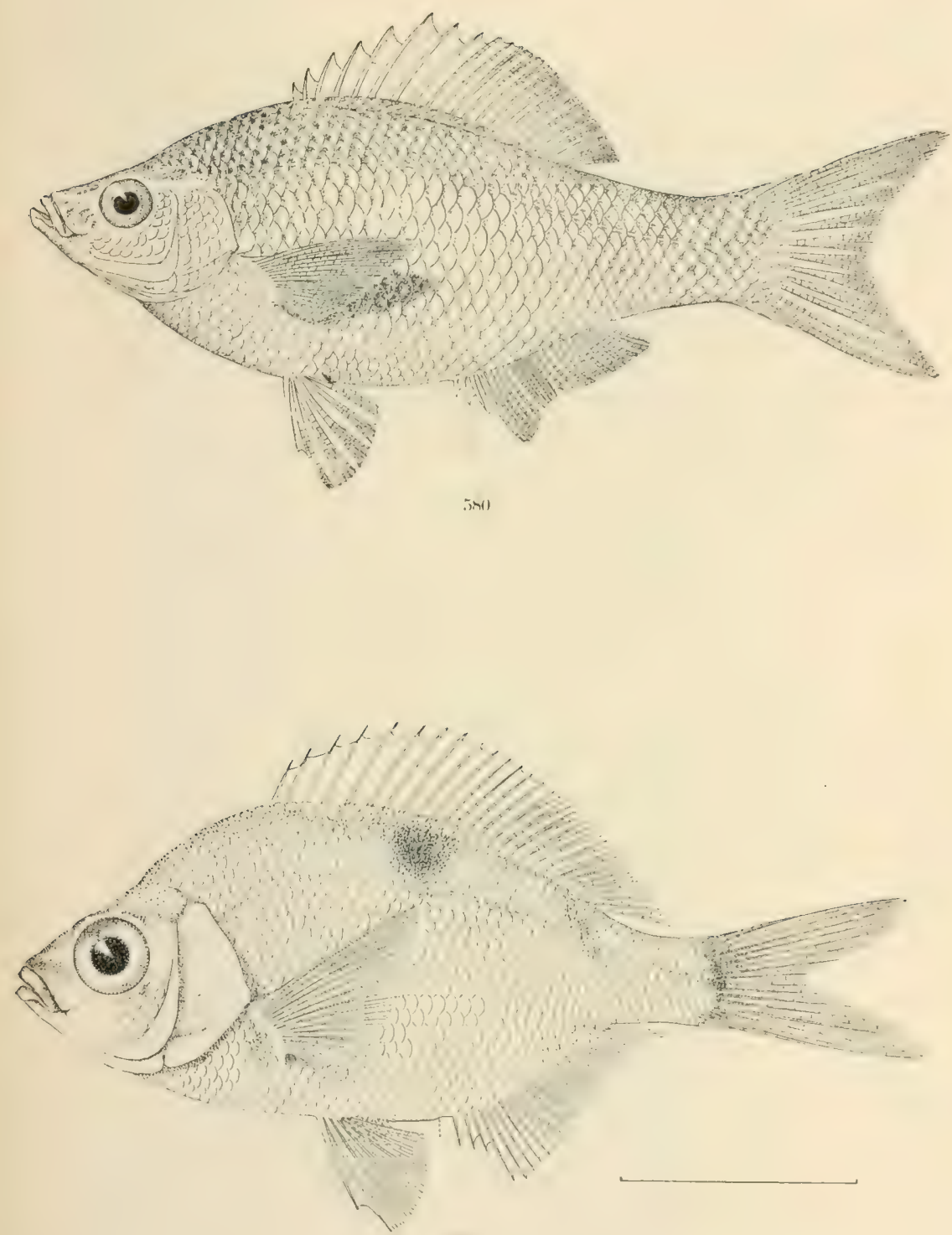

51

580. IBraciristius frexatus. (P. 1499.)

581. ZaleabiUs rosacels. (P. 1500.) 



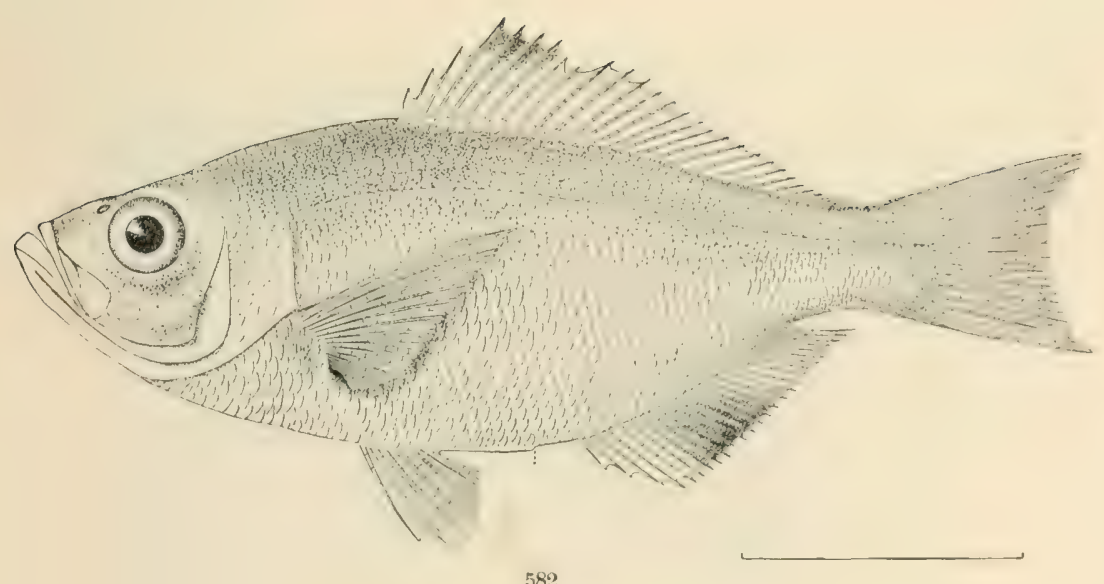

582

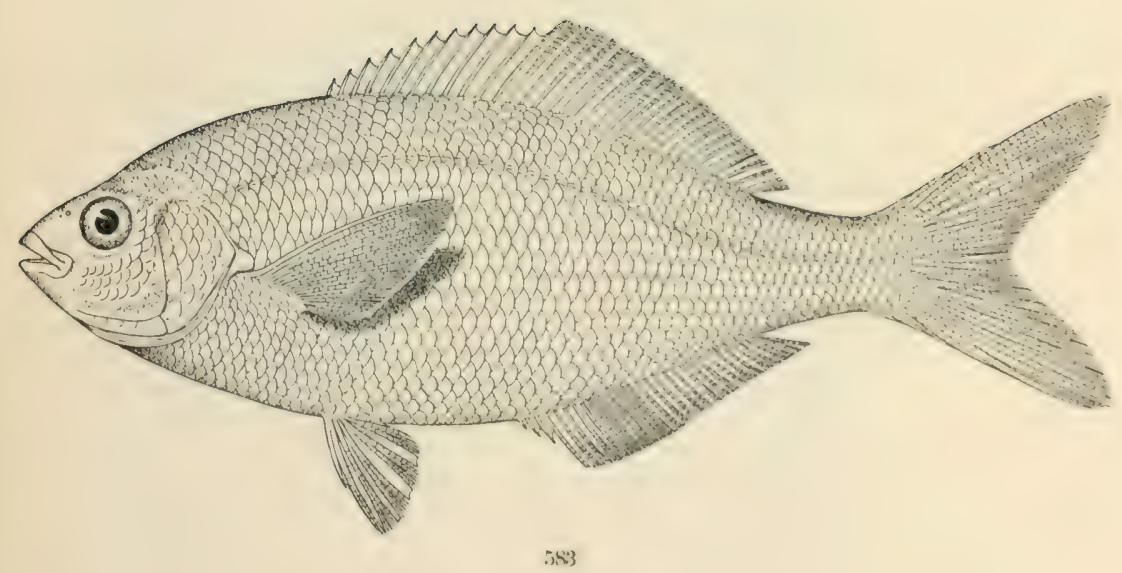

582. HYPocRitichthY' Axalis. (1'. 1500.)

583. PHaNerodon furcatus. (P. 1506.) 


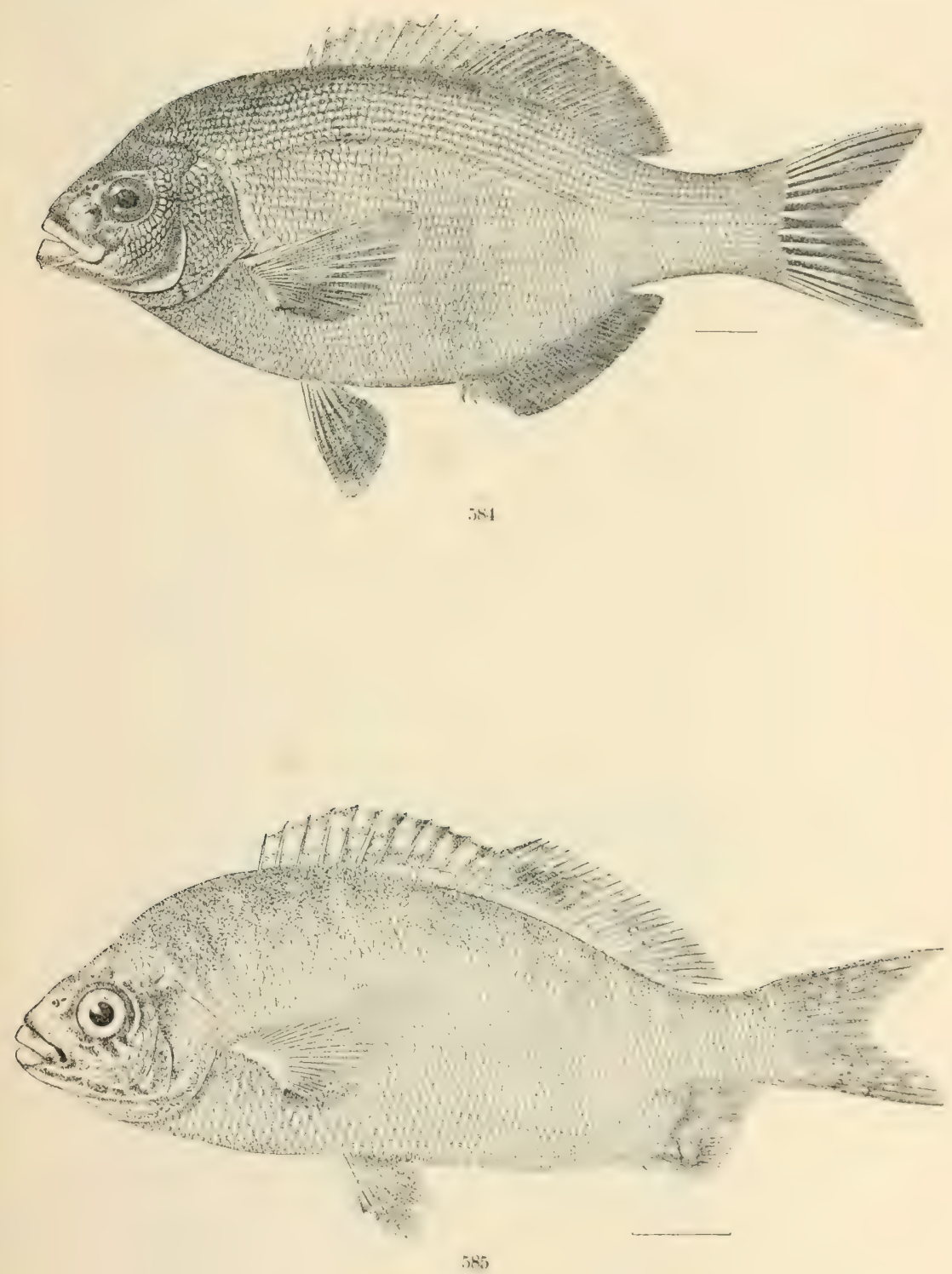

584. RHacochilus toxotes. (P, 1507.)

585. HYPSURUS CARY. (P. 1508.) 


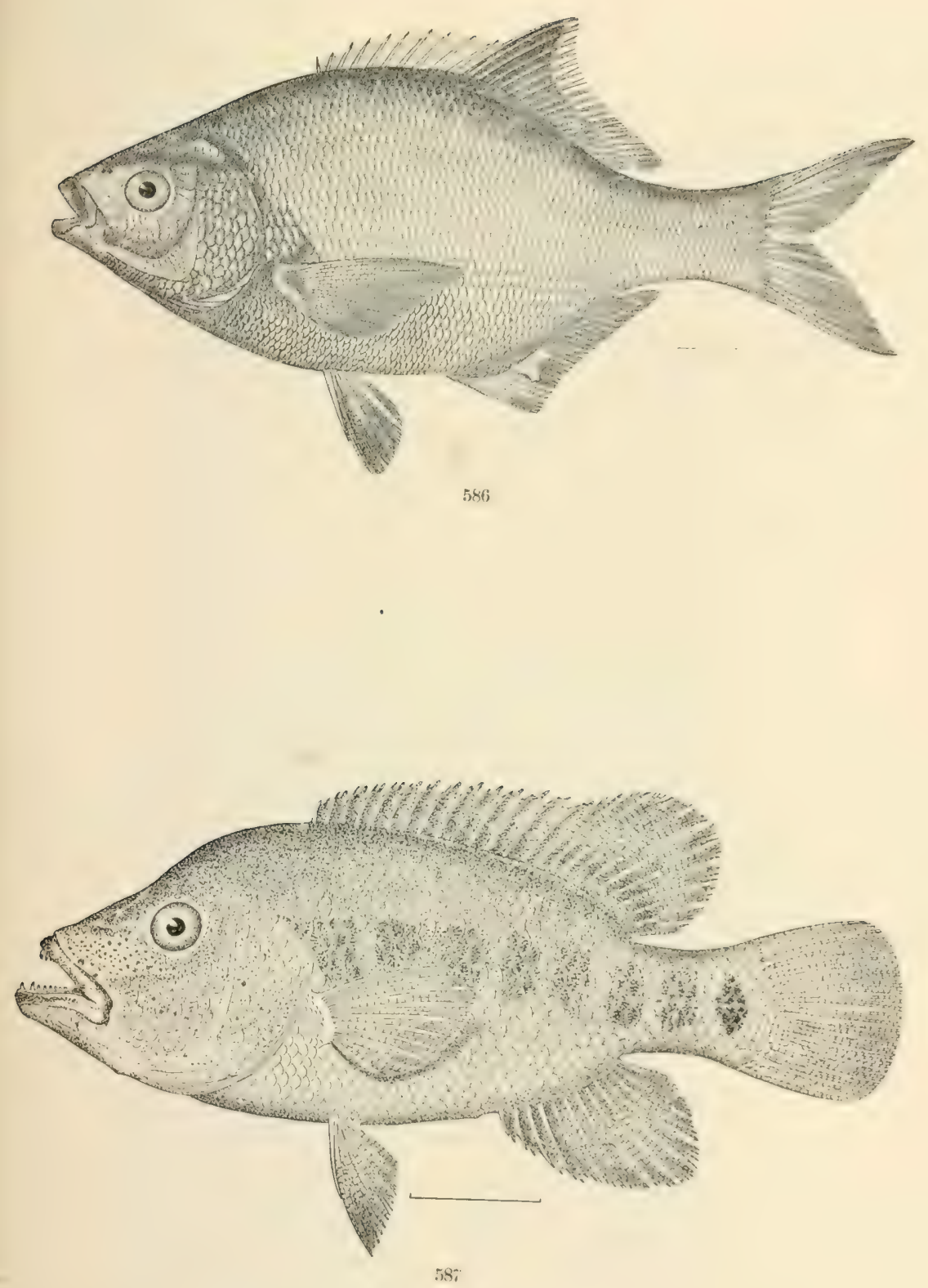

586. Damalichtily argrrosonus. (P. 1509.)

587. Cichlasoma bartoni. (P. 1515.) 1509.) 


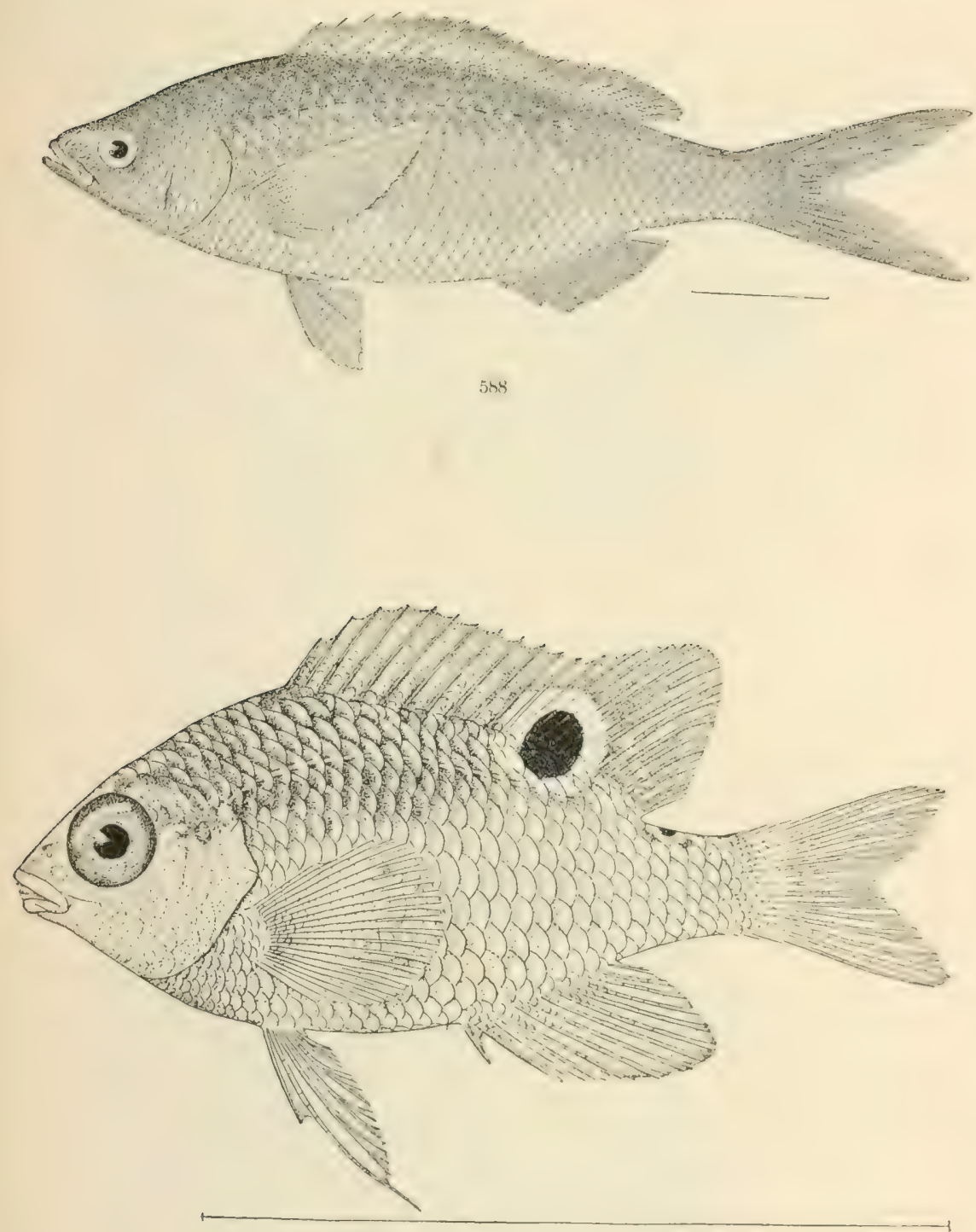

ist!

588. AZURINA IIIRUNDO. (P. 1544.)

589. EuponaCeNTrus Flavilatus. (P. 1557.) 


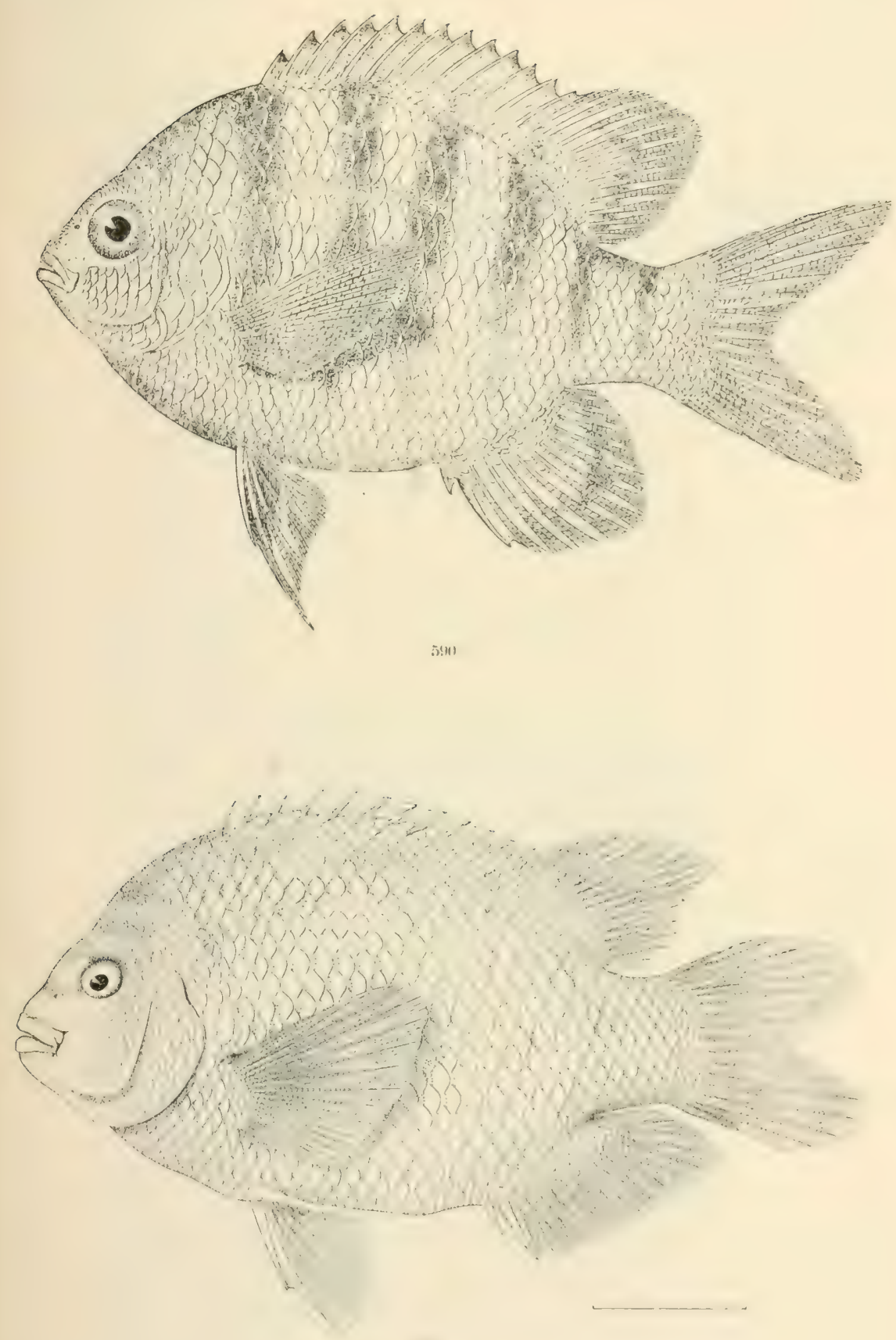

i1

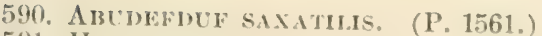

591. Hrisviops I3UHICUNits. (I'. 1564.) 



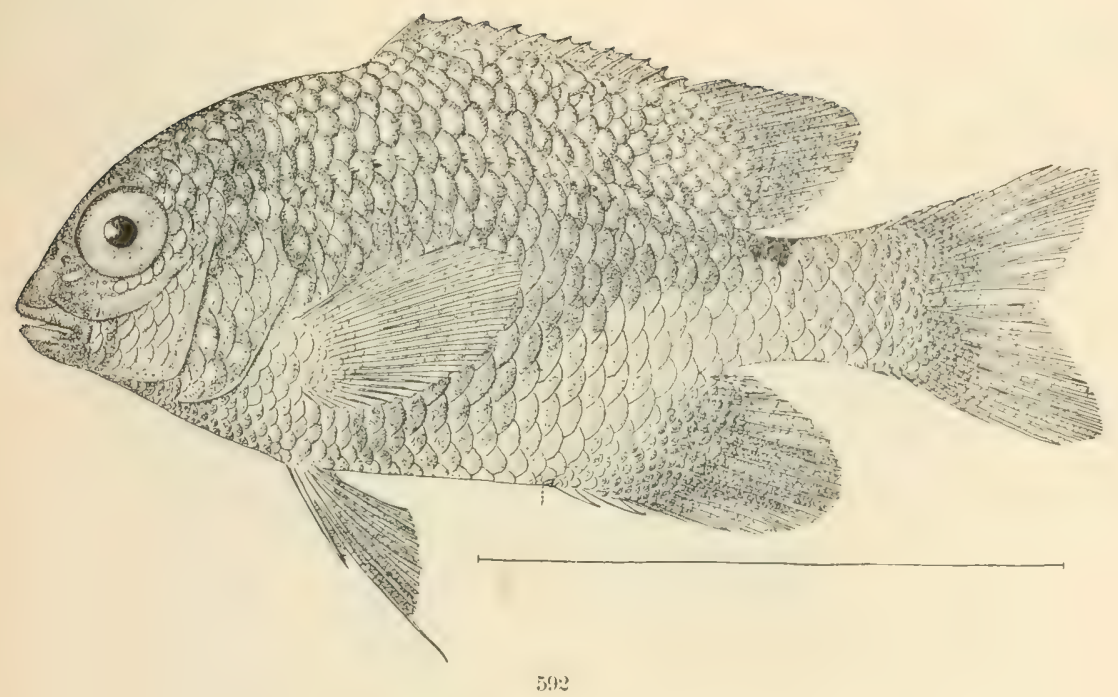

592

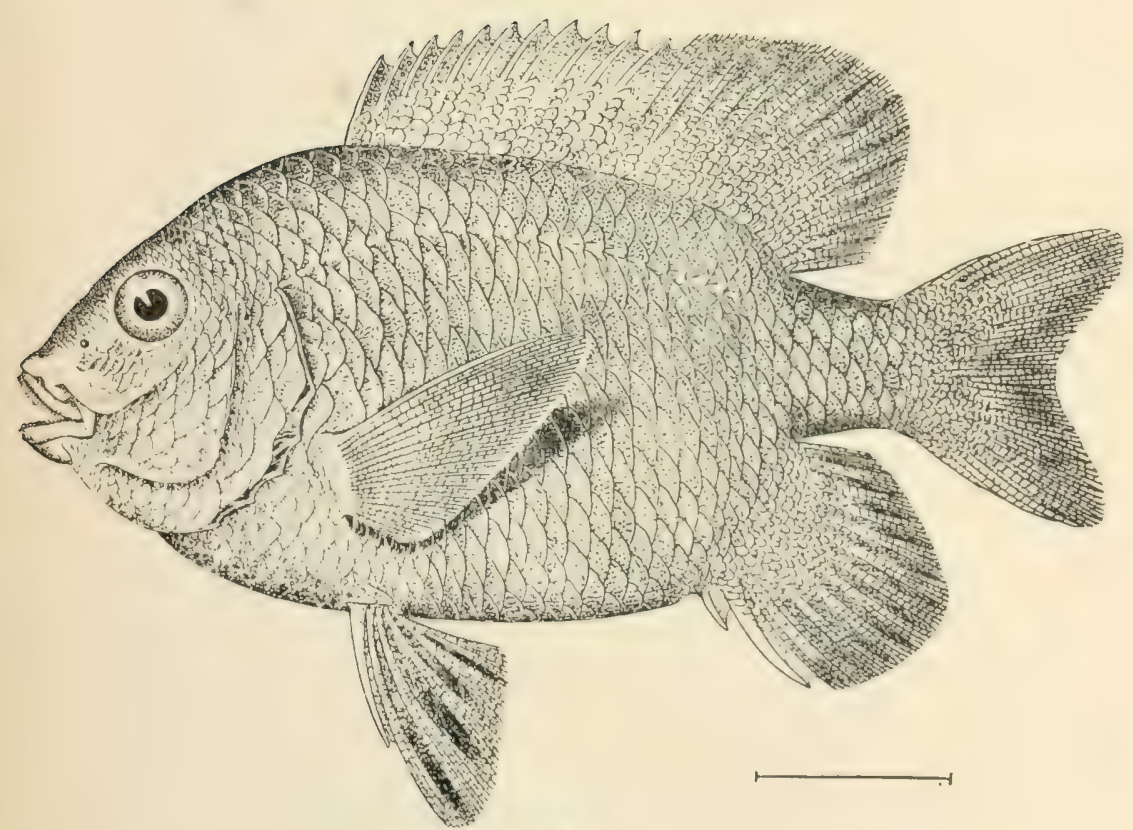

592. Microspatmodon Bairdit. (P. 1566.)

593. Michospathodon chrysurus. (P. 1567.) 


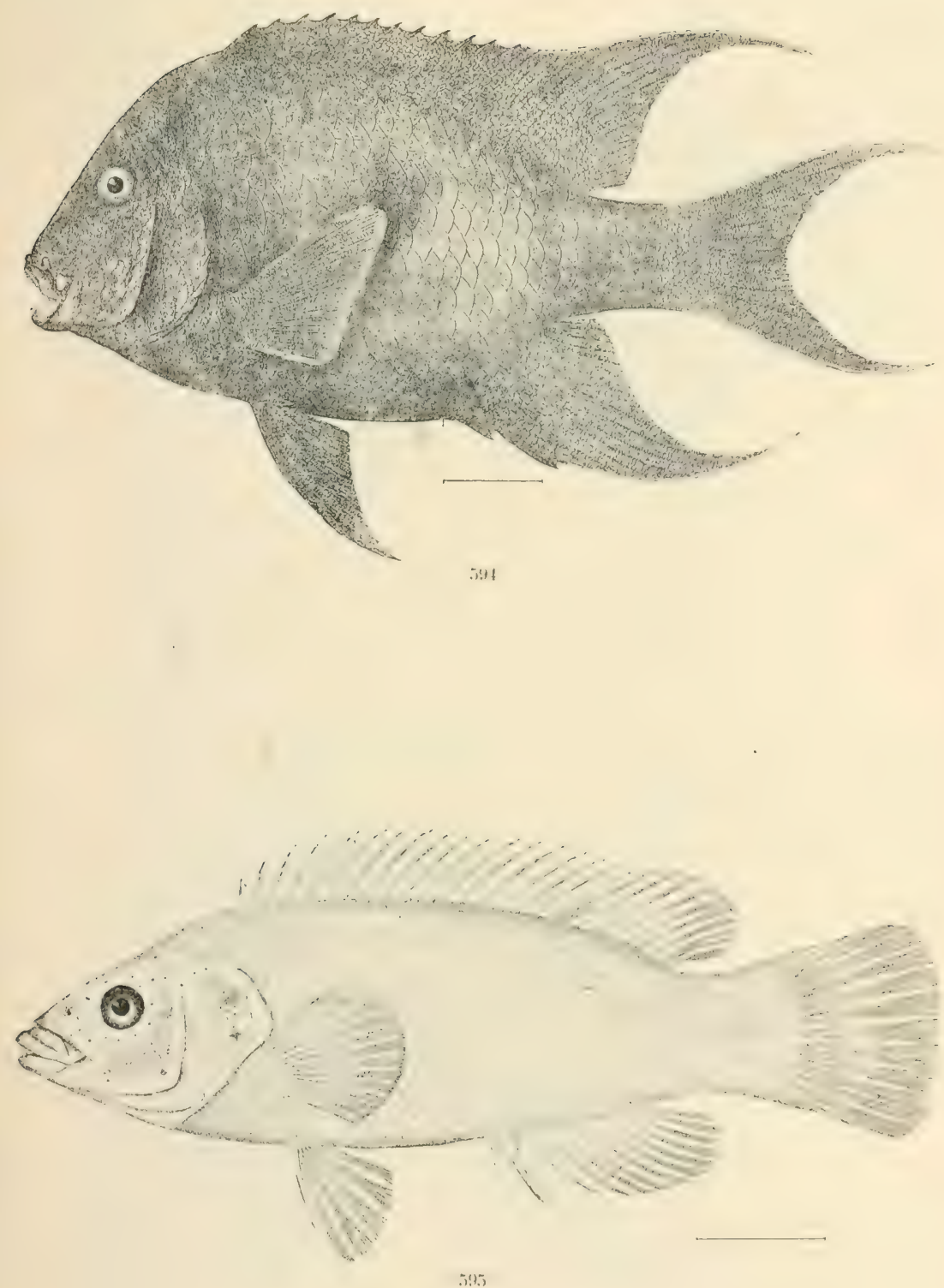

594. Microspationon domsalis, (P. 1568.)

595. 'Tautogolabrus ainsierisus. (P. 157T.) 


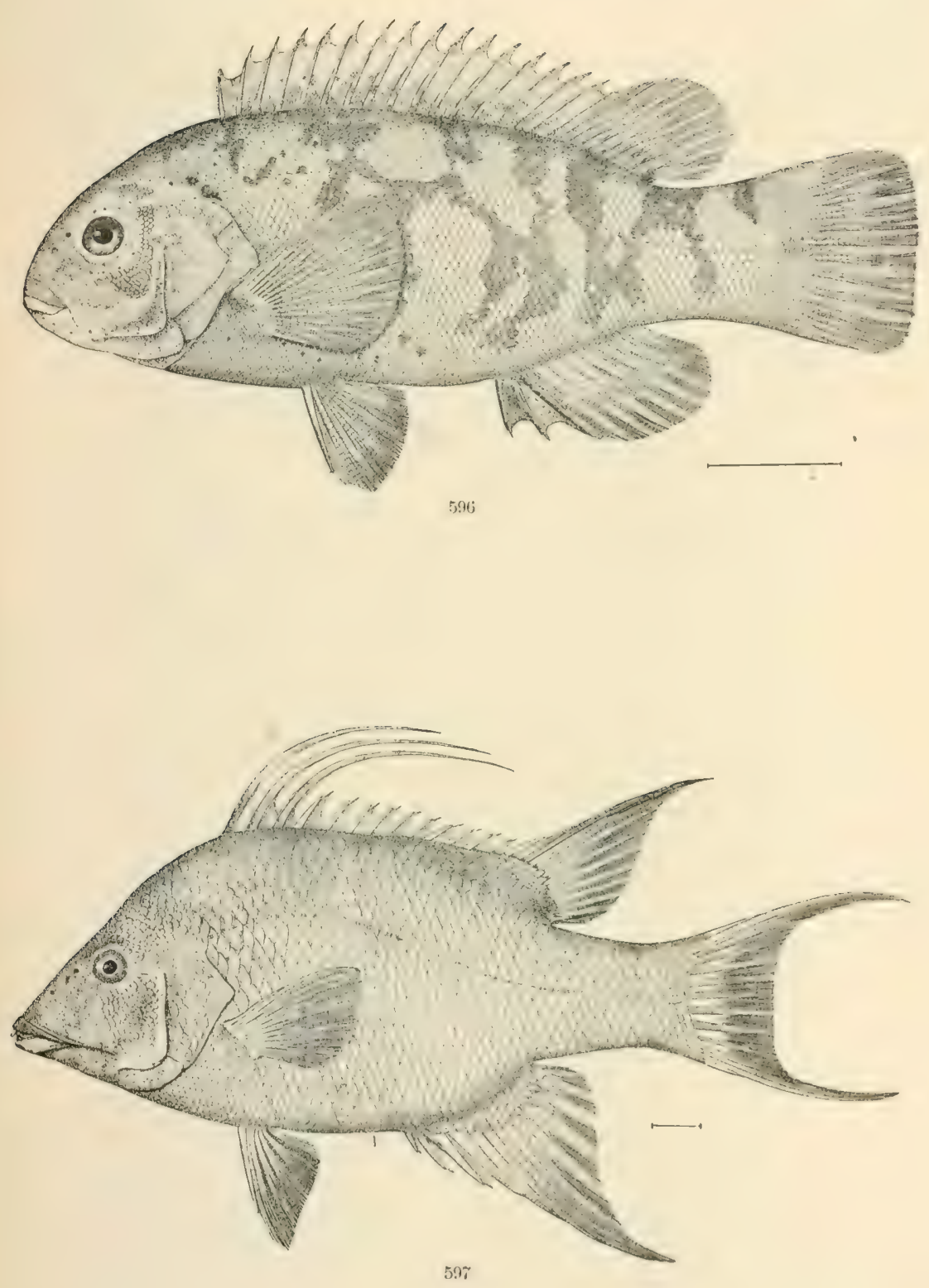

596. TAUTOGA ONITIS. (P. 1578.)

597. LACHNOLAIMLS MANIMLS. (1'. 1579.) 



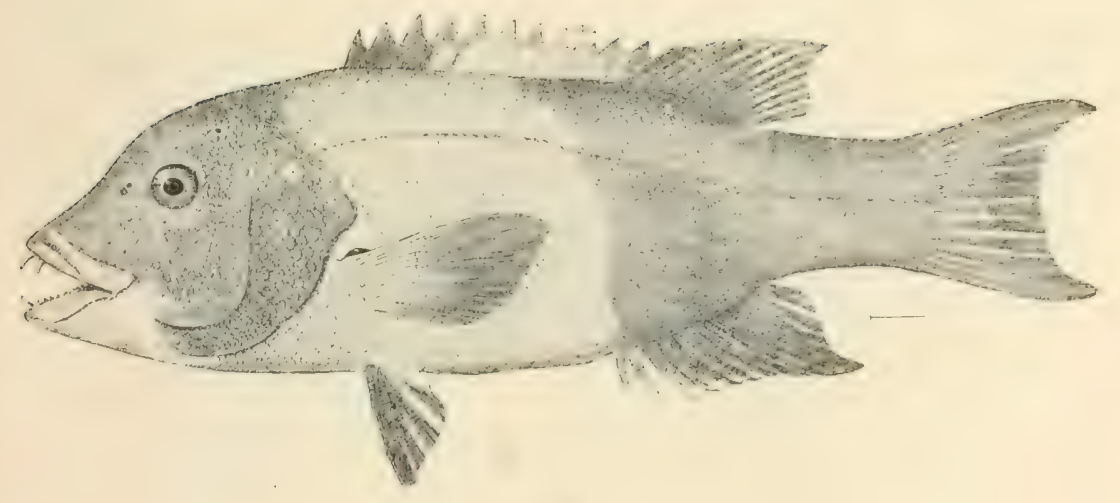

598

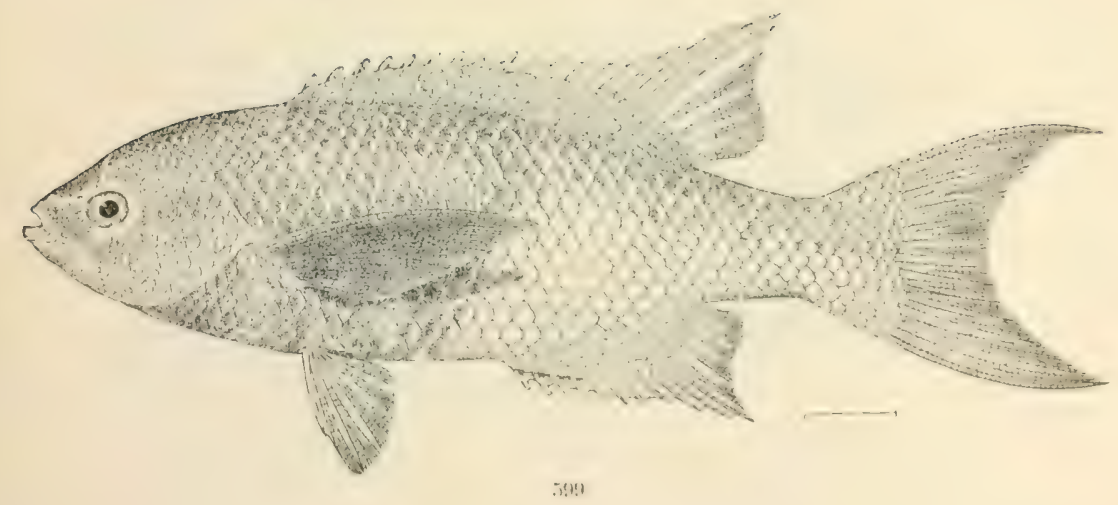

598. PMHLOMETOPON I'TCHER, (1'. 1585.)

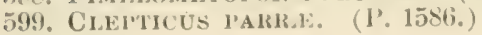





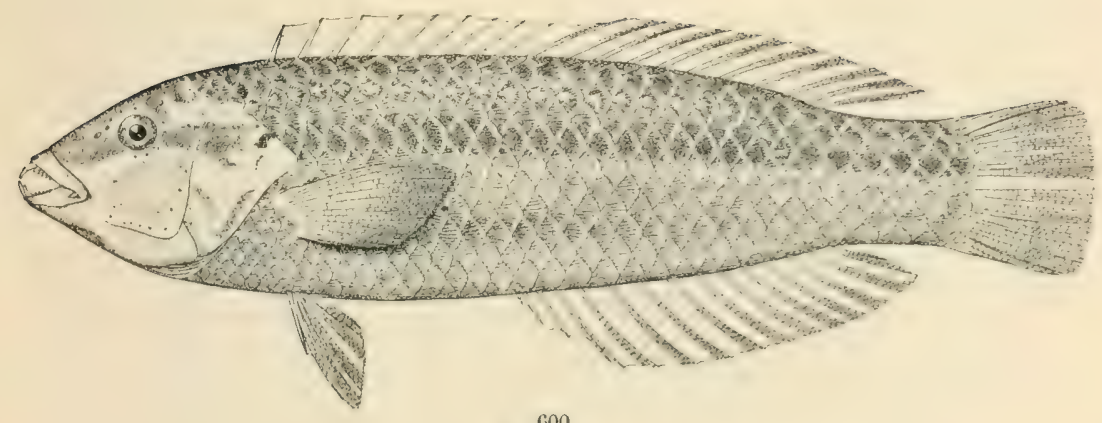

600

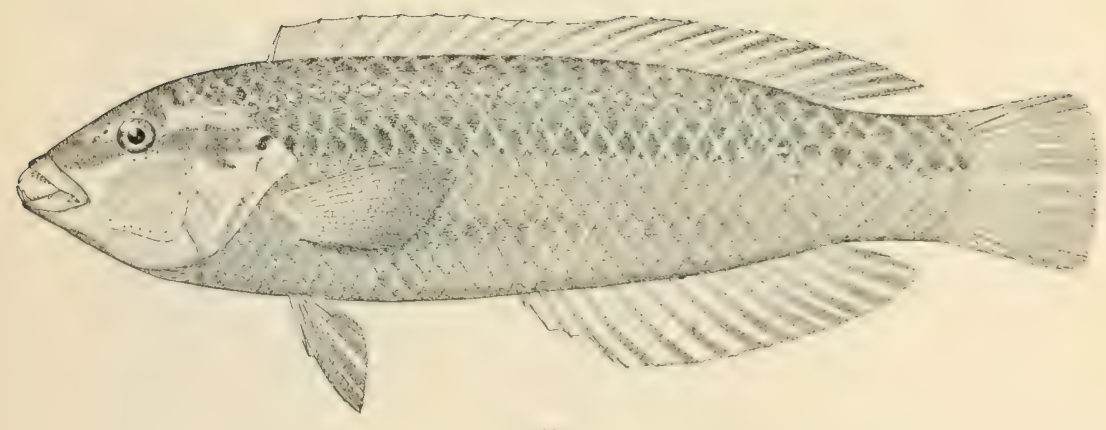

601

600. IrIdio Radiatus. (P. 1590.)

601. Inidio isivitatús. (P. 1595.) 


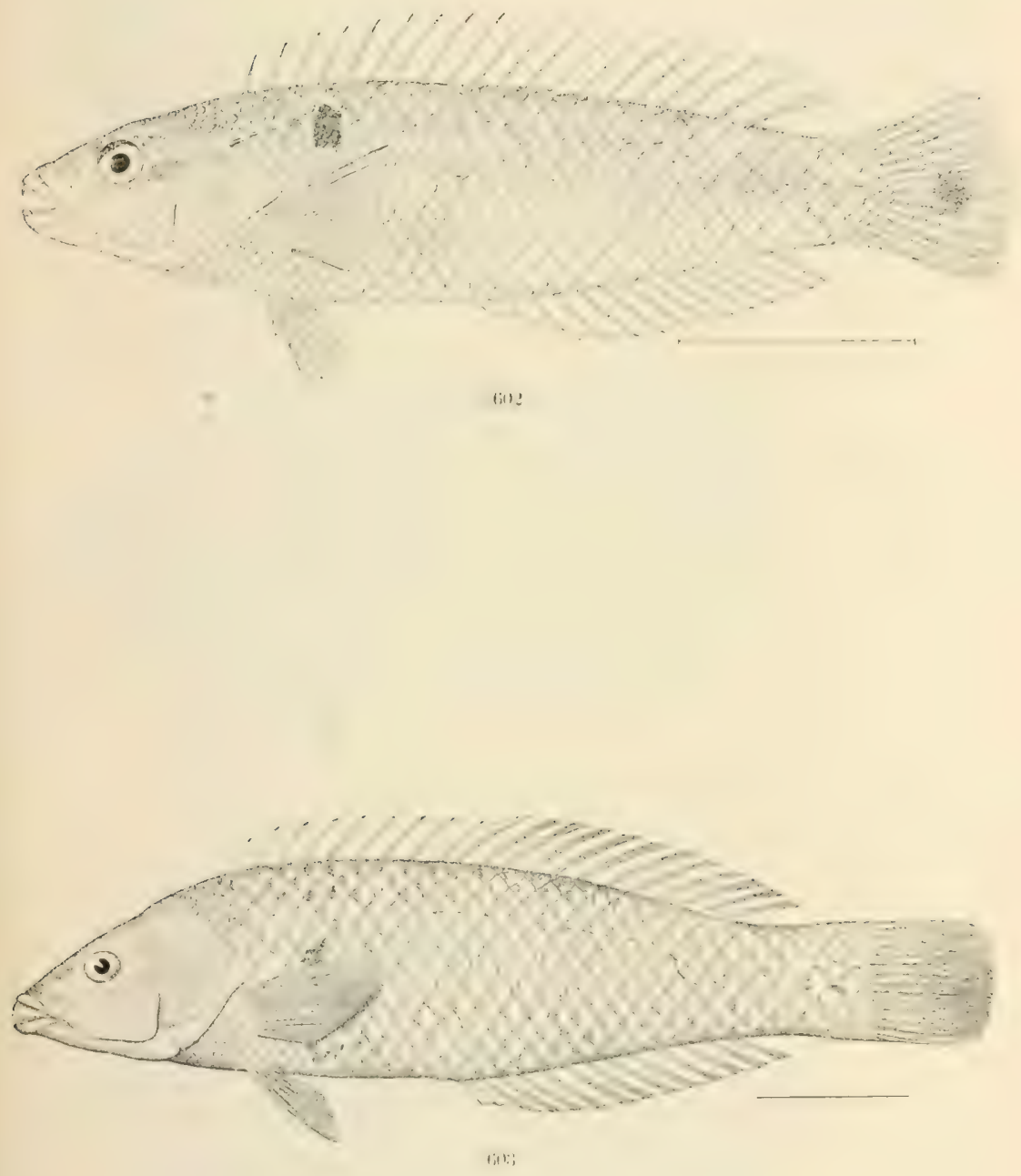

602. IRIMIO DISIILUS. ( $\mathrm{l}, 1597$.

603. EMMEEKIA VENUSTA. (ए'. 1602.) 


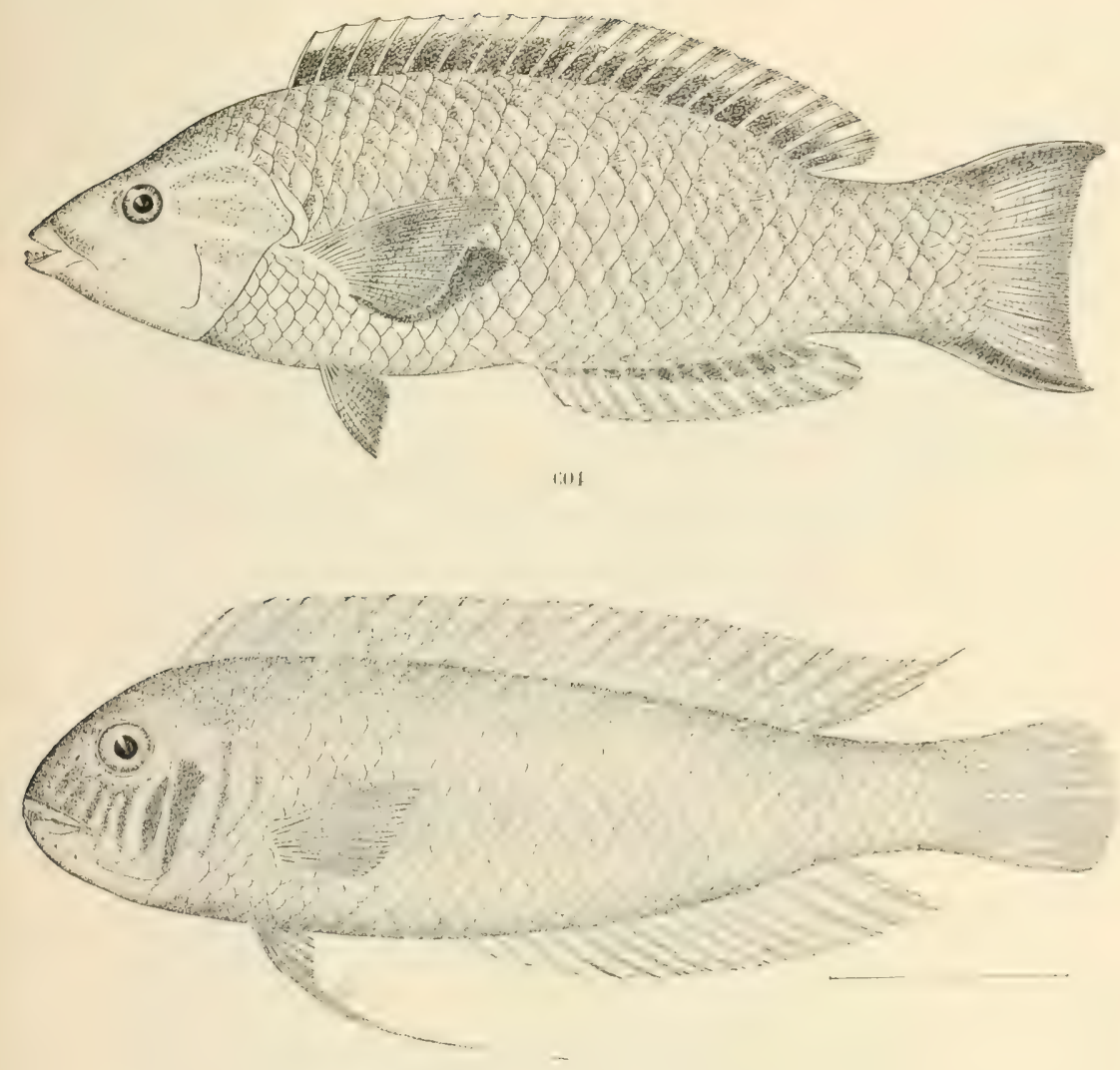

(it):5

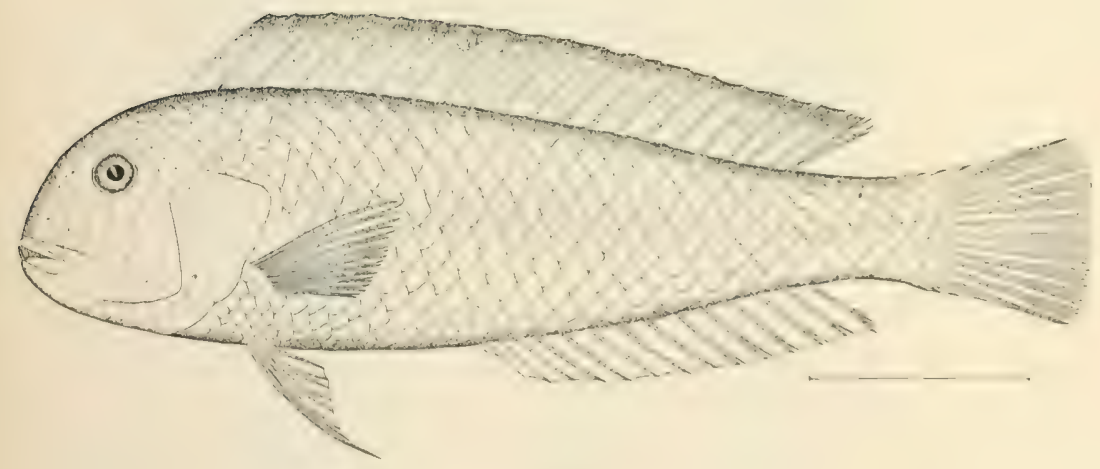

init

604. C'Horichthys gramaticus. (P. 1610.)

605. Novaculathins ventralis. (P. 1615.)

606. Novaclichthys infirmes. (P. 1616.) 


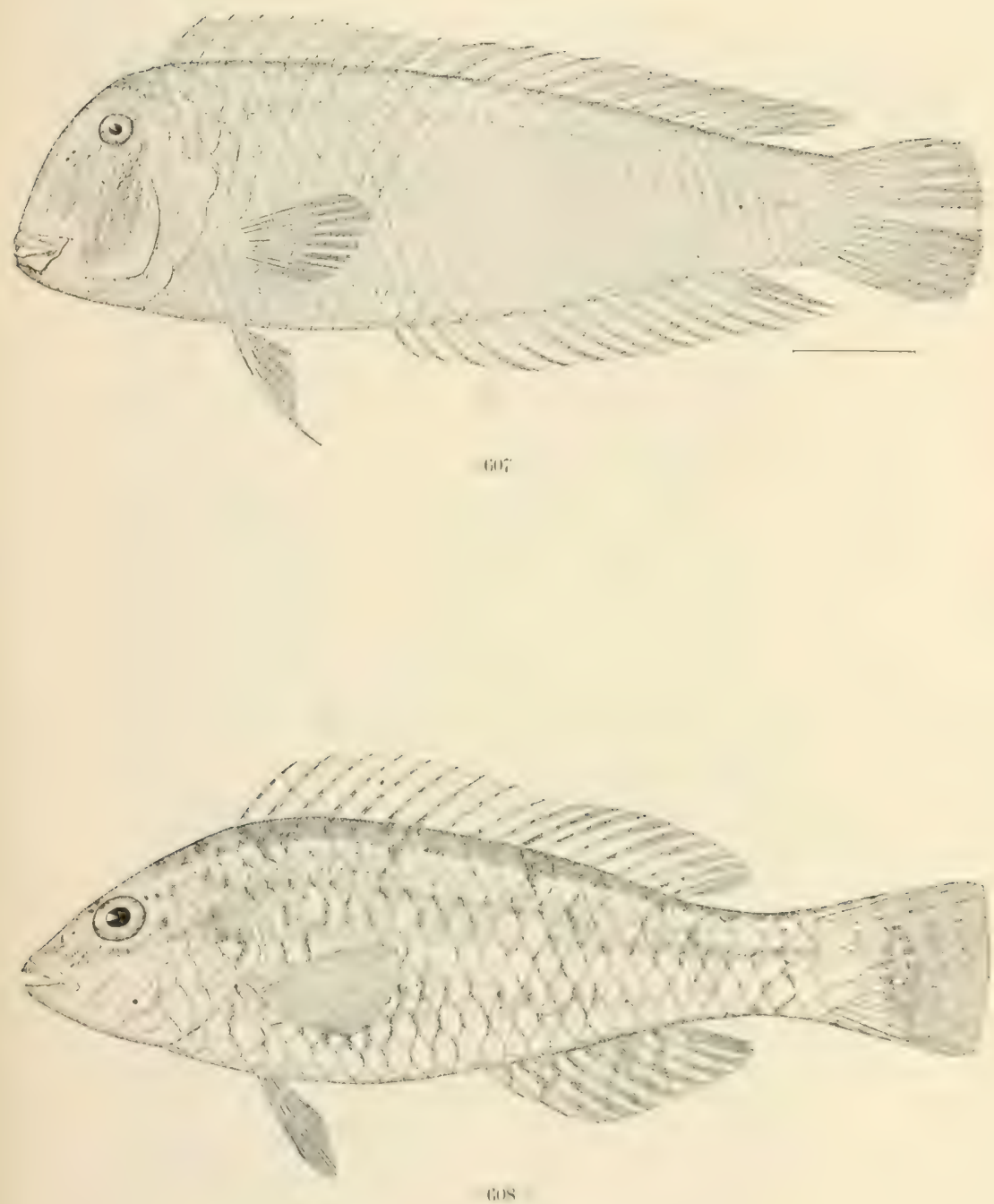

607. XYRichthys pisittacus. (P. 1618.)

608. CRY'PTOTOMUS BERYLLINES, (1', 1625.) 




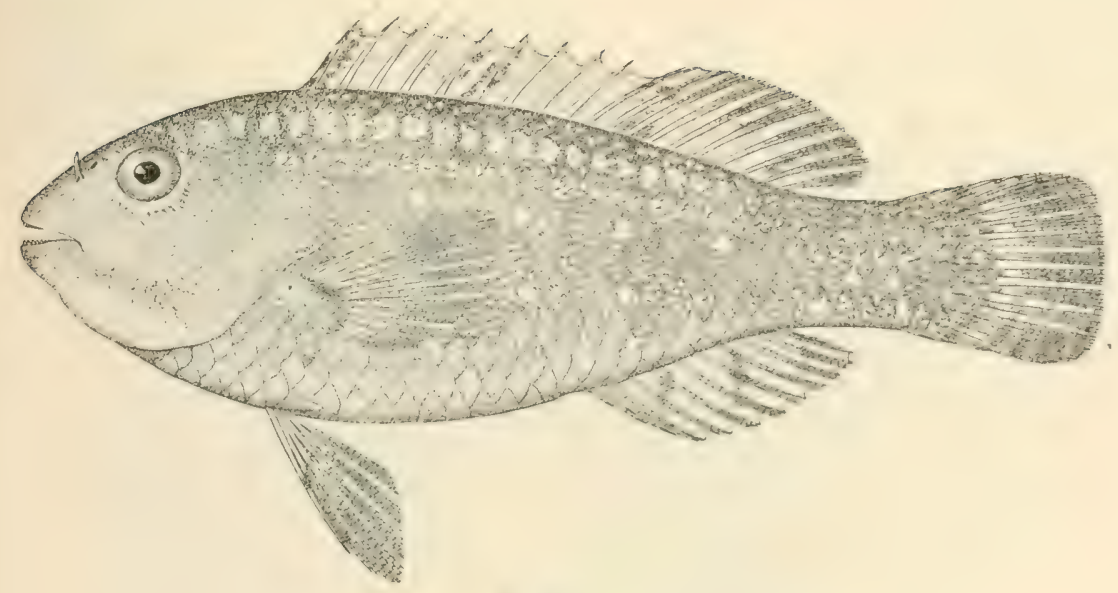

611

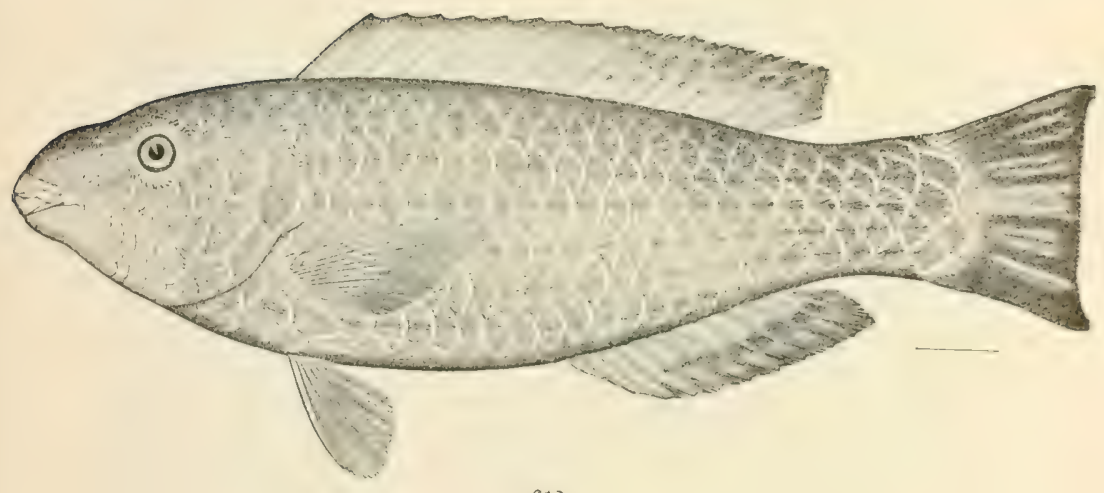

612

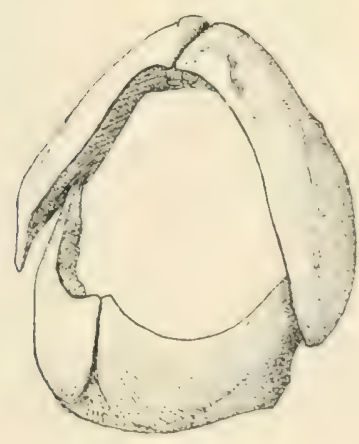

613

611. SPARISOMA nOLLOMYSTAX. (P. 1632.)

612. Scarus CUZAMILE. (P. 16.48.)

613. Jaws OF Scarus cerulets. (P. 165'2.) 



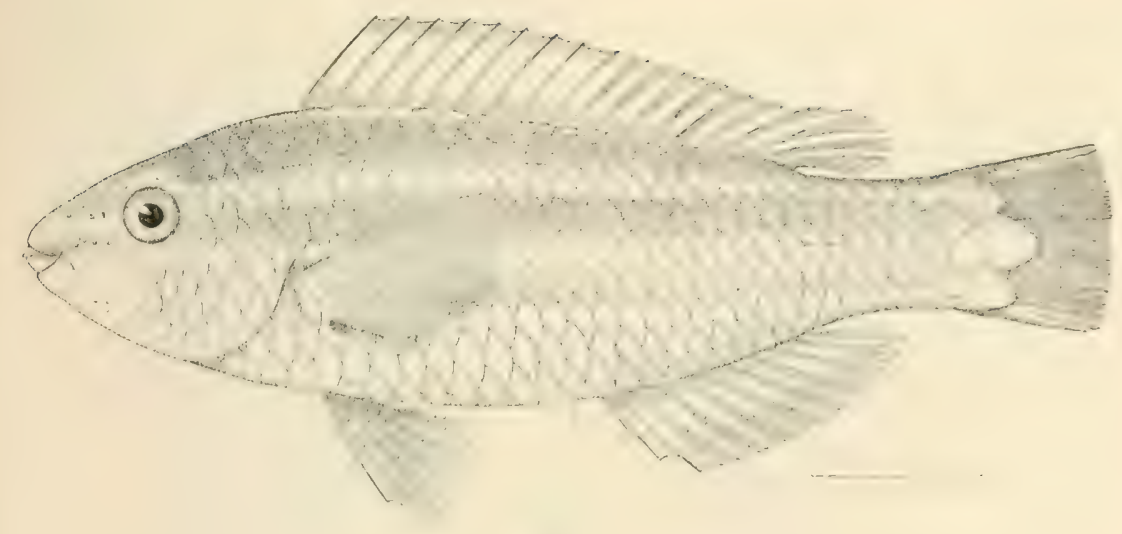

611
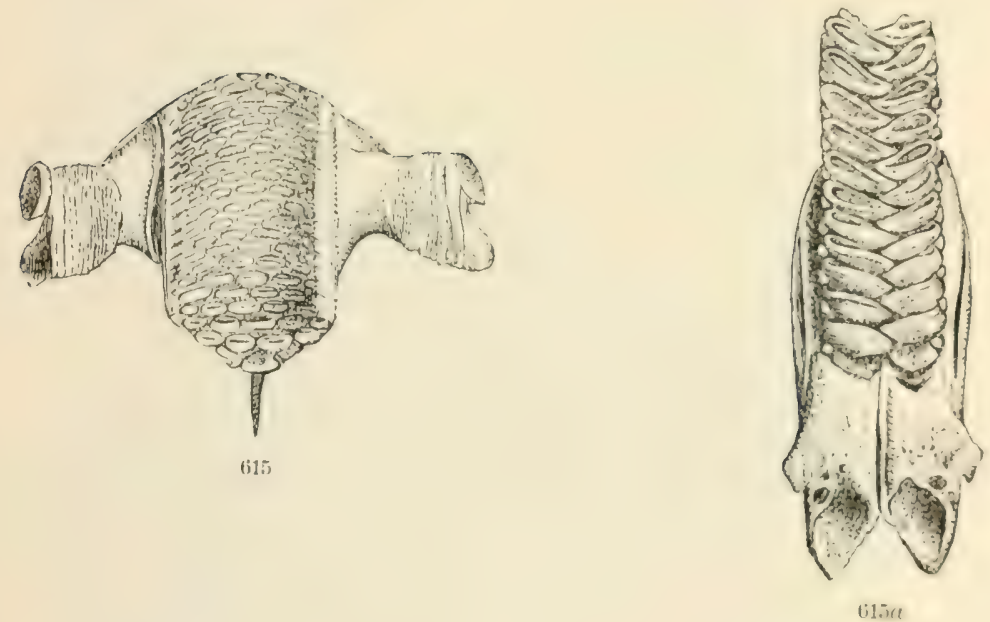

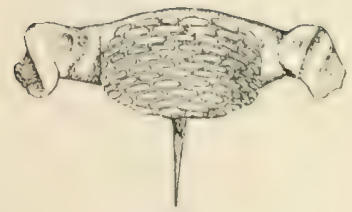

(i)i

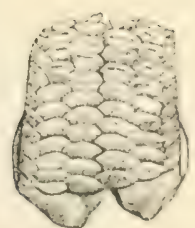

bier

611. Scarus miblmaticls. (P. 1654.)

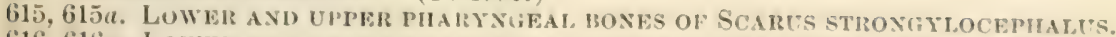
$616,616 a$. LOWER AND UPPER PHARYNIEAR BGNIS OF SPARISOMA CRETFNSF. 



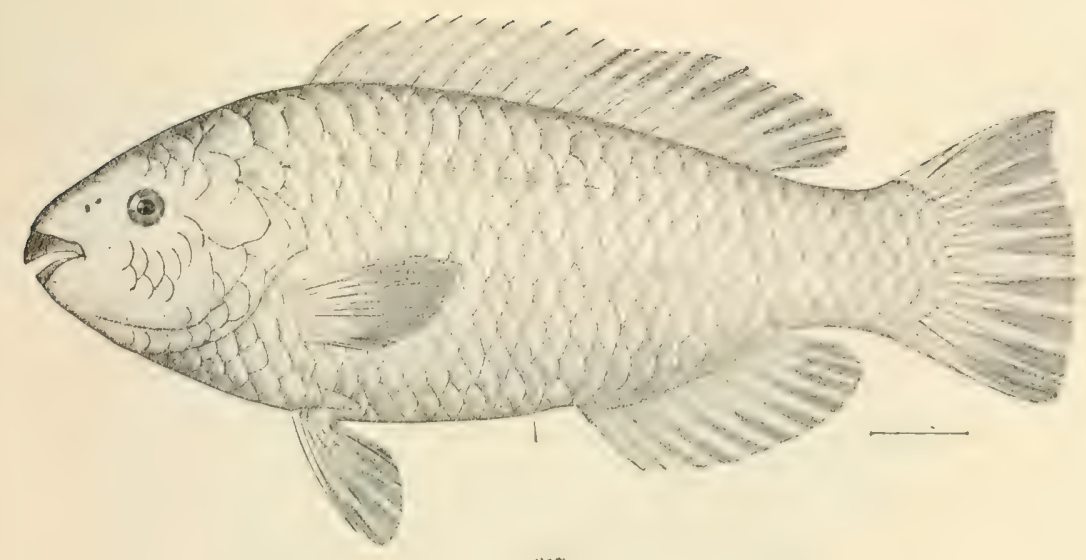

(iti)

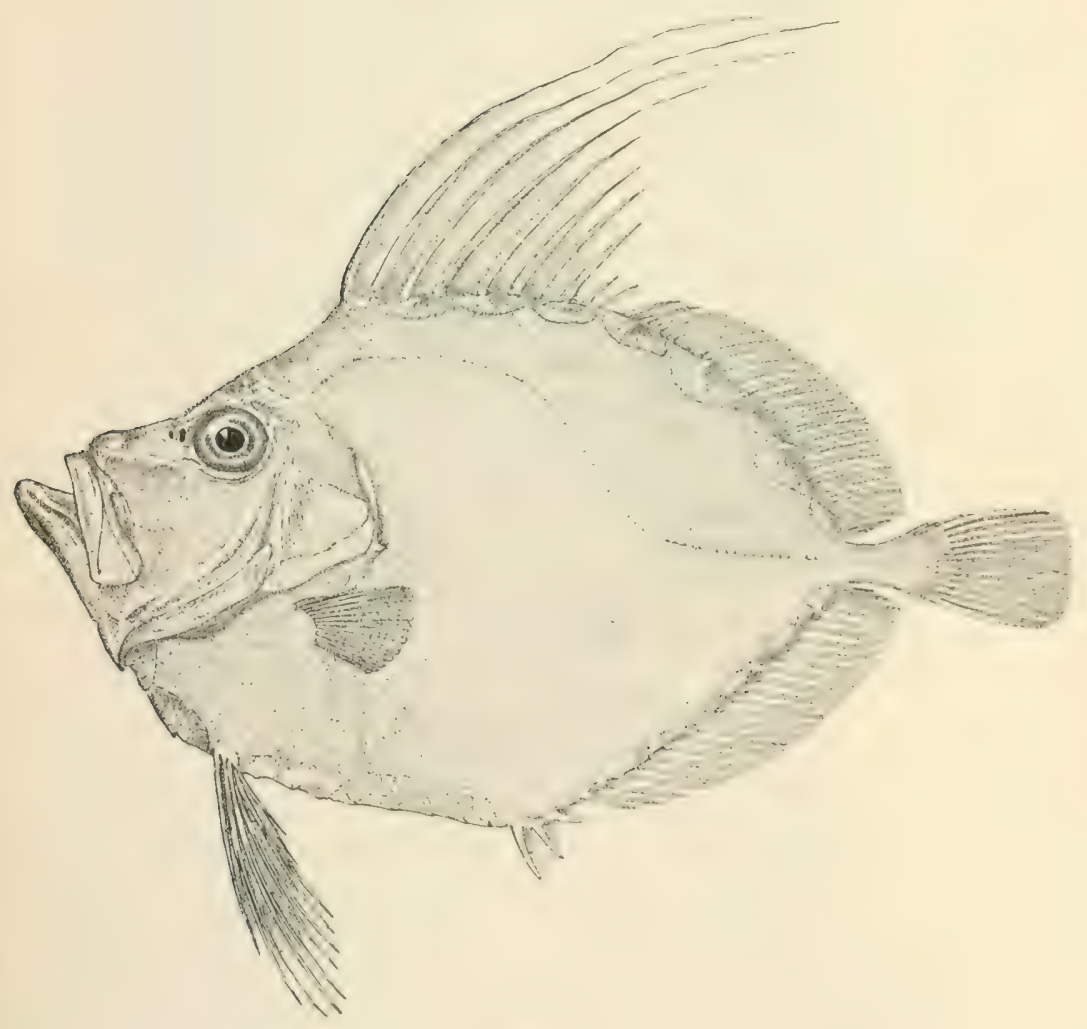

618

617. Pseudoscartes guacamaia, (P. 1657.)

618. ZENOPSIS OCELLATUS. (P. 1660.) 



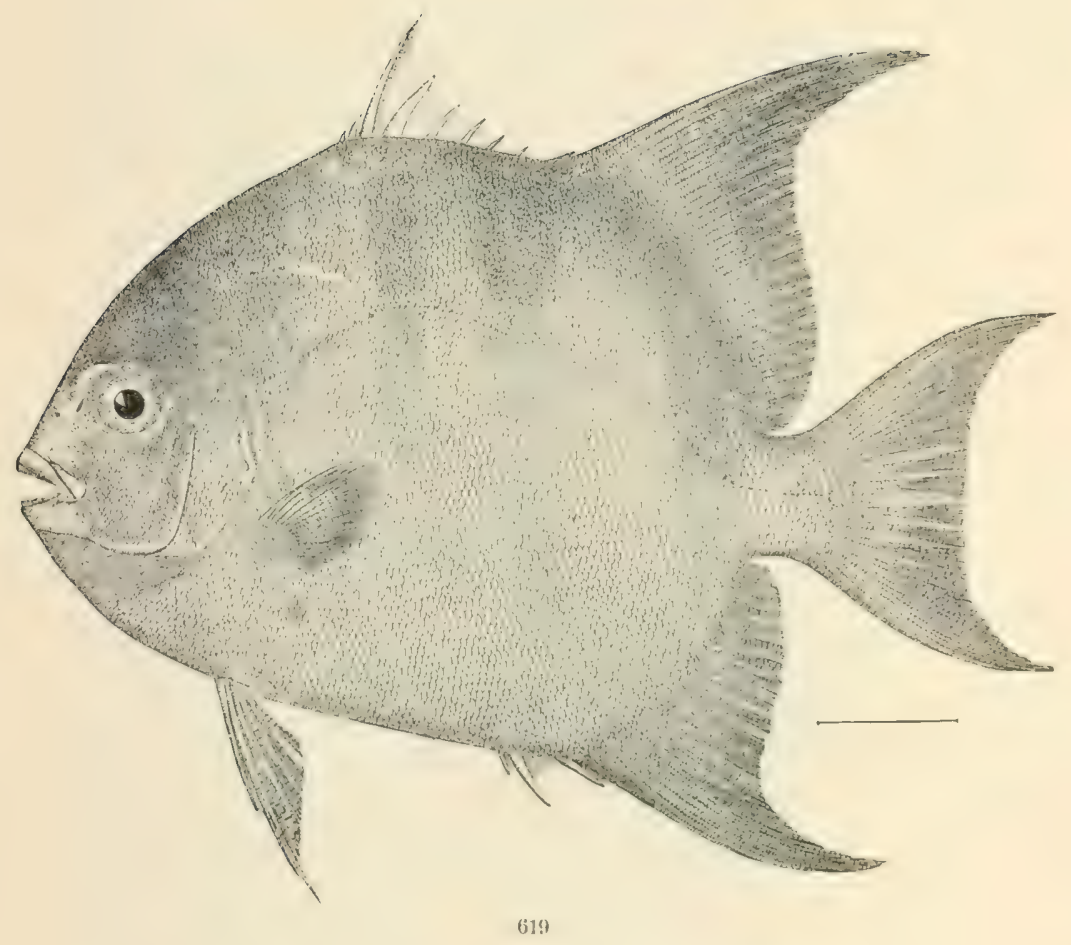

619. Chatodipterus Fabre. (P. 1668.) 



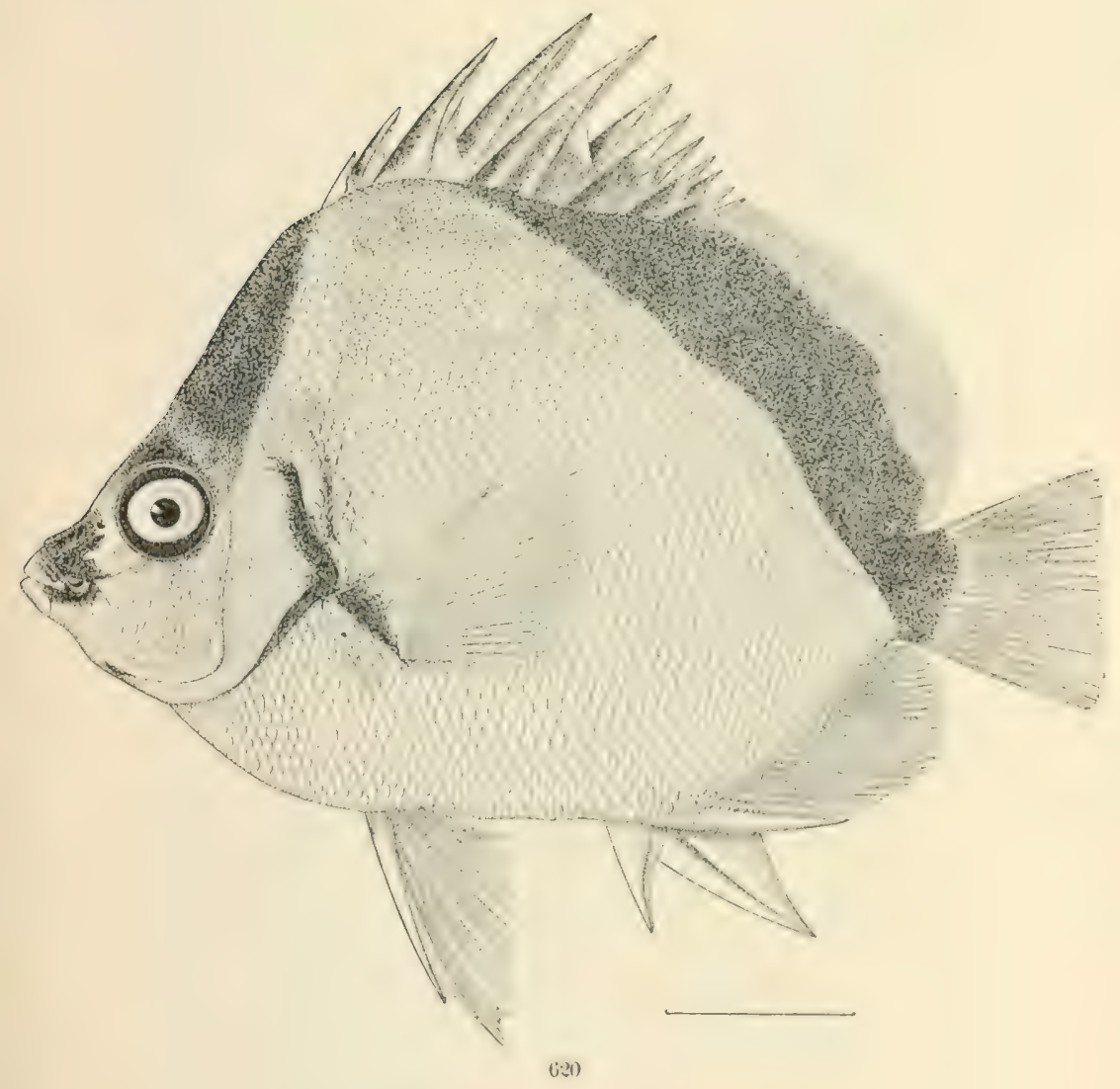

620. Ciletodon nighirostris. (P. 1673.) 



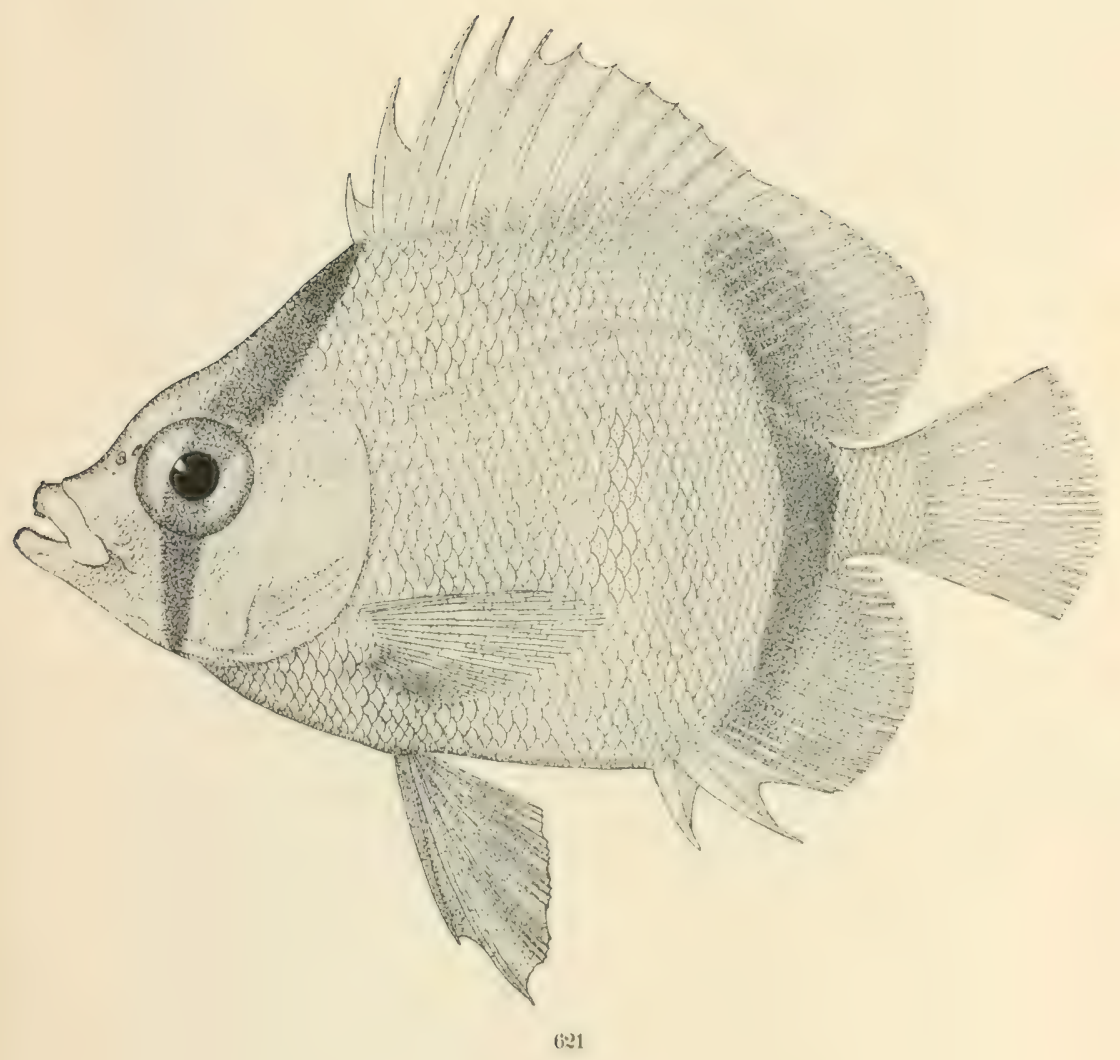

621. Chitodon oceinatus. (P.1674.) 



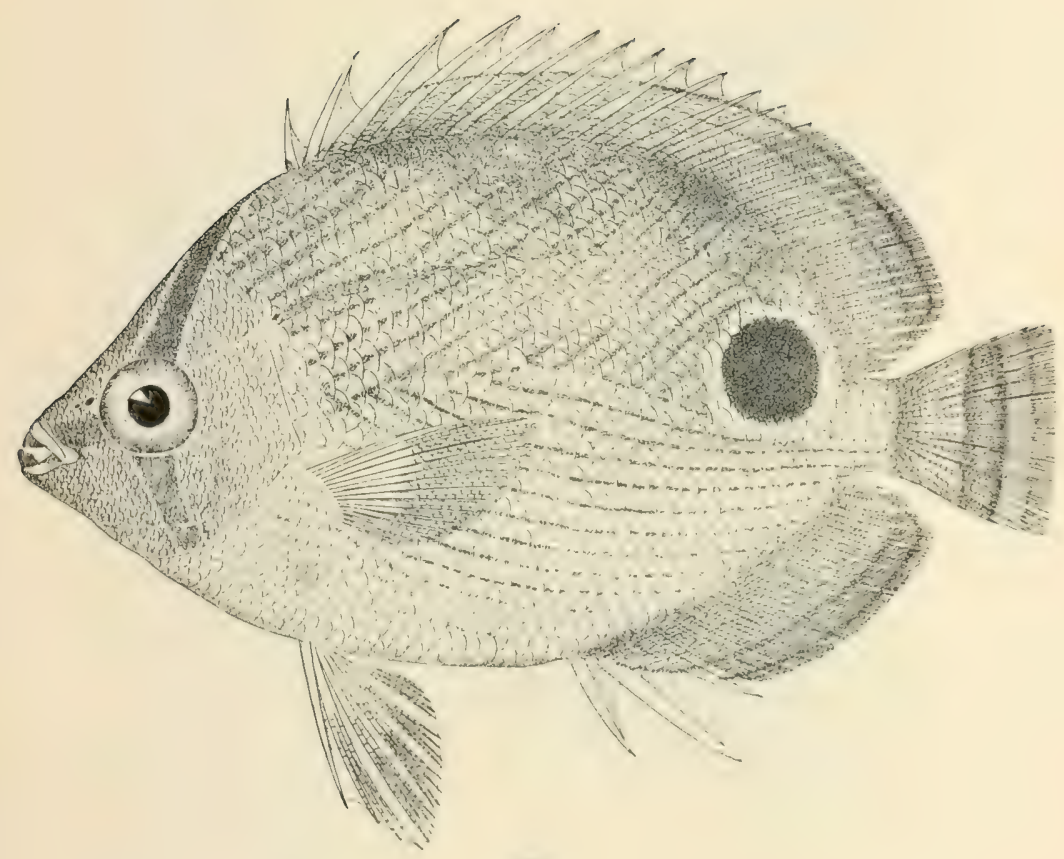

6:2:2

622. Chrtomon calistiates. (I'. 167\%, 



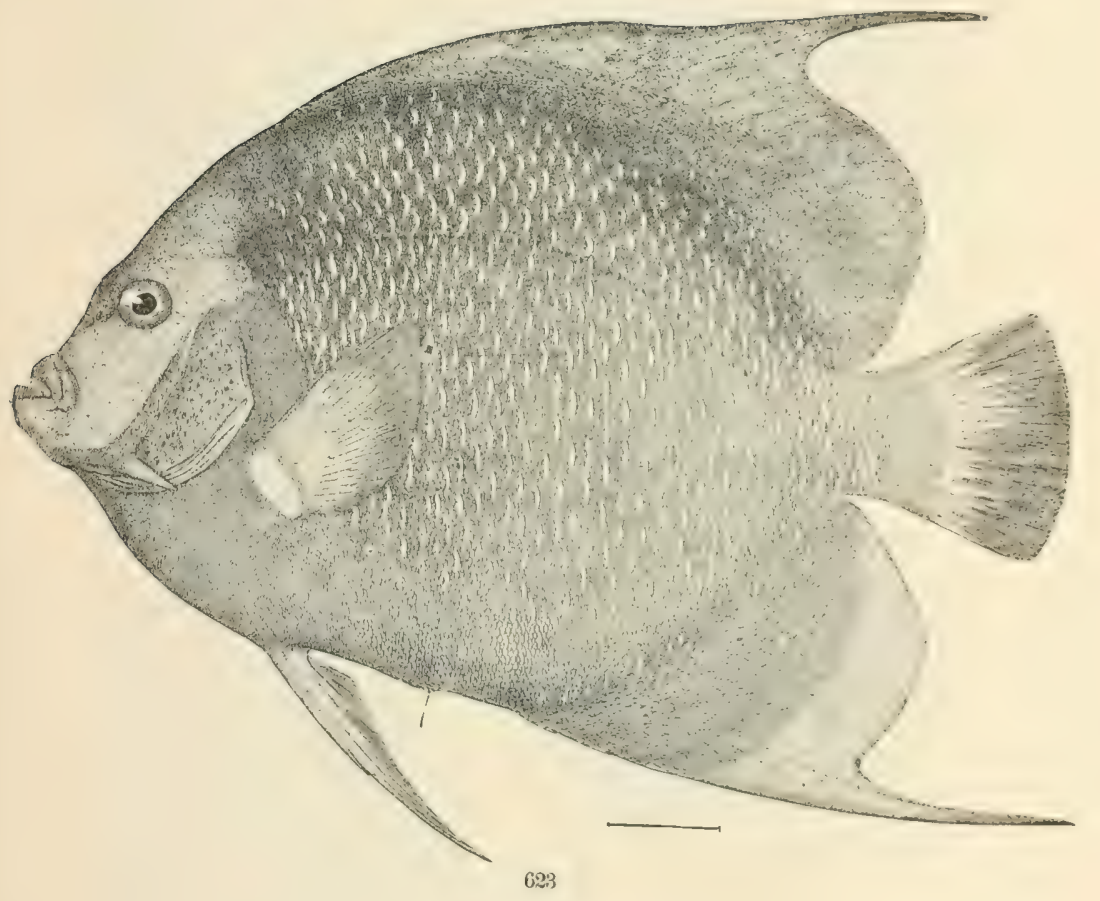

623. Pomacantuus arcuatus. (P. 1679.) 



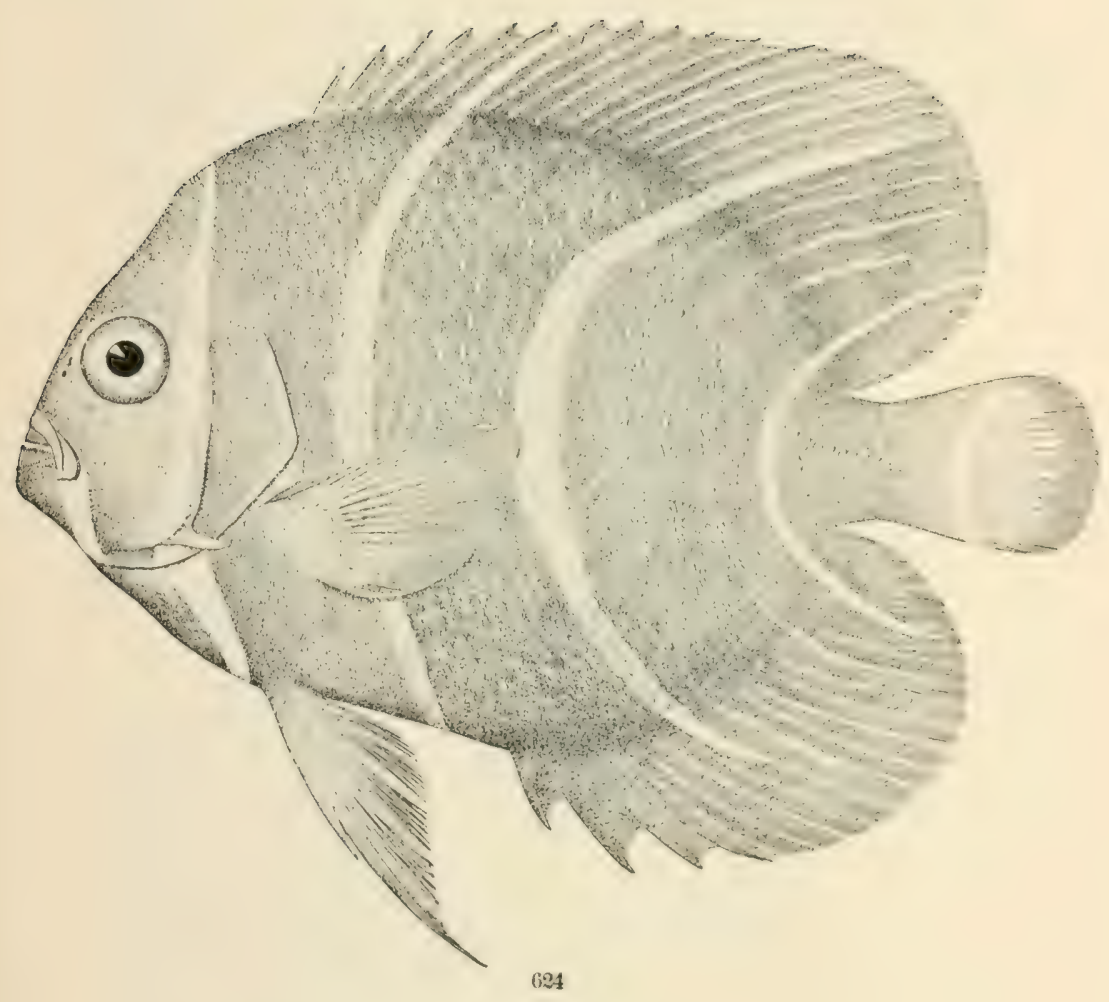

624. Pomacanthus zonilectes; young, (P. 1681.) 



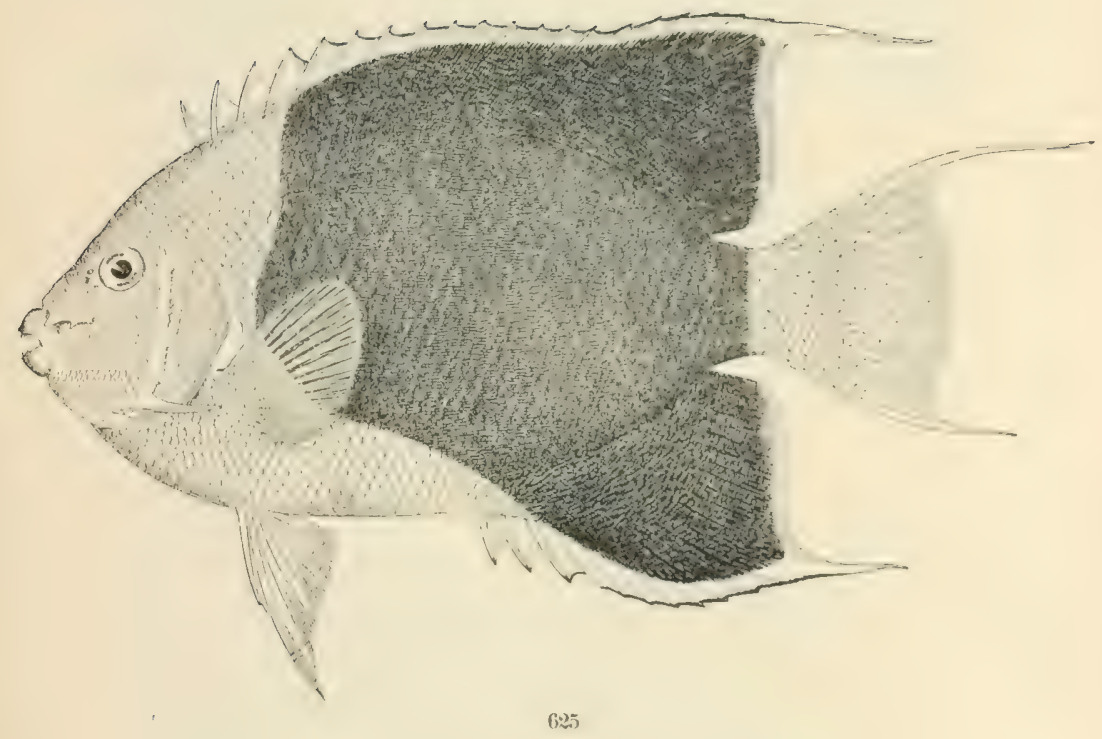

625. Holacanthus thicolor. (P. 1684.) 



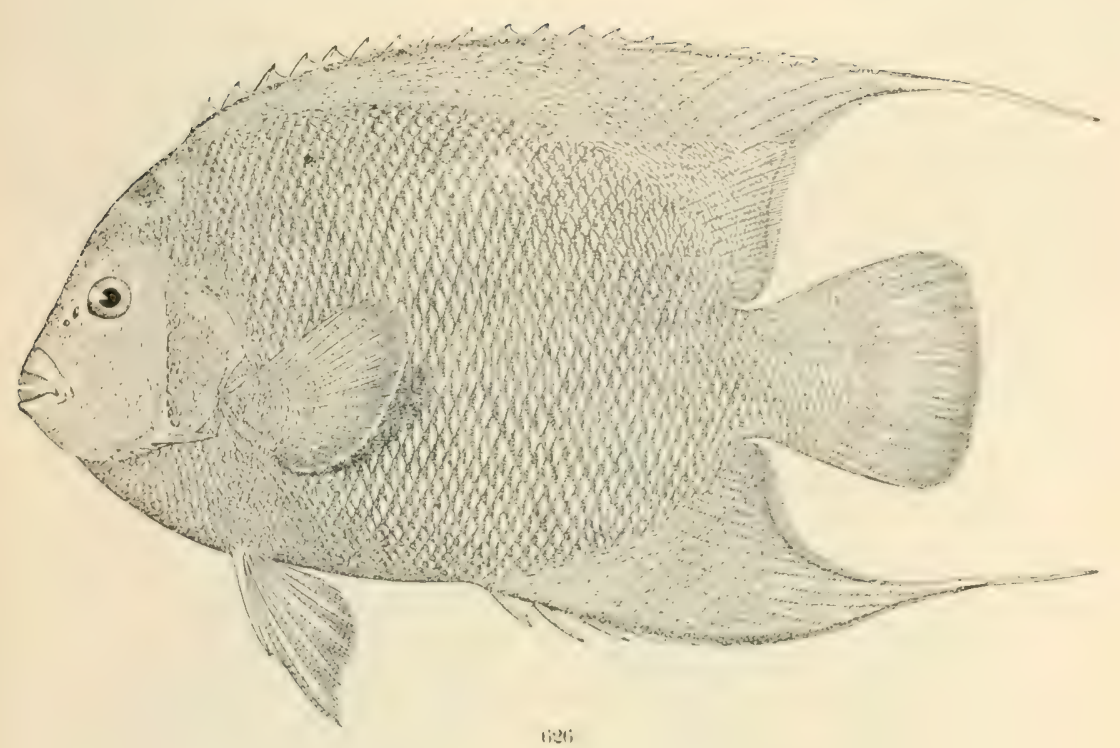

1i:2;

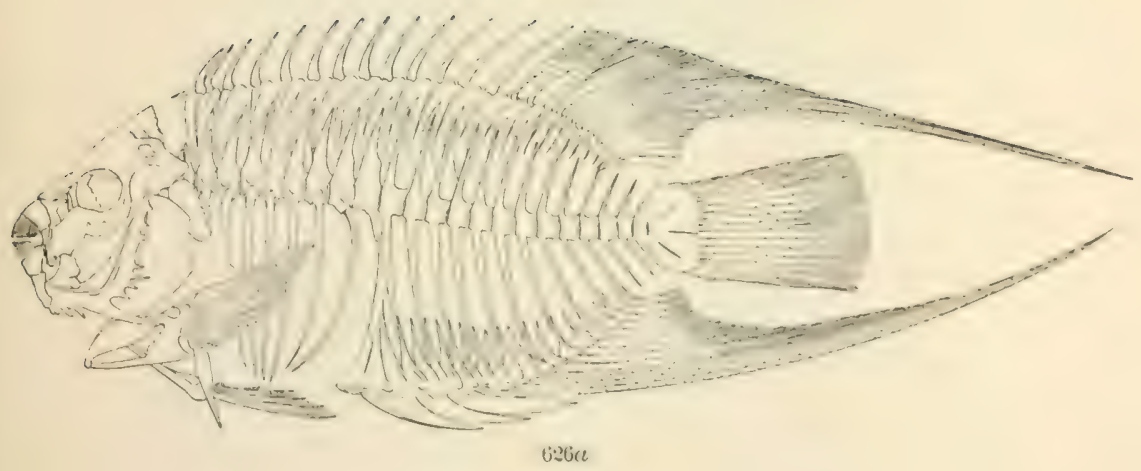

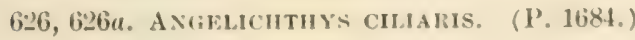





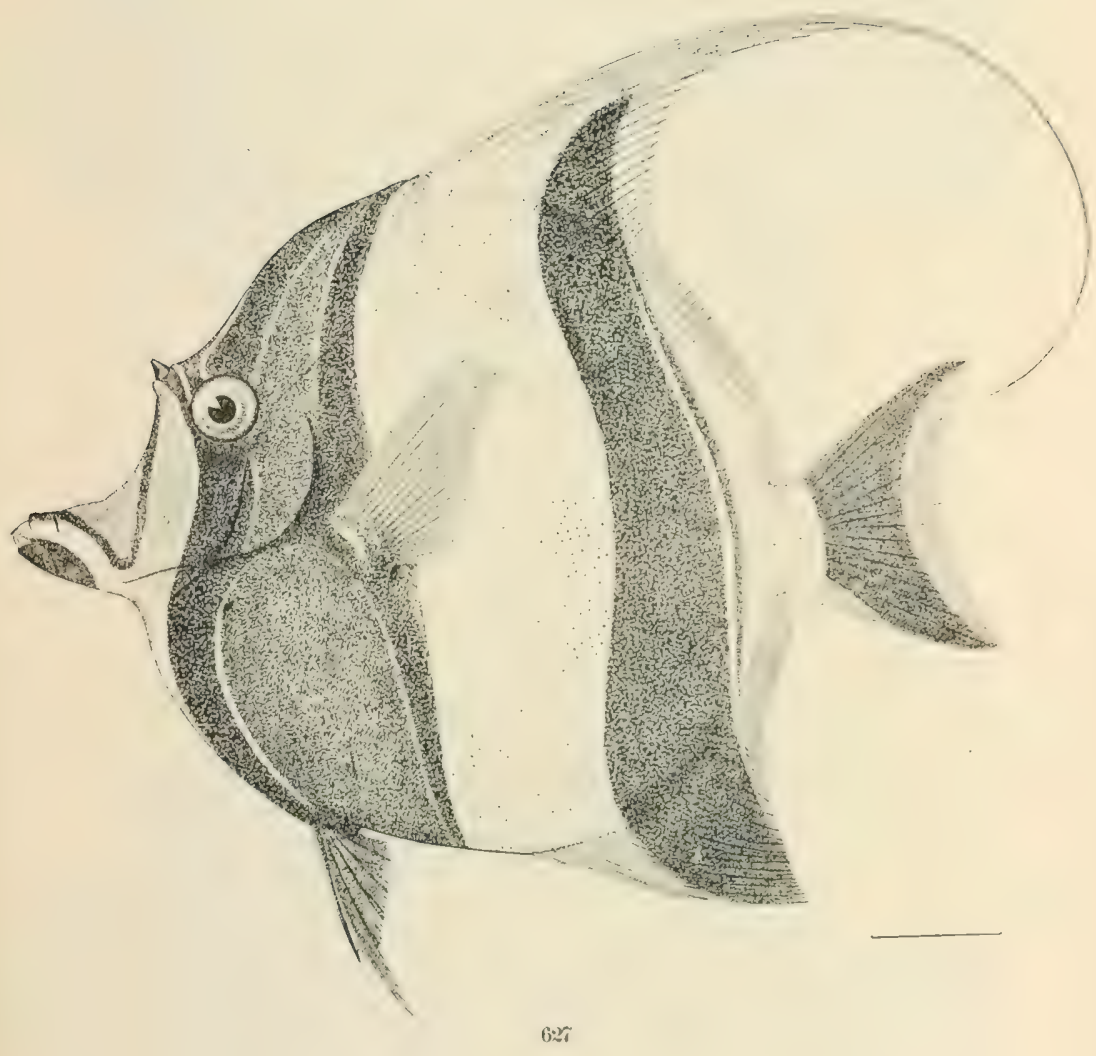

627. Zanclus connutus. (P. 1687.) 


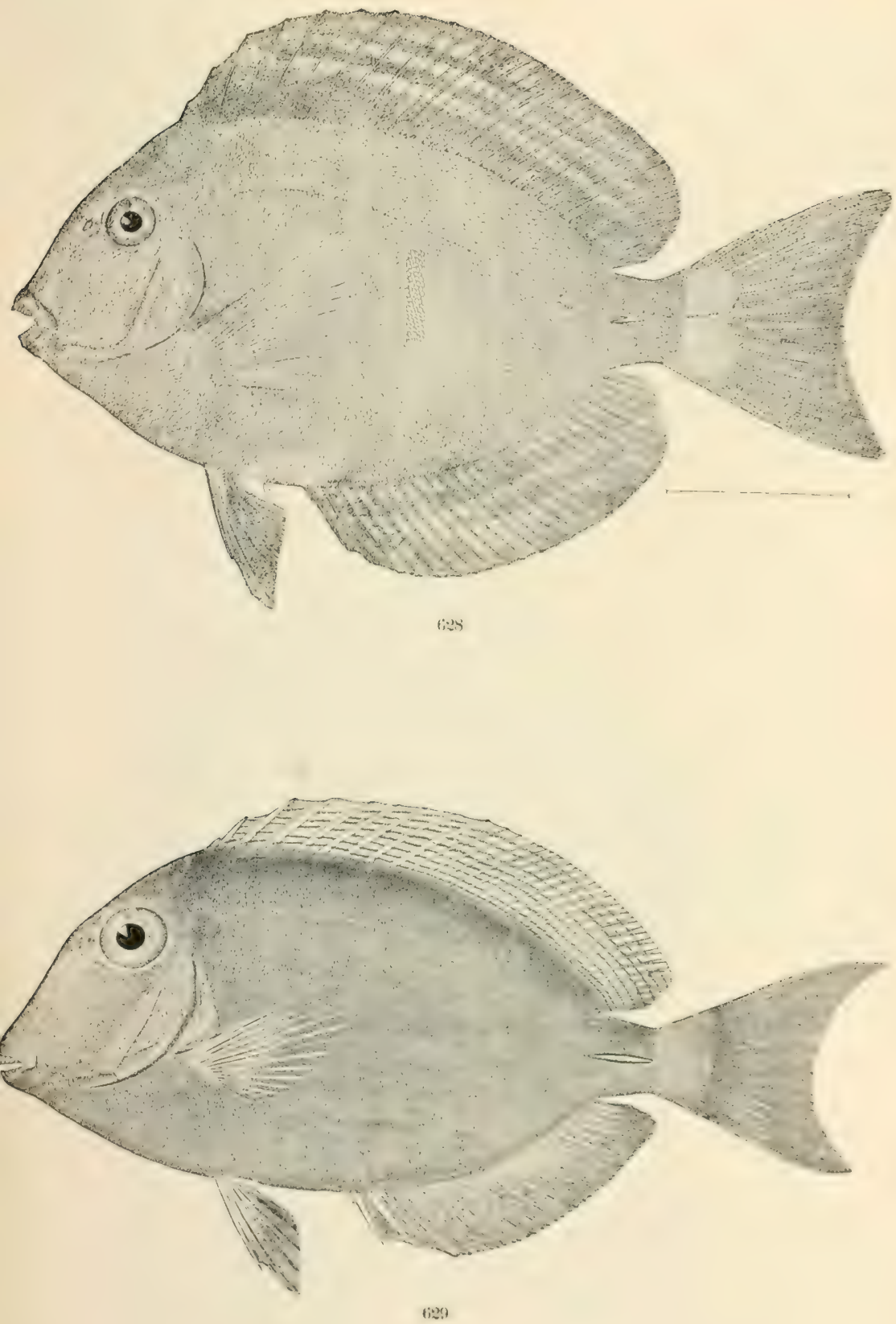

628. TeUthIS CizETTONIS, (P. 1692.$)$

629. Teuturs Bamianus. (P. 1693.) 



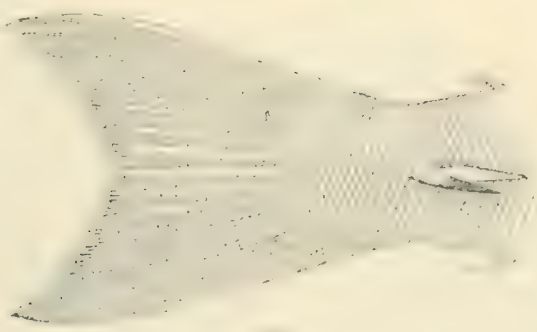

$\because 0$

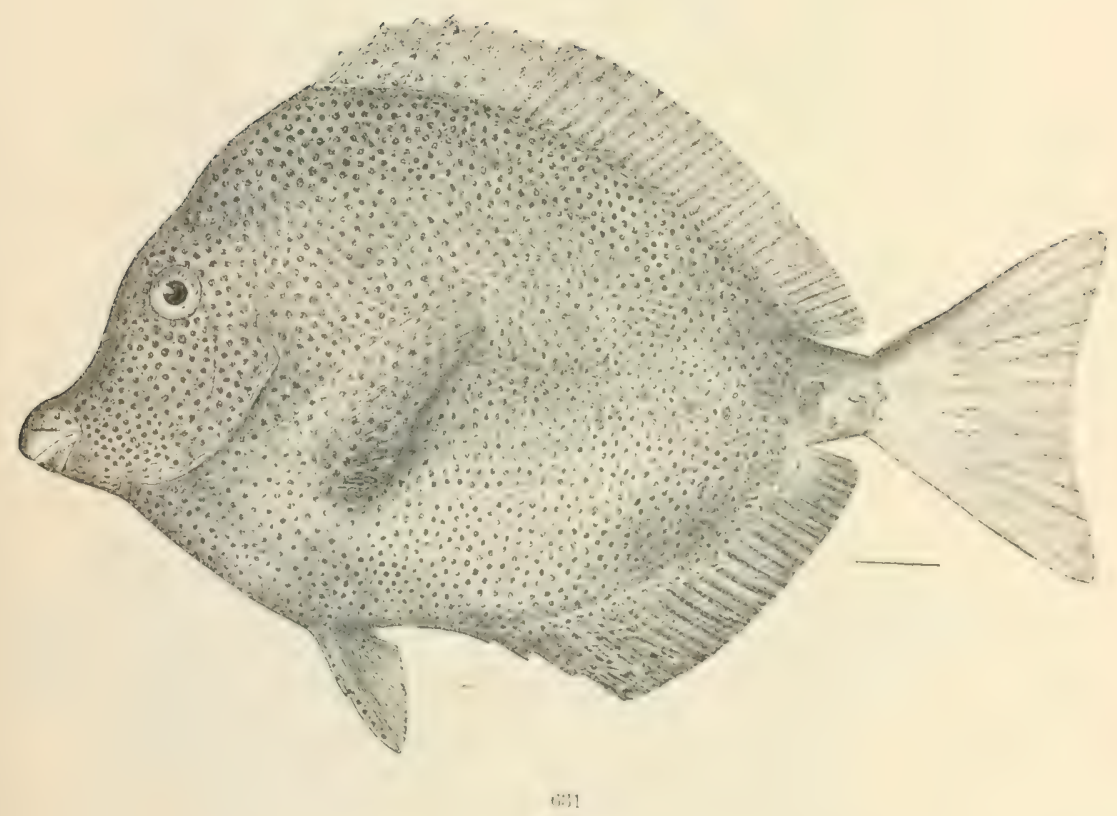

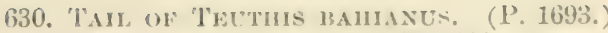

631. Xesurt's puxetates. (1'. 1694.) 


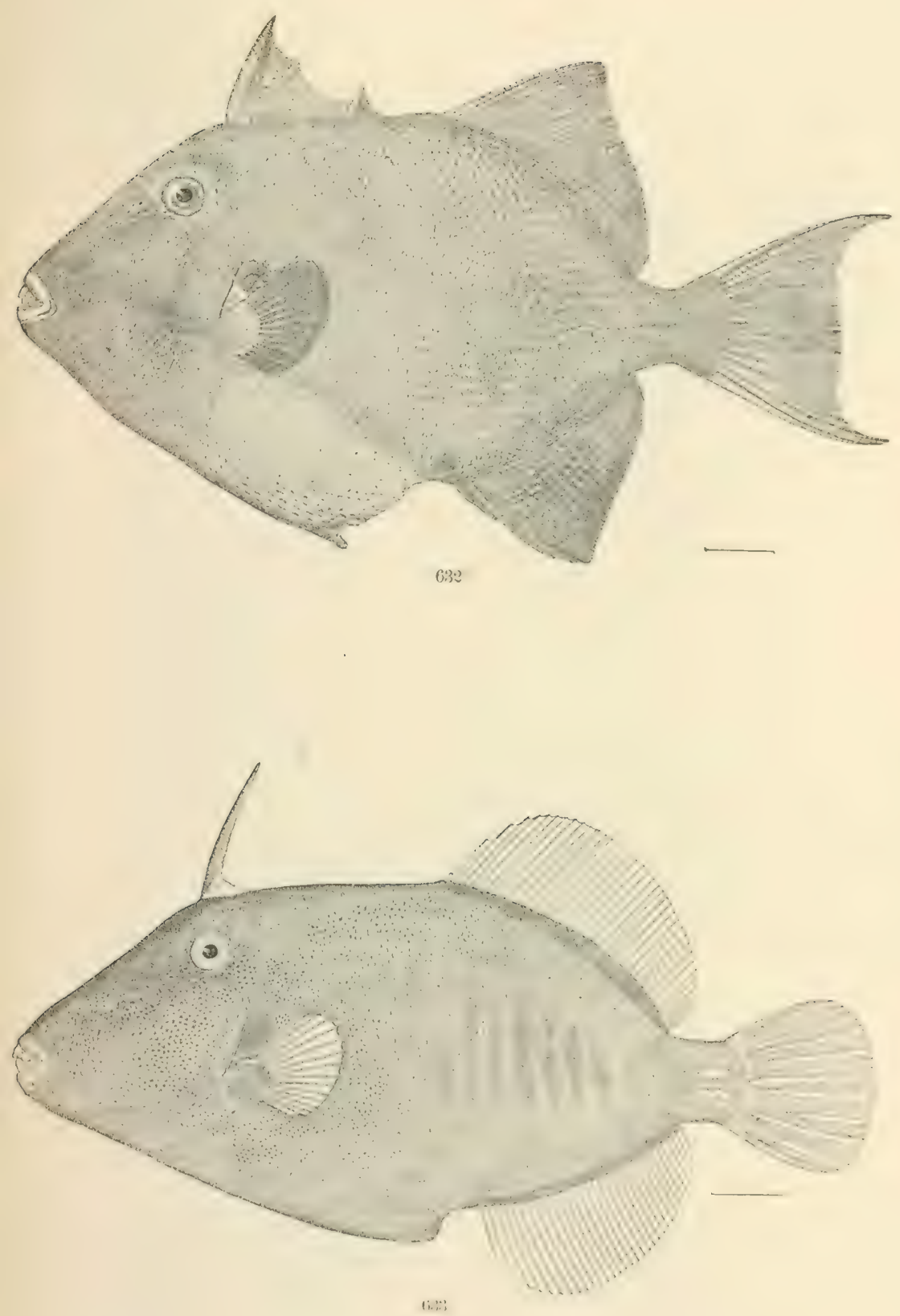

632. Maidstes Camoliningis. (P. 1701.)

633. Canthemines caliol.2. (P. 1713.) 


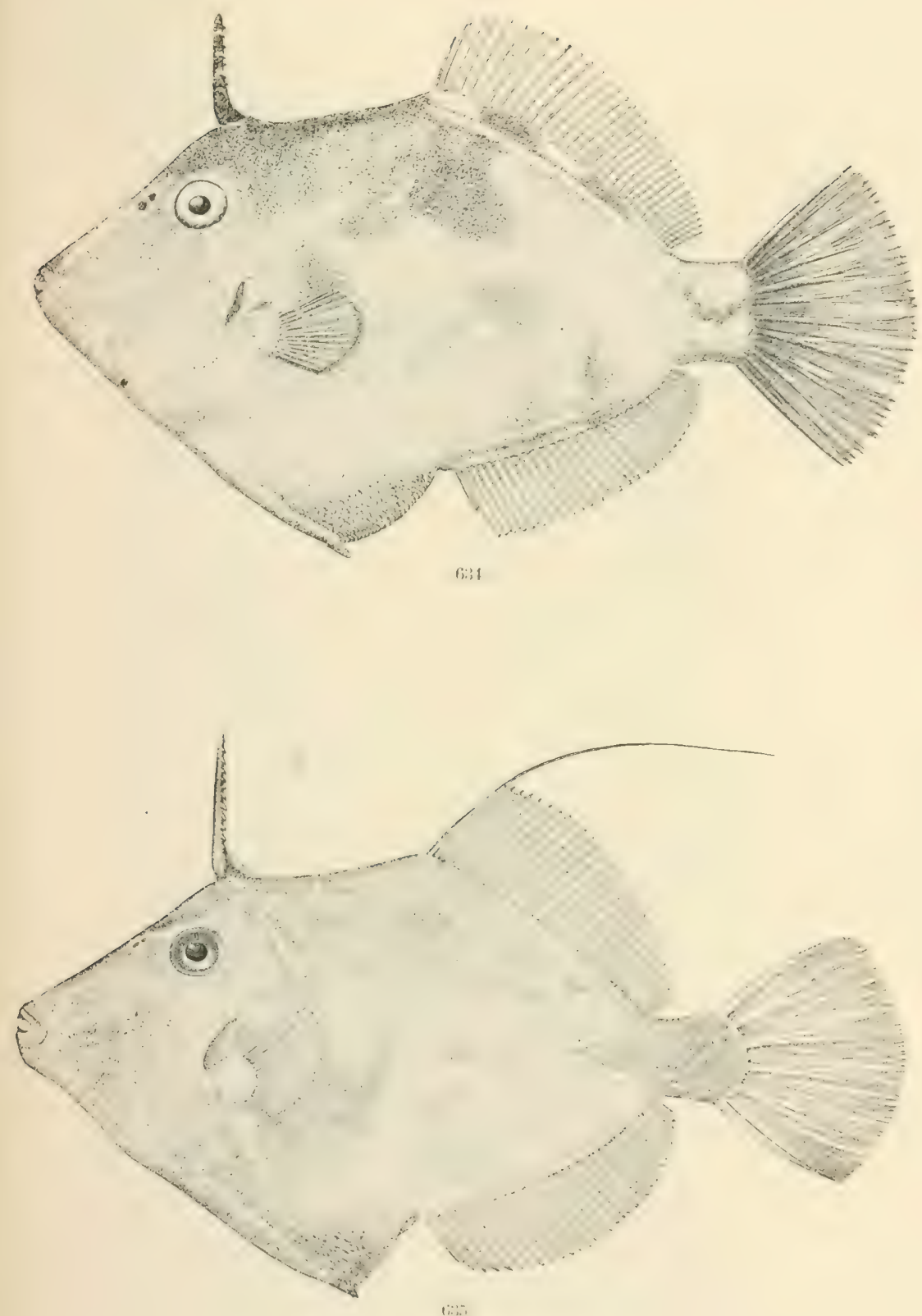

631. Munucanthes chlatus. (P. 171\%. 635. MovocanthI'S HISPIDUS. P. 1715. 



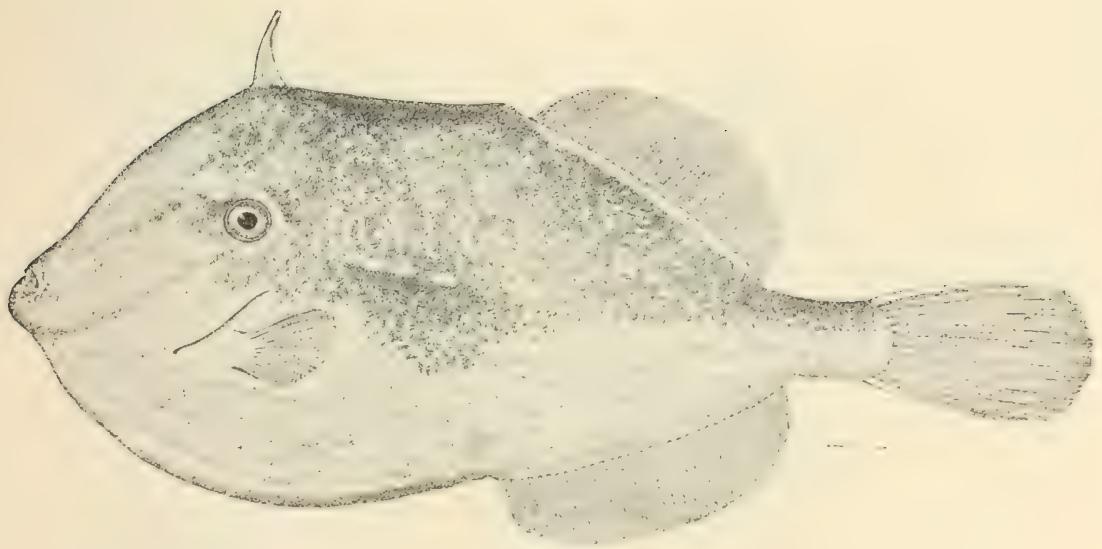

636

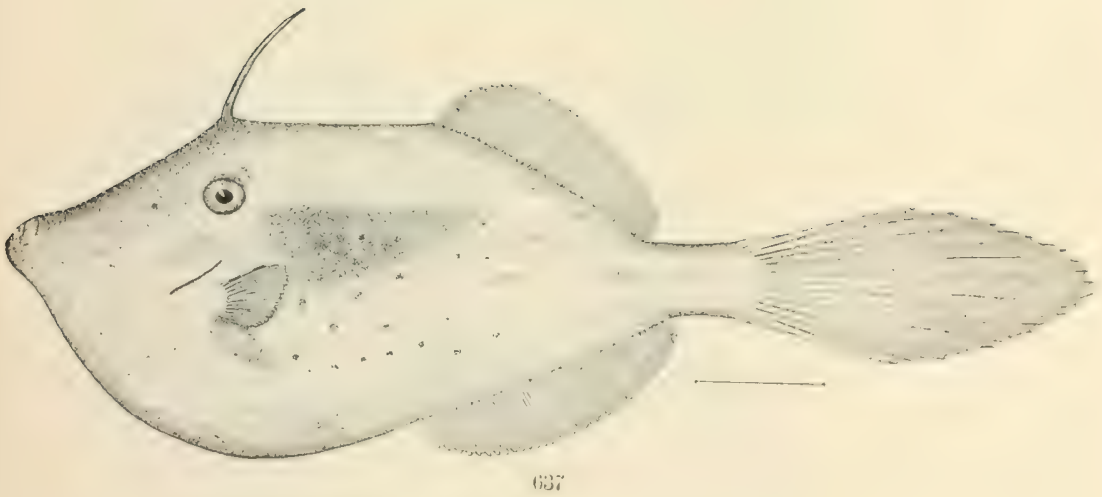

636. Alutelia sCHelphi. (P. 1718.)

637. AlUtera sCrIPTA. (P. 1719.) 



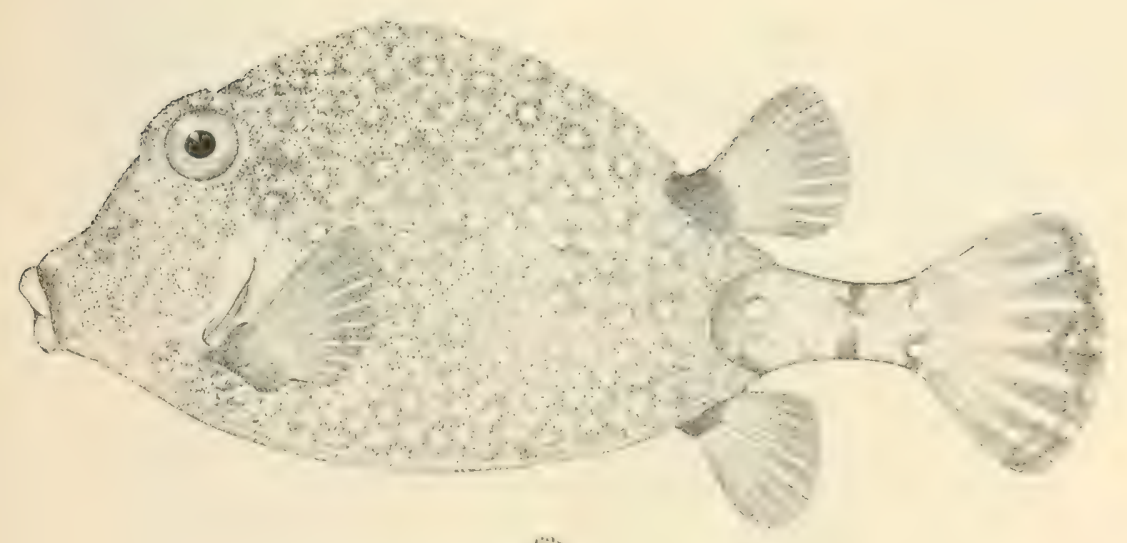

1;:-

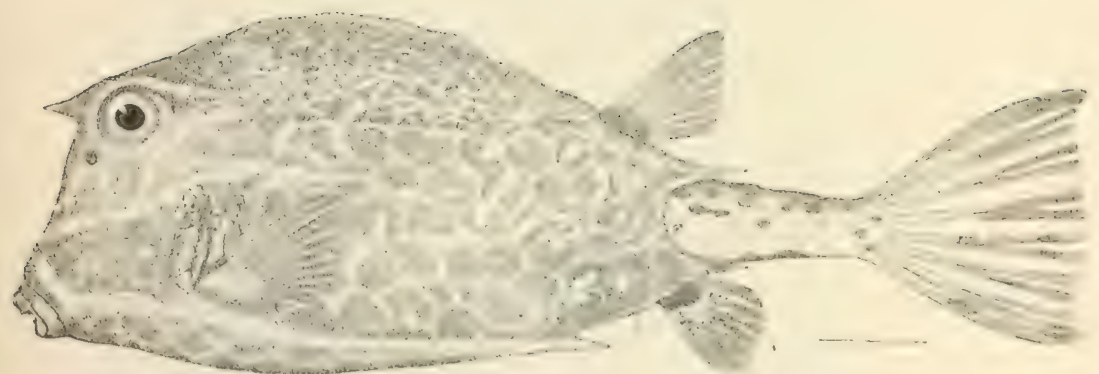

1*,

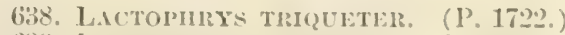

639. IAACTOLHRY'S THICURNAs. (I'. 1724.) 

U. S. NATIONAL MUSEUM

BULLETIN NO. 47, PL. CCLXII
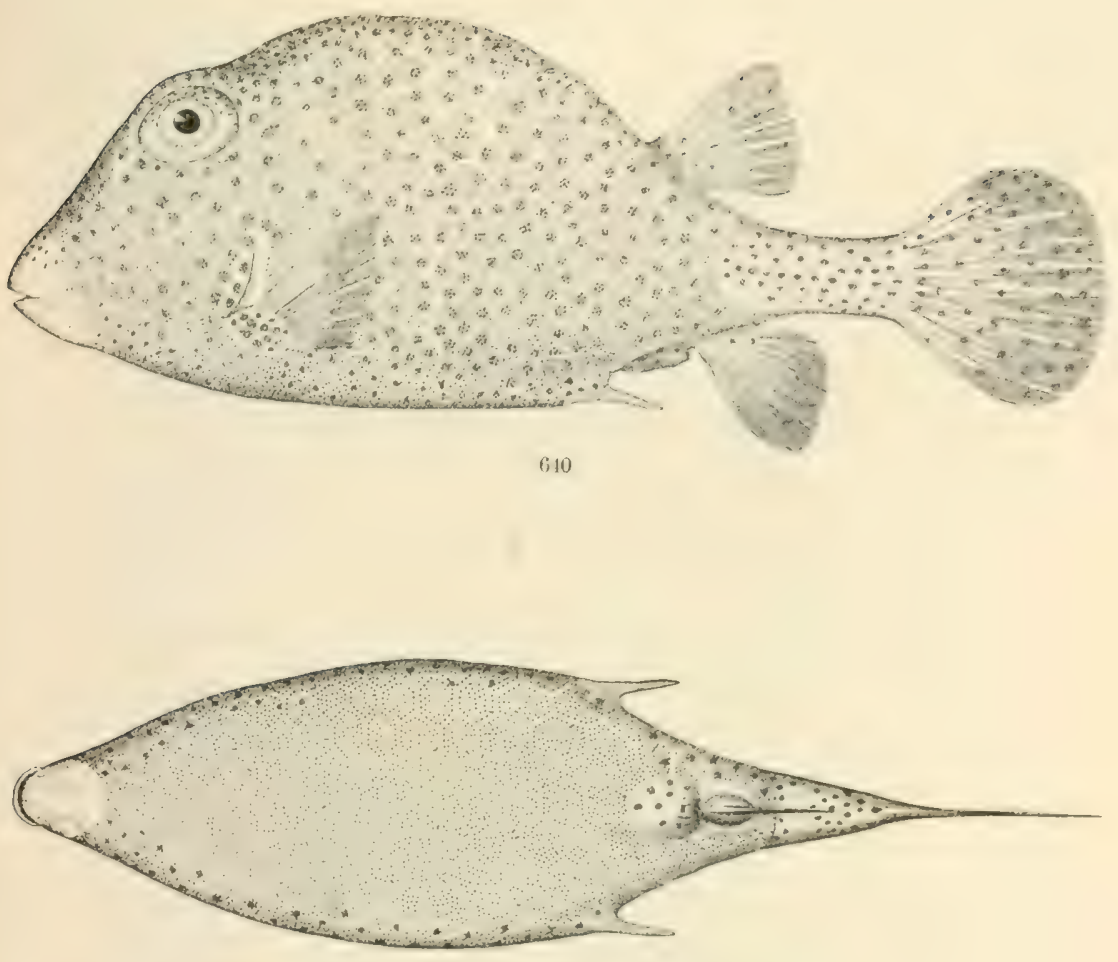

sAles

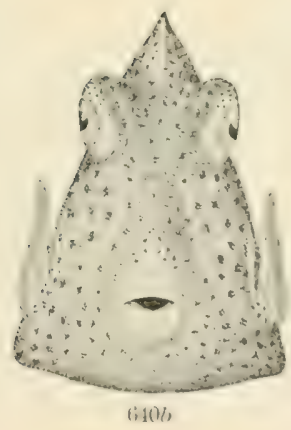

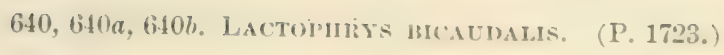





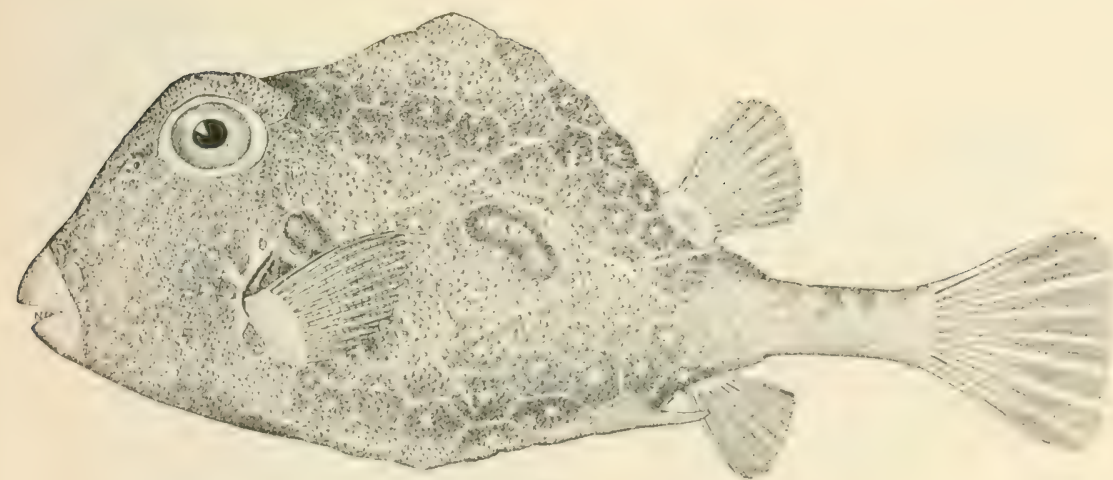

tit
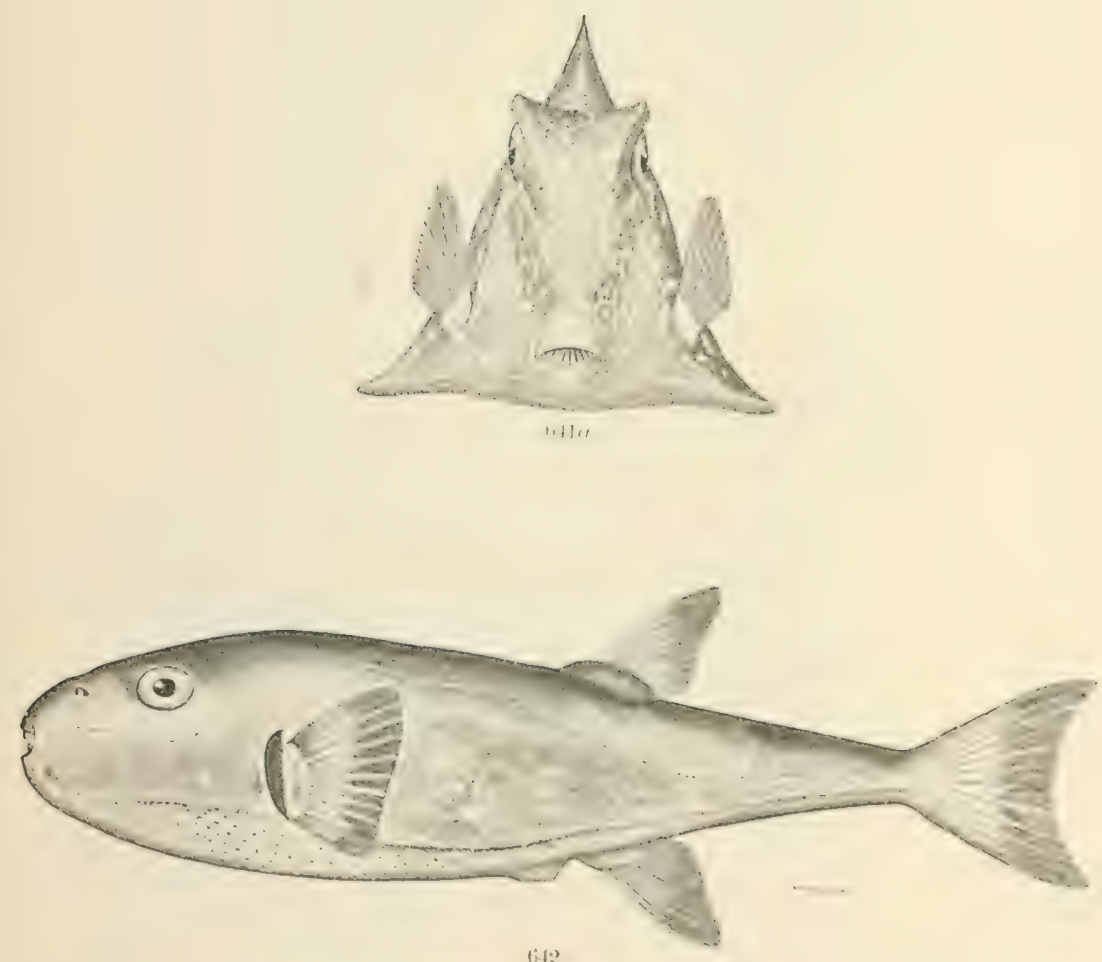

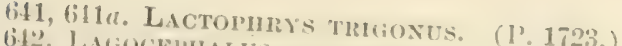

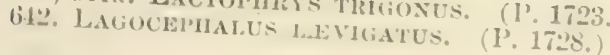





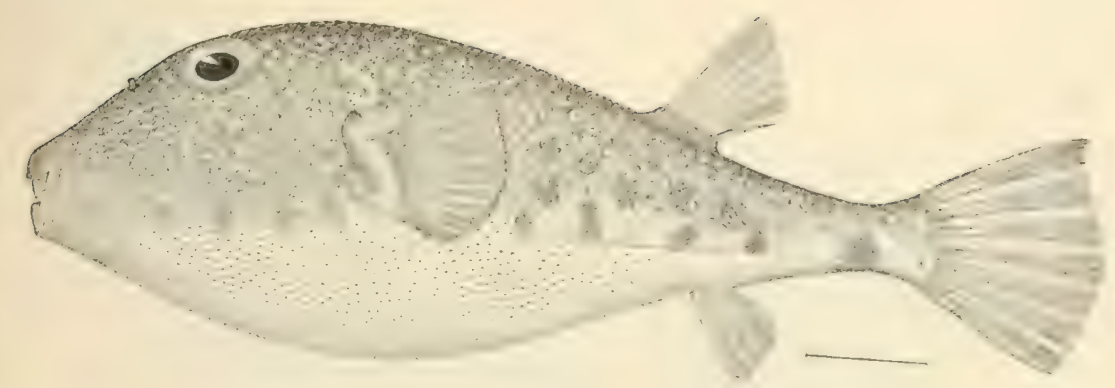

(ii)
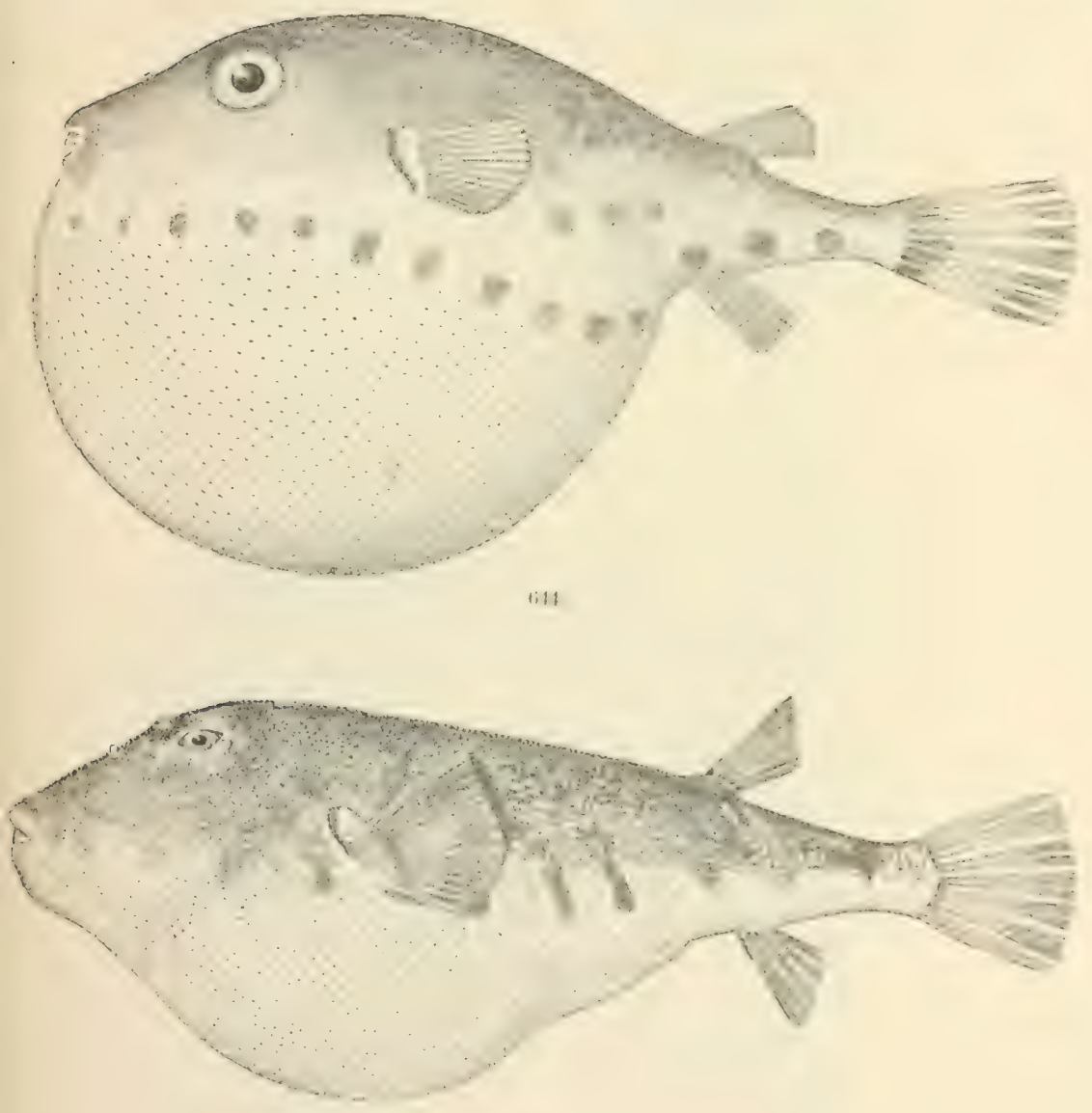

(i):

643. SHIFROIDS NEMHELUS. (P. 1732.)

614. SPIIROIDES SPEXGLIR. (P, 1732.)

61\%. SIIIEROIDES M.LCULATUS, (P. 1733. 



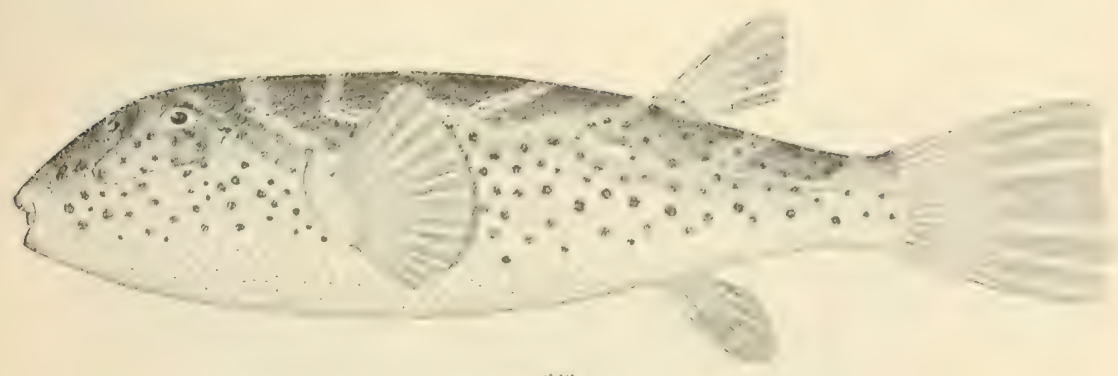

linti
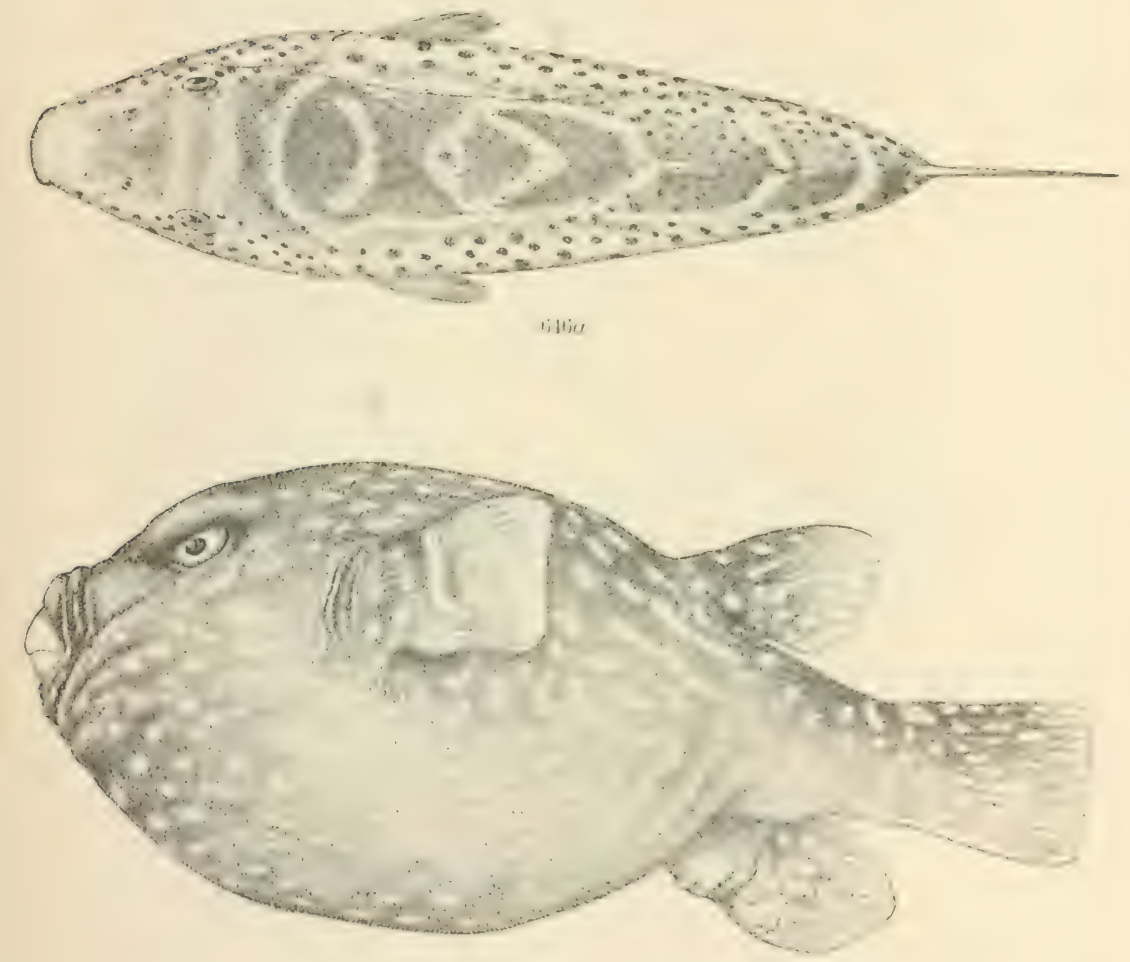

iiti

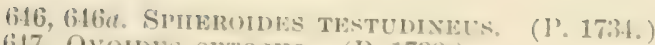

617. OvOLDE shTOSUs, (P. 1739.) 


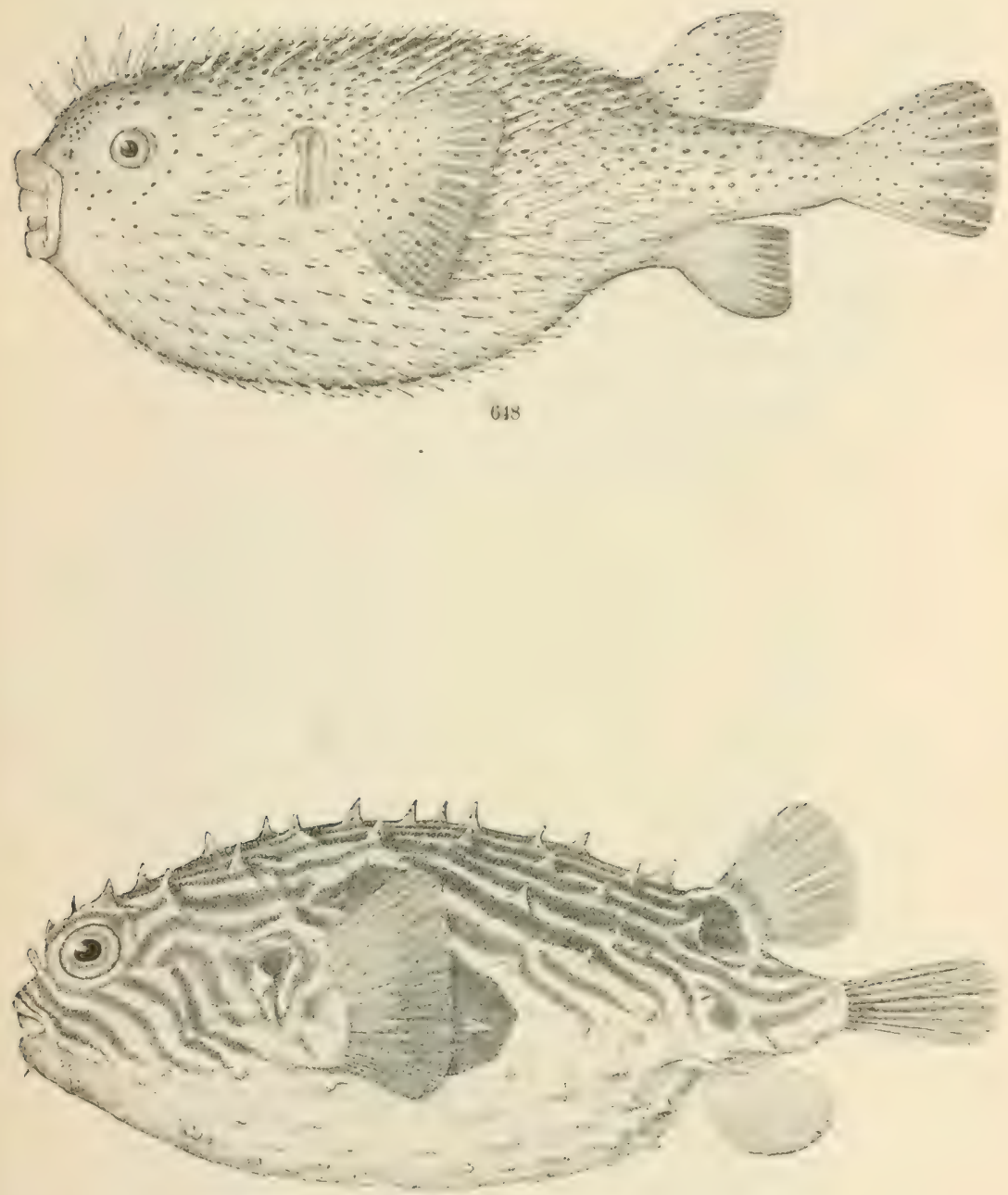

$61 !$

618. Dronox mystrix, (1', 1745.)

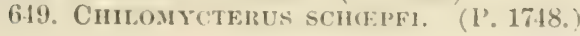




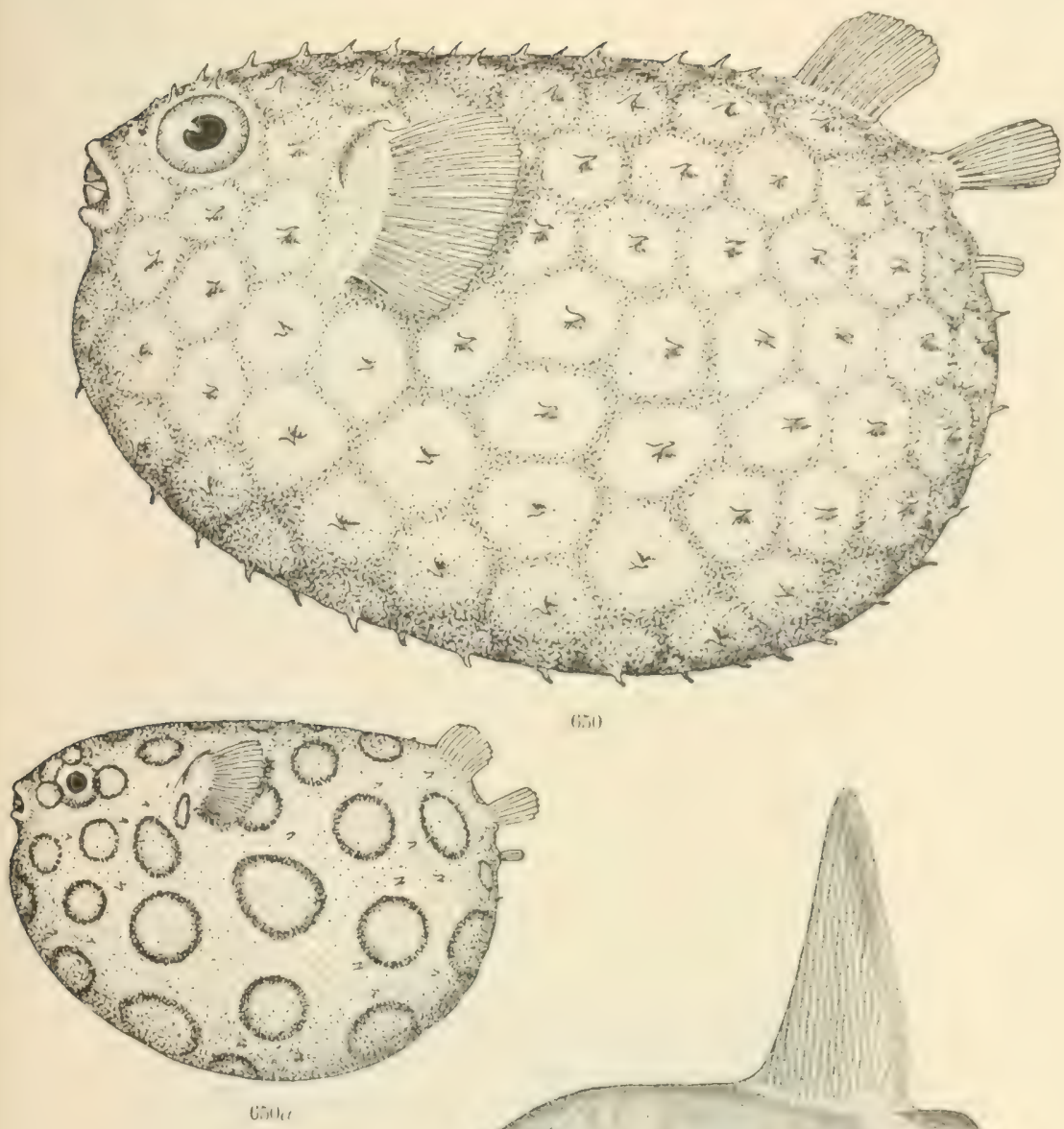

(is,i)

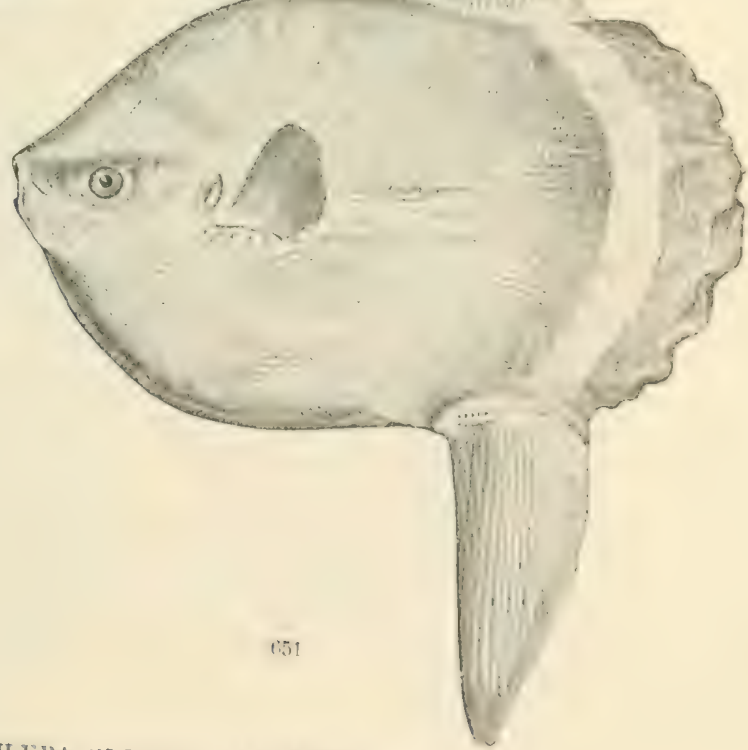

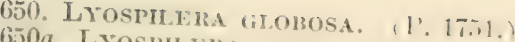

650a. LYOSPIIRKA GLOBOSA; youmg. (I'. 1751. 



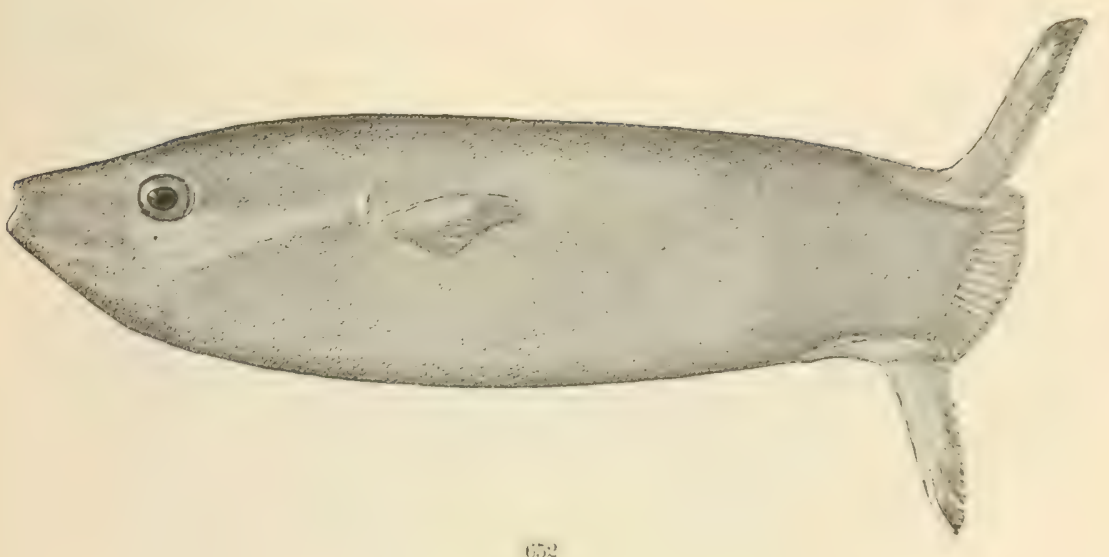

(ii)

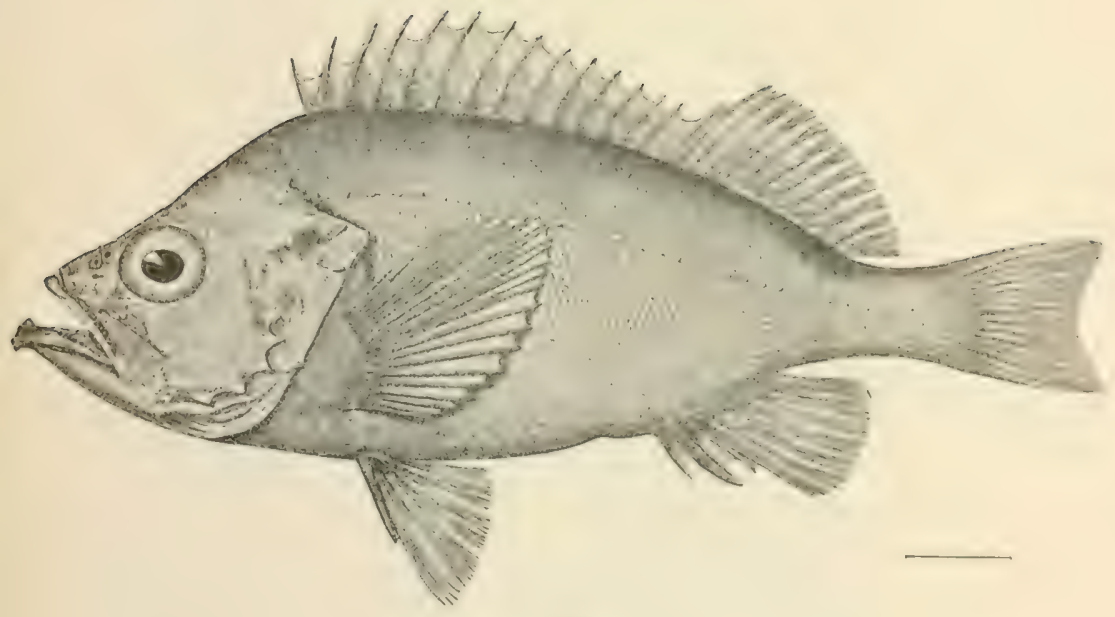

(6):

652. RANZANIA TIUNCATA. (P. 1755.)

653. SEBASTES MARINES. (P. 1760.) 


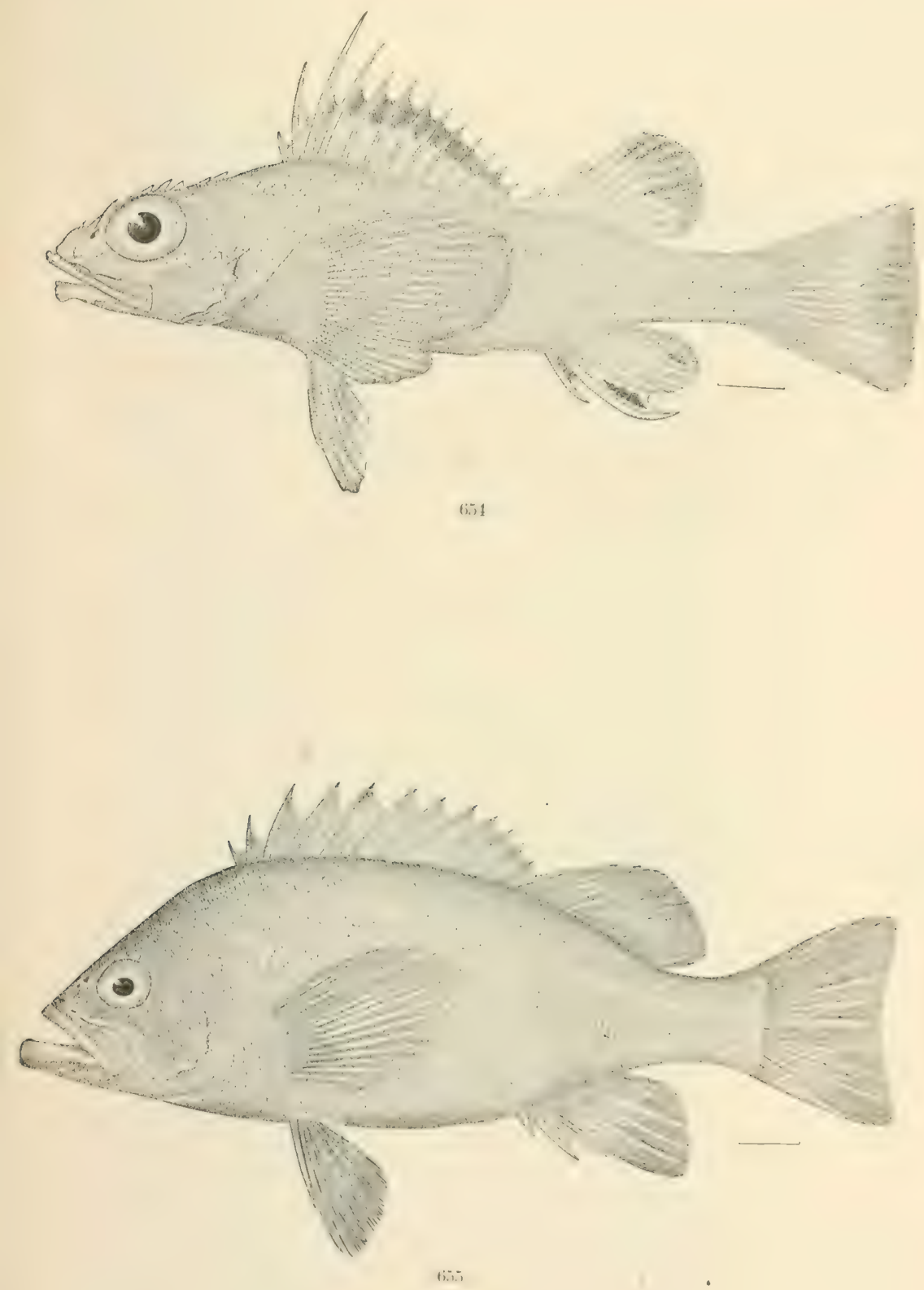

654. SEBASTOLOBUS AITIVELIS. (P. 1763.)

655. SE1BASTODES MELANOI'S, (P. 1782.) 


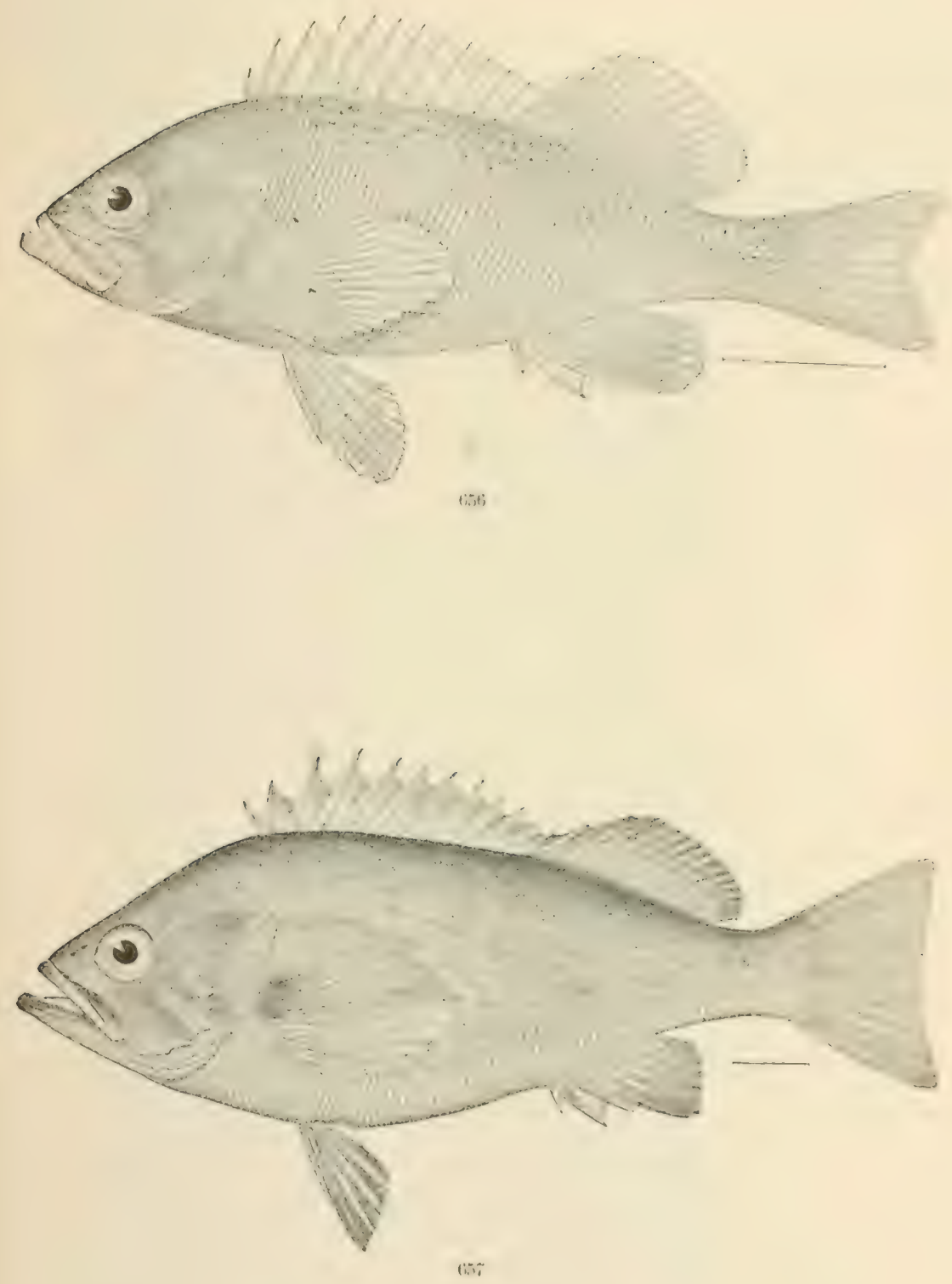

656. Sibastones cil.iatus. (P. 1783.)

657. Selastodes mistives. (P'. 1781.) 



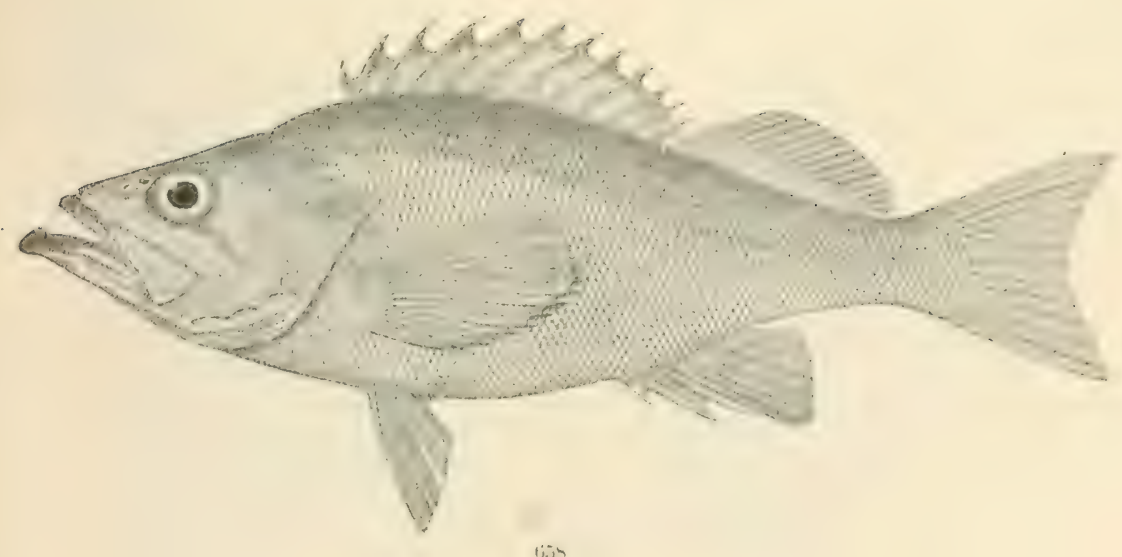

inis

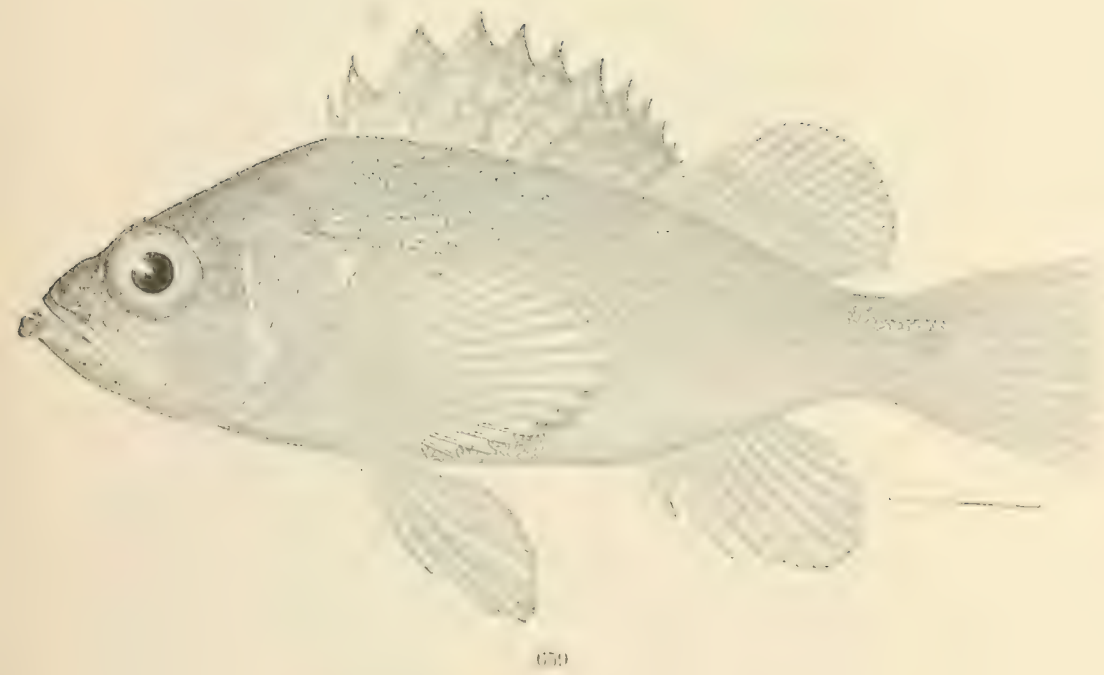

658. SEBASTODES BREVISPINIS, (P. 1787.)

659 . SEBASTODIS FIGENMANNI. ( $1,1789$. 


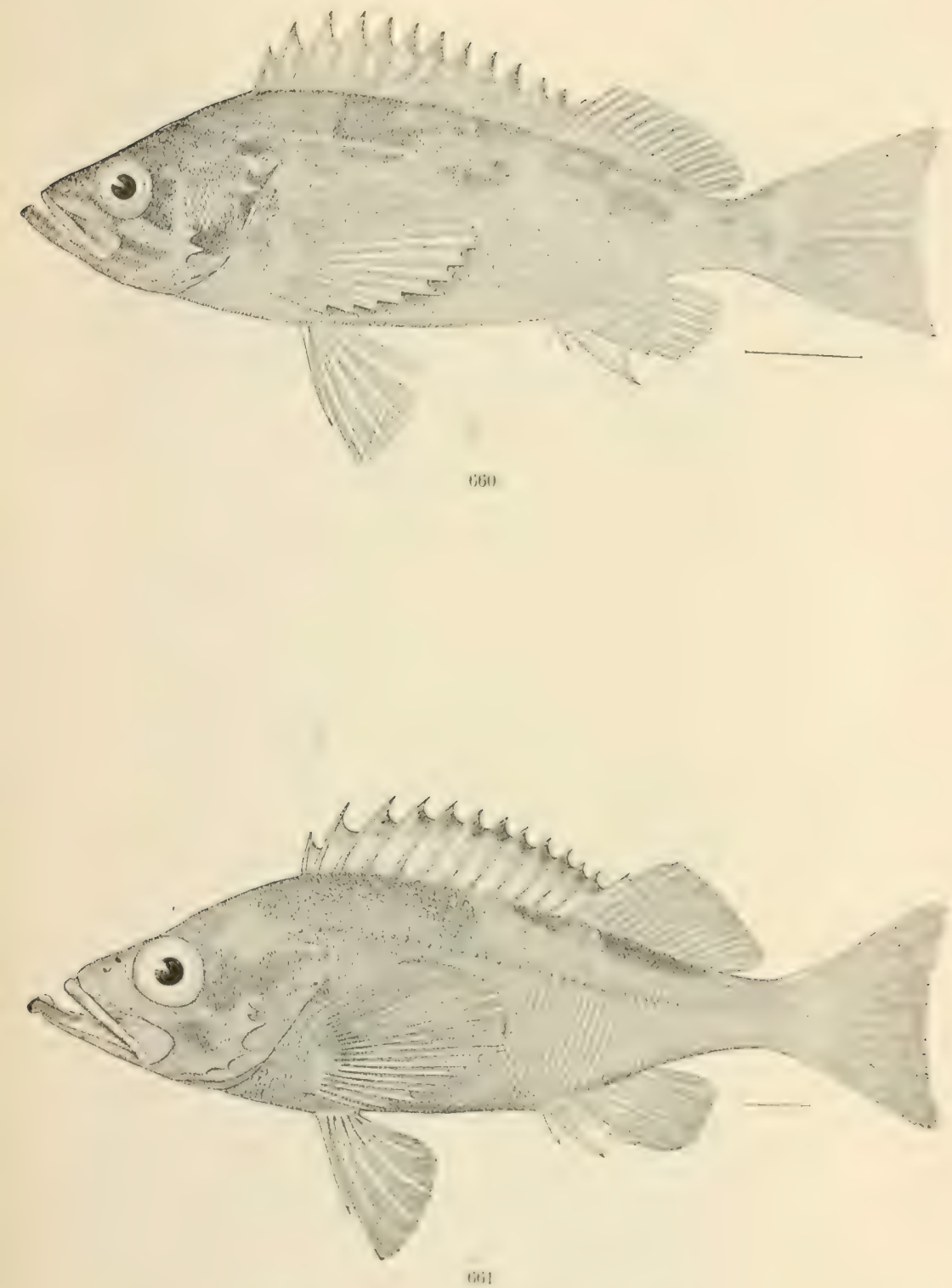

660. SEBASTODES HOP'KINSI, (P', 1789.)

661. SEHASTODES ALUTCS. (I’. 17\%0, 



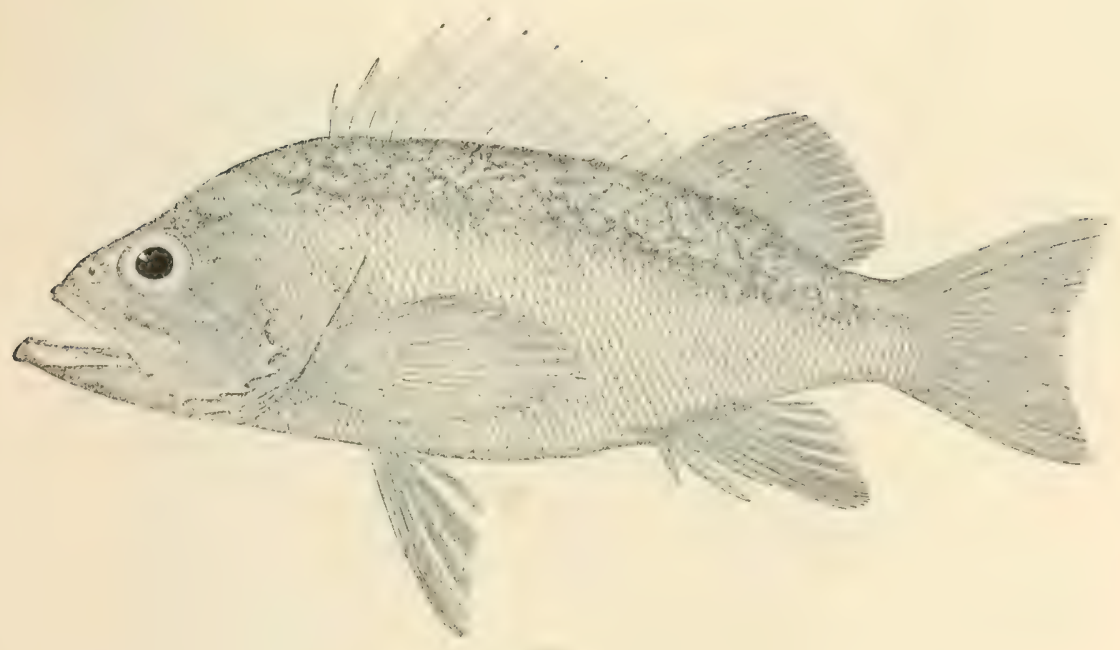

tiv

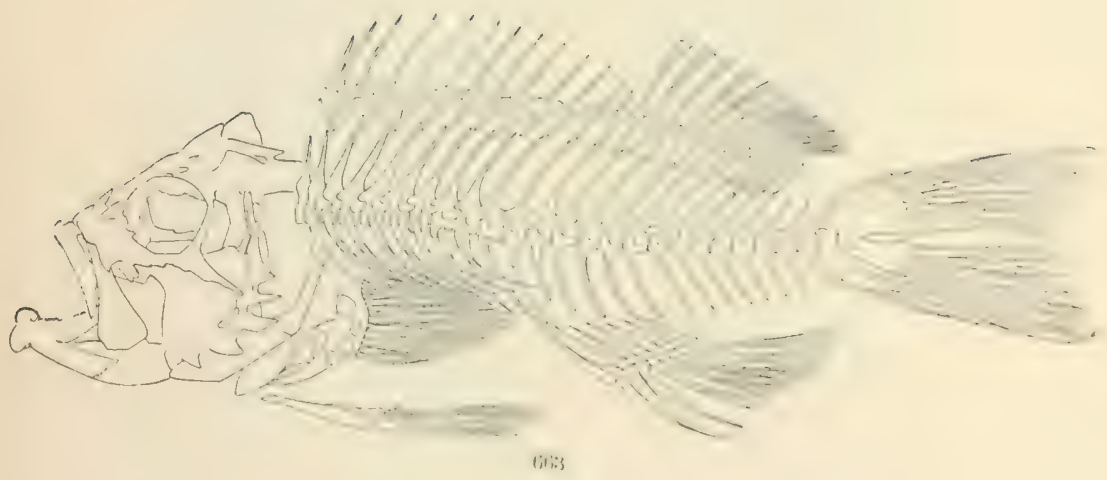

662. SFIBASTODES PINXIGEli. (P. 1793.)

663. Sebastomes minitus. (1? 1794.) 


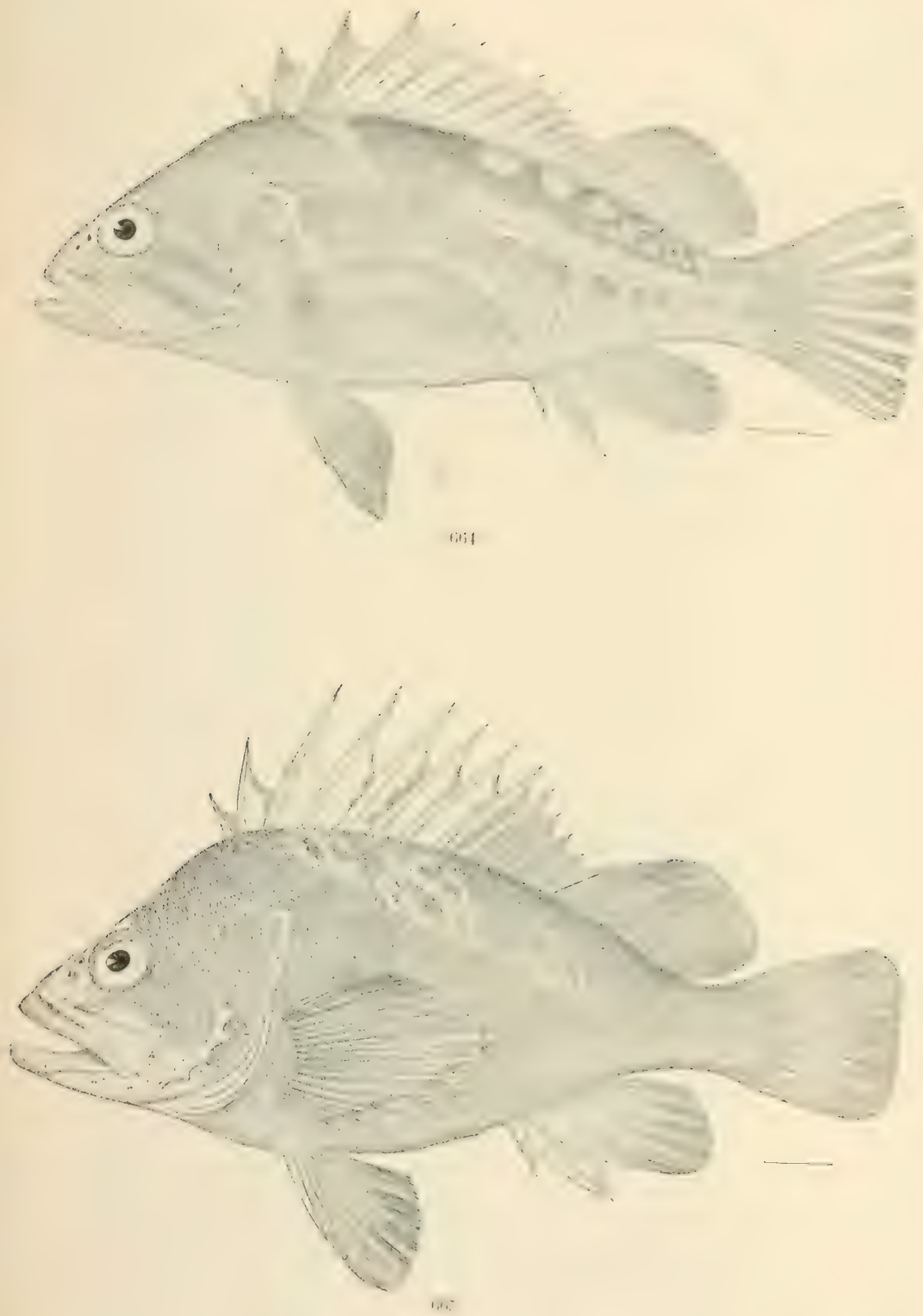

664. SFabstones cal'mines. (I', 1820.)

665. SEBASTODES MALIGER. (1', 1822.) 


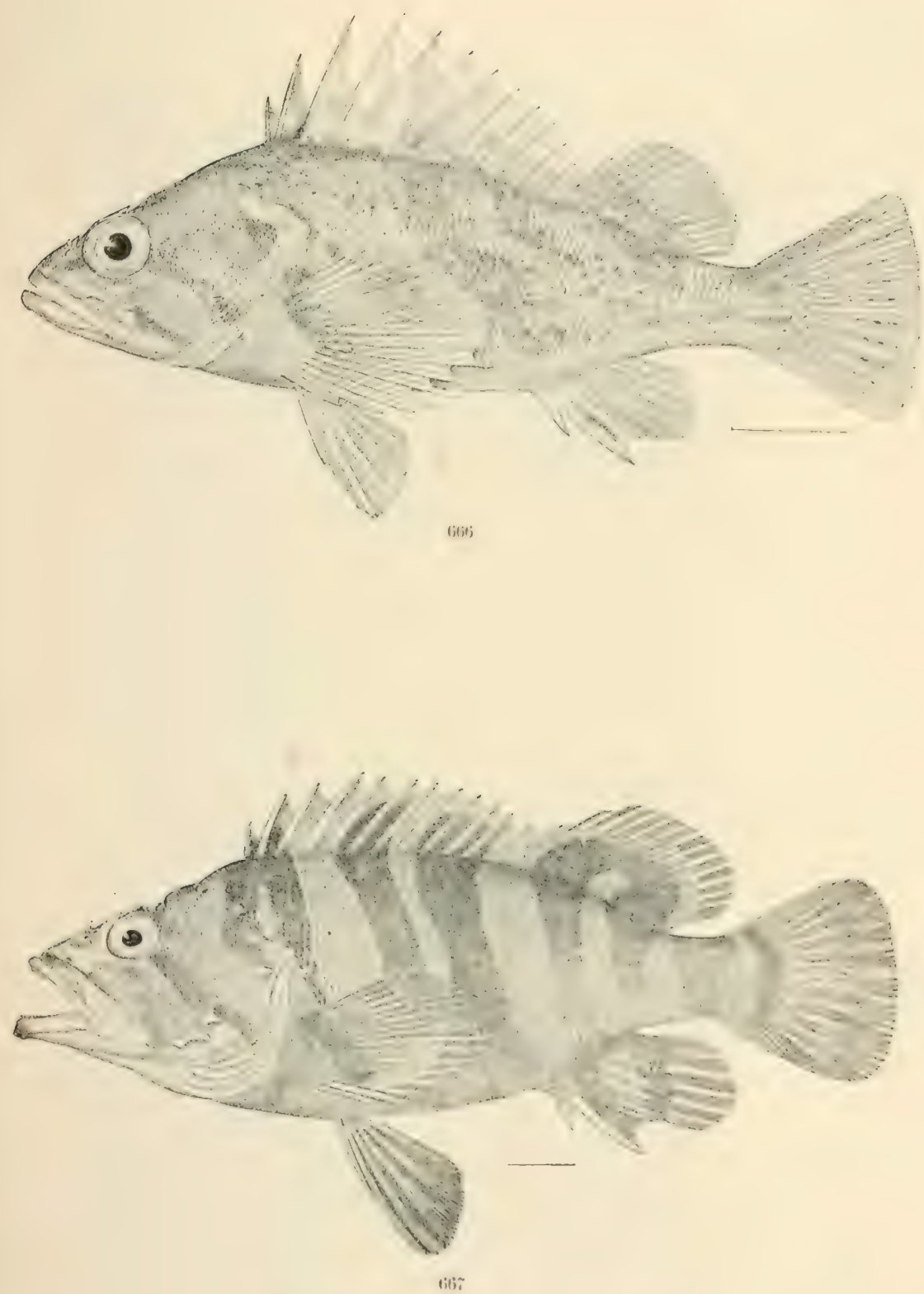

666. Sebastones mlibrti. (l’. 1823.)

667. SEBASTODES SERRICER, ( ${ }^{\prime}, 18 \%$. 


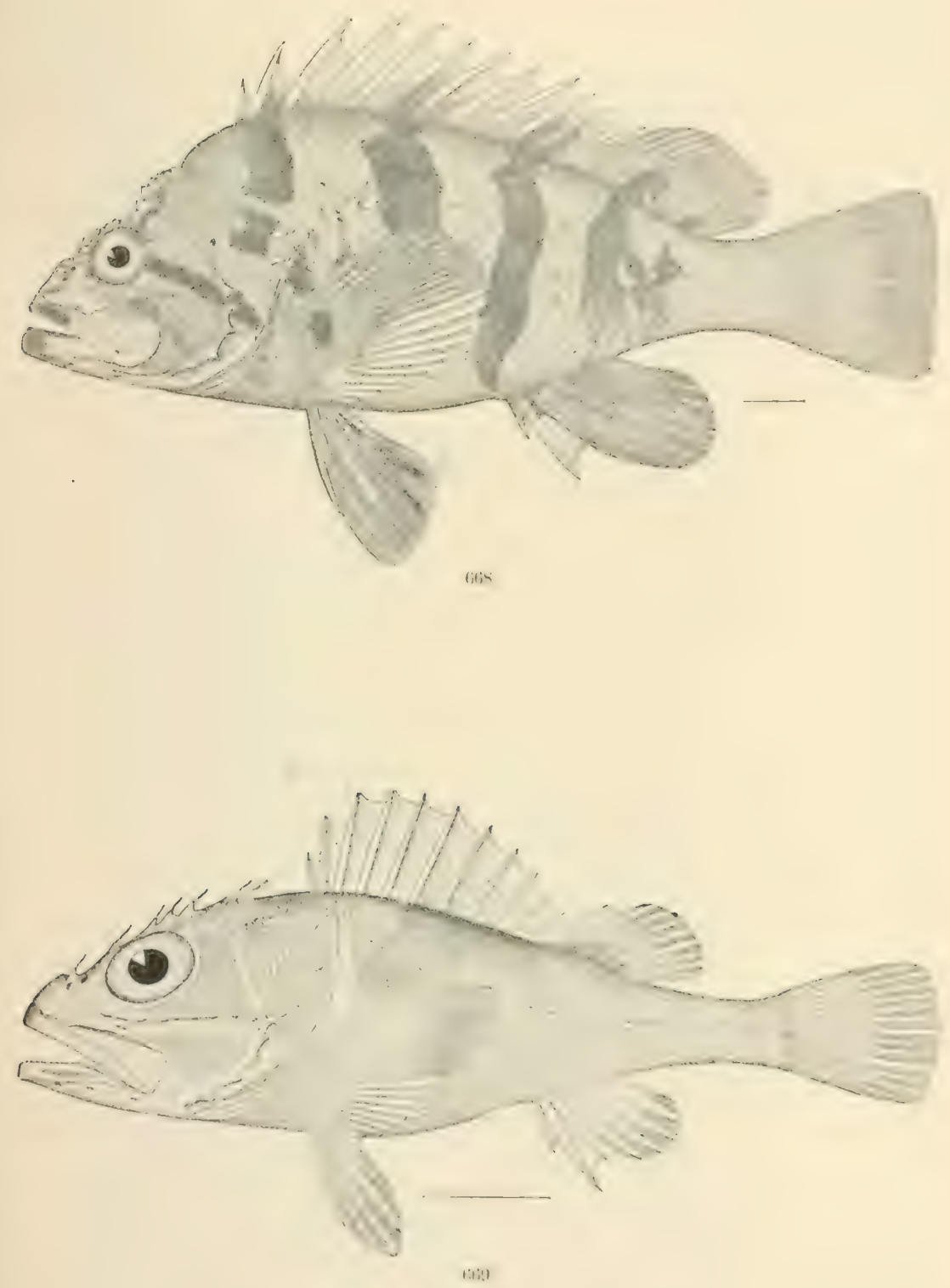

668. Ser.astomes Nigrocinctes. (P. 1827.)

669. SCORPAEA CIBISTULATA. (P. 1811.) 



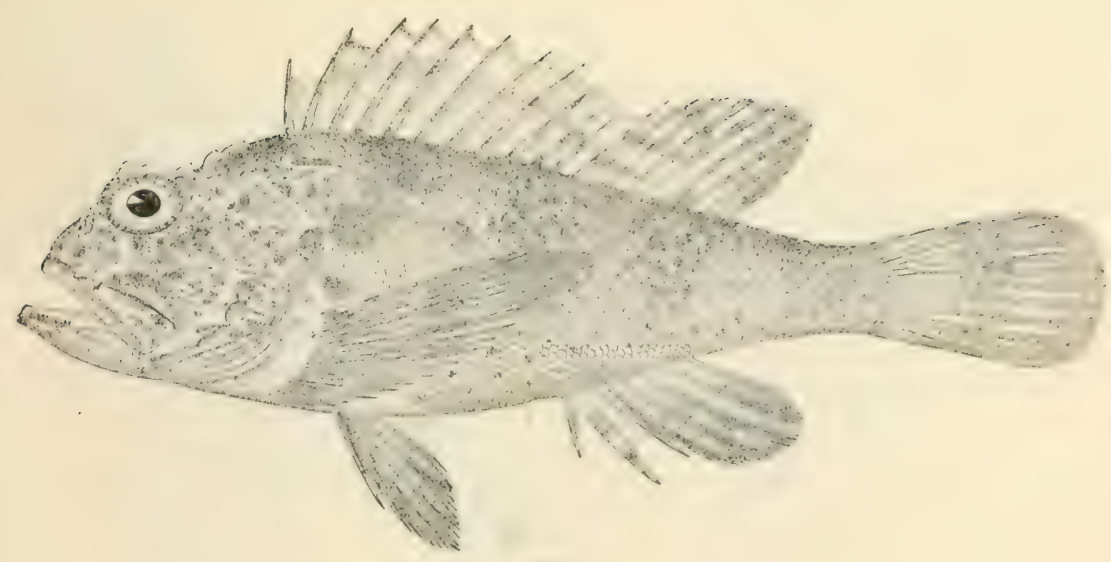

(6)

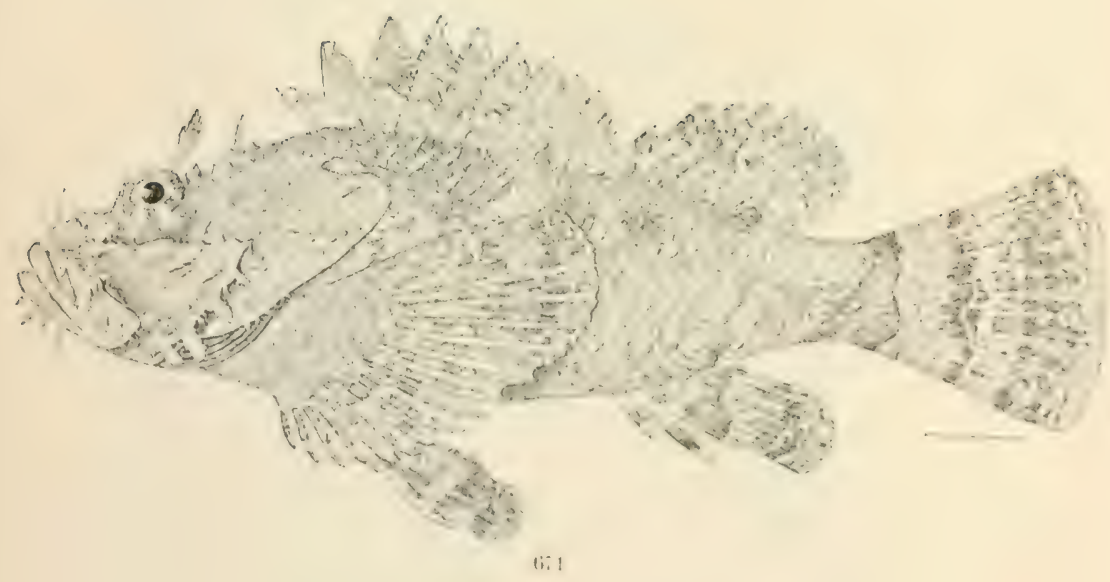

670. SCORI ENA BRASHIWXEIS, (1'. 1842.)

671. SCORP.IXA MYSTLS, (1'. 1819.) 



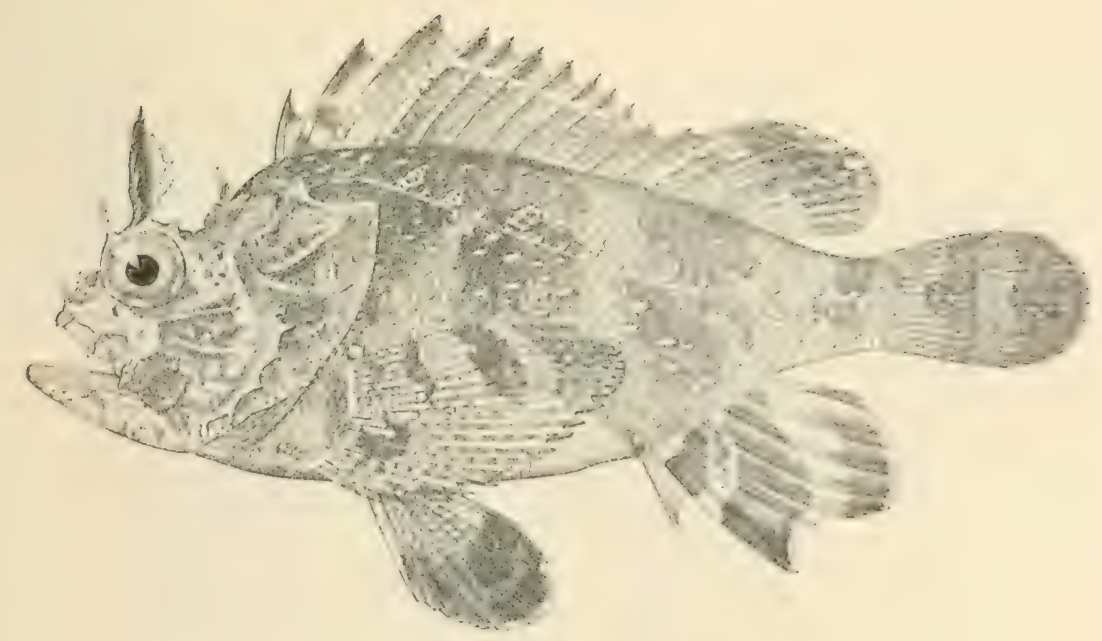

(ii)

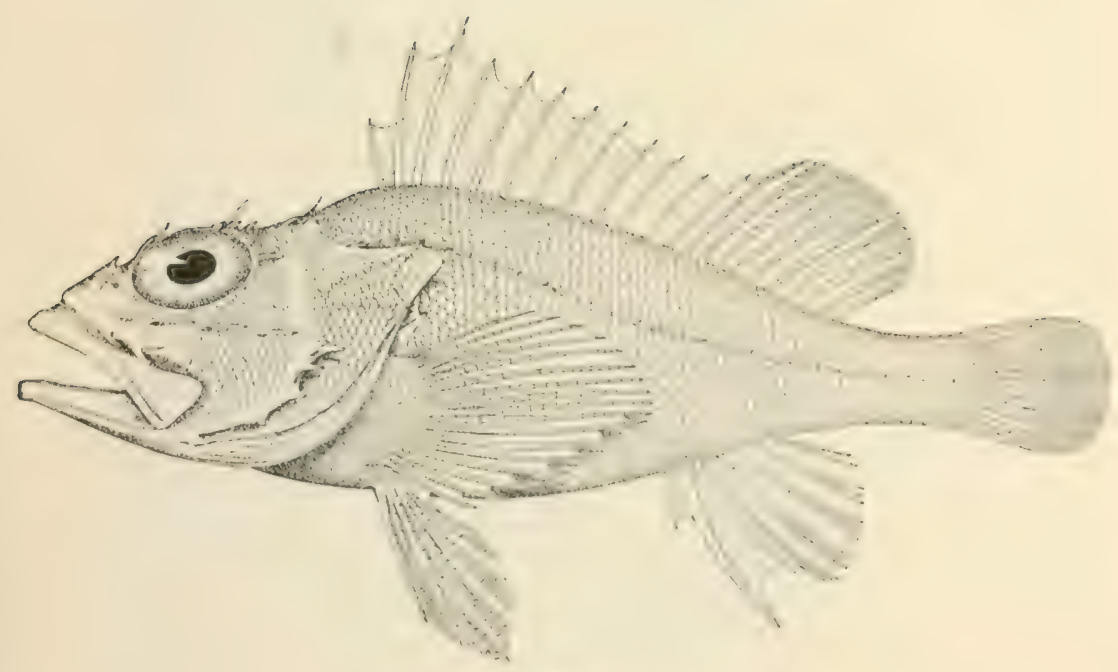

1i.:3

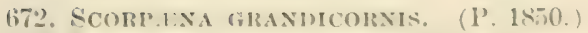

673. P'ONTINUS MACRGLEPI'. (P. 1-55.) 



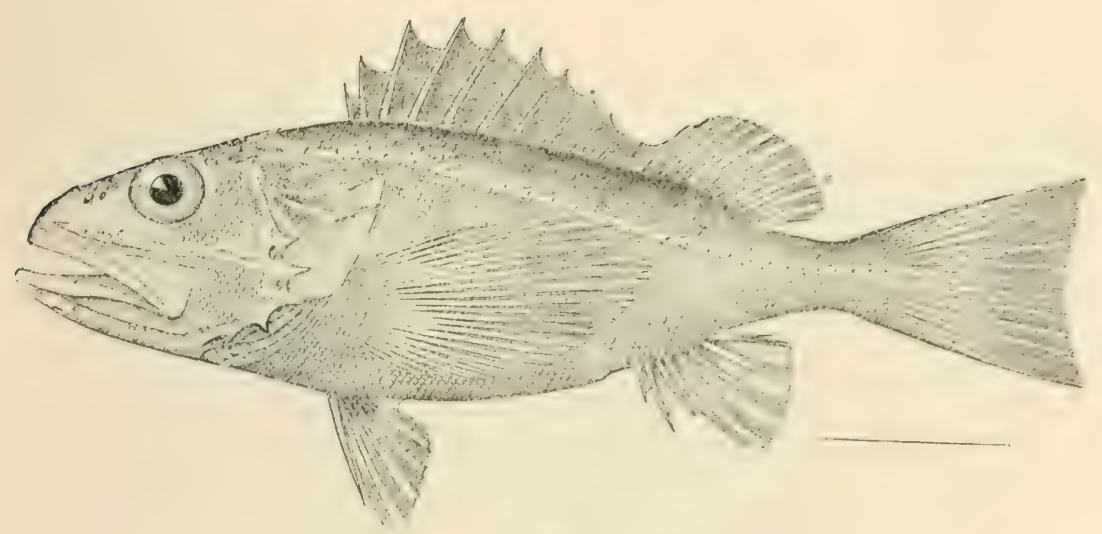

$6 \% 4$

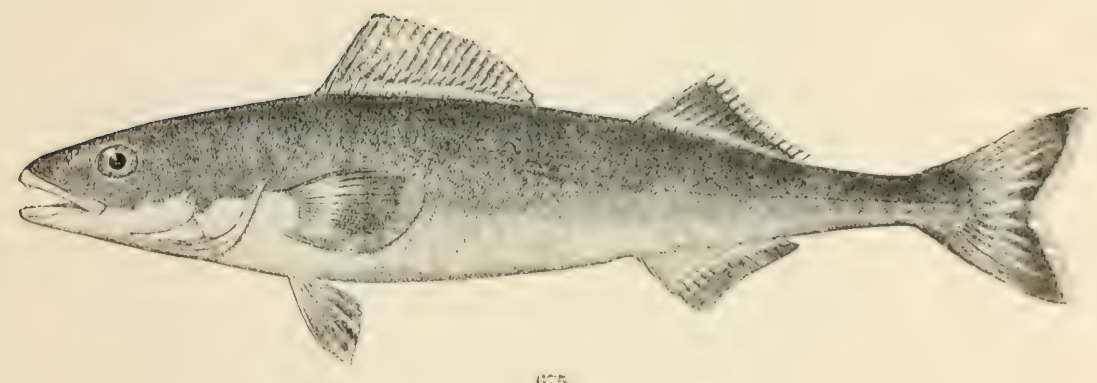

iiis

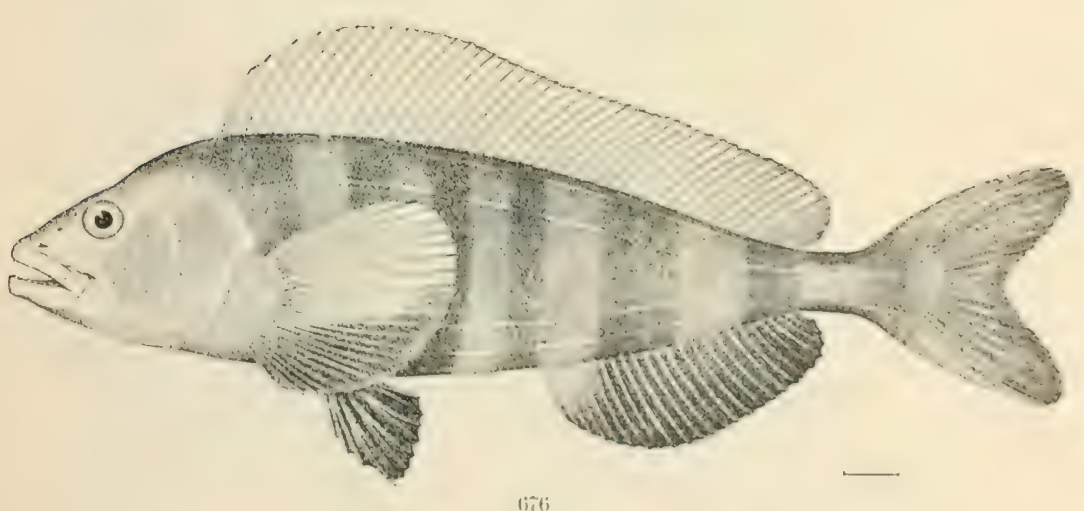

biiti

674. SMTARCHES PARMATUS, (P. 1860.)

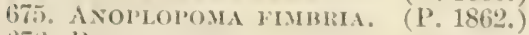

676. Plevrogimames moxomterygivs, (P. 186\%.) 


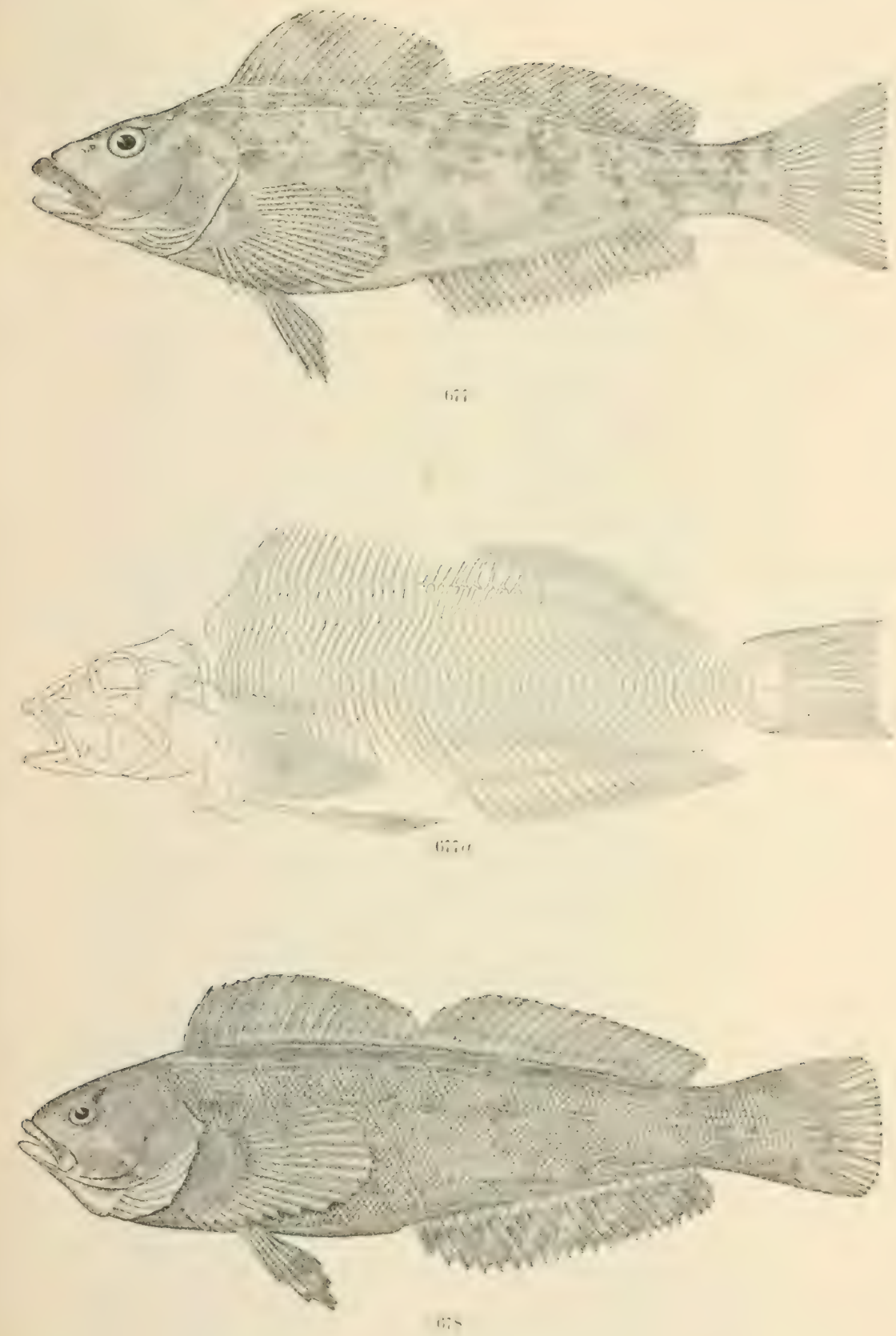

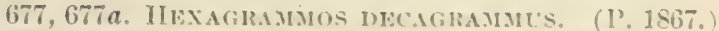

678. IIEXAGRAMOS OCTOGRMMI's, (1', 1869.) 


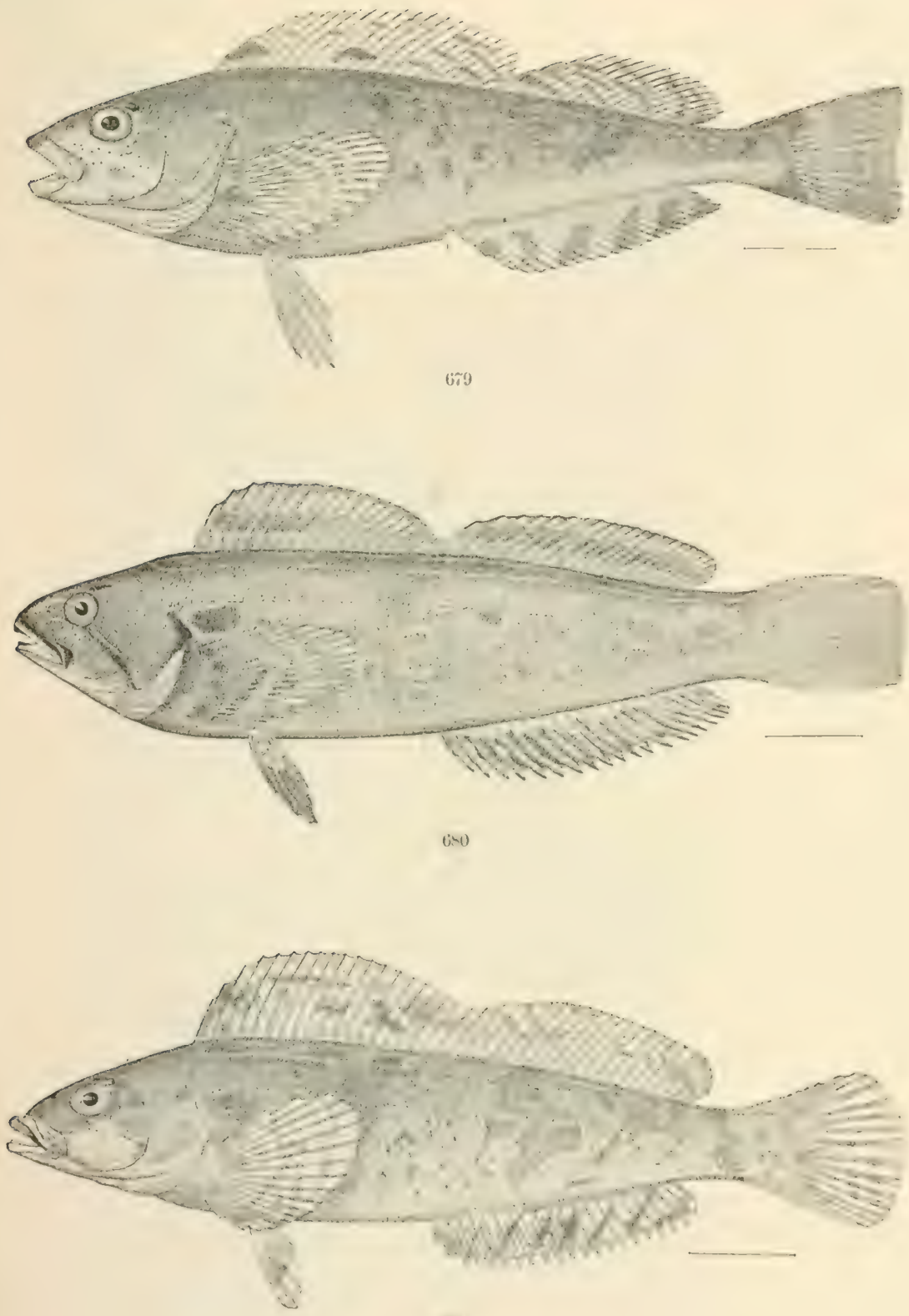

in:

679. Hzxagkammos stellert. (1’.1871.)

680. Hexagramos superchlosts. (I'. 1872.)

681. HExagRaMMOS OTAKII. (P.1867.) 



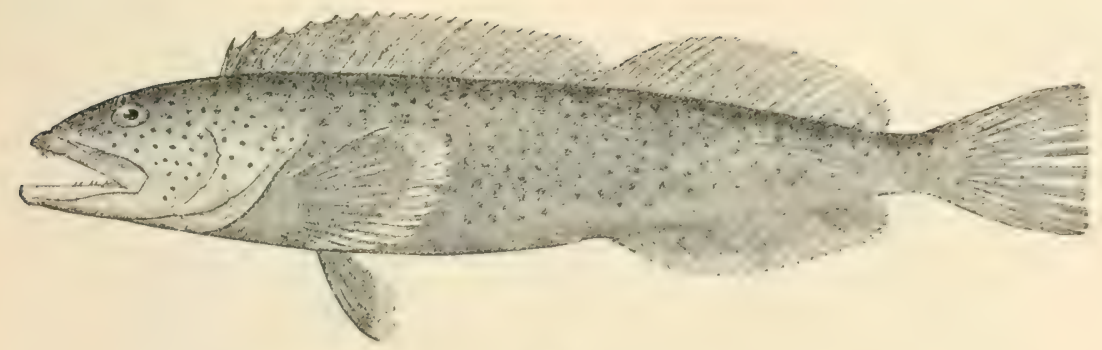

68:

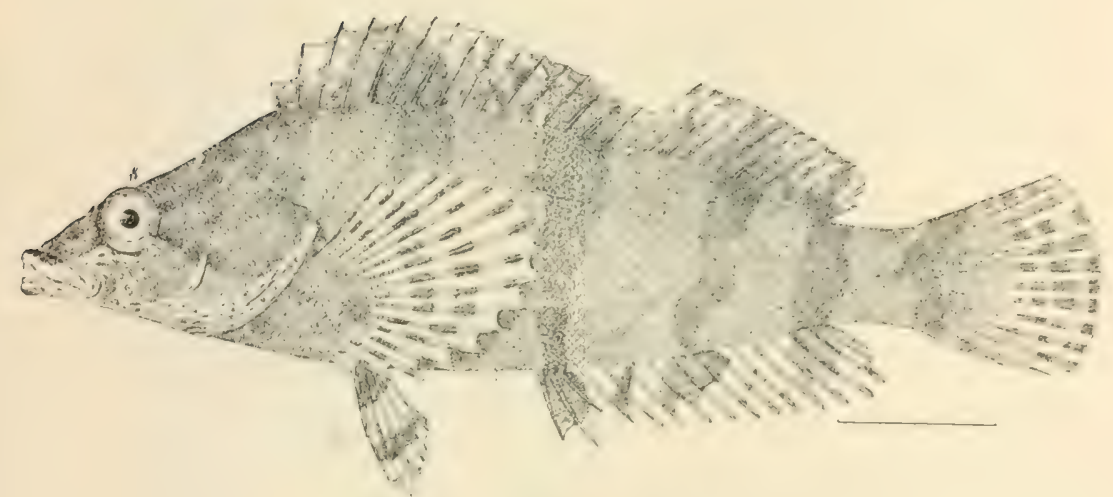

65:3

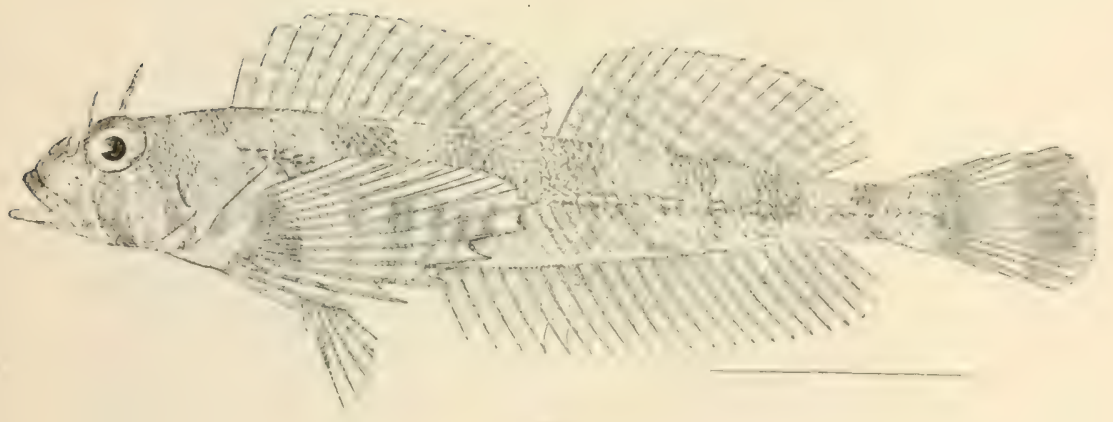

tist

682. OrHIHON ELANGATUA (J., 1875,)

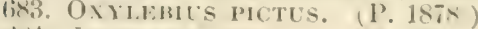

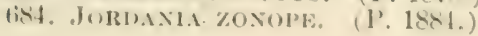




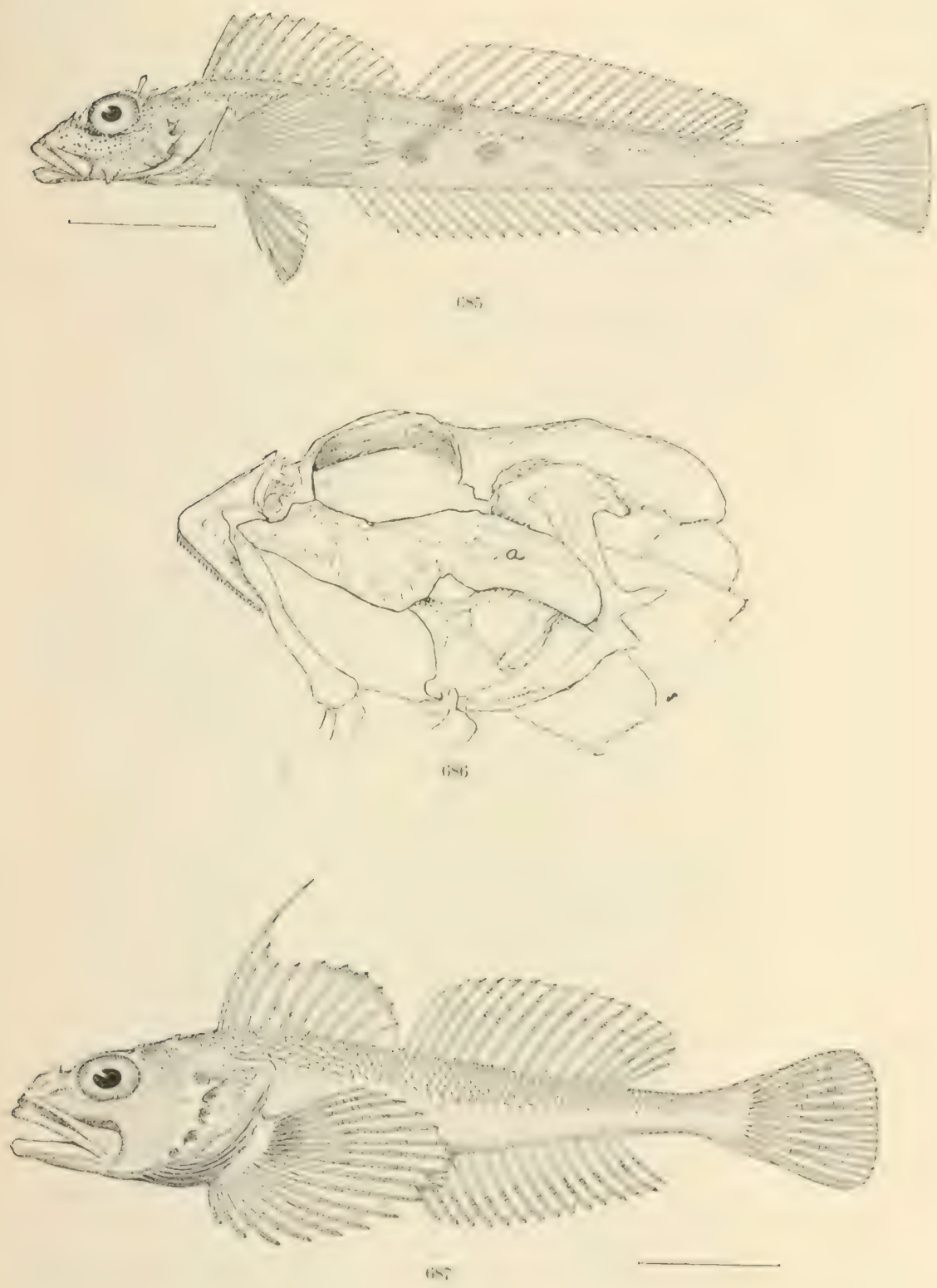

685. AlCidEA THOBURNi. (1', 1887.)

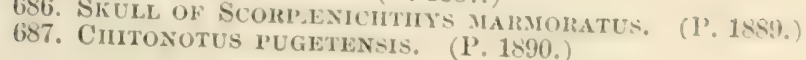




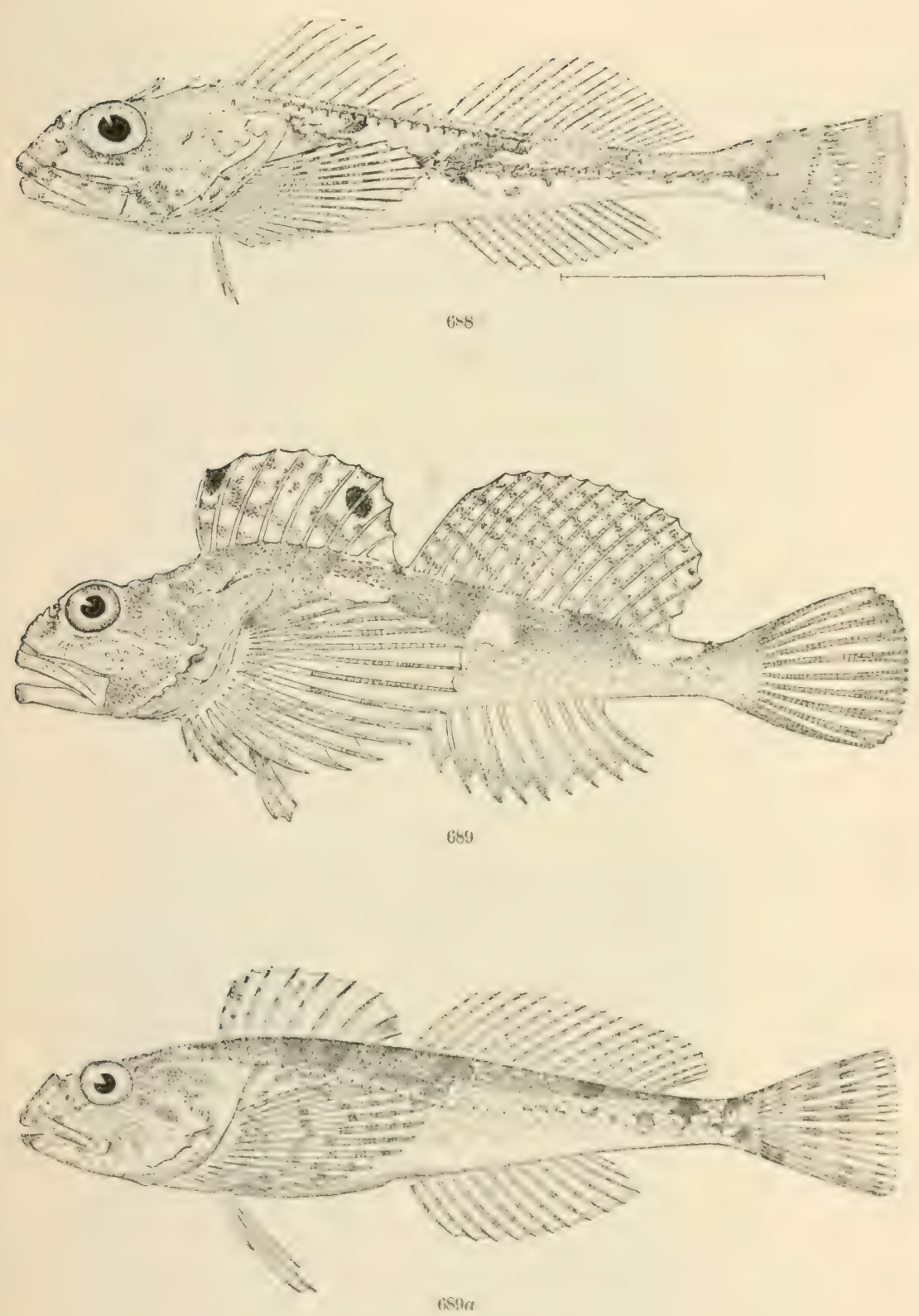

688. ICELMUS HORFALIS. (1'. 1896.)

689. AsTROLYTES NOTOSPLOTCS, (1', 1899.)

689a. Astrolytes xotospllotes; young? (ऐ. 1899.) 


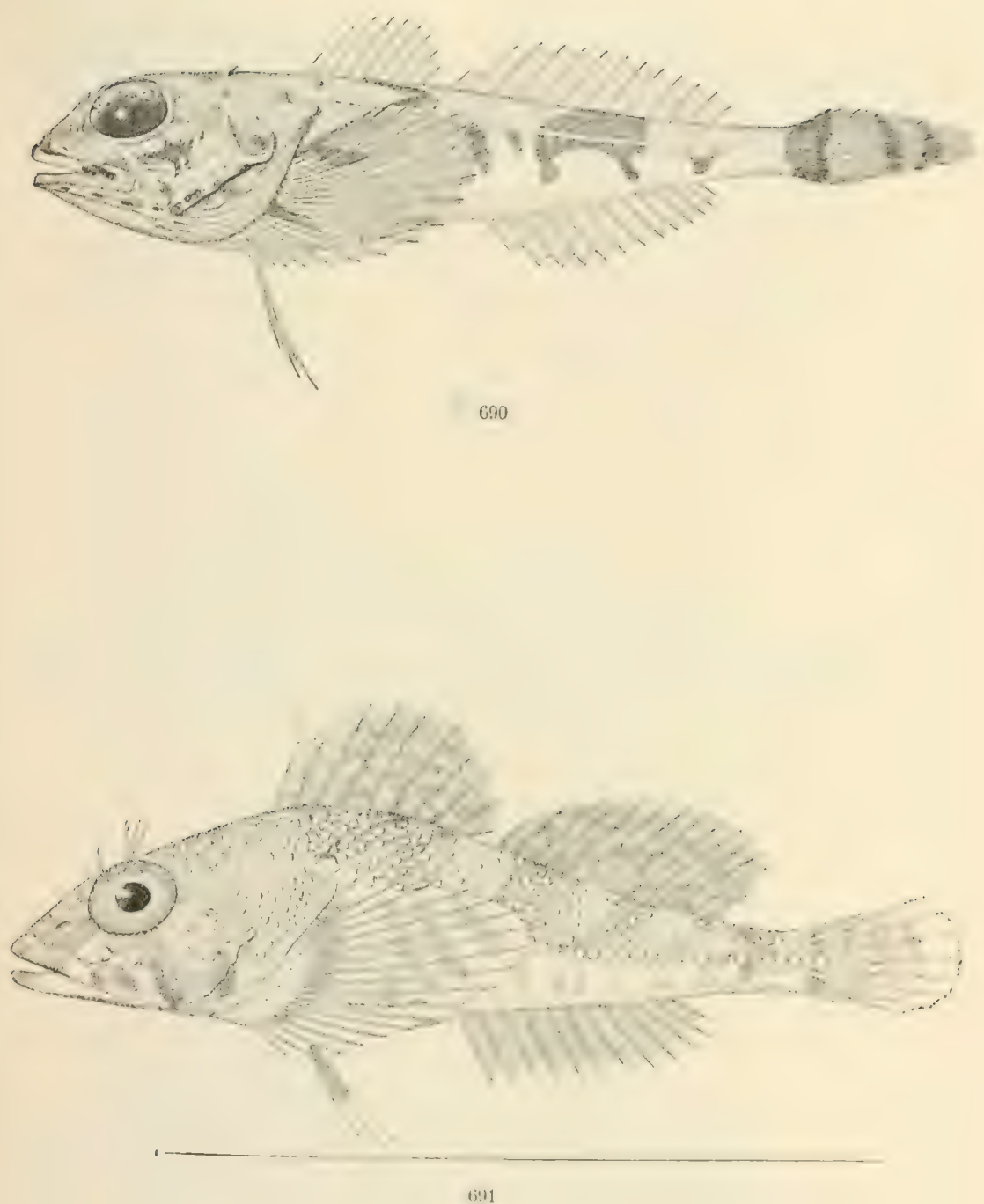

690. Artediellus atlanticus. (P. 1906.)

691. RUSCariUs Meaxit. (P. 1908.) 



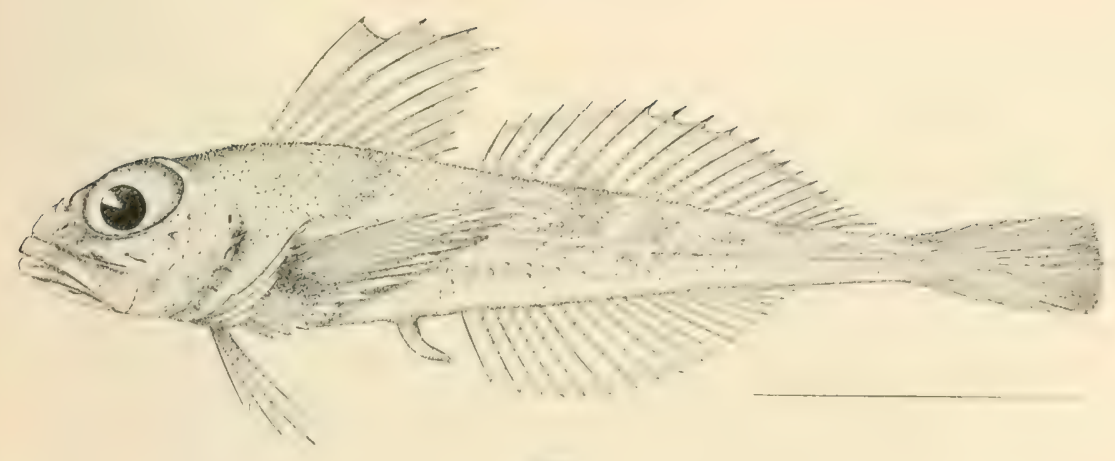

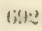
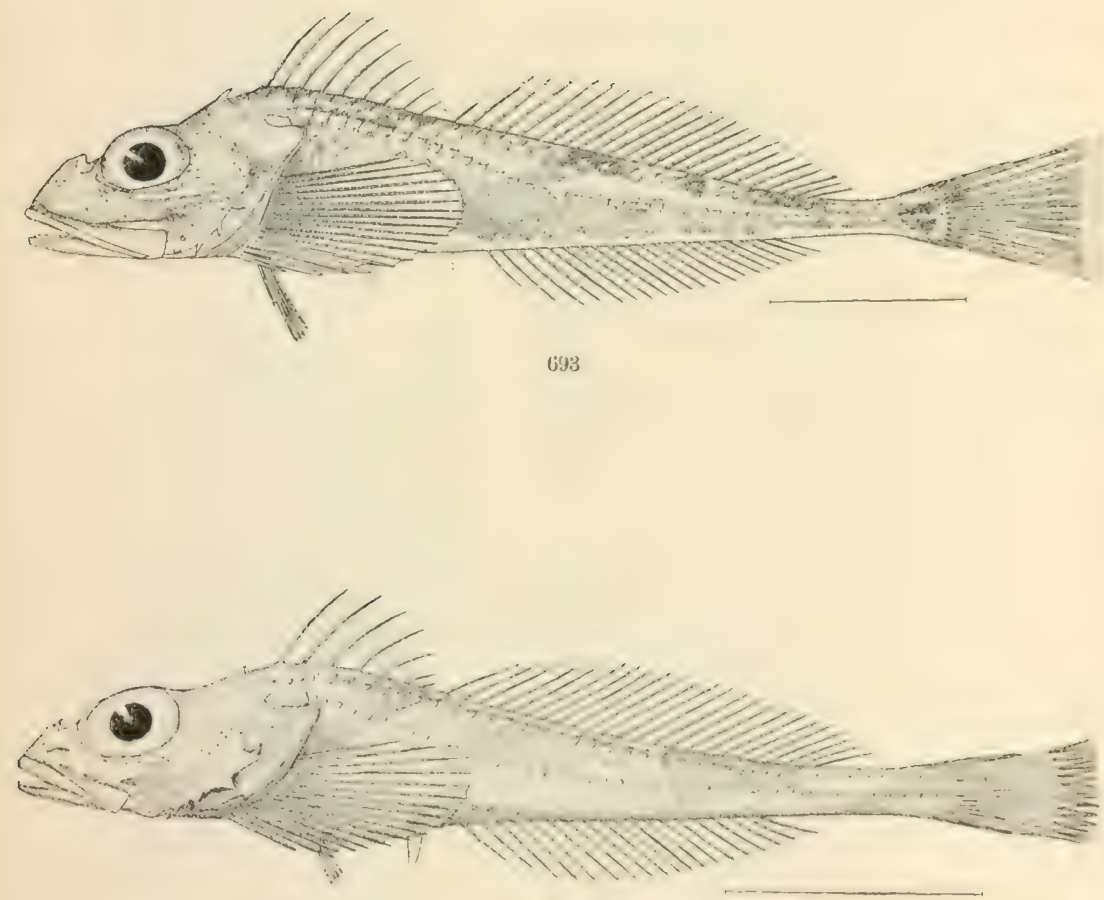

(4)!

692. Rastrixes scutigler. (IP. 1909.)

693. ICELUS SPNIGER. (P'. 1914.)

694. ICELe's Casidictlatus. (P. 1917.) 



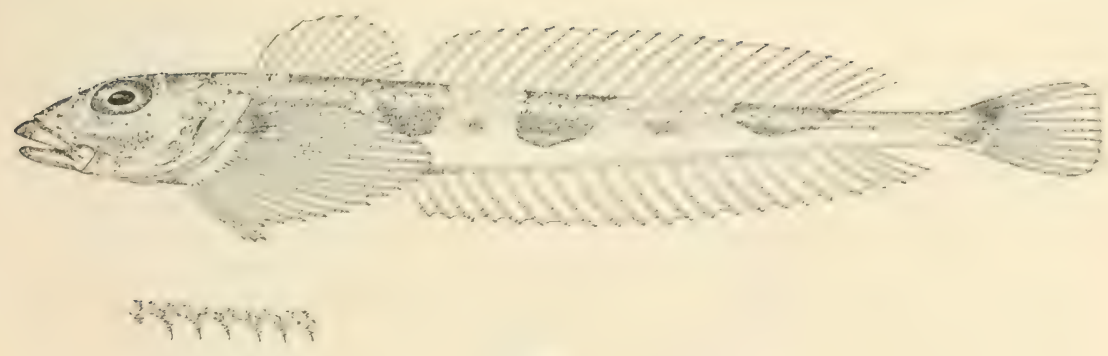

695

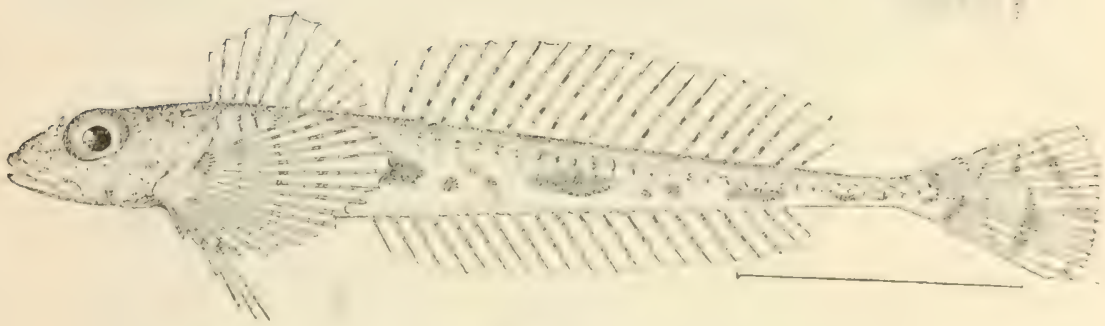

696

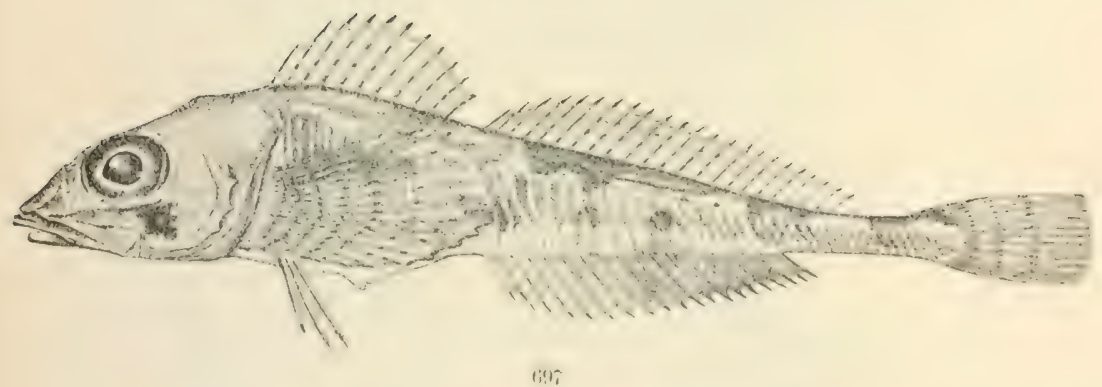

695. RaDUliNuS BOLEOIDIS. (P. 1919.)

696. RADULINUS ASPRELLUS. (P. 19:0.)

697. 'T'RIgLOPS PINGELI. (P. 1923.) 



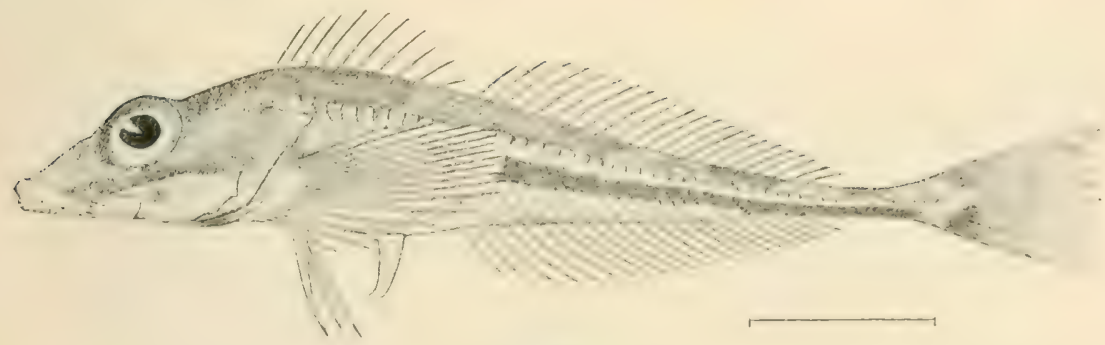

698
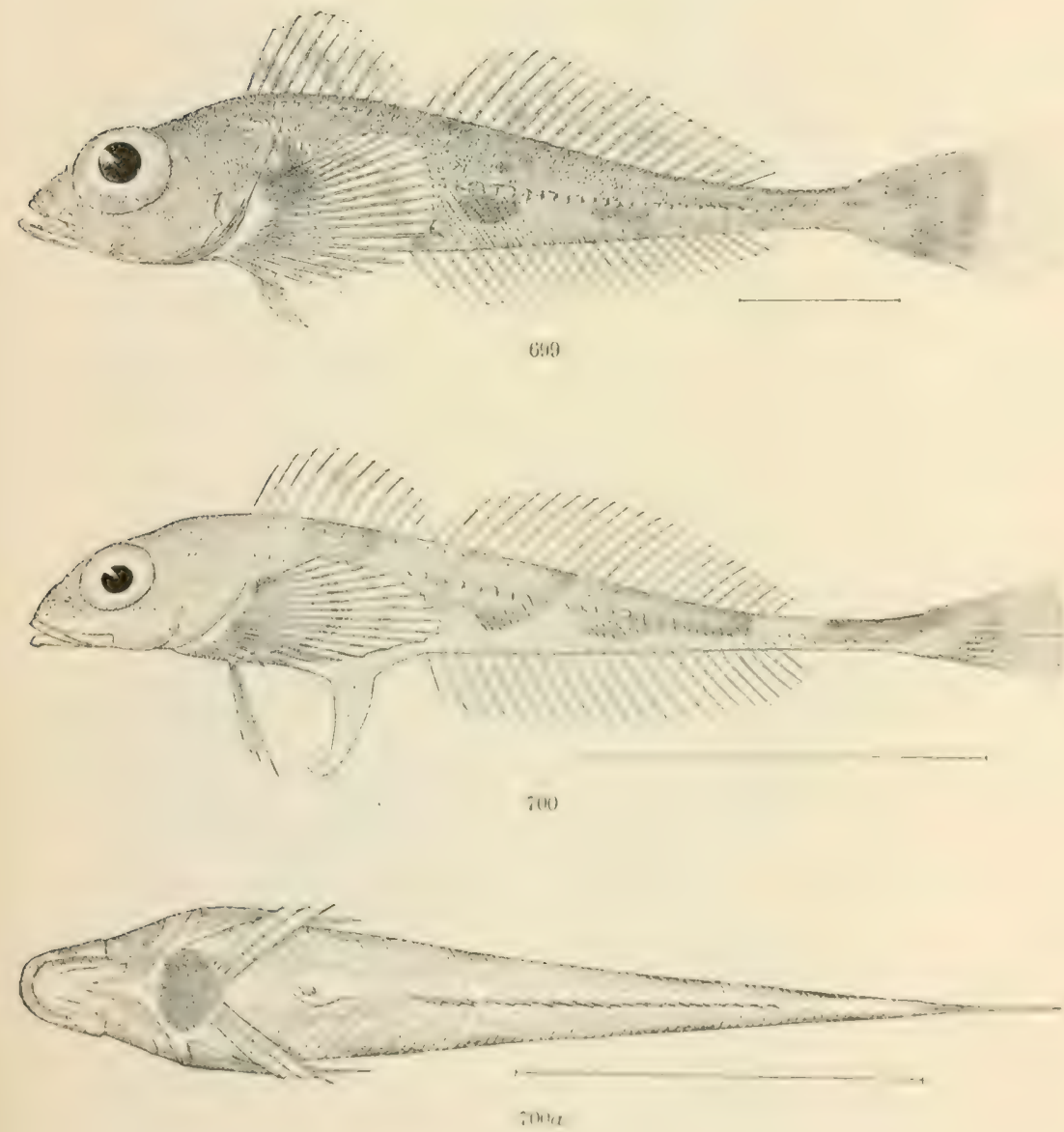

698. 'TRIGLOPS BEANY. (P. 1924.)

699. 'Triglors scerticts. (P, 1925.)

700, 700a. Stersias xexostminus. (P. 1927.) 


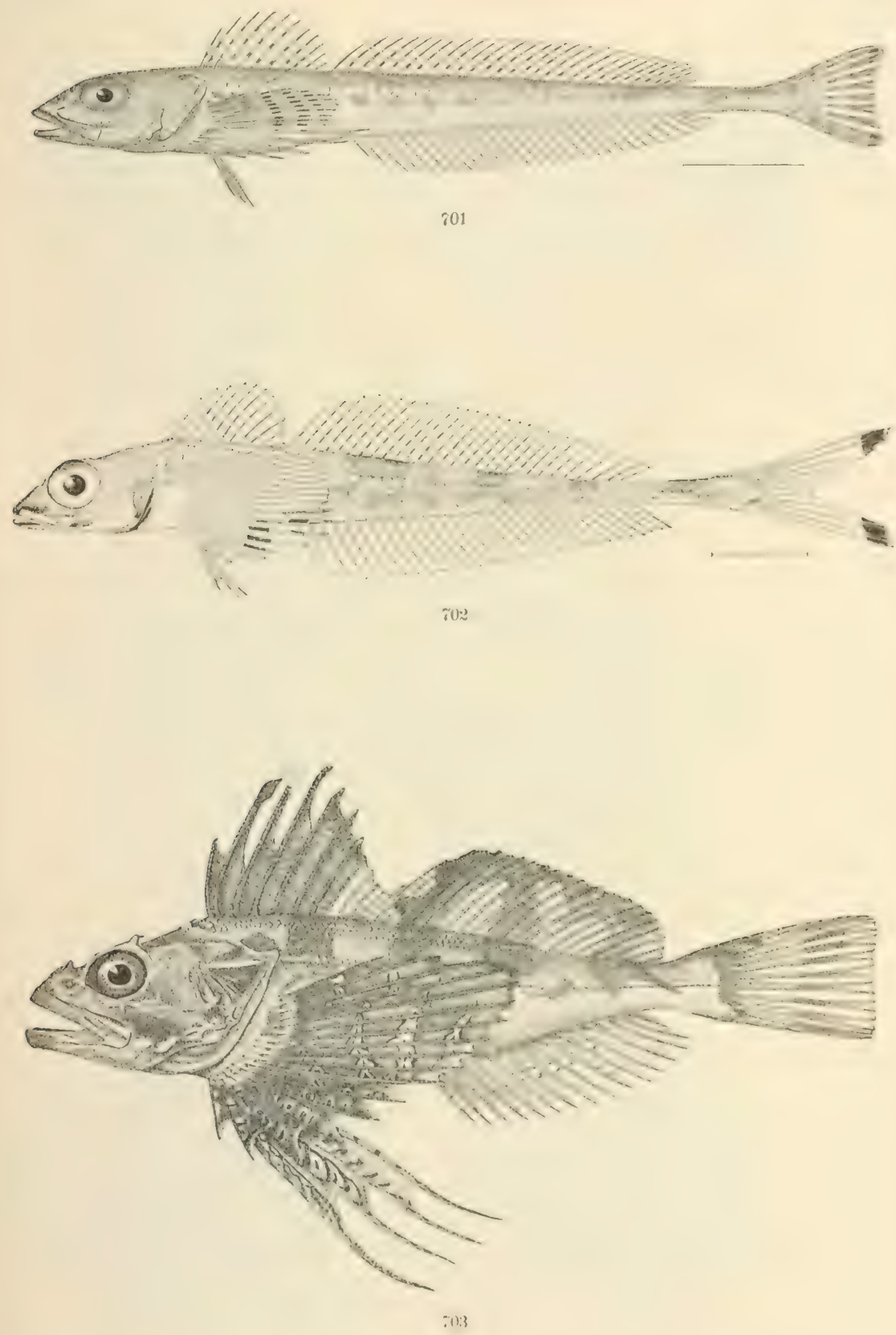

701. PRIONISTIUS MACILLUS. (l'. 1928.)

702. ÉlaxuRa FORICATA, (P.1930.)

703. MELLETES PAPILIO. (P. 1932.) 



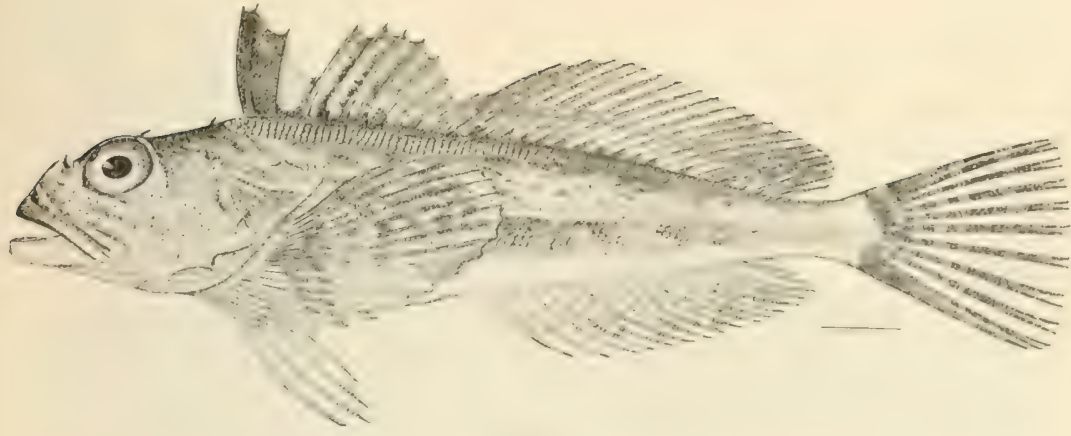

701

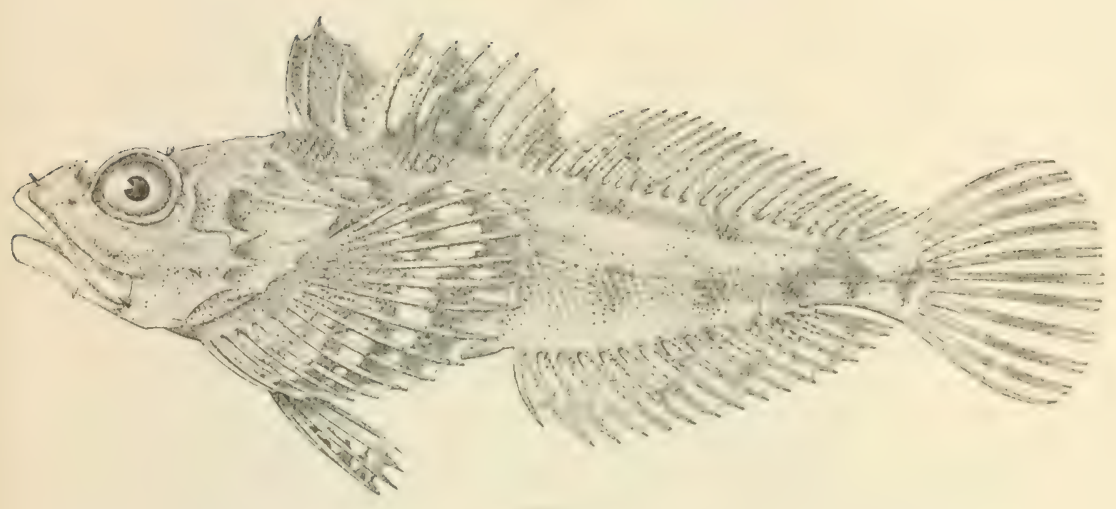

i11;
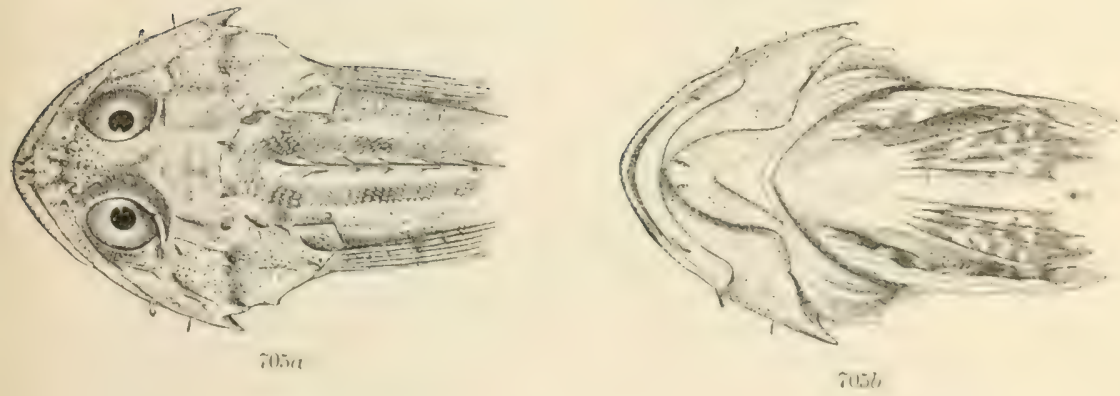

704. HFMILEIHOTUS JORDANI. (I'. 1931.)

705, 705a, 705b. HEMILEMHOTU'S HEMILEIDOTE's. I'. 1935.) 


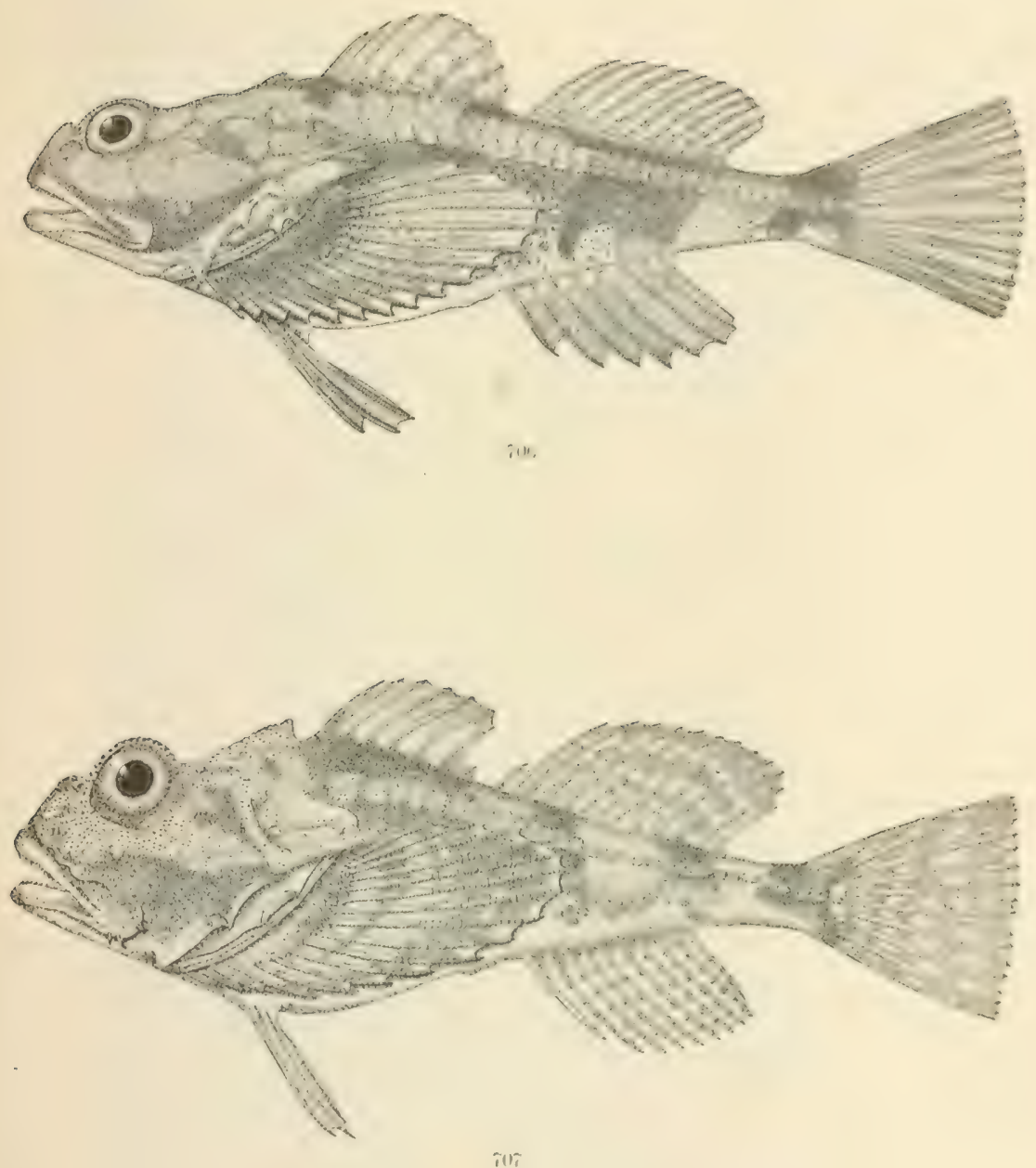

706. ENOPIIT'S 131sox. (P. 1938,

707. Ceratocottús micriaus. (P. 1940.) 


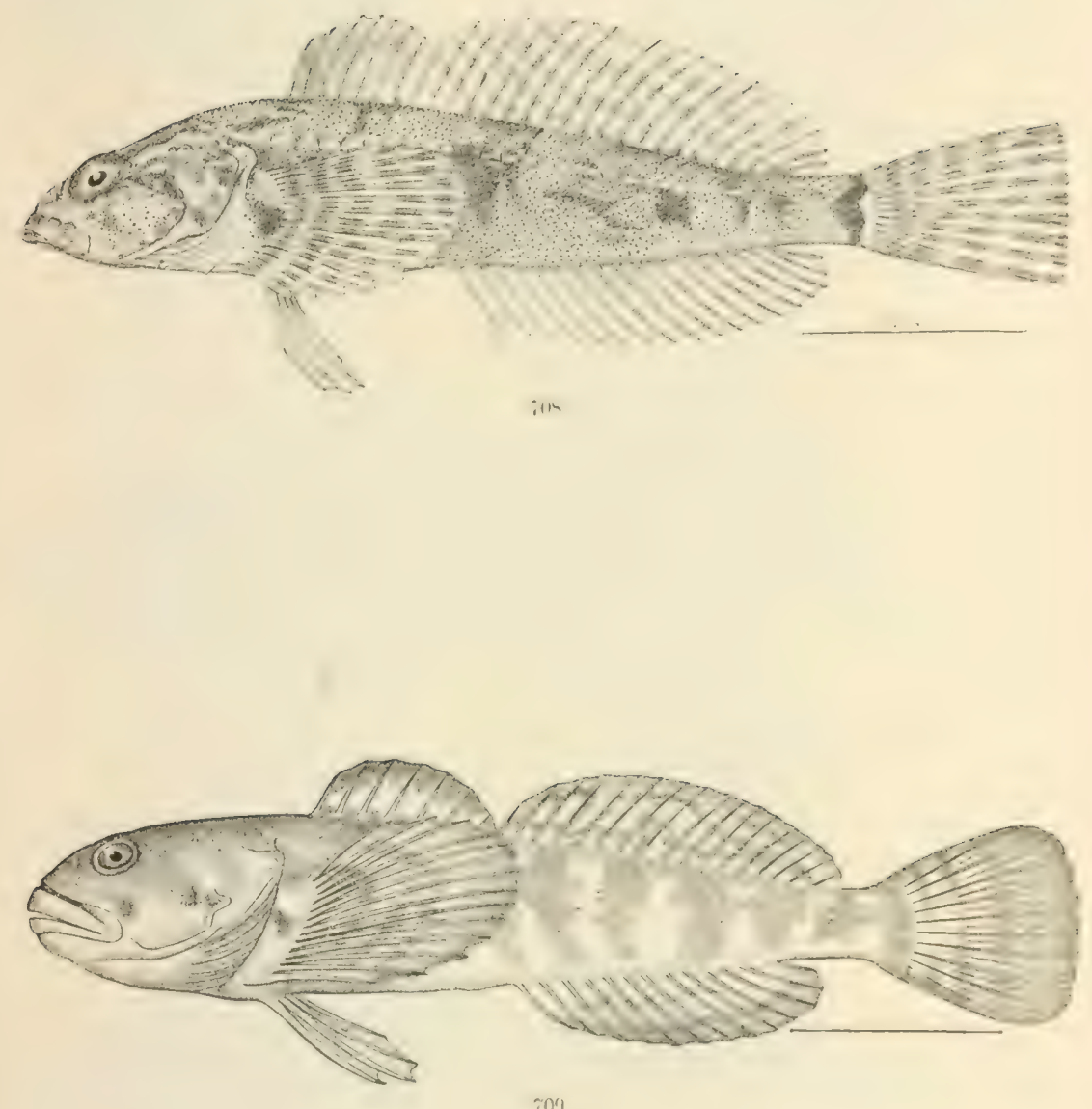

708. Cottus EvirMaxis. (P. 19.5.)

709. Cottus I'ixctilates. ( $\left.\mathrm{I}^{\prime}, 1948.\right)$ 


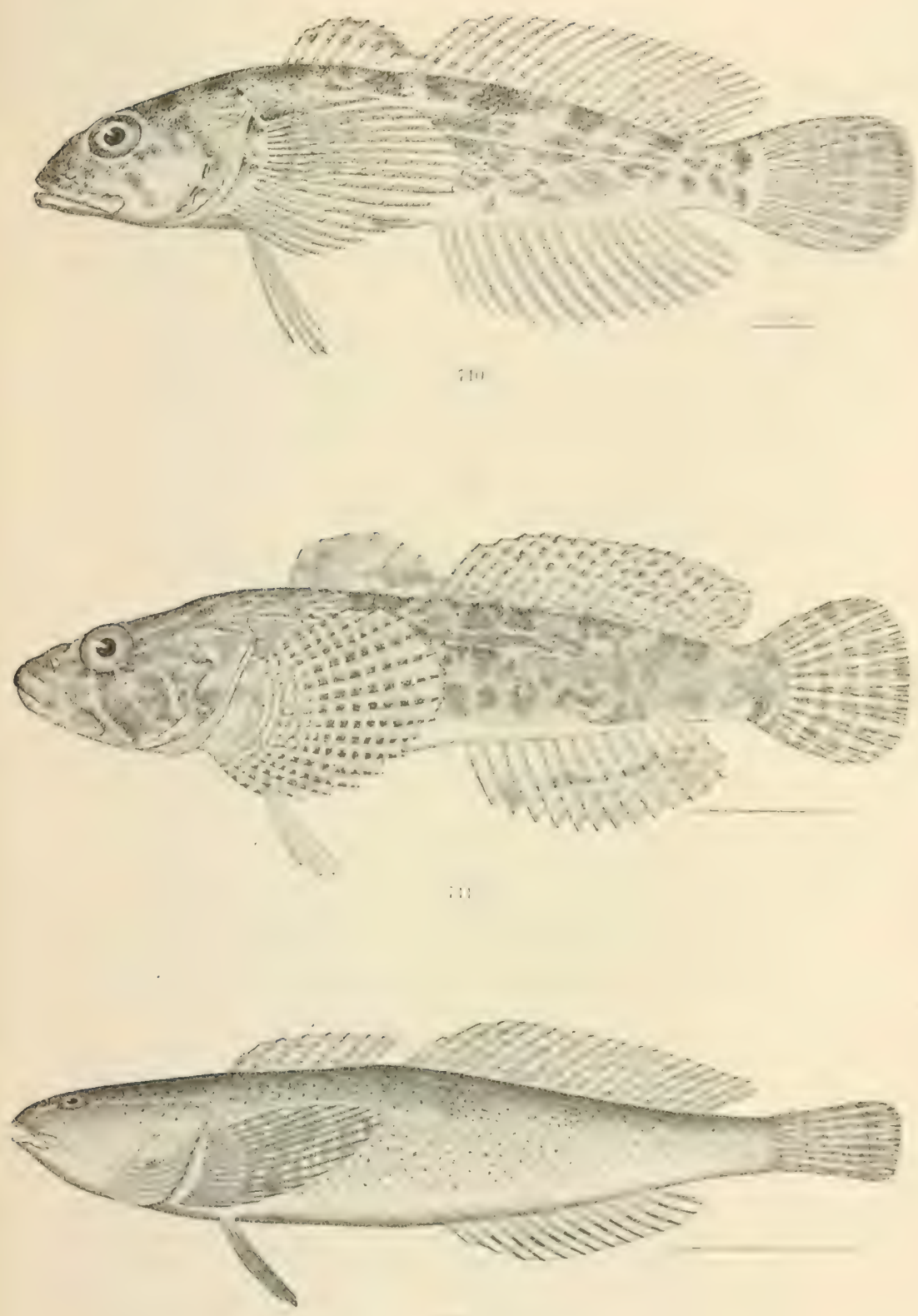

:1:

710. Cottus Prorlaxes, (1', 19.5.)

711. CotTus kilaMathexsis. (1', 1955.)

712. Cottus aleuticl's. ( 1 , $199 \overline{7}$. 



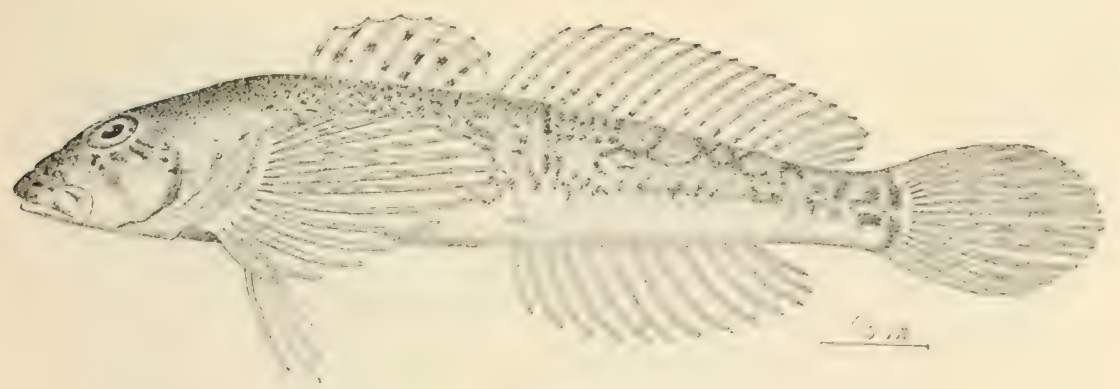

i:?

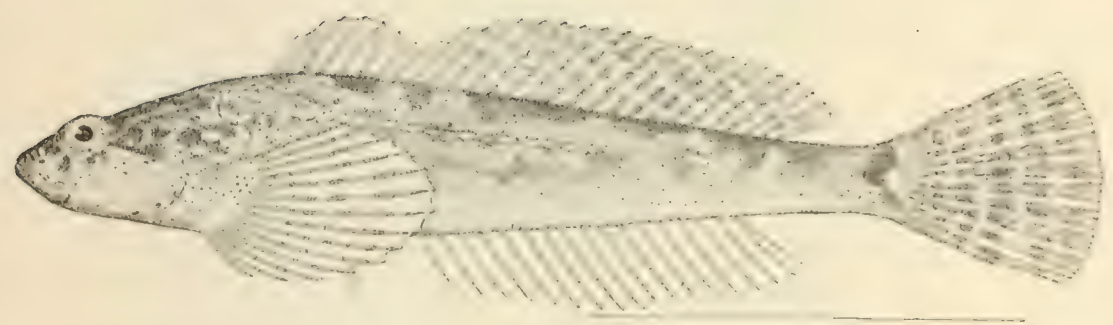

i11

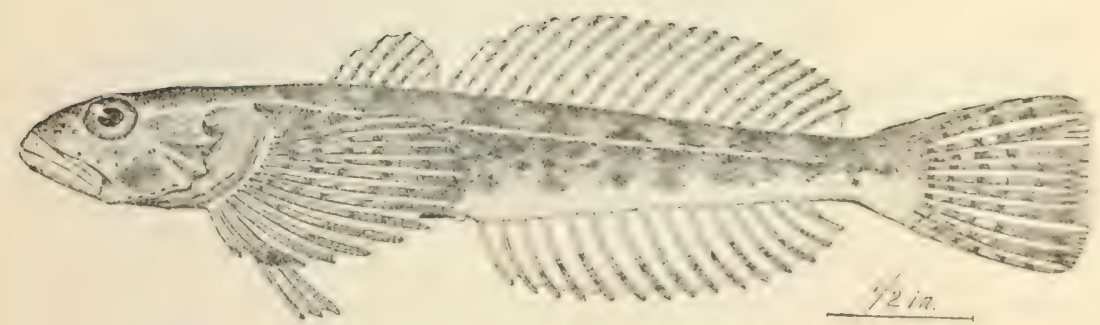

715

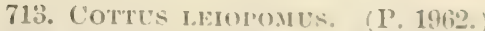

714. COTTUS l'RIXicERs. (P., 196.2.)

715. URANIDEA TEXUIS. ( 1 , 1966.) 



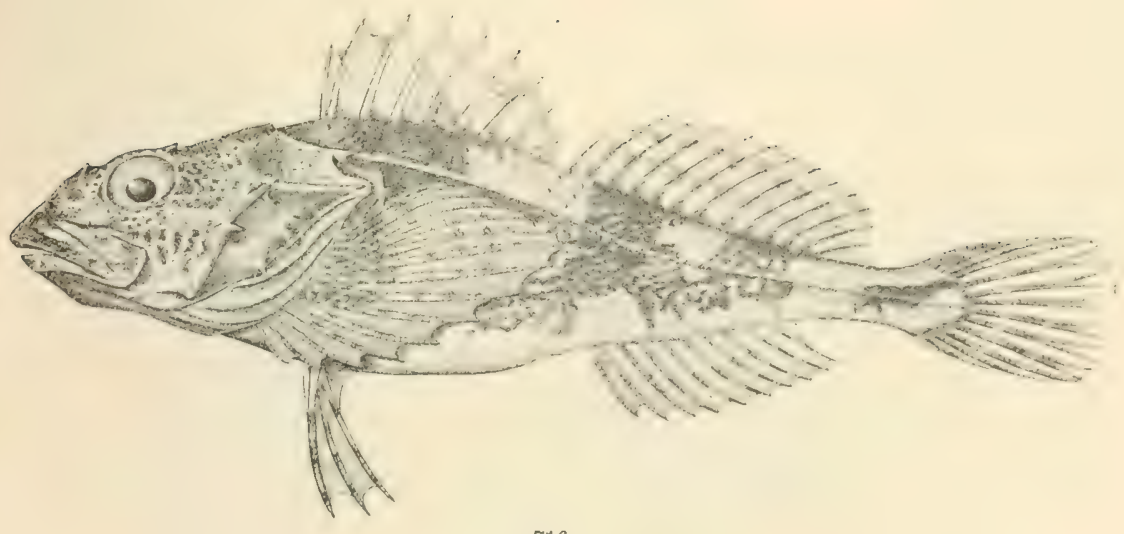

716

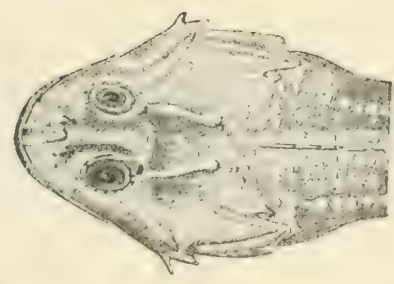

8106

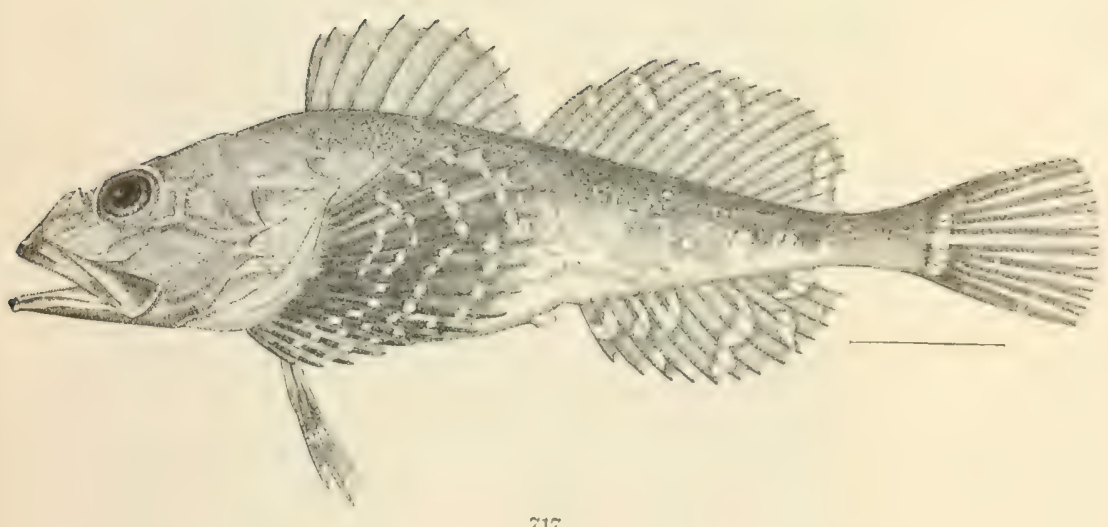

210

716, 716a. Myoxocephalus eneus. (P. 1972.)

717. If Yoxocepialus scorpius. (P. 1974.) 



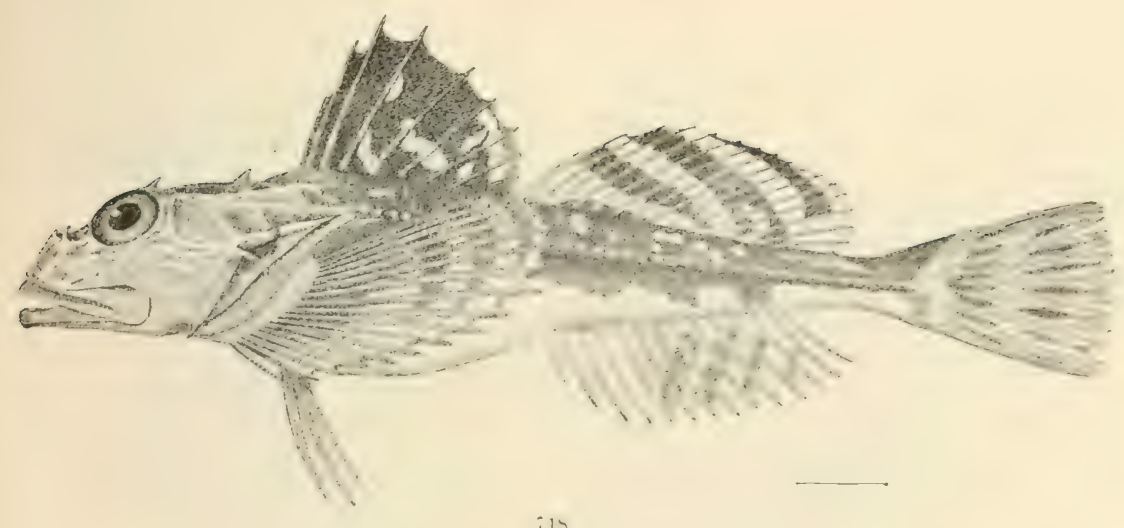

i1

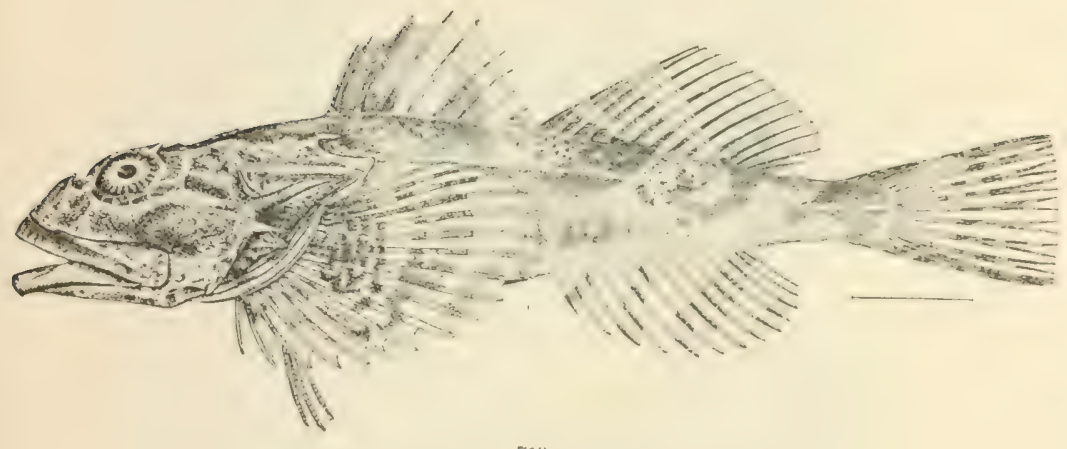

;1!

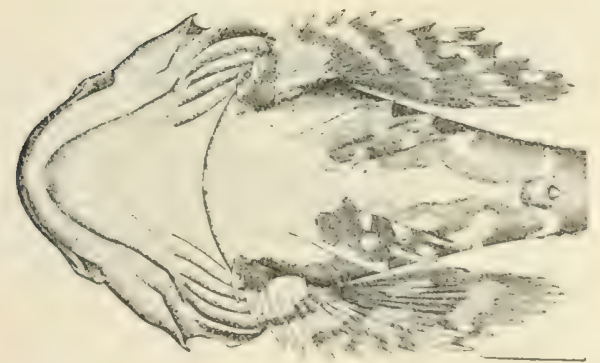

$7 \because 0$

718. MYOXOCEMHAUS OCTODECMLI'IXOSUS. (P. 1976.)

719. MYOXOCEPHALUS POLYACANTHOCEPHLUS, (P. 1976.)

720. MYOXOCEPHALU'S JAOK. (P, 1977.) 


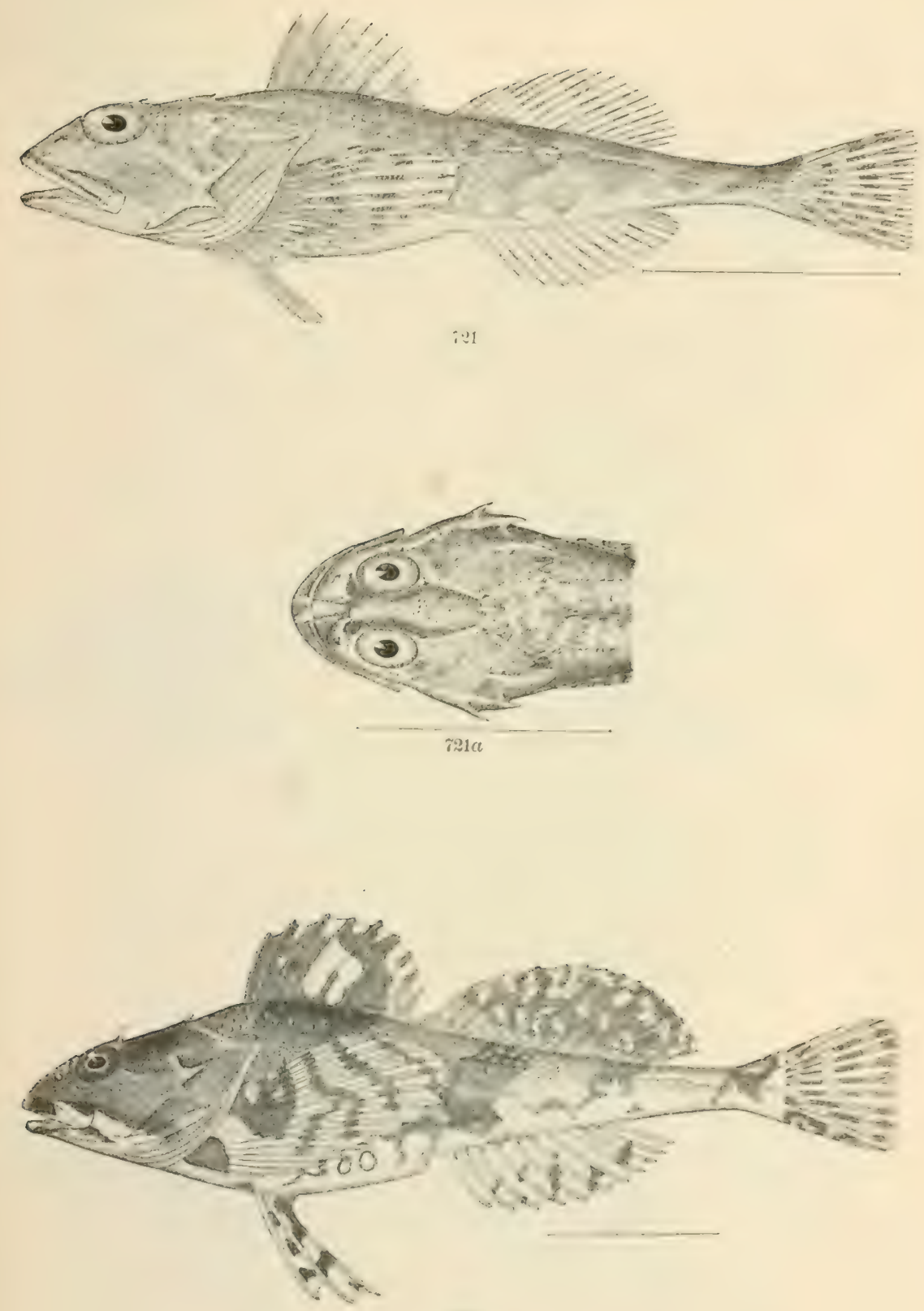

i*n

721, 721a. Myoxocepinalus veriucoses. (P. 1979.)

722. MroxocEPHAL'S AXILLARIS. (P. 1980.) 



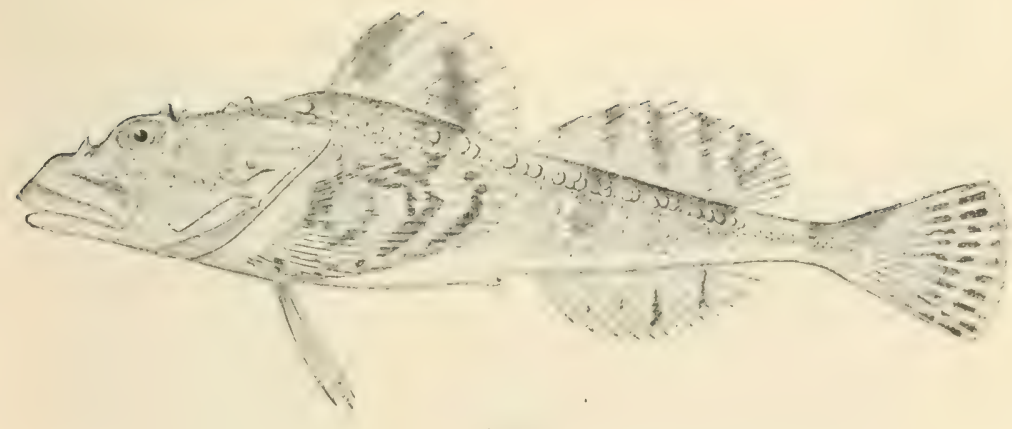

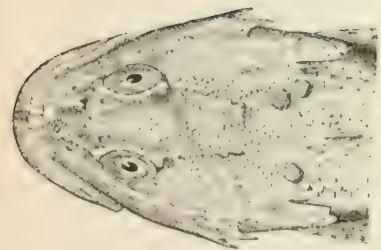

$723 a$

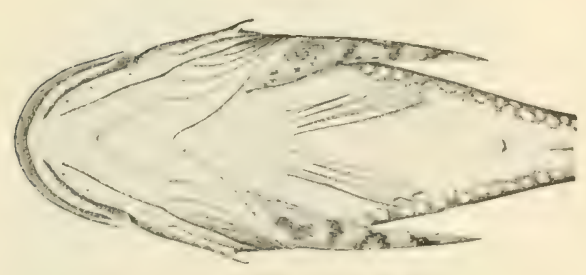

is31

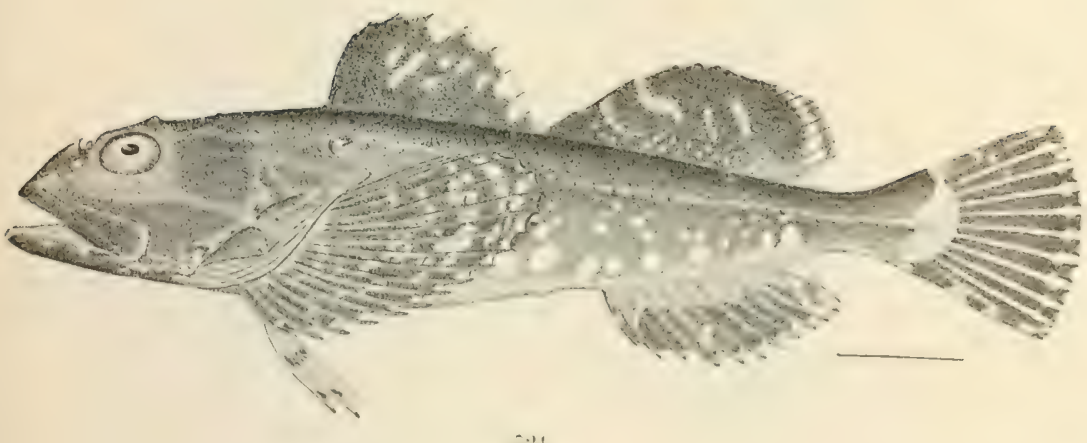

$\because \because 1$

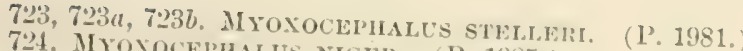

724. MYoxocephalus Nigen. (P. 1985.) 



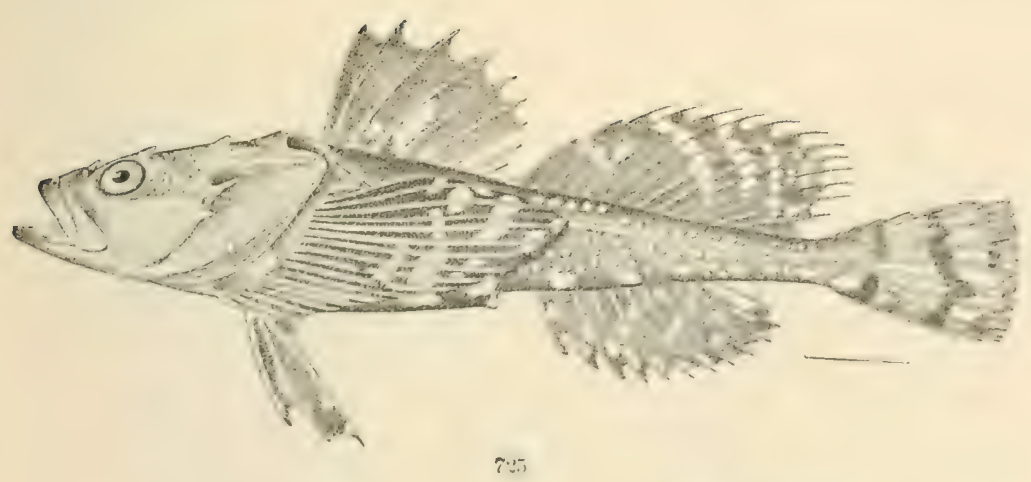

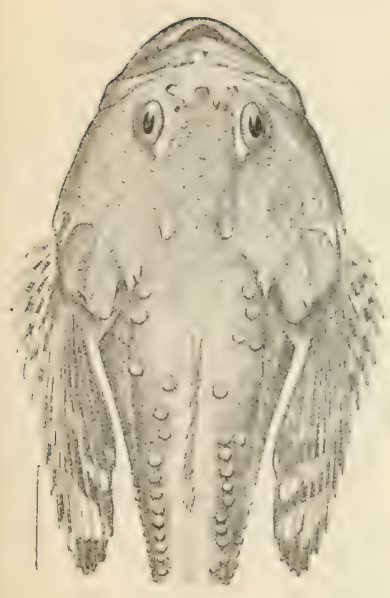

ระงัย

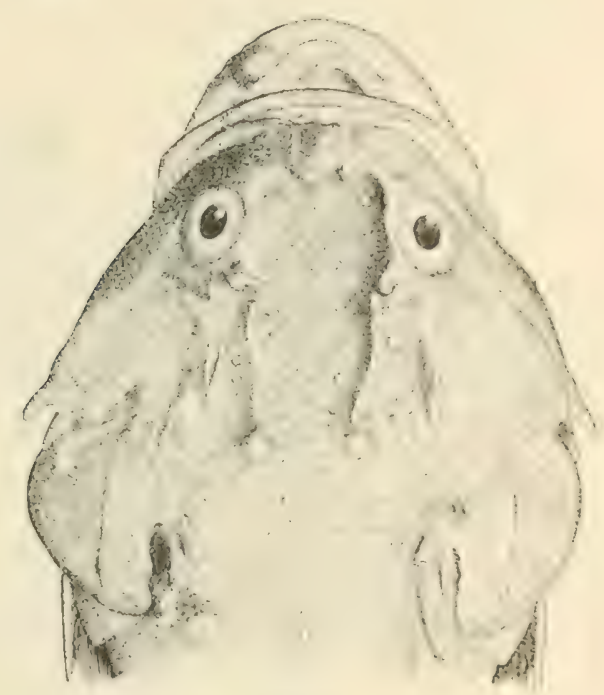

i

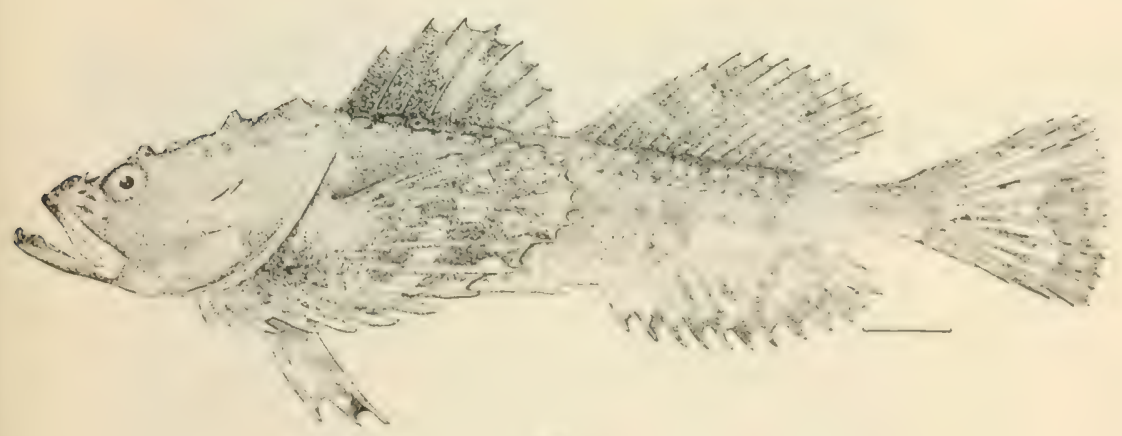

725, 725 $a$. MEGAMOCOTTUS PLATYCEPHALUS. (1'. 1987.)

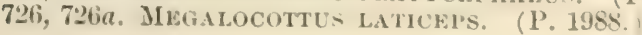




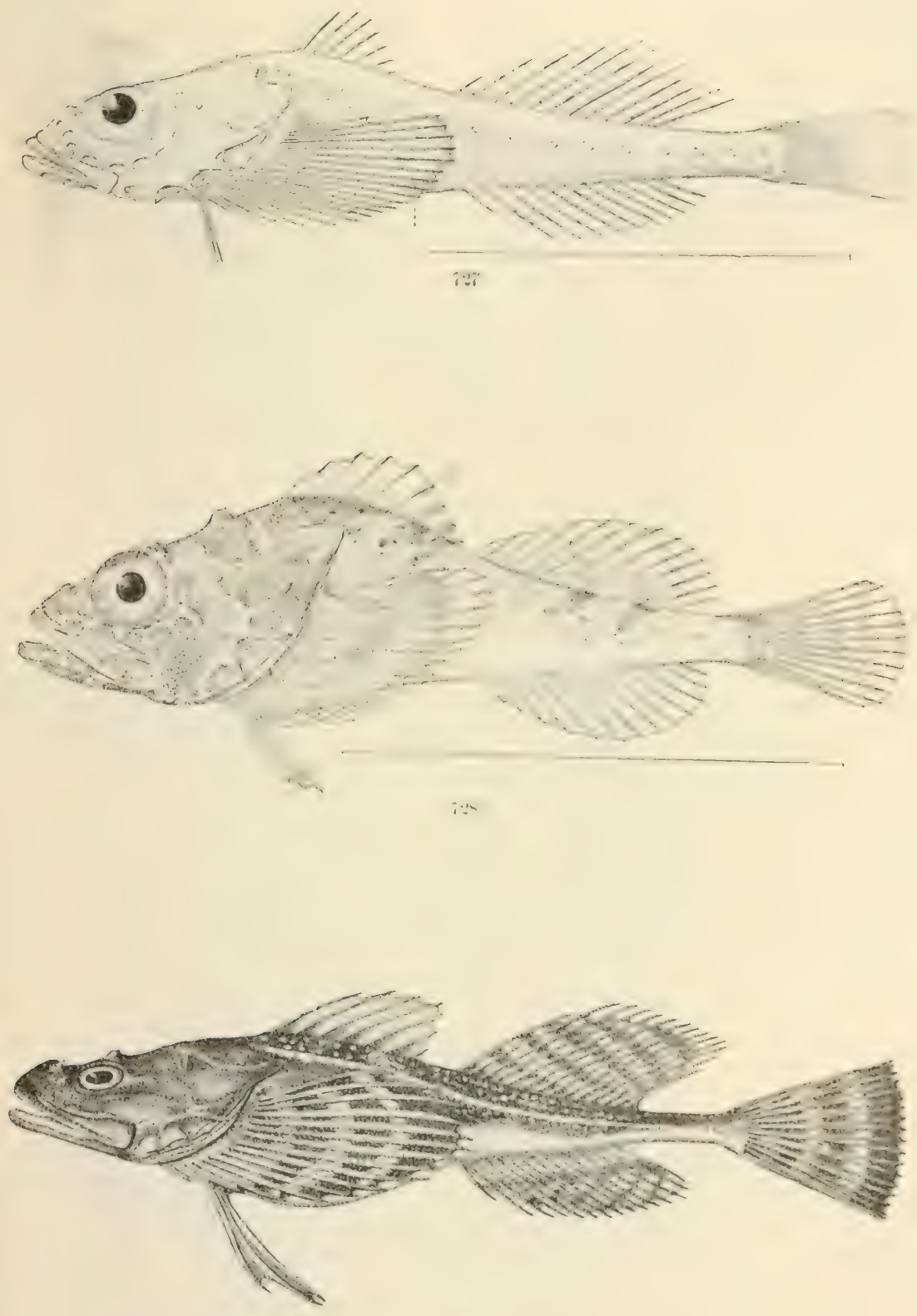

$\because \because 1$

727. Zesticelus PliofunioneMr. (P, 1990.)

728. DAsYCOTTUS SETIGER. (P. 1991.)

729. ONCuCotTUS QUaDkicoksis. (P. 2001.) 



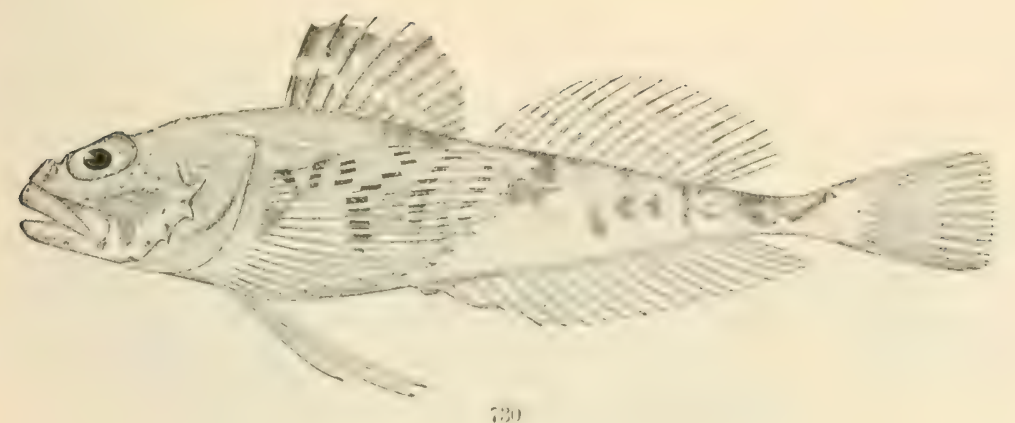

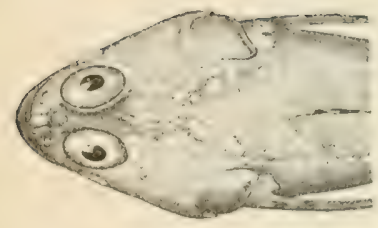

ii)

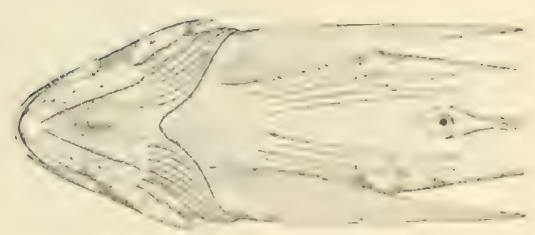

:20'

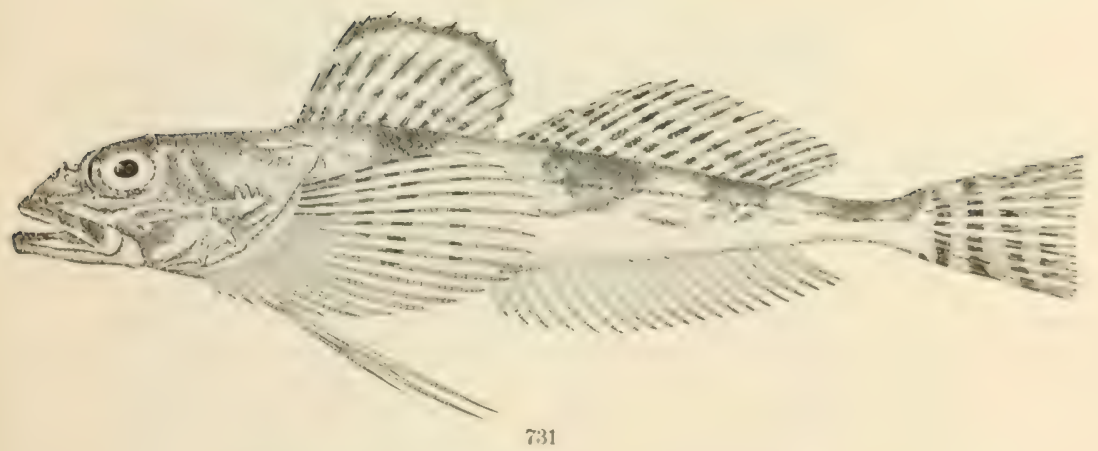

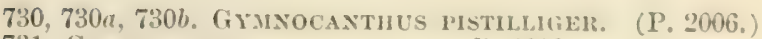

731. GYMNOCANTHUS GALE.ITUS. (P.2010.) 



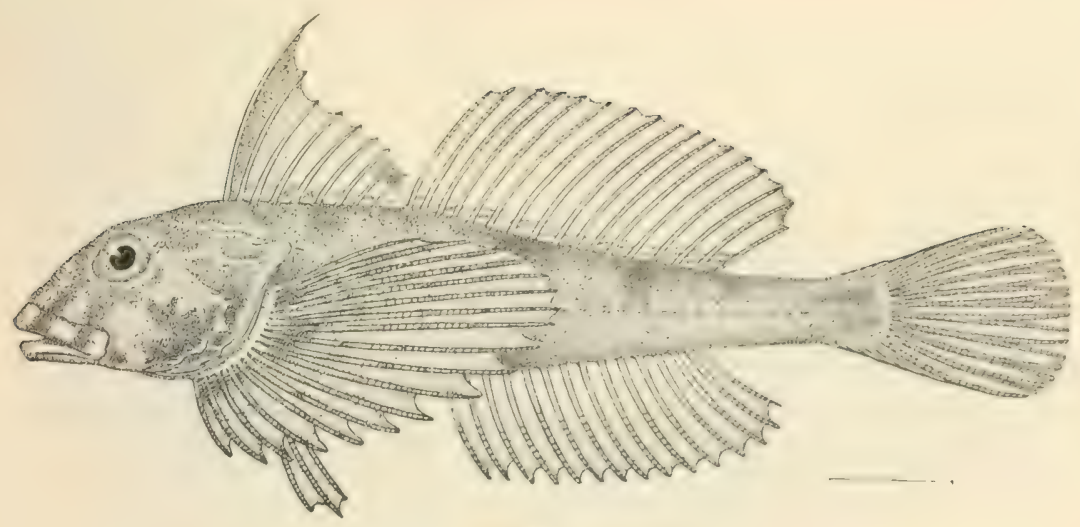

73

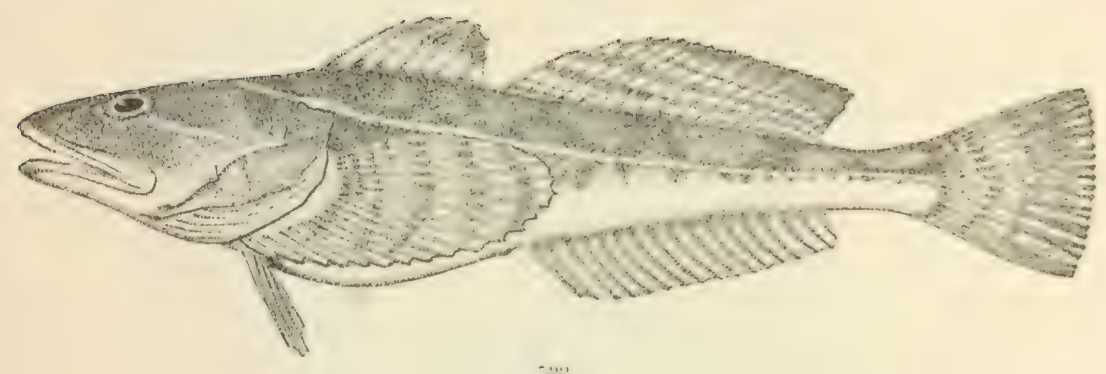

i:3:;

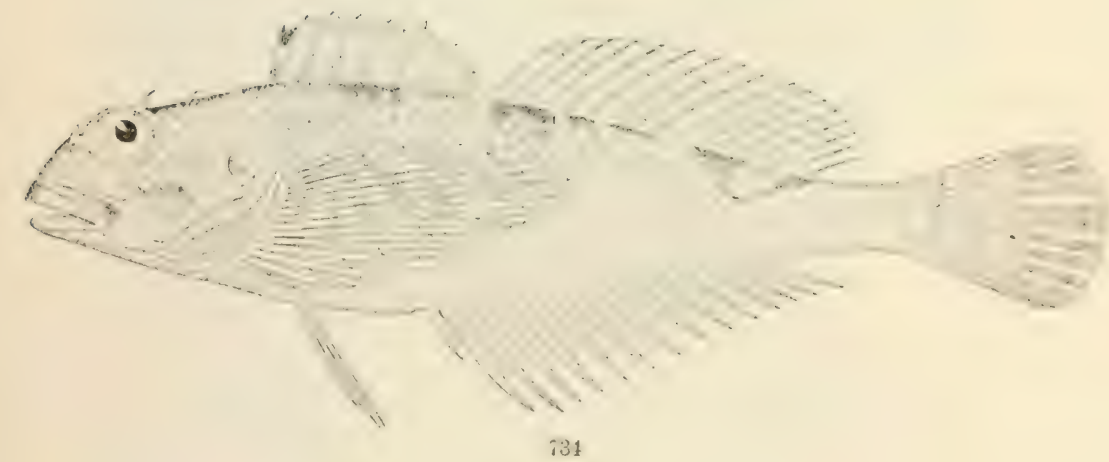

732. Leiocottus minundo. (P. 2011.)

733. LEPTOCOTTUS ARMATUS. (P. 2012.)

734. Oligocottes Maculosus. (P. 2013.) 


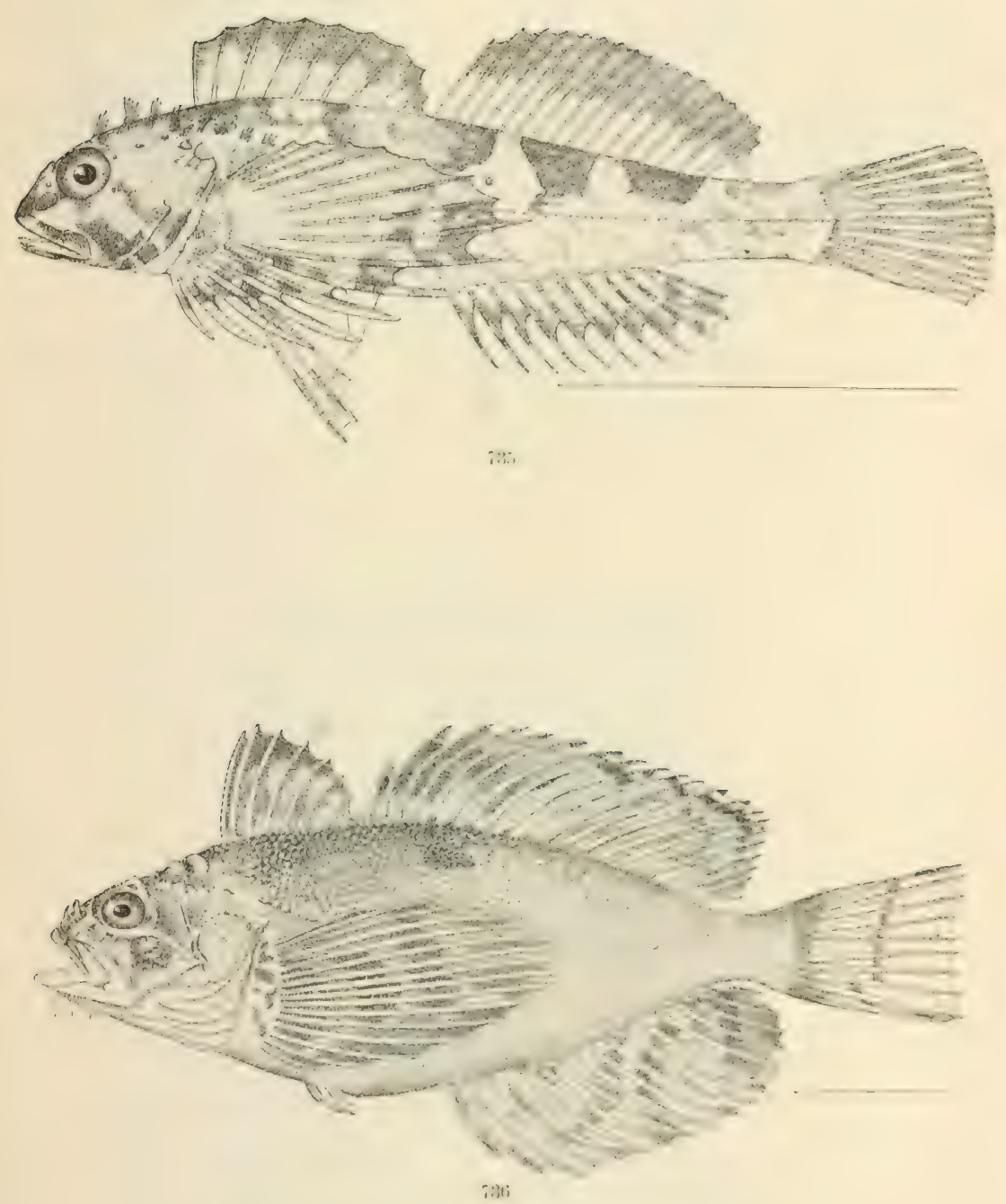

735. Blenvicottus emrirum. (Pp. 2016, 2861.)

736. Histiocotrus im.olsus. (P. 2018.) 


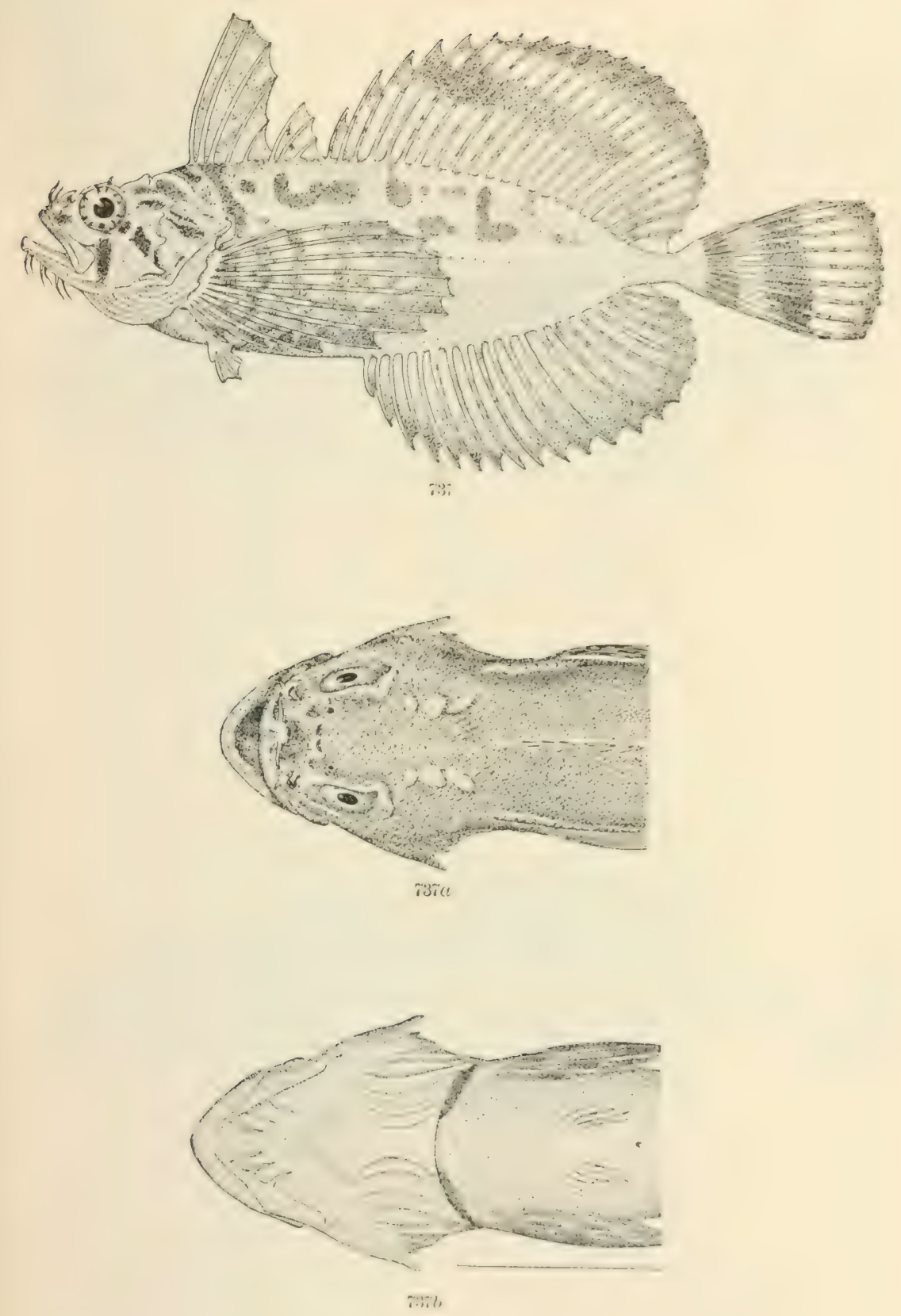

737, 737 $a, 737 b$. BLEL'SMS CMmHOSUS. (1'.2018.) 


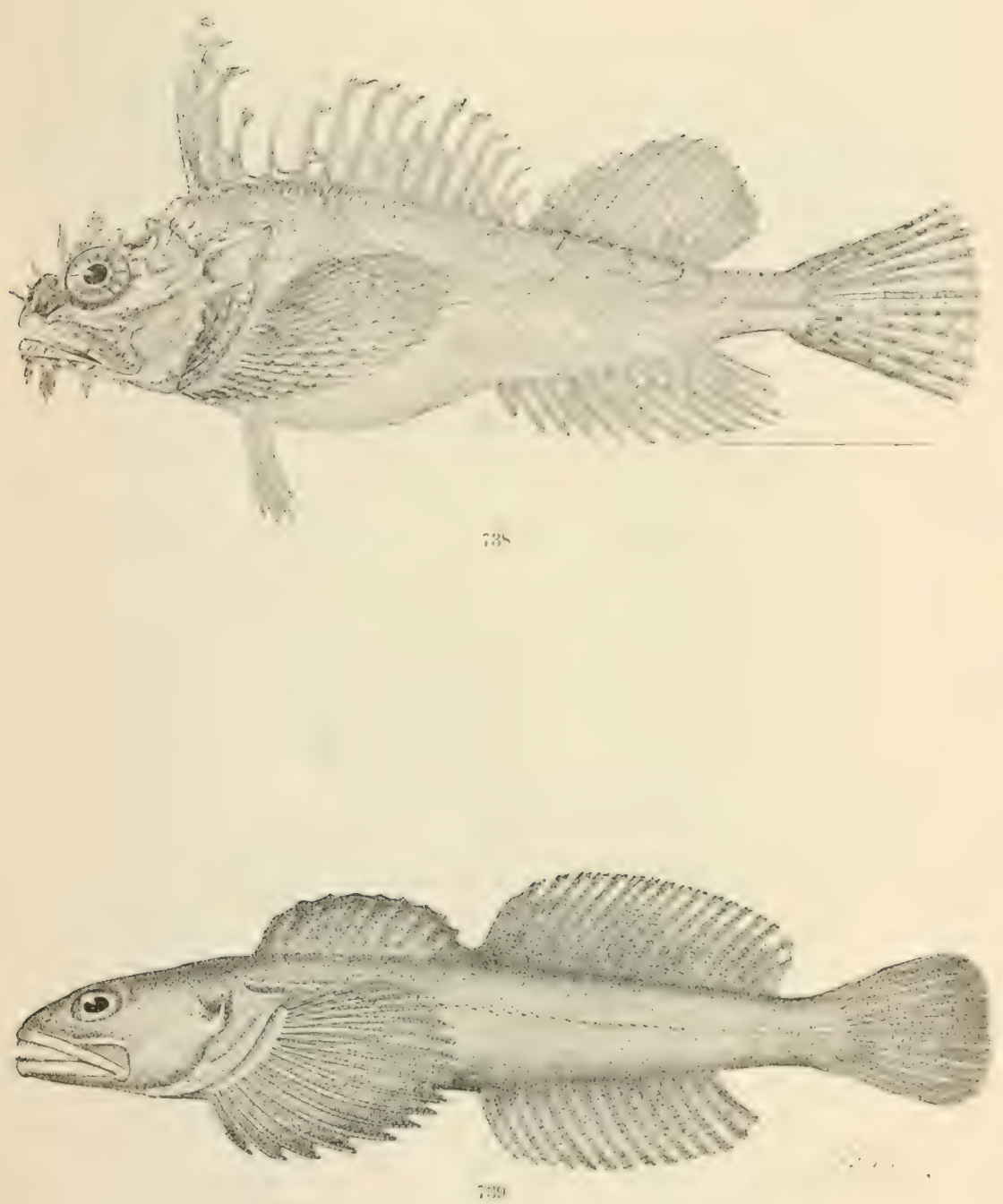

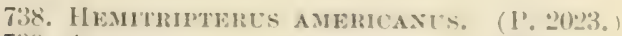

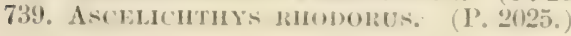





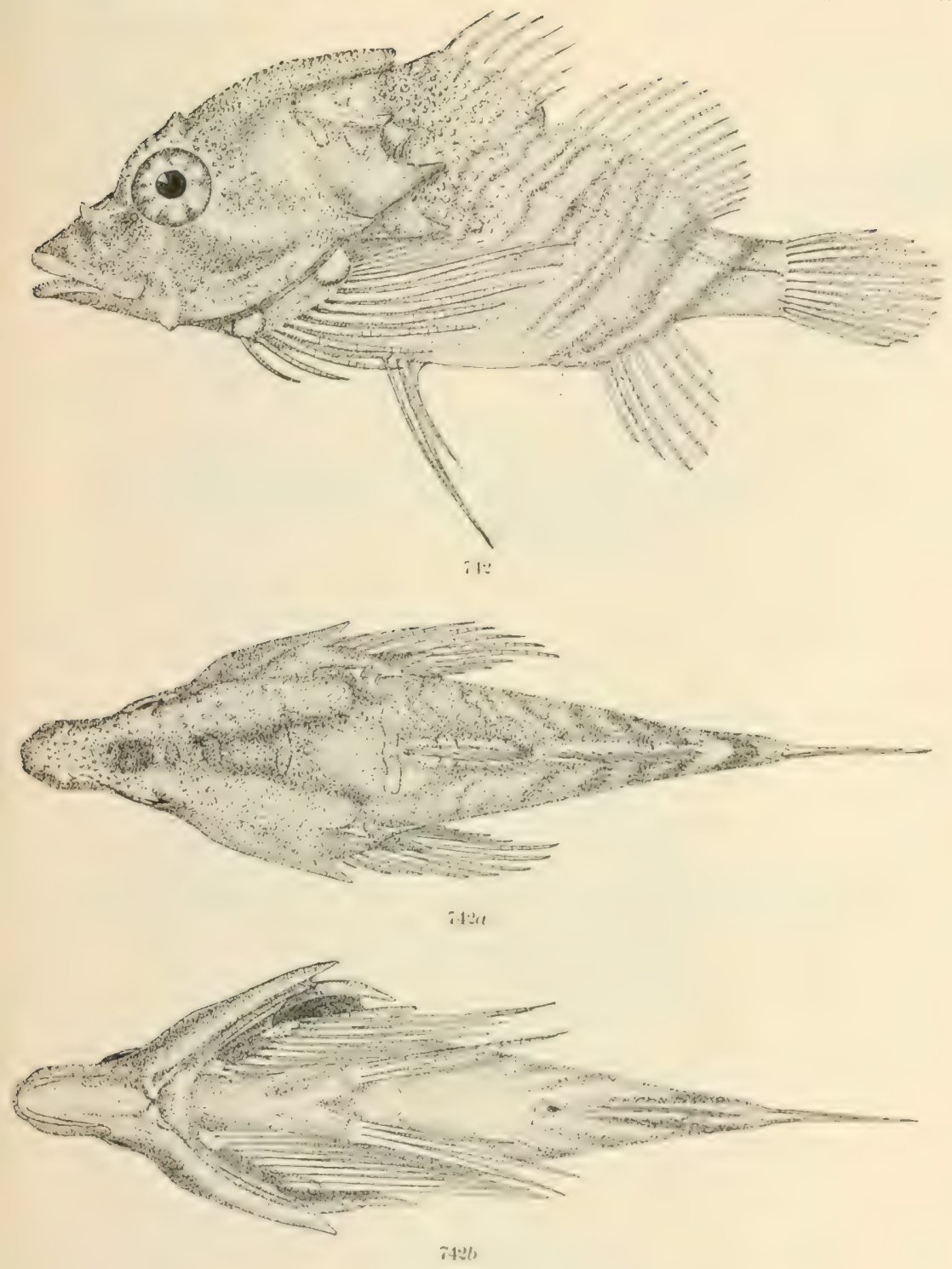

$74: 36$
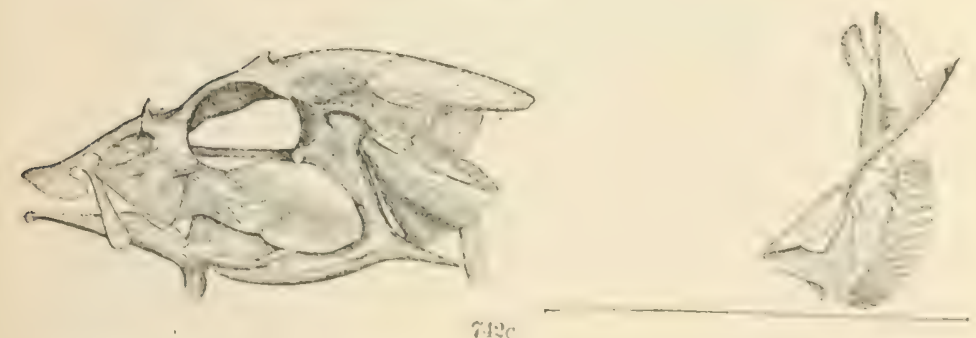

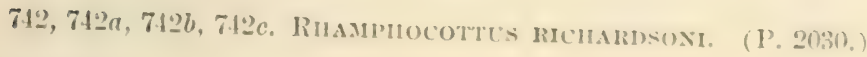




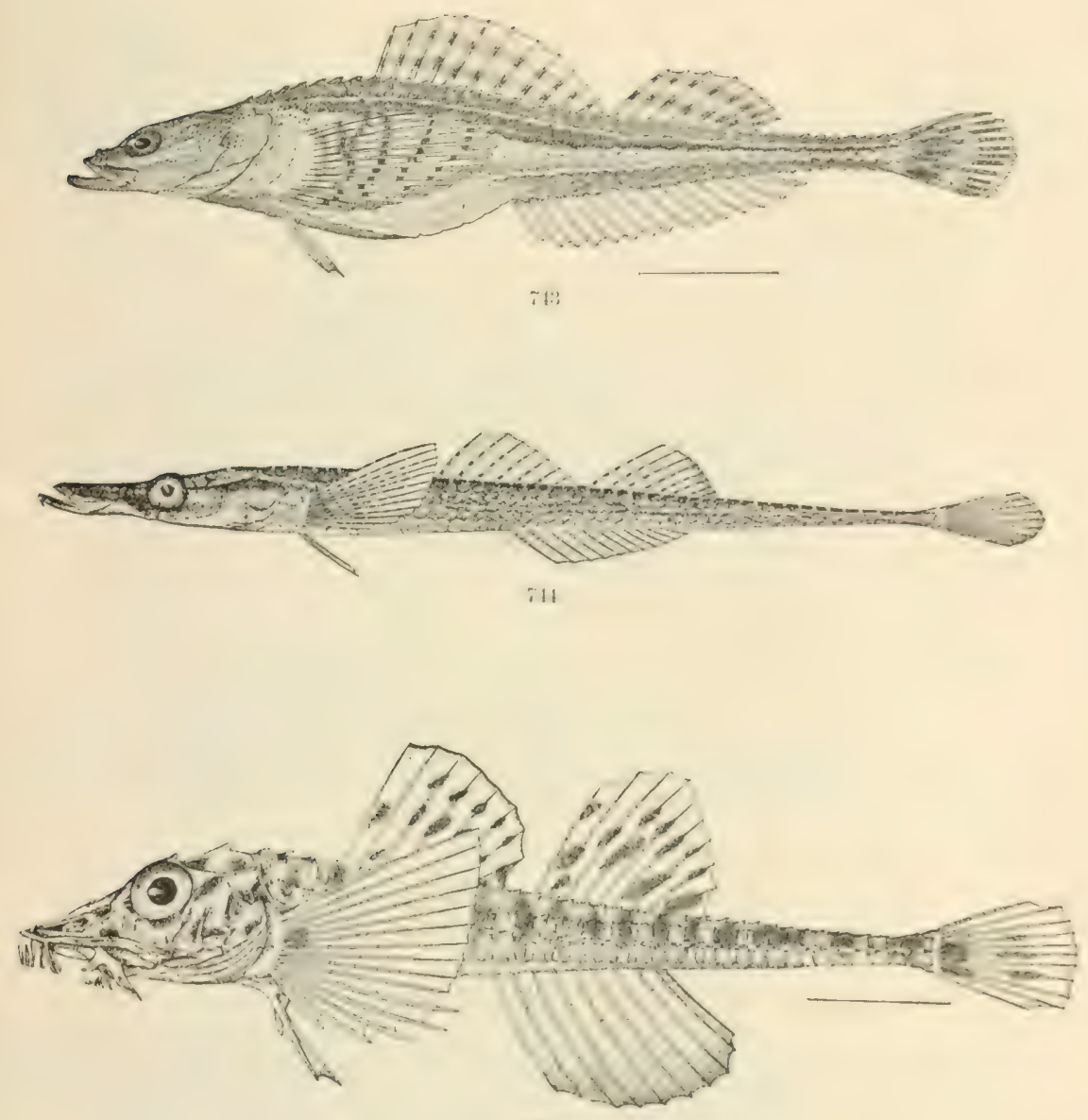

i.15

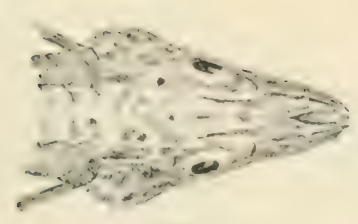

ilini,

743. OCCA DODECAEIRON. (P. 204.)

714. Pallasina barbata. (1', 20.19.

745, 74\%. P'ODOTHECUS ACCH'1ER. (1'.2055.) 



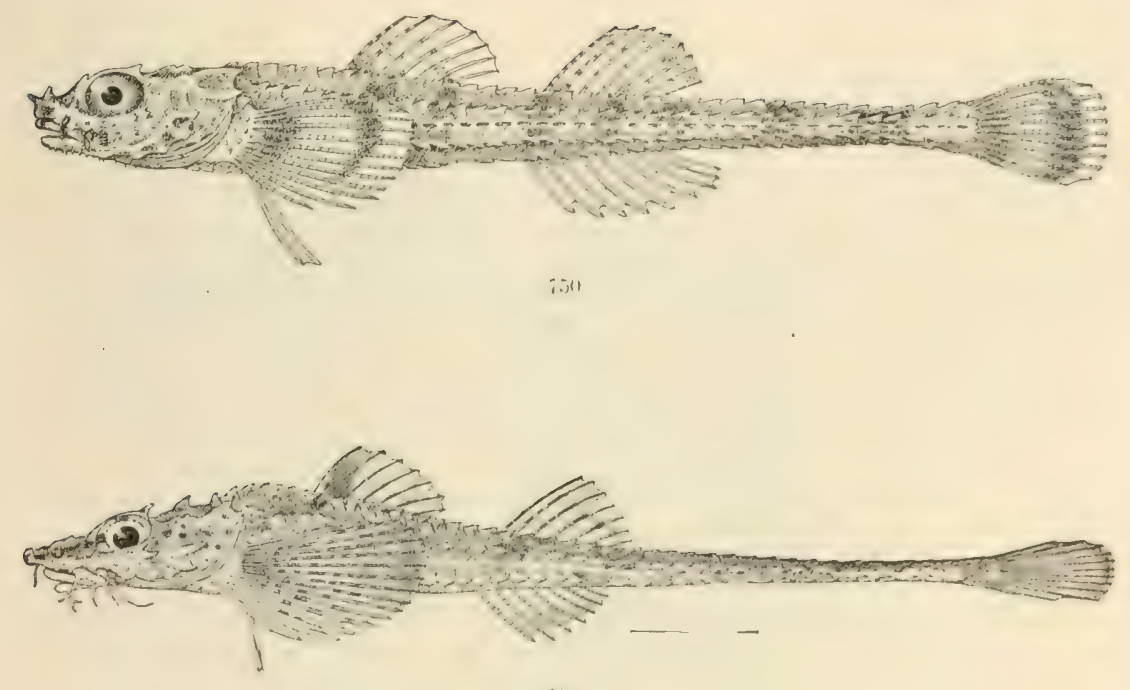

is 1
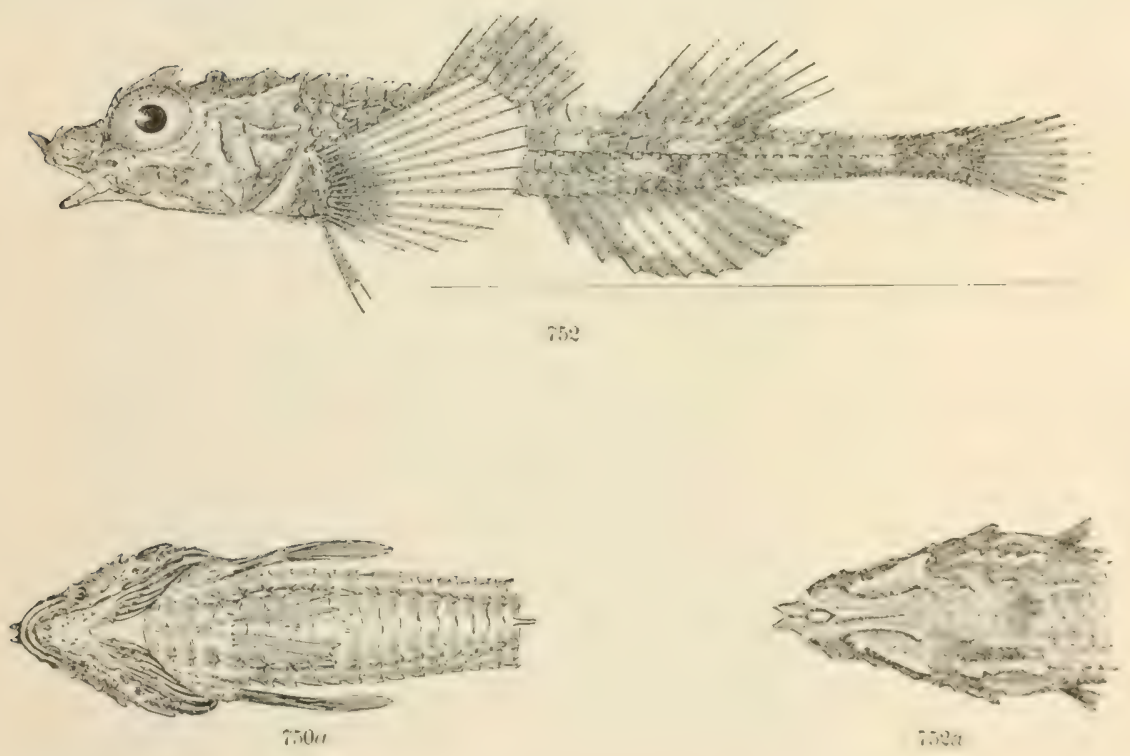

750, 750a. AVEIRUNCI'S STERTITS, (P. 2071.)

751. SalBITOR FREXatus. (P', 2073.)

752, 752 $\because$. IXSTES ANINOPHRYS. (P. 2076。) 




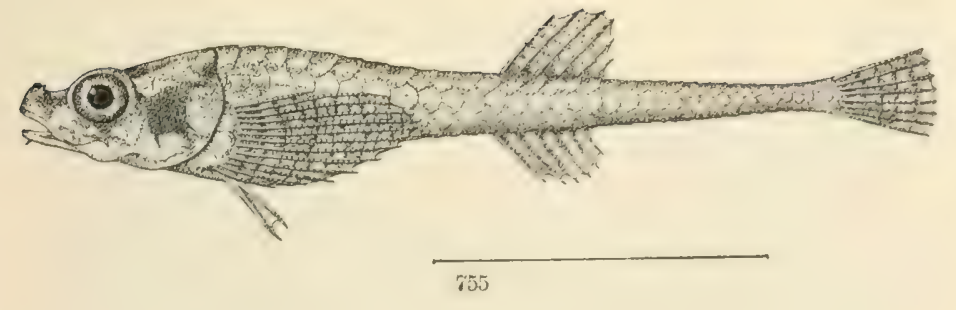

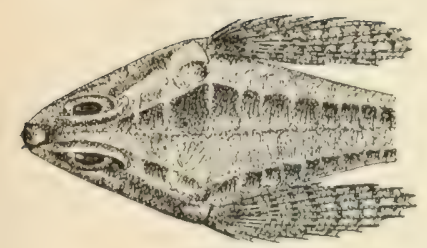

$755 a$

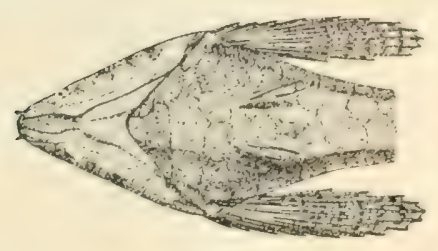

$\% 556$

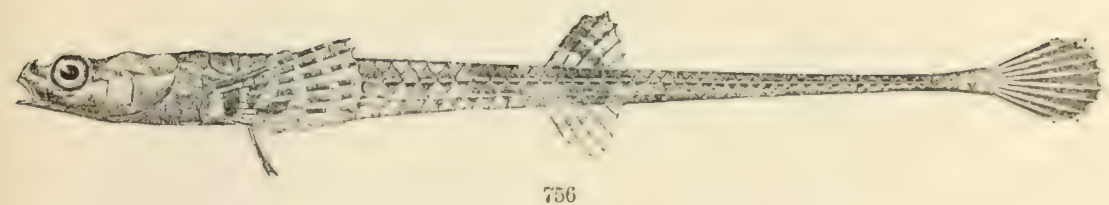

756

nitict

755, 755a, 755b. ASHIDOPHOROHDES GUNTIERT. (P. 2090.)

756, 756a. AspidolionoIDEs Moxopterygius. (P.2091.) 

U. S. NATIONAL MUSEUM

BULLETIN NO. 47, PL. CCCXIII
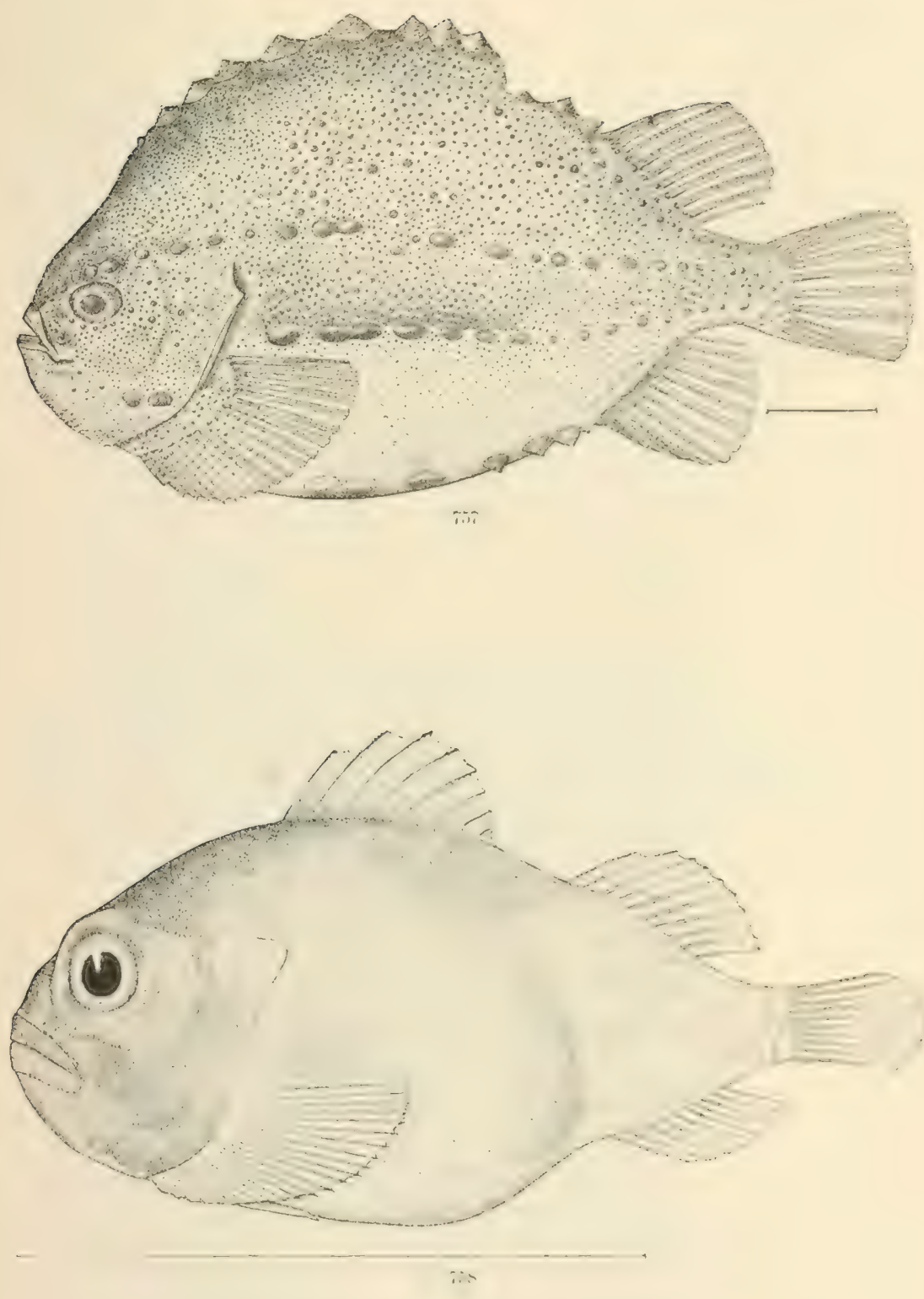

757. Crclopteres lumus. (P. 2096.)

758. LeTHOTREMU's MUTICUS. (P.2101.) 



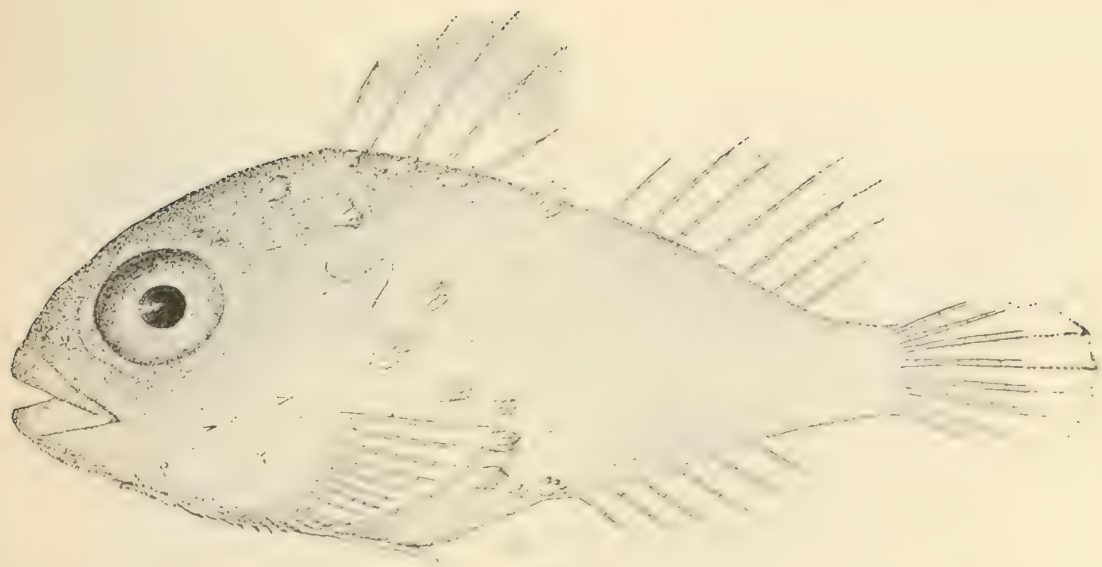

$\% 59$

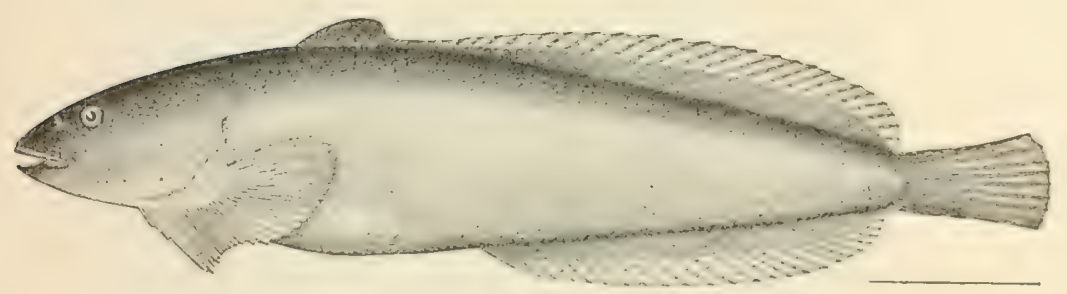

i(i)

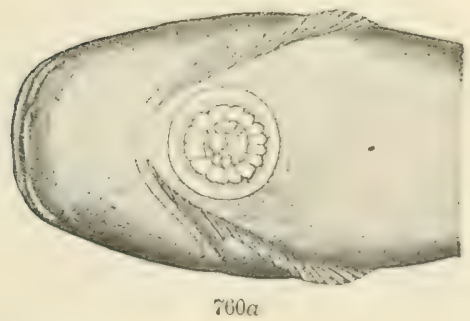

759. LeTHOTreat'S vixolExtUS. (1'.2101.)

760, 760a. NEOLIPAIIS CALLYODON. (1'.2110.) 



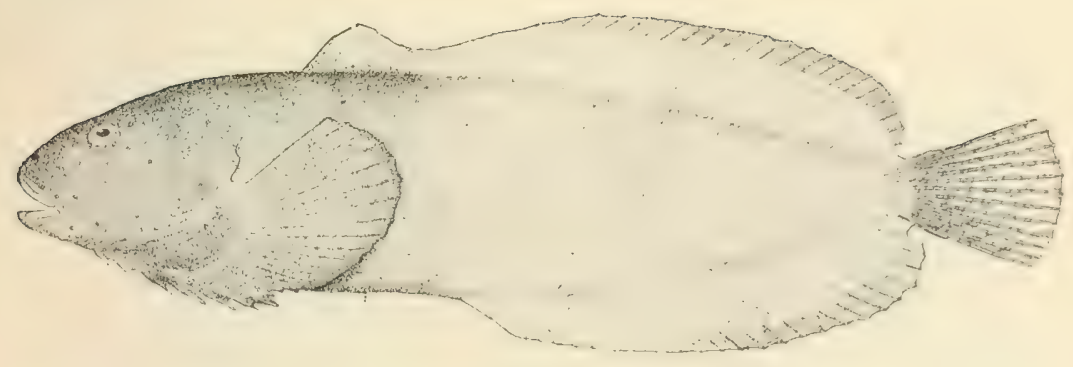

$\tau 61$

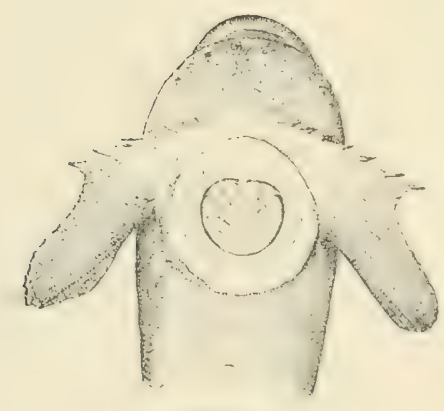

itila

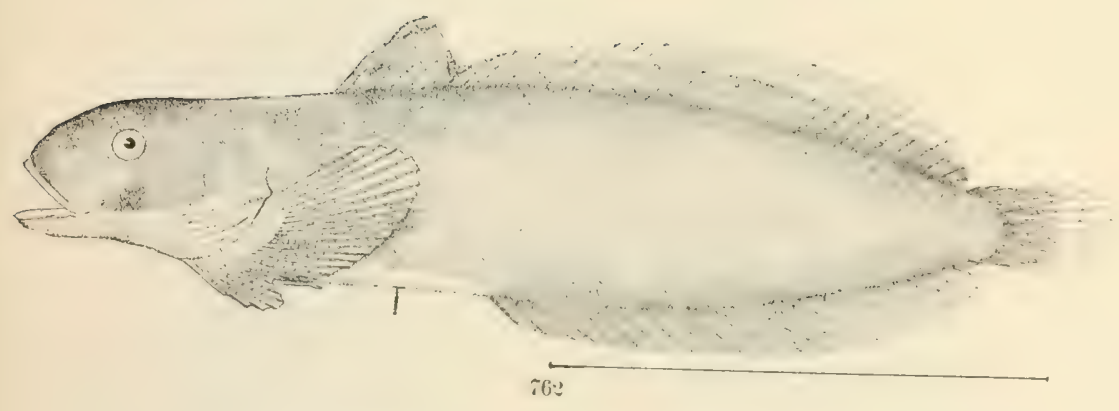

761, 76ia. NEOLHARIS MUCOSUS. (1'. 2111.)

762. NEOLIPARIS FLORE. (P. 2111.) 


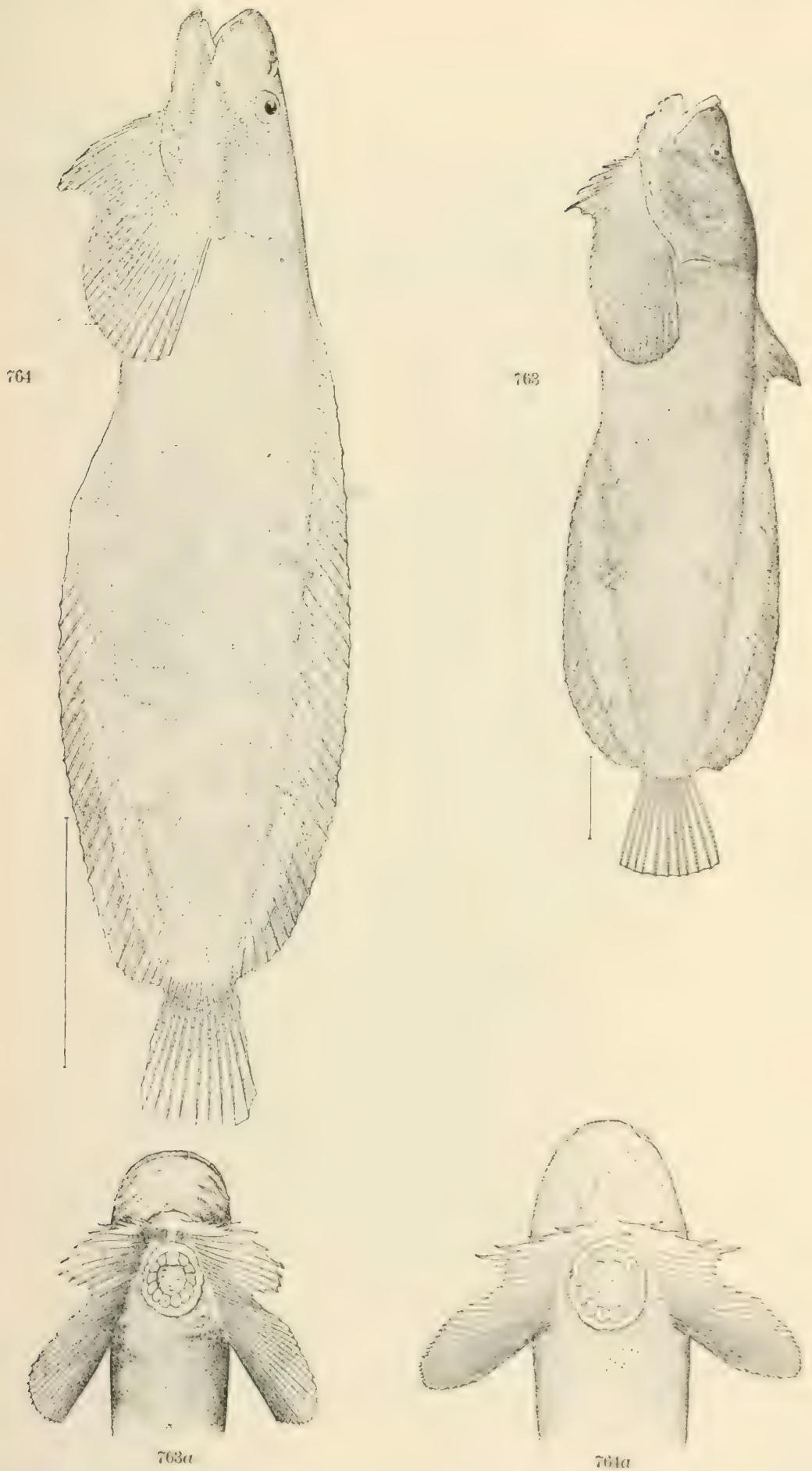

763, 763a. NEOLHAIIS GREENI. (P. 2112.)

764, 764a. LIPARIS CYCLOPUS. (P. 2118.) 



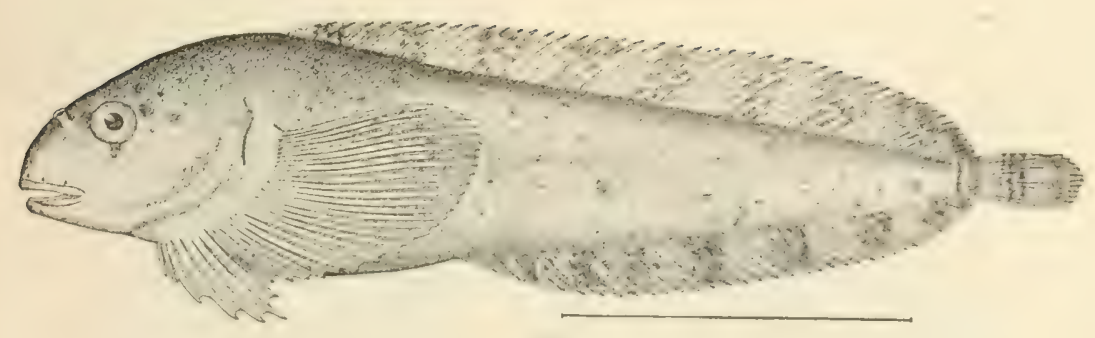

iti:
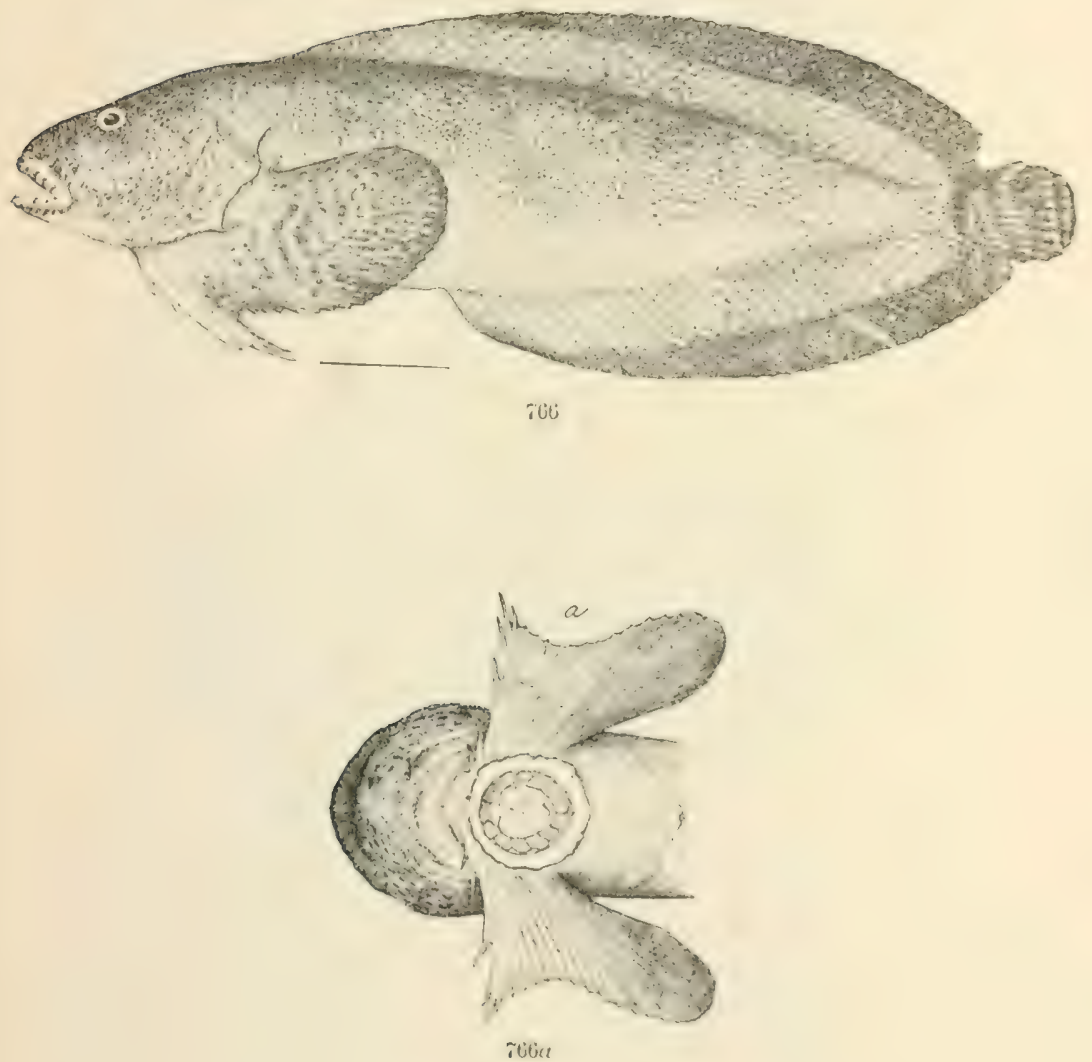

765. Liparis afaAsizil. (1'.2121.)

766, 766a. LIPAIRIS BENNTI. (Y. 2124.) 



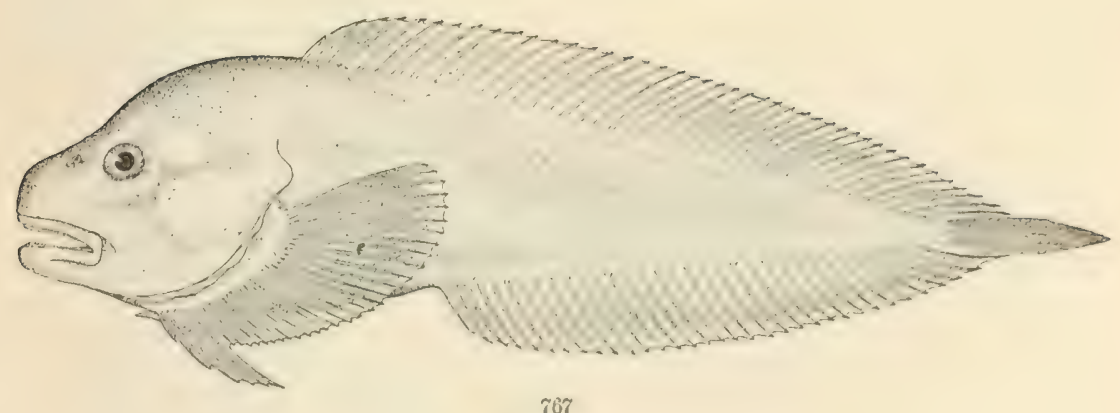

767

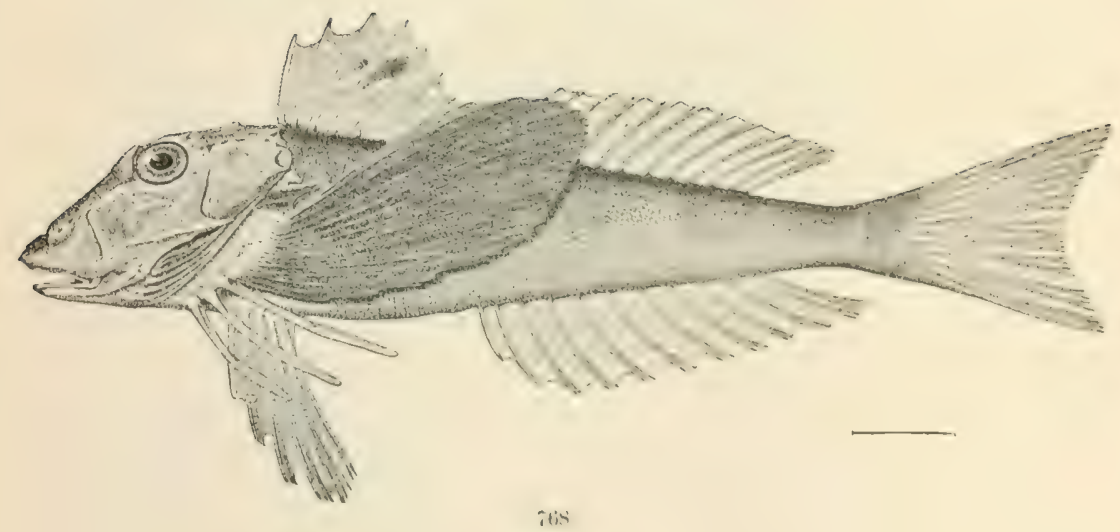

767. BATHYPIASMA OVIGERUM. (P. 2128.)

768. Prionotus carolinus. (P. 2156.) 


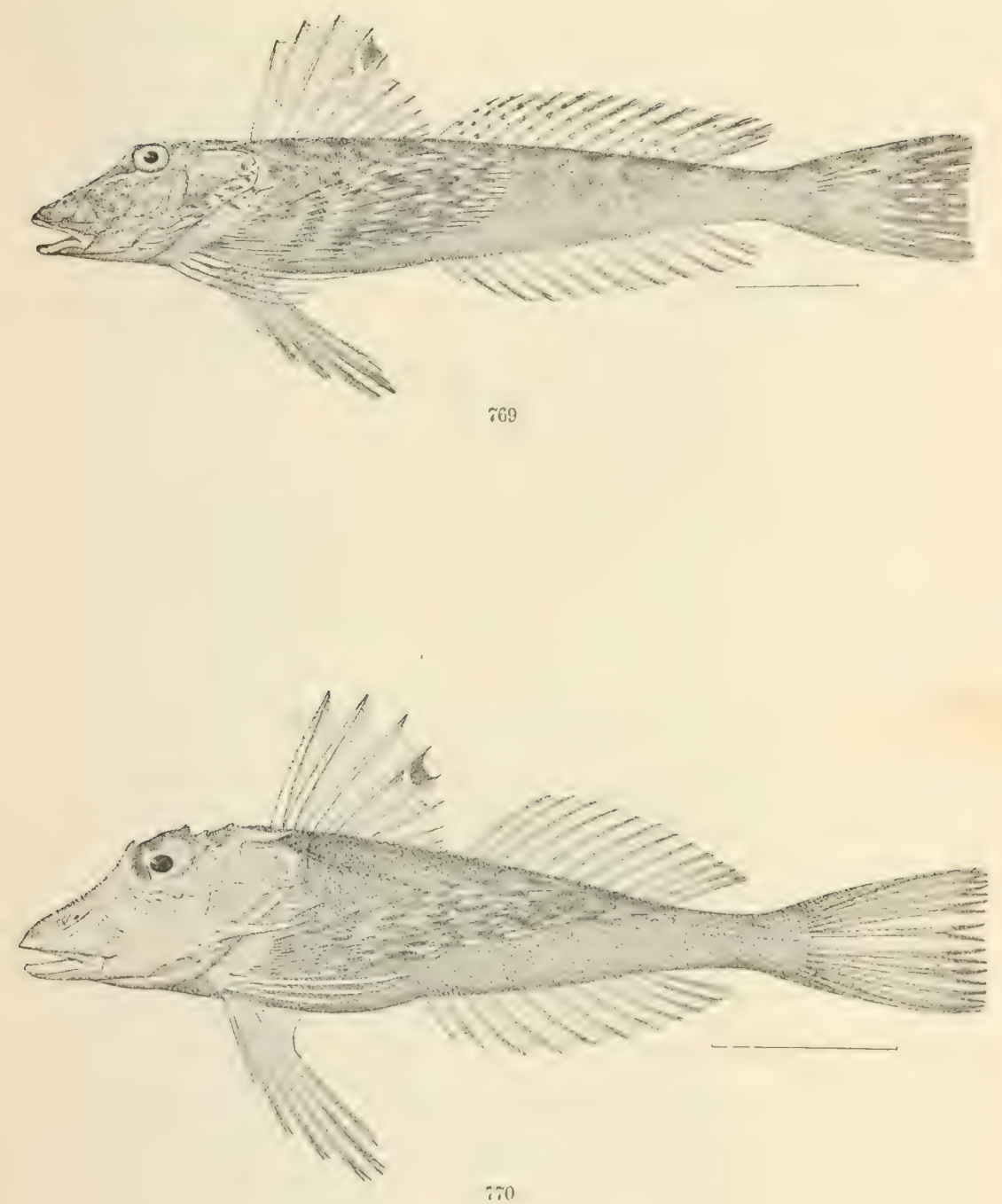

769. Prionotus scitulus. (P. 2157.)

770. Prioxotus alates. (P. 2159.) 



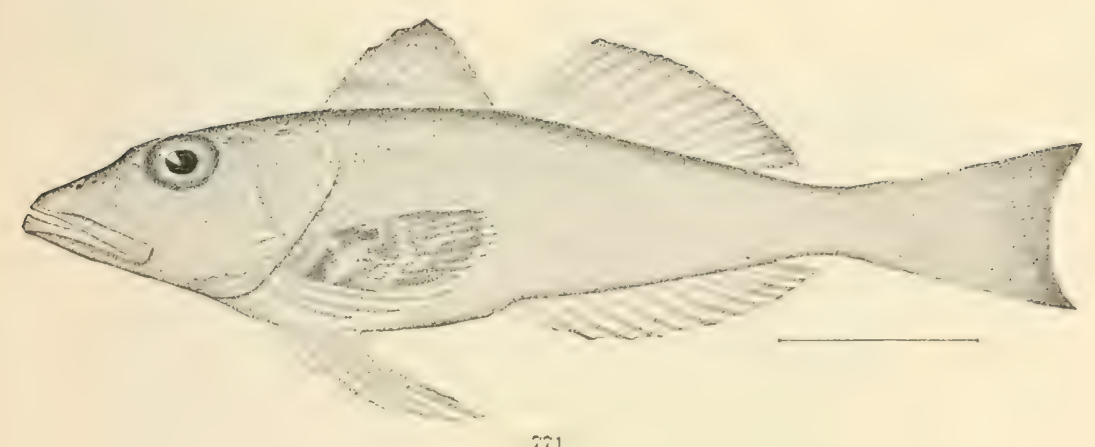

ii

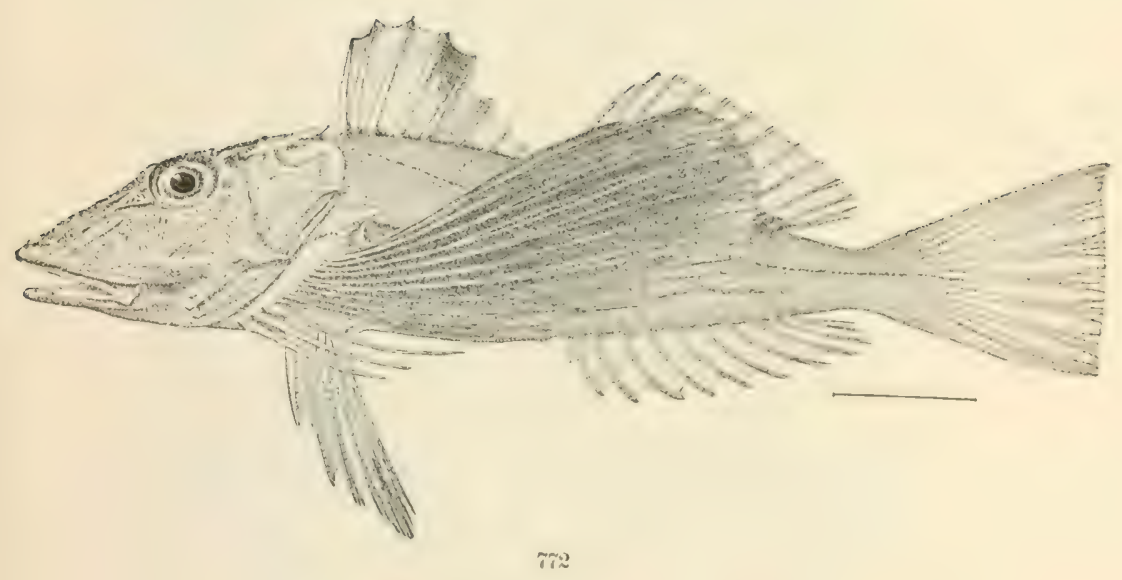

771. Phionotus Stealisi. (

772. Prionotus evolaxs. (P. 2168.) 



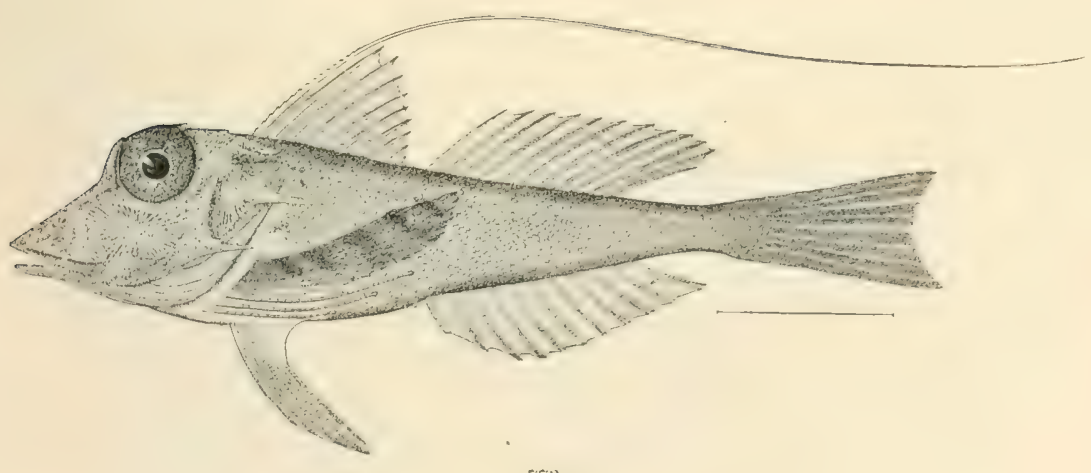

;:3

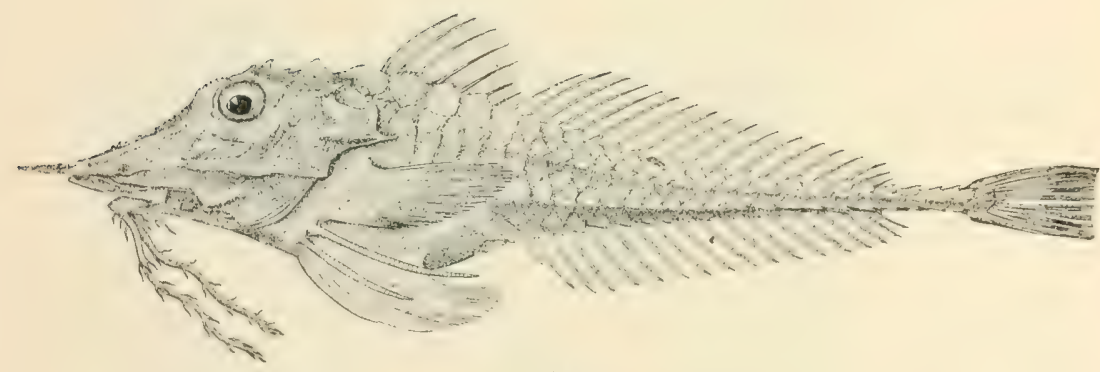

it

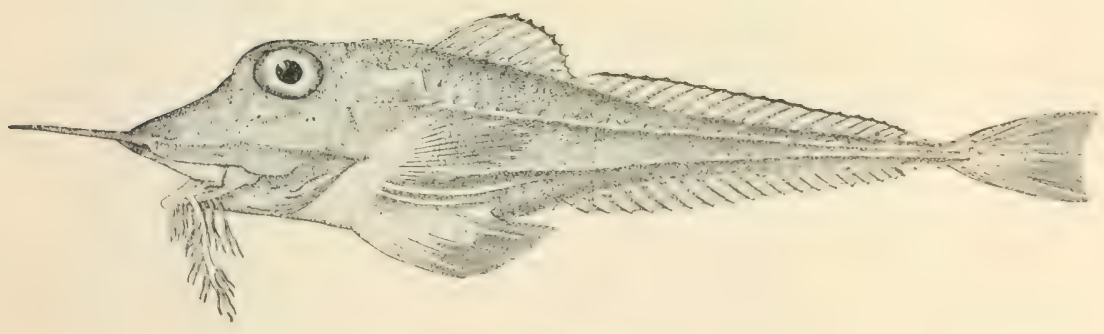

iis

773. BELLATOR EGRETTA. (P. 217!.)

774. PERIsteDION MINITUM. (P. 2178.)

775. PEBISTLDION 1.ONGISPATHUM. (P.2178.) 


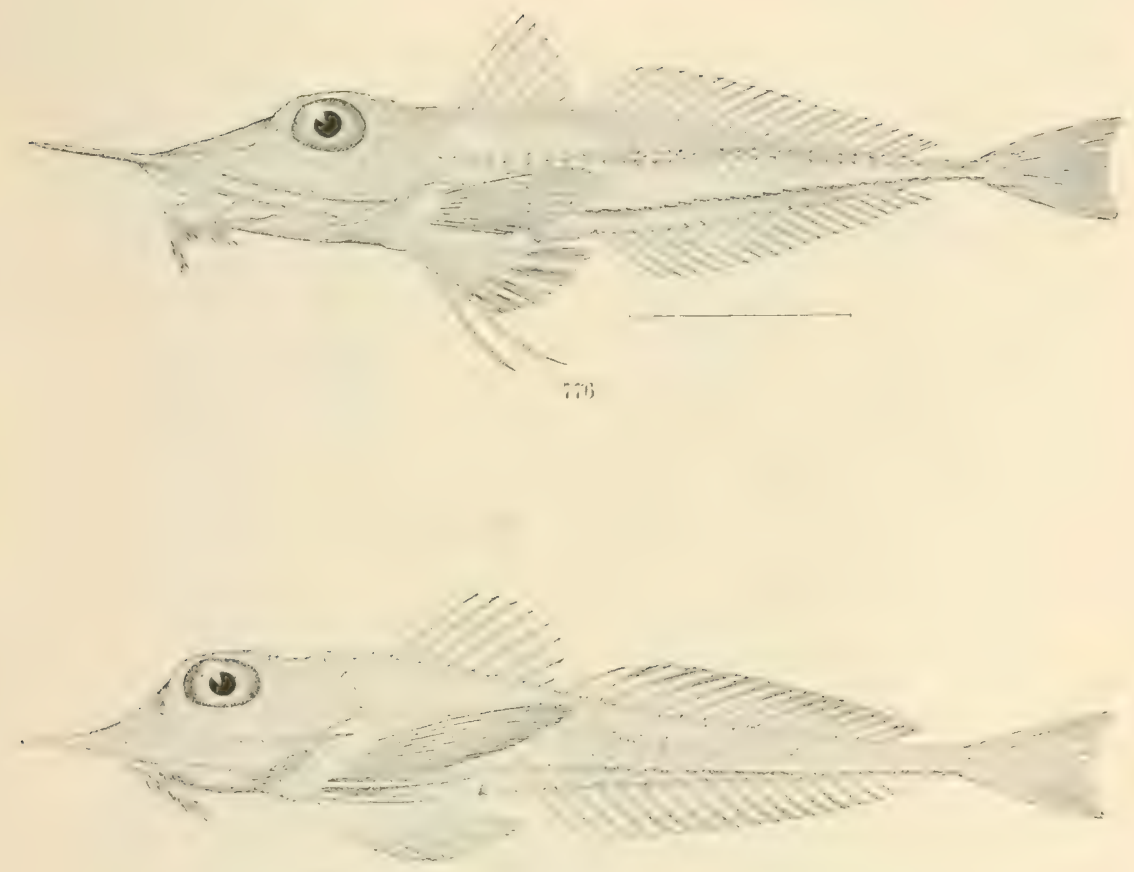

i氵

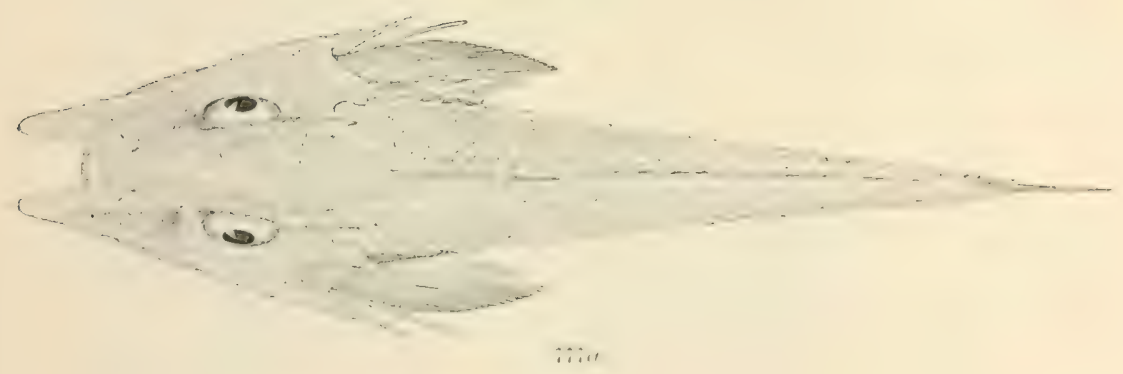

776. Perrstudion Gracile. (P. 2179.)

777, 777a. Peristedon l'Latycephal.cir. (P'.2180.) 



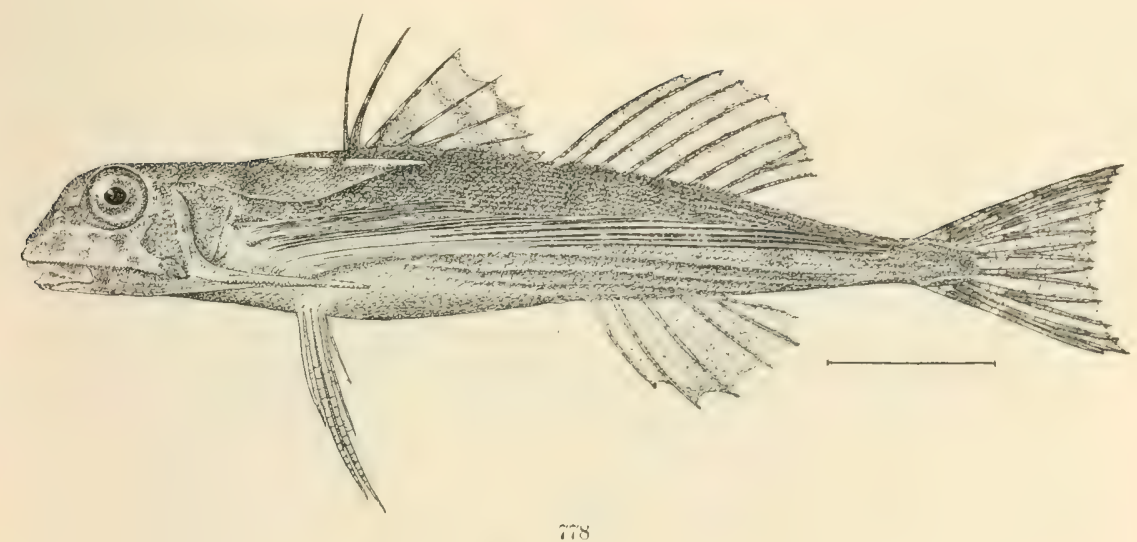

iro

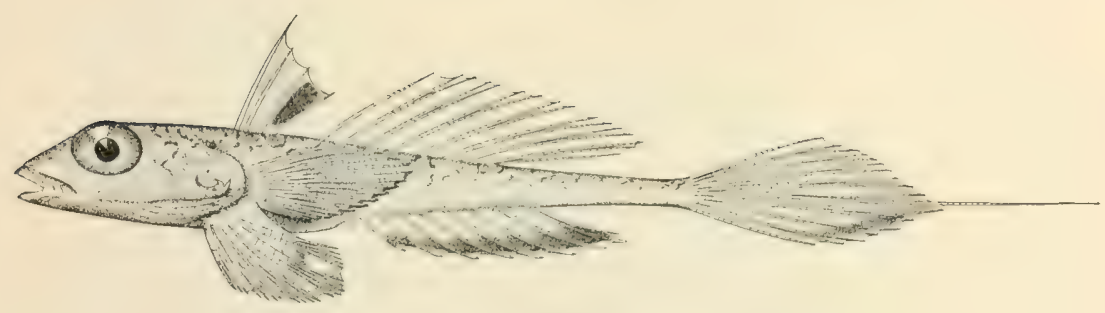

$\because 9$

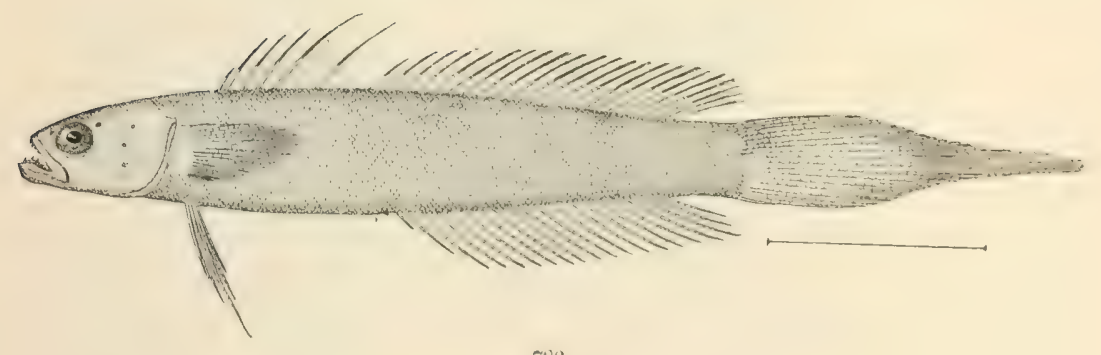

$\pi 81$

778. Cepilalacanthus VolitaNs. (P. 2183.)

779. Callongues agassizit. (P. 2186.)

780. IOglossus CaldiliuUs. (P. 2193.) 



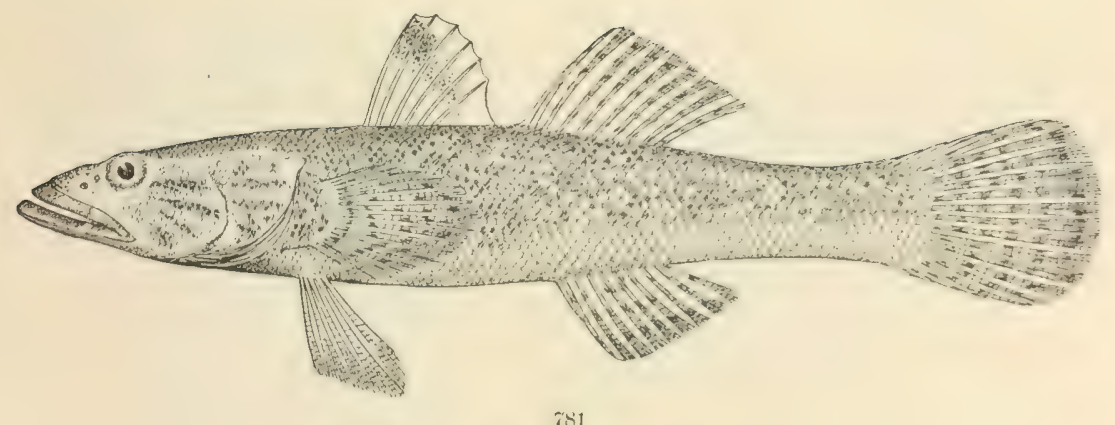

$7 \times 1$

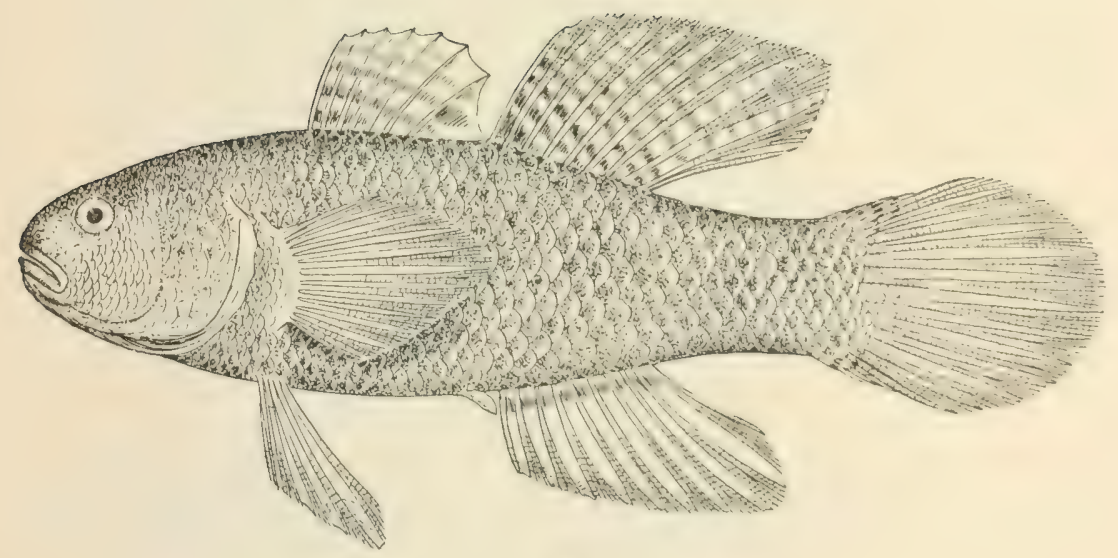

$78 \%$

781. Phinypens Dormitor. (P, 2194.)

78.2. Dommtator maculates. (P. 2196.) 



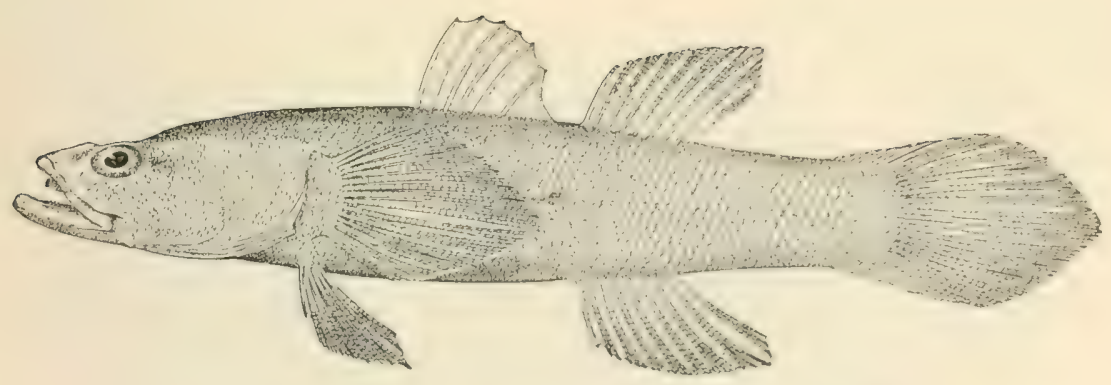

(x)
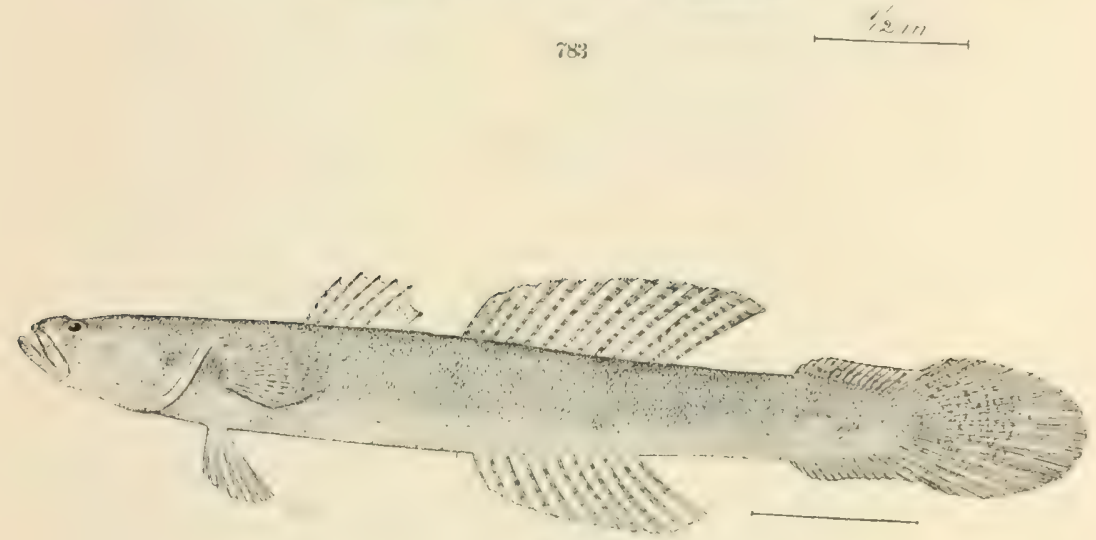

781

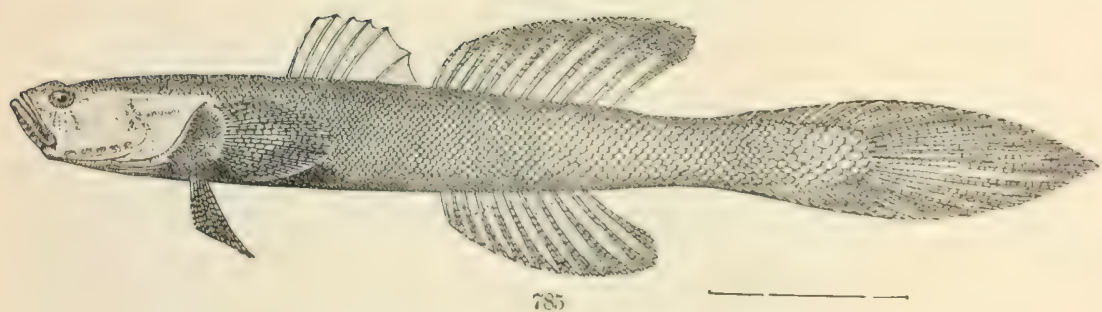

783. ELEOTIS PISONIS, (P. 2200.)

785. EROTELIS AMaMAER (P. 2203.) 



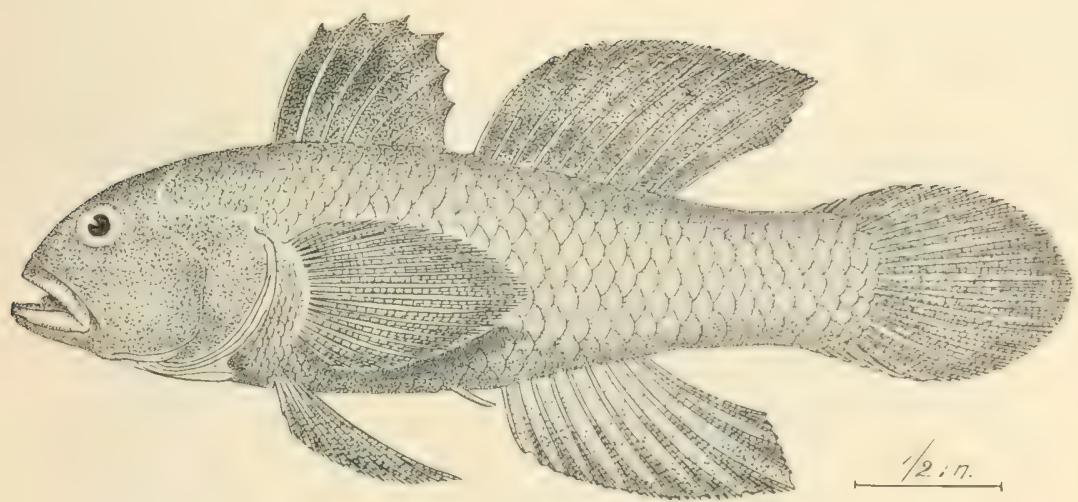

786
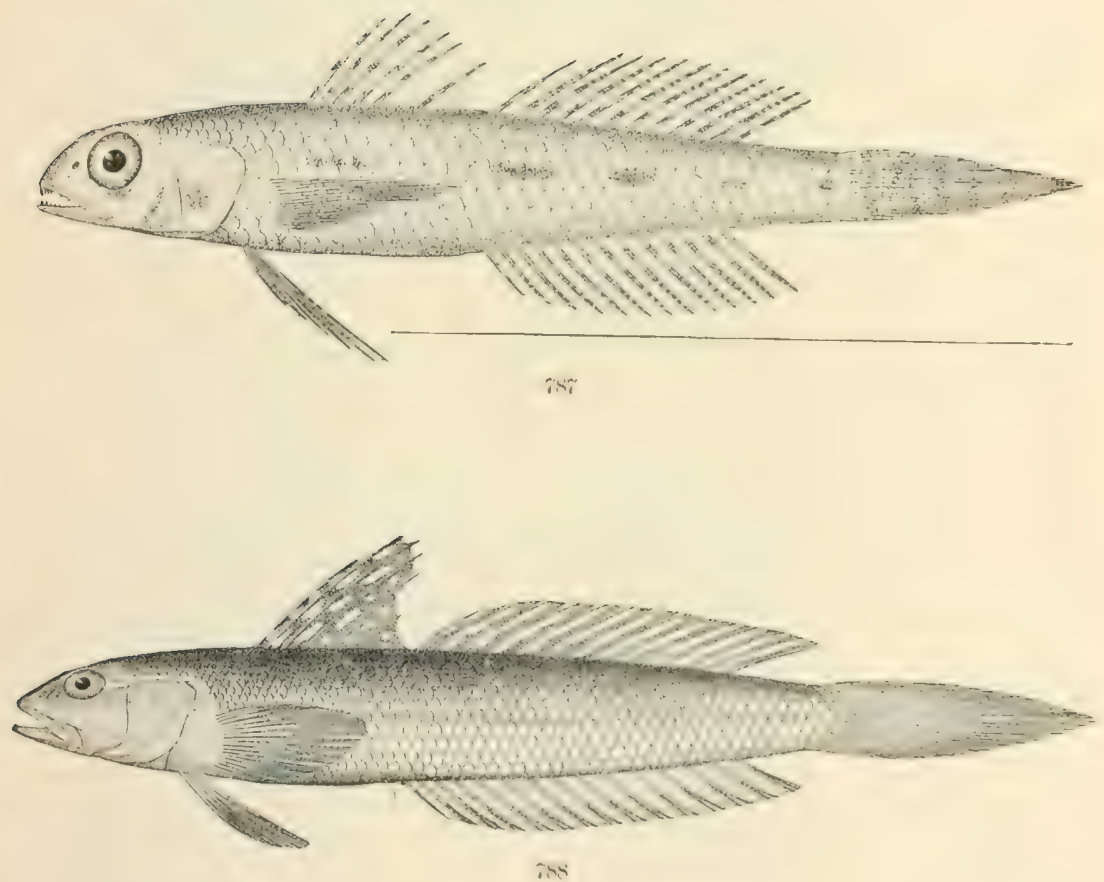

786. LOPHOGOHIUS CYPRINOIDES. (P. 2209.)

787. Gomus stignaticus, (P. 2224.)

788. Gomius hastatus. (P. 2229.) 


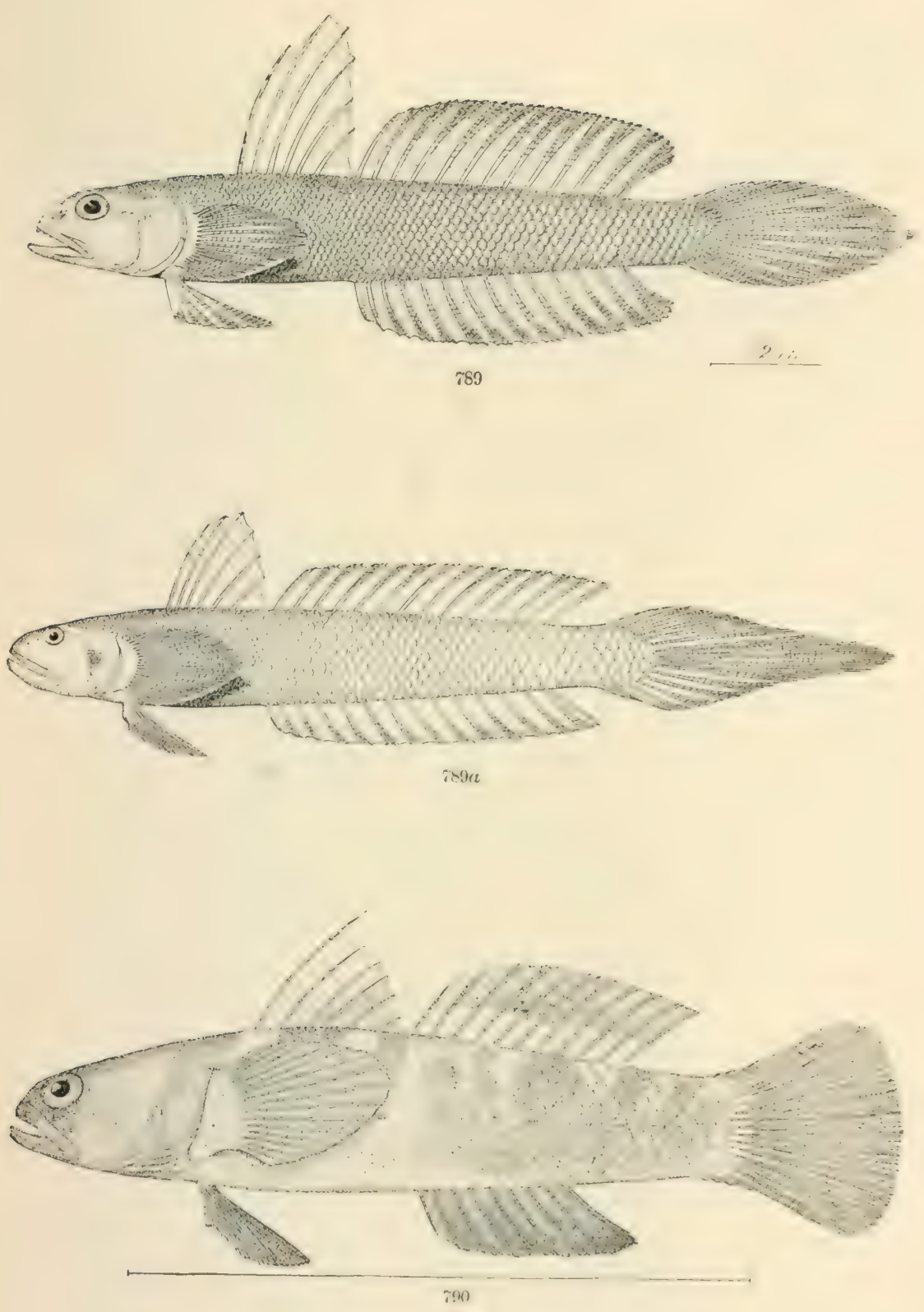

789. 789a. GobIts Ockaxicus. (1'. 20230.)

790 GakMaNita Baradoxa. (1'.2232.) 



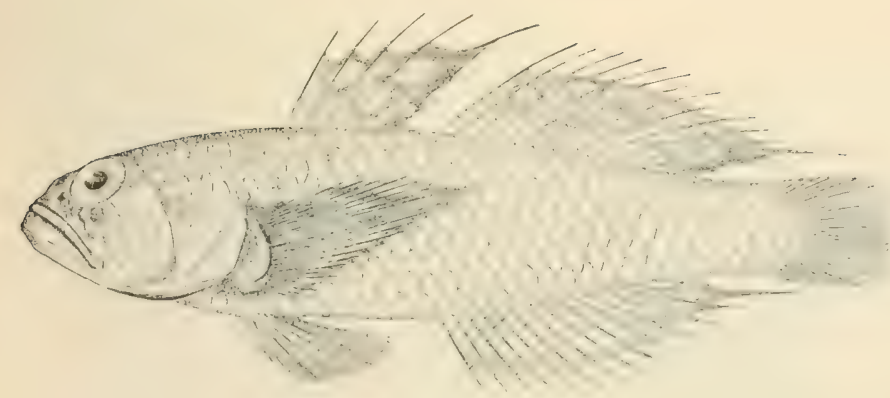

i!n

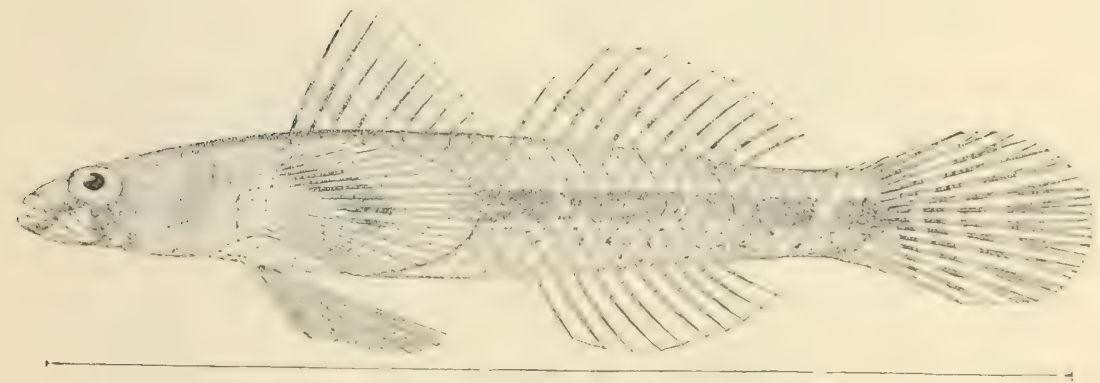

792

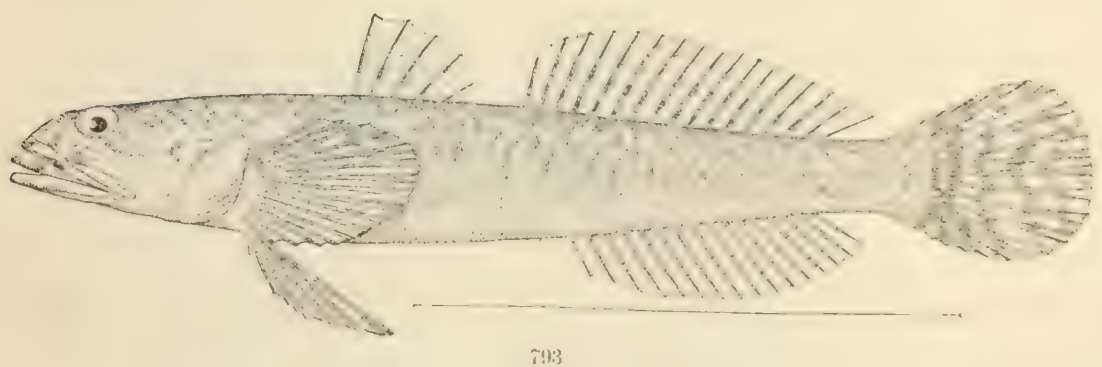

791. BOLLMANNA CIILAMINES, (P. 2238.)

792. A BOMA ETIKOSTOMA. (P. 2.240.

793. ClevelaAinA IOS. (P.2.254.) 


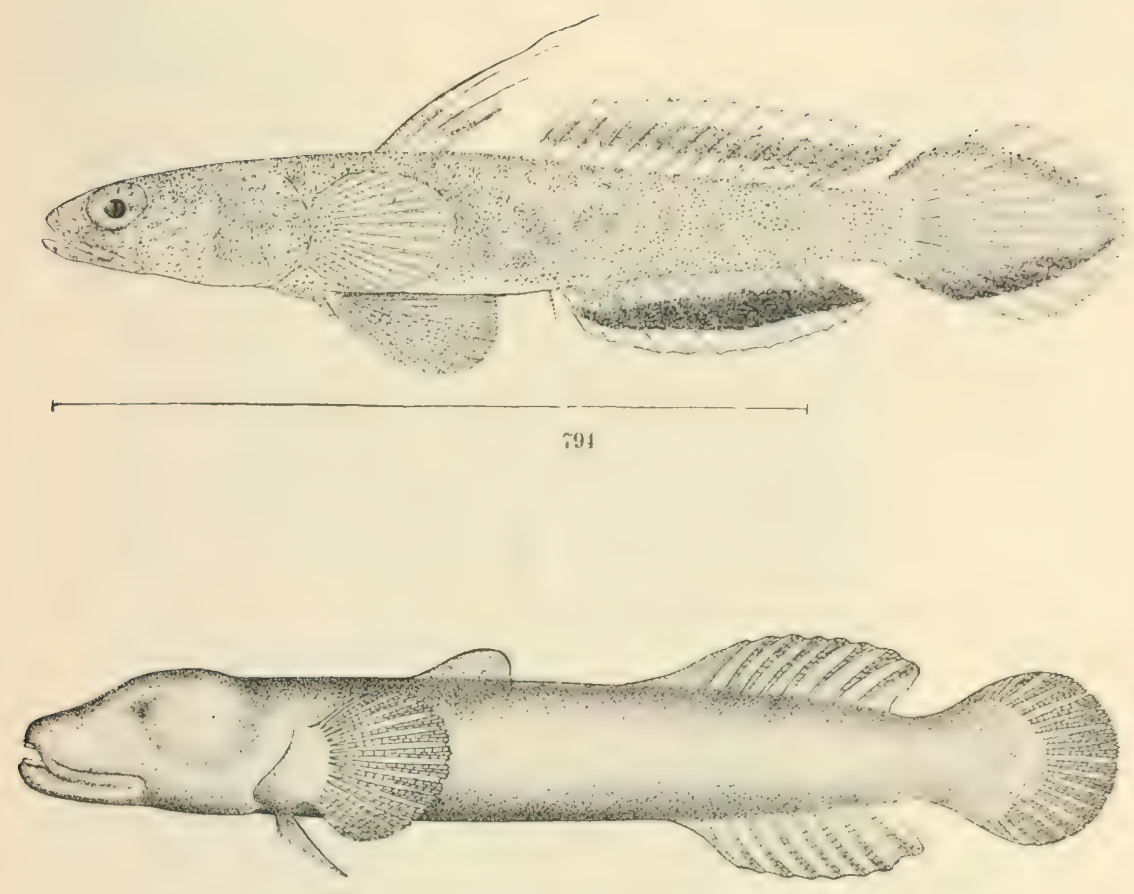

795

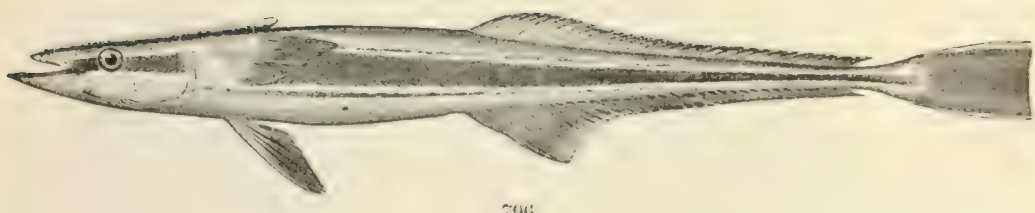

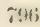

794. Evermania zosterura. (P. 2256.)

795. TYRHLOGOBIUS CALIFORNIENSIS. (1, 2262.)

796. Echeneis naucrates. (P. 2269.) 


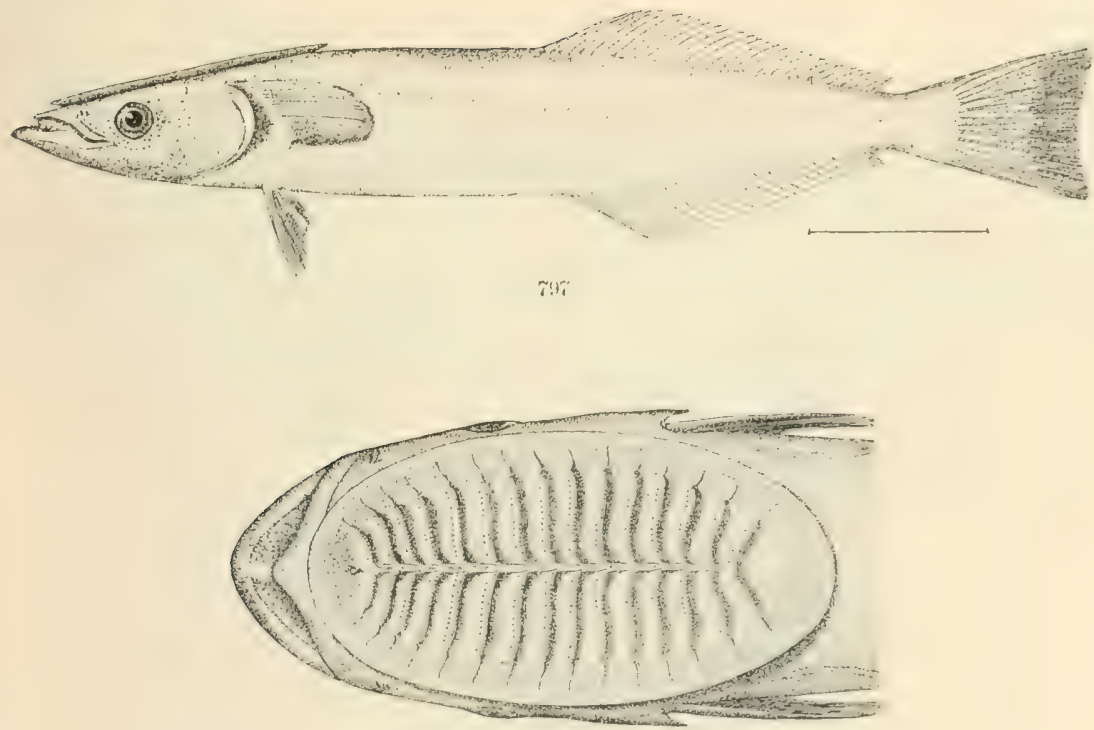

*⿻i"

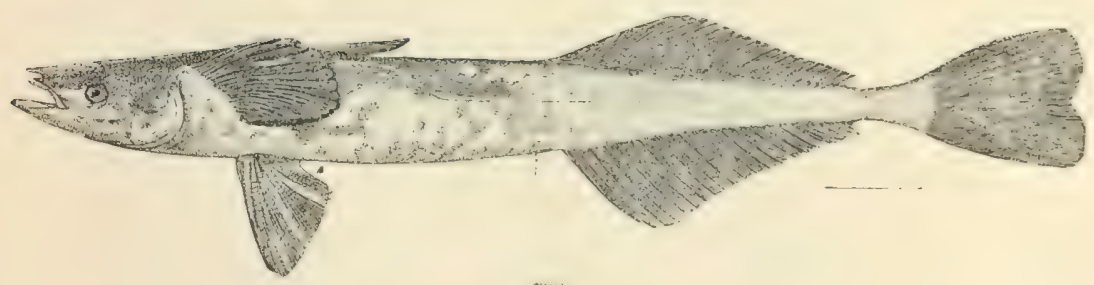

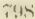

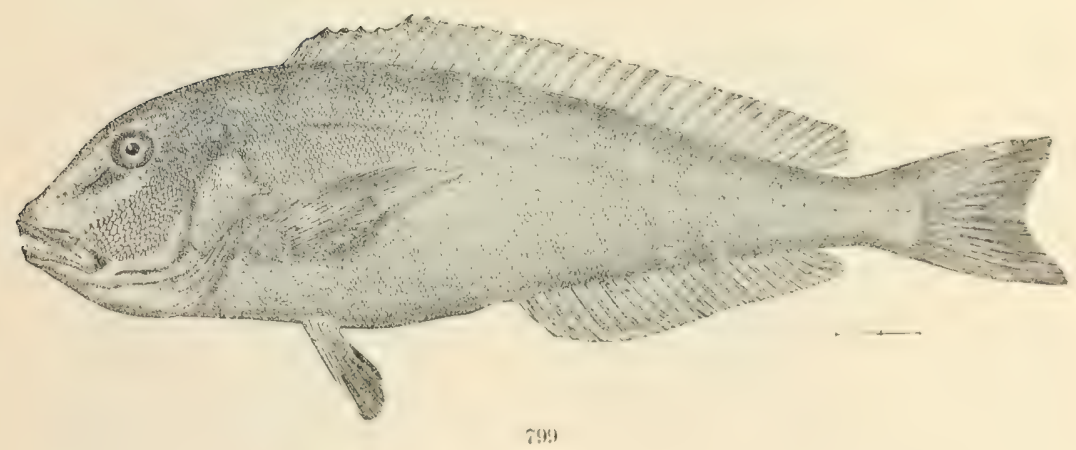

797, 797 $\alpha$. REMORA IHACHYTERA. (P, 2272.

798. RHOMHOCHIRCS OSTEOCHIR. (P. 2273.)

799. CACLOLATILUS MICBOP'S. (1',2277.) 



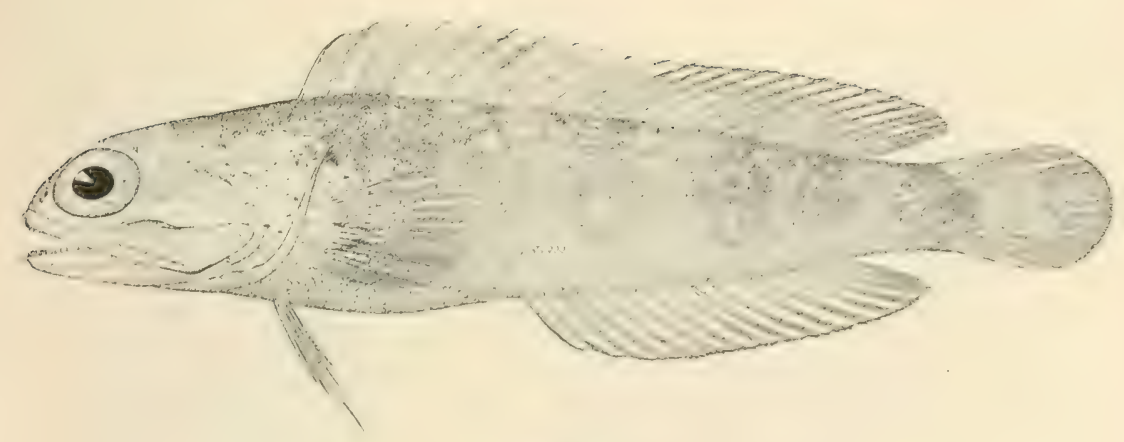

(ini)

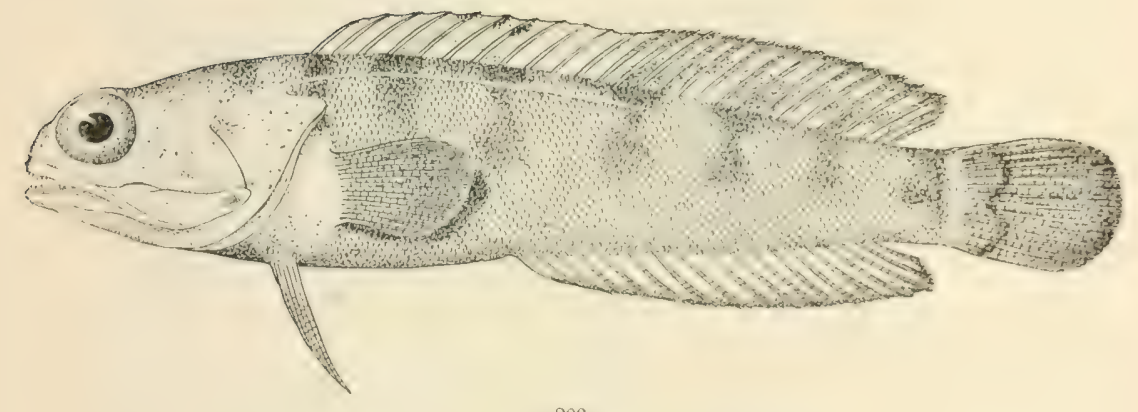

$800 x$

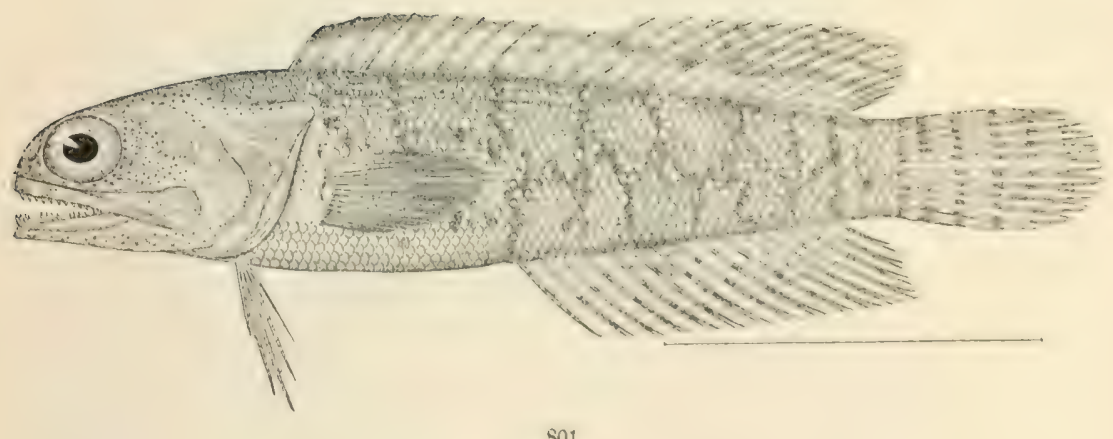

S01

800, 800a. Opisthognathus macrogitutum. (P.2281.)

801. GNATHYPops Maxillosa. (P.2281.) 


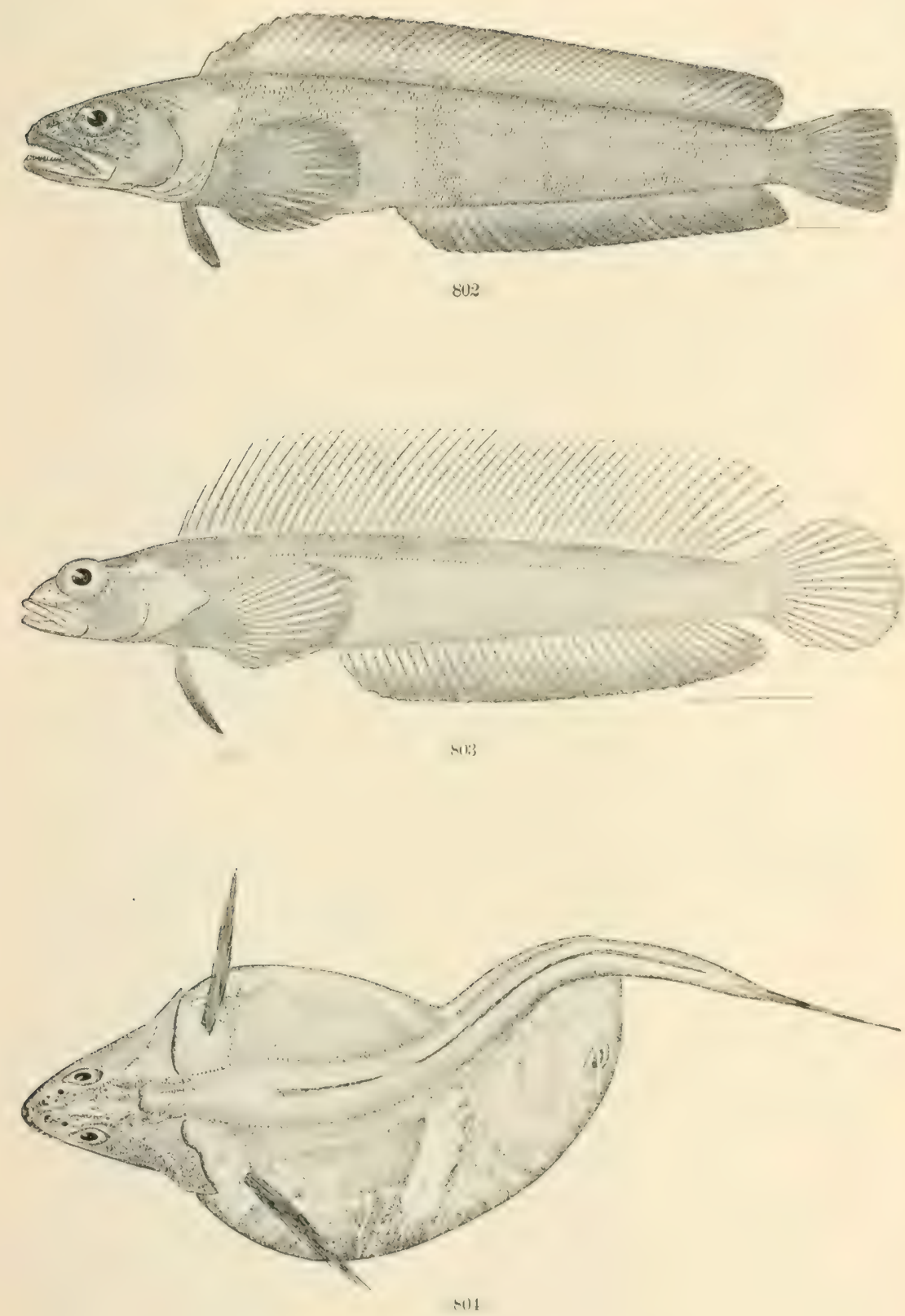

802. Bathyaster signatus. (P. 2288.)

803. Rosiuitus JORDAxI. (P. 2289.)

804. CIILSMODON NIGER. (1', 2291.) 



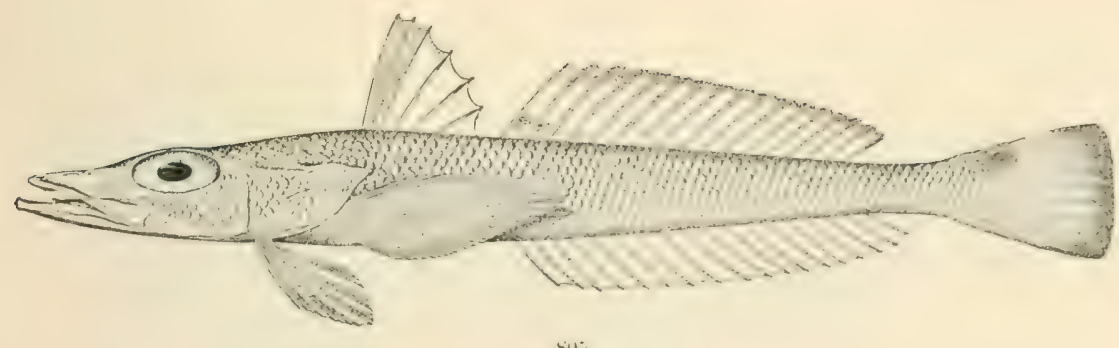

sus

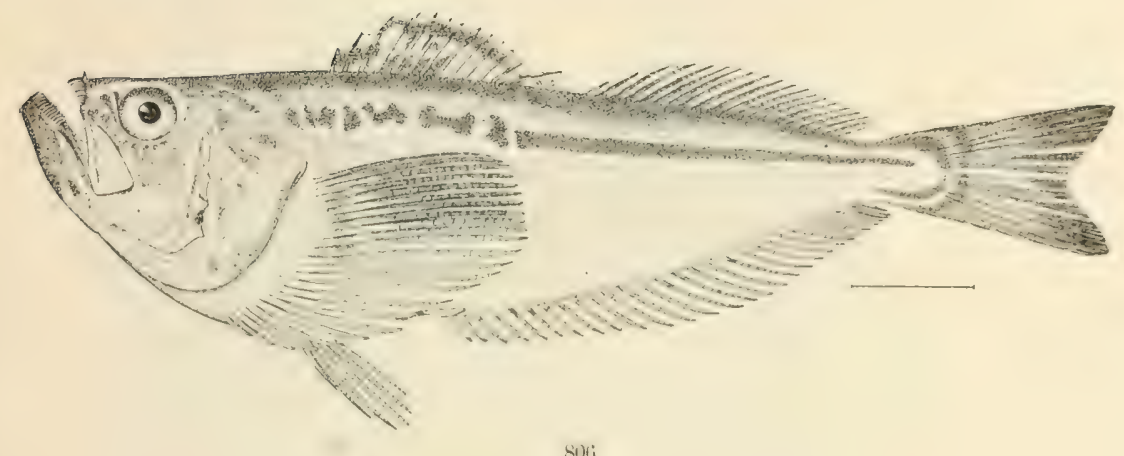

$80) ;$

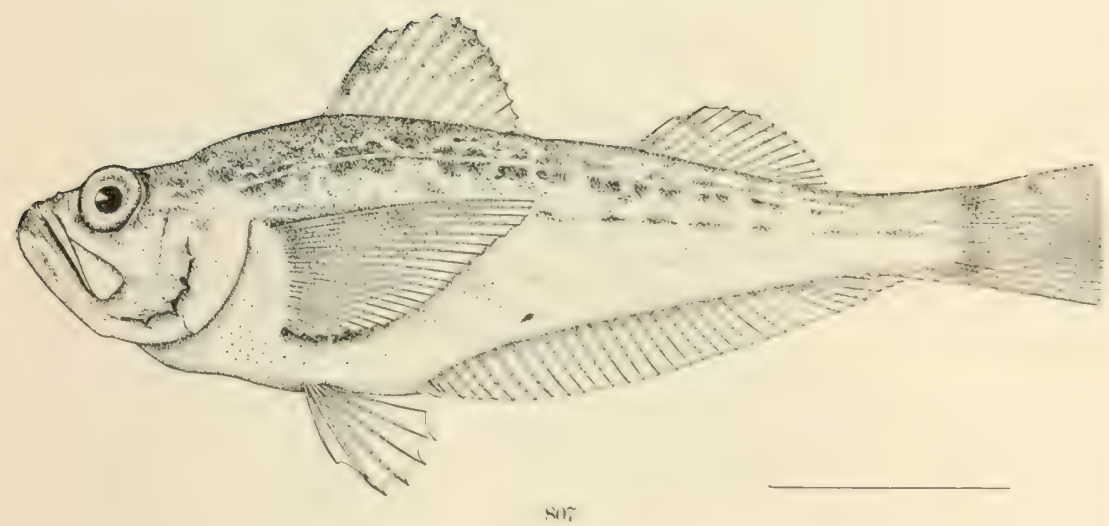

805. Hypsicometes GOHIOIDES. (P. 2294.)

806. TRICHODON TRICHODON. (P. 2295.)

807. Arctoscopus Japonicus. (I'.2297.) 


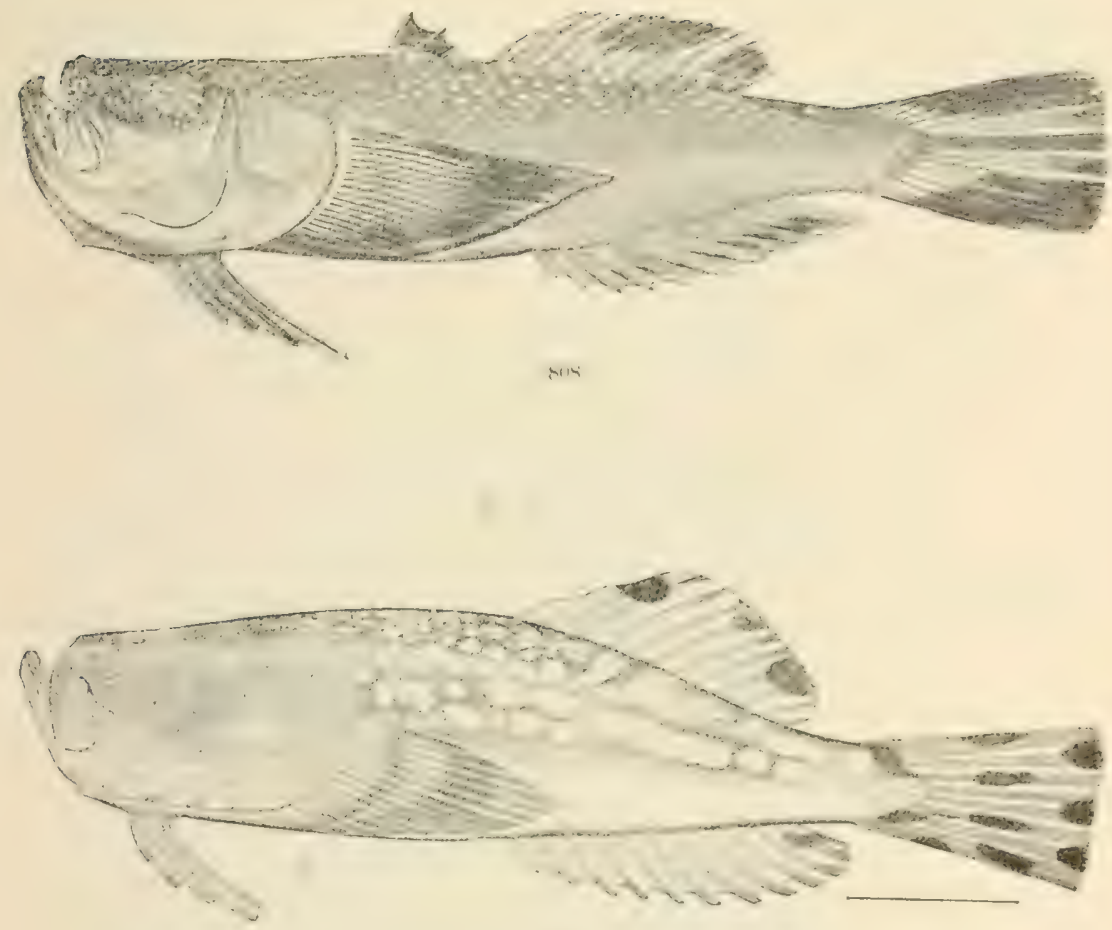

si!)

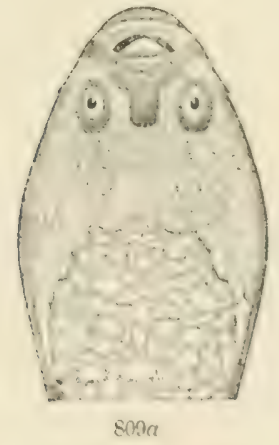

808. Astroscortus y-cir.scus. (1'2307.)

809, 809a. КАTHETOSTOMA ALHigUTTA. (P. 2312.) 



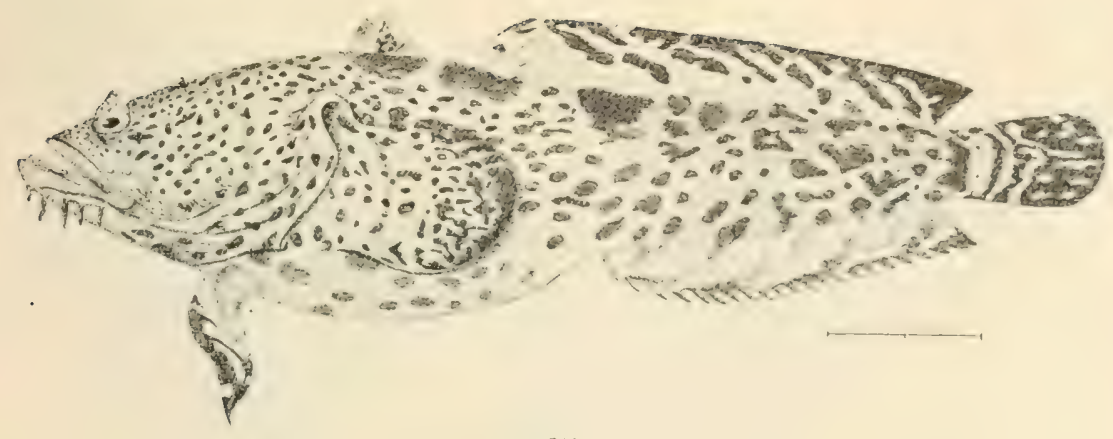

810

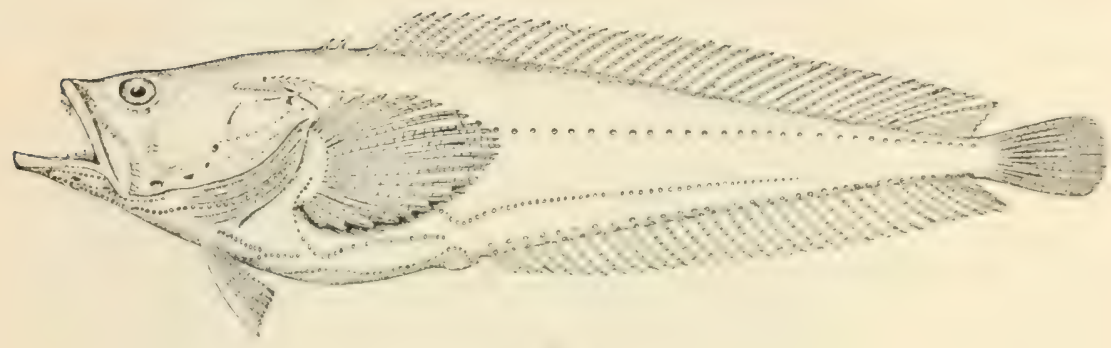

$-11$
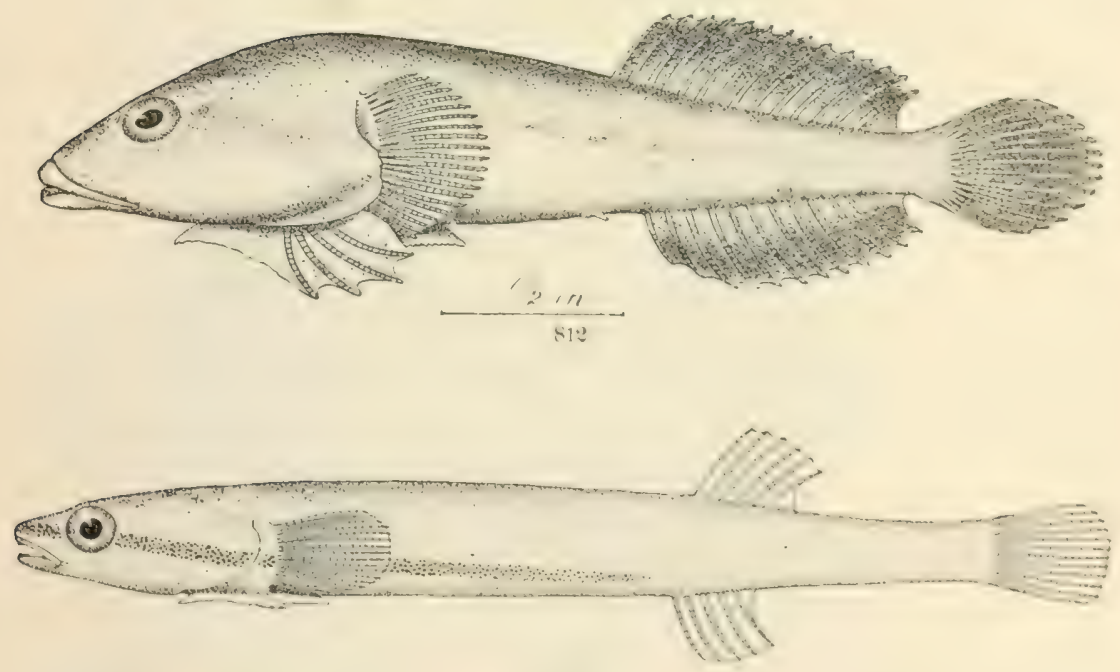

810. OPSANUS MaRDES. (P. 2316.)

811. P'ORICHTIY' p'OROSISAMI's. (P. 2319.)

812. CaUlarchus M.kaxnRICUS. (P. 2325.)

813. RIMICOLA MUSCARUM. (T. 23is.) 



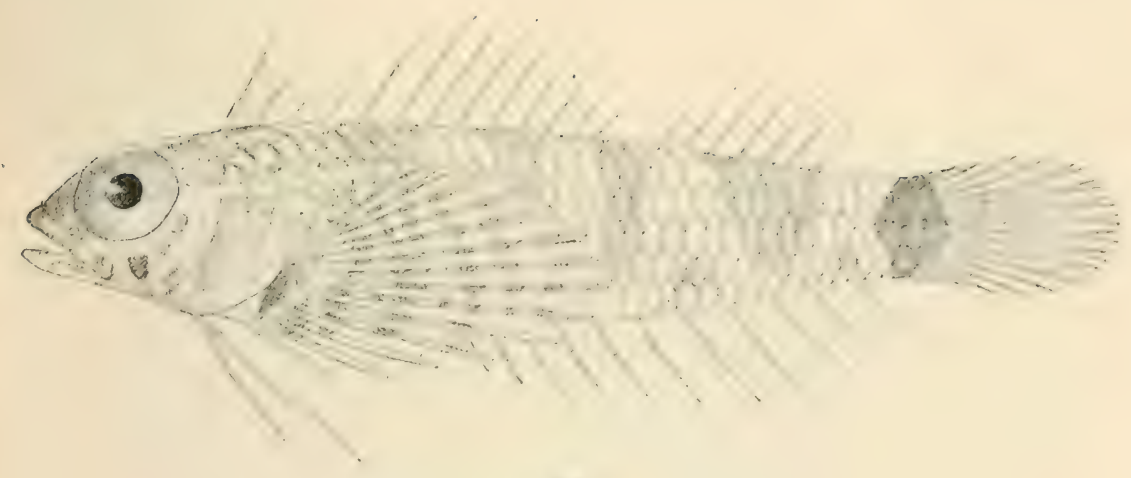

411
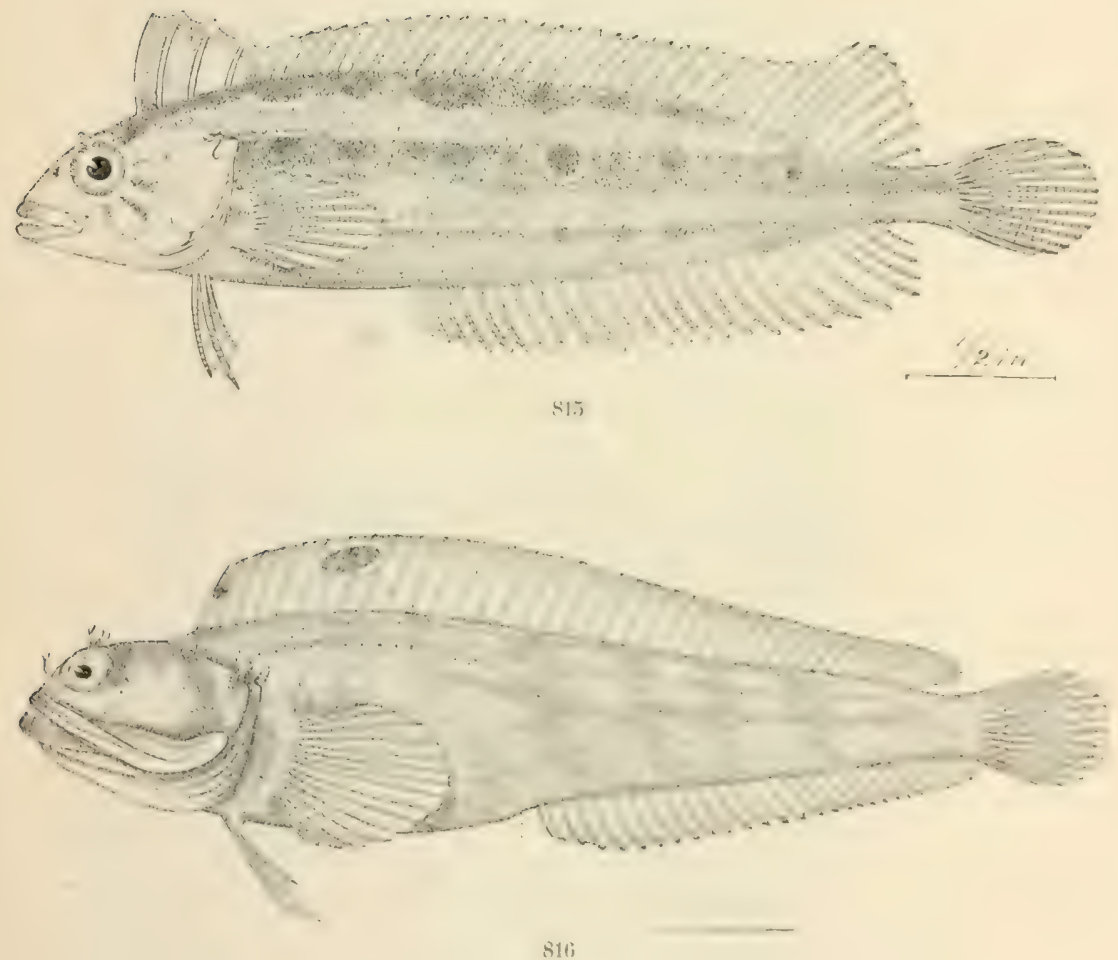

814. ENNIANECTLS CAMMINALS (1'. 2350.)

815. GIBBUNSIA ELEGANS EVIDES. (1'.235\%)

811. Neoclinus satiricus. (P.235\%) 


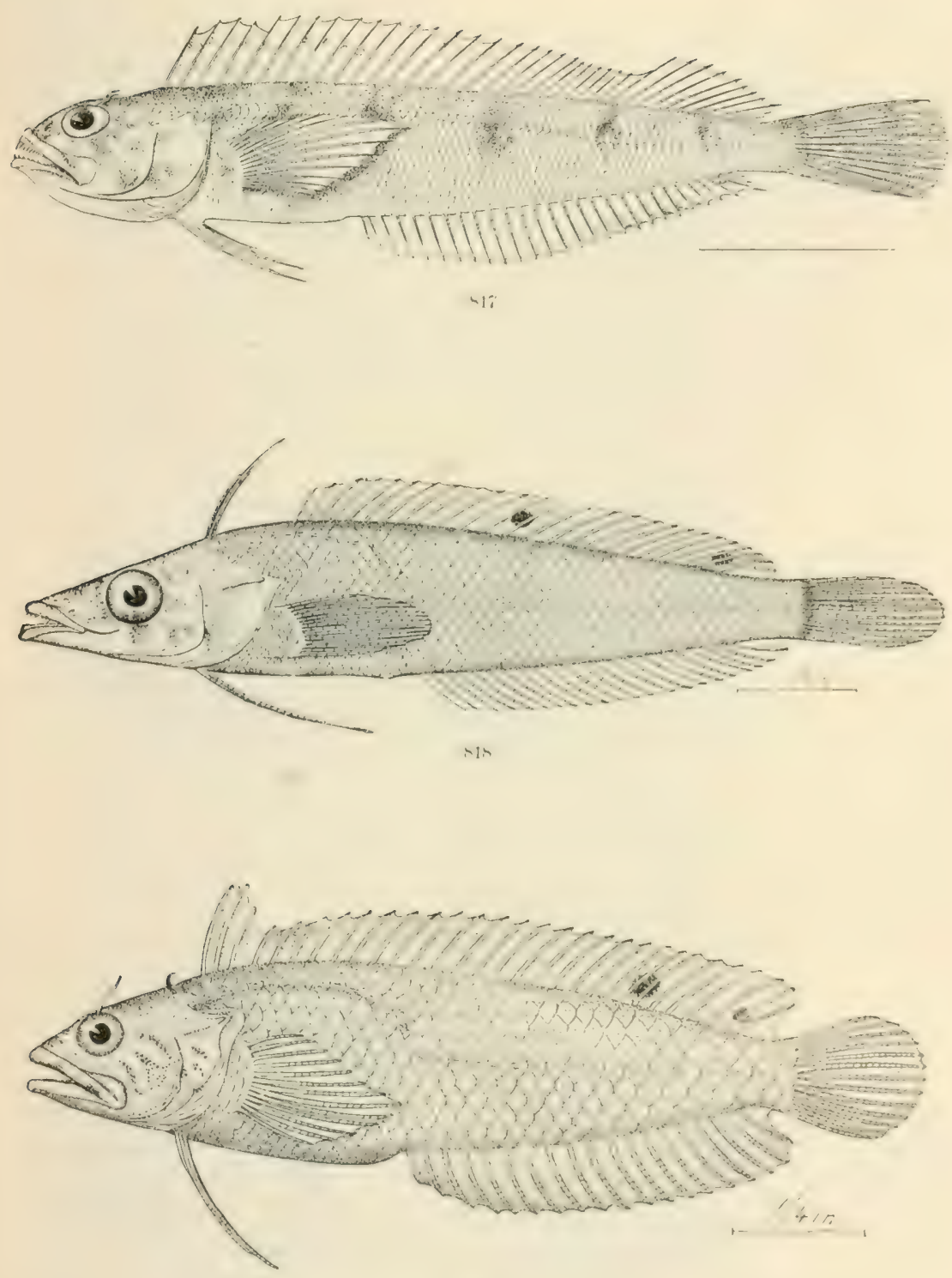

819

817. Crytototiema coliallixum. (I', 2366.)

818. EXERTS ASPER. (P. 2367.)

819. AUCHEXOPTERCS Nox. (P. 2373.) 



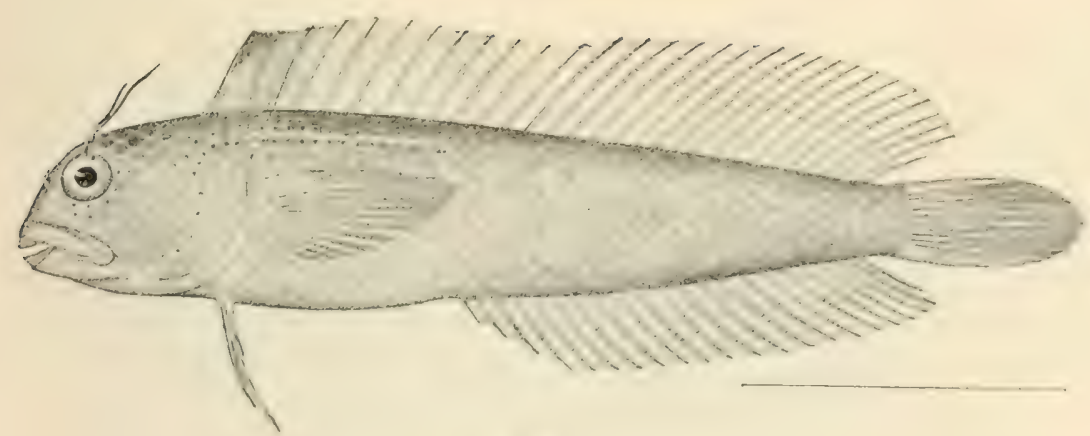

$x-31$

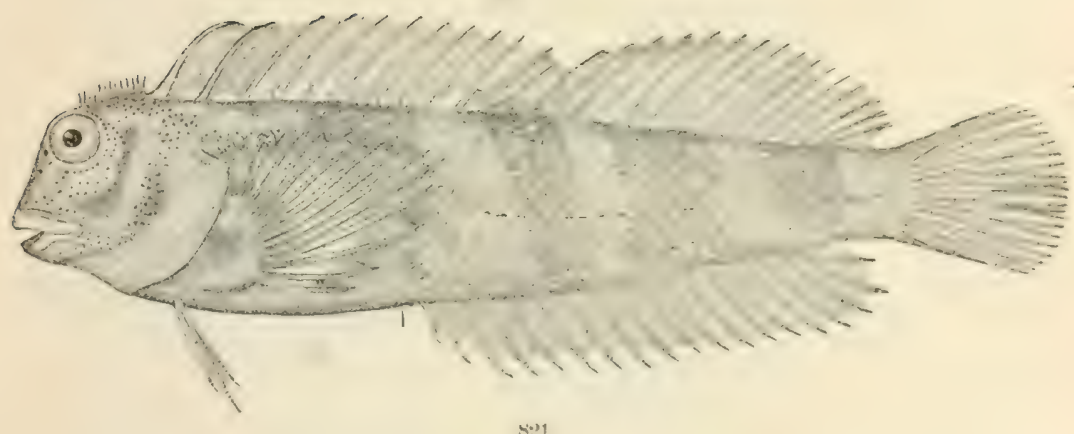

$x \div 1$

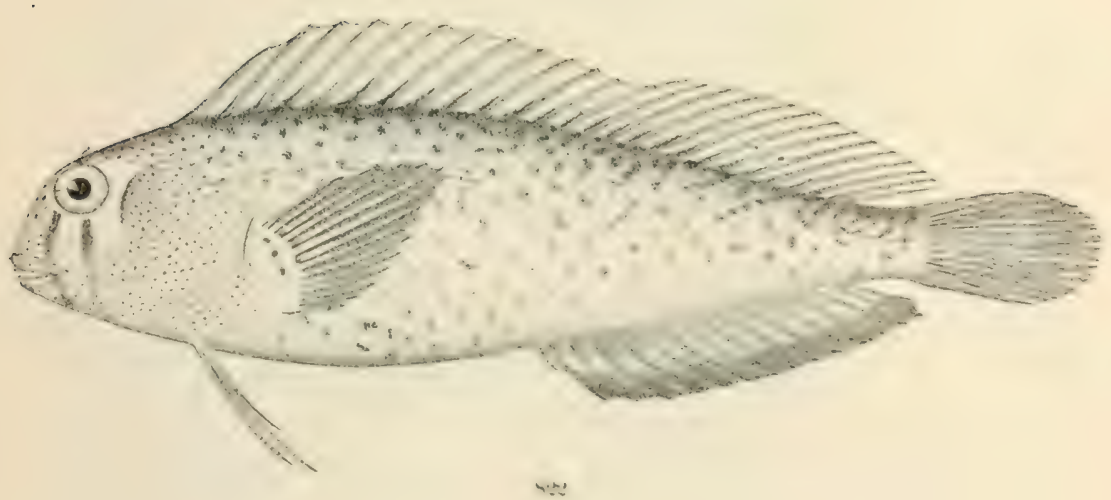

820. Blaxites favosus, (I’, 2380,)

821. BLENYIUS CIRTATUS. (P.238.)

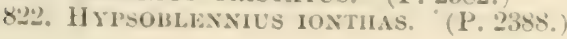




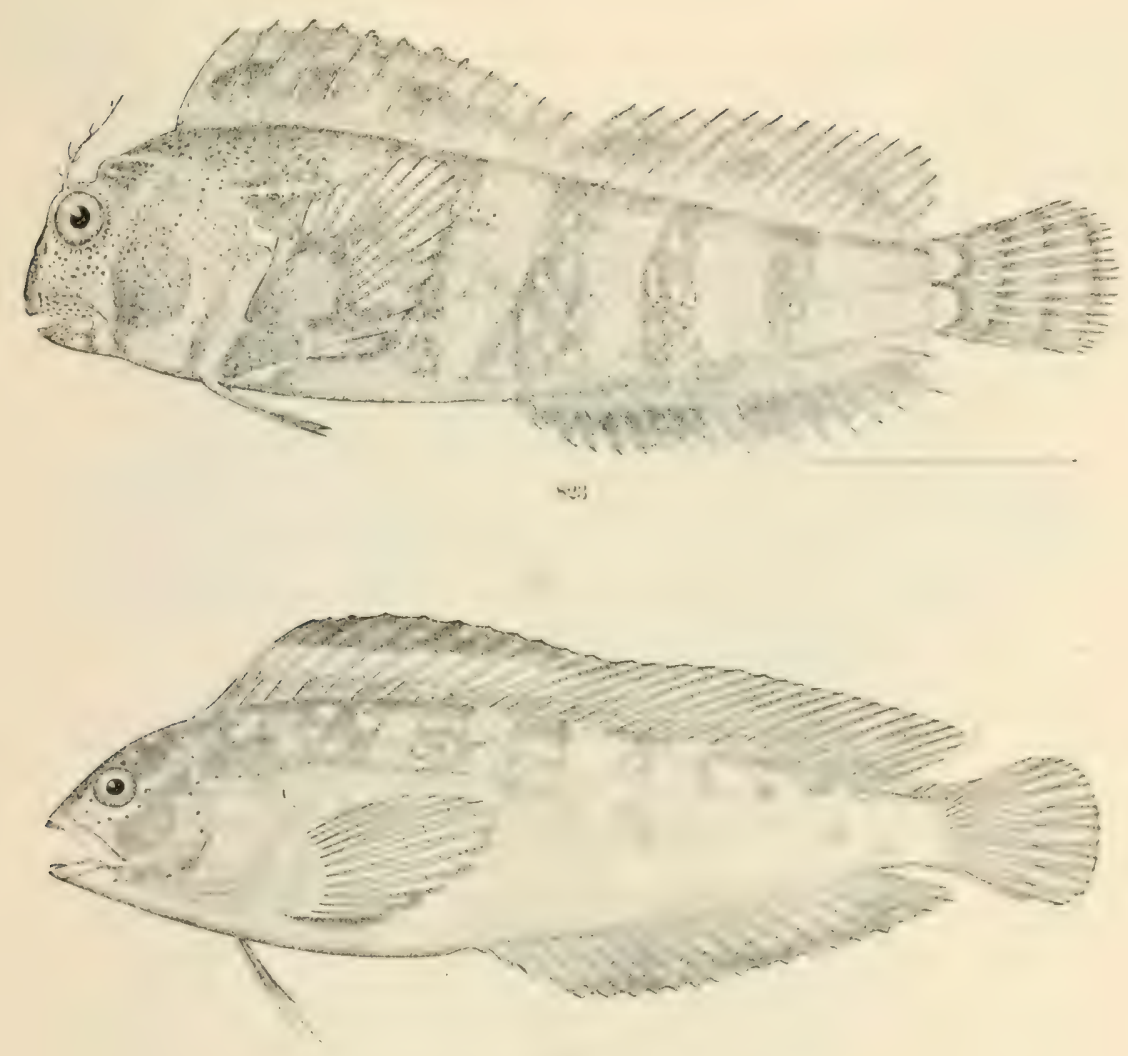

N:14

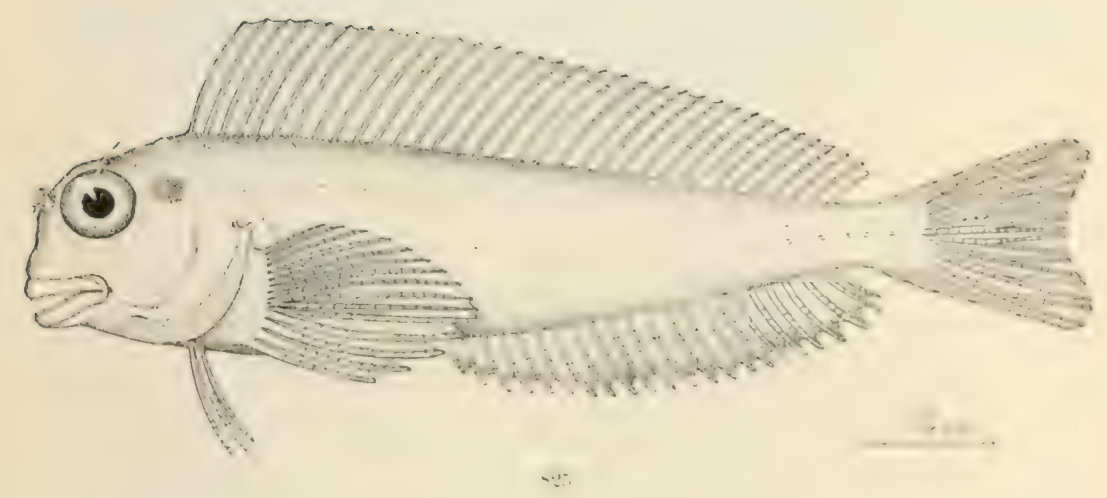

823. HYHAOHIFXITS 1H:XT\% (1'. 2390.)

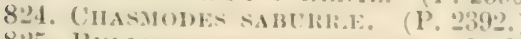

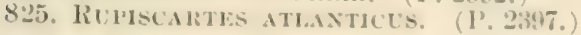





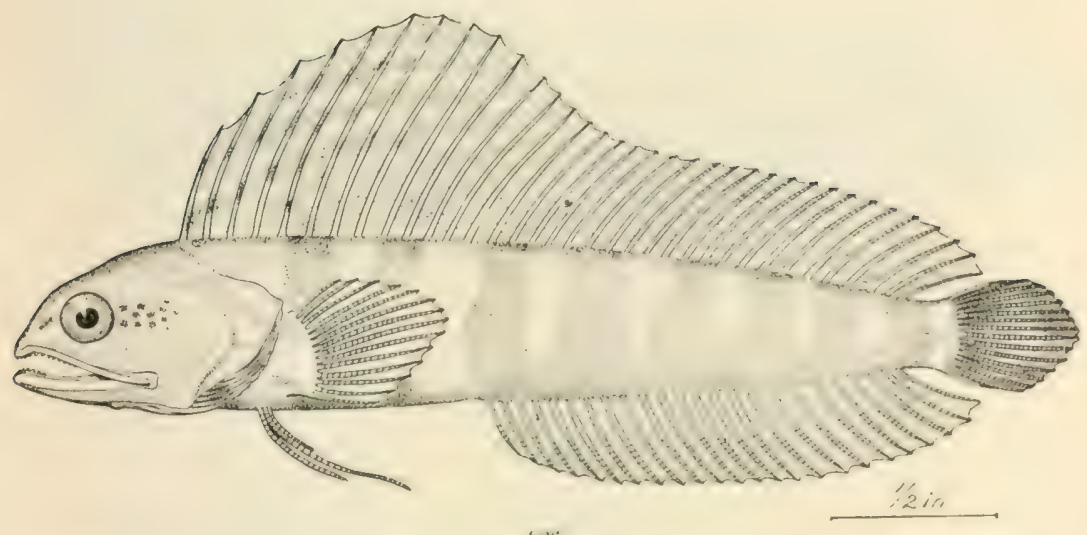

s.3t;

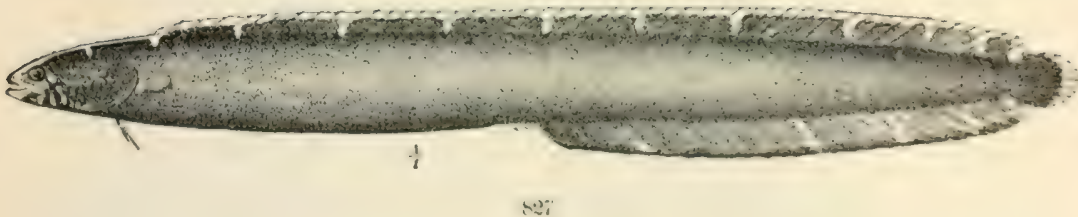

826. EMBLEMaria atlantica. (P. 240:.)

827. Stathionotus HeMPHLLI. (P. 2407.) 

U. S. NATIONAL MUSEUMI

BULLETIN NO. 47. PL. CCCXLI
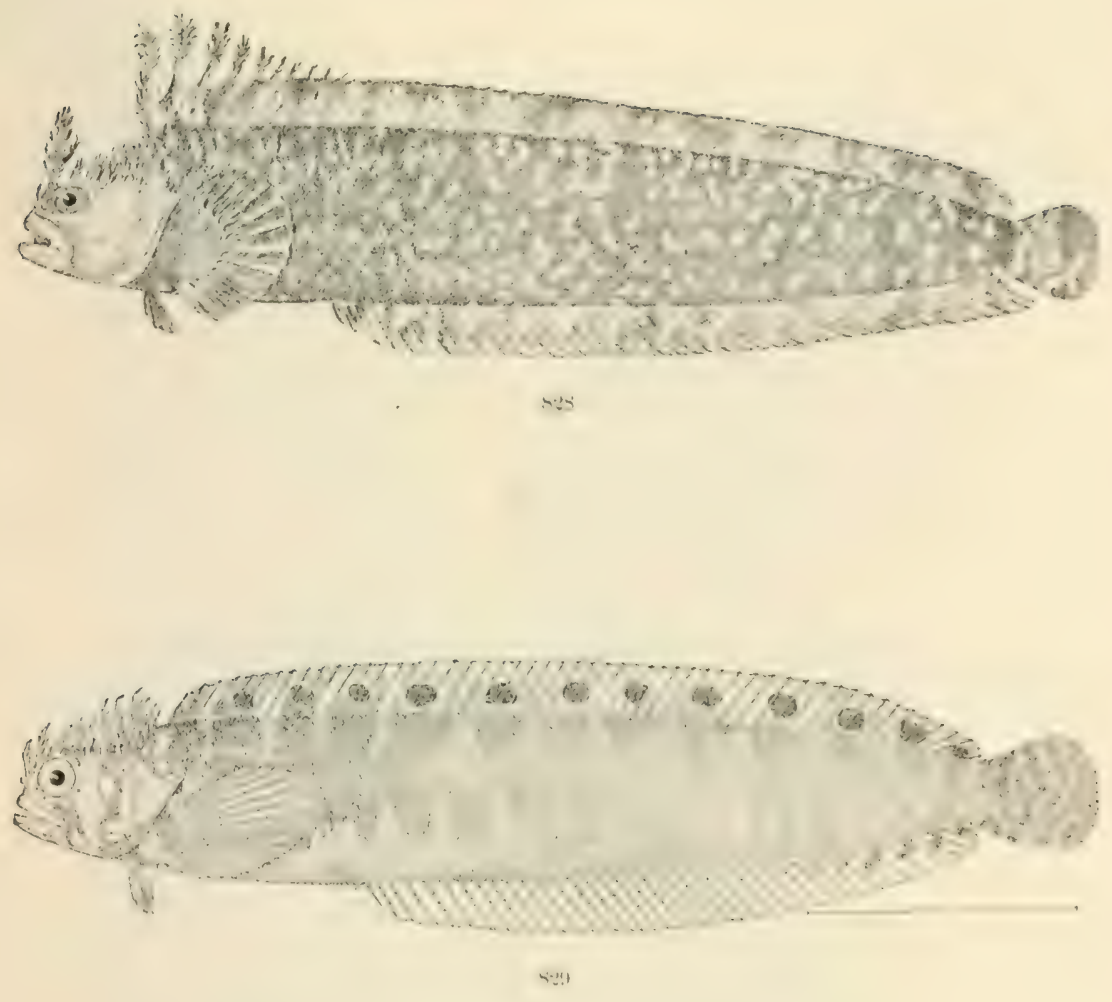

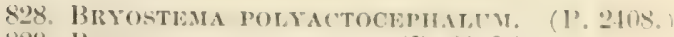

829. BRYOSTEMA NL(TATOR. (1.2110.)

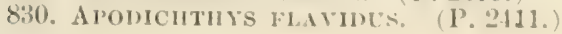




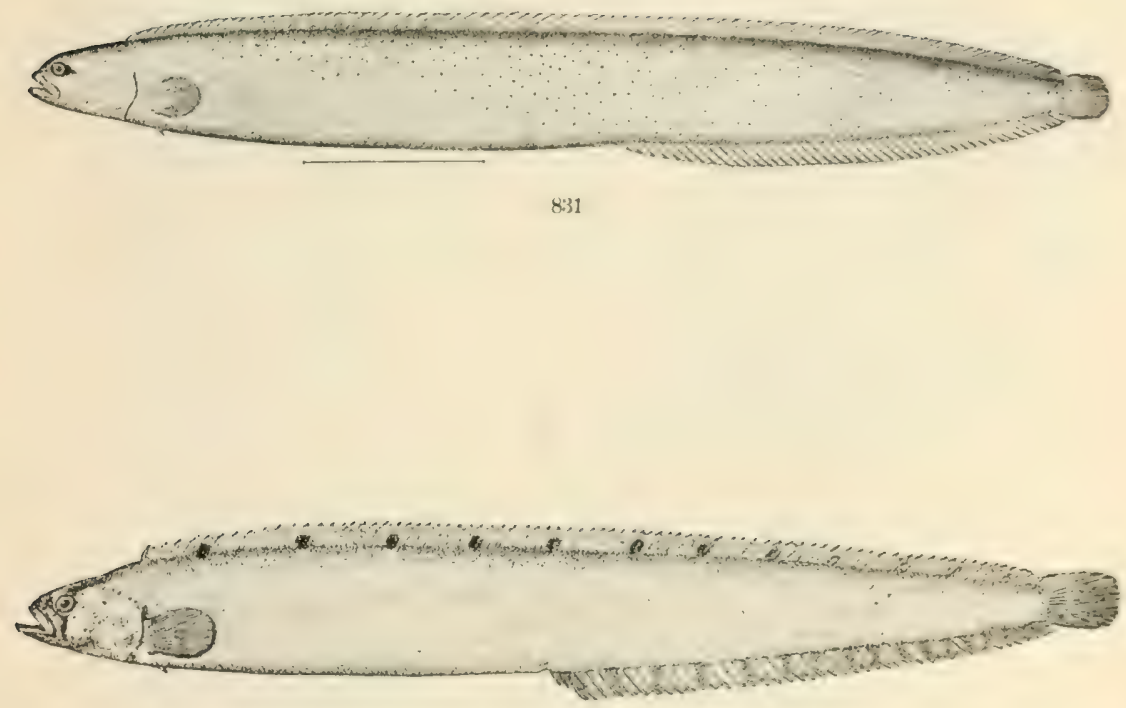

8i3:

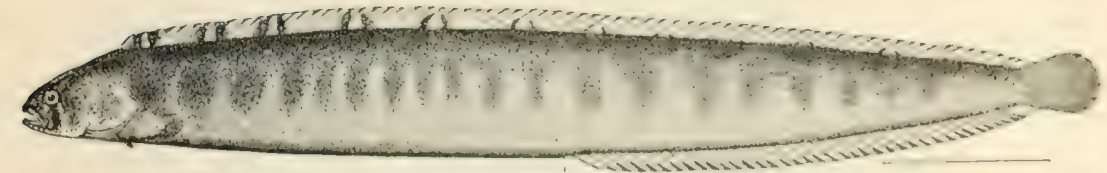

(3):

831. Pilolis dolichogaster. (P.2416.)

832. Pilolis gunnellus. ( $(1.2419$.

833. Pholis ornatus. ( $P .2419$. 


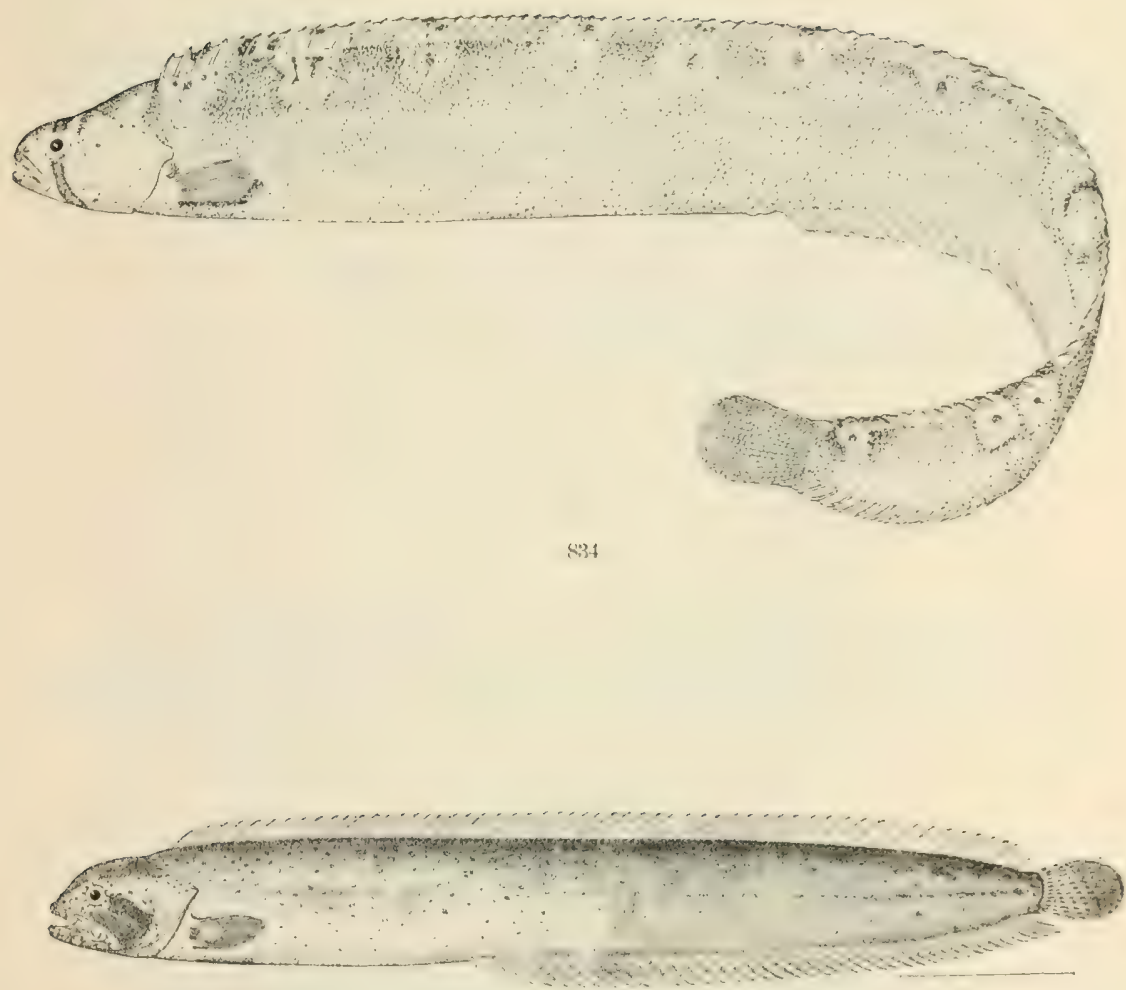

S:15

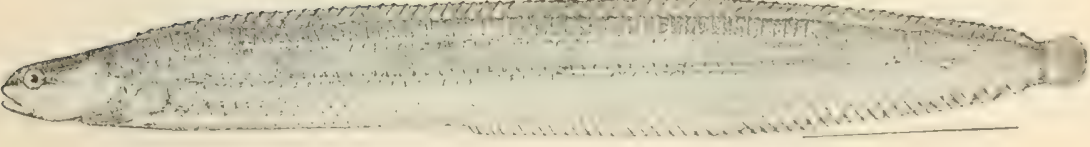

Bi31;

831. AsthinoptTERT GUNXELIFORMS. (P.24?0)

835. ANOPLARCITS ATROPULPUEUS. (P. 212.2.)

836. XIPHITES ULY.. (P. 



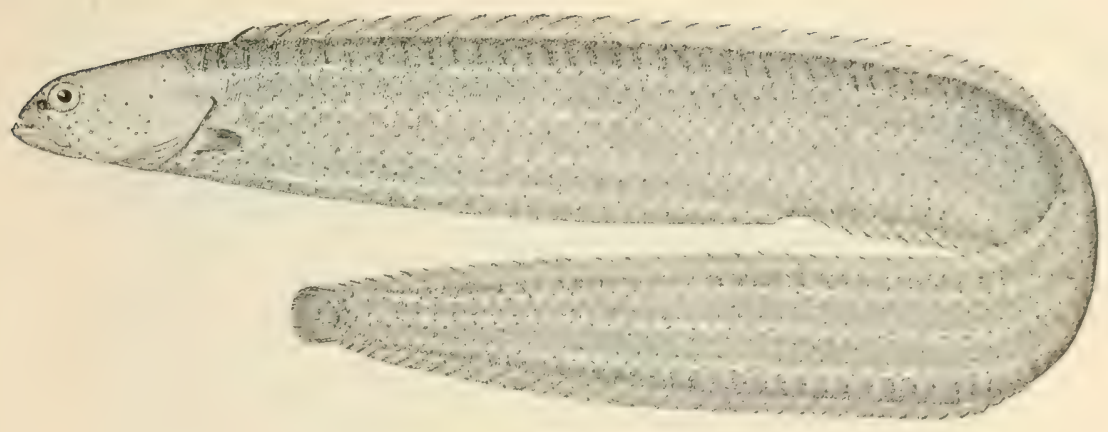

หล:

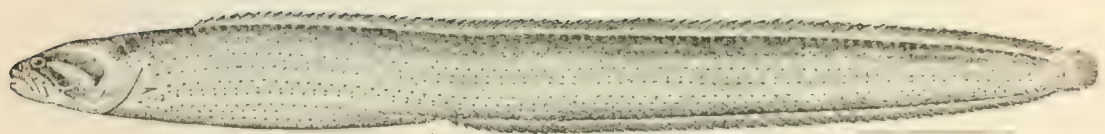

Nis

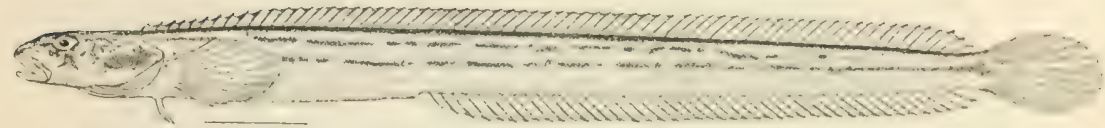

S35

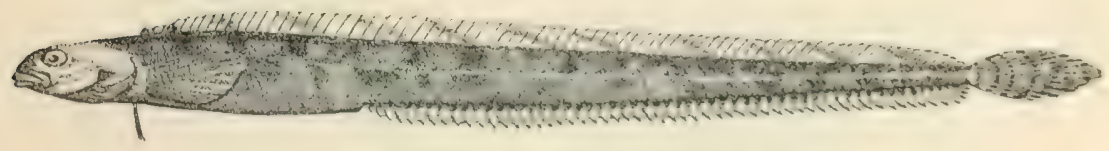

(1)

837. Ximplistes chirus, (1', 2124.)

838. NIIHIDION RUPESTRL. (P. 2426.)

839. LUMPLSUS MACKYT. (P. 2136.)

840. LUMLENUS LAMIPETREFORMIS. (P. 2438.) 


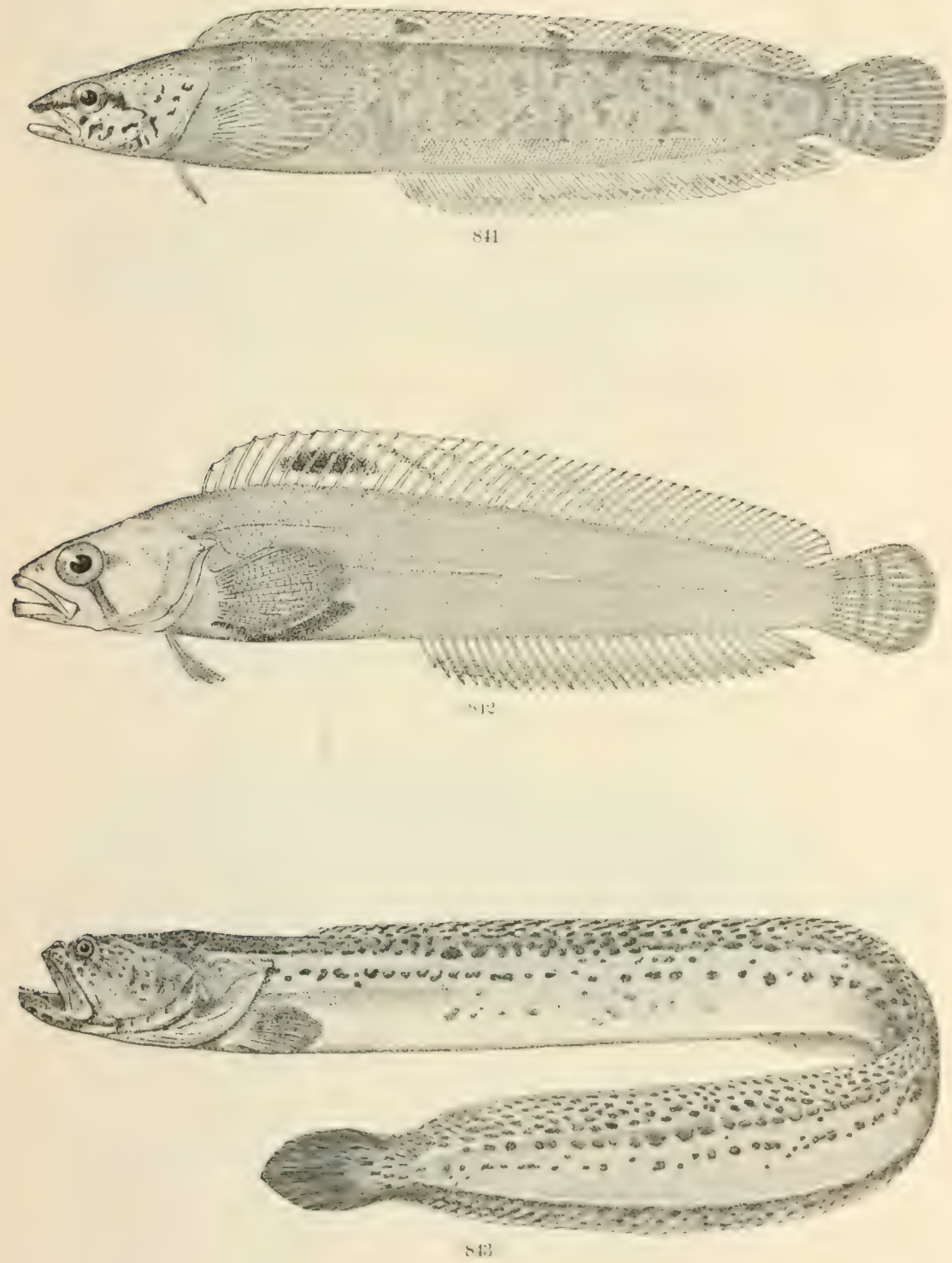

811. Strcheus puxctatus, (P. 2439.)

842. Ulvaria SUmifircata. (P. 24.40.

843. Cryptacanthotes anculatus, (P. 2443.) 



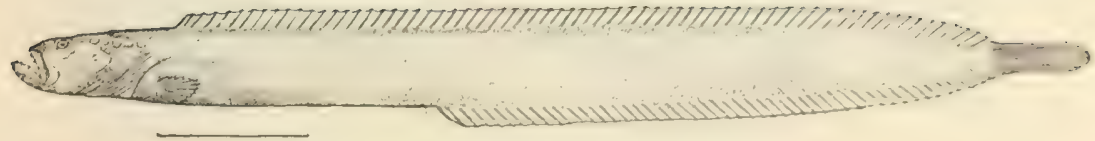

841

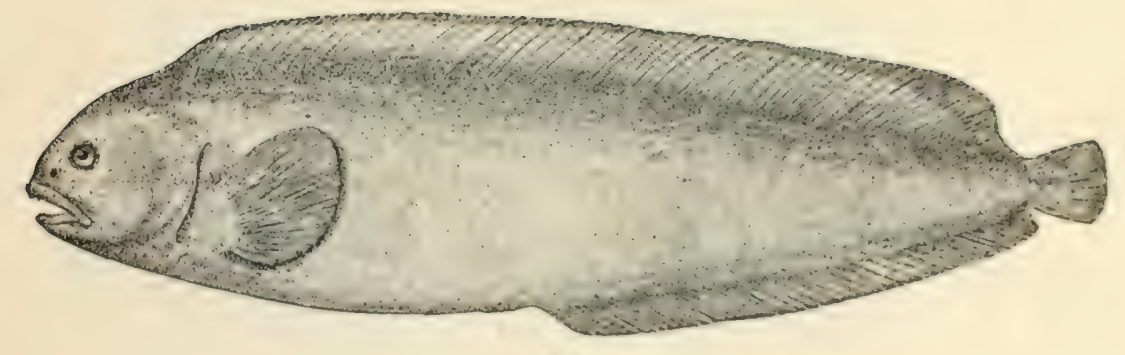

sis

841. LYCONECTES ALEUTENSIS. (1'.2414.)

845. ANarimcilas latifroxs. (P. 2446.) 

U. S. NATIONAL MUSEUM

BULLETIN NO. 47 , PL. CCCXLVII
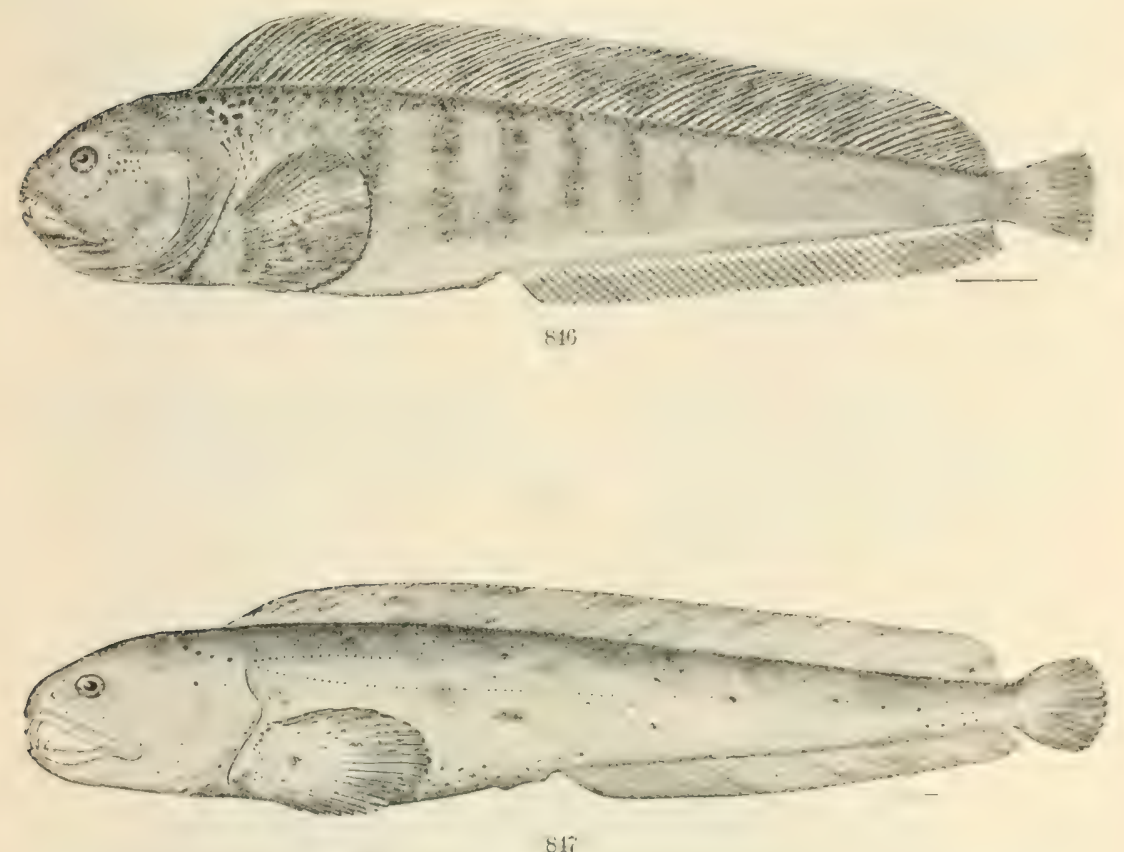

817

846. AxarHCHAS ITTUS. (I’. 2416.)

847. ANarHCHAS LEHTLUS. (1'.2447.)

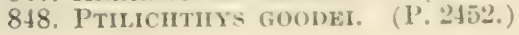



849

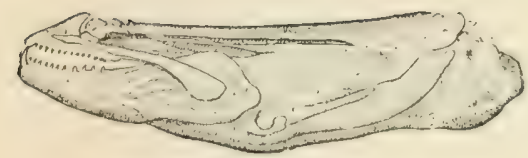

स.4.

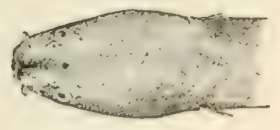

$849 b$

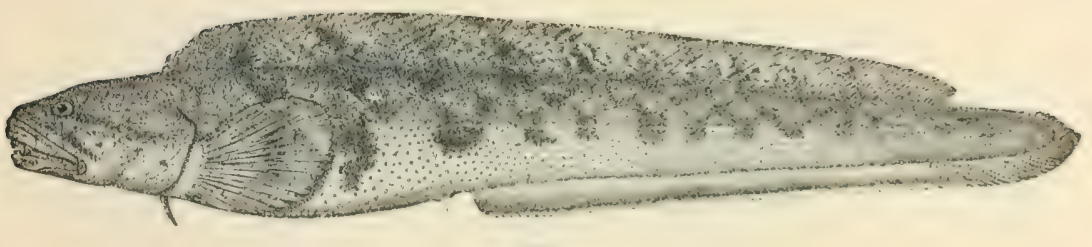

850

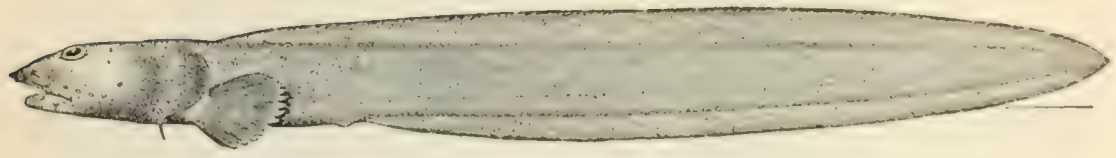

851

849, $849 a, 849 b$. SCYTALINA CERDAIE. (P. 2454.)

850. KOARCES ANGUILLARIS. (P. 2457.)

851. EMBryx crotalinus. ( 


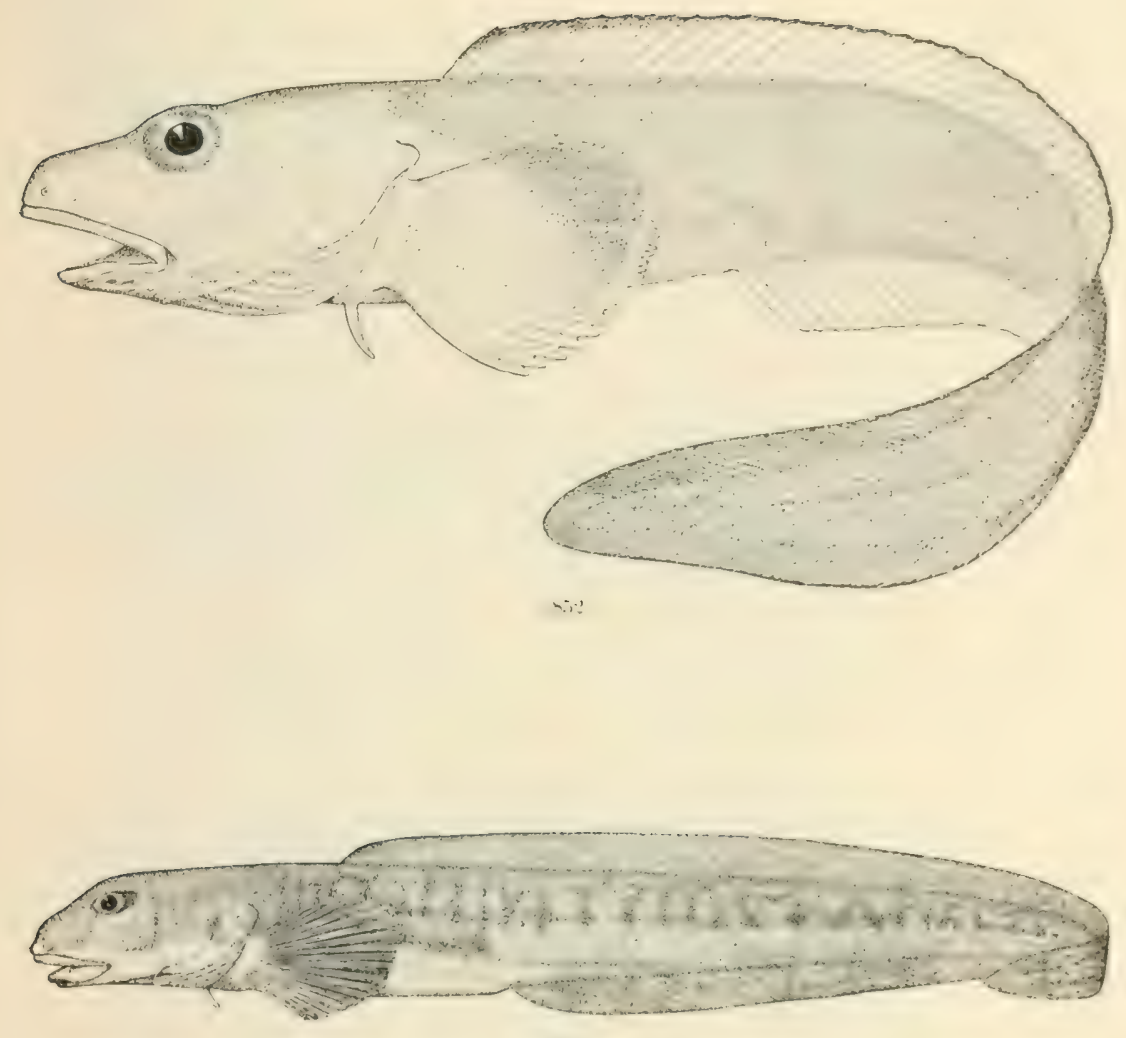

$(-i)$

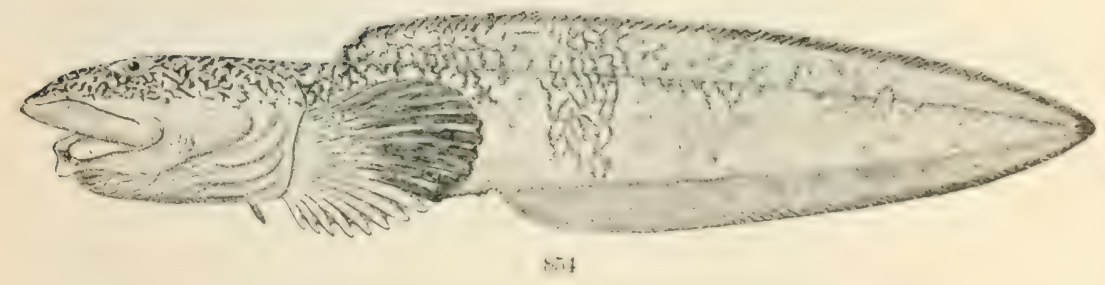

852. APRODON CORTMZIANUS. (P. 2461.)

853. LYCODIA 70ARCHUS. (P. 2464.)

854. Liconle RETICULATCS. (P, 2465.) 


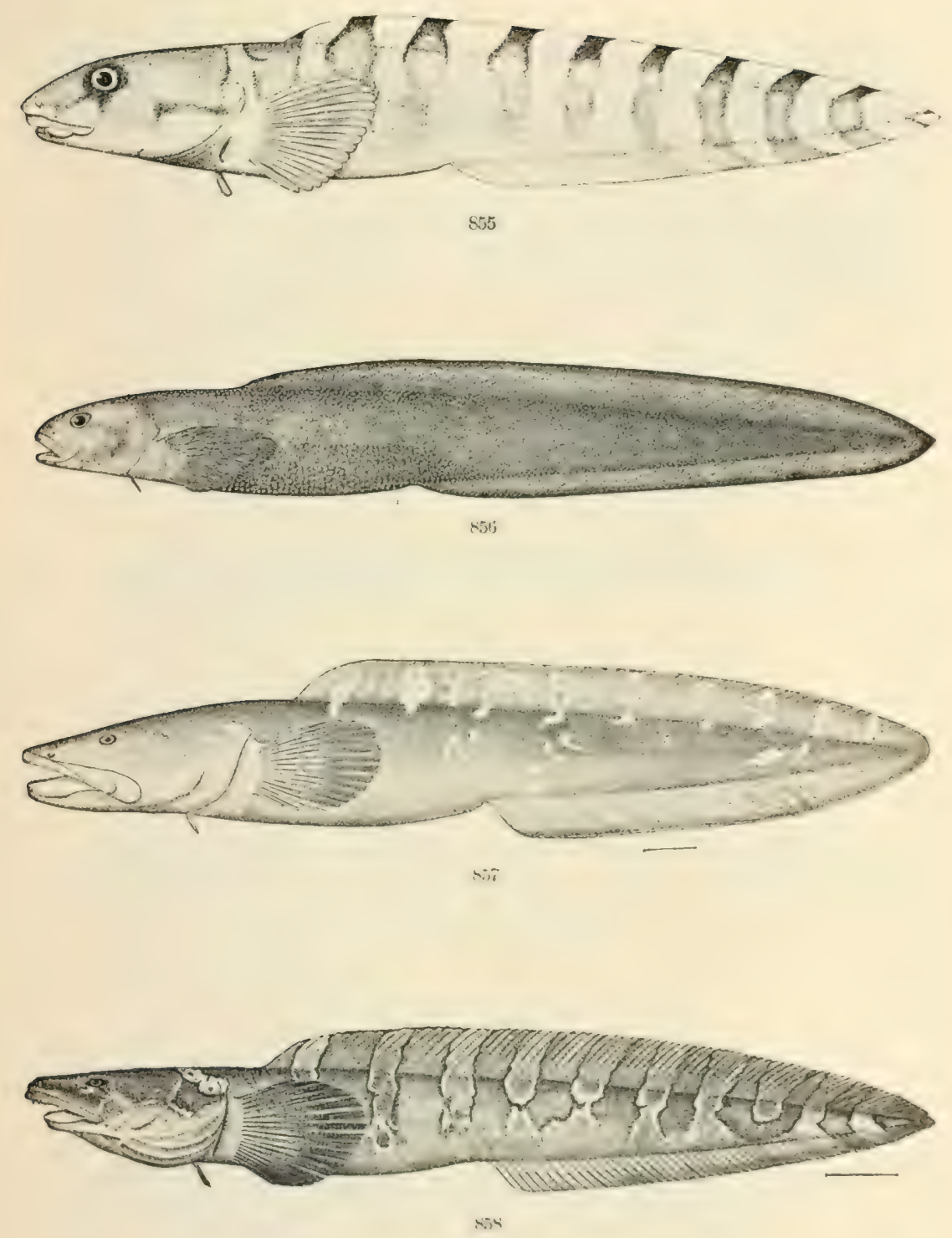

85. LyCOMES PERSPICILLUM. (P. 2165.)

856. LYCODES FRMIIDUS. (1', 2465.)

857. LYCODALEPIS POLARIS. (1', 2468.$)$

858. LYCODAIERIS TURXERI. (1'.2468.) 



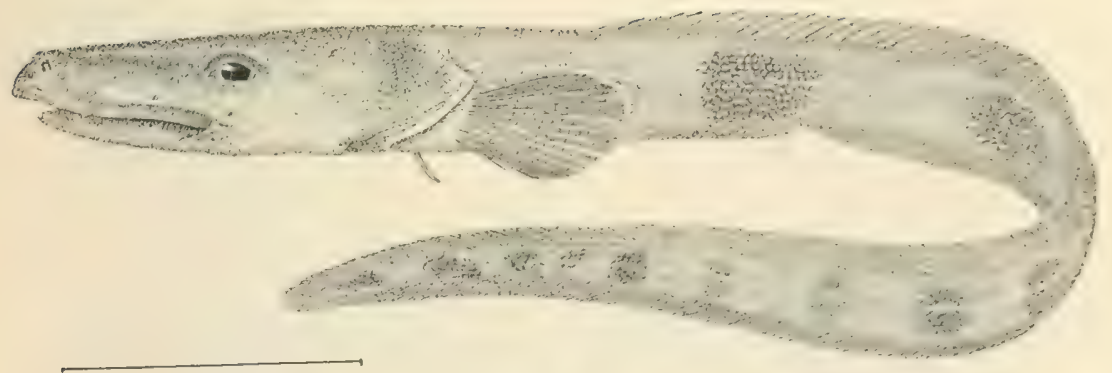

Nis

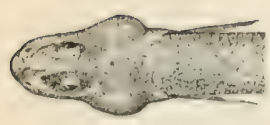

S60a

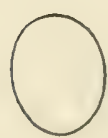

$860 c^{\circ}$

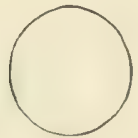

scod

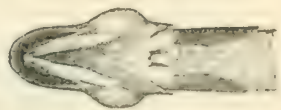

$860 b$

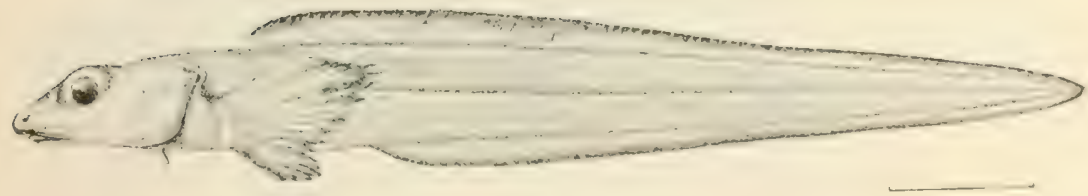

Sti1

859. LYCLXCHFLYS VERnMLIY. (P. 2470.)

$860,860 a, 860 b, 860 c, 860$ d. LYCENCHELYS PAXILLUS. (1'. 2471.)

861. Furchinus diaterus. ( $\mathrm{P} .2472$. 



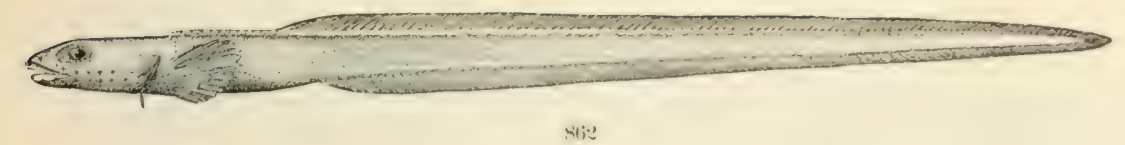

sii:

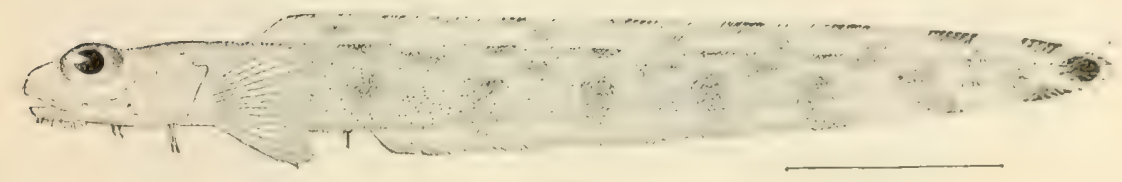

86:3

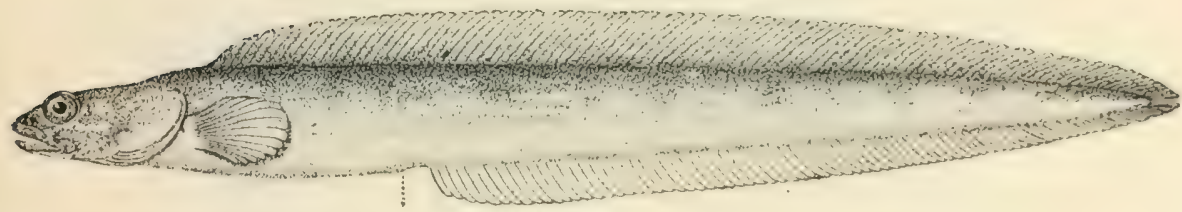

861

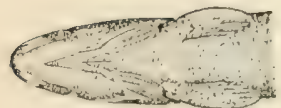

$861 u$

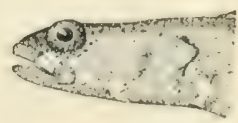

8616

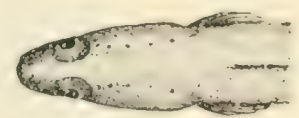

site

Siti.

869. LyCODONUS MIRABLLS. (P. 2474.)

863. LYCONFMA BARBATEM. (P. 2474.)

86t, 864a, 864b, 864c. GYMNELIS VIRIDIS. (P. 2477.)

865. Mriajostigan PAMMELAs. (P. 2479.) 

U. S. NATIONAL MUSEUM

BULLETIN NO. 47, PL. CCCLIII
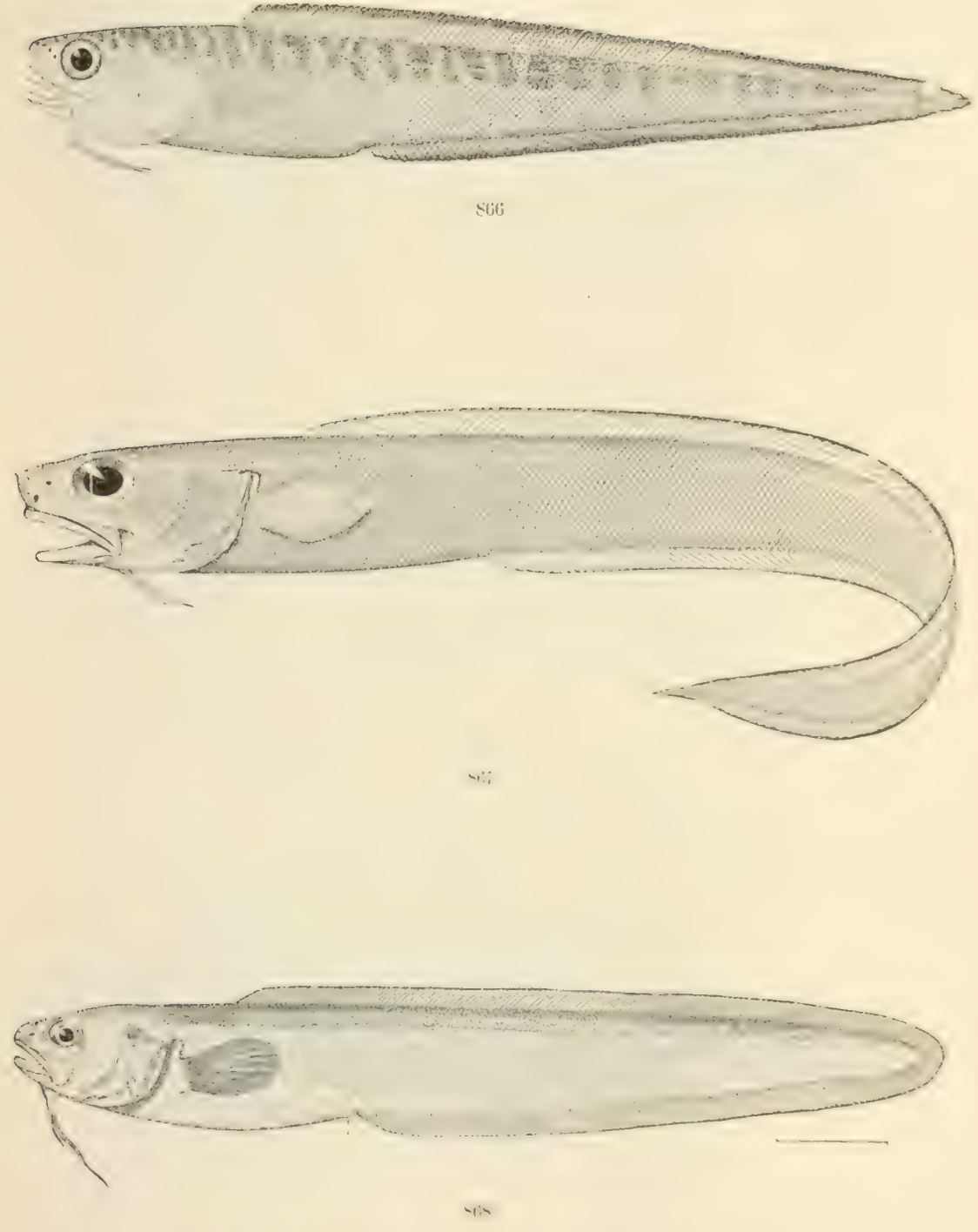

866. Lipophint:y marmoratrit. (P. 2482.)

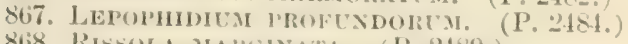

868. Rissola malikinata. (P.2489.) 

U. S. NATIONAL MUSEUM.

BULLETIN NO. 47, PL. CCCLIV
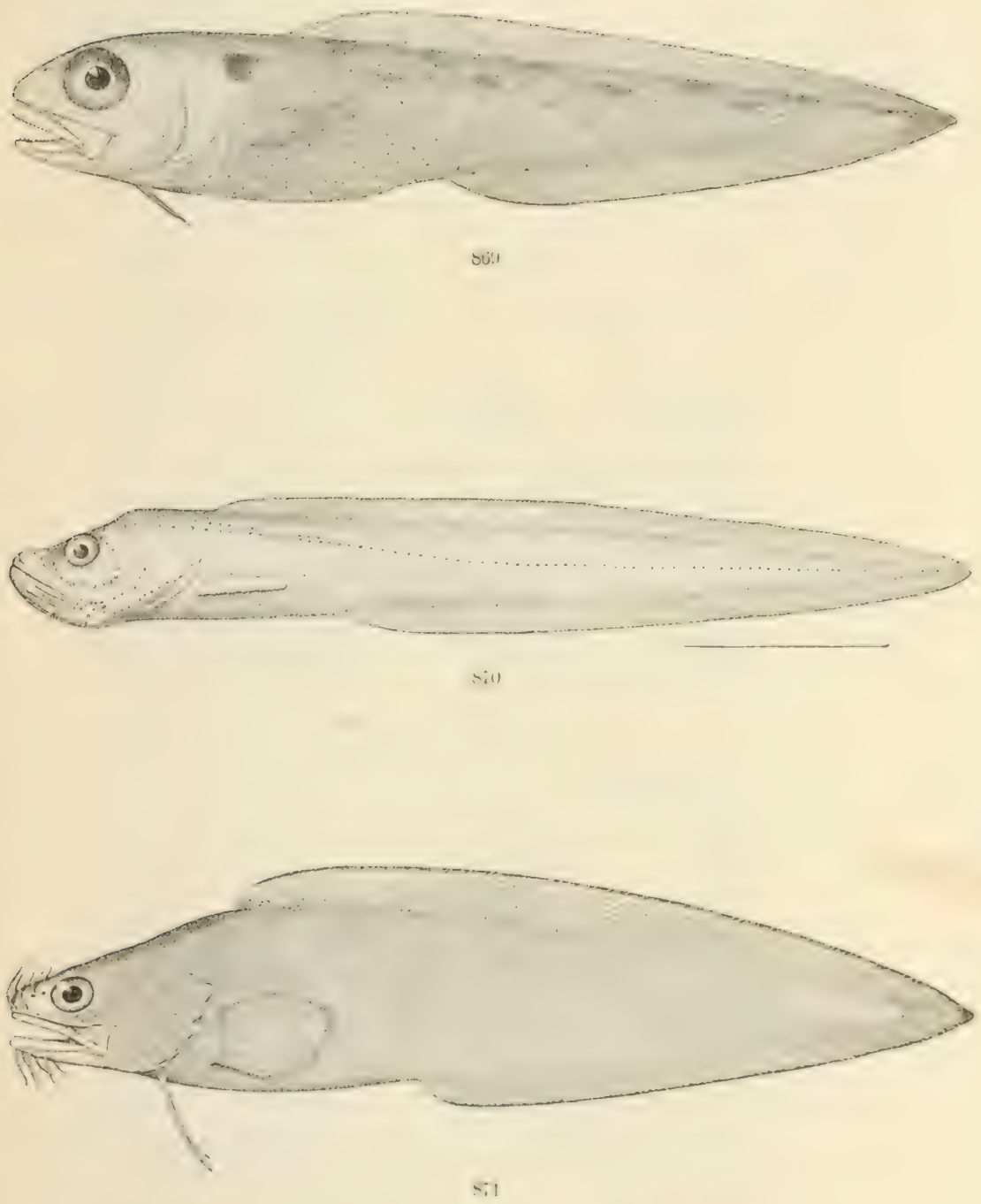

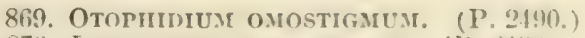

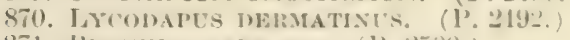

871. BHOTLLA BARIBATA. (1'. 2500.) 


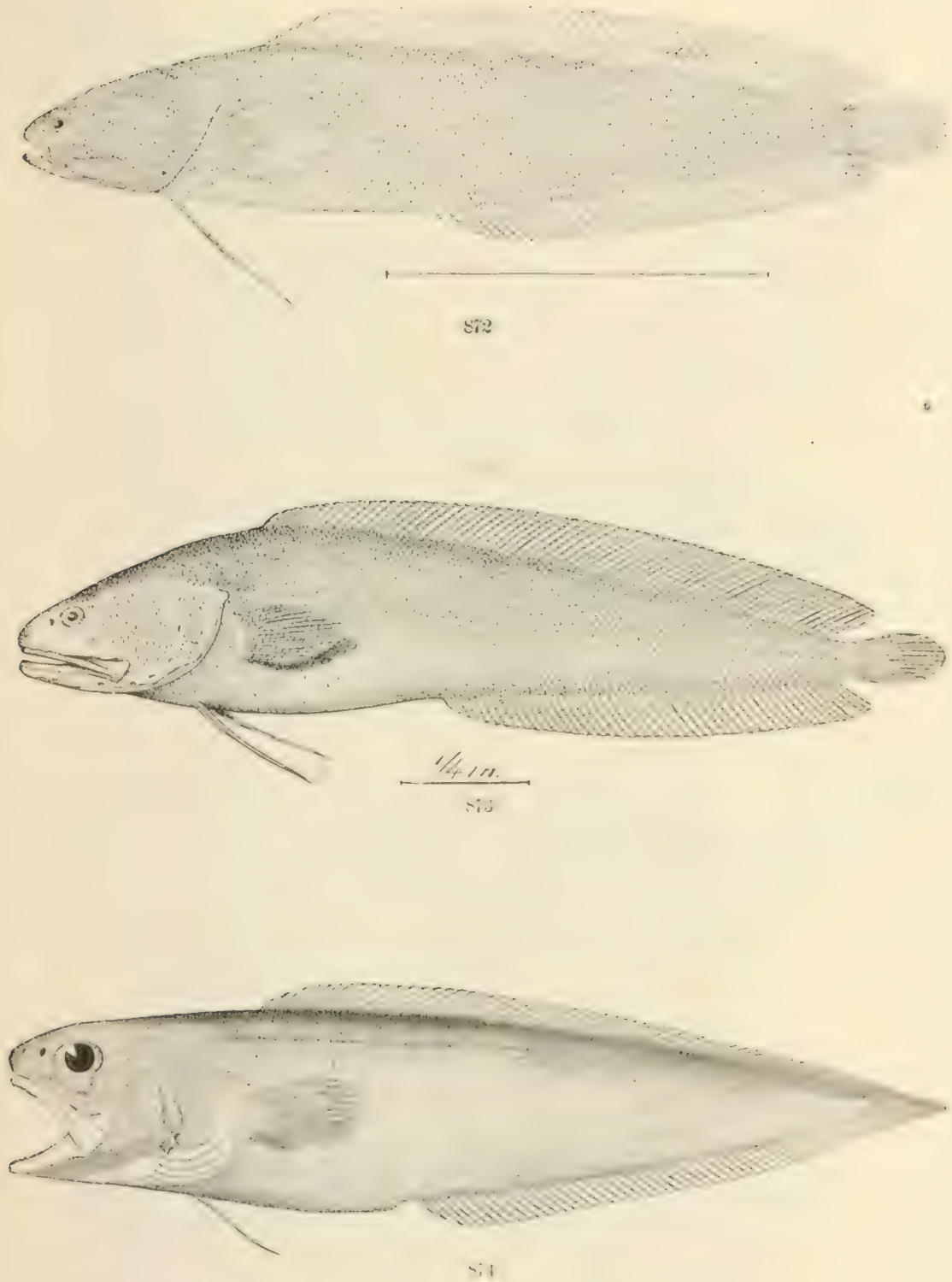

87.2. O(illura VExTIALIs. (1'. 250)3.)

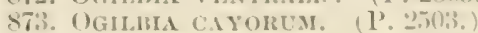

87. J)1: 


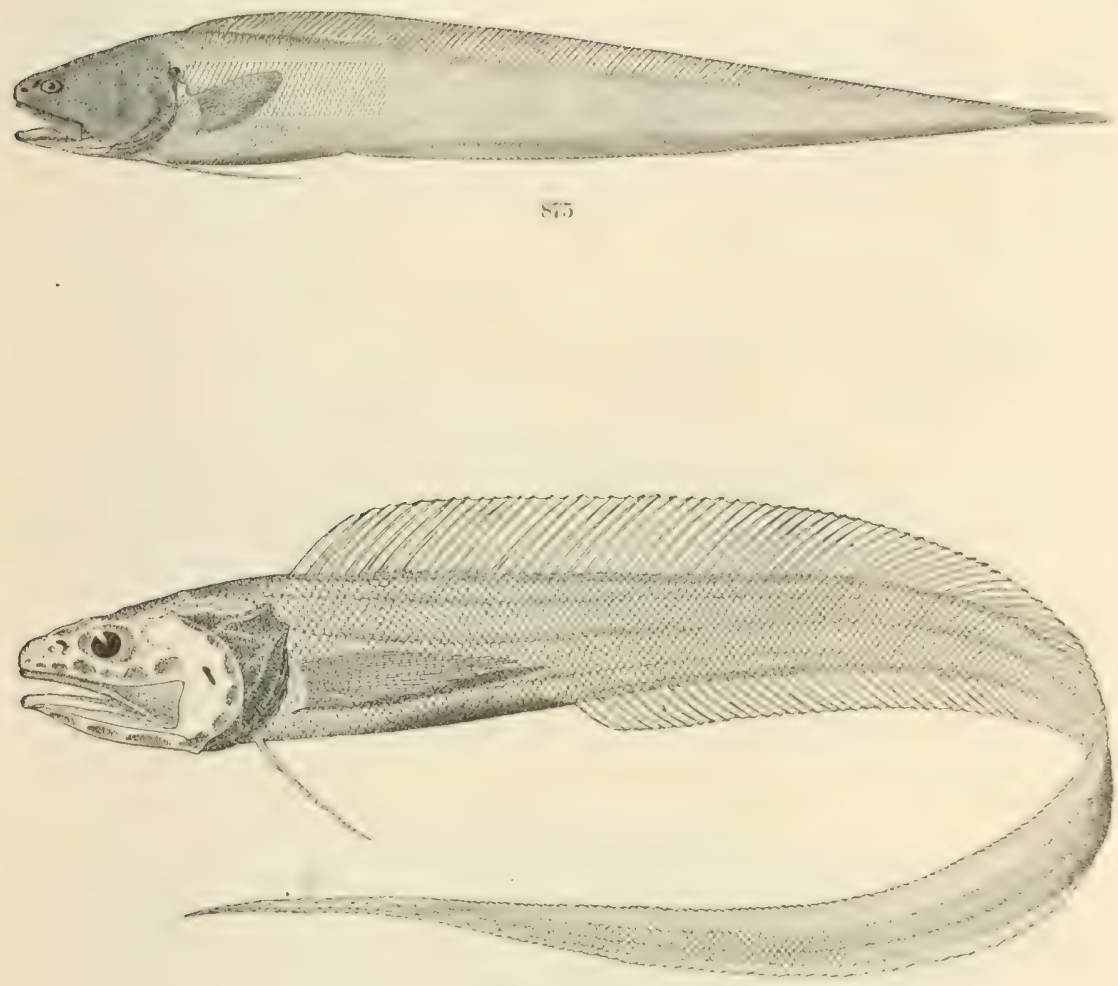

riti

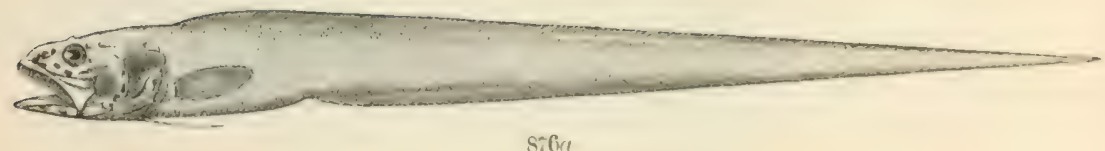

875. 13assozetus Noliarals. (1'. 2507.)

$876,876 a$. BAsiozETCS CATENA. (1'. 2509.) 


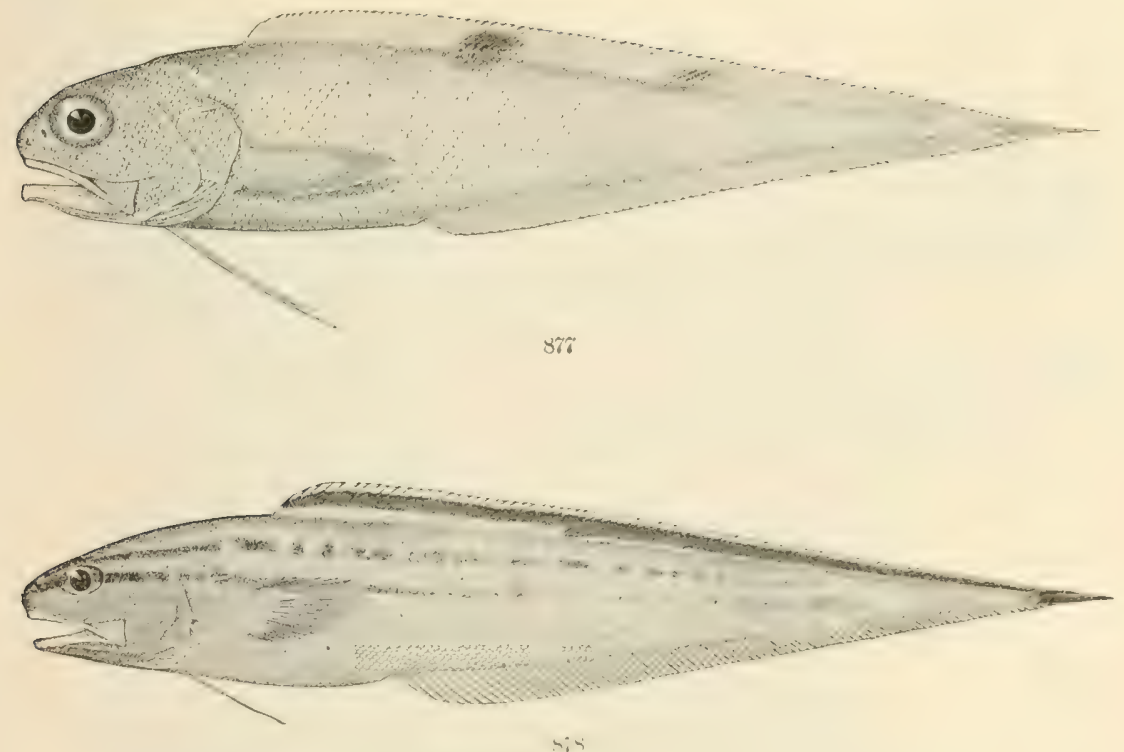

sis
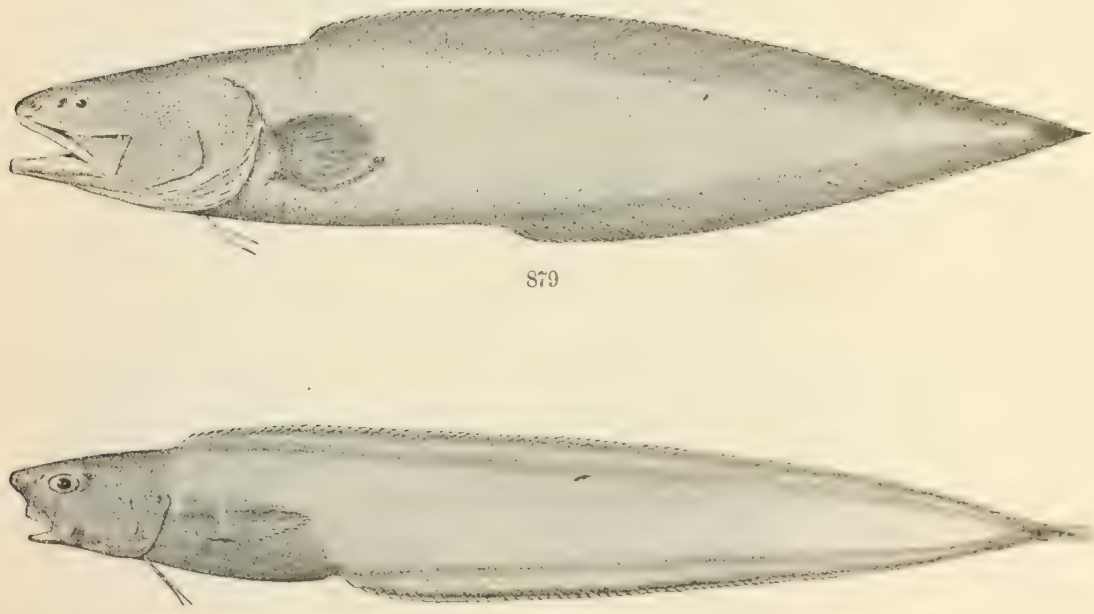

$m-1$

877. NEOHYTIITES GIIII. (1'. 251\%.)

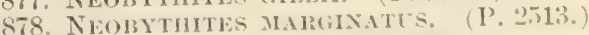

879. Bastogigas (illi.11. (1'. 2515.)

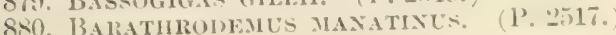




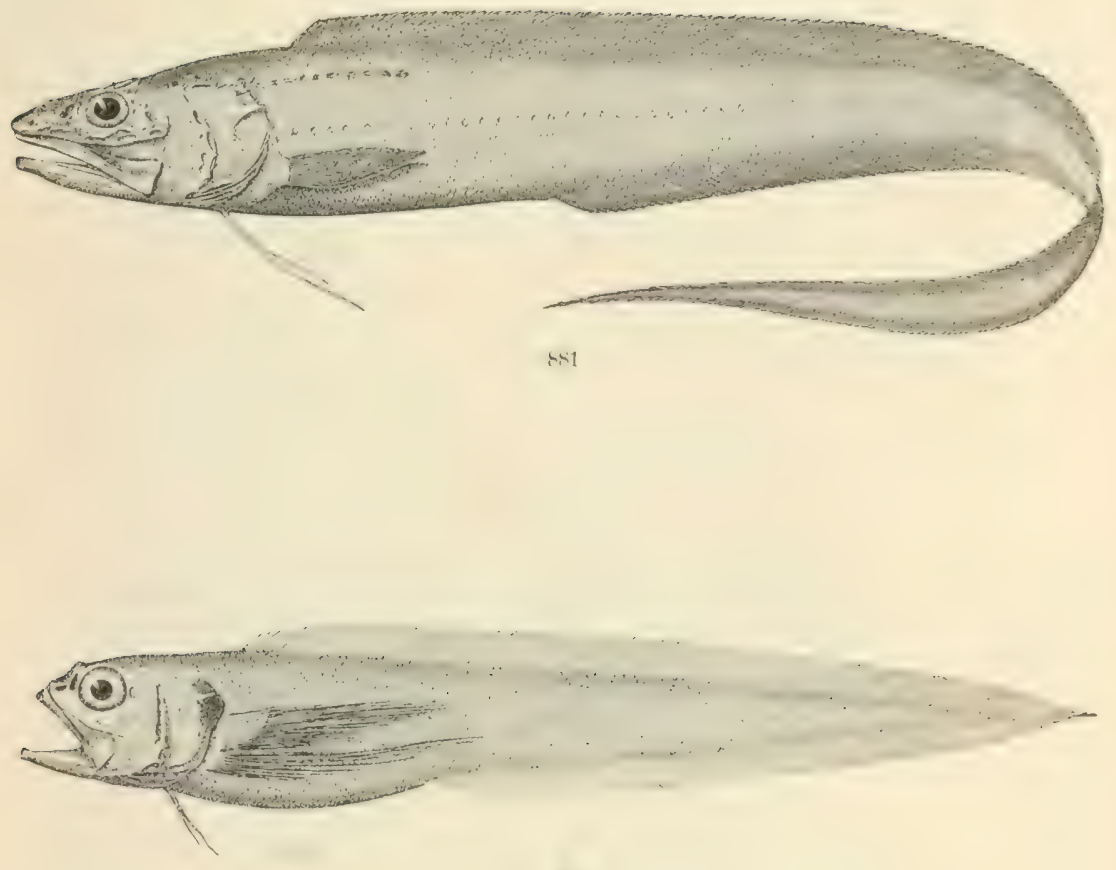

sis

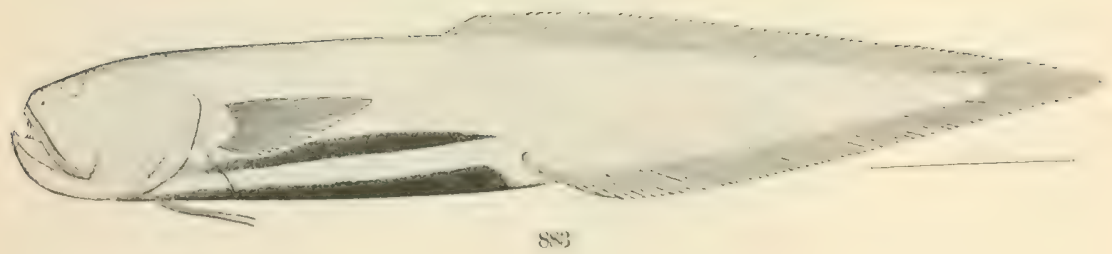

881. Poluciands Mr.es, (1'. 2520.)

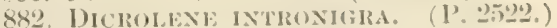

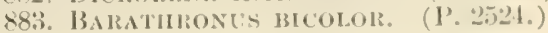




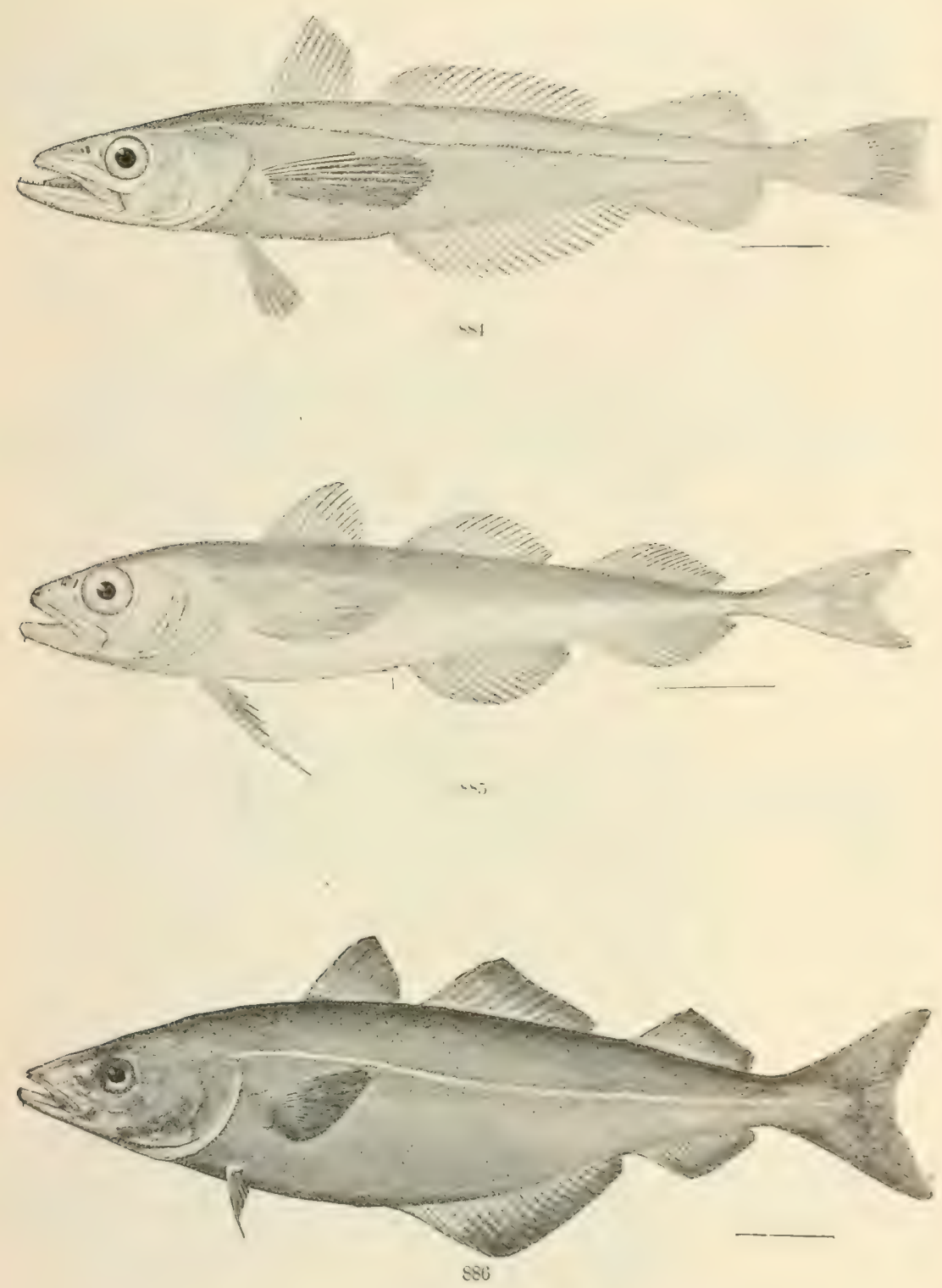

884. Merluccius plioductés. (1.2531.)

885. Borogadus SAInA. (1'. 2533.)

886. Pollachies viless. ( 


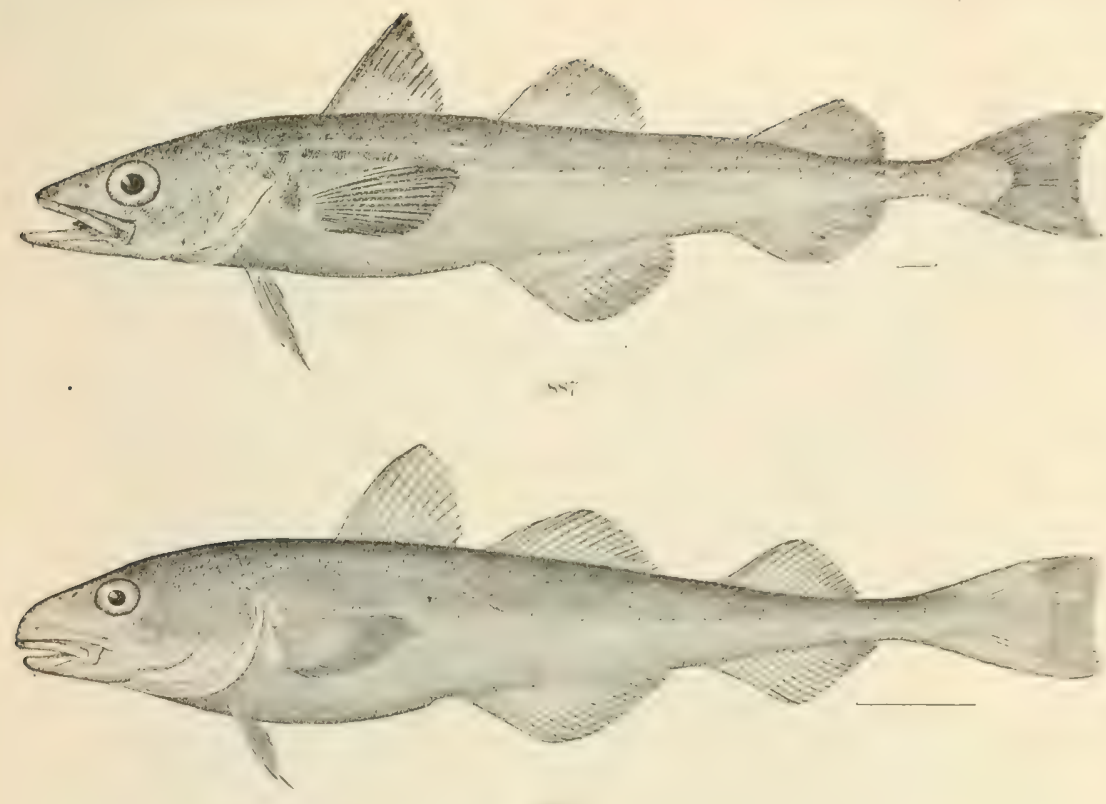

ins
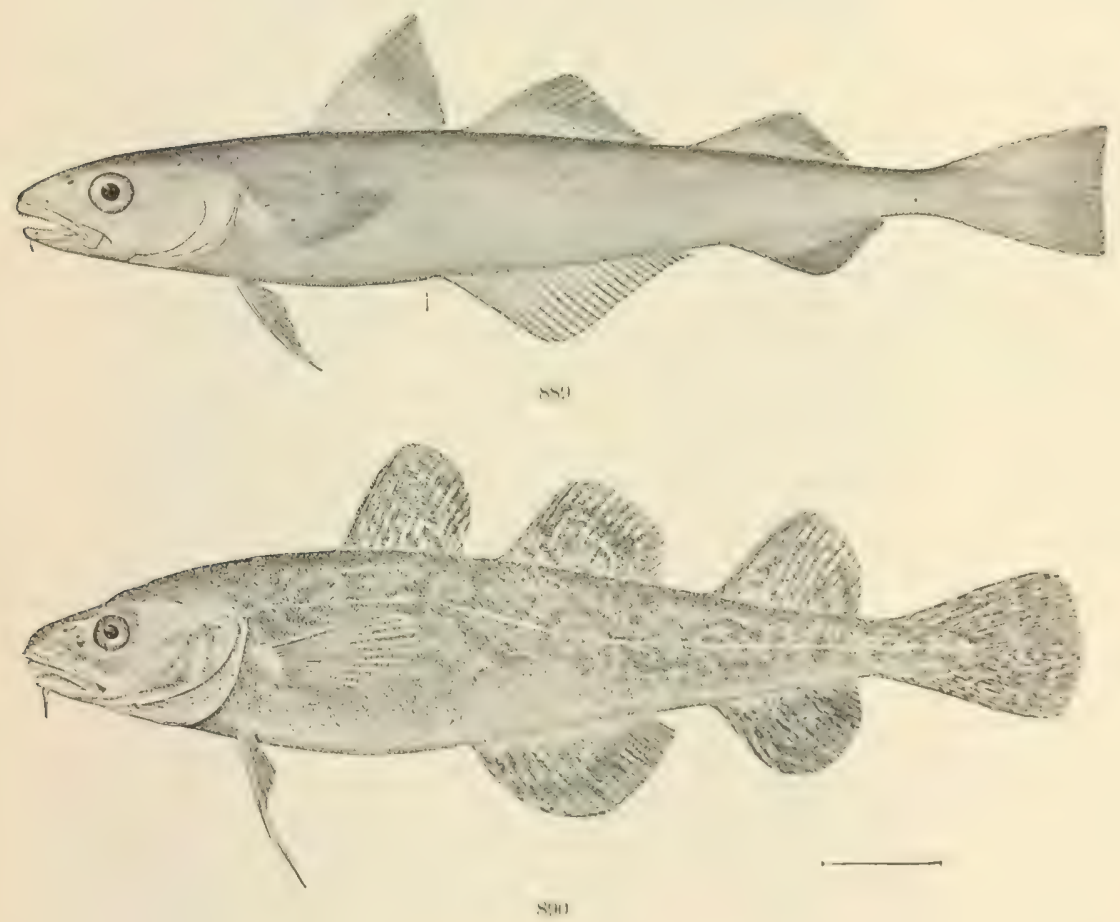

887. 'THeragra chalcograma. (P. 2535.)

888. ElEGINUS NAVAGA. (1', 2537.)

889. MIClzogades proximus. (P. 2539.)

890. MICROGADLS TOMCOI), (P. 2510.) 


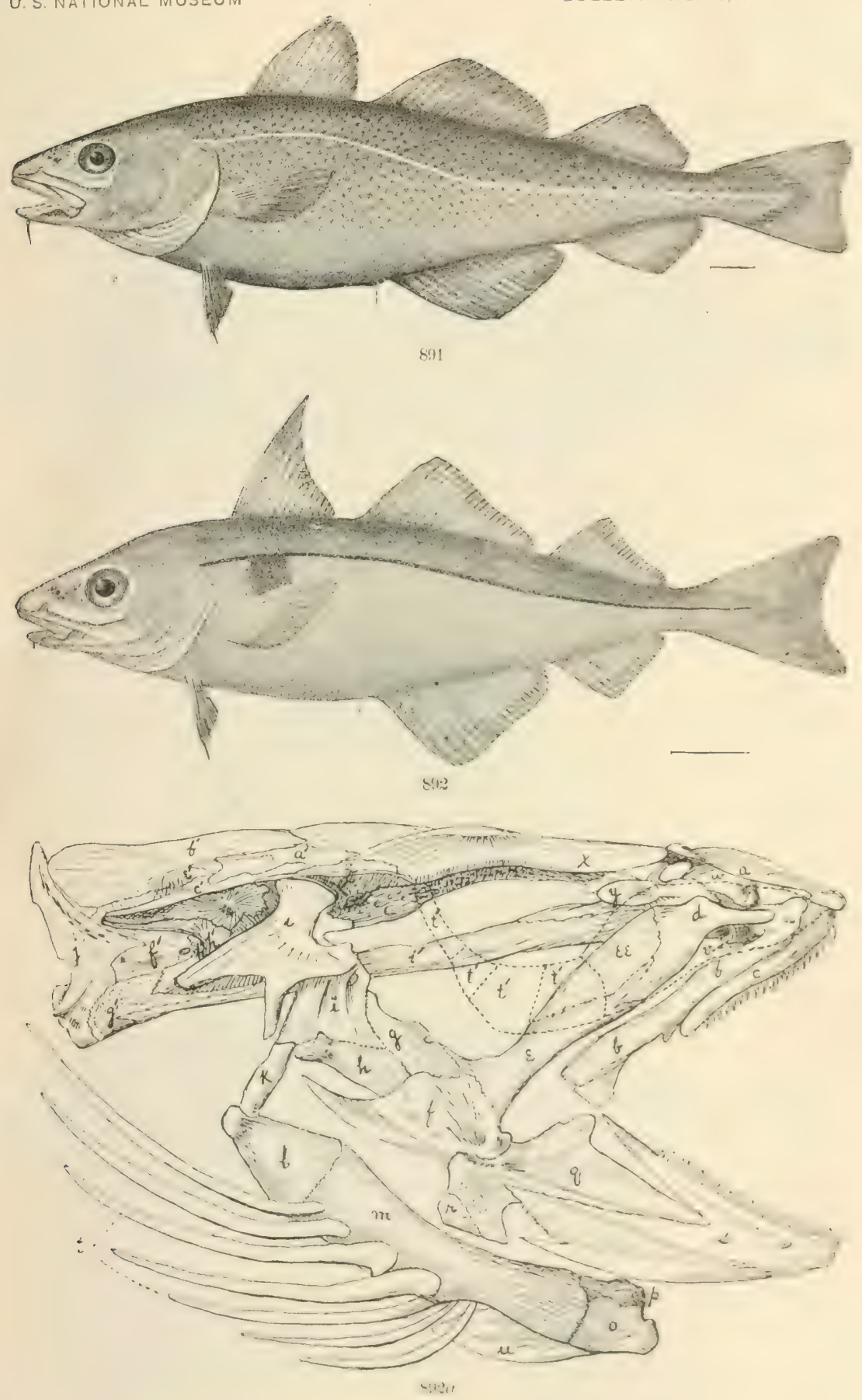

891. G.MIUS COLLARIAS. (1'. 25.11.)

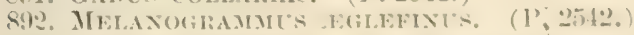

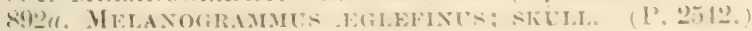





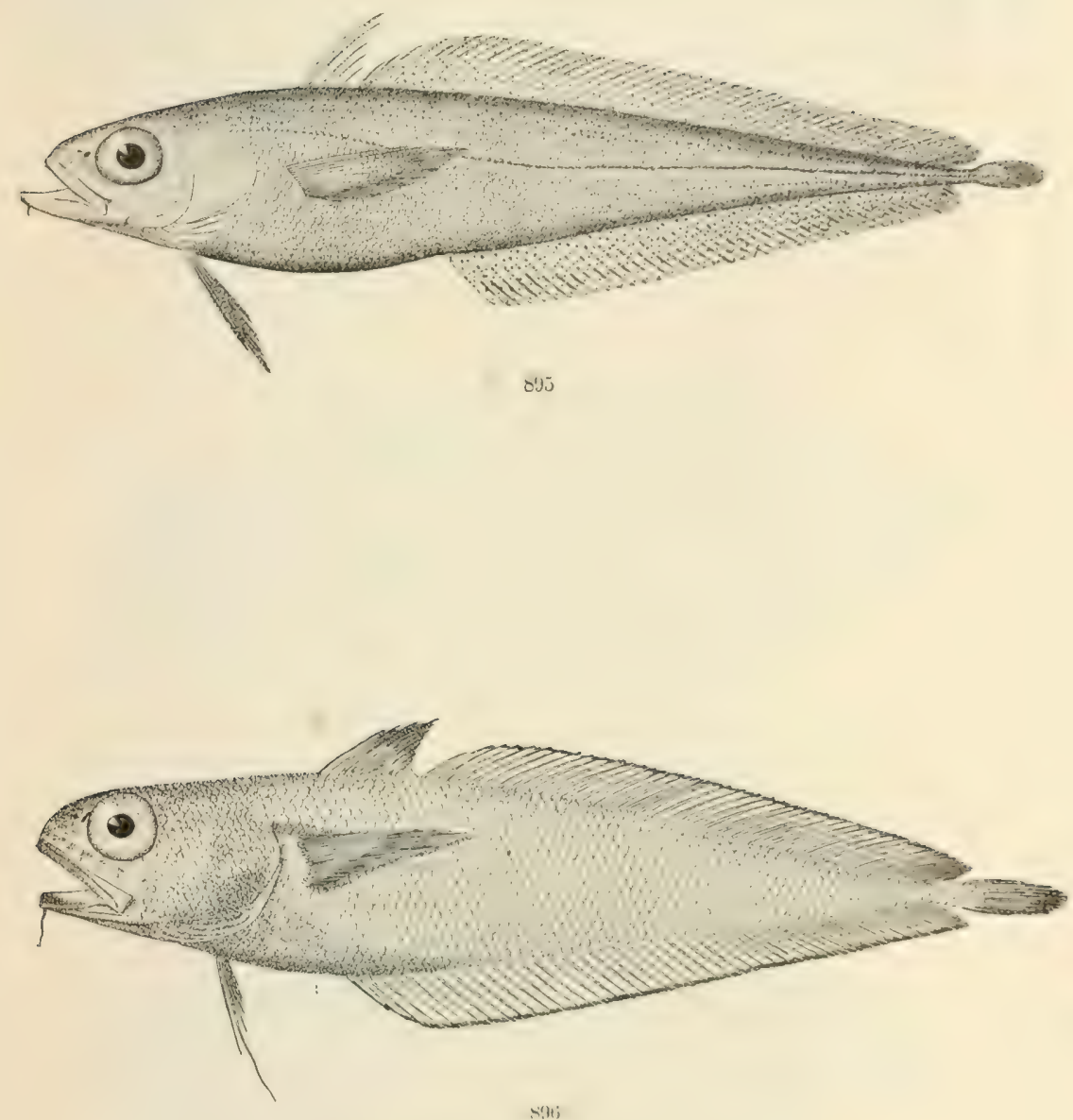

895. Lotella Maxillaris. (P. 2546.)

896. Physiculus fulves. (P. 2547.) 


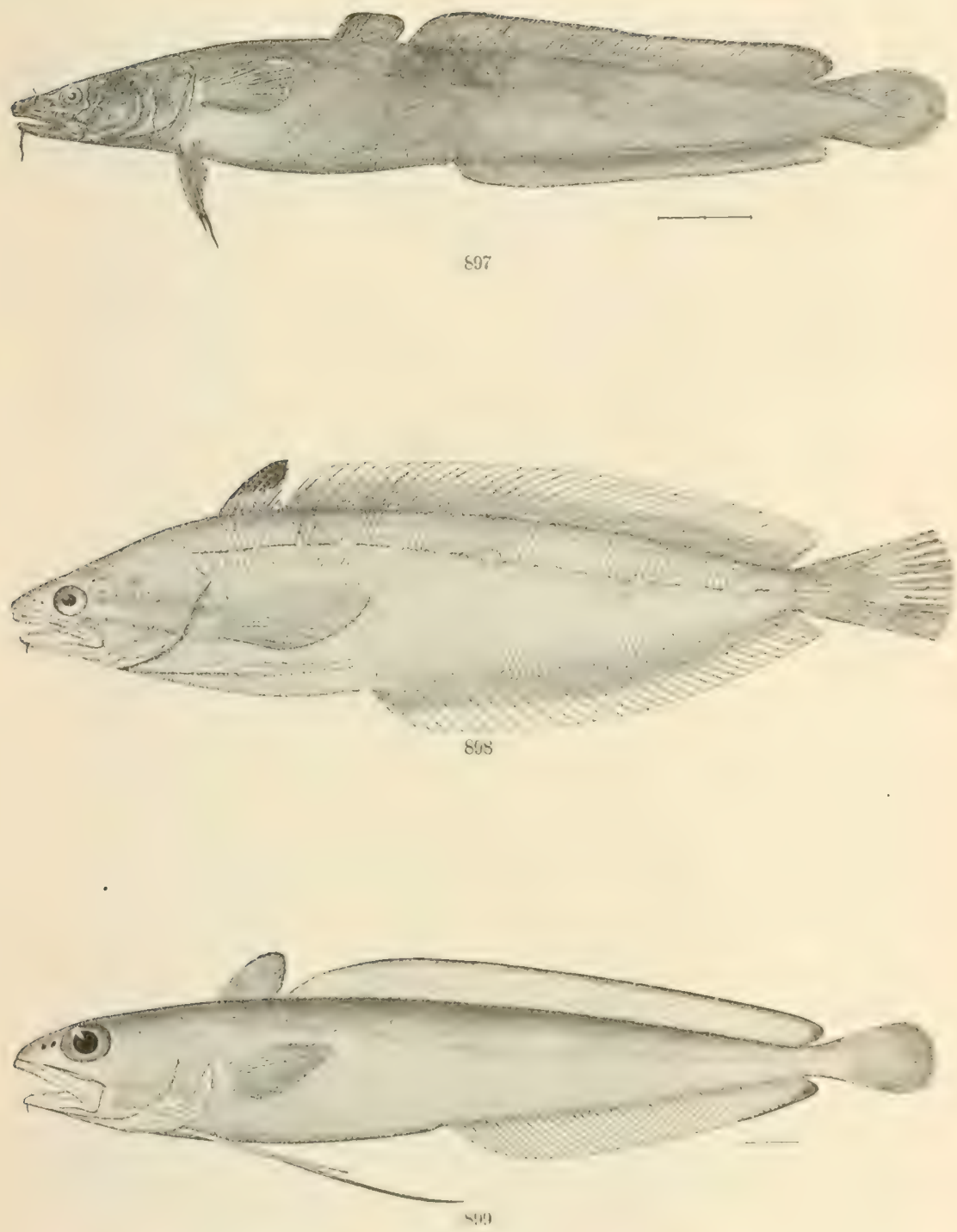

897. Lota Maculosa. (P. 2550.)

898. UROPHYCIS REAIUS. (I'. 2553.)

899. Urophycis chrRatus. (1'.2553.) 



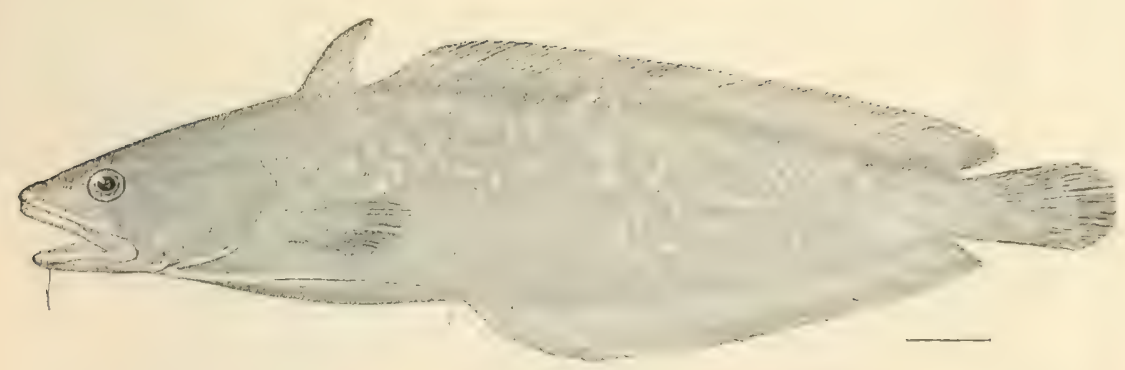

300

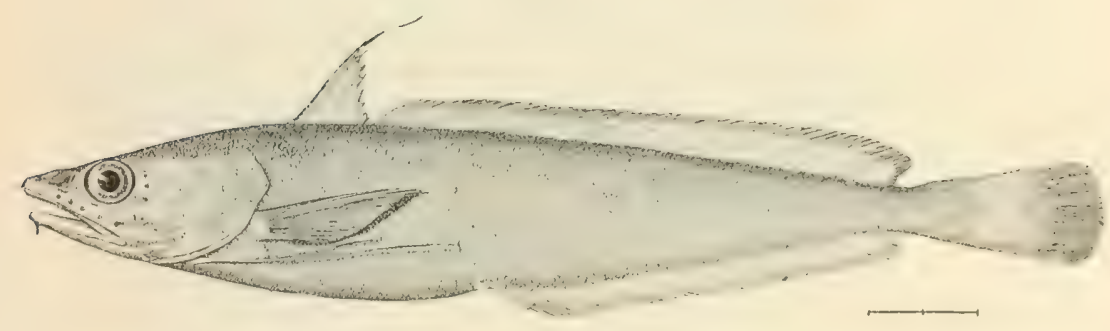

901

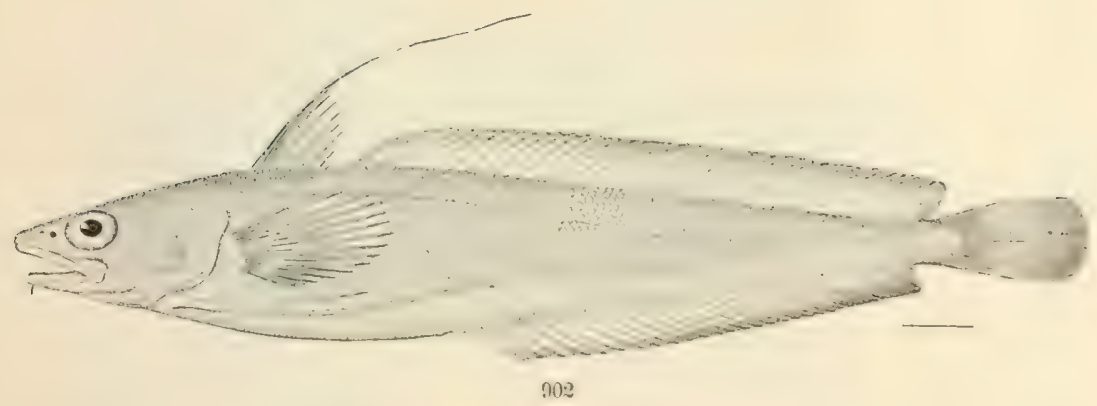

900. UROPHYCIS EARLLI. (P. 255̆.)

901. UROLHYCIS TENUIS. (P. 25\%).)

902. Unorilycis chuss. (1'.2555.) 


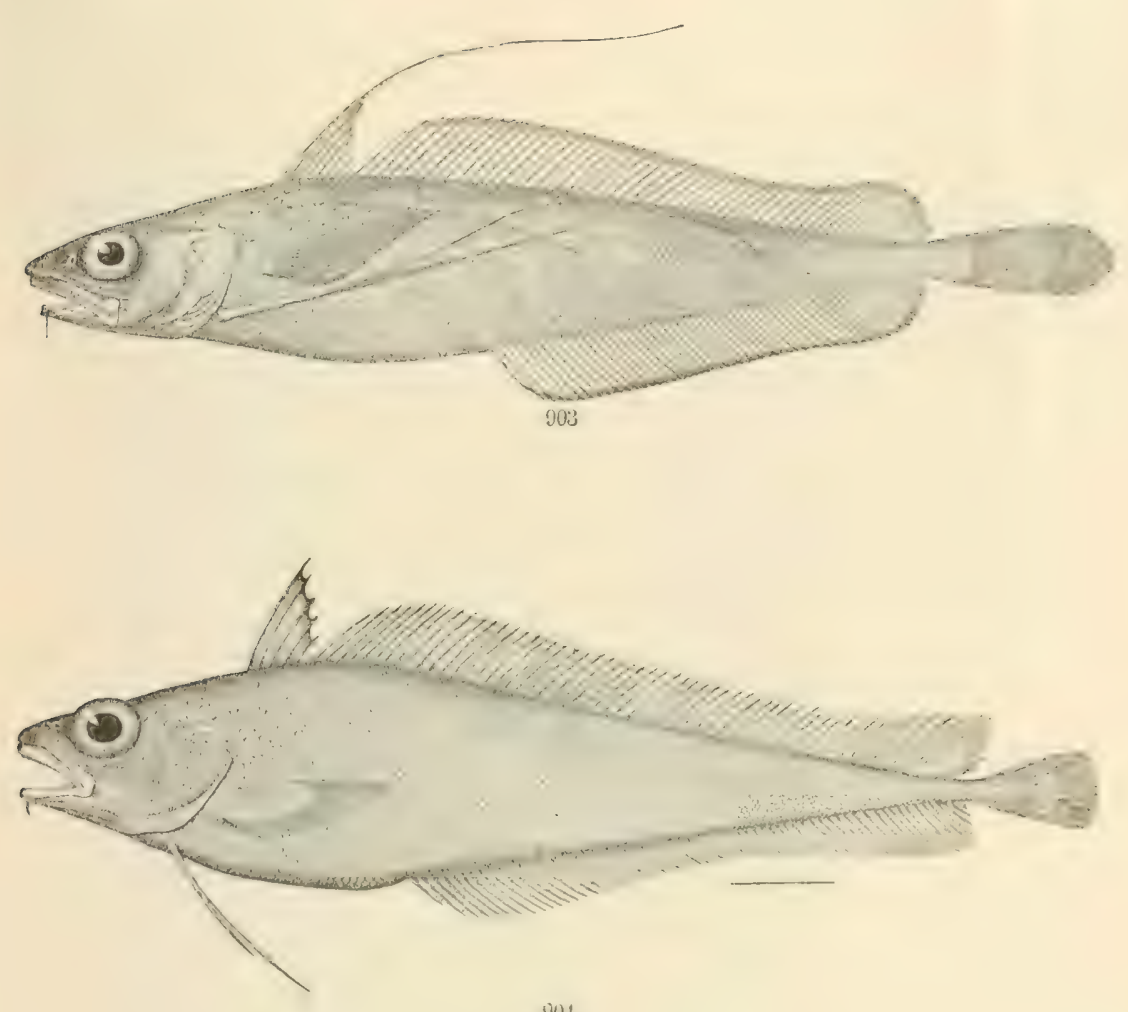

(3)!

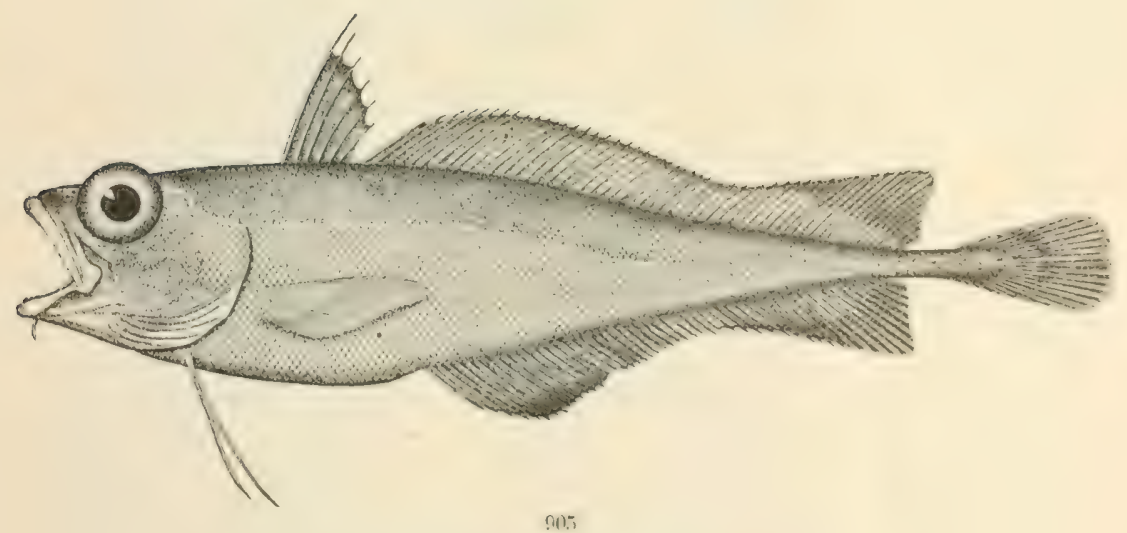

903. UROPHYCIS CHESTERI. (P. 2556.)

904. LeMONEMA BarbatUlum. (P. 2556.)

905. Lemonema Melanurum. (P. 2557.) 



$$
\equiv
$$




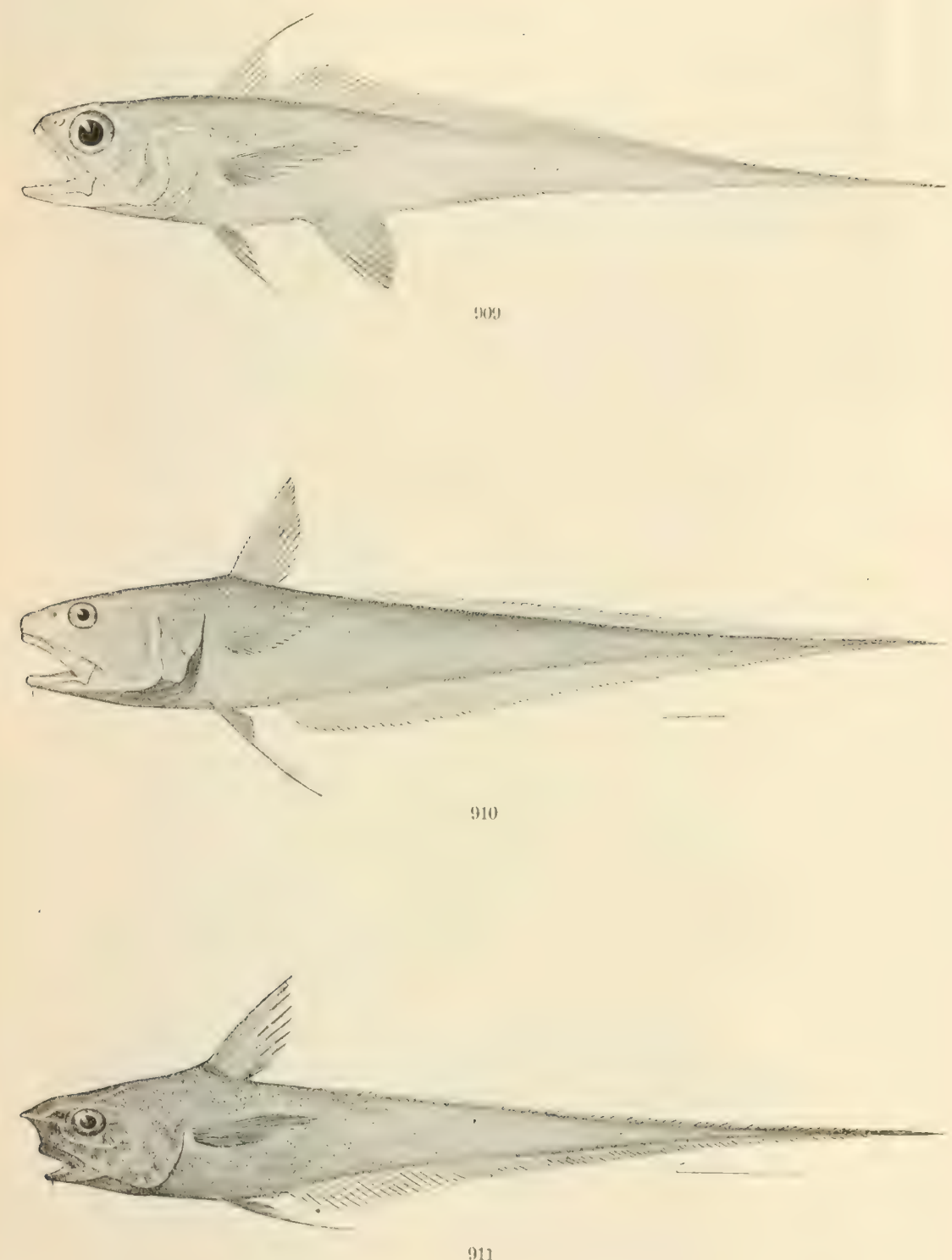

a1]

909. Steind

910. Chalinura smila. (P. 2578.)

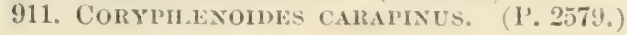





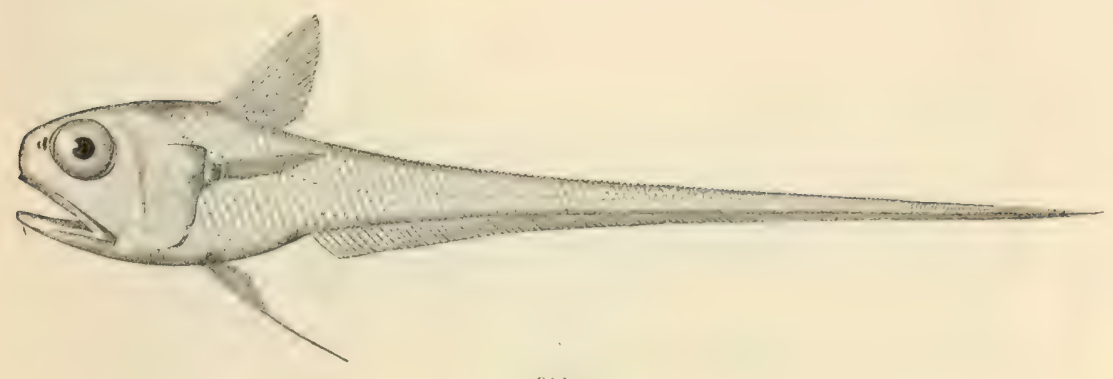

912
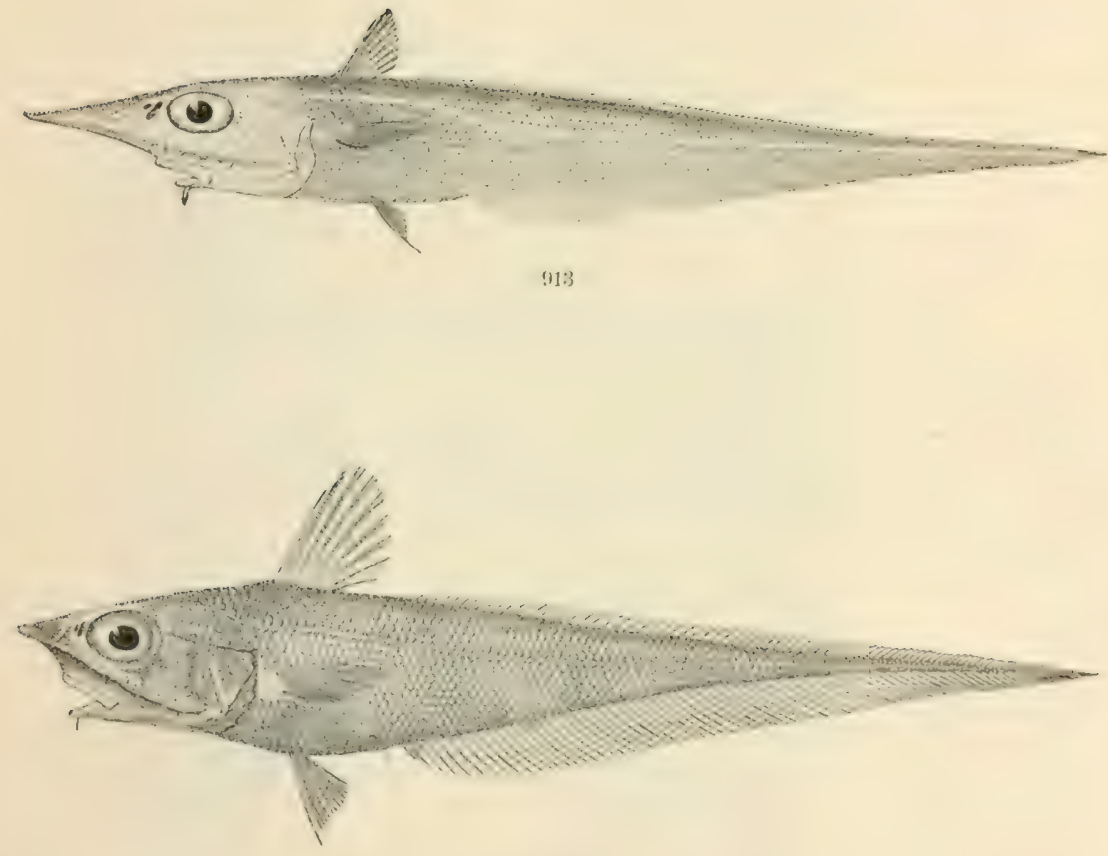

914

912. HyMexocephale's CAVERNosus, (P. 2580.)

913. CELorhýNCHUS OCCA. (P.2588.)

914. Cqioriyxchus caleminatus. (P. 2588.) 



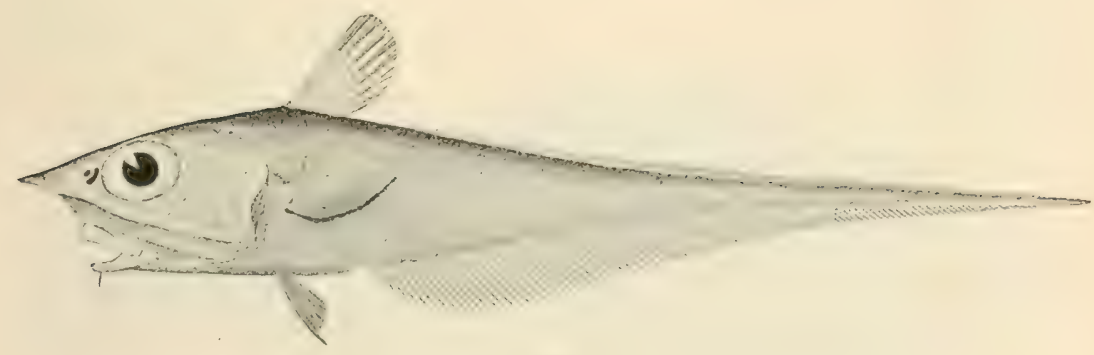

31:

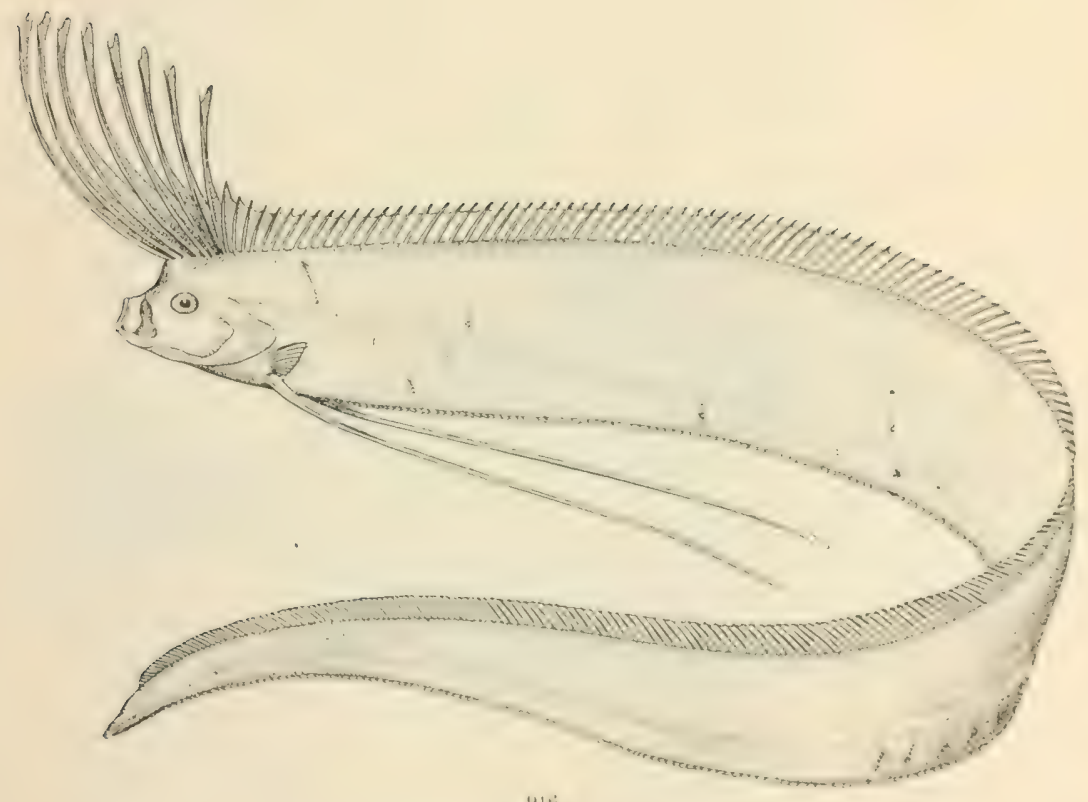

914

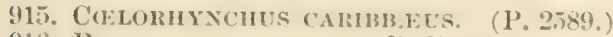

916. ReGallec's GLESNE. (I'.25!6.) 


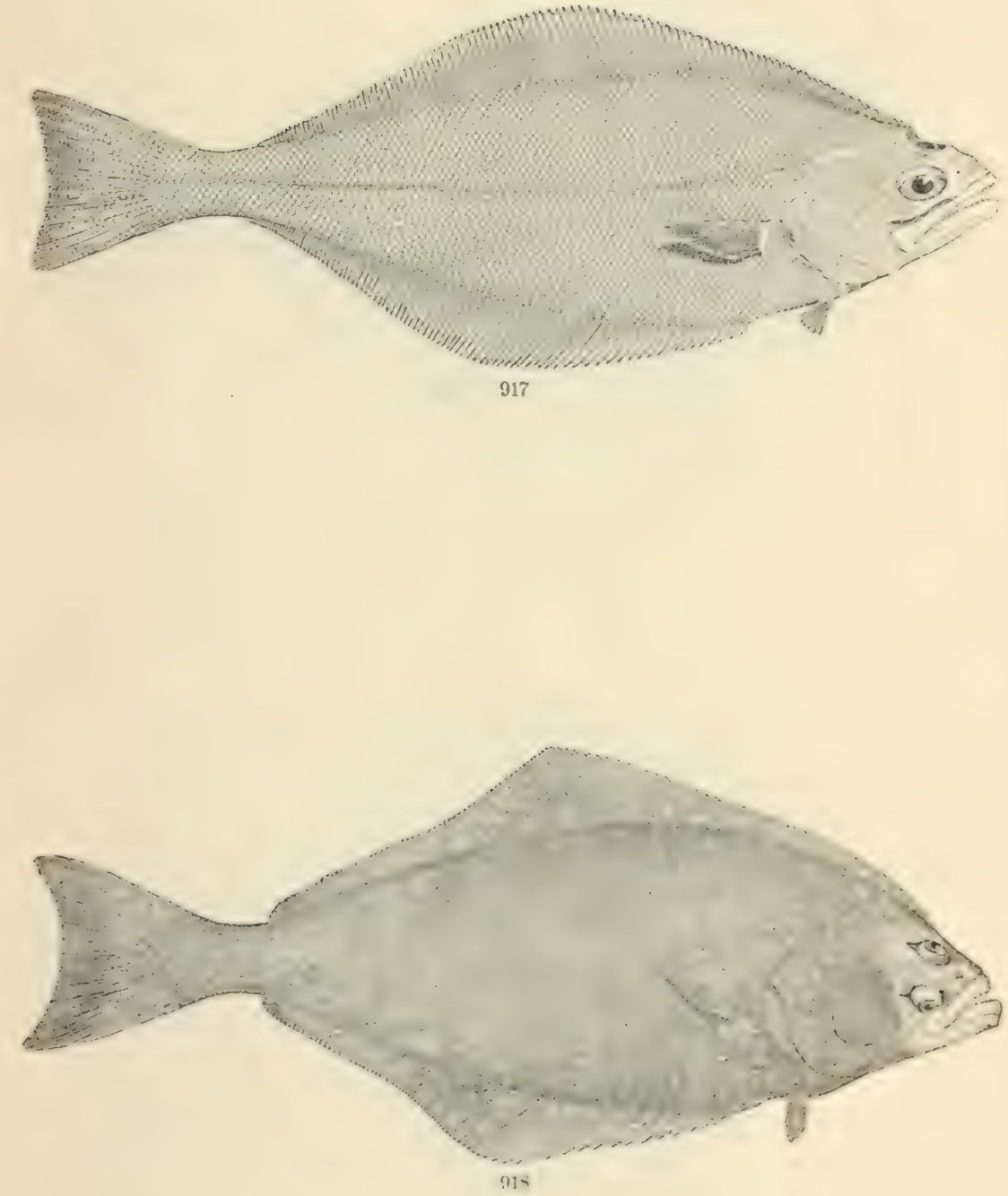

917. AtHenlisthes stomas, (P. 2609.)

918. Hippoglosit's hirpoglossus. (P', 2611.) 


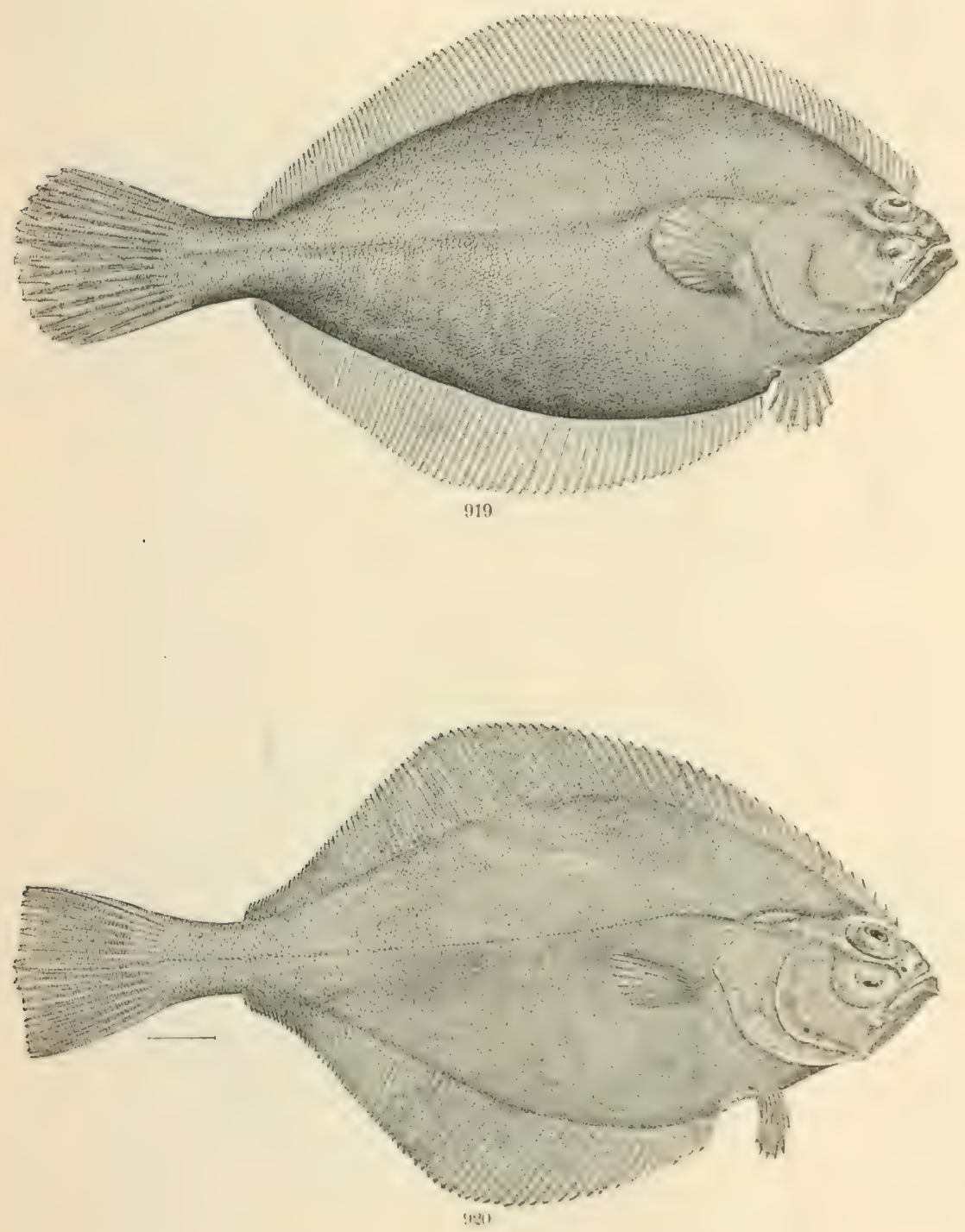

919. HIPOGLOSSOHDS P'LATESOHDES. (P. 2614.)

920. HXPPOGLOSFOINES ELASSODON. (1',2615.) 



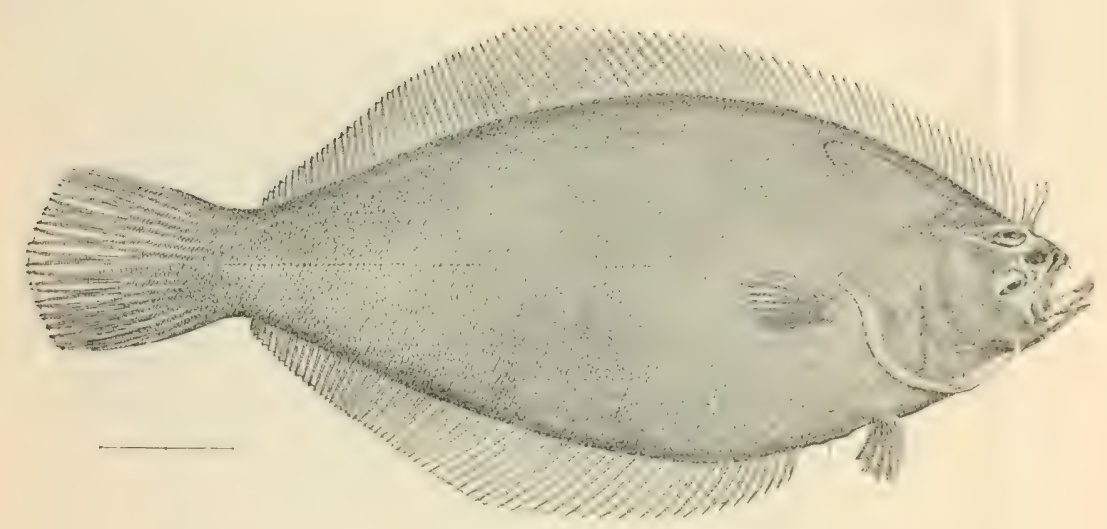

221

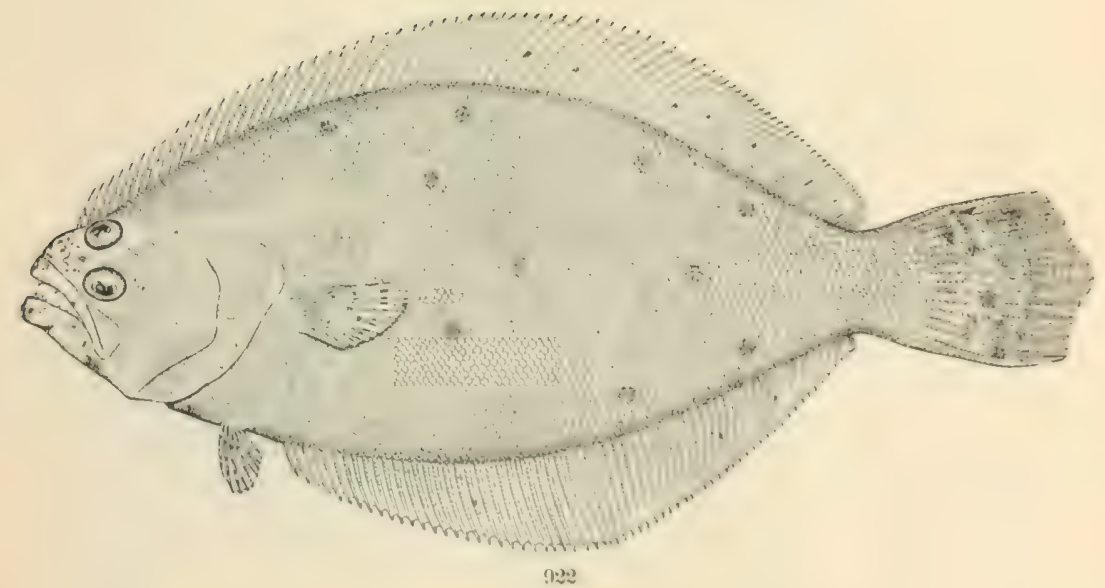

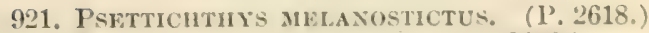

922. Paralichtiys dentatus. ( 


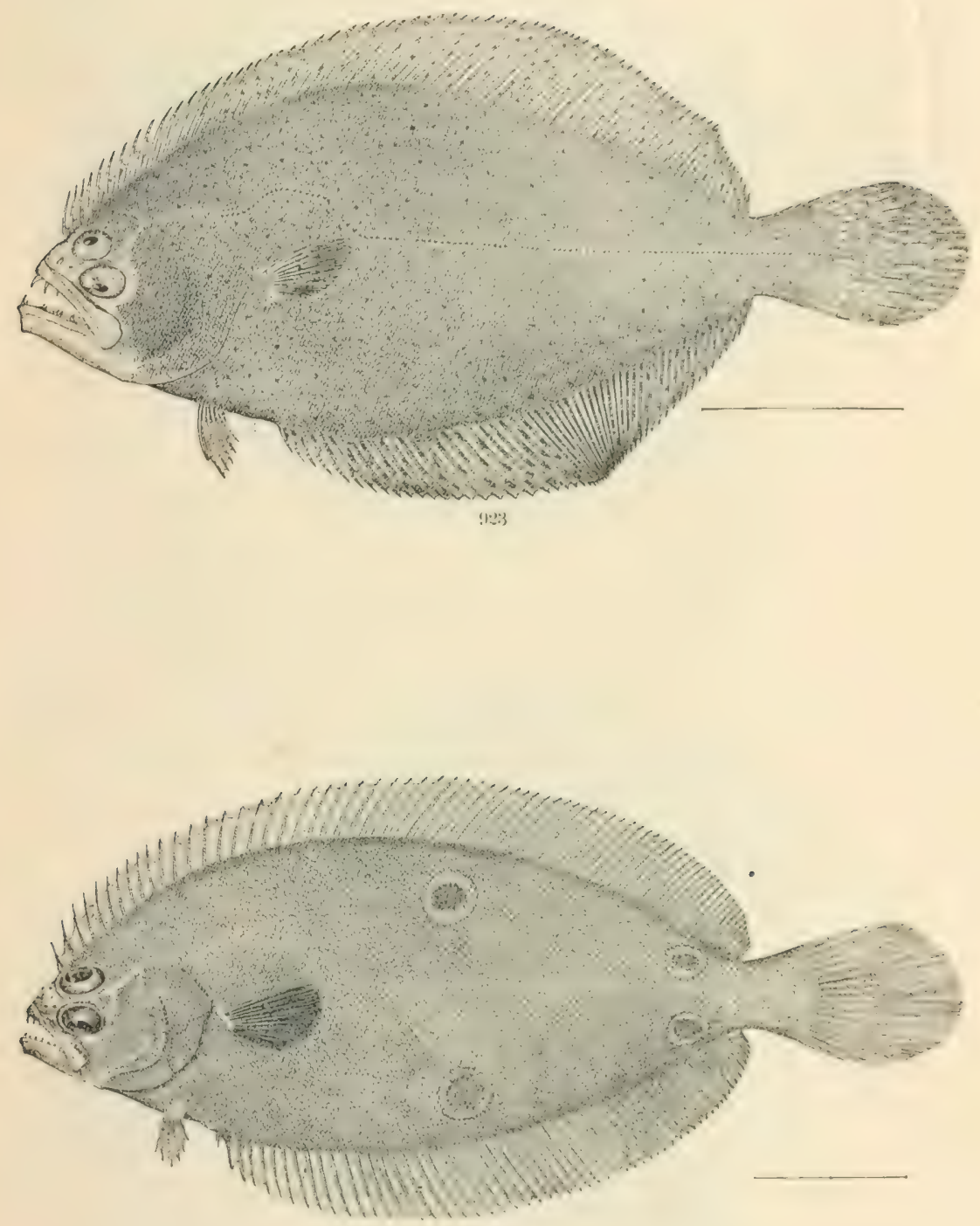

!w4

923. Paralichith's squamLlext's. (P'.2631.)

924. P'aralichtiY's OhLONGUS. (P. 2632.) 


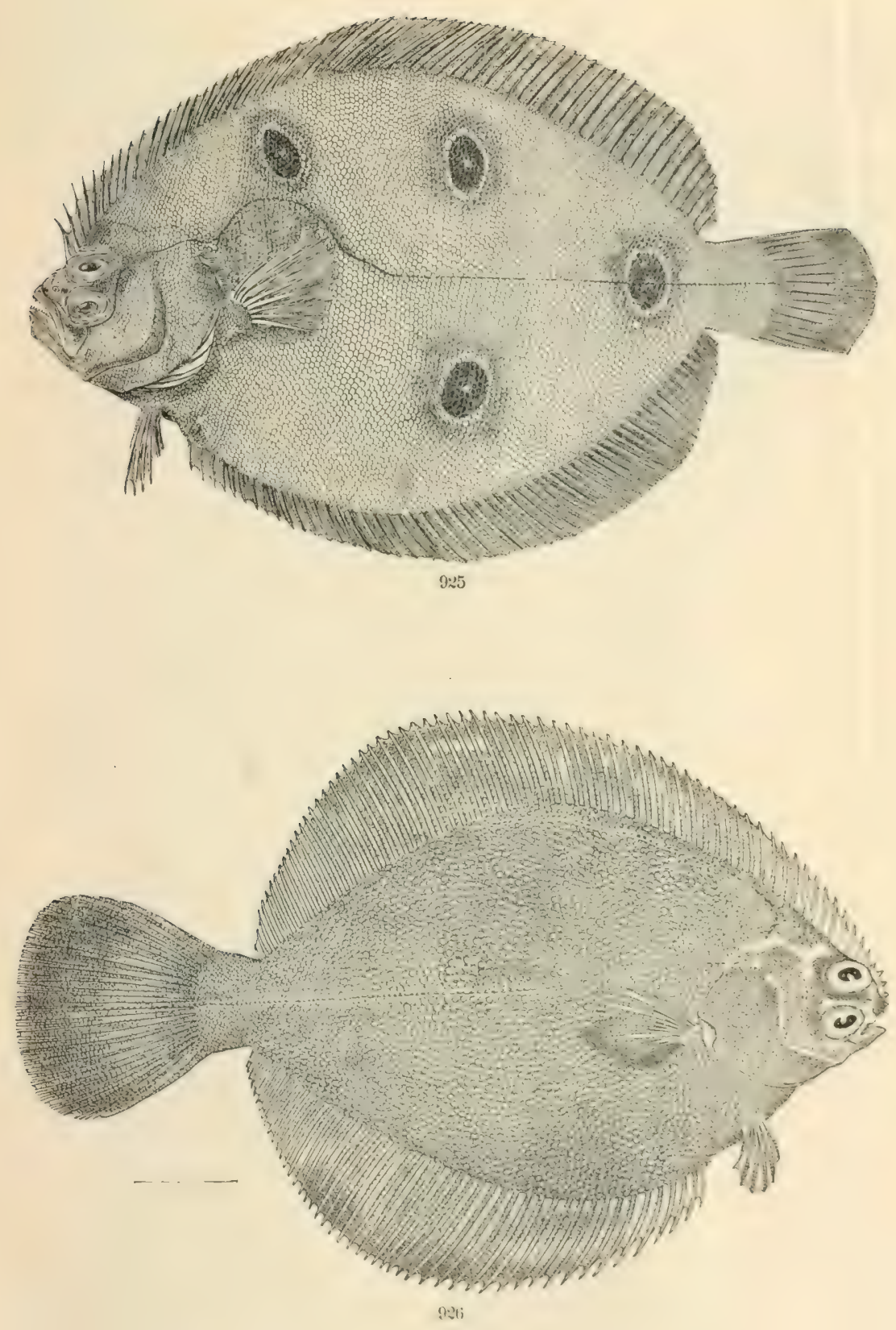

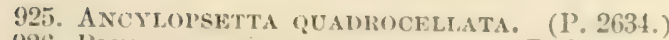

926. PleuronichtiYYS DECIRREXS. (P. 2637.) 



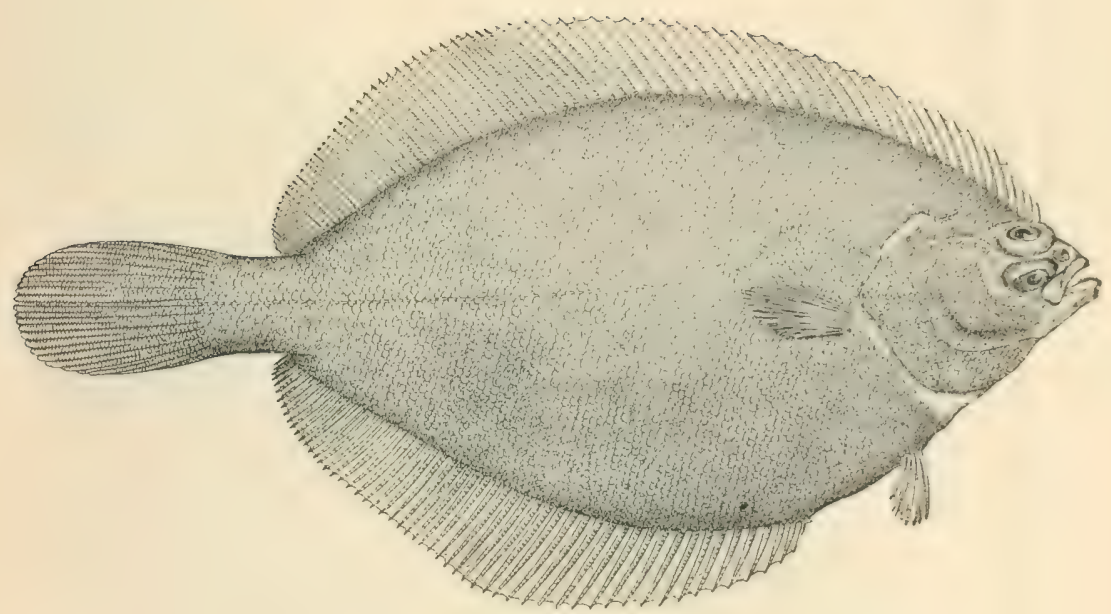

$92 \hat{i}$

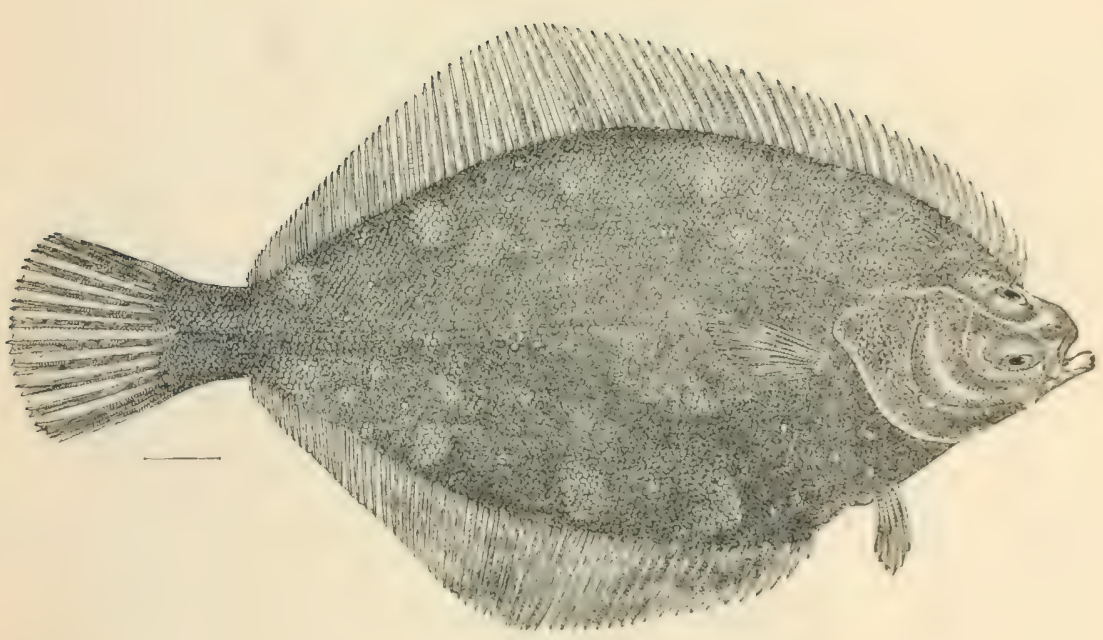

923

927. INOPSETTA ISCHYRA. (Р. 2641.)

928. LEIDOPSETTA BLINEATA. (P.2643.) 


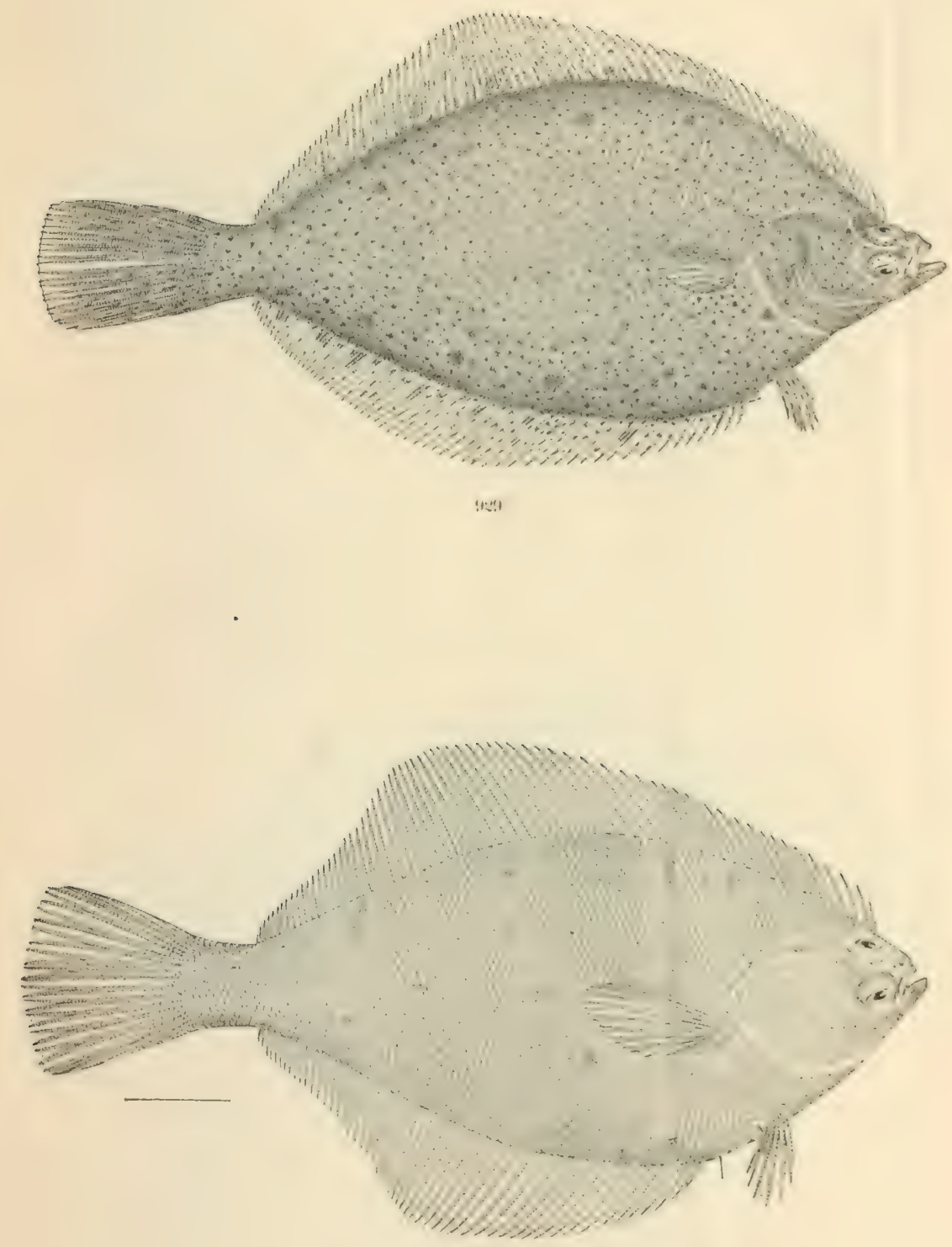

$\$ 30$

929. LmaNda FerRUginea. (P. 264.)

930. Limanda aspera. (P. 2645.) 



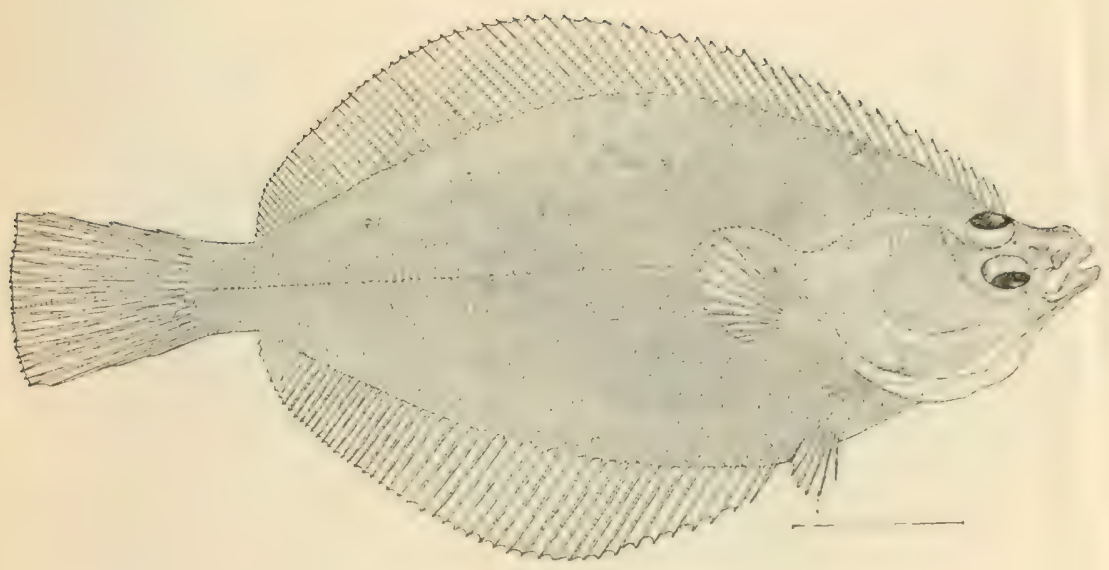

4:31

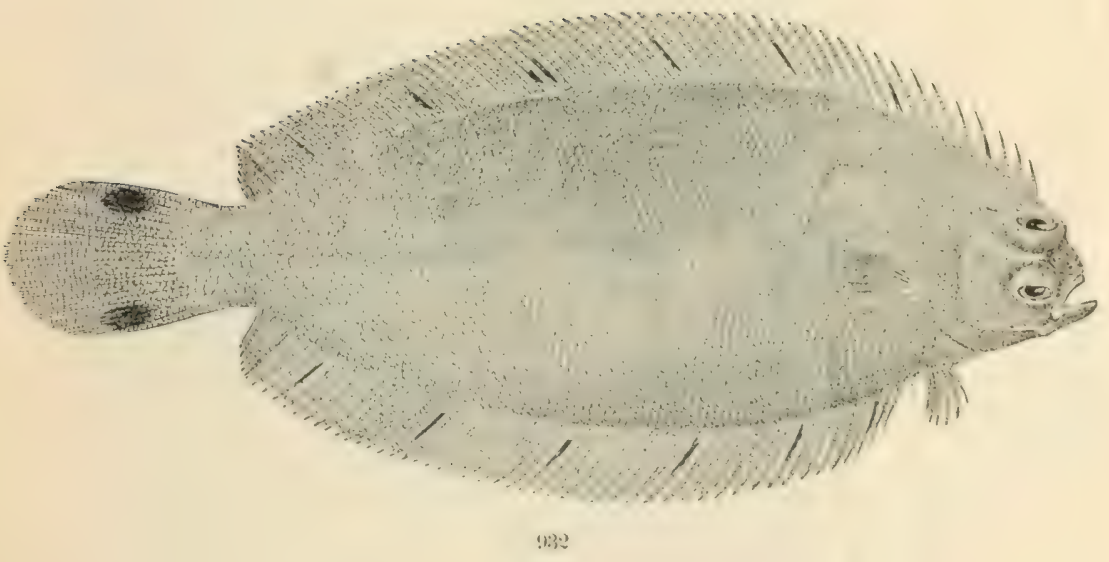

931. Limaxida promoscinlia. (P. 2645.)

932. LIMANIAA BEANII. (P. 2646.) 



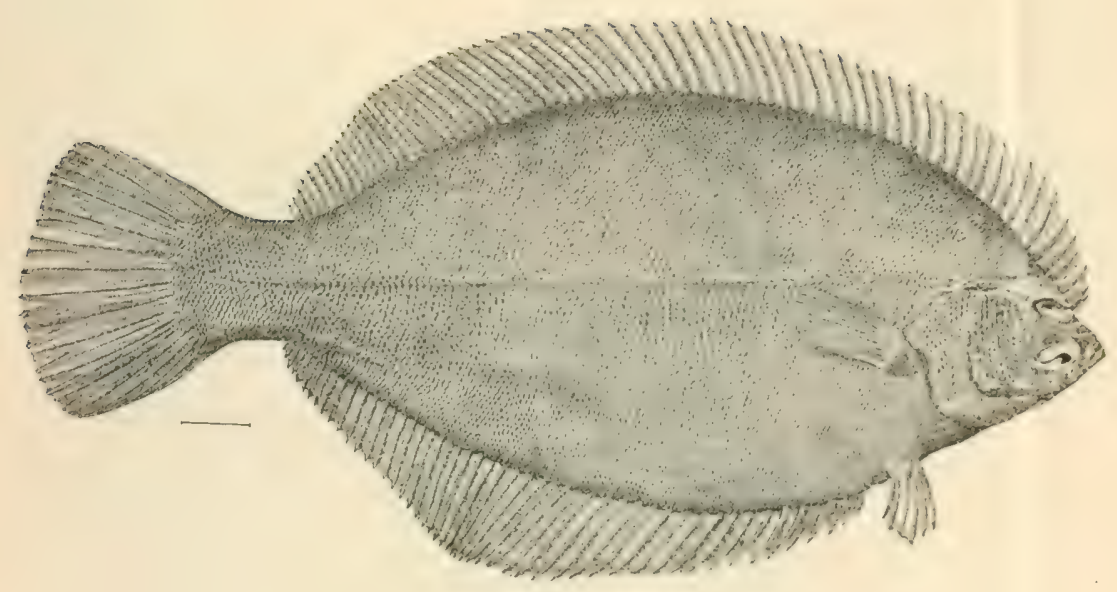

(1):3

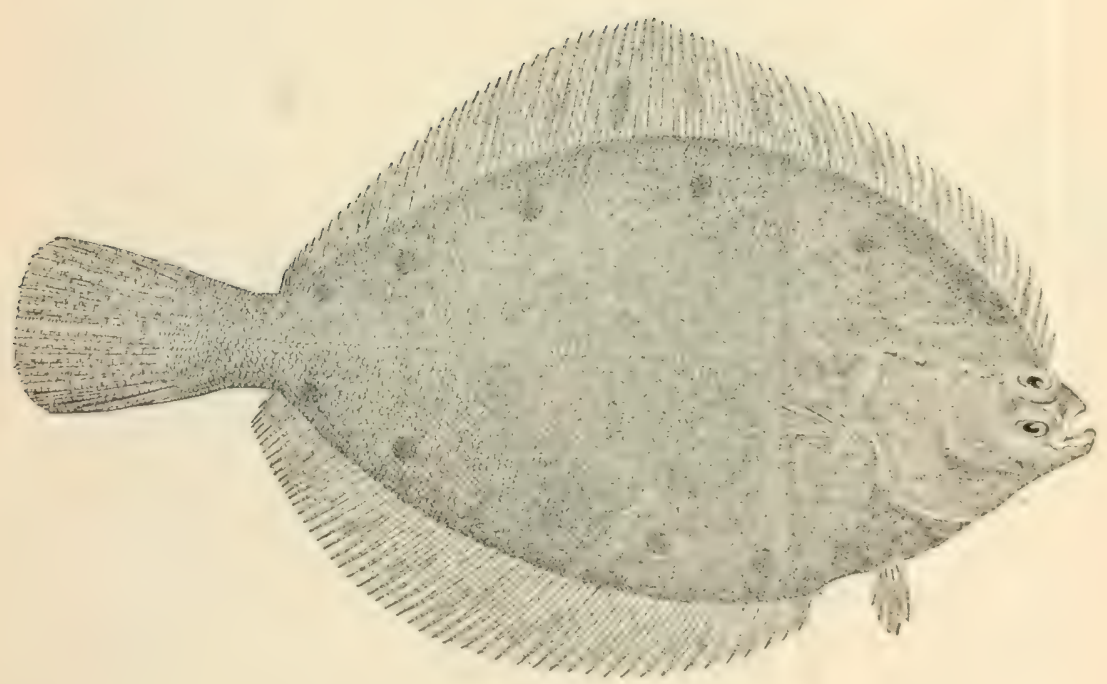

931

933. Pseudorleuronectes anericanus. (P. 2617.)

934. Pleuronectes quadritulemculatus. ( $P$. 2618.) 



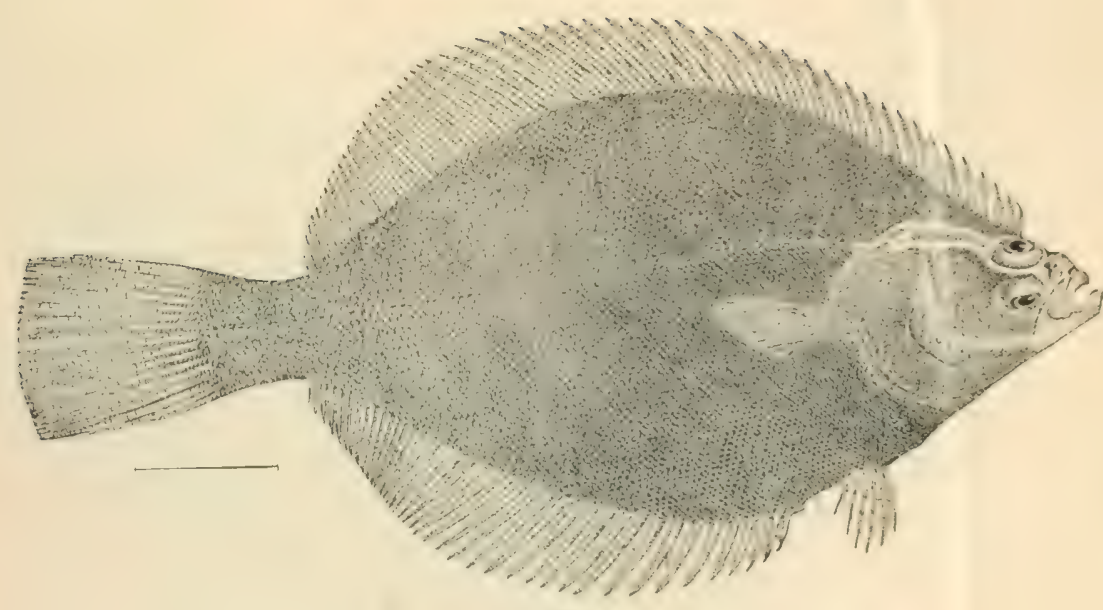

435

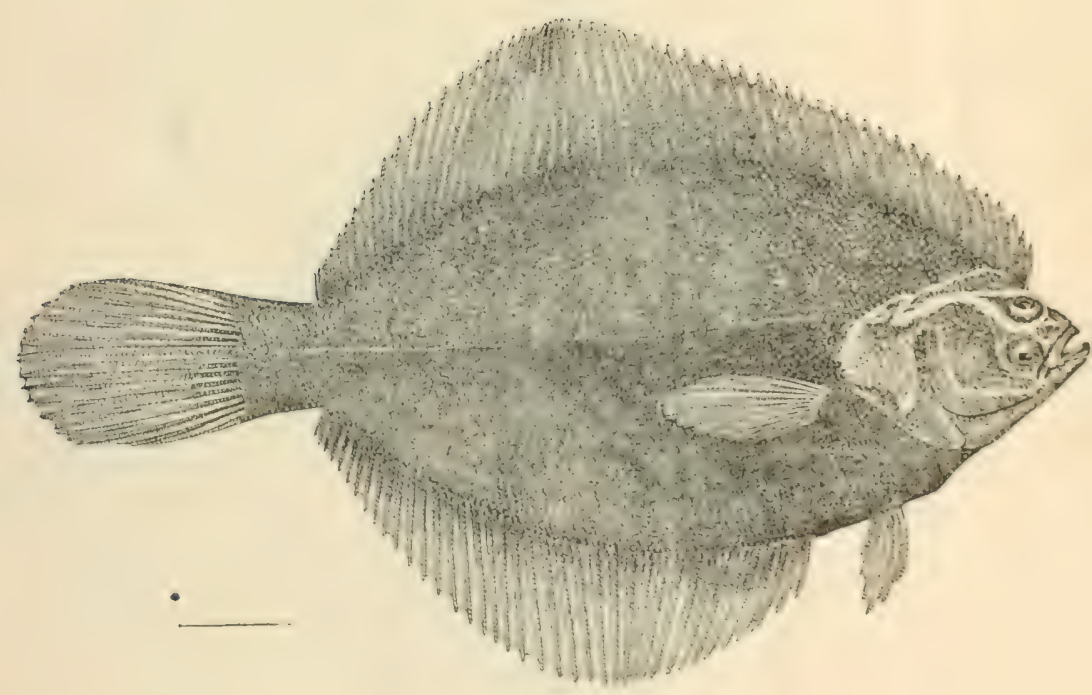

(3) i

935. LIOPSETTA GLACIAIS. (P. 2649.)

936. LIOPSETTA PUTNAMI. (P. 2650.) 


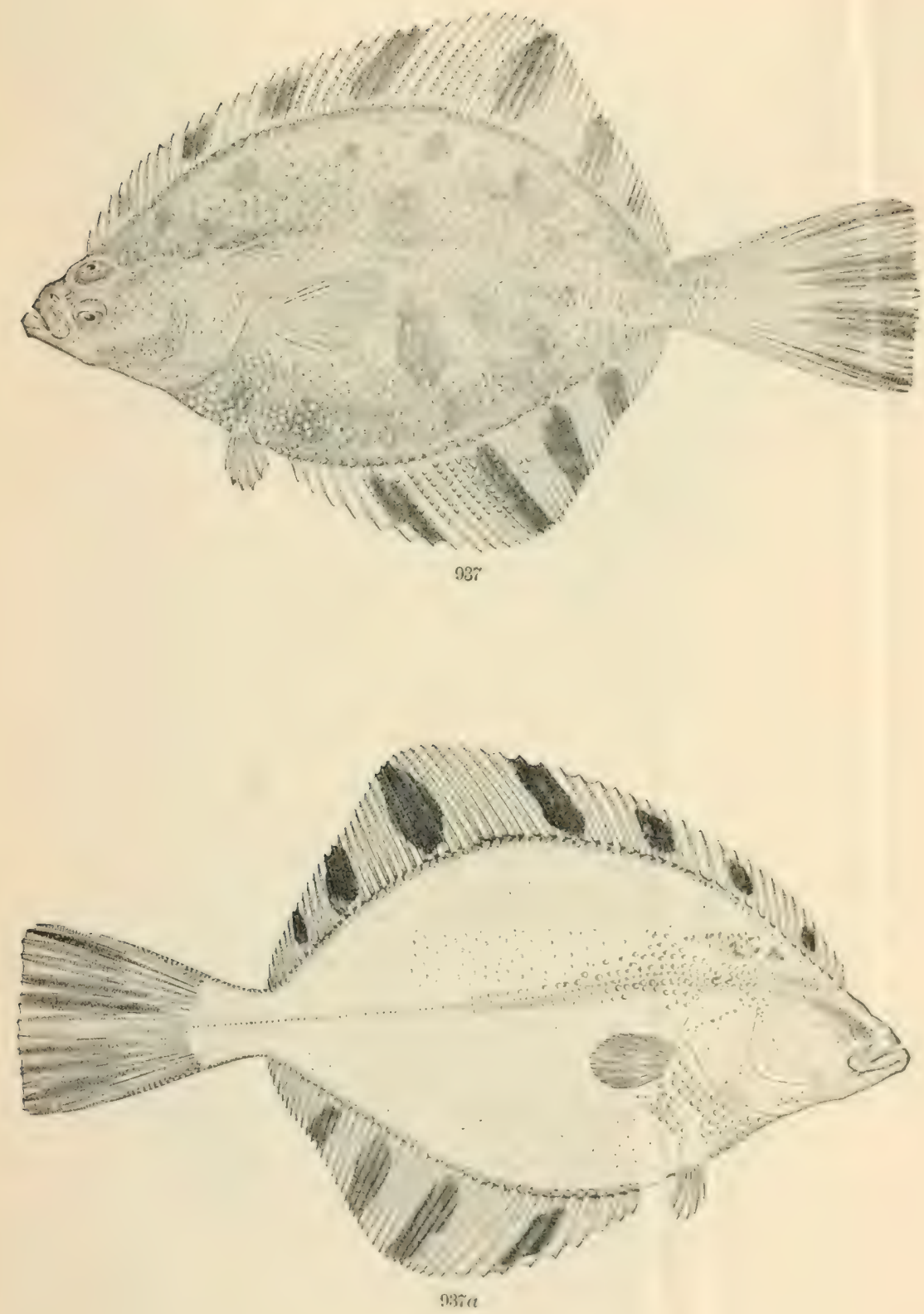

937, 937a. PlatichtuYs stellatus, (P. 2652.) 



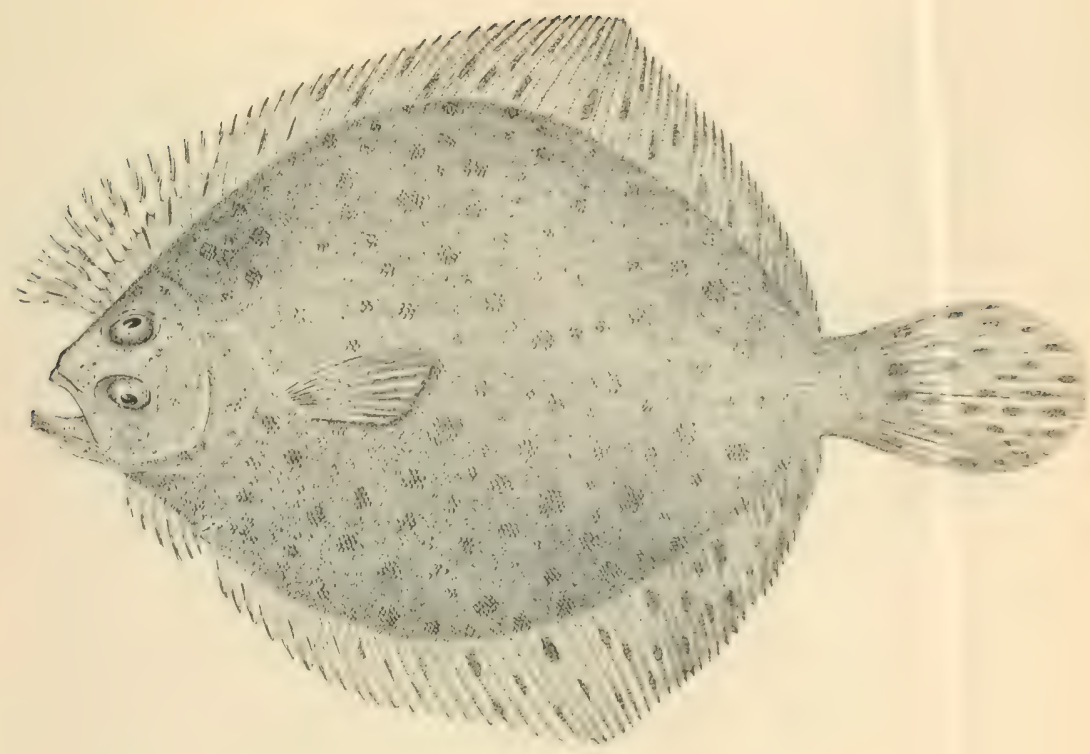

(1:34)

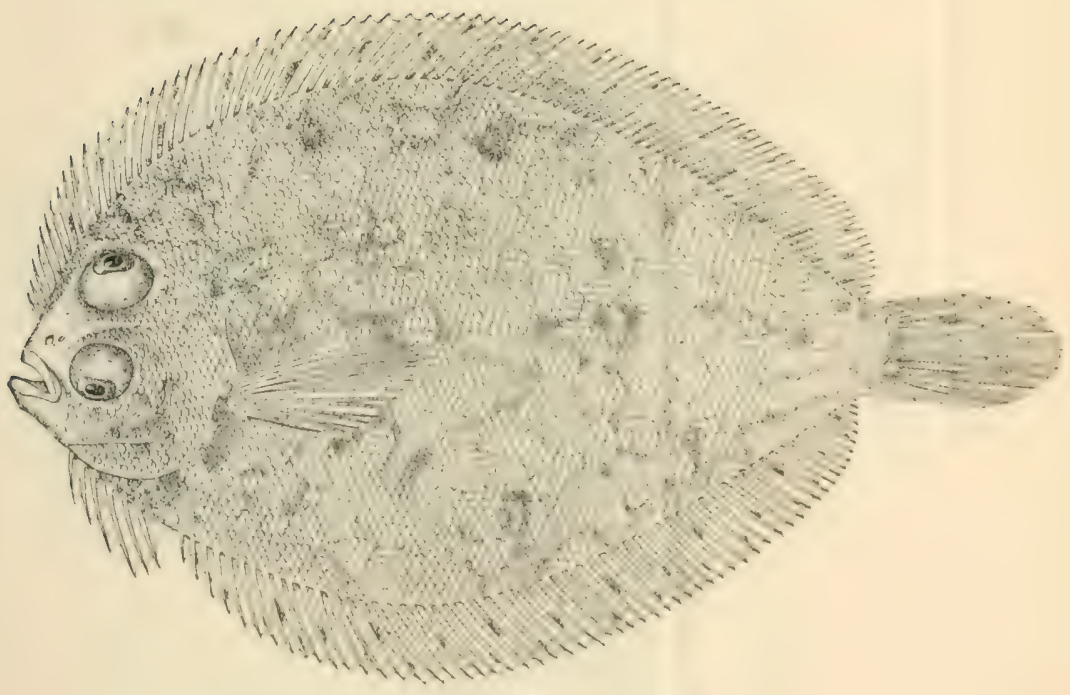

(2:3!)

938. LOMHOPEVTA MACVATA. (P. 2660.)

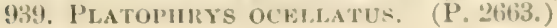



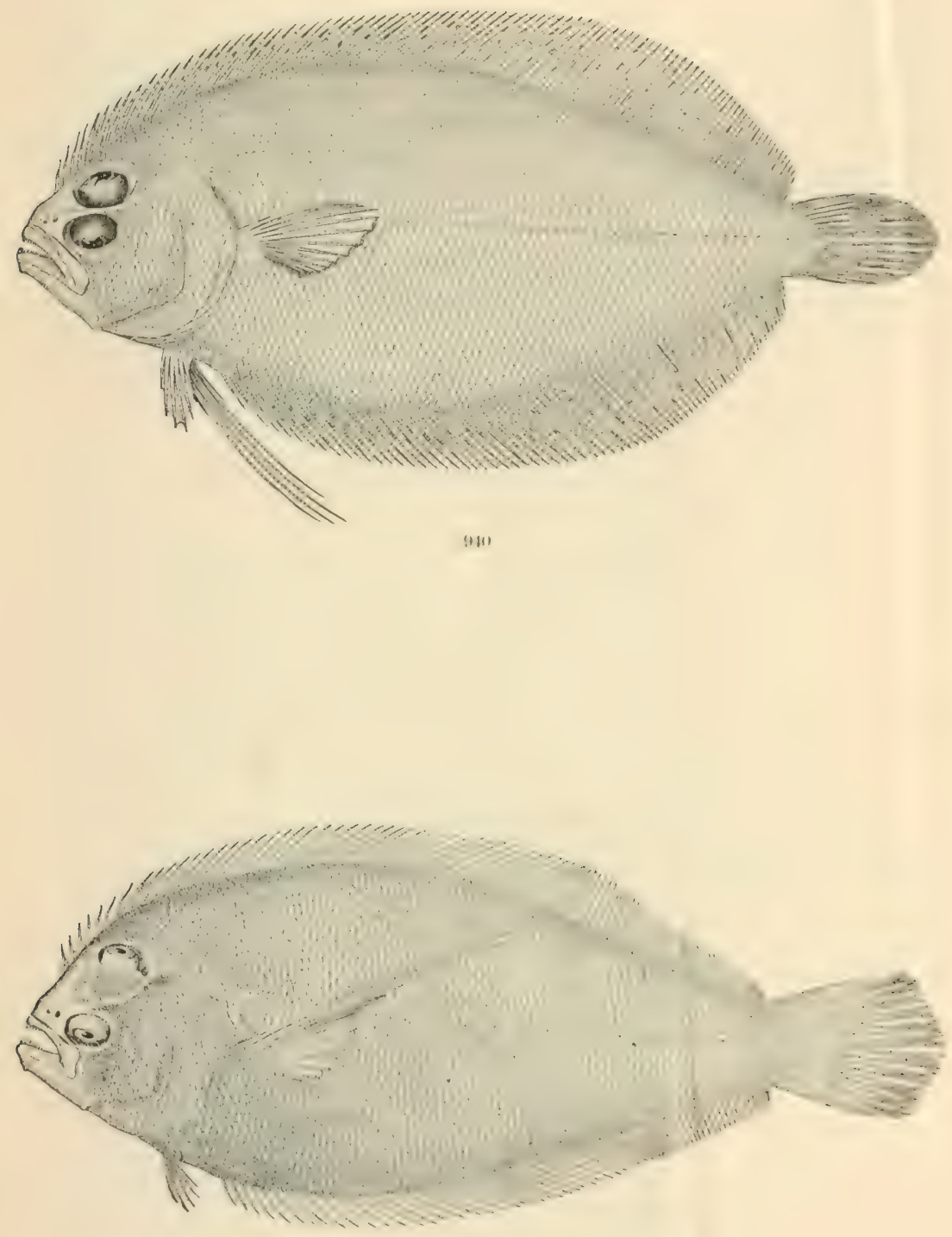

$9+1$

940. TRichopserta vexthalis. (P. 2669.)

941. Syacium paillosem, (P'.2671.) 



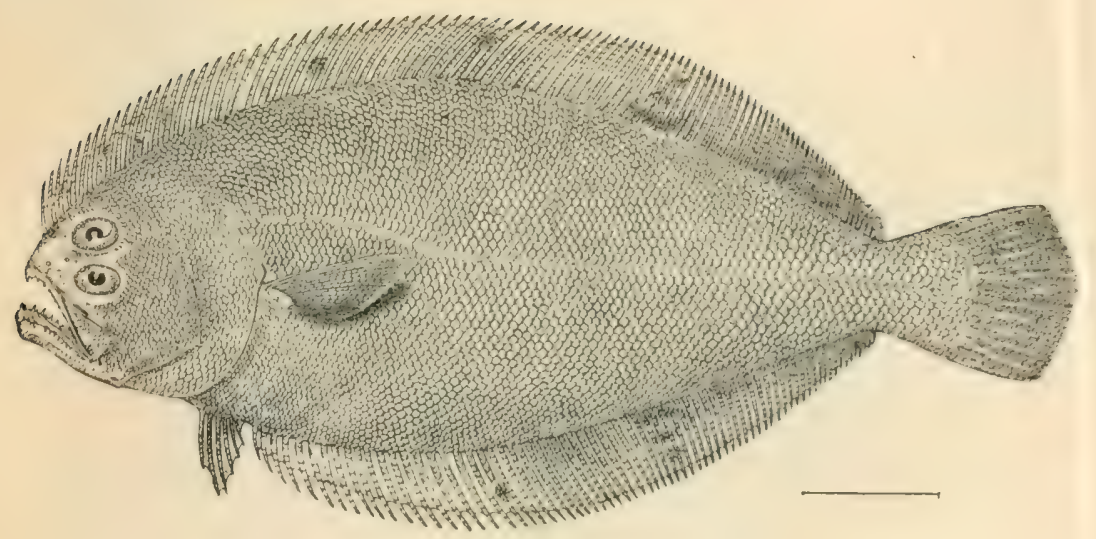

113

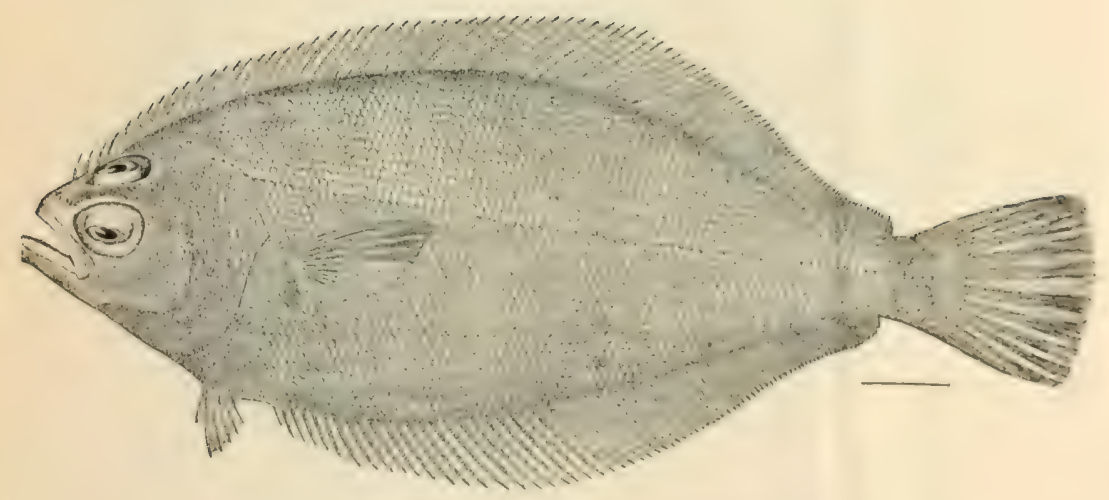

913

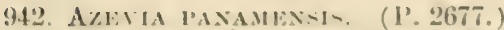

943. CITHARICHTins sokm)tis (1'.2679.) 


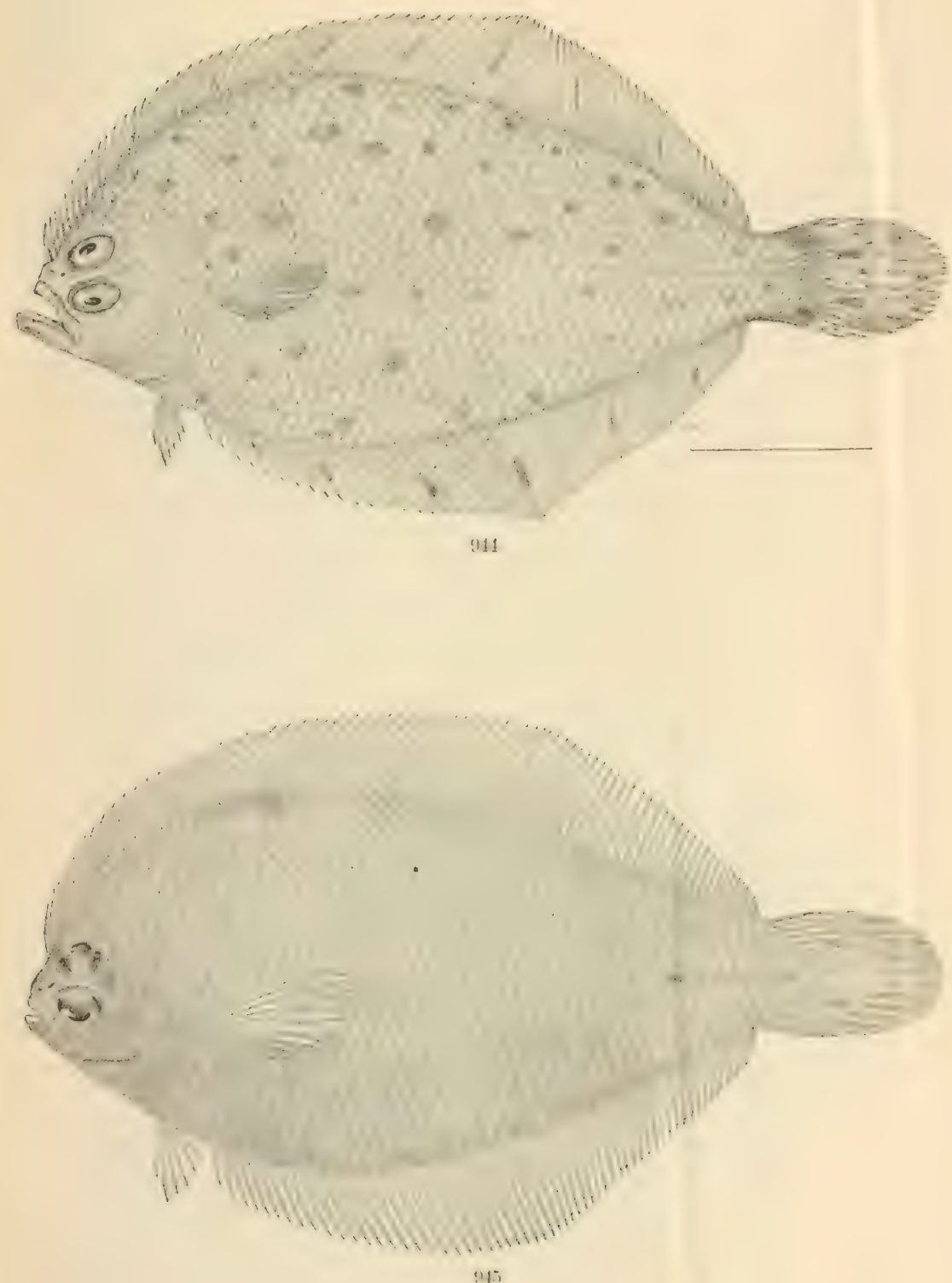

944. CitharichtuYs Mackops. (I'. 2684.)

945. Etrop's mmoses. (P. 268s.) 



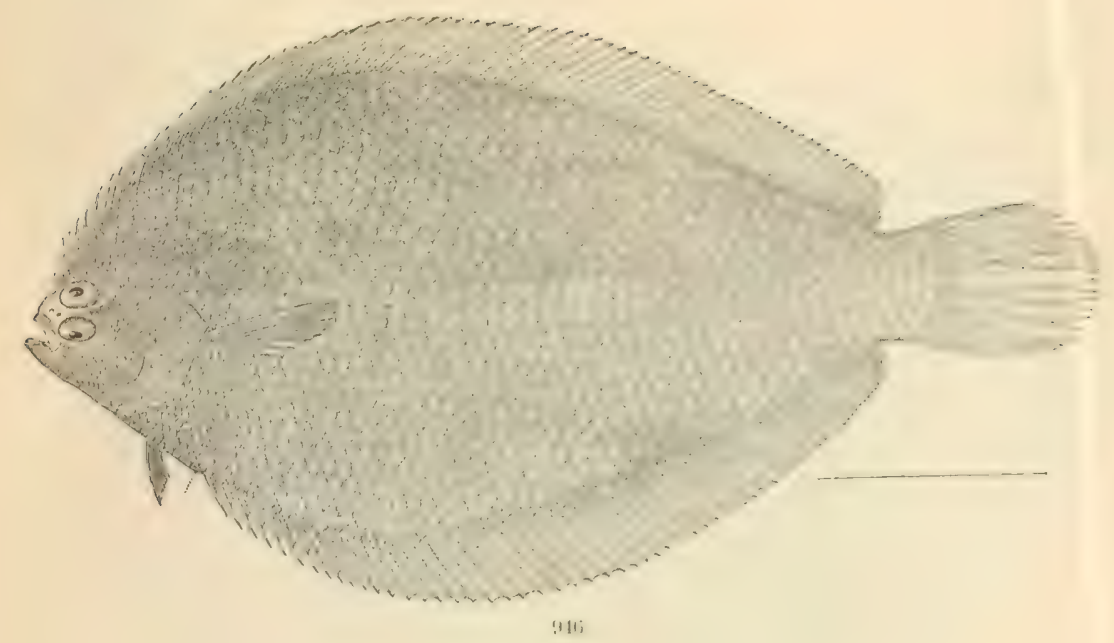

!11:

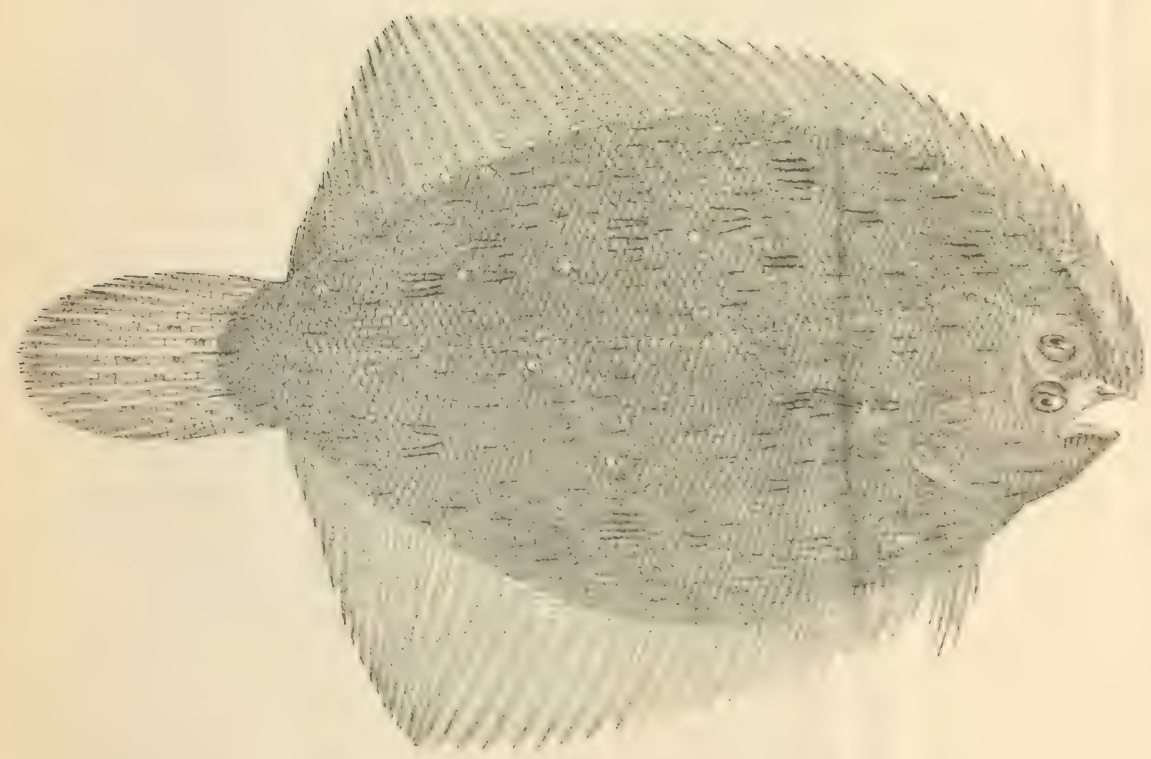

$! 4:$

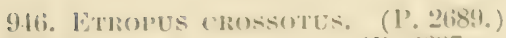

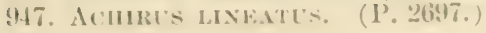




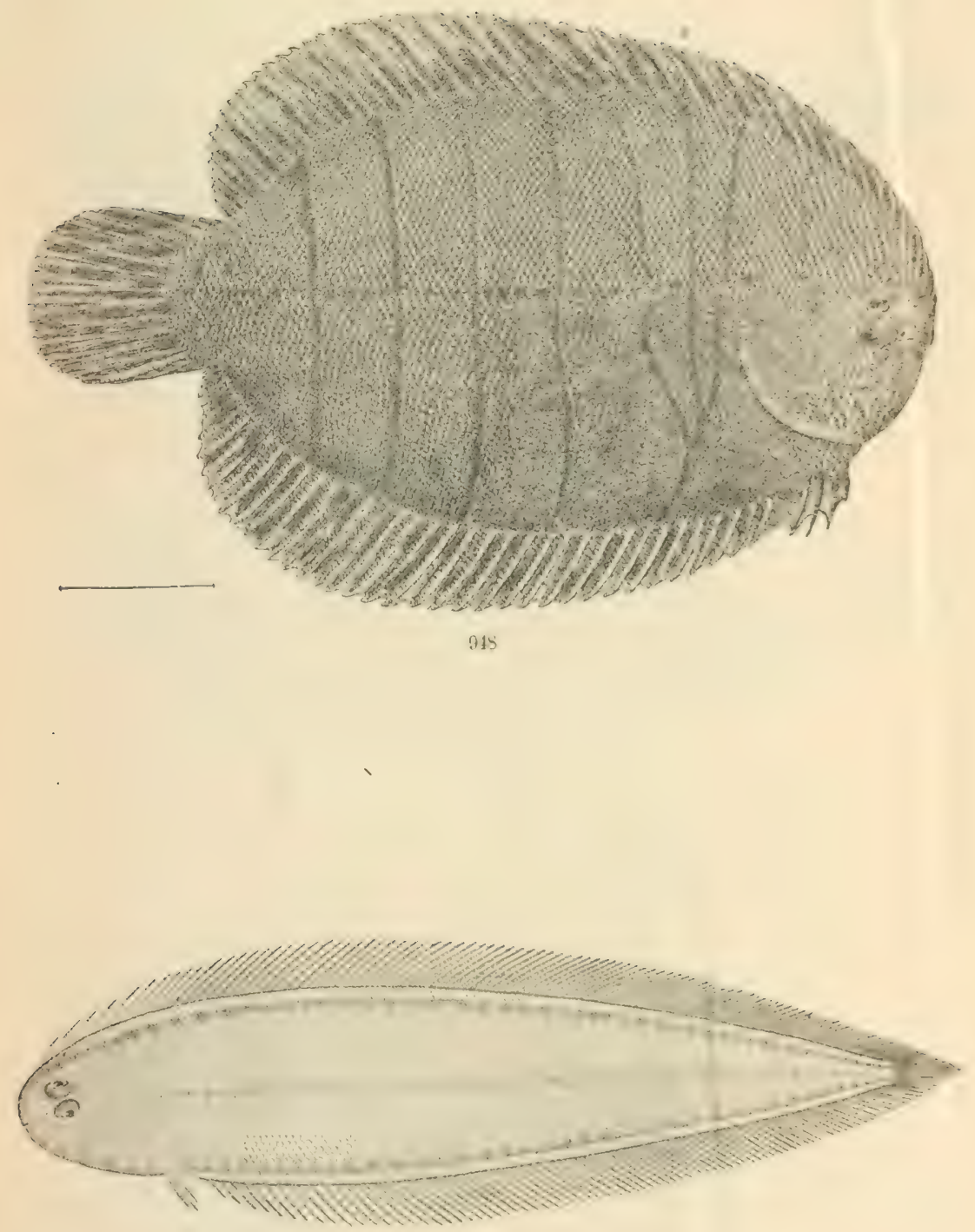

919

948. Achmos faschatus. (P. 2700.$)$

949. SYMPHURUS MARGiNatus. (I’,706.) 



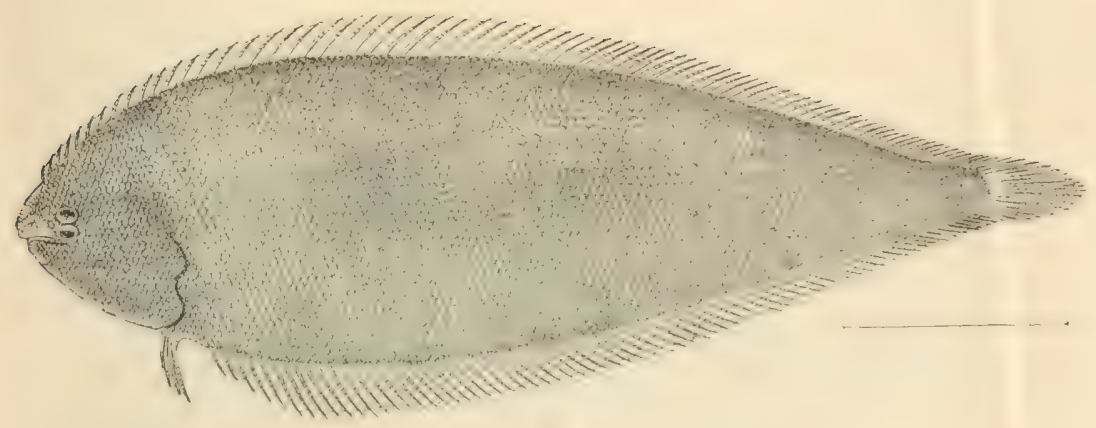

9.50
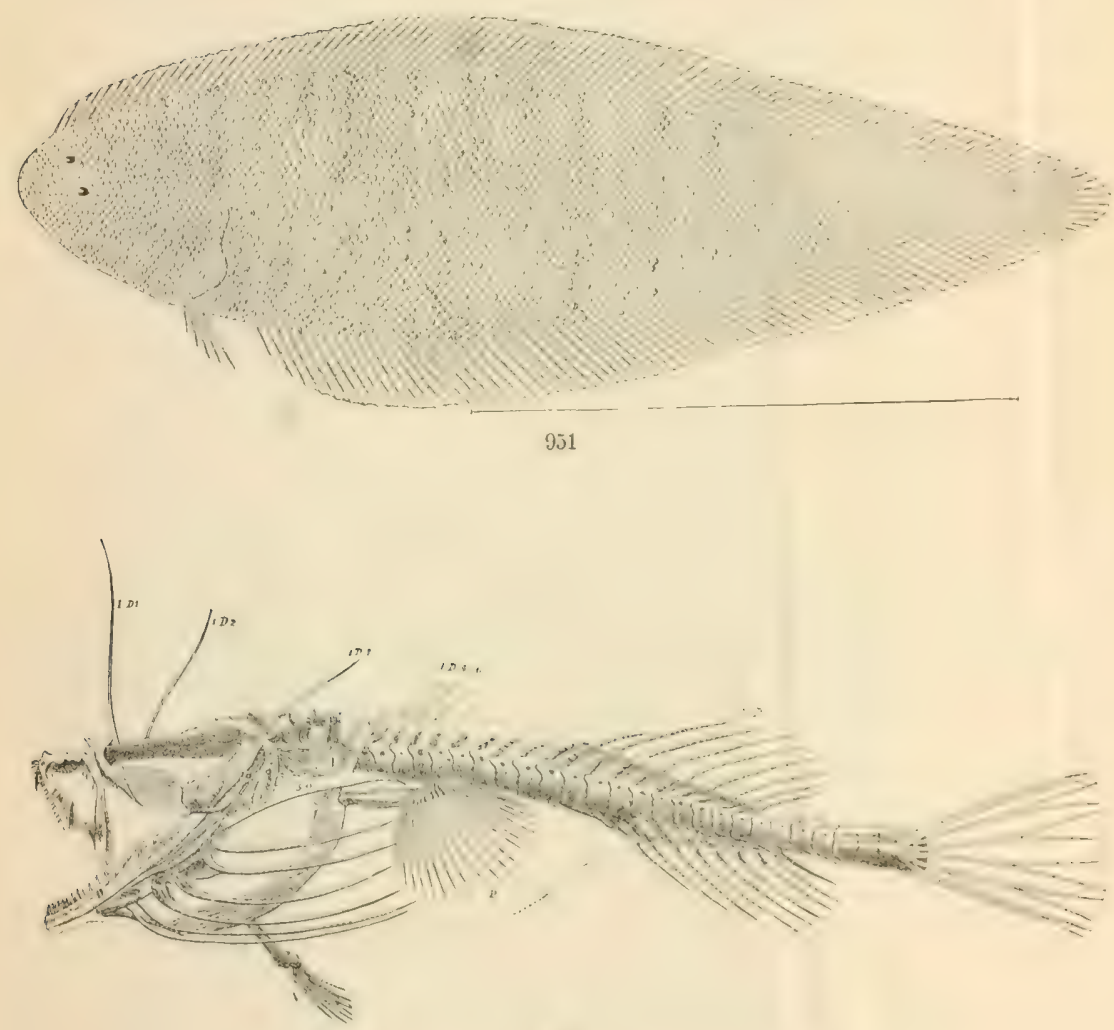

90

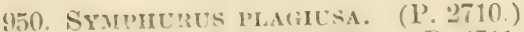

9.1. SyMPHURI's WILIMMSI. (P. 2711.)

952. LoPHI - PINCATORIUS. (I'. 2713.) 


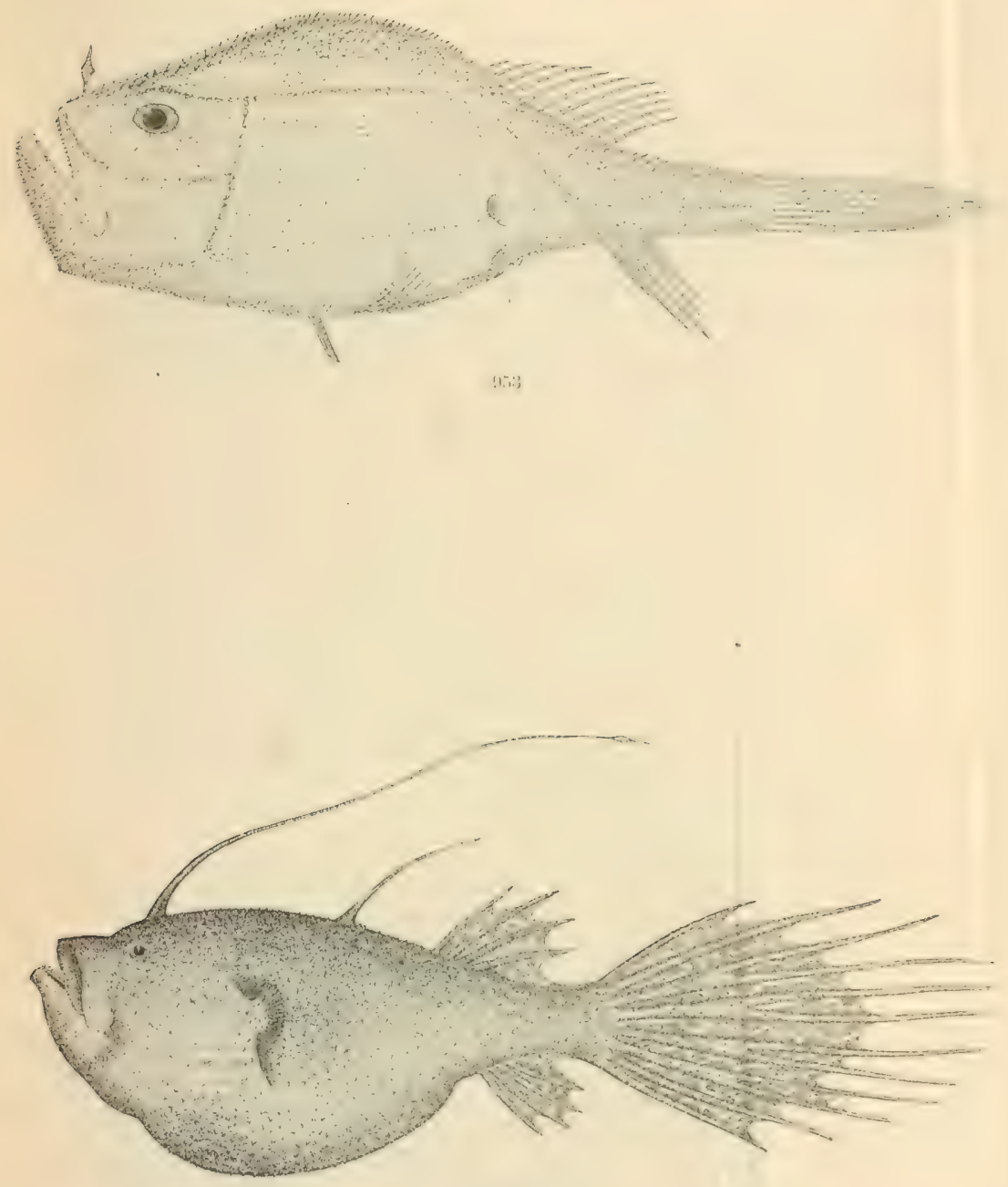

4.5

953. ChaUNax pictes. (1'. 27\%6.)

954. Ceratias holboll. (P. 2729.) 



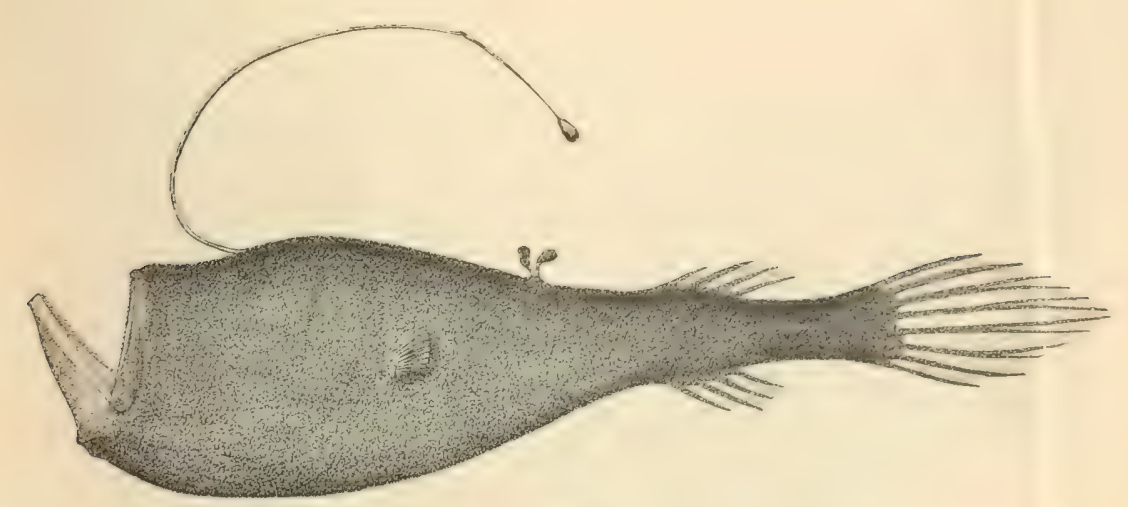

(5).5

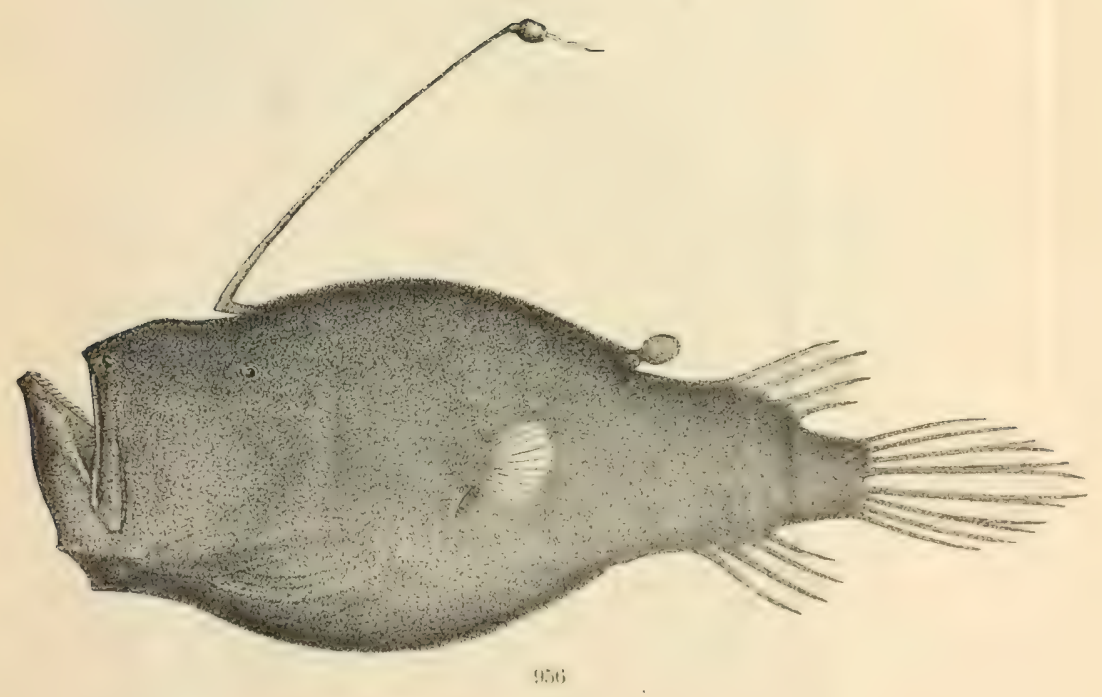

955. Mancalias smeremdti. (P. 2730.)

956. CIrYPTOPSARAS COUESII. (P. 2731.) 



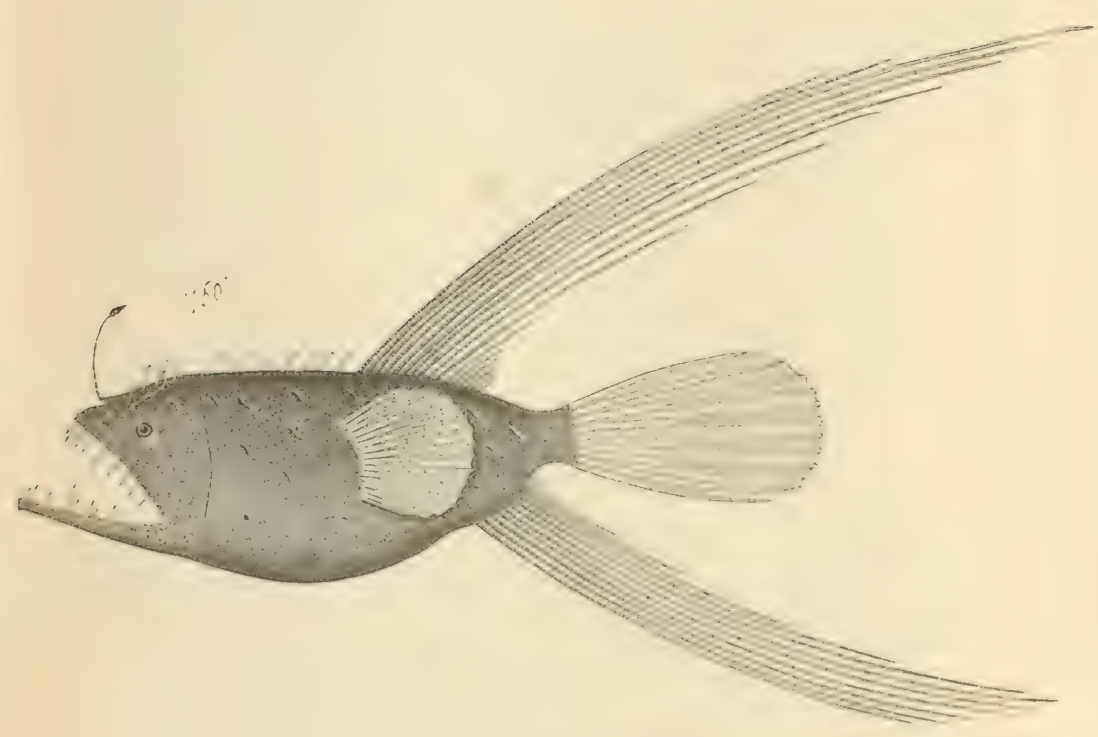

$\therefore$

957. CAUlopirrye JORDANI. (1'.2735.) 



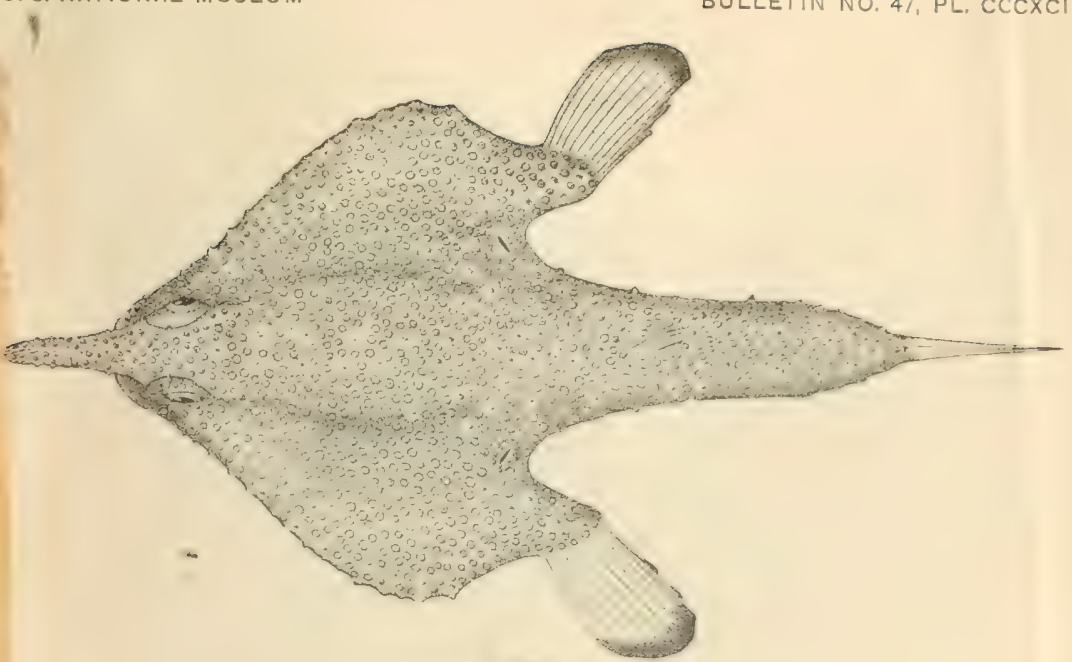

95
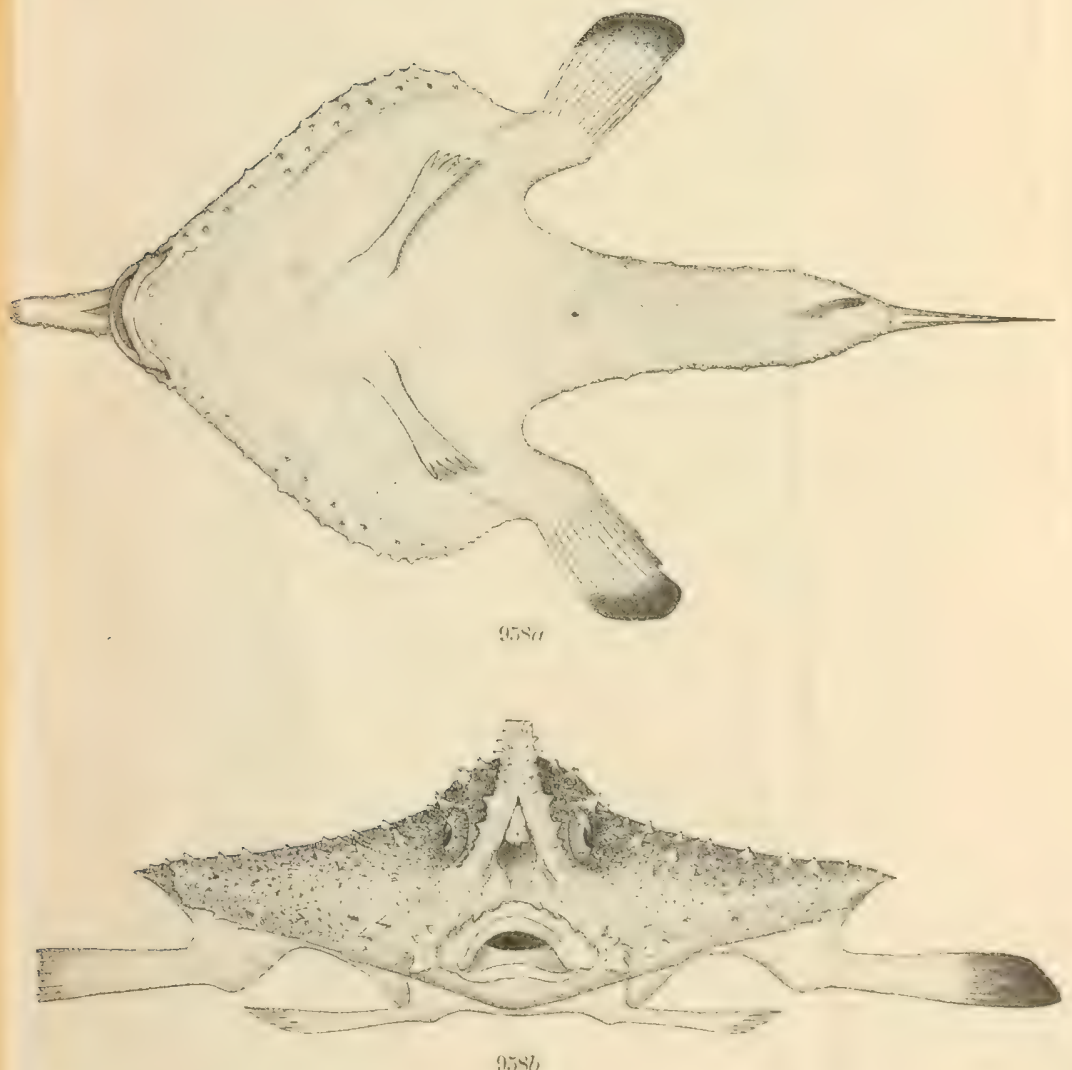

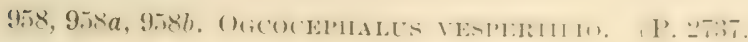




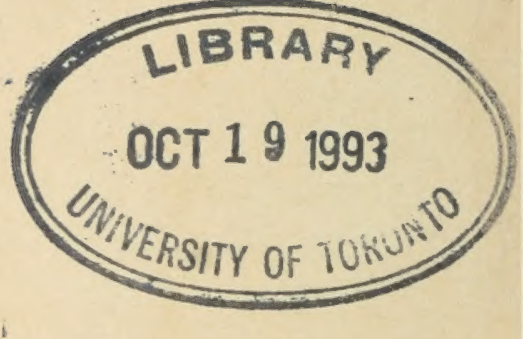

,

DEPARTAENT OF ZOOLOGY 
